Prepared for the U.S. Department of Energy

under Contract DE-AC05-76RL01830

\title{
Large-Scale Spray Releases: Additional Aerosol Test Results
}

RC Daniel

PA Gauglitz

CA Burns

MS Fountain

RW Shimskey

JM Billing

JR Bontha

DE Kurath

JJ Jenks

PJ MacFarlan

LA Mahoney

August 2013

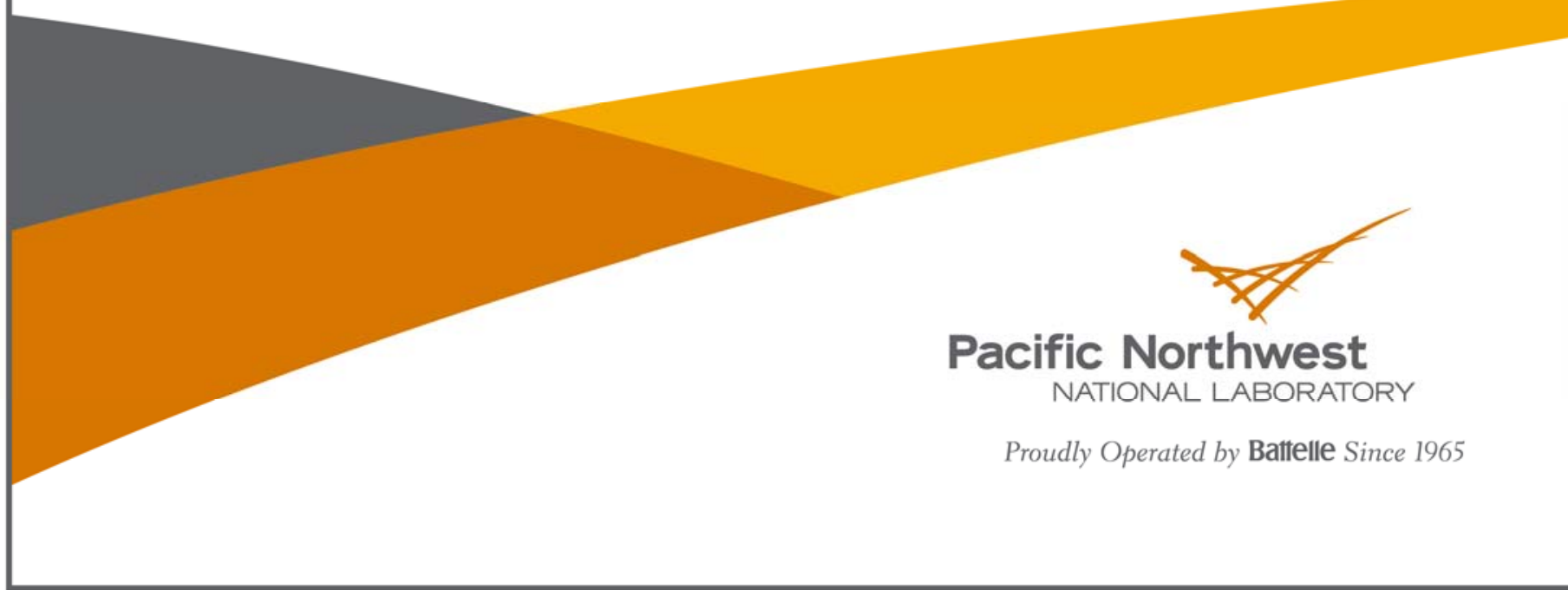




\title{
DISCLAIMER
}

This report was prepared as an account of work sponsored by an agency of the United States Government. Neither the United States Government nor any agency thereof, nor Battelle Memorial Institute, nor any of their employees, makes any warranty, express or implied, or assumes any legal liability or responsibility for the accuracy, completeness, or usefulness of any information, apparatus, product, or process disclosed, or represents that its use would not infringe privately owned rights. Reference herein to any specific commercial product, process, or service by trade name, trademark, manufacturer, or otherwise does not necessarily constitute or imply its endorsement, recommendation, or favoring by the United States Government or any agency thercof, or Battelle Memorial Institute. The views and opinions of authors expressed herein do not necessarily state or reflect those of the United States Government or any agency thereof.

\author{
PACIFIC NORTHWEST NATIONAI, I.ABORATORY \\ operated by \\ BAI'I'ELLE \\ for the \\ UNITED STATES DFPARTMENT OF FNFRGY \\ under Contract $D E-A C 05-76 R L 01830$ \\ Printed in the United States of America \\ Available to DOE and DOE contractors from the \\ Office of Scientific and Technical Information, \\ P.O. Box 62, Oak Ridge, TN 37831-0062; \\ ph: (865) 576-8401 \\ fax: (865) 576-5728 \\ email: reports@adonis.osti.gov
}

\begin{abstract}
Available to the public from the National Technical Information Service, U.S. Department of Commerce, 5285 Port Roy al Rd., Springfield, VA 22161 ph: $(800) 553-6847$ fax: $(703) 605-6900$ email: orders(a) ntis.fedworld.gov online ordering: http://www.ntis.gov/ordering.htm
\end{abstract}

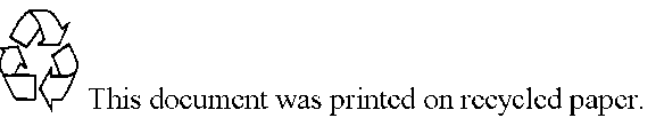

(9/2003) 


\title{
Large-Scale Spray Releases: Additional Aerosol Test Results
}

\author{
RC Daniel \\ PA Gauglitz \\ CA Burns \\ MS Fountain \\ RW Shimskey \\ JM Billing \\ JR Bontha \\ DE Kurath \\ JJ Jenks \\ PJ MacFarlan \\ LA Mahoney
}

August 2013

Test Specification: None

Work Authorization: WA42AM01

Test Plan: TP-WTPSP-031, Rev. 1.0

Test Exceptions: None

Prepared for

the U.S. Department of Energy

under Contract DE-AC05-76RL01830

Pacific Northwest National Laboratory

Richland, Washington 99352 



\section{Completeness of Testing}

This report describes the results of work and testing specified by Test Plan TP-WTPSP-031, Rev. 1.0. The work followed the quality assurance requirements outlined in the Test Plan. The descriptions provided in this report are an accurate account of both the conduct of the work and the data collected. Test plan results are reported. Also reported are any unusual or anomalous occurrences that are different from expected results. The test results and this report have been reviewed and verified.

\section{Approved:}

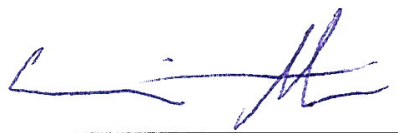

Reid A. Peterson, Manager WTP R\&T Support Project
$3 / 2 / 20 / 3$

Date 



\section{Testing Summary}

One of the events postulated in the hazard analysis for the Waste Treatment and Immobilization Plant (WTP) and other U.S. Department of Energy (DOE) nuclear facilities is a breach in process piping that produces aerosols with droplet sizes in the respirable range. The current approach for predicting the size and concentration of aerosols produced in a spray leak event involves extrapolating from correlations reported in the literature. These correlations are based on results obtained from small engineered spray nozzles using pure liquids that behave as a Newtonian fluid. The narrow ranges of physical properties on which the correlations are based do not cover the wide range of slurries and viscous materials that will be processed in the WTP and in processing facilities across the DOE complex.

To expand the data set upon which the WTP accident and safety analyses were based, an aerosol spray leak testing program was conducted by Pacific Northwest National Laboratory (PNNL). PNNL's test program addressed two key technical areas to improve the WTP methodology (Larson and Allen 2010). The first technical area was to quantify the role of slurry particles in small breaches where slurry particles may plug the hole and prevent high-pressure sprays. The results from an effort to address this first technical area can be found in Mahoney et al. (2012). The second technical area was to determine aerosol droplet size distribution and total droplet volume from prototypic breaches and fluids, including sprays from larger breaches and sprays of slurries for which literature data are mostly absent. To address the second technical area, the testing program collected aerosol generation data at two scales, commonly referred to as small-scale and large-scale testing. The small-scale testing and resultant data are described in Mahoney et al. (2013), and the large-scale testing and resultant data are presented in Schonewill et al. (2012). In tests at both scales, simulants were used to mimic the relevant physical properties projected for actual WTP process streams.

Examination of the results from the initial (subsequently referred to as Phase I) aerosol spray leak testing described in the preceding paragraph highlighted some uncertainties in evaluating aerosol generation that remained after the original scope of work was completed. Specifically, there was uncertainty with respect to aerosol behavior (namely aerosol release fraction and generation rates) for:

- simulant(s) near the WTP rheological boundaries of (6 Pa/6 mPa $\cdot \mathrm{s}$ and $30 \mathrm{~Pa} / 30 \mathrm{mPa} \cdot \mathrm{s})$

- simulant(s) with solids loadings greater than $20 \mathrm{wt} \%$

- simulant(s) with a small solids fraction of particles that have a relatively high density

- lower spray pressures (i.e., specifically 100 and 200 psig in the small-scale tests)

- in-spray measurements.

The need for additional (subsequently referred to as Phase II) aerosol spray leak testing was identified. The purpose of the study described in this report is to provide experimental data and analyses to supplement the results obtained during Phase I testing and reduce uncertainty in the remaining technical areas by performing large-scale tests with a range of orifice sizes and orientations representative of the WTP typical conditions. The companion study describing the Phase II small-scale testing is available in Schonewill et al. (2013). 


\section{S.1 Objectives}

Table S.1 provides a summary of each Phase II specific large-scale aerosol test objective, whether the objective was met, and discussions of the test results. Test Plan TP-WTPSP-031, Rev. 1.0 identifies four objectives relevant to Phase II large-scale spray leak testing. These are test objectives 10, 12, 13, and 14 (as listed in Table S.1). Other objectives identified in Test Plan TP-WTPSP-031, Rev. 1.0, are not discussed in this report as they apply either to Phase I test objectives completed in previous testing and discussed in Mahoney et al. (2012, 2013) and Schonewill et al. (2012) or to objectives addressed by Phase II small-scale testing and discussed in Schonewill et al. (2013). 
Table S.1. Summary of Phase II Large-Scale Test Objectives and Results

\begin{tabular}{|c|c|c|}
\hline Test Objective & $\begin{array}{c}\text { Objectiv } \\
\text { Met? }\end{array}$ & Discussion \\
\hline $\begin{array}{l}\text { Test Objective 10: Assess the } \\
\text { capability of the Malvern Insitec-S } \\
\text { in-process particle size analyzer, which } \\
\text { is the instrument used in the aerosol } \\
\text { testing, to measure accurately the } \\
\text { concentration and size distribution of } \\
\text { samples. This will be accomplished by } \\
\text { measuring carefully controlled dilute } \\
\text { aqueous slurries of known } \\
\text { concentration and particle size } \\
\text { distribution and comparing the } \\
\text { Malvern result to the known values. } \\
\text { Testing will include mono- and } \\
\text { poly-disperse suspensions and will } \\
\text { evaluate all four Malvern } \\
\text { configurations of 1) Phase I } \\
\text { small-scale spacers and old lens, } \\
\text { 2) Phase II small-scale spacers and } \\
\text { new lens, 3) Phase I large-scale } \\
\text { spacers and old lens, and 4) Phase II } \\
\text { large-scale spacers and new lens (old } \\
\text { lens } 100 \text { mm, new lens 500 mm). }\end{array}$ & Yes & $\begin{array}{l}\text { Validation testing was performed to assess the ability of the } \\
\text { Malvern Insitec-S aerosol analyzer to accurately measure } \\
\text { dispersion/aerosol concentration and particle size distribution } \\
\text { (PSD) for all instrument lenses and spacer configurations } \\
\text { relevant to Phase I and Phase II aerosol testing. In addition, the } \\
\text { ability of the instrument to accurately measure distributed } \\
\text { dispersions across } \sim 400 \text { and } ~ 900 \mathrm{~mm} \text { path lengths was tested } \\
\text { and verified. The validation testing was aimed at assessing } \\
\text { accuracy for in-chamber test configurations used in Phase I and } \\
\text { Phase II testing. Test configurations and laser obscurations } \\
\text { relevant to in-spray testing were not evaluated. }\end{array}$ \\
\hline $\begin{array}{l}\text { Test Objective 12: Determine the size } \\
\text { distribution of aerosol droplets and the } \\
\text { total droplet volume concentration as a } \\
\text { fraction of the total spray volume for } \\
\text { water sprays for a range of different } \\
\text { chamber sizes and for sprays traveling } \\
\text { different distances within the largest } \\
\text { chamber. Also determine the droplet } \\
\text { size distribution directly in the spray } \\
\text { (in-spray measurement) for the largest } \\
\text { sprays that can be measured. Testing } \\
\text { will be conducted using at least one } \\
\text { circular hole and multiple rectangular } \\
\text { breaches up to the } 1 \times 76 \text { mm breach } \\
\text { at target pressures between } 100 \text { and } \\
380 \text { psi. The chamber length will be } \\
\text { varied from } 39 \text { ft to } 10 \text { ft and perhaps } \\
\text { as small as } 5 \text { ft if the data show this } \\
\text { will help the extrapolation to } \\
\text { long-distance sprays. }\end{array}$ & Yes & $\begin{array}{l}\text { In-Chamber Release. Fraction testing has determined the } \\
\text { droplet volume concentration and size distribution for water in } \\
\text { large-scale test chambers of different chamber volume. } \\
\text { Chamber size was controlled by changing the total length of the } \\
\text { chamber while maintaining the same cross-sectional area as } \\
\text { tested in Phase I spray leak testing. Chamber lengths of } 10,20 \text {, } \\
30 \text {, and } 39 \text { ft were evaluated. Release fraction results from } \\
\text { these four chamber lengths were sufficient to establish a basis } \\
\text { for extrapolation to different spray lengths, and as such, testing } \\
\text { in chambers shorter than } 10 \text { ft was not needed. In addition, } \\
\text { in-chamber release fraction testing in the } 39 \text { ft chamber also } \\
\text { evaluated sprays of different length by adding spool extension } \\
\text { pieces to the spray header. These extensions reduced the } \\
\text { distance between the spray header, where high-pressure spray is } \\
\text { generated through an engineered orifice, and the chamber wall } \\
\text { downstream of the spray and upon which the spray impacts } \\
\text { (called the splash wall). Using this method, spray lengths (i.e., } \\
\text { the distance between the spray source and splash wall) of } 3.5 \text {, } \\
\text { 19, } 29 \text {, and } 38 \mathrm{ft} \text { were tested. The in-chamber release fraction } \\
\text { results were supplemented with in-spray testing, which } \\
\text { measured the size distribution of droplets generated by the spray } \\
\text { directly by placing the aerosol analyzers in-line with the spray } \\
\text { (i.e., in the direct path of the spray such that the spray passed } \\
\text { directly through the aerosol analyzer's measuring volume). All } \\
\text { Phase II, in-chamber and in-spray testing evaluated droplet } \\
\text { volume concentration and size distribution from sprays } \\
\text { generated from four orifices (a } 2 \text { mm circular hole, a } 1 \times 10 \text { mm } \\
\text { slot, a } 1 \times 20 \text { mm slot, and a } 1 \times 76 \text { mm slot) at three test } \\
\text { pressures ( } 100,200 \text {, and } 380 \text { psig). }\end{array}$ \\
\hline
\end{tabular}


Table S.1. (contd)

\begin{tabular}{|c|c|c|}
\hline Test Objective & $\begin{array}{l}\text { Objectiv } \\
\text { Met? }\end{array}$ & Discussion \\
\hline $\begin{array}{l}\text { Test Objective 13: Determine the size } \\
\text { distribution of aerosol droplets and the } \\
\text { total droplet volume concentration as a } \\
\text { fraction of the total spray volume for } \\
\text { non-Newtonian clay slurries. Also } \\
\text { determine the droplet size distribution } \\
\text { directly in the spray (in-spray } \\
\text { measurement) for these simulants. The } \\
\text { rheology of the simulant will be } \\
\text { adjusted so that one slurry, at the } \\
\text { beginning of testing, has at least one } \\
\text { Bingham parameter near } 30 \text { Pa/30 cP } \\
\text { (target range is } 30 \pm 4 \text { Pa or cP) and } \\
\text { the second Bingham parameter should } \\
\text { be less than or equal to the upper } \\
30 \pm 4 \text { Pa or cP target. The second } \\
\text { simulant will be adjusted so that one } \\
\text { slurry has at least one Bingham } \\
\text { parameter near } 6 \text { Pa/6 cP (target } \\
\text { range is } 6 \pm 2 \text { Pa or cP) and the } \\
\text { second Bingham parameter should be } \\
\text { greater than or equal to the lower } \\
6 \pm 2 \text { Pa or cP target. Testing will be } \\
\text { conducted using at least one circular } \\
\text { hole and multiple rectangular breaches } \\
\text { up to the } 1 x 76 \text { mm breach at target } \\
\text { pressures between } 100 \text { and } 380 \text { psi. }\end{array}$ & Yes & $\begin{array}{l}\text { Phase II spray leak testing evaluated release fraction results } \\
\text { (i.e., droplet volume concentration and size distribution) for two } \\
\text { non-Newtonian simulants composed of a mixed dry clay } \\
\text { powder ( } 80 \mathrm{wt} \% \text { kaolin and } 20 \mathrm{wt} \% \text { bentonite) slurried in water. } \\
\text { To meet the rheological targets specified in the test objective, } \\
\text { two mixed clay slurries were prepared at average total solids } \\
\text { concentrations of } \sim 27 \mathrm{wt} \% \text { and } \sim 32 \mathrm{wt} \% \text {. The rheological } \\
\text { properties of both slurries were characterized using a Bingham } \\
\text { Plastic rheology model for consistency and yield stress using a } \\
\text { controlled rate concentric cylinder rheometer. The } \sim 27 \mathrm{wt} \% \\
\text { clay slurry exhibited an average Bingham yield stress and } \\
\text { consistency of } 6.7 \mathrm{~Pa} \text { and } 12 \mathrm{mPa} \cdot \mathrm{s} \text {, respectively (note that } \\
1 \mathrm{mPa} \cdot \mathrm{s}=1 \mathrm{cP}) \text {. The rheology target was met for all testing } \\
\text { performed with the } \sim 27 \mathrm{wt} \% \text { clay slurry. The } \sim 32 \mathrm{wt} \% \text { clay } \\
\text { slurry exhibited an average Bingham yield stress and } \\
\text { consistency of } 26 \mathrm{~Pa} \text { and } 30 \mathrm{mPa} \cdot \mathrm{s} \text {, respectively. The rheology } \\
\text { target was met for all testing performed with the } \sim 32 \mathrm{wt} \% \text { clay } \\
\text { slurry. As with water testing, all in-chamber and in-spray } \\
\text { testing with clay slurries examined sprays generated through } \\
\text { four orifices (a } 2 \mathrm{~mm} \text { circular hole, a } 1 \times 10 \mathrm{~mm} \text { slot, a } \\
1 \times 20 \mathrm{~mm} \text { slot, and a } 1 \times 76 \mathrm{~mm} \text { slot) at three test pressures } \\
\text { ( } 100,200 \text {, and } 380 \mathrm{psig} \text { ). All testing with the clay simulants } \\
\text { was conducted with a chamber length of } 20 \mathrm{ft} \text {. }\end{array}$ \\
\hline $\begin{array}{l}\text { Test Objective 14: Compare the } \\
\text { aerosol results from the Malvern } \\
\text { Insitec-S using the new } 500 \text { mm lens } \\
\text { that has a nominal measurement range } \\
\text { of } 2.5 \text { to } 2500 \mu \text { m (Malvern } \\
\text { Instruments, Ltd. 2010) to aerosol } \\
\text { results using the } 100 \mathrm{~mm} \text { lens } \\
\text { employed in Phase I that provided a } \\
\text { nominal range of } 0.5 \text { to } 200 \mu \mathrm{m} \\
\text { (Schonewill et al. } 2012 \text { ). Tests will use } \\
\text { one or more orifices. }\end{array}$ & $\mathrm{Ye}$ & $\begin{array}{l}\text { During Phase II analyses, functional testing of both } 100 \text { and } \\
500 \mathrm{~mm} \text { lenses was performed to compare lens performance. } \\
\text { Primary lens comparison tests were performed using a water } \\
\text { spray generated through a } 1 \times 76 \mathrm{~mm} \text { rectangular slot at } \\
380 \text { psig and indicate that despite the difference in working } \\
\text { range for both lenses, the } 500 \mathrm{~mm} \text { lens provides comparable (if } \\
\text { not fully equivalent) results to the } 100 \mathrm{~mm} \text { lens results within } \\
\text { expected and measured uncertainty limits. Additional tests that } \\
\text { evaluated all configuration changes made to the large-scale test } \\
\text { chamber and aerosol instruments (such as the expansion of } \\
\text { chamber size, alteration of aerosol instrument hanging } \\
\text { mechanisms, use of the } 500 \mathrm{~mm} \text { lens, and use of longer } 1 \text { meter } \\
\text { rods for the aerosol instrument spacers) also indicate that } \\
\text { Phase II release fraction results can be compared directly to } \\
\text { Phase I release fraction results for equivalent orifice and test } \\
\text { pressure combinations. }\end{array}$ \\
\hline
\end{tabular}




\section{S.2 Results and Performance Against Success Criteria}

The success criteria for achieving the large-scale aerosol test objectives are discussed in Table S.2. These test criteria were established in Test Plan TP-WTPSP-031, Rev. 1.0.

Table S.2. Success Criteria for Large-Scale Aerosol Tests

\begin{tabular}{l} 
Success Criteria \\
\hline Criterion for \\
Measure the concentration and size distribution of \\
known aqueous suspensions with the Malvern used \\
for aerosol measurements and quantitatively \\
compare the Malvern (Insitec-S) results with the \\
known values to estimate the Malvern (Insitec-S) \\
accuracy for measuring aerosols.
\end{tabular}

How Testing Did or Did Not Meet Success Criteria

Test Objectives 10

Met: The concentration and size distribution of eight

powder systems was measured and compared to known values based on material balance or list/reference properties. The eight powders included mono- and poly-disperse glass bead powders and irregular non-spherical powders. Tests with these powders evaluated concentration and size distribution accuracy under different instrument configurations (i.e., lenses and spacer bars) relevant to Phase I and Phase II testing. Furthermore, tests evaluated the accuracy of corrections made when analyzing aerosols distributed over $\sim 400$ and $\sim 900 \mathrm{~mm}$ path lengths.

\section{Criteria for Test Objectives 12 and 13}

Measure the droplet size distribution, total volume concentration of droplets, and total volume flow rate sprayed for each of the breaches and simulants tested.

For in-spray measurements, measure the droplet size distribution for each of the breaches and simulants tested.

Characterize the viscosity or rheology, PSD, bulk density, and surface tension of each simulant tested.
Met: Up to three Malvern Insitec-S aerosol analyzers were used to continuously measure the droplet volume concentration and size distribution during spray leak testing in the large-scale test chamber for all orifice and pressure combinations. The total volumetric flow rate of spray generated through each orifice was determined by monitoring differential loss of simulant mass from the simulant feed and storage tanks as a function of time during active spray.

Met: Three Malvern-Insitec-S aerosol analyzers were used to measure the "in-spray" size distribution of water and clay simulant sprays for all test pressure and orifice combinations. In-spray measurements involved placing the aerosol analyzers in the direct path of the spray (before it impinged on the wall downstream of the spray header and engineered breach) such that the spray passed directly through the measuring volume of the Malvern Insitec-S. This contrasts with the normal "in-chamber" aerosol configuration where the analyzers are positioned above the spray and outside of its direct path.

Met: Phase II tests evaluated water and two concentrations of mixed dry clay powders (composed of $80 \mathrm{wt} \%$ kaolin and $20 \mathrm{wt} \%$ bentonite) slurried water. For these three simulants, the rheology, PSD, bulk density, and surface tension where characterized through direct measurement or through use of standard chemical references (for common properties such as the viscosity of water). 
Table S.2. (contd)

\begin{tabular}{l} 
Success Criteria \\
\hline For the 30 Pa/30 cP simulant at the beginning of \\
testing, at least one Bingham parameter is \\
$30 \pm 4$ Pa or cP and the second Bingham parameter \\
should be less than or equal to this upper target of \\
$30 \pm 4$ Pa or $\mathrm{cP}$.
\end{tabular}

How Testing Did or Did Not Meet Success Criteria

Met: A concentrated clay slurry (32.1 wt $\%)$ was used to represent the upper test range of non-Newtonian simulant rheology. It exhibited an average Bingham yield stress and consistency of $26 \mathrm{~Pa}$ and $30 \mathrm{mPa} \cdot \mathrm{s}$, respectively. A target consistency criterion of $30 \pm 4 \mathrm{mPa}$ s and yield stress $\leq 30 \mathrm{~Pa}$ was met for all testing performed with this concentrated clay slurry.

For the 6 Pa/ $6 \mathrm{cP}$ simulant at the beginning of Met: A dilute clay slurry (27.1 wt $\%$ ) was used to represent testing, at least one Bingham parameter is $6 \pm 2 \mathrm{~Pa}$ or $\mathrm{CP}$ and the second Bingham parameter should be greater than or equal to this lower target of $6 \pm 2 \mathrm{~Pa}$ or $\mathrm{cP}$. the lower test range of non-Newtonian simulant rheology. It exhibited an average Bingham yield stress and consistency of $6.7 \mathrm{~Pa}$ and $12 \mathrm{mPa} \cdot \mathrm{s}$, respectively. A target yield stress criterion of $6 \pm 2 \mathrm{~Pa}$ and consistency $\geq 6 \mathrm{mPa} \cdot \mathrm{s}$ was met for all release fraction testing performed with the dilute clay slurry.

Measure the pressure and flow in the piping.

Met: A data acquisition system was used to continuously monitor and record recirculation loop pressure and flow during Phase II spray leak testing.

Calculate the large-scale test chamber volume from internal dimensions for chamber configurations different from Phase I testing.

Met: For all test configurations evaluated in Phase II, including different chamber lengths, different spool configurations, and different hanging mechanisms for aerosol instrumentation, internal dimensions were recorded and used to determine the internal chamber volume which can be occupied by aerosol.

Criterion for Test Objective 14

Quantitative comparison demonstrates that the old and new lenses give equivalent results for aerosol conditions that have droplet sizes in a range appropriate for both lenses.

Met: During Phase II analysis, functional testing of both 100 and $500 \mathrm{~mm}$ lens has been performed to compare lens performance. Primary lens comparison tests were performed using a water spray generated through a $1 \times 76 \mathrm{~mm}$ rectangular slot at $380 \mathrm{psig}$ and indicate that despite the difference in working range for both lenses, the $500 \mathrm{~mm}$ lens provides comparable (if not fully equivalent) results to the $100 \mathrm{~mm}$ lens results within expected and measured uncertainty limits. Additional tests that evaluated all configuration changes made to the large-scale test chamber and aerosol instruments (such as the expansion of chamber size, alteration of aerosol instrument hanging mechanisms, use of the $500 \mathrm{~mm}$ lens, and use of longer 1 meter rods for the aerosol instrument spacers) also indicate that Phase II release fraction results can be compared directly to Phase I release fraction results for equivalent orifice and test pressure combinations. 


\section{S.3 Quality Requirements}

The Quality Assurance (QA) program at PNNL is based on the requirements defined in the DOE Order 414.1D, Quality Assurance, and 10 CFR 830, Energy/Nuclear Safety Management, and Subpart A-Quality Assurance Requirements (a.k.a., the Quality Rule). PNNL has chosen to implement the following consensus standards in a graded approach:

- ASME NQA-1-2000, Quality Assurance Requirements for Nuclear Facility Applications, Part 1, Requirements for Quality Assurance Programs for Nuclear Facilities

- ASME NQA-1-2000, Part II, Subpart 2.7, Quality Assurance Requirements for Computer Software for Nuclear Facility Applications

- ASME NQA-1-2000, Part IV, Subpart 4.2, Graded Approach Application of Quality Assurance Requirements for Research and Development.

The procedures necessary to implement the requirements are documented through PNNL's "How Do I...?" (HDI) ${ }^{1}$ system.

The Waste Treatment Plant Support Project (WTPSP) implements an NQA-1-2000 Quality Assurance program, graded on the approach presented in NQA-1-2000, Part IV, Subpart 4.2. The WTPSP Quality Assurance Manual (QA-WTPSP-0002) describes the technology life cycle stages under the WTPSP Quality Assurance Plan (QA-WTPSP-0001). The technology life cycle includes the progression of technology development, commercialization, and retirement in process phases of basic and applied research and development $(R \& D)$, engineering and production and operation until process completion. The life cycle is characterized by flexible and informal QA activities in basic research, which becomes more structured and formalized through the applied R\&D stages.

The work described in this report has been completed under the QA technology level of Developmental Work. WTPSP addresses internal verification and validation activities by conducting an Independent Technical Review of the final data report in accordance with WTPSP's procedure QA-WTPSP-601, Document Preparation and Change. This review verifies that the reported results are traceable, that inferences and conclusions are soundly based, and that the reported work satisfies the test plan objectives.

\section{S.4 Simulant Use}

A broad range of simulants have been developed for spray leak testing (Mahoney et al. 2013; Schonewill et al. 2012). However, because of the hazards and costs associated with some of the simulants developed, only a small subset of simulants was used in the large-scale test stand during Phase II testing. Table S.3 presents a list of the simulants tested in the large-scale test stand, their physical and rheological property ranges, and their relevance to the WTP operations. The properties important to aerosol generation include PSD, viscosity, rheology, bulk density, weight percent undissolved solids, and surface tension. Target property ranges are listed in Table S.3; the actual physical properties of simulants tested during Phase II large-scale spray leak studies are reported in Chapter 3 of this report.

\footnotetext{
${ }^{1} \mathrm{HDI}$ is a web-based system for managing the delivery of PNNL policies, requirements, and procedures.
} 
Table S.3. Target Simulants Tested in Phase II Large-Scale Test Stand and the WTP Process Stream Categories

\begin{tabular}{|c|c|c|c|}
\hline Material/Description & Alias & Target Property Range & $\begin{array}{c}\text { WTP Process } \\
\text { Stream Categories }\end{array}$ \\
\hline Water & Water & $\begin{array}{l}\text { Viscosity } 1 \mathrm{mPa} \cdot \mathrm{s}(1 \mathrm{cP}) \\
\text { Density } 1000 \mathrm{~kg} / \mathrm{m}^{3} \\
\text { Surface tension } 73 \mathrm{mN} / \mathrm{m}\end{array}$ & $\begin{array}{l}\text { - Ultrafilter permeate/treated low } \\
\text { activity waste } \\
\text { - Cs ion exchange eluate } \\
\text { - Recycle streams }\end{array}$ \\
\hline $\begin{array}{l}80 / 20 \text { solids blend of a } \\
\text { kaolin/bentonite clay } \\
\text { slurry, } 32 \mathrm{wt} \%\end{array}$ & 30 Pa Clay & $\begin{array}{l}\text { The solids loading was adjusted to } \\
\text { meet target Bingham yield stress of } \\
30 \mathrm{~Pa}\end{array}$ & Non-Newtonian slurries \\
\hline $\begin{array}{l}80 / 20 \text { solids blend of a } \\
\text { kaolin/bentonite clay } \\
\text { slurry, } 27 \mathrm{wt} \%\end{array}$ & $30 \mathrm{~Pa}$ Clay & $\begin{array}{l}\text { The solids loading was adjusted to } \\
\text { meet the target Bingham yield stress of } \\
6 \mathrm{~Pa}\end{array}$ & Non-Newtonian slurries \\
\hline
\end{tabular}

\section{S.5 Summary of Phase II Results}

Phase II aerosol generation tests were performed to quantify the release fraction and generation rate for a range of non-Newtonian clay slurries that represent expected WTP process stream properties, chamber lengths, spray lengths, and initial chamber humidity. Results were collected from transient concentration measurements in the chambers and from in-spray measurements. PNNL also conducted extrapolations of orifice data to larger postulated breaches in 3 and $10 \mathrm{in}$. process piping and to longer-distance sprays. Both the test results and extrapolations were compared with the WTP model predictions. In addition, testing was conducted to evaluate instrument performance, the role of initial chamber humidity, and experimental variation in repeat tests. These results were used to estimate the bias in the in-chamber release fraction measurements and to estimate reasonably conservative aerosol generation rate predictions as a function of orifice area, test pressure, and aerosol diameter. Below are summaries of the key findings.

- Correlation Development for Comparing Test Results to the WTP Model. A reasonably conservative correlation for aerosol generation rate was developed based on the in-chamber test data and extrapolations of the in-chamber data to $100 \mathrm{ft}$ chambers. The correlation was developed for water, but it is appropriate for all the liquids and slurries tested because the aerosol generation from the other fluids is overwhelmingly always the same or less than water sprays. The results for the non-Newtonian chemical slurry simulants are the primary exceptions, but these slurries had unusual rheologies in comparison to actual waste and the clay simulants, making the applicability of these results questionable. The correlation was compared to in-spray data and was found to match the in-spray data for the range of orifices and spray pressures tested. The good comparisons in the regions of overlap for different size orifices and different spray pressures confirm that the conservative correlation has orifice area, spray pressure, and droplet size dependences that agree with in-spray data. Because the conservative correlation matches the in-spray results, this demonstrates that the conservative correlation has accounted for the potential biases (humidity and method bias) with the in-chamber method without actually quantifying them. A comparison of the WTP model for generation rate to the test results, as represented by the conservative correlation, shows that the WTP model has the correct dependence for droplet size, under-represents the effect of increasing generation rate with orifice area, and under-represents the effect of increasing generation rate with spray pressure. Accordingly, the greatest under-estimation in generation rate from the WTP model occurs for the highest postulated pressure and the largest postulated breach, which is consistent with 
the conclusions from previous testing (Schonewill et al. 2012). One specific process stream of interest for the WTP is a 540 psig spray from a $290 \mathrm{~mm}^{2}$ orifice. For this example, the WTP model under-predicts the generation rate compared to the conservative correlation by about a factor of 20 .

- Performance of Malvern Insitec-S Aerosol Analyzers Using Phase II Chamber and Instrument Measuring Configurations. Functional tests were performed to verify that changes made to both large-scale test chamber and aerosol instruments for Phase II testing did not impact release fraction measurements and prevent direct comparison with Phase I data. The most significant change made to aerosol instruments was installation of the new focusing lens to change the working range of the Malvern Insitec-S from 0.5 to $200 \mu \mathrm{m}$ (100 mm lens, Phase I) to 2.5 to $2500 \mu \mathrm{m}$ (500 mm lens, Phase II). Side-by-side comparison of 100 and $500 \mathrm{~mm}$ lens release fractions measured for water sprayed through a $1 \times 76 \mathrm{~mm}$ orifice at 380 psig indicated that both lenses produced equivalent results at aerosol diameters between 23 and $100 \mu \mathrm{m}$. Instrument sensitivity prevented comparison of lens performance at release fractions corresponding to lower aerosol sizes. Additional functional tests were conducted to determine optimal mixing fan settings and instrument purge air rate settings for Phase II analysis and their influence on release fraction measurements. These tests affirmed the adequacy of Phase I mixing fan selections and helped define the range of purge air settings for Phase II analyses.

- Validation of Malvern Insitec-S Aerosol Analyzer Performance. The performance of the Malvern Insitec-S analyzer has been evaluated extensively. Tests examined the accuracy of Malvern Insitec-S concentration and size distribution measurements by testing carefully controlled dilute aqueous slurries of known concentration and PSD and comparing the Malvern Insitec-S result to the known values. Testing included mono- and poly-disperse suspensions and evaluated six Malvern Insitec-S configurations relevant to Phase I and Phase II testing:

- Phase I small-scale configuration: lens - $100 \mathrm{~mm}$; spacer bar length - $150 \mathrm{~mm}$

- Phase II small-scale configuration: lens - $500 \mathrm{~mm}$; spacer bar length - $150 \mathrm{~mm}$

- Phase I large-scale configuration: lens - $100 \mathrm{~mm}$; spacer bar length - $500 \mathrm{~mm}$

- Phase II large-scale configuration: lens - $500 \mathrm{~mm}$; spacer bar length - $1000 \mathrm{~mm}$

- Dispersions uniformly distributed across $\sim 400 \mathrm{~mm}$ analyzed by the $100 \mathrm{~mm}$ lens (Phase I in-chamber configurations)

- Dispersions uniformly distributed across $\sim 900 \mathrm{~mm}$ analyzed by the $500 \mathrm{~mm}$ lens (Phase II in-chamber configurations).

Testing indicates that under the best measuring circumstances, the Malvern Insitec-S can measure concentration and median dispersion size to approximately 2 and 3 percent of list values, respectively. Particle shape was observed to increase the apparent concentration measured by the Malvern Insitec-S (relative to the actual value); however, at the sizes of interest for spray leak analysis (10 to $100 \mu \mathrm{m}$ ), aerosol droplets are expected to be spherical so concentration readings will not be impacted. Evaluation of the $500 \mathrm{~mm}$ lens results indicates that it is subject to loss of accuracy when used to analyze dispersions that fall between primarily 2.5 and $20 \mu \mathrm{m}$. Aerosols observed in Phase I and Phase II spray leak testing, which always contain significant fractions of aerosols greater than $20 \mu \mathrm{m}$ in size, are not expected to be significantly impacted. Spray geometry corrections used to interpret concentration and size measurements for distributed aerosols in Phase I and Phase II have been evaluated and found to provide reasonable and appropriate corrections for laser attenuation. Overall 
evaluation of the validation results suggests that no bias correction in either aerosol concentration or size distribution measurements is needed for Malvern Insitec-S configurations used in Phase I and Phase II testing.

- Assessment of Chamber Uniformity. Testing was performed to evaluate chamber uniformity (with respect to aerosol concentration and size distribution) and its impact on accuracy of release fraction measurements and their associated uncertainty. Tests employed the smallest Phase II test orifice (i.e., the $2 \mathrm{~mm}$ hole) to evaluate $39 \mathrm{ft}$ test enclosure uniformity under conditions approaching the least turbulent, and therefore least uniform, spray conditions evaluated in Phase II large-scale testing. These measurements indicate that standard in-chamber test locations (which employ aerosol sampling locations $21 \mathrm{in}$. above the orifice) will be subject to loss of large droplets as a result of settling, leading to inaccurate assessment of release fraction and generation rate for droplets $\sim 100 \mu \mathrm{m}$ and greater. Droplet loss is worst above the spray header and becomes less severe at downstream positions and closer to the spray. Release fraction estimates for smaller droplets (i.e., 10 to $\sim 80 \mu \mathrm{m}$ ) do not appear to be impacted and are relatively insensitive to measurement location as long as the direct path of the spray is avoided.

- Evaluation of In-Spray Release Fractions as a Function of Position. In-spray measurements evaluate release fraction in the direct path (which is defined as the most optically dense region) of the spray. These measurements provide a direct means of assessing the full size distribution of aerosol droplets produced by spray without incurring significant loss of large aerosols from droplet settling or inertial wall capture. Phase II testing evaluated in-spray release fractions for water and two non-Newtonian clay slurries as a function of downstream distance from the orifice. For many test pressure, orifice, and simulant combinations, the in-spray release fraction did not change substantially with increasing distance from the orifice. For test cases where significant change did occur, the release fraction contributions were typically shifted to larger aerosol diameters. This increase in overall aerosol size has been attributed to droplet coalescence or to preferential sampling of large aerosols that settle to the bottom of the chamber. Relative to in-spray behavior for the majority of spray configurations and simulants tested, the in-spray trends for water sprays generated by the $1 \times 76 \mathrm{~mm}$ slot were anomalous and indicated that aerosol size decreased with increasing distance from the orifice.

- Assessment of In-Chamber Measurement Basis. In-chamber release fractions are expected to under-estimate true fractional aerosol release because of bias introduced by evaporation (caused by low relative humidity at the start of testing) and aerosol sampling bias introduced by settling of large droplets and mixing limitations. In-spray measurements are not subject to these limitations and are expected to over-estimate (and thus be conservative) actual release fractions in the size range of interest (10 to $100 \mu \mathrm{m}$ ) because of spatial measurement bias. However, measurement of in-spray release fractions in the size range of interest is frequently impossible because of loss of size distribution measurement accuracy at cumulative fractional volumes below $0.5 \times 10^{-2}$. In practical terms, this prevents measurement of size distribution information below $50 \mu \mathrm{m}$. Because in-spray measurements are expected to be conservative (i.e., larger than) the actual release, they can be used as a guide for correcting in-chamber release fractions such that they are "as-conservative" as in-spray measurements. Comparison of in-spray and in-chamber release fraction measurements indicates a multiplicative correction factor of three to in-chamber data will typically render them as conservative as in-spray measurements (see Section 7.4). This "method bias" correction contains size distribution and concentration measurements that impacts both in-spray and in-chamber measurements and also 
contains, in part, impacts to release fraction for humidity. However, the multiplicative factor of three does not fully account for all humidity effects, and additional correction is necessary.

- Determination of Fractional Aerosol Release from Non-Newtonian Slurries. The effect of non-Newtonian rheology for clay slurry sprays was evaluated within the WTP rheological limits for the Bingham yield stress and consistency of $6 \mathrm{~Pa} / 6 \mathrm{cP}$ and $30 \mathrm{~Pa} / 30 \mathrm{cP}$. Spray tests were conducted for measuring the generation rate and release fraction from concentration increase in the chamber and directly in-spray. For the in-chamber measurements, the release fraction is the same as water or decreases with increasing Bingham parameters. For in-spray measurements, the release fraction for the clay slurries at $380 \mathrm{psig}$ is the same as water. At 100 and $200 \mathrm{psig}$, the release fraction for the clay slurries decreases with increasing solids content (and increasing rheology as characterized by the Bingham yield stress and consistency). These results show that water results are typically the same or larger than (i.e., conservative relative to) release fraction measured for non-Newtonian clay slurries.

- Evaluation of the Effects of Spray Length and Confinement Size on Fractional Aerosol Release. The effect of spray length within a chamber was investigated for a range of sprays within a $39 \mathrm{ft}$ chamber. Similar to the Phase I results in a $20 \mathrm{ft}$ chamber, the release fraction always increased with spray length in the $39 \mathrm{ft}$ chamber. Accordingly, for a given size orifice, fluid, spray pressure, and chamber size, the highest release fraction and aerosol generation rate are given by the test results when the spray travels the full length of the chamber. The role of chamber length, when sprays travel the full length of the chamber, was investigated for chambers ranging from 10 to $39 \mathrm{ft}$. For the largest slot tested $(1 \times 76 \mathrm{~mm})$, the release fraction increased with chamber length for all droplet sizes at 380 psig. The $1 \times 76 \mathrm{~mm}$ slot also gave higher generation rates than the smaller orifices, and as a result, it is significant in terms of the worst-case spray for accident analyses. For other orifices and pressures, increasing chamber length typically caused the cumulative release fraction to increase for larger droplets (above roughly $30 \mu \mathrm{m}$ ) and decrease for smaller droplets (below roughly $30 \mu \mathrm{m}$ ).

\section{S.6 Discrepancies and Recommended Follow-On Tests}

No discrepancies associated with the large-scale testing were identified, all planned objectives for Phase II testing were completed, and all listed success criteria from Test Plan TP-WTPSP-031, Rev. 1.0, were met. Expert reviews of the spray release results indicate that additional work would improve the technical basis and application of the results at the WTP. As such, several follow-on tests and analyses are suggested:

- Chemical Slurry Simulant. Several attempts have been made with the small-scale test stand to obtain aerosol generation rates with a chemical slurry simulant representing WTP slurries with limited success. The tests were compromised by atypical behavior of the simulant, which exhibited a rheology that thickened with shear (dilatant) and was time dependent (rheopectic). The available literature and discussion with subject matter experts indicates that no other Hanford tank waste simulant or actual waste samples have exhibited this type of rheological behavior. Moreover, the undissolved solids concentration, which ranged from 35 to $40 \mathrm{wt} \%$, exceeded the current WTP solids concentration upper limit of $27 \mathrm{wt} \%$ and, thus, was too concentrated to be truly representative. The unstable rheology of the simulant forced modifications to the test equipment and procedures to allow completion of the majority of the aerosol tests. Release fractions measured from chemical simulant tests were typically greater than the release fractions measured for water or clay. Because of the unusual rheology, elevated undissolved solids concentration, and the operational difficulties, direct 
comparison of the results between the chemical simulant and other simulants is questionable. If results using a chemical slurry simulant are needed it is recommended that the cause of the unusual rheology be identified and perhaps a new simulant formulation be developed. Use of the large-scale test stand should be considered for further testing of the chemical slurry simulant to allow a wider range of orifice sizes and in-spray measurements to be obtained.

- Experimental Method Validation Testing for In-Chamber Configurations. The method and model used for evaluating in-chamber generation rates makes several assumptions with respect to the time-scale and completeness of aerosol mixing inside the test chambers and with respect to the functionality of aerosol loss in the test chamber. Phase I and Phase II experimental work has not fully evaluated the appropriateness of these assumptions and the impact of chamber heterogeneity on the inferred generation rates. This issue may be addressed by using a suitably conservative correlation or by developing and implementing an experimental validation method. Experimental validation studies would be performed using an aerosol source that produces a known aerosol generation rate with a well characterized PSD. These experiments would be conducted in the same manner that the in-chamber spray release results are being obtained. The collected data would be analyzed in the same manner and the results compared to the known aerosol source. The experiments would be conducted over a range of conditions including various injection locations, injection rates and simulants. Proposed materials to be aerosolized would include water, at least one other liquid that has a low vapor pressure such that it does not evaporate, and if possible a particulate slurry.

- Validation of Malvern Insitec-S In-Spray Measurement Accuracy. The Malvern Insitec-S validation activities described in Chapter 6 of this report addressed concerns related to aerosol analyzer performance in Phase I testing. The analyzer configurations and concentrations tested strongly reflect those associated with in-chamber testing, which represented the bulk of Phase I large-scale testing. In anticipation of similar concerns, Phase II in-chamber configurations also were evaluated in validation testing. Configurations and dispersion concentrations relevant to in-spray testing were not tested as part of Malvern Insitec-S validation. With the increased importance of in-spray configurations in Phase II analyses, additional follow-on validation work may be necessary to address similar concerns with instrument performance and accuracy with respect to in-spray measurements. Specific issues that may need to be addressed include 1) performance of the Malvern Insitec-S at high laser obscurations, 2) accuracy of corrections for multiple light scattering at high dispersion/aerosol concentrations, and 3) accuracy of spray geometry corrections for spray geometries relevant to in-spray testing and that simulate the core of the spray.

- Evaluation of Irregular Breach Morphology and Breaches that Occur in Thin Pipes. To date, PNNL spray release testing indicates that aerosol generation rate and release fractions from pipeline breaches depends only on the breach area and not on the morphology of the breach (Schonewill et al. 2012). However, PNNL spray leak studies have used engineered orifices with well-defined and uniform dimensions using a pipe wall thickness representative of new pipe, and there is concern that actual line breaks (e.g., at welds), breaches due to corrosion (e.g., bowl shaped pits), or large radial (along the pipe circumference) breaches (known as fan spray releases) will result in aerosol generation behavior that is different from the orifices tested. Furthermore, erosion (or corrosion) of the pipe wall could lead to breaches with smaller aspect ratios and result in different aerosol generation behaviors. To address the first concern regarding irregular breaches, PNNL suggests follow-on testing with more complex breach geometries. These might encompass irregular breach geometries such as cracks of varying length and multiple pinhole breaches at irregular overlapping and non-overlapping spacing. Of particular concern to this study is the need to define the range of 
"typical" or "representative" transfer line breaches and their morphologies. To address concerns related to wall thickness, PNNL suggests evaluation of breaches that derive from $3 \mathrm{in}$. transfer lines at a reduced pipe wall thickness. Consideration should also be given to testing a large radial breach, which could result in a large aerosol fan and increased aerosol generation. These studies can be accomplished in existing test equipment by creating additional test spools with the complex breach geometries and test spools with smaller wall thicknesses. Testing can be limited to selected orifice areas similar to those previously tested.

- Testing at Additional Hydraulic Orifice Diameters. While a significant range of orifice areas have been tested, the range of hydraulic diameters tested in Phase II was limited. Hydraulic diameter is thought to be an important parameter in aerosol formation. Additional testing with a larger range of hydraulic diameters would expand the applicability of the existing results. An emphasis in this additional testing would be to obtain additional in-spray measurements using water and both in-chamber and in-spray measurements with a non-Newtonian slurry.

- Additional Data Analyses. The available aerosol data for water collected in Phase II of the PNNL testing has been used to develop a reasonably conservative correlation over an aerosol particle size range of 10 to $100 \mu \mathrm{m}$ for application at the WTP. While this correlation may be suitable for application, the technical basis could be improved by incorporating data from Phase I as well as examining data from other simulants. This range $(10$ to $100 \mu \mathrm{m})$ is suitable for assessing the hazards associated with the respirable fraction. To address toxicological impacts the full range of aerosol diameters (e.g., in-spray results) needs to be considered. At this point no correlation or analysis of the in-spray data has been attempted. While the in-spray results are largely consistent with the in-chamber results the two data sets are not entirely consistent. Follow-on work would consist of using the existing data (and perhaps new data) to expand the correlation to be more generally applicable over the entire range of aerosol diameters. The development of a correlation based on dimensionless groups that include the physical properties may improve the basis for extrapolation to other conditions.

- Improve Conservative Correlation to Account for Non-Newtonian Fluid Properties and to Include Phase I Test Results. A conservative correlation was developed to represent the in-chamber test results for all chamber sizes, including the extrapolation to $100 \mathrm{ft}$ chambers. A useful result would be an equivalent evaluation of just the $20 \mathrm{ft}$ chamber data to determine how to quantitatively adjust a correlation based on $20 \mathrm{ft}$ chamber data to match the conservative correlation that represented all of the different chamber sizes. With this information, testing results collected in only the $20 \mathrm{ft}$ chamber could be used to quantitatively modify the current conservative correlation. One specific application of this approach would be to determine how the correlation could be adjusted to account for the general decrease in aerosol generation from non-Newtonian slurries with progressively higher Bingham yield stress and consistency. A second application would be to incorporate the Phase I data (Schonewill et al. 2012), which were collected in a $20 \mathrm{ft}$ chamber, and these data include results for a broader range of orifices areas and slot shapes. 



\section{Acknowledgments}

The authors would like to thank all the Pacific Northwest National Laboratory staff who assisted with this test program. Development of this report was supported by many dedicated PNNL staff involved in laboratory experiments, sampling, data acquisition, data processing, data quality confirmation, technical reviews, and data analysis. These staff included:

$\begin{array}{lll}\text { Harold Adkins } & \text { Jim Davis } & \text { Amanda Casella } \\ \text { Nathan Bauman } & \text { Dennese Smith } & \text { Craig Lukins } \\ \text { Garrett Brown } & \text { Chris Fischer } & \text { Lenna Mahoney } \\ \text { Bill Buchmiller } & \text { Jesse Lang } & \text { Diana Tran } \\ \text { Paul Eslinger } & & \end{array}$

The careful and thorough technical review of this report by Philip Schonewill was instrumental in shaping the document. We also thank Kirsten Meier for her quality assurance support. The technical editing and formatting of this report by Cary Counts, Mike Parker, and Kathy Neiderhiser was greatly appreciated.

We also sincerely appreciate the support, advice, and guidance from Mike Epstein (Fauske and Associates), Ameer Hassan (WTP Technical Lead), Susan Omberg-Carro (WTP), Chris Harrington (DOE-ORP), Joel Fox (DOE-ORP), Ralph Crowe (Sludge Treatment Project - K-Basin) and Bruce Zimmerman (Hanford Tank Farms).

We would also like to acknowledge the technical support and advice provided by Alon Vaisman and Jeff Denigris of Malvern Instruments, Ltd. We appreciate the outstanding customer service provided by Alon Vaisman and Malvern Instruments which enabled us to meet the challenging Phase II test schedule with minimal aerosol instrumentation downtime.

Funding for this effort was provided by the U.S. Department of Energy's Hanford Waste Treatment and Immobilization Plant Project. 



\section{Acronyms and Abbreviations}

6 Pa Clay

30 Pa Clay

APEL

ASME

CSTR

DAS

DOE

HDI

LAW

LRB

ORP

PEP

PLC

PNNL

PPC

PPM

PPMV

PSD

QA

$\mathrm{R} \& \mathrm{D}$

RI

RPP-WTP

STR

VFD

WTP

WTPSP short-hand simulant notation for the $\sim 27-\mathrm{wt} \%$ mixed clay

(80:20 kaolin:bentonite) slurry in water meeting the $6 \mathrm{~Pa} / 6 \mathrm{cP}$ rheological target for Phase II testing (also called Clay, $6 \mathrm{~Pa}$ )

short-hand simulant notation for the $\sim 32-\mathrm{wt} \%$ mixed clay

(80:20 kaolin:bentonite) slurry in water meeting the $30 \mathrm{~Pa} / 30 \mathrm{cP}$ rheological target for Phase II testing (also called Clay, $30 \mathrm{~Pa}$ )

Applied Process and Engineering Laboratory

American Society of Mechanical Engineers

continuously stirred tank reactor

data acquisition system

U.S. Department of Energy

"How Do I," the standards-based management system for PNNL

high-volume low-activity waste

Laboratory Record Book

DOE Office of River Protection

Pretreatment Engineering Platform

programmable logic controller

Pacific Northwest National Laboratory

process particle counter

parts per million

parts per million volume

particle size distribution

Quality Assurance

research and development

refractive index

River Protection Project - Waste Treatment Plant

small treated

variable frequency drive

Hanford Tank Waste Treatment and Immobilization Plant

Waste Treatment Plant Support Project 



\section{Contents}

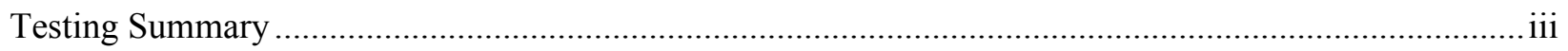

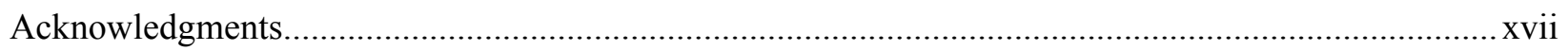

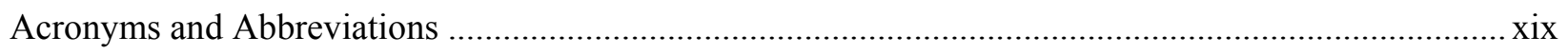

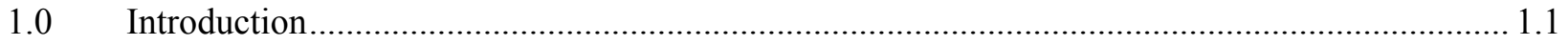

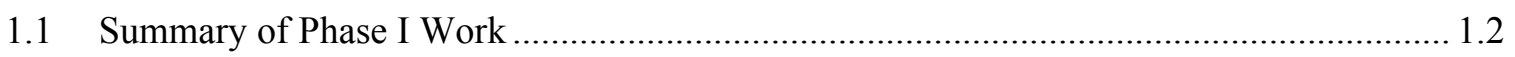

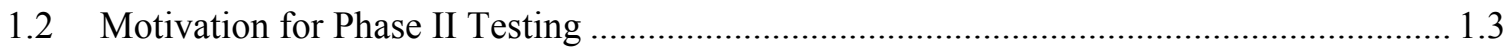

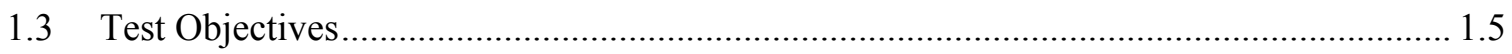

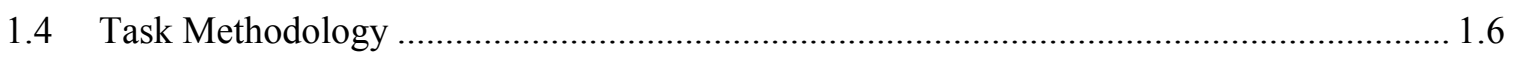

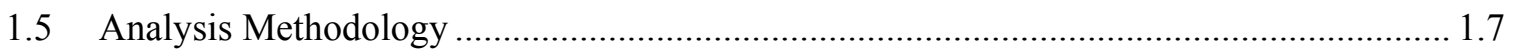

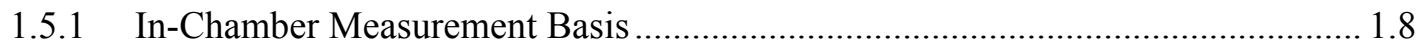

1.5.2 In-Spray Measurement Basis .................................................................. 1.10

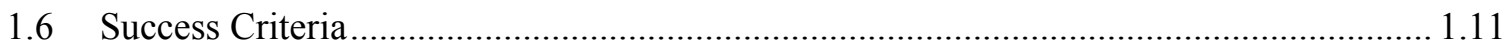

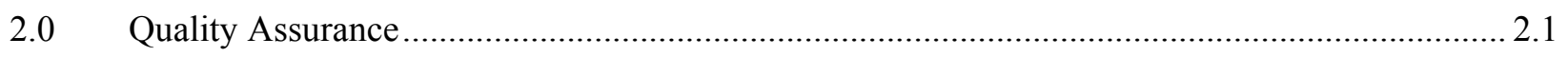

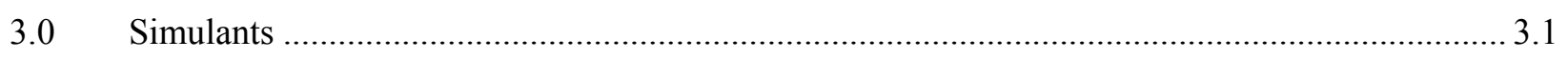

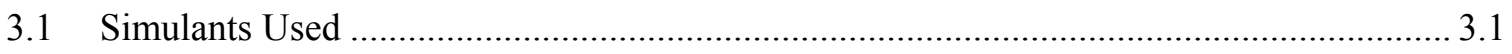

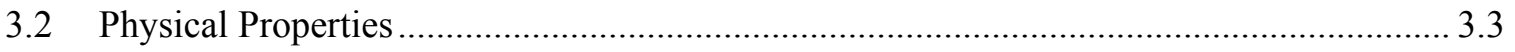

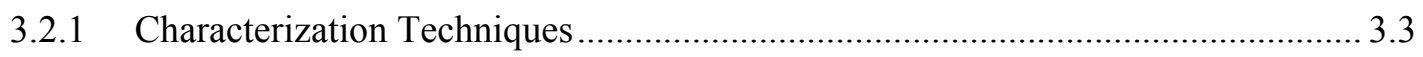

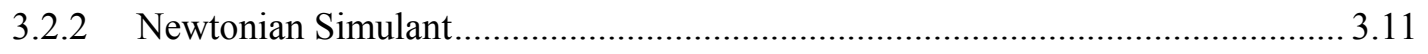

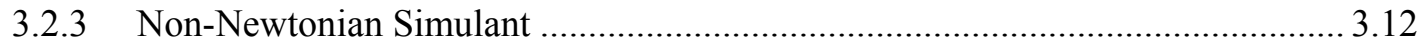

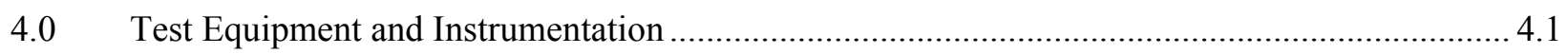

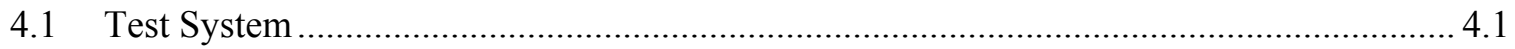

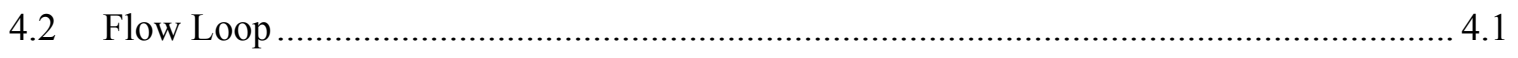

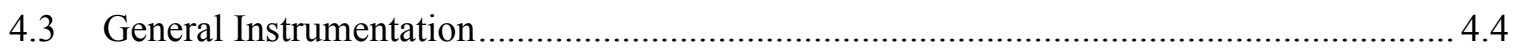

4.4 Phase II Test Chamber Configurations and Spools............................................................ 4.6

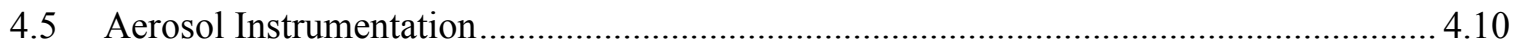

4.5.1 Malvern Insitec-S Operating Principle....................................................... 4.10

4.5.2 Phase II Malvern Insitec-S Frame Configuration and Installation Location........ 4.11

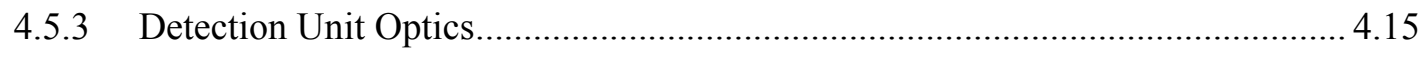

4.5.4 Aerosol Purge and Spray Shrouds................................................................. 4.17

4.5.5 Aerosol Instrumentation Control and Data Acquisition..................................... 4.18

4.5.6 Total Impact of Phase II Malvern Insitec-S Configuration Changes ................... 4.19

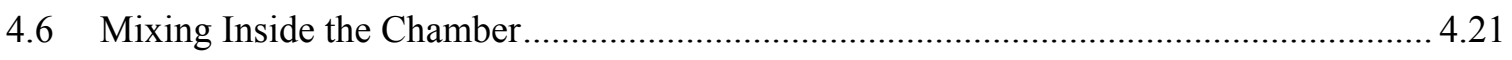

4.7 Summary of Phase II Changes to Test Equipment ....................................................... 4.21

5.0 Test Approach, System Operations, and Data Analysis ....................................................... 5.1

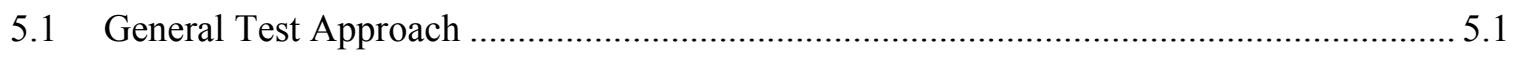

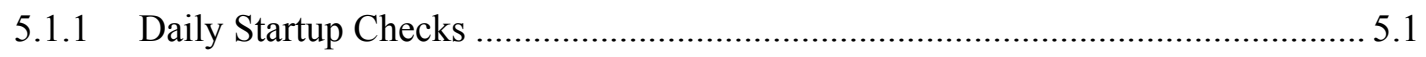




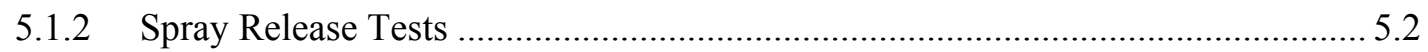

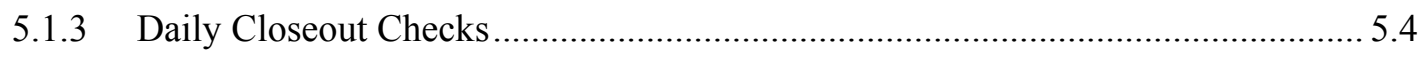

5.1.4 Verification of Malvern Insitec-S Performance …................................................. 5.4

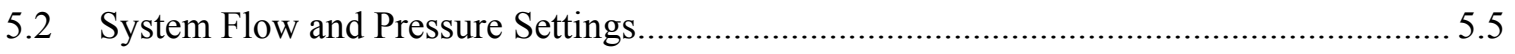

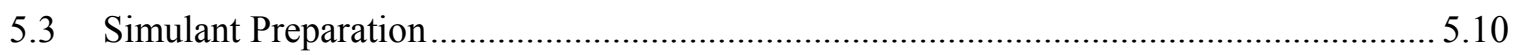

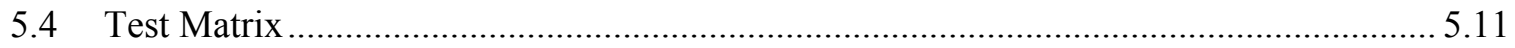

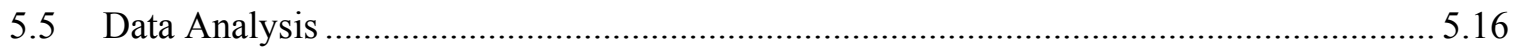

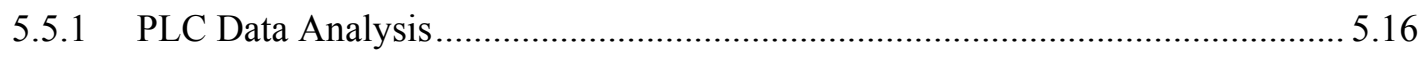

5.5.2 Determination of In-Chamber Generation Rate and Release Fraction................. 5.18

5.5.3 Determination of In-Spray Release Fraction.................................................. 5.20

5.5.4 Averaging of Replicate Results................................................................... 5.21

5.5.5 Determination of WTP Model Release Fraction Curves ....................................5.21

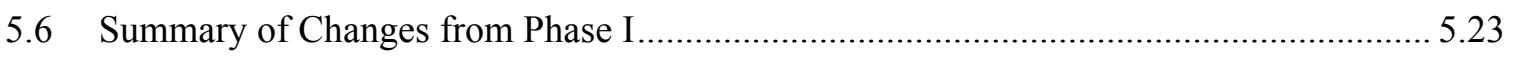

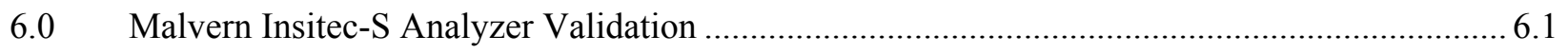

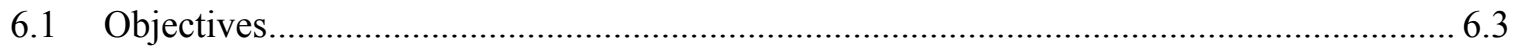

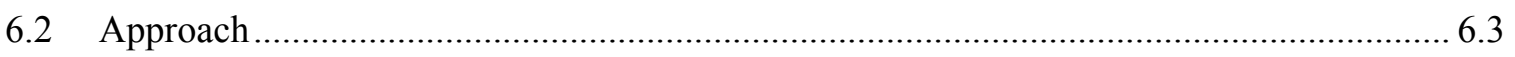

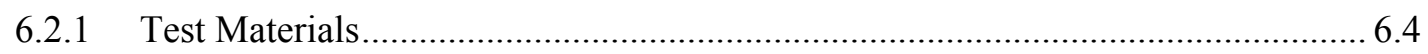

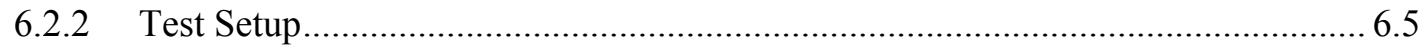

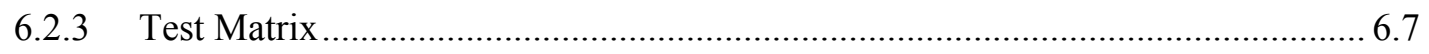

6.2.4 Test Methodology and Data Analysis …............................................................. 6.9

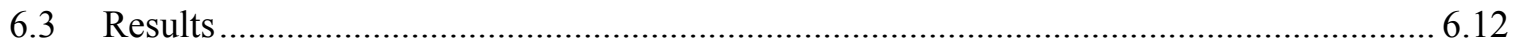

6.3.1 Tests of the Standard Instrument Configuration and Performance ....................... 6.12

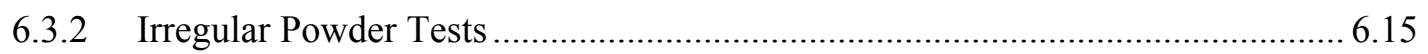

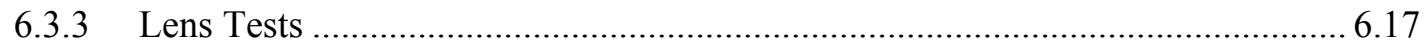

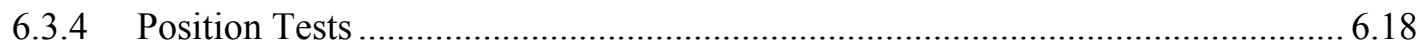

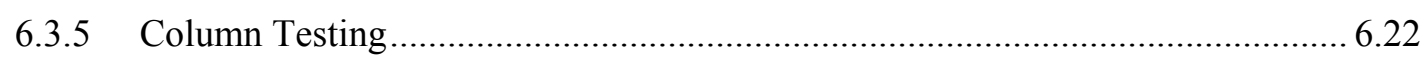

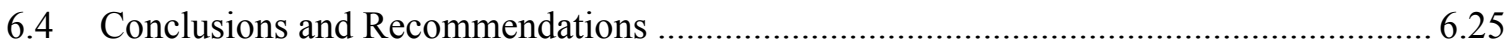

7.0 Impact of Aerosol Instrument Position on Measured Release Fractions ................................... 7.1

7.1 Presentation of Release Fraction Data ............................................................................ 7.3

7.2 In-Chamber Aerosol Instrument Position Dependence ..................................................... 7.3

7.3 In-Spray Aerosol Instrument Position Dependence ...................................................... 7.11

7.4 Comparison of In-Spray and In-Chamber Measurements............................................ 7.17

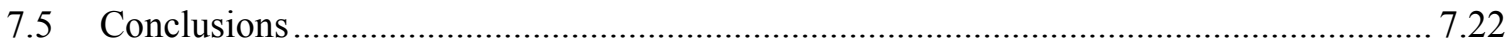

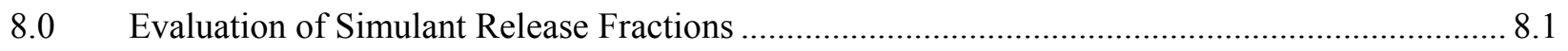

8.1 Evaluation of Phase II Simulant Release Fractions ........................................................ 8.2

8.2 Parametric Variation of Simulant Release Fraction Data ........................................... 8.10

8.2.1 Impact of Solids on Test Pressure Dependence of Release Fraction ................... 8.11

8.2.2 Impact of Solids on Orifice Area Dependence of Release Fraction...................... 8.12

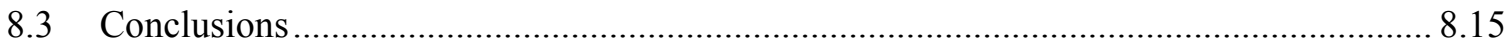




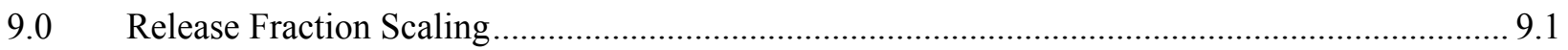

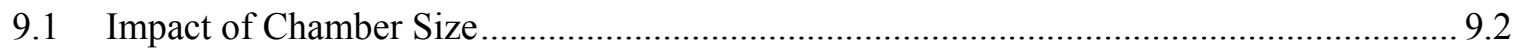

9.1.1 Impact of Chamber Length on the Pressure Functionality of Release

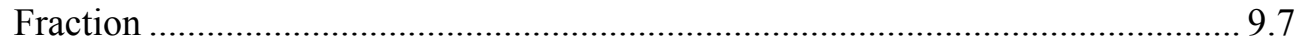

9.1.2 Impact of Chamber Length on the Orifice Area Functionality of Release

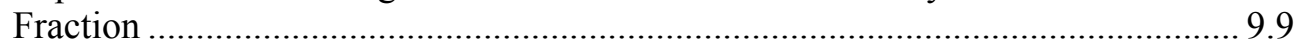

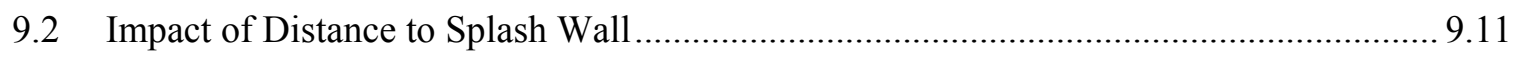

9.2.1 Impact of Distance to Splash Wall on the Pressure Functionality of Release

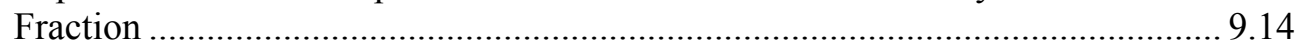

9.2.2 Impact of Distance to Splash Wall on the Orifice Area Functionality of

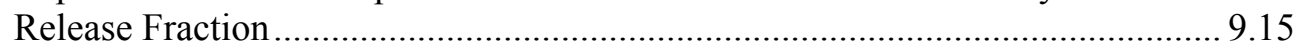

9.3 In-Chamber Sprays of Equivalent Length in Different Chambers ................................... 9.15

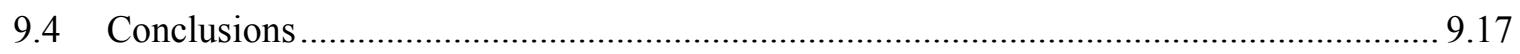

10.0 Generation Rate Estimates for Longer Chambers and Correlation Development for

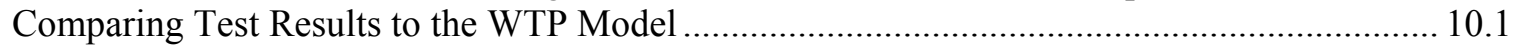

10.1 Extrapolation of Generation Rate Results to Longer Chambers and Sprays..................... 10.2

10.2 Worst-Case Fluids and Spray Lengths Within a Chamber ......................................... 10.15

10.3 Correlation Development for Estimating Reasonably Conservative Aerosol

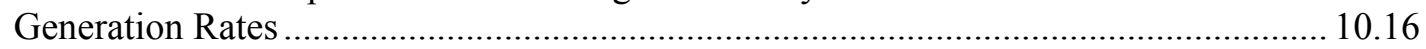

10.4 Comparison of Conservative Generation Rate Correlation to In-Spray Results and Humidity-Corrected (FIO) Generation Rates........................................................... 10.23

10.5 Comparison of Conservative Correlation and In-Spray Results to WTP Model and Selected Test Data.

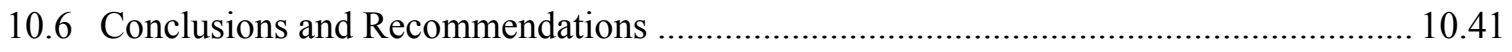

10.6.1 Recommendations for Future Work........................................................... 10.42

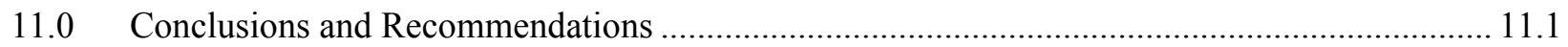

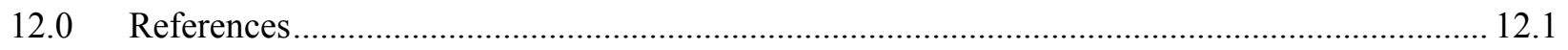

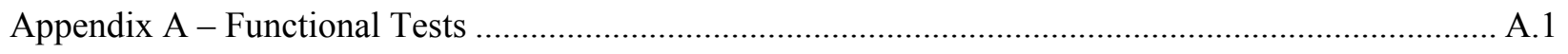

Appendix B - Phase II Large-Scale Test Run Log ....................................................................... 


\section{Figures}

3.1 Rotor and Cup Geometry Used in Rotational Viscometry Testing in Phase II.......................... 3.7

3.2 Summary of Flow Curve Behaviors Typically Observed for Concentrated Slurries, Including Common Time-Independent Behaviors, Static and Dynamic Yield Stress, and

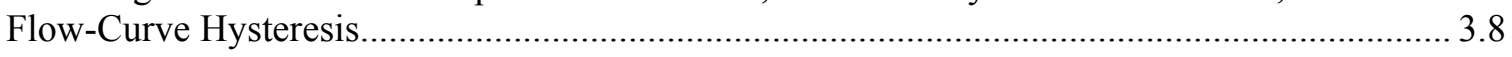

3.3 Flow Curve of the 30 Pa Clay Batch 1Simulant Prior to Testing ............................................ 3.13

3.4 Flow Curve of the 30 Pa Clay Batch 2 Reclaimed Simulant .................................................. 3.14

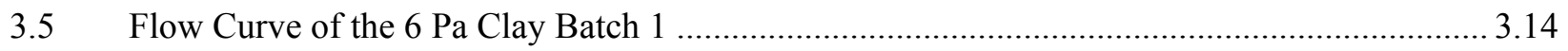

3.6 Flow Curve of the 6 Pa Clay Batch 2 Reclaimed Simulant ................................................... 3.15

3.7 Cumulative Volume Percent Undersize for Unsonicated and Post Sonication 30 Pa Clay

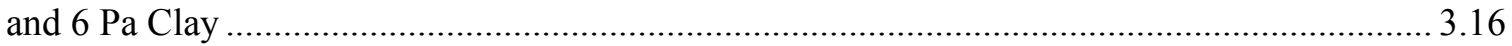

3.8 Differential Particle Size Distribution for Unsonicated and Post Sonication 30 Pa Clay

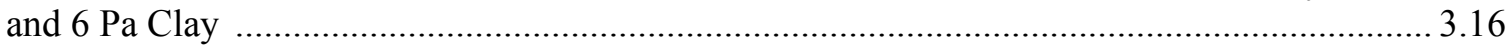

4.1 Schematic of the Large-Scale Test System............................................................................ 4.2

$4.2 \quad$ P\&ID Diagram of the Large-Scale Spray Release Test System .............................................. 4.3

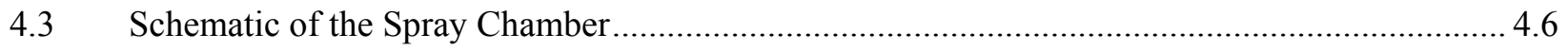

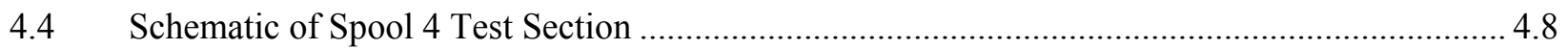

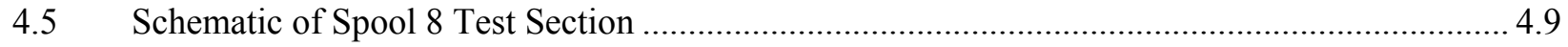

4.6 Model of the Release Assembly Used for Initiating a Spray .................................................... 4.9

4.7 Schematic of the Basic Malvern Insitec-S Aerosol Analyzer Setup and Operating Principle

4.8 Malvern Insitec-S Aerosol Analyzers Configurations ......................................................... 4.12

4.9 Two Schematic Views Showing Key Dimensions that Define the "In-Chamber" Measuring Locations of the Malvern Insitec-S Analyzers....................................................... 4.13

4.10 Comparisons of Release Fraction Measurements Made Using the $100 \mathrm{~mm}$ Lens and the $500 \mathrm{~mm}$ Lens

4.11 Comparison of Release Fraction Measurements Made During Phase I to Those Made During Phase II using the Standard Phase II Malvern Insitec-S Locations as Well as the Standard Malvern Insitec-S Locations Used in Phase I and the $500 \mathrm{~mm}$ Lens ........................ 4.20

4.12 Top View of Malvern Insitec-S Instruments and Fan Arrangement ....................................... 4.21

5.1 Pump Performance Curve for Pumps 1, 2, and 3 .............................................................. 5.6

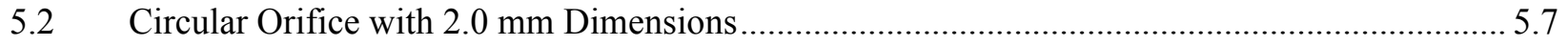

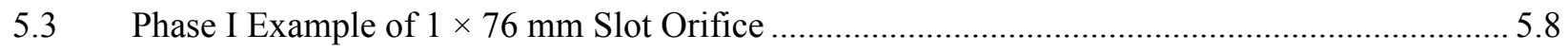

5.4 Example Upstream and Downstream Flow Meter Readings for the $1 \times 76 \mathrm{~mm}$ Slot

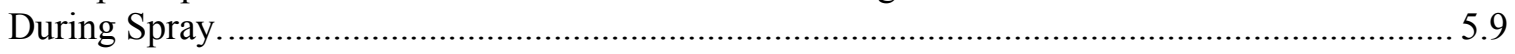

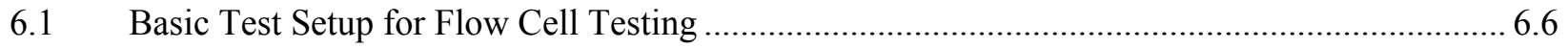

6.2 Diagram for $400 \mathrm{~mm}$ Flow Column Testing used to Simulate the Phase I Malvern Insitec-

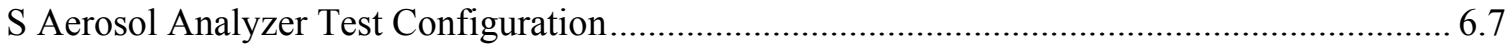

6.3 Diagram for $900 \mathrm{~mm}$ Flow Column Testing used to Simulate the Phase II Malvern Insitec-S Aerosol Analyzer Test Configuration. 
6.4 Expected Concentration Curve Result Obtained from a Series of Step-Wise Mass

Additions and Measurements to the Flow Cell Test System ................................................... 6.10

6.5 Concentration Curve for a $10 \mu \mathrm{m}$ Mono-Disperse Glass Bead Powder ..................................... 6.14

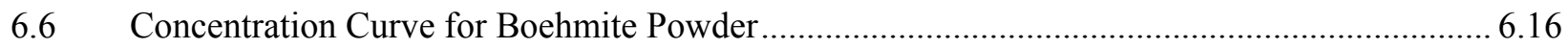

6.7 Impact of Distance from Detector on the Measured PSD for a 3 to 30 Poly-Disperse Glass Bead and an XLSciTech Glass Powder .....

6.8 PSD Data from Figure 6.7 After Spray Geometry Corrections Were Applied......................... 6.21

6.9 PSD Results for the $400 \mathrm{~mm}$ Column Test and the $900 \mathrm{~mm}$ Column Tests ............................. 6.24

6.10 Concentration PSDs for the $400 \mathrm{~mm}$ Column Test and the $900 \mathrm{~mm}$ Column Tests.................. 6.25

7.1 Side Profile of the $39 \mathrm{ft}$ Test Chamber Illustrating Vertical and Downstream Measuring Positions Assessed During In-Chamber Position Tests

7.2 Cumulative Size Distribution of Aerosol Droplets as a Function of Downstream Distance from the Spray Header at $21 \mathrm{in.}$. Above the Orifice, 13 in. Above the Orifice, and 5 in. Below the Orifice.

7.3 Cumulative Concentration Undersize of Aerosol Droplets as a Function of Downstream Distance from the Spray Header at $<10 \mu \mathrm{m},<32 \mu \mathrm{m}$, and $<102 \mu \mathrm{m}$.

7.4 Cumulative Generation Rate Undersize of Aerosol Droplets as a Function of Downstream

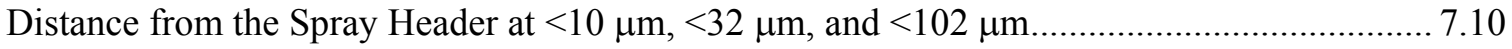

7.5 In-Spray Measurement Locations for Water Testing .......................................................... 7.12

7.6 In-Spray Release Fractions Measured for Water as a Function of Distance from the Back Wall for a $2 \mathrm{~mm}$ Hole, a $1 \times 10 \mathrm{~mm}$ Slot, a $1 \times 20 \mathrm{~mm}$ Slot, and a $1 \times 76 \mathrm{~mm}$ Slot......

7.7 In-Spray Release Fractions Measured for 6 Pa Clay as a Function of Distance from the Back Wall for a $2 \mathrm{~mm}$ Hole, a $1 \times 10 \mathrm{~mm}$ Slot, a $1 \times 20 \mathrm{~mm}$ Slot, and a $1 \times 76 \mathrm{~mm}$ Slot........ 7.15

7.8 In-Spray Release Fractions Measured for $30 \mathrm{~Pa}$ Clay as a Function of Distance from the Back Wall for a $2 \mathrm{~mm}$ Hole, a $1 \times 10 \mathrm{~mm}$ Slot, a $1 \times 20 \mathrm{~mm}$ Slot, and a $1 \times 76 \mathrm{~mm}$ Slot........ 7.16

7.9 Comparison of In-Spray and In-Chamber Release Fractions Measured for Water for a 2 $\mathrm{mm}$ Hole, a $1 \times 10 \mathrm{~mm}$ Slot, a $1 \times 20 \mathrm{~mm}$ Slot, and a $1 \times 76 \mathrm{~mm}$ Slot.....

7.10 Comparison of In-Spray and In-Chamber Release Fractions Measured for 6 Pa Clay for a $2 \mathrm{~mm}$ Hole, a $1 \times 10 \mathrm{~mm}$ Slot, a $1 \times 20 \mathrm{~mm}$ Slot, and a $1 \times 76 \mathrm{~mm}$ Slot

7.11 Comparison of In-Spray and In-Chamber Release Fractions Measured for $30 \mathrm{~Pa}$ Clay for a $2 \mathrm{~mm}$ Hole, a $1 \times 10 \mathrm{~mm}$ Slot, a $1 \times 20 \mathrm{~mm}$ Slot, and a $1 \times 76 \mathrm{~mm}$ Slot $\ldots$

8.1 Release Fraction as a Function of Aerosol Size for Water, $6 \mathrm{~Pa}$ Clay, and $30 \mathrm{~Pa}$ Clay for a $2 \mathrm{~mm}$ Round Hole.

8.2 Release Fraction as a Function of Aerosol Size for Water, $6 \mathrm{~Pa}$ Clay, and $30 \mathrm{~Pa}$ Clay for a $1 \times 10 \mathrm{~mm}$ Slot...

8.3 Release Fraction as a Function of Aerosol Size for Water, $6 \mathrm{~Pa}$ Clay, and $30 \mathrm{~Pa}$ Clay for a $1 \times 20 \mathrm{~mm}$ Slot......

8.4 Release Fraction as a Function of Aerosol Size for Water, 6 Pa Clay, and $30 \mathrm{~Pa}$ Clay for a $1 \times 76 \mathrm{~mm}$ Slot......

8.5 Example Fit of Release Fraction at $10 \mu \mathrm{m}, 32 \mu \mathrm{m}$, and $102 \mu \mathrm{m}$ as a Function of Test Pressure.

8.6 Example Fit of Release Fractions at $10 \mu \mathrm{m}, 32 \mu \mathrm{m}$, and $102 \mu \mathrm{m}$ as a Function of Orifice Area...... 
8.7 Example Fit of Release Fractions at $10 \mu \mathrm{m}, 32 \mu \mathrm{m}$, and $102 \mu \mathrm{m}$ as a Function of Orifice Area Showing Potential Orifice Shape Effects

9.1 In-Chamber Release Fraction as a Function of Aerosol Size for Different Chamber Lengths and for Sprays Generated at 100 psig

9.2 In-Chamber Release Fraction as a Function of Aerosol Size for Different Chamber Lengths and for Sprays Generated at 200 psig

9.3 In-Chamber Release Fraction as a Function of Aerosol Size for Different Chamber Lengths and for Sprays Generated at 380 psig

9.4 Example Fit of In-Chamber Release Fraction at 10, 32, and $102 \mu \mathrm{m}$ as a Function of Test Pressure

9.5 Example Fit of In-Chamber Release Fraction at 10, 32, and $102 \mu \mathrm{m}$ as a Function of Orifice Area .....

9.6 In-Chamber Release Fraction as a Function of Aerosol Size for Different Spray Lengths and for Sprays Generated at $100 \mathrm{psig}$ in the $39 \mathrm{ft}$ Chamber

9.7 In-Chamber Release Fraction as a Function of Aerosol Size for Different Spray Lengths and for Sprays Generated at 200 psig in the $39 \mathrm{ft}$ Chamber

9.8 In-Chamber Release Fraction as a Function of Aerosol Size for Different Spray Lengths and for Sprays Generated at 380 psig in the $39 \mathrm{ft}$ Chamber

9.9 Comparison of In-Chamber Release Fraction as a Function of Aerosol Size for Equivalent $30 \mathrm{ft}$ Sprays in the 30 and $39 \mathrm{ft}$ Chambers and for Sprays Generated at 380 psig.

9.10 Comparison of In-Chamber Release Fraction as a Function of Aerosol Size for Equivalent $20 \mathrm{ft}$ Sprays in the 20 and $39 \mathrm{ft}$ Chambers and for Sprays Generated at 380 psig.

10.1 Generation Rate Estimates for Extrapolation to a $100 \mathrm{ft}$ Chamber and Spray Length for Water Sprays from a $2 \mathrm{~mm}$ Hole at 380 psig in Chambers of Different Length 10.3

10.2 Generation Rate Estimates for Extrapolation to a $100 \mathrm{ft}$ Chamber and Spray Length for Water Sprays from a $2 \mathrm{~mm}$ Hole at 200 psig in Chambers of Different Length

10.3 Generation Rate Estimates for Extrapolation to a $100 \mathrm{ft}$ Chamber and Spray Length for Water Sprays from a $2 \mathrm{~mm}$ Hole at 100 psig in Chambers of Different Length

10.4 Generation Rate Estimates for Extrapolation to a $100 \mathrm{ft}$ Chamber and Spray Length for Water Sprays from a $1 \times 10 \mathrm{~mm}$ Slot at 380 psig in Chambers of Different Length ...... 10.6

10.5 Generation Rate Estimates for Extrapolation to a $100 \mathrm{ft}$ Chamber and Spray Length for Water Sprays from a $1 \times 10 \mathrm{~mm}$ Slot at 200 psig in Chambers of Different Length

10.6 Generation Rate Estimates for Extrapolation to a $100 \mathrm{ft}$ Chamber and Spray Length for Water Sprays from a $1 \times 10 \mathrm{~mm}$ Slot at $100 \mathrm{psig}$ in Chambers of Different Length ......

10.7 Generation Rate Estimates for Extrapolation to a $100 \mathrm{ft}$ Chamber and Spray Length for Water Sprays from a $1 \times 20 \mathrm{~mm}$ Slot at $380 \mathrm{psig}$ in Chambers of Different Length

10.8 Generation Rate Estimates for Extrapolation to a $100 \mathrm{ft}$ Chamber and Spray Length for Water Sprays from a $1 \times 20 \mathrm{~mm}$ Slot at 200 psig in Chambers of Different Length

10.9 Generation Rate Estimates for Extrapolation to a $100 \mathrm{ft}$ Chamber and Spray Length for Water Sprays from a $1 \times 20 \mathrm{~mm}$ Slot at 100 psig in Chambers of Different Length

10.10 Generation Rate Estimates for Extrapolation to a $100 \mathrm{ft}$ Chamber and Spray Length for Water Sprays from a $1 \times 76 \mathrm{~mm}$ Slot at $380 \mathrm{psig}$ in Chambers of Different Length 
10.11 Generation Rate Estimates for Extrapolation to a $100 \mathrm{ft}$ Chamber and Spray Length for Water Sprays from a $1 \times 76 \mathrm{~mm}$ Slot at 200 psig in Chambers of Different Length

10.12 Generation Rate Estimates for Extrapolation to a $100 \mathrm{ft}$ Chamber and Spray Length for Water Sprays from a $1 \times 76 \mathrm{~mm}$ Slot at $100 \mathrm{psig}$ in Chambers of Different Length ....

10.13 Conceptual Example of Correlation that Represents the Highest Generation Rate of All Chamber Sizes and $100 \mathrm{ft}$ Extrapolation for a $2 \mathrm{~mm}$ Hole at $380 \mathrm{psig}$ as a Selected Example

10.14 Comparison of Measured Generation Rates and the Generation Rate Correlation Given by Equation for the Individual Test Results Used for Developing the Correlation

10.15 Individual Test Examples Comparing the Measured Generation Rates with the Generation Rate Correlation Given by Equation (10.2)

10.16 Comparison of Measured Upper Confidence Interval Values for Individual Tests with the Correlation for the Upper Confidence Interval Generation Rates ....

10.17 Cross Plot Comparison of the Correlations for Generation Rate and Upper Confidence Interval for Generation Rate for the Range of Data Used in Developing the Correlations

10.18 Comparison of the Measured Generation Rate and Upper Confidence Interval Values for the Data Used in Developing the Correlations.

10.19 Comparison of Measured Upper Confidence Interval of Generation Rate Values for Individual Tests and the $100 \mathrm{ft}$ Extrapolation with the Conservative Correlation for Generation Rate

10.20 Comparison of Measured Generation Rate for Individual Tests and the $100 \mathrm{ft}$

Extrapolations with the Conservative Correlation

10.21 Comparison of Conservative Correlation with In-Spray Results for Water Sprays at 380 psig for the Four Orifices Tested

10.22 Comparison of Conservative Correlation with In-Spray Results for Water Sprays at 200 psig for the Four Orifices Tested

10.23 Comparison of Conservative Correlation with In-Spray Results for Water Sprays at 100 psig for the Four Orifices Tested

10.24 Comparison of Upper Confidence Interval Generation Rate Values, Corrected for Humidity Based on FIO Data, Compared to the Conservative Correlation. 10.26

10.25 Comparison of Measured Generation Rate Data, Corrected for Humidity Based on FIO Data, Compared to the Conservative Correlation

10.26 Generation Rate Results for a Water Spray from a $2 \mathrm{~mm}$ Hole at 380 psig Showing a Comparison of the Conservative Correlation, In-Spray Data, and WTP Model

10.27 Generation Rate Results for a Water Spray from a $1 \times 10 \mathrm{~mm}$ Slot at 380 psig Showing a Comparison of the Conservative Correlation, In-Spray Data, and WTP Model

10.28 Generation Rate Results for a Water Spray from a $1 \times 20 \mathrm{~mm}$ Slot at 380 psig Showing a Comparison of the Conservative Correlation, In-Spray Data, and WTP Model

10.29 Generation Rate Results for a Water Spray from a $1 \times 76 \mathrm{~mm}$ Slot at 380 psig Showing a Comparison of the Conservative Correlation, In-Spray Data, and WTP Model

10.30 Generation Rate Results for a Water Spray from a $2 \mathrm{~mm}$ Hole at 200 psig Showing a Comparison of the Conservative Correlation, In-Spray Data, and WTP Model.

10.31 Generation Rate Results for a Water Spray from a $1 \times 10 \mathrm{~mm}$ Slot at 200 psig Showing a Comparison of the Conservative Correlation, In-Spray Data, and WTP Model 
10.32 Generation Rate Results for a Water Spray from a $1 \times 20 \mathrm{~mm}$ Slot at 200 psig Showing a Comparison of the Conservative Correlation, In-Spray Data, and WTP Model.

10.33 Generation Rate Results for a Water Spray from a $1 \times 76 \mathrm{~mm}$ Slot at 200 psig Showing a Comparison of the Conservative Correlation, In-Spray Data, and WTP Model.

10.34 Generation Rate Results for a Water Spray from a $2 \mathrm{~mm}$ Hole at 100 psig Showing a Comparison of the Conservative Correlation, In-Spray Data, and WTP Model......

10.35 Generation Rate Results for a Water Spray from a $1 \times 10 \mathrm{~mm}$ Slot at 100 psig Showing a Comparison of the Conservative Correlation, In-Spray Data, and WTP Model

10.36 Generation Rate Results for a Water Spray from a $1 \times 20 \mathrm{~mm}$ Slot at 100 psig Showing a Comparison of the Conservative Correlation, In-Spray Data, and WTP Model

10.37 Generation Rate Results for a Water Spray from a $1 \times 76 \mathrm{~mm}$ Slot at 100 psig Showing a Comparison of the Conservative Correlation, In-Spray Data, and WTP Model.

10.38 Generation Rate Results for a Water Spray from a $120 \mathrm{~mm}^{2}$ Orifice at 380 psig Showing a Comparison of the Conservative Correlation and WTP Model

10.39 Generation Rate Results for a Water Spray from a $209 \mathrm{~mm}^{2}$ Orifice at 184 psig Showing a Comparison of the Conservative Correlation and WTP Model

10.40 Generation Rate Results for a Water Spray from a $290 \mathrm{~mm}^{2}$ Orifice at 540 psig Showing a Comparison of the Conservative Correlation and WTP Model

10.41 Example of the Aerosol Generation Behavior Predicted by the Conservative Correlation and Four Other Models.

10.42 Comparison of Measured Generation Rate of Individual Test Performed in the $20 \mathrm{ft}$ Chamber with Water and Clay Simulants with the Conservative Correlation Derived from In-Chamber Water Testing....

\section{Tables}

S.1 Summary of Phase II Large-Scale Test Objectives and Results ................................................. v

S.2 Success Criteria for Large-Scale Aerosol Tests....................................................................... vii

S.3 Target Simulants Tested in Phase II Large-Scale Test Stand and the WTP Process

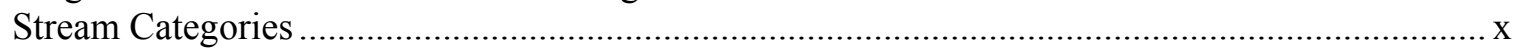

3.1. WTP Process Stream Categories and Representative Fluid Properties ..................................... 3.1

3.2. Target Simulants and the WTP Process Stream Categories...................................................... 3.2

3.3. Summary of Malvern Mastersizer 2000 Instrument Information .............................................. 3.5

3.4. Refractive Indices for Simulants Used in Large-Scale Spray Leak Testing ............................ 3.11

3.5. Newtonian Simulant Properties for Large-Scale Aerosol Tests .............................................. 3.12

3.6. Non Newtonian Simulant Properties for Aerosol Tests ........................................................... 3.13

3.7. Summary of Selected Percentile Values for Measurements Taken at 1 min Recirculation Time, for Unsonicated, $30 \mathrm{~Pa}$ Clay and $6 \mathrm{~Pa}$ Clay Simulants................................................ 3.15

4.1. Instrumentation Used for Process Parameter Measurements.................................................... 4.5

4.2. Spray Chamber Configurations and Chamber Volumes .......................................................... 4.7

4.3. Orifice ID and Geometries Used for Phase II Large-Scale Testing ............................................ 4.8 
4.4. Values of Key Dimensions Listed in Figure 4.9 for In-Chamber Testing ................................ 4.13

4.5. Values of Key Dimensions Listed in Figure 4.9 for In-Spray Testing .................................... 4.14

4.6. Malvern Insitec-S Instrument Configuration and Software Parameters Used in Typical Phase II Measurements

4.7. Summary of Instrument and Test Chamber Changes Between Phase I and Phase II Testing ......

5.1. Group 6 Tests to Establish Effect of Spray Orifice Distance from Splash Wall ..................... 5.13

5.2. Group 7 Tests to Establish Effect of Chamber Size.................................................................. 5.14

5.3. Group 8 Tests to Evaluate Effect of the WTP Non-Newtonian Rheology Limits on Aerosol Generation Rates

5.4. Eight Primary Instruments Used in Phase II Large-Scale Data Analysis ................................ 5.17

5.5. Summary of Key Changes Made in the General Test Approach and Data Analysis Methodologies for Phase II Spray Leak Testing.

6.1. Selected Properties of Powder Systems Used in Malvern Insitec-S Validation Testing .............. 6.4

6.2. Flow Cell Test Matrix for Malvern Insitec-S Validation........................................................... 6.8

6.3. Column Test Matrix for Malvern Insitec-S Validation.............................................................. 6.8

6.4. Summary of Baseline Concentration Response for Individual Concentration Points ............... 6.13

6.5. Results of Linear Regression for Tests Involving Stepwise Addition of Mass ........................ 6.14

6.6. Evaluation of Size Distribution Accuracy for Spherical Systems .......................................... 6.15

6.7. Summary of Irregular Powder Concentration Response for Individual Concentration

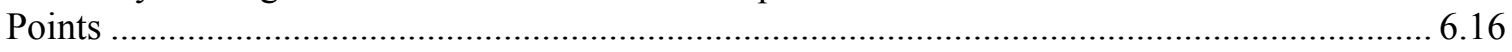

6.8. Evaluation of Size Distribution Accuracy for Irregular Particles .......................................... 6.17

6.9. Summary of Lens Test Concentration Results and Comparisons ............................................ 6.18

6.10. Evaluation of Size Distribution Accuracy for Spherical Systems ............................................ 6.18

6.11. Summary of Uncorrected Position Test Concentration Measurements .................................... 6.20

6.12. Summary of Position Test Concentration Measurements after Correction for Laser Attenuation.

6.13. Summary of Irregular Powder Concentration Response for Individual Concentration Points

6.14. Comparison of Column Test Size Distributions to Baseline Size Distributions........................ 6.24

7.1. Polynomial Coefficients for Trajectories Shown in Figure 7.5 and Fit to Equation (7.1) ......... 7.13

8.1. Best-Fit Pressure Scaling Factors for Simulant Release Fractions in $20 \mathrm{ft}$ Chambers .............. 8.12

8.2. Best-Fit Orifice Area Scaling Factors for Simulant Release Fractions in $20 \mathrm{ft}$ Chambers........ 8.14

9.1. Best-Fit Pressure Scaling Factors for Water In-Chamber Release Fractions for Different Chamber Sizes and Orifice Combinations.

9.2. Best-Fit Orifice Area Scaling Factors for Water In-Chamber Release Fractions for Different Chamber Size and Test Pressure Combinations.

9.3. Best-Fit Pressure Scaling Factors for Water Release Fractions for Different Spray Length and Orifice Size Combinations.

9.4. Best-Fit Orifice Area Scaling Factors for Water Release Fractions for Different Spray Length and Test Pressure Combinations. 



\subsection{Introduction}

One of the events postulated in the hazard analyses for the Waste Treatment and Immobilization Plant (WTP) and other U.S. Department of Energy (DOE) nuclear facilities is a breach in process piping that produces aerosols with droplet sizes in the respirable range. The current approach for predicting the size and concentration of aerosols produced in a spray leak involves extrapolating from correlations reported in the literature. These correlations are based on results obtained from small engineered spray nozzles using pure liquids that behave as Newtonian fluids. The narrow ranges of physical properties on which the correlations are based do not cover the wide range of slurries and viscous materials that will be processed in the WTP and in other processing facilities across the DOE complex.

Two key technical areas were identified for which testing results were needed to improve the technical basis by reducing uncertainty due to extrapolating existing literature results. The first technical need was to quantify the role of slurry particles in small breaches where the slurry particles may plug and result in substantially reduced, or even negligible, respirable fraction formed by high-pressure sprays. The second technical need was to determine the aerosol droplet size distribution and volume from prototypic breaches and fluids, specifically including sprays from larger breaches with slurries where data from the literature are scarce.

To address these technical areas, small- and large-scale test stands were constructed and operated with simulants to determine aerosol release fractions and generation rates from a range of breach sizes and geometries. The properties of the simulants tested represented the range of properties expected in the WTP process streams. An extensive test campaign was conducted to address the two key technical areas outlined in the preceding paragraph. The objective of this first phase of testing (hereafter called Phase I testing) was to improve the technical basis for predicting the size and concentration of aerosols produced from a spray leak by reducing the uncertainty introduced by extrapolating existing literature results. The results of Phase I testing (which are documented in Mahoney et al. (2012), Mahoney et al. (2013), and Schonewill et al. (2012) and which are briefly summarized in Section 1.1) led to a conclusion that a second phase of testing was needed, with the primary goal of improving confidence of the release fraction results when extrapolating (i.e., scaling) those results to postulated spray leak scenarios in the WTP. In addition to this primary goal for testing in the large-scale test stand, the Phase II spray leak testing was conducted to address areas of uncertainty remaining from Phase I testing with respect to aerosol behavior (namely aerosol release fraction and generation rates) for:

- simulant(s) near the WTP rheological boundaries of (6 Pa/6 mPa·s and $30 \mathrm{~Pa} / 30 \mathrm{mPa} \cdot \mathrm{s})$

- simulant(s) with solids loadings greater than $20 \mathrm{wt} \%$

- simulant(s) with a small solids fraction of particles that have a relatively high density

- lower spray pressures (i.e., specifically 100 and 200 psig in the small-scale tests)

- in-spray measurements.

The following sections provide the basis for executing the Phase I follow-on testing. First, a summary of Phase I work is provided. This is followed by a discussion of the motivations for follow-on (Phase II) spray leak testing. Finally, a list of test objectives, the task methodology, the technical basis, and success criteria are given for this second phase of spray leak testing. 


\subsection{Summary of Phase I Work}

Initial testing to obtain aerosol release fraction and generation rates was completed during Phase I in small- and large-scale test stands and presented in the large-scale aerosol report (Schonewill et al. 2012) and the small-scale plugging and aerosol test reports (Mahoney et al. 2012 and Mahoney et al. 2013). These tests were conducted with simulants representing the expected WTP process stream properties over a range of orifice sizes, geometries, and line pressures. The objective of Phase I work was to improve the technical basis for predicting the size and concentration of aerosols produced from a spray leak by reducing uncertainty introduced by extrapolating existing literature results As such, the functionality of spray with respect to key operating parameters, such as spray pressure, orifice size, and fluid properties, was evaluated and compared to a predictive aerosol generation model employed in WTP design evaluations (hereafter referred to simply as "the WTP model"). From these evaluations, the behavior of sprays generated from both small and large breaches of fluids representative of WTP wastes was evaluated both on an absolute basis and relative to model predictions. The discussion that follows summarizes the key findings of these evaluations as determined during Phase I spray leak testing.

Overall, the Phase I results indicated a straightforward functionality of the cumulative aerosol release fraction and generation rate on pressure and orifice area. Viscosity and the weight fraction of solids had a negligible effect on release fraction and generation rate. The configuration and geometry of the spray was important, as evidenced by the splash wall distance and in-spray aerosol tests. In particular, Phase I tests that evaluated the impact of distance between the transfer line breach (which is the point of origin for the spray) and the splash wall (which is the downstream wall on which jets with sufficient force and travel distance impinge) demonstrated that longer distance sprays yield a higher release fraction (unless the spray was very close to the splash wall [i.e., 1 in.]). In-spray data provided a conservative measurement of the release fraction and generation rates for selected breaches relative to in-chamber data. Phase I in-spray data was subject to uncertainty because the in-spray release fraction depended on the location at which the in-spray measurement was taken and because it was a strong function of the Malvern Insitec-S analyzer setting used to interpret the in-spray light scattering data. In-spray data obtained in the small-scale test stand indicated the majority of the data fell below or was equivalent to the release fraction estimates generated using the WTP model. In-spray data obtained in the large-scale test stand indicated the release fractions could be greater than the release fraction estimates from the WTP model by as much as a factor of 50. The largest orifice evaluated in Phase I in-spray testing was a $2 \mathrm{~mm}$ hole, and as such, Phase I in-spray measurements did not include testing of larger orifices whose in-chamber release fraction results challenge the WTP model. For this reason, additional in-spray test results with larger orifices were needed. The Phase I large-scale test results showed clear trends with increasing orifice area for both the release fraction and generation rate. The small-scale results did not show much of a trend with increasing orifice area, but they did generally agree with the large-scale results within the range of orifice areas that were tested in Phase I. Comparison of small- and large-scale test results confirmed the overall trend of increasing generation rate with increasing orifice area for both circular holes and slots. The results also showed that the Phase I large-scale test results, which span a wider range of orifice areas than the small-scale tests, provided the best data for extrapolating to larger breaches.

In general, Phase I testing found that the WTP model has the correct qualitative behavior, but either does not have a quantitatively correct dependence on a quantity (pressure, orifice areas) or ignores a quantity altogether (distance from a splash wall). Likewise, Phase I data indicate that the model may not be conservative for all sprays. Assuming the WTP model uses the fluid properties of water for all the 
sprays, the combined extrapolations indicated that the cumulative release fractions from long-distance sprays from the largest postulated orifice of a Bingham plastic fluid at the upper rheological boundary will exceed the WTP model by factor of two to four for droplets that are $<10 \mu \mathrm{m}$. For larger droplets, the release fraction will exceed the WTP model, but the magnitude is more uncertain. The most significant uncertainty in this estimated release fraction is due to the role of chamber size and an assumed $100 \mathrm{ft}$ spray distance. An upper-bound release fraction from an in-spray particle size distribution (PSD) is slightly more than the extrapolated large-scale chamber result for droplets $<10 \mu \mathrm{m}$. However, the difference becomes progressively larger with increasing droplet size, and the upper bound is as much as a factor of 50 more than that for the WTP model for droplets $<100 \mu \mathrm{m}$. The uncertainty of these Phase I estimates was addressed with additional Phase II testing of non-Newtonian slurries sprayed through large orifices in the large-scale chamber.

Phase I testing indicated that a number of factors affected the overall uncertainty in the measured aerosol generation rates and release fractions. While a systematic quantitative evaluation of all the combined factors was not performed, a qualitative estimate of release fraction and generation rate uncertainty was determined. For Phase I large-scale chamber testing, overall uncertainty in the reported release fractions and generation rates for the Phase I large-scale test chamber was estimated to be approximately a factor of two to three (the reported Phase I results were not adjusted to account for this uncertainty). The comparison of release fraction results for the small and large test chambers (in Phase I) showed the difference in release fraction varying from none to as much as fivefold. This is important for understanding the uncertainty in extrapolations to larger chambers and spray distances. However, not enough data was available in Phase I tests, with only two chamber sizes, to estimate the uncertainty in extrapolations to larger chambers and served as a primary motivation for Phase II testing with larger chamber lengths (i.e., 30 and $39 \mathrm{ft}$ ).

\subsection{Motivation for Phase II Testing}

Phase II large-scale testing was motivated primarily by the need to improve understanding of the impact of test enclosure size (scaling) and unobstructed spray length on release fraction and to better understand the aerosolization behavior of non-Newtonian simulants from orifices larger than those tested in Phase I. In addition, Phase II testing sought to reduce, or at least improve understanding of, uncertainty with respect to release fraction and generation rate measurements and bias in release fraction measurements from test methodology and instrument sources. As stated in the preceding section, Phase I test results indicated that the largest postulated breaches in the largest test chamber would challenge release fraction and aerosol generation rate estimates provided by the WTP model. However, because Phase I testing involved only two test enclosure sizes, there was insufficient test scale information to reliably extrapolate release fractions to different chamber sizes and spray lengths. To address this weakness, Phase II large-scale testing evaluated aerosol generation rate and release fraction in several chamber sizes. Chamber size was varied by changing the length of the chamber while maintaining the same cross-section of chamber area used in Phase I testing. Chamber lengths evaluated were 10, 20, 30, and $39 \mathrm{ft}$. Furthermore, spool extension pieces were used to vary the length of the spray (at a fixed chamber length of $39 \mathrm{ft}$ ) in order to isolate the effects of changing unobstructed spray length from changing chamber volume.

Phase I testing was focused on evaluating the influence of a broad range of parameters on aerosolization of simulants representing WTP process streams. The parameters evaluated in Phase I 
included, but were not limited to, orifice area and morphology, simulant viscosity, density, surface tension, spray pressure, and test scale. Because of the need to evaluate a wide range of parameters, less emphasis was placed on replicate testing and assessments of uncertainty. For Phase II testing, an increased emphasis was placed on obtaining replicates at all test conditions to better estimate uncertainty and improve statistical confidence in trends with pressure, orifice area, chamber size, and spray length. To this end, all configurations were tested and the results screened to provide two or more acceptable measurements. Replicate testing allows application of statistical analysis to determine the significance of release fraction and generation rate scaling with key test parameters such as orifice area and spray pressure.

Phase I testing indicated that non-Newtonian slurry, and in particular, chemical simulant slurries that closely mimic the actual chemical make-up of WTP process streams, yield the greatest fractional release and generation of aerosols of consequence to safety analysis. Consequence is expected to be increased when non-Newtonian slurries are released through the largest postulated breaches. However, no non-Newtonian simulants were tested in the large-scale test enclosure during Phase I to verify this assertion. For this reason, Phase II large-scale operations emphasized testing of non-Newtonian simulants. Because the large-scale test chamber did not have the appropriate permits to handle hazardous simulants, the chemical simulant slurry was not tested. Instead, two non-Newtonian (and non-hazardous) mixed clay slurries were prepared with rheologies that bound the range expected for waste treatment operations in the WTP.

Phase I large-scale testing focused primarily on in-chamber measurement of release fraction, which evaluates aerosol generation by measuring the change in aerosol concentration with time at fixed locations above the spray. Only limited attention was given to in-spray measurements, which provide a direct and conservative measurement of release fraction, in Phase I. As stated in the preceding section, in-spray measurements made in Phase I were subject to uncertainty that derived from a locational dependence on the in-spray release fraction with distance from orifice and variation of the in-spray release fraction with analyzer settings that correct the light scattering pattern based on assumed spray geometry. Further uncertainty derived from the selection of detection unit optics, which had a working range of 0.5 to $200 \mu \mathrm{m}$ that, while capable of detecting aerosols in the size range of interest (10 to $100 \mu \mathrm{m}$ ) to WTP safety analyses, was not capable of detecting aerosol droplet sizes encountered in in-spray measurements. Finally, Phase I in-spray testing was limited to a very small set of orifice size and test pressure. Phase II provided an opportunity to address these uncertainties. First, a new detection lens was purchased. This new lens allowed measurement of aerosols between 2.5 and $2500 \mu \mathrm{m}$ and was capable of detecting aerosols in the size range anticipated for in-spray measurements. Furthermore, use of the $500 \mathrm{~mm}$ lens was observed to virtually eliminate the dependence of the in-spray release fraction on analyzer settings used to interpret the diffraction pattern, leading to improved confidence in the measurement. Next, in-spray testing was conducted for all orifice sizes, test pressures, and simulant combinations evaluated in the in-chamber configuration for Phase II testing. This allowed for a comprehensive side-by-side assessment of in-spray and in-chamber test configurations, which in turn, facilitated improved evaluation of the uncertainty in each of the two methods. Finally, additional evaluation of the dependence of in-spray release fractions on position was performed to reduce uncertainty with respect to the behavior of in-spray measurement as a function of distance from the orifice. The expanded test scope allowed evaluation of position dependence for all test conditions and simulants, providing a large data set against which the reasonableness of in-spray position dependence could be evaluated. 
The issue of aerosol analyzer detection unit optics also impacted in-chamber testing results. All Phase I testing was accomplished with a $100 \mathrm{~mm}$ lens with a working range of 0.5 to $200 \mu \mathrm{m}$. While Phase II testing employed a $500 \mathrm{~mm}$ lens to facilitate improved in-spray testing, it also provided an opportunity to assess the presence of larger aerosols in the in-chamber test locations. Phase I in-chamber test results indicated few aerosols larger than $200 \mu \mathrm{m}$. There was uncertainty as to whether this resulted from a physical mechanism (such as settling) that prevented observation of greater than $200 \mu \mathrm{m}$ aerosols in the in-chamber location, whether there were few greater than $200 \mu \mathrm{m}$ aerosols produced by spray, or whether the lens selection prevented observation of larger aerosols. Use of the $500 \mathrm{~mm}$ lens in in-chamber testing resolved this issue.

An unrelated but important uncertainty outstanding from the Phase I aerosol testing was the performance of the Malvern Insitec-S instrument. Early in the Phase I testing, the Malvern Insitec-S was identified as the primary aerosol instrument and all subsequent data analysis was derived from Malvern Insitec-S measurements. Because the experimental method depends on an accurate and well-resolved measure of droplet concentration and size distribution with time, understanding the performance of the Malvern Insitec-S is an issue that applies to both the small- and large-scale testing. Reticle studies presented in the Phase I reports suggested that the cumulative particle size distribution (by volume) measured by the Malvern Insitec-S instrument becomes less precise below volume fractions of approximately $5 \times 10^{-3}$. Furthermore, the error in the concentration measurement was estimated to be \pm 40 percent by performing an evaluation of the laser diffraction technique (see Section 5.3.2 in Schonewill et al. 2012). The measurement of precision in Phase I was based on a significant number of data sets, but the assessment of accuracy was anecdotal: it was based on a single data set and was not conducted using the Malvern Insitec-S, but a related instrument using the same analysis principle. A more rigorous study to validate the Malvern Insitec-S performance and assess its accuracy was conducted in Phase II to address this uncertainty.

\subsection{Test Objectives}

Phase II work presented in this report addresses four test objectives (Test Objectives 10, 12, 13, and 14 of TP-WTPSP-031, Rev. 1.0 ${ }^{1}$ ). These four objectives from TP-WTPSP-031, Rev 1.0 are restated below:

- Test Objective 10: Assess the capability of the Malvern Insitec-S in-process particle size analyzer, which is the instrument used in the aerosol testing, to measure accurately the concentration and size distribution of samples. This will be accomplished by measuring carefully controlled dilute aqueous slurries of known concentration and PSD and comparing the Malvern [Insitec-S] result to the known values. Testing will include mono- and poly-disperse suspensions and will evaluate all four Malvern [Insitec-S] configurations of 1) Phase I small-scale spacer bars and old lens, 2) Phase II small-scale spacer bars and new lens, 3) Phase I large-scale spacer bars and old lens, and 4) Phase II large-scale spacer bars and new lens (old lens $100 \mathrm{~mm}$, new lens $500 \mathrm{~mm}$ ).

- Test Objective 12: Determine the size distribution of aerosol droplets and the total droplet volume concentration as a fraction of the total spray volume for water sprays for a range of different chamber sizes and for sprays traveling different distances within the largest chamber. Also determine the droplet size distribution directly in the spray (in-spray measurement) for the largest sprays that can be

\footnotetext{
${ }^{1}$ Gauglitz PA. 2012. Test Plan for Spray Leak Quantification to Support WTP Spray Release Methodology.
} TP-WTPSP-031, Rev. 1, Pacific Northwest National Laboratory, Richland, Washington. 
measured. Testing will be conducted using at least one circular hole and multiple rectangular breaches up to the $1 \times 76 \mathrm{~mm}$ breach at target pressures between 100 and 380 psi. The chamber length will be varied from 39 to $10 \mathrm{ft}$ and perhaps as small as $5 \mathrm{ft}$ if data show this will help the extrapolation to long distance sprays.

- Test Objective 13: Determine the size distribution of aerosol droplets and the total droplet volume concentration as a fraction of the total spray volume for non-Newtonian clay slurries. Also determine the droplet size distribution directly in the spray (in-spray measurement) for these simulants. The rheology of the simulant will be adjusted so that one slurry, at the beginning of testing, has at least one Bingham parameter near $30 \mathrm{~Pa} / 30 \mathrm{cP}$ (target range is $30 \pm 4 \mathrm{~Pa}$ or $\mathrm{cP}$ ) and the second Bingham parameter should be less than or equal to the upper $30 \pm 4 \mathrm{~Pa}$ or $\mathrm{cP}$ target. The second simulant will be adjusted so that one slurry has at least one Bingham parameter near $6 \mathrm{~Pa} / 6 \mathrm{cP}$ (target range is $6 \pm 2 \mathrm{~Pa}$ or $\mathrm{cP}$ ) and the second Bingham parameter should be greater than or equal to the lower $6 \pm 2 \mathrm{~Pa}$ or $\mathrm{cP}$ target. Testing will be conducted using at least one circular hole and multiple rectangular breaches up to the $1 \times 76 \mathrm{~mm}$ breach at target pressures between 100 and 380 psi.

- Test Objective 14: Compare the aerosol results from the Malvern Insitec-S using the new $500 \mathrm{~mm}$ lens that has a nominal measurement range of 2.5 to $2500 \mu \mathrm{m}$ (Malvern Instruments, Ltd. 2010) to aerosol results using the $100 \mathrm{~mm}$ lens employed in Phase I that provided a nominal range of 0.5 to $200 \mu \mathrm{m}$ (Schonewill et al. 2012). Tests will use one or more orifices.

\subsection{Task Methodology}

Project Plan PP-WTPSP-091, Rev. 0, ${ }^{1}$ defines the task methodology to be applied to Phase II testing. The project plan text that outlines Phase II task methodology is reproduced in this section. It states that testing will be conducted using the large-scale spray release test stand that was designed and commissioned during the Phase I scope of the Spray Release Methodology testing project. The task methodology will be similar to that used during Phase I portion of the work (Schonewill et al. 2012) with the following changes:

- Chamber size will be expanded from $20 \mathrm{ft}$ to $39 \mathrm{ft}$ to facilitate evaluation of the effect of chamber size and spray length on the size distribution of aerosol droplets and the total droplet volume concentration as a fraction of the total spray volume for water sprays for a range of different chamber sizes and for sprays traveling different distances within the largest chamber.

- Malvern Insitec-S instruments with $500 \mathrm{~mm}$ lenses will be used to measure the aerosol size distribution and total droplet concentration in the spray chamber. The change in lens size from the previously used $100 \mathrm{~mm}$ lenses to the $500 \mathrm{~mm}$ lenses was made because previous in-spray measurements with $100 \mathrm{~mm}$ lenses could not quantify droplets larger than $200 \mu \mathrm{m}$, and this made the PhaseI1 in-spray results over-estimate the release fractions. ${ }^{2}$ Using $500 \mathrm{~mm}$ lenses helps get a more accurate representation of PSD during in-spray measurements while including the PSD range of

\footnotetext{
${ }^{1}$ Bontha J. 2012. Project Plan for Phase II Large Scale Spray Release Aerosol Testing. PP-WTPSP-091, Rev. 0, Pacific Northwest National Laboratory, Richland, Washington.

${ }^{2}$ A Malvern Insitec-S instrument with $500 \mathrm{~mm}$ lens has a nominal particle size measurement range of 2.5 to $2500 \mu \mathrm{m}$ compared to the $100 \mathrm{~mm}$ lens employed in Phase I, which provided a nominal range of 0.5 to $200 \mu \mathrm{m}$ (Malvern Instruments, Ltd. 2010). Droplets above the instrument range are interpreted as droplets within the instrument range leading to over-estimates.
} 
interest (e.g., 10 to $100 \mu \mathrm{m}$ ) and, thus, a better estimate of the cumulative release fractions from the in-spray measurements.

- Approximately $1000 \mathrm{~mm}$ spacers will be installed between the Malvern Insitec-S transmitter and detector to 1) improve the measurable particle size concentration and 2) enable in-spray measurements with larger orifices.

- Testing will be conducted with non-Newtonian (clay) slurries with two target rheologies (yield stress and consistency combinations) of 1) $6 \mathrm{~Pa}$ and $6 \mathrm{cP}$ and 2) $30 \mathrm{~Pa}$ and $30 \mathrm{cP}$.

NOTE: The expectation is that some parameters that were established during the Phase I testing such as using fans and fan speed to enhance mixing in the chamber, instrument purge air rates to prevent Malvern Insitec-S flooding particularly during the in-spray measurements, and other similar system parameters may need to be adjusted to improve reliability and repeatability of the data collected. Proposed changes to these parameters, if any, will be investigated during shakedown of the system.

\subsection{Analysis Methodology}

The analysis methodology for Phase II testing is adapted directly from the Phase I large-scale data analysis methodology. The technical basis for large-scale analysis of the in-chamber results is discussed both in the main body of and in the Appendix C of Schonewill et al. (2012). It is reproduced below using an alternate approach that better defines the assumptions inherent in the methodology approach applied to determination of spray leak generation rates and release fractions. The analysis methodology for the in-spray results is much more direct and is discussed briefly at the end of this section.

In the current report, spray of liquids or slurries into an enclosure is evaluated using either in-chamber or in-spray methods. Because each method samples a different population of droplets, the two methods do not generally yield the same result. When material is released from the orifice, it can travel freely as an unbroken jet until it impacts an obstruction and pools in the catch pans at the bottom of the chamber. High-velocity release causes break-up of the spray jet resulting in the formation of a broad size distribution of droplets or solid particulate (Mahoney et al. 2013; Schonewill et al. 2012). It is expected that large droplets will follow a trajectory governed by the inertia imparted at initial release, drag, and settling. Small aerosols are subject to similar forces, but can become entrained in the carrier phase (air) and mixed throughout the chamber by turbulence created by the high-velocity spray.

In-chamber measurements employ aerosol analyzers installed several feet above the spray at both upstream and downstream measurement locations. In this configuration, the aerosol analyzers preferentially observe the smaller aerosols capable of being suspended and mixed throughout the chamber. Large droplets may still be observed in in-chamber configurations, but require that the analyzers be located in the downstream trajectory of the spray (or a portion thereof). For this reason, determination of generation rate and release fraction from in-chamber measurements use mixing models such as the continuously stirred tank reactor (CSTR) model used in Phase I analysis, for example see Schonewill et al. (2012). In contrast, in-spray measurements employ aerosol analyzers that are located in the direct downstream path of the jet. Specifically, measurements track the most optically dense region of the spray at fixed downstream locations. In-spray measurements include both large and small droplets generated by the spray, which allows direct association of measured droplet size distribution and spray leak flow rate with release fraction and generation rate measurements. 


\subsubsection{In-Chamber Measurement Basis}

For spray leak analysis of the in-chamber results, the large-scale test chamber is modeled as bounded control volume where aerosol is introduced by spray of a high-pressure liquid from a breach at a fixed location. Aerosol is "lost" from the bounded control volume through deposition onto chamber surfaces (primarily the chamber walls, ceiling, and floor). Other processes, such as evaporation or droplet coalescence, can alter the size of aerosol. Deposition will cause the spray material to pool and collect with the test enclosure and slowly displace free chamber volume over time. Because the largest sprays tested in Phase II introduced at most 150 gal into test chambers with total free volumes of 3600 gal (for the $10 \mathrm{ft}$ chamber) or more, displacement will not result in a significant change in free aerosol volume during individual spray tests and can be neglected. A basic material balance (Deen 1998) of the aerosolized fraction of the spray within the chamber that falls within a size range bounded by diameters $\mathrm{d}_{\mathrm{i}}$ and $\mathrm{d}_{\mathrm{i}+1}$ yields the time rate equation for the aerosol concentration in size bin $i$ :

$$
\frac{d}{d t} \int_{V} \hat{c}_{i} d V=\int_{S} R_{S, i} d S+\int_{V} R_{V, i} d V+\int_{V} R_{N, i} d V
$$

where $\quad \hat{c}_{i}=$ the local (position dependent) aerosol concentration for size bin $i$

$R_{s, i}=$ the rate of loss of aerosol size $i$ as a result of deposition at the chamber surface $S$

$R_{V, i}=$ the generation of aerosol size $i$ caused by aerosolization of the spray

$R_{N, \mathrm{i}}=$ non-spray generation/consumption that impact the apparent generation of aerosol.

Other variables not defined above include the time $t$ and the total chamber volume $V$. For the overall mass balance, the terms associated with the net generation rate can be replaced by:

$$
\int_{V} R_{V, i} d V=f_{s, i} Q_{S}
$$

where $Q_{s}$ is the volumetric flow rate of liquid generated by pressure through the engineered orifice and $f_{s, i}$ is the fraction of that spray aerosolized to produce aerosol in size bin $i$. Equation (1.2) treats droplet formation as a fractional aerosolization process and is the product of the total leak flow rate $\left(Q_{s}\right)$ and the fractional aerosolization of the spray $\left(f_{s, i}\right)$, which is equivalent to the aerosol release fraction (see Equation (1.16) below). In terms of aerosol generation rate, Equation (1.2) is simply:

$$
\int_{V} R_{V, i} d V=g_{S, i}
$$

where $g_{S, i}$ is the droplet generation rate.

Aerosol material balance is impacted by non-spray terms that account for additional loss or generation of aerosol in the chamber as a result of evaporation, downstream droplet break-up, and/or coalescence with other droplets. These phenomena depend both on droplet concentration and on other system parameters, such as the rate of turbulent mixing and relative humidity. The impact of these processes can be approximated by zero and first-order concentration terms, such that

$$
\int_{V} R_{N, i} d V=g_{N, i}-h_{i} \int_{V} \hat{c}_{i} d V
$$

Here, $g_{N, i}$ accounts for zeroth-order processes such as apparent generation by droplet break-up far downstream of the orifice, and $h_{i}$ is a generic loss coefficient for first-order processes. The negative sign 
convention is adopted to treat first-order process terms as aerosol loss mechanisms. Higher-order effects, such as droplet coalescence, have a net effect but are not explicitly accounted for in the droplet balance as they are considered small relative to the dominant first-order effect of wall loss (discussed later).

As discussed in the Phase I large-scale report, deposition loss mechanisms can be expressed as first-order loss mechanisms with respect to concentration such that:

$$
\int_{S} R_{S, i} d S=-\int_{S} \hat{c}_{S, i} \boldsymbol{v}_{S, i} \cdot \boldsymbol{n} d S
$$

where, $\boldsymbol{v}_{s, i}$ (a vector quantity) represents the convective, settling, diffusive, or inertial aerosol velocity that deposits aerosols of size $i$ onto the chamber surface or transfers them across the permeable system boundary and $\hat{c}_{s, i}$ is the concentration of aerosol at the surface of the chamber. Both parameters are position dependent, and determining their exact values requires full solution of the convective equations for aerosol motion in the chamber. Combining Equations (1.1), (1.3), (1.4), and (1.5) yields:

$$
\frac{d}{d t} \int_{V} \hat{c}_{i} d V=-\int_{S} \hat{c}_{S, i} \boldsymbol{v}_{S, i} \cdot \boldsymbol{n} d S+g_{S, i}+g_{N, i}-h_{i} \int_{V} \hat{c}_{i} d V
$$

Equation (1.4) can be simplified by using chamber averages for the time rate change in aerosol and for aerosol loss terms. The average chamber concentration for aerosols in size bin $i$ (denoted by $c_{i}$ ) is defined as:

$$
c_{i}=\frac{1}{V} \int_{V} \hat{c}_{i} d V
$$

Likewise, the deposition terms can be similarly averaged to yield an expression for the average surface concentration of aerosol:

$$
c_{S, i}=\frac{1}{\alpha_{i} A} \int_{\mathrm{S}} \hat{c}_{S, i} \boldsymbol{v}_{S, i} \cdot \boldsymbol{n} d S
$$

where the term $\alpha_{i}$ represents an average velocity for events that bring aerosol into contact with the surface and $c_{s, i}$ is the average surface aerosol concentration. Substitution of these averages into Equation (1.6) produces:

$$
V \frac{d c_{i}}{d t}=g_{S, i}+g_{N, i}-V h_{i} c_{i}-\alpha_{i} A c_{s, i}
$$

If we define a net generation rate $g_{i}$ and deposition loss coefficient $h_{s, i}$ for size bin $i$ as:

$$
\begin{gathered}
g_{i}=g_{S, i}+g_{N, i} \\
h_{S, i}=\frac{A}{V} \alpha_{i}
\end{gathered}
$$

Here, the first term indicates the net generation of aerosol and includes aerosol produced by initial or in-flight break-up of spray. Substituting Equations (1.10) and (1.11) into Equation (1.9) yields Equation (1.12): 


$$
\frac{d c_{i}}{d t}=\frac{g_{i}}{V}-h_{i} c_{i}+h_{s, i} c_{s, i}
$$

This equation is similar, but not identical, to the average chamber aerosol concentration rise curve equation derived in Appendix $\mathrm{C}$ of Schonewill et al. (2012). The primary difference is that one of the loss terms depends on the average surface concentration of aerosol, $c_{s, i}$, which reflects that a portion of aerosol within the chamber must reach a surface before it is removed from the system. If the aerosol is considered to be immediately and uniformly mixed by chamber turbulence, there will be no difference between the average chamber and average surface concentration of aerosols. In this case, Equation (1.12) reduces to:

$$
\frac{d c_{i}}{d t}=\frac{g_{i}}{V}-\lambda_{i} c_{i}
$$

where $\lambda_{i}=h_{i}+h_{s, i}$. This can be integrated analytically, and yields the following standard exponential rise equation, which is used throughout Phase I aerosol generation rate analysis for differential aerosol concentration:

$$
c_{i}=\frac{g_{i}}{\lambda_{i} V}\left(1-e^{-\lambda_{i} t}\right)
$$

For spray leak analysis, aerosol quantities are usually expressed and worked with on a cumulative basis. Two key parameters evaluated in spray leak analysis are the cumulative aerosol concentration $\left(C_{k}\right)$ and generation rate $\left(G_{k}\right)$ undersize, which are defined as $C_{k}=\sum_{i=1}^{k} c_{i}$ and $G_{k}=\sum_{i=1}^{k} g_{i}$. The concentration rise expression defined by Equation (1.14) is best suited for analyzing differential concentration, as several generation and loss terms are size specific. However, Phase I testing indicates that this expression also can be used to describe the dynamics of cumulative quantities. In terms of cumulative aerosol concentration, Equation (1.14) becomes

$$
C_{k}=\frac{G_{k}}{\lambda_{k} V}\left(1-e^{-\Lambda_{k} t}\right)
$$

where $G_{k}$ and $\Lambda_{k}$ are the cumulative analogues of the generation rate and release fraction. Large-scale aerosol testing is expected to produce in-chamber measurements of aerosol concentration and size distribution that can be used to determine the concentration rise curves for aerosols as a function of size. These data are interpreted using Equations (1.14) or (1.15) to determine the aerosol generation rate, $G_{k}$, as a function of test chamber configuration such as chamber length, size of breach, and pressure driving the spray leak. From generation rates, the cumulative aerosol release fraction $R_{k}$ can be determined by:

$$
R_{k}=\frac{G_{k}}{Q_{s}}
$$

This approach defines release fraction calculations for in-chamber measurements, which represent the majority of the spray release measurements that were conducted in Phase II.

\subsubsection{In-Spray Measurement Basis}

In-spray measurements, which make up the remainder of spray leak measurements, employ a direct approach for release fraction analysis, whereby the size distribution of the spray is measured in-flight 
before dispersing through the chamber or incurring loss from inertial impact on the splash wall. Under this operating method, the in-spray size distribution may be assumed to be equivalent to the release fraction (with a total aerosolized fraction of one). In-spray size distribution measurements can be made using either flux or spatial based measurements. Flux measurements nominally observe particles that pass through a measurement surface and determine aerosol size, velocity, and concentration. Spatial measurements observe aerosol particles within a defined measurement volume and determine only droplet size distribution and concentration. Flux-based methods are advantageous in that they are free of size distribution measurement bias that results from differences in aerosol particle velocity as the measurement area is typically set up so that only one particle at a time is evaluated and because any bias from velocity effects can be corrected using the measured particle velocity. In contrast, spatial measurements will be biased toward the slowest moving particles as these particles remain in the fixed spatial measurement volume for greater periods of time relative to faster moving particles. Because particle velocity is not measured by spatial techniques, no correction for this bias is possible. Despite this limitation, spatial techniques are often superior to flux techniques because of their ability to measure an ensemble of particles in a single measurement, producing a statistically significant and representative measurement of size distribution in a very short period of time $(\sim 1 \mathrm{~s})$. Flux techniques require small measurement volumes and typically count and size aerosols on a particle-by-particle basis. As a consequence, acquisition of statistically significant and representative size distributions with flux-based measurements requires long integration times (typically 10 to $100 \mathrm{~s}$ ). For the current report, in-spray measurements are made using a spatial aerosol analyzer to facilitate rapid analysis. The impact of this selection may be to over-estimate release fractions in the droplet size range of interest to WTP spray leak consequence analysis (10 to $100 \mu \mathrm{m})$

\subsection{Success Criteria}

At the start of Phase II testing, several success criteria were proposed to assess and help meet the overall success of follow-on testing. These criteria are documented in Test Plan TP-WTPSP-031, Rev. 1.0.

Tests aimed as satisfying Test Objective 10 will be considered successful if testing:

- Measure[s] the concentration and size distribution of known aqueous suspensions with the Malvern used for aerosol measurements and quantitatively compare[s] the Malvern (Insitec-S) results with the known values to estimate the Malvern (Insitec-S) accuracy for measuring aerosols.

Tests aimed at satisfying Test Objectives 12 and 13 (see Section 1.3) will be considered successful if the following criteria are met:

- Measure the droplet size distribution, total volume concentration of droplets, and total volume flow rate sprayed for each of the breaches and simulants tested.

- For in-spray measurements, measure the droplet size distribution for each of the breaches and simulants tested.

- Characterize the viscosity or rheology, PSD, bulk density, and surface tension of each simulant tested.

- For the $30 \mathrm{~Pa} / 30 \mathrm{cP}$ simulant at the beginning of testing, at least one Bingham parameter is $30 \pm 4 \mathrm{~Pa}$ or $\mathrm{cP}$ and the second Bingham parameter should be less than or equal to this upper target of $30 \pm 4 \mathrm{~Pa}$ or $\mathrm{cP}$. 
- For the $6 \mathrm{~Pa} / 6 \mathrm{cP}$ simulant at the beginning of testing, at least one Bingham parameter is $6 \pm 2 \mathrm{~Pa}$ or $\mathrm{cP}$ and the second Bingham parameter should be greater than or equal to this lower target of $6 \pm 2 \mathrm{~Pa}$ or $\mathrm{cP}$.

- Measure the pressure and flow in the piping.

- Calculate the large-scale test chamber volume from internal dimensions for chamber configurations different from Phase I testing.

Finally, tests aimed at satisfying Test Objective 14 will be considered successful if:

- Quantitative comparison demonstrates that the old and new lenses give equivalent results for aerosol conditions that have droplet sizes in a range appropriate for both lenses. 


\subsection{Quality Assurance}

The Pacific Northwest National Laboratory (PNNL) Quality Assurance (QA) program is based upon the requirements defined in the DOE 414.1D, Quality Assurance, and 10 CFR 830, Energy/Nuclear Safety Management, and Subpart A-Quality Assurance Requirements (a.k.a., the Quality Rule). PNNL has chosen to implement the following consensus standards in a graded approach:

- ASME NQA- $1^{1}$-2000, Quality Assurance Requirements for Nuclear Facility Applications, Part 1, Requirements for Quality Assurance Programs for Nuclear Facilities

- ASME NQA-1-2000, Part II, Subpart 2.7, Quality Assurance Requirements for Computer Software for Nuclear Facility Applications

- ASME NQA-1-2000, Part IV, Subpart 4.2, Graded Approach Application of Quality Assurance Requirements for Research and Development.

The procedures necessary to implement the requirements are documented through PNNL's "How Do I...?" (HDI) system, which is a web-based system for managing delivery of PNNL policies, requirements, and procedures.

The Waste Treatment Plant Support Project (WTPSP) implements an NQA-1-2000 Quality Assurance program, graded on the approach presented in NQA-1-2000, Part IV, Subpart 4.2. The WTPSP Quality Assurance Manual (QA-WTPSP-0002) describes the technology life cycle stages under the WTPSP Quality Assurance Plan (QA-WTPSP-0001). The technology life cycle includes the progression of technology development, commercialization, and retirement in process phases of basic and applied research and development $(\mathrm{R} \& \mathrm{D})$, engineering and production, and operation until process completion. The life cycle is characterized by flexible and informal QA activities in basic research, which becomes more structured and formalized through the applied R\&D stages.

The work described in this report has been completed under the QA technology level of Developmental Work. WTPSP addresses internal verification and validation activities by conducting an Independent Technical Review of the final data report in accordance with WTPSP procedure QA-WTPSP-601, Document Preparation and Change. This review verifies that the reported results are traceable, that inferences and conclusions are soundly based, and that the reported work satisfies the test plan objectives.

\footnotetext{
${ }^{1}$ NQA-1 is the abbreviation for Nuclear Quality Assurance-1, a regulatory standard created and maintained by the American Society of Mechanical Engineers.
} 



\subsection{Simulants}

This chapter describes the waste simulants used in the large-scale aerosol tests, states the basis for their selection, describes the physical property measurement methods used, and reports the measured physical properties of the simulants. The simulants and the basis for selection are discussed in Section 3.1. The physical property measurement methods and the resulting measurements are reported in Section 3.2.

\subsection{Simulants Used}

Table 3.1 summarizes the WTP process streams and typical ranges for important fluid properties. ${ }^{1}$ The ranges of properties and descriptions are only representative, and specific waste examples may vary from these generalizations. These are the process streams that were represented by simulants in the spray release tests. The non-Newtonian simulants represent slurries that are expected to be in the vessels commonly referred to as the non-Newtonian vessels. These include the ultrafiltration feed vessels (UFP-VSL-00002 A/B) and the high-level waste lag storage and blend vessels (HLP-VSL-0027 A/B and HLP-VSL-0028). During some of the process steps, the slurries in the ultrafiltration feed vessel are expected to exhibit a Newtonian rheology. Some of the other vessels are expected to contain Newtonian slurries and include, but are not limited to, the high-level waste receipt vessel (HLP-VSL-00022) and the ultrafiltration feed preparation vessels (UFP-VSL-0001 A/B).

Table 3.1. WTP Process Stream Categories and Representative Fluid Properties

\begin{tabular}{llll}
\hline \multicolumn{1}{c}{ WTP Process Stream Categories } & Particles & Composition & Viscosity Rheology \\
\hline $\begin{array}{l}\text { Ultrafilter permeate treated low activity } \\
\text { waste (LAW) }\end{array}$ & Negligible & $\begin{array}{l}\text { Caustic solution } \\
5-10 \mathrm{M} \mathrm{Na}\end{array}$ & $\begin{array}{l}\text { Newtonian } \\
2-3 \mathrm{cP}\end{array}$ \\
$\begin{array}{lll}\text { Cs ion exchange eluate } \\
\text { Recycle streams }\end{array}$ & Negligible & $\begin{array}{l}\mathrm{Na}, \mathrm{K}, \mathrm{Cs} \text { ions with } \\
0.5 \mathrm{M} \mathrm{HNO}\end{array}$ & $\begin{array}{l}\text { Newtonian } \\
0.5 \mathrm{cP} \text { and above }\end{array}$ \\
& $<2 \mathrm{wt} \%$ & $0.2-2 \mathrm{M} \mathrm{Na}$ & $\begin{array}{l}\text { Newtonian } \\
0.5 \mathrm{cP} \text { and above }\end{array}$ \\
Newtonian slurries & Approximately & Up to $8 \mathrm{M} \mathrm{Na}$ & $\begin{array}{l}\text { Newtonian } \\
\text { about } 1-3 \mathrm{cP}\end{array}$ \\
& $2-16 \mathrm{wt} \%$ & & Non-Newtonian \\
Non-Newtonian slurries & Up to & $0.2-2 \mathrm{M} \mathrm{Na}$ & $6 \mathrm{cP} / 6 \mathrm{~Pa}$ to \\
& $\sim 20 \mathrm{wt} \%$ & & $30 \mathrm{cP} / 30 \mathrm{~Pa}$ \\
\hline
\end{tabular}

(a) The upper limit of $\sim 16 \mathrm{wt} \%$ corresponds to a limit of $200 \mathrm{~g} / \mathrm{L}$ in the waste acceptance criteria (ICD-19 $2011^{2}$ ). A new upper limit of $144 \mathrm{~g} / \mathrm{L}$ in a $7 \mathrm{M} \mathrm{Na}$ feed has been recommended, and this corresponds to about $10 \mathrm{wt} \%$ solids (Campbell et al. 2010).

(b) This category also could be a weak non-Newtonian fluid based on the feed acceptance criterion that allows up to $1 \mathrm{~Pa}$ Bingham yield stress slurries to be delivered to the WTP (ICD-19 2011).

\footnotetext{
${ }^{1}$ These categories and ranges of process parameters were provided by the WTP client as guidance for proposal preparation.

${ }_{2}^{2}$ ICD 19. 2011. ICD 19 - Interface Control Document for Waste Feed. 24590-WTP-ICD-MG-01-19, Rev 5, River

Protection Project, Waste Treatment Plant, Richland, Washington.
} 
Table 3.2 lists the target simulants presented in the test plan (TP-WTPSP-031, Rev 1.0) for the spray release testing effort. ${ }^{1}$ The four simulant classes and materials were chosen to represent the range of waste categories shown in Table 3.1. The last column in Table 3.2 shows how each simulant represents one or more of the WTP process stream categories. Of the four classes of wastes listed, three were tested during the Phase I large-scale tests. Water was used for shakedown and baseline testing during both Phases I and II. One aqueous sodium thiosulfate solution was chosen during Phase I testing to represent process streams in the WTP that are Newtonian fluids but with a higher viscosity. These include the ultrafilter permeate/treated low activity waste (LAW), Cs ion exchange eluate, and recycle streams. During Phase I, the non-hazardous boehmite slurry at 8 and $20 \mathrm{wt} \%$ in water was tested in the large-scale system but no simulants exhibiting a significant non-Newtonian rheology were tested. The types of simulants tested in both Phase I and Phase II were selected carefully to provide test data for each simulant class within the schedule, budget, and safety considerations associated with 500 gal batches of simulant. These constraints included chemical cost, disposal cost, time for preparation, time for cleaning, and chemical hazards. A greater number of simulants were tested in the small-scale spray leak tests, including the chemical slurry simulant (Mahoney et al. 2012, 2013).

Table 3.2. Target Simulants and the WTP Process Stream Categories

\begin{tabular}{|c|c|c|c|}
\hline Simulant Class & Material & Target Property Range & $\begin{array}{c}\text { WTP Process } \\
\text { Stream Categories }\end{array}$ \\
\hline Baseline & Water & $\begin{array}{l}\text { Viscosity } 1 \mathrm{mPa} \cdot \mathrm{s}(1 \mathrm{cP}) \\
\text { Density } 1000 \mathrm{~kg} / \mathrm{m}^{3} \\
\text { Surface tension } 73 \mathrm{mN} / \mathrm{m}\end{array}$ & Rinse waters \\
\hline $\begin{array}{l}\text { Range of } \\
\text { Newtonian } \\
\text { viscosity }\end{array}$ & $\begin{array}{l}\text { Solutions of water and } \\
\text { non-hazardous salts (sodium } \\
\text { nitrate and sodium thiosulfate) }\end{array}$ & $\begin{array}{l}\text { Viscosities of } \sim 1.5, \\
\sim 2.5 \mathrm{mPa} \cdot \mathrm{s}(1.5,2.5 \mathrm{cP})\end{array}$ & $\begin{array}{l}\text { Ultrafilter permeate/ } \\
\text { treated LAW } \\
\text { Cs ion exchange eluate } \\
\text { recycle streams }\end{array}$ \\
\hline $\begin{array}{l}\text { Range of } \\
\text { slurries (non- } \\
\text { hazardous) }\end{array}$ & $\begin{array}{l}\text { Gibbsite, boehmite, or other } \\
\text { non-hazardous particulates in } \\
\text { water or dilute salt solutions }\end{array}$ & $\begin{array}{l}\text { PSDs of slurries were selected } \\
\text { to match Hanford waste PSDs } \\
8 \text { and } 20 \mathrm{wt} \% \text { solids }\end{array}$ & $\begin{array}{l}\text { Newtonian and } \\
\text { non-Newtonian slurries }\end{array}$ \\
\hline $\begin{array}{l}\text { Washed and } \\
\text { leached } \\
\text { chemical slurry } \\
\text { simulant }\end{array}$ & $\begin{array}{l}\text { A washed and leached version of } \\
\text { the simulant used in } \\
\text { Pretreatment Engineering } \\
\text { Platform (PEP) testing } \\
\text { (Kurath et al. 2009) }\end{array}$ & $\begin{array}{l}\text { The solids loading was } \\
\text { adjusted in an attempt to meet } \\
\text { target Bingham yield stresses } \\
\text { of } 6 \text { and } 30 \mathrm{~Pa}\end{array}$ & Non-Newtonian slurries \\
\hline
\end{tabular}

The non-hazardous slurry simulant chosen for Phase II large-scale testing was an 80/20 solids blend of a kaolin/bentonite clay slurry simulant. The kaolin/bentonite mixture was chosen because it satisfied the simulant selection criteria for a non-hazardous, non-Newtonian simulant that could be used in both the small- and large-scale test systems during Phase II testing as outlined in Gauglitz 2011. The 80/20 kaolin/ bentonite clay simulant has been used historically in a variety of studies such as Poloski et al. (2004), Bamberger et al. (2005), and Russell et al. (2005). A wide range of Bingham rheological parameters can be obtained for an $80 / 20$ blend of these two clay materials by varying the total clay fraction in water (wt $\%$ solids). For Phase II, clay slurry simulants were tested in both the small- and large-scale test systems in

\footnotetext{
${ }^{1}$ Gauglitz PA. 2011. Test Plan for Spray Leak Quantification to Support WTP Spray Release Methodology. TP-WTPSP-031, Rev 0.2, Pacific Northwest National Laboratory, Richland, Washington.
} 
order to obtain a direct side-by-side comparison with the chemical slurry simulants that were tested solely in the small-scale system and for scaling comparisons between the small-scale and large-scale test results. Two clay simulants were used during Phase II testing to quantify the release fraction results at the rheology limits of $30 \mathrm{~Pa} / 30 \mathrm{cP}$ and $6 \mathrm{~Pa} / 6 \mathrm{cP}$, respectively, for non-Newtonian slurries. The total solids concentration of the $80 / 20$ clay mixture was adjusted to achieve the Bingham parameter boundaries of $30 \mathrm{~Pa} / 30 \mathrm{cP}$ for the first simulant, depicted as "30 Pa Clay," and a $6 \mathrm{~Pa} / 6 \mathrm{cP}$ clay simulant for the second as simulant, known "6 Pa Clay." The rheological properties of the clay simulants were continuously monitored during testing to ensure that the Bingham parameters fell within the targeted range as defined in TP-WTPSP-031, Rev. 1.0.

Simulant samples were taken at the start of each test day and the rheological properties were evaluated, and if necessary adjustments were made and subsequent samples taken until the rheological properties were acceptable. For the simulant $30 \mathrm{~Pa}$ Clay, at the beginning of testing at least one Bingham parameter was required near $30 \mathrm{~Pa} / 30 \mathrm{cP}$ (target range was $30 \pm 4 \mathrm{~Pa}$ or $\mathrm{cP}$ ) and the second Bingham parameter was less than or equal to the upper $30 \pm 4 \mathrm{~Pa}$ or $\mathrm{cP}$ target. The second clay simulant, $6 \mathrm{~Pa}$ Clay, was adjusted by dilution so that at least one Bingham parameter was near $6 \mathrm{~Pa} / 6 \mathrm{cP}$ (target range was $6 \pm 2 \mathrm{~Pa}$ or $\mathrm{cP}$ ) and the second Bingham parameter was greater than or equal to the lower $6 \pm 2 \mathrm{~Pa}$ or $\mathrm{cP}$ target, at the beginning of testing. For all test performed with both clay simulants the target rheological properties were met.

\subsection{Physical Properties}

The physical properties were measured from samples collected before, during, and after testing for the $30 \mathrm{~Pa}$ Clay and the $6 \mathrm{~Pa}$ Clay simulants. The measurement techniques are described in Section 3.2.1, and the results are presented in Section 3.2.2 and Section 3.2.3.

\subsubsection{Characterization Techniques}

Samples were characterized for physical and rheological properties including PSD, bulk density, surface tension, solids content, yield stress, and consistency (viscosity for Newtonian fluids). Sample analyses were performed according to procedures RPL-COLLOID-02, Rev. 2, Measurement of Physical and Rheological Properties of Solutions, Slurries and Sludges; OP-WTPSP-004, Rev. 1, Operation of the Mettler Moisture Analyzer; OP-WTPSP-035, Rev. 0, Measurement of Static Surface Tension of Liquids, Dispersions, and Slurries; and OP-WTPSP-003, Rev. 1, Size Analysis Using Malvern MS2000

(re-issuance of TPR-RPP-WTP-626).

\subsubsection{Solids Content}

The total solids content of slurry samples by weight were determined using a Mettler-Toledo Halogen Moisture analyzer (Model HR83, Serial No. 1129192189) in accordance with OP-WTPSP-004, Rev. 1, Operation of the Mettler Moisture Analyzer. Approximately 5 to 10 grams of slurry material were introduced into the moisture analyzer and a preprogrammed drying program was run. The program consisted of an initial hold period of $30 \mathrm{~min}$ at $95^{\circ} \mathrm{C}$ and then a second temperature of $105^{\circ} \mathrm{C}$ was used to complete the drying process. The criterion for ending the program was set to a mean weight loss of $1 \mathrm{mg}$ over a $140 \mathrm{~s}$ period. The results were recorded as dry content (i.e., total solids content) by the moisture analyzer, the definition of this result is given by Equation (3.1). 


$$
\text { Dry Content }=\frac{d r y \text { weight }}{\text { wet weight }} \times 100 \%
$$

\subsubsection{Surface Tension}

Supernatant samples were obtained from both the $30 \mathrm{~Pa}$ Clay and the $6 \mathrm{~Pa}$ Clay simulants for surface tension analysis by centrifugation at 20,200 RCF using an RC5C Sorvall centrifuge coupled with a fixed angle rotor model SS-34.

Surface tension measurements were performed using a commercial force-balance K-12 MK6 Tensiometer (Kruss USA) in accordance with OP-WTPSP-035, Rev 0, Measurement of Static Surface Tension of Liquids, Dispersions, and Slurries. The tensiometer consists of a K-12 MK6 tensiometer processor unit, a force measuring unit (the balance), a Wilhelmy platinum plate, and a quartz sample vessel. Static surface tension of the supernatant obtained from the two clay simulants was measured using the Wilhelmy method, which is based on a force measurement. A platinum plate with a known geometry was vertically suspended above the sample liquid. The lower edge of the plate then was brought into contact with the sample liquid surface. The sample liquid wets the plate and pulls it slightly into the liquid by the Wilhelmy force, which results from the wetting. The Wilhelmy force is measured by moving the plate up to the level of the sample liquid surface. The resulting force was determined from the weight measured by the balance. Surface tension measurements were carried out at room temperature, which was recorded at the time of measurement with a calibrated thermocouple.

\subsubsection{Density}

Bulk density of the clay simulant samples were measured using certified glass pycnometers (Wilmad LabGlass) in accordance with RPL-COLLOID-02 Rev 2.0. All density measurements were performed at a sample temperature of $20^{\circ} \mathrm{C}$; this was achieved by equilibrating the samples in a $20^{\circ} \mathrm{C}$ re-circulating water bath. Daily balance checks were performed with a calibrated $50 \mathrm{~g}$ weight when the balance was in use. After the balance performance check, the tare weight of the pycnometer to be used was obtained and recorded in the density measurement bench sheet. The pycnometer was then filled with the simulant fluid to be measured. The gross weight of the pycnometer containing the simulant fluid was obtained and recorded in the density measurement bench sheet. The net weight of the simulant fluid was calculated by subtracting the pycnometer tare weight from the gross weight of the pycnometer containing the simulant. The bulk density of the simulant fluid was calculated using Equation (3.2). Unless specified otherwise, all density measurements were carried out at room temperature. Room temperature associated with each density measurement was also measured using a calibrated thermocouple, and the thermocouple readout was recorded in the density measurement bench sheet.

$$
\rho=\frac{M}{V}
$$

where $\quad \rho=$ bulk density in $\mathrm{g} / \mathrm{mL}$

$\mathrm{M}=$ net weight of the simulant fluid in $\mathrm{g}$

$\mathrm{V}=$ volume of the simulant fluid in $\mathrm{mL}$. 


\subsubsection{Particle Size Analysis}

Particle size characterization was accomplished using a Mastersizer 2000 (Malvern Instruments, Inc., Southborough, Massachusetts) with a Hydro G wet dispersion accessory (equipped with a continuously variable and independent pump, stirrer, and ultrasound). The Mastersizer 2000 has a nominal size measurement range of 0.02 to $2000 \mu \mathrm{m}$. The actual range is dependent on the accessory and the properties of the solids being analyzed. When coupled with the Hydro G wet dispersion accessory, the nominal measuring range is 0.02 to $2000 \mu \mathrm{m}$ (this is dependent on material density). Table 3.3 provides a summary of basic information regarding the analyzer and accessory. A NIST traceable particle size standard is used to evaluate the performance of the particle size analyzer in accordance with OP-WTPSP-003, Rev. 1.0.

Table 3.3. Summary of Malvern Mastersizer 2000 Instrument Information

\begin{tabular}{l|l}
\hline Analyzer & Malvern Mastersizer 2000 (Serial No. MAL 1019545) \\
Measurement principle & $\begin{array}{l}\text { Laser Diffraction (Mie scattering) } \\
\text { Hydro G, } 800 \mathrm{~mL} \text { capacity }\end{array}$ \\
Analyzer accessory & $\begin{array}{l}0.02 \text { to } 2000 \mu \mathrm{m} \text { nominal } \\
\text { Measurement range } \\
\text { Type }\end{array}$ \\
\hline
\end{tabular}

Small aliquots of the simulant samples ( $<1 \mathrm{~mL}$ for slurries, $\sim 0.2$ to $1 \mathrm{~g}$ for dry simulants) were diluted with de-ionized water in the Hydro $G$ dispersion unit with pump and stirrer speed set typically at 2000 and $8000 \mathrm{rpm}$, respectively, for $60 \mathrm{~s}$ before making the particle size measurements. Appropriate dilutions were determined by the amount of light passing through the diluted material (obscuration), which was measured by the particle size analyzer. Samples were analyzed on the same aliquot initially without sonication and then during sonication (100 percent, $20 \mathrm{~W}$ ) after an initial sonication period of $60 \mathrm{~s}$ and also post-sonication.

All simulant samples were well mixed before taking aliquots for PSD measurements. Measurements were made on duplicate samples to confirm the mixing and sub-sampling technique; all PSD data reported in this report are averages of six measurements taken from two different aliquots.

\subsubsection{Rheology}

Rheological characterizations were performed in accordance with RPL-COLLOID-02, Rev. 2.0 using a Haake RS600 rheometer operated with RheoWin Pro 2.96 Software (Thermo Electron Corporation). The RS600 rheometer is equipped with a low-inertia torque motor and a coaxial cylinder measurement geometry. The drive shaft of the motor is centered by an air bearing that ensures an almost frictionless transmission of the applied torque to the sample. Unless specified otherwise, all rheological analyses were conducted at $25^{\circ} \mathrm{C}$. Samples were gently shaken by hand to ensure complete mixing before introducing them into the measuring device. Before any flow curves were measured, a pre-shear at a constant $250 \mathrm{~s}^{-1}$ rate for 3 min was performed on all simulant samples. Flow curves were obtained by shearing the sample at a controlled rate from zero to $1000 \mathrm{~s}^{-1}$ for $5 \mathrm{~min}$, holding constant at $1000 \mathrm{~s}^{-1}$ for 
$1 \mathrm{~min}$, and then shearing at a controlled rate from $1000 \mathrm{~s}^{-1}$ to zero for $5 \mathrm{~min}$. This procedure was performed twice, and the second measurement was used for data analysis. Where possible, the down ramp from 800 to $250 \mathrm{~s}^{-1}$ was used to evaluate the flow curves.

Understanding and interpreting the flow curves is critical to assessing the rheology of the non-Newtonian simulants used in Phase II. For these measurements, non-elastic flow of non-Newtonian materials is characterized with rotational viscometry. The goal of rotational viscometry is measurement of a material's flow curve, which describes the shear stress response, $\tau$, as a function of applied shear rate, $\dot{\gamma}$ (also called the rate-of-strain). The result of a flow curve measurement is a set of $\tau$ versus $\dot{\gamma}$ measurements, which are called flow curve data. Flow curve data can be interpreted with several constitutive equations that relate viscous stress to shear rate. Such analysis allows the flow behavior over a broad range of conditions to be described with just a few rheological descriptors (e.g., viscosity, yield stress, consistency, and flow index).

Flow curves were measured using a concentric cylinder rotational viscometer operated in controlled rate mode. These types of viscometers operate by placing a given volume of test sample into a measurement cup of known geometry. A cylindrical rotor attached to a torque sensor is lowered into the sample until the slurry is even with, but does not cover, the top of the rotor. For a given concentric cylinder geometry, both the radius and height of the rotor are known such that the gap distance between cup and rotor and surface area of fluid contact can be determined. In addition, the top and bottom of the rotor have recessed surfaces such that the fluid only contacts the radial surfaces of the rotor. A filled rotor-in-cup test geometry is shown in Figure 3.1. Fluid flow properties of a sample are determined by spinning the rotor at a known rotational speed, $\Omega$, and measuring the resisting torque, $M$, acting on the rotor. Because fluid only contacts the rotor on the radial surfaces of rotation, all of the force resisting steady-state rotation can be ascribed to shearing of the fluid in the cup-rotor gap. Assuming an isotropic fluid and cup and rotor dimensions as shown in Figure 3.1, the torque acting on the rotor can be directly related to the shear stress at the rotor using the equation,

$$
\tau=\frac{M}{2 \pi H R_{I}^{2}}
$$

Shear stress is measured in units of force per area $\left[\mathrm{N} / \mathrm{m}^{2}\right]$. Calculation of the fluid shear rate at the rotor is complicated by the fact that shear rate depends on both on the measurement system geometry and the fluid rheological properties. For the simplest fluids (i.e., Newtonian fluids) the shear rate of the fluid at the rotor can be calculated given the geometry of the cup rotor shear (see Figure 3.1) by using the equation,

$$
\dot{\gamma}=\left(\frac{2 R_{O}^{2}}{R_{O}^{2}-R_{I}^{2}}\right) \Omega
$$




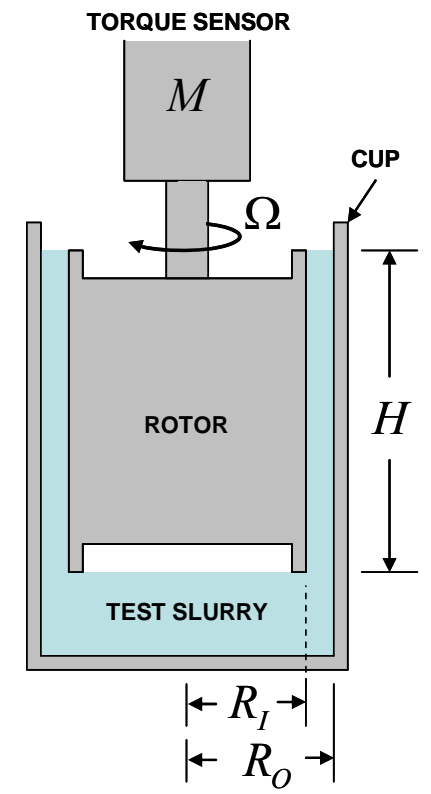

Figure 3.1. Rotor and Cup Geometry Used in Rotational Viscometry Testing in Phase II

Here, shear rate has units of inverse seconds [1/s]. Calculation of shear rate for materials showing more complex shear stress versus shear rate behavior (i.e., non-Newtonian fluids) requires input of flow curve parameters (e.g., yield stress and degree of shear-thinning or shear-thickening). Typically, because the required input parameters are not known prior to measurement, this requirement is circumvented by using a cup and rotor system with a small gap $(\sim 1 \mathrm{~mm})$ such that shear rate effects introduced by fluid properties are minimized. For these systems, Equation (3.4) provides an accurate determination of shear rate for non-Newtonian materials.

The resistance of a fluid to flow can also be described in terms of the fluid's apparent viscosity, $\eta_{\text {app}}$, which is defined as the ratio of the shear stress to shear rate:

$$
\eta_{a p p}=\frac{\tau}{\dot{\gamma}}
$$

The units of apparent viscosity are $\mathrm{Pa} \cdot \mathrm{s}$; however, viscosity is typically reported in units of centipoise (cP; where $1 \mathrm{cP}=1 \mathrm{mPa} \cdot \mathrm{s})$.

The flow curve data provided in this section and in Appendix E are presented in plots of $\tau$ as a function of $\dot{\gamma}$. As stated above, flow curve data can be interpreted with several constitutive equations (i.e., flow curves), allowing characterization of that data with just a few rheological descriptors. The behaviors of the slurries have been described by the Bingham plastic flow curve equation for the simulants used in Phase II testing. 
Bingham plastics are fluids that show finite yield points. This stress (i.e., the yield stress) must be exceeded before these types of materials flow. Once flow is initiated, the stress response of the material is linear over the rest of the shear rate range. Bingham plastics are described by the expression

$$
\tau=\tau_{o}^{B}+k_{B} \dot{\gamma}
$$

where $\tau_{o}^{B}$ is the Bingham yield index (or stress) and $k_{B}$ is the Bingham consistency index.

Concentrated slurries can show complex flow curve phenomena, including both time-independent and time-dependent behaviors. Figure 3.2 outlines flow behaviors typical of sludge materials. With respect to time-independent behaviors, yield stress materials can be classified by changes in the slope of the equilibrium flow curve after material yield (see Figure 3.2a). Materials that show increasing slope with applied shear rate are considered "shear-thickening" yield materials, whereas materials with decreasing slope are considered to be "shear thinning." Materials with constant slope after yield are referred to as Bingham plastics. These types of flow behavior are time-independent and do not depend on the direction the flow curve is being measured (i.e., the stress response is the same when measured with increasing shear rate or decreasing shear rate). As such, time-independent changes are reversible.

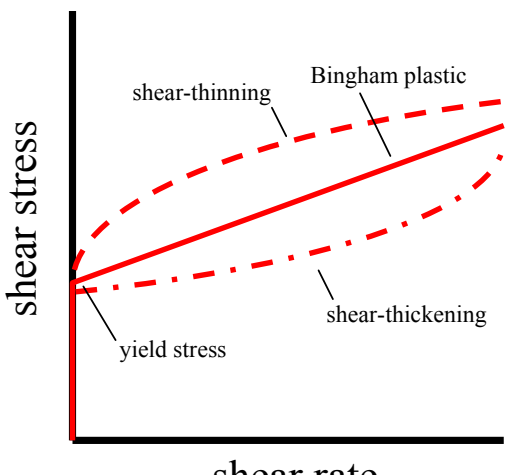

shear rate

(a)

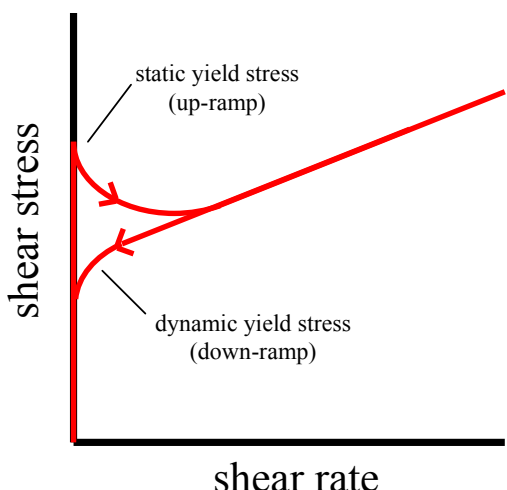

(b)

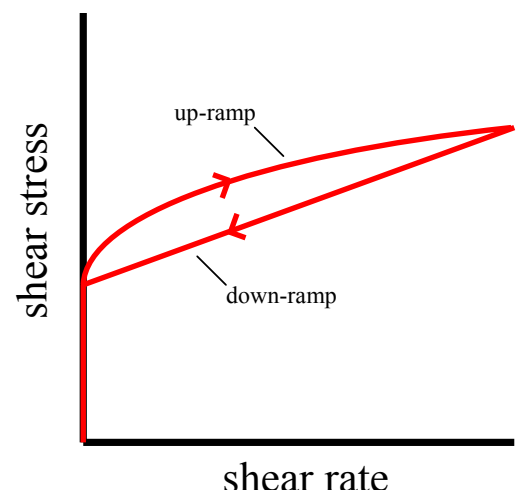

(c)

Figure 3.2. Summary of Flow Curve Behaviors Typically Observed for Concentrated Slurries, Including

(a) Common Time-Independent Behaviors, (b) Static and Dynamic Yield Stress, and

(c) Flow-Curve Hysteresis

Time-dependent flow curve phenomena refer to immediately irreversible (i.e., either short-term or permanent) changes in the stress response of a material. These changes can be caused by the application of shear or may simply occur over time. Time-dependent phenomena can be attributed to breakage of slurry structure, settling of dispersed solids, or changes in the chemistry of slurry components. As shown in Figure 3.2b and Figure 3.2c, time-dependent phenomena can manifest as the different static and dynamic slurry yield stresses and as flow curve hysteresis.

\subsubsection{Selection of Refractive Index for Laser Diffraction Analysis}

The simulants tested in the current application consist of both pure liquids or solutions and suspensions of solid particles in pure liquids or solutions. For the majority of aerosols generated from 
pure liquids or suspensions, determination of aerosol refractive index (RI) is straightforward because RIs for the test solutions are available in the literature. In particular, the RI of water can be easily obtained from general chemistry references.

While RIs are generally available for the liquids and supernates tested, assignment of appropriate values for RI for aerosols generated during spray of slurries is not straightforward. Assigning RIs for slurry systems is difficult because 1) aerosols generated by slurry sprays are heterogeneous composite particles of solids either fully or partially encapsulated in water and 2) the composition of the composite particles is not necessarily equal to that of the test slurry as confinement and jamming of particles at the point of release may alter the composition and in-flight drag or evaporation may strip the aerosol of water content or particles. For the purpose of estimating release fractions, a single RI may be assigned to the system with the understanding that the concentration and size distribution derived from the laser diffraction aerosol instrumentation used in the current testing may not be accurate. One of the following three basic RIs can be assigned: 1) that of the solid component, 2) that of the liquid component, or 3) an effective RI for a particular concentration of solid in liquid. For simple simulants, solid and liquid RIs are generally available in the literature. These values can be used to bound the RI of aerosol composites and are necessary inputs for estimating the effective RI of the composite liquid-solid aerosol.

Estimates of RI for composite solids can be made by using one of several mixing rules available in the literature. These rules are derived for binary mixtures where optical properties of the pure components are known. These rules are:

- Bruggeman Rule (Bruggeman 1935)

$$
f \frac{\epsilon_{1}-\epsilon_{e f f}}{\epsilon_{1}+2 \epsilon_{e f f}}+(1-f) \frac{\epsilon_{2}-\epsilon_{e f f}}{\epsilon_{2}+2 \epsilon_{e f f}}=0
$$

- Maxwell-Garnett Rule (Garnett 1904)

$$
\frac{\epsilon_{e f f}-\epsilon_{2}}{\epsilon_{e f f}+2 \epsilon_{2}}=f \frac{\epsilon_{1}-\epsilon_{2}}{\epsilon_{1}+2 \epsilon_{2}}
$$

- Looyenga Rule (Looyenga 1965)

$$
\epsilon_{e f f}^{1 / 3}=f \epsilon_{1}^{1 / 3}+(1-f) \epsilon_{2}^{1 / 3}
$$

- Monecke Rule (Monecke 1994)

$$
\epsilon_{e f f}=\frac{2\left\{f \epsilon_{1}+(1-f) \epsilon_{2}\right\}^{2}+\epsilon_{1} \epsilon_{2}}{(1+f) \epsilon_{1}+(2-f) \epsilon_{2}}
$$

- Hollow sphere equivalent (Bohren and Huffman 1983)

$$
\epsilon_{e f f}=\epsilon_{1} \frac{(3-2 f) \epsilon_{2}+2 f \epsilon_{1}}{f \epsilon_{2}+(3-f) \epsilon_{1}}
$$


Here, $f$ is the volume fraction of component $1, \varepsilon_{\text {eff }}$ is the effective dielectric constant on the medium, and $\varepsilon_{1}$ and $\varepsilon_{2}$ are the dielectric constants of component 1 and 2, respectively. The dielectric constant is related to the complex refractive index (denoted by $n$ ) by:

$$
\epsilon=n^{2}
$$

Of these five mixing rules, two appear amenable to extension beyond binary systems. These are the Bruggeman and Looyenga mixing rules. From these rules, the following extension to multicomponent systems is proposed:

- Extended Bruggeman Rule

$$
\sum_{i} f_{i} \frac{\epsilon_{i}-\epsilon_{e f f}}{\epsilon_{i}+2 \epsilon_{e f f}}=0
$$

- Extended Looyenga Rule

$$
\epsilon_{\text {eff }}^{1 / 3}=\sum_{i} f_{i} \epsilon_{i}^{1 / 3}
$$

Here, $f_{i}$ and $\varepsilon_{i}$ are the volume fraction and dielectric constant of component $i$, respectively. These multicomponent rules produce complex RIs that are in reasonable agreement with results produced by nesting the binary rules to produce RI estimates for complex mixtures of particles.

The test matrix for the large-scale Phase II effort includes water and two double component solid slurries. The following approach was used to determine RIs for this diverse array of test mixtures:

1. Using standard chemical references, determine the RI for all solid components, pure liquids, and salt solutions. For the salt solutions, the dissolved component concentration was measured directly or determined by mass balance.

2. Determine the composition of all test mixtures in terms of volume fraction by mass balance or available documentation of mixture chemical makeup.

3. Based on the composition determined in step 2 and the pure component and suspending phase RIs assigned in step 1, calculate the RI of the mixture using the appropriate set of mixing rules (i.e., Equations (3.7) to (3.14)). The general approach used was to apply all of these mixing rules (five for binary systems and two for multicomponent mixtures) to a given system. It should be noted that each mixing rule produces a slightly different RI result; however, differences in the RI produced by application of different mixing rules to the same chemical mixture typically occur in the third decimal place, and as such, analysis of Malvern Insitec-S data using the different RI will not produce a noticeable difference in final aerosol concentration or PSD results. To produce a single RI result for analysis, the final composite RI for the mixture was calculated as the arithmetic average of the RI results produced by the individual mixing rules.

RIs for all pure liquid and solid components and test slurries are presented in Table 3.4. In general, the absorption index (i.e., the imaginary component) of pure solids is not available in the literature. However, unless the solid particles are spherical, the imaginary component may be generally taken to be non-zero. Likewise, even spherical aerosol droplets of supernatant that contain non-spherical insoluble 
particles also will have a non-zero absorption index. Because the exact value of solid particle absorption index is not known, it has been set to 1.000 for all solid particles, such that they are represented (in terms of Mie theory) as being completely opaque. Proper assignment of the solid particle absorption index is not expected to strongly impact the size distribution result, as the diffraction result above $1 \mu \mathrm{m}$ is expected to be robust with respect to order-of-magnitude changes in absorption index, as long as the RI is reasonable for non-spherical particles (i.e., non-zero and on the order of 0.01 to 1 ).

Table 3.4. Refractive Indices for Simulants Used in Large-Scale Spray Leak Testing. The RI of pure components has been taken from reference sources available in the literature. RIs for mixtures have been calculated using both binary and multicomponent mixing rules. Both the real (RI) and the imaginary (i.e., the absorption index) components of the complex RI are given. All RIs are listed to three decimal places to demonstrate the value of RIs provided to the Malvern Insitec-S software. Real RI values are typically significant to two figures, whereas imaginary RI values are significant to one figure.

\begin{tabular}{|c|c|c|c|}
\hline Component & Method $^{(a)}$ & $\begin{array}{l}\text { Refractive } \\
\text { Index (Real) }\end{array}$ & $\begin{array}{l}\text { Absorption Index } \\
\text { (Imaginary Refractive } \\
\text { Index Component) }\end{array}$ \\
\hline \multicolumn{4}{|c|}{ Pure Liquids and Solutions } \\
\hline Water & Reference & 1.330 & 0.000 \\
\hline \multicolumn{4}{|c|}{ Pure and Mixed Solids } \\
\hline Kaolin-Bentonite Solids & Calculated & 1.592 & 1.000 \\
\hline \multicolumn{4}{|c|}{ Slurry Systems } \\
\hline 30 Pa Clay & Calculated & 1.378 & 0.148 \\
\hline 6 Pa Clay & Calculated & 1.389 & 0.119 \\
\hline \multicolumn{4}{|c|}{ Slurry System Suspending Phase } \\
\hline 30 Pa Clay & Reference & 1.330 & 0.000 \\
\hline 6 Pa Clay & Reference & 1.330 & 0.000 \\
\hline $\begin{array}{l}\text { (a) Method: 1) Reference means } \\
\text { and 2) calculated means the ir } \\
\text { mixing rules (two for binary s }\end{array}$ & $\begin{array}{l}\text { m a referen } \\
\text { known con } \\
\text { icomponent }\end{array}$ & $\begin{array}{l}\text { hemical handb } \\
\text { nent indices an } \\
\text { tems). }\end{array}$ & $\begin{array}{l}\text { ok or literature source } \\
\text { averaging of all }\end{array}$ \\
\hline
\end{tabular}

\subsubsection{Newtonian Simulant}

The Newtonian simulant used in large-scale testing was tap water from the Richland, Washington, municipal water system. Table 3.5 documents the physical properties of water; these properties were included in all data collected corresponding to Newtonian aerosol testing. During Phase I testing, the surface tension of the tap water was measured as $72.25 \pm 0.07 \mathrm{mN} \cdot \mathrm{m}^{-1}$, which agrees with values documented in the literature. Surface tension measurements for tap water were not repeated for the Phase II tests. 
Table 3.5. Newtonian Simulant Properties for Large-Scale Aerosol Tests

\begin{tabular}{|c|c|}
\hline Property & Water \\
\hline Target solution density $(\mathrm{kg} / \mathrm{L})$ & $0.998^{(\mathrm{a})}$ \\
\hline Reference surface tension $(\mathrm{mN} / \mathrm{m})$, at $25^{\circ} \mathrm{C}$ & $71.99 \pm 0.36^{(\mathrm{b})}$ \\
\hline Tap water surface tension $(\mathrm{mN} / \mathrm{m})$, at $25^{\circ} \mathrm{C}$ & $72.25 \pm 0.07^{(\mathrm{c})}$ \\
\hline Target viscosity $(\mathrm{mPa} \cdot \mathrm{s})$ & $1.002^{(a)}$ \\
\hline \multicolumn{2}{|c|}{$\begin{array}{l}\text { (a) Water bulk density and viscosity at } 20^{\circ} \mathrm{C} \text { from the } C R C \text { Handbook, } 56^{\text {th }} \text { Edition } \\
\text { pp. F- } 11 \text { and F- } 47.1 \mathrm{mPa} \cdot \mathrm{s}=1 \mathrm{cP} \text {. } \\
\text { (b) J.Phys. Chem. Ref. Data Vol. } 12 \text {, No. } 3,1983 \text {. } \\
\text { (c) Measured during Phase I testing, error given is the standard deviation. }\end{array}$} \\
\hline
\end{tabular}

\subsubsection{Non-Newtonian Simulant}

The physical properties for both non-Newtonian clay simulants used during large-scale aerosol testing are given in Table 3.6. The clay simulants were prepared in water provided by the City of Richland as described in Chapter 5 of this report.

The rheological targets for both the clay simulants were readily achieved during large-scale aerosol testing with minor adjustments usually resulting from simulant dehydration due to tank head space or dilution resulting from clay recycling activities. The average values reported in Table 3.6 have been generated from the daily samples that were collected each day of clay testing to ensure that the targeted rheological properties were being met. Ten samples were taken and averaged for the $30 \mathrm{~Pa}$ Clay, while nine samples were averaged for the 6 Pa Clay. The error values reported in Table 3.6 are the tolerance limits, which for the purposes of this report are defined as twice the standard deviation of the data set. Typical rheograms obtained for the $30 \mathrm{~Pa}$ Clay simulant are given in Figure 3.3 and Figure 3.4. Figure 3.3 shows the initial rheogram of the unused $30 \mathrm{~Pa}$ Clay simulant for the first batch of clay used during testing. The second rheogram shown in Figure 3.4 was taken from the second batch of clay simulant. The second batch of clay was reclaimed from the test chamber and reused for subsequent tests after its initial use. Typical rheograms for the $6 \mathrm{~Pa}$ Clay simulant have also been given in Figure 3.5 and Figure 3.6, again Batch 2 was made from reclaimed clay slurry.

The measured bulk densities for both clay simulants are in agreement with the calculated theoretical densities and are consistent with the measured weight percent of total solids. The surface tension of the simulant supernates was measured after centrifuging at 20,200 relative centrifugal force (RCF). The resulting supernates were yellow, which suggests that soluble organic compounds leached from the clay simulants. The surface tension measured for the supernates from both clay simulants was found to be slightly less than that of tap water. The values appear to be within the method error. 
Table 3.6. Non Newtonian Simulant Properties for Aerosol Tests

\begin{tabular}{lcc}
\hline \multicolumn{1}{c}{ Property } & 30 Pa Clay & 6 Pa Clay \\
\hline Measured wt \% TS $(\%)$ & $32.32 \pm 0.64^{(\mathrm{a}, \mathrm{b})}$ & $27.10 \pm 1.40^{(\mathrm{a}, \mathrm{c})}$ \\
Calculated slurry density $(\mathrm{kg} / \mathrm{L}), 20^{\circ} \mathrm{C}$ & 1.242 & 1.195 \\
Measured slurry density $(\mathrm{kg} / \mathrm{L}), 20^{\circ} \mathrm{C}$ & $1.249 \pm 0.009^{(\mathrm{a}, \mathrm{b})}$ & $1.201 \pm 0.012^{(\mathrm{a}, \mathrm{c})}$ \\
Surface tension, Supernate $(\mathrm{mN} / \mathrm{m}), 25^{\circ} \mathrm{C}$ & $71.91 \pm 0.10^{(\mathrm{a}, \mathrm{d})}$ & $71.66 \pm 0.03^{(\mathrm{a}, \mathrm{d})}$ \\
Bingham yield stress $(\mathrm{Pa}), 25^{\circ} \mathrm{C}$ & $25.51 \pm 3.12^{(\mathrm{a}, \mathrm{b})}$ & $6.72 \pm 1.49^{(\mathrm{a}, \mathrm{c})}$ \\
Bingham consistency $(\mathrm{mPa} \cdot \mathrm{s}), 25^{\circ} \mathrm{C}$ & $29.72 \pm 6.01^{(\mathrm{a}, \mathrm{b})}$ & $12.012 \pm 1.71^{(\mathrm{a}, \mathrm{c}))}$ \\
\hline
\end{tabular}

(a) Error analysis is calculated at the tolerance limit, defined as twice the standard deviation.

(b) Average of ten samples, one sample collected each day of testing.

(c) Average of nine samples, one sample collected on each day of testing.

(d) Average of two measurements on the same sample aliquot.

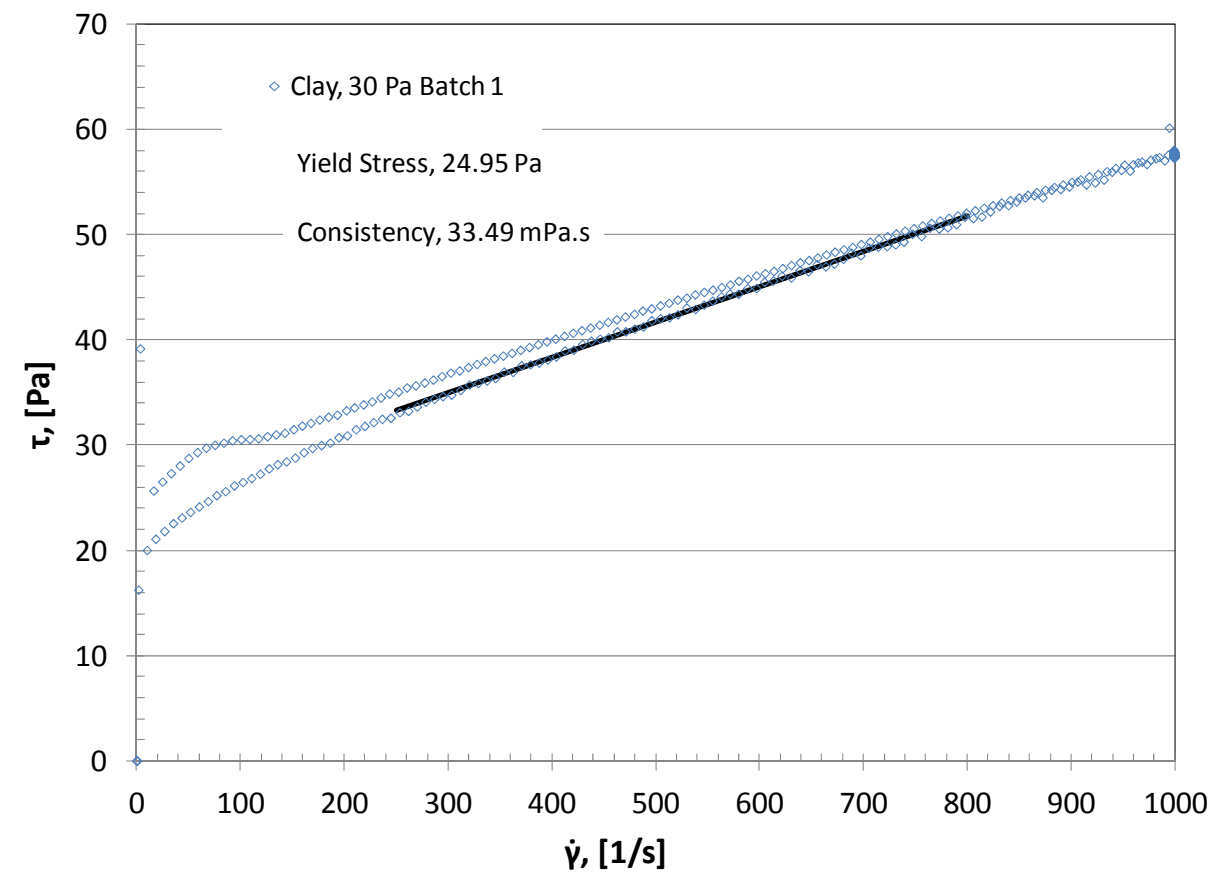

Figure 3.3. Flow Curve of the $30 \mathrm{~Pa}$ Clay Batch 1Simulant Prior to Testing. The Bingham parameters were analyzed from the down-ramp between 250 and $800 \dot{\gamma}$. 


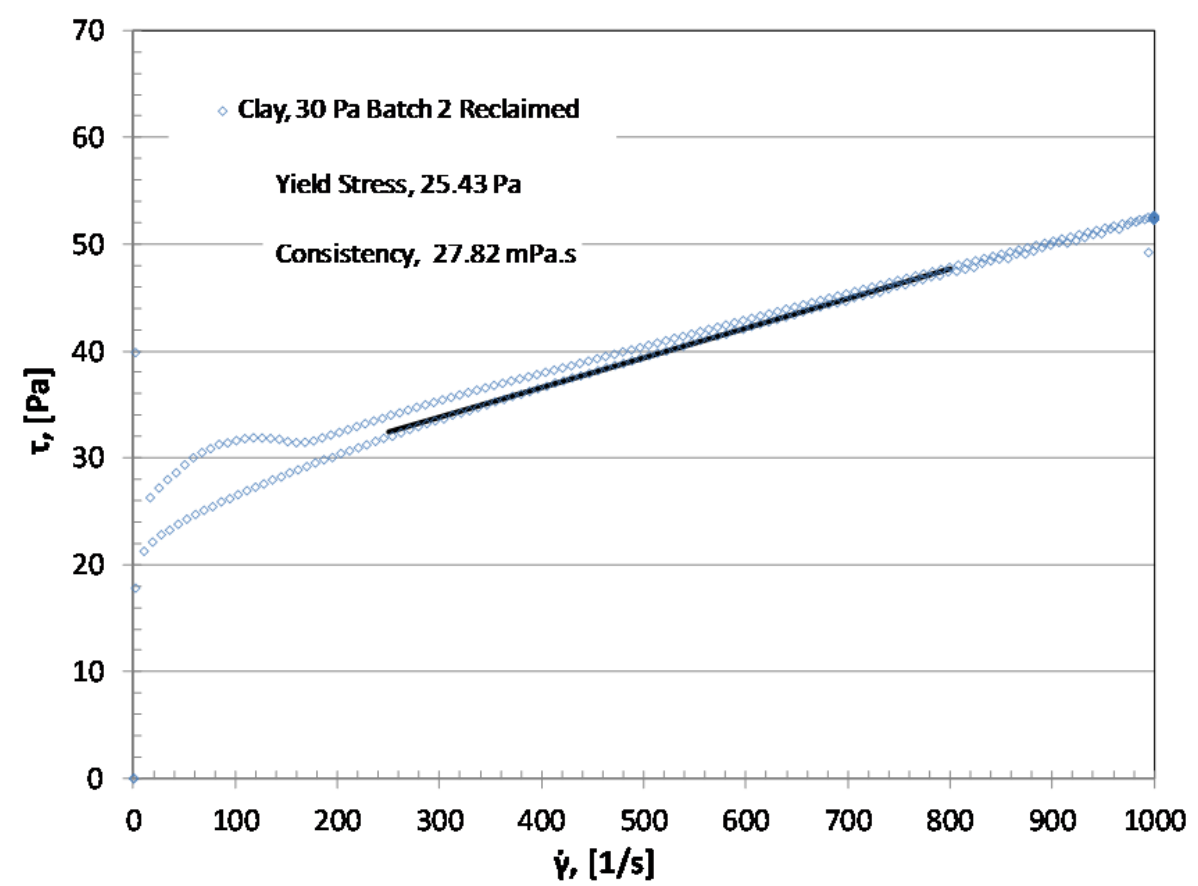

Figure 3.4. Flow Curve of the 30 Pa Clay Batch 2 Reclaimed Simulant. The Bingham parameters were analyzed from the down-ramp between 250 and $800 \dot{\gamma}$.

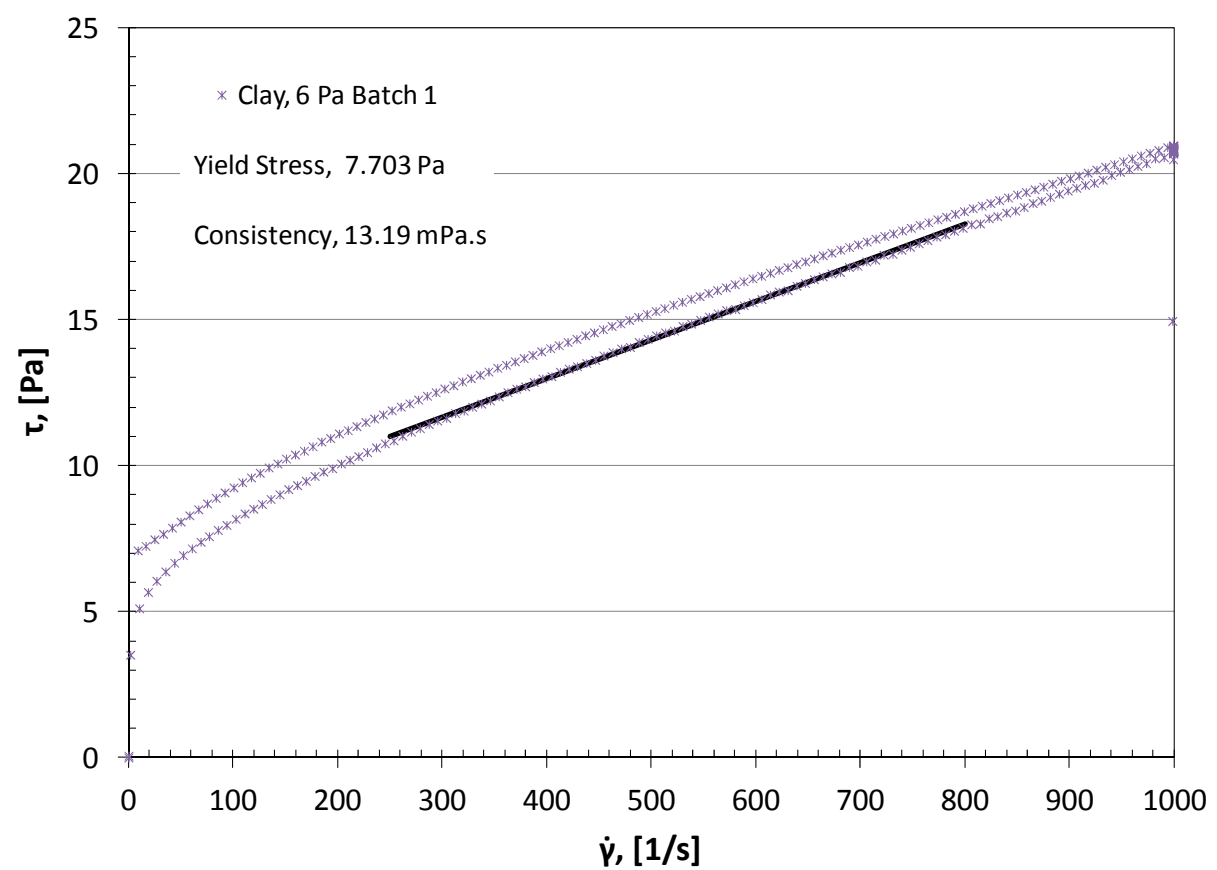

Figure 3.5. Flow Curve of the 6 Pa Clay Batch 1. The Bingham parameters were analyzed from the down-ramp between 250 and $800 \dot{\gamma}$. 


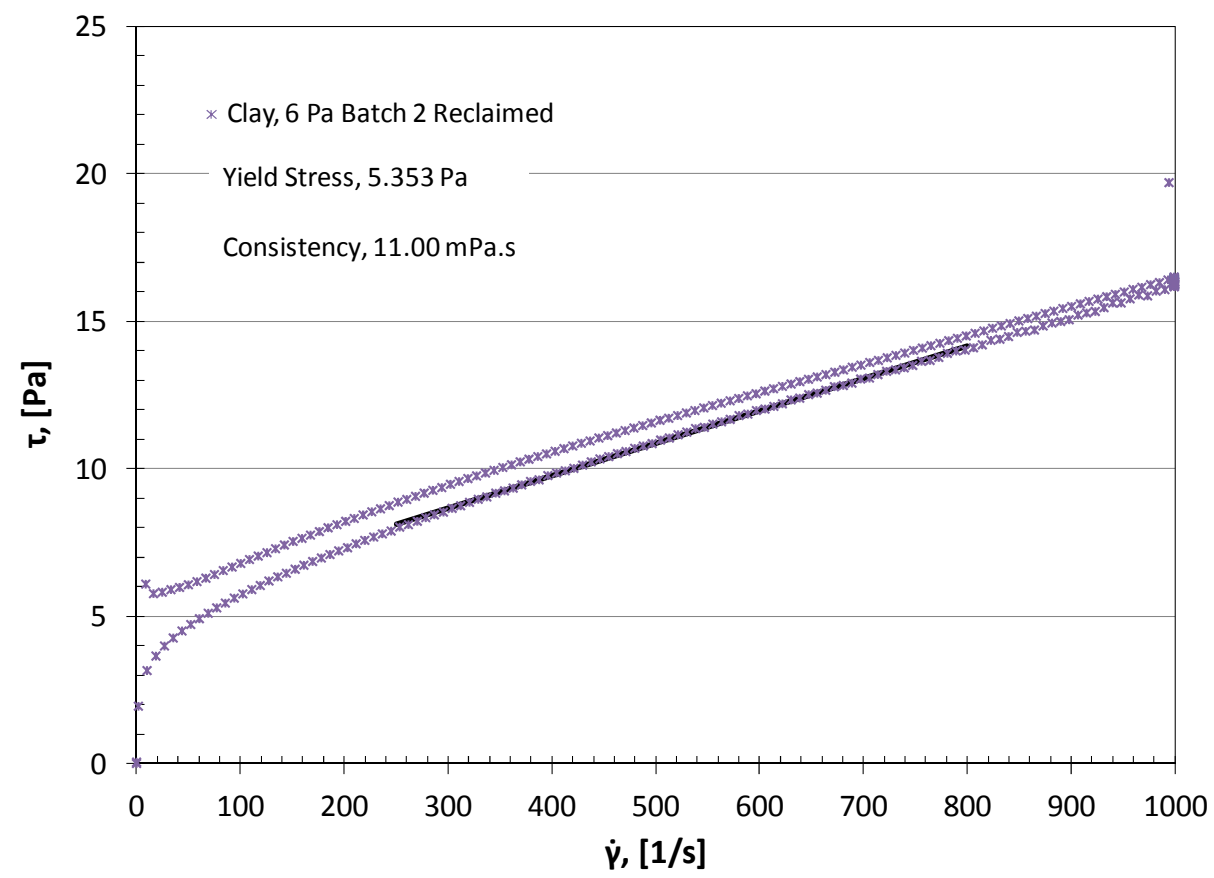

Figure 3.6. Flow Curve of the 6 Pa Clay Batch 2 Reclaimed Simulant. The Bingham parameters were analyzed from the down-ramp between 250 and $800 \dot{\gamma}$.

A select number of percentiles are presented in Table 3.7 for both unsonicated clay simulants. The cumulative volume percent undersize PSD for both simulants unsonicated and post sonication is shown in Figure 3.7 and the differential PSD of the unsonicated material is given in Figure 3.8. The PSD analysis was measured under conditions of flow and sonication, as well as unsonicated and post-sonicated.

Sonication did not appear to have any impact on the PSD of either clay simulant, suggesting that the clay was well hydrated and the PSD results represent primary particle sizes. The particle size data presented here is the average of 12 and 15 measurements for the $30 \mathrm{~Pa}$ Clay and $6 \mathrm{~Pa}$ Clay, respectively, taken from multiple samples collected on different days.

Table 3.7. Summary of Selected Percentile Values for Measurements Taken at 1 min Recirculation Time, for Unsonicated, 30 Pa Clay and 6 Pa Clay Simulants

\begin{tabular}{|c|c|c|}
\hline Percentiles & $\begin{array}{c}30 \text { Pa Clay } \\
\sim 32 \mathrm{wt}^{\circ} \% \\
\text { Unsonicated }^{(\mathrm{a})}\end{array}$ & $\begin{array}{c}6 \text { Pa Clay } \\
\sim 27 \mathrm{wt}^{\circ} \% \\
\text { Unsonicated }^{(\mathrm{a})}\end{array}$ \\
\hline $\mathrm{d}(0.01)$ & 0.35 & 0.35 \\
\hline $\mathrm{d}(0.05)$ & 0.60 & 0.60 \\
\hline $\mathrm{d}(0.10)$ & 0.92 & 0.92 \\
\hline $\mathrm{d}(0.25)$ & 2.06 & 2.07 \\
\hline $\mathrm{d}(0.50)$ & 5.13 & 5.13 \\
\hline $\mathrm{d}(0.75)$ & 13.0 & 12.9 \\
\hline $\mathrm{d}(0.90)$ & 28.3 & 27.6 \\
\hline $\mathrm{d}(0.95)$ & 44.4 & 42.8 \\
\hline $\mathrm{d}(0.99)$ & 93.4 & 88.4 \\
\hline
\end{tabular}

(a) Average of 12 measurements taken from four samples.

(b) Average of 15 measurements taken from five samples. 


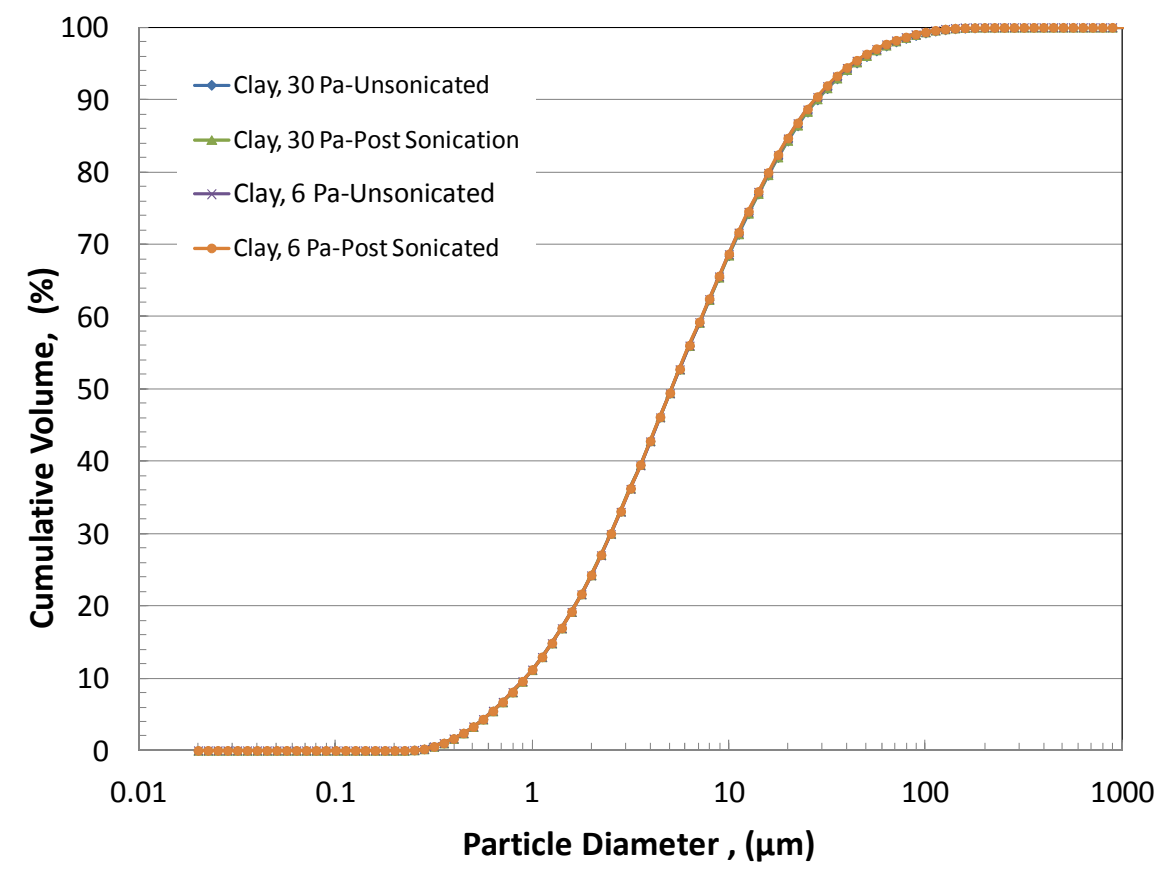

Figure 3.7. Cumulative Volume Percent Undersize for Unsonicated and Post Sonication $30 \mathrm{~Pa}$ Clay and 6 Pa Clay

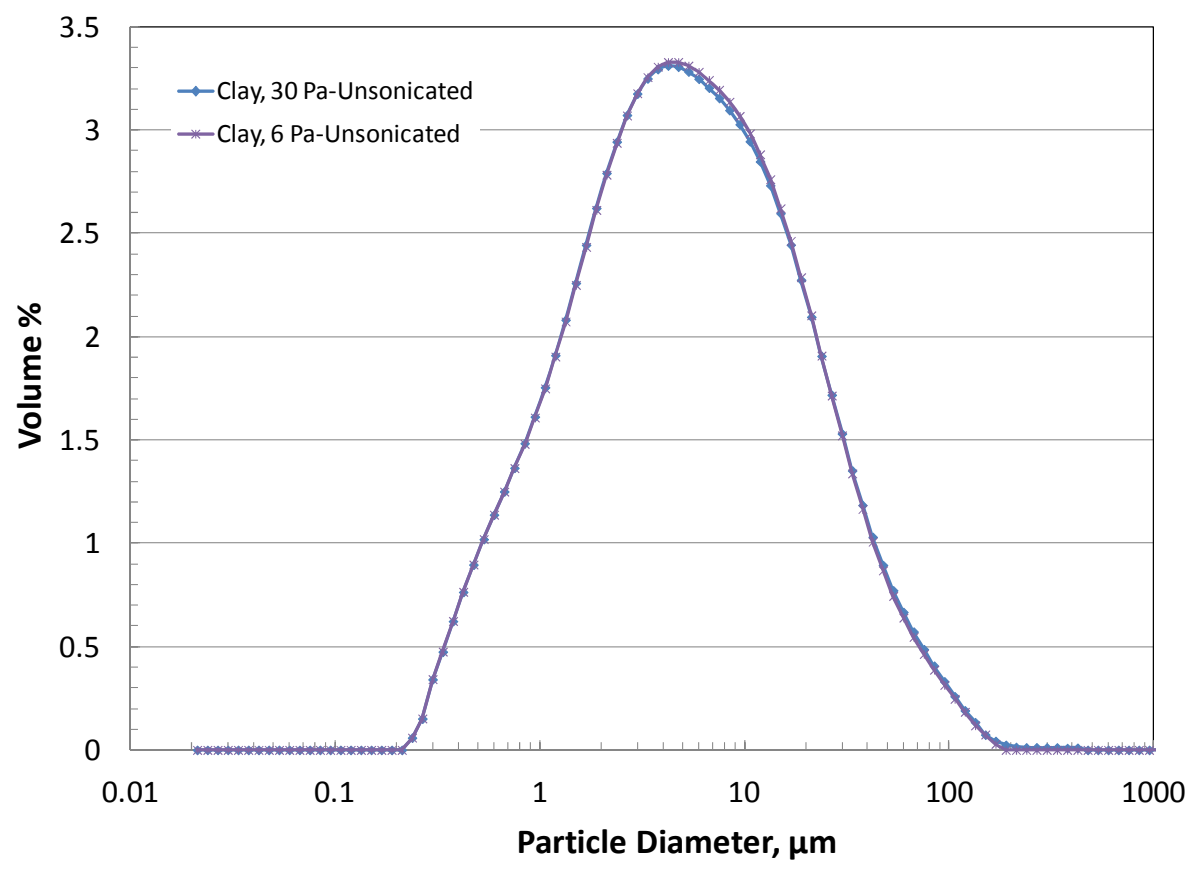

Figure 3.8. Differential Particle Size Distribution for Unsonicated and Post Sonication 30 Pa Clay and 6 Pa Clay 


\subsection{Test Equipment and Instrumentation}

This chapter briefly describes the large-scale test system equipment and the key instrumentation used to measure aerosol concentration and size distribution during Phase II testing. Where applicable, we refer readers to the Phase I large-scale spray release testing report (Schonewill et al. 2012) for detailed descriptions of test equipment and instrumentation.

\subsection{Test System}

The large-scale test system was designed and built to accomplish the test objectives discussed in Section 1.3 of this report. The Phase II design provided additional flexibility in chamber size relative to the Phase I test configuration, allowing chamber lengths of 5 to $39 \mathrm{ft}$ in total length while maintaining the same cross-sectional chamber area used in Phase I testing. Phase II tests employed the same spool system to accommodate a broad range of test orifices. In addition, two measurement approaches to accurately measure the volumetric spray rate generated by high-pressure flows through the test orifices were implemented by collecting data from 1) load cells on the test simulant feed and storage vessels to monitor loss of fluid from the circulation loop as a result of the spray and 2) Coriolis flow meters upstream and downstream of the orifice to directly measure the loss of fluid through an orifice by flow rate differences during testing.

A schematic of the large-scale test system designed and built in Laboratory 184 of the Applied Process and Engineering Laboratory (APEL) is shown in Figure 4.1. The system consists of the following major components: 1) flow loop, 2) test chamber, 3) aerosol instrumentation, 4) general instrumentation, and 5) data acquisition systems (DAS). This section briefly summarizes the design and operating characteristics of these large-scale test system components; a full description of these components may be found in Schonewill et al. (2012).

\subsection{Flow Loop}

The flow loop and associated instrumentation, detailed through the Process and Instrumentation Diagram (P\&ID) in Figure 4.2, is composed of the primary loop section, which includes the breached pipe, three variable speed centrifugal pumps in series, two Coriolis flow meters, pressure sensors (PSs), resistance temperature detectors (RTDs), load cells, and ancillary equipment such as 500-gal simulant feed/storage tanks, transfer pump, and agitators. The loop was designed to produce sprays from prefabricated spool pieces, called "test sections," with prototypic breach sizes varying from the smallest to the largest postulated breach in a 3 in. schedule 40 pipe. Except for the largest breach/orifice $(2.74 \times 76 \mathrm{~mm})$, the loop can maintain fluid velocities at or above $6.5 \mathrm{ft} / \mathrm{s}$ with pressures from 100 to 380 psig during sprays.

During spray release tests, simulant was delivered into the closed loop via a 3 in. flexible hose linking the bottom of the feed tank (TK-2) to the inlet of the first pump (P-1), through the upstream Coriolis flow meter (FE-1), the next two pumps in series (P-2 and P-3), passing through the test section (bypass leg not used) before entering the downstream Coriolis flow meter (FE-2), and returning to the feed tank (TK-2). 


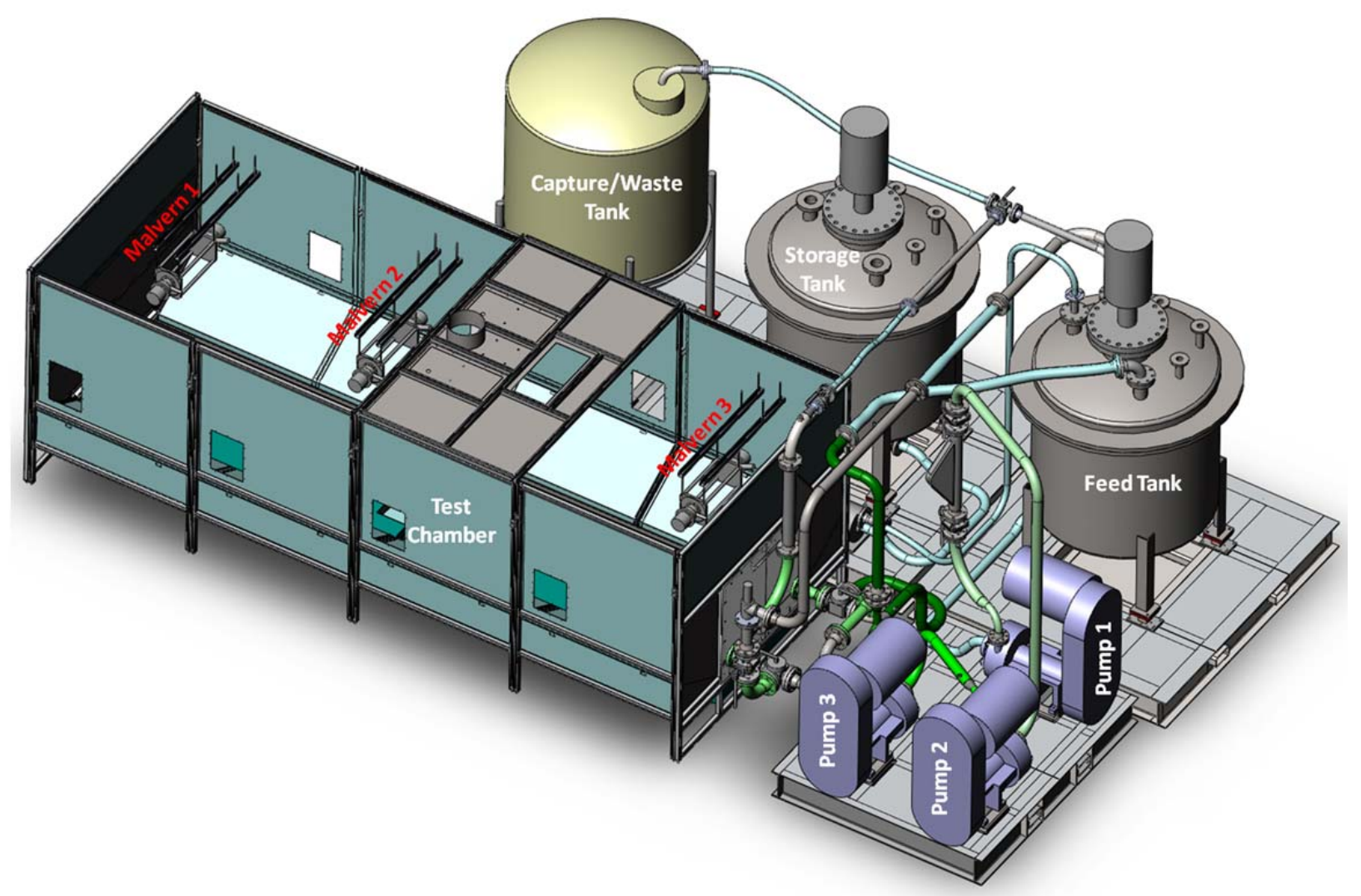

Figure 4.1. Schematic of the Large-Scale Test System

In addition to the primary flow system, varying lengths of extension piping were added to the flow loop to achieve $3.5,19,28$, and $38 \mathrm{ft}$ nominal distances from the spray orifice to the back wall (or splash wall) in the $39 \mathrm{ft}$ chamber to evaluate the effect of spray distance and distance from the splash wall on the aerosol concentration and PSDs.

Fluid velocities greater than $6.5 \mathrm{ft} / \mathrm{sec}$ and pressures of up to $380 \mathrm{psig}$ at the test section were achieved using three Krebs millMAX centrifugal pumps connected in series and controlled using Honeywell variable frequency drives (VFDs) in a slave/master configuration to match the frequency of the upstream or master pump. Pressure in the loop was regulated using two globe valves (GV-1 and GV-2 in Figure 4.2) that were located downstream of the test/bypass sections. Multiple RTDs and absolute pressure transducers were installed in the system to monitor temperature and pressure conditions, respectively.

The flow rate through the recirculation loop was measured both upstream and downstream of the spray orifice using two Coriolis mass flow meters (FE-1 and FE-2 in Figure 4.2). The location of the flow meters allowed the spray leak flow rate to be determined from the differences in the flow rates during the spray release tests. Alternatively, the spray leak flow rate also was determined by measuring the feed tank (TK-2) mass change indicated by load cells. The feed tank (TK-2), the storage tank (TK-1), and the capture/waste tank (TK-3) all were mounted on individual load cells so mass changes in each tank could be monitored. 


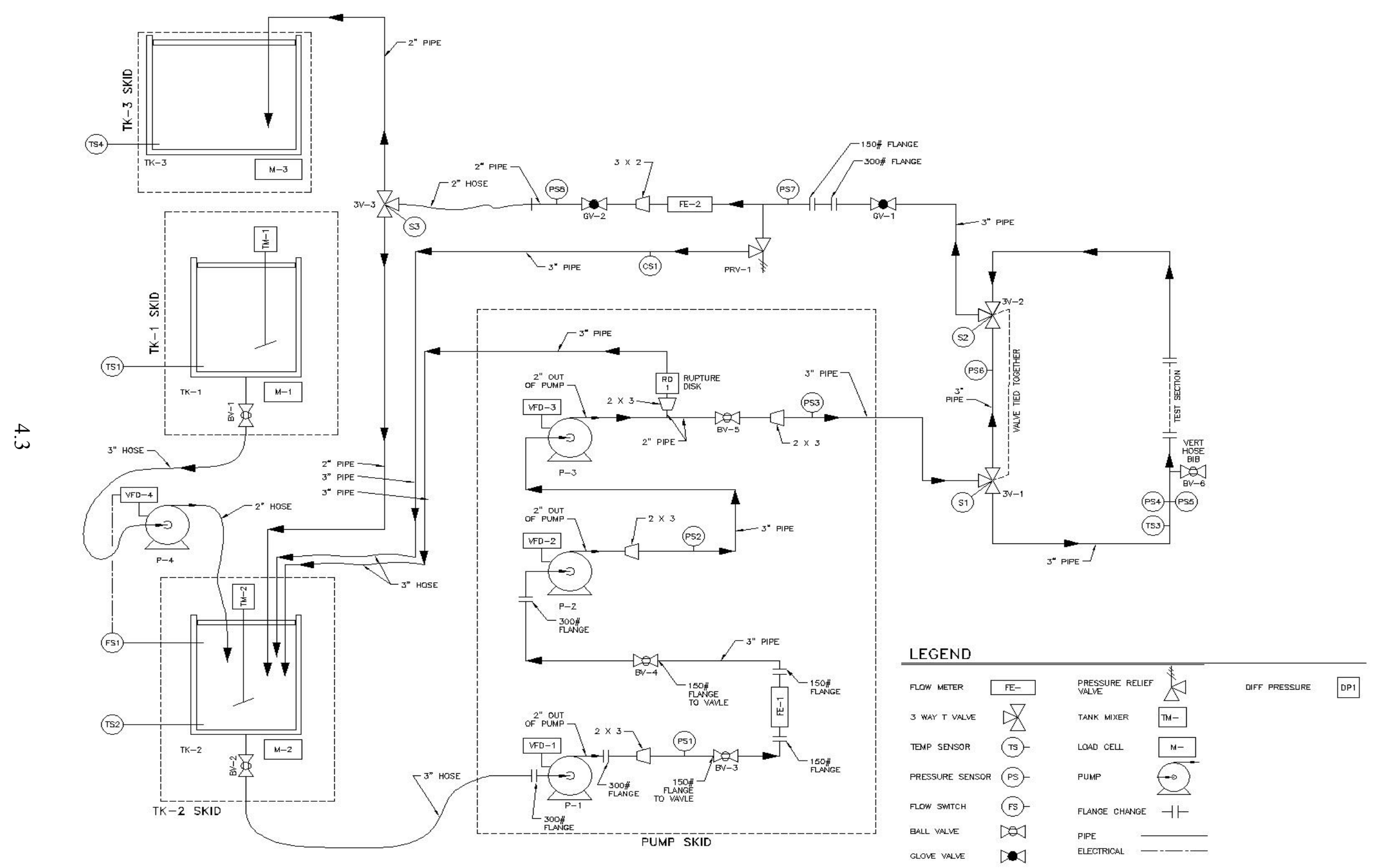

Figure 4.2. P\&ID Diagram of the Large-Scale Spray Release Test System 
The working capacity of the feed (TK-2) and storage (TK-1) tanks was approximately 500 gal each, and $2 \mathrm{hp} \mathrm{mixers} \mathrm{each} \mathrm{connected} \mathrm{to} \mathrm{a} \mathrm{VFD} \mathrm{provided} \mathrm{agitation} \mathrm{for} \mathrm{each} \mathrm{tank.} \mathrm{These} \mathrm{two} \mathrm{tanks} \mathrm{included}$ diffusers designed to spread the returning fluid horizontally and minimize vortexing, were baffled to improve mixing, and contained internal cooling coils to maintain a constant temperature during the tests. The feed tank (TK-2) served as the primary simulant feed source to the recirculation loop during testing, while the storage tank (TK-1) supplied makeup fluid to the feed tank (TK-2) via a transfer pump. The makeup fluid from the storage tank allowed operators to maintain the hydrostatic head in the feed tank (TK-2) within a few inches, thereby providing a constant net positive suction head to the pumps. This operational requirement in Phase I testing was relaxed during Phase II work.

The main function of the capture/waste (TK-3) tank was to support simulant capture and recirculation loop flush operations. The working capacity of the tank was approximately 1000 gal. Also, this vessel could receive the recirculation loop discharge when testing with an open flow loop configuration (if required). Although the open loop configuration was never used during actual test runs, it was always used during flush operations when cleaning the recirculation loop.

\subsection{General Instrumentation}

Several instruments were used to acquire data for the test operating conditions. A P\&ID for the large-scale test configuration showing the various instruments and their locations is provided in Figure 4.2. These instruments also are listed in Table 4.1 along with the relative location, manufacturer, model, and accuracy specifications. The data quality associated with each of these instruments also is listed in Table 4.1. In the table, the entry "NQA-1" in the Data Quality column implies that the data meets the NQA-1 requirements for the project by ensuring that the instrument has been either calibrated by an approved vendor or through approved PNNL procedures. Instruments that are listed under the category "For Information Only" are those that are used to provide information to the test operator.

The type of instrumentation used in Phase II testing did not change from Phase I. However, the manufacturer and model of pressure transducers was changed from an Omega PX440E0-500GI to a Cole-Parmer C206/68073-14 for PS1, PS2, PS4, and PS5 (see Figure 4.2 for locations). Note that the instruments listed in Table 4.1 and the P\&ID in Figure 4.2 do not show the aerosol concentration measurement devices that are included in the spray chamber. These aerosol concentration measurement devices are discussed in Section 4.5. A measuring and test equipment (M\&TE) list that includes calibration information was maintained separately for the project. This list tracked any changes to the instruments that were added or removed from the large-scale test system. Data from the sensors listed in Table 4.1 were logged on a DAS running Allen Bradley programmable logic controller (PLC) data acquisition and control software. The PLC-based DAS was chosen for this application because of its robustness and versatility; that is, it would allow expansion to an automated control system if desired. 
Table 4.1. Instrumentation Used for Process Parameter Measurements

\begin{tabular}{|c|c|c|c|c|c|}
\hline Parameter Measured & Relative Test Location & Manufacturer & Model & Accuracy & Data Quality \\
\hline \multirow{3}{*}{ Weight } & Storage Tank (M-1) & \multirow{3}{*}{ Hardy Instruments } & HI HLPS (18000\#) & $\pm 4.5 \mathrm{lb}$ & NQA-1 \\
\hline & Feed Tank (M-2) & & HI HLPS (18000\#) & $\pm 4.5 \mathrm{lb}$ & NQA-1 \\
\hline & Capture/Waste Tank (M-3) & & HI HLPS (9000\#) & $\pm 2.25 \mathrm{lb}$ & NQA-1 \\
\hline \multirow{7}{*}{ Pressure } & Feed Pump 1 (PS1) & \multirow{4}{*}{ Cole Parmer } & C206/68073-14 & \pm 2.5 PSI & For Information Only \\
\hline & Feed Pump 2 (PS2) & & C206/68073-14 & \pm 2.5 PSI & For Information Only \\
\hline & Test Section (Absolute) (PS4) & & C206/68073-14 & \pm 2.5 PSI & NQA-1 \\
\hline & Test Section (Backup) (PS5) & & C206/68073-14 & \pm 2.5 PSI & NQA-1 \\
\hline & Feed Pump 3 (PS3) & \multirow{3}{*}{ Omega } & PX44E0-500GI & \pm 2.5 PSI & For Information Only \\
\hline & Flow Meter 2 (PS6 and PS7) & & PX44E0-500GI & \pm 2.5 PSI & For Information Only \\
\hline & Globe Valve 2 (PS8) & & PX44E0-1KGI & \pm 5.0 PSI & For Information Only \\
\hline \multirow{3}{*}{ Humidity } & Test Chamber (RH1) & \multirow{3}{*}{ Omega } & \multirow{3}{*}{ HX93DAC-RP1-F } & \multirow{3}{*}{ $\pm 2.5 \% \mathrm{RH}$} & For Information Only \\
\hline & Test Chamber (RH2) & & & & For Information Only \\
\hline & Ambient (RH3) & & & & For Information Only \\
\hline \multirow{6}{*}{ Temperature } & Test Chamber (TS1) & \multirow{6}{*}{ Omega } & \multirow{6}{*}{ PR-13-2-100-1/4 } & \multirow{6}{*}{ $\pm 1^{\circ} \mathrm{F}$} & NQA-1 \\
\hline & Ambient (TS2) & & & & NQA-1 \\
\hline & Storage Tank (TS3) & & & & NQA-1 \\
\hline & Feed Tank (TS4) & & & & NQA-1 \\
\hline & Capture/Waste Tank (TS5) & & & & NQA-1 \\
\hline & Test Section (TS6) & & & & NQA-1 \\
\hline \multirow[b]{2}{*}{ Flow Rate } & Downstream of Pump 1 (FE-1) & \multirow[b]{2}{*}{ MicroMotion } & \multirow[b]{2}{*}{ F300S } & \multirow[b]{2}{*}{ \pm 0.027 gal } & NQA-1 \\
\hline & $\begin{array}{l}\text { Downstream of Primary Pres. } \\
\text { Reg. Valve (FE-2) }\end{array}$ & & & & For Information Only \\
\hline \multirow[b]{2}{*}{ Density } & Downstream of Pump 1 (FE-1) & \multirow[b]{2}{*}{ MicroMotion } & \multirow[b]{2}{*}{ F300S } & \multirow[b]{2}{*}{ $\pm 0.0001 \mathrm{~g} / \mathrm{cm}^{3}$} & NQA-1 \\
\hline & $\begin{array}{l}\text { Downstream of Primary Pres. } \\
\text { Reg. Valve (FE-2) }\end{array}$ & & & & For Information Only \\
\hline
\end{tabular}




\subsection{Phase II Test Chamber Configurations and Spools}

The test/spray chamber design used in Phase I, shown schematically in Figure 4.3, also was used in Phase II testing. However, the chamber length for Phase II tests was extended to include options for 25, 30,34 , and $39 \mathrm{ft}$ total chamber lengths. Aerosols generated from the test section are contained in the test chamber and are characterized to determine the size distribution of aerosol droplets and the total droplet volume concentration as a fraction of the total spray volume.

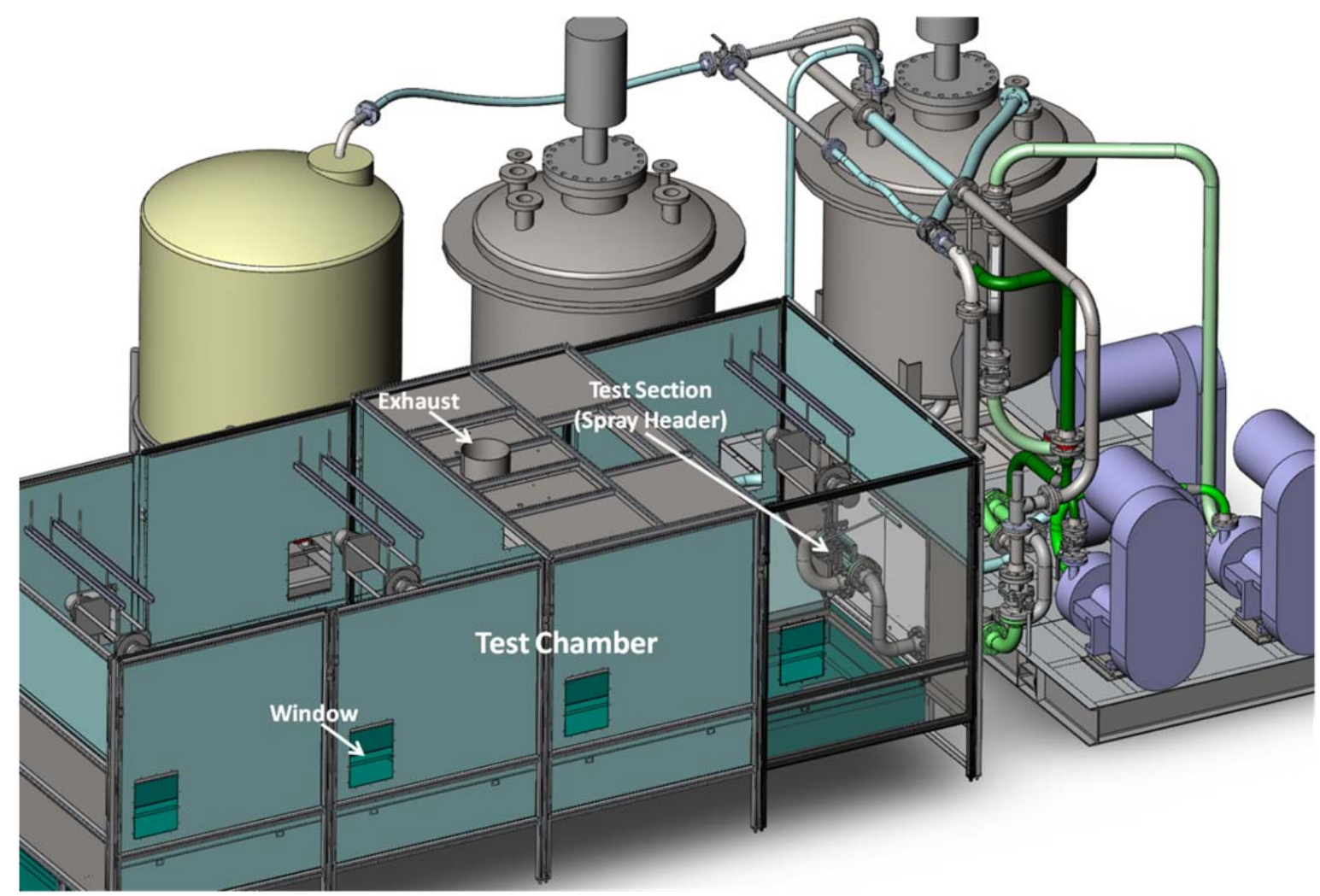

Figure 4.3. Schematic of the Spray Chamber

The spray chamber was designed to meet the following requirements:

- It is adjustable from a minimum size of $\sim 4 \mathrm{ft}$ wide $\times 8 \mathrm{ft}$ high $\times 5 \mathrm{ft}$ long $\left(\sim 160 \mathrm{ft}^{3}\right.$ volume $)$ to a maximum size of $\sim 8 \mathrm{ft}$ wide $\times 8 \mathrm{ft}$ high $\times 39 \mathrm{ft}$ long. The test chamber volume is incrementally adjustable in increments of $5 \mathrm{ft}$ in length (one section is $4 \mathrm{ft}$ for the $39 \mathrm{ft}$ chamber) and $2 \mathrm{ft}$ in width.

- It is constructed of materials (primarily stainless steel sheets) that have minimal affinity for attracting or interacting with aerosols (e.g., through high static charges) generated during the testing.

- Non-aerosolized liquid is directed to a collection vessel.

- It is accessible for mounting aerosol characterization instruments.

- It has viewing ports for visually observing and video/still camera for recording the discharging spray.

- The enclosed volume is calculated easily. 
- It is easily cleaned of simulant materials when not in use.

- It has an exhaust system for clearing/evacuating aerosols between tests.

Throughout testing, the fluid volume that collects at the bottom of the chamber was minimized via sump pump transfer from the test chamber to a tank, tote, or drain. The windows were covered during testing to preclude any potential light interference with the aerosol measuring instruments (discussed in Section 4.5). In the absence of sprays, the back wall (i.e., the splash wall) could be removed to allow easy access to the chamber by operators if needed.

The various spray chamber configurations used during Phase II testing and corresponding chamber volumes are summarized in Table 4.2.

Table 4.2. Spray Chamber Configurations and Chamber Volumes

\begin{tabular}{|c|c|c|c|c|c|}
\hline $\begin{array}{c}\text { Spray } \\
\text { Chamber } \\
\text { Identifier }^{(a)}\end{array}$ & $\begin{array}{c}\text { Spray } \\
\text { Chamber } \\
\text { Length (ft) }\end{array}$ & $\begin{array}{c}\text { Malvern Insitec-S } \\
\text { Instrument Support } \\
\text { Type [Frames(F) or } \\
\text { Spacer Bars (S)] }\end{array}$ & $\begin{array}{c}\text { Number of Malvern } \\
\text { Insitec-S } \\
\text { Instruments in } \\
\text { Chamber }\end{array}$ & $\begin{array}{l}\text { Spray Header } \\
\text { Extension } \\
\text { Configuration }\end{array}$ & $\begin{array}{c}\text { Spray } \\
\text { Chamber } \\
\text { Volume } \\
\left(\mathrm{m}^{3}\right)\end{array}$ \\
\hline $10 \mathrm{~S} 2 \mathrm{~A}$ & 10 & Spacer Bars & 2 & No Extension & 13.7 \\
\hline $20 \mathrm{~S} 3 \mathrm{~A}$ & 20 & Spacer Bars & 3 & No Extension & 27.5 \\
\hline $20 \mathrm{~F} 3 \mathrm{~A}$ & 20 & Frames & 3 & No Extension & 27.5 \\
\hline $30 \mathrm{~S} 3 \mathrm{~A}$ & 30 & Spacer Bars & 3 & No Extension & 41.4 \\
\hline $39 \mathrm{~S} 3 \mathrm{~A}$ & 39 & Spacer Bars & 3 & No Extension & 53.9 \\
\hline 39S3B & 39 & Spacer Bars & 3 & 120" Extension & 53.9 \\
\hline $39 \mathrm{~S} 3 \mathrm{C}$ & 39 & Spacer Bars & 3 & 228" Total Extension & 53.8 \\
\hline 39S3D & 39 & Spacer Bars & 3 & 412" Total Extension & 53.8 \\
\hline $39 \mathrm{~F} 3 \mathrm{~A}$ & 39 & Frames & 3 & No Extension & 53.9 \\
\hline
\end{tabular}

(a) The spray chamber identifier (i.e., chamber configuration) includes five characters that is generically presented as \#\#X\#Y. The first two characters represent chamber length $(10,20,30$, or $39 \mathrm{ft})$. The third character (X) represents the Malvern Insitec-S instrument support type and is either spacer bars (S) or frames (F). The fourth character represents the number of Malvern Insitec-S instruments installed in the chamber $(1,2$, or 3$)$. The fifth character represents the spray header extension configuration with the following definitions: $\mathrm{A}=$ no extension, $B=120$ in. extension, $C=120$ in. +108 in. extensions, and $D=120$ in. +108 in. +107 in. +77 in. extensions.

The test section is a spool piece that has spray orifices (i.e., circular holes and slots) through which the fluid is discharged to create the spray leak for characterization (i.e., release fraction and PSD). For the testing discussed in this section, a $2 \mathrm{~mm}$ circular hole and three different slots (ranging from $1.0 \mathrm{~mm} \times 10 \mathrm{~mm}$ to $1.0 \mathrm{~mm} \times 76.2 \mathrm{~mm}$ ) were tested to establish the release fraction and PSD. The various spray orifices used during testing are detailed in Table 4.3. 
Table 4.3. Orifice ID and Geometries Used for Phase II Large-Scale Testing

\begin{tabular}{cccccc}
\hline \multicolumn{1}{c}{ Orifice } & ID & Shape & $\begin{array}{c}\text { Nominal Orifice Size } \\
\text { Diameter or Length } \\
\text { Width }(\mathrm{mm})\end{array}$ & $\begin{array}{c}\text { Measured Dimensions } \\
(\mathrm{mm})\end{array}$ & $\begin{array}{c}\text { Orifice Area } \\
\left(\mathrm{mm}^{2}\right)\end{array}$ \\
\hline $1 \times 76 \mathrm{~mm}$ slot & S4A & Slot & $\begin{array}{c}1.0 \times 76.2 \text { horizontal } \\
\text { (axial) }\end{array}$ & $0.96 \times 76.11$ & 73.14 \\
$2 \mathrm{~mm}$ hole & S8A & Circular & $\begin{array}{c}1.0 \times 10 \text { horizontal } \\
\text { (axial) }\end{array}$ & $0.95 \times 10.02$ & 3.14 \\
$1 \times 10 \mathrm{~mm}$ slot & S8B & Slot & $\begin{array}{c}1.0 \times 20 \text { horizontal } \\
\text { (axial) }\end{array}$ & $0.99 \times 20.01$ & 9.53 \\
$1 \times 20 \mathrm{~mm}$ slot & S8C & Slot & &
\end{tabular}

Both spool 4 (S4A) and spool 8 (S8A, S8B, and S8C) test sections are shown in Figure 4.4 and Figure 4.5, respectively. These test sections typically consists of an 18.24-in.-long 3-in.-diameter, schedule 40 pipe spool. As shown in Figure 4.5, smaller spray orifices allowed multiple orifices to be machined on one spool piece.

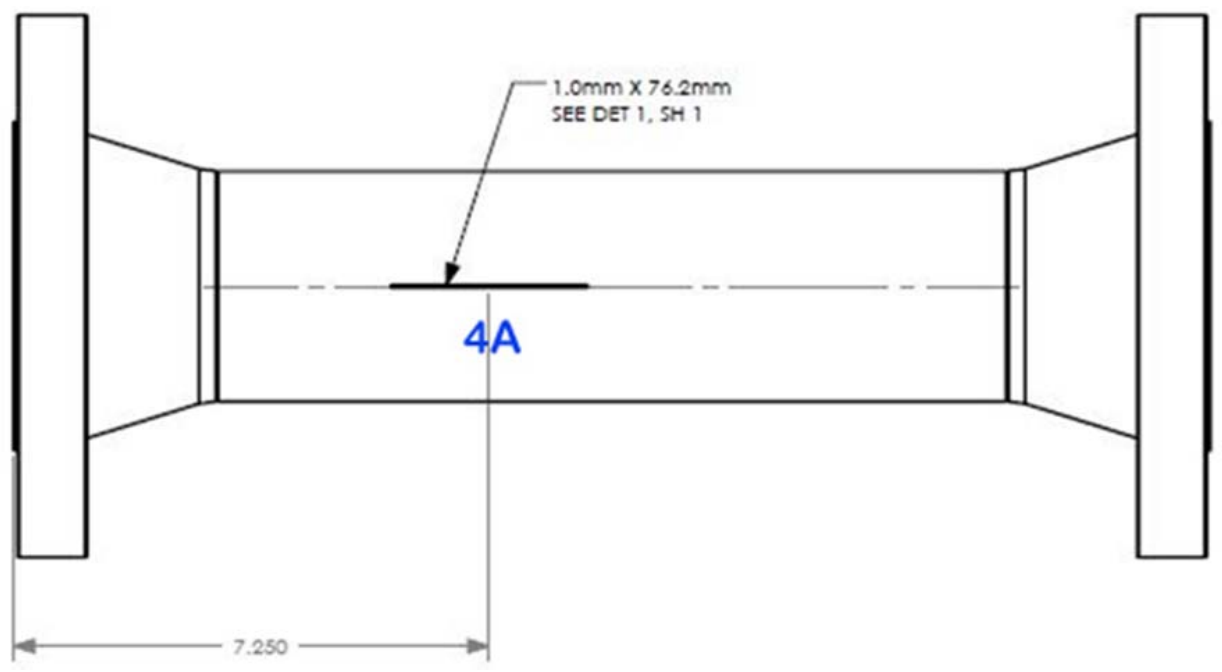

Figure 4.4. Schematic of Spool 4 Test Section

To facilitate spray initiation and cessation for each test, individual orifices were sealed with a specially designed release assembly that allowed the orifice to be opened when the desired conditions in the recirculation loop were met. The spray release assembly consisted of an over-center lever positioned at each orifice. Each release lever was opened using a manual pull-rod from outside the test chamber. A test spool with the spray release assembly attached is shown in Figure 4.6. 


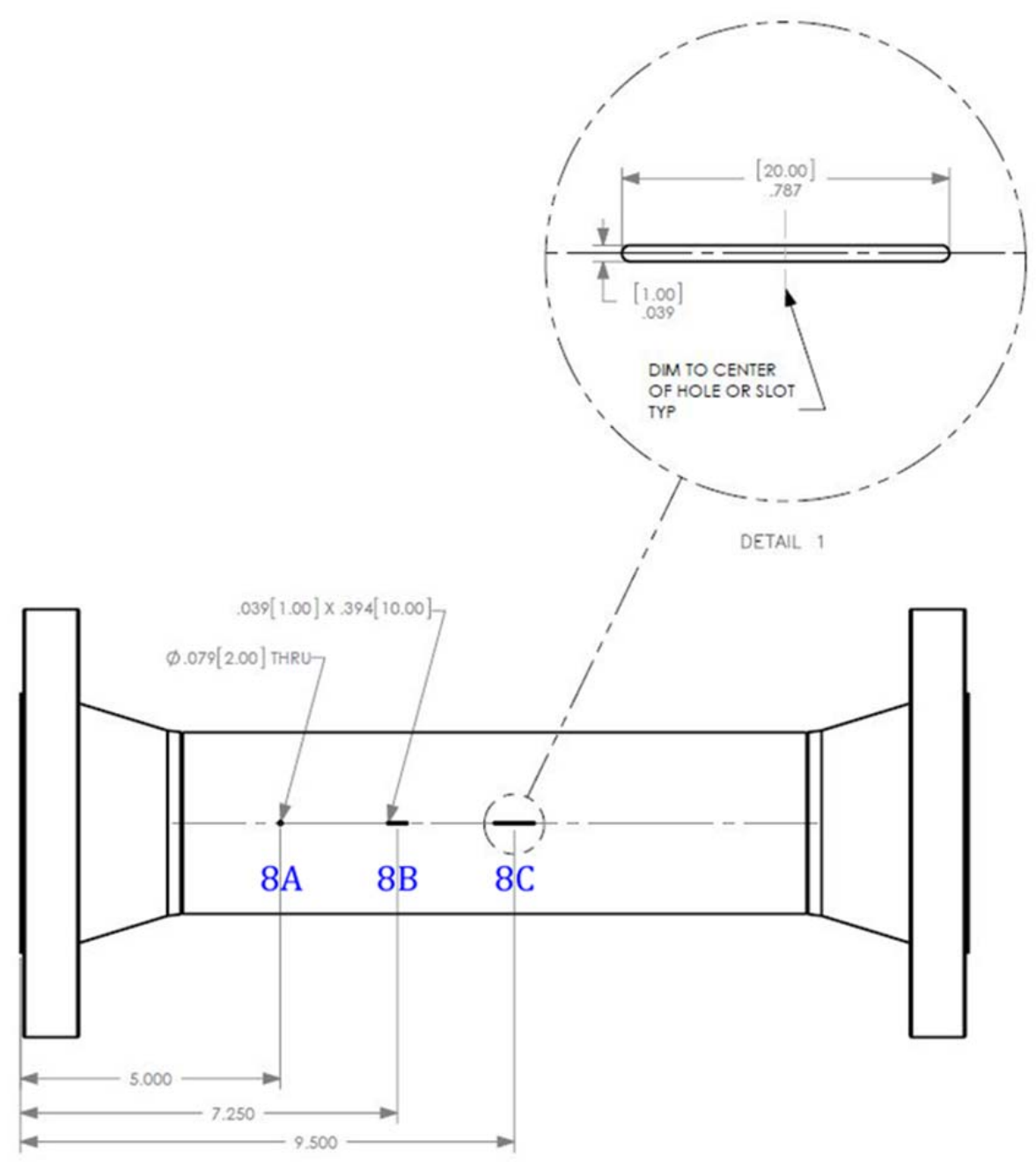

Figure 4.5. Schematic of Spool 8 Test Section

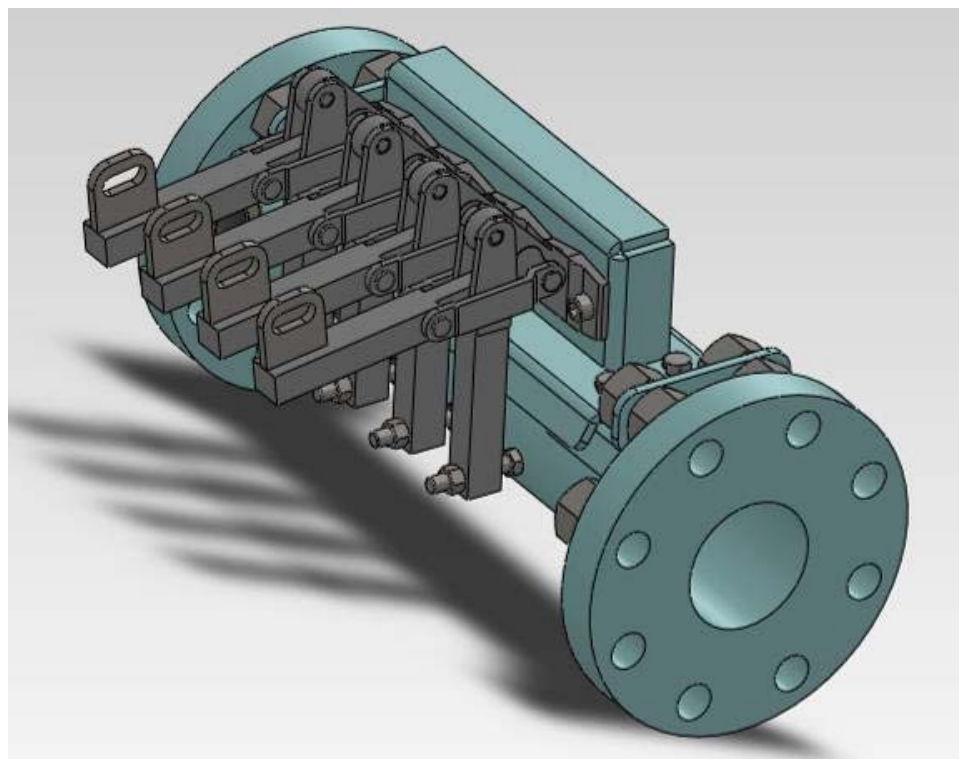

Figure 4.6. Model of the Release Assembly Used for Initiating a Spray 


\subsection{Aerosol Instrumentation}

During Phase II testing, only the Malvern Insitec-S open-frame process aerosol analyzers (Malvern Instruments, Ltd.) were used for measuring aerosol concentration and size distribution. In contrast, Phase I work used both the Malvern Insitec-S and a Process Metrix process particle counter (PPC) (Process Metrix). During Phase I testing, the results, which are discussed in Chapter 8 of Schonewill et al. (2012), indicated that the Malvern Insitec-S instrument was best suited to be the primary aerosol measuring instrument; therefore, the Process Metrix PPC was not used in Phase II testing. In the sections that follow, the principles of measurement and operational specifications for the Malvern Insitec-S analyzer are described in detail. These discussions include information on instrument locations and installation configurations in the large-scale test chamber, data collection/sampling rates, and the primary measurement input parameters (e.g., refractive index).

\subsubsection{Malvern Insitec-S Operating Principle}

Three Malvern Insitec-S aerosol analyzers were purchased for use during large-scale spray leak testing. The Malvern Insitec-S analyzer is an open-frame aerosol size analyzer that uses laser diffraction to determine aerosol size and concentration. The basic instrument setup and operating principle are illustrated in Figure 4.7. The Malvern Insitec-S consists of a relatively broad $10 \mathrm{~mm}$-diameter laser source positioned opposite a photodetector assembly. The volume swept by the laser beam as it passes from the laser source to the photodetector assembly defines the Malvern Insitec-S measuring volume. The distance between the laser source and the photodetector is set by spacer bars that typically range in length from 150 to $500 \mathrm{~mm}$. For 150 and $500 \mathrm{~mm}$ spacer bar lengths, the measuring volume swept by the laser is $\sim 5.5$ and $\sim 33 \mathrm{~cm}^{3}$, respectively. For Phase II testing, the spacer bar length was increased to $1000 \mathrm{~mm}$ to increase aerosol concentration sensitivity by increasing the measuring volume swept by the laser to $\sim 74 \mathrm{~cm}^{3}$.

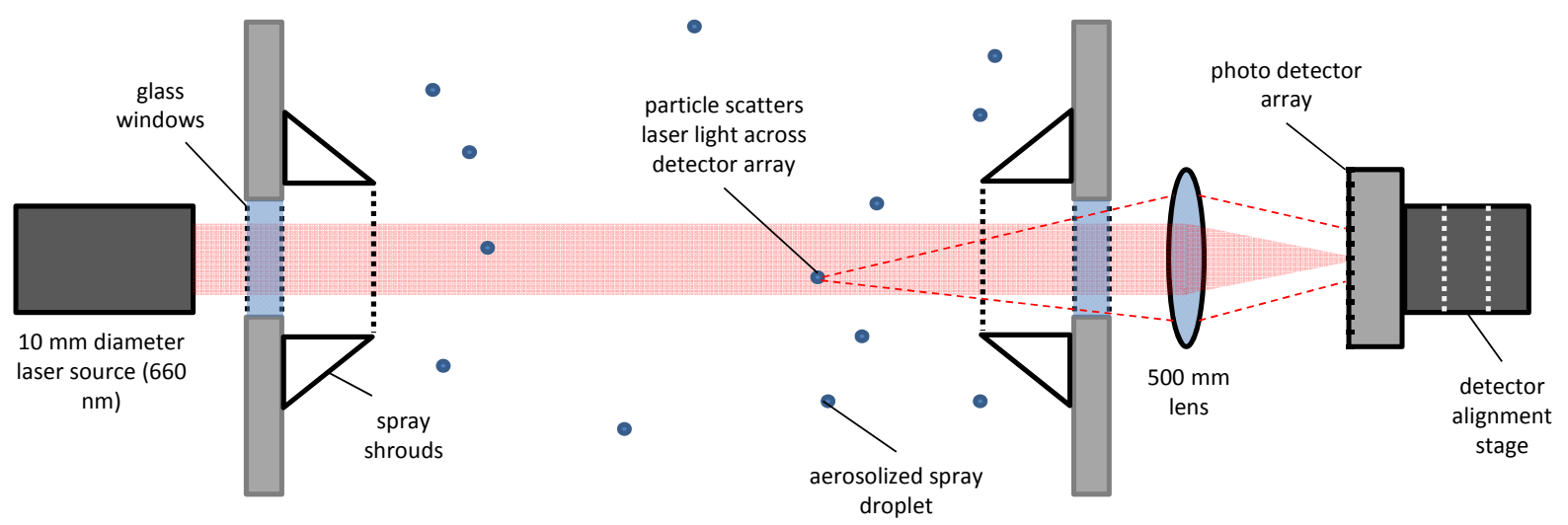

Figure 4.7. Schematic of the Basic Malvern Insitec-S Aerosol Analyzer Setup and Operating Principle

The photodetector assembly consists of a Fourier lens that focuses the laser onto a ring photodetector array. During operation, undeflected laser light is focused onto a laser power detector at the center of the ring detector. Deflected laser light is focused onto one of 32 photodetector rings on the surface of the photodetector assembly. The displacement of the deflected light from the center of the ring detector is proportional to the angle at which the laser light has been diffracted. 
The Malvern Insitec-S functions by continuously monitoring and recording both the intensity of undeflected laser light striking the central detector relative to the background intensity when no aerosol is present (termed the transmission) and the light scattering pattern generated by scattering of laser light by aerosol in the measuring zone. When an aerosol particle enters the measuring volume, it scatters light in all directions. However, the intensity of scattered light varies as a function of scattering angle and generates a scattered light pattern that is unique to particles of that size and morphology. As the measuring volume of the Malvern Insitec-S is large, the instrument is capable of simultaneously measuring multiple particle scattering events in a single measurement. Using Mie scattering theory and given the nominal optical properties of the aerosol and the suspending phase, the scattered light pattern generated by the particle ensemble can be analyzed to determine the size distribution of aerosol particles. Diffraction of the laser beam yields a loss of intensity of the laser beam that strikes the central detector relative to the intensity measured when no aerosol is present (termed the background laser intensity). This decrease in intensity, along with the size distribution estimated from the scattered light pattern, can be used to determine aerosol concentration.

The specific size and concentration range of the Malvern Insitec-S as configured for Phase II large-scale aerosol testing are as follows:

- Size Range. The nominal size range that can be measured by the Malvern Insitec-S is defined by the focal length of the Fourier lens used to focus laser light on the photodetector. A $500 \mathrm{~mm}$ lens was employed for all Phase II large-scale matrix testing. This lens yields a nominal measuring range of 2.5 to $2500 \mu \mathrm{m}$. Select functional tests conducted before and during primarily Phase II matrix testing employed a $100 \mathrm{~mm}$ lens with a nominal measuring range of 0.5 to $200 \mu \mathrm{m}$.

- Concentration. The nominal range of concentration that can be detected by the Malvern Insitec-S analyzer strongly depends on the instrument setup and spray configuration. In general, the Malvern Insitec-S can measure aerosols in the range of 0.01 to 1000 parts per million volume (ppmv). The specific range achievable in a given experimental setup is determined primarily by the length of the spacer bars and the geometry of the spray. Longer spacer bars allow lower aerosol concentrations to be probed, but also reduces the aerosol concentration at which the detector saturates. The geometry of the spray also has a strong impact on the range of aerosols that can be detected by the instrument. For example, high aerosol concentrations can be measured if the aerosol is confined to a limited portion of the measurement volume, such as fan sprays. However, correct interpretation of aerosol concentration for such sprays requires knowledge of the spray geometry relative to the measurement volume. The Malvern Insitec-S measurement software allows input of spray geometry and spray concentration profiles to correctly determine the concentration of localized sprays.

\subsubsection{Phase II Malvern Insitec-S Frame Configuration and Installation Location}

In Phase II testing, the $1000 \mathrm{~mm}$ spacer bar and frame configuration was used for "in-chamber" and "in-spray" measurements of release fraction, respectively. In the "in-chamber" configuration, all Malvern Insitec-S analyzers were positioned above the spray at approximately half the distance between the path of the spray and the top of the chamber. Figure 4.8a shows three Malvern Insitec-S instruments in their in-chamber measurement configuration. All three instruments employed $1000 \mathrm{~mm}$ spacer bars and were installed above the expected path of the spray. During "in-spray" testing, measurements were made in which the Malvern Insitec-S analyzers were placed directly in the path of the spray. Customized $1000 \mathrm{~mm}$ rectangular hanging frames were fabricated for "in-spray" measurements; these rectangular 
frames enabled "in-spray" measurements to be taken without the spray hitting the spacer bars, thus eliminating or minimizing impact to aerosol concentration and size measurements made at downstream Malvern Insitec-S analyzers. Figure $4.8 \mathrm{~b}$ shows three Malvern Insitec-S aerosol analyzers set up for in-spray measurements on the custom in-spray frames.

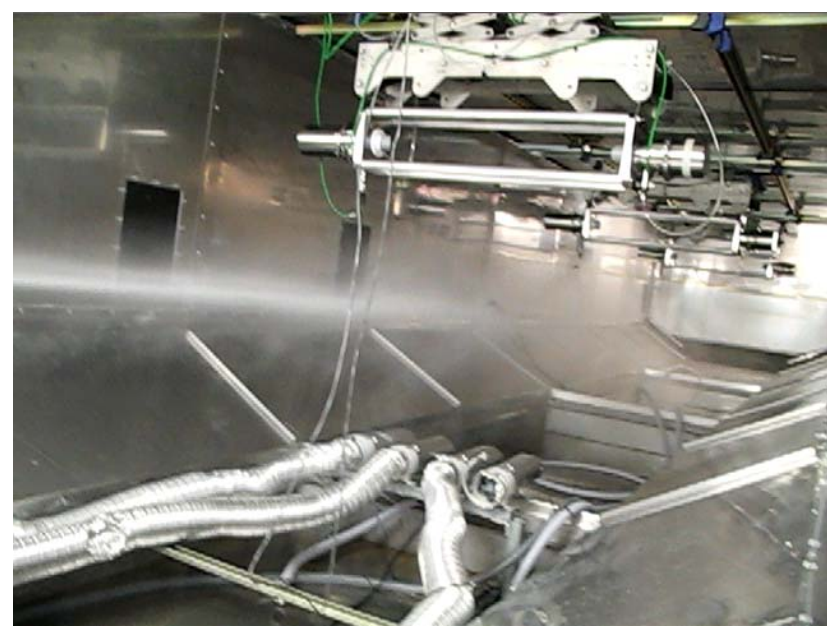

(a) - In-Chamber Malvern Insitec-S Configuration

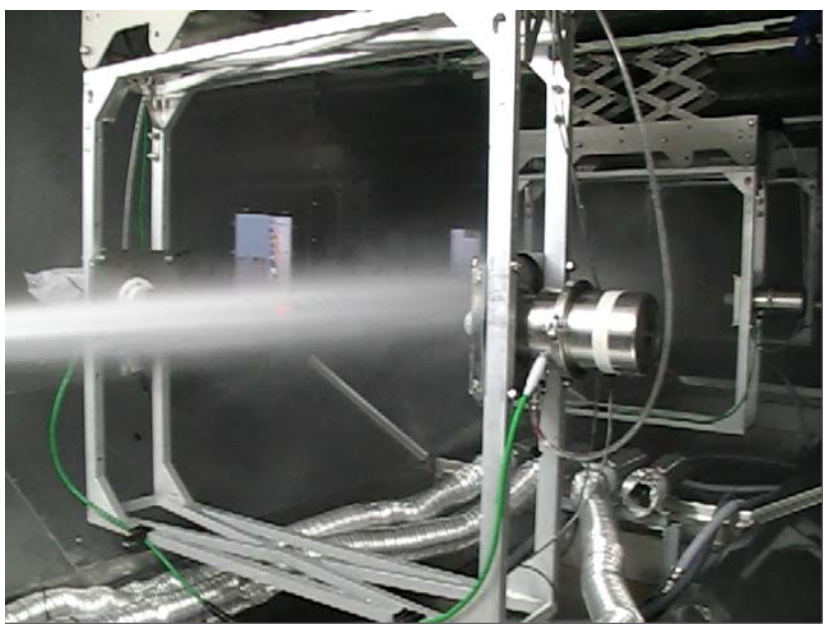

(b) - In-Spray Malvern Insitec-S Configuration

Figure 4.8. Malvern Insitec-S Aerosol Analyzers Configurations. (a) The "in-chamber" configuration employing $1000 \mathrm{~mm}$ spacer bars, and (b) the "in-spray" configuration using custom built hanging frames that limit impingement of the spray on the Malvern Insitec-S support frame.

For in-chamber measurements, the Malvern Insitec-S analyzers were installed at different locations in the large-scale test chamber to allow profiling of the aerosol concentration as a function of test position. Key instrument locations and spacing dimensions used for in-chamber testing are illustrated in Figure 4.9; their values are listed in Table 4.4. Dimension A measurements are referenced from the spray header wall to the leading edge of the Malvern Insitec-S mounting end-plate. To convert Dimension A to the actual horizontal distance between the spray orifice face and the Malvern Insitec-S laser beam centerline requires the subtraction of 13 in. (spray orifice face to spray header wall distance) and addition of $4.8 \mathrm{in}$. (Malvern Insitec-S mounting end-plate edge to laser beam centerline).

When testing in chambers longer than $10 \mathrm{ft}$ in length, three Malvern Insitec-S analyzers were used. Analyzers were spaced evenly through the chamber and were placed 1) near the spray header, 2) at the "middle" of the test chamber, and 3) near the splash wall. It should be noted that the in-chamber spacing of Malvern Insitec-S analyzers used in Phase II differs from that used in Phase I, in which two of the aerosol analyzers were more closely spaced near the spray. Phase I testing located the Malvern Insitec-S aerosol analyzers at 3.3, 6.2, and $13.3 \mathrm{ft}$ from the wall near the spray header (as measured by Key Dimension A). The change in Malvern Insitec-S spacing for Phase II testing was done to more closely match the physical location (and associated chamber volume) of each analyzer to assumptions made for analysis of in-chamber data. 


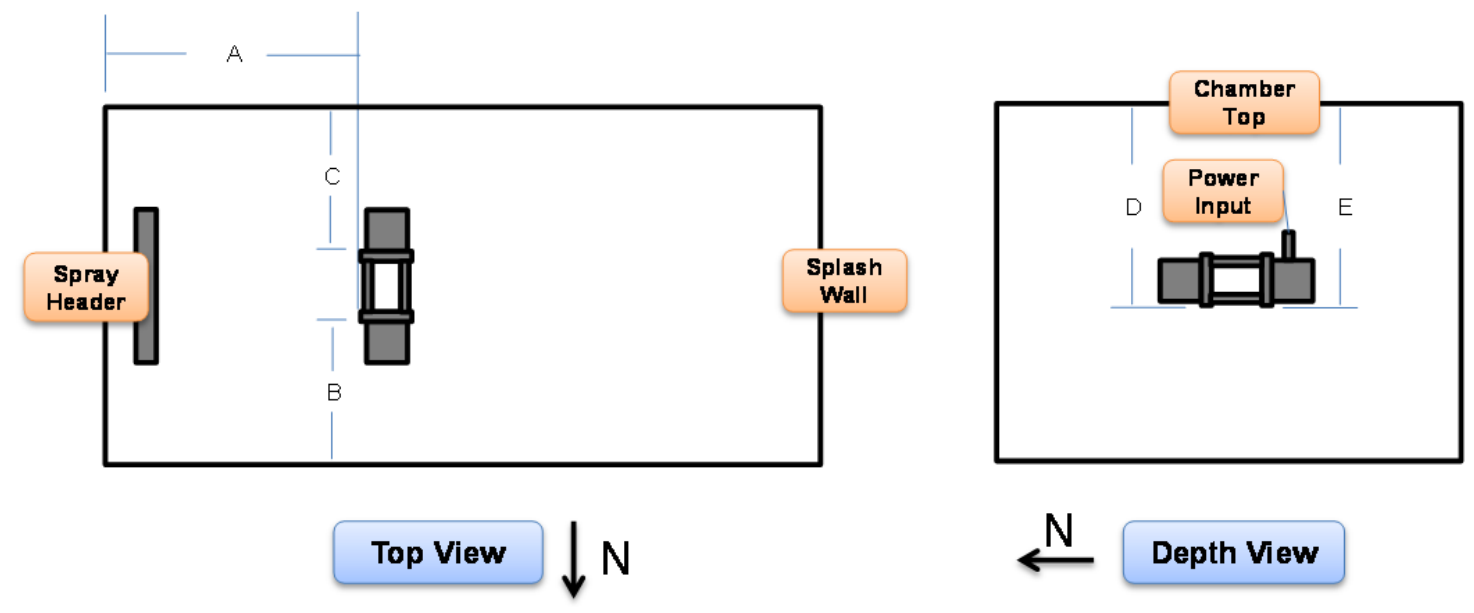

Figure 4.9. Two Schematic Views Showing Key Dimensions that Define the "In-Chamber" Measuring Locations of the Malvern Insitec-S Analyzers. The top view is shown in the left-hand figure and a depth (front to back) view is shown in the right-hand figure. For reference, north (N) is indicated on the figure.

Table 4.4. Values of Key Dimensions Listed in Figure 4.9 for In-Chamber Testing

\begin{tabular}{|c|c|c|c|c|c|c|c|}
\hline Chamber Length $(\mathrm{ft})$ & $\begin{array}{c}\text { Malvern } \\
\text { Insitec-S } \\
\text { Analyzer ID }\end{array}$ & $\begin{array}{l}\text { Insitec-S Analyzer } \\
\text { Chamber Position }^{(a)}\end{array}$ & $\mathrm{A}(\mathrm{ft})$ & $\mathrm{B}(\mathrm{ft})$ & $\mathrm{C}(\mathrm{ft})$ & $\mathrm{D}(\mathrm{ft})$ & $\mathrm{E}(\mathrm{ft})$ \\
\hline \multirow[t]{3}{*}{10} & $\mathrm{n} / \mathrm{a}$ & 3 & $\mathrm{n} / \mathrm{a}$ & $\mathrm{n} / \mathrm{a}$ & $\mathrm{n} / \mathrm{a}$ & $\mathrm{n} / \mathrm{a}$ & $\mathrm{n} / \mathrm{a}$ \\
\hline & MAL1855 & 2 & 3.3 & 2.3 & 2.3 & 2.3 & 2.3 \\
\hline & MAL1854 & 1 & 6.6 & 2.3 & 2.3 & 2.3 & 2.3 \\
\hline \multirow[t]{3}{*}{20} & MAL1852 & 3 & 5 & 2.3 & 2.3 & 2.3 & 2.3 \\
\hline & MAL1855 & 2 & 10 & 2.3 & 2.3 & 2.3 & 2.3 \\
\hline & MAL1854 & 1 & 15 & 2.3 & 2.3 & 2.3 & 2.3 \\
\hline \multirow[t]{3}{*}{30} & MAL1852 & 3 & 7.5 & 2.3 & 2.3 & 2.3 & 2.3 \\
\hline & MAL1855 & 2 & 15 & 2.3 & 2.3 & 2.3 & 2.3 \\
\hline & MAL1854 & 1 & 22.5 & 2.3 & 2.3 & 2.3 & 2.3 \\
\hline \multirow[t]{3}{*}{39} & MAL1852 & 3 & 10 & 2.3 & 2.3 & 2.3 & 2.3 \\
\hline & MAL1855 & 2 & 20 & 2.3 & 2.3 & 2.3 & 2.3 \\
\hline & MAL1854 & 1 & 30 & 2.3 & 2.3 & 2.3 & 2.3 \\
\hline
\end{tabular}

(a) The Malvern Insitec-S analyzer order in proximity to the spray header and respective identification number is illustrated in Figure 4.12.

When executing in-chamber testing in the $10 \mathrm{ft}$ chamber configuration, only two Malvern Insitec-S analyzers were used because of limited chamber space. These analyzers where spaced evenly in the $10 \mathrm{ft}$ chamber, with one analyzer near the spray header and the other near the splash wall. Regardless of chamber length, the Malvern Insitec-S instruments were always centered (Dimension B and C) in the chamber and the Malvern Insitec-S laser beams were always vertically positioned midway between the spray orifice and the top of the chamber, at approximately $23.1 \mathrm{in}$. from the chamber top/ceiling. The spray header orifices were also fixed at approximately $44 \mathrm{in.} \mathrm{from} \mathrm{the} \mathrm{chamber} \mathrm{top/ceiling.} \mathrm{Therefore,}$ conversion of Dimension D and E (in Table 4.4) to distances between the spray orifice and the Malvern 
Insitec-S laser beam location requires the following arithmetic: 44 in. (chamber ceiling to spray orifice) minus 28 in. (Dimension D or E) plus 4.8 in. (Malvern Insitec-S mounting end-plate edge to laser beam centerline).

In-spray testing was conducted using Malvern Insitec-S analyzer locations that differed from those used for in-chamber testing. The most notable difference is the height of Malvern Insitec-S analyzers with respect to the spray. Fixed heights were not used for in-spray testing; instead a pre-measurement spray was used to identify the nominal path of the spray through the chamber. The analyzers then were centered directly in the path of the spray. Depending on the path of the spray, each of the analyzers had slightly different positions depending on how far downstream of the spray they were located. All in-spray testing employed three Malvern Insitec-S analyzers using the in-spray frames shown in Figure 4.8b. In-spray testing with water was done in the $39 \mathrm{ft}$ chamber configuration, whereas in-spray testing with clay simulants was performed in the $20 \mathrm{ft}$ chamber to avoid cost and schedule loss associated with having to reconfigure the chamber during a single campaign of clay testing or having to thoroughly clean the test chamber and flow system if switching between simulants. The key dimension recorded for in-spray testing was the distance of each Malvern Insitec-S analyzer from the chamber wall located near the spray header (i.e., Dimension A in Table 4.4); this distance is presented for in-spray measurements in Table 4.5.

Table 4.5. Values of Key Dimensions Listed in Figure 4.9 for In-Spray Testing

\begin{tabular}{lccrc}
\hline & Malvern & Malvern Insitec-S & & \\
Test Simulant/Configuration & Ansitec-S & $\begin{array}{c}\text { Analyzer Chamber } \\
\text { Position }^{(\mathrm{a})}\end{array}$ & $\mathrm{A}(\mathrm{ft})$ & $\mathrm{X}^{(\mathrm{b})}(\mathrm{ft})$ \\
\hline Water/1 $\times$ 76 mm slot & MAL1852 & 3 & 5.0 & 4.3 \\
& MAL1855 & 2 & 12.5 & 11.8 \\
& MAL1854 & 1 & 20.0 & 19.3 \\
Water/All Other Orifices & MAL1852 & 3 & 5.0 & 4.3 \\
& MAL1855 & 2 & 8.5 & 7.8 \\
& MAL1854 & 1 & 12.0 & 11.3 \\
Clay/All Orifices & MAL1852 & 3 & 5.0 & 4.3 \\
& MAL1855 & 2 & 8.5 & 7.8 \\
& MAL1854 & 1 & 12.0 & 11.3 \\
\hline
\end{tabular}

(a) The Malvern Insitec-S analyzer order in proximity to the spray header and respective identification number is illustrated in Figure 4.12.

(b) Horizontal distance from the orifice to the aerosol analyzer measuring location (i.e., the laser centerline).

In addition to in-chamber and in-spray testing, several additional series of spray leak tests were conducted. Some of these tests used non-standard aerosol instrument locations. One such set of tests evaluated chamber uniformity and involved testing with the Malvern Insitec-S in 18 unique chamber locations. While these tests are not discussed in detail in the main body of this report, the test approach, location, and results for these additional tests are documented in Appendix A. Furthermore, a detailed list of large-scale chamber aerosol instrument configurations, for both standard in-spray and in-chamber tests as well as additional tests (such as chamber uniformity and humidity testing), is provided in Appendix B of this report. 


\subsubsection{Detection Unit Optics}

The Malvern Insitec-S aerosol analyzers were equipped with a $500 \mathrm{~mm}$ lens for all of aerosol tests conducted in Phase II. The lens is a part of the Malvern Insitec-S detection unit and functions to translate (i.e., focus) fixed angles of scattered light to fixed light displacement on the photodetector rings. The focal length of the lens determines the range and resolution of light scattering angles that can be interpreted by the photodetector and, thus, the range of aerosol sizes accessible to the Malvern Insitec-S analyzer.

Phase I testing used a $100 \mathrm{~mm}$ lens. This lens had a nominal aerosol size measurement range of 0.5 to $200 \mu \mathrm{m}$ and was selected for Phase I testing because it gave the greatest sensitivity (in terms of size resolution) for aerosols in the range of interest (i.e., 10 to $100 \mu \mathrm{m}$ ). Use of the $500 \mathrm{~mm}$ lens, which has a nominal working range of 2.5 to $2500 \mu \mathrm{m}$, for Phase II testing was driven by concerns that aerosols larger than $200 \mu \mathrm{m}$ had not been adequately measured during Phase I testing. This concern was greatest for in-spray measurements because in-spray testing conducted at the end of Phase I with the $100 \mathrm{~mm}$ lens demonstrated clear clipping of the size distribution at the upper end of the range. In addition, there also was concern that in-chamber measurements conducted during Phase I testing might not have representatively measured aerosols greater than $200 \mu \mathrm{m}$ in size, although there was no obvious clipping of the size distribution in Phase I in-chamber measurements. With respect to the latter concern, it should be noted that Phase II in-spray testing with the $500 \mathrm{~mm}$ lens evidences a significant fraction of large aerosols $(>200 \mu \mathrm{m})$ while similar Phase II in-chamber tests are consistent with Phase I and do not evidence aerosols larger than $200 \mu \mathrm{m}$. Thus, it can be tentatively concluded that large aerosols were not "missed" in Phase I in-chamber measurements as a result of using the $100 \mathrm{~mm}$ lens. The lack of measured aerosols greater than $200 \mu \mathrm{m}$ in in-chamber measurements most likely results from sampling issues associated with the location of aerosol analyzers in both Phase I and Phase II testing. This is discussed in more detail in Section 7 of this report, where direct comparisons of in-spray and in-chamber release fraction measurements are presented.

Comparative testing of the 100 and $500 \mathrm{~mm}$ lenses was performed before the start of primary Phase II testing to evaluate the impact of the lenses on measured release fractions. Testing was performed in the $39 \mathrm{ft}$ large-scale chamber configuration (with no spray header extensions) using a nominal $1 \times 76 \mathrm{~mm}$ slot with sprays at $380 \mathrm{psig}$. The results of lens testing are shown in Figure 4.10. Comparisons of the release fraction results indicated that both lenses gave equivalent results (based on expected uncertainty and reproducibility of release fractions determined during Phase I testing).

Because use of the $500 \mathrm{~mm}$ lens did not appear to affect the ability to measure release fraction in the size range of interest, it was selected for use for the entirety of Phase II water and clay matrix tests. Exclusive use of the $500 \mathrm{~mm}$ lens avoided the need to frequently switch out Malvern Insitec-S optical lenses (which requires realignment and has the potential to damage the instrument) and allowed access to sizes larger than $200 \mu \mathrm{m}$ for the bulk of Phase II testing (which eliminated speculation regarding the existence of larger aerosols in both in-chamber and in-spray measurements). 


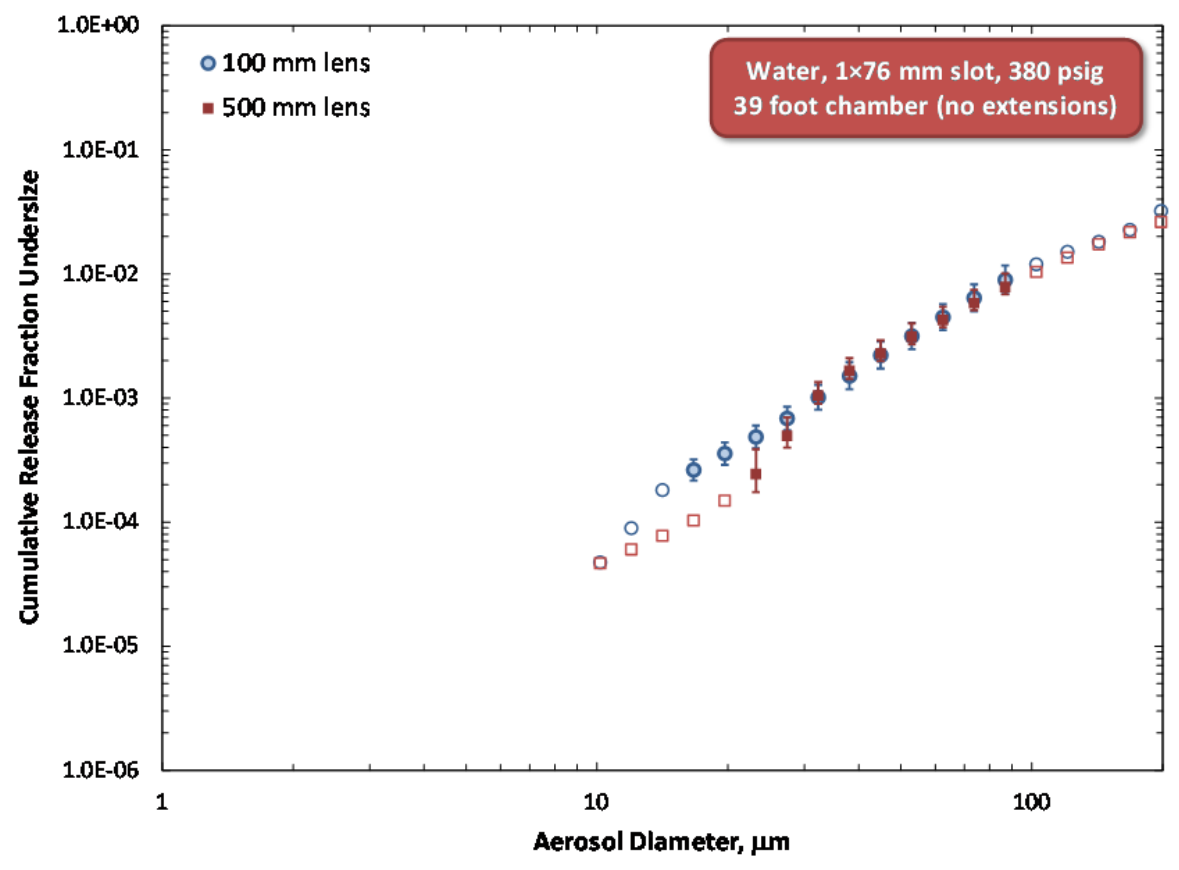

Figure 4.10. Comparisons of Release Fraction Measurements Made Using the $100 \mathrm{~mm}$ Lens (blue circles) and the $500 \mathrm{~mm}$ Lens (red squares). Data correspond to release fractions measured for water spray through a nominal $1 \times 76 \mathrm{~mm}$ orifice at $380 \mathrm{psig}$. Each measurement curve represents the average of at least two repeat tests; error bars correspond to the maximum and minimum release fractions measured in individual tests. Open circles represent points at aerosol diameters that fall outside the nominal Malvern Insitec-S measuring range for in-chamber measurements and, as a result, are suspect. Criteria for determining suspect points for in-chamber measurements are described in Section 7.1 of this report.

The favorable comparison between 100 and $500 \mathrm{~mm}$ lens results found in large-scale testing differs from the conclusions derived from similar lens studies carried out in the small-scale test stand. When comparing the performance of 100 and $500 \mathrm{~mm}$ lenses, small-scale studies found that “... the $500-\mathrm{mm}$ lens gave higher release fractions in the size range between 10 and $100 \mu \mathrm{m}$, with release fractions obtained with the 500-mm lens being two to three times the values obtained with the 100 -mm lens" (Schonewill et al. 2013). It is important to note that the factor of two to three identified in small-scale testing is an overall (i.e., typical) difference in 100 and $500 \mathrm{~mm}$ lens results observed across multiple tests involving several different orifices and simulants. In selected tests, good agreement between 100 and $500 \mathrm{~mm}$ lens tests in the small-scale studies was observed. The lens tests conducted in large-scale studies were limited in scope and involved one test orifice, pressure, and simulant combination. It is possible that large-scale lens agreement is simply a coincidence of limited testing and that more extensive testing would produce findings in line with those observed in small-scale tests. Likewise, the agreement between 100 and $500 \mathrm{~mm}$ lens in large-scale tests could result from an orifice or chamber size scaling effect. If this issue warrants additional attention, efforts should be made to collect additional lens comparisons in large-scale tests for a more diverse range of spray geometries and chamber scales. 


\subsubsection{Aerosol Purge and Spray Shrouds}

Because the Malvern Insitec-S aerosol analyzers are located inside the test chamber, the aerosol generated by the spray directly contacts the instrument surfaces. While the instruments have been positioned above the spray for in-chamber measurements and employ a specialized open frame that avoids jet impingement during in-spray measurements, jet formation and break-up is generally a turbulent process, and there may be times when the spray passes through the Malvern Insitec-S measuring volume or strikes the analyzer itself. Although the electronic components are protected inside specialized enclosures for the laser and detector, the laser and scattered light still pass through optical windows to generate the measuring volume (see Figure 4.7). Deposition of aerosol or the presence of contaminants will impact the quality of the measurement. Any minor surface contamination or surface imperfections, such as scratches, can be taken into account by measuring the "background" scattering intensity generated by the laser prior to aerosol generation (i.e., when the measurement volume is free of aerosol). Gross contamination or damage to the windows found during these background measurements may be corrected by cleaning the windows with a lens paper and an appropriate solvent, or new windows may be installed. During the course of Phase II testing, simple cleaning operations with water or an alcohol-based lens cleaner and compressed air generally restored window cleanliness to a level acceptable for aerosol measurement. In the one instance when cleaning did not restore window cleanliness, the windows were replaced in all three instruments.

Contamination of the window during aerosol generation is of significant concern because particles and aerosol collected on the window will yield a static scattering signal that will remain through the measurement. To prevent contamination of the windows by direct spray impact, all Malvern Insitec-S instruments are equipped with a $40 \mathrm{~mm}$ cylindrical spray shroud. In addition to the factory supplied spray shrouds, a 2 in. extension piece cut on a $45^{\circ}$ angle was added to the end of the shrouds to protect the lenses from directional spray or rebound (whichever was evaluated as having the greatest chance of impacting data collection). Even with these protective extension pieces in place, lens contamination was observed periodically. As such, real-time and post-measurement evaluations of all aerosol results were performed by the aerosol instrument operator, the lead aerosol engineer, and aerosol data analysts. If a review of aerosol data found or suspected contamination and that contamination event impacted data necessary for release fraction analysis (such as the data collected during the initial concentration rise of aerosol at the start of spray), the tests impacted by contamination were repeated.

The spray shroud protects the optics from direct impact, but does not provide protection against diffusive or convective transport of aerosol particles. As such, the Malvern Insitec-S analyzers also are equipped with a purge system that passes air across the windows and transports aerosol out of the volume enclosed by the spray shrouds. The purge air flow rate was generally set at $\sim 1 \mathrm{SCFH} /$ window (a total of $\sim 2 \mathrm{SCFH} /$ instrument) for the nominal $2 \mathrm{~mm}$ and $1 \times 10 \mathrm{~mm}$ orifice, $\sim 50 \mathrm{SCFH} /$ window for the nominal $1 \times 20 \mathrm{~mm}$ orifice, and $\sim 100 \mathrm{SCFH} /$ window for the nominal $1 \times 76 \mathrm{~mm}$ orifice during in-chamber testing. For in-spray and clay simulant testing, when window contamination was highly likely, purge rates between 50 and $100 \mathrm{SCFH}$ were generally used for the $2 \mathrm{~mm}, 1 \times 10 \mathrm{~mm}$, and $1 \times 20 \mathrm{~mm}$ orifice while even larger purge rates of $70 \mathrm{SCFH}$ (for Malvern Insitec-S positions 1 and 2) and $150 \mathrm{SCFH}$ (for Malvern Insitec-S position 3) were used for the $1 \times 76 \mathrm{~mm}$ orifice. The purge air setting was found to have no effect on the observed release fraction and was kept at the minimum value that enabled data collection without lens contamination. A discussion of the effect of purge air settings can be found in Appendix A of this report. 


\subsubsection{Aerosol Instrumentation Control and Data Acquisition}

All Malvern Insitec-S analyzers are interfaced to a single control computer through a multiport local communications interface box (part MPS2991-OP10045353). Each instrument is operated through a separate installation of Malvern's RTSizer software (Version 7.40). The software collects, analyzes, and reports the aerosol data collected by the instrument. The primary program outputs are aerosol size and concentration; other parameters - including raw data, such as the raw light-scattering signal, laser transmission, and background, and other calculated parameters, such as the Sauter Mean Diameter (SMD) - also are recorded. For Phase II testing, the RTSizer software required a software patch (purchased from Malvern Instruments, Ltd.) to accommodate the larger $1000 \mathrm{~mm}$ spacer bars used in Phase II. This patch was necessary because the original (unpatched) software did not allow for values greater than $255 \mathrm{~mm}$ for the spray radius and consequently limited analysis of collected aerosol data to an optical path length of $510 \mathrm{~mm}$ or less.

Measurements made with the Malvern Insitec-S analyzer were set up and controlled through the RTSizer software. The typical instrument configuration and software settings used for analysis are listed in Table 4.6. A complete description of these parameters is given in the RTSizer user manual (Malvern Instruments, Ltd. 2010). The first three parameters - the lens, gain, and update period - must be set before any data are collected because these parameters cannot be changed after collection of data. The lens must be set to match the focal length of the lens installed in the instrument; for all large-scale Phase I testing, the focal length of the lens was $100 \mathrm{~mm}$. For Phase II, the optical lens was changed to a $500 \mathrm{~mm}$ focal length lens for both in-spray and in-chamber measurements. Use of the $500 \mathrm{~mm}$ lens, which has a nominal working range of 2.5 to $2500 \mu \mathrm{m}$, for Phase II testing was driven by concerns that aerosols larger than $200 \mu \mathrm{m}$ had not been adequately measured during Phase I testing (see Section 4.5.3 for additional discussion). The gain setting is a photodiode multiplier that determines the instrument response (sensitivity) to scattered light. The highest gain setting of $2 \times$ was used for large-scale testing because aerosol concentrations were expected to be low (i.e., $\leq 10$ ppmv based on initial estimates). The update period (or accumulation period) determines the time period over which results are integrated. Longer update periods tend to smooth variations in aerosol concentration with time, thus yielding smoother data; however, data may be time-averaged over periods when aerosol concentration transience is of interest (such as the initial increase in concentration from which release fraction estimates are made). Shorter accumulation periods can be selected to capture fast transients; however, this also tends to yield an increase in noise-to-signal ratio. For large-scale testing, two update periods were employed. Initial testing used a 1-s update period, which adequately captured dynamics on sprays generated on most smallto medium-sized orifices. For larger orifices, the initial increase in concentration typically occurred on time scales of order $1 \mathrm{~s}$, and as such, a lower update period of $0.25 \mathrm{~s}$ was used in later testing. 
Table 4.6. Malvern Insitec-S Instrument Configuration and Software Parameters Used in Typical Phase II Measurements

\begin{tabular}{|c|c|}
\hline Parameter & Setting \\
\hline Lens & $500 \mathrm{~mm}$ \\
\hline Gain & $2 \times$ \\
\hline Update period & $0.25 \mathrm{~s}$ \\
\hline Particulate RI & $\begin{array}{l}1.33+0.00 \mathrm{i} \text { (for water), } \\
1.389+0.148 \mathrm{i} \text { (for } 30 \mathrm{~Pa} \text { Clay), or } \\
1.378+0.119 \mathrm{i} \text { (for } 6 \mathrm{~Pa} \text { Clay) }\end{array}$ \\
\hline Media RI & Air: $1.00+0.00 \mathrm{i}$ \\
\hline Particle density, $\mathrm{g} / \mathrm{cm}^{3}$ & 0.998 (water), 1.195 (6 Pa Clay) and 1.242 (30 Pa Clay) \\
\hline Scattering threshold & 2 \\
\hline Minimum size & 2.5 \\
\hline Maximum size & 2500 \\
\hline First scatter & 1 \\
\hline Multiple scatter & On \\
\hline Spray properties & $\begin{array}{l}\text { Measured: } \\
\text { - Uniform spray concentration in measurement volume } \\
\text { - Path length for } 1000 \mathrm{~mm} \text { spacers is } 920 \mathrm{~mm} \\
\text { - Path length for } 500 \mathrm{~mm} \text { spacers is } 420 \mathrm{~mm}\end{array}$ \\
\hline Air Purge rate & 1 or $50 \mathrm{SCFH}$ per side \\
\hline
\end{tabular}

Other properties, such as the particulate and media RIs and the scattering threshold, may be changed by post-analysis processing to evaluate the effect of these parameters on the instrument results. From the set of parameters that can be changed post-process, two important parameter selections are RI and the spray properties. The RI is a complex number that specifies how light refracts through a material (real component) and how the material attenuates or absorbs light (imaginary component). As all aerosols are tested in air, the media RI is always set to that of air $(1.00+0 \mathrm{i})$. The particulate RI depends on the material being tested. Selection of particulate RI values for measuring large-scale aerosols is discussed in Chapter 3 of this report. The spray properties allow the user of the RTSizer software to define the shape, size, and concentration profile of the spray as it passes through the measurement volume. The shape and profile options are outlined in the RTSizer software manual (Malvern Instruments, Ltd. 2010).

\subsubsection{Total Impact of Phase II Malvern Insitec-S Configuration Changes}

Phase II spray leak testing implemented several changes to the configuration of the Malvern Insitec-S. The three most significant changes include use of the $500 \mathrm{~mm}$ lens rather than the $100 \mathrm{~mm}$ lens (Section 4.5.3), use of $1000 \mathrm{~mm}$ spacer bars (as opposed to the $500 \mathrm{~mm}$ spacer bars used in Phase I), and keeping the same horizontal chamber spacing between the three Malvern Insitec-S instruments (Section 4.5.2). In addition, there were several changes made to the test chamber, such as the mechanisms used to hang the Malvern Insitec-S analyzers to allow test operators to more easily relocate instrument locations during chamber size changes and during transitions from in-chamber to in-spray measurements. There was concern that the combined impact of all these changes would produce a significant difference in release fraction measurements derived from the Phase II configuration relative to those made in Phase I. To address this issue, direct comparisons of Phase I and Phase II test results were 
performed. The data compared are derived from Phase I and Phase II testing of a 380 psig spray generated through a $2 \mathrm{~mm}$ round hole in the $20 \mathrm{ft}$ chamber. Phase II testing of this spray configuration involved one set of tests with the aerosol analyzers installed at the standard Phase II locations (as listed in Table 4.4) and another set of tests in which the analyzers were returned to the Phase I locations (which were 3.3, 6.2, and $13.3 \mathrm{ft}$ downstream from the wall near the spray header). These tests allowed isolation of changes to release fraction measurements that occur as a result of configuration changes to the Malvern Insitec-S analyzers (such as the use of the $500 \mathrm{~mm}$ lens) and those that result from changes in measurement positions. The result of this comparison, which is shown in Figure 4.11, indicates that Phase II release fractions are comparable (equivalent) to those collected in Phase I under the same spray conditions (e.g., pressure/orifice). It should be noted that configuration changes to the Malvern Insitec-S analyzers and chamber do appear to reduce release fractions as measured in the standard Phase I configuration. However, this change appears to be offset by keeping the equal spacing between the three Malvern Insitec-S instruments in Phase II testing. As such, there appears to be no overall impact to release fraction measurements.

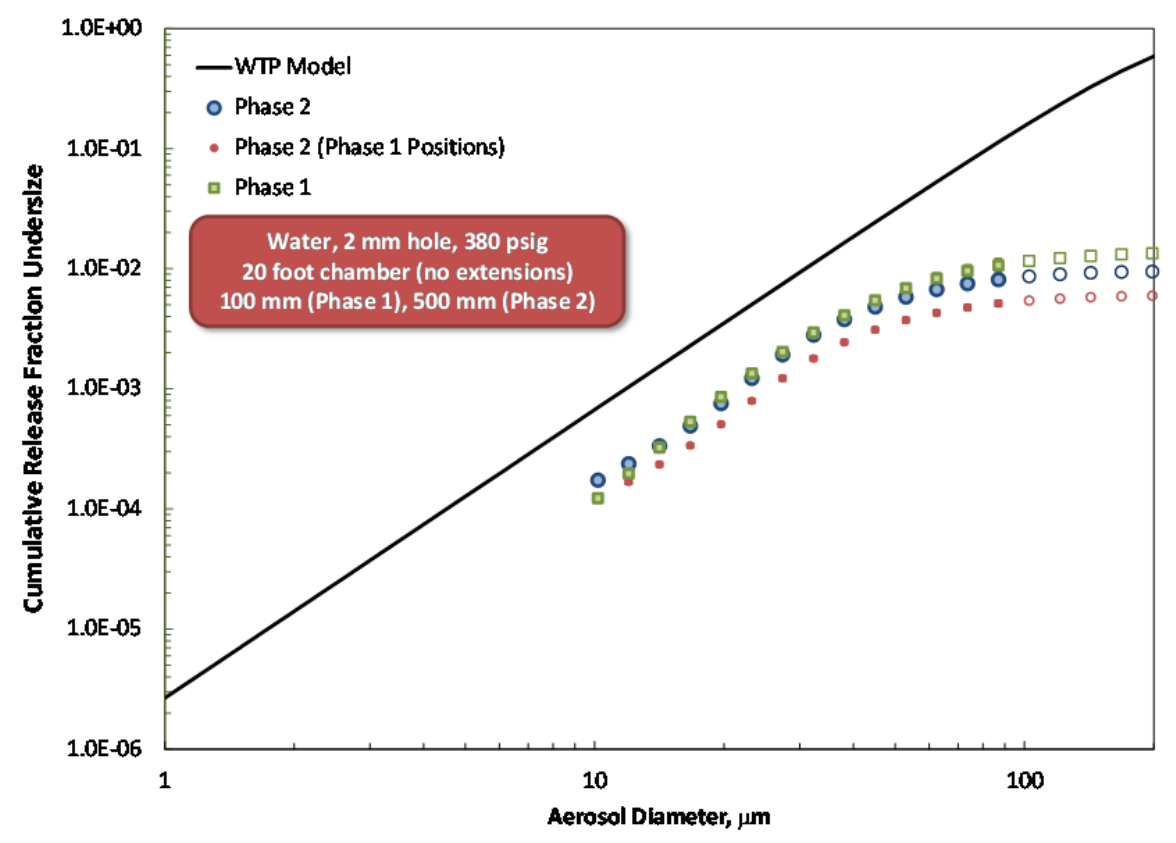

Figure 4.11. Comparison of Release Fraction Measurements Made During Phase I (green squares) to Those Made During Phase II using the Standard Phase II Malvern Insitec-S Locations (blue circles) as Well as the Standard Malvern Insitec-S Locations Used in Phase I (red dots) and the $500 \mathrm{~mm}$ Lens (red circles). Data correspond to release fractions measured for water spray through a $2 \mathrm{~mm}$ hole at $380 \mathrm{psig}$. Each measurement curve represents the average of at least two repeat tests; error bars correspond to the maximum and minimum release fractions measured in individual tests. Test-to-test reproducibility was excellent during comparative testing, and as such, the error bars are mostly hidden behind the measurement symbols. Open circles represent points at aerosol diameters that fall outside the nominal Malvern Insitec-S analyzer measuring range for in-chamber measurements and, as a result, are suspect. Criteria for determining suspect points for in-chamber measurements are described in Section 7.1 of this report. 


\subsection{Mixing Inside the Chamber}

To create a more homogenous aerosol concentration, particularly within the larger chamber lengths (20 to $39 \mathrm{ft}$ ), an array of four "shrouded" Detmar fans was installed $\sim 10 \mathrm{ft}$ downstream of the spray header and just above the collection pan of the chamber. These 4 in. fans were installed with flexible ducting that moved the inlet closer to the bottom of the chamber and spray header to preclude any influence on the jet. Phase I testing included a series of spray release tests conducted using a $2 \mathrm{~mm}$ hole to identify the fan configuration and settings that provided the best mixing of aerosol in the test chamber and best aerosol measurement reproducibility. The results, discussed in Chapter 8 of Schonewill et al. (2012), indicated that use of only the two central fans in the shrouded Detmar array shown in Figure 4.12 was necessary to achieve "adequate" chamber mixing and measurement reproducibility. Before the start of primary Phase II testing, these mixing fan optimization tests were repeated to verify that changes to the chamber configuration (namely the increase in chamber length from 20 to $39 \mathrm{ft}$ ) did not require increased mixing power or use of additional fans. Phase II mixing fan optimization tests confirmed that the fan configuration and operational parameters identified in Phase I were adequate for tests conducted in the $39 \mathrm{ft}$ chamber. As such, no changes were made to fan configuration or operations for Phase II. The results of optimization testing are provided in Appendix A of this report.

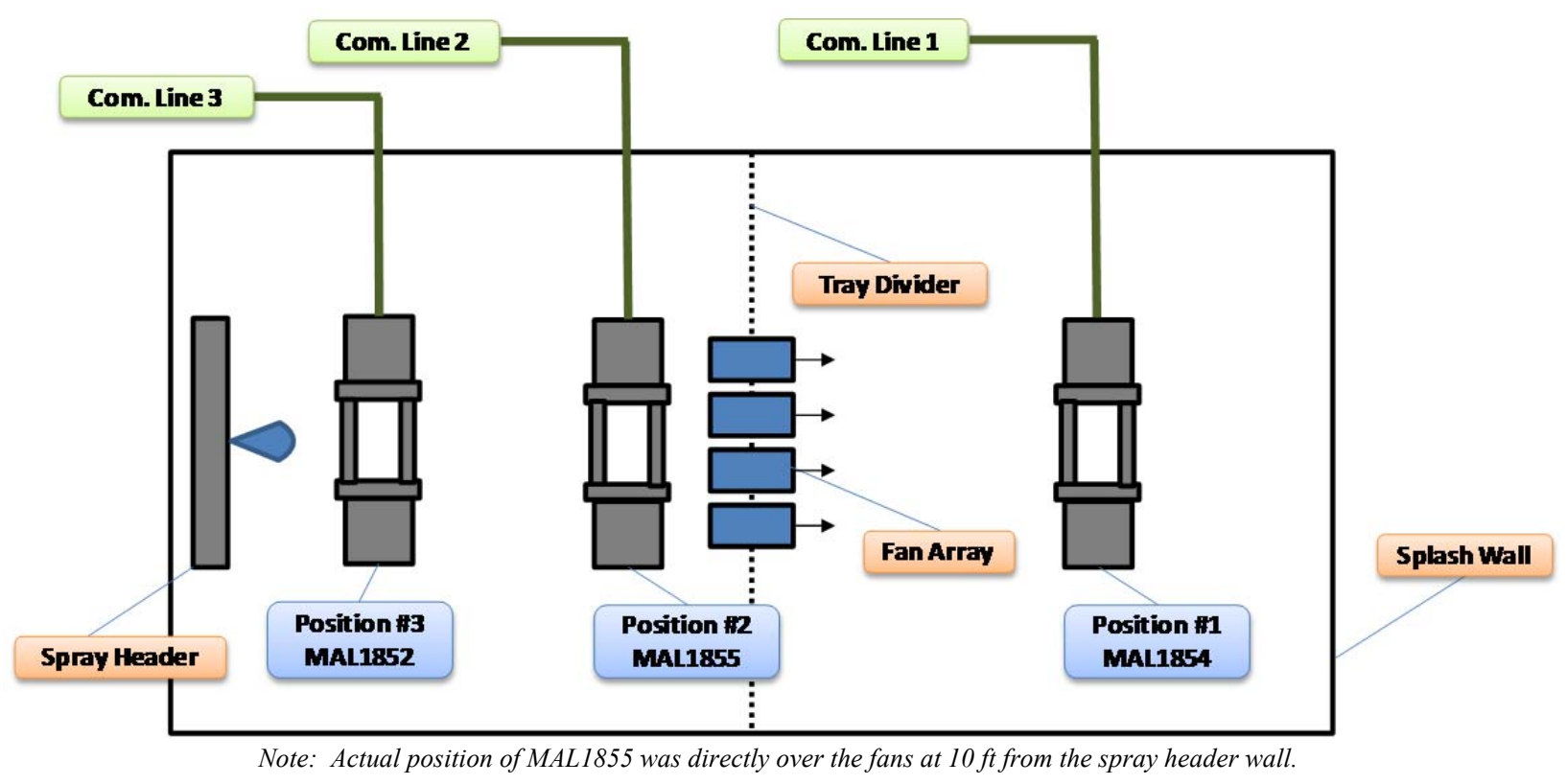

Figure 4.12. Top View of Malvern Insitec-S Instruments and Fan Arrangement

\subsection{Summary of Phase II Changes to Test Equipment}

The test equipment used in Phase II spray release testing is described in this chapter. Much of the equipment used in Phase I testing was adapted without change for use in Phase II testing. However, several key changes were made to the test chamber and aerosol instruments to facilitate successful completion of the Phase II test objective. A summary of these changes is provided in Table 4.7. 
Table 4.7. Summary of Instrument and Test Chamber Changes Between Phase I and Phase II Testing

\begin{tabular}{|c|c|c|}
\hline Parameter/Configuration & Phase I & Phase II \\
\hline Minimum Chamber Length Tested & $20 \mathrm{ft}$ & $10 \mathrm{ft}$ \\
\hline Maximum Chamber Length Tested & $20 \mathrm{ft}$ & $39 \mathrm{ft}$ \\
\hline Aerosol Detection Instruments Used & $\begin{array}{l}\text { (3) Malvern Insitec-S analyzers and } \\
\text { (1) Process Metrix PPC }\end{array}$ & $\begin{array}{l}\text { (Up to three) Malvern Insitec-S } \\
\text { analyzers }\end{array}$ \\
\hline Malvern Insitec-S Horizontal Spacing & $\begin{array}{l}\text { Not uniform: } 20 \mathrm{ft} \text { test chamber } \\
\text { positions were } 13.3,6.2 \text {, and } 3.3 \mathrm{ft} \\
\text { (as measured by key dimension } \mathrm{A} \text { in } \\
\text { Figure 4.9) }\end{array}$ & $\begin{array}{l}\text { Uniform: } 20 \mathrm{ft} \text { test chamber } \\
\text { positions were } 15,10 \text {, and } 5 \mathrm{ft} \\
\text { (as measured by key dimension } \mathrm{A} \\
\text { in Figure } 4.9 \text { ) }\end{array}$ \\
\hline Malvern Insitec-S Lens Used & $100 \mathrm{~mm}$ & $500 \mathrm{~mm}$ \\
\hline Malvern Insitec-S Spacer Length & $500 \mathrm{~mm}$ & $1000 \mathrm{~mm}$ \\
\hline Special Frames for In-Spray Testing & No & Yes \\
\hline $\begin{array}{l}\text { Spray Orifice Distance from Splash Wall at } \\
\text { Maximum Chamber Length Tested }\end{array}$ & $3.5,9.9$ and $19 \mathrm{ft}$ & $3.5,19,28$, and $38 \mathrm{ft}$ \\
\hline Constant Feed Tank Hydrostatic Head & Yes & No \\
\hline Feed Tank Diffuser on Return Leg & Yes - All simulants & $\begin{array}{l}\text { Yes - Water } \\
\text { No-Clay }\end{array}$ \\
\hline
\end{tabular}




\subsection{Test Approach, System Operations, and Data Analysis}

Chapter 4 presented a detailed description of the large-scale test configuration and equipment. In this chapter, the experimental approach used for large-scale testing, test operations, Malvern Insitec-S performance check, simulant preparation, and data analysis are discussed in detail. Sections 5.1 and 5.2 present the general methodology that was used throughout all of the testing and also provide representative pressure and flow profiles to demonstrate system operation. In Sections 5.3 and 5.4, the approach used to prepare test simulant and a matrix of the tests that were performed is presented. Finally, Section 5.5 presents the data analysis methodology used for Phase II testing.

\subsection{General Test Approach}

Each test was conducted in accordance with an approved Test Instruction, and all data and necessary information were recorded electronically, in Laboratory Record Books (LRBs) or in the Test Instruction itself. Operationally, each test consisted of the following checks:

- Daily startup checks

- Spray release tests

- Daily closeout checks.

In addition, daily functional checks and periodic (approximately weekly) performance checks were conducted to verify that each Malvern Insitec-S instrument was performing within acceptable ranges in accordance with the Malvern Insitec-S operating procedure (OP-WTPSP-047, Rev. $2^{1}$ ). The details of these routine checks are provided in Section 5.1.2.

\subsubsection{Daily Startup Checks}

Daily startup checks involved turning on the instruments, configuring the recirculation loop for testing, checking the functionality of all components, and allowing the entire system to warm up. Key tasks that were performed during daily startup checks are identified below:

- Turning on all instruments and DASs

- Synchronizing all DAS and, if needed, video/still camera clocks

- Performing a functional check of all key instruments

- Verifying that ancillary equipment to the aerosol instruments are operating

- Mixing feed/storage tank contents, as needed

- Verifying valve configurations

- Configuring chamber, internal components, and instrumentation as required and recording any changes in the LRB

\footnotetext{
${ }^{1}$ Burns CA. 2012. Malvern Insitec-S Operating Procedure. OP-WTPSP-047, Rev. 2, Pacific Northwest National Laboratory, Richland, Washington.
} 
- Wetting the test chamber walls and, when the chamber is closed, humidifying it to $>80$ percent relative humidity $(\mathrm{RH})$ before collection of baseline test data and subsequent test execution.

\subsubsection{Spray Release Tests}

Spray release tests involved the actual test runs during which the spray was initiated and aerosols were measured in the chamber. The start of each test day began with verifying sufficient simulant inventory was available in the simulant feed tank (TK-2), followed by a subsequent pre-test simulant mixing step that typically only required the use of tank agitators. However, the agitator did not provide adequate tank mixing (i.e., in the space behind the feed tank cooling coils) for the $30 \mathrm{~Pa} \mathrm{Clay}^{1}$ slurry simulant. To assure adequate mixing of the $30 \mathrm{~Pa}$ Clay simulant, the feed tank contents were recirculated through the recirculation loop at $\sim 250$ gpm while both globe valves (GV-1 and GV-2) were fully open. The loop return flow was split equally at valve $3 \mathrm{~V}-3$ to deliver flow to both the storage and feed tanks. When the feed tank mass reached $\sim 1850 \mathrm{lb}(\sim 176 \mathrm{gal})$, the storage tank transfer pump was started to deliver $30 \mathrm{~Pa}$ Clay simulant to the feed tank until the storage tank inventory was depleted. This process was repeated at least three times to assure adequate blending of the $30 \mathrm{~Pa}$ Clay. The $6 \mathrm{~Pa}$ Clay simulant did not require this special mixing protocol and was mixed adequately with the feed tank agitators. Water from the City of Richland did not require agitation, but was agitated to improve feed tank temperature uniformity and control. The feed tank was under constant agitation from the beginning to the end of the test.

To prevent potential Malvern Insitec-S measurement interference from external light sources during background and aerosol data collection, the chamber windows were covered in black plastic and the chamber lights were turned off. The center two shrouded chamber fans were verified to be on and set to $12 \mathrm{~V}$, which was the voltage determined during Phase I shakedown tests to attain good mixing within the chamber (see Chapter 8 in Schonewill et al. (2012)). The chamber RH was verified to be $>75$ percent and the feed tank (TK-2) temperature within the range of 65 to $85^{\circ} \mathrm{F}$ prior to the start of each test. At this point, baseline data needed for the Malvern Insitec-S aerosol analyzers were collected, and then, data logging was initiated on the PLC and the Malvern Insitec-S DASs.

The desired test fluid pressure (100, 200, or 380 psig) and flow (143 to $\sim 300$ gpm) were achieved by bringing the system up to operating conditions via the Pump 1 VFD (Pump 2 and 3 VFDs were slaves) and adjusting globe valves GV-1 and GV-2 (see Figure 4.2). In all tests, the fluid velocity was set to be $>6.5 \mathrm{ft} / \mathrm{s}(>143 \mathrm{gpm}$ ) in the Coriolis meter (FE-2) downstream of the test section. In addition, the Coriolis flow meter (FE-2) backpressure was maintained at $>50$ psig using GV-2 to preclude formation of micro-bubbles that could lead to erroneous readings.

Upon achieving the required operating conditions, the appropriate spray release lever was actuated to release spray from the orifice being evaluated. Simultaneously, the DAS operators were notified to record the time when the spray was initiated. The spray release duration typically lasted 2 min during

\footnotetext{
${ }^{1}$ Throughout this report, discussion of mixed clay ( $80 \mathrm{wt} \%$ kaolin and $20 \mathrm{wt} \%$ bentonite) slurries will be denoted using one of several notations that indicate the target rheological conditions for these simulants. Discussion will primarily refer to the mixed clay simulants as "6 Pa Clay" for mixtures made up to meet a target yield stress of $6 \mathrm{~Pa}$ or as "30 Pa Clay" for mixtures that target a yield stress of $30 \mathrm{~Pa}$. Other identifiers may be used to maintain consistency with test plan and test instruction simulant or sample names. Such identifiers will be defined in the "Acronyms and Abbreviations" section in the front of this report.
} 
water testing, but was reduced to $\sim 70 \mathrm{~s}$ during for both $6 \mathrm{~Pa}$ and $30 \mathrm{~Pa}$ Clay testing to conserve clay simulant and minimize mechanical heat generation. During the time the lever was open, the test engineer constantly monitored the fluid pressure at the test section (PS4 and PS5, see Figure 4.2) to assure that the pressure did not drop by more than 10 psi because of the alternate flow path created by the spray orifice. In contrast to the Phase I tests, no pump leakage (from Pumps 2 and 3 at $<200$ psig) was observed during Phase II testing with water. However, slight pump leakage from the mechanical seal in Pumps 2 and 3 at high pressure (200 to 380 psig) during clay testing was observed, and the leaking fluid was collected and weighed for each test during $30 \mathrm{~Pa}$ Clay testing. ${ }^{1}$ The mass of the leaked fluid coupled with the mass change in the feed tank during a test was used to determine the amount of fluid lost through the spray orifice. After the $\sim 70 \mathrm{~s}$ or 2 min spray duration, the spray release lever was closed to prevent fluid from further leaking through the orifice. Simultaneously, the DAS operators were notified to record the time at which the spray release lever was closed.

After the spray was terminated, the PLC DAS operator would typically stop logging data ( $\sim 1 \mathrm{~min}$ from the end of the spray). The Malvern Insitec-S DAS data logging continued for at least $30 \mathrm{~s}$ to $1 \mathrm{~min}$ after the "end of aerosol detection" condition was observed, and data often was logged during chamber evacuation. The "end of aerosol detection" condition is defined as the point at which the measureable aerosol concentration was zero or very close to zero depending on background/instrument stability. After this, the residual aerosol in the chamber was evacuated, as necessary, by forced ventilation of the chamber. Chamber evacuation was sometimes necessary to clear the test chamber of fine particles that lingered sometimes up to 5 to $10 \mathrm{~min}$ after the spray was stopped. These particles obscured the Malvern Insitec-S laser, but because of their small size, they did not generate a measurable diffraction pattern (and hence did not generate a measurable PSD) on the $500 \mathrm{~mm}$ lens optical detector used in the Malvern Insitec-S for Phase II analysis. If not evacuated, the lingering particles caused an apparent (artificial) increase in the concentration of particles in measurable size ranges at the start of the following test and would impact determination of the initial concentration for that test. This effect was observed predominately after testing with the $30 \mathrm{~Pa}$ Clay simulant, and the generation of lingering particulate matter varied with the orifice used and the fluid pressure.

Because internal reflection from external light sources can manifest as abnormally high signals in the raw light scattering pattern, still images and video recordings of the sprays were collected separately from the spray release tests to avoid impacting the Malvern Insitec-S data. The lights in the chamber were turned on and the plastic window coverings were removed to allow sufficient light into the chamber for the still and video image captures. Still images were captured using a digital camera typically positioned above the spray header. Video images were captured by opening the northeast chamber window port along the side of the spray header. During clay simulant testing, the video images were typically taken from behind the test section (or spray header) looking down the length of the test chamber.

The feed tank temperature was maintained at $75 \pm 10^{\circ} \mathrm{F}$, prior to the start of a test, to minimize any effects that may be caused by condensation or evaporation. During clay simulant testing, density was monitored at the Coriolis flow meters to make sure no significant changes occurred throughout the course of testing with a given simulant.

\footnotetext{
${ }^{1}$ The maximum recorded fluid leak from the Pump 2 and Pump 3 mechanical seals was during test S757. The percent of mass leaked divided by the total sprayed mass was 1.3 percent. Fluid leaks from the seals steadily decreased during $30 \mathrm{~Pa}$ Clay testing to negligible masses by test S793 during which a mass leak percent of 0.03 percent was recorded. The fluid leak rate was not measured after test S793 as it was negligible; however, the lead test engineer continued to visually monitor for any leak mass rate increase.
} 
Samples were collected from the feed tank at the end of each test day during water testing. During clay testing, samples were collected from the PS1 sample port at the start and end of each test day. The clay simulant sample collected at the start of the day was evaluated for consistency and yield stress. Testing was started only when target rheological properties guidelines, as provided in the Test Plan, were met. Clay simulant samples collected at the end of the day were archived. The associated Test Instructions defined the naming conventions used for designating the grab samples collected during testing. In general, the file naming convention allowed easy identification of the test conditions for which the samples were collected. For tests with water, no sample analyses were performed on the samples collected, but samples were archived in case an analysis need was identified later. For the clay simulants, analysis for consistency and yield stress, bulk density, and weight percent of total solids was conducted for samples representing each day of testing. Four $30 \mathrm{~Pa}$ Clay and six $6 \mathrm{~Pa}$ Clay samples were analyzed for particle size. Surface tension measurements were also performed on the supernatant obtained from a representative sample for both clay slurry simulants.

\subsubsection{Daily Closeout Checks}

At the completion of testing each day, a series of daily closeout checks were performed to verify that the testing was completed as planned, video/still images were taken (as necessary), data were backed up, data sheets were collected into test data packages, and system valves were placed in a safe mode.

\subsubsection{Verification of Malvern Insitec-S Performance}

Routine checks of all Malvern Insitec-S aerosol analyzers were conducted to verify acceptable and optimal performance of the instruments during the course of Phase II testing. These checks included alignment of the optical components and verification that the detection system and its electrical subcomponents were working properly before each day of testing (and throughout each day if needed). For example, each Malvern Insitec-S analyzer, for a given combination of components (i.e., optical lens, detector, and laser), has a unique, fingerprint scatter pattern that enables the user to quickly identify window contamination or internal shine resulting from scattered light from either an external light source or the laser. If a shine peak was identified by aerosol engineers, the windows were cleaned, rotated, or replaced until the detector signal was within the normal range for the instrument. In addition, routine (approximately weekly) checks of the Malvern Insitec-S analyzers were performed using an RS-3 Mark II reticle, which is a physical standard purchased directly from Malvern Instruments Ltd. for the purpose of validating instrument performance. The reticle consists of a fixed number of micrometer-sized opaque circles that range in size from 4 to $95 \mu \mathrm{m}$ and that are deposited onto a glass plate. The reticle provides a fixed physical standard against which instrument performance can be assessed. For all performance checks, each Malvern Insitec-S instrument met the accuracy requirements specified by the reticle certificate of analysis. These requirements specify that the diameters corresponding to the $10^{\text {th }}, 50^{\text {th }}$, and $90^{\text {th }}$ cumulative volume percent undersize must be within \pm 3 percent of $33.36 \mu \mathrm{m}, \pm 2$ percent of $44.37 \mu \mathrm{m}$, and \pm 10 percent of $60.94 \mu \mathrm{m}$, respectively. These performance checks provide a baseline measure of instrument accuracy with respect to PSD against which data may be assessed. During the course of large-scale testing, approximately 20 performance checks were conducted on each of the three Malvern Insitec-S analyzers. During Phase I, the reproducibility of the Malvern Insitec-S was evaluated and has been discussed in detail in Chapter 5 of Schonewill et al. (2012). The combined verification of performance against the reticle and checks of optical alignment and detection units maintained the fidelity of aerosol concentration and size measurements made throughout the Phase II testing campaign. 


\subsection{System Flow and Pressure Settings}

This section presents a brief discussion of the pressure/flow variations for the range of orifices that were evaluated during the testing. When a spray was initiated for the largest orifices, the fluid pressure and flow rate exiting the pumps decreased and increased, respectively. For all of the orifices tested in Phase II, the pressure decrease was typically $<5$ psig and on the order of $\sim 10$ psig for the largest orifice (i.e., S4A, $1 \times 76 \mathrm{~mm}$ slot) if not mitigated by restricting the flow path with a globe valve (GV-1) just downstream of the orifice at the initiation of a test spray. However, the flow rate increased noticeably depending on the area of the orifice. The reason for this is directly related to changing the system pressure (or head) curve when the spray is initiated. When the orifice is open to the atmosphere, the system curve shifts to the right, and the point at which this intersects the pump curve shifts to the right. This is tantamount to a decrease in frictional loss and the pressure drop needed to achieve a given flow. This intersection is the operating point. This condition is illustrated in Figure 5.1, which is the pump performance curve supplied by Krebs for the slurry pumps As an example, referring to the red arrow in the figure and using the best efficiency point (BEP) as the system curve, shifting the curve to the right is representative of initiating the spray. Note that the head (and pressure drop) decreases slightly from 160 to $150 \mathrm{ft}$, while the flow rate increases from approximately 280 to $350 \mathrm{gpm}$. The result is a relatively small pressure change, and an increase in the flow rate commensurate with the spray leak rate. This behavior provided stable test pressures that were very close to the target pressures. 


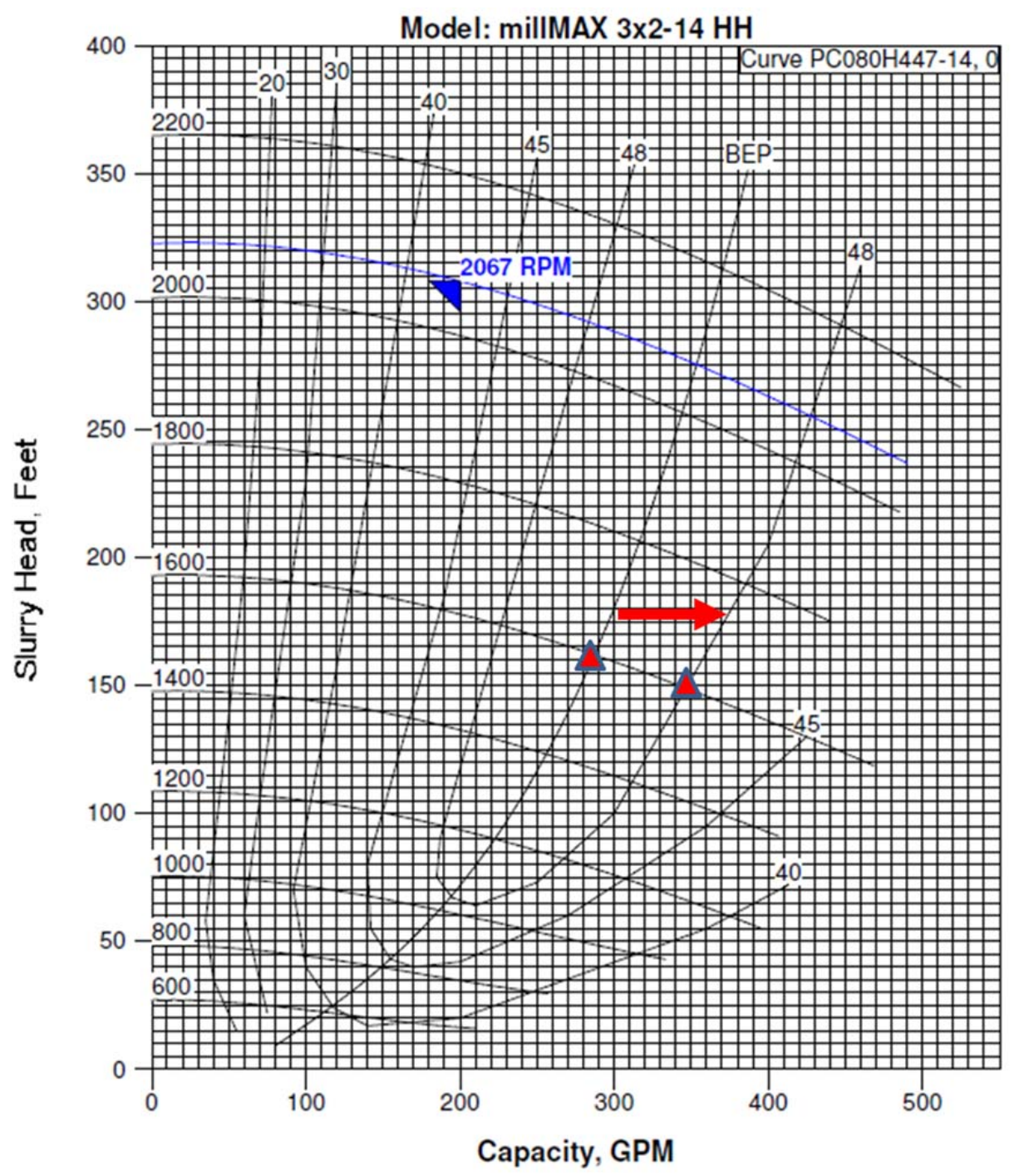

Figure 5.1. Pump Performance Curve for Pumps 1, 2, and 3

A difference in the effect of orifice size can be seen by comparing Figure 5.2 and Figure 5.3.

Figure 5.2 shows the pressure and flow rate versus time for a small orifice $(2 \mathrm{~mm})$ at a relatively low pressure, while Figure 5.3 (an example Phase I dataset) shows a large slot orifice $(1 \times 76 \mathrm{~mm})$ at a high pressure. Note that the change in pressure $(\sim 0 \mathrm{psig})$ and flow rate $(\sim 2 \mathrm{gpm})$ at spray initiation $(\mathrm{t}=0 \mathrm{~s})$ is very small for the $2 \mathrm{~mm}$ case; whereas, for the larger slot, this change is much larger ( $\sim 10 \mathrm{psig}, 55 \mathrm{gpm})$. As discussed above, the pressure change is not as large in magnitude as the flow rate change upon spray initiation. 

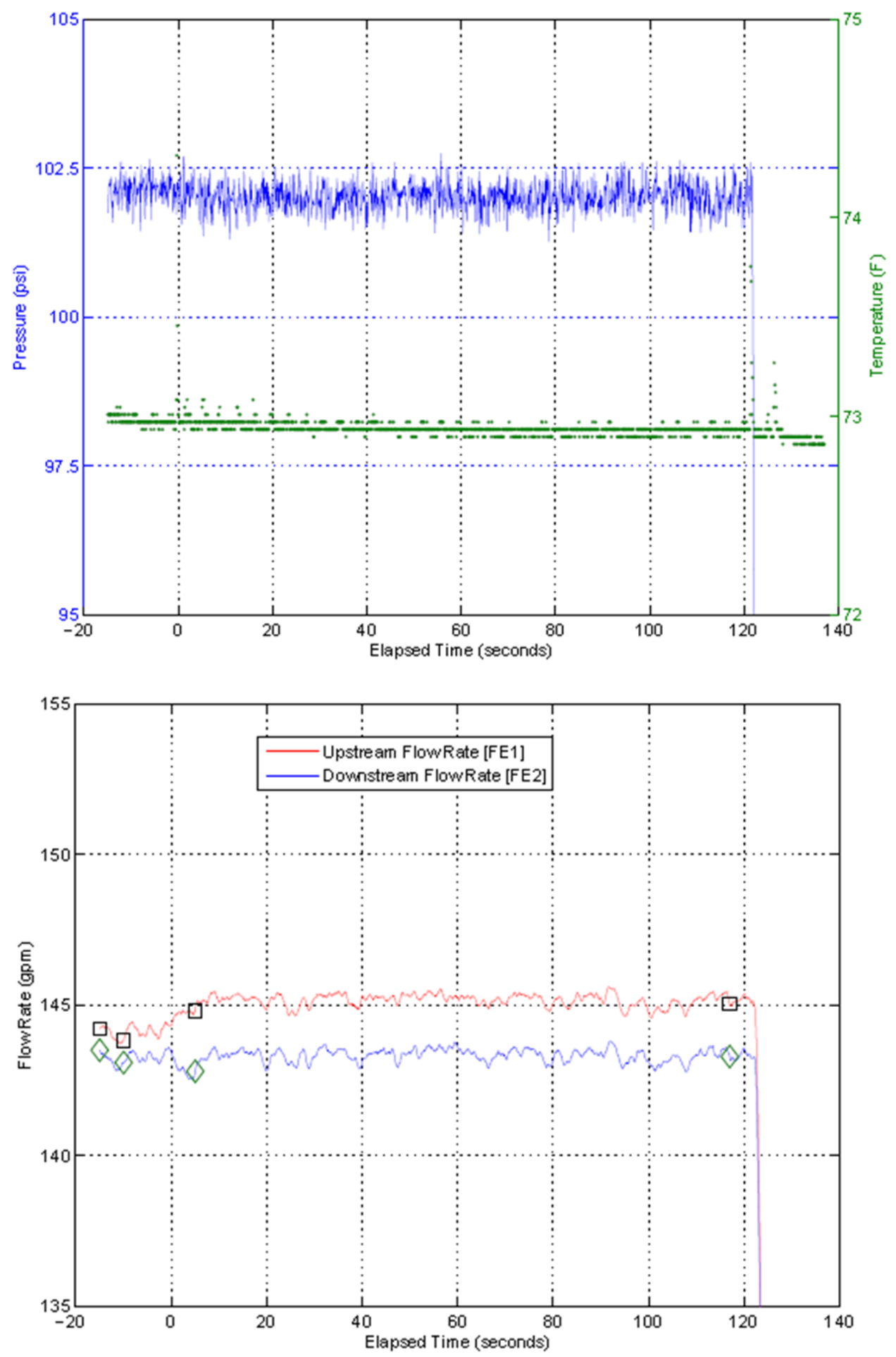

Figure 5.2. Circular Orifice with $2.0 \mathrm{~mm}$ Dimensions (100 psig water test) 

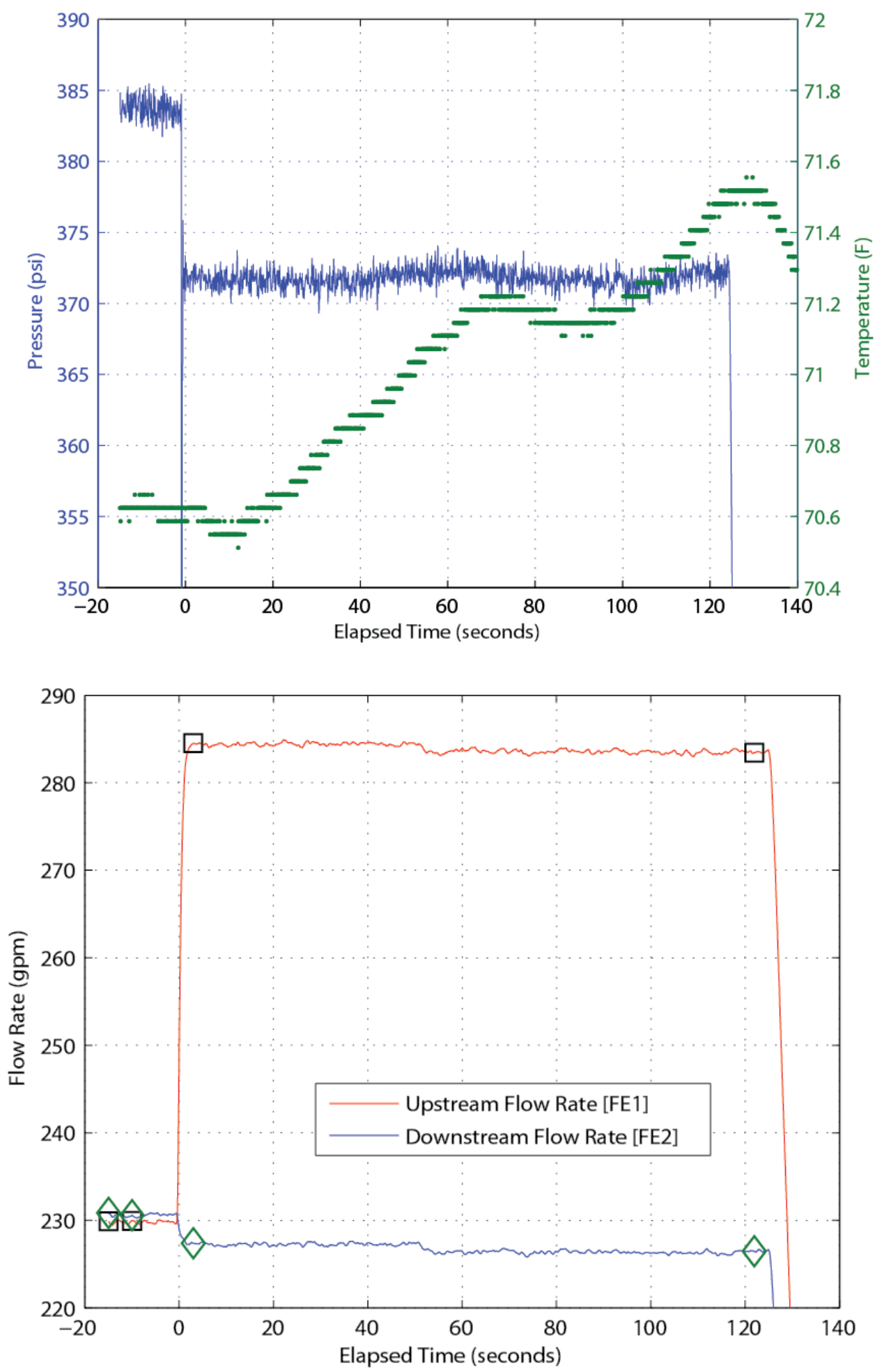

Figure 5.3. Phase I Example of $1 \times 76 \mathrm{~mm}$ Slot Orifice ( 380 psig water test) 
The pressure decrease, as shown in Figure 5.4, can be small ( $\sim 2$ psig) for this largest slot orifice at the highest test pressure upon spray initiation if mitigated. This condition existed because, at the same time the spray was initiated, the flow path was purposely restricted via a globe valve (GV-1) just downstream of the test section. If the flow path remained fully open, the pressure would drop well below the target pressure of $380 \mathrm{psig}$. The reason for adjusting the globe valve $(\mathrm{GV}-1)$ downstream of the test section at the exact time the spray was initiated was to prevent over-pressurization of the recirculation loop, which would cause a pressure relief flow path to open. This was a special case that occurred in tests involving the largest orifice $(1 \times 76 \mathrm{~mm})$ and highest operating pressure $(380 \mathrm{psig})$.
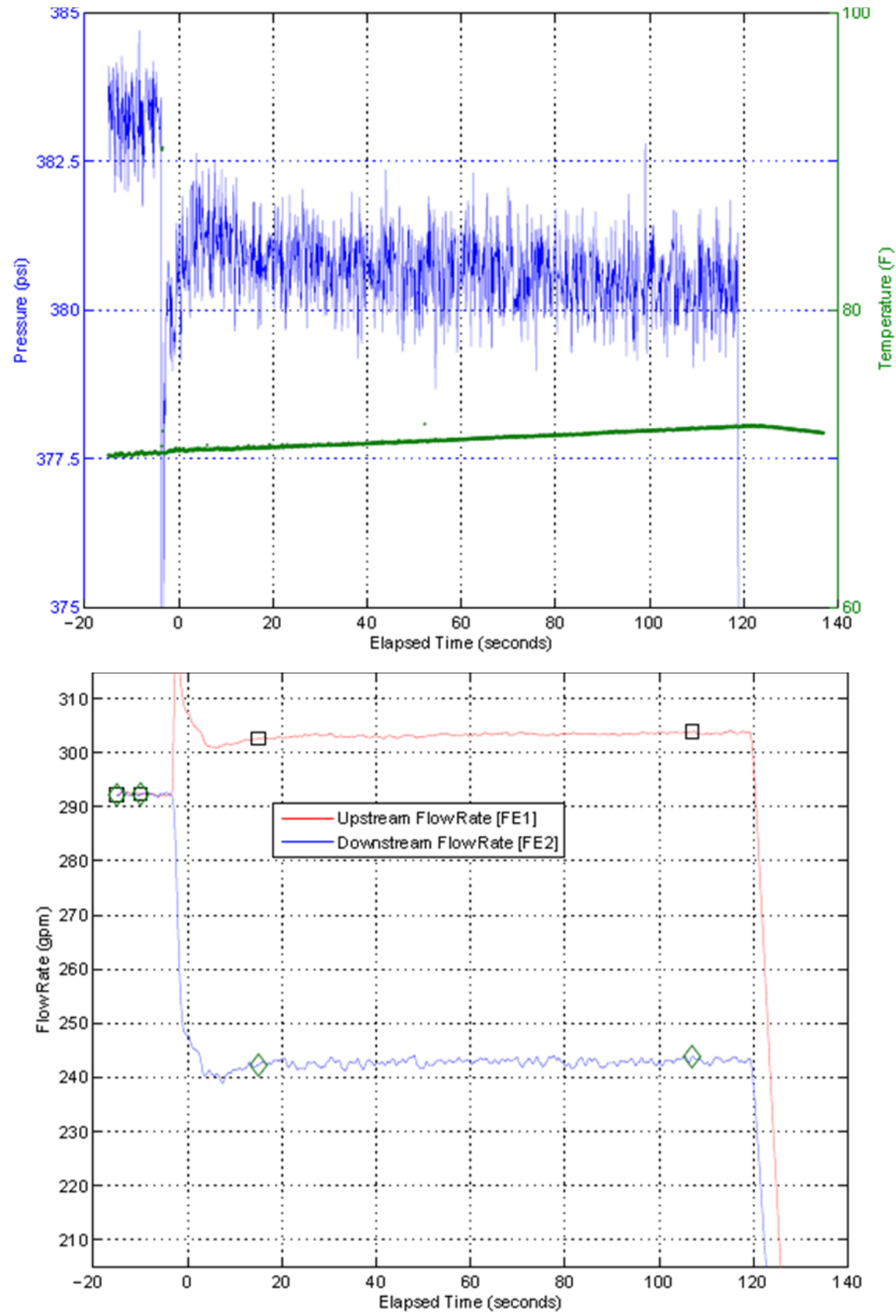

Figure 5.4. Example Upstream and Downstream Flow Meter Readings for the $1 \times 76 \mathrm{~mm}$ Slot During Spray (380 psig, water) - Flow Meter Readings are "For Information Only" 


\subsection{Simulant Preparation}

As discussed in Chapter 3 two clay simulant slurries, each composed of an 80:20 mixture (by weight) of kaolin and bentonite powder in water, were used during large-scale testing,. The first clay simulant had target rheological properties of $30 \mathrm{~Pa} / 30 \mathrm{cP}$; this simulant is otherwise known as the $30 \mathrm{~Pa}$ Clay simulant. The second clay slurry target rheological properties were $6 \mathrm{~Pa} / 6 \mathrm{cP}$; this simulant is referred to as the $6 \mathrm{~Pa}$ Clay simulant. The total $\mathrm{wt} \%$ solids for the final $30 \mathrm{~Pa}$ Clay was $\sim 32 \mathrm{wt} \%$ solids while the 6 Pa Clay simulant was $\sim 27 \mathrm{wt} \%$ solids. Both simulants were used to evaluate the effect of non-Newtonian rheology on aerosol generation rates and release fractions. The basis for the simulant selection and the physical properties of the simulants used are presented in Chapter 3. This section only presents the approach used to prepare and maintain the two clay simulants used during large-scale testing.

To prepare the $30 \mathrm{~Pa}$ Clay simulant, a 1:1 ratio of kaolin and bentonite were pre-mixed before introducing them into the mixing vessel. To complete the $30 \mathrm{~Pa}$ Clay test matrix, a total volume of $\sim 500$ gal of $30 \mathrm{~Pa}$ Clay simulant was prepared. To allow target rheological properties to be evaluated and controlled during large-scale simulant preparation, small, 60-gal batches of $30 \mathrm{~Pa}$ Clay were prepared using an 80-gal mixing vessel equipped with a variable speed overhead impeller mixer and a diaphragm pump. The pneumatic diaphragm pump drew simulant material from the bottom of the mixing vessel, thus inducing recirculation. This approach was required because the $30 \mathrm{~Pa}$ Clay simulant was very difficult to mix after the addition of $\sim 90$ percent of the dry powders needed for its makeup.

For each 60-gal batch of $30 \mathrm{~Pa}$ Clay simulant, the mixing vessel was preloaded with the required amount of City of Richland water to obtain a targeted total weight percent of solids. The initial mass of water varied from batch to batch and was adjusted as the rheological properties of the initial batches of clay slurry were evaluated. Before the addition of any clay powder, Mt Hood 480 Biocide was added and dispersed throughout the mixing tank at a concentration of 5 parts per mission (ppm) to avoid any biological activity that could potentially impact the physical properties (notably the rheology) of the simulant mixture. The 1:1 kaolin-to-bentonite powder blend was added slowly to the mixing tank to assure that the material was well dispersed and able to hydrate without clumping. After all of the 1:1 blended powder was added, the system was allowed to mix for a minimum of 10 min before adding the remainder of the dry kaolin powder needed to achieve the desired 80:20 kaolin-to-bentonite ratio. After the final addition of kaolin, the simulant was mixed for an additional $15 \mathrm{~min}$ to assure that the clay was well-homogenized. Archive samples were taken at the completion of each batch.

When preparation of each batch was complete, the $30 \mathrm{~Pa}$ Clay simulant was transferred using a pneumatic diaphragm pump to an intermediate bulk container, commonly referred to as a "tote." Four $\sim 60$-gal batches were placed in each tote and multiple totes were filled. The feedstock simulant was transferred from totes into the large feed tank (TK-2) in 350 to 400 gal batches. The 30 Pa Clay simulant material was sheared and recirculated using the recirculation loop and the tank agitators, as discussed in Section 5.1.2, to provide adequate tank mixing (i.e., in the space behind the feed tank cooling coils). Once the rheological properties were considered quasi-stable and the material was well-mixed, the total solids of the simulant were modified to achieve and maintain the targeted rheological properties, in this case $30 \mathrm{~Pa} / 30 \mathrm{cP}$. This usually required dilution of the simulant with City of Richland water (to counteract any upward drift in simulant rheology from shear or evaporation) or blending of different simulant batches of different total weight percent of solids. 
The 6 Pa Clay simulant was generated by diluting the remaining 30 Pa Clay simulant inventory stored in totes and the feed tank at the end of $30 \mathrm{~Pa}$ Clay simulant testing. It should be noted that at least one full tote of $30 \mathrm{~Pa}$ Clay simulant inventory used to produce the $6 \mathrm{~Pa}$ Clay simulant was simulant that was tested (i.e., sprayed into the test chamber) and reclaimed from the test chamber catch pans via a 1 in. diaphragm pump. During initial dilution of the clay simulant, the same blending/mixing protocol (i.e., at least three storage tank transfers) discussed previously was followed. Unlike with the $30 \mathrm{~Pa}$ Clay simulant, once the targeted rheological properties were achieved, the $6 \mathrm{~Pa}$ Clay simulant was adequately mixed by the feed tank agitator alone, and as a result, no additional mixing was required for testing.

\subsection{Test Matrix}

Two distinct series of tests were included in Phase II testing-functional tests and aerosol release fraction tests, commonly referred to as matrix tests. The functional tests were focused on determining the effect of Malvern Insitec-S instrument changes (i.e., 100 to $500 \mathrm{~mm}$ lens and 500 to $1000 \mathrm{~mm}$ spacer bars), chamber humidity, Malvern Insitec-S vertical and horizontal position, Malvern Insitec-S detector lens purge rate (with air), and chamber mixing fan configurations (e.g., number of bilge fans, fan voltage, etc.). These functional tests are discussed in detail in Appendix A and only summarized in groups 0 through 5 below. The scope of the matrix tests, summarized in groups 6 through 8 below, was provided in TP-WTPSP-031 ${ }^{1}$ and focused on measuring the aerosol release fractions to evaluate 1) the effect of spray header orifice distance from the splash wall, 2) the effect of chamber size and spray wall distance, and 3) the effect of the WTP non-Newtonian rheology limits on aerosol generation. In total, a series of 620 tests were completed. These test groups are:

\section{Functional Tests (Groups 0 through 5)}

- Group 0 focused on establishing the impact of the mixing fan configuration on release fraction and size distribution in the $39 \mathrm{ft}$ chamber with the $2 \mathrm{~mm}$ orifice and at $380 \mathrm{psig}$. Conditions varied from no fans, to four fans at $12 \mathrm{~V}$, two center fans at $6 \mathrm{~V}$, and two center fans at $12 \mathrm{~V}$.

- Group 1 focused on establishing the impact of Malvern Insitec-S position (vertical and horizontal), in relation to the spray orifice, on release fraction and aerosol size distribution measurements. Malvern Insitec-S laser heights were varied from $1.7 \mathrm{ft}$ above the spray orifice (standard position), $1.1 \mathrm{ft}$ above the spray orifice, and $0.4 \mathrm{ft}$ "below" the spray orifice. The horizontal lengths, laser distance from the spray orifice, were varied from the standard lengths of 29.3, 19.3, and $9.3 \mathrm{ft}$ for Malvern Insitec-S positions 1,2, and 3, respectively, to alternative horizontal lengths of 34.3, 24.3, and $14.3 \mathrm{ft}$ for Malvern Insitec-S positions 1, 2, and 3, respectively.

- Group 3 focused on establishing the effect of Malvern Insitec-S detector lenses on size distribution and concentration measurements when using a $500 \mathrm{~mm}$ lens during Phase II and a $100 \mathrm{~mm}$ lens during Phase I.

- Group 4 focused on establishing the impact of Malvern Insitec-S purge air rate (varied from 1 to $100 \mathrm{SCFH}$ ) on Malvern Insitec-S measurements for a range of test conditions (orifice, fluid pressure, Malvern Insitec-S position).

\footnotetext{
${ }^{1}$ Gauglitz PA. 2012. Test Plan for Spray Leak Quantification to Support WTP Spray Release Methodology. TP-WTPSP-031, Rev 1.0, Pacific Northwest National Laboratory, Richland, Washington.
} 
- Group 5 focused on establishing the effect of chamber RH, ranging from 50 to 100 percent, on the release fraction and size distribution.

\section{Matrix Tests (Groups 6 through 8)}

- Group 6 matrix tests, summarized in Table 5.1, focused on establishing the impact of the effect of spray header distance from the splash wall on release fraction and aerosol size distribution. In the $39 \mathrm{ft}$ chamber, all four orifices and three fluid pressures were evaluated with the spray header orifice at $38,28,19$, and $3.5 \mathrm{ft}$ from the chamber splash wall.

- Group 7 matrix tests, summarized in Table 5.2, focused on establishing the impact of the chamber size $(10,20,30$, and $39 \mathrm{ft}$ wall-to-wall) and spray orifice-to-splash-wall distance on release fraction and size distribution.

- Group 8 matrix tests, summarized in Table 5.3, focused on establishing the impact of using the kaolin-bentonite clay simulant with non-Newtonian rheology $(\sim 30 \mathrm{~Pa} / 30 \mathrm{cP}$ and $\sim 6 \mathrm{~Pa} / 6 \mathrm{cP})$ on aerosol generation, release fraction, and aerosol size distribution in a $20 \mathrm{ft}$ chamber with all four orifices and three fluid pressures.

Initial testing was primarily focused on functional tests, Groups 0 through 5, and provided the foundation for spray release test configuration and conditions on parameters such as Malvern Insitec-S lens and spacer bars, Malvern Insitec-S purge rate, mixing fan configuration, Malvern Insitec-S laser position in relation to spray orifice, and chamber RH. The number of functional tests was dictated by the observed results and need for additional insight into potential effects of the particular test condition. The matrix tests, described by Groups 6 through 8, encompassed the bulk of spray release testing and went as planned, with any additional tests being conducted only because of operational mishaps, test parameter errors, or measurement equipment malfunctions. Table 5.1 through Table 5.3 summarizes these matrix tests and provides both the actual and accepted number of tests. 
Table 5.1. Group 6 Tests (Planned and Actual) to Establish Effect of Spray Orifice Distance from Splash Wall

\begin{tabular}{|c|c|c|c|c|c|c|c|c|c|}
\hline \multirow[b]{2}{*}{ Simulant } & \multirow[b]{2}{*}{$\begin{array}{c}\text { Chamber } \\
\text { Configuration }^{(a)}\end{array}$} & \multirow{2}{*}{$\begin{array}{l}\text { Spray Orifice } \\
\text { Distance from } \\
\text { Splash Wall (ft) }\end{array}$} & \multicolumn{2}{|c|}{ Malvern Insitec-S Configuration $^{(\mathrm{b})}$} & \multirow[b]{2}{*}{$\begin{array}{c}\text { Mixing Fan } \\
\text { Conditions }\end{array}$} & \multirow[b]{2}{*}{$\begin{array}{l}\text { Nominal Orifice } \\
(\mathrm{mm})\end{array}$} & \multirow{2}{*}{$\begin{array}{l}\text { Test Fluid } \\
\text { Pressure } \\
\text { (psig) }\end{array}$} & \multicolumn{2}{|c|}{ Number of Tests } \\
\hline & & & Purge Rate $(\mathrm{SCFH})^{(\mathrm{c})}$ & Locations & & & & Planned $^{(\mathrm{e})}$ & $\begin{array}{c}\text { Actual/ } \\
\text { Accepted }\end{array}$ \\
\hline Water & 39S3A & 38 & $\begin{array}{c}1 \text { [S8A, S8B (100 psig)] } \\
50[\text { S8B (200, 380 psig), S8C }] \\
100(\text { S4A })\end{array}$ & Standard $^{(d)}$ & $\begin{array}{l}12 \mathrm{~V}, \text { two } \\
\text { center fans }\end{array}$ & $\begin{array}{l}1.0 \times 10 \text { Slot } \\
1.0 \times 20 \text { Slot } \\
1.0 \times 76.2 \text { Slot } \\
\text { Circular, } 2.0\end{array}$ & $100,200,380$ & 36 & $47 / 40$ \\
\hline Water & 39S3B & 28 & $\begin{array}{c}1(\mathrm{~S} 8 \mathrm{~A}, \mathrm{~S} 8 \mathrm{~B}) \\
50(\mathrm{~S} 8 \mathrm{C}, \mathrm{S} 4 \mathrm{~A})\end{array}$ & Standard $^{(\mathrm{d})}$ & $\begin{array}{l}12 \mathrm{~V} \text {, two } \\
\text { center fans }\end{array}$ & $\begin{array}{l}1.0 \times 10 \text { Slot } \\
1.0 \times 20 \text { Slot } \\
1.0 \times 76.2 \text { Slot } \\
\text { Circular, } 2.0\end{array}$ & $100,200,380$ & 36 & $44 / 40$ \\
\hline Water & $39 \mathrm{~S} 3 \mathrm{C}$ & 19 & $\begin{array}{c}1(\mathrm{~S} 8 \mathrm{~A}, \mathrm{~S} 8 \mathrm{~B}) \\
50(\mathrm{~S} 8 \mathrm{C}, \mathrm{S} 4 \mathrm{~A})\end{array}$ & Standard $^{(\mathrm{d})}$ & $\begin{array}{l}12 \mathrm{~V} \text {, two } \\
\text { center fans }\end{array}$ & $\begin{array}{c}1.0 \times 10 \text { Slot } \\
1.0 \times 20 \text { Slot } \\
1.0 \times 76.2 \text { Slot } \\
\text { Circular, } 2.0\end{array}$ & $100,200,380$ & 36 & $53 / 50$ \\
\hline Water & 39S3D & $3.6^{(\mathrm{f})}$ & $\begin{array}{c}1(\mathrm{~S} 8 \mathrm{~A}, \mathrm{~S} 8 \mathrm{~B}) \\
50(\mathrm{~S} 8 \mathrm{C}, \mathrm{S} 4 \mathrm{~A})\end{array}$ & Standard $^{(d)}$ & $\begin{array}{l}12 \mathrm{~V}, \text { two } \\
\text { center fans }\end{array}$ & $\begin{array}{c}1.0 \times 10 \text { Slot } \\
1.0 \times 20 \text { Slot } \\
1.0 \times 76.2 \text { Slot } \\
\text { Circular, } 2.0\end{array}$ & $100,200,380$ & 36 & $39 / 38$ \\
\hline & & & & & & & Total & 144 & $183 / 168$ \\
\hline
\end{tabular}

(a) The five-character chamber configuration code (e.g., 39S3A) represents: $39=$ total chamber length in feet, $\mathrm{S}=$ spacer bars (in-chamber testing), $3=\mathrm{Malvern}$ Insitec-S analyzers used, and the last character represents lengths of spray header extensions where A = no spray header extension, B $=10 \mathrm{ft}, \mathrm{C}=19 \mathrm{ft}$, and $\mathrm{D}=34.3 \mathrm{ft}$ spray header extension.

(b) The Malvern Insitec-S instruments were configured with $500 \mathrm{~mm}$ lens and $1000 \mathrm{~mm}$ spacers for all matrix tests.

(c) Malvern Insitec-S purge air rates were varied depending on the spray orifice and test fluid pressure.

(d) Standard In-Chamber Malvern Insitec-S Locations: (Position 1) A = 75\% of chamber length (360 in.), B\&C = 28 in., D\&E $=28$ in.; (Position 2) A = 50\% of chamber length (240 in.), $\mathrm{B} \& \mathrm{C}=28$ in., D\&E = 28 in.; (Position 3) $\mathrm{A}=25 \%$ of chamber length (120 in.), B\&C = 28 in., D\&E = 28 in. Dimensions A, B, C, D, and E are defined and illustrated in Figure 4.9 .

(e) Triplicate runs were planned for each orifice at each fluid pressure for in-chamber tests.

(f) A target distance of $3.5 \mathrm{ft}$ was selected to match small-scale spray distances. The as-fabricated distance for large-scale was $3.6 \mathrm{ft}$. Throughout this report, the target distance of $3.5 \mathrm{ft}$ is used when referring to this dimension. 
Table 5.2. Group 7 Tests (Planned and Actual) to Establish Effect of Chamber Size

\begin{tabular}{|c|c|c|c|c|c|c|c|c|c|}
\hline \multirow[b]{2}{*}{ Simulant } & \multirow[b]{2}{*}{$\begin{array}{c}\text { Chamber } \\
\text { Configuration }^{(a)}\end{array}$} & \multirow{2}{*}{$\begin{array}{l}\text { Spray Orifice } \\
\text { Distance from } \\
\text { Splash Wall (ft) }\end{array}$} & \multicolumn{2}{|c|}{ Malvern Insitec-S Configuration $^{(b)}$} & \multirow[b]{2}{*}{$\begin{array}{c}\text { Mixing Fan } \\
\text { Conditions }\end{array}$} & \multirow[b]{2}{*}{$\begin{array}{c}\text { Nominal } \\
\text { Orifice }(\mathrm{mm})\end{array}$} & \multirow{2}{*}{$\begin{array}{l}\text { Test Fluid } \\
\text { Pressure } \\
\text { (psig) }\end{array}$} & \multicolumn{2}{|c|}{ Number of Tests } \\
\hline & & & Purge Rate $(\mathrm{SCFH})^{(\mathrm{c})}$ & Locations & & & & Planned $^{(\mathrm{e})}$ & $\begin{array}{c}\text { Actual/ } \\
\text { Accepted }\end{array}$ \\
\hline Water & $39 \mathrm{~S} 3 \mathrm{~A}^{(\mathrm{f})}$ & 38 & $\begin{array}{c}1 \text { [S8A, S8B (100 psig)] } \\
50[\text { S8B (200, 380 psig), S8C }] \\
100(\mathrm{~S} 4 \mathrm{~A})\end{array}$ & Standard $^{(\mathrm{d})}$ & $\begin{array}{l}12 \mathrm{~V} \text {, two } \\
\text { center fans }\end{array}$ & $\begin{array}{c}1.0 \times 10 \text { Slot } \\
1.0 \times 20 \text { Slot } \\
1.0 \times 76.2 \text { Slot } \\
\text { Circular, } 2.0\end{array}$ & $100,200,380$ & 36 & $47 / 40$ \\
\hline Water & $39 \mathrm{~F} 3 \mathrm{~A}$ & 38 & 100 (S8A, S8B, S8C, S4A) & In-spray ${ }^{(\mathrm{f})}$ & No fans & $\begin{array}{l}1.0 \times 10 \text { Slot } \\
1.0 \times 20 \text { Slot } \\
1.0 \times 76.2 \text { Slot } \\
\text { Circular, } 2.0\end{array}$ & $100,200,380$ & 12 & $21 / 20$ \\
\hline Water & $30 \mathrm{~S} 3 \mathrm{~A}$ & 29 & $\begin{array}{c}1 \text { [S8A, S8B (100 psig)] } \\
50[\text { S8B (200, 380 psig), S8C }] \\
50(\mathrm{~S} 4 \mathrm{~A})\end{array}$ & Standard $^{(\mathrm{d})}$ & $\begin{array}{l}12 \mathrm{~V} \text {, two } \\
\text { center fans }\end{array}$ & $\begin{array}{c}1.0 \times 10 \text { Slot } \\
1.0 \times 20 \text { Slot } \\
1.0 \times 76.2 \text { Slot } \\
\text { Circular, } 2.0\end{array}$ & $100,200,380$ & 36 & $38 / 34$ \\
\hline Water & $20 \mathrm{~S} 3 \mathrm{~A}$ & 19 & $\begin{array}{c}1[\mathrm{~S} 8 \mathrm{~A}, \mathrm{~S} 8 \mathrm{~B}(100 \mathrm{psig})] \\
50[\mathrm{~S} 8 \mathrm{~B}(200,380 \mathrm{psig}), \mathrm{S} 8 \mathrm{C}] \\
50[\mathrm{~S} 4 \mathrm{~A}(100 \mathrm{psig})] \\
100[\mathrm{~S} 4 \mathrm{~A}(200,380 \mathrm{psig})]\end{array}$ & Standard $^{(\mathrm{d})}$ & $\begin{array}{l}12 \mathrm{~V} \text {, two } \\
\text { center fans }\end{array}$ & $\begin{array}{c}1.0 \times 10 \text { Slot } \\
1.0 \times 20 \text { Slot } \\
1.0 \times 76.2 \text { Slot } \\
\text { Circular, } 2.0\end{array}$ & $100,200,380$ & 36 & $37 / 37$ \\
\hline Water & $10 \mathrm{~S} 2 \mathrm{~A}$ & 9 & $\begin{array}{c}1 \text { [S8A, S8B (100 psig) }] \\
50[\mathrm{~S} 8 \mathrm{~B}(200,380 \mathrm{psig}), \mathrm{S} 8 \mathrm{C}] \\
100(\mathrm{~S} 4 \mathrm{~A})\end{array}$ & Standard $^{(d)}$ & No fans & $\begin{array}{c}1.0 \times 10 \text { Slot } \\
1.0 \times 20 \text { Slot } \\
1.0 \times 76.2 \text { Slot } \\
\text { Circular, } 2.0\end{array}$ & $100,200,380$ & 36 & $38 / 37$ \\
\hline
\end{tabular}

(a) The five-character chamber configuration code (e.g., 30S3A) represents: $30=$ total chamber length in feet, $\mathrm{S}=$ spacer bars (in-chamber testing) or $\mathrm{F}=$ frames (in-spray testing), $3=$ Malvern Insitec-S analyzers used, and $\mathrm{A}=$ no spray header extension.

(b) The Malvern Insitec-S instruments were configured with $500 \mathrm{~mm}$ lens and $1000 \mathrm{~mm}$ spacers for all matrix tests.

(c) Malvern Insitec-S purge air rates were varied depending on the spray orifice and test fluid pressure.

(d) Standard In-Chamber Malvern Insitec-S Locations: (Position 1) A = 75\% of chamber length (360 in.), B\&C = 28 in., D\&E = 28 in.; (Position 2) A = 50\% of chamber length (240 in.), $\mathrm{B} \& \mathrm{C}=28$ in., $\mathrm{D} \& \mathrm{E}=28$ in.; (Position 3) $\mathrm{A}=25 \%$ of chamber length (120 in.), $\mathrm{B} \& \mathrm{C}=28$ in., D\&E $=28$ in. Dimensions A, B, C, D, and E are defined and illustrated in Figure 4.9.

(e) Triplicate runs were planned for each orifice at each fluid pressure for in-chamber tests and single runs were planned for each orifice at each fluid pressure for in-spray tests.

(f) The test data set for the 39S3A chamber configuration is duplicated from Table 5.1 to illustrate the complete test data package used in establishing the effect of chamber length.

(g) For in-spray tests: The vertical position of Malvern Insitec-S instrument laser beam varied and was always centered in the spray jet majority. Similarly, the spray jet was always centered between the Malvern Insitec-S laser source and detector. The Malvern Insitec-S laser beam to spray orifice distance was: MAL1854 (Position 1) = 11.3 ft, MAL1852 $($ Position 2) $=7.8 \mathrm{ft}$, and MAL1855 (Position 3) $=4.3 \mathrm{ft}$. 
Table 5.3. Group 8 Tests (Planned and Actual) to Evaluate Effect of the WTP Non-Newtonian Rheology Limits on Aerosol Generation Rates

\begin{tabular}{|c|c|c|c|c|c|c|c|c|c|c|}
\hline \multirow[b]{2}{*}{ Simulant } & \multirow{2}{*}{$\begin{array}{c}\text { Simulant } \\
\text { Rheology } \\
\text { Target }\end{array}$} & \multirow[b]{2}{*}{$\begin{array}{c}\text { Chamber } \\
\text { Configuration }^{(a)}\end{array}$} & \multirow{2}{*}{$\begin{array}{c}\text { Spray } \\
\text { Orifice } \\
\text { Distance } \\
\text { from Splash } \\
\text { Wall (ft) }\end{array}$} & \multicolumn{2}{|c|}{ Malvern Insitec-S Configuration $^{(b)}$} & \multirow[b]{2}{*}{$\begin{array}{c}\text { Mixing Fan } \\
\text { Conditions }\end{array}$} & \multirow[b]{2}{*}{$\begin{array}{c}\text { Nominal } \\
\text { Orifice }(\mathrm{mm})\end{array}$} & \multirow{2}{*}{$\begin{array}{c}\text { Test Fluid } \\
\text { Pressure } \\
\text { (psig) }\end{array}$} & \multicolumn{2}{|c|}{ Number of Tests } \\
\hline & & & & Purge Rate $(\mathrm{SCFH})^{(\mathrm{c}, \mathrm{d})}$ & Locations & & & & Planned $^{(f)}$ & $\begin{array}{c}\text { Actual/ } \\
\text { Accepted }\end{array}$ \\
\hline \multirow{2}{*}{ Clay } & \multirow{2}{*}{$\begin{array}{c}30 \mathrm{~Pa} / \\
30 \mathrm{mPa} \cdot \mathrm{s}\end{array}$} & $20 \mathrm{~S} 3 \mathrm{~A}$ & \multirow{2}{*}{19} & $\begin{array}{c}50(\mathrm{~S} 8 \mathrm{~A}, \mathrm{~S} 8 \mathrm{~B}, \mathrm{~S} 8 \mathrm{C}) \\
100(\mathrm{~S} 4 \mathrm{~A})\end{array}$ & Standard $^{(\mathrm{e})}$ & $\begin{array}{l}12 \mathrm{~V} \text {, two } \\
\text { center fans }\end{array}$ & $\begin{array}{c}1.0 \times 10 \text { Slot } \\
1.0 \times 20 \text { Slot } \\
1.0 \times 76.2 \text { Slot } \\
\text { Circular, } 2.0\end{array}$ & $\begin{array}{l}100,200 \\
380\end{array}$ & 36 & $37 / 35$ \\
\hline & & $20 \mathrm{~F} 3 \mathrm{~A}$ & & $\begin{array}{c}150,70,60(\mathrm{~S} 8 \mathrm{~A}, \mathrm{~S} 8 \mathrm{~B}, \mathrm{~S} 8 \mathrm{C}) \\
150,70,70(\mathrm{~S} 4 \mathrm{~A})\end{array}$ & In-spray ${ }^{(\mathrm{g})}$ & No fans & $\begin{array}{c}1.0 \times 10 \text { Slot } \\
1.0 \times 20 \text { Slot } \\
1.0 \times 76.2 \text { Slot } \\
\text { Circular, } 2.0\end{array}$ & $\begin{array}{c}100,200 \\
380\end{array}$ & 12 & $15 / 13$ \\
\hline \multirow{3}{*}{ Clay } & \multirow{2}{*}{$\begin{array}{c}6 \mathrm{~Pa} / \\
6 \mathrm{mPa} \cdot \mathrm{s}\end{array}$} & $20 \mathrm{~S} 3 \mathrm{~A}$ & \multirow{2}{*}{19} & $\begin{array}{c}50(\mathrm{~S} 8 \mathrm{~A}, \mathrm{~S} 8 \mathrm{~B}, \mathrm{~S} 8 \mathrm{C}) \\
150,70,70(\mathrm{~S} 4 \mathrm{~A})\end{array}$ & Standard $^{(\mathrm{e})}$ & $\begin{array}{l}12 \mathrm{~V} \text {, two } \\
\text { center fans }\end{array}$ & $\begin{array}{c}1.0 \times 10 \text { Slot } \\
1.0 \times 20 \text { Slot } \\
1.0 \times 76.2 \text { Slot } \\
\text { Circular, } 2.0\end{array}$ & $\begin{array}{l}100,200 \\
\quad 380\end{array}$ & 36 & $41 / 35$ \\
\hline & & $20 \mathrm{~F} 3 \mathrm{~A}$ & & $\begin{array}{c}100(\mathrm{~S} 8 \mathrm{~A}, \mathrm{~S} 8 \mathrm{~B}, \mathrm{~S} 8 \mathrm{C}) \\
150,70,70(\mathrm{~S} 4 \mathrm{~A})\end{array}$ & In-spray ${ }^{(\mathrm{g})}$ & No fans & $\begin{array}{c}1.0 \times 10 \text { Slot } \\
1.0 \times 20 \text { Slot } \\
1.0 \times 76.2 \text { Slot } \\
\text { Circular, } 2.0\end{array}$ & $\begin{array}{l}100,200 \\
380\end{array}$ & 12 & $12 / 12$ \\
\hline & & & & & & & & TOTAL & 96 & $102 / 95$ \\
\hline
\end{tabular}

(a) The five-character chamber configuration code (e.g., 20S3A) represents: $20=$ total chamber length in feet, $\mathrm{S}=$ spacer bars (in-chamber testing) or $\mathrm{F}=$ frames (in-spray testing), 3 = Malvern Insitec-S analyzers used, and A = no spray header extension.

(b) The Malvern Insitec-S instruments were configured with $500 \mathrm{~mm}$ lens and $1000 \mathrm{~mm}$ spacers for all matrix tests.

(c) Malvern Insitec-S purge air rates were varied depending on the spray orifice.

(d) When a series of three Malvern Insitec-S air purges rates are listed these correspond to MAL1854 (Position 1), MAL1852 (Position 2), and MAL1855 (Position 3) with position 1 being the farthest from the spray orifice header (horizontally).

(e) Standard in-chamber Malvern Insitec-S locations: (Position 1) A $=75 \%$ of chamber length (360 in.), B\&C $=28$ in., D\&E $=28$ in.; (Position 2$) \mathrm{A}=50 \%$ of chamber length (240 in.), $\mathrm{B} \& \mathrm{C}=28$ in., D\&E = 28 in.; (Position 3) $\mathrm{A}=25 \%$ of chamber length (120 in.), B\&C = 28 in., D\&E = 28 in. Dimensions A, B, C, D, and E are defined and illustrated in Figure 4.9.

(f) Triplicate runs were planned for each orifice at each fluid pressure for in-chamber tests and single runs were planned for each orifice at each fluid pressure for in-spray tests.

(g) For in-spray tests: The vertical position of Malvern Insitec-S instrument laser beam varied and was always centered in the spray jet majority. Similarly, the spray jet was always centered between the Malvern Insitec-S laser source and detector. The Malvern Insitec-S laser beam to spray orifice distance was: MAL1854 (Position 1) $=11.4 \mathrm{ft}$, MAL1852 $($ Position 2) $=7.8 \mathrm{ft}$, and MAL1855 (Position 3) $=4.3 \mathrm{ft}$. 


\subsection{Data Analysis}

Analysis of large-scale PLC and Malvern Insitec-S data in Phase II testing followed the same basic approach employed in Phase I large-scale testing (Schonewill et al. 2012). Slight changes in analytical methods were incorporated to accommodate changes to the large-scale test instrumentation (in particular, configuration changes to the Malvern Insitec-S analyzers) and changes in the test methodology (such as the widespread use of replicate and in-spray testing). Aerosol tests on the large-scale spray release test skids produce a set of PLC data (corresponding to test skid measurements such as test fluid pressure, recirculation loop volumetric flow rate, and chamber $\mathrm{RH}$ ), a set of aerosol analyzer data (including, but not limited to, total aerosol concentration and PSD), and a set of process meta-data such as timestamps corresponding to the start and stop of spray releases. After data collection, the analytical method applied to data depends on whether the data collected derive from "in-chamber" measurements or "in-spray" measurements. For an "in-chamber" measurement, the analytical approach involves 1) analyzing "raw" PLC data and 2) determining the "in-chamber" generation rate and release fraction from Malvern Insitec-S size distribution and concentration data and the processed PLC data. Analysis of "in-spray" data is more direct and involves only determination of the "in-spray" release fraction from Malvern Insitec-S size measurements. In the sections that follow, a brief review of data analysis methods used in the treatment of PLC and Malvern Insitec-S data is given. For a more detailed review of the basis for and approach to spray release data analysis, the reader is referred to the initial test reports for small- and large-scale (Mahoney et al. 2013 and Schonewill et al. 2012, respectively).

\subsubsection{PLC Data Analysis}

PLC data sources include sensors located throughout the large-scale test apparatus; information derived from these test sensors is used primarily in the analysis of "in-chamber" measurements. Eight key instruments are employed in Phase II large-scale data analysis; these instruments are listed in Table 5.4.

During testing, load cell and Coriolis meter readings are used to assess the spray leak rate developed at the engineered orifice. The first step involved in calculating volumetric spray rate from tank mass is to determine the difference measured between the initial test fluid mass (at the start of spray), $M_{i}$, and the final test fluid mass (and the end of spray), $M_{f}$, and from the measured spray duration using:

$$
\Delta M=\left(M_{f}-M_{i}\right)-M_{\text {loss }}
$$

where

$$
\begin{gathered}
M_{i}=M 1_{i}+M 2_{i} \\
M_{f}=M 1_{f}+M 2_{f}
\end{gathered}
$$

Here, $M 1$ and $M 2$ refer to the masses from the storage and feed tanks, respectively, and $M_{\text {loss }}$ is the mass lost through pump leakage. As before, the subscripts $i$ and $f$ define the initial (before the spray is initiated) and final (after the spray is stopped) masses. The spray start and stop times were used to specify 
Table 5.4. Eight Primary Instruments Used in Phase II Large-Scale Data Analysis

\begin{tabular}{|c|c|c|c|c|}
\hline Instrument Type & Description/Location & $\begin{array}{l}\text { Data } \\
\text { Quality }\end{array}$ & Identifier & Units \\
\hline Load cell/scale & Mass of storage tank contents & NQA-1 & M1 & $\mathrm{lb}_{\mathrm{m}}$ \\
\hline Load cell/scale & Mass of feed tank contents & NQA-1 & M2 & $1 b_{m}$ \\
\hline Coriolis flow sensor & $\begin{array}{l}\text { Located upstream of test section, pre-spray } \\
\text { flow rate }\end{array}$ & NQA-1 & FE1 & gpm \\
\hline Coriolis flow sensor & $\begin{array}{l}\text { Located downstream of test section, } \\
\text { post-spray flow rate }\end{array}$ & FIO & FE2 & gpm \\
\hline Pressure transducer & $\begin{array}{l}\text { Test section pressure at elbow just upstream of } \\
\text { orifice(s) }\end{array}$ & NQA-1 & PS4 & psig \\
\hline $\begin{array}{l}\text { Resistance temperature } \\
\text { detector }\end{array}$ & $\begin{array}{l}\text { Test section temperature, at elbow just } \\
\text { upstream of orifice(s) }\end{array}$ & NQA-1 & TS6 & ${ }^{\circ} \mathrm{F}$ \\
\hline Relative humidity sensor & $\begin{array}{l}\text { Test chamber RH, } 33 \text { in. from wall near spray } \\
\text { header }\end{array}$ & $\mathrm{FIO}^{1}$ & RH1 & $\%$ \\
\hline Relative humidity sensor & $\begin{array}{l}\text { Test chamber RH, } 101 \text { in. from wall near } \\
\text { spray header }\end{array}$ & FIO & RH2 & $\%$ \\
\hline
\end{tabular}

a spray duration $\Delta t_{\text {spray }}$. Then, this information can be combined with the mass loss and simulant density ( $\rho$ ) (listed in Table 3.6 in Chapter 3 of this report) to determine the tank-mass-based volumetric spray rate $\left(Q_{m}\right)$ :

$$
Q_{m}=\frac{1}{\rho}\left(\frac{\Delta M}{\Delta t_{\text {spray }}}\right)
$$

To calculate the volumetric spray rate from Coriolis flow meter readings, $Q_{c}$, the following equation is used:

$$
Q_{c}=(\langle F E 1\rangle-\langle F E 2\rangle)+\left(F E 1_{p s}-F E 2_{p s}\right)
$$

Here, the first term in parentheses represents the difference between the time-averaged Coriolis flow rates as measured by FE1 (upstream of the orifice) and FE2 (downstream of the orifice) during the spray. The second term in parentheses represents a correction for any pre-spray (thus the $p s$ subscript) difference in sensor readings. In Phase I testing, both upstream (FE1) and downstream (FE2) Coriolis meters were qualified as NQA-1 instruments; however, because of difficulties with the downstream Coriolis meter FE2 during Phase II testing, this meter output was marked as "For Information Only" (FIO). ${ }^{2}$ As a result, volumetric spray rates determined by Coriolis meter readings, although still calculated for all Phase II tests, must also be marked FIO and cannot be used for release fraction determination. Instead, the

\footnotetext{
${ }^{1}$ The relative humidity sensors were procured with a factory calibration but this did not meet the stringent NQA-1 quality requirements of this work. Performance checks of the sensors near the end of testing indicate that the values obtained from these sensors are reasonably accurate.

${ }^{2}$ The FE2 Coriolis meter, downstream of the orifice, was tagged out of service/FIO on July 18, 2012, (at W254) due to the zero point reading was outside the Test Instruction acceptance criterion of $\pm 0.1 \mathrm{gpm}$ and could not be corrected. A calibrated replacement Coriolis meter was placed into service at the FE2 location on July 27, 2012, (at W312); however, the meter was designated as FIO since it did not have a project recognized NQA-1 pedigree. The zero point reading acceptance criterion was met for FE2 for the remainder of testing and the meter output was in agreement with the FE1 output.
} 
volumetric spray rate determined from tank mass difference (Equation (5.4)) is used in the determination of release fractions. Use of the tank-mass-based spray rate in the calculation of Phase II release fractions is not expected to impact comparability of Phase I and Phase II results, as Phase I release fraction determinations also used volumetric flow spray rates derived from the tank mass.

Pressure transducer readings are captured for use in the WTP model, which requires knowledge of pressure to estimate the exit velocity of liquid from the orifice. The remaining sensors listed in Table 5.4 including the temperature probe and RH sensors, are used for informational purposes. In particular, humidity sensor readings were used to assess the initial humidity at the start of sprays and, as described in Appendix A, also were used in a set of information studies aimed at determining the impact of RH on the release fraction results. Temperature sensor readings (which are NQA-1) were used to verify that temperature fell within the appropriate control boundaries before the start of spray.

\subsubsection{Determination of In-Chamber Generation Rate and Release Fraction}

For each test, measurements from Malvern Insitec-S analyzers yield aerosol concentration and size distribution measurements as a function of the period immediately before, during, and after termination of spray releases. Before use, all Malvern Insitec-S transmission data were evaluated and corrected for laser drift. To correct for drift, the laser intensity, $S$, measured as a function of time during each test was re-normalized to a pre-spray averaged background level $\left\langle S_{p s}\right\rangle$ obtained by averaging the laser intensity readings over a period of $2 \mathrm{~s}$ immediately before spray. The corrected laser transmission, $\tau$, is thus:

$$
\tau=\frac{s}{\left\langle S_{p s}\right\rangle}
$$

Corrected total aerosol concentrations, denoted by $c_{v}$, are then calculated using the Beer-Lambert law:

$$
c_{v}=\frac{2 D_{3,2}}{3 b} \ln \tau
$$

where $D_{3,2}$ is the SMD (which is reported by the Malvern Insitec-S software) and $b$ is a proportionality constant determined by evaluation of how the uncorrected concentration data reported by the Malvern Insitec-S software vary with $\tau$. Determination of $b$ is accomplished by using linear regression analysis of $\ln (\tau)$ versus $c_{v} / D_{3,2}$ with available uncorrected data, where the resulting best-fit slope is $1.5 \cdot b$.

Determination of $b$ is only possible when sufficient (i.e., greater than five) measurements of uncorrected $c_{v}$ (and corresponding $D_{3,2}$ and $\tau$ ) are reported by the Malvern Insitec-S software. After $b$ is determined, there is sufficient information to determine a corrected $c_{v}$ in cases where old (uncorrected) $c_{v}$ and $D_{3,2}$ measurements are available from the raw Malvern Insitec-S output. In certain cases, laser drift can prevent direct reporting of $\underline{\mathbf{c}}_{\underline{V}}$ and $D_{3,2}$ measurements while still allowing the Malvern Insitec-S to report a size distribution. In these cases, the Sauter mean diameter must be calculated from the size distribution information before the corrected aerosol concentration can be calculated using Equation (5.7).

Generation rates and release fractions are determined by evaluating the rate of concentration rise immediately after the start of a spray and are typically calculated on a cumulative or differential basis as a function of particle size. The cumulative and differential approaches differ in only the format of the concentration size distribution (i.e., the set of data that describes the cumulative or differential concentration of aerosol in ppmv as a function of aerosol diameter) used during analysis. The approach 
outlined below shows the cumulative approach; the reader is referred to Schonewill et al. (2013) for a description of the differential approach. If we denote $F_{k}$ as the cumulative volume fraction that falls below a particle diameter, $d_{k}$, the concentration distribution by size is given by:

$$
c_{k}=c_{v} F_{k}
$$

Here, $c_{k}$ represents the cumulative concentration that falls under particle diameter, $d_{k}$. Both $c_{v}$ and $F_{k}$, and as a result $c_{k}$, are functions of time, and are converted to an elapsed test time scale, $t$, by using:

$$
t=t_{m}-t_{s}
$$

where $t_{m}$ is the measurement timestamp and $t_{s}$ is the timestamp corresponding to the start of spray. For release fraction analysis, a chamber volume averaged concentration is determined from the concentration distribution curves corresponding to each of the two (for the $10 \mathrm{ft}$ test configuration) or three (for chamber configurations longer than $10 \mathrm{ft}$ ) Malvern Insitec-S analyzers. Because Phase II Malvern Insitec-S aerosol analyzers are evenly spaced across the length of the test chamber, the average volume concentration of the chamber is calculated as the arithmetic average:

$$
\bar{c}_{k}=\frac{1}{n_{m}} \sum_{j}^{n_{m}} c_{k, j}
$$

Here, $n_{m}$ represents the number of individual Malvern Insitec-S measurements ( $n_{m}=3$ for all but the $10 \mathrm{ft}$ chamber configuration, where $n_{m}=2$ ). The average transient concentration data, $\bar{c}_{k}$, are used to determine the best-fit cumulative generation rate, $G_{k}$, and loss coefficient, $\lambda_{\mathrm{k}}$. Specifically, non-linear least-squares analysis is used to fit the transient aerosol concentration distribution data, $\bar{c}_{k}(t)$, to:

$$
\bar{c}_{k}=\frac{G_{k}}{\lambda_{k} V}\left(1-e^{-\lambda_{k} t}\right)
$$

Here, $V$ is the volume of the test chamber. Least-squares analysis yields $G_{k}$, which is the cumulative generation rate that occurs under particle diameter $d_{k}$. The cumulative release fraction undersize, $\mathrm{R}_{\mathrm{k}}$, is then calculated by:

$$
R_{k}=\frac{G_{k}}{Q_{m}}
$$

It should be recognized that this analysis incorporates assumptions made in the derivation of Equation (5.11) (see Section 1.5). The most significant of these is that mixing of aerosol droplets within the chamber is rapid with respect to the rate of accumulation of aerosol within the test chamber.

Furthermore, the average volumetric chamber concentration $\bar{c}_{k}$ needed to interpret data in terms of the exponential model is associated with the arithmetic average of Malvern Insitec-S concentration readings. The aerosol analyzers employed in most test configurations measure aerosol concentration at three fixed locations above the spray and sample a total volume of $\sim 220 \mathrm{~cm}^{3}(\sim 0.057$ gal). Even relative to the minimum chamber volume of $\sim 3600$ gal, the sampling volume represents less than 0.002 percent of the region occupied by aerosol. Because the actual aerosol volume sampled is significantly smaller than the chamber volume, consideration must be given to the representativeness of aerosol measured relative to the bulk aerosol. Processes that can change or alter the aerosol before it reaches the measuring location, such as evaporation or coalescence, can impact apparent generation rates as determined by Equation (5.11). 


\subsubsection{Determination of In-Spray Release Fraction}

In-spray measurements are made with the Malvern Insitec-S aerosol analyzers installed directly in the path of the spray. Passage of the leading edge of the jet through the measurement zone is essentially instantaneous relative to the maximum aerosol data collection rate $(4 \mathrm{~Hz})$. As a result, in-jet concentration profiles (with time) are essentially step functions instead of gradual increases to some equilibrium concentration. The time at which the step change in concentration occurs for in-spray measurements is dictated by the jet velocity and the downstream distance of the analyzer from the orifice (as well as jet trajectory to a lesser extent). As such, interpretation of in-spray concentration rise with the exponential model does not produce a physically meaningful result. For this reason, determination of in-spray release fractions use the alternate approach outlined below. Schonewill et al. (2012) demonstrated that release fraction can be expressed as:

$$
R_{k}=f_{s} F_{k, o}
$$

where $f_{s}$ is the fraction of spray aerosolized and $F_{k, o}$ is the size distribution of aerosol produced by the spray. For in-spray measurements, the measurement location of the Malvern Insitec-S instruments is such that 1) the size distribution should closely resemble that produced by aerosolization of the high-pressure spray and 2) the measured spray is not yet impacted by aerosol loss mechanisms. The latter assertion means that in-spray measurements can treat the aerosolization process as complete with the caveat that such aerosolization includes particles that would not be considered aerosolized in "in-chamber" measurements because of losses due to inertial impact against a wall or gravitational settling. For complete aerosolization,

$$
f_{s}=1
$$

Then, if the in-spray size distribution measured by the Malvern Insitec-S analyzer is denoted by $F_{k}^{(I N S)}$, then the corresponding in-spray release fraction must be (from Equation (5.13)):

$$
R_{k}^{(I N S)}=\left\langle F_{k}^{(I N S)}\right\rangle
$$

The size brackets in the right-side term reflect that Malvern Insitec-S size distribution measurements are time-averaged (typically over a period of $4 \mathrm{~s}$ ) to improve result confidence and to capture any variances resulting from natural transients (such as spray turbulence). Once flow is established through the engineered orifice, the in-spray size distribution is not expected to exhibit transience beyond turbulence. However, in-spray measurements may be impacted by buildup of smaller particles in the test chamber and re-entrained into the spray. For these reasons, time averaging of in-spray data for release fraction analysis is taken near the start of spray (typically between 8 and $12 \mathrm{~s}$ ).

Once the in-spray release fraction is determined, the cumulative generation rate undersize (i.e., below a particle diameter, $d_{k}$ ) for in-spray measurements may then be calculated as:

$$
G_{k}^{(I N S)}=Q_{m} R_{k}^{(I N S)}
$$

For Phase II analysis of in-spray measurements, in-spray generation rates were not calculated. 


\subsubsection{Averaging of Replicate Results}

In Phase II spray leak testing, significant effort was made to perform at least one (but typically two or more) replicate "in-chamber" tests for each test configuration and orifice. Replicate tests were performed to provide a better estimate of overall "in-chamber" release fraction and generation rate measurement variability. To reduce the complexity of presenting measurement results from several replicate tests and to provide a clearer picture of data trends and functionality, the geometric mean of both release fraction and generation rates were determined for each set of replicate tests. Specifically, for a given test configuration $L$, the mean release fraction and generation rates were determined using

$$
\begin{aligned}
& \bar{R}_{k}^{(L)}=\sqrt[n]{\prod_{j}^{n} R_{k, i}} \\
& \bar{G}_{k}^{(L)}=\sqrt[n]{\prod_{j}^{n} G_{k, i}}
\end{aligned}
$$

Here, $\bar{R}_{k}^{(L)}$ and $\bar{G}_{k}^{(L)}$ represent the geometric mean release fraction and generation rates, respectively, of $n$ individual release fraction, $R_{k, i}$, and generation rate measurements, $G_{k, i}$. As before, the subscript $k$ associates each result with its corresponding aerosol diameter $d_{k}$. Based on the limited number of replicate tests employed in analysis for each unique test configuration (i.e., simulant, orifice and pressure combination) and the difficulty in quantitatively combining uncertainty from separate test conditions, a statistical presentation of the uncertainty (i.e., the 95 percent confidence limit) is not provided when presenting mean release fraction results for a given test configuration. Instead, the uncertainty presented with the mean values derived from Equations (5.17) and (5.18) is simply taken as the maximum and minimum values of the individual measurements. Statistical tests will be applied when interpreting trends in larger sets of measurement data, such as release fraction variability with pressure or orifice area. Geometric averaging of in-chamber data was selected based on the current presentation of results, which presents release fractions on a logarithmically scaled axis. Furthermore, geometric averaging is applied only to in-chamber results. Similar test-by-test averaging is not needed for in-spray measurements because those measurements typically involved a time-averaged size distribution measurement derived from a single test.

\subsubsection{Determination of WTP Model Release Fraction Curves}

Larson and Allen (2010) summarize the methodology used by WTP for estimating the aerosol release fraction and generation rate of spray releases, and McAllister (2010) provides additional details on the equations and method. The method uses the theoretically-based correlation by Dombrowski and Johns (1963) for estimating the Sauter mean diameter, $D_{3,2}$, and then estimates the aerosol droplet size distributions using the Rosin and Rammler (1933) distribution. The Dombrowski and Johns (1963) equations used in the WTP methodology for estimating the Sauter mean diameter are

$$
\begin{gathered}
d_{l}=0.9614\left(\frac{\mathrm{K}^{2} \sigma^{2}}{\rho_{l} \rho_{a} U^{4}}\right)^{1 / 6}\left[1+2.6 \mu\left(\frac{\mathrm{K} \rho_{a}^{4} U^{7}}{72 \rho_{l}^{2} \sigma^{5}}\right)^{1 / 3}\right]^{1 / 5} \\
d_{d}=1.882 d_{l}\left[1+\frac{3 \mu}{\left(\rho_{l} d_{l} \sigma\right)^{1 / 2}}\right]^{1 / 6}
\end{gathered}
$$




$$
D_{3,2}=0.63 d_{d}
$$

where $\quad d_{l}=$ the theoretical ligament diameter $(\mathrm{m})$

$d_{d}=$ the theoretical droplet diameter $(\mathrm{m})$

$\mu=$ the liquid viscosity $(\mathrm{Pa} \mathrm{s})^{1}$

$\rho_{l}=$ the liquid density $\left(\mathrm{kg} \mathrm{m}^{-3}\right)$

$\rho_{a}=$ the air density $\left(\mathrm{kg} \mathrm{m}^{-3}\right)$

$\sigma=$ the surface tension of the liquid $\left(\mathrm{N} \mathrm{m}^{-1}\right)$

$\mathrm{U}=$ the fluid velocity at the orifice $\left(\mathrm{m} \mathrm{s}^{-1}\right)$

$\mathrm{K}=$ the spray nozzle parameter $\left(\mathrm{m}^{2}\right)$.

The K parameter is determined with the (McAllister 2010) relationship

$$
\mathrm{K}=\frac{0.5 \mathrm{~A}_{b}}{\sin (\theta / 2)}
$$

where $A_{b}$ is the area of the orifice for all shapes and $\theta$ is the full spray angle and assumed to be the maximum value of $150^{\circ}$ for a fan spray. For use in Equation (5.19), the velocity of the liquid leaving the orifice can be determined from the pressure differential $(\Delta P)$ with an orifice flow equation (e.g., see Denn 1980), and McAllister (2010) uses the following orifice flow equation with a typical value of 0.62 for the orifice coefficient:

$$
\mathrm{U}=0.62\left(\frac{2 \Delta P}{\rho_{l}}\right)^{1 / 2}
$$

The Sauter mean diameter for a particular spray can be determined using Equations (5.19) to (5.22). To determine the fraction of a spray contained in droplets below any particular size for a spray release accident analysis, a relationship is needed for the droplet size distribution. For the WTP methodology, Larson and Allen (2010) use the Rosin and Rammler (1933) distribution and further assume that the release fraction of a spray is equal to the droplet size distribution. As determined by the WTP methodology, the cumulative release fraction $R_{k}^{(W T P)}$ of droplets with diameters equal to or smaller than $d_{k}$ is given by:

$$
\begin{gathered}
R_{k}^{(W T P)}=1-\exp \left[-\left(\frac{D_{3,2}}{D_{X}} \frac{d_{k}}{D_{3,2}}\right)^{q}\right] \\
\frac{D_{3,2}}{D_{X}}=[\Gamma(1-1 / q)]^{-1}
\end{gathered}
$$

The WTP superscript has been included to differentiate release fractions determined by Equation (5.24) from other uses of release fraction in this report. Here, $q$ and $D_{X}$ are a constant and characteristic diameter, respectively, that determine the median of and spread in the droplet size distribution. The ratio of Sauter mean diameter to characteristic diameter $D_{X}$ is defined by Equation (5.25), where $\Gamma$ is the gamma function. Larson and Allen (2010) recommend $q=2.4$ when using Equations (5.24) and (5.25)

\footnotetext{
${ }^{1}$ McAllister (2010) has a typographical error and shows incorrect units for viscosity (kinematic viscosity units are used), but correctly uses the correct viscosity and units in the example calculation.
} 
for WTP release fraction calculations. Once release fraction is calculated, the cumulative generation rate predicted by the WTP model, $G_{k}^{(W T P)}$, may be determined as:

$$
G_{k}^{(W T P)}=Q_{s} R_{k}^{(W T P)}
$$

where $Q_{s}$ is the volumetric flow of the spray $\left(\mathrm{m}^{3} \mathrm{~s}^{-1}\right)$. In the WTP model, the volumetric flow for calculating the total release with Equation (1.7) is simply the spray velocity times the area of the orifice.

$$
Q_{s}=U A_{b}
$$

\subsection{Summary of Changes from Phase I}

In this chapter, we outline the general test approach, system operations, and test configurations along with the data analysis approach employed in the Phase II spray release testing. The overall approach and test methodology were adapted from Phase I testing; however, several key changes were made to address unresolved test issues that remain from Phase I testing and to address new issues identified. A summary of these changes, along with brief discussion of each, is provided in Table 5.5. 
Table 5.5. Summary of Key Changes Made in the General Test Approach and Data Analysis Methodologies for Phase II Spray Leak Testing

\begin{tabular}{|c|c|}
\hline Phase II Change & Rationale \\
\hline Testing of non-Newtonian simulants & $\begin{array}{l}\text { Phase I large-scale tested only Newtonian simulants. Phase II testing } \\
\text { included tests of non-Newtonian slurry composed of mixed clay ( } 80 \mathrm{wt} \% \\
\text { kaolin and } 20 \mathrm{wt} \% \text { bentonite) in water. The ratio of mixed clay } \\
\text { solids-to-water was selected to produce slurry with one of two target } \\
\text { slurry yield stresses ( } 6 \mathrm{~Pa} \text { or } 30 \mathrm{~Pa} \text { ). }\end{array}$ \\
\hline $\begin{array}{l}\text { In-Spray testing for all Phase II test } \\
\text { pressure, orifice, and simulant } \\
\text { combinations }\end{array}$ & $\begin{array}{l}\text { Phase I did not perform in-spray testing for all test combinations. } \\
\text { However, because it is believed that in-spray measurements provide an } \\
\text { upper-bound estimate for release fractions, Phase II testing evaluated } \\
\text { in-spray release fractions for Phase II test configurations. }\end{array}$ \\
\hline $\begin{array}{l}\text { Widespread use of replicate testing for } \\
\text { in-chamber tests }\end{array}$ & $\begin{array}{l}\text { For the in-chamber testing, at least one or more replicate tests were } \\
\text { conducted in an attempt to better understand experimental variability. }\end{array}$ \\
\hline $\begin{array}{l}\text { Averaging of in-chamber release fraction } \\
\text { (and generation rate) replicates }\end{array}$ & $\begin{array}{l}\text { The approach and methods for determining in-chamber release fractions } \\
\text { and generation rates were adapted from Phase I testing without } \\
\text { substantial change. However, replicate testing was performed for all } \\
\text { Phase II in-chamber test configurations and yielded two or more repeat } \\
\text { measurements of release fraction for each simulant, test pressure and } \\
\text { orifice combination. To reduce the complexity and improve the } \\
\text { readability of data presentations, repeat measurements were uniformly } \\
\text { averaged (using a geometric mean) in Phase II testing. Replicate testing } \\
\text { helped improve confidence when evaluating trends in release fraction } \\
\text { data with changing test pressure and orifice area and facilitated improved } \\
\text { extrapolation of large-scale results to larger postulated breaches. }\end{array}$ \\
\hline $\begin{array}{l}\text { Exclusion of release fraction data } \\
\text { impacted by measurement bias }\end{array}$ & $\begin{array}{l}\text { Both small- and large-scale Phase I reports typically provided all release } \\
\text { fraction data between } 1 \text { and } 200 \mu \text { m. Release fraction data were } \\
\text { highlighted when the fit of the exponential concentration rise was poor } \\
\left(R^{2}<0.5\right) \text {. For Phase II reporting, release fraction data were subjected to } \\
\text { more stringent reporting requirements. For the current report, both } \\
\text { in-spray and in-chamber release fraction data below } 10 \mu \mathrm{m} \text { are not } \\
\text { reported, as they fall outside the primary size range of interest ( } 10 \text { to } \\
100 \mu \mathrm{m}) \text {. In additional, both in-spray and in-chamber release fraction } \\
\text { data that fall below } 0.5 \% \text { of the total fractional release are not reported } \\
\text { because of loss measurement accuracy. Finally, in-chamber release } \\
\text { fraction data above } 80 \mu \mathrm{m} \text { are highlighted because of concerns } \\
\text { associated with poor sampling of large aerosols in in-chamber } \\
\text { measurement configurations. These guidelines are established in } \\
\text { Section } 7 \text { of this report. }\end{array}$ \\
\hline $\begin{array}{l}\text { Chamber RH requirement of }>80 \% \text { before } \\
\text { collection of baseline test data and } \\
\text { subsequent test execution spray release }\end{array}$ & $\begin{array}{l}\text { During functional tests, when investigating the influence of chamber RH } \\
\text { on test results, preliminary results suggested that maintaining }>80 \% \\
\text { chamber RH had no detectable influence on aerosol concentrations and } \\
\text { size distributions The most conservative release fraction results are } \\
\text { captured under a } 100 \% \text { RH chamber condition. }\end{array}$ \\
\hline
\end{tabular}




\subsection{Malvern Insitec-S Analyzer Validation}

In both Phase I and Phase II testing, the Malvern Insitec-S aerosol analyzer was used as the primary means for measuring aerosol concentrations and size distributions. The Malvern Insitec-S employs open frame laser diffraction, whereby aerosol size and concentration are measured by time averaging laser power and scattered light intensities from diffraction events that occur in the measurement volume defined by the path of the $10 \mathrm{~mm}$ diameter laser beam across the 70 to $920 \mathrm{~mm}$ open path between the laser source and detector. As discussed below, the measurement technique and configuration pose several difficulties when interpreting aerosol results:

- PSD is not measured directly. Instead, the scattered light pattern produced by the aerosol or dispersion is measured. Then, the size distribution of that dispersion is inferred by calculating the size distribution of a dispersion of spherical particles that produces the best match of the measured diffraction pattern (Jillavenkatesa et al. 2001). The process requires calculation of scattered light intensity as a function of particle size and scattering angle through Mie theory (van de Hulst 1981). To reduce the computational power needed to invert the diffraction pattern, inversion algorithms may invoke a model (Jillavenkatesa et al. 2001) that assumes size distribution functionality. Example inversion models for a Malvern Instruments Mastersizer 2000 particle size analyzer (laboratory equivalent to the Insitec-S; Malvern Instruments Ltd.) include mono-disperse single peak distributions, mono-disperse multiple peak distributions, or poly-disperse (broad) size distributions. The distribution of sizes is typically assumed to be log-normal. The inversion model can have a dramatic impact on the measured size distribution, and examples of this impact are given Appendix $G$ of Wells et al. (2011). In one example, a mixture of two mono-disperse powders of distinct size (i.e., with minimal overlap in their size distributions) was analyzed using laser diffraction. The mixture size distribution was properly characterized when a multi-modal mono-disperse model was selected. However, when a general purpose (broad) size distribution model was selected, the result was a single broad distribution of particles that showed no indication of two distinct size distributions. In many cases, especially when dealing with dispersions created from aerosolization, the size distribution is continuous and broad. For poly-disperse mixtures, application of a broad general purpose size distribution model tends to smooth the distribution and will broaden the size distribution (i.e., increase the lower and upper size boundaries of the distribution). The inversion algorithm is typically instrument specific, and as is the case with the Malvern Insitec-S aerosol analyzer used in PNNL's spray leak analyses, the user is not always able to select or alter the inversion method.

- Aerosol concentration is determined using the Beer-Lambert law, which relates the loss of laser power (or conversely, the obscuration of the laser intensity) resulting from the presence of aerosol in the Malvern Insitec-S analyzer measuring volume to the concentration of aerosol in the measuring volume. Use of the Beer-Lambert law requires knowledge of the aerosol size distribution (as determined by the inversion of the measured diffraction pattern) and determination of the scattering efficiency of aerosol particles (which requires both knowledge of the size distribution and optical properties of the aerosol). As such, the concentration measurement is tied directly to the determination of particle size, and errors in the measured size distribution may carry through to concentration determination. Like size measurements, the Beer-Lambert formulation used by the Malvern Insitec-S analyzer assumes spherical particles. Interpretation of concentration measurements for non-spherical particles may be subject to bias that depends on the surface area to volume ratio. With respect to aerosol measurements conducted in Phase I and Phase II spray release studies, the assumption of spherical droplets is reasonable for water droplets in the size range of interest (10 to 
$100 \mu \mathrm{m}$ ), as capillary forces are expected to dominate the drop shape. On the other hand, aerosols formed by high velocity spray of slurries may contain non-spherical particulate that will be subject to particle shape measurement bias.

- Diffraction events may occur at any point along the measurement zone. Because diffraction path length for the open frame (70 to $920 \mathrm{~mm}$ ) is long relative to typical laser diffraction techniques (which employ short path lengths on the order of 1 to $10 \mathrm{~mm}$ ), diffraction events collected through a single lens (100 mm in Phase I and $500 \mathrm{~mm}$ in Phase II) must consider that diffraction events occurring far from the collection optics will have a much lower range of accepted scattering angles than events occurring near the optics. As such, scattering events that occur far from the detector will show attenuation or complete loss of scattered light at high angles relative to events that occur near to the detector. And as a result, the overall diffraction pattern also will be attenuated at large scattering angles. Because the diffraction signal at large scattering angles typically correspond to light scattered by small particles, the resulting PSD will either under-estimate or completely exclude the volume contribution of those small particles. If the concentration profile of the aerosol (or geometry of the spray) within the measurement zone is known, the algorithm for determining the size distribution can attempt to correct for large scattering angle attenuation. The Malvern Insitec-S analyzer implements corrections for laser attenuation; however, this correction is based on first principles, and the accuracy should be evaluated to determine their overall accuracy.

Despite the difficulties outlined above, the Malvern Insitec-S is still a far superior tool for aerosol characterization and analysis because it allows rapid in-process assessment (i.e., complete measurements at data rates up to $4 \mathrm{~Hz}$ ) of statistically significant populations of aerosol particles (ensemble diffraction) whose size range can span several orders of magnitude $(0.5$ to $2500 \mu \mathrm{m})$. Other aerosol sizing techniques, such as phase Doppler and optical image capture and analysis, require much longer observation times (i.e., on the order of $1 \mathrm{~min}$ ) to build statistically significant size distribution results. Optical techniques are further hindered by field-of-view constraints and with respect to the overall range of particle sizes that can be captured at a single magnification level.

The difficulties outlined above pose a challenge to those attempting to assess overall instrument accuracy with respect to size and concentration measurements. As stated previously, the Malvern Insitec-S analyzers are the primary means of aerosol characterization for spray leak characterization in both the Phase I and Phase II test campaigns. As such, it is important to understand the accuracy of these instruments for measuring aerosol concentrations and size distributions. Phase II test activities included studies focused on determining the accuracy of the Malvern Insitec-S size and concentration measurements under various test configurations. In this chapter, the test approach and results for these Malvern Insitec-S validation studies are described in detail, and an overall assessment of measurement accuracy with respect to the standard aerosol test configurations used during Phase I and Phase II is provided. 


\subsection{Objectives}

Malvern Insitec-S validation testing addressed Test Objective \#10 of TP-WTPSP-031, Rev. 1.0 1 ). As stated in TP-WTPSP-031, Rev 1.0, this test objective required that PNNL:

[A]ssess the capability of the Malvern Insitec-S in-process particle size analyzer, which is the instrument used in the aerosol testing, to measure accurately the concentration and size distribution of samples. This will be accomplished by measuring carefully controlled dilute aqueous slurries of known concentration and PSD and comparing the Malvern result to the known values. Testing will include mono- and poly-disperse suspensions and will evaluate all four Malvern configurations of 1) Phase I small-scale spacers and old lens, 2) Phase II small-scale spacers and new lens, 3) Phase I large-scale spacers and old lens, and 4) Phase II large-scale spacers and new lens (old lens $100 \mathrm{~mm}$, new lens $500 \mathrm{~mm}$ ).

In more simple terms, the aim of Malvern Insitec-S validation testing was to:

1. Evaluate the accuracy of concentration, the linearity of concentration response, and the accuracy of size distribution measurements under instrument configurations where there is no need to correct the diffraction pattern for distance effects

2. Evaluate the validity of diffraction pattern corrections that account for distance effects.

According to TP-WTPSP-031, Rev. 1.0, the success criterion that governed validation testing was:

$[\mathrm{M}]$ easure[s] the concentration and size distribution of known aqueous suspensions with the Malvern used for aerosol measurements and quantitatively compare[s] the Malvern (Insitec-S) results with the known values to estimate the Malvern (Insitec-S) accuracy for measuring aerosols.

Validation testing evaluated if it was necessary to change analyzer settings for analysis of Phase I and Phase II data to improve measurement accuracy. To meet the objectives, a series of validation tests that evaluated Malvern Insitec-S analyzer performance against known or certified materials and that parametrically evaluated the impact of instrument settings on the result were conducted. These tests were used to determine if any corrections for measurement bias should be made and if any optimal instrument settings existed to improve the overall aerosol measurements used for both Phase I and Phase II release fraction analysis. As discussed in detail below, validation testing observed no systematic bias in the Malvern Insitec-S analyzer's concentration or size results that required correction.

\subsection{Approach}

Phase II Malvern Insitec-S validation activities represent an extension of Phase I validation activities reported in Section 5.3.2.1 in Schonewill et al. (2012). Phase II validation studies employ solid-particle-in-liquid dispersion testing in both liquid flow cell and column configurations. Use of solid-in-liquid dispersions rather than airborne liquid or solid particulates simplifies mass balance considerations by eliminating significant loss of dispersed particulates to walls through condensation,

\footnotetext{
${ }^{1}$ Gauglitz PA. 2012. Test Plan for Spray Leak Quantification to Support WTP Spray Release Methodology. TP-WTPSP-031, Rev. 1.0, Pacific Northwest National Laboratory, Richland, Washington.
} 
adsorption, or settling. Testing activities examine the impact of test material, dispersion configuration, and software settings that affect interpretation of the diffraction pattern. Key differences between Phase I and Phase II testing activities are described below:

- Phase II validation activities represent a significant expansion of validation tests, including development of concentration curves for mono-disperse and poly-disperse materials of difference size and optical properties.

- Tests evaluate not only concentration accuracy, but size accuracy as well.

- Phase I validation examined the performance of the Malvern Mastersizer 2000 instrument, which is a laboratory equivalent of the in-process Malvern Insitec-S analyzer. Phase II validation studies employed the Malvern Insitec-S analyzer directly and eliminated the need to consider differences in the operating specifications of the Malvern Mastersizer 2000 and the Malvern Insitec-S analyzer.

In the following subsections, the test materials and methodology employed in Phase II validation activities are discussed in detail.

\subsubsection{Test Materials}

Phase II validation activities employed a range of test materials to evaluate the impact of material properties on measurement accuracy. Materials were selected to fall within the range of interest for spray leak testing (e.g., 10 to $100 \mu \mathrm{m}$ in size). A summary of materials tested is given in Table 6.1 along with key material specifications.

Table 6.1. Selected Properties of Powder Systems Used in Malvern Insitec-S Validation Testing

\begin{tabular}{lccccccc}
\hline \multicolumn{1}{c}{ Material } & Shape & $\begin{array}{c}\text { Refractive } \\
\text { Index }\end{array}$ & Distribution & $\begin{array}{c}\mathrm{d}(10), \\
\mu \mathrm{m}\end{array}$ & $\begin{array}{c}\mathrm{d}(50), \\
\mu \mathrm{m}\end{array}$ & $\begin{array}{c}\mathrm{d}(90), \\
\mu \mathrm{m}\end{array}$ & $\begin{array}{c}\text { PSD } \\
\text { Source }\end{array}$ \\
\hline Glass beads, $10 \mu \mathrm{m}$ & Spherical & $1.520+0.000 \mathrm{i}$ & Mono-disperse & 8.1 & 9.2 & 10.2 & Cert $^{\mathrm{a}}$ \\
Glass beads, $20 \mu \mathrm{m}$ & Spherical & $1.520+0.000 \mathrm{i}$ & Mono-disperse & 21.8 & 22.8 & 23.8 & Cert $^{\mathrm{a}}$ \\
Glass beads, $50 \mu \mathrm{m}$ & Spherical & $1.520+0.000 \mathrm{i}$ & Mono-disperse & 47.4 & 49.2 & 51.0 & Cert $^{\mathrm{a}}$ \\
\hline Glass beads, 3 to $30 \mu \mathrm{m}$ & Spherical & $1.520+0.000 \mathrm{i}$ & Poly-disperse & 9.14 & 13.4 & 20.3 & Cert $^{\mathrm{a}}$ \\
Glass beads, XLSciTech & Spherical & $1.520+0.000 \mathrm{i}$ & Poly-disperse & 49.4 & 68.1 & 93.7 & Ref $^{\mathrm{b}}$ \\
Glass beads, QAS3001B & Spherical & $1.520+0.000 \mathrm{i}$ & Poly-disperse & 37.1 & 60.0 & 95.5 & Cert $^{\mathrm{a}}$ \\
$\begin{array}{l}\text { Boehmite, APRYAL } \\
\text { AOH20 }\end{array}$ & Irregular & $1.655+1.000 \mathrm{i}$ & Poly-disperse & 1.68 & 8.04 & 20.1 & Ref $^{\mathrm{b}}$ \\
Gibbsite, NOAH R6011 & Irregular & $1.580+1.000 \mathrm{i}$ & Poly-disperse & 1.8 & 9.0 & 21.3 & Ref $^{\mathrm{b}}$ \\
\hline
\end{tabular}

(a) Certificate of analysis based on Andreasen Pipette, Coulter Counter, and Optical Microscopy.

(b) Reference measurement (Malvern Mastersizer 2000).

(c) Certificate of analysis based on laser diffraction.

For activities focused on verifying Malvern Insitec-S size measurements, several mono-disperse and poly-disperse glass beads were purchased from Whitehouse Scientific, Ltd., (Waverton, Chester, United Kingdom). These materials included 10, 20, and $50 \mu \mathrm{m}$ mono-disperse glass powders and a 3 to $30 \mu \mathrm{m}$ poly-disperse glass powder. All materials purchased from Whitehouse Scientific came with a 
National Institute of Standards and Technology (NIST) traceable certified size distribution that is based on methods independent of laser diffraction, including Andreasen Pipette, Coulter Counter, and Optical Microscopy. These materials allow any size distribution bias introduced by laser diffraction to be evaluated directly. To evaluate instrument bias, a separate size standard, QAS3001B (which is a 15 to $150 \mu \mathrm{m}$ poly-disperse glass bead), was purchased from Malvern Instruments. This standard has a certified (NIST-traceable) size distribution based on laser diffraction measurements and is intended to check performance of the Malvern Mastersizer 2000 (and other Malvern Instruments Ltd. size analyzers).

Activities related to evaluating concentration accuracy employed the certified glass beads purchased from Whitehouse Scientific and Malvern Instruments. These standards were further supplemented by three additional test powders: 1) XLSciTech mono-disperse glass beads, 2) Gibbsite NOAH R6011 powder, and 3) Boehmite APYRAL AOH20 powder. The gibbsite powder was manufactured by BASF but was procured from a U.S. supplier, NOAH Technologies Corporation. The boehmite powder was manufactured by Nabaltec GmbH but also was procured from NOAH Technologies Corporation. The XLSciTech glass powder is XLSciTech product XLSL063075. This powder was manufactured by and purchased from XL Science and Technology (Richland, Washington). Unlike the Whitehouse Scientific and Malvern Instruments glass bead standard, the XLSciTech glass beads and gibbsite and boehmite powders are not NIST traceable. Their reference size distributions have been measured using a Malvern Mastersizer 2000 particle size analyzer whose performance was verified against NIST traceable standards.

\subsubsection{Test Setup}

Malvern Insitec-S validation testing employed one of two standard test configurations: 1) flow cell testing and 2) column testing. These tests differ primarily in how the solid-in-liquid dispersion was presented to the instrument. The aim of flow cell tests was to evaluate baseline instrument accuracy; they also included limited testing of software correction of the diffraction pattern for distance. Column testing was intended to provide a limited evaluation of the accuracy of concentration and size distribution measurements in instrument configurations that replicate Phase I and Phase II aerosol setups. As such, these tests provided an assessment of the accuracy of diffraction pattern corrections made on aerosols distributed across long $(>150 \mathrm{~mm})$ path lengths.

A basic schematic for flow cell testing is shown in Figure 6.1. As indicated in the diagram, the test particle dispersion was pumped from an agitated storage vessel through a liquid flow cell and back through a return line to the storage vessel. For validation testing, a Hydro G dispersion unit (purchased from Malvern Instruments) was adapted for use in dispersion storage, mixing, and pumping. The Hydro $\mathrm{G}$ dispersion unit can hold up to $800 \mathrm{~mL}$ of dispersion and is equipped with an overhead mixer for the dispersion reservoir and a built-in pump to circulate the dispersion. This dispersion unit is intended for analysis of standard materials (such as glass beads) with particle diameters as large as $2000 \mu \mathrm{m}$ and can uniformly disperse the test materials identified in Table 6.1. The dispersion was presented to the Malvern Insitec-S measuring region using a liquid flow cell (part number MPS2628) purchased from Malvern Instruments. The flow cell creates an optical path of variable length through which a test dispersion can be flowed and is attached to the Malvern Insitec-S frame using a tri-clamp. The flow cell path length can adjusted using stainless steel spacers of variable thickness. Spacers can be used individually or can be combined to create a range of optical path lengths from 1 to $10 \mathrm{~mm}$; a standard $3 \mathrm{~mm}$ spacer was employed for all flow cell analysis. For the majority of validation tests, the flow cell 
was installed on the faceplate of the detection unit, as this eliminates the need to correct for laser attenuation from lowered angle of acceptance. The small optical path combined with the proximity of the flow cell to the detector is typical of most laboratory laser diffraction setups, and as such, this configuration provided a baseline assessment of Malvern Insitec-S performance. For select test cases, the flow cell was installed at the laser unit, and its distance from the detection unit varied by installation of 150,500 , and $1000 \mathrm{~mm}$ spacers, to evaluate the impact of distance from detector on the interpretation of the diffraction pattern. In addition, the majority of flow cell tests employed the $100 \mathrm{~mm}$ lens because its measurement range was best suited for the test dispersions listed in Table 6.1. Select flow cell tests also examined the Malvern Insitec-S performance using the $500 \mathrm{~mm}$ lens. The flow cell test matrix (which is discussed in detail in the next subsections) employs all materials listed in Table 6.1.

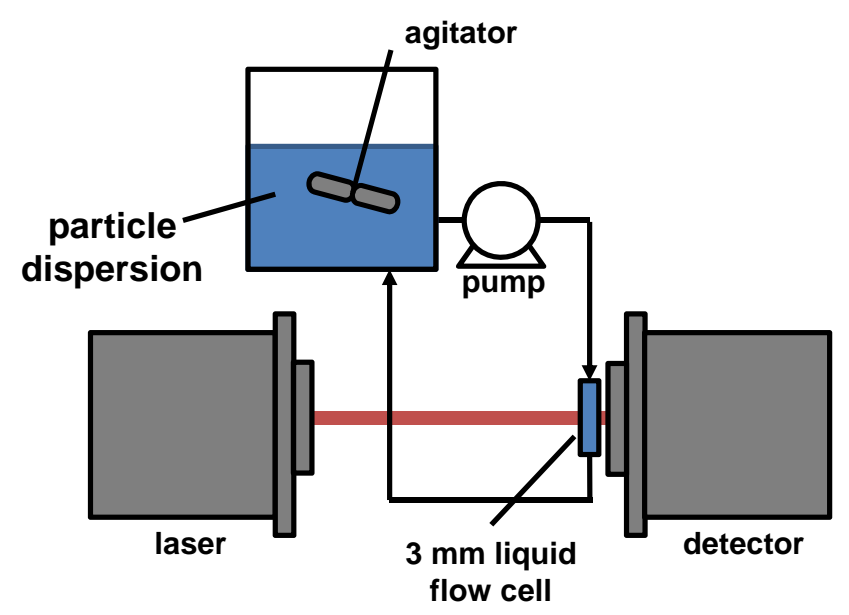

Figure 6.1. Basic Test Setup for Flow Cell Testing

In column testing, the flow cell is replaced with a flow column. Two column lengths, $400 \mathrm{~mm}$ and $900 \mathrm{~mm}$, were employed in testing. The basic configuration for each column length is shown in Figure 6.2 and Figure 6.3. Flow columns are intended to simulate aerosols uniformly distributed over a long (>150 mm) path length. The $400 \mathrm{~mm}$ column configuration represents the typical Phase I large-scale Malvern Insitec-S analyzer configuration in which the aerosol measurement path length was $420 \mathrm{~mm}$. In keeping with the Phase I Malvern Insitec-S analyzer configuration, $400 \mathrm{~mm}$ flow column testing employed a $100 \mathrm{~mm}$ lens. ${ }^{1}$ Likewise, $900 \mathrm{~mm}$ column testing employed an optical path length and lens choice consistent with Phase II large-scale testing, in which the optical path length was $920 \mathrm{~mm}$ and a $500 \mathrm{~mm}$ lens was installed on the Malvern Insitec-S detection unit. Both column configurations employed a closed loop flow system, which consisted of a 50 gal dispersion reservoir and a stainless steel rotary lobe pump capable of flow rates up to $10 \mathrm{gpm}$. The dispersion reservoir was agitated with an overhead mixer. Both 400 and $900 \mathrm{~mm}$ columns are fabricated of 1.5 in. inner diameter black PVC tubing. The inner diameter of the tube is rough to prevent specular reflections of scattered light. Column testing only employed the 3 to $30 \mu \mathrm{m}$ poly-disperse glass bead powder purchased from Whitehouse Scientific.

\footnotetext{
${ }^{1}$ For the $400 \mathrm{~mm}$ column test configuration (shown in Figure 6.2), the distance between laser and detection units was $1000 \mathrm{~mm}$. This distance has no functional impact on the results of column testing, as only the dispersion configuration (i.e., the location of the $400 \mathrm{~mm}$ column relative to the detection unit) is relevant.
} 


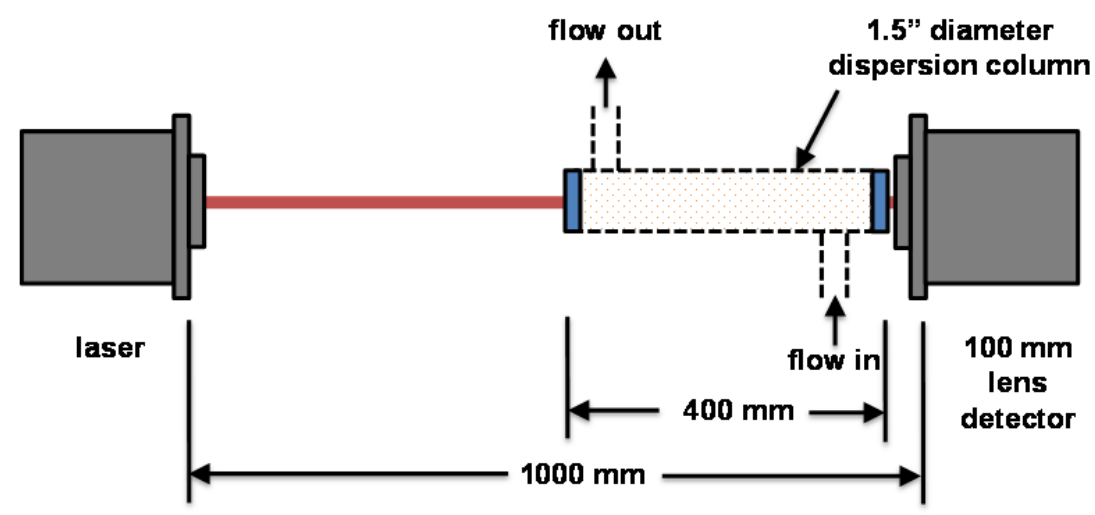

Figure 6.2. Diagram for $400 \mathrm{~mm}$ Flow Column Testing Used to Simulate the Phase I Malvern Insitec-S Aerosol Analyzer Test Configuration

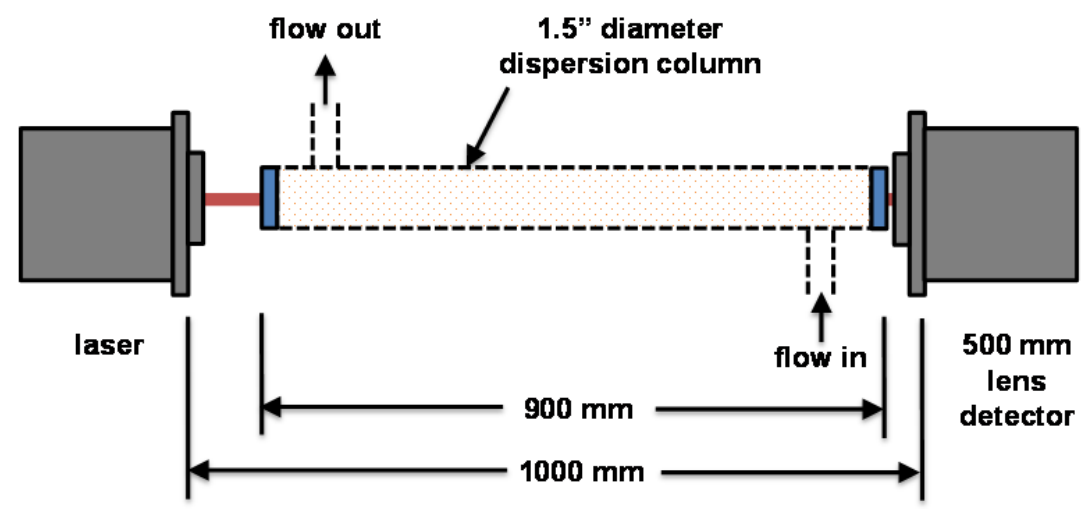

Figure 6.3. Diagram for $900 \mathrm{~mm}$ Flow Column Testing Used to Simulate the Phase II Malvern Insitec-S Aerosol Analyzer Test Configuration

\subsubsection{Test Matrix}

Flow cell tests employed a diverse matrix of tests to evaluate 1) concentration accuracy, 2) linearity of concentration response, and 3) accuracy of particle size. As described previously, several particle systems were tested and several instrument configurations assessed. Table 6.2 outlines the matrix of flow cell tests performed during Malvern Insitec-S validation activities; it does not include column tests.

The flow cell test matrix lists several tests with each having one of the following test goals:

- Concentration and Size Response Tests are evaluations of concentration and size distribution accuracy for several different test powders in the standard Malvern Insitec-S flow cell configuration (i.e., $100 \mathrm{~mm}$ lens, $150 \mathrm{~mm}$ spacers, and flow cell installed near the detection unit).

- Lens Tests are evaluations of the impact of the $500 \mathrm{~mm}$ lens on the concentration and size distribution result relative to the standard instrument configuration using the $100 \mathrm{~mm}$ lens.

- Position Tests evaluate the impact of distance of the flow cell from the detection unit. The flow cell is installed at the laser unit, and different spacer bars $(150 \mathrm{~mm}, 500 \mathrm{~mm}$, and $1000 \mathrm{~mm})$ are used to alter the distance between flow cell and detection unit. 
- Irregular Particle Evaluations examine the impact of irregular powders on the diffraction results. While standard tests employ spherical glass beads, irregular particle tests use either boehmite or gibbsite powders. These are industrial materials, and are expected to have broad size distribution and irregular shapes with rough surfaces.

Table 6.2. Flow Cell Test Matrix for Malvern Insitec-S Validation

\begin{tabular}{|c|c|c|c|c|c|}
\hline Test No. & Test Goal & Test Dispersion & Lens & Spacer Bars & $\begin{array}{c}\text { Flow Cell } \\
\text { Location }\end{array}$ \\
\hline 1 & $\begin{array}{l}\text { Concentration and } \\
\text { Size Response }\end{array}$ & $10 \mu \mathrm{m}$ glass bead & $100 \mathrm{~mm}$ & $150 \mathrm{~mm}$ & Detector \\
\hline 2 & $\begin{array}{l}\text { Concentration and } \\
\text { Size Response }\end{array}$ & $20 \mu \mathrm{m}$ glass bead & $100 \mathrm{~mm}$ & $150 \mathrm{~mm}$ & Detector \\
\hline 3 & $\begin{array}{l}\text { Concentration and } \\
\text { Size Response }\end{array}$ & $50 \mu \mathrm{m}$ glass bead & $100 \mathrm{~mm}$ & $150 \mathrm{~mm}$ & Detector \\
\hline 4 & $\begin{array}{l}\text { Concentration and } \\
\text { Size Response }\end{array}$ & 3 to $30 \mu \mathrm{m}$ glass bead & $100 \mathrm{~mm}$ & $150 \mathrm{~mm}$ & Detector \\
\hline 5 & $\begin{array}{l}\text { Concentration and } \\
\text { Size Response }\end{array}$ & XLSciTech & $100 \mathrm{~mm}$ & $150 \mathrm{~mm}$ & Detector \\
\hline 6 & $\begin{array}{l}\text { Concentration and } \\
\text { Size Response }\end{array}$ & QAS3001B & $100 \mathrm{~mm}$ & $150 \mathrm{~mm}$ & Detector \\
\hline 7 & Lens Test & 3 to $30 \mu \mathrm{m}$ glass bead & $500 \mathrm{~mm}$ & $150 \mathrm{~mm}$ & Detector \\
\hline 8 & Lens Test & XLSciTech & $500 \mathrm{~mm}$ & $150 \mathrm{~mm}$ & Detector \\
\hline 9 & Position Test & 3 to $30 \mu \mathrm{m}$ glass bead & $100 \mathrm{~mm}$ & $150 \mathrm{~mm}$ & Laser \\
\hline 10 & Position Test & 3 to $30 \mu \mathrm{m}$ glass bead & $100 \mathrm{~mm}$ & $500 \mathrm{~mm}$ & Laser \\
\hline 11 & Position Test & 3 to $30 \mu \mathrm{m}$ glass bead & $100 \mathrm{~mm}$ & $1,000 \mathrm{~mm}$ & Laser \\
\hline 12 & Position Test & XLSciTech & $100 \mathrm{~mm}$ & $150 \mathrm{~mm}$ & Laser \\
\hline 13 & Position Test & XLSciTech & $100 \mathrm{~mm}$ & $500 \mathrm{~mm}$ & Laser \\
\hline 14 & Position Test & XLSciTech & $100 \mathrm{~mm}$ & $1,000 \mathrm{~mm}$ & Laser \\
\hline 15 & $\begin{array}{l}\text { Irregular Particle } \\
\text { Evaluation }\end{array}$ & Boehmite powder & $100 \mathrm{~mm}$ & $150 \mathrm{~mm}$ & Detector \\
\hline 16 & $\begin{array}{l}\text { Irregular Particle } \\
\text { Evaluation }\end{array}$ & Gibbsite powder & $100 \mathrm{~mm}$ & $150 \mathrm{~mm}$ & Detector \\
\hline
\end{tabular}

While flow cell tests assessed a variety of instrument configurations (including changes in spacer bar length and flow cell location) and test powders, column tests focused solely on examining the impact of Phase I and Phase II test configurations on measurement accuracy. As such, the test matrix consisted of only two tests: 1) a single $400 \mathrm{~mm}$ column evaluation and 2) a single $900 \mathrm{~mm}$ column evaluation. The test configurations for these two tests are listed in Table 6.3.

Table 6.3. Column Test Matrix for Malvern Insitec-S Validation

\begin{tabular}{clcccc}
\hline Test No. & Purpose & Test Dispersion & Lens & Spacer Bars & Column Length \\
\hline 17 & $\begin{array}{l}\text { Phase I Configuration } \\
\text { Validation }\end{array}$ & $\begin{array}{c}3 \text { to } 30 \mu \mathrm{m} \text { glass } \\
\text { bead }\end{array}$ & $100 \mathrm{~mm}$ & $1,000 \mathrm{~mm}$ & $\begin{array}{l}400 \mathrm{~mm} \\
\text { (at detector) }\end{array}$ \\
\hline 18 & $\begin{array}{l}\text { Phase II Configuration } \\
\text { Validation }\end{array}$ & $\begin{array}{c}3 \text { to } 30 \mu \mathrm{m} \text { glass } \\
\text { bead }\end{array}$ & $500 \mathrm{~mm}$ & $1,000 \mathrm{~mm}$ & $\begin{array}{l}900 \mathrm{~mm} \\
\text { (at detector) }\end{array}$ \\
\hline
\end{tabular}




\subsubsection{Test Methodology and Data Analysis}

For each test (including both flow cell and column tests), a known mass of de-ionized or distilled water was added to the flow loop dispersion reservoir and circulated to remove entrained air. After the water was added, the Malvern Insitec-S analyzer was aligned, and baseline measurements of background laser and diffraction intensities were taken to prepare the instrument for measurements. Next, a known mass of dry test powder is added to the dispersion reservoir and mixed (by overhead agitator) and circulated through the system for at least $1 \mathrm{~min}$ or until a stable laser obscuration reading was observed in the Malvern Insitec-S software readout. The target mass of dry powder added was selected in some cases to yield a range of laser obscuration that bound those typically seen in in-chamber aerosol testing (i.e., 1 to 15 percent). On this basis, mass additions target a final (concentration endpoint) test obscuration of 20 percent. Achievement of this target in actual testing was constrained by test material limits. Specifically, standards procured from Whitehouse Scientific and Malvern Instruments Ltd. are provided in pre-measured $0.1,0.5$, or $1 \mathrm{~g}$ aliquots. To eliminate potential size bias from fractionating individual aliquots (which would hamper size verification efforts), the entire pre-measured aliquots were added. The need to add fixed amounts of mass limited the control over the test obscuration. As result, some flow cell tests saw obscurations up to 30 percent. It should be noted that because the path length used in flow cell testing is small ( $3 \mathrm{~mm}$ ) relative to those used in chamber testing ( 400 to $900 \mathrm{~mm}$ ), the dispersion concentrations employed in validation testing ( $\sim 100$ to $1000 \mathrm{ppmv})$ are by necessity much higher than those observed in aerosol testing ( $\sim 0.1$ to $1 \mathrm{ppmv})$. After addition and circulation, the dispersion obscuration, concentration, and size were measured to verify dispersion stability and sonication or additional mixing/circulation were used as needed to address poor dispersion stability. It should be noted that no dispersing agents were used to aide particle stability. Once a stable dispersion was achieved, the dispersion concentration and size distribution were measured.

For certain tests, a series of mass additions were made to the same dispersion to yield a set of increasing concentration measurements for a single test system. In other tests, a single mass addition was made. For all tests and/or concentration test points, each mass addition has a corresponding Malvern Insitec-S size and concentration measurement. The "actual" volumetric concentration of solid in the dispersion for concentration point $i, y_{s, i}$, is determined by mass balance and the standard reference density of the powder $\left(\rho_{\mathrm{s}}\right)$ and dispersing phase (water, $\left.\rho_{\mathrm{w}}\right)$ using Equation (6.1).

$$
y_{s, i}=\frac{\frac{1}{\rho_{S}} \sum_{j}^{i} m_{s, j}}{\frac{m_{w}}{\rho_{w}}+\frac{1}{\rho_{s}} \sum_{j}^{i} m_{s, j}}
$$

Here, $m_{s, j}$ represents the sequence of mass additions up to concentration point $i$. These are compared to the total volumetric solid concentration of measurement, $c_{v, i}$, made by the Malvern Insitec-S analyzer for concentration point $i$. The ratio of the "actual" concentration to the concentration, $\lambda_{\mathrm{c}, \mathrm{i}}$ measured by the Malvern Insitec-S analyzer is calculated using Equation (6.2).

$$
\lambda_{s, i}=\frac{c_{v, i}}{y_{s, i}}
$$

The result of the calculation from of Equation (6.2) can be used to assess the accuracy of the Malvern Insitec-S total concentration result on a measurement-by-measurement basis, with a perfect match between the actual concentration and measured concentration yielding $\lambda_{c, i}=1$. For a series of tests in which the mass of a given powder is added and subsequently characterized in step-wise additions, the 
resulting set of concentration data form a "concentration curve," an example of which is shown in Figure 6.4. For such data, the accuracy of the concentration measurement can be assessed by linear regression analysis where the data are fit to:

$$
y_{s}=\bar{\lambda}_{s} c_{v}
$$

This analysis results in a best-fit slope, $\bar{\lambda}_{S}$, for a set of measurements that are functionally equivalent to $\lambda_{\mathrm{c}, \mathrm{i}}$ (derived from a single measurement), in that it provides a measure of agreement between the "actual" concentration and the concentration measured by the Malvern Insitec-S analyzer (with $\bar{\lambda}_{s}=1$ indicating perfect agreement). Regression analysis also provides a coefficient of determination, $R^{2}$, for the data set, which can be used to assess the quality of correlation and the linearity of the concentration response. When $R^{2} \sim 1$, the data indicate close correlation between changes in actual and measured concentration. If $R^{2}$ is significantly less than 1, it may indicate a deficiency in how the instrument responds to increases in concentration after various mass additions. Assessments of both $\bar{\lambda}_{s}$ and $R^{2}$ are necessary, as it is possible to have $\overline{\lambda_{s}}=1$ and $R^{2}<1$ when there is significant scatter in the data or to have $\overline{\lambda_{s}} \neq 1$ and $R^{2}=1$ when the concentration response is linear but the slope is not unity.

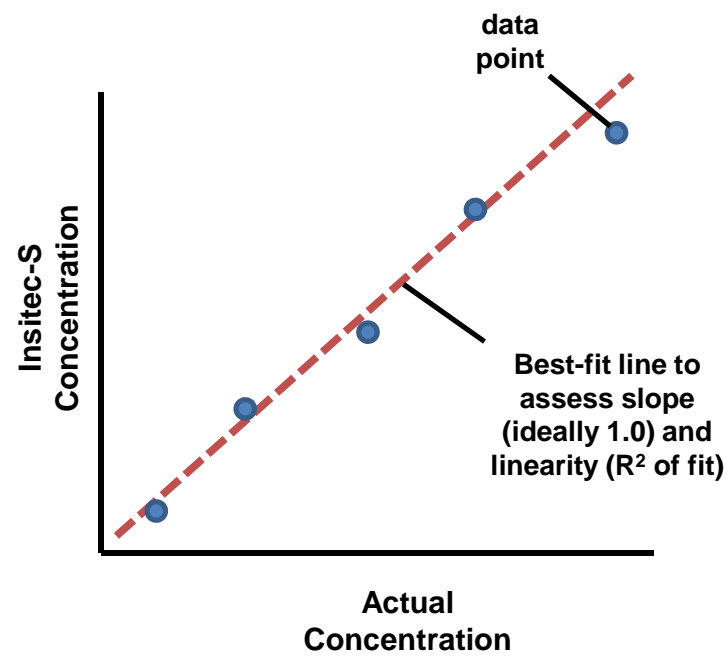

Figure 6.4. Expected Concentration Curve Result Obtained from a Series of Step-Wise Mass Additions and Measurements to the Flow Cell Test System

To assess the accuracy of the PSD determination, the measured size distribution was compared to either the list size distribution (for certified size standards such as those purchased from Whitehouse Scientific or Malvern Instruments, Ltd.) or to a size distribution measured on a Malvern Mastersizer 2000 (which is a laboratory particle size analyzer that uses laser diffraction). The primary means of assessing size distribution accuracy is to evaluate the ratio of measured-to-list values for the $10^{\text {th }}, 50^{\text {th }}$, and $90^{\text {th }}$ size percentiles:

$$
\begin{aligned}
& \delta(10)=\frac{d_{m}(10)}{d_{r e f}(10)} \\
& \delta(50)=\frac{d_{m}(50)}{d_{r e f}(50)}
\end{aligned}
$$




$$
\delta(90)=\frac{d_{m}(90)}{d_{r e f}(90)}
$$

Here, $\delta(10), \delta(50)$, and $\delta(90)$ represent the ratio of measured-to-list value for the $10^{\text {th }}, 50^{\text {th }}$, and $90^{\text {th }}$ size percentiles. The subscripts " $m$ " and "ref" on the diameter $d(X)$ at the $X^{\text {th }}$ percent refer to the measured and list values, respectively. These three diameter ratios provide similar information to $\lambda_{c, i}$, such as values close to unity indicate good agreement.

For column testing, the concentration and size distribution results obtained from the dispersion distributed over the entire column length was compared to reference test cases. The reference test cases for 400 and $900 \mathrm{~mm}$ column tests correspond to concentration PSDs made for the same test dispersion ( 3 to $30 \mu \mathrm{m}$ glass powder) with the flow cell. Reference flow cell measurements employed the same lenses used for column testing ( $100 \mathrm{~mm}$ lens for the $400 \mathrm{~mm}$ column and the $500 \mathrm{~mm}$ lens for the $900 \mathrm{~mm}$ column). Reference measurements employed "at-detection-unit" flow cell configurations to allow direct comparison of the column test, which requires distance corrections for laser attenuation, to results requiring no distance correction.

The concentration PSD indicates the cumulative concentration $C_{k}$ for all particle sizes under a particle diameter, $d_{k}$. To calculate the concentration PSD, the measured total dispersion concentration, $c_{v, i}$, provided by the Malvern Insitec-S analyzer is multiplied by the cumulative volume fraction, $F_{\mathrm{k}}$, for all particle sizes under $d_{k}$. For a concentration test point $i$, the concentration PSD is formed by the set of:

$$
C_{k, i}=c_{v, i} F_{k}
$$

for all $k$.

The analysis approach outlined above occurs after data from the Malvern Insitec-S analyzer have been processed by the Malvern Insitec-S data collection and analysis software. Because several of the software settings can impact analysis, the correct settings must be implemented before the data are exported for use in Equations (6.1) to (6.7). Several key software settings must be applied to correctly analyze the data. These include the material RI and whether not to apply spray geometry (laser attenuation) corrections. The particle RI listed in Table 6.1 was used for analysis; an RI of $1.330+0 \mathrm{i}$ was used for the dispersing phase (water). For flow cell testing, the application of spray geometry corrections depends on where the flow cell is installed. For tests in which the flow cell is installed at the detection unit, no spray correction is needed, and the "Unity" calculation approach may be used. The "Unity" calculation refers to diffraction analysis with no correction for distance or attenuation. For tests in which the flow cell is installed at the laser unit, the result is determined by using both the "Unity" approach and applying spray geometry corrections, which require input of the flow cell location (e.g., $500 \mathrm{~mm}$ away from the detection unit when the $500 \mathrm{~mm}$ spacers are used) and optical path length of the dispersion (e.g., $3 \mathrm{~mm}$ ). Finally, column tests are also examined with both the "Unity" analysis and the analysis that corrects for spray geometry. The spray geometry correction inputs for dispersion location and optical path length are selected to be consistent with the setup shown in Figure 6.2 for the $400 \mathrm{~mm}$ column test and Figure 6.3 for the $900 \mathrm{~mm}$ column test. 


\subsection{Results}

In the following sections, test results for Malvern Insitec-S validation testing are presented and discussed in detail. Baseline performance tests (i.e., flow cell tests in the standard instrument configuration) are discussed first. Next, the impact of changes in the Malvern Insitec-S configuration, such as flow cell installation location, is evaluated. Finally, the results of column testing are presented and the impact of spray geometry corrections discussed.

\subsubsection{Tests of the Standard Instrument Configuration and Performance}

The Malvern Insitec-S is a highly configurable in-process particle size analyzer. Before evaluating instrument configurations relevant to spray leak testing, tests were performed that evaluated the instrument in a "standard" configuration (with respect to the laser diffraction method) which avoids complications associated with distance of scattering events from the detection unit, non-spherical particles, and distributed aerosol. As defined herein, the "standard" instrument configuration consists of the $100 \mathrm{~mm}$ lens, the $150 \mathrm{~mm}$ spacer bars, and a liquid flow cell installed directly against the detection unit. This configuration is hereafter referred to as the "baseline" configuration, as it serves as a point of reference for different configurations that evaluate changes in lens selection or the consequence of long dispersion path lengths (i.e., column testing). All tests assessing instrument performance in this baseline configuration employ spherical glass bead powders, as these systems do not violate the spherical particle assumption made by the Malvern Insitec-S software to interpret the light scattering pattern, and as such, should not be impacted by uncertainty when using this assumption to interpret irregular particle systems.

Baseline assessments of performance include all tests falling under "Concentration and Size Response" heading in Table 6.2. The results of concentration and size response tests are presented in Table 6.4. When all tests shown in Table 6.4 are considered, the ratio of measured-to-test concentration ranges from 0.92 to 1.11 . This range suggests a typical accuracy of concentration of approximately \pm 10 percent for spherical particles. For the systems tested, the broadness of the size distribution (i.e., mono-disperse or poly-disperse) does not appear to have a significant impact on agreement between measured and test concentrations. An overall average of the data yields an average concentration ratio of 1.02, suggesting excellent overall agreement within 2 percent between Malvern Insitec-S concentration measurements and those measured by mass balance. Application of tolerance analysis to the measured data indicates that 95 percent of all concentration measurements should fall within a concentration ratio range of 0.87 to 1.17 (assuming a 95 percent confidence level). On this basis, the estimated uncertainty in concentration measurements appears to be \pm 15 percent for spherical particles under "ideal" measuring circumstances (that is with the flow cell near the detection unit, for the $100 \mathrm{~mm}$ lens).

Tests for the 10, 20, and $30 \mu \mathrm{m}$ mono-disperse glass powders, as well as the 3 to $30 \mu \mathrm{m}$ poly-disperse glass powder and XLSciTech glass beads, included several step-by-step mass additions that allow analysis of mass addition and concentration rise linearity. The sample result shown for the $10 \mu \mathrm{m}$ glass bead powder in Figure 6.5 indicates a best-fit slope $\bar{\lambda}_{s}$ of 1.032 and an $\mathrm{R}^{2}$ of 0.998 . These results suggest good (i.e., 3 percent) agreement between measured and actual concentrations and excellent linearity of the concentration response for the $10 \mu \mathrm{m}$ glass powder system. The full set of linearity testing results is provided in Table 6.1, and generally indicates good agreement between measured and actual concentrations (with a range of -5 percent to +9 percent relative difference) and excellent overall linearity (with all $\mathrm{R}^{2}$ coefficients greater than 0.99 ). 
Table 6.4. Summary of Baseline Concentration Response for Individual Concentration Points

\begin{tabular}{|c|c|c|c|c|}
\hline Glass Bead System & Test Point & $\begin{array}{c}\text { Measured (Malvern } \\
\text { Insitec-S) } \\
\text { Concentration, } \\
\text { ppmv }\end{array}$ & $\begin{array}{c}\text { Actual } \\
\text { Concentration, } \\
\text { ppmv }\end{array}$ & Ratio $\left(\lambda_{c, i}\right)$ \\
\hline \multirow[t]{8}{*}{$10 \mu \mathrm{m}$ mono-disperse } & $1-1$ & 46 & 43 & 1.08 \\
\hline & $1-2$ & 91 & 85 & 1.07 \\
\hline & $1-3$ & 139 & 130 & 1.07 \\
\hline & $1-4$ & 186 & 180 & 1.03 \\
\hline & $1-5$ & 227 & 224 & 1.01 \\
\hline & $1-6$ & 214 & 224 & 0.96 \\
\hline & $1-7$ & 213 & 224 & 0.95 \\
\hline & Average & -- -- & -- -- & 1.02 \\
\hline \multirow[t]{7}{*}{$20 \mu \mathrm{m}$ mono-disperse } & $1-1$ & 109 & 102 & 1.06 \\
\hline & $1-2$ & 201 & 196 & 1.02 \\
\hline & $1-3$ & 298 & 289 & 1.03 \\
\hline & $1-4$ & 410 & 393 & 1.04 \\
\hline & $1-5$ & 524 & 505 & 1.04 \\
\hline & $1-6$ & 437 & 443 & 0.99 \\
\hline & Average & -- -- & -- -- & 1.03 \\
\hline \multirow[t]{6}{*}{$50 \mu \mathrm{m}$ mono-disperse } & $1-1$ & 199 & 213 & 0.94 \\
\hline & $1-2$ & 394 & 416 & 0.95 \\
\hline & $1-3$ & 586 & 616 & 0.95 \\
\hline & $1-4$ & 762 & 809 & 0.94 \\
\hline & $1-5$ & 969 & 1002 & 0.97 \\
\hline & Average & -- -- & -- -- & 0.95 \\
\hline \multirow[t]{10}{*}{3 to $30 \mu \mathrm{m}$ poly-disperse } & $1-1$ & 227 & 207 & 1.10 \\
\hline & $1-2$ & 447 & 411 & 1.09 \\
\hline & $1-3$ & 454 & 411 & 1.11 \\
\hline & $2-1$ & 43 & 46 & 0.95 \\
\hline & $2-2$ & 94 & 90 & 1.04 \\
\hline & $2-3$ & 143 & 134 & 1.07 \\
\hline & $2-4$ & 233 & 216 & 1.08 \\
\hline & $2-5$ & 322 & 293 & 1.10 \\
\hline & $3-1$ & 534 & 492 & 1.08 \\
\hline & Average & -- -- & -- -- & 1.07 \\
\hline \multirow[t]{2}{*}{ QAS3001B } & $1-1$ & 171 & 186 & 0.92 \\
\hline & Average & -- -- & -- -- & 0.92 \\
\hline \multirow[t]{4}{*}{ XLSciTech } & $1-1$ & 224 & 235 & 0.95 \\
\hline & $1-2$ & 455 & 467 & 0.97 \\
\hline & $1-3$ & 947 & 961 & 0.99 \\
\hline & Average & -- -- & -- -- & 0.97 \\
\hline Overall Average & -- -- & -- -- & -- -- & 1.02 \\
\hline Lower 95/95 Tolerance & -- -- & -- -- & ---- & 0.87 \\
\hline Upper 95/95 Tolerance & -- -- & -- -- & ---- & 1.17 \\
\hline
\end{tabular}




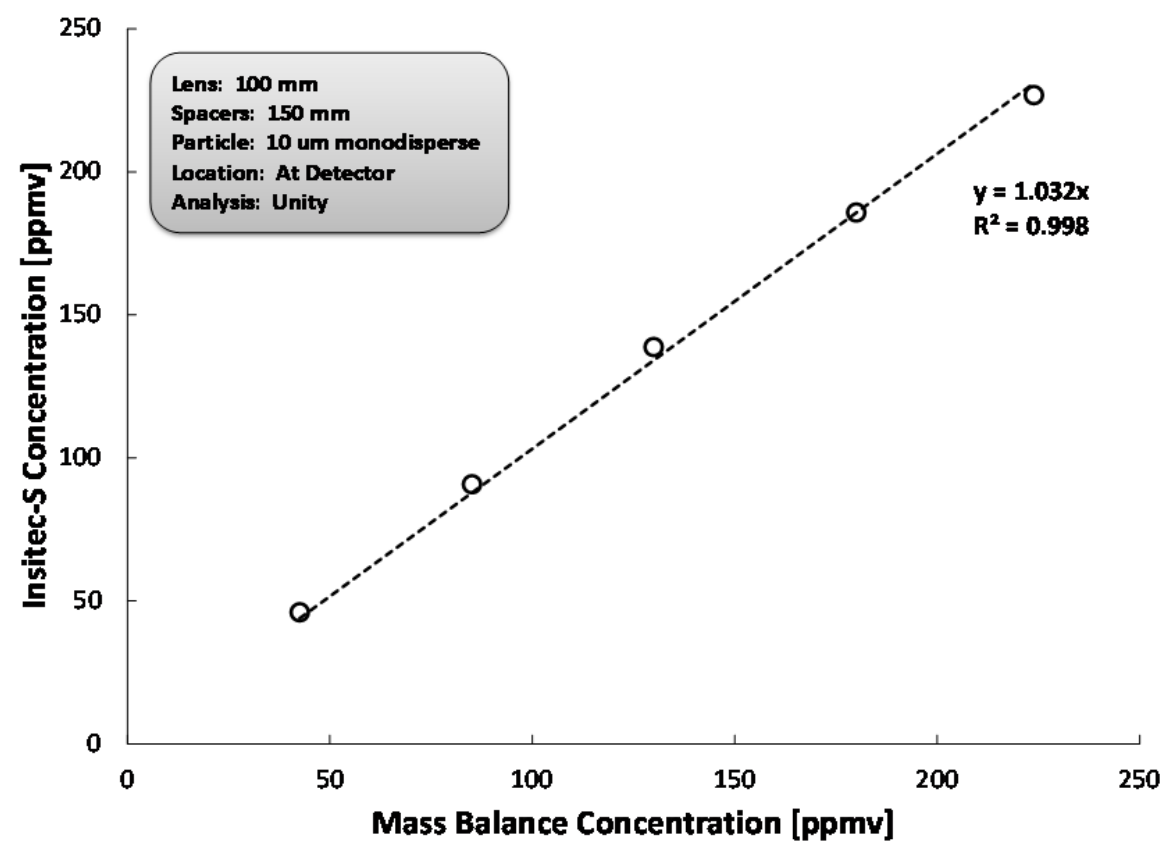

Figure 6.5. Concentration Curve for a $10 \mu \mathrm{m}$ Mono-Disperse Glass Bead Powder. The test configuration and analysis methodology are listed in the figure legend. Linear regression analysis reports a best fit slope $\left(\overline{\lambda_{s}}\right)$ of 1.032 and a $R^{2}$ of 0.998 .

The test results presented in Table 6.4 and Table 6.5 demonstrate the expected accuracy of the Malvern Insitec-S measuring system for spherical particles measured. The results correspond to "ideal" measuring configurations for the $100 \mathrm{~mm}$ lens where the flow cell is located near the detection unit such that no spray geometry correction is required. Analysis of the test data generally indicates good agreement between the concentration measured by the Malvern Insitec-S and that determined by material balance. On average, individual test points are only 2 percent higher than the expected concentration. Furthermore, tests where step-wise additions of material to the dispersion reservoir indicate excellent linearity of the concentration response. Specifically, increases in concentration are linear over the entire range of test concentrations (and obscurations) examined. An overall evaluation of expected concentration tolerance was applied to determine an expected working accuracy for the Malvern Insitec-S analyzer. This analysis indicates that 95 percent of all aerosol concentration measurements are expected to fall within \pm 15 percent of the actual concentration (with 95 percent confidence) under ideal test circumstances (i.e., spherical particles and no correction for spray geometry).

Table 6.5. Results of Linear Regression for Tests Involving Stepwise Addition of Mass. These results show the best-fit slope, $\bar{\lambda}_{\boldsymbol{s}}$, for each series and correlation coefficient $R^{2}$.

\begin{tabular}{lcc}
\hline \multicolumn{1}{c}{ Test System } & Series Ratio, $\bar{\lambda}_{s}$ & $R^{2}$ \\
\hline $10 \mu \mathrm{m}$ mono-disperse & 1.032 & 0.998 \\
$20 \mu \mathrm{m}$ mono-disperse & 1.037 & 1.000 \\
$50 \mu \mathrm{m}$ mono-disperse & 0.955 & 0.999 \\
3 to $30 \mu \mathrm{m}$ poly-disperse & 1.087 & 0.998 \\
XLSciTech & 0.982 & 1.000 \\
\hline
\end{tabular}


Evaluation of the accuracy of PSDs measured by the Malvern Insitec-S analyzer is presented in Table 6.6. The results indicate variations in the quality of the particle size measurement. For the $10 \mu \mathrm{m}$ and 3 to $30 \mu \mathrm{m}$ glass bead powders, the measured $\mathrm{d}(10)$ and $\mathrm{d}(50)$ values both show good agreement with their respective list values (as indicated by a $\delta$ value close to 1 ), while the $\mathrm{d}(90)$ value for both is larger than expected. For the 20 and $50 \mu \mathrm{m}$ powders, the measured d(10) values are about $\sim 20$ percent too small, whereas the measured $d(90)$ values are $\sim 20$ to 30 percent too large. This result indicates that the measured distributions for the 20 and $50 \mu \mathrm{m}$ particles are too broad, which is typically observed when comparing size distributions measured by laser diffraction to reference values from optical methods. The QAS3001B and XLSciTech beads show reasonable agreement with all measured size percentiles within $\sim 10$ percent of their list values. This result likely reflects the fact that the reference size distributions for these two powders were measured by laser diffraction.

Table 6.6. Evaluation of Size Distribution Accuracy for Spherical Systems

\begin{tabular}{lcccccc}
\hline \multicolumn{1}{c}{ Glass Bead System } & $\begin{array}{c}\text { Measured } \\
\mathrm{d}(10), \mu \mathrm{m}\end{array}$ & $\begin{array}{c}\text { Measured } \\
\mathrm{d}(50), \mu \mathrm{m}\end{array}$ & $\begin{array}{c}\text { Measured } \\
\mathrm{d}(90), \mu \mathrm{m}\end{array}$ & $\begin{array}{c}\text { Ratio } \\
\delta(10)\end{array}$ & $\begin{array}{c}\text { Ratio } \\
\delta(50)\end{array}$ & Ratio $\delta(90)$ \\
\hline $10 \mu \mathrm{m}$ mono-disperse & 7.9 & 9.9 & 13.5 & 0.98 & 1.08 & 1.33 \\
$20 \mu \mathrm{m}$ mono-disperse & 18.0 & 22.6 & 31.4 & 0.82 & 0.99 & 1.32 \\
$50 \mu \mathrm{m}$ mono-disperse & 39.4 & 49.2 & 61.8 & 0.83 & 1.00 & 1.21 \\
3 to $30 \mu \mathrm{m}$ poly-disperse & 8.9 & 13.5 & 22.7 & 0.97 & 1.01 & 1.11 \\
QAS3001B & 40.3 & 64.7 & 98.5 & 1.09 & 1.08 & 1.03 \\
XLSciTech & 55.6 & 69.2 & 86.5 & 1.12 & 1.02 & 0.92 \\
Overall Average & ---- & ---- & ---- & 0.97 & 1.03 & 1.15 \\
\hline
\end{tabular}

\subsubsection{Irregular Powder Tests}

The goal of the irregular powder tests was to examine the performance of the Malvern Insitec-S instrument for analyzing non-spherical particles. The results of concentration analysis are shown in Table 6.7.

The results for gibbsite powder show close agreement between the measured and actual concentrations. In contrast, the results for boehmite powder indicate that the measured concentrations all are approximately a factor of two larger than their corresponding actual concentrations. This disagreement likely reflects particle shape effects, as the correlations used to interpret the laser diffraction pattern assume that particles are spherical. Deviation of the particle shape from spherical introduces error into both the concentration and size measurement. A step-wise mass addition was performed for the boehmite powder. The resulting concentration curve for this step-wise addition is shown in Figure 6.6 and indicates that each addition produces a proportional gain in the concentration measured by the Malvern Insitec-S analyzer over the range of actual concentrations tests. Linear regression analysis indicates an excellent correlation between Malvern Insitec-S and measured concentration with an $R^{2} \sim 1$. 
Table 6.7. Summary of Irregular Powder Concentration Response for Individual Concentration Points

\begin{tabular}{ccccc}
\hline Powder System & Test Point & $\begin{array}{c}\text { Measured (Malvern Insitec-S) } \\
\text { Concentration ppmv }\end{array}$ & $\begin{array}{c}\text { Actual Concentration } \\
\text { ppmv }\end{array}$ & $\begin{array}{c}\text { Ratio } \\
\left(\lambda_{c, i}\right)\end{array}$ \\
\hline Boehmite & $1-1$ & 16.3 & 8.3 & 1.97 \\
& $1-2$ & 34.0 & 16.6 & 2.05 \\
& $1-3$ & 52.1 & 24.9 & 2.10 \\
& $1-4$ & 69.9 & 33.1 & 2.11 \\
& $1-5$ & 87.6 & 41.3 & 2.12 \\
& $1-6$ & 105.3 & 49.6 & 2.13 \\
& $2-1$ & 117.2 & 54.3 & 2.16 \\
& Average & ---- & --- & 2.09 \\
& $1-1$ & 87.4 & 84.9 & 1.03 \\
\hline
\end{tabular}

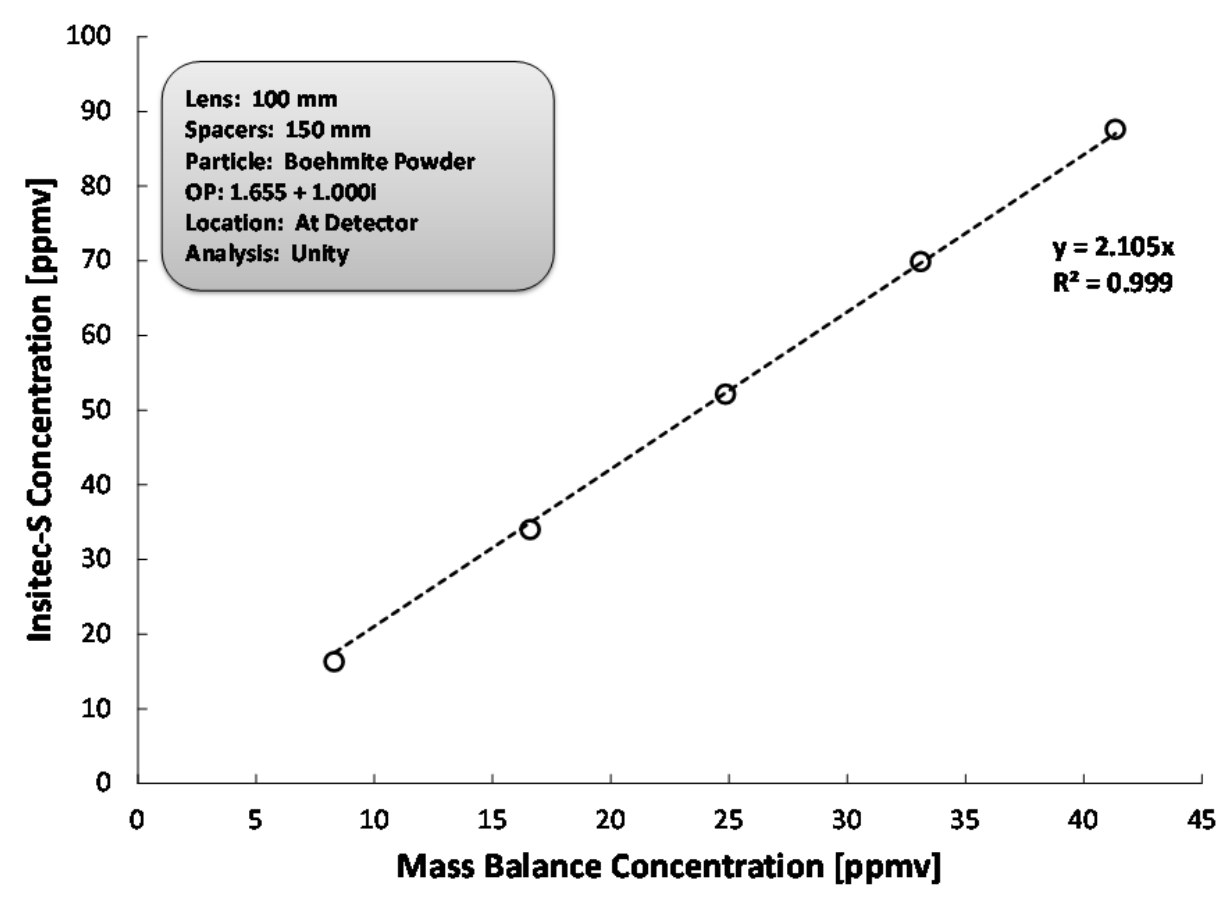

Figure 6.6. Concentration Curve for Boehmite Powder. The test configuration and analysis methodology are listed in the figure legend. Linear regression analysis reports a best fit slope $\left(\overline{\lambda_{s}}\right)$ of 2.105 and a $R^{2}$ of 0.999 .

Size distribution analysis results for the two irregular powder tests are shown in Table 6.8. The results indicate the Malvern Insitec-S analyzer over-estimates the d(50) by less than 10 percent and over-estimates the $\mathrm{d}(10)$ and $\mathrm{d}(90)$ for both powders by approximately 20 percent relative to measurements made on a Malvern Mastersizer 2000. This is not unexpected, as the Mastersizer 2000 is more sensitive to particulate fines because it employs a dual blue $(470 \mathrm{~nm})$ and red $(633 \mathrm{~nm})$ laser system. For boehmite, the Mastersizer 2000 detects a significant fraction of particulate ( $\sim 7$ percent by volume) 
below $1 \mu \mathrm{m}$. This fraction is not detected by the Malvern Insitec-S, which employs a single $660 \mathrm{~nm}$ laser. The gibbsite powder, which has a size distribution nearly identical to that of the boehmite powder, is similarly impacted.

Table 6.8. Evaluation of Size Distribution Accuracy for Irregular Particles

\begin{tabular}{ccccccc}
\hline & Measured & Measured & Measured & Ratio & Ratio & Ratio \\
Glass Bead System & $\mathrm{d}(10), \mu \mathrm{m}$ & $\mathrm{d}(50), \mu \mathrm{m}$ & $\mathrm{d}(90), \mu \mathrm{m}$ & $\delta(10)$ & $\delta(50)$ & $\delta(90)$ \\
\hline Boehmite & 2.1 & 8.8 & 24.4 & 1.26 & 1.09 & 1.21 \\
Gibbsite & 1.9 & 9.6 & 25.9 & 1.10 & 1.07 & 1.22 \\
\hline
\end{tabular}

\subsubsection{Lens Tests}

In lens testing, a $500 \mathrm{~mm}$ Malvern Insitec-S lens was installed and used to measure concentration and size results for the 3 to $30 \mu \mathrm{m}$ poly-disperse glass beads and the XLSciTech glass beads. The $500 \mathrm{~mm}$ lens has a nominal working range of 2.5 to $2500 \mu \mathrm{m}$, whereas the $100 \mathrm{~mm}$ lens used in baseline testing (see Section 6.3.1) has a nominal working range of 0.5 to $200 \mu \mathrm{m}$. While the $100 \mathrm{~mm}$ lens working range is much better suited to in-chamber testing and the size range of interest (10 to $100 \mu \mathrm{m})$ for spray leak testing, the $500 \mathrm{~mm}$ lens also covers the size range of interest and can be used for in-spray testing (where large 100 to $1000 \mu \mathrm{m}$ particles are expected). Because the working range of the $500 \mathrm{~mm}$ lens is large, the particle systems selected for $100 \mathrm{~mm}$ testing may challenge the measuring limit of the $500 \mathrm{~mm}$ lens. As such, it is important to test the accuracy of the $500 \mathrm{~mm}$ lens when measuring such systems.

The results of concentration testing with the $500 \mathrm{~mm}$ lens for 3 to $30 \mu \mathrm{m}$ and XLSciTech glass beads are shown in Table 6.9. In this table, the measured, actual, and concentration ratio are given for each series test with the $500 \mathrm{~mm}$ lens along with a performance observed in $100 \mathrm{~mm}$ lens testing. Two separate tests were performed on 3 to $30 \mu \mathrm{m}$ poly-disperse glass beads. Both of these tests indicate that the Malvern Insitec-S with a $500 \mathrm{~mm}$ lens under-estimates concentration by $\sim 23$ percent relative to the actual concentration and by about $\sim 30$ percent relative to the average $100 \mathrm{~mm}$ lens performance. The source for this difference may derive from the Malvern Insitec-S $500 \mathrm{~mm}$ lens particle size measurement shown in Table 6.10, which indicates that the $500 \mathrm{~mm}$ lens over-estimates the broadness of the size distribution, which in turn, could bias the concentration measurement low. In contrast, the concentration and size distribution measurements for the XLSciTech powder made using the $500 \mathrm{~mm}$ lens show excellent agreement with the $100 \mathrm{~mm}$ lens. This result may indicate that the working size range of the $500 \mathrm{~mm}$ lens is challenged by the 3 to $30 \mu \mathrm{m}$ powder, which has a d(50) value of $\sim 13 \mu \mathrm{m}$ that is less than one order of magnitude higher than the lower working limit of the lens (i.e., $\sim 25 \mu \mathrm{m}$ ). We speculate that working this close to the lower range limit impacts the accuracy of the PSD measurements. 
Table 6.9. Summary of Lens Test Concentration Results and Comparisons

\begin{tabular}{|c|c|c|c|c|}
\hline Powder System & Test Point & $\begin{array}{l}\text { Measured (Malvern } \\
\text { Insitec-S) } \\
\text { Concentration, ppmv }\end{array}$ & $\begin{array}{l}\text { Actual Concentration, } \\
\text { ppmv }\end{array}$ & $\begin{array}{l}\text { Ratio } \\
\left(\lambda_{c, i}\right)\end{array}$ \\
\hline \multirow[t]{4}{*}{3 to $30 \mu \mathrm{m}$ poly-disperse } & $1-1$ & 165 & 213 & 0.77 \\
\hline & $2-1$ & 166 & 212 & 0.78 \\
\hline & Ave: $500 \mathrm{~mm}$ lens & -- -- & -- -- & 0.78 \\
\hline & Ref: $100 \mathrm{~mm}$ lens ${ }^{(a)}$ & -- -- & -- -- & 1.07 \\
\hline \multirow[t]{3}{*}{ XLSciTech } & $1-1$ & 1110 & 1150 & 0.97 \\
\hline & Ave: 500 mm lens & ---- & -- -- & 0.97 \\
\hline & Ref: 100 mm lens ${ }^{(a)}$ & -- -- & -- -- & 0.97 \\
\hline
\end{tabular}

(a) Reference $100 \mathrm{~mm}$ lens measurement (averages values from Table 6.4).

Table 6.10. Evaluation of Size Distribution Accuracy for Spherical Systems

\begin{tabular}{llccc}
\hline \multirow{2}{*}{ Glass Bead System } & Test System & $\begin{array}{c}\text { Meas. } \\
\mathrm{d}(10), \mu \mathrm{m}\end{array}$ & $\begin{array}{c}\text { Meas. } \\
\mathrm{d}(50), \mu \mathrm{m}\end{array}$ & $\begin{array}{c}\text { Meas. } \\
\mathrm{d}(90), \mu \mathrm{m}\end{array}$ \\
\hline 3 to $30 \mu \mathrm{m}$ poly-disperse & $500 \mathrm{~mm}$ lens & 5.4 & 14.4 & 26.4 \\
& $100 \mathrm{~mm}$ lens & 8.9 & 13.5 & 22.7 \\
& Ratio (500/100) & 0.61 & 1.07 & 1.17 \\
XLSciTech & $500 \mathrm{~mm}$ lens & 57.5 & 71.0 & 88.4 \\
& $100 \mathrm{~mm}$ lens & 55.6 & 69.2 & 86.5 \\
& Ratio (500/100) & 1.04 & 1.03 & 1.02 \\
\hline
\end{tabular}

\subsubsection{Position Tests}

Position tests were conducted to evaluate the ability of the Malvern Insitec-S analyzer to appropriately correct for laser attenuation caused by the increased optical distance between scattering centers and the detection unit optics. For flow cell testing, the position of the scattering events are highly localized (i.e., confined to a single distance away from the detector) and, as a result, do not represent the configurations employed in actual aerosol testing. Instead, these flow cell position tests represent a first-order assessment of the accuracy of distance corrections by the Malvern Insitec-S software (which is based on first principles). It should be noted that, in addition to assessing the accuracy of the software corrections for distance, the impact of distance on the uncorrected results is considered to better highlight why corrections are needed. As the distance of scattering event most strongly impacts the angle of acceptance, the measured PSD is impacted most directly and will be the focus of discussion in this subsection.

Figure 6.7 shows the uncorrected size distributions for the 3 to $30 \mu \mathrm{m}$ and XLSciTech glass bead powders. For comparison, the list (for the 3 to $30 \mu \mathrm{m}$ ) and reference (for the XLSciTech) size distributions are included. In addition, the size distribution measured at-detector is shown to facilitate direct comparison to a result not impacted by laser attenuation. The results in Figure 6.7 show a gradual loss of fines from the size distribution as the flow cell is moved progressively farther (i.e., $150 \mathrm{~mm}$, $500 \mathrm{~mm}$, and $1000 \mathrm{~mm}$ ) from the detection unit for the 3 to $30 \mu \mathrm{m}$ poly-disperse material. The impact at 
$150 \mathrm{~mm}$ is minor above the $10^{\text {th }}$ size percentile; however, at $1000 \mathrm{~mm}$, there is almost a complete attenuation of particles below $20 \mu \mathrm{m}$. This means that for scattering events that occur $\sim 1000 \mathrm{~mm}$ from the detector, the analyzer cannot detect particles below $20 \mu \mathrm{m}$ at all using the optical configuration employed by Malvern Insitec-S. This attenuation is primarily related to the size of particle. This assertion is demonstrated by the flow cell position tests for the XLSciTech powder, which has a size distribution that falls almost entirely above $50 \mu \mathrm{m}$ and which shows very little (if any) attenuation of the PSD with distance.

Attenuation of the fines fraction of the PSD will impact concentration measurement, as the uncorrected instrument size will show an apparent increase resulting from the apparent loss of fines from the diffraction measurement. This increase in apparent size in the uncorrected result yields an increase in apparent (uncorrected) concentration in systems impacted by attenuation. The concentration test results for the 3 to $30 \mu \mathrm{m}$ system are shown in Table 6.11 and demonstrate the expected effect. That is, the apparent concentration increases monotonically with increasing distance from the detector from 1.07 when the flow cell is installed at the detector to 2.12 when the flow cell is installed $\sim 1000 \mathrm{~mm}$ from the detector. This trend was not observed in the concentration points for increased distance in the XLSciTech glass beads system. However, there does appear to be a minor impact of the initial move of the flow cell from the detector to the laser side for the XLSciTech beads, as the concentration ratio jumps from 0.97 to about 1.1 as a result of the configuration change.

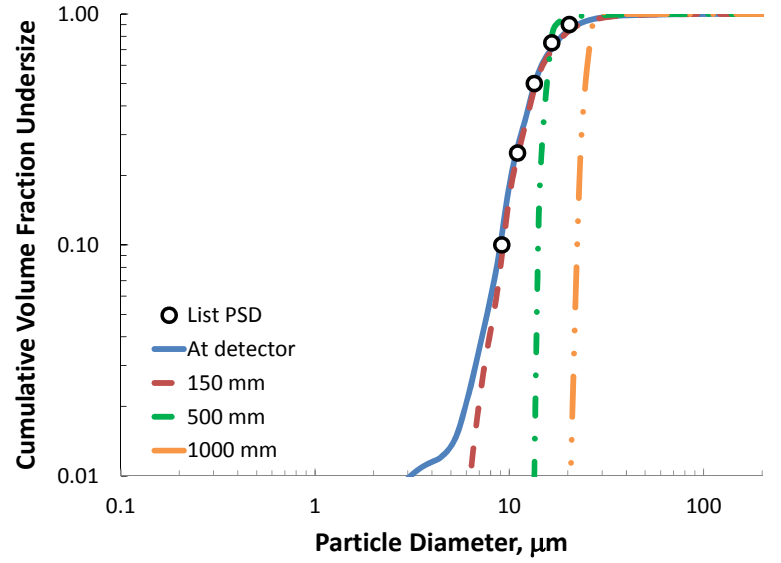

(a)

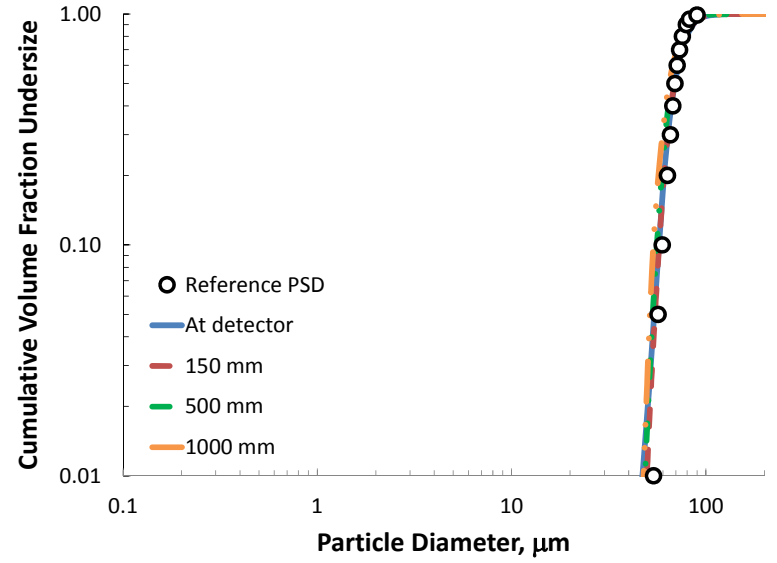

(b)

Figure 6.7. Impact of Distance from Detector on the Measured PSD for (a) a 3 to 30 Poly-Disperse Glass Bead and (b) an XLSciTech Glass Powder. Measurements were made using a flow cell with a $3 \mathrm{~mm}$ optical path. The measured size distributions have not been corrected for spray geometry effects. 
Table 6.11. Summary of Uncorrected Position Test Concentration Measurements

\begin{tabular}{llccc}
\hline Powder System & \multicolumn{1}{c}{$\begin{array}{c}\text { Position } \\
\text { (Spacer Length) }\end{array}$} & $\begin{array}{c}\text { Measured (Malvern } \\
\text { Insitec-S) } \\
\text { Concentration, ppmv }\end{array}$ & $\begin{array}{c}\text { Actual } \\
\text { Concentration, ppmv }\end{array}$ & $\begin{array}{c}\text { Ratio } \\
\left(\lambda_{c, i}\right)\end{array}$ \\
\hline 3 to $30 \mu$ m poly-disperse & Detector & ---- & --- & 1.07 \\
& Laser $(150 \mathrm{~mm})$ & 239 & 215 & 1.11 \\
& Laser $(500 \mathrm{~mm})$ & 269 & 195 & 1.38 \\
& Laser $(1,000 \mathrm{~mm})$ & 407 & 192 & 2.12 \\
XLSciTech & Detector & $--1)$ & ---- & 0.97 \\
& Laser $(150 \mathrm{~mm})$ & 1280 & 1110 & 1.15 \\
& Laser $(500 \mathrm{~mm})$ & 1240 & 1110 & 1.12 \\
& Laser $(1,000 \mathrm{~mm})$ & 1230 & 1110 & 1.11 \\
\hline
\end{tabular}

(a) Reference at-detector measurement is the average of the $100 \mathrm{~mm}$ baseline tests from Table 6.4.

The overall impact of increasing distance on the uncorrected result is twofold: 1) the uncorrected total concentration reported by the Malvern Insitec-S increases, while 2) the uncorrected PSD shows attenuation of the fractional contribution of below a certain threshold diameter (which depends on distance from the detector). As a result, both effects must be considered when trying to assess the overall impact of distance on the uncorrected result. At particle sizes in which there is no attenuation of the volume contribution, the concentration of aerosol particles in that size range will increase. However, for particle sizes in which attenuation of the volume fraction does occur, the concentration of aerosol can be partially or entirely reduced. Because of the potential impact to the result, the particle size and concentration results must be corrected when this attenuation is significant. Based on the results in Figure 6.7 and Table 6.11, attenuation for systems that best represent the lower limit of size interest (i.e., the 3 to $30 \mu \mathrm{m}$ ) appears to become significant when scattering events occur farther than $150 \mathrm{~mm}$ from the detection unit. This means that the impact of attenuation need only be considered and corrected for configurations where scattering events occur beyond $150 \mathrm{~mm}$. Because both Phase I and Phase II small-scale aerosol tests employ a maximum $70 \mathrm{~mm}$ optical path length, small-scale aerosol data are not expected to be subject to increased uncertainty associated with attenuation. On the other hand, both Phase I and Phase II large-scale configurations employ path lengths in excess of $150 \mathrm{~mm}$. As a result, the impact of attenuation and corrections made to address attenuation must be considered.

Figure 6.8 and Table 6.12 show the impact of corrections for laser attenuation on the measured PSD and concentration result, respectively. As before, the at-detector result (which requires no correction) and the list/reference PSD are included for comparison. The impact of correction on concentration and PSD results measured at distances of $150 \mathrm{~mm}$ or greater appears to depend on both the test system (i.e., powder) and distance from the spray. Likewise, the correction does not appear to adjust the result in a uniform manner. For example, corrections to PSDs measured for the 3 to $30 \mu \mathrm{m}$ powder at $150 \mathrm{~mm}$ yield a small population of 1 to $7 \mu \mathrm{m}$ particles that is not present in the uncorrected result and that over-estimates the fractional contribution of a similar population seen in the at-detector result. Still, the corrected result for $150 \mathrm{~mm}$ shows excellent agreement with the at-detector data above 3 percent of the total population, and the concentration result in Table 6.12 is only 7 percent low relative to the actual concentration. In contrast, the correction of the 3 to $30 \mu \mathrm{m}$ powder data at $500 \mathrm{~mm}$ yields a PSD result that grossly over-estimates the population fraction of fines and under-estimates concentration by a factor 
of $\sim 20$. Finally, correction of $1000 \mathrm{~mm}$ data for the 3 to $30 \mu \mathrm{m}$ powder does not appear to appreciably change the PSD or concentration results relative to the uncorrected data, suggesting insufficient signal on the diffraction channels that need to be corrected. For the XLSciTech powder system, correction of diffraction data produces a better size and concentration result for the $150 \mathrm{~mm}$ test position (relative to uncorrected data). Correction of the $500 \mathrm{~mm}$ data set yields a significant (10 percent by volume) and anomalous size population at $1 \mu \mathrm{m}$ and produces a concentration that is a factor of 10 too low. Similar to the 3 to $30 \mu \mathrm{m}$ system, corrections of the XLSciTech data collected at $1000 \mathrm{~mm}$ produce no noticeable change relative to uncorrected data.

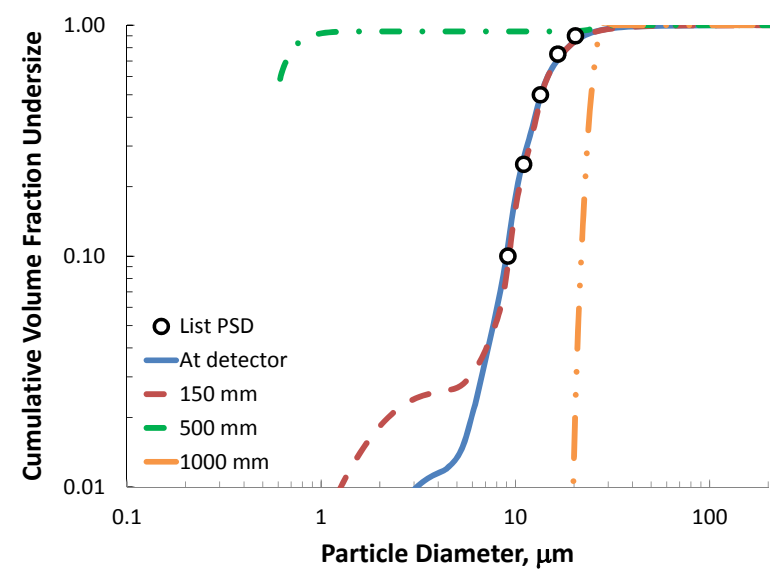

(a)

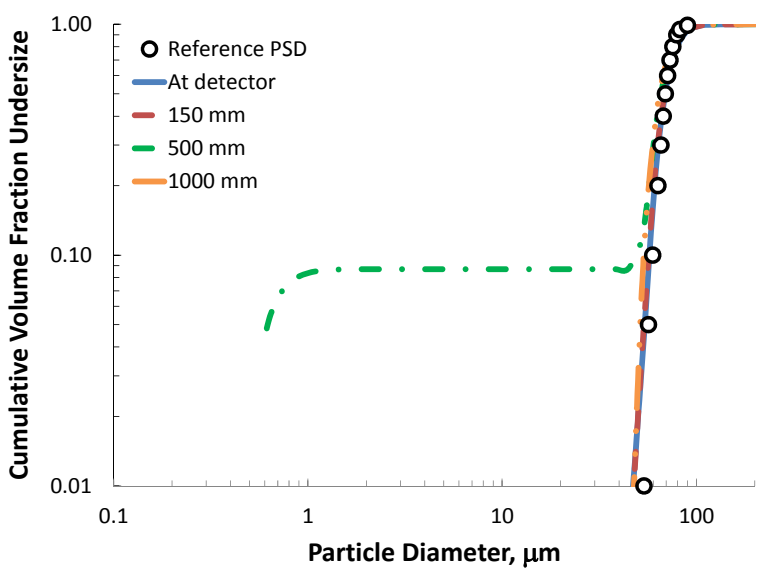

(b)

Figure 6.8. PSD Data from Figure 6.7 After Spray Geometry (laser attenuation) Corrections Were Applied. Data correspond to corrected distributions for (a) a 3 to $30 \mu \mathrm{m}$ poly-disperse glass bead and (b) an XLSciTech glass powder.

Table 6.12. Summary of Position Test Concentration Measurements after Correction for Laser Attenuation

\begin{tabular}{llccc}
\hline \multicolumn{1}{c}{ Powder System } & \multicolumn{1}{c}{$\begin{array}{c}\text { Position } \\
\text { (Spacer Length) }\end{array}$} & $\begin{array}{c}\text { Measured (Malvern } \\
\text { Insitec-S) Concentration, } \\
\text { ppmv }\end{array}$ & $\begin{array}{c}\text { Actual Concentration, } \\
\text { ppmv }\end{array}$ & $\begin{array}{c}\text { Ratio } \\
\left(\lambda_{c, i}\right)\end{array}$ \\
\hline 3 to $30 \mu$ m poly-disperse & Detector & ---- & 1.07 \\
& Laser $(150 \mathrm{~mm})$ & 201 & 215 & 0.93 \\
& Laser $(500 \mathrm{~mm})$ & 9.0 & 195 & 0.046 \\
& Laser $(1,000 \mathrm{~mm})$ & 405 & 192 & 2.11 \\
XLSciTech & Detector & --- & ---- & 0.97 \\
& Laser $(150 \mathrm{~mm})$ & 1120 & 1110 & 1.00 \\
& Laser $(500 \mathrm{~mm})$ & 300 & 1110 & 0.27 \\
& Laser $(1,000 \mathrm{~mm})$ & 1230 & 1110 & 1.11 \\
\hline
\end{tabular}

(a) Reference at-detector measurement is the average of the $100 \mathrm{~mm}$ baseline tests from Table 6.4.

The results outlined above demonstrate significant difficulty in appropriately correcting laser diffraction patterns corresponding to localized scattering far from the detector. This result is not entirely 
unexpected, as the lowered angle of acceptance may attenuate the diffraction signal down to levels comparable to but still above measurement and electronic noise, yielding anomalous PSD and concentration results after signal amplification. In cases in which the attenuation is severe or nearly complete, the signal may fall below the noise thresholds, leaving no signal to correct. This would yield diffraction data only on channels that do not need correction such that the corrected and uncorrected results would not differ.

As stated previously, the physical configuration tested in flow cell position tests do not reflect an actual aerosol configuration tested in Phase I or Phase II. While the results inform the need to test the accuracy of aerosol configurations in which aerosol is sampled at distances greater than $150 \mathrm{~mm}$, they do not reflect on the validity of corrections for optical path lengths longer than $3 \mathrm{~mm}$. Specifically, the flow cell configuration tested here demonstrates the impact of severe or complete attenuation on high-angle diffraction channels that will not occur for configurations in which aerosols are distributed across a long path length between laser and detector. In the latter configuration, the detector will still receive high-angle diffraction signals from scattering events that occur close to the detector. The diffraction pattern resulting from this "distributed" aerosol will only suffer partial attenuation rather than the complete attenuation suffered from a "localized" aerosol far from the detector. In the next section, the ability of the Malvern Insitec-S software to correct for partial attenuation in "distributed" systems is tested.

\subsubsection{Column Testing}

Column testing was performed to evaluate the accuracy of aerosol concentration and size measurements for distributed systems with optical path lengths in excess of $150 \mathrm{~mm}$. As discussed at the end of the preceding section, such systems are subject to partial (but not complete) attenuation of the high-angle diffraction pattern and, as a result, may not be subject to the same difficulties observed in the correction of complete attenuation in localized diffraction events. Two column tests were performed: 1) a $400 \mathrm{~mm}$ column (optical path length) test and 2) a $900 \mathrm{~mm}$ column (optical path length) test. The test configurations for 400 and $900 \mathrm{~mm}$ column tests were selected to match Phase I and Phase II aerosol instrument and measuring configurations, respectively. The purpose of these tests is to evaluate the accuracy of size and concentration "correction" for partial attenuation.

The concentration and PSD results for the 400 and $900 \mathrm{~mm}$ columns are shown in Table 6.13 and Figure 6.9. For the $400 \mathrm{~mm}$ column test, the uncorrected concentration result is approximately 30 percent too large relative to the actual value and about 25 percent too large relative to "at-detector" measurements requiring no correction. This over-estimate in concentration results from an under-estimate in the volume fraction of fine material in the PSD (which yields a larger apparent particle size for the dispersion). Similar trends appear in the $900 \mathrm{~mm}$ column test results. The "uncorrected" concentration is $\sim 25$ percent larger than the actual concentration and 50 percent larger than "at-detector" measurements; as before, the over-estimate in uncorrected concentration results from underestimating the fractional contribution of particles below approximately $30 \mu \mathrm{m}$.

Application of corrections for laser attenuation generally improves agreement between the actual and measured size and concentration results. For the $400 \mathrm{~mm}$ column test, correction yields a dispersion concentration result that is 13 percent larger than the actual result and that is only 5 percent larger than the "at-detector" measurement. There is still disagreement between the corrected and "at-detector" size 
distribution measurements, but the corrected result now shows a population shoulder below $7 \mu \mathrm{m}$ that is consistent with the at-detector measurement. As shown in Table 6.14, analysis of the differences in corrected and reference particle sizes at the $10^{\text {th }}, 50^{\text {th }}$, and $90^{\text {th }}$ percentiles suggests that the corrected size distribution is uniformly larger than the expected (i.e., at-detector) measurement by 20-25 percent. The results of the $900 \mathrm{~mm}$ column test suggest that correction of the data yields a concentration and size distribution result that are generally comparable to the "at-detector" measurement. The corrected $900 \mathrm{~mm}$ concentration measurement is 17 percent too low relative to the actual concentration; however, it compares well to the at-detector measurement which is itself low by 23 percent relative to the actual concentration. The accuracy of the corrected $900 \mathrm{~mm}$, PSD result relative to the "at-detector" result, which is shown in Table 6.14, appears variable. The $\mathrm{d}(10)$ value matches for corrected and reference results, but the $\mathrm{d}(50)$ and $\mathrm{d}(90)$ values are too large by $\sim 20$ and $\sim 60$ percent, respectively. The increasing uncertainty at larger particle diameters for corrections made to $900 \mathrm{~mm}$ column data may result from 1) aggregation of the 3 to $30 \mu \mathrm{m}$ particles in the larger 50 gal test system used for column testing (which is not equipped with a sonicator to disrupt particle agglomerates and has lower mixing power relative to the flow cell dispersion unit) or 2) the combined effect of PSD broadening and a baseline size uncertainty of 20 percent. The latter is proposed given that the $\mathrm{d}(50)$ values for both the 400 and $900 \mathrm{~mm}$ columns is 20 percent too large.

Table 6.13. Summary of Irregular Powder Concentration Response for Individual Concentration Points

\begin{tabular}{|c|c|c|c|}
\hline Test System & Measurement & $\begin{array}{l}\text { Measured (Malvern Insitec-S) } \\
\text { Concentration, ppmv }\end{array}$ & $\begin{array}{l}\text { Ratio } \\
\left(\lambda_{c, i}\right) \\
\end{array}$ \\
\hline \multirow{5}{*}{$\begin{array}{l}400 \mathrm{~mm} \text { column } \\
\text { ( } 3 \text { to } 30 \mu \mathrm{m} \text { powder) }\end{array}$} & Actual Concentration & 2.42 & -- -- \\
\hline & Reference Measurement $^{(\mathrm{a})}$ & 2.62 & 1.08 \\
\hline & Uncorrected Column Measurement & 3.20 & 1.32 \\
\hline & Corrected Column Measurement & 2.73 & 1.13 \\
\hline & Ratio (Column/At-Detector) & 1.04 & -- -- \\
\hline \multirow{5}{*}{$\begin{array}{l}900 \mathrm{~mm} \text { column } \\
\text { ( } 3 \text { to } 30 \mu \mathrm{m} \text { powder) }\end{array}$} & Actual Concentration & 0.56 & -- -- \\
\hline & Reference Measurement ${ }^{(b)}$ & 0.44 & 0.77 \\
\hline & Uncorrected Column Measurement & 0.70 & 1.23 \\
\hline & Corrected Column Measurement & 0.47 & 0.83 \\
\hline & Ratio (Column/At-Detector) & 1.07 & -- -- \\
\hline
\end{tabular}

(a) $100 \mathrm{~mm}$ lens, 3 to $30 \mu \mathrm{m}$ powder test concentration scaled to an actual concentration of $2.42 \mathrm{ppmv}$.

(b) $500 \mathrm{~mm}$ lens, 3 to $30 \mu \mathrm{m}$ powder test concentration scaled to an actual concentration of $0.56 \mathrm{ppmv}$. 


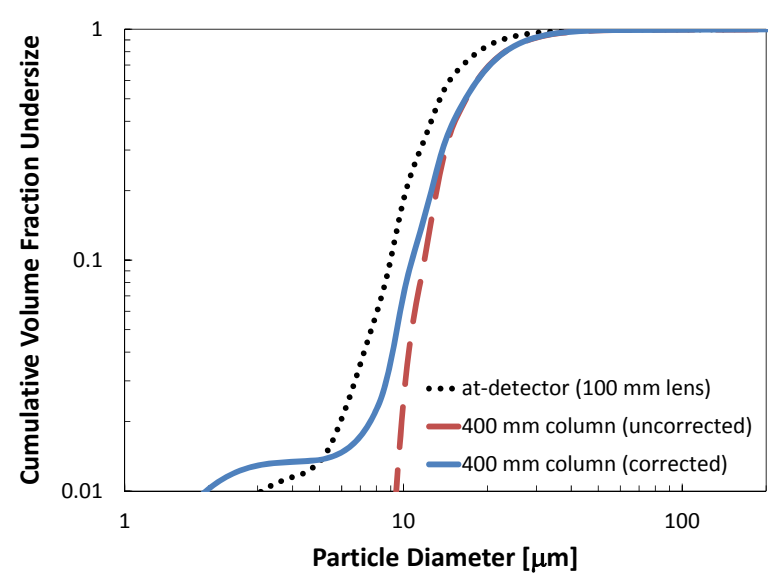

(a)

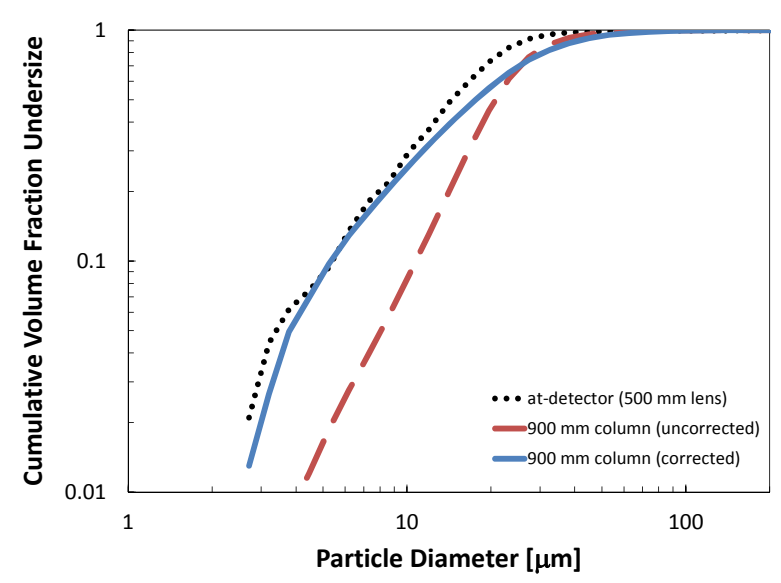

(b)

Figure 6.9. PSD Results for (a) the $400 \mathrm{~mm}$ Column Test and (b) the $900 \mathrm{~mm}$ Column Tests. PSD results are shown both when uncorrected (dashed red curve) and when corrected (solid blue curve) for laser attenuation. For reference, the PSD measurement from at-detector flow cell testing is included and is shown as the black dotted curve. Laser attenuation corrections should yield a result that agrees with the "at-detector" measurement.

Table 6.14. Comparison of Column Test Size Distributions to Baseline Size Distributions

\begin{tabular}{|c|c|c|c|c|}
\hline Test System & Measurement & $\begin{array}{l}\text { Measurement } \\
\mathrm{d}(10), \mu \mathrm{m}\end{array}$ & $\begin{array}{c}\text { Measurement } \\
\mathrm{d}(50), \mu \mathrm{m}\end{array}$ & $\begin{array}{c}\text { Measurement } \\
\mathrm{d}(90), \mu \mathrm{m}\end{array}$ \\
\hline \multirow{3}{*}{$\begin{array}{l}400 \mathrm{~mm} \text { column } \\
\text { ( } 3 \text { to } 30 \mu \mathrm{m} \text { powder) }\end{array}$} & Reference Measurement $^{(\mathrm{a})}$ & 8.9 & 13.5 & 22.7 \\
\hline & Corrected Column Measurement & 10.7 & 16.7 & 28.3 \\
\hline & Ratio (Column/At-Detector) & 1.20 & 1.23 & 1.25 \\
\hline \multirow{3}{*}{$\begin{array}{l}900 \mathrm{~mm} \text { column } \\
\text { ( } 3 \text { to } 30 \mu \mathrm{m} \text { powder) }\end{array}$} & Reference Measurement ${ }^{(b)}$ & 5.4 & 14.5 & 26.4 \\
\hline & Corrected Column Measurement & 5.3 & 17.7 & 41.5 \\
\hline & Ratio (Column/At-Detector) & 0.99 & 1.22 & 1.57 \\
\hline
\end{tabular}

(a) $100 \mathrm{~mm}$ lens measurement for 3 to $30 \mu \mathrm{m}$ powder (see Table 6.6).

(b) $500 \mathrm{~mm}$ lens measurement for 3 to $30 \mu \mathrm{m}$ powder (see Table 6.9 ).

The results presented in the preceding paragraphs for column testing indicate that the corrections generate an improved result relative to the uncorrected data and do not cause the anomalous overcorrections observed in the localized data. The latter observation is expected, as corrections to distributed data employ partially attenuated diffraction patterns rather than fully attenuated patterns that can occur for localized systems. While the corrections improve the data, they do not yield size and concentration measurements that are in full agreement with the "at-detector" measurements, which represent size and concentration measurements not impacted by attenuation (but still subject to the baseline uncertainties associated with the Malvern Insitec-S analyzer measuring system).

To understand how uncertainty in concentration and size measurements impact release fraction analysis, it is necessary to examine their combined effect on the concentration PSD (see Equation (6.7)), which is the starting point for release fraction analysis. The corrected concentration PSD for both the 400 and 900 mm column tests is shown in Figure 6.10 along with the uncorrected concentration PSD and "at-detector" measurements for reference. Correction of the concentration PSD should yield a 
concentration size distribution that matches the "at-detector" reading. The result of corrections to the $400 \mathrm{~mm}$ data produce a reasonable match between corrected and "at-detector" readings, with the main difference being that the corrected data appear about 20 percent larger relative to the "at-detector" reading. Correction of $900 \mathrm{~mm}$ data produces a concentration PSD that is in good agreement with the "at-detector" reading, with the only defect being a slight divergence near the bounds of the distribution. Overall, application of spray geometry (laser attenuation) corrections to dispersions that are distributed over long path lengths appears to produce a result that is in reasonable agreement to reference results measured in configurations in which corrections are not needed.

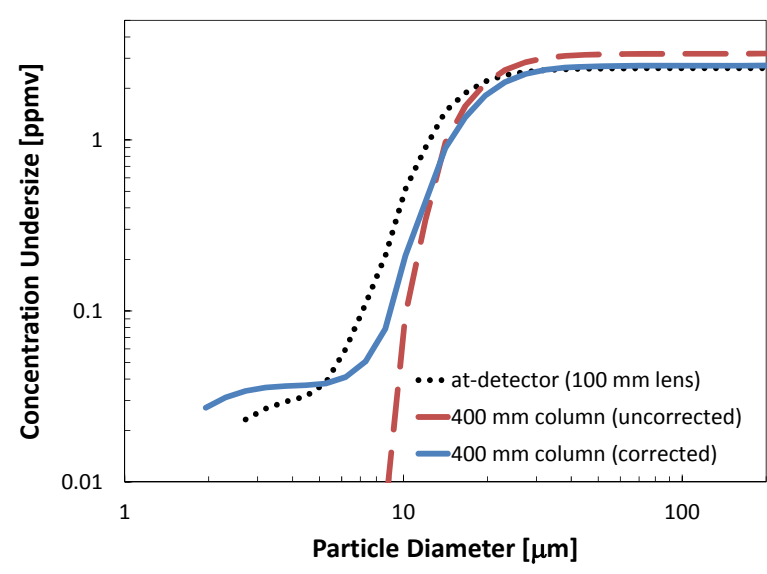

(a)

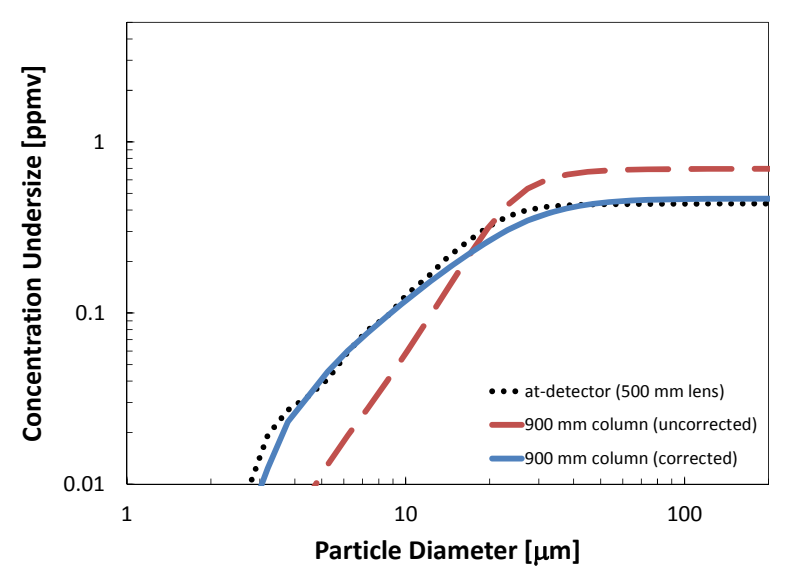

(b)

Figure 6.10. Concentration PSDs for (a) the $400 \mathrm{~mm}$ Column Test and (b) the $900 \mathrm{~mm}$ Column Tests. Concentration PSD results are shown both when uncorrected (dashed red curve) and when corrected (solid blue curve) for laser attenuation. For reference, the concentration PSD measurement from at-detector flow cell testing is included and is shown as the black dotted curve. Laser attenuation corrections should yield a result that agrees with the "at-detector" measurement.

\subsection{Conclusions and Recommendations}

A series of validation tests were performed to examine the accuracy of size and concentration measurements made by the Malvern Insitec-S aerosol analyzer and to assess the accuracy of corrections made by the Malvern Insitec-S measuring system software to account for loss of diffraction signal expected when measuring aerosol in the test configurations employed in Phase I and Phase II spray leak testing. Tests were conducted to isolate and evaluate:

- The accuracy of size and concentration measurements for the Malvern Insitec-S aerosol analyzer in a "standard" configuration (i.e., $100 \mathrm{~mm}$ lens, $150 \mathrm{~mm}$ spacer bars, and flow cell installed next to the detector to avoid distance effects) for ideal (spherical) particulate systems in the size range of interest for aerosol testing (i.e., 10 to $100 \mu \mathrm{m}$ )

- The impact of irregular particles on measurement accuracy

- Differences in the size and concentration result when measuring with a $500 \mathrm{~mm}$ lens (used in Phase II testing) relative to the $100 \mathrm{~mm}$ lens (used in Phase I testing) 
- The impact of laser attenuation from long optical path lengths or increased distance from detector on "localized" and "distributed" aerosol size distribution and concentration measurements

- The appropriateness of corrections made to compensate for laser attenuation.

From these tests, general guidelines and limitations for the accuracy of Malvern Insitec-S aerosol measurements can be postulated. These are:

- Accuracy for Spherical Particulate Systems in the "Standard" Malvern Insitec-S Configuration. The Malvern Insitec-S aerosol analyzer, like many laser diffraction analyzers, treats all particulate systems as spherical when calculating a size distribution and concentration result from measured diffraction patterns. Validation test results indicate that, on average, the Malvern Insitec-S analyzer measures the concentration of spherical systems to within 2 percent of the actual value. Statistical analysis of the concentration results suggest that within a 95 percent confidence level, 95 percent of all concentration measurements for spherical systems will fall within a range of -13 percent to +17 percent of the actual value. Baseline testing also found that the concentration response of the Malvern Insitec-S measuring system is highly linear. Step-wise additions of concentration yield proportional increases in the concentration measured by the Malvern Insitec-S analyzer. With respect to measurement of size distribution, on average the Malvern Insitec-S can measure the expected $\mathrm{d}(50)$ to within 3 percent. Less accuracy is observed toward the tails of the distributions as reflected by increased uncertainty in the $\mathrm{d}(10)$ ( 6 percent low on average) and $\mathrm{d}(90)$ (18 percent high on average). Loss of accuracy in the tails of the distribution may result from difficulty in dispersing the sample or from increased broadness in the laser diffraction PSD because of inversion method assumptions. The latter assertion is supported by the fact that differences in Malvern Insitec-S and reference PSD measurements are greatest when the reference distribution derives from methods other than laser diffraction (such as optical microscopy).

Impact of Irregular Particle Shape. Because the Malvern Insitec-S assumes spherical particles when interpreting the diffraction pattern, increased concentration and size distribution uncertainty is expected when working with irregular particles. Analysis of two powder systems finds that concentration inaccuracy can result from particle shape. In particular, for boehmite powder, step-wise addition of boehmite powder to the test dispersion produced a proportional (linear) response in concentration measured by the Malvern Insitec-S analyzer. However, all concentration measurements were approximately a factor of two too large relative to actual concentrations determined by mass-balance. The difference results in part from application of a spherical particle assumption to non-spherical particles and in part from inaccuracy associated with the size measurement. Evaluation of size accuracy for irregular particles could only be performed on a comparative basis, as the reference PSDs for both irregular powders (boehmite and gibbsite) were measured on laser diffraction systems. The Malvern Insitec-S PSD measurement uniformly overestimates the size of the boehmite powder; this is not unexpected, as the boehmite powder contains a fraction of fine particles (below $1 \mu \mathrm{m}$ ) that cannot be accurately observed by the Malvern Insitec-S instrument as configured for testing. The Malvern Insitec-S over-estimated the broadness of the gibbsite distribution relative to its reference measurement, but was able to measure the $\mathrm{d}(50)$ to within less than 1 percent of the reference value. Overall, particle shape does appear to impact the accuracy of the size result. For current spray leak application, the potential impact of this uncertainty on the result of water is minimal, as aerosols produced by water sprays are generally expected to be spherical for the aerosol sizes observed in in-chamber testing. Likewise, aerosolization of slurries 
may produce irregular particles, but only at aerosol sizes approaching the primary particle size (which is below $50 \mu \mathrm{m}$ for the kaolin-bentonite clay mixture tested).

- Impact of Lens Selection. The working range of the $100 \mathrm{~mm}$ lens (i.e., 0.5 to $200 \mu \mathrm{m}$ ) is well suited for in-chamber measurements in the range of size interest to spray release (10 to $100 \mu \mathrm{m})$. For Phase II testing, a $500 \mathrm{~mm}$ lens with a 2.5 to $2500 \mu \mathrm{m}$ working range was used for both in-spray and in-chamber measurements. It was therefore necessary to evaluate impacts of lens selection on concentration and size analysis in the range of interest (i.e., 10 to $100 \mu \mathrm{m}$ ). Baseline Malvern Insitec-S testing employed a $100 \mathrm{~mm}$ lens; as such, lens testing used a $500 \mathrm{~mm}$ lens and compared the size results to those obtained using the $100 \mathrm{~mm}$ lens. Two systems were tested: 1) a 3 to $30 \mu \mathrm{m}$ poly-disperse glass bead powder and 2) a larger $(\sim 70 \mu \mathrm{m})$ XLSciTech glass bead. The 3 to $30 \mu \mathrm{m}$ powder was found to challenge the measuring ability of the $500 \mathrm{~mm}$ lens. Specifically, when configured with the $500 \mathrm{~mm}$ lens, the concentration measured by the Malvern Insitec-S analyzer for the 3 to $30 \mu \mathrm{m}$ was approximately 25 percent low, and the size result was broader than expected. In contrast, $500 \mathrm{~mm}$ lens tests with the XLSciTech beads did not have similar difficulties and compared well to their $100 \mathrm{~mm}$ lens counterparts. From this observation, we postulate that the difficulties encountered when measuring the 3 to $30 \mu \mathrm{m}$ powder with the $500 \mathrm{~mm}$ lens derive from the proximity of the $\mathrm{d}(50)$, which is $\sim 14 \mu \mathrm{m}$, to the lower measuring range $(2.5 \mu \mathrm{m})$ of the lens. With respect to Phase II testing, no significant impact on the accuracy of either in-spray or in-chamber aerosol measurements is expected because none of the aerosols exhibited size distributions that fell primarily within one order of magnitude of lower measuring range of the $500 \mathrm{~mm}$ lens. Indeed, aerosols measured exhibited broad distributions, with the majority of particles falling between 20 and $100 \mu \mathrm{m}$. Based on available large-scale data, it appears that the only consequence of $500 \mathrm{~mm}$ lens use may be loss of accuracy at sizes below $20 \mu \mathrm{m}$. It should be noted that for many test cases, this coincides with detection limits associated with resolving the fraction contribution aerosols with diameters that fall below cumulative concentrations of $0.5 \times 10^{-2}$ (see Section 5.3.2.2 in Schonewill et al. (2012)). These statements should be approached with caution, as the presence of fine ( $1 \mu \mathrm{m}$ and below) aerosol can increase the apparent concentration measured by the Malvern Insitec-S system (Schonewill et al. 2013). It is therefore difficult to fully rationalize the lower-than-actual concentration observed in measurements of the 3 to $30 \mu \mathrm{m}$ system with respect to the expected behavior of fine solids. While this discrepancy must be recognized, the $500 \mathrm{~mm}$ lens test results do not provide strong arguments for correcting all $500 \mathrm{~mm}$ lens data based on the single observation of performance in the challenging 3 to $30 \mu \mathrm{m}$ system.

- Impact of Distance of Scattering Center from Detection Unit. As the distance between the particles being imaged and the detection unit where scattered light is collected and analyzed increases, the portion of the diffraction pattern that reaches the detector decreases. Specifically, the diffraction pattern associated with large-angle scattering is attenuated, yielding an apparent decrease in the volume fractions of fines in the size distribution produced by the analyzer. The overall effect is to yield an apparent increase in the average particle size of the dispersion and an increase in the total dispersion concentration measured. Malvern Insitec-S position testing has verified that this effect can be observed for particles present at distances greater than $150 \mathrm{~mm}$ from the detection unit. Localized dispersions at test distances of $1000 \mathrm{~mm}$ showed complete attenuation (i.e., loss of measurement signal) for particles smaller than $20 \mu \mathrm{m}$, leading to inaccurate/ incomplete assessment of PSDs in systems that contain fractions $<20 \mu \mathrm{m}$. Attempts to correct for distance from detector in localized dispersions using the Malvern Insitec-S software were not successful and, for the case of the $500 \mathrm{~mm}$ 
position test, led to a PSD that grossly over-estimated the contribution of particles below $20 \mu \mathrm{m}$, which in turn result in concentrations that were at least a factor of 10 low. This latter result is not surprising, as correction of localized dispersions is expected to be difficult because of either complete attenuation of diffraction signal on certain detector channels or amplification of noise on channels where attenuation reduces signal to near noise levels. The poor performance observed in the correct algorithm observed in localized dispersions does not carry over to corrections for dispersions distributed over long optical path lengths, like those tested in Phase I and Phase II aerosol tests, where only partial attenuation of the signal occurs. On the other hand, position testing of localized dispersions does indicate that concentration and size results occurring in configurations with optical path lengths shorter than $150 \mathrm{~mm}$ (such as both Phase I and Phase II small-scale aerosol configuration) are not significantly impacted by laser attenuation.

- Impact of Corrections to Distributed Dispersions. To assess the accuracy of spray geometry (laser attenuation) corrections for distributed aerosols, tests that simulated the Phase I and II distributed aerosol configurations were conducted. In these tests, distributed dispersions were presented to the Malvern Insitec-S measuring volume by flowing them through a column with optical glass at both ends. Two "column" tests were conducted: one using a $400 \mathrm{~mm}$ column and $100 \mathrm{~mm}$ detection lens (to simulate the Phase I large-scale aerosol configuration) and another using a $900 \mathrm{~mm}$ column and $500 \mathrm{~mm}$ detection lens (to simulate the Phase II large-scale aerosol configuration). Both tests were performed using the 3 to $30 \mu \mathrm{m}$ powder, which has a primary size distribution that falls below $20 \mu \mathrm{m}$ and which is expected to be challenged by laser attenuation as a result. The results of column testing indicate that for distributed dispersions, where distance affects only results in partial attenuation of the diffraction pattern, the corrections to the diffraction pattern made by the Malvern Insitec-S software are able to compensate for attenuation without yielding the size distribution and concentration anomalies observed in localized testing. Uncorrected concentration and size distribution measurements from distribution dispersion measurements show the expected impact of partial attenuation of the diffraction pattern, namely the apparent loss of volume contribution from the fines fraction of the size distribution and an over-estimation of total aerosol concentration. Software correction of the distributed dispersion concentration result generally produces a concentration measurement that is slightly (about 5 percent) higher than expected from measurements not needing correction. Likewise, correction of the distributed measurement data yield a size distribution that is comparable to, but about 20 percent larger, than that when measured in configurations where laser attenuation does not occur. The overall impact of these observations on Phase I and Phase II release fraction data is difficult to assess but is likely small. Because aerosols generated by spray typically have a broad size distribution (relative to the 3 to $30 \mu \mathrm{m}$ powder tested in the current study), the decrease in release fraction resulting from a 2 percent increase in size will generally be offset by the 5 percent increase in apparent concentration. On this basis, no systematic correction for column test biases is recommended.

The results of Malvern Insitec-S validation testing outlined above inform on the overall instrument accuracy with respect to measurement of concentration and size distribution for the instrument configurations tested in Phase I and Phase II spray release programs. Based on the validation test results, the following recommendations for analysis of Malvern Insitec-S aerosol data are given:

- No correction for bias in the overall Malvern Insitec-S aerosol concentration is needed for spray release data. The only correction considered was to account for irregular particle shape for dried particulates resulting from aerosolization of clays (or particles completely striped of water by 
high-pressure spray release). This was deemed unnecessary because the chamber humidity was generally elevated (i.e., greater than 80 percent relative humidity) to minimize the effects of evaporation, and furthermore, the irregular shape affected an increase in the observed concentration (making any release fraction result derived from this concentration larger and therefore more conservative). For the majority of test cases and aerosol size ranges, the aerosol particles generated in spray release testing are expected to be spherical. When the $500 \mathrm{~mm}$ lens is used, loss of concentration accuracy may occur for dispersion systems with the bulk of their sizes falling within one order-of-magnitude of the lower working range of the lens of $2.5 \mu \mathrm{m}$. This is not expected to be a problem for spray release testing, as Phase II aerosol test configurations generally yield a majority of aerosol particles in sizes above $20 \mu \mathrm{m}$.

- No correction for bias in PSD is needed. Loss of accuracy in the size measurement may result from several factors that include 1) the general broadening of the PSD as a result of light scattering inversion techniques and their assumptions used by laser diffraction, 2) irregular particle shapes, and 3) particulate systems that challenge the size working limit of the lens. As with inaccuracies impacting concentration measurements, these biases are typically system specific and do not directly apply to aerosols generated in spray testing.

- The treatment of aerosol data in Phases I and II data analyses, which applies the Malvern Insitec-S software correction for spray geometry effects (laser attenuation), is appropriate. No change to the way in which the Malvern Insitec-S size and concentration data are handled is necessary.

- No correction bias introduced by correction for spray geometry (laser attenuation) is recommended at this time. The corrected total dispersion concentration slightly over-estimates the actual concentration (and is thus conservative). The corrected size distribution is generally about 20 percent too large. This bias is not expected to significantly impact concentration results for spray leak analyses given the broadness of typical aerosol size distributions produced by sprays in large-scale testing.

The Malvern Insitec-S validation testing described above was performed to address concerns related to aerosol analyzer performance in Phase I testing. For this reason, the analyzer configurations and concentrations tested strongly reflect those associated with in-chamber testing, which represented the bulk of Phase I large-scale chamber testing. In anticipation of similar concerns, Phase II in-chamber configurations were also evaluated in validation testing. Configurations and dispersion concentrations relevant to in-spray testing were not tested as part of Malvern Insitec-S validation testing. With the increased importance of in-spray testing in Phase II analysis, additional follow-on validation work may be necessary to address similar concerns with instrument performance and accuracy with respect to in-spray measurements. Specific issues that may need to be addressed include:

- Performance of analyzer at high laser obscurations

- Accuracy of corrections of multiple light scattering at high dispersion / aerosol concentrations

- Accuracy of spray geometry corrections for spray geometries relevant to in-spray testing and that simulate the core of the spray. 



\subsection{Impact of Aerosol Instrument Position on Measured Release Fractions}

This chapter discusses tests undertaken to evaluate the impact of aerosol analyzer location on both in-chamber and in-spray release fraction and generation rate measurements. The studies were motivated by the need to better understand accuracy and bias in in-chamber generation rate measurements and how the in-spray size distribution of droplets formed by jet break-up evolves downstream from the orifice. In-spray and in-chamber position tests provide a basis for estimating uncertainty in Phase II large-scale test results that derive from position effects.

The method for determining in-chamber release fractions discussed in Section 1.5 of this report makes several assumptions with respect to uniformity of aerosol concentration in the test chamber. The most significant and restricting of these assumptions is the requirement for instantaneous mixing, such that the chamber is uniformly mixed at all times. Furthermore, the analysis methodology for determining in-chamber generation rate and release fraction requires knowledge of the average chamber volume concentration. The aerosol analyzers employed in most test configurations measure aerosol concentration at three fixed locations above the spray and sample with a total volume of $\sim 220 \mathrm{~cm}^{3}$ ( $\left.\sim 0.057 \mathrm{gal}\right)$. Even relative to the minimum chamber volume of $\sim 3600$ gal, the sampling volume represents less than 0.002 percent of the region occupied by aerosol. For this reason, there is significant cause for concern that the average aerosol concentration may not be representative of the actual bulk aerosol concentration.

There are several observations that inform against the assumption of instantaneous mixing and overall chamber uniformity. First, aerosol concentration does vary with sampling location. The most dramatic difference is observed when comparing in-chamber concentrations (where the aerosol analyzers are located above the spray and typically measure on the order of $1 \mathrm{ppmv}$ aerosol) to in-spray concentrations (where the aerosol analyzers measure the concentration in the direct path of the spray and can measure up to $\sim 100 \mathrm{ppmv}$ aerosol). Second, aerosol concentration rise curves at the start of spray exhibit finite mixing times, such that there is a delay (on the order of 1 to $10 \mathrm{~s}$ ) between the start of spray and the first detection of aerosol. This delay is most severe for low pressure sprays through small orifices, such as 100 psig water sprays generated through the $2 \mathrm{~mm}$ round-hole orifice. Figure 9.1 in Schonewill et al. (2012) indicates a $\sim 5 \mathrm{~s}$ delay for a $1 \mathrm{~mm}$ hole, $380 \mathrm{psig}$ spray in the $20 \mathrm{ft}$ large-scale test chamber. This delay is position dependent, and is generally worse for aerosol analyzers located farther away from the direct path of the spray or for analyzers located upstream of the spray.

In-spray measurements provide a direct measurement of aerosol size distribution by placing the aerosol analyzers in the direct downstream path of the spray. For these measurements, aerosol analyzers were placed in the most optically dense region of the spray. Because in-spray measurements evaluate spray as it exits the orifice, they are not subject to assumptions about the rate and overall degree of aerosol mixing in the large-scale chamber. In this regard, in-spray measurements at first appear to be the better choice for gaging the aerosol release fractions and generation rates; however, the usefulness of in-spray release fraction data is limited by several factors. The first of these limitations is the potential inclusion of a partially aerosolized liquid core. Non-spherical jet fragments or droplets larger than the upper limit of size analysis $(\sim 2500 \mu \mathrm{m})$ can confound size analysis by laser diffraction and produce anomalous, inaccurate results. For this reason, in-spray measurements must consider jet break-up distances when selecting aerosol sampling locations and avoid regions where liquid cores may potentially 
cause difficulties with size analysis. Even when precautionary measures are taken, difficulties with in-spray analysis may be encountered as a result of liquid core inclusion (Schonewill et al. 2013).

A second, and more significant, limitation derives from the fact that in-spray measurements will generally include most (if not all) of the droplets generated by spray. Phase I test results suggest that the vast majority of spray does not aerosolize or is contained in large droplets that cannot be dispersed inside the enclosure by turbulence and that will quickly settle to the floor or be deposited on downstream surfaces (Schonewill et al. 2012). Inclusion of these droplets "blinds" the aerosol analyzer and prevents it from measuring droplets in the size range of interest (i.e., 10 to $100 \mu \mathrm{m}$ ). Loss of sensitivity impacts any aerosols that make up less than $0.5 \times 10^{-2}$ fractional aerosol volume (Schonewill et al. 2012). Because of this effect, in-spray measurements of aerosols are typically limited to a lower working range of $50 \mu \mathrm{m}$ (at best), below which aerosols cannot be accurately assessed. In contrast, in-chamber measurements can routinely observe aerosols down to $10 \mu \mathrm{m}$ (or lower).

Other factors that impact the quality of in-spray release fractions include:

1. The evolution of the size distribution of droplets in the jet as the spray moves downstream as a result of droplet coalescence (Schonewill et al. 2012) and the ambiguity with respect to which downstream location is "best" for measuring the "true" in-spray droplet size distribution

2. High (or near complete) obscuration of the laser and/or multiple light scattering

3. Spatial (as opposed to flux-based) measurement of the size distribution (Bartell et al. 1991), which causes bias in size distribution measurements of droplets with a distribution of velocity

4. The identification of the most optically dense region of the spray becomes more difficult for large sprays and as sprays fan out farther downstream from the orifice and can be ambiguous for sprays that bifurcate (i.e., show two or more optically dense regions).

The first issue deals exclusively with determining if a "best" downstream in-spray measurement location exists and its location. With respect to the second issue, in-spray measurements evaluate regions where aerosols are concentrated and can yield multiple scattering phenomena that increase the complexity of laser diffraction analysis. This concern has been addressed by applying multiple scattering corrections available in the control software for the aerosol analyzers. The third issue regards the manner in which size distribution is measured. The aerosol analyzers used in Phase II testing make spatial measurements of aerosol that analyze all aerosols within a fixed control volume. Aerosols that tend to linger in the measurement will be "counted" more frequently than those that pass rapidly through the sampling volume, resulting in a size distribution bias that favors the slower-moving aerosols. This bias cannot be directly assessed without knowledge of the droplet velocity distribution as a function of size. However, the impact of size bias can be inferred by assuming that the smallest droplets will move with the carrier fluid while large droplets will retain inertia and move more quickly than the carrier fluid. Under this assumption, it follows that spatial measurement bias yields an increase in the contribution of fine aerosols. In terms of release fraction analysis, this bias will increase conservatism in the release fraction range of interest (10 to $100 \mu \mathrm{m})$.

Of the sources for in-spray measurement uncertainty identified above, all but uncertainty due to the Malvern Insitec-S position can be mitigated or accepted through consideration of spray physics and/or computational correction. In this respect, in-spray measurements share uncertainty that derives from measurement location also found in in-chamber measurements, although the physical mechanism 
responsible for this uncertainty is different in both. Specifically, in-chamber measurement position uncertainty derives from concerns involving the rate and extent of mixing and the representativeness of aerosol sampling, both of which could bias generation rate measurements. In contrast, in-spray measurement position uncertainty derives from expected changes in the droplet size distribution as the spray travels downstream from the point of release.

To evaluate the consequence of these uncertainties, PNNL performed a series of functional tests that evaluated the impact of measurement position on in-chamber aerosol size, concentration, and release fraction results and on in-spray release fraction results. Because the goal in this set of functional tests was to evaluate how aerosol measurements change with instrument position, the test and data analysis methodologies differ slightly from those used elsewhere in this report. The test methodology used to conduct these functional tests and the results obtained are discussed in detail in this chapter. In-chamber and in-spray position testing are discussed separately. Comparisons of in-spray and in-chamber data also are given to highlight differences in how each method interprets fractional release of aerosol. These comparisons allow assessment of release fraction uncertainty in both in-spray and in-chamber test measurements and form the basis for guidelines (i.e., measurement filtering rules) used when reporting release fraction and generation rate measurements in later sections of this report.

\subsection{Presentation of Release Fraction Data}

As discussed in Chapter 5 of this report, the Malvern Insitec-S aerosol analyzers used to determine both in-spray and in-chamber release fractions do not measure aerosol size and concentration directly, but instead determine them from mathematical treatment (based on first-principles) of light scattering patterns. The fractional volume contribution component of size distribution reported by the Malvern Insitec-S software spans several orders of magnitude. Depending on the time-averaging period over which the size distribution is measured, fractional contributions as low as $10^{-30}$ can be reported by the instrument. Phase I Malvern Insitec-S validation testing indicated that fractional contributions reported by the Malvern Insitec-S software are only reproducible at cumulative volume fraction undersize of $\sim 0.5 \times 10^{-2}$ (i.e., the $0.5^{\text {th }}$ percentile) or greater. As such, attempts to measure aerosols at sizes below 0.5 percent of the total aerosol volume are subject to significant uncertainty. For this reason, release fraction data presented in this report do not typically report size and aerosol concentration data below $\sim 0.5 \times 10^{-2}$. When presenting release fraction data in this section, in-spray measurements (which are strictly size measurements) are not reported when the cumulative release fractions are less than $0.5 \times 10^{-2}$. Because in-chamber release fractions derive from size distribution measurements, they also are subject to similar reproducibility limits. Treatment of uncertainty in in-chamber release fractions can be simplified by recalling that release fractions can be expressed as the product of the initial size distribution of aerosol with the fraction of spray aerosolized (see Equation (5.13)). This means that impacts to size measurement reproducibility can be accounted for by not reporting in-chamber release fractions below the product of $0.5 \times 10^{-2}$ and the total fraction of spray aerosolized. The latter is approximated by using the maximum cumulative release fraction measured for the test under consideration. The guidelines for data established above are applied to presentation of data in this section.

\subsection{In-Chamber Aerosol Instrument Position Dependence}

In-chamber aerosol instrument position testing was performed with the primary goal of assessing how non-uniform concentrations affect release fraction results and the uncertainty in those release fraction 
results. This was accomplished by evaluating aerosol concentration, size distribution, and release fraction uniformity as a function of test position. To this end, a specialized matrix of six release fraction functional tests, collectively referred to as "chamber uniformity tests," were conducted in the $39 \mathrm{ft}$ chamber. For each of the six tests, the three Malvern Insitec-S analyzers were placed at unique measurement locations within the test chamber. Figure 7.1 provides a side profile of the test chamber and depicts the Malvern Insitec-S measuring locations, which include three tests heights (28, 36, and 54 in., as measured from the top of the chamber to the lowest point on the Malvern Insitec-S analyzer) and six downstream positions $(10,15,20,25,30$, and $35 \mathrm{ft}$ from the chamber wall adjacent to the spray header). All chamber homogeneity tests were conducted with water, using the $2 \mathrm{~mm}$ circular orifice and $380 \mathrm{psig}$ fluid pressure. ${ }^{1}$ The test matrix yielded a set of measurements that define the aerosol concentration and size distribution profiles as a function of time for 18 different measurement locations.

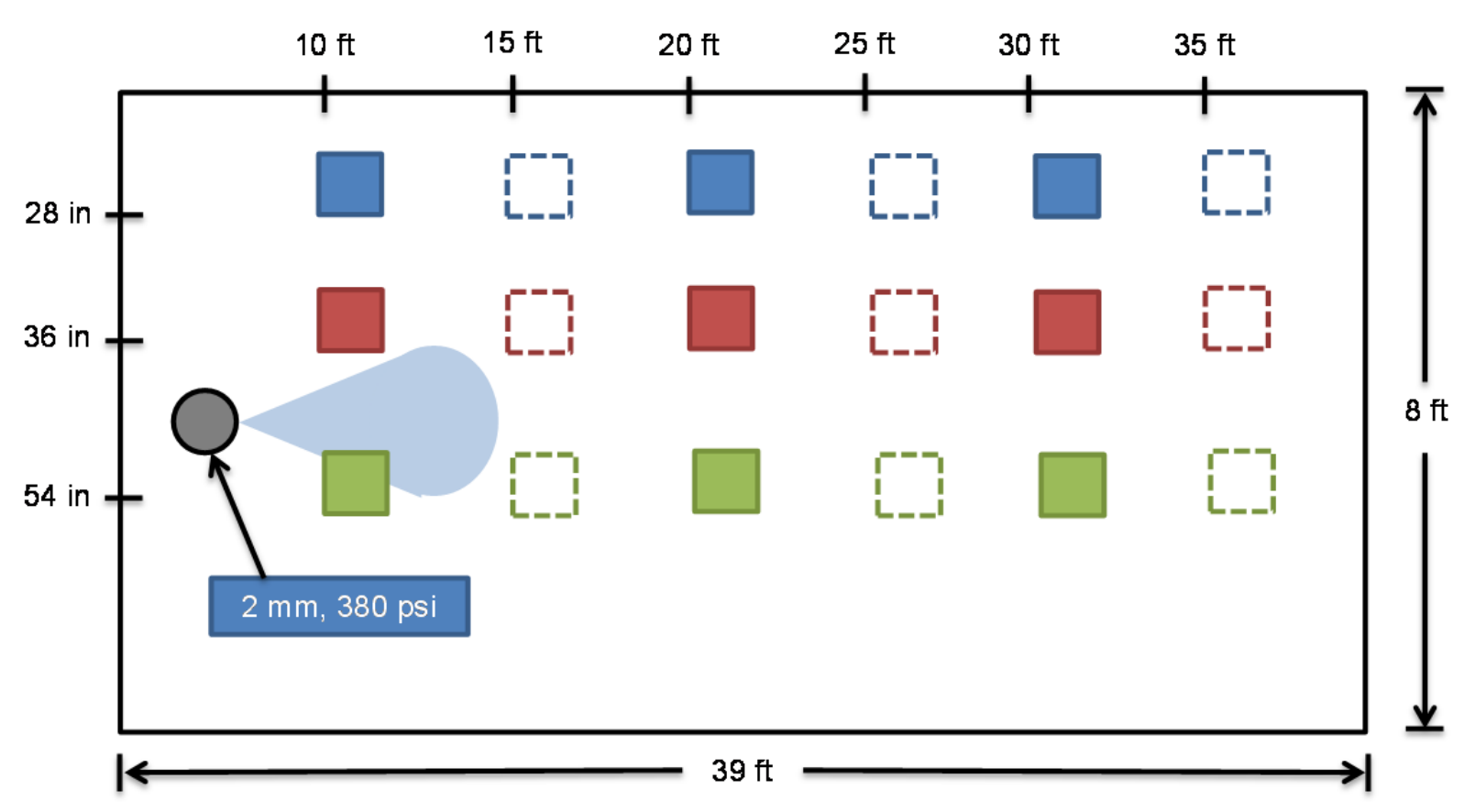

Figure 7.1. Side Profile of the $39 \mathrm{ft}$ Test Chamber Illustrating Vertical and Downstream Measuring Positions Assessed During In-Chamber Position Tests

By varying the height of the measuring position, the position of the Malvern Insitec-S measuring zone was changed relative to the spray. The exact location of the aerosol instruments relative to the spray depends on the path the spray takes; for the current studies, the path of the spray was not mapped. To provide a more meaningful measurement of position relative to the spray, the heights listed in Figure 7.1 are recast in terms of the height differential between the Malvern Insitec-S sampling volume (i.e., the laser centerline) and the orifice. In these terms, the 28, 36, and 54 in. measuring positions correspond to

\footnotetext{
${ }^{1}$ The $2 \mathrm{~mm}$ hole generates the lowest spray volumes of all Phase II large-scale orifices, and when coupled with the $39 \mathrm{ft}$ chamber test chamber (the largest tested), should present a "worst-case" configuration with respect to mixing and chamber homogeneity. The test pressure selected (380 psig) is not optimal for assessing a "worst-case" heterogeneity in that it provides more convective energy than $100 \mathrm{psig}$. However, $100 \mathrm{psig}$ sprays challenge the concentration detection limits of the aerosol analyzers. For this reason, the highest test pressure is selected for uniformity testing to improve resolution of trends in the concentration, size distribution, and generation rate data.
} 
21 in. above, 13 in. above, and 5 in. below the orifice, respectively. Selection of the lowest test location was made to facilitate aerosol below the spray (as was done in Phase I testing). Reconfiguration of the aerosol analyzers to accommodate $1 \mathrm{~m}$ spacer bars prevented testing at locations more than $5 \mathrm{in}$. below the spray. This test height is sufficiently near the orifice that it could be considered an in-spray measurement (assuming minimal impact to the spray trajectory from gravity). For the purpose of this and later sections, the $54 \mathrm{in}$. test position is referred to as a near-spray measurement to indicate its proximity to the orifice and to differentiate it from in-spray measurements, for which the measuring volume was placed at true centerline path of the spray. In practice, the trajectory of all sprays tested is influenced by gravity (see Section 7.3). For this reason, near-spray measurements are further differentiated from in-spray measurements in that measuring height of the analyzer does not change with downstream distance. Thus, even though the near-spray measuring position is $5 \mathrm{in}$. below the orifice, the measuring location will encompass positions below the spray (near the orifice) and above the spray (at downstream locations).

The impact of test location on fractional aerosolization measurements was determined by evaluating the position dependence of three parameters: 1) the cumulative concentration at select aerosol diameters $(10,32$, and $102 \mu \mathrm{m}), 2)$ the size distribution of the aerosol, and 3$)$ the generation rate of aerosol. The in-chamber test method and analysis used for these studies is unique in that it examines aerosol parameters on an instrument-by-instrument basis, rather than using the average chamber concentration. Because single instrument measurements are used for analysis rather than an average of up to three typically used for in-chamber testing, the results derived from these position tests are subject to greater uncertainty. To minimize some of this uncertainty, concentration and aerosol size data presented in this section are time-averaged. The averaging period includes data collected between 8 to $12 \mathrm{~s}$ after start of spray (with a median time of $10 \mathrm{~s}$ ) and was selected to be consistent with the time averaging period for in-spray and to capture the aerosol behavior before significant buildup of aerosol in the chamber.

Figure 7.2 shows the aerosol size distribution as a function of position. Each sub-figure shows data from two test configurations: one with aerosol analyzers at 10,20, and $30 \mathrm{ft}$, and another with analyzers at 15,25 , and $35 \mathrm{ft}$. The horizontal position dependence is shown for each of the three test heights (21 and 13 in. above the orifice and $5 \mathrm{in}$. below the orifice). Aerosol size distributions $21 \mathrm{in}$. above the orifice (Figure 7.2a) generally indicate that PSD becomes slightly more coarse downstream of the spray. Likewise, aerosol size distributions 13 in. above the orifice (Figure 7.2b) also show this trend, although the overall change in aerosol size from 10 to $35 \mathrm{ft}$ is smaller at $13 \mathrm{in}$. above the orifice than that observed at $21 \mathrm{in}$. above the orifice. As such, both sets of in-chamber results for which the analyzers are positioned above the orifice indicate that larger aerosols are more prevalent near the splash wall than at measurement locations near the spray header, or conversely, that fine aerosols are more prevalent at the spray header. This spray behavior can be attributed to several causes:

- A decrease in droplet size with increasing radial distance from the center of the jet, such that the finest droplets are located near the periphery of the jet

- Preferential back-transport of fine aerosol and removal of larger droplets in the back-transport current by settling. Here, back-transport of aerosol results from circulation currents and counter-currents set up by the spray as it passes through the center of the chamber and entrains air during break-up 


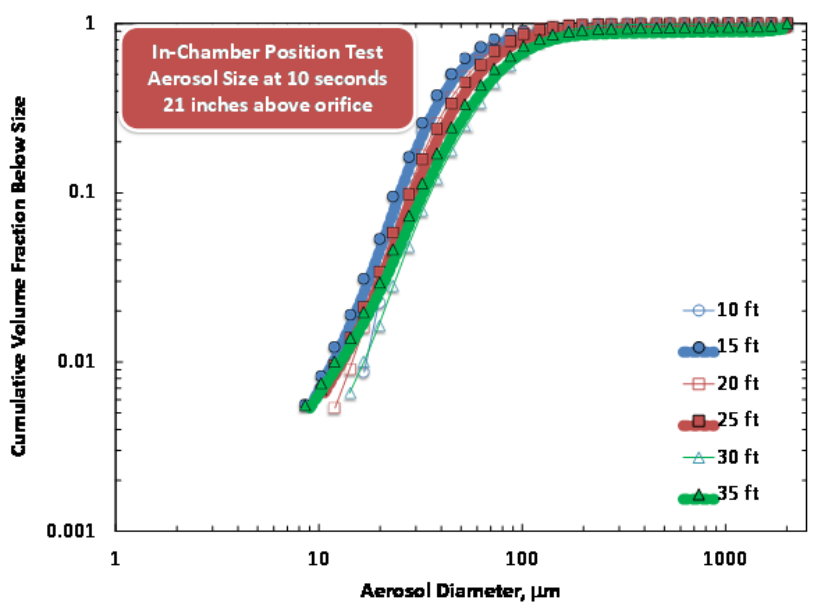

(a) - In-chamber aerosol size distribution, 21 in. above the orifice

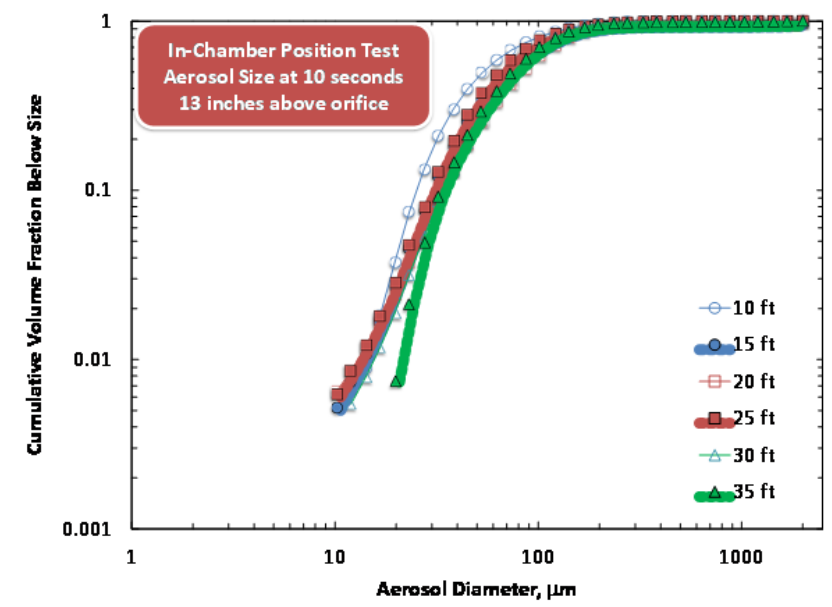

(b) - In-chamber aerosol size distribution, 13 in. above the orifice

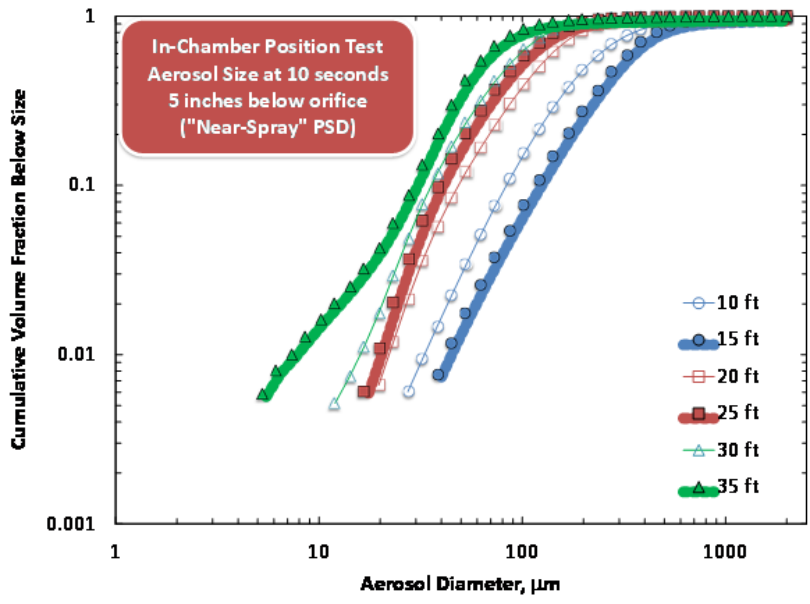

(c) - In-chamber (near-spray) aerosol size distribution, 5 in. below the orifice

Figure 7.2. Cumulative Size Distribution of Aerosol Droplets as a Function of Downstream (horizontal) Distance from the Spray Header at (a) 21 in. Above the Orifice, (b) 13 in. Above the Orifice, and (c) 5 in. Below the Orifice. PSDs are time-averages. Each sub-figure shows data from two test configurations: one with aerosol analyzers at 10,20, and $30 \mathrm{ft}$, and another with analyzers at 15,25 , and $35 \mathrm{ft}$. 
- Coalescence of droplets as they travel down the chamber and subsequent inertial capture (which prevents or limits back-transport, as described in the previous bullet)

- Increased sampling of the core of the spray as it fans out and engulfs analyzers in downstream positions.

Each of these mechanisms relates directly to how the spray changes at downstream measurement locations, and as such, also is given consideration in later chapters of this report (see Chapters 8 and 9). The measured size distributions at different chamber locations are not sufficient to eliminate all potential causes for the increased relative prevalence of large particles with increasing downstream distance. Overall, the change in aerosol size distribution at test locations above the orifice is relatively small. Direct comparison of the 10 and $35 \mathrm{ft}$ locations at $21 \mathrm{in}$. above the orifice generally finds only a $\sim 100$ percent increase in the median [i.e., $\mathrm{d}(50)$ ] aerosol diameter. Relative to changes observed in the near-spray data (discussed below), this overall change is not substantial.

As shown in Figure 7.2c, the size distributions collected 5 in. below the orifice show significant variation with increasing downstream distance from the orifice. From 10 to $15 \mathrm{ft}$, an increase in aerosol size is observed. Beyond $15 \mathrm{ft}$, the aerosol size distribution becomes progressively finer. The decrease in total aerosol size between 15 and $35 \mathrm{ft}$ is significant, with the median (i.e., $50^{\text {th }}$ ) size percentile dropping from $\sim 300$ to $60 \mu \mathrm{m}$ (a factor of five decrease). As such, the size distribution appears to strongly depend on position for near-spray measurements. There appears to be a two-step behavior with increasing distance from the orifice in near-spray measurements. Immediately near the orifice, the aerosol size increases with increasing distance. The suspected cause for this increase is coalescence of fine droplets produced by high-pressure spray. This behavior is consistent with Phase I in-spray observations at downstream distances of 2 to $5 \mathrm{ft}$ that showed an increase in droplet size with increasing distance (Schonewill et al. 2012). After passing through a maximum, the aerosol size progressively decreases with increasing distance from orifice. The most plausible cause for this increase is gravitational settling of large droplets. Indeed, evaluation of the in-spray trajectories (see Section 7.3) indicates the spray passes through the near-spray measuring height ( $5 \mathrm{in}$. below the orifice) somewhere between 10 and $15 \mathrm{ft}$ from the orifice. It seems reasonable that near-spray measurements at 10 and $15 \mathrm{ft}$ would be representative of in-spray measurements as both are within several inches of the spray centerline. Furthermore, based on trajectory information, the near-spray measurement location at $20 \mathrm{ft}$ could be as much as $1 \mathrm{ft}$ above the spray centerline (making it more representative of an in-chamber measurement).

The near-spray size distribution trends, coupled with the observations made with respect to spray trajectory, inform on several potential impacts to the current analysis. First, the near-spray measurements at 10 and $15 \mathrm{ft}$ fall within the spray and are not suitable for in-chamber analysis (although an attempt will be made to analyze their generation rates using in-chamber methods for completeness). Next, all chamber uniformity tests considered valid for in-chamber and uniformity analyses fall above the spray. This prevents making any conclusions with respect to uniformity below the spray or with respect to how these positions compare to above spray positions.

Figure 7.3 shows the variation in cumulative concentration undersize (for 10, 32, and $102 \mu \mathrm{m}$ droplets) as a function of test position. Similar aerosol concentration trends are observed at each of the three aerosol sizes evaluated. Beyond $20 \mathrm{ft}$, there does not appear to be a statistically significant change in aerosol concentration with position, regardless of test height. This observation is consistent with observations made with respect to aerosol size, in that all test locations (including the lowest) transition to 
in-chamber measurement beyond $15 \mathrm{ft}$ for the $2 \mathrm{~mm}$ hole, $380 \mathrm{psig}$ spray. As expected, measurements at test locations that are functionally in-spray (specifically, those $5 \mathrm{in}$. below and 10 and $15 \mathrm{ft}$ downstream from the orifice), indicate concentrations significantly higher than those taken at 13 and $21 \mathrm{in}$. above the spray.

It should be noted that the standard measurement location used for $39 \mathrm{ft}$ in-chamber analysis correspond to the locations that are $21 \mathrm{in}$. above the spray and 10, 20, and $30 \mathrm{ft}$ downstream of the orifice. In the 20 and $30 \mathrm{ft}$ locations, the chamber concentration appears to be relatively uniform for 10 and $32 \mu \mathrm{m}$ droplets within the measurement uncertainty that can be achieved using measurements from a single instrument. At $10 \mathrm{ft}$, cumulative concentrations for the 32 droplets are reduced relative to the downstream measuring locations. The concentration of $102 \mu \mathrm{m}$ droplets does appear to decrease with increasing test height at the 10 to $20 \mathrm{ft}$ test locations. This decrease most probably results from there being insufficient turbulent energy (from the jet) to suspend larger droplets at the highest test positions. This observation is not surprising, as in-chamber measurements generally show limited populations of aerosols above $200 \mu \mathrm{m}$. As such, there is a size limit to uniformity, and large aerosols may not be representatively sampled by aerosol instruments in their standard in-chamber test locations.

As presented in Figure 7.3, trends in the aerosol concentration measurements taken $\sim 10 \mathrm{~s}$ after the start of spray generally support chamber uniformity of drops in the size range of interest (10 to $100 \mu \mathrm{m})$, with the limitation that droplets near $102 \mu \mathrm{m}$ may not be representatively sampled at the standard test locations. For the current tests using the $2 \mathrm{~mm}$ hole at $380 \mathrm{psig}$, impact to $102 \mu \mathrm{m}$ droplet concentrations is limited and will likely become smaller with larger test orifices. Overall, the droplet concentration trends support model assumptions with respect to uniformity.

When determining release fractions, the requirement of chamber uniformity should nominally be met at time zero. Specifically, if mixing of aerosols is fast relative to the generation of aerosol, then the generation rate measured at positions throughout the test enclosure should be uniform. This condition is evaluated by determining differences in generation rate as a function of measurement position. The result of this analysis is shown in Figure 7.4 and indicates a uniform generation rate for the majority of test locations (within the limited statistical uncertainty afforded by position testing). Generation rates are generally similar for 10 and $32 \mu \mathrm{m}$ droplets at all test locations 21 and $13 \mathrm{in}$. above the spray. It should be noted that there does not appear to be a reduction of similar magnitude in generation rate of droplets less than $32 \mu \mathrm{m}$ at the $10 \mathrm{ft}$ measurement location similar to that observed in aerosol concentration (cf. Figure $7.3 \mathrm{~b}$ and $7.4 \mathrm{~b}$ ). For $102 \mu \mathrm{m}$ droplets, the generation rate appears to show small, but statistically significant, differences between the 13 and $21 \mathrm{in}$. test heights. This observation is consistent with arguments made with respect to the ability of the $2 \mathrm{~mm}$ hole, 380 psig spray to suspend large droplets. Near-spray testing indicates uniform generation rates approximately 25 to $35 \mathrm{ft}$ downstream of the $2 \mathrm{~mm}$ hole, 380 psig spray (where the measurements are functionally in-chamber). At distances shorter than $25 \mathrm{ft}$, the near-spray generation rate shows significant order-of-magnitude increases. These increases are the result of incorrectly applying the in-chamber model to aerosol regions that are functionally "in-spray." 


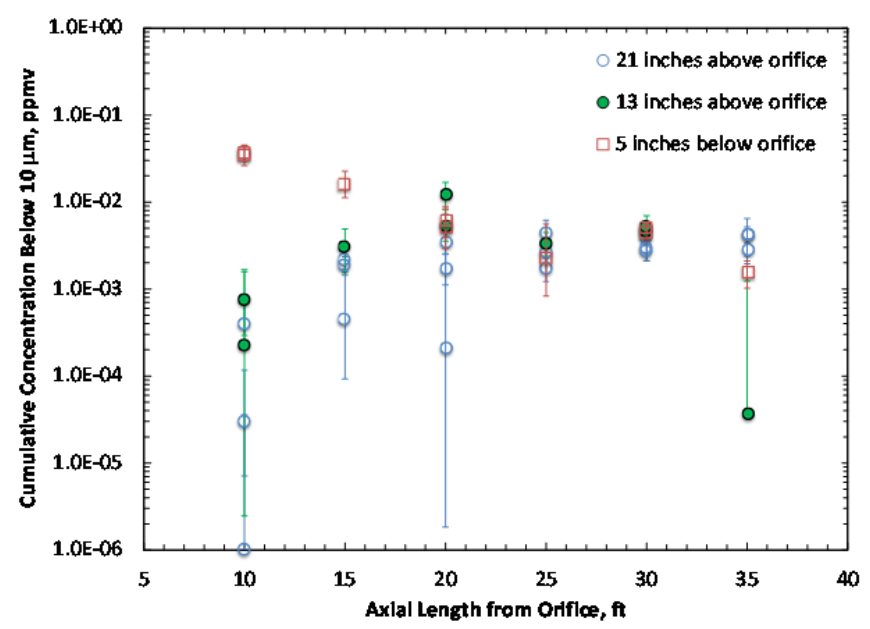

(a) - Cumulative concentration of droplets $<10 \mu \mathrm{m}$

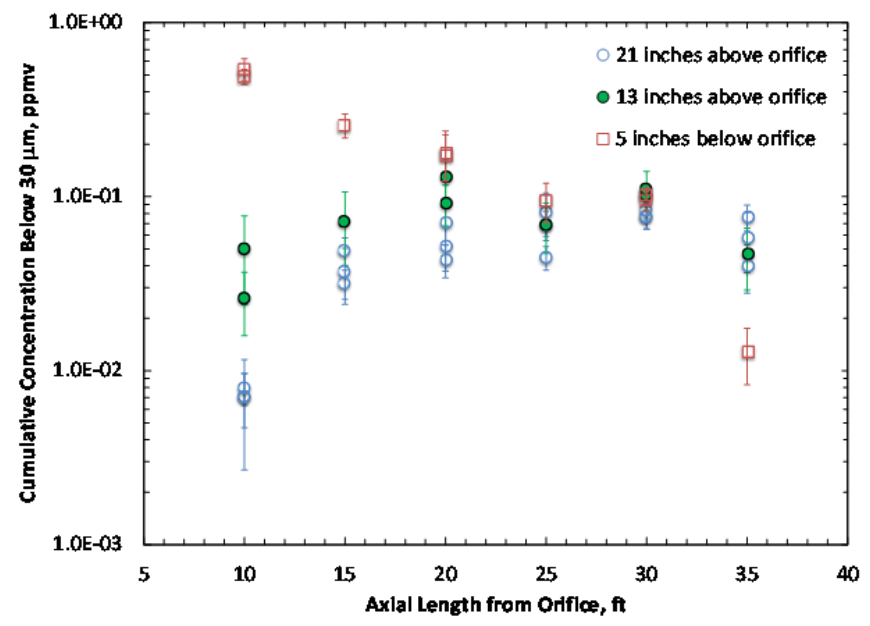

(b) - Cumulative concentration of droplets $<32 \mu \mathrm{m}$

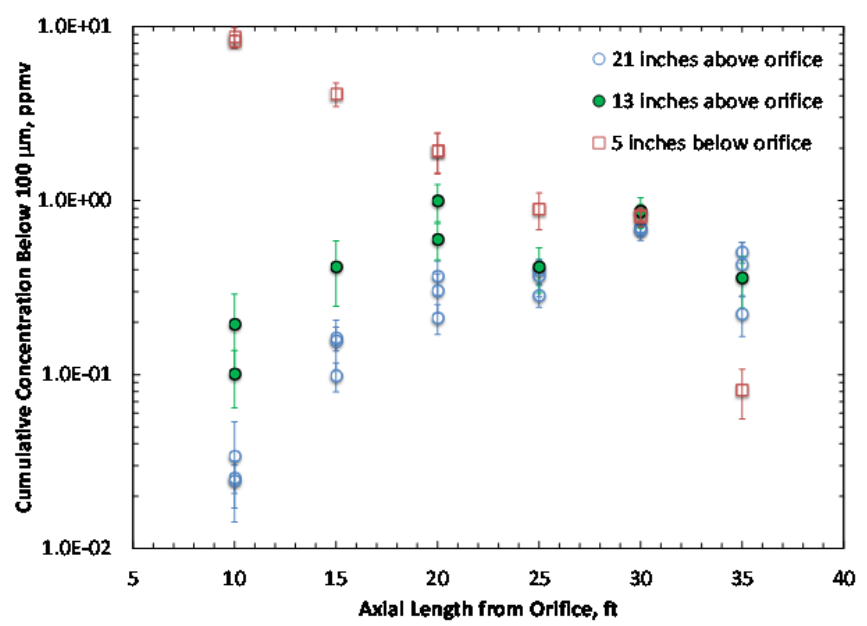

(c) - Cumulative concentration of droplets $<102 \mu \mathrm{m}$

Figure 7.3. Cumulative Concentration Undersize of Aerosol Droplets as a Function of Downstream (horizontal) Distance from the Spray Header at (a) $<10 \mu \mathrm{m}$, (b) $<32 \mu \mathrm{m}$, and (c) $<102 \mu \mathrm{m}$. Error bars shown correspond to 95 percent confidence limits. 


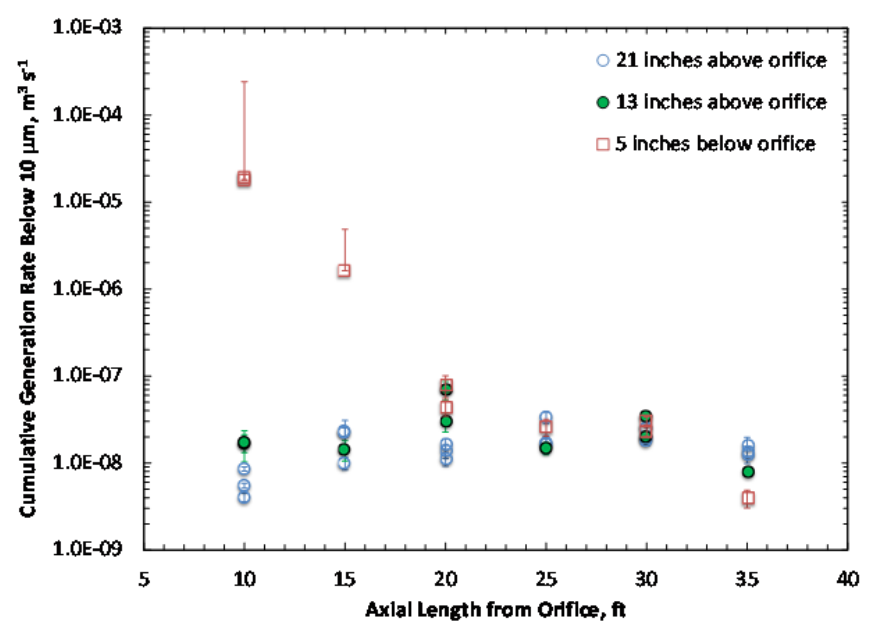

(a) - Generation rate of droplets $<10 \mu \mathrm{m}$

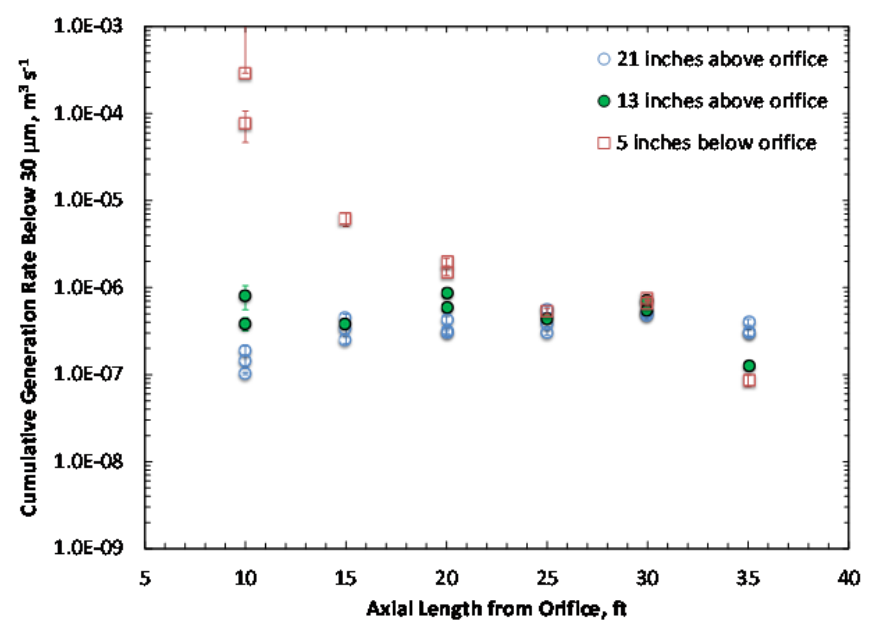

(b) - Generation rate of droplets $<32 \mu \mathrm{m}$

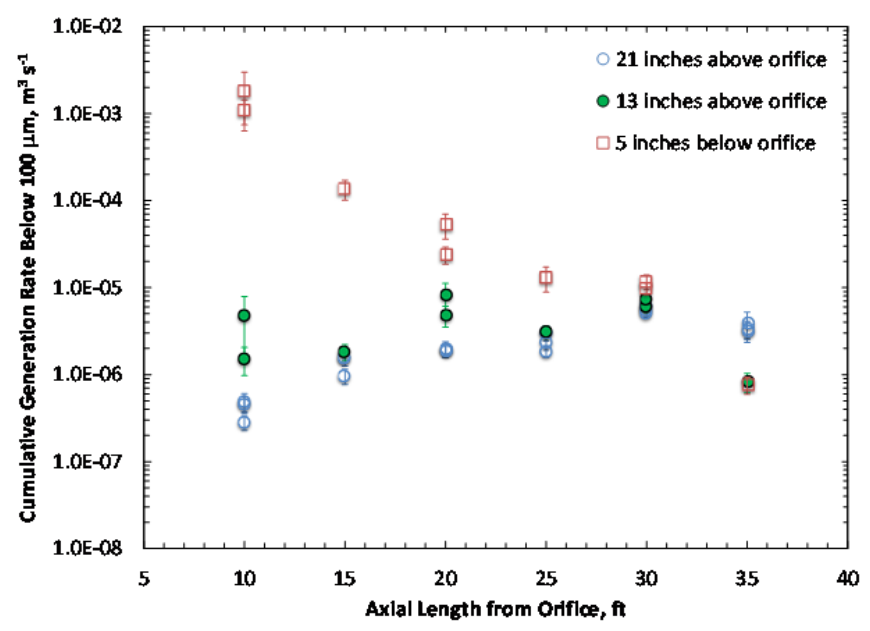

(c) - Generation rate of droplets $<102 \mu \mathrm{m}$

Figure 7.4. Cumulative Generation Rate Undersize of Aerosol Droplets as a Function of Downstream (horizontal) Distance from the Spray Header at (a) $<10 \mu \mathrm{m}$, (b) $<32 \mu \mathrm{m}$, and (c) $<102 \mu \mathrm{m}$. Error bars shown correspond to 95 percent confidence limits. 
When considered in their entirety, the results of position testing indicate a relatively uniform and well-mixed region above and downstream from the spray orifice in which the concentration, size distribution, and generation rate determined from release fraction analysis do not vary significantly with position for most droplets in the size range of interest. Furthermore, uniformity test results suggest that the $2 \mathrm{~mm}$ hole, 380 psig spray could not fully suspend $102 \mu \mathrm{m}$ droplets to heights corresponding to the standard in-chamber aerosol locations. This result is consistent with the range of aerosol sizes typically observed for in-chamber release fraction results in Phase I testing (which included sizes typically no larger than $200 \mu \mathrm{m}$ ). It is expected that sampling of $102 \mu \mathrm{m}$ droplets (and larger droplets) will be improved when using larger orifices, as they provide more turbulent mixing energy. This should manifest as an increase in in-chamber release fraction associated with poorly mixed (i.e., large) aerosols.

\subsection{In-Spray Aerosol Instrument Position Dependence}

Large-scale in-spray measurements were performed at three test locations in the direct path of the spray for all simulant materials tested (i.e., water, 6 Pa Clay, and 30 Pa Clay). As discussed in Section 4.5.2, the tests locations were typically 5, 8.5, and $12 \mathrm{ft}$ as measured by the distance between the back wall (i.e., the wall adjacent to the spray header) with exception of the $1 \times 76 \mathrm{~mm}$ slot tests for water, which used test locations of 5, 12.5, and $20 \mathrm{ft}$ downstream of the back wall. For the purpose of discussion, these distances will be used to define the in-spray test locations in this section. These distances can also be posed in terms of distances from the orifice to the sampling volume (laser centerline) of each of the aerosol analyzers. These distances are 4.3, 7.8, and $11.3 \mathrm{ft}$, respectively, for the typical test locations, and 4.3,11.8, and $19.3 \mathrm{ft}$, respectively, for water tests with the $1 \times 76 \mathrm{~mm}$ slot. All in-spray testing with water was executed in the $39 \mathrm{ft}$ test chamber, whereas all testing with clay simulants was done in the $20 \mathrm{ft}$ test chamber.

Selection of test locations described in Section 4.5.2 was based on a desire to measure the core of the spray with increasing distance from the orifice. At each distance, the aerosol analyzers were positioned in the most optically dense region of the spray. Figure 7.5 shows the in-spray measuring positions for water (in terms of height above the floor of the chamber) as a function of distance from the orifice. All distances shown are with respect to the sampling volume (laser centerline) of each Malvern Insitec-S aerosol analyzer. Similar measuring positions were employed in clay testing. The figure indicates that the trajectory of all sprays tested is impacted by gravity, as all sprays arc toward the chamber floor. As expected, increasing pressure increases the distance the spray travels (by virtue of an increase in orifice exit velocity for the spray). The impact of orifice is difficult to categorize. At $380 \mathrm{psig}$, little if any difference is observed in the trajectories of sprays generated with the $2 \mathrm{~mm}$ hole, $1 \times 10 \mathrm{~mm}$ slot, and $1 \times 20 \mathrm{~mm}$ slot, whereas the $1 \times 76 \mathrm{~mm}$ slot sprays travel substantially farther. It is possible that resolution of differences in spray trajectory may not be possible with the increase in orifice area that occurs between the $2 \mathrm{~mm}$ hole and the $1 \times 20 \mathrm{~mm}$ slot. 


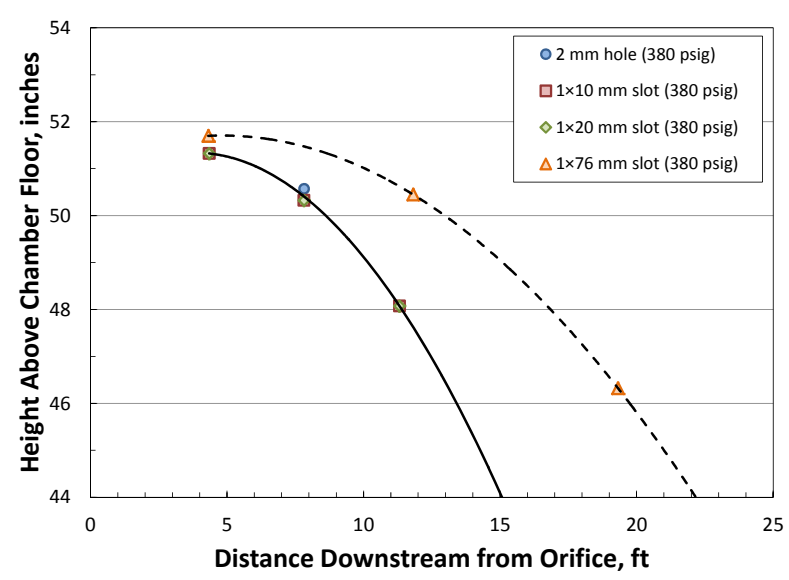

(a)

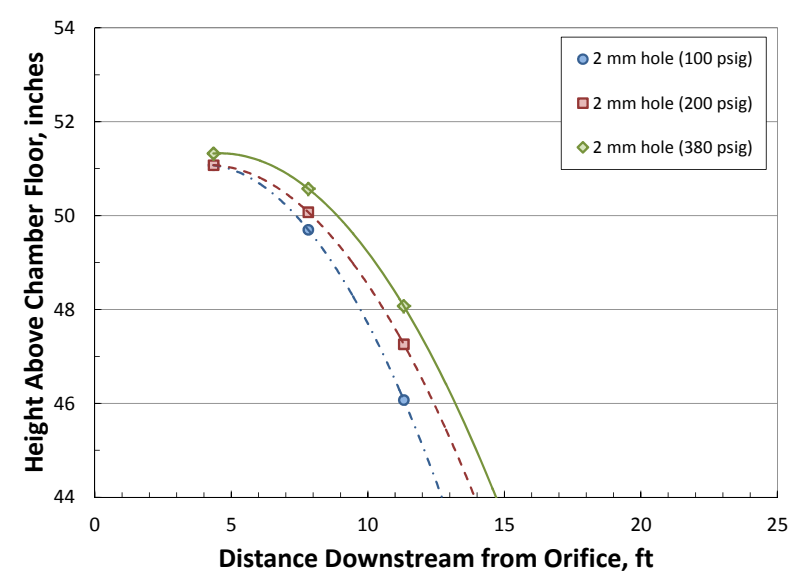

(b)

Figure 7.5. In-Spray Measurement Locations for Water Testing. Measuring locations correspond to the most optically dense region of the spray. Marked points indicate the position of the sampling volume (laser centerline) in terms of estimated (to within \pm 0.5 in.) height above the chamber floor as a function of downstream distance from the orifice. The left-hand figure (a) shows positions for all slots at 380 psig, while the right-hand figure (b) shows positions for the $2 \mathrm{~mm}$ hole at different test pressures. Solid curves correspond to estimated trajectory projections (based on a second-order polynomial fit). The orifice is located $52 \mathrm{in}$. above the chamber floor.

In-spray positions show that the spray has fallen below the lowest achievable measuring position (which is $\sim 46$ in. for Phase II Malvern Insitec-S frame and spacer bar configurations) at $\sim 13.5 \mathrm{ft}$ for the three smaller of the four test orifices and at $\sim 20 \mathrm{ft}$ for the $1 \times 76 \mathrm{~mm}$ slot. For this reason, measurement of in-spray data at distances much farther than those tested here was not possible with the Phase II Malvern Insitec-S configuration. To estimate trajectories, the position data (i.e., height of spray above chamber floor versus downstream distance) were fit to a second-order polynomial:

$$
y_{m}=b+m_{1} x_{m}+m_{2} x_{m}^{2}
$$

Here, $y_{m}$ is the vertical height of the spray from the floor (in inches), and $x_{m}$ is the downstream distance from the orifice (in feet). The fits are shown as the solid and dashed curves in Figure 7.5. Because of the similarity in trajectories, the $2 \mathrm{~mm}, 1 \times 10 \mathrm{~mm}$, and $1 \times 20 \mathrm{~mm}$ orifices have been grouped for trajectory analysis. The polynomial coefficients corresponding to the trajectories shown in Figure 7.5 are listed in Table 7.1. Some caution should be used when extrapolating beyond the measured position because selection of the polynomial model is arbitrary. Although this model can be appropriate for evaluating trajectories of objects not affected by drag, it does not account for the complexities of droplet drag and settling (which are both functions of particle size) and droplet suspension by turbulence and may lead to gross misrepresentations of the true spray trajectory. With these limitations in mind, extrapolation of the trajectory curves indicates that 380 psig sprays travel anywhere from $30 \mathrm{ft}$ (for the $2 \mathrm{~mm}$ hole and $1 \times 10$ and $1 \times 20 \mathrm{~mm}$ slots) to $50 \mathrm{ft}$ (for the $1 \times 76 \mathrm{~mm}$ slot) downstream of the orifice before hitting the chamber floor. These results indicate that only the $1 \times 76 \mathrm{~mm}$ spray travels the full length of all chamber sizes tested (although again, this may be true for the core of the spray). This observation is somewhat in line with visual observations of large-scale chamber spray behavior, which indicate that the $2 \mathrm{~mm}$ hole 
does dissipates before contacting the splash wall and that the $1 \times 76 \mathrm{~mm}$ spray strikes the splash wall with significant force. Visual observation suggests that intermediate orifices (i.e., the $1 \times 10$ and $1 \times 20 \mathrm{~mm}$ slots) show intermediate behavior.

Table 7.1. Polynomial Coefficients for Trajectories Shown in Figure 7.5 and Fit to Equation 7.1

\begin{tabular}{|c|c|c|c|c|c|}
\hline Orifice(s) & Pressure & Points Fit & $\begin{array}{c}b \\
{[\mathrm{in} .]}\end{array}$ & $\begin{array}{c}\boldsymbol{m}_{1} \\
\text { [inch } \mathrm{ft}^{-1} \text { ] }\end{array}$ & $\begin{array}{c}\boldsymbol{m}_{2} \\
{\left[\mathrm{inch}^{2} \mathrm{ft}^{-2}\right]}\end{array}$ \\
\hline $2 \mathrm{~mm}$ hole & 100 & 3 & 49.7 & $7.25 \times 10^{-1}$ & $-9.22 \times 10^{-2}$ \\
\hline $2 \mathrm{~mm}$ hole & 200 & 3 & 49.8 & $6.08 \times 10^{-1}$ & $-7.39 \times 10^{-2}$ \\
\hline $2 \mathrm{~mm}$ hole & 380 & 3 & 49.8 & $6.50 \times 10^{-1}$ & $-7.14 \times 10^{-2}$ \\
\hline $2 \mathrm{~mm}$ hole, $1 \times 10 \mathrm{~mm}$ slot, $1 \times 20 \mathrm{~mm}$ slot & 380 & 9 & 50.5 & $4.38 \times 10^{-1}$ & $-5.78 \times 10^{-2}$ \\
\hline $1 \times 76 \mathrm{~mm}$ slot & 380 & 3 & 51.1 & $2.45 \times 10^{-1}$ & $-2.56 \times 10^{-2}$ \\
\hline
\end{tabular}

As discussed previously, the in-spray trajectories provide a physical basis for describing trends observed in the near-spray chamber uniformity test data. Specifically, trajectory data indicate that $2 \mathrm{~mm}$ hole, $380 \mathrm{psig}$ sprays (Figure $7.5 \mathrm{~b}$ ) pass through the near-spray test height (which is $\sim 47 \mathrm{in}$. above the chamber floor) between 10 and $15 \mathrm{ft}$ downstream from the orifice. Near-spray measurement locations at 10 and $15 \mathrm{ft}$ occur at heights that are within several inches of the spray trajectory, and as a result, their behavior (in terms of aerosol concentration and size distribution) can be expected to be similar to that of in-spray measurements. Near-spray measuring locations at and beyond $20 \mathrm{ft}$ fall well above spray and, as indicated by chamber uniformity test results, behave similarly to in-chamber measurements.

Figure 7.5 shows a direct effect of gravity on the spray, as the spray height decreases with increasing downstream distance from the orifice. As will be shown in the in-spray release fraction results below (Figure 7.6 to Figure 7.8), the core of the spray contains droplets ranging from $50 \mu \mathrm{m}$ (if not smaller) up to $\sim 2000 \mu \mathrm{m}$. Whether or not these particles may be suspended depends on their size and the amount of turbulence generated by high-pressure spray and mixing fans placed in the chamber to improve homogeneity. It is reasonable to expect that chamber turbulence will not be sufficient to suspend all droplets (particularly large droplets). Large droplets are also more strongly impacted by inertial capture and deposition on downstream surfaces. As such, there are several mechanisms by which aerosols can size segregate. Such segregation can be observed in in-chamber uniformity testing, where the large aerosols are increasingly removed from the near-spray measurement location beyond $15 \mathrm{ft}$ and in loss of $102 \mu \mathrm{m}$ droplets at the highest test locations (21 in. above the spray) directly above the spray header. Such size segregation could also bias in-spray measurements. Because in-spray measurements track the trajectory of the spray to locations closer to the floor of the chamber, there is the potential that measurements may preferentially include those aerosols that settle (i.e., large droplets). Schonewill et al. (2012) found an increase in the average droplet size with increasing downstream distance from the orifice between $\sim 2$ and $5 \mathrm{ft}$. Based on trajectory data in Figure 7.5, the cause of this increase is most probably droplet coalescence (as the spray does not drop more than 1 in. over 2 to $5 \mathrm{ft}$ ). For current data, a similar increase in droplet size with distance could also result from droplet coalescence; however, any trends observed could also be subject to bias from settling of large droplets.

In-spray release fraction data for water as a function of position for all orifices at $380 \mathrm{psig}$ are shown in Figure 7.6. The curves show measured release fraction data for the three test positions (indicated in the figure legend) as well as the release fraction calculated by the WTP model for the corresponding orifice and test pressure. The WTP model is included here for reference only; the significance of in-spray results 
with respect to the WTP will be discussed in detail in Chapter 10 of this report. With the exception of the $1 \times 76 \mathrm{~mm}$ data, in-spray release fractions show small changes in release fraction with the locations tested. The data for the $2 \mathrm{~mm}$ hole and $1 \times 10 \mathrm{~mm}$ slot indicate that the overall size of aerosol increases (release fraction decreases) with increasing downstream distance from the orifice (up to the maximum test distance of $12 \mathrm{ft}$ ). Of the two orifices, the $2 \mathrm{~mm}$ hole shows the greatest increase in size. In contrast, size distribution trends from the $1 \times 20 \mathrm{~mm}$ slot are unclear; while there is a change in overall aerosol size, they are slight and appear to reverse with increasing distance. Release fraction data for the $1 \times 76 \mathrm{~mm}$ show a clear decrease in the size of in-spray aerosols as the distance from the spray source increases, such that, in contrast with the smaller orifice data, the most coarse PSD corresponds to the $5 \mathrm{ft}$ data. It should be noted that the $5 \mathrm{ft}, 1 \times 76 \mathrm{~mm}$ in-spray data challenged the Malvern Insitec-S aerosol analyzers as the core of the spray obscured approximately 95 percent of the instrument laser power (which is the maximum operating limit of the analyzers). As such, measurements of sprays from the $1 \times 76 \mathrm{~mm}$ may be subject to significant uncertainty.

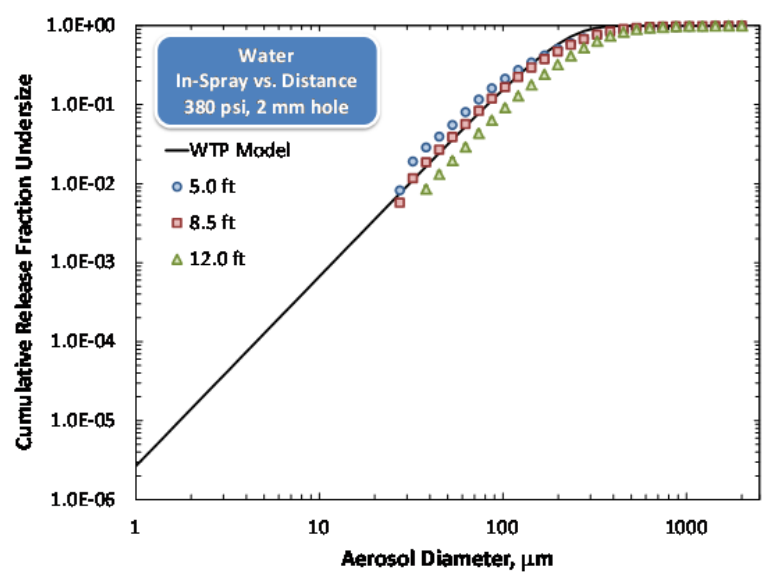

(a) $-2 \mathrm{~mm}$ hole

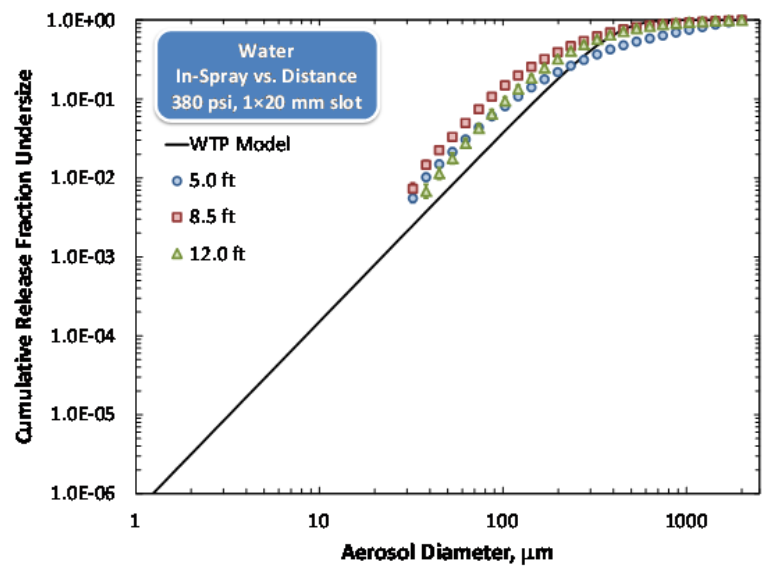

(c) $-1 \times 20 \mathrm{~mm} \mathrm{slot}$

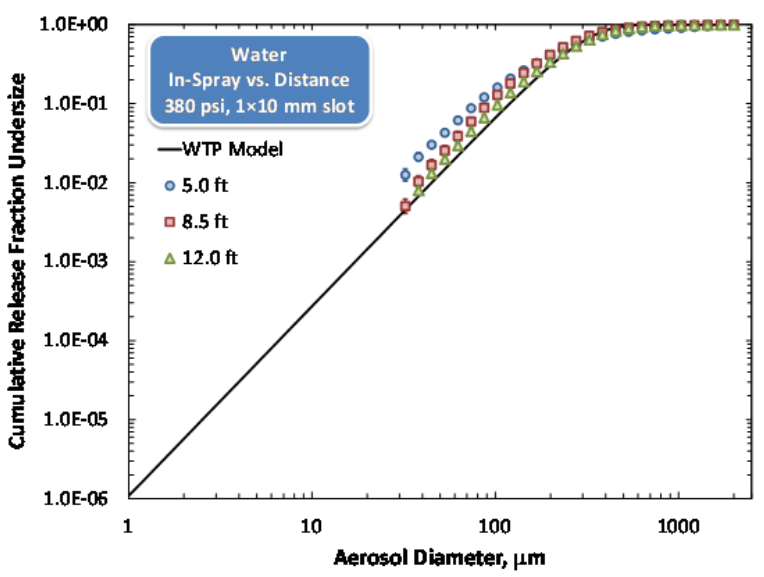

(b) $-1 \times 10 \mathrm{~mm}$ slot

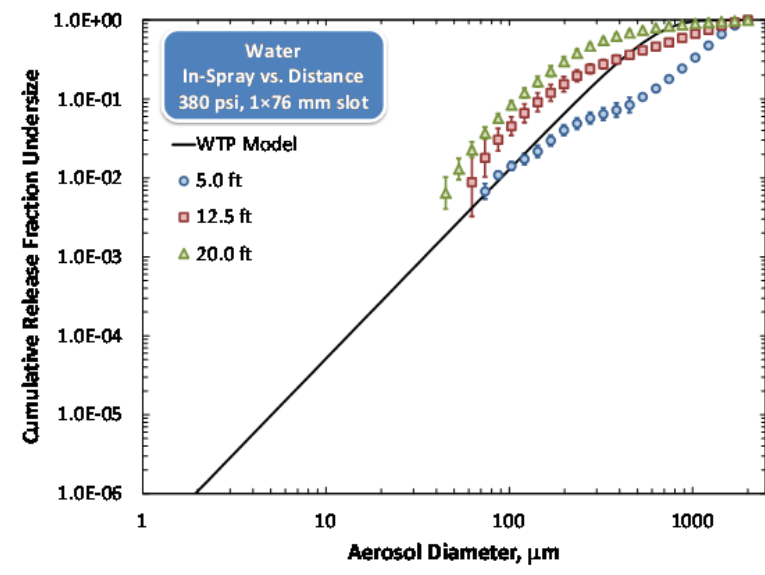

(d) $-1 \times 76 \mathrm{~mm}$ slot

Figure 7.6. In-Spray Release Fractions Measured for Water as a Function of Distance from the Back Wall (i.e., that next to the spray header) for (a) a $2 \mathrm{~mm}$ Hole, (b) a $1 \times 10 \mathrm{~mm}$ Slot, (c) a $1 \times 20 \mathrm{~mm}$ Slot, and (d) a $1 \times 76 \mathrm{~mm}$ Slot. All sprays were generated at $380 \mathrm{psig}$. 
Figure 7.7 and Figure 7.8 show in-spray release fraction curves as a function of position for the $6 \mathrm{~Pa}$ Clay and $30 \mathrm{~Pa}$ Clay test simulants, respectively. Relative to water data, the change in in-spray clay simulant PSD is uniform, with the in-spray aerosol size typically increasing with increasing distance.

In some cases (such as the $2 \mathrm{~mm}$ hole data for both the $6 \mathrm{~Pa}$ Clay and the $30 \mathrm{~Pa}$ Clay), there is little if any change with increasing distance. In other cases, the increase is significant, with the $6 \mathrm{~Pa}$ Clay for the $1 \times 76 \mathrm{~mm}$ slot showing the largest change in in-spray size distribution with distance. The general uniformity of trends of the in-spray clay data may derive from the fact that all testing for clay examined similar test positions, namely $5,8.5$, and $12 \mathrm{ft}$.
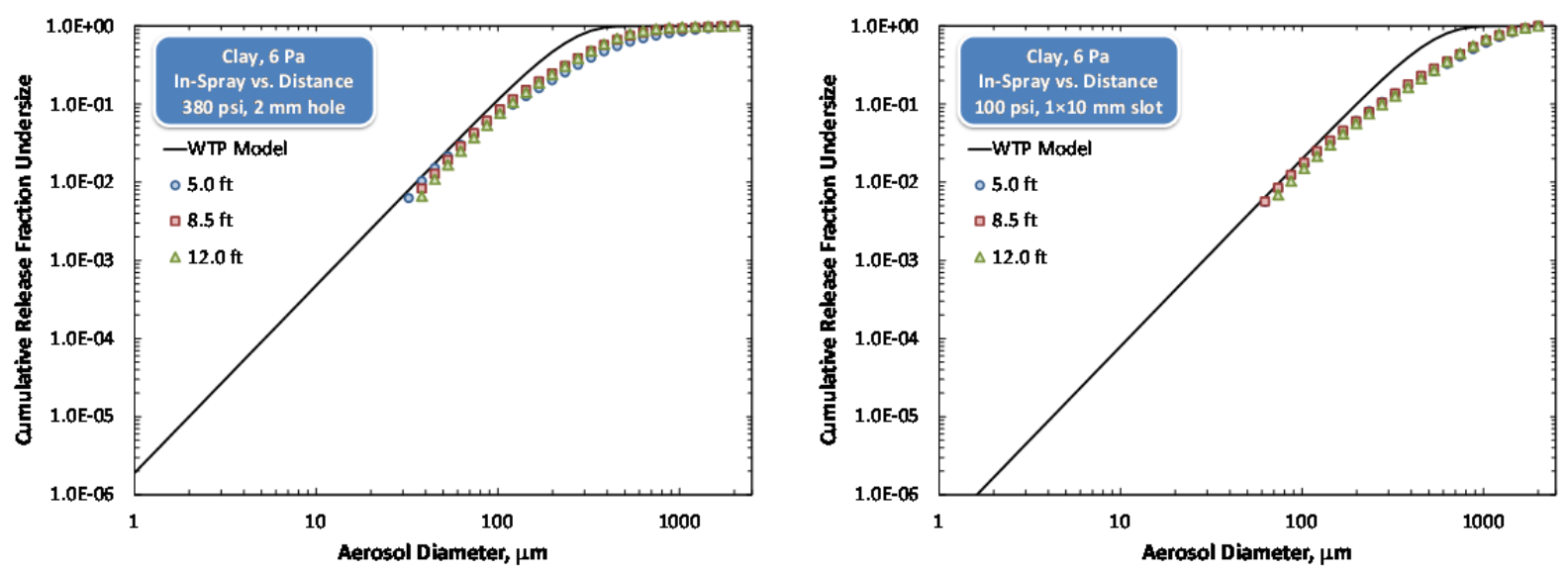

(a) $-2 \mathrm{~mm}$ hole
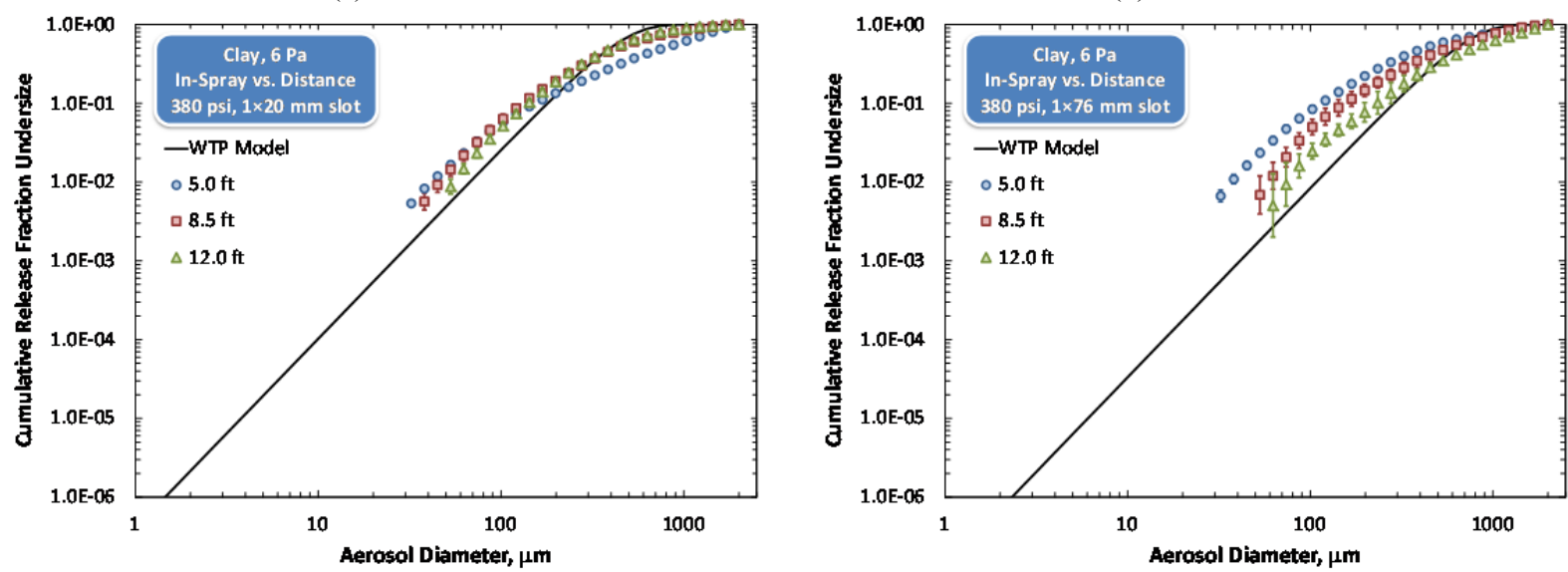

(c) $-1 \times 20 \mathrm{~mm}$ slot

(d) $-1 \times 76 \mathrm{~mm}$ slot

Figure 7.7. In-Spray Release Fractions Measured for 6 Pa Clay as a Function of Distance from the Back Wall (i.e., next to the spray header) for (a) a $2 \mathrm{~mm}$ Hole, (b) a $1 \times 10 \mathrm{~mm}$ Slot, (c) a $1 \times 20 \mathrm{~mm}$ Slot, and (d) a $1 \times 76 \mathrm{~mm}$ Slot. All sprays were generated at $380 \mathrm{psig}$. 


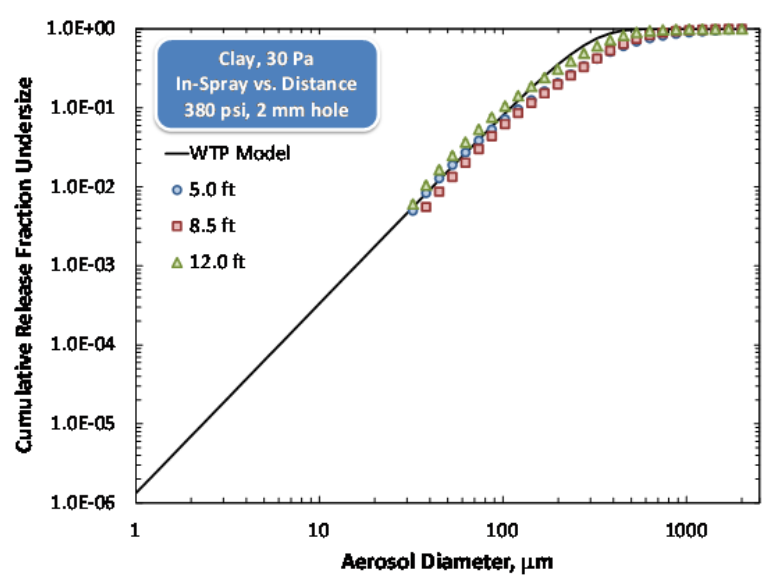

(a) $-2 \mathrm{~mm}$ hole

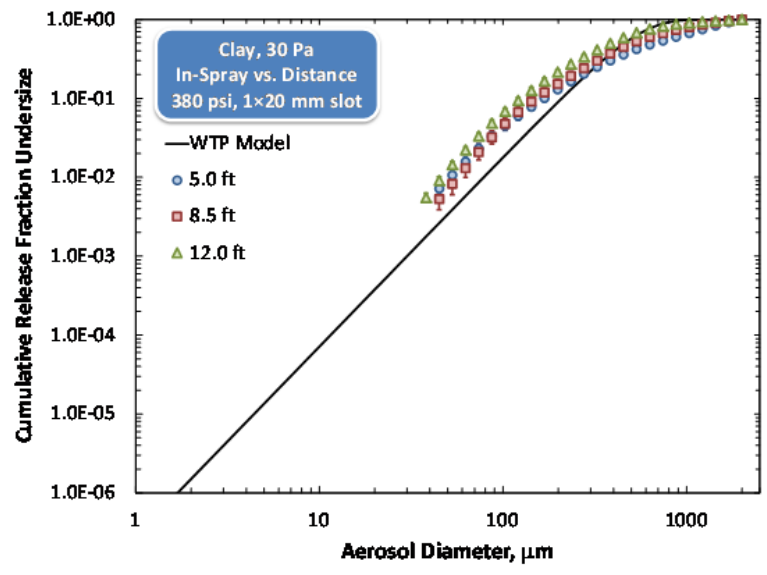

(c) $-1 \times 20 \mathrm{~mm}$ slot

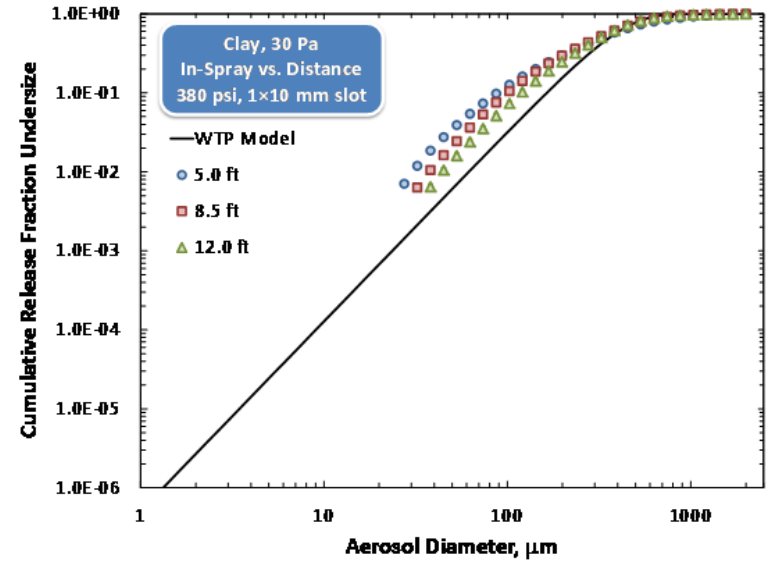

(b) $-1 \times 10 \mathrm{~mm}$ slot

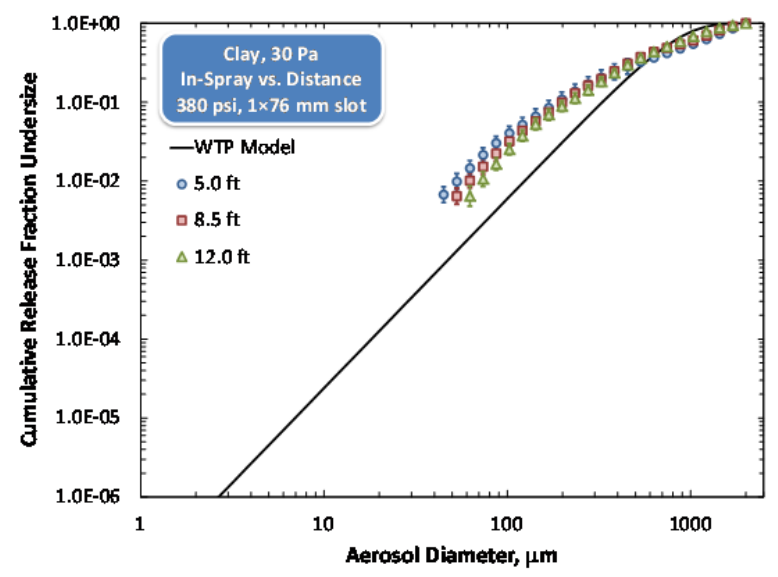

(d) $-1 \times 76 \mathrm{~mm}$ slot

Figure 7.8. In-Spray Release Fractions Measured for $30 \mathrm{~Pa}$ Clay as a Function of Distance from the Back Wall (i.e., next to the spray header) for (a) a $2 \mathrm{~mm}$ Hole, (b) a $1 \times 10 \mathrm{~mm}$ Slot, (c) a $1 \times 20 \mathrm{~mm}$ Slot, and (d) a $1 \times 76 \mathrm{~mm}$ Slot. All sprays were generated at $380 \mathrm{psig}$.

Although not shown here, the 100 and 200 psig in-spray test data for water and both clay simulants exhibit comparable behavior with distance to that identified above. The full set of in-spray measurement data for clay and water, including in-spray release fraction as a function of distance, may be found in Appendix B. The aerosol size behavior described above can be summarized as follows:

- For water, sprays generated from the $2 \mathrm{~mm}$ orifice exhibit increasing droplet size with increasing distance from the orifice. The in-spray droplet size distribution generated by spraying water from the $1 \times 10$ and $1 \times 20 \mathrm{~mm}$ slots exhibit very little change with increasing distance from orifice (with no clear trend toward larger or smaller droplets). Water sprays generated through the $1 \times 76 \mathrm{~mm}$ orifice show an in-spray size distribution that becomes finer with increasing distance from orifice.

- For clay, in-spray release fraction (i.e., the droplet size distribution) generally becomes coarser with increasing distance. An initial break-up of droplets, which produces a fine, concentrated distribution of aerosol immediately near the orifice at positions close to the spray header. 
It is difficult to rationalize the overall behavior observed. In general, water and clay in-spray release fractions measured at 5,8.5, and $12 \mathrm{ft}$ show either little change in overall size or became coarser with increasing downstream distance from the orifice. The simplest explanation for these trends is coalescence of droplets as they travel downstream through the chamber or from preferential sampling of large aerosols because of settling at the in-spray measurement position (which get progressively closer to the chamber floor at distances far from the orifice). Phase II in-spray water data for the $2 \mathrm{~mm}$ hole show significant increase in aerosol size at downstream locations that is consistent both with expectations (based on coalescence and sampling) and with observations made during Phase I in-spray tests (Schonewill et al. 2012). The difficultly with Phase II in-spray measurements lies primarily with the $1 \times 76 \mathrm{~mm}$ slot release fractions, which are unique in that they are the only release fractions that show a significant decrease in aerosol size with increasing distance from the orifice and in that they are the only data collected at 5, 12.5, and $20 \mathrm{ft}$. The trends observed in the $1 \times 76 \mathrm{~mm}$ in-spray release fractions could thus derive from either the change in measuring location or the increase in orifice area. Other factors that also contribute to uncertainty in the $1 \times 76 \mathrm{~mm}$ data include:

- Near complete (95 percent) obscuration of the spray during water measurements

- Spatial bias in the size distribution measurement, which could reasonably impact all in-spray measurements, but which could be more significant far downstream ( $>10 \mathrm{ft})$ if the spray slows substantially.

Overall, the in-spray release fractions can show significant variation with respect to measurement location. In some cases, in-spray measurements do not change significantly over the downstream distances tested herein, while in other cases, the in-spray droplet size distributions can evolve with distance. Selection of a "best" measurement location for in-spray size distributions (with respect to their use for analyses in this report and elsewhere) is not straightforward when the entire set of in-spray data is considered - proper selection requires consideration of the specific scenario being evaluated (and potentially the spray distances involved). For the purpose of comparison and analysis in later chapters of this report, the in-spray PSD measured $\sim 12 \mathrm{ft}$ downstream of the spray header is taken as the representative aerosol size distribution generated by high-pressure spray. This selection is based on the assumption that spray at this distance allows for the greatest maturation of the size distribution (as a result of droplet coalescence). As mentioned previously, $1 \times 76 \mathrm{~mm}$ in-spray water data show unique behavior and, as such, are handled differently than the bulk of in-spray measurements. For performing simulant comparisons, the $12.5 \mathrm{ft} 1 \times 76 \mathrm{~mm}$ slot data are used; for scaling and bounding release fraction analyses presented in Chapter 10 of this report, $20 \mathrm{ft} 1 \times 76 \mathrm{~mm}$ data are used (because they provide the most conservative measurement of in-spray release fraction for the given condition).

\subsection{Comparison of In-Spray and In-Chamber Measurements}

In-spray and in-chamber testing methods provide two approaches for assessing fractional release of aerosol from high-pressure spray leaks. Both methods are subject to limiting assumptions that impact the quality and uncertainty associated with the release fraction results derived from each approach. With respect to in-chamber measurements, mixing and aerosol sampling limitations are expected to bias the apparent rate of generation evaluated using the exponential aerosol concentration rise model relative to the true generation rate. The in-spray method overcomes this weakness by directly measuring the size distribution generated aerosol; however, as indicated by the test results presented in previous sections, the presence of large aerosols in in-spray measurements does not allow access to aerosols at sizes lower than 
$\sim 50 \mu \mathrm{m}$, which greatly reduces the usefulness of in-spray measurements for evaluating release fraction behavior in the size range of interest (10 to $100 \mu \mathrm{m})$. Because large particles are removed from in-chamber measurement locations by inertial impact or gravitational settling, in-chamber measurements do not suffer from this limitation and generally provide release data over the range of interest (10 to $100 \mu \mathrm{m})$. Thus, in-chamber measurements provide the only assessment of release fraction in the size range of interest for the majority of test configurations, and it is necessary to evaluate the quality of these measurements to determine what, if any, bias exists in the data relative to more direct measurements of fractional release. To this end, comparison of in-spray and in-chamber test data for equivalent test configurations is conducted in this section.

Figure 7.9, Figure 7.10, and Figure 7.11 compare large-scale in-spray to in-chamber measurements made for the four Phase II test orifices for water, $6 \mathrm{~Pa}$ Clay, and $30 \mathrm{~Pa}$ Clay, respectively. The results show release fraction as a function of aerosol diameter for in-chamber (blue circles) and in-spray (green triangles) measurement for 380 psig. To simplify comparison, in-chamber test results for a single chamber length are shown. These results correspond to a full-length spray (i.e., generated from a test spool with no extension added). The chamber length selected for comparison corresponds to the length tested during in-spray measurements. Water release fraction comparisons in Figure 7.9 use $39 \mathrm{ft}$ in-chamber data because in-spray tests were conducted in a $39 \mathrm{ft}$ chamber. In-chamber release fraction data for clay simulants shown in Figure 7.10 and Figure 7.11 use the $20 \mathrm{ft}$ chamber data because in-spray testing was only performed in the $20 \mathrm{ft}$ chamber. These selections were done to avoid the need to talk about chamber length effects on the in-chamber release fraction result in the current section. Chamber length discussions are provided in Chapters 9 and 10 of this report.

To further simplify discussion, only 380 psig data are presented below. Results for lower test pressures show similar trends and are not discussed (but may be found in Appendix B of this report). For all test results shown below, a curve (black dashed line) that bounds the in-spray measurements has been added to help facilitate comparison of curves at diameters where only in-chamber data exist. This bounding in-spray release fraction curve $R_{k}^{\text {(bound) }}$ is based upon Rosin-Rammler (1933) distribution, such that:

$$
R_{k}^{(\text {bound })}=1-\exp \left[-\left(\frac{d_{k}}{d_{o}}\right)^{q}\right]
$$

where a $q$ of 2.4 is used (for consistency with WTP model calculations) and $d_{o}$ is selected such that the resulting $R_{k}^{\text {(bound) }}$ falls just to the left of the in-spray release fraction data. This curve is (and should be) used for comparative purposes only.

Comparisons of in-spray and in-chamber release fraction measurements for water are shown in Figure 7.9. The results for all orifices show similar differences between the two measurement methods and indicate that, at least for the smallest aerosol diameters where there is measurement overlap $(\sim 70 \mu \mathrm{m})$, the in-spray measurements yield a release fraction that is approximately three times higher than the corresponding in-chamber measurement. The difference in in-spray and in-chamber results increases significantly at larger sizes, with the difference at the largest in-chamber aerosols tested (i.e., $\sim 200 \mu \mathrm{m}$ ) being one to two orders of magnitude. The results indicate a significant loss of large aerosol from the in-chamber measurements. Loss is most significant for the $2 \mathrm{~mm}$ hole data, which show an in-chamber measurement plateau (i.e., show no particles larger than $\sim 100 \mu \mathrm{m}$ whereas in-spray data evidence aerosols up to $\sim 600 \mu \mathrm{m}$ ). Based on previous discussion concerning $102 \mu \mathrm{m}$ droplet uniformity (see Section 7.2), it 
is postulated that the difference in maximum aerosol sizes observed in the two measurements derives from inertial capture (deposition) or settling of large aerosols before they reach the in-chamber measurement locations. The current data suggest that mechanism does not become significant until aerosols reach $\sim 100 \mu \mathrm{m}$.

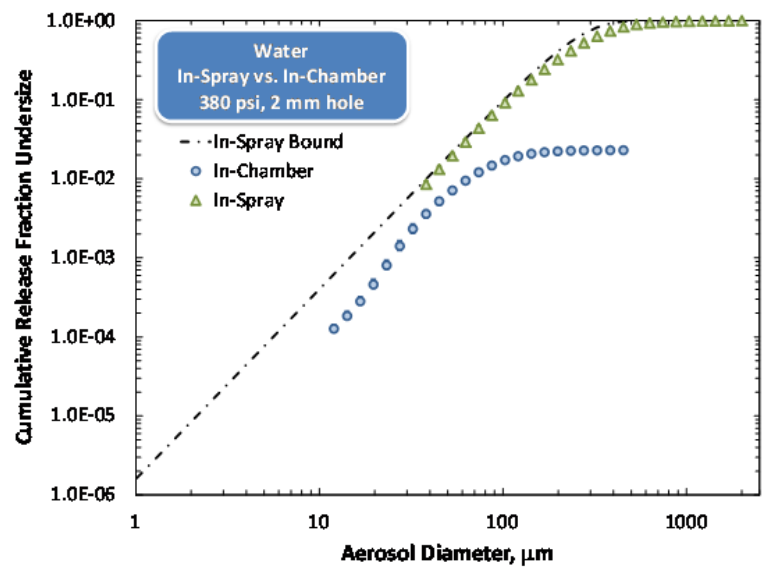

(a) $-2 \mathrm{~mm}$ hole

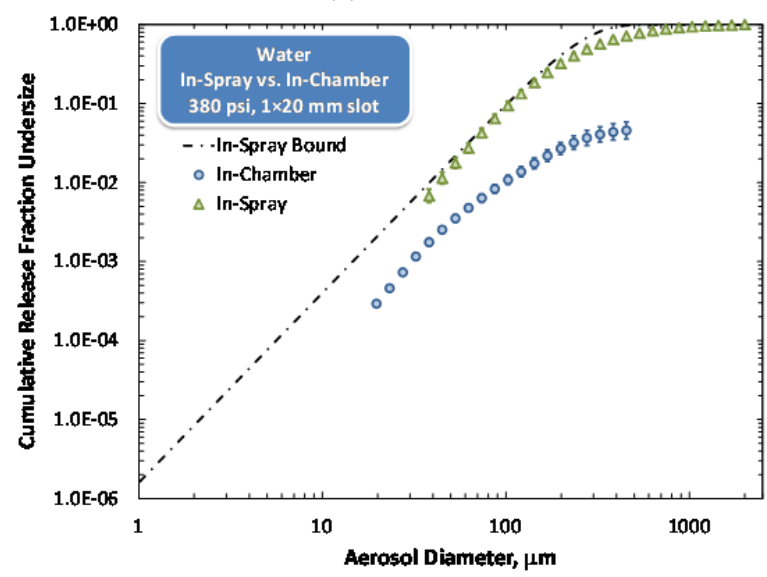

(c) $-1 \times 20 \mathrm{~mm}$ slot

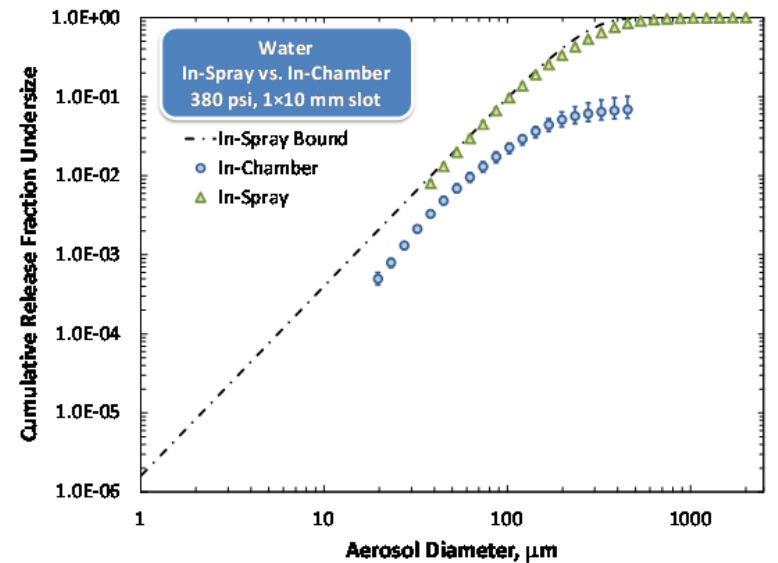

(b) $-1 \times 10 \mathrm{~mm}$ slot

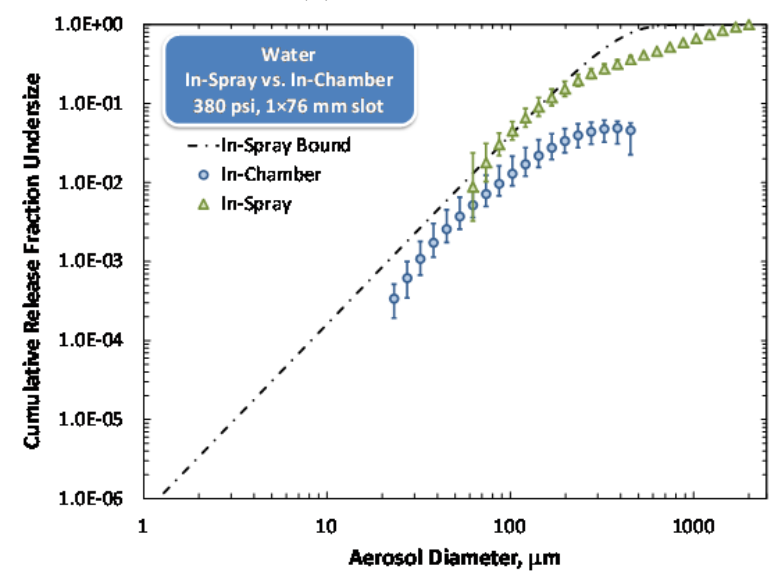

(d) $-1 \times 76 \mathrm{~mm}$ slot

Figure 7.9. Comparison of In-Spray (at $\sim 12 \mathrm{ft}$ from the spray header) and In-Chamber Release Fractions Measured for Water for (a) a $2 \mathrm{~mm}$ Hole, (b) a $1 \times 10 \mathrm{~mm}$ Slot, (c) a $1 \times 20 \mathrm{~mm}$ Slot, and (d) a $1 \times 76 \mathrm{~mm}$ Slot. All sprays were generated at $380 \mathrm{psig}$. Both in-spray and in-chamber measurements shown were captured in the $39 \mathrm{ft}$ chamber (no spray extensions).

Despite the difference in release fraction magnitude, the slope of in-chamber and in-spray release fraction data (i.e., the change in release fraction with size) generally appears to approach the same limit at small aerosol size. Similar slopes indicate similar fractionations of aerosol size for both in-chamber and in-spray aerosol (which should be expected if the measurement methods are correctly evaluating the generation, as both size distributions derive from the same spray). As stated above, there is approximately a factor of three difference in the in-chamber and in-spray release fractions when results are compared at equivalent aerosol diameters below $102 \mu \mathrm{m}$. The potential causes for this difference include under-estimation of in-chamber release fractions as a result of aerosol sampling and mixing limitations, over-estimation of aerosol fines in the in-spray data as a result of differences in small- and 
large-aerosol velocities, or some combination thereof. Furthermore, the under-estimate may also derive in part from humidity bias (where a fraction of droplets are lost to evaporation before they reach the analyzer measuring zone - see Appendix A). Because the data on which this correction is proposed are impacted by humidity effects, the correction factor of three also partially (but not completely) corrects for humidity bias. An additional correction factor is needed to completely account for the lowering of release fraction from humidity effects. If all errors and biases associated with in-chamber and in-spray measurements could be properly accounted for (including spatial measurement bias, loss of droplets to gravitational settling or inertial capture, loss of droplets to evaporation, etc.), then it is expected that inchamber and in-spray release fraction results would be equivalent over the full range of aerosol diameters observable by the analyzers.

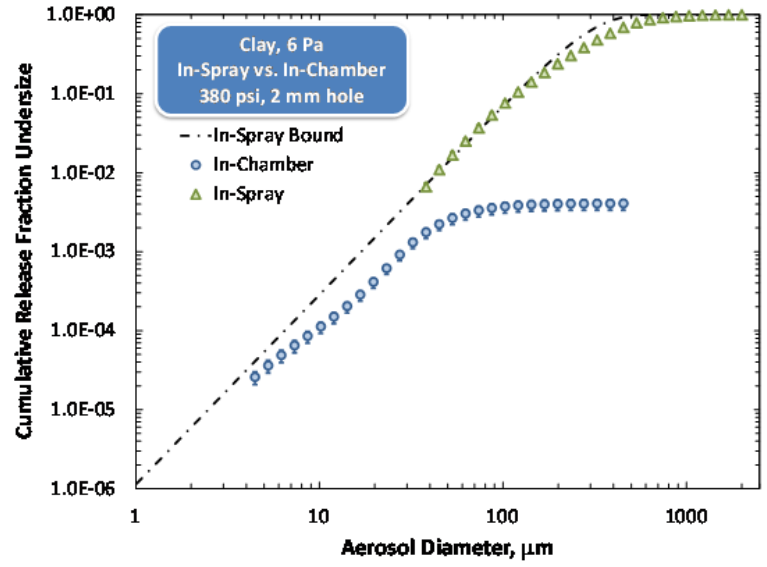

(a) $-2 \mathrm{~mm}$ hole

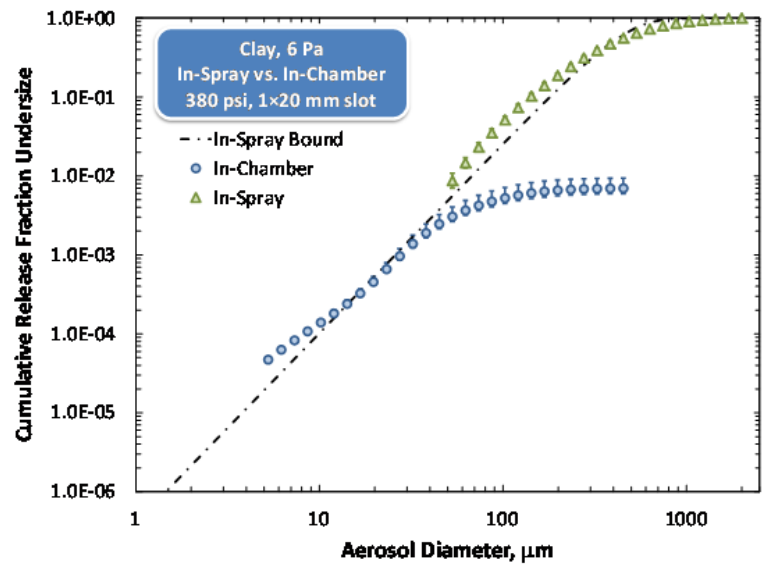

(c) $-1 \times 20 \mathrm{~mm}$ slot

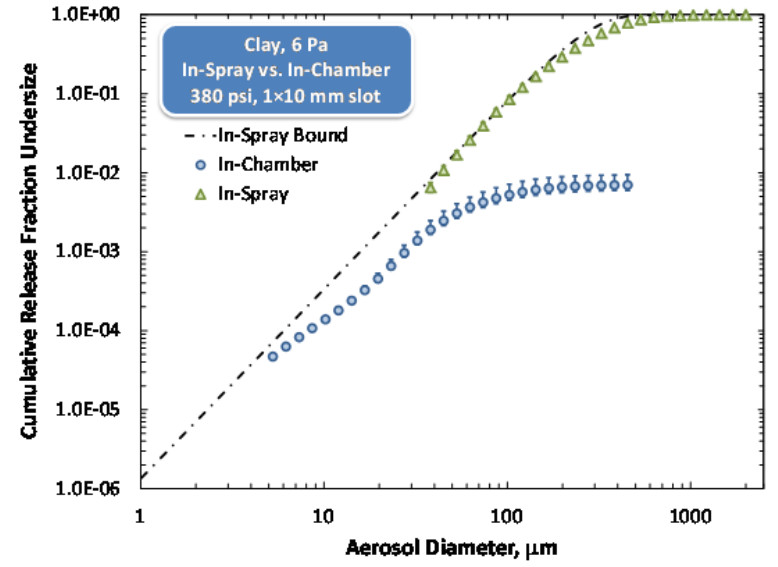

(b) $-1 \times 10 \mathrm{~mm}$ slot

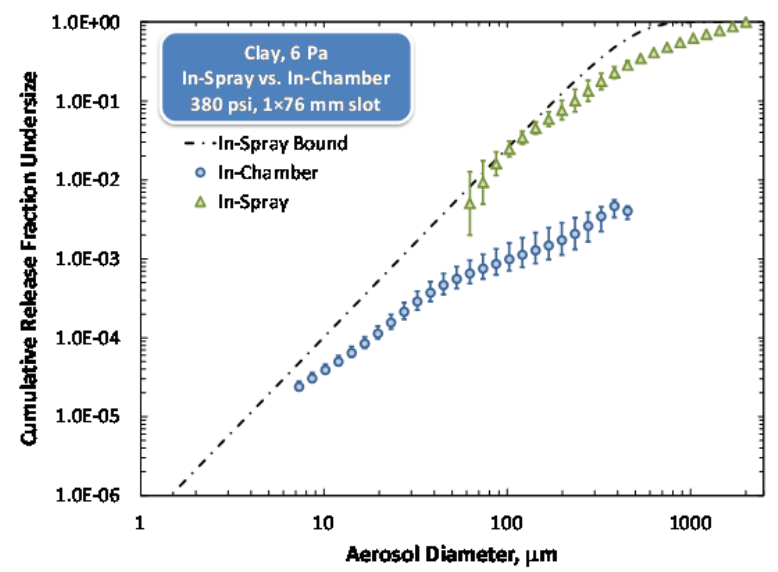

(d) $-1 \times 76 \mathrm{~mm}$ slot

Figure 7.10. Comparison of In-Spray (at $\sim 12 \mathrm{ft}$ from the spray header) and In-Chamber Release Fractions Measured for $6 \mathrm{~Pa}$ Clay for (a) a $2 \mathrm{~mm}$ Hole, (b) a $1 \times 10 \mathrm{~mm}$ Slot, (c) a $1 \times 20 \mathrm{~mm}$ Slot, and (d) a $1 \times 76 \mathrm{~mm}$ Slot. All sprays are generated at $380 \mathrm{psig}$. Both in-spray and in-chamber measurements shown were captured in the $20 \mathrm{ft}$ chamber (no spray extensions).

Comparisons of in-spray and in-chamber release fraction data for the $6 \mathrm{~Pa}$ Clay and $30 \mathrm{~Pa}$ Clay simulants are shown in Figure 7.10 and Figure 7.11, respectively. The results are similar in many respects to those found for the water data. There is still significant difference in the in-spray and 
in-chamber release fraction data at large aerosol diameters; however, divergence appears to become significant above $50 \mu \mathrm{m}$. This change may be a result of increased inertia and settling rates because of increased aerosol bulk density (relative to water). This, and other such simulant effects, are discussed in Chapter 8 of this report. Another key difference is that the slopes of the in-chamber and in-spray release fractions do not appear to approach the same value at low aerosol sizes. This difference derives from an apparent change in slope in the in-chamber data around $40 \mu \mathrm{m}$; discussion in Chapter 9 attributes this change in slope to the presence of clay particulates, as the slope inflection point occurs in the region where the clay particle size becomes comparable to the aerosol size. Extrapolation of the Rosin-Rammler curve that bounds in-spray data to small droplet sizes $(\sim 10 \mu \mathrm{m})$ suggests improved in-chamber performance relative to in-spray data such that there is less difference between the two methods. Caution should be taken when interpreting this result as the in-spray bound is projected from data where the aerosol size is much larger than the clay particulate. If actual in-spray measurements were possible in the range of 10 to $50 \mu \mathrm{m}$, it is expected that they would show a similar inflection (if inflection does indeed result from the presence of clay particles).

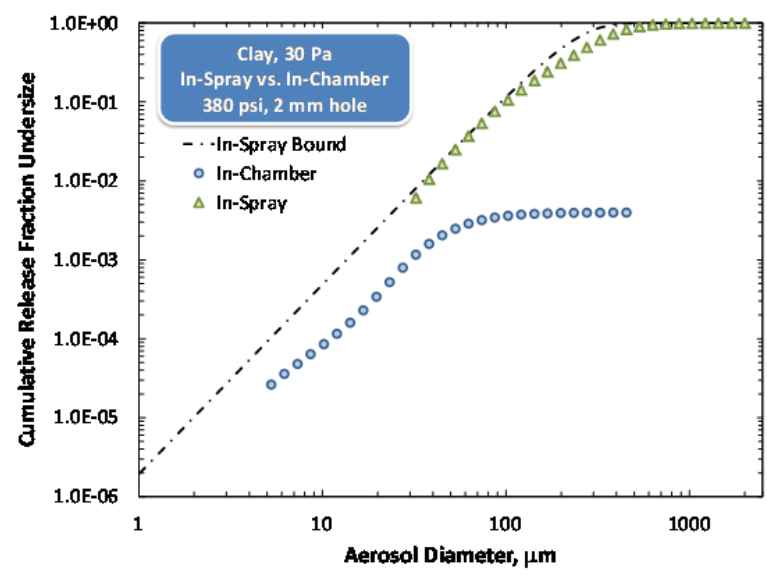

(a) $-2 \mathrm{~mm}$ hole

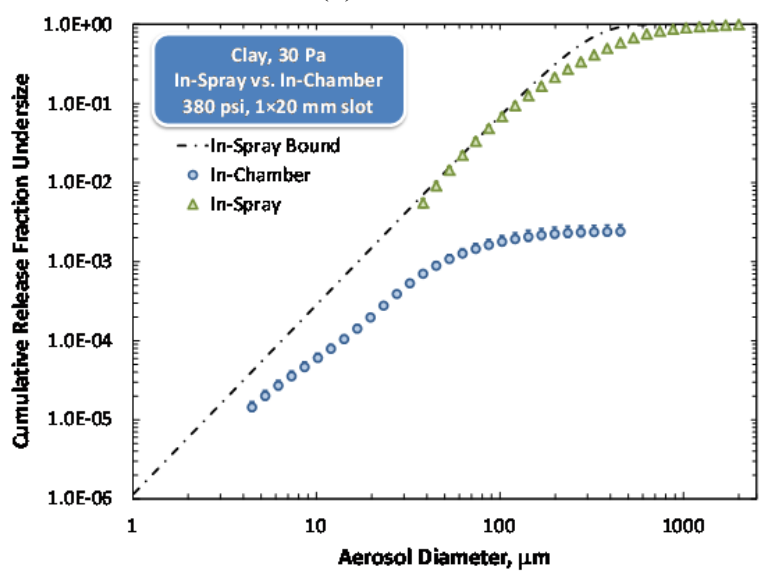

(c) $-1 \times 20 \mathrm{~mm}$ slot

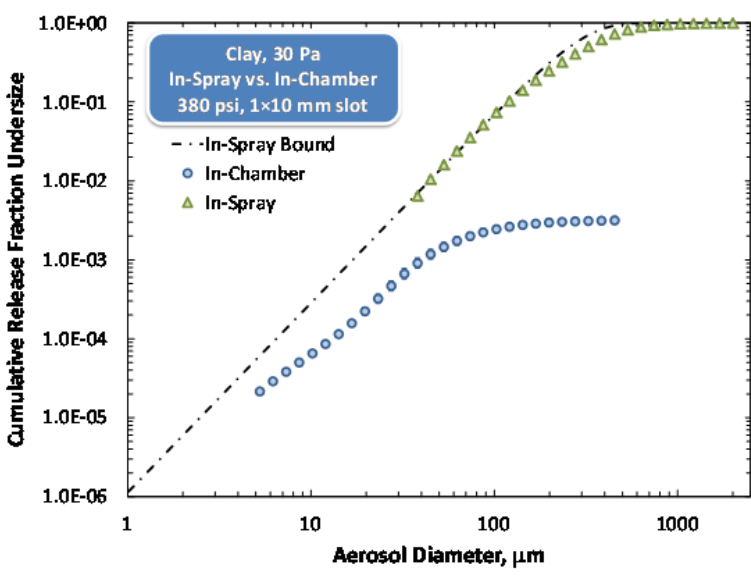

(b) $-1 \times 10 \mathrm{~mm}$ slot

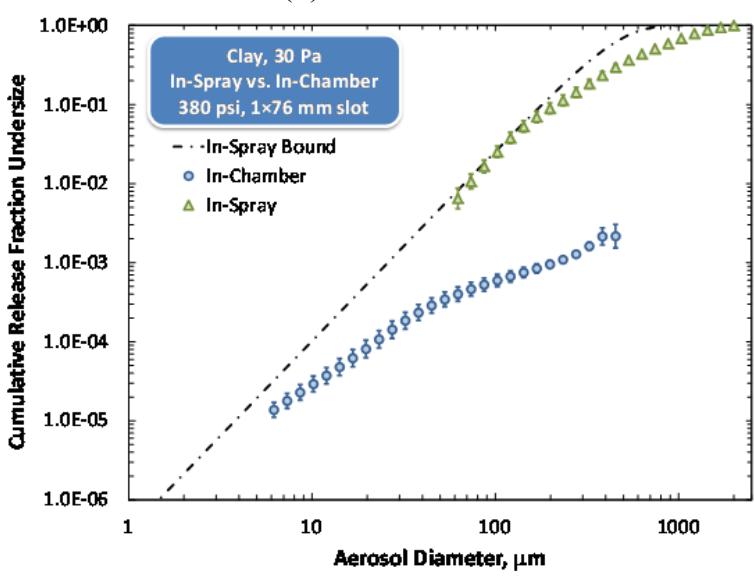

(d) $-1 \times 76 \mathrm{~mm}$ slot

Figure 7.11. Comparison of In-Spray (at $\sim 12 \mathrm{ft}$ from the spray header) and In-Chamber Release Fractions Measured for 30 Pa Clay for (a) a $2 \mathrm{~mm}$ Hole, (b) a $1 \times 10 \mathrm{~mm}$ Slot, (c) a $1 \times 20 \mathrm{~mm}$ Slot, and (d) a $1 \times 76 \mathrm{~mm}$ Slot. All sprays are generated at $380 \mathrm{psig}$. Both in-spray and in-chamber measurements shown were captured in the $20 \mathrm{ft}$ chamber (no spray extensions). 
The water and clay simulant in-spray and in-chamber comparisons presented above show significant differences in release fraction measurements made by the two methods. As stated previously, in-spray release fractions are expected to over-estimate actual release. However, in-spray measurements cannot typically measure droplets in the size range of interest because of instrument precision limitations below cumulative volume fractions of $0.5 \times 10^{-2}$. Relative to in-spray configurations, release fraction measurements made in in-chamber configurations appear to be subject to significant loss and under-estimation of aerosol fractions at droplets sizes $>100 \mu \mathrm{m}$. However, because large aerosols are not present at in-chamber aerosol sampling locations, the release fraction of smaller (10 to $100 \mu \mathrm{m})$ aerosols can be accurately assessed. At aerosol sizes where in-spray and in-chamber measurements overlap and are not subject to significant loss from settling or inertia, comparison indicates that in-spray release fraction measurements are approximately three times higher than their corresponding in-chamber measurements. This difference probably results from measurement biases that reduce in-chamber release fractions (humidity and sampling bias) while increasing in-spray release fractions (spatial size distribution bias).

Correction of measurement bias for either in-chamber or in-spray release fraction results to yield a true "un-biased" result is not possible, because the information needed to correct for biases is physically complex to model (such as chamber turbulence, which impacts aerosol settling) or was not measured during testing (such as the droplet velocity distribution needed to correct for spatial bias). This information is not necessarily needed to yield a conservative release fraction result over the size range of interest $(10$ to $100 \mu \mathrm{m})$. As stated previously, in-spray release fraction measurements are expected to over-estimate actual release. If the in-spray measurement is used as a guide for correcting in-chamber release fractions, then such correction should render in-chamber measurements as-conservative as in-spray measurements and would eliminate the need to determine the true aerosol release fraction. Comparison of in-spray and in-chamber measurements indicates a multiplicative correction factor of three to in-chamber data will typically render them as conservative as in-spray measurements. This assertion forms the basis of worst-case release fraction analyses performed in Section 10. It should be noted that this correction does not account for humidity bias, which requires its own correction factor (see Appendix A).

\subsection{Conclusions}

In this chapter, tests evaluating the impact of position on in-chamber and in-spray release fractions were presented. The dependence of in-chamber release fraction on measuring position was evaluated through uniformity testing. Chamber uniformity was assessed through aerosol size distribution, concentration, and apparent generation rate. Uniformity testing indicates that aerosol size distribution varies as a function of test position. Several mechanisms were postulated to explain changes observed in aerosol size distribution with position. These were:

- A decrease in droplet size with increasing radial distance from the center of the jet, such that the finest droplets are located near the periphery of the jet

- Preferential back-transport of fine aerosol and removal of larger droplets in the back-transport current by settling. Here, back-transport of aerosol results from circulation currents (and counter-currents) set up by the spray as it passes through the center of the chamber and entrains air during break-up 
- Coalescence of droplets as they travel down the chamber and subsequent inertial capture (which prevents or limits back-transport, as described in the previous bullet)

- Increased sampling of the core of the spray as it fans out and engulfs analyzers in downstream positions.

These mechanisms are not necessarily exclusive, and may contribute individually to the overall trends observed. Aerosol concentration and generation rate data generally suggest that the chamber is uniformly mixed at test locations above the spray for 10 and $32 \mu \mathrm{m}$ droplets. The concentration and generation rate of larger $(102 \mu \mathrm{m})$ droplets was uniform downstream of the spray, but was reduced (relative to downstream locations) above the spray header at the largest test height ( $21 \mathrm{in}$. above the orifice). All uniformity tests were performed with the $2 \mathrm{~mm}$ hole at $380 \mathrm{psig}$. The location trends observed in the $102 \mu \mathrm{m}$ droplets suggests that the turbulent mixing energy provided by uniformity test spray was insufficient to suspend droplets near the top of the chamber. These measurements indicate that standard in-chamber test locations (which employ aerosol sampling locations 21 in. above the orifice) will be subject to loss of large droplets, leading to inaccurate assessments of release fractions for droplets larger than $100 \mu \mathrm{m}$ (based on available data). The exact size range impacted by large-droplet settling is expected to be dependent on the available turbulence, such that sprays larger than that tested in uniformity evaluations may not observe loss until droplet sizes are larger than $102 \mu \mathrm{m}$. This limitation only impacts generation rate estimates for large droplets. For smaller droplets (i.e., 10 to $\sim 80 \mu \mathrm{m}$ ), generation rate (and thus release fraction) appears to be relatively insensitive to measurement location. These conclusions are consistent with findings from similar uniformity tests performed in Phase I large-scale testing (Schonewill et al., 2012).

Tests aimed at evaluating changes in in-spray release fraction as a function of measuring location observed the following behavior:

- For water, sprays generated from the $2 \mathrm{~mm}$ orifice exhibit increasing droplet size with increasing distance from the orifice. The in-spray droplet size distribution generated by spraying water from the $1 \times 10$ and $1 \times 20 \mathrm{~mm}$ slots exhibit very little change with increasing distance from orifice (with no clear trend toward larger or smaller droplets). Water sprays generated through the $1 \times 76 \mathrm{~mm}$ orifice show an in-spray size distribution that becomes finer with increasing distance from orifice.

- For clay, in-spray release fraction (i.e., the droplet size distribution) generally becomes coarser with increasing distance. An initial break-up of droplets, which produces a fine, concentrated distribution of aerosol immediately near the orifice at positions close to the spray header.

For most tests cases, in-spray release fraction either does not change substantially with distance, or if it does change, the release fraction contribution is shifted to larger aerosol diameters. This increase in overall aerosol size can be attributed to droplet coalescence or preferential sampling of large aerosols that settle to the bottom of the chamber (which is a result of following the downward trajectory of the jet). In-spray trends are anomalous for water sprays generated by the $1 \times 76 \mathrm{~mm}$ slot and indicate that the water droplets generated by this spray decrease significantly in size with increasing distance from the orifice. From available data it is not clear if this behavior is associated with the $1 \times 76 \mathrm{~mm}$ orifice itself, the unique test locations employed in testing of the $1 \times 76 \mathrm{~mm}$ slot with water, or near complete obscuration of the laser by the $1 \times 76 \mathrm{~mm}$ sprays. 
In-spray release fractions can show significant variation with respect to measurement location. Selection of a "best" measurement location for in-spray size distributions (with respect to their use for analyses in this report and elsewhere) is not straightforward. Proper selection requires consideration of the specific scenario being evaluated (and potentially the spray distances involved). For the purpose of comparison and analysis in later chapters of this report, the in-spray PSD measured $\sim 12 \mathrm{ft}$ downstream of the spray header is taken as the representative aerosol size distribution generated by high-pressure spray. This selection is based on the assumption that spray at this distance allows for the greatest maturation of the size distribution (as a result of droplet coalescence). As mentioned previously, $1 \times 76 \mathrm{~mm}$ in-spray water data show unique behavior and, as such, are handled differently than the bulk of in-spray measurements. For performing simulant comparisons, the $12.5 \mathrm{ft}, 1 \times 76 \mathrm{~mm}$ slot data are used; for scaling and bounding release fraction analyses presented in Chapter 10 of this report, $20 \mathrm{ft}, 1 \times 76 \mathrm{~mm}$ data are used (because they provide the most conservative measurement of in-spray release fraction for the given condition).

Finally, direct comparison of in-spray and in-chamber release fraction results demonstrates difficulties with both sets of data. In-spray release fractions may be considered conservative relative to the true release because of spatial size distribution measurement bias (Bartell et al. 1991); however, in-spray measurements cannot reproducibly measure the contribution of aerosols that fall below cumulative volume fractions of $0.5 \times 10^{-2}$. In terms of spray leak analysis, this limits the usable size range of in-spray measurements to $50 \mu \mathrm{m}$ and greater (in the best circumstances observed in Phase II testing). Because large aerosol droplets are not present in regions of the large scale test chamber sampled for in-chamber measurements, in-chamber release fractions are not subject to this limitation and typically provide release fraction measurements for the entire size range of interest (10 to $100 \mu \mathrm{m}$ ). In-chamber measurements are subject to bias from droplet mixing and sampling limitations, which render them less conservative than in-spray measurements in regions where the two methods overlap. Because in-spray measurements are expected to be conservative (i.e., larger than) the actual release, they can be used as a guide for correcting in-chamber release fractions such that they are "as-conservative" as in-spray measurements. Comparison of in-spray and in-chamber measurements indicates a multiplicative correction factor of three to in-chamber data will typically render them as conservative as in-spray measurements. This assertion forms the basis of worst-case release fraction analyses performed in Section 10. It should be noted that this correction does not account for humidity bias, which requires its own correction factor (see Appendix A). 


\subsection{Evaluation of Simulant Release Fractions}

The WTP model, as described in Section 5.5.5 of this report, was developed to evaluate the consequences of breaches of transfer lines that carry radioactive tank waste slurries at the Waste Treatment and Immobilization Plant. While its intended use is to analyze aerosolization of waste slurries, the WTP model does not have any terms that directly account for the solid content of the transfer material. Instead, the impact of increased solids content is captured indirectly through terms that account for changes in bulk fluid density and viscosity. Application of the WTP model to Newtonian liquids is straightforward, as the interpretation of the fluid properties needed for model calculations is more-or-less unchanged. For example, a single value for fluid viscosity can be determined and input to the model for a Newtonian slurry. Evaluation of non-Newtonian slurries, however, introduces challenges, as there are no model terms that account explicitly for finite fluid yield stress (or other impacts that derive from structuring of particles or colloidal interactions). The impact of fluid can be assessed by using an apparent viscosity, which will depend on the shear rate experienced by the fluid as it leaves the breach, and we postulate that, at sufficiently high breach pressures, the apparent viscosity will approach the fluid consistency (i.e., the infinite shear apparent viscosity).

Because the WTP model does not lend itself to direct application to slurry systems, evaluations of fraction aerosolization of both Newtonian and non-Newtonian systems were performed in Phase I spray release testing at PNNL. Small-scale testing (Mahoney et al. 2013) evaluated dispersions composed of boehmite and gibbsite solids in water at varying concentrations and of a chemical simulant representative of leach-and-washed WTP waste streams. Large-scale testing (Schonewill et al. 2012) evaluated dispersions of boehmite solids in water at two concentrations ( 8 and $20 \mathrm{wt} \%$ ). Definitive change in the fractional aerosolization with increased solids loadings was not observed during Phase I testing. Tests with $8 \mathrm{wt} \%$ slurry indicated that the presence of solids generally reduced release fraction, whereas tests with more concentrated $20 \mathrm{wt} \%$ slurry showed a slight increase in the release fraction. Likewise, testing of the non-Newtonian chemical simulant failed to show a definite trend, with the release fraction sometimes increasing and at other times decreasing with increasing release pressure. Because of the variable response observed in the release fraction to changes in solids content and fluid rheology, PNNL recommended additional spray release testing to better define the functionality of release fraction with increasing solids content for non-Newtonian simulants. To this end, Phase II large-scale testing included

release fraction evaluations for two non-Newtonian simulants composed of an 80:20 mixture by weight of kaolin and bentonite dry clay powders slurried in water at different concentrations to create test fluids with different rheologies.

In this chapter, we discuss the results of simulant testing with non-Newtonian materials, and describe their consequence for safety-basis analysis with the WTP model. Evaluations of non-Newtonian simulants included both "in-chamber" and "in-spray" testing with sprays generated at 100, 200, and 380 psig through $2 \mathrm{~mm}, 1 \times 10 \mathrm{~mm}, 1 \times 20 \mathrm{~mm}$, and $1 \times 76 \mathrm{~mm}$ orifices. Tests were accompanied by extensive release fraction testing with water using the same orifice and driving pressures to better define "baseline" release fraction functionality with pressure and orifice size/configuration. This chapter begins with a comparison of $6 \mathrm{~Pa}$ Clay and $30 \mathrm{~Pa}$ Clay release fractions to water release fractions for equivalent in-chamber and in-spray configurations. Next, the response of non-Newtonian simulant release fraction to changing solids concentration and fluid rheology is evaluated. Throughout this section, predictions of release fraction for the materials tested are made using the WTP model. These model predictions can be used to evaluate how the WTP model captures trends in the data with changing process variables (such as 
pressure or rheology). However, care should be taken when evaluating the performance of the WTP model with respect to the magnitude of results (and thus inferring how "conservative" the WTP is with respect to safety analyses), because the release fraction results determined using in-chamber methods are subject to method and humidity bias (see Section 7 and Appendix A for details). In overall terms, assessment of in-chamber measurement accuracy indicates that the in-chamber results may be low relative to the true release fraction. As such, comparisons showing the WTP model prediction as being conservative relative to in-chamber measurements of release fraction should be treated as inconclusive. On the other hand, cases where the WTP predictions fall below measured in-chamber release fractions can indicate under-performance of the WTP model, with the caveat that model performance may be worse than is shown.

\subsection{Evaluation of Phase II Simulant Release Fractions}

Phase II large-scale testing activities evaluated the release fraction of three different simulants: water, $6 \mathrm{~Pa}$ Clay, and $30 \mathrm{~Pa}$ Clay. Simulant evaluations included in-chamber testing in the $20 \mathrm{ft}$ chamber configuration and in-spray testing. For both in-chamber and in-spray testing, PNNL evaluated simulant release fractions for a $2 \mathrm{~mm}$ hole and $1 \times 10 \mathrm{~mm}, 1 \times 20 \mathrm{~mm}$, and $1 \times 76 \mathrm{~mm}$ horizontal slot orifices at release pressures of 100,200 , and $380 \mathrm{psig}$. The results of simulant release fraction testing are shown in Figure 8.1 through Figure 8.4, with each figure showing the results for testing of a given orifice. Individual figures show in-chamber release fractions (on the left-hand side of the figure) and in-spray release fractions (on the right-hand-side of the figure) for each of the three test pressures. All in-spray data shown in this section were taken at $\sim 12 \mathrm{ft}$ downstream of the spray orifice and correspond to the in-spray aerosol state approximately $10 \mathrm{~s}$ after the start of spray. ${ }^{1}$ For all cases, the WTP model is calculated as described in Section 5.5.5 of this report using the appropriate values for simulant density and viscosity/consistency. Presentation of both in-chamber and in-spray data has been limited to avoid size fractions for which the precision of Malvern Insitec-S analyzer measurements is reduced. Analysis of Malvern Insitec-S analyzer performance in Phase I (Schonewill et al. 2012) found that particle size measurements were reproducible at diameters corresponding to cumulative volume fractions of $0.5 \times 10^{-2}$ or greater. As a result, in-spray data are only presented for cumulative release fractions greater than or equal to $0.5 \times 10^{-2}$. In-chamber measurements of release fraction correspond to the initial size distribution of the spray multiplied by the fractional aerosolization of the total spray release. For this reason, presentation of in-chamber data is not subject to the same requirements as in-spray data. When presenting in-chamber data in this report, the following criteria have been applied:

\footnotetext{
${ }^{1}$ In-spray data have been time averaged over $4 \mathrm{~s}$ to reduce measurement uncertainty. The averaging period ranges from 8 to $12 \mathrm{~s}$ after the start of spray. The elapsed time associated with in-spray data is the mid-point of this range $(10 \mathrm{~s})$.
} 


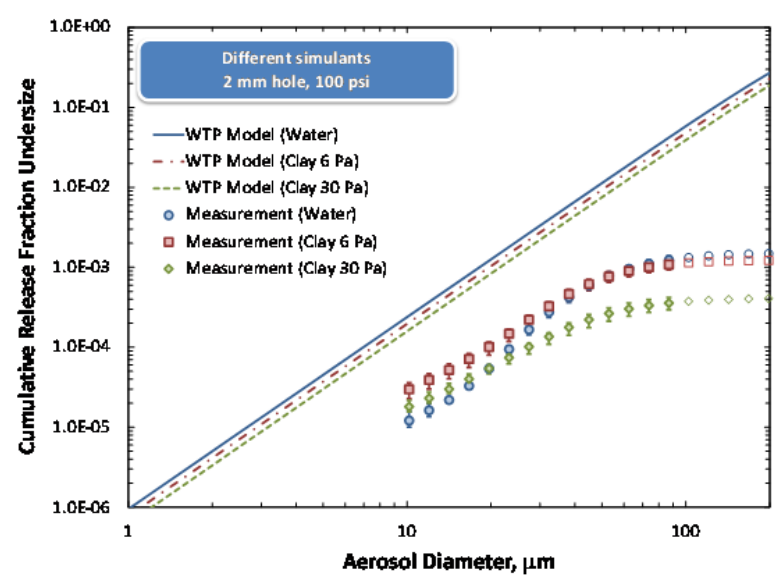

(a) - In-Chamber 100 psig, $2 \mathrm{~mm}$ hole

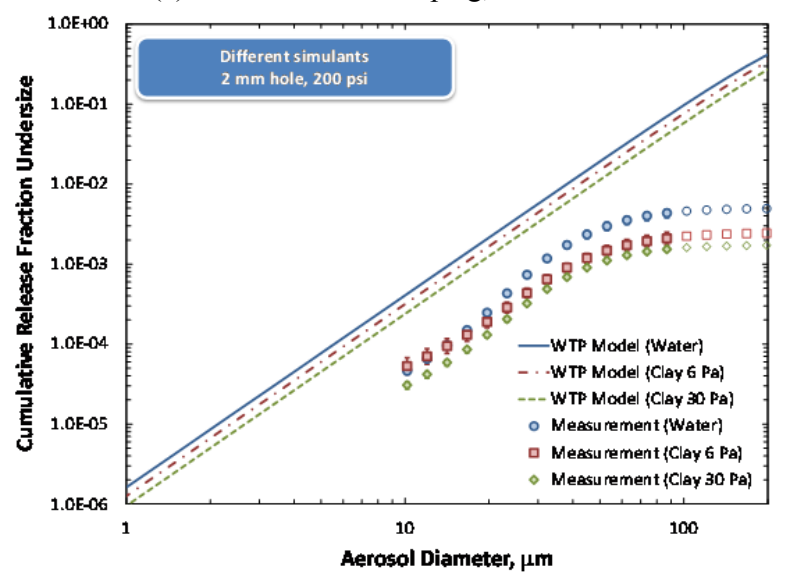

(c) - In-Chamber 200 psig, $2 \mathrm{~mm}$ hole

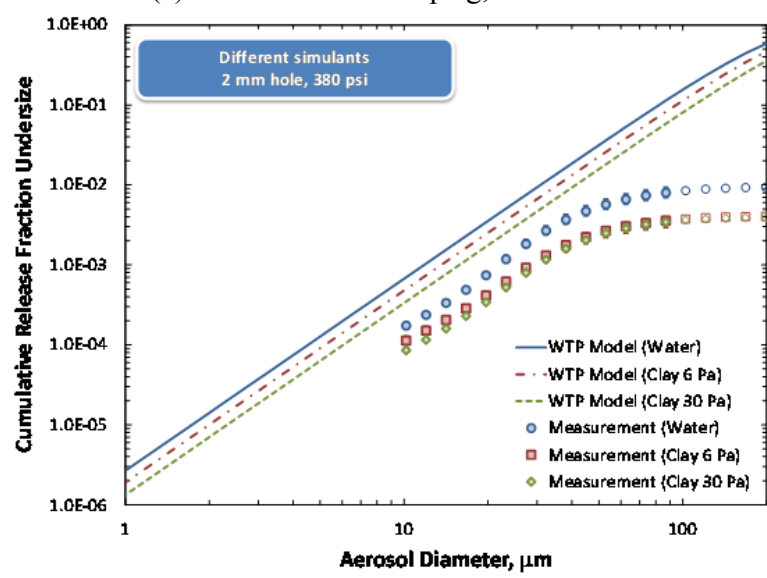

(e) - In-Chamber 380 psig, $2 \mathrm{~mm}$ hole

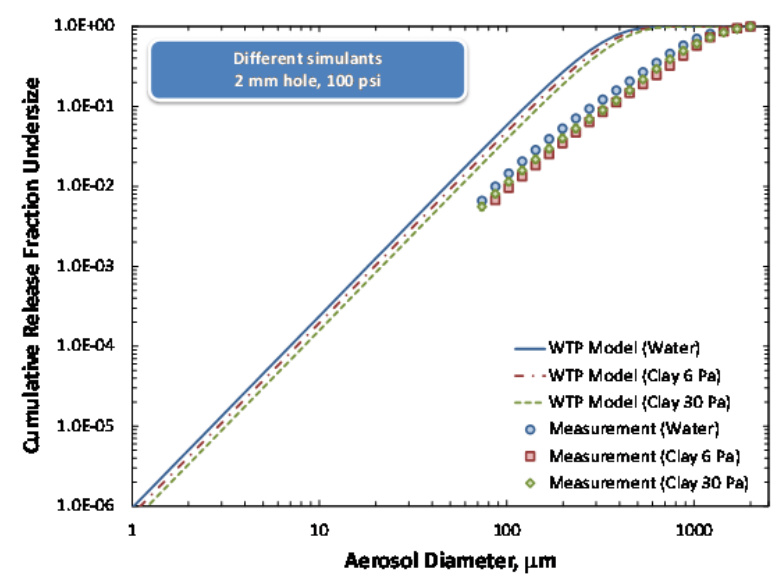

(b) - In-Spray 100 psig, $2 \mathrm{~mm}$ hole

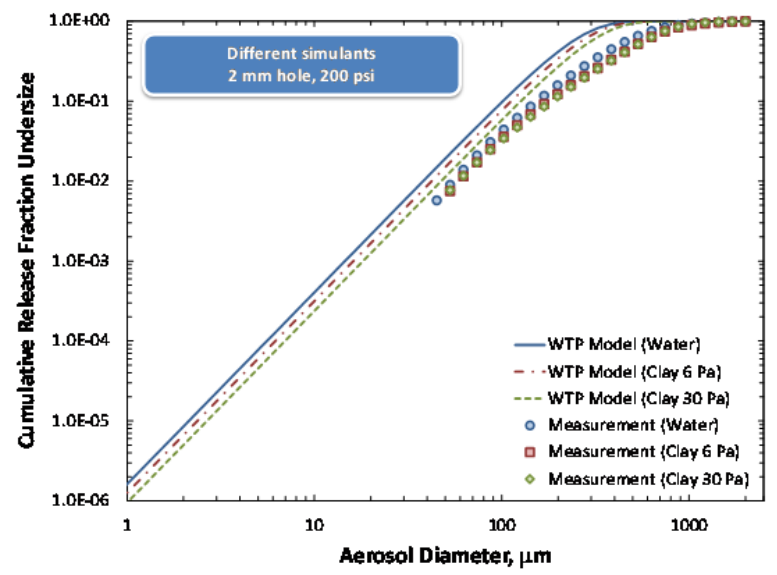

(d) - In-Spray 200 psig, $2 \mathrm{~mm}$ hole

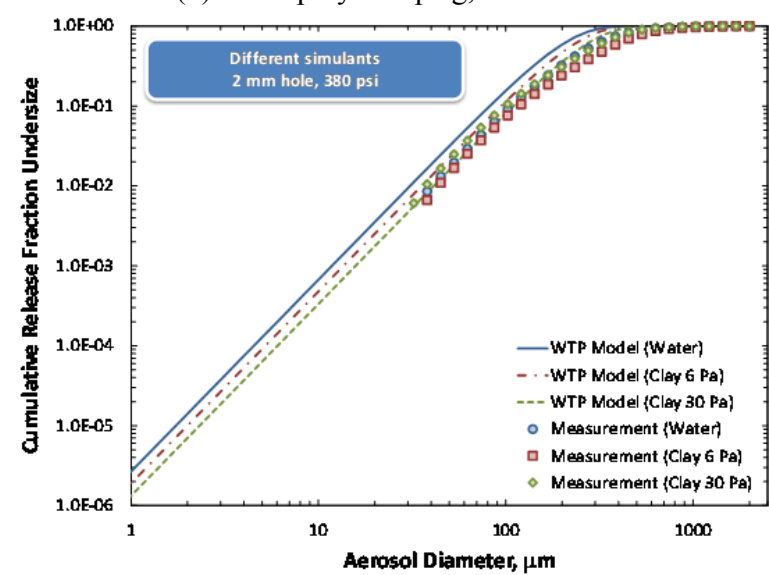

(f) - In-Spray 380 psig, $2 \mathrm{~mm}$ hole

Figure 8.1. Release Fraction as a Function of Aerosol Size for Water, $6 \mathrm{~Pa}$ Clay, and $30 \mathrm{~Pa}$ Clay for a $2 \mathrm{~mm}$ Round Hole. In-chamber results are shown for (a) $100 \mathrm{psig}$, (c), $200 \mathrm{psig}$, and (e) 380 psig on the left-hand side of the figure. In-spray results are shown for (b) 100 psig, (d) $200 \mathrm{psig}$, and (f) $380 \mathrm{psig}$ on the right-hand side of the figure. Water was tested in the $39 \mathrm{ft}$ chamber; both clay simulants were tested in the $20 \mathrm{ft}$ chamber. 


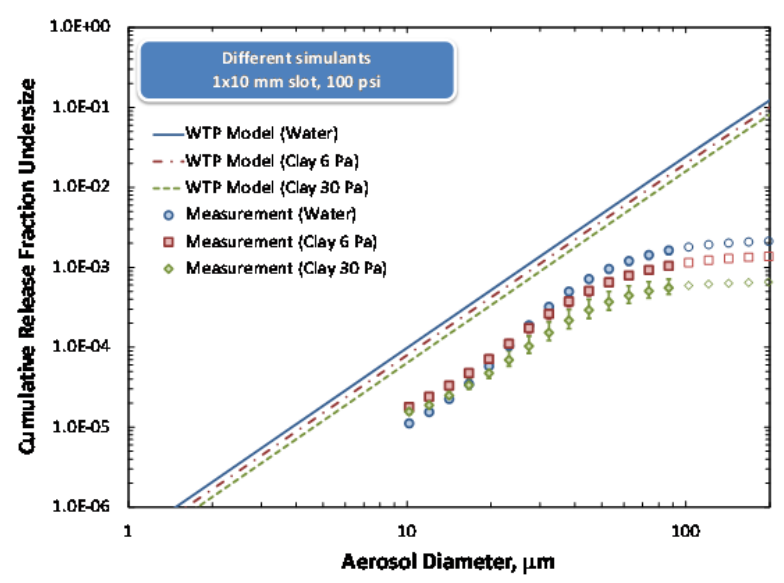

(a) - In-Chamber 100 psig, $1 \times 10 \mathrm{~mm}$ slot

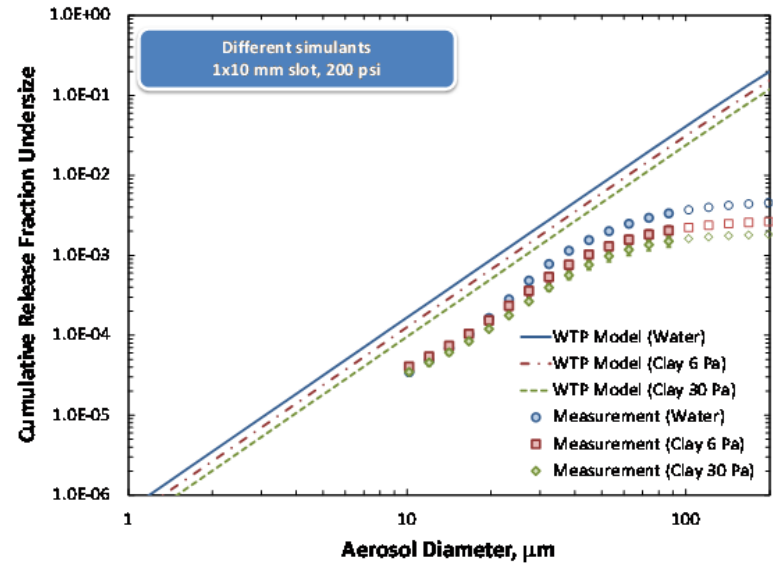

(c) - In-Chamber 200 psig, $1 \times 10 \mathrm{~mm}$ slot

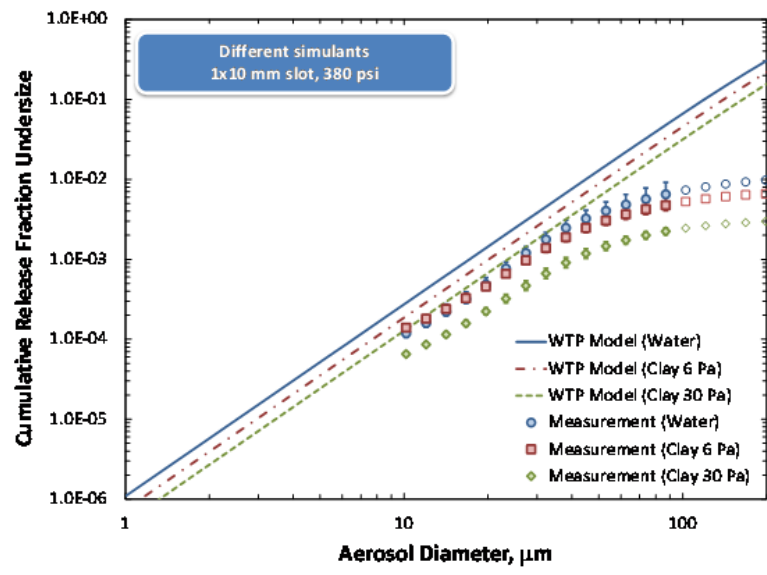

(e) - In-Chamber $380 \mathrm{psig}, 1 \times 10 \mathrm{~mm}$ slot

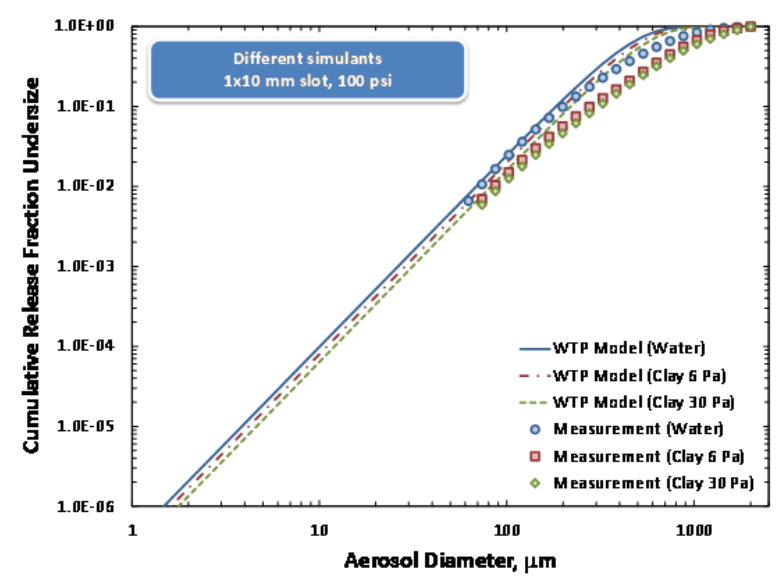

(b) - In-Spray 100 psig, $1 \times 10 \mathrm{~mm}$ slot

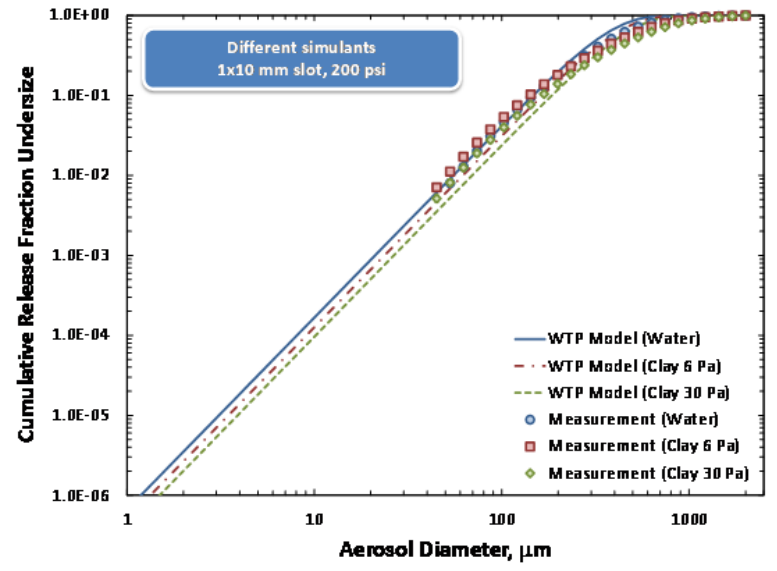

(d) - In-Spray 200 psig, $1 \times 10 \mathrm{~mm}$ slot

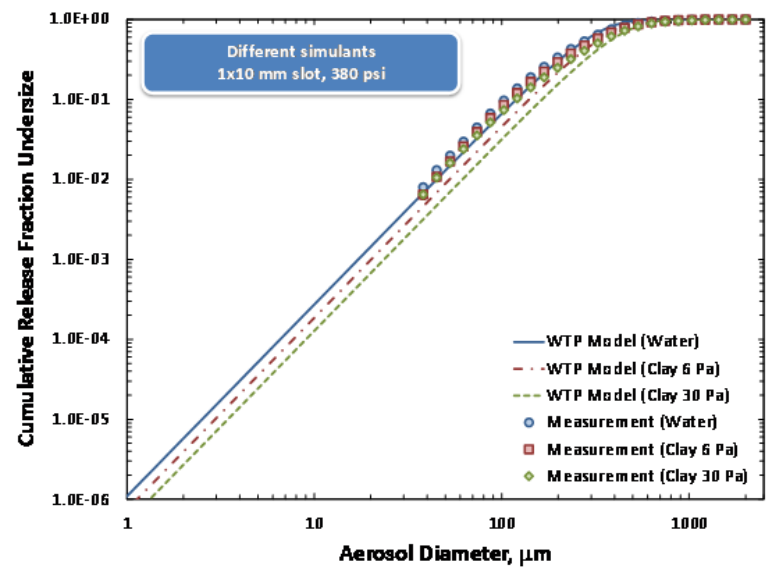

(f) - In-Spray 380 psig, $1 \times 10 \mathrm{~mm}$ slot

Figure 8.2. Release Fraction as a Function of Aerosol Size for Water, $6 \mathrm{~Pa}$ Clay, and $30 \mathrm{~Pa}$ Clay for a $1 \times 10 \mathrm{~mm}$ Slot. In-chamber results are shown for (a) $100 \mathrm{psig}$, (c) $200 \mathrm{psig}$, and

(e) $380 \mathrm{psig}$ on the left-hand side of the figure. In-spray results are shown for (b) $100 \mathrm{psig}$, (d) $200 \mathrm{psig}$, and (f) $380 \mathrm{psig}$ on the right-hand side of the figure. Water was tested in the $39 \mathrm{ft}$ chamber; both clay simulants were tested in the $20 \mathrm{ft}$ chamber. 


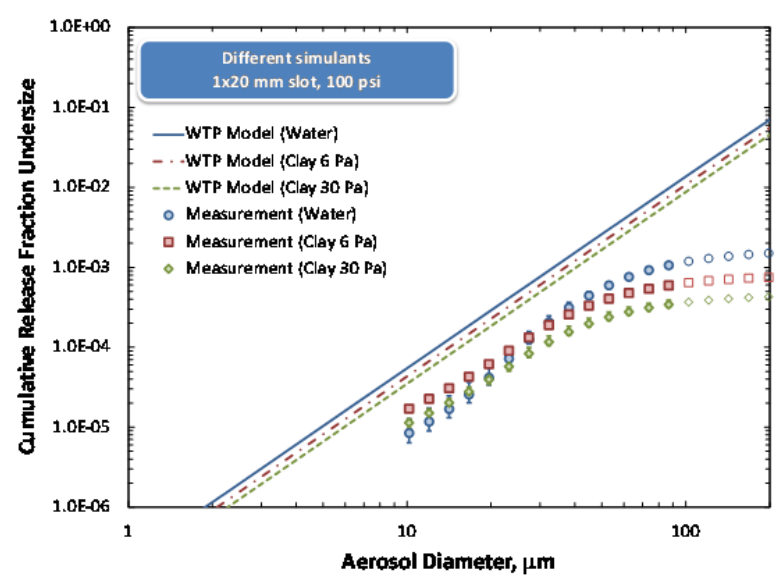

(a) - In-Chamber 100 psig, $1 \times 20 \mathrm{~mm}$ slot

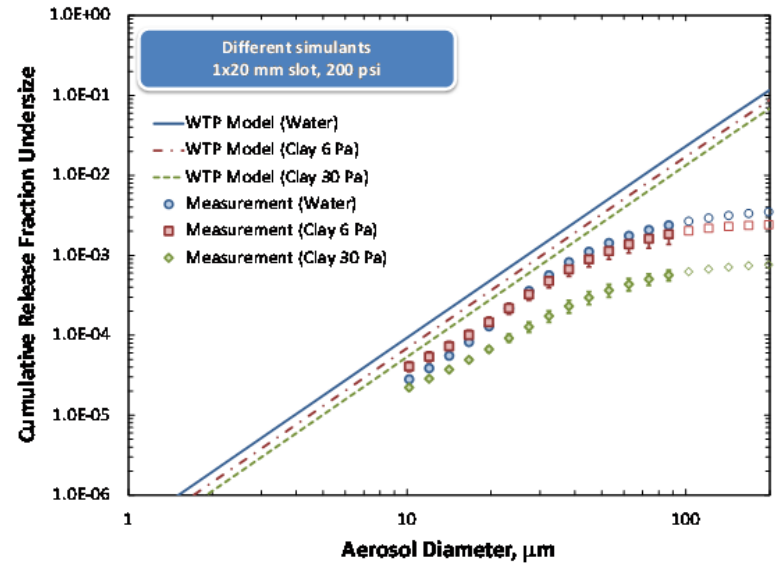

(c) - In-Chamber 200 psig, $1 \times 20 \mathrm{~mm}$ slot

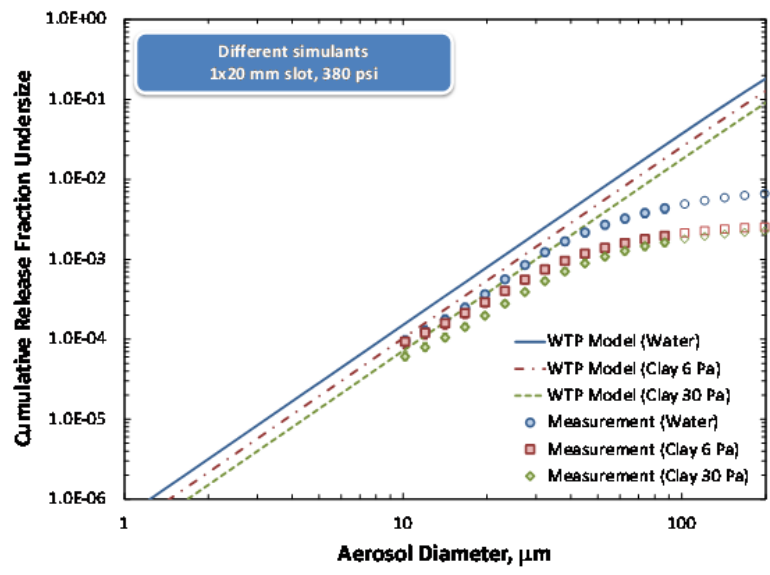

(e) - In-Chamber $380 \mathrm{psig}, 1 \times 20 \mathrm{~mm}$ slot

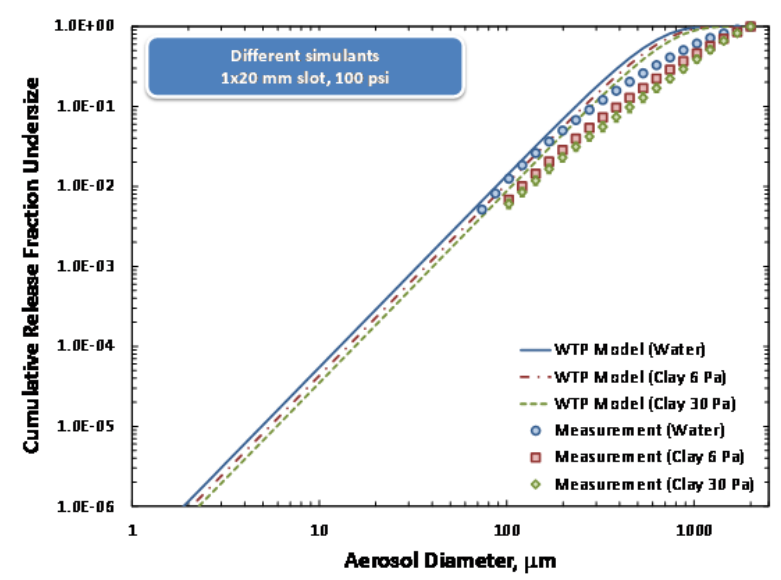

(b) - In-Spray 100 psig, $1 \times 20 \mathrm{~mm}$ slot

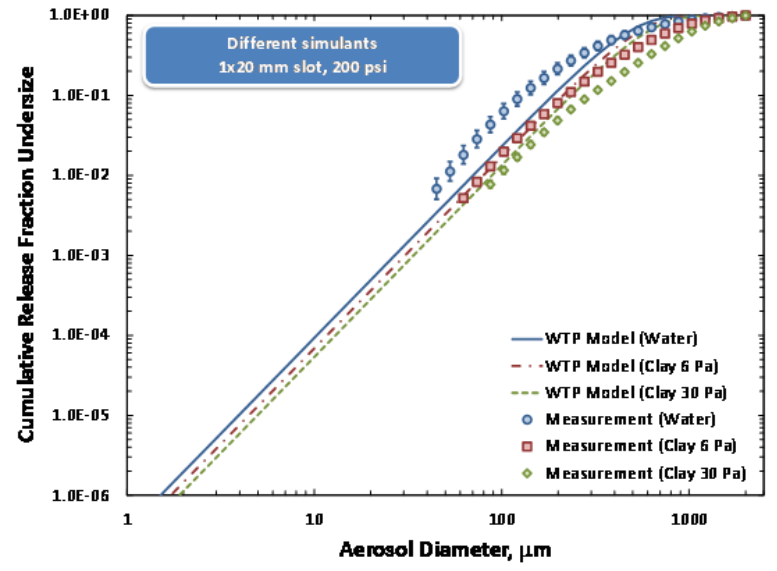

(d) - In-Spray 200 psig, $1 \times 20 \mathrm{~mm}$ slot

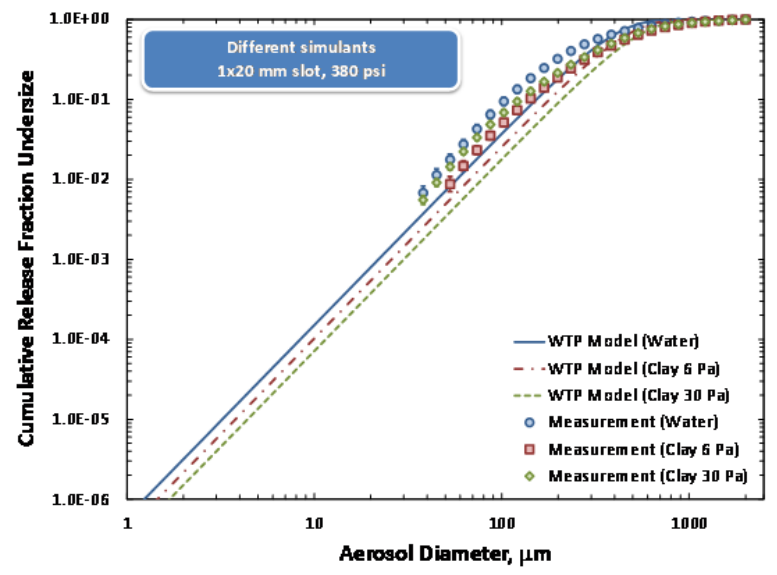

(f) - In-Spray 380 psig, $1 \times 20 \mathrm{~mm}$ slot

Figure 8.3. Release Fraction as a Function of Aerosol Size for Water, $6 \mathrm{~Pa}$ Clay, and $30 \mathrm{~Pa}$ Clay for a $1 \times 20 \mathrm{~mm}$ Slot. In-chamber results are shown for (a) $100 \mathrm{psig}$, (c), $200 \mathrm{psig}$, and (e) $380 \mathrm{psig}$ on the left-hand side of the figure. In-spray results are shown for (b) $100 \mathrm{psig}$, (d) $200 \mathrm{psig}$, and (f) $380 \mathrm{psig}$ on the right-hand side of the figure. Water was tested in the $39 \mathrm{ft}$ chamber; both clay simulants were tested in the $20 \mathrm{ft}$ chamber. 


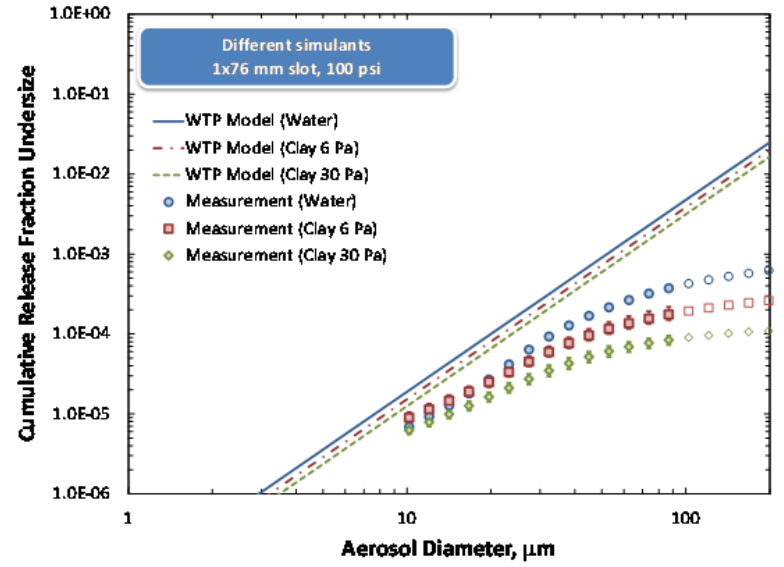

(a) - In-Chamber 100 psig, $1 \times 76 \mathrm{~mm}$ slot

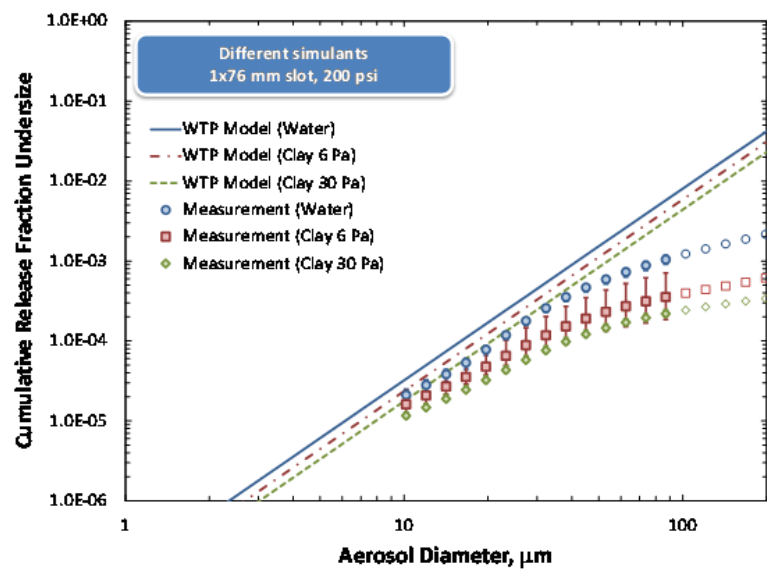

(c) - In-Chamber $200 \mathrm{psig}, 1 \times 76 \mathrm{~mm}$ slot

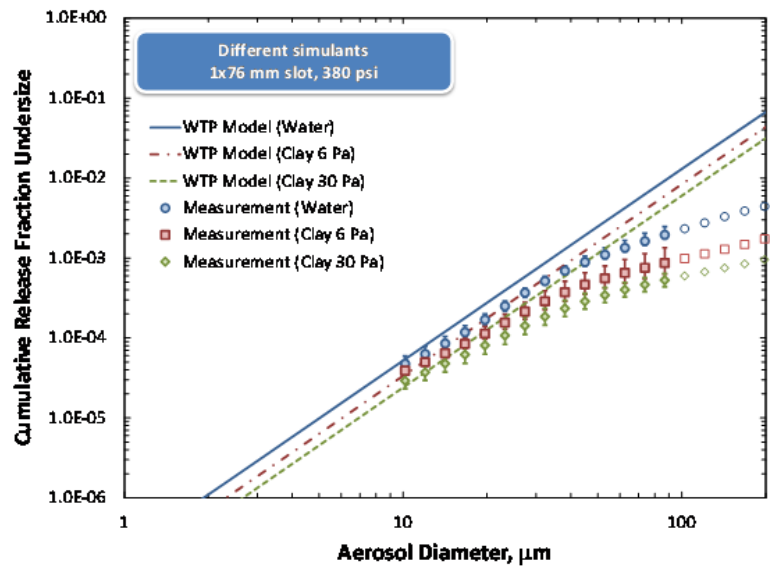

(e) - In-Chamber 380 psig, $1 \times 76 \mathrm{~mm}$ slot

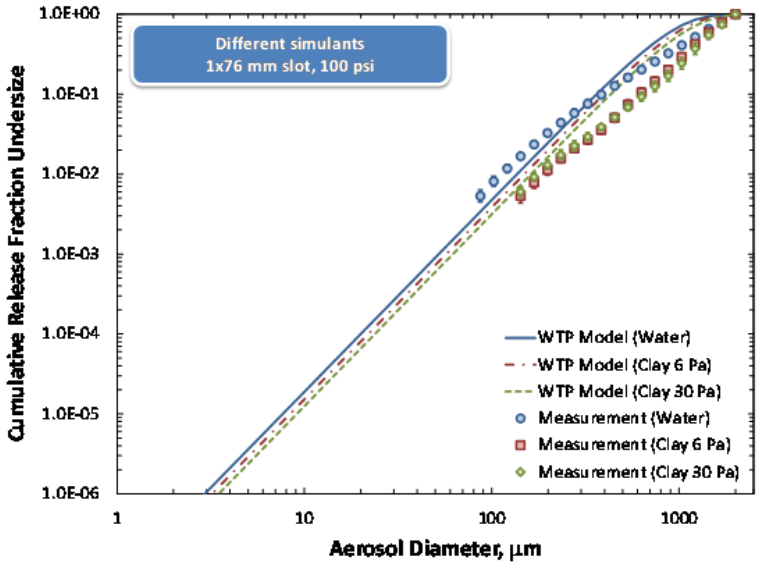

(b) - In-Spray $100 \mathrm{psig}, 1 \times 76 \mathrm{~mm}$ slot

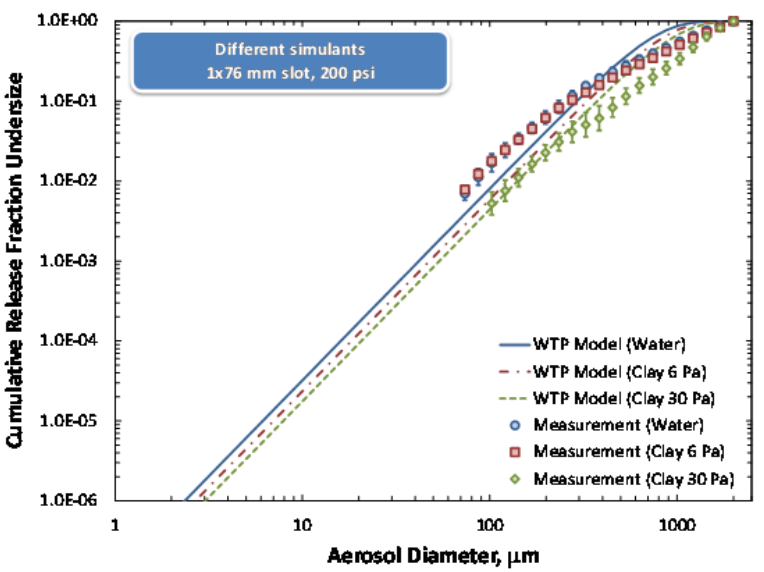

(d) - In-Spray 200 psig, $1 \times 76 \mathrm{~mm}$ slot

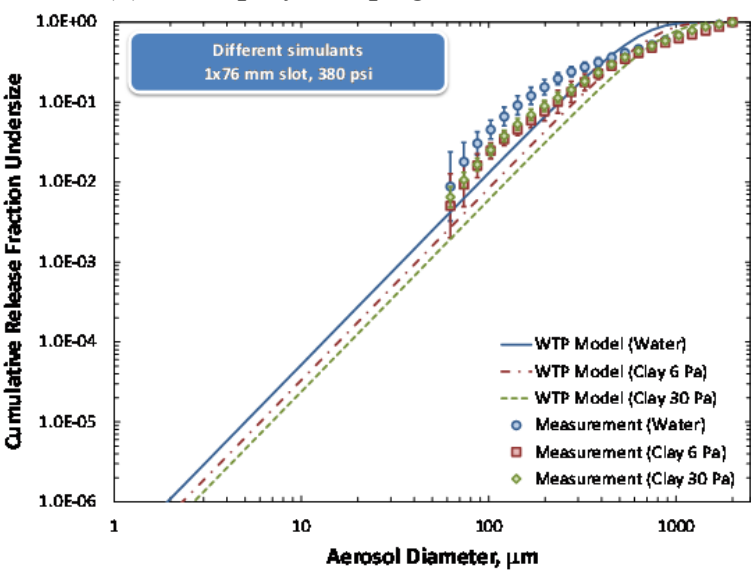

(f) - In-Spray $380 \mathrm{psig}, 1 \times 76 \mathrm{~mm}$ slot

Figure 8.4. Release Fraction as a Function of Aerosol Size for Water, $6 \mathrm{~Pa}$ Clay, and $30 \mathrm{~Pa}$ Clay for a $1 \times 76 \mathrm{~mm}$ Slot. In-chamber results are shown for (a) $100 \mathrm{psig}$, (c) $200 \mathrm{psig}$, and

(e) 380 psig on the left-hand side of the figure. In-spray results are shown for (b) 100 psig, (d) $200 \mathrm{psig}$, and (f) $380 \mathrm{psig}$ on the right-hand side of the figure. Water was tested in the $39 \mathrm{ft}$ chamber; both clay simulants were tested in the $20 \mathrm{ft}$ chamber. 
- Release fractions for diameters below $10 \mu \mathrm{m}$ are not presented. It should be noted that even though release fractions below $10 \mu \mathrm{m}$ are not reported all measureable results are included in the cumulation.

- Release fractions between 10 and $200 \mu \mathrm{m}$ are reported, but with the following caveats:

- Release fractions for diameters $>87 \mu \mathrm{m}$ are presented using open (unfilled) symbols to reflect the fact that these results may be under-estimated as a result of gravitational settling. ${ }^{1}$

- Release fractions between 10 and $100 \mu \mathrm{m}$ that fall below the product of $0.5 \times 10^{-2}$ and the maximum release fraction measured for that test are marked with open (unfilled) symbols to reflect loss of Malvern Insitec-S analyzer precision and reproducibility.

- All other release fractions are reported with filled symbols.

It should be noted that these guidelines differ from those applied in Section 7 in that additional criteria are applied: 1) that $<10 \mu \mathrm{m}$ data are not reported (as they fall outside the range of interest) and 2) that in-chamber release fractions at diameters $>87 \mu \mathrm{m}$ are reported with open symbols (to indicate loss of aerosol as a result of settling or sampling effects). Section 7 employed different guidelines to help assess measurement uncertainty, particularly at larger aerosol diameters. Finally, in-chamber data presented in Figure 8.1 through Figure 8.4 represent the average release fraction values from at least two repeat tests, with uncertainty bars showing the maximum and minimum release fraction values measured for those repeat tests. In-spray data typically represent time-averaged data from a single in-spray test. The averaging period for in-spray data shown in these figures is 8 to $12 \mathrm{~s}$ after the start of spray (with a median time of $10 \mathrm{~s}$ ). The error bars shown on in-spray data are 95 percent confidence intervals. The different approaches used to estimate uncertainty for in-chamber and in-spray data results from the difference in the number of repeat measurements for each set. The limited number of replicates for in-chamber testing does not provide a strong statistical foundation for estimating uncertainty in the mean release fraction. In-spray release fractions typically represent the average of 15 individual in-spray measurements, and as such, there is a stronger basis for applying statistical tests based on observed measurement variation.

If in-chamber data for all orifice and test pressure combinations shown in Figure 8.1 through Figure 8.4 are considered, the same overall behavior of release fraction is observed as the fluid rheology increases (i.e., as the simulant transitions from water to $6 \mathrm{~Pa}$ Clay to $30 \mathrm{~Pa}$ Clay), namely that release fraction is depressed by the increase in rheology or, similarly, the increase in solids loading of the simulant. Specifically, slurry systems generally show cumulative release fractions that are equal to or lower than those for water. This assertion holds for all in-chamber release fraction measurements at

\footnotetext{
${ }^{1}$ Comparison of in-spray and in-chamber data show that in-chamber measurements infrequently see aerosols $>87$ to $200 \mu \mathrm{m}$, even when the Insitec-S system is equipped with the appropriate lens. This indicates that large particles may not be representatively observed by the Malvern Insitec-S analyzer when these instruments are installed in the standard in-chamber measurement locations. Failure to observe large aerosols during in-chamber measurements likely derives from physical mechanisms, such as gravitational settling, not accounted for when considering the average chamber concentration. Evaluation of size measurements from Malvern Insitec-S analyzers located below the spray confirms this assertion (Schonewill et al. 2012). The overall impact is that in-chamber measurements are expected to under-estimate the release fraction contribution at aerosol diameters $>100 \mu \mathrm{m}$. This bias derives from the method in which in-chamber measurements are made, and are lumped into an overall uncertainty category associated with method bias. When presenting results in this report, in-chamber release fraction measurements at diameters greater than $100 \mu \mathrm{m}$ are marked with different (open or unfilled) symbols to reflect loss of accuracy associated with method bias.
} 
particle diameters $\geq 50 \mu \mathrm{m}$. For in-chamber release fractions $<50 \mu \mathrm{m}$, there are several examples where slurry release fractions are higher than those for water, with the majority of cases that show this reversal occurring at lower operating pressures such as 100 or 200 psig. The increased fraction of fine particles observed in the slurry system release fractions relative to water, and more strongly in the $6 \mathrm{~Pa}$ Clay, is attributed to solid clay particles or agglomerates that modify the slope of the release fraction curve at particle sizes comparable to the primary size distribution of the clay solids. In two cases (Figure 8.1A and Figure 8.3A), the reversal is sufficiently large to be considered significant. When considering the majority of in-chamber test cases, water release fraction measurements generally provide the most conservative value for aerosol release fraction at aerosol diameters $>10 \mu \mathrm{m}$. None of the in-chamber measurements exceed the WTP model calculations based on water physical properties.

When all in-spray release fraction measurements are considered, the trends observed confirm the conclusions drawn from in-chamber measurements that increasing slurry rheology or solids content depresses release fraction. For in-spray measurements, the water release fraction curve always is equal to or greater than that for either test slurry (i.e., $6 \mathrm{~Pa}$ Clay or $30 \mathrm{~Pa}$ Clay). The precision cutoff for in-spray measurements (at release fraction less than $0.5 \times 10^{-2}$ ) is typically $\geq 50 \mu \mathrm{m}$, and as a result, in-spray measurements do not evaluate release fractions at aerosol diameters that approach the primary PSD of the clay. Thus, any reversal of release fraction trends with increasing solid content (like those observed for select in-chamber tests) with aerosol diameter $<50 \mu \mathrm{m}$ cannot be assessed using in-spray data. Relative to in-chamber measurements, in-spray release fractions do not show significant decrease with increasing rheology or solids for either $2 \mathrm{~mm}$ hole or $1 \times 10 \mathrm{~mm}$ slot. Release fractions for the larger two slots $(1 \times 20$ and $1 \times 76 \mathrm{~mm})$ tend to show greater decrease with increasing rheology/solids content, yet the decrease is not universal for all test pressures.

As stated previously, in-spray release fractions measured for water are always equal to or greater than those measured for slurry systems, regardless of aerosol size considered. It can be tentatively concluded that in-spray water measurements may be considered bounding and conservative for slurry systems. This conclusion should be approached with caution, as the test simulants employed in large-scale testing are relatively simple (in terms of chemical composition). While the test simulants (water, 6 Pa Clay, and $30 \mathrm{~Pa}$ Clay) employed in large-scale testing have rheologies that define the operating range of the WTP, the chemical makeup of the simulants is simple (single component) and does not accurately reflect the complex chemical speciation (in both solid particulate and suspending phase) expected in real wastes handled by the WTP. The impact of complex chemistry has been evaluated in small-scale chamber aerosol testing, and the results of that testing should be considered when evaluating if water data truly bound release fractions determined from spray leak testing.

It should be noted that in-spray release fractions challenge (i.e., match or exceed) predictions made by the WTP model. The only in-spray data that fall well below WTP model predictions at all aerosol diameters are low-pressure (i.e., 100 and $200 \mathrm{psig}$ ) release fraction measurements for a $2 \mathrm{~mm}$ hole. With both increased orifice area and increased pressure, the release fraction data first match and then exceed WTP model estimates. This indicates that the WTP model, as currently implemented, does not accurately capture release fraction trends with increasing pressure and orifice area. This result will be evaluated in more detail in sections that examine directly the impact of pressure and orifice area on release fraction.

A reduction in in-chamber release fraction for larger aerosols as solids content of the sprayed fluid is increased is expected for several reasons. As solids content increases, increases in both yield stress and 
consistency increase the energy needed to break the core of the spray into droplets. Furthermore, an increase in solids content yields an increase in the bulk density of large droplets. Any loss or apparent loss of droplets that impacts in-chamber measurements will be augmented by an increase in droplet density. Examples of such loss mechanisms include, but are not limited to, inertial impact of large droplets against the splash wall and gravitational settling. It also is important to note that these latter mechanisms should only impact in-chamber measurements, meaning that in-chamber and in-spray measurements should not be expected to show similar changes in size distribution with increased solids content unless the mechanisms driving those changes derive solely from the initial spray break-up mechanism.

The increase in release fraction seen for some in-chamber tests at small aerosol sizes is interesting in that it differs from the large aerosol behavior described in the preceding paragraph. It suggests that some care should be taken in applying inertial and rheological arguments to small aerosol sizes. It seems reasonable that a transition in aerosol generation and loss behavior for simulants containing solid particles would occur when aerosol diameters approach those of the slurry particulate. And indeed, the change in aerosol behavior observed in clay simulants occurs around $50 \mu \mathrm{m}$, which is roughly equivalent to the $\mathrm{d}(95)$ of both $6 \mathrm{~Pa}$ Clay and $30 \mathrm{~Pa}$ Clay simulants (which is $\sim 45 \mu \mathrm{m}$ ). At this and smaller sizes, individual aerosol droplets produced by spraying the clay slurry will become less representative of the bulk clay material and more representative of individual clay particulate or agglomerates.

It should be noted that the primary particle size only limits aerosolization for solids, and that liquid droplets can still be produced in the size range encompassed by the solid PSD and lower. The solid particle size does represent an important limit with respect to evaporation. Indeed, it is reasonable to expect that evaporation of liquid from bulk slurry droplets or partially wet slurry particles will be limited by the size of the primary particle and solid agglomerate. One consequence of this behavior is that dependence of in-chamber release fraction on relative humidity (and as such, the magnitude of humidity bias) for slurries should be different from that for water. Humidity testing (described in Appendix A of this report) confirms this assertion.

Overall, the differences in clay and water behavior at small aerosol sizes indicate a need to consider not only the bulk physical properties of the slurry but also the size distribution of slurry particles that make up the aerosolized slurry. For the current set of large-scale test results, the increase in release fraction observed at small $(<50 \mu \mathrm{m})$ aerosol diameters is generally largest for the $6 \mathrm{~Pa}$ Clay. When the $30 \mathrm{~Pa}$ Clay is considered, the release fraction generally is reduced at all diameters relative to water. Thus, while there is a size and primary particle effect, it should be stressed that solid particulate size is not the only consideration governing slurry aerosolization.

In conclusion, for the majority of pressures and orifices tested, in-chamber and in-spray test results indicate that the presence of solids and/or increased system rheology either reduces or does not impact cumulative release fractions at aerosol diameters $>50 \mu \mathrm{m}$. In some test cases (in particular those at low test pressures), a slight increase in cumulative release fraction $<50 \mu \mathrm{m}$ was observed when testing simulant slurries. This increase was most notable for size ranges that encompass the primary PSD of the clay solids tested. Given the limited number of repeat measurements for each test condition and that a significant increase in small $(<50 \mu \mathrm{m})$ aerosol release fractions was not observed in all test conditions, it is difficult to evaluate the significance of this increase. Overall, the majority of simulant release fraction 
data indicates that water release fractions are conservative and bound the behavior of release fractions in slurry systems.

\subsection{Parametric Variation of Simulant Release Fraction Data}

In this section, we present the results of our evaluation of the parametric variation of simulant release fraction data for water, $6 \mathrm{~Pa}$ Clay, and $30 \mathrm{~Pa}$ Clay with changes in spray leak pressure and orifice size. Specifically, the focus of this section is to observe how changes in simulant impact the relationship between release fraction and the test pressure and orifice size. For this reason, release fraction measurements in the $20 \mathrm{ft}$ chamber configuration are considered only; a more complete evaluation of the functionality of water data with pressure, orifice size, chamber length, and spray length is provided in Chapter 9 . The complexity of comparative and parametric analysis is reduced by only considering in-chamber release fraction measurements at three aerosol diameters $(10 \mu \mathrm{m}, 32 \mu \mathrm{m}$, and $102 \mu \mathrm{m})$. In-spray data are excluded because the precision of in-spray analysis is severely reduced at aerosol sizes $<50 \mu \mathrm{m}$. The second limitation, which dictates that analysis only consider aerosol sizes of $10 \mu \mathrm{m}, 32 \mu \mathrm{m}$, and $102 \mu \mathrm{m}$, is set to simply reduce the number of data sets to three sizes that bound and represent the size range of interest in release fraction analysis (i.e., 10 to $100 \mu \mathrm{m}$ ). Even with these two limitations, there are still 36 parametric combinations of orifice size, pressure, and aerosol diameter. Presentation and discussion of these combinations is difficult without making the discussion overly simplistic or qualitative. In an attempt to make a quantitative argument about the trends observed in the simulant release fraction tests, parametric trends in cumulative release fraction measurements, $R_{k}$, are fit to a power-law equation of the form:

$$
R_{k}=R_{k, o}\left(\frac{z}{z_{o}}\right)^{n_{z}}
$$

Here, $z$ represents the parameter varied (such as pressure or orifice area), and $\mathrm{R}_{\mathrm{k}, \mathrm{o}}$ is a reference release fraction value at reference value of $z$, denoted by $z_{o}$. Choice of $z_{o}$ is arbitrary, as the ultimate goal of the analysis is determination of the power-law exponent, $n_{z}$, which determines how release fraction scales with the parameter $z$. As such, $z_{o}$ is set to unity and Equation (8.1) becomes:

$$
R_{k}=R_{k, o} z^{n_{z}}
$$

For a given set of release fraction data (e.g., the cumulative release fraction $<10 \mu \mathrm{m}$ as a function of pressure), linear regression of Equation (8.2) can provide a best-fit determination of the power-law exponent (and of the best fit $R_{k, o}$ corresponding to $z_{o}=1$ although this result is not usually reported). Along with statistical analysis of the data, the significance of trends can be quantified. It should be noted that selection of power-law functionality is done for the purpose of providing a semi-quantitative evaluation of trends and their significance. Limiting cases of the WTP model suggest power-law functionality of release fraction and generation rate with respect to both pressure and orifice area. The goal of the current analysis is not to validate pressure and orifice area functionality, but simply to determine if these functionalities change with increased solids loading or rheology. In the subsections that follow, the impact of controlled test parameters, including pressure and orifice area, will be presented and quantified using statistical analysis of Equation (8.2). 


\subsubsection{Impact of Solids on Test Pressure Dependence of Release Fraction}

Section 8.1 evaluated the impact of simulant solids on overall release fraction. It is expected that the presence of solids also may affect how release fraction varies with pressure. To test this assertion, the pressure functionality of simulant release fraction data for water, $6 \mathrm{~Pa}$ Clay, and $30 \mathrm{~Pa}$ Clay, is quantified using power-law fits to:

$$
R_{k}=R_{k, o} P^{n_{P}}
$$

where $P$ is the test pressure and $n_{P}$ is the pressure exponent. Linear regression analysis provides best-fit values for $n_{P}$ and statistical confidence limits. It should be noted that individual (i.e., un-averaged) release fraction data are used in fitting analysis to provide better estimates of result confidence. Example fits of release fraction results as a function of test pressure for 6 Pa Clay sprayed through a $1 \times 10 \mathrm{~mm}$ orifice are shown in Figure 8.5. These data indicate that for the test case shown, increasing pressure yields an increase in the in-chamber release fraction for all sizes considered. The correlation obtained from the power-law model is reasonable, with $R^{2}$ values of 0.93 or greater.

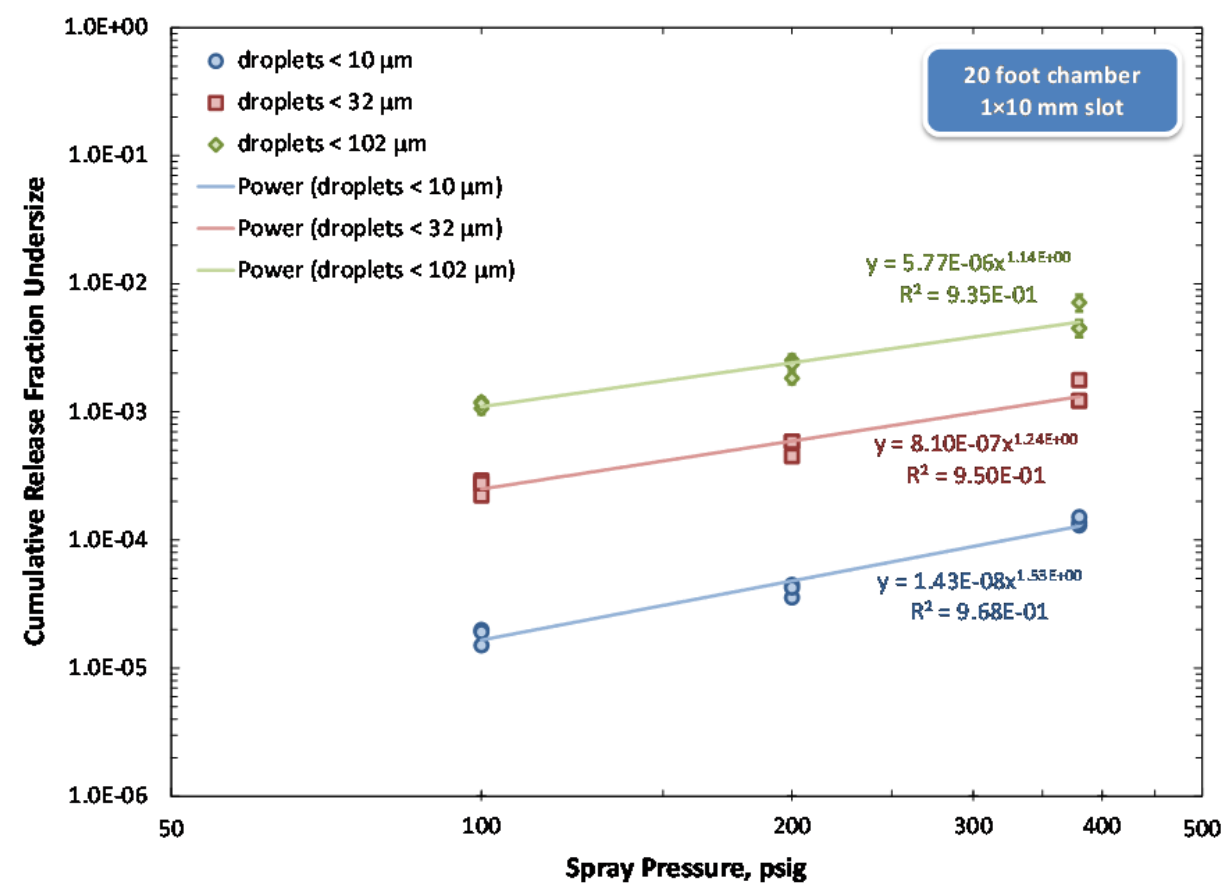

Figure 8.5. Example Fit of Release Fraction at $10 \mu \mathrm{m}, 32 \mu \mathrm{m}$, and $102 \mu \mathrm{m}$ as a Function of Test Pressure. Data correspond to release fraction measurements of $6 \mathrm{~Pa}$ Clay sprayed through a $1 \times 10 \mathrm{~mm}$ slot in the $20 \mathrm{ft}$ test chamber. Individual test measurements are shown as solid points, with error bars corresponding to the uncertainty (95 percent confidence limit of generation rate fit) with individual release fraction measurements. The curves correspond to a best fit of the individual measurements using the power-law relationship defined by Equation (8.3).

Evaluation of release fraction as a function of test pressure for other simulant orifice combinations yield fits of similar quality. The power-law exponents (i.e., the pressure scaling factors) derived from 
these fits are shown in Table 8.1 for all test fluid, pressure, and orifice combinations. The scaling factors for all simulant, orifice, and pressure combinations indicate a statistically significant dependence of release fraction on pressure. The pressure scaling factor generally varies between 1 and 2, indicating that release fraction uniformly increases with increasing pressure. For water, the pressure-scaling factor depends on aerosol size, with smaller aerosols showing greater increase in release fraction with increasing pressure than larger aerosol droplets. The presence of solids (or increased slurry rheology) generally appears to weaken the pressure dependence of release fraction at small particle diameters. For example, pressure scaling factors for the $30 \mathrm{~Pa}$ Clay generally fall at 1.1 for all size diameters considered. As such, slurry release fraction is not only lower than most comparable water data (see Section 7.1), but also appears to show a weaker dependence on pressure for the test conditions considered.

Table 8.1. Best-Fit Pressure Scaling Factors for Simulant Release Fractions in $20 \mathrm{ft}$ Chambers (from Equation (8.3)). The uncertainty reported for each scaling factor is a 95 percent confidence limit.

\begin{tabular}{cccc}
\hline Aerosol Diameter, $\mu \mathrm{m}$ & $n_{P}$, Water & $n_{P}, 6$ Pa Clay & $n_{P}, 30$ Pa Clay \\
\hline 10 & $2 \mathrm{~mm}$ hole & \\
32 & $2.0 \pm 0.2$ & $1.0 \pm 0.3$ & $1.1 \pm 0.2$ \\
102 & $1.7 \pm 0.3$ & $1.0 \pm 0.2$ & $1.6 \pm 0.2$ \\
10 & $1.4 \pm 0.3$ & $0.9 \pm 0.3$ & $1.7 \pm 0.2$ \\
\hline 32 & $1 \times 10 \mathrm{~mm}$ slot & \\
102 & $1.8 \pm 0.2$ & $1.5 \pm 0.2$ & $1.1 \pm 0.2$ \\
10 & $1.3 \pm 0.2$ & $1.2 \pm 0.3$ & $1.1 \pm 0.4$ \\
32 & $1.1 \pm 0.3$ & $1.1 \pm 0.3$ & $1.1 \pm 0.4$ \\
102 & $1 \times 20 \mathrm{~mm}$ slot \\
10 & $1.8 \pm 0.3$ & $1.3 \pm 0.2$ & $1.3 \pm 0.2$ \\
32 & $1.3 \pm 0.1$ & $1.0 \pm 0.3$ & $1.1 \pm 0.4$ \\
102 & $1.1 \pm 0.1$ & $0.9 \pm 0.6$ & $1.2 \pm 0.3$ \\
\hline & $1 \times 76 \mathrm{~mm}$ slot \\
& $1.5 \pm 0.3$ & $1.1 \pm 0.3$ & $1.1 \pm 0.3$ \\
\hline & $1.3 \pm 0.2$ & $1.2 \pm 0.4$ & $1.2 \pm 0.3$ \\
& $1.3 \pm 0.2$ & $1.2 \pm 0.6$ & $1.4 \pm 0.2$ \\
\hline
\end{tabular}

\subsubsection{Impact of Solids on Orifice Area Dependence of Release Fraction}

To evaluate the impact of solids content on the functionality of simulant release fraction with orifice size, release fraction data for water, $6 \mathrm{~Pa}$ Clay, and $30 \mathrm{~Pa}$ Clay are fit to the following power-law expression:

$$
R_{k}=R_{k, o} A_{b}^{n_{A}}
$$

Here, $A_{b}$ is the area of the orifice, and $n_{A}$ is the orifice-area scaling exponent that describes how release fraction responds to changes in area. As in Section 8.2.1, linear regression analysis is used to determine the scaling exponent for orifice area for all test fluid and pressure combinations for $10 \mu \mathrm{m}, 32 \mu \mathrm{m}$, and $102 \mu \mathrm{m}$ aerosol droplets. This approach only considers orifice area. Differences in release fraction resulting from difference in orifice shape (a hole versus a slot) are not considered because of the limited number of orifices tested in Phase II. However, Phase I testing indicated that release fraction did not 
appear to depend on orifice shape (Schonewill et al. 2012). Best-fit orifice area scaling factor results are given in Table 8.2. An example of how release fraction varies with pressure and of the quality of fit of data to Equation (8.4) is shown in Figure 8.6. The quality of correlation between release fraction and orifice area shown in this example is typical of most pressure/simulant combinations. In some cases, data appear to show a dependence on orifice shape. Such an example is shown in Figure 8.7, where release fraction results for the $2 \mathrm{~mm}$ hole, which has the smallest area of orifices tested in Phase II, appears to fall below release fraction extrapolations based only on the slot data (i.e., data corresponding to the three highest test areas).

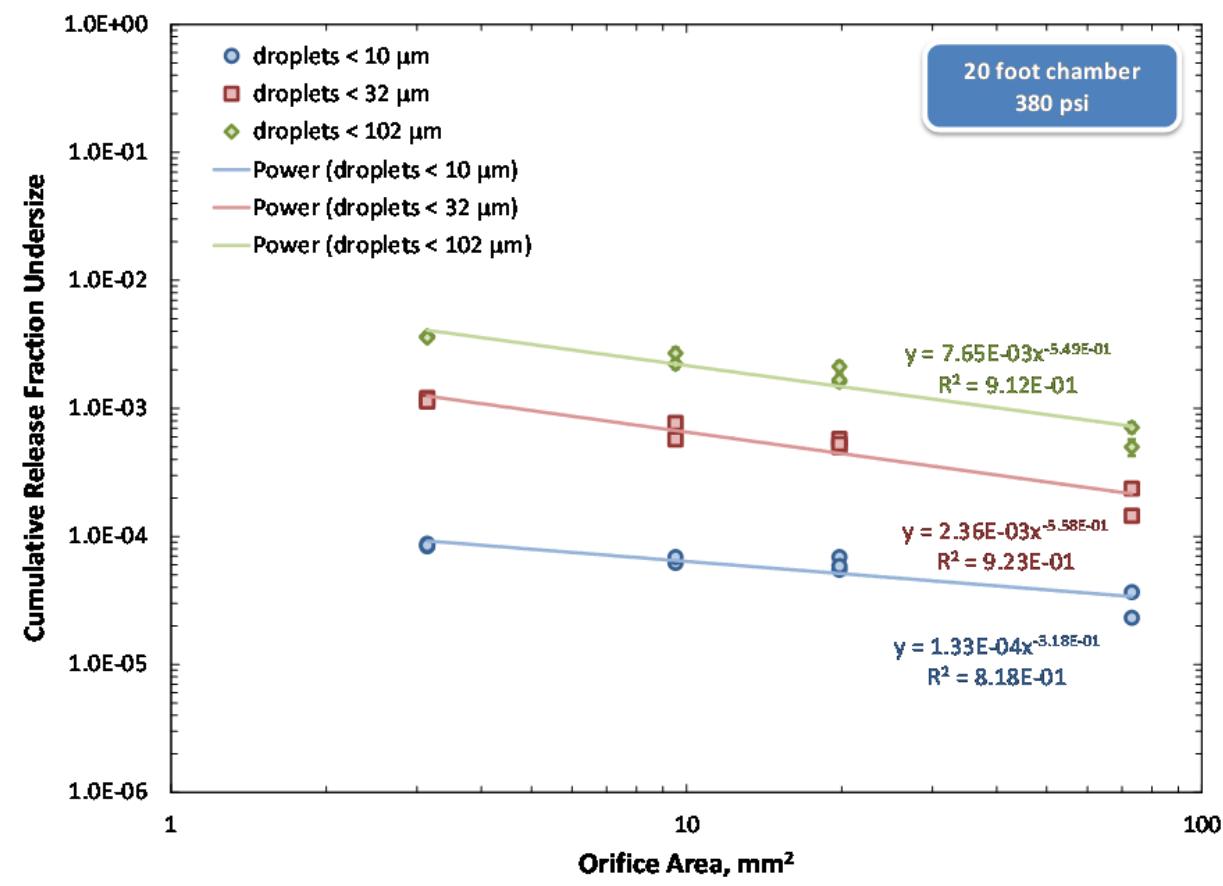

Figure 8.6. Example Fit of Release Fractions at $10 \mu \mathrm{m}, 32 \mu \mathrm{m}$, and $102 \mu \mathrm{m}$ as a Function of Orifice Area. Data correspond to release fraction measurements of $30 \mathrm{~Pa}$ Clay at a release pressure of 380 psig in the $20 \mathrm{ft}$ test chamber. Individual test measurements are shown as solid points, with error bars corresponding to the uncertainty (95 percent confidence limit of generation rate fit) with individual release fraction measurements. The curves correspond to a best fit of the individual measurements using the power-law relationship defined by Equation (8.4). 


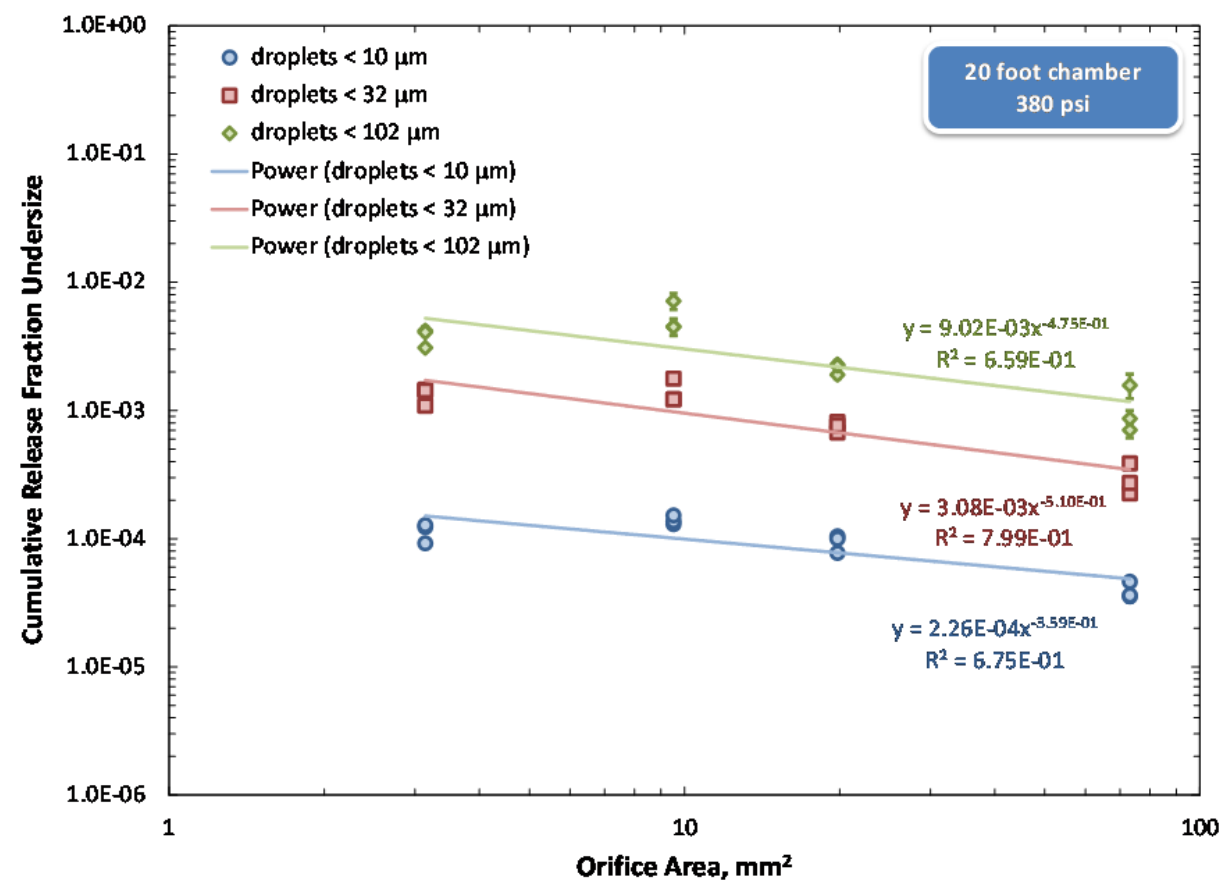

Figure 8.7. Example Fit of Release Fractions at $10 \mu \mathrm{m}, 32 \mu \mathrm{m}$, and $102 \mu \mathrm{m}$ as a Function of Orifice Area Showing Potential Orifice Shape Effects. Data correspond to release fraction measurements of $6 \mathrm{~Pa}$ Clay at a release pressure of $380 \mathrm{psig}$ in the $20 \mathrm{ft}$ test chamber. Individual test measurements are shown as solid points, with error bars corresponding to the uncertainty (95 percent confidence limit of generation rate fit) with individual release fraction measurements. Data for the $2 \mathrm{~mm}$ hole at $\sim 3 \mathrm{~mm}^{2}$ appear to differ (i.e., fall below) expected trends based on slot data at 10, 20, and $76 \mathrm{~mm}^{2}$.

Table 8.2. Best-Fit Orifice Area Scaling Factors for Simulant Release Fractions in $20 \mathrm{ft}$ Chambers (from Equation (8.4)). The uncertainty reported for each scaling factor is a 95 percent confidence limit.

\begin{tabular}{|c|c|c|c|}
\hline Aerosol Diameter, $\mu \mathrm{m}$ & $n_{A}$, Water & $n_{A}, 6 \mathrm{~Pa}$ Clay & $n_{A}, 30 \mathrm{~Pa}$ Clay \\
\hline \multicolumn{4}{|c|}{100 psig } \\
\hline 10 & $-0.2 \pm 0.1$ & $-0.4 \pm 0.1$ & $-0.3 \pm 0.1$ \\
\hline 32 & $-0.4 \pm 0.2$ & $-0.6 \pm 0.1$ & $-0.4 \pm 0.2$ \\
\hline 102 & $-0.4 \pm 0.2$ & $-0.6 \pm 0.2$ & $-0.4 \pm 0.3$ \\
\hline \multicolumn{4}{|c|}{200 psig } \\
\hline 10 & $-0.3 \pm 0.1$ & $-0.4 \pm 0.2$ & $-0.3 \pm 0.1$ \\
\hline 32 & $-0.5 \pm 0.1$ & $-0.6 \pm 0.3$ & $-0.6 \pm 0.1$ \\
\hline 102 & $-0.4 \pm 0.1$ & $-0.6 \pm 0.4$ & $-0.6 \pm 0.2$ \\
\hline \multicolumn{4}{|c|}{380 psig } \\
\hline 10 & $-0.4 \pm 0.1$ & $-0.4 \pm 0.2$ & $-0.3 \pm 0.1$ \\
\hline 32 & $-0.5 \pm 0.1$ & $-0.5 \pm 0.2$ & $-0.6 \pm 0.1$ \\
\hline 102 & $-0.4 \pm 0.1$ & $-0.5 \pm 0.2$ & $-0.5 \pm 0.1$ \\
\hline
\end{tabular}


The orifice-area scaling factors listed in Table 8.2 range from -0.6 to -0.2 and indicate a slight but statistically significant decrease in release fraction with increasing orifice area (based on the Phase II test data) for all simulant materials tested. The most significant conclusion that can be drawn from Table 8.2 is that the dependence of release fraction on orifice area is statistically similar for all simulants tested, regardless of solids content. This means that the presence of solids (or increased rheology) does not significantly alter the functionality of release fraction with respect to orifice size. The results do indicate a slight aerosol size dependence of the area scaling factor, with $10 \mu \mathrm{m}$ droplets exhibiting an average scaling factor of about -0.3 and $32 \mu \mathrm{m}$ and $102 \mu \mathrm{m}$ exhibiting an average scaling factor of about -0.5 .

\subsection{Conclusions}

In this chapter, the release fraction of non-Newtonian simulants composed of mixed clay powder in water was evaluated and compared to water release fraction data. The goal of non-Newtonian simulant testing was to better define the impact of the presence of solids and increased system rheology on release fractions for aerosols generated by high-pressure spray through large orifices. The results of Phase I testing indicate that the worst-case spray in terms of aerosol release from an accidental pipe breach occurs when the breach is large, when the spray is not obstructed (and can fully break up), and when the material aerosolized had the highest Bingham parameters of the non-Newtonian fluids tested (Mahoney et al. 2013; Schonewill et al. 2012). During Phase I, non-Newtonian simulants were tested only in the small-scale test system and the increase in release fraction tended to be for droplets less than $10 \mu \mathrm{m}$. Due to the limited orifice size and spray length in the small-scale system, there was a need to confirm the conditions leading to the "worst-case" spray release in large-scale, where the large orifice and test chamber configurations considered by PNNL could be directly evaluated. Further testing also was warranted by the fact that Phase I spray leak simulant testing with both Newtonian and non-Newtonian slurries was inconsistent with model predictions for the impact of solids concentration on release fraction.

Phase II spray leak testing of non-Newtonian simulants evaluated fractional aerosolization of two clay slurries (with target yield stresses of 6 and $30 \mathrm{~Pa}$ ) from four orifice sizes at three release pressures. Evaluation of the release fraction results from clay testing yields the following conclusions:

- The presence of solids (or increased rheology) typically reduces the release fraction of aerosols $>50 \mu \mathrm{m}$ in diameter or does not impact release fraction at all (relative to the fractional aerosolization of water).

- The presence of solids can sometimes yield a slight increase in the release fraction of aerosols that are $<50 \mu \mathrm{m}$ in diameter, but the increase is typically not statistically significant (relative to the fractional aerosolization of water).

- The presence of solids typically reduces the dependence of release fraction on pressure, such that increasing pressure produces less of an increase in release fraction for slurries relative to the increase seen for water.

- The presence of solids does not change the functional dependence of release fraction on orifice area relative to that observed for water.

- In-spray measurements of simulant release fraction frequently challenge (and in some cases exceed) release fraction predictions obtained using the WTP model, especially for the largest orifices and highest pressures tested. 
From these observations, it can be concluded that release fraction data are impacted to some extent by the presence of solids such that any absolute evaluations of release fractions for slurries must account for the solids content (or its impact on physical properties). On the other hand, the comparison of release fraction for simulants indicate that for the majority of test configuration and aerosol diameters considered, release fractions are bounded by measurements for water. This suggests that it may be possible to provide a bounding value for release fraction using water data, thus avoiding the need to consider fractional releases from slurries altogether. This last assertion should be approached with caution, as the simulants tested in Phase II large-scale activities bound only the expected range of operating rheologies for the WTP and were not representative of either the chemical complexity or the size distribution of solids found in actual wastes. Small-scale testing activities conducted spray tests with a chemically representative simulant in Phase I (Mahoney et al. 2013) and Phase II (Schonewill et al. 2012), but the direct comparison of test results for clay slurries and chemical simulant slurries was complicated by several factors with the primary factor being the shear thickening (dilatant) and time dependent (rheopectic) rheology of the chemical slurry simulants. While these difficulties reduce the significance of the small-scale chemical simulant test results, Phase II testing of the chemical simulant confirms that sprays from the chemical slurry simulant produce release fractions that are larger than those produced by water sprays. Until a more representative chemical slurry simulant can be developed and tested, conclusions about release fraction of wastes and waste materials representative of non-Newtonian WTP process streams will need to be made based on testing results for clay slurries. With respect to the systems tested in Phase II large-scale operations, use of water data still appears to provide a basis for conservative estimates of release fraction. 


\subsection{Release Fraction Scaling}

During Phase I in-chamber testing, it was observed that a significant fraction of the droplets generated by high-pressure spray through an engineered orifice were captured by impact on the chamber wall downstream of the spray (i.e., the splash wall). Further changes were observed when the distance between orifice and splash wall was shortened by adding extension pieces to the spray header. These Phase I observations reveal that release consequences evaluated outside the spray depend on the configuration of boundaries that impinge or contain the spray (and the aerosol generated by that spray) and also suggest the presence of spray length effects on the overall degree of aerosolization. For these reasons, follow-on spray release testing conducted in Phase II included two studies designed to evaluate the impacts to apparent fractional aerosolization from chamber size and distance to spray impingement. These two studies are described below:

- Chamber Size Evaluation. Spray leak testing through four engineered orifices at three operating pressures was conducted to evaluate the combined impact of chamber size and spray length. The chamber length was varied from 10 to $39 \mathrm{ft}$ in length, while maintaining the same cross-sectional chamber area and keeping the spray header and point of spray release at a fixed location. This approach yielded a set of tests where both chamber volume and spray length varied (with the latter changing from 9 to $38 \mathrm{ft}^{1}$ ). For these tests, two to three Malvern Insitec-S aerosol analyzers were placed in the test chamber at equal spacing. As chamber size increased, the distance between aerosol analyzers also increased, and the analyzers sampled aerosols farther downstream of the point of release.

- Spray Length Evaluations. Spray leak testing through four engineered orifices at three operating pressures was conducted to evaluate the impact of spray length alone. Spray length testing was performed in the $39 \mathrm{ft}$ chamber configuration. The "length" of the spray (i.e., the distance between the source and splash wall) was changed by moving the location of the spray header within the chamber. This was accomplished by adding extension pieces to the supply and return legs of the test spool. Spray lengths of $38 \mathrm{ft}$ (with no extension piece added) to $3.5 \mathrm{ft}$ (with the full-length extension added) were tested. The locations of the aerosol analyzers were fixed at their $39 \mathrm{ft}$ chamber positions $(10,20$, and $30 \mathrm{ft}$ from the wall next to the spray header).

In the sections that follow, the release fraction test results from the chamber size and spray length evaluations are discussed in detail. The format for analysis and discussion uses a format similar to that used in our discussion of simulant solids effects in Section 8. The impact of chamber and spray length on release fraction curves showing cumulative release fractions as a function of aerosol size will be discussed first. Then, the impact of chamber length on the pressure and orifice size functionality of the release fraction results will be discussed. Discussion will be limited primarily to the results derived from Phase II testing and will not reference Phase I results. However, the data presented in this section will form the basis for more extensive evaluations and extrapolations of data to longer spray lengths and larger breaches, which are described in Chapter 10.

\footnotetext{
${ }^{1}$ The term "spray length", as it is used throughout this report, refers to the distance between the orifice and the downstream wall of the chamber (i.e., the "splash wall"). Both in-spray measurements, which track the most optically dense region of the spray, and direct observation of sprays indicate that this region of the spray (also referred to as the "core" of the spray) does not necessarily travel the full distance between the orifice and splash wall.
} 
As in Chapter 8, predictions of release fraction for the materials tested are made using the WTP model. These model predictions can be used to evaluate how the WTP model captures trends in the data with changing process variables (such as pressure or rheology). However, care should be taken when evaluating the performance of the WTP model with respect to the magnitude of results (and thus inferring how "conservative" the WTP is with respect to safety analyses), because the release fraction results determined using in-chamber methods are subject to method and humidity bias (see Section 7 and Appendix A for details). In overall terms, assessment of in-chamber measurement accuracy indicates that it is likely low relative to the true release fraction. As such, comparisons showing the WTP model prediction as being conservative relative to in-chamber measurements of release fraction should be treated as inconclusive. On the other hand, cases where the WTP predictions fall below measured in-chamber release fractions can indicate under-performance of the WTP model, with the caveat that model performance may be worse than is shown.

\subsection{Impact of Chamber Size}

The impact of chamber size on release fraction results from the four tests orifices $(2 \mathrm{~mm}$ hole, $1 \times 10 \mathrm{~mm}$ slot, $1 \times 20 \mathrm{~mm}$ slot, and $1 \times 76 \mathrm{~mm}$ slot) is shown in Figure 9.1, Figure 9.2, and Figure 9.3. The three figures show cumulative release fraction undersize curves at various orifice and chamber size combinations for spray pressures of 100,200, and 380 psig, respectively. Data are subject to the same rejection criteria identified in Section 8.1 of this report. These criteria are that 1) data below $10 \mu \mathrm{m}$ are not reported, 2) data are marked with open symbols to indicate increased uncertainty at cumulative release fractions below $0.5 \times 10^{-2}$ times the maximum cumulative release fraction of the data set, and 3 ) points above $87 \mu \mathrm{m}$ have been marked with open symbols to indicate release fractions where droplet loss (method bias) has been observed (see Section 7).

The release fraction data presented in Figure 9.1 to Figure 9.3 show functionality relationships between release fractions and chamber sizes. Specifically, the manner in which release fraction varies with chamber size depends on the orifice size and test pressure, and there is no single trend that describes the behavior seen in the entire set of results. If the 100 psig data are considered, data for the $2 \mathrm{~mm}$ hole appear to show a monotonic decrease in release fraction with increasing chamber size. For the $1 \times 10 \mathrm{~mm}$ orifice, a reversal of this trend is observed at diameters greater than $\sim 50 \mu \mathrm{m}$, such that the release fraction increases with increasing chamber size for the largest aerosol diameters shown $(200 \mu \mathrm{m})$. Below $50 \mu \mathrm{m}$, the $1 \times 10 \mathrm{~mm}$ release fraction data still increases with increasing chamber size. For larger orifices $(1 \times 20$ and $1 \times 76 \mathrm{~mm}$ slots), the reversal appears to be pushed to smaller aerosol diameters, such that no significant change in release fraction is observed at small aerosol diameters, whereas larger aerosols exhibit increasing release fraction with increasing chamber size.

Release fraction trends at higher pressures are generally similar to those observed at $100 \mathrm{psig}$. For 200 psig test data (shown in Figure 9.2), the primary difference is that trend reversal is observed in the $2 \mathrm{~mm}$ data. Here, release fractions appear to decrease with increasing chamber length below $50 \mu \mathrm{m}$ and appear to increase with increasing chamber length above $50 \mu \mathrm{m}$. Trend reversal in the $200 \mathrm{psig}$ $1 \times 10 \mathrm{~mm}$ slot data is depressed to $30 \mu \mathrm{m}$ (50 $\mu \mathrm{m}$ for 100 psig data). For larger slots, 200 psig release fraction data generally show no significant increase at small aerosol diameters and indicate an increase in release fractions with chamber size at larger diameters. Release fractions measured at $380 \mathrm{psig}$ (Figure 9.3) show similar results to the $200 \mathrm{psig}$ data. Trend reversal in the $2 \mathrm{~mm}$ hole is depressed to $30 \mu \mathrm{m}$ (versus $50 \mu \mathrm{m}$ at $200 \mathrm{psig}$ ). Trend reversal in the $1 \times 10 \mathrm{~mm}$ slot data is almost entirely depressed 
relative to that seen at lower pressures $(100$ and $200 \mathrm{psig})$. The $1 \times 20$ and $1 \times 76 \mathrm{~mm}$ slots show similar behavior with respect to chamber size as their lower pressure counterparts.
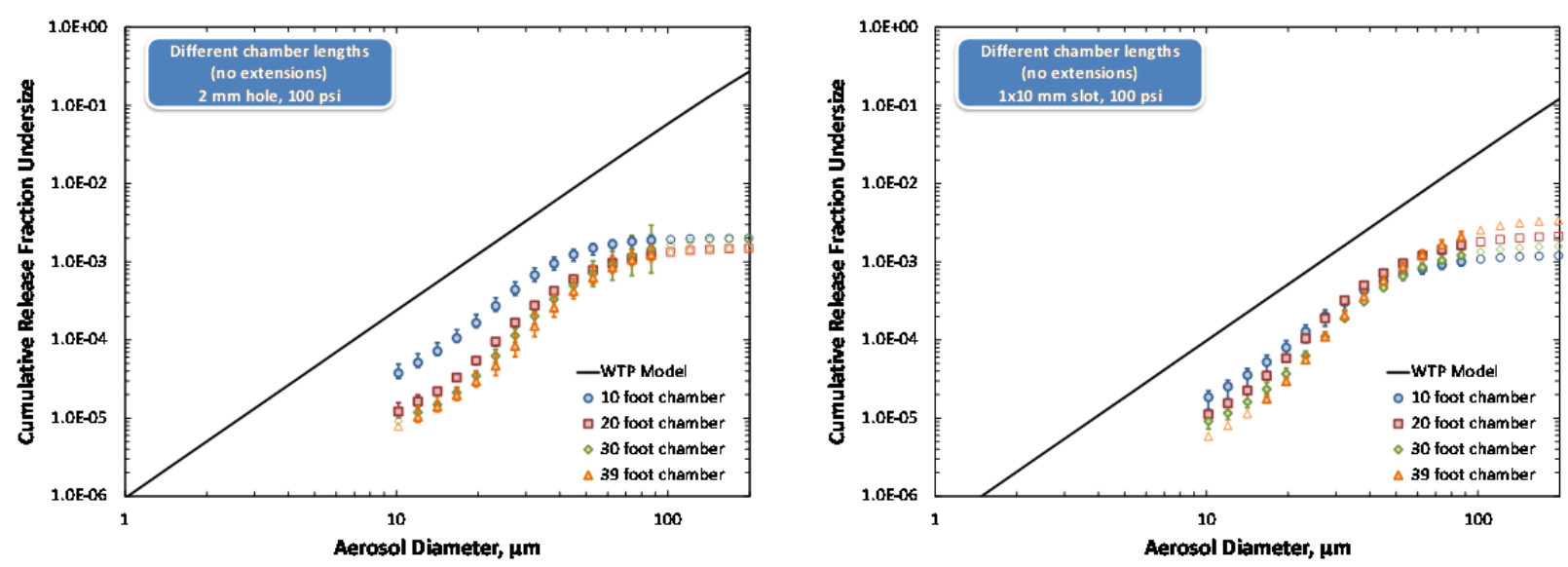

(a) $-2 \mathrm{~mm}$ hole

(b) $-1 \times 10 \mathrm{~mm}$ slot

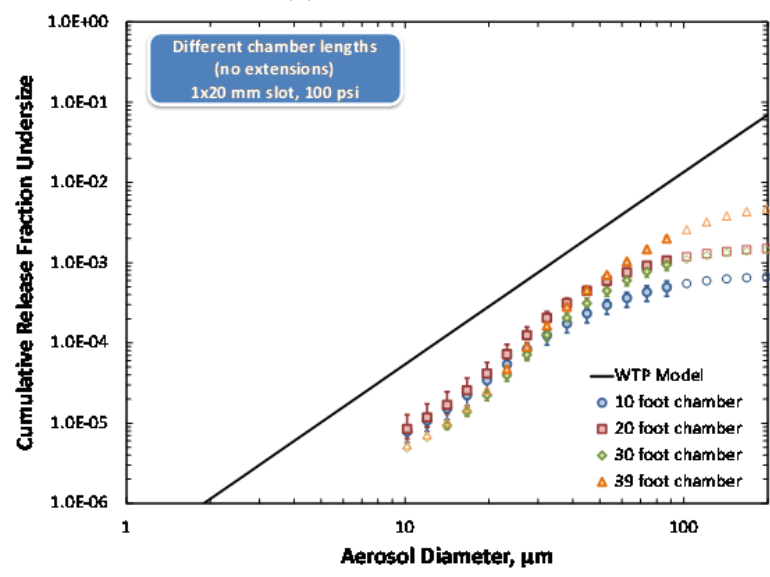

(c) $-1 \times 20 \mathrm{~mm}$ slot

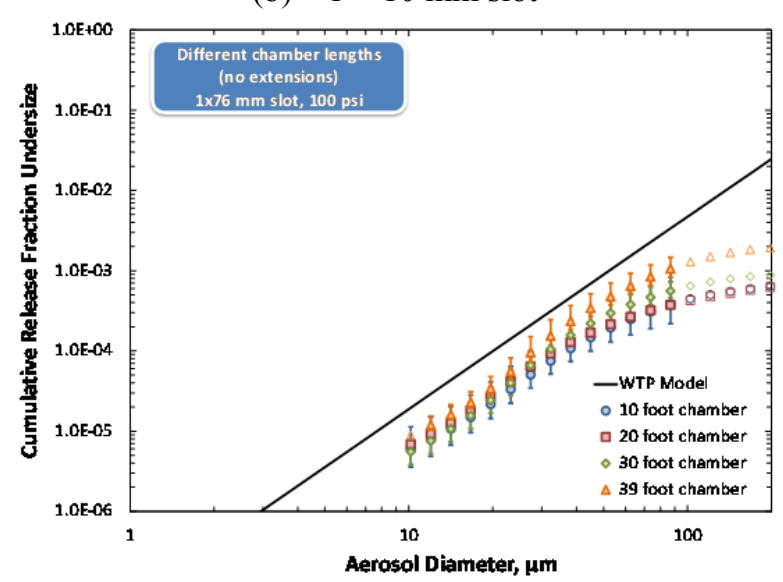

(d) $-1 \times 76 \mathrm{~mm}$ slot

Figure 9.1. In-Chamber Release Fraction as a Function of Aerosol Size for Different Chamber Lengths and for Sprays Generated at $100 \mathrm{psig}$. Test results are shown for a (a) $2 \mathrm{~mm}$ hole, (b) $1 \times 10 \mathrm{~mm}$ slot, (c) $1 \times 20 \mathrm{~mm}$ slot, and (d) $1 \times 76 \mathrm{~mm}$ slot. Release fraction curves are the mean of two or more replicate measurements; error bars correspond to the minimum and maximum release fractions measured in replicate testing. 


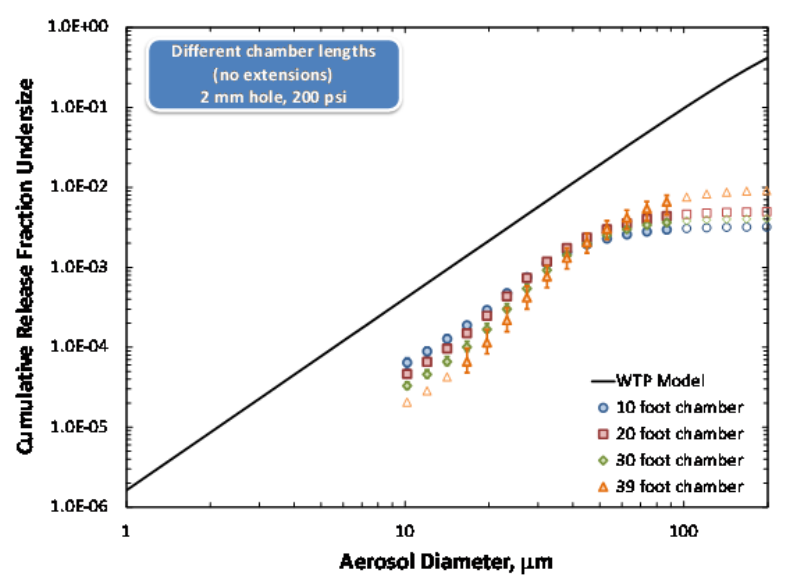

(a) $-2 \mathrm{~mm}$ hole

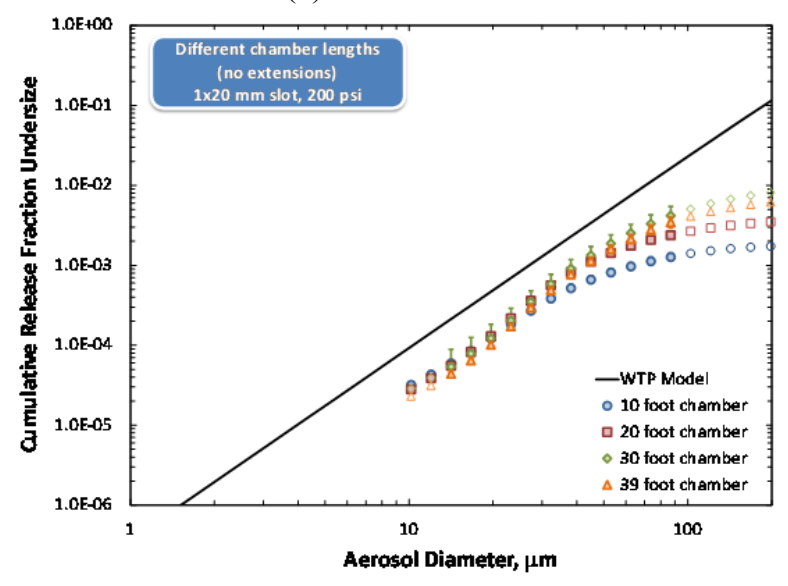

(c) $-1 \times 20 \mathrm{~mm}$ slot

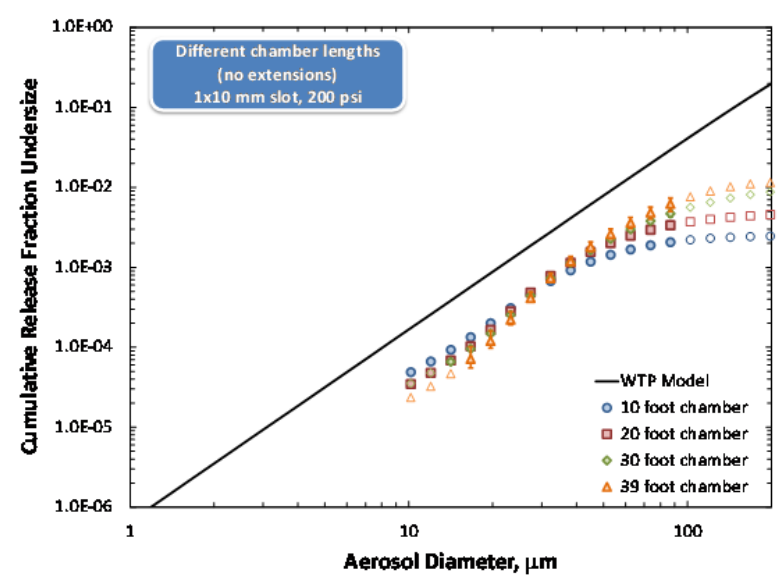

(b) $-1 \times 10 \mathrm{~mm}$ slot

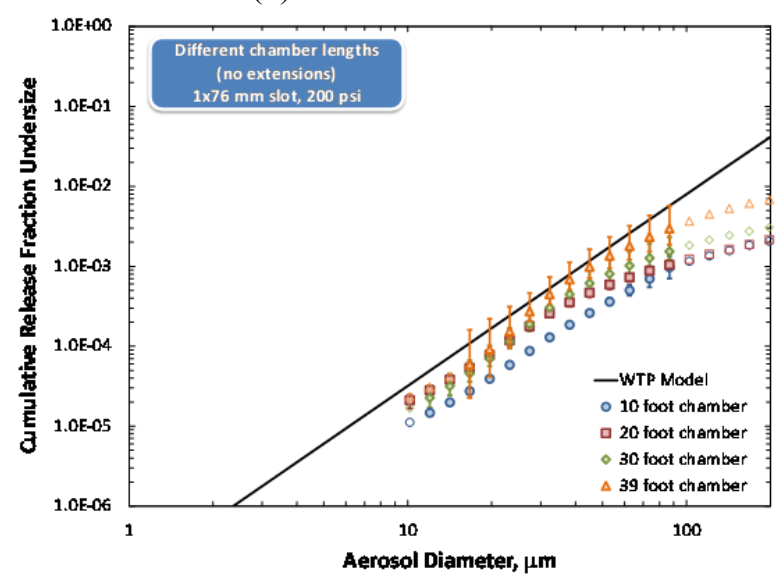

(d) $-1 \times 76 \mathrm{~mm}$ slot

Figure 9.2. In-Chamber Release Fraction as a Function of Aerosol Size for Different Chamber Lengths and for Sprays Generated at 200 psig. Test results are shown for a (a) $2 \mathrm{~mm}$ hole, (b) $1 \times 10 \mathrm{~mm}$ slot, (c) $1 \times 20 \mathrm{~mm}$ slot, and (d) $1 \times 76 \mathrm{~mm}$ slot. Release fraction curves are the mean of two or more replicate measurements; error bars correspond to the minimum and maximum release fractions measured in replicate testing. 


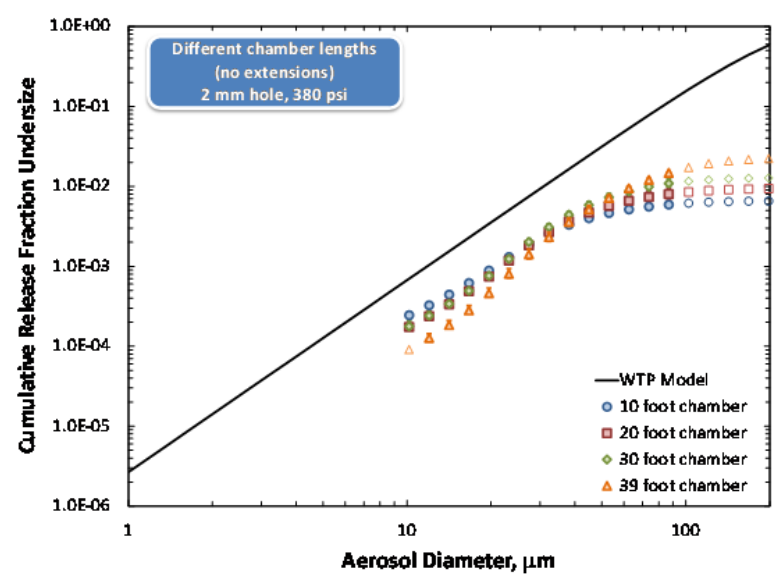

(a) $-2 \mathrm{~mm}$ hole

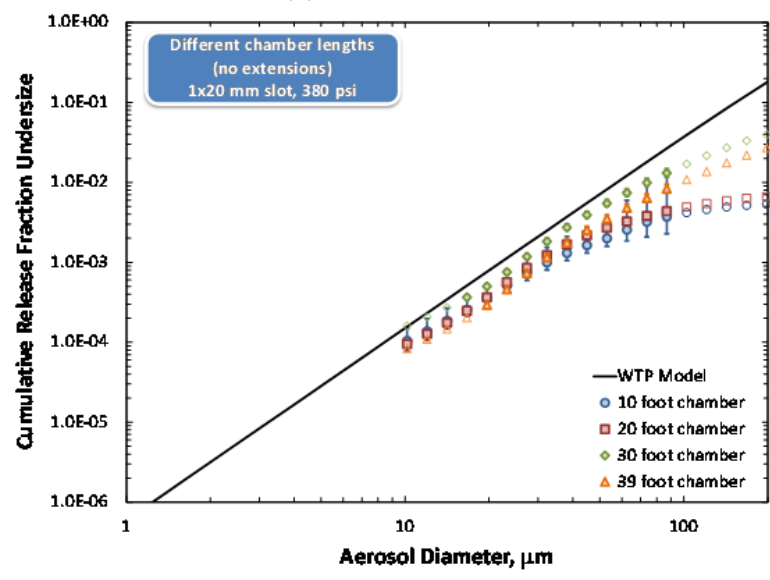

(c) $-1 \times 20 \mathrm{~mm}$ slot

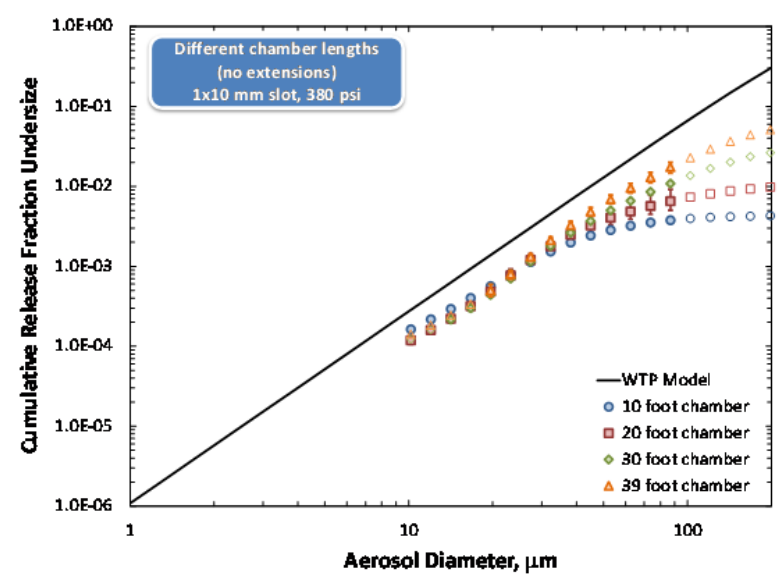

(b) $-1 \times 10 \mathrm{~mm}$ slot

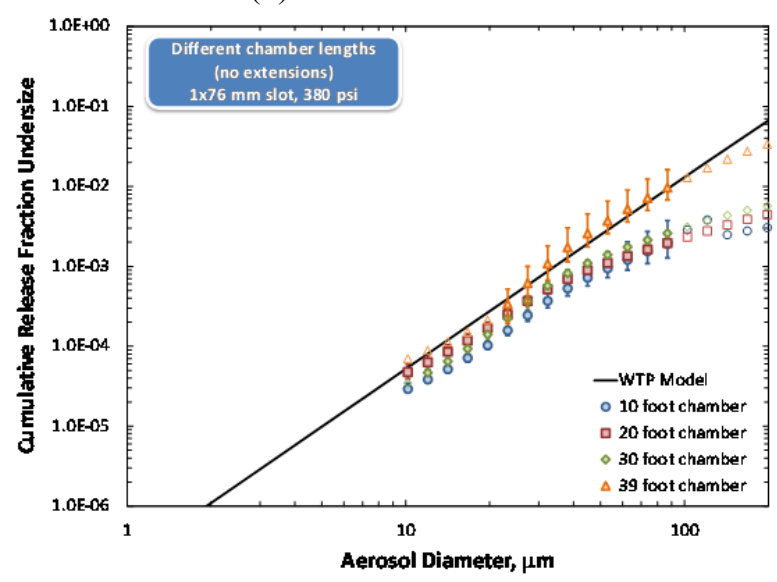

(d) $-1 \times 76 \mathrm{~mm}$ slot

Figure 9.3. In-Chamber Release Fraction as a Function of Aerosol Size for Different Chamber Lengths and for Sprays Generated at 380 psig. Test results are shown for a (a) $2 \mathrm{~mm}$ hole, (b) $1 \times 10 \mathrm{~mm}$ slot, (c) $1 \times 20 \mathrm{~mm}$ slot, and (d) $1 \times 76 \mathrm{~mm}$ slot. Release fraction curves are the mean of two or more replicate measurements; error bars correspond to the minimum and maximum release fractions measured in replicate testing.

Based on trends observed in Figure 9.1 to Figure 9.3, it may be concluded that chamber size significantly impacts the in-chamber release fractions. The observations made in the preceding paragraph made be summarized as follows:

- For the smallest orifice ( $2 \mathrm{~mm}$ hole) at the lowest pressure (100 psig), the total aerosolization of the spray does not change with increasing distance; instead, the release fraction contributions are shifted to larger droplets

- For many small orifice and low test pressure combinations, an increase in chamber length yields both an increase in the total fraction aerosolized and a shift in the release fraction curve to larger aerosols (such that the small aerosol fraction is reduced while the large aerosol fraction is increased). Examples include the $2 \mathrm{~mm}$ hole at $200 \mathrm{psig}$ (Figure 9.2a) and the $1 \times 10 \mathrm{~mm}$ slot at $100 \mathrm{psig}$ (Figure 9.1b). 
- For several high-pressure and large orifice combinations, the increase in chamber length is accompanied by increase in the cumulative release fraction for large aerosols only, with the small aerosols remaining unchanged. Examples include release fraction data for the $1 \times 10 \mathrm{~mm}$ and $1 \times 20 \mathrm{~mm}$ slots at $380 \mathrm{psig}$ (Figure 9.3c and Figure 9.3d, respectively).

These trends should be interpreted in terms of the in-spray behavior described in Chapter 7 of this report. From in-spray position testing, it is known that sprays generated from the $2 \mathrm{~mm}$ orifice exhibit increasing droplet size with increasing distance from the orifice. The in-spray droplet size distribution generated from the $1 \times 10$ and $1 \times 20 \mathrm{~mm}$ slots exhibit very little change with increasing distance from orifice (with no clear trend toward larger or smaller droplets). Sprays generated through the $1 \times 76 \mathrm{~mm}$ orifice show an in-spray size distribution that becomes finer with increasing distance from orifice. The in-spray trajectory for all sprays indicates that a portion of the droplets are subject to gravitational settling such that the spray trajectory is not straight but arcs toward the floor of the chamber. As expected, increasing pressure appears to cause sprays to travel farther down the chamber. There is no apparent difference in the trajectories of sprays generated through the $2 \mathrm{~mm}$ hole, $1 \times 10 \mathrm{~mm}$ slot, and $1 \times 20 \mathrm{~mm}$ slot. Only jets exiting the $1 \times 76 \mathrm{~mm}$ slot appear to travel farther downstream. With this in mind, the several potential physical explanations can be postulated to explain in-chamber trends with chamber length.

The release fraction result for the $2 \mathrm{~mm}$ hole at $100 \mathrm{psig}$ is unique. It is the only measurement that does not show an appreciable increase in overall release fraction with increasing length and exhibits a shift in the release fraction contribution to larger aerosols in larger chambers. Both trends seem reasonable. The $2 \mathrm{~mm}, 100$ psig spray is expected to be the shortest (and carry the least momentum) of all sprays tested during Phase II large-scale operations. The lack of increase in total aerosolization in larger chambers can be attributed to near or almost complete aerosolization at $10 \mathrm{ft}$. However, this observation is not entirely consistent with the in-spray trajectories, which indicate that $2 \mathrm{~mm}$ hole sprays travel farther than $10 \mathrm{ft}$ at $100 \mathrm{psig}$. The shift in release fraction contributions to larger aerosols is likely an artifact of an increased opportunity for droplet coalescence in larger chambers before aerosol is removed from system by deposition on solid surfaces. Coalescence is generally a second-order aerosol concentration reaction that is not explicitly accounted for in the exponential model used to determine generation rate. As such, changes in the overall frequency of coalescence can yield changes in the apparent generation rate. Coalescence has been observed in $2 \mathrm{~mm}$ hole in-spray data and seems reasonable for in-chamber measurements.

For intermediate test pressure and orifice size combinations, an increase in the total aerosolization and a shift of the release fraction contributions to larger droplet sizes is observed when chamber length is increased. The combined effect of these two phenomena causes a reversal in the release fraction trends (discussed above). The increase in total aerosolization (or large droplet cumulative release fraction) can be tentatively attributed to three causes: 1) increased opportunity for jet break-up in larger chambers, 2) lessened opportunity for inertial capture of large droplets at the splash wall, and 3 ) increased sampling of inertial droplets as the analyzers move progressively downstream and more directly into the spray core. The first and second causes result from an increase in the distance before the jet and associated aerosols can travel before impinging on the downstream obstruction. However, the increase in total release does not appear to abate, even as the $30 \mathrm{ft}$ threshold is exceeded. In-spray jet trajectories suggest that all sprays except those generated by the $1 \times 76 \mathrm{~mm}$ slot should not travel much beyond $30 \mathrm{ft}$. Visual observation confirms this finding for the $2 \mathrm{~mm}$ hole, and yet the $2 \mathrm{~mm}$ hole data for 200 and 380 psig still show increase in release fraction between chamber lengths of 30 and $39 \mathrm{ft}$. For this reason, part of the 
increase in release fraction with chamber length is attributed to sampling effects. The aerosol analyzers are evenly spaced in the large-scale test chamber. For larger chambers, the analyzers are placed farther downstream of the orifice but are still installed $28 \mathrm{in}$. from the top of the chamber. Visual observation of large-scale test chambers indicates that sprays typically fan out as they travel through the chamber. As such, placing the analyzers farther downstream also pushes them toward the path of the spray core. This argument is mitigated to some extent by observations that release fraction measurements made in the $39 \mathrm{ft}$ chamber do not depend strongly on aerosol analyzer placement.

The shift in the release fraction contribution to larger droplet sizes also observed in intermediate sprays can be attributed to droplet coalescence. However, this behavior is not entirely consistent with observations of in-spray data. In particular, in-spray data for slots either show no change in droplet size or show increasingly finer droplets with increasing distance from the orifice. The shift toward larger aerosols could also be attributed to the change in Malvern Insitec-S sampling location. Because of the inconsistent behavior of the in-chamber and in-spray data sets for slots, determination of the exact cause for the shift in release fraction is not possible from the current data set.

At 380 psig, all slots show an increase in total release fraction with increasing distance; however, there is no loss in the contribution of fines like that observed for intermediate slots and test pressures. It should be noted that this behavior is observed in all $1 \times 76 \mathrm{~mm}$ slot data, regardless of test pressure. As before, the increase in total release fraction is tentatively attributed to increased opportunity for droplet break-up, reduced opportunity for droplet capture, and increased downstream sampling of large aerosols. The overall change in release fraction at high pressure yields an increased contribution of large aerosols. Again, this behavior is not consistent with in-spray observations of slots, which typically show either no change or a decrease in droplet size with increasing distance from the orifice.

When considered against the WTP model, the results only begin to challenge the model estimates for high-pressure sprays from large orifices in large chamber sizes. This is significant, since in-chamber data are expected to under-estimate the true value of release fraction. In addition, comparison of the current in-chamber data against WTP model performance affirms Phase I findings that sprays in chambers longer than those tested in the current study may exceed WTP model predictions. This concern is addressed in Chapter 10, in which the chamber and orifice size data are used to estimate larger chamber and breach sizes relative to WTP model predictions.

\subsubsection{Impact of Chamber Length on the Pressure Functionality of Release Fraction}

In Section 9.1, we presented our evaluation of the impact of chamber length on release fraction. We expect that changes in chamber length may also impact how release fraction varies with pressure. To test this assertion, we used the same approach employed in Section 8.2.1 of this report. Specifically, the pressure functionality of water release fraction for different chamber lengths is quantified using power-law fits to:

$$
R_{k}=R_{k, o} P^{n_{P}}
$$

where $P$ is the test pressure and $n_{P}$ is the pressure exponent. Linear regression analysis provides best-fit values for $n_{P}$ and statistical confidence limits. Example fits of release fraction results for water sprayed through a $1 \times 20 \mathrm{~mm}$ orifice in the $39 \mathrm{ft}$ chamber (with no spool extensions) as a function of test pressure 
are shown in Figure 9.4. These data indicate that for the test case shown, increasing pressure yields an increase in the in-chamber release fraction for all sizes considered. Correlation using the power-law model is reasonable for $R^{2}$ values of 0.91 or greater.

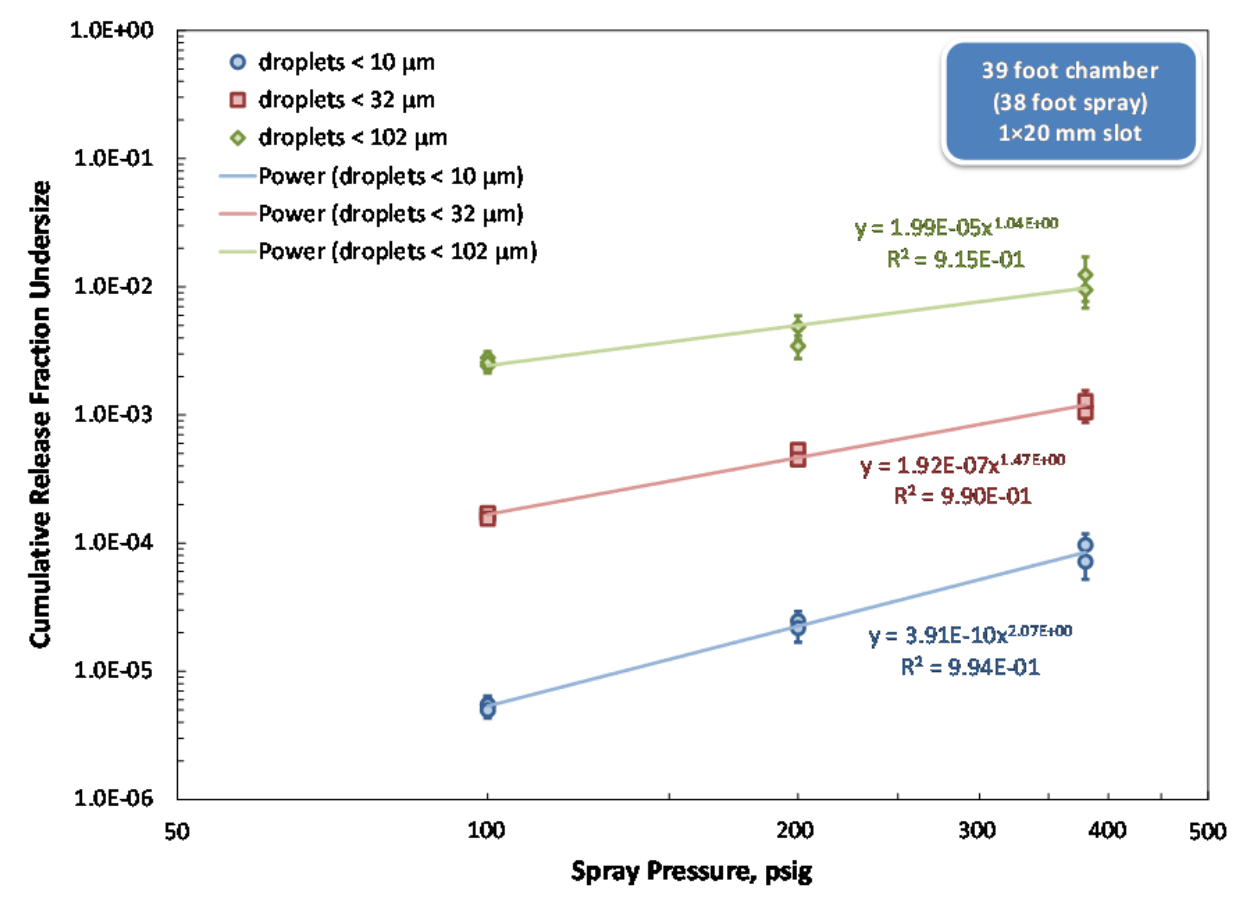

Figure 9.4. Example Fit of In-Chamber Release Fraction at 10, 32, and $102 \mu \mathrm{m}$ as a Function of Test Pressure. Data correspond to release fraction measurements of water sprayed through a $1 \times 20 \mathrm{~mm}$ slot in the $39 \mathrm{ft}$ test chamber (with no spray extensions added). Individual test measurements are shown as solid points, with error bars corresponding to the uncertainty with individual release fraction measurements. The curves correspond to a best fit of the individual measurements using the power-law relationship defined by Equation (9.1).

The pressure scaling factors for all chamber length and orifice size combinations are shown in Table 9.1 and generally range from 1 to 2, indicating that increasing pressure always yields an increase in release fraction regardless of chamber length or orifice tested. Individual results vary greatly in quality (i.e., in terms of the fit statistics provided, which are expressed as 95 percent confidence intervals), but generally indicate a greater pressure dependence (higher scaling factors) for $10 \mu \mathrm{m}$ droplets relative to 32 and $102 \mu \mathrm{m}$ droplets. For the smallest two orifices (i.e., the $2 \mathrm{~mm}$ hole and $1 \times 10 \mathrm{~mm}$ slot), the pressure scaling factor shows chamber length dependence. Longer chambers show greater pressure dependence (higher pressure scaling factor) than smaller chambers. Pressure scaling factors for larger orifices also suggest similar functionality, but the trend is not statistically significant. 
Table 9.1. Best-Fit Pressure Scaling Factors for Water In-Chamber Release Fractions for Different Chamber Sizes and Orifice Combinations (from Equation (9.1)). The uncertainty reported for each scaling factor is a 95 percent confidence limit.

\begin{tabular}{rcccc}
\hline $\begin{array}{c}\text { Aerosol Diameter, } \\
\mu \mathrm{m}\end{array}$ & $\begin{array}{c}n_{P} \\
10 \mathrm{ft} \text { Chamber }\end{array}$ & $\begin{array}{c}n_{P} \\
20 \mathrm{ft} \text { Chamber }\end{array}$ & $\begin{array}{c}n_{P} \\
30 \mathrm{ft} \text { Chamber }\end{array}$ & $\begin{array}{c}n_{P} \\
39 \mathrm{ft} \text { Chamber }\end{array}$ \\
\hline \multicolumn{5}{c}{$2 \mathrm{~mm}$ hole } \\
32 & $1.4 \pm 0.4$ & $2.0 \pm 0.2$ & $2.2 \pm 0.4$ & $1.8 \pm 0.4$ \\
102 & $1.0 \pm 0.2$ & $1.7 \pm 0.3$ & $2.0 \pm 0.3$ & $2.1 \pm 0.4$ \\
10 & $0.9 \pm 0.2$ & $1.4 \pm 0.3$ & $1.5 \pm 0.8$ & $1.9 \pm 0.4$ \\
\hline 32 & $1.6 \pm 0.3$ & $1.8 \pm 0.2$ & $2.0 \pm 0.3$ & $2.4 \pm 0.5$ \\
102 & $1.2 \pm 0.2$ & $1.3 \pm 0.2$ & $1.7 \pm 0.2$ & $1.7 \pm 0.2$ \\
10 & $1.0 \pm 0.1$ & $1.1 \pm 0.3$ & $1.8 \pm 0.3$ & $1.6 \pm 0.2$ \\
\hline 32 & $1.9 \pm 0.3$ & $1.8 \pm 0.3$ & $2.6 \pm 0.4$ & $2.1 \pm 0.2$ \\
102 & $1.6 \pm 0.3$ & $1.3 \pm 0.1$ & $2.0 \pm 0.3$ & $1.5 \pm 0.2$ \\
10 & $1.5 \pm 0.7$ & $1.1 \pm 0.1$ & $2.1 \pm 0.3$ & $1.0 \pm 0.4$ \\
\hline 32 & $1.2 \pm 0.5$ & $1 \times 76 \mathrm{~mm} \mathrm{slot}$ & \\
102 & $1.2 \pm 0.6$ & $1.5 \pm 0.3$ & $1.4 \pm 0.4$ & $1.5 \pm 0.6$ \\
\hline & $1.4 \pm 1.1$ & $1.3 \pm 0.2$ & $1.3 \pm 0.4$ & $1.5 \pm 0.4$ \\
\hline & & $1.2 \pm 0.4$ & $1.8 \pm 0.4$ \\
\hline
\end{tabular}

\subsubsection{Impact of Chamber Length on the Orifice Area Functionality of Release Fraction}

To evaluate the effect of chamber length on the functionality of release fraction with orifice area, cumulative release fraction undersize for aerosol diameters at 10,32, and $102 \mu \mathrm{m}$ are fit to the following power-law expression:

$$
R_{k}=R_{k, o} A_{b}^{n_{A}}
$$

Here, $A_{b}$ is the area of the orifice, and $n_{A}$ is the orifice-area scaling exponent that describes how release fraction responds to changes in area. As in the previous section, linear regression analysis is used to determine the scaling exponent for orifice area for all test fluid and pressure combinations for 10, 32, and $102 \mu \mathrm{m}$ aerosol droplets. Best-fit orifice area scaling factor results are given in Table 9.2. An example of how release fraction varies with area and of the quality of fit of data to Equation (9.2) is given in Figure 9.5. The quality of the results and fits depends on the combination of orifice and chamber length tested. Those shown in the example figure indicate a relatively low correlation coefficient $\left(R^{2}\right)$ ranging from 0.01 to 0.5 and indicate little to no correlation between release fraction and orifice size for the data shown. Overall, smaller chambers show a decrease in release fraction with increasing orifice area. As chamber size increases, the dependence of release fraction on orifice area gradually disappears and is not significant in the $39 \mathrm{ft}$ chamber. 


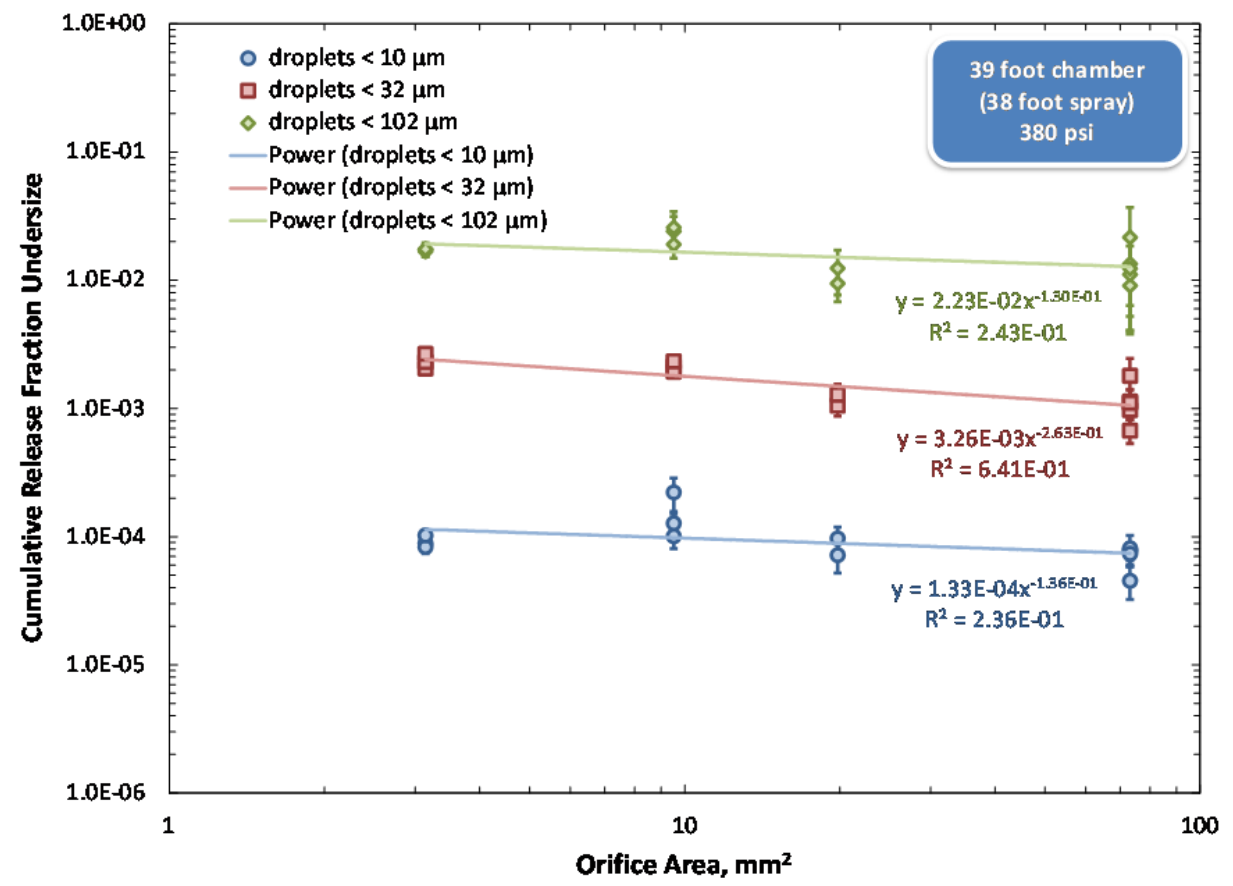

Figure 9.5. Example Fit of In-Chamber Release Fraction at 10, 32, and $102 \mu \mathrm{m}$ as a Function of Orifice Area. Data correspond to release fraction measurements of water at a release pressure of $380 \mathrm{psig}$ in the $39 \mathrm{ft}$ test chamber (with no spray header extensions added, yielding a $38 \mathrm{ft}$ spray). Individual test measurements are shown as solid points, with error bars corresponding to the uncertainty in individual release fraction measurements. The curves correspond to a best fit of the individual measurements using the power-law relationship defined by Equation (9.2).

Table 9.2. Best-Fit Orifice Area Scaling Factors for Water In-Chamber Release Fractions for Different Chamber Size and Test Pressure Combinations (from Equation (9.2)). The uncertainty reported for each scaling factor is a 95 percent confidence limit.

\begin{tabular}{|c|c|c|c|c|}
\hline $\begin{array}{c}\text { Aerosol Diameter, } \\
\mu \mathrm{m}\end{array}$ & $\begin{array}{c}n_{A}, \\
10 \mathrm{ft} \text { chamber }\end{array}$ & $\begin{array}{c}n_{A}, \\
20 \mathrm{ft} \text { chamber }\end{array}$ & $\begin{array}{c}n_{A}, \\
30 \mathrm{ft} \text { chamber }\end{array}$ & $\begin{array}{c}n_{A}, \\
39 \mathrm{ft} \text { chamber }\end{array}$ \\
\hline \multicolumn{5}{|c|}{$100 \mathrm{psig}$} \\
\hline 10 & $-0.6 \pm 0.2$ & $-0.2 \pm 0.1$ & $-0.2 \pm 0.2$ & $0.0 \pm 0.2$ \\
\hline 32 & $-0.7 \pm 0.2$ & $-0.4 \pm 0.2$ & $-0.2 \pm 0.1$ & $0.0 \pm 0.2$ \\
\hline 102 & $-0.5 \pm 0.2$ & $-0.4 \pm 0.2$ & $-0.3 \pm 0.3$ & $0.0 \pm 0.2$ \\
\hline \multicolumn{5}{|c|}{200 psig } \\
\hline 10 & $-0.5 \pm 0.1$ & $-0.3 \pm 0.1$ & $-0.2 \pm 0.2$ & $0.0 \pm 0.2$ \\
\hline 32 & $-0.7 \pm 0.1$ & $-0.5 \pm 0.1$ & $-0.4 \pm 0.1$ & $-0.2 \pm 0.1$ \\
\hline 102 & $-0.3 \pm 0.1$ & $-0.4 \pm 0.1$ & $-0.2 \pm 0.2$ & $-0.2 \pm 0.1$ \\
\hline \multicolumn{5}{|c|}{$380 \mathrm{psig}$} \\
\hline 10 & $-0.7 \pm 0.1$ & $-0.4 \pm 0.1$ & $-0.5 \pm 0.2$ & $-0.1 \pm 0.2$ \\
\hline 32 & $-0.6 \pm 0.1$ & $-0.5 \pm 0.1$ & $-0.5 \pm 0.1$ & $-0.3 \pm 0.1$ \\
\hline 102 & $-0.2 \pm 0.3$ & $-0.4 \pm 0.1$ & $-0.4 \pm 0.3$ & $-0.1 \pm 0.2$ \\
\hline
\end{tabular}




\subsection{Impact of Distance to Splash Wall (Spray Length)}

The impacts of spray length (at constant chamber length) on cumulative release fraction as a function of aerosol diameter are shown in Figure 9.6, Figure 9.7, and Figure 9.8 for all Phase II orifice and test pressure combinations. As noted in previous sections, spray length refers to the distance between the orifice and splash wall and does not necessarily correspond to the true length of the spray. Spray length tests provide a more direct evaluation of spray length impacts, but the results derived from spray length testing may be subject to bias caused by the increased distance between the spray and upstream aerosol analyzers. Specifically, as the spray header is moved farther down the length of the $39 \mathrm{ft}$ chamber, the mixing energy imparted by high-pressure spray and auxiliary chamber fans decreases, thus possibly reducing convection (and as a result, reducing apparent generation of aerosol) to the upstream aerosol analyzers.

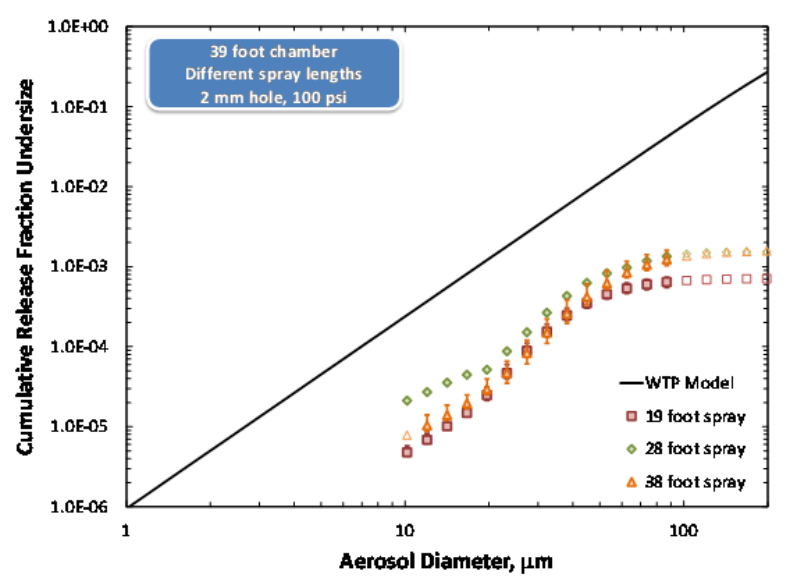

(a) $-2 \mathrm{~mm}$ hole

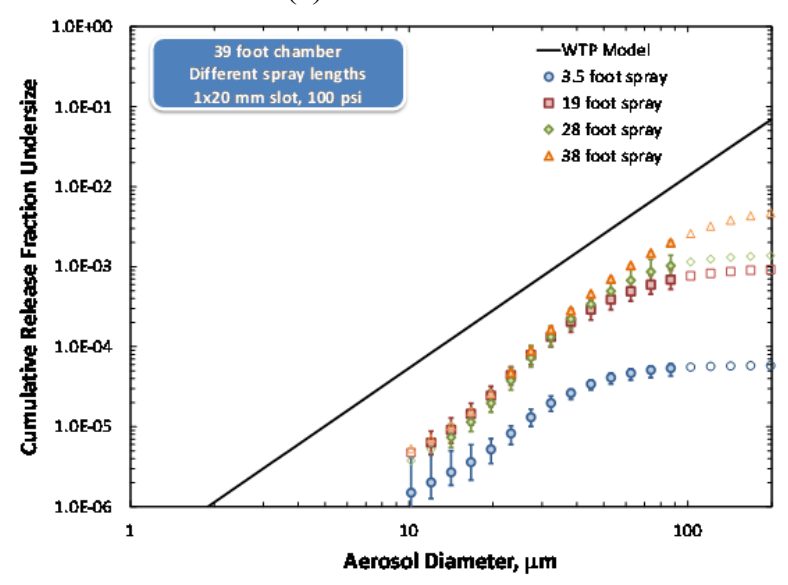

(c) $-1 \times 20 \mathrm{~mm}$ slot

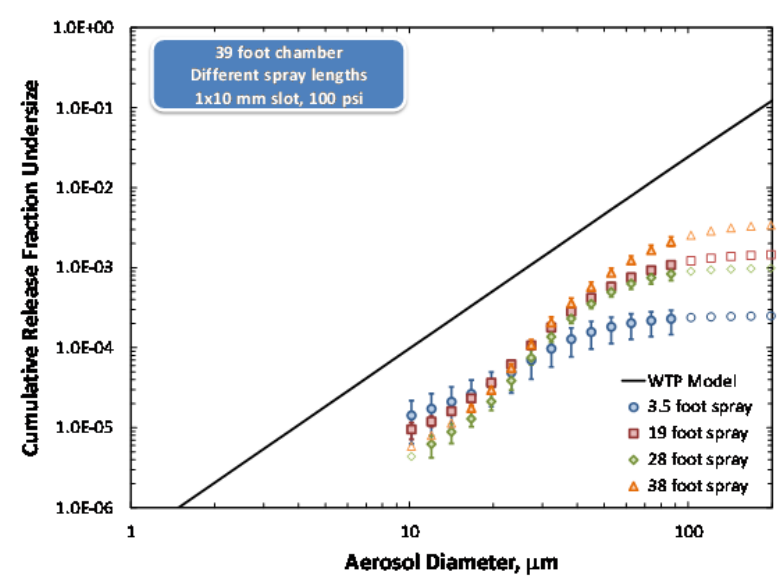

(b) $-1 \times 10 \mathrm{~mm}$ slot

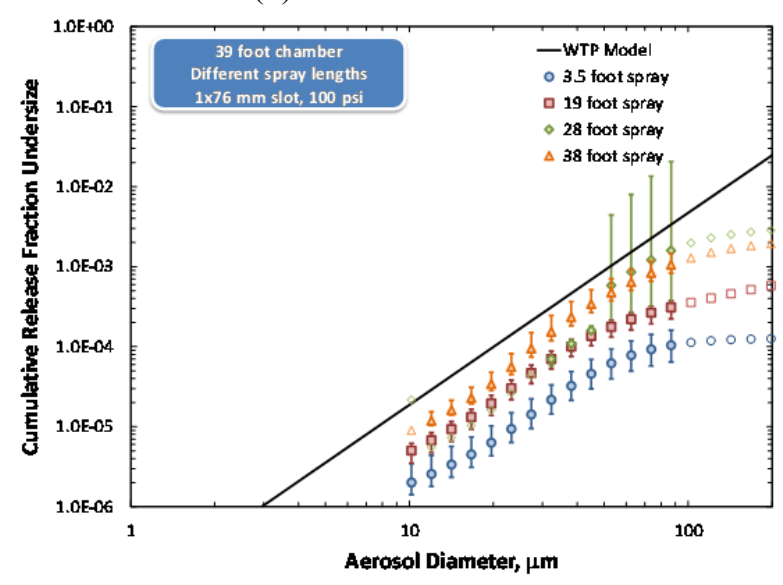

(d) $-1 \times 76 \mathrm{~mm}$ slot

Figure 9.6. In-Chamber Release Fraction as a Function of Aerosol Size for Different Spray Lengths and for Sprays Generated at 100 psig in the $39 \mathrm{ft}$ Chamber. Test results are shown for a (a) $2 \mathrm{~mm}$ hole, (b) $1 \times 10 \mathrm{~mm}$ slot, (c) $1 \times 20 \mathrm{~mm}$ slot, and (d) $1 \times 76 \mathrm{~mm}$ slot. Release fraction data for the $3.5 \mathrm{ft}, 2 \mathrm{~mm}$ hole, $100 \mathrm{psig}$ spray is not included because data quality concerns (see text on the next page for details). 
The trends observed in release fraction with changing spray length (as shown in Figure 9.6, Figure 9.7, and Figure 9.8) are functionally similar to those observed for increasing chamber length. In general, the majority of orifice area and test pressure combinations show either that release fraction increases or remains relatively unchanged at all aerosol diameters with increasing spray length for all aerosol diameters. It should be noted that the $3.5 \mathrm{ft}$ data for the $2 \mathrm{~mm}$ hole, $100 \mathrm{psig}$ spray are not included in Figure 9.6 because concentration rise data associated with this spray are extremely noisy and are not fit meaningfully by the exponential concentration rise model (relative to the majority of other in-chamber test data, whose behavior are approximated reasonably well by the exponential model). Poor data quality results from the difficulty in detecting aerosols for this configuration, which are present at extremely low concentrations because all analyzers are located upstream of the spray (i.e., behind the spray header) and because the $2 \mathrm{~mm}$ hole, 100 psig spray is the smallest tested during Phase II large-scale operations. Release fraction data for the $1 \times 10 \mathrm{~mm}$ slot exhibit trend reversal at small aerosol diameters $(<30 \mu \mathrm{m})$, indicating that release fraction increases as the spray length decreases. This observation is consistent with trends observed in chamber length data for small orifice areas and low test pressures. Decreases in small aerosol release fraction contributions with increasing chamber length were attributed to scavenging of small aerosols by large aerosols.
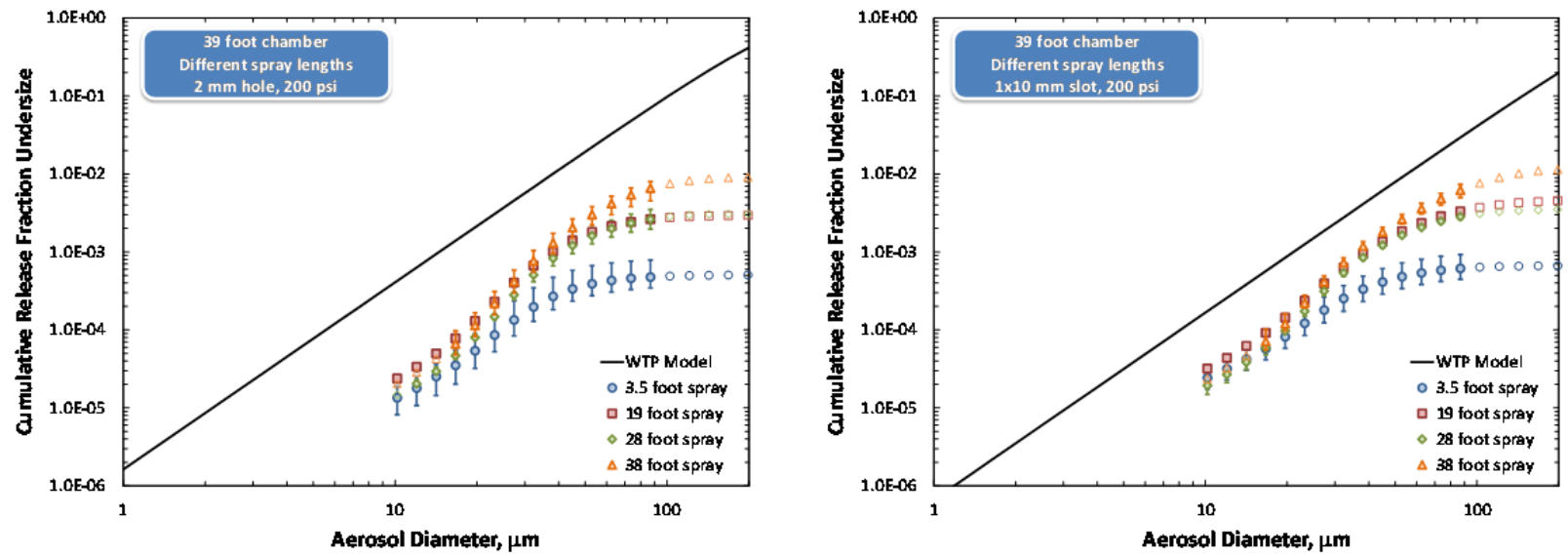

(a) $-2 \mathrm{~mm}$ hole

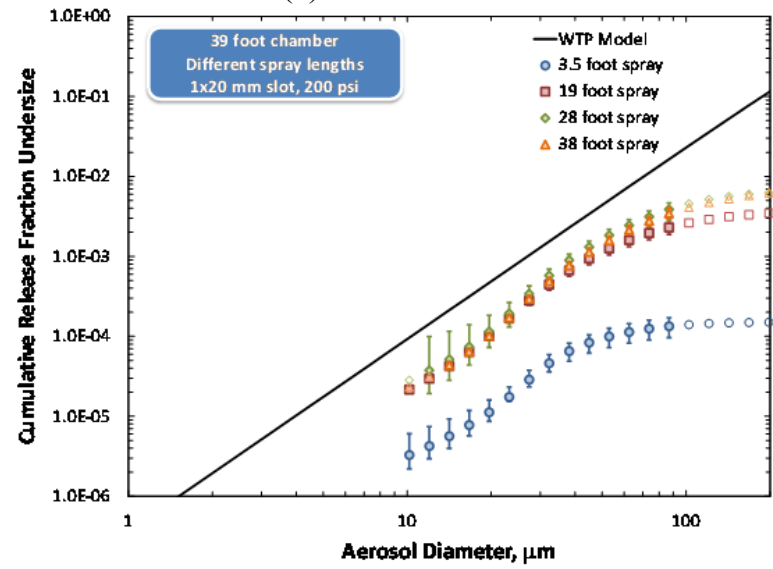

(c) $-1 \times 20 \mathrm{~mm}$ slot

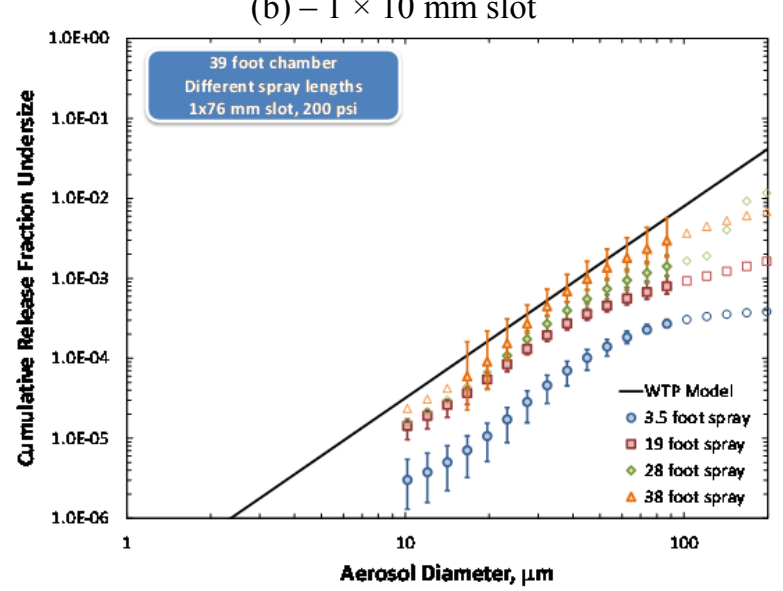

(d) $-1 \times 76 \mathrm{~mm}$ slot

Figure 9.7. In-Chamber Release Fraction as a Function of Aerosol Size for Different Spray Lengths and for Sprays Generated at 200 psig in the $39 \mathrm{ft}$ Chamber. Test results are shown for a (a) $2 \mathrm{~mm}$ hole, (b) $1 \times 10 \mathrm{~mm}$ slot, (c) $1 \times 20 \mathrm{~mm}$ slot, and (d) $1 \times 76 \mathrm{~mm}$ slot. 
Beyond the two exceptions identified above, the release fraction trends observed in the data appear to uniformly support observations that release fraction is either not impacted by spray length or it decreases with decreasing spray length. The actual trend observed and the amount of change in release fraction with spray length appears to vary with the orifice and test pressure combination. In many test cases, the $38 \mathrm{ft}$ spray yields the highest release fractions. In other cases, the release fraction for the $38 \mathrm{ft}$ spray is similar to that of the 20 and $30 \mathrm{ft}$ sprays. In most cases, the $3.5 \mathrm{ft}$ spray yields release fractions that fall below that observed for longer sprays. For chamber length tests, we postulate that increased spray length allows for a more complete break-up and dispersion of the spray droplets before impact with the splash wall. Secondary formation of aerosol by impact of high-pressure sprays on the splash wall does not appear to yield an increase in release fraction, for either large or small droplet sizes, over that observed for longer sprays. As such, the longest sprays tested (i.e., $38 \mathrm{ft}$ ) generally yield the largest release fractions. As stated previously, this observation affirms the Phase I conclusion that the longest postulated spray likely represents a "worst-case" spray from a safety analysis perspective.

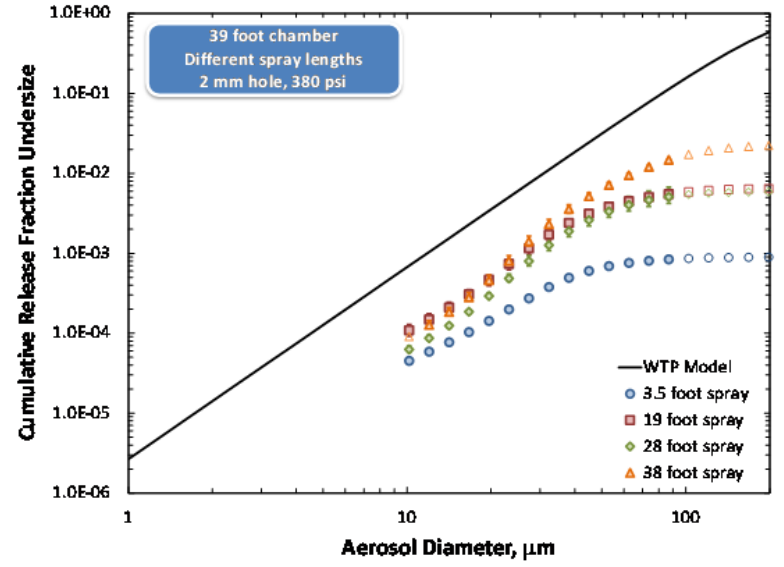

(a) $-2 \mathrm{~mm}$ hole

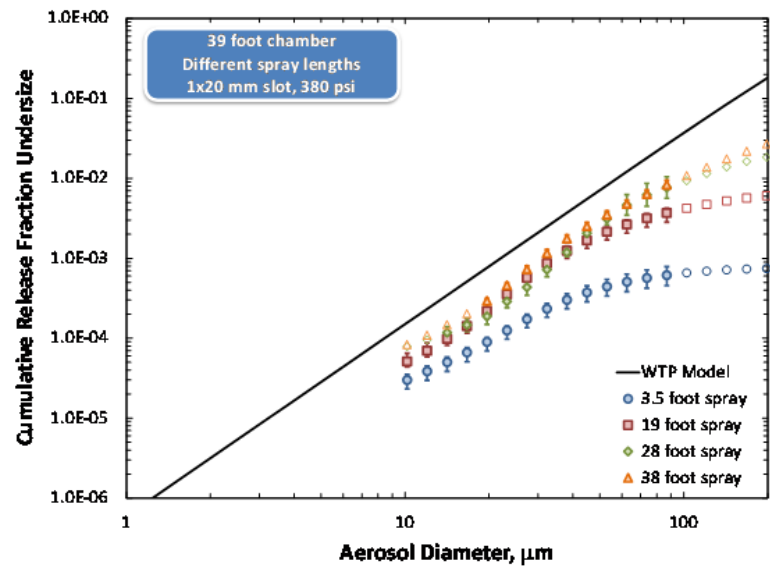

(c) $-1 \times 20 \mathrm{~mm}$ slot

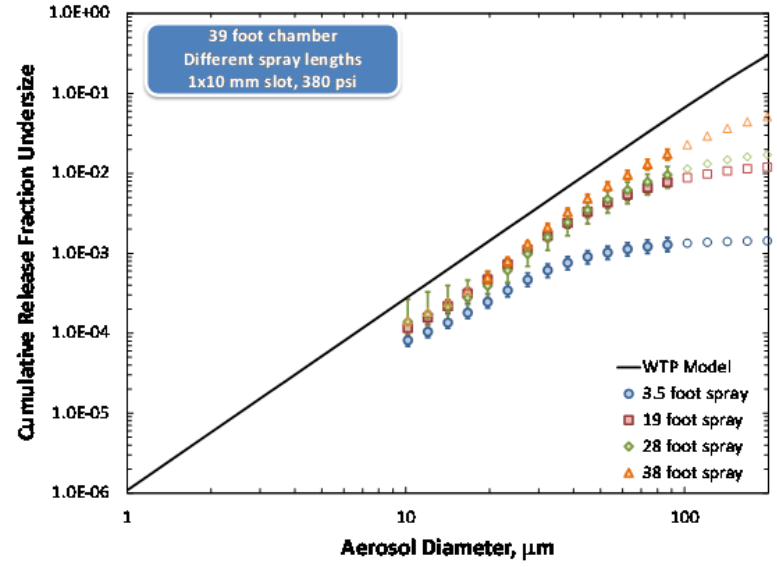

(b) $-1 \times 10 \mathrm{~mm}$ slot

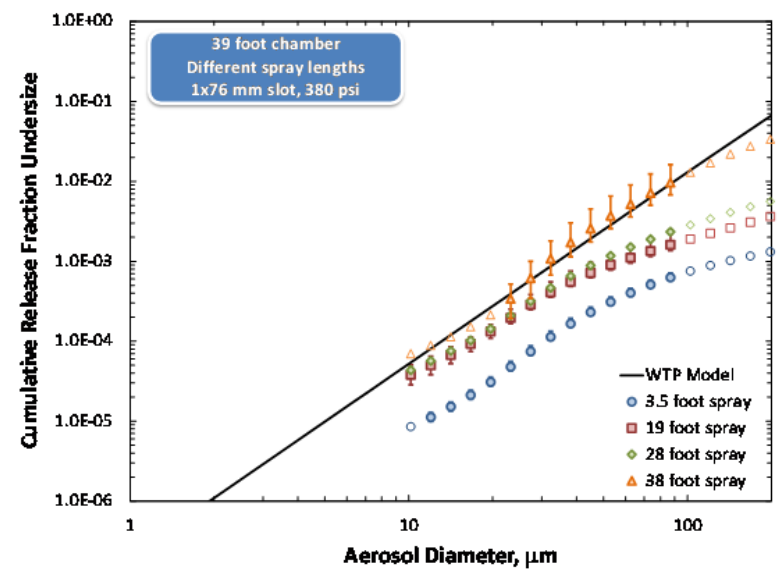

(d) $-1 \times 76 \mathrm{~mm}$ slot

Figure 9.8. In-Chamber Release Fraction as a Function of Aerosol Size for Different Spray Lengths and for Sprays Generated at 380 psig in the $39 \mathrm{ft}$ Chamber. Test results are shown for a (a) $2 \mathrm{~mm}$ hole, (b) $1 \times 10 \mathrm{~mm}$ slot, (c) $1 \times 20 \mathrm{~mm}$ slot, and (d) $1 \times 76 \mathrm{~mm}$ slot. 


\subsubsection{Impact of Distance to Splash Wall on the Pressure Functionality of Release Fraction}

The impact of spray length on release fraction at constant chamber length was evaluated above. Changes in spray length also might affect how release fraction varies with pressure. Pressure data corresponding to spray length tests were fit using Equation (9.1); the results are shown in Table 9.3. It should be stressed that the pressure scaling factors indicate general trends (i.e., increase or decrease) in the release fraction data with increasing pressure. For each orifice and spray length combination tested, the pressure scaling factor is accompanied by a 95 percent confidence limit to provide a measure of uncertainty in the result.

The pressure scaling factors shown in Table 9.3 vary greatly in terms of fit quality and result significance. Results for the $2 \mathrm{~mm}$ hole show significant uncertainty at spray lengths of 3.5 and $30 \mathrm{ft}$. This increased uncertainty is driven by significant variations in the individual (test-to-test) release fraction measurements for these two test conditions, thus complicating evaluation of trends in the $2 \mathrm{~mm}$ data set. When the entire set of pressure scaling factors are considered, the pressure scaling factor generally appears to fall between 1 and 2. This dependence indicates that release fraction increases with increasing test pressure and is consistent with observations made from test data evaluating the impact of the simulant used (Chapter 8) and chamber size (Section 9.1). Increased pressure dependence (i.e., higher scaling factors) is observed for $10 \mu \mathrm{m}$ aerosols relative to larger 32 and $102 \mu \mathrm{m}$ aerosols, but the most significant observations are generally limited to the $1 \times 10$ and $1 \times 20 \mathrm{~mm}$ slot data for $20 \mathrm{ft}$ sprays and longer and do not appear to be significant for short sprays or for any scaling factors derived from the $2 \mathrm{~mm}$ hole and $1 \times 76 \mathrm{~mm}$ slot configurations. In general, it is difficult to identify any strong trend in how release fraction scales with pressure for different length sprays.

Table 9.3. Best-Fit Pressure Scaling Factors for Water Release Fractions for Different Spray Length and Orifice Size Combinations (from Equation (9.1)). The uncertainty reported for each scaling factor is a 95 percent confidence limit.

\begin{tabular}{ccccc}
\hline $\begin{array}{c}\text { Aerosol Diameter, } \\
\mu \mathrm{m}\end{array}$ & $\begin{array}{c}n_{P} \\
3.5 \mathrm{ft} \text { spray }\end{array}$ & $\begin{array}{c}n_{P} \\
20 \mathrm{ft} \text { spray }\end{array}$ & $\begin{array}{c}n_{P} \\
30 \mathrm{ft} \text { spray }\end{array}$ & $\begin{array}{c}n_{P} \\
38 \mathrm{ft} \text { spray }\end{array}$ \\
\hline \multicolumn{5}{c}{$2 \mathrm{~mm}$ hole } \\
32 & $1.9 \pm 1.6$ & $2.3 \pm 0.2$ & $1.1 \pm 1.1$ & $1.8 \pm 0.4$ \\
102 & $1.0 \pm 1.9$ & $1.8 \pm 0.3$ & $1.2 \pm 0.4$ & $2.1 \pm 0.4$ \\
10 & $0.9 \pm 1.6$ & $1.6 \pm 0.3$ & $1.0 \pm 0.5$ & $1.9 \pm 0.4$ \\
\hline 32 & $1.3 \pm 0.7$ & $1.9 \pm 0.2$ & $2.6 \pm 0.5$ & $2.4 \pm 0.5$ \\
102 & $1.4 \pm 0.5$ & $1.7 \pm 0.1$ & $1.8 \pm 0.3$ & $1.7 \pm 0.2$ \\
10 & $1.3 \pm 0.5$ & $1.5 \pm 0.1$ & $1.9 \pm 0.2$ & $1.6 \pm 0.2$ \\
\hline 32 & $2.3 \pm 0.8$ & $1.8 \pm 0.3$ & $2.4 \pm 0.8$ & $2.1 \pm 0.2$ \\
102 & $1.9 \pm 0.4$ & $1.4 \pm 0.2$ & $1.4 \pm 0.6$ & $1.5 \pm 0.2$ \\
10 & $1.9 \pm 0.4$ & $1.3 \pm 0.3$ & $1.6 \pm 0.5$ & $1.0 \pm 0.4$ \\
\hline 32 & $1.1 \pm 0.7$ & $1.5 \pm 0.3$ & $0.5 \pm 1.4$ & $1.5 \pm 0.6$ \\
102 & $1.2 \pm 0.5$ & $1.3 \pm 0.2$ & $1.4 \pm 0.4$ & $1.5 \pm 0.4$ \\
\hline & $1.4 \pm 0.4$ & $1.2 \pm 0.2$ & $0.3 \pm 1.7$ & $1.8 \pm 0.4$ \\
\hline
\end{tabular}




\subsubsection{Impact of Distance to Splash Wall on the Orifice Area Functionality of Release Fraction}

The impact of spray length on release fraction at constant chamber length was evaluated above. Changes in spray length also might affect how release fraction varies with orifice area. Release fraction data for different test orifices corresponding to spray length tests were fit using Equation (9.2). The orifice area scaling factors for test pressure and spray length combinations are shown in Table 9.4. Scaling factors derived from the 100 psig data set show significant uncertainty, thus limiting evaluation of trends with spray length at low pressure; this uncertainty is reduced at higher test pressures. Overall, the test results suggest that for spray lengths of $20 \mathrm{ft}$ and longer, release fractions show limited (i.e., marginally significant) variation with orifice area. In these cases, the direction in which release fraction varies is not consistent: $38 \mathrm{ft}$ data indicate a very slight decrease, whereas $20 \mathrm{ft}$ data suggest a very slight increase. As stated previously, both trends are near the edge of uncertainty, and the variability might simply reflect poor correlation between release fraction and orifice area for the spray length test data. Orifice area scaling factors for $3.5 \mathrm{ft}$ data suggest a decrease in release fraction with increasing orifice area, with the absolute value of scaling factor being significantly larger than most other test pressure and spray length configurations. The significance of the $3.5 \mathrm{ft}$ area scaling factors is still marginal, as the uncertainty associated with $3.5 \mathrm{ft}$ data also is increasingly large. Overall, data indicate poor correlation of release fraction with the power-law model used to evaluate scaling factors and do not support a strong dependence of release fraction with orifice area under test conditions evaluated in spray length testing.

Table 9.4. Best-Fit Orifice Area Scaling Factors for Water Release Fractions for Different Spray Length and Test Pressure Combinations (from Equation (9.2)) in the $39 \mathrm{ft}$ Test Chamber. The uncertainty reported for each scaling factor is a 95 percent confidence limit.

\begin{tabular}{ccccc}
\hline $\begin{array}{c}\text { Aerosol Diameter, } \\
\mu \mathrm{m}\end{array}$ & $\begin{array}{c}n_{A}, \\
3.5 \mathrm{ft} \text { spray }\end{array}$ & $\begin{array}{c}n_{A}, \\
20 \mathrm{ft} \text { spray }\end{array}$ & $\begin{array}{c}n_{A}, \\
30 \mathrm{ft} \text { spray }\end{array}$ & $\begin{array}{c}n_{A}, \\
38 \mathrm{ft} \text { spray }\end{array}$ \\
\hline \multicolumn{5}{c}{$100 \mathrm{psig}$} \\
32 & $-0.8 \pm 1.0$ & $0.0 \pm 0.2$ & $0.3 \pm 0.9$ & $0.0 \pm 0.2$ \\
102 & $-0.6 \pm 0.6$ & $0.1 \pm 0.2$ & $-0.4 \pm 0.2$ & $0.0 \pm 0.2$ \\
10 & $-0.3 \pm 0.7$ & $0.2 \pm 0.2$ & $0.2 \pm 0.8$ & $0.0 \pm 0.2$ \\
\hline 32 & $-0.6 \pm 0.4$ & $200 \mathrm{psig}$ \\
102 & $-0.6 \pm 0.4$ & $0.2 \pm 0.1$ & $0.0 \pm 0.3$ & $0.0 \pm 0.2$ \\
10 & $-0.3 \pm 0.4$ & $0.4 \pm 0.2$ & $-0.2 \pm 0.2$ & $-0.2 \pm 0.1$ \\
32 & $-0.7 \pm 0.3$ & $380 \mathrm{psig}$ & $-0.2 \pm 0.2$ & $-0.2 \pm 0.1$ \\
\hline 102 & $-0.5 \pm 0.2$ & $0.4 \pm 0.2$ & $-0.2 \pm 0.3$ & $-0.1 \pm 0.2$ \\
\hline & $-0.1 \pm 0.2$ & $0.5 \pm 0.3$ & $-0.4 \pm 0.2$ & $-0.3 \pm 0.1$ \\
\hline
\end{tabular}

\subsection{In-Chamber Sprays of Equivalent Length in Different Chambers}

The results discussed in Sections 9.1 and 9.2 evaluate the impact of chamber and spray length on the release fraction for various Phase II orifice and test pressure combinations. The two studies considered the effect of changing the distance between the source of spray and the surface upon which the spray impinges (i.e., the splash wall). In one study, the chamber volume (and length) was held constant, 
whereas in the other study, the chamber size was changed. Both evaluation approaches show similar trends in how release fraction varies with increasing spray length, and we expect that both studies should show similar release fraction values at equivalent spray length if the impact of chamber volume on release fraction is appropriately accounted for by the method.

The large-scale test matrix allows for $\sim 30 \mathrm{ft}$ sprays in the $30 \mathrm{ft}$ test chamber and in the $39 \mathrm{ft}$ test chamber (with spool extensions added). Likewise, the large-scale test matrix allows for $\sim 20 \mathrm{ft}$ sprays in both the $20 \mathrm{ft}$ test chamber and the $39 \mathrm{ft}$ chamber (with spool extensions added) comparison. Comparison of release fractions for sprays of equivalent length for 30 and $20 \mathrm{ft}$ spray lengths is shown for all orifices at a test pressure of 380 psig in Figure 9.9 and Figure 9.10, respectively. For both 20 and $30 \mathrm{ft}$ sprays, the $1 \times 10 \mathrm{~mm}$ and $1 \times 76 \mathrm{~mm}$ slot data show excellent agreement between sprays of equivalent length but in different chamber sizes. In contrast, data from the $2 \mathrm{~mm}$ hole and the $1 \times 20 \mathrm{~mm}$ slot indicate higher release fractions for both 20 and $30 \mathrm{ft}$ sprays in the shorter chamber relative to the spray of equivalent
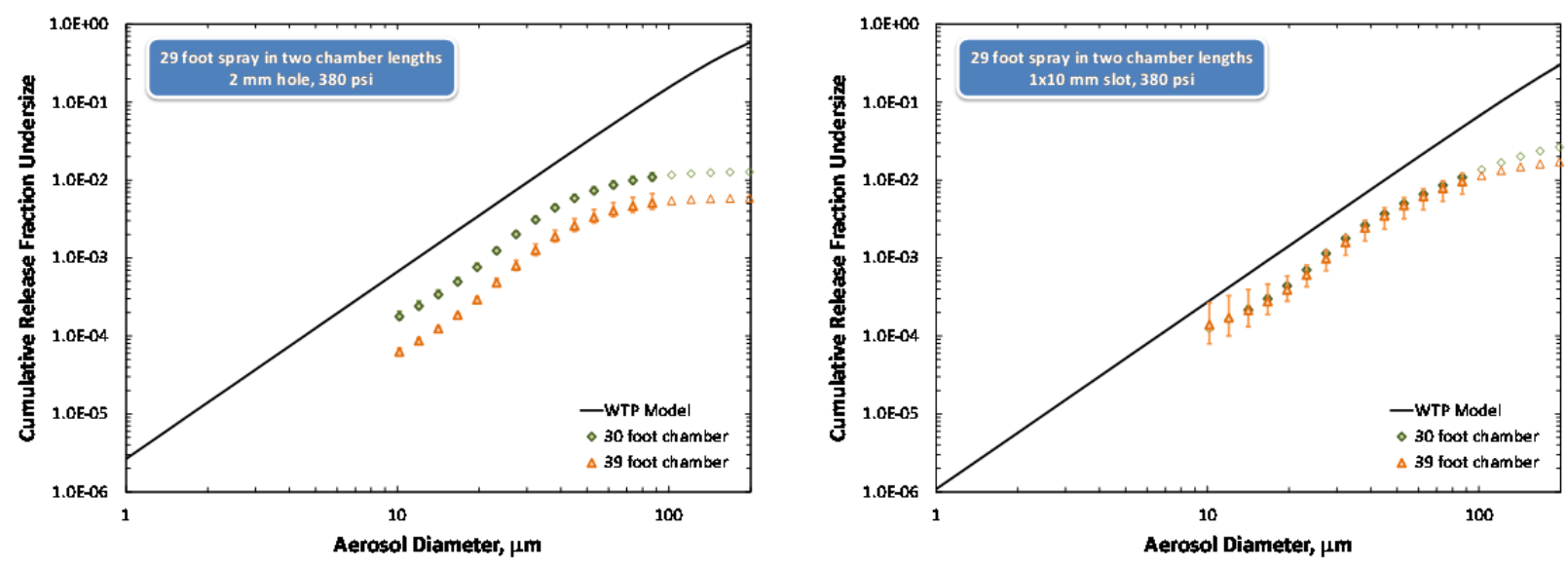

(a) $-2 \mathrm{~mm}$ hole

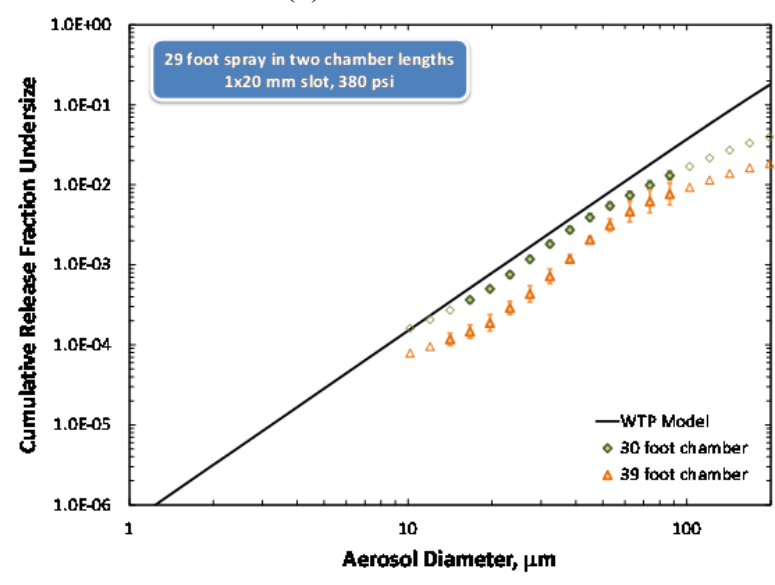

(c) $-1 \times 20 \mathrm{~mm}$ slot

(b) $-1 \times 10 \mathrm{~mm}$ slot

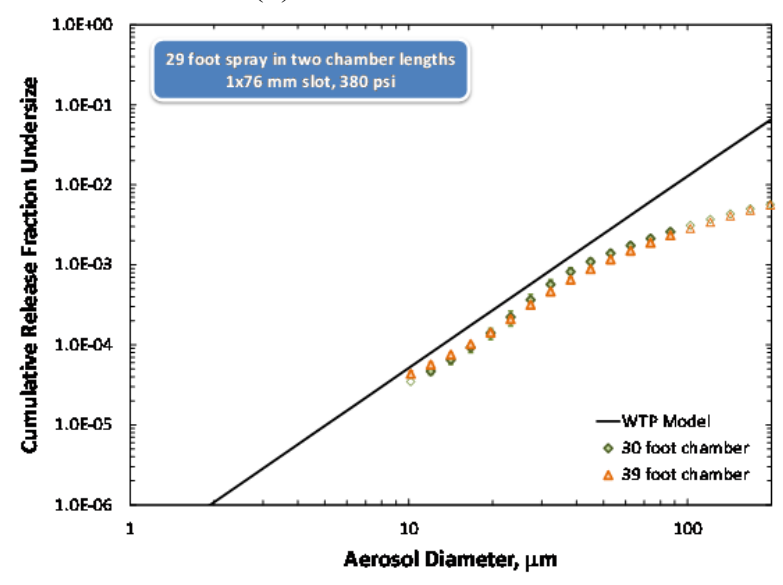

(d) $-1 \times 76 \mathrm{~mm}$ slot

Figure 9.9. Comparison of In-Chamber Release Fraction as a Function of Aerosol Size for Equivalent $30 \mathrm{ft}$ Sprays in the 30 and $39 \mathrm{ft}$ Chambers and for Sprays Generated at $380 \mathrm{psig}$. Test results are shown for a (a) $2 \mathrm{~mm}$ hole, (b) $1 \times 10 \mathrm{~mm}$ slot, (c) $1 \times 20 \mathrm{~mm}$ slot, and (d) $1 \times 76 \mathrm{~mm}$ slot. 
length in the $39 \mathrm{ft}$ chamber. With respect to the latter spray results, the difference is greatest in the $30 \mathrm{ft}$ chamber. It is difficult to identify potential cause that yields higher release fractions in smaller chambers for the $2 \mathrm{~mm}$ hole and $1 \times 20 \mathrm{~mm}$ slot but not for equivalent data for the $1 \times 10 \mathrm{~mm}$ and $1 \times 76 \mathrm{~mm}$ slot. The difference could simply represent test method uncertainty associated with changes in the chamber configuration itself (although Chapter 7 indicates that exact placement of the aerosol analyzer should not matter) or may derive from differences in chamber relative humidity at the start of test. It should be noted that only the 380 psig are discussed here for brevity; data corresponding to sprays generated at 100 and 200 psig show similar trends.
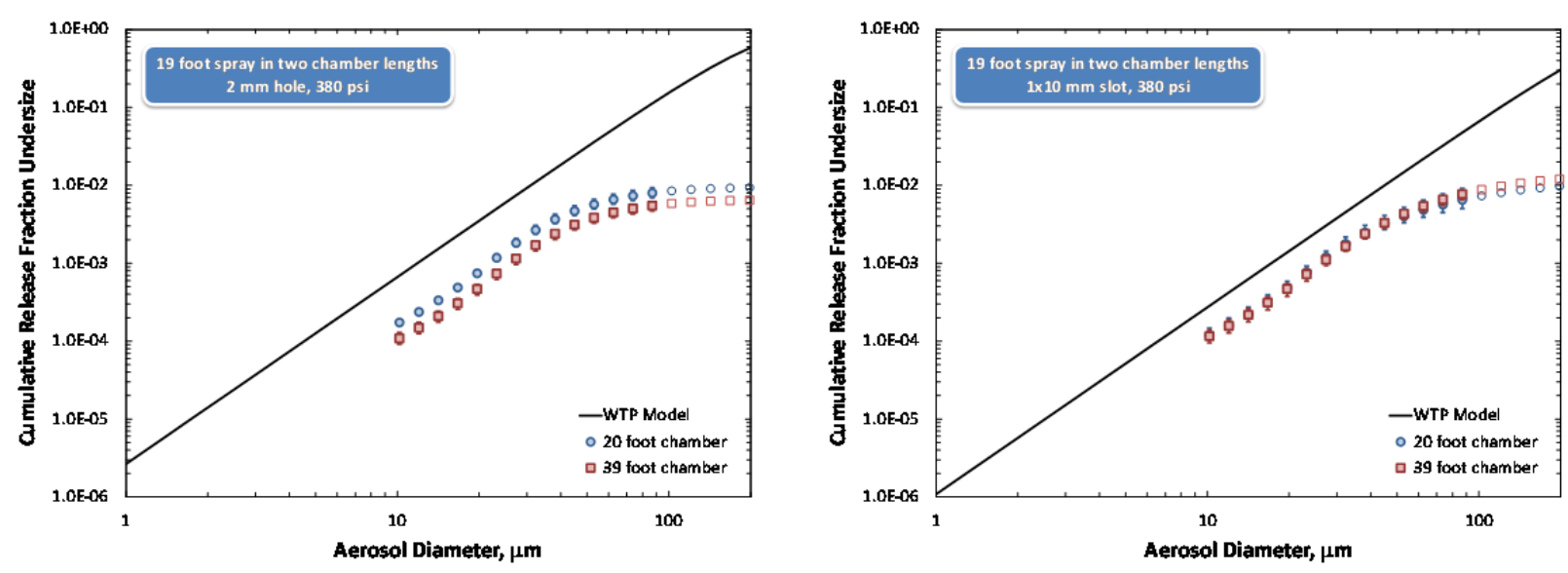

(a) $-2 \mathrm{~mm}$ hole

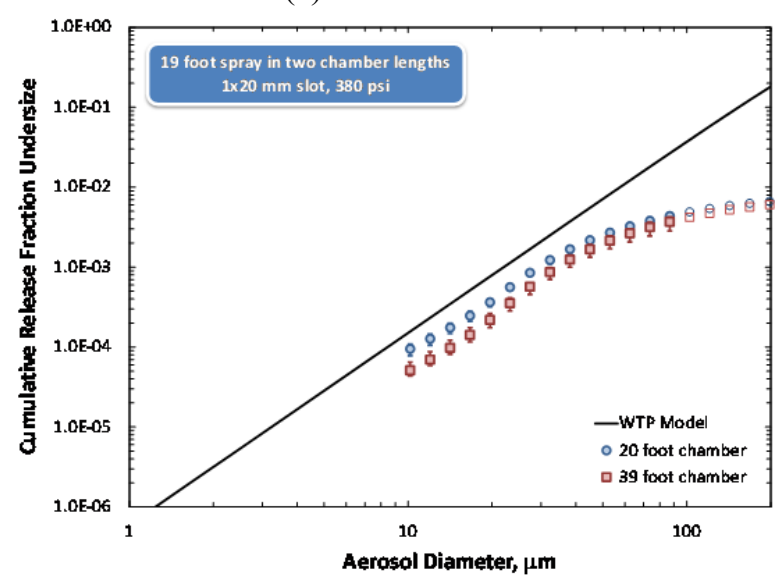

(c) $-1 \times 20 \mathrm{~mm}$ slot

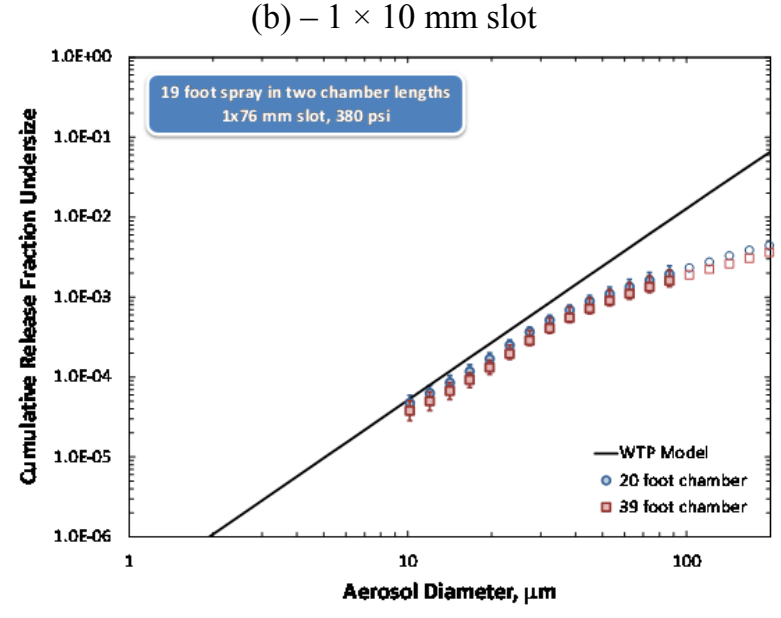

(d) $-1 \times 76 \mathrm{~mm}$ slot

Figure 9.10. Comparison of In-Chamber Release Fraction as a Function of Aerosol Size for Equivalent $20 \mathrm{ft}$ Sprays in the 20 and $39 \mathrm{ft}$ Chambers and for Sprays Generated at $380 \mathrm{psig}$. Test results are shown for a (a) $2 \mathrm{~mm}$ hole, (b) $1 \times 10 \mathrm{~mm}$ slot, (c) $1 \times 20 \mathrm{~mm}$ slot, and (d) $1 \times 76 \mathrm{~mm}$ slot.

\subsection{Conclusions}

In this chapter, the results of studies that evaluated the impact of chamber size and spray length on release fraction for Phase II test orifice and pressure combinations were presented and discussed. These results indicate that changes in the distance from the source of the spray (i.e., the breach) to surfaces on 
which the spray impinges can have a significant impact on the release fraction, as measured above the spray in the test chamber. The change in cumulative release fraction undersize with changes in spray length is complex, and there is not a single overreaching statement that can explain the trends observed in the data for all test orifice and pressure combinations. However, several broad conclusions can be made with respect to the release fraction trends with increasing spray length. These conclusions are summarized below:

- For large aerosol (typically $>50 \mu \mathrm{m}$ ) particles, cumulative release fraction undersize typically (but not always) increases with increasing length of spray (i.e., as the distance between the spray source and splash wall increases). One postulated mechanism by which the total cumulative release fraction (i.e., the value of cumulative release fraction at the largest aerosol sizes examined) increases is a greater opportunity for aerosol break-up and entrainment throughout the chamber before impingement and collection on the splash wall for aerosols generated by long sprays (relative to that expected for aerosols generated by "short" sprays). However, the increase may also derive from changes in aerosol analyzer locations. In larger chambers, the analyzers are placed farther downstream from the orifice and into sampling locations that contain a portion of the spray as it fans out. Sampling concerns are mitigated to some extent by the findings presented in Section 7, which indicate that the in-chamber generation rate result should not strongly depend on position. The increase in total release fraction (i.e., that for large aerosols) becomes more significant at the highest pressures and largest orifices tested in Phase II, but is noticeable at most test conditions. The only orifice for which total release fraction does not increase with chamber length occurs in sprays generated using the $2 \mathrm{~mm}$ hole at $100 \mathrm{psig}$. These sprays exhibit no change in total release fraction with increasing chamber length. This test condition represents the smallest orifice and smallest test pressure, and as a result, it should yield a short spray that is expected to completely break up before impingement. It may be postulated that the spray from this orifice is completely aerosolized by $10 \mathrm{ft}$ such that longer chamber lengths do not allow for improved aerosolization; however, spray trajectories measured during in-spray testing indicate that the $2 \mathrm{~mm}, 100 \mathrm{psig}$ spray can travel farther than $10 \mathrm{ft}$. This does not necessarily negate arguments made with respect to complete aerosolization, but it requires that no further aerosolization take place at distances longer than $10 \mathrm{ft}$.

- In larger chambers, release fraction contributions are shifted to larger diameters. This yields a result in small $(<50 \mu \mathrm{m})$ aerosol release fraction and an increase in large particle aerosolization $(>50 \mu \mathrm{m})$ for intermediate sprays (i.e., those at intermediate orifice areas or intermediate pressure). For the largest orifice tested, the release fraction of large particles increased while those for small particles were unchanged. The shift in release fraction to larger aerosols is attributed primarily to increased opportunity for droplet coalescence in large chambers, but it could also result from improved sampling of large aerosols in downstream chamber positions. For $2 \mathrm{~mm}$ holes, in-spray data confirm a general increase in size distribution with increasing downstream distance from the orifice. However, the shift in in-chamber release fraction for larger orifices $(1 \times 10 \mathrm{~mm}, 1 \times 20 \mathrm{~mm}$, and $1 \times 76 \mathrm{~mm}$ ) does not agree with in-spray behavior, which shows either no change or a decrease in aerosol size with distance. Overall, the shift in release fraction contribution to larger particles appears to be an effect of chamber size rather than spray length, as the effect is observed most strongly in tests where chamber size is varied, and is not observed to the same extent in tests where the spray length, but not the chamber size, is varied. 
- Based on the two observations outlined above, consideration of bounding release fractions for safety analysis should consider the combination of release fractions associated with small $(<50 \mu \mathrm{m})$ aerosols generated by spray in confined spaces (short/small chambers) and large $(>50 \mu \mathrm{m})$ aerosol generated by spray in large/open spaces (long/large chambers).

- All results indicate that release fraction shows a statistically significant increase with increases in spray test pressure over the range tested in Phase II studies (100 to 380 psig). Evaluation of the pressure dependence of the results with a power-law model indicates that release fraction scales with pressure raised to a power that generally ranges from 1 to 2 . Results for tests where chamber size was varied indicate that this pressure scaling depends on chamber and aerosol size. Release fractions for smaller aerosols show greater increase with increasing pressure relative to larger aerosols. Likewise, release fractions for aerosols generated in large chambers shows greater pressure dependence than those generated in confined spaces. Analysis of pressure scaling factors for tests in which spray length was varied in a chamber of fixed length indicate similar aerosol size dependence but do not suggest a statistically significant variation in scaling factors with actual spray length.

- The response of release fraction to changes in orifice area appears to demonstrate chamber size dependence. For smaller test chambers, release fraction appears to show a slight (but statistically significant) decrease in release fraction with increasing orifice area. Analysis of this dependence with a power-law model indicates an orifice area scaling factor near -0.5. For the largest test chamber length studied, the orifice area scaling factor is small and, in many cases, not statistically different than zero (indicating no dependence on release fraction with orifice area). Evaluation of orifice area scaling factors for different spray lengths in the $39 \mathrm{ft}$ test chamber indicates poor correlation between release fraction and orifice area data derived from spray length testing. While the scaling factor results indicate an orifice area dependence for the shortest sprays tested (3.5 ft), release fractions for longer sprays typically did not show significant variation with orifice area (relative to the uncertainty in the scaling factor results).

- Comparison of release fraction results for sprays of equivalent length in different chamber sizes generally indicates excellent agreement. In several cases, however, the release fraction for shortened sprays measured in the $39 \mathrm{ft}$ chamber was substantially smaller than sprays of similar length in smaller chambers. The occurrence of such differences does not correlate with test pressure or orifice area/morphology, which suggests that the differences result from test bias associated with operating conditions (such as test humidity). 



\subsection{Generation Rate Estimates for Longer Chambers and Correlation Development for Comparing Test Results to the WTP Model}

Previous (Schonewill et al. 2012) and current (see Chapter 9) test results show that the generation rate $^{(1)}$ of aerosol droplets can increase or decrease with increasing chamber length depending on the droplet size, orifice, and spray pressure. In general, the highest generation rate for any specific orifice and spray pressure is needed for use in spray release accident analysis calculations. The purpose of the tests summarized in Chapter 9 was to estimate, by extrapolation, the aerosol generation rate and release fraction for a plausible long-distance spray because this can represent the highest generation rate for a specific orifice and spray pressure. The results for extrapolations of the test data to longer chambers and sprays are presented in this chapter. A distance of $100 \mathrm{ft}$ was used previously as a reasonable distance for a long-distance spray (Schonewill et al. 2012), and we used this length in our work.

As described in the DOE standard for preparing safety analyses (DOE-3009-94), reasonably conservative aerosol generation rate estimates are needed for use in accident analysis calculations. Accordingly, reasonably conservative aerosol generation rate estimates based on the test data are needed for comparison to the WTP model and, potentially, for use in estimating aerosol generation rates for accident analysis calculations. The current test results do not match each potential accident condition for spray releases in the WTP (orifice, spray pressure, fluid) and also do not include the largest postulated sprays, such as the largest postulated breach in a 10 in. pipe. A method for using the current test data to estimate aerosol generation rates for droplets between 10 and $100 \mu \mathrm{m}$, which is the size range needed for accident analyses, for any particular spray of interest in an accident analysis in the WTP also would be beneficial.

The purpose of this chapter is to develop a correlation, based on the in-chamber data, for the cumulative generation rate of aerosol droplets for the size range of interest that is reasonably conservative. This correlation can then be used to extrapolate to larger orifices that were not tested, can be used to interpolate to other orifices and test conditions that were not specifically tested, and can be used to compare with the WTP model. The intent is to develop a correlation that adequately represents all the testing data, including the in-spray results that are typically considered to give conservative (i.e., high) generation rate values. Ideally, a reasonably conservative correlation will also account for potential bias in the test results and will also overcome the limitation of the in-chamber method to provide accurate generation rate measurements of droplets roughly $87 \mu \mathrm{m}$ and larger and the limitation of the in-spray method to provide accurate generation rate measurements of droplets smaller than about $50 \mu \mathrm{m}$.

In Section 10.1, results for extrapolating the generation rate test data to a $100 \mathrm{ft}$ chamber and spray for each orifice and spray pressure are presented. Section 10.2 summarizes the test results showing that water sprays always give the same or higher generation rates than the other liquids and slurries tested, which is the technical basis for why water spray results represent conservative values for other liquids and slurries used in the testing. Section 10.3 presents the approach for developing a correlation of the generation rate data for water sprays and the method for selecting a reasonably conservative correlation. Section 10.4 evaluates the correlation to determine if it is reasonably conservative by comparing it to the

\footnotetext{
${ }^{1}$ The generation rate referred to is a net generation rate: that is, the in-spray generation rate minus losses from settling, wall impact, etc.
} 
in-spray data for the different orifices and spray pressures and also comparing the conservative correlation to humidity-corrected data. The humidity correction is based on humidity measurements obtained from sensors that did not meet the project calibration requirements, so this comparison is For Information Only (FIO) and is focused on evaluating if the conservation correlation is sufficient to account for an FIO estimate of the humidity correction for the test data. Section 10.5 shows a comparison of the conservative correlation and in-spray data to the WTP model. This comparison is done for the orifices and spray pressures tested and comparisons are also shown for three specific orifices and spray pressures for process streams that are of interest to the WTP for accidental spray release scenarios. The conservative correlation also is compared to three other correlations from the literature, and finally, a comparison is made between the conservative correlation and clay and water spray data. Finally, Section 10.6 gives a summary of the conclusions and recommendations based on the discussions presented in this section. For the figures in this chapter, the criteria for when to show test data in a figure, and when to use open and closed symbols, follows the conventions given in Section 8.1.

\subsection{Extrapolation of Generation Rate Results to Longer Chambers and Sprays}

As shown in Section 9, the aerosol generation rates and release fractions determined from the transient concentrations depend on the chamber length. Depending on the droplet size, orifice and test conditions, the aerosol generation rate and release fractions sometimes increase and other times decrease with increasing chamber length. To provide estimates of the highest generation rates and release fractions, extrapolation to longer distances is needed, and the test results in different length chambers can be used for this purpose. There is no specific length spray that is defined for the WTP, but a distance of $100 \mathrm{ft}$ will be selected as a reasonable distance for extrapolation to a long-distance spray and is the same distance assumed previously (Schonewill et al. 2012). The smallest chamber tested, which was $10 \mathrm{ft}$ long, is proposed as a reasonable selection to represent the smallest volume where an accidental spray might occur within the WTP.

The approach for extrapolating the measured generation rates to longer chambers is to fit the results for the cumulative generation rate as a function of chamber size for each droplet size with a power-law fit and then extrapolate to a $100 \mathrm{ft}$ chamber. Figure 10.1 (upper) shows the generation rates for each chamber length and the $100 \mathrm{ft}$ extrapolation. Figure 10.1 (lower) shows examples of the power-law fits and extrapolations of power-law fits for three droplet sizes. These results are for a $380 \mathrm{psig}$ water spray from a $2 \mathrm{~mm}$ hole. For the smaller droplets, such as $10 \mu \mathrm{m}$ droplets, the generation rate decreases with increasing chamber length. For the larger droplets, such as $100 \mu \mathrm{m}$ droplets, the generation rate increases with increasing chamber length. The extrapolated estimates follow the trend in the test data. Figure 10.2 and Figure 10.3 show the results for the $2 \mathrm{~mm}$ hole at 200 and 100 psig. The effect of chamber length, where the generation rate decreases with chamber length for smaller droplets and increases for larger droplets, is generally similar at the lower spray pressures. Figure 10.4 through Figure 10.12 show the additional results for the other orifices $(1 \times 10,1 \times 20$, and $1 \times 76$ slots $)$ at the three spray pressures $(380$, 200 , and $100 \mathrm{psig}$ ) used in the testing. The general trend with increasing orifice area is that the effect of chamber length progressively shifts to where the generation rate increases with chamber length for all droplet sizes and not just for the larger droplets. 

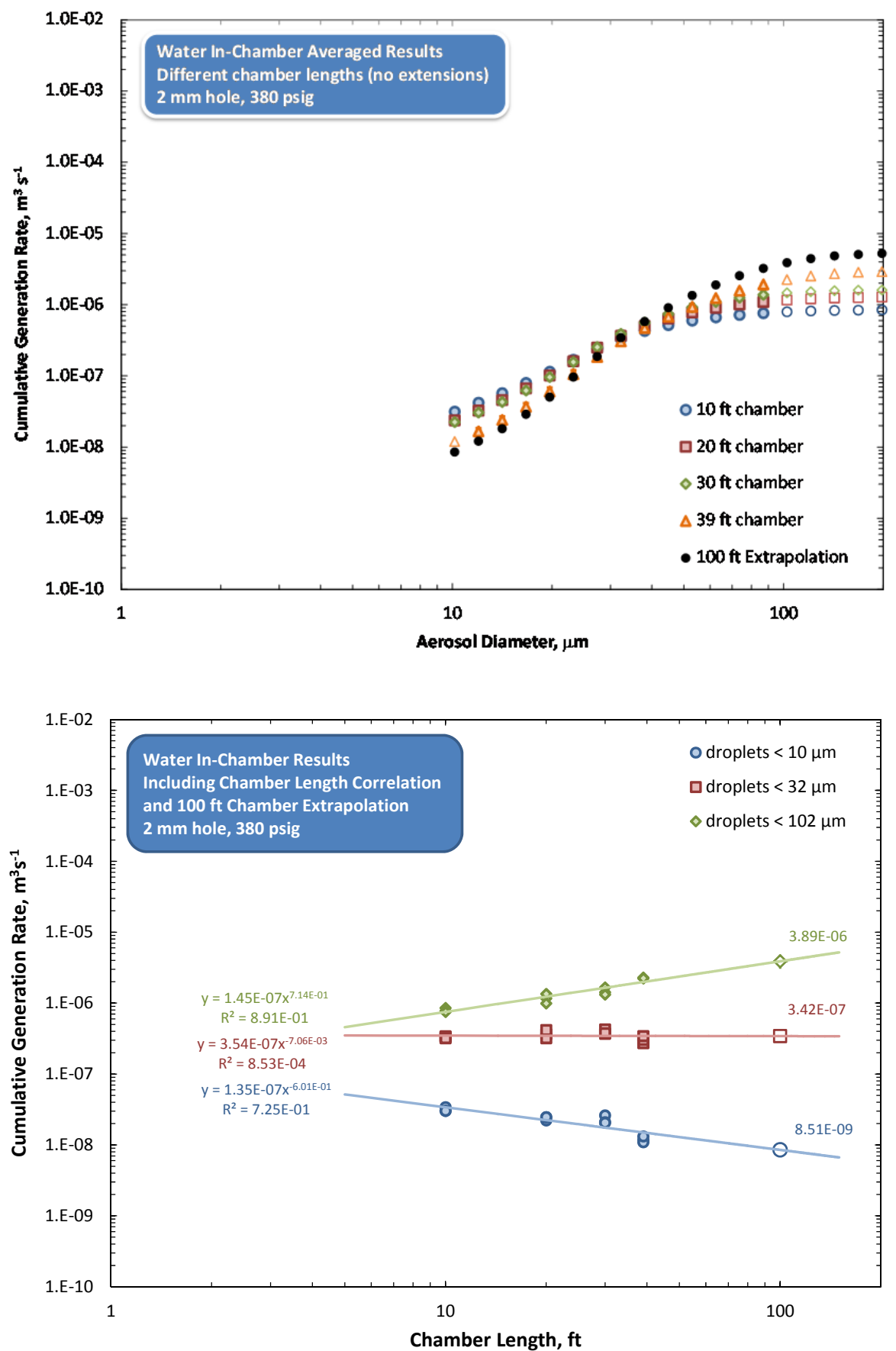

Figure 10.1. Generation Rate Estimates for Extrapolation to a $100 \mathrm{ft}$ Chamber and Spray Length for Water Sprays from a $2 \mathrm{~mm}$ Hole at $380 \mathrm{psig}$ in Chambers of Different Length. The upper plot shows generation rate, and the lower plot shows examples of extrapolation behavior for select droplet diameters. In the lower plot, the open symbols and values at $100 \mathrm{ft}$ chamber length show the extrapolation of the power-law fits for the selected droplet diameters. 

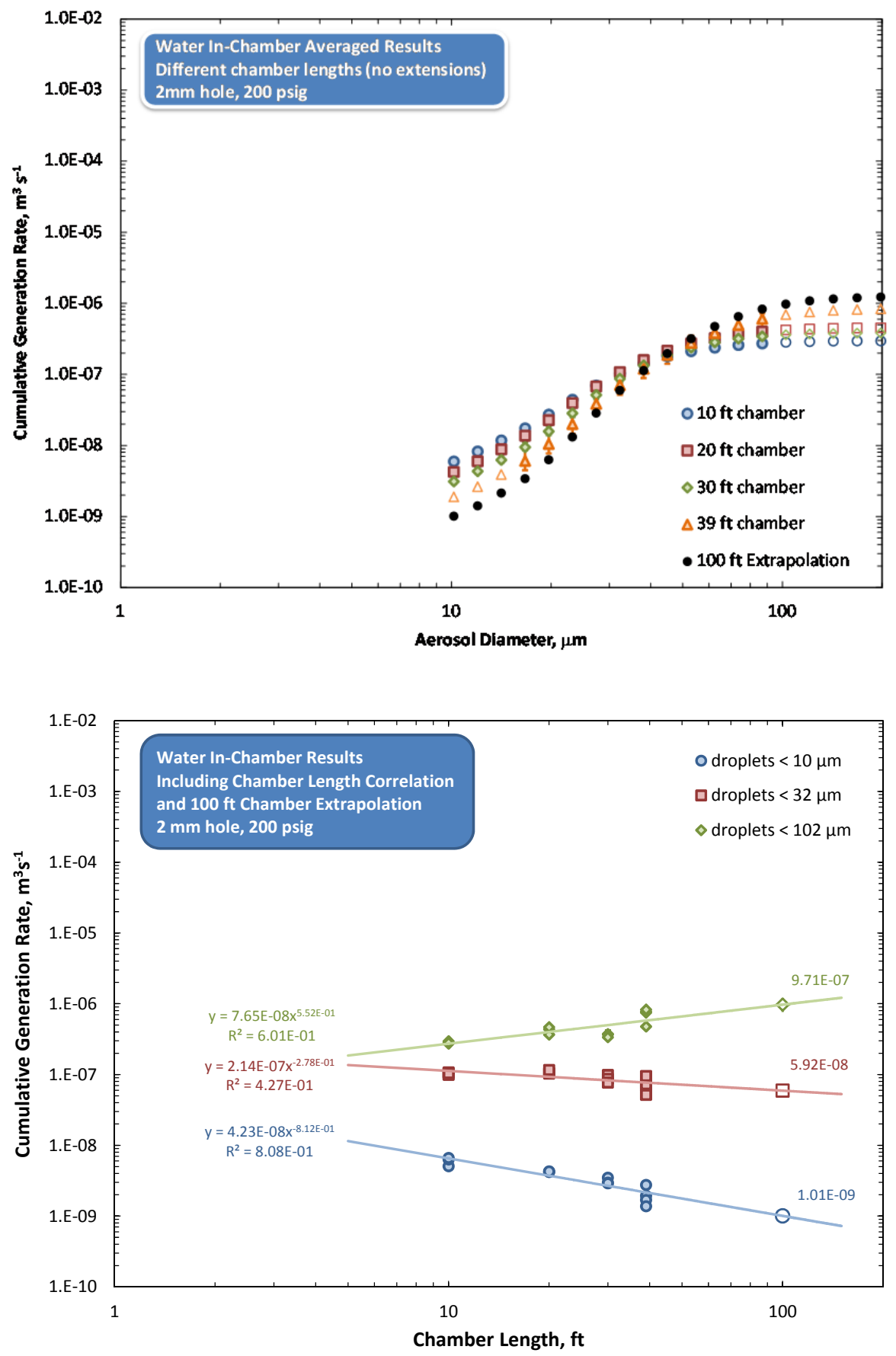

Figure 10.2. Generation Rate Estimates for Extrapolation to a $100 \mathrm{ft}$ Chamber and Spray Length for Water Sprays from a $2 \mathrm{~mm}$ Hole at 200 psig in Chambers of Different Length. The upper plot show shows generation rate, and the lower plot shows examples of extrapolation behavior for select droplet diameters. In the lower plot, the open symbols and values at $100 \mathrm{ft}$ chamber length show the extrapolation of the power-law fits for the selected droplet diameters. 

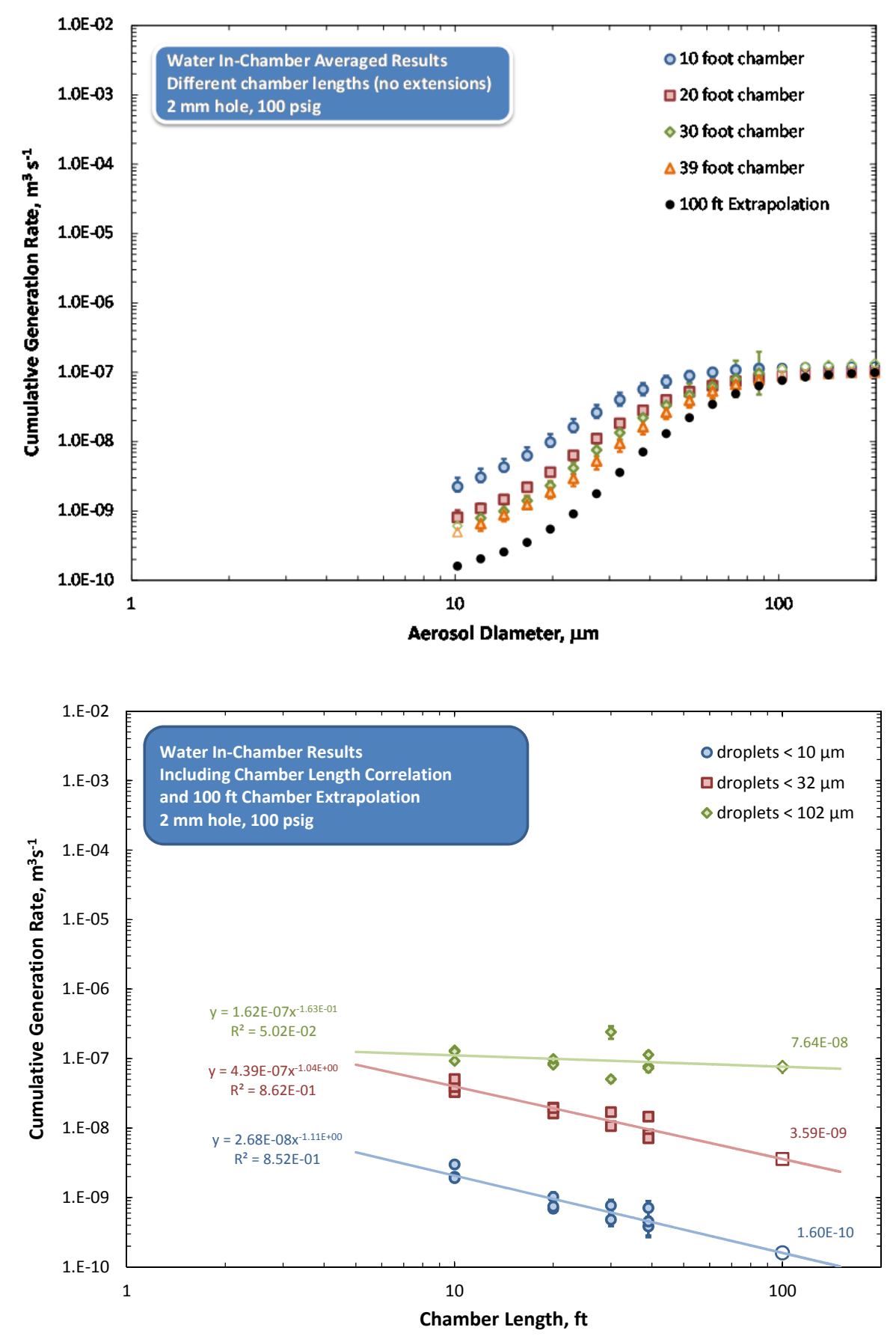

Figure 10.3. Generation Rate Estimates for Extrapolation to a $100 \mathrm{ft}$ Chamber and Spray Length for Water Sprays from a $2 \mathrm{~mm}$ Hole at $100 \mathrm{psig}$ in Chambers of Different Length. The upper plot shows generation rate, and the lower plot shows examples of extrapolation behavior for select droplet diameters. In the lower plot, the open symbols and values at $100 \mathrm{ft}$ chamber length show the extrapolation of the power-law fits for the selected droplet diameters. 

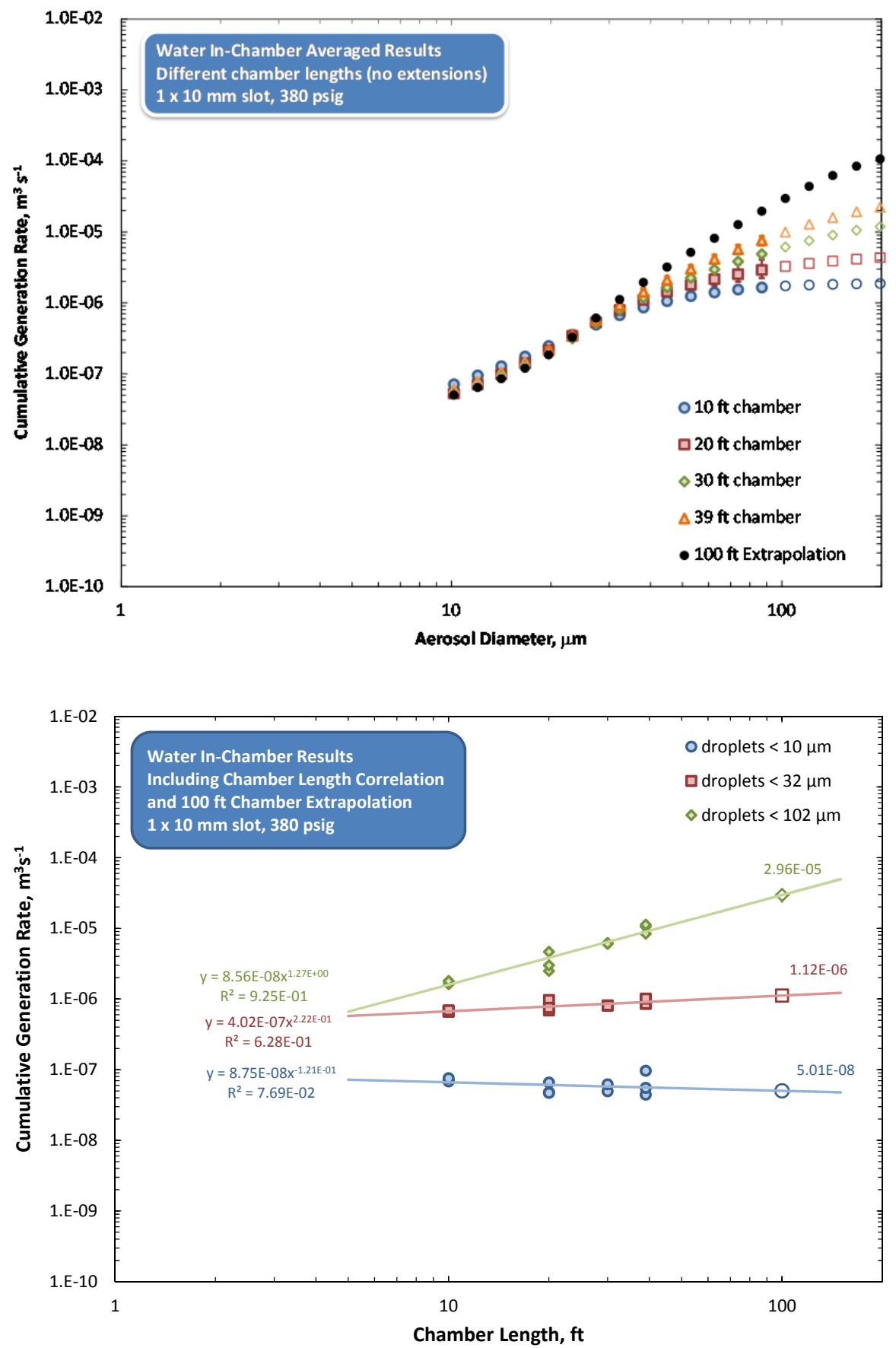

Figure 10.4. Generation Rate Estimates for Extrapolation to a $100 \mathrm{ft}$ Chamber and Spray Length for Water Sprays from a $1 \times 10 \mathrm{~mm}$ Slot at 380 psig in Chambers of Different Length. The upper plot shows generation rate, and the lower plot shows examples of extrapolation behavior for select droplet diameters. In the lower plot, the open symbols and values at $100 \mathrm{ft}$ chamber length show the extrapolation of the power-law fits for the selected droplet diameters. 

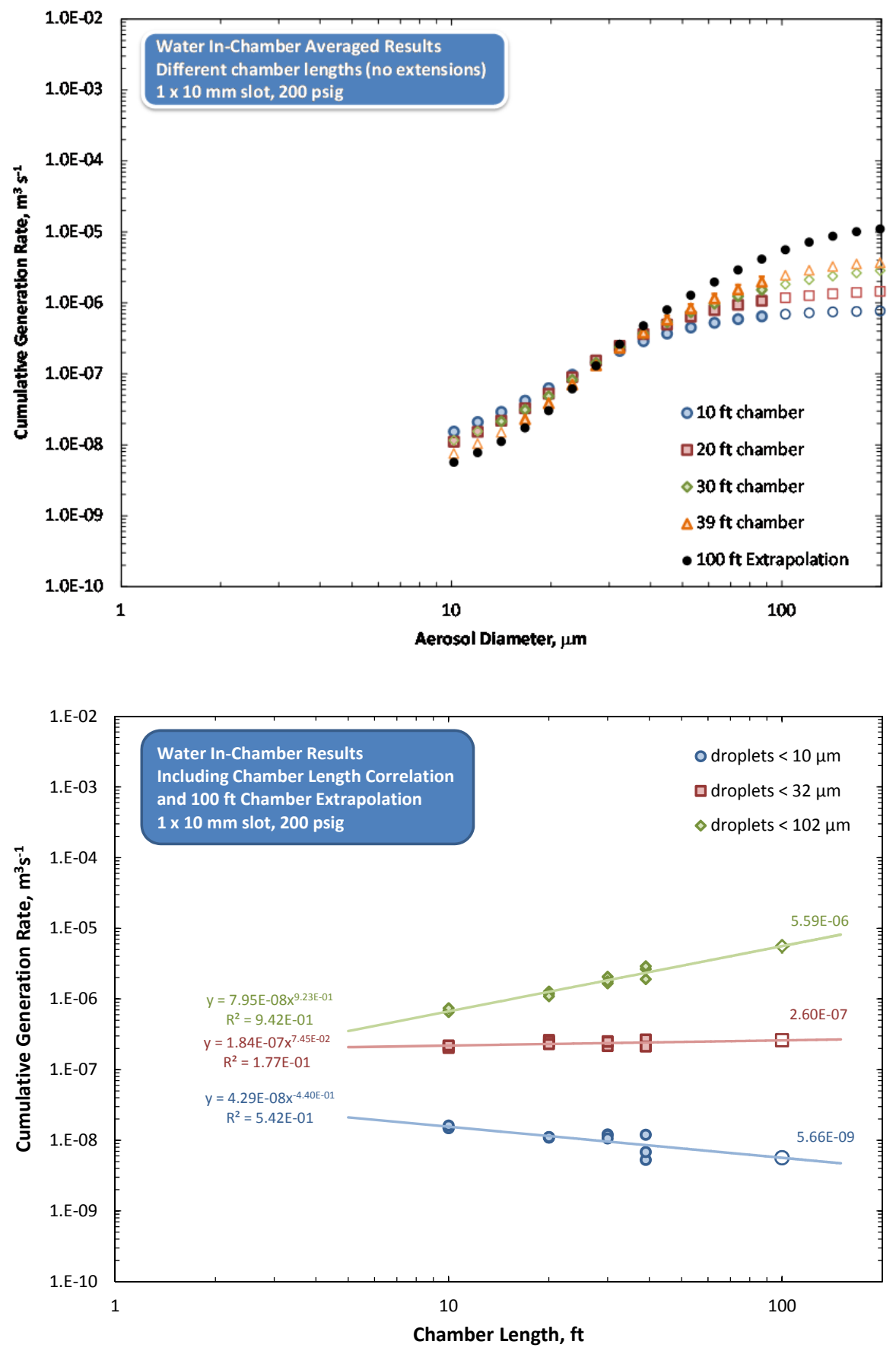

Figure 10.5. Generation Rate Estimates for Extrapolation to a $100 \mathrm{ft}$ Chamber and Spray Length for Water Sprays from a $1 \times 10 \mathrm{~mm}$ Slot at 200 psig in Chambers of Different Length. The upper plot shows generation rate, and the lower plot shows examples of extrapolation behavior for select droplet diameters. In the lower plot, the open symbols and values at $100 \mathrm{ft}$ chamber length show the extrapolation of the power-law fits for the selected droplet diameters. 

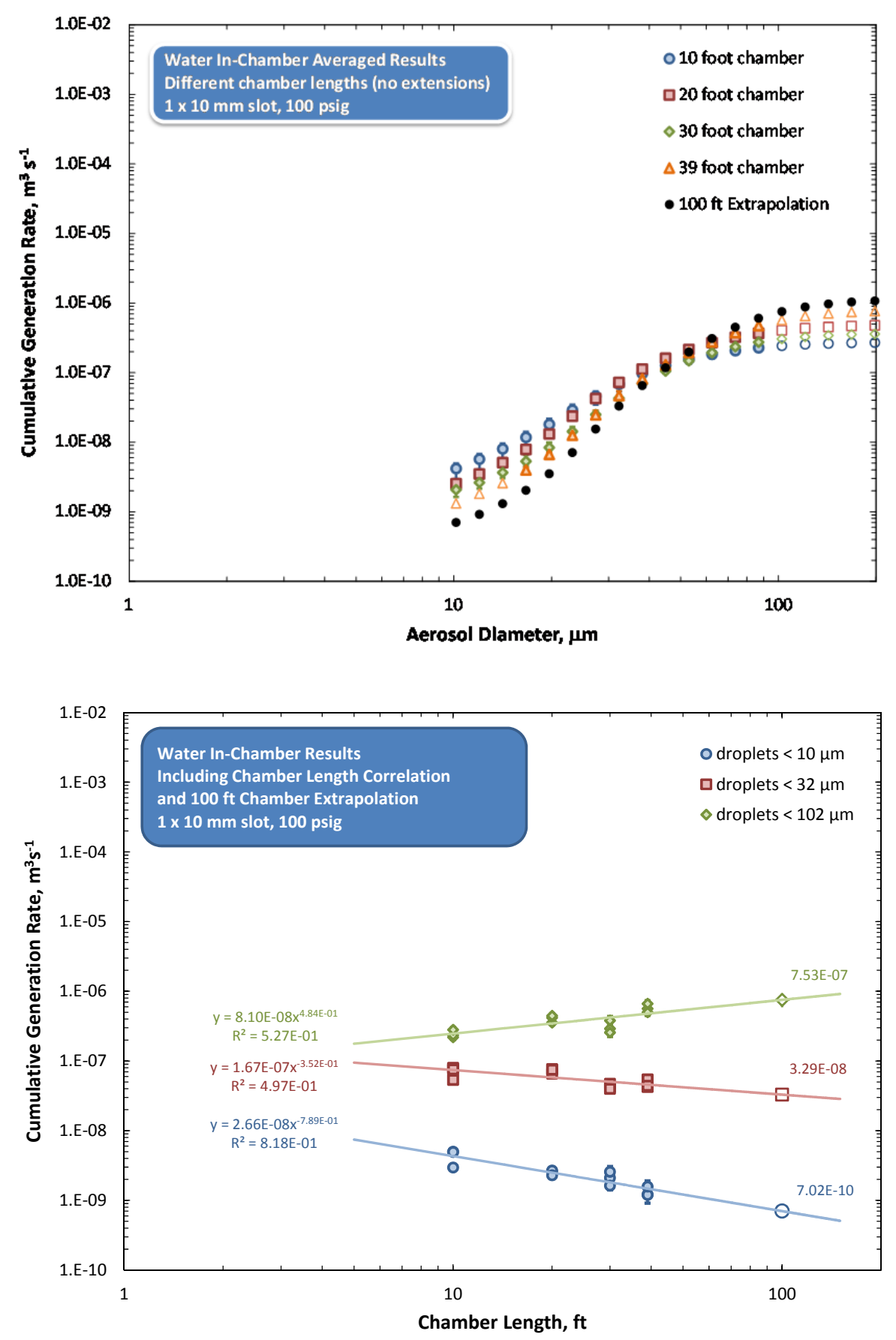

Figure 10.6. Generation Rate Estimates for Extrapolation to a $100 \mathrm{ft}$ Chamber and Spray Length for Water Sprays from a $1 \times 10 \mathrm{~mm}$ Slot at 100 psig in Chambers of Different Length. The upper plot shows generation rate, and the lower plot shows examples of extrapolation behavior for select droplet diameters. In the lower plot, the open symbols and values at $100 \mathrm{ft}$ chamber length show the extrapolation of the power-law fits for the selected droplet diameters. 

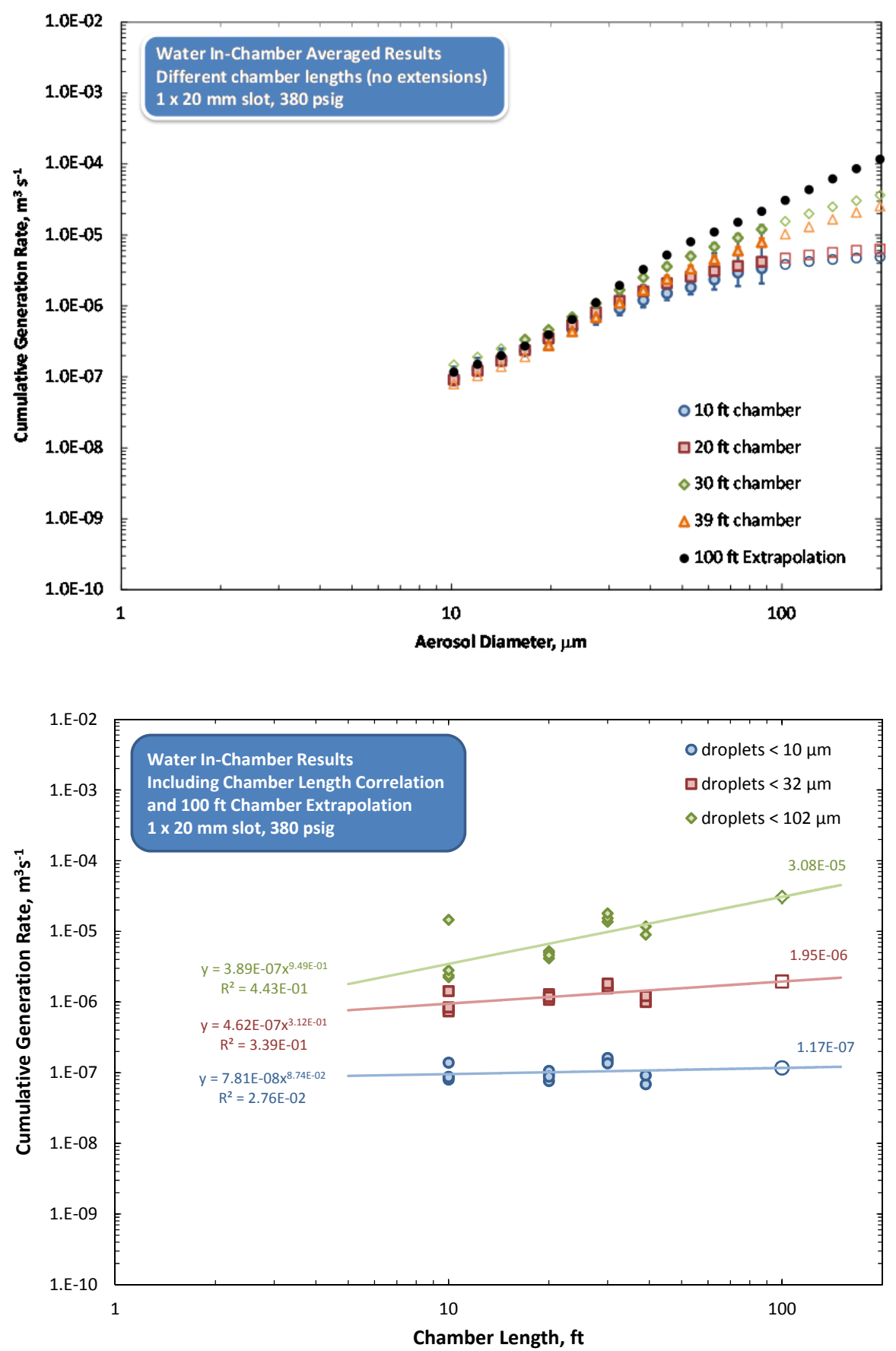

Figure 10.7. Generation Rate Estimates for Extrapolation to a $100 \mathrm{ft}$ Chamber and Spray Length for Water Sprays from a $1 \times 20 \mathrm{~mm}$ Slot at 380 psig in Chambers of Different Length. The upper plot shows generation rate, and the lower plot shows examples of extrapolation behavior for select droplet diameters. In the lower plot, the open symbols and values at $100 \mathrm{ft}$ chamber length show the extrapolation of the power-law fits for the selected droplet diameters. 

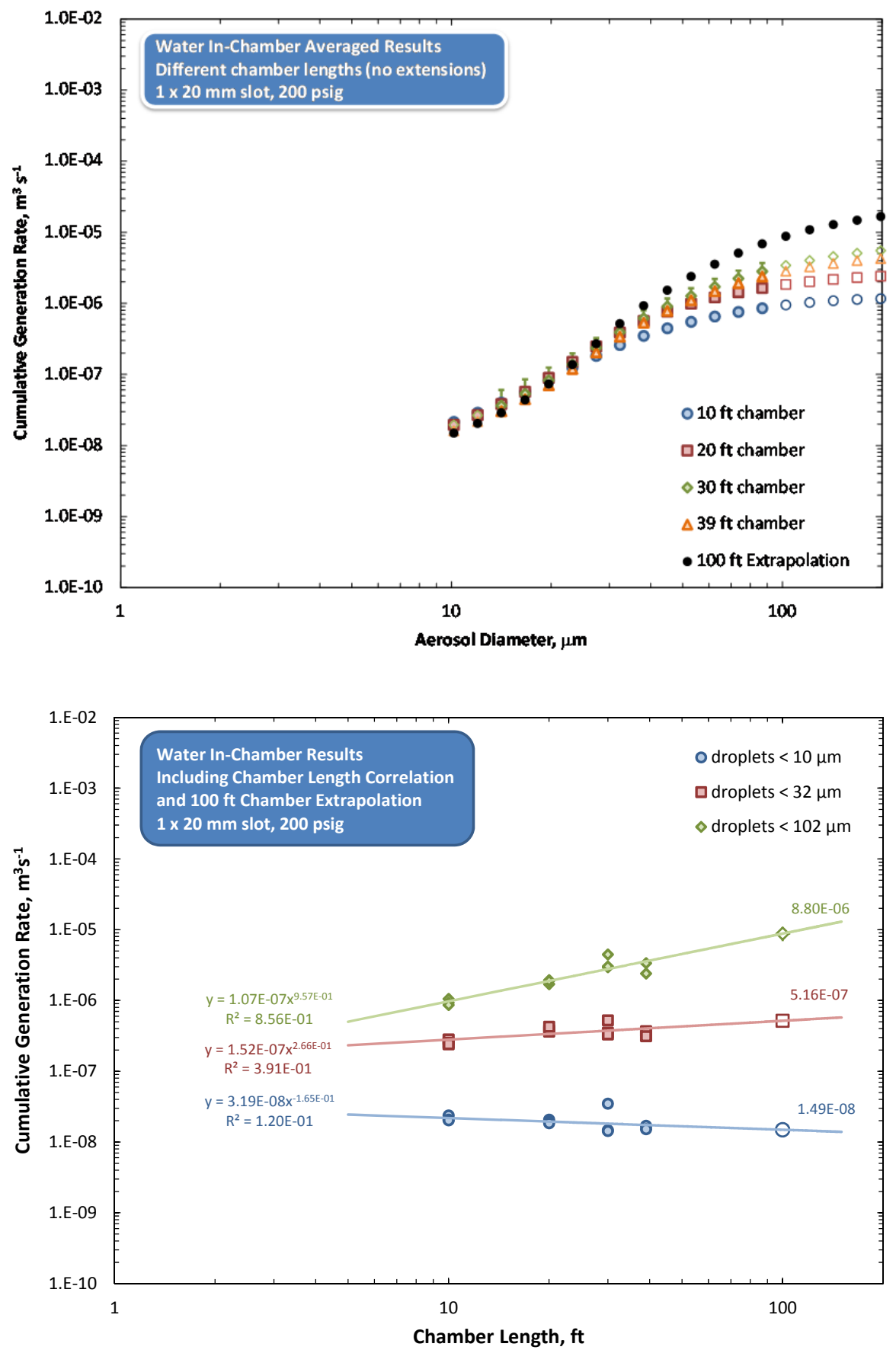

Figure 10.8. Generation Rate Estimates for Extrapolation to a $100 \mathrm{ft}$ Chamber and Spray Length for Water Sprays from a $1 \times 20 \mathrm{~mm}$ Slot at 200 psig in Chambers of Different Length. The upper plot shows generation rate, and the lower plot shows examples of extrapolation behavior for select droplet diameters. In the lower plot, the open symbols and values at $100 \mathrm{ft}$ chamber length show the extrapolation of the power-law fits for the selected droplet diameters. 

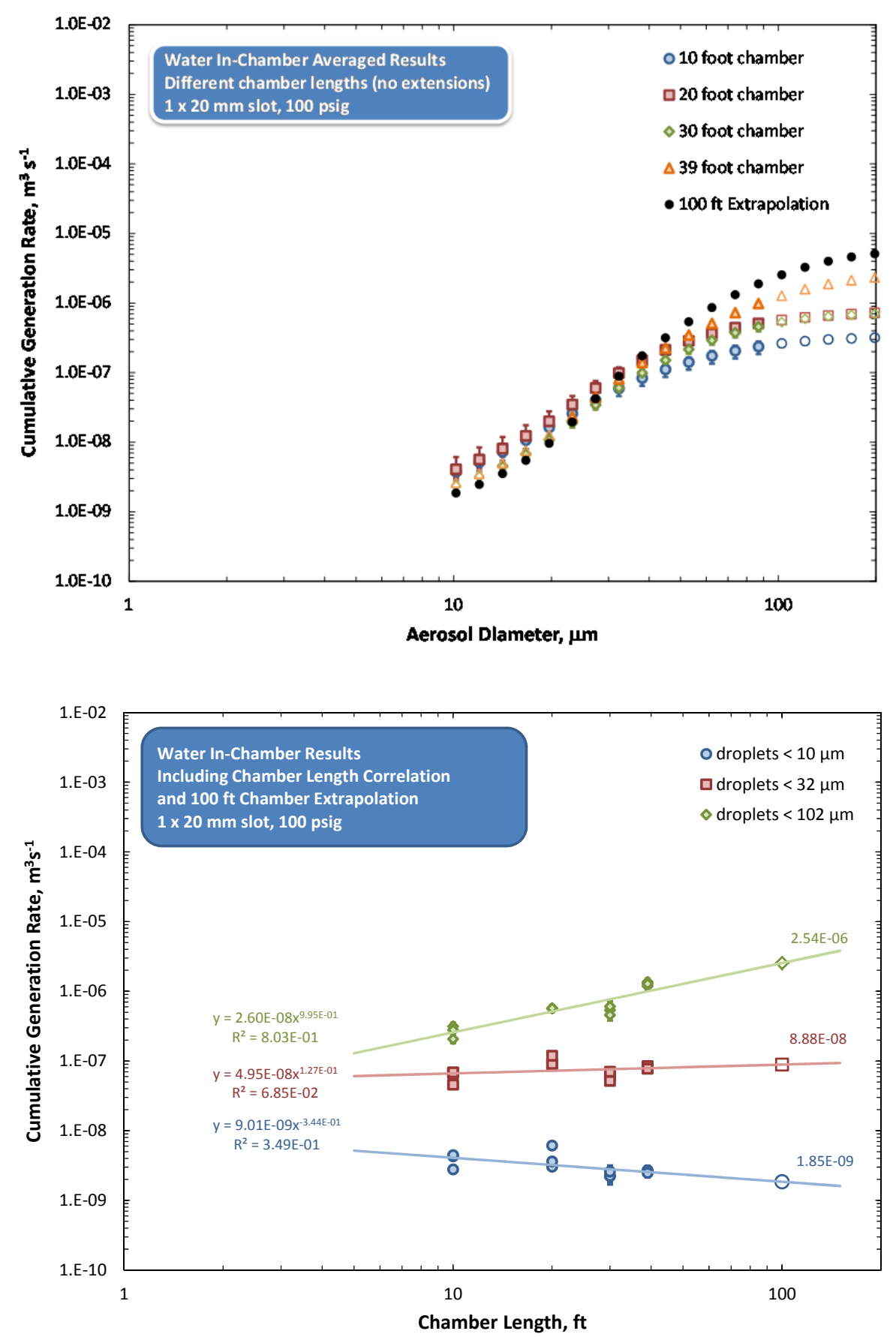

Figure 10.9. Generation Rate Estimates for Extrapolation to a $100 \mathrm{ft}$ Chamber and Spray Length for Water Sprays from a $1 \times 20 \mathrm{~mm}$ Slot at 100 psig in Chambers of Different Length. The upper plot shows generation rate, and the lower plot shows examples of extrapolation behavior for select droplet diameters. In the lower plot, the open symbols and values at $100 \mathrm{ft}$ chamber length show the extrapolation of the power-law fits for the selected droplet diameters. 

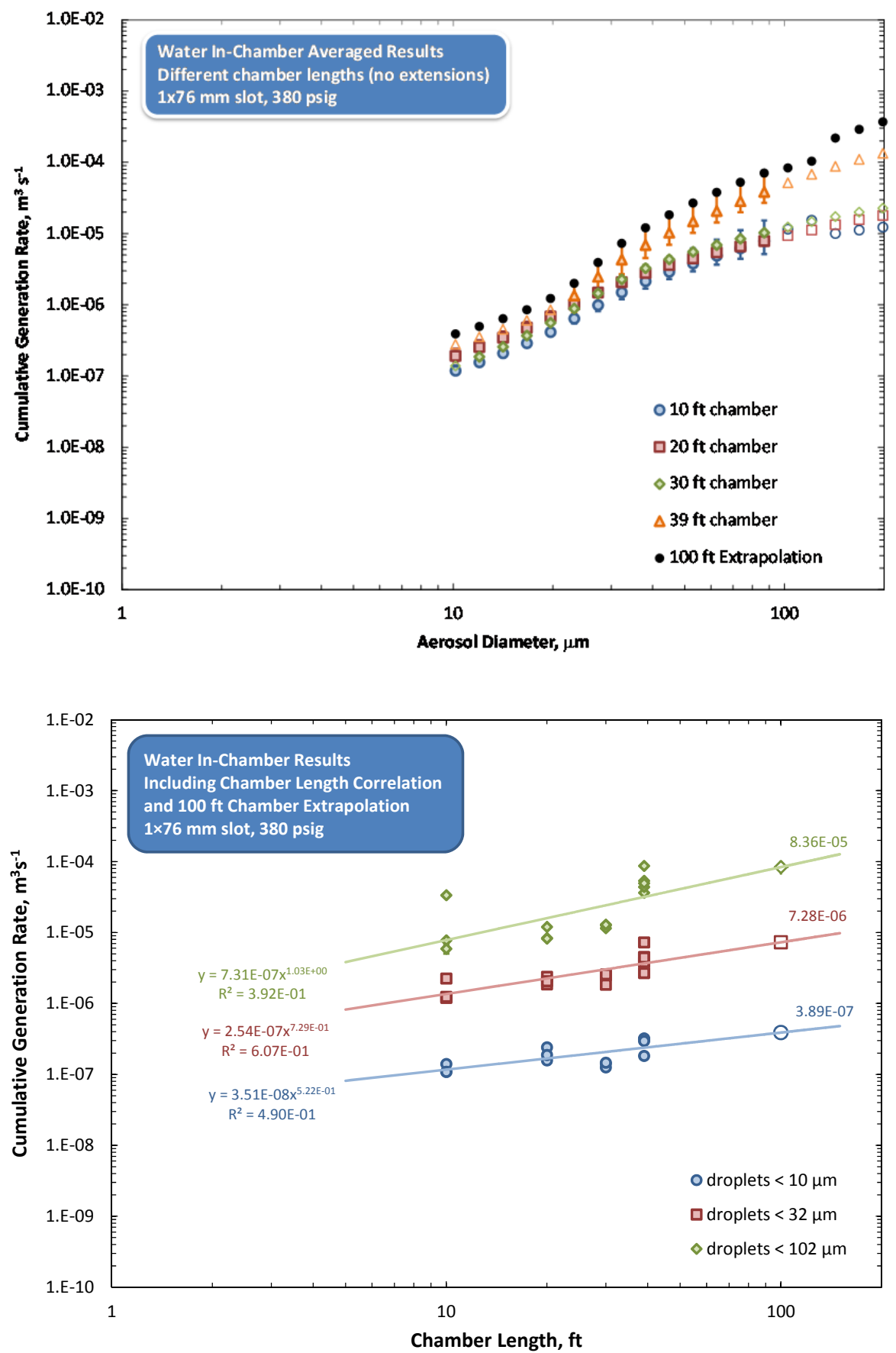

Figure 10.10. Generation Rate Estimates for Extrapolation to a $100 \mathrm{ft}$ Chamber and Spray Length for Water Sprays from a $1 \times 76 \mathrm{~mm}$ Slot at $380 \mathrm{psig}$ in Chambers of Different Length. The upper plot shows generation rate, and the lower plot shows examples of extrapolation behavior for select droplet diameters. In the lower plot, the open symbols and values at $100 \mathrm{ft}$ chamber length show the extrapolation of the power-law fits for the selected droplet diameters. 

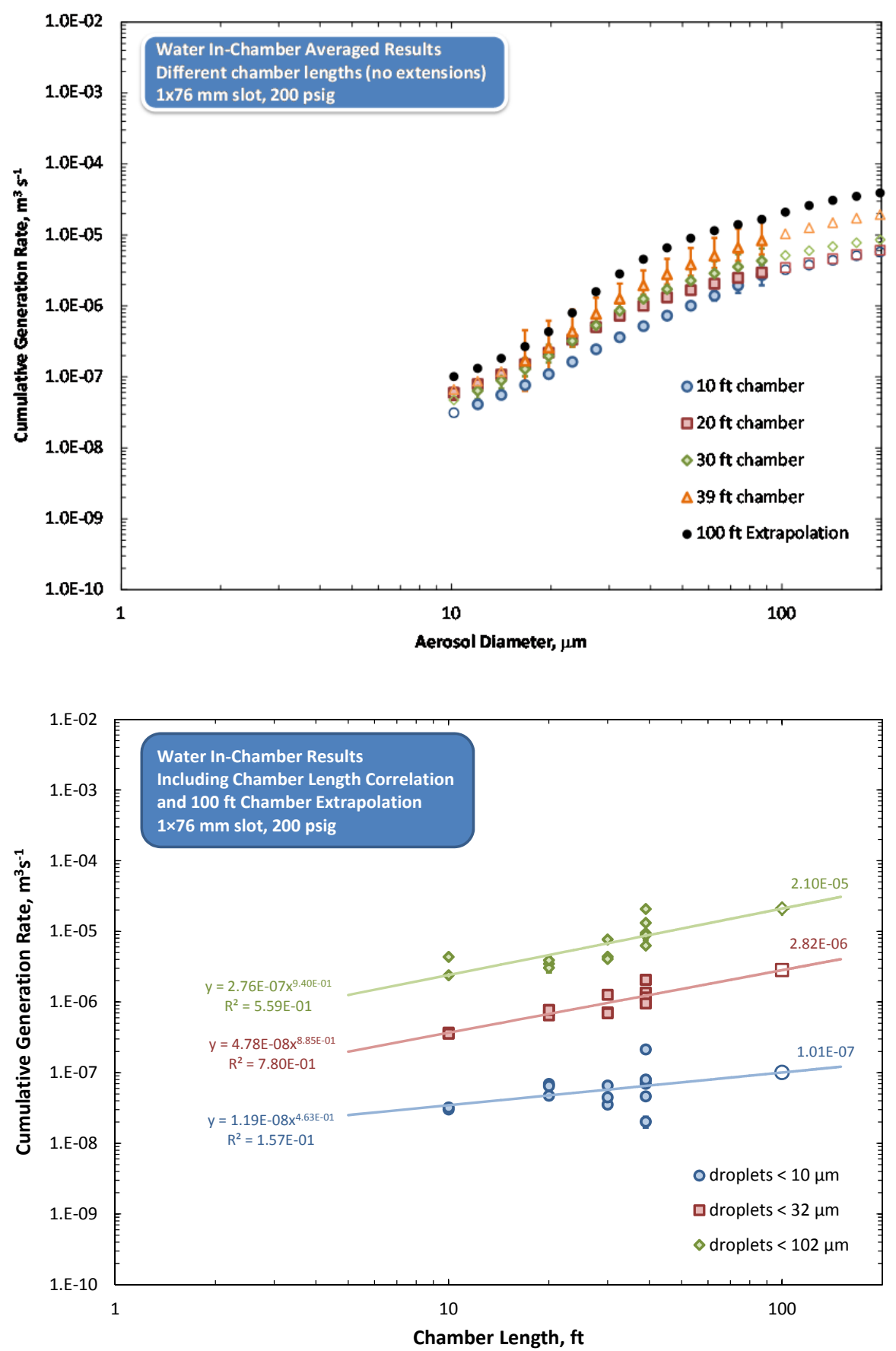

Figure 10.11. Generation Rate Estimates for Extrapolation to a $100 \mathrm{ft}$ Chamber and Spray Length for Water Sprays from a $1 \times 76 \mathrm{~mm}$ Slot at 200 psig in Chambers of Different Length. The upper plot shows generation rate, and the lower plot shows examples of extrapolation behavior for select droplet diameters. In the lower plot, the open symbols and values at $100 \mathrm{ft}$ chamber length show the extrapolation of the power-law fits for the selected droplet diameters. 

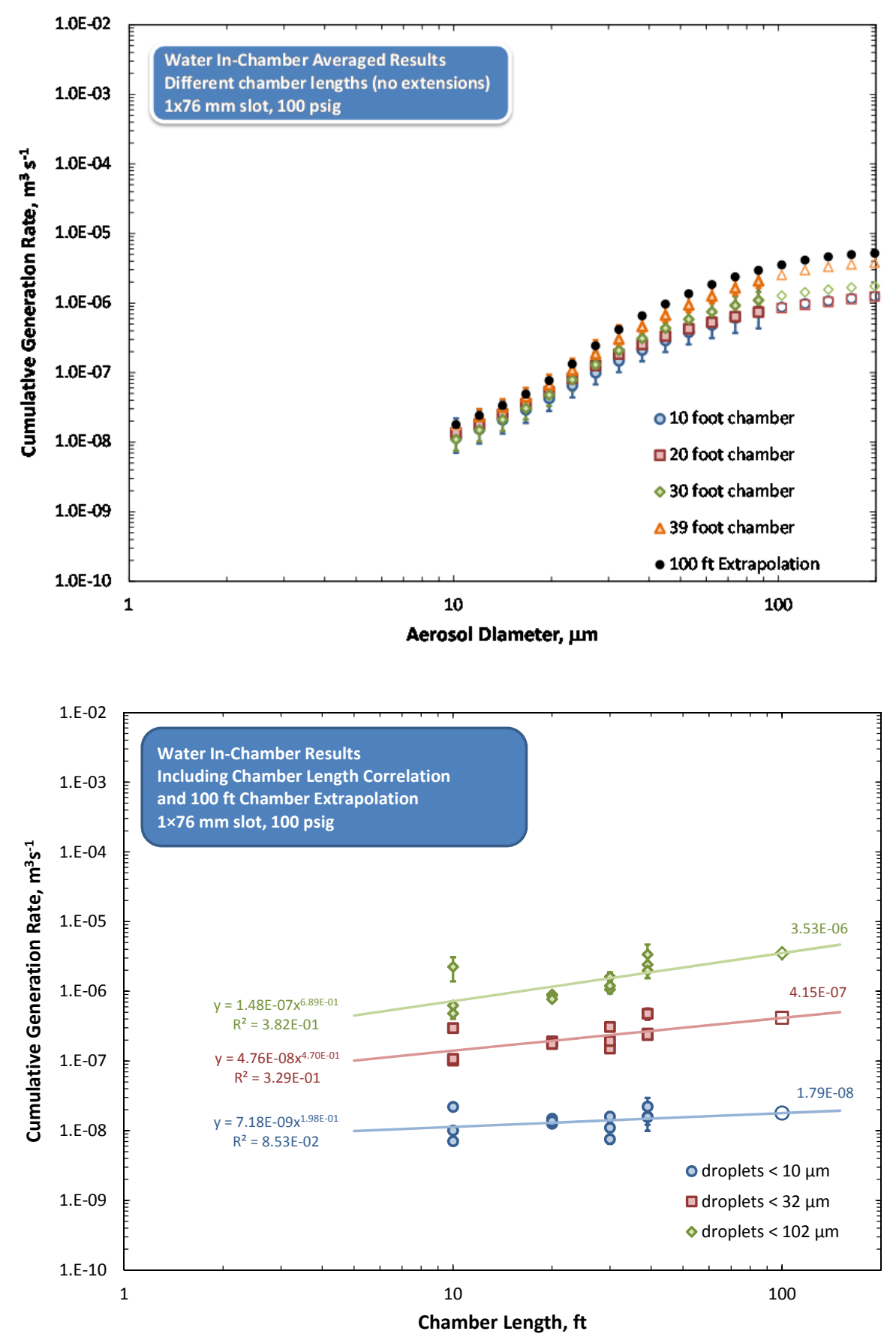

Figure 10.12. Generation Rate Estimates for Extrapolation to a $100 \mathrm{ft}$ Chamber and Spray Length for Water Sprays from a $1 \times 76 \mathrm{~mm}$ Slot at 100 psig in Chambers of Different Length. The upper plot shows generation rate, and the lower plot shows examples of extrapolation behavior for select droplet diameters. In the lower plot, the open symbols and values at $100 \mathrm{ft}$ chamber length show the extrapolation of the power-law fits for the selected droplet diameters. 


\subsection{Worst-Case Fluids and Spray Lengths Within a Chamber}

In both the large- and small-scale chambers, a series of tests were conducted to evaluate how fluid properties affect aerosol droplet generation and how sprays from this range of fluids compare with water sprays. The fluids tested include Newtonian salt solutions with increased density and viscosity, slurries of gibbsite or boehmite in water that are essentially Newtonian fluids, and non-Newtonian slurries of clays and a chemical simulant with rheology targets for Bingham yield stress and consistency of $6 \mathrm{~Pa} / 6 \mathrm{cP}$ and $30 \mathrm{~Pa} / 30 \mathrm{cP}$ (Schonewill et al. 2012, 2013; Mahoney et al. 2013). In general, the aerosol generation rates from the water sprays are always the same or higher than each of these other fluids. As summarized in Section 8.3 for the evaluation of the role of non-Newtonian fluid properties on release fraction, for clay slurries the presence of slurry particles typically reduces the release fraction of aerosols $>50 \mu \mathrm{m}$ in diameter or does not impact release fraction at all (relative to the fractional aerosolization of water). The presence of slurry particles can sometimes yield a slight increase in the release fraction of aerosols that are $<50 \mu \mathrm{m}$ in diameter, but the increase is typically not statistically significant (relative to the fractional aerosolization of water). For the clay slurries, the primary test conditions where the clay slurries gave higher release fractions in comparison to water spray are the 100 psig sprays (see Section 8.1). For aerosol generation rate, rather than release fraction, the difference between non-Newtonian slurry and water sprays is largely the same as for release fraction. This same comparison occurs because for the same orifice and test pressure, Equation (5.23) shows that the flow rate, and hence generation rate, for the non-Newtonian slurries is generally a bit smaller than water (the orifice coefficients are about the same (see Appendix B) and the density of the non-Newtonian slurries is higher [see Section 3.2.3]).

The primary exception where the test data show that water sprays may not be the same or higher than all other fluids are the results for the non-Newtonian chemical slurry simulants used in the small-scale chamber (Schonewill et al. 2013; Mahoney et al. 2013). These slurries did, in some cases, give higher release fractions than water, but these slurries had quite unusual rheology, and much different than our knowledge of actual waste, making the applicability of these results questionable. The release fractions from chemical slurry simulant sprays were also different from non-Newtonian clay simulant sprays with equivalent Bingham rheology parameters, which also support these results being questionable.

Accordingly, the test results for water are believed to give the worst-case (highest) aerosol generation rates. In the following section, a correlation for aerosol generation rate is developed for water sprays. This water-spray correlation represents a reasonably conservative spray release estimate for these other fluids.

Sprays of different lengths within a chamber of fixed length also were tested to evaluate whether aerosol generation increases or decreases as the orifice moves closer to a wall where the sprays impact the wall. Previous studies in a $20 \mathrm{ft}$ chamber (see Figure 9.18 of Schonewill et al. 2012) showed that the aerosol generation rate always increased with increasing spray length, and the largest aerosol generation occurred when the sprays travel essentially the full length of the chamber. These previous tests used spray pressures of 380 and 200 psig and included the same orifices being tested in this study. The results in Chapter 9 of this report include results for 100 psig spray pressures in addition to 380 and 200 psig sprays. These results show that the aerosol generation again increases with increasing spray length, with the exception being sprays at 100 psig for the two smaller orifices $(2 \mathrm{~mm}$ hole and $1 \times 10 \mathrm{~mm}$ slot at smaller droplet sizes). Readers should note that the 100 psig spray data for the $2 \mathrm{~mm}$ hole gave an unusual increase in release fraction at $100 \mathrm{psig}$ in comparison to higher pressure sprays. The other exception to the longest spray giving the highest generation rate are tests in the small-scale chamber that 
showed an increase in aerosol generation when the length of the spray decreased to $1 \mathrm{in}$. from a splash wall (Mahoney et al. 2013). The correlation developed in the following section uses spray data for tests in which the sprays traveled essentially the full length of the chambers. Accordingly, this correlation represents a reasonably conservative spray release estimate for any shorter spray length.

\subsection{Correlation Development for Estimating Reasonably Conservative Aerosol Generation Rates}

Reasonably conservative aerosol generation rate estimates are needed for comparison to the WTP model and, potentially, for use in estimating aerosol generation rates for accident analysis calculations. Depending on the intended use of the result, an analyst may prefer values reflecting the measured values or may choose to use a higher estimate (conservative) that accounts for the uncertainty in the measurements and test variability. The purpose of this section is to develop a correlation, based on the in-chamber data, for the generation rate of aerosol droplets that is reasonably conservative. This correlation can then be used to extrapolate to larger orifices and higher pressures that were not tested and can also be used to interpolate to other conditions that were not specifically tested. The correlation will be developed using the test data from all chamber lengths and the extrapolations to a $100 \mathrm{ft}$ chamber presented in Section 10.1.

The experimental result calculated from the in-chamber aerosol concentration measurement is the generation rate of aerosol droplets, and the generation rate is what is needed for spray release accident analyses. For these two reasons, the correlation was developed for generation rate rather than release fraction. In this section, a correlation is developed from the individual test data based on both the generation rate and upper confidence interval for the generation rate determined from the method used to fit the transient aerosol concentration data within the chamber (see Schonewill et al. 2012 for a discussion of determining the upper confidence interval value from the test data). The correlation developed from the upper confidence interval values will then be adjusted to be reasonably conservative for all of the test data.

The primary parameters needed for correlating the generation rate data are the effect of spray pressure, orifice area, and droplet diameter. The role of fluid properties also is important. As discussed in Section 10.2, for a specific spray pressure and orifice, the aerosol generation for water sprays is always the same, or higher, than any of other fluids or slurries tested (with the exception of the non-Newtonian chemical slurry simulants as discussed in Section 10.2). Accordingly, the correlation will be developed for water and this correlation will be reasonably conservative (i.e., higher) for any of the other fluids tested. The length of the spray within a chamber also affects the measured generation rate, and the data used for the correlation are from sprays that travel essentially the full length of the chambers, because this is the highest generation rate and appropriate for developing a conservative correlation.

The role of chamber size is also important. The generation rate can both increase and other times decrease with increasing chamber length depending on the droplet size, orifice, and test pressure. To account for the effect of chamber size, test data for all chamber sizes and the extrapolated values for a $100 \mathrm{ft}$ chamber are used in developing the correlation, but a specific parameter for chamber length will not be included in the correlation. Figure 10.13 shows a conceptual example of how the correlation will account for the role of chamber size. For this example, a straight line is shown that is above all of the test data from individual chambers. In this sense, the intent of the correlation is to represent the highest 
generation rate at any droplet size for all of the chamber sizes and the $100 \mathrm{ft}$ extrapolation. While the correlation might be improved by explicitly including the role of chamber and spray length, the ultimate use of the correlation will be to estimate the generation rate from sprays where the configuration of the surrounding environment may not be known, so a correlation that provides an upper bound without needing to specify a chamber length will be most generally applicable.

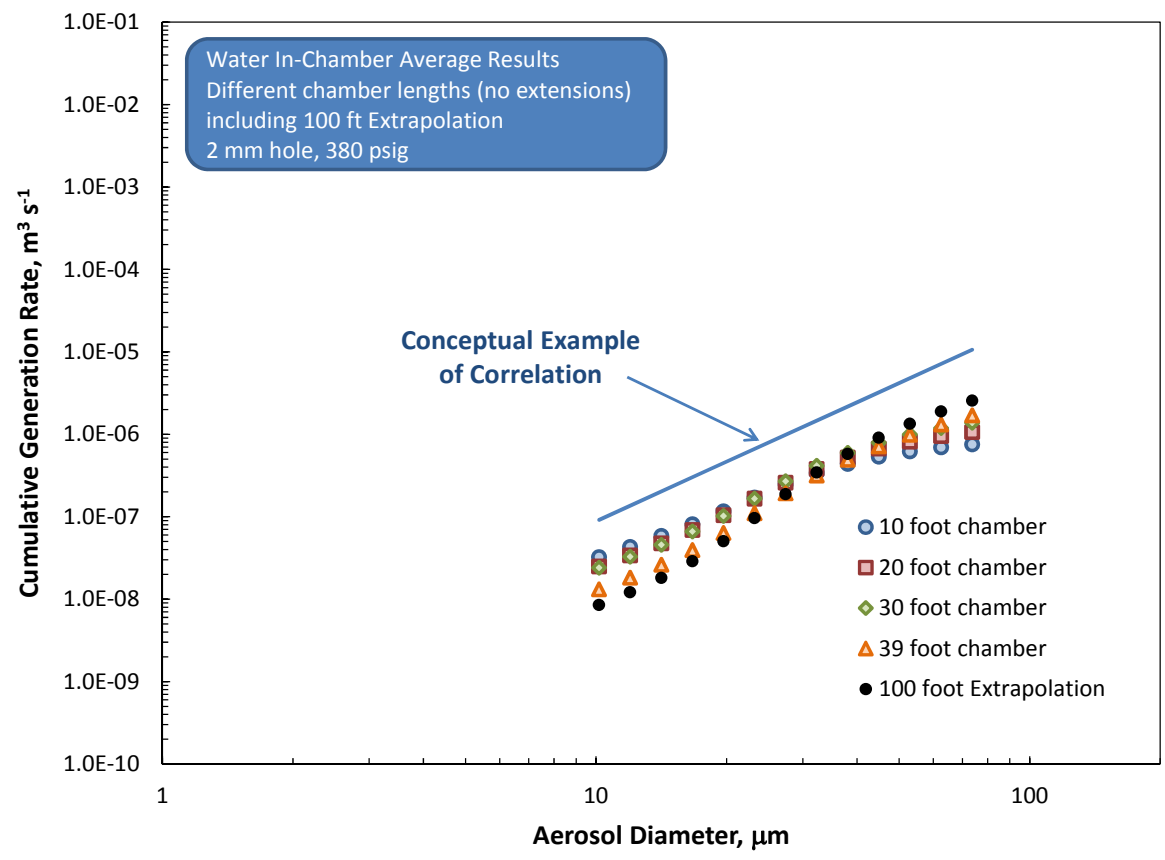

Figure 10.13. Conceptual Example of Correlation that Represents the Highest Generation Rate of All Chamber Sizes and $100 \mathrm{ft}$ Extrapolation for a $2 \mathrm{~mm}$ Hole at $380 \mathrm{psig}$ as a Selected Example

An empirical correlation, with power-law dependences for each term, was selected as the approach for representing the test data. Previous test results (Schonewill et al. 2012, Figure 8.19 as an example) and the current results in Sections 8.2.2 and 9.1.2 show the role of orifice area can be represented by a power-law dependence. The results given in Schonewill et al. (2012) are for the cumulative generation rate while the results in Chapters 8 and 9 are given as cumulative release fractions. Because the release fraction results were determined by dividing the generation rate results by the measured flow rate of the spray (see Section 1.5) and because the flow rate is essentially proportional to orifice area (see Equation (5.27)), a power-law dependence of orifice area for release fraction implies a power-law dependence for generation rate. For the effect of spray pressure, a power-law dependence is also suitable based on previous test results (Schonewill et al. 2012) and the current results in Sections 8.2.1 and 9.1.1 (again, a power-law dependence for release fraction implies a power-law dependence in generation rate; see Equations (5.23) and (5.27)). Finally, the conceptual example given in Figure 10.13 shows that a power-law dependence is reasonable for representing the role of droplet diameter within the droplet size range of importance, which is 10 to $100 \mu \mathrm{m}$. The empirical correlation, assuming power-law dependences for each term, has the following form:

$$
G R=c(A)^{a 1}\left(P_{S}\right)^{a 2}\left(d_{p}\right)^{a 3}
$$


where $\quad G R=$ cumulative droplet generation rate $\left(\mathrm{m}^{3} / \mathrm{s}\right)$

$A=$ orifice areas $\left(\mathrm{mm}^{2}\right)$

$P_{S}=$ spray pressure (psig)

$d_{p}=$ aerosol droplet diameter $(\mu \mathrm{m})$

$c=$ leading constant

$a 1=$ coefficient for orifice area

$a 2=$ coefficient for spray pressure

$a 3=$ coefficient for droplet diameter.

Test data for both the generation rate and the upper confidence interval values for all the test runs were fit with Equation (10.1) using a least-squares method where the error for each measurement was taken as the difference between the log of the measured value and the log of the correlation value. This approach was used to accommodate the generation rate values that spanned more than five orders of magnitude. By using data from all of the test runs, rather than averages of multiple runs at each spray pressure and orifice, the test-to-test variability is represented. The generation rates determined by extrapolation to a $100 \mathrm{ft}$ chamber and spray, which were shown in Section 10.1, also are included as representing an individual test. For the upper confidence interval values, the same general approach was used for extrapolating to a $100 \mathrm{ft}$ chamber as was used for extrapolating the generation rate data. The location of the Malvern Insitec-S instruments for these tests is above the spray. As discussed in Schonewill et al. (2012) and in Chapter 7, this location gives results that under-represent the aerosol generation rate for larger droplets, specifically droplets $\sim 87 \mu \mathrm{m}$ and larger. Accordingly, only droplets in the size range from 10 to $74 \mu \mathrm{m}$ are used for the purpose of determining the exponents in the correlation (74 $\mu \mathrm{m}$ is the next smaller bin size reported by the Malvern Insitec-S). For the generation rate test results, the following correlation was obtained from the least-squares fit.

$$
G R=6.76 \times 10^{-17}(A)^{0.772}\left(P_{S}\right)^{2.16}\left(d_{p}\right)^{2.41}
$$

Figure 10.14 shows a cross plot comparison of the measured generation rate and this correlation with different symbols for the different size chambers. For points above the diagonal line, the measured result exceeds the correlation, and for points below the line, the measured result is less than the correlation. The different size chambers align differently on the plot with the smaller chamber having the highest measured values in the lower range of generation rates and the $100 \mathrm{ft}$ extrapolation having the highest measured values for the larger generation rates. Figure 10.15 shows an example of four specific tests for different orifices at $380 \mathrm{psig}$ to highlight how individual tests align on the plots comparing the data and correlation. For each test, the individual data points represent the cumulative generation rate for the individual droplet size bins reported by the Malvern Insitec-S instrument, beginning with $10 \mu \mathrm{m}$ droplets for the lowest generation rate and increasing to $74 \mu \mathrm{m}$ droplets. Figure 10.15 also shows how a smaller orifice, such as the $2 \mathrm{~mm}$ hole, has a lower generation rate for the range of droplet sizes (indicated by the individual points) and the larger orifices have higher generation rates for this same size range of droplets. 


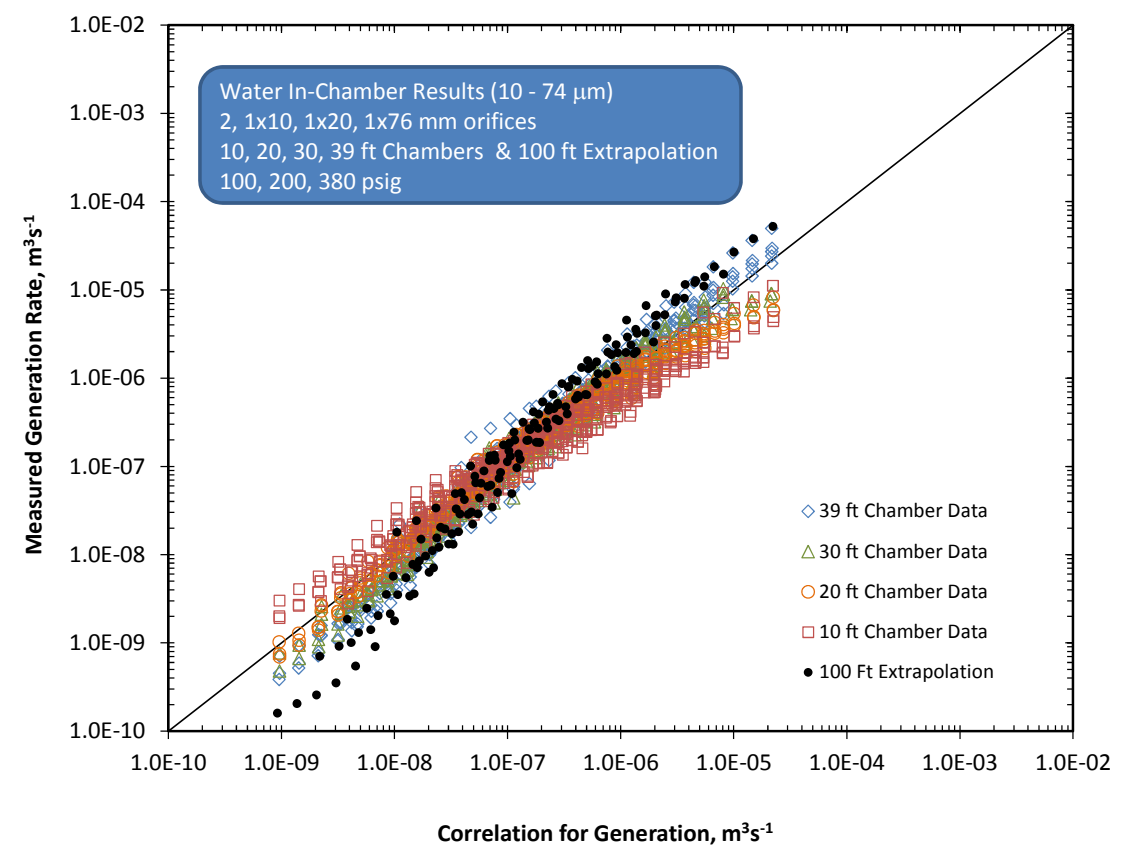

Figure 10.14. Comparison of Measured Generation Rates and the Generation Rate Correlation Given by Equation (10.2) for the Individual Test Results Used for Developing the Correlation

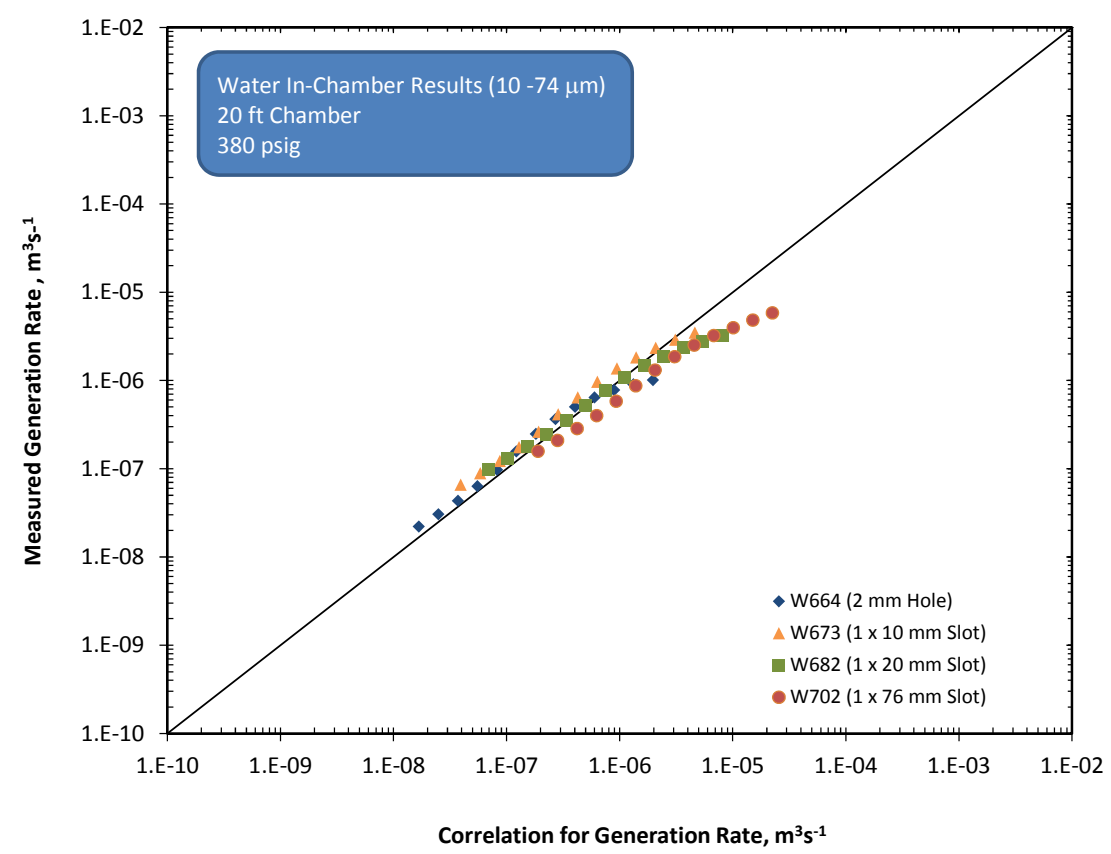

Figure 10.15. Individual Test Examples Comparing the Measured Generation Rates with the Generation Rate Correlation Given by Equation (10.2)

The upper confidence interval values for each test, which represent a $95^{\text {th }}$ percentile confidence interval in fitting the transient droplet concentration data with an exponential function, provide an alternate data set that can be fit with Equation (10.1). Here, the correlation accounts for this fit 
uncertainty and, thus, is more appropriate for developing a conservative correlation. The result from the least-squares fit gives the following result, which is the correlation for the upper confidence interval of the generation rate, $G R_{U C I}$.

$$
G R_{U C I}=6.52 \times 10^{-17}(A)^{0.793}\left(P_{S}\right)^{2.18}\left(d_{p}\right)^{2.40}
$$

Figure 10.16 shows a cross plot comparison of the measured upper confidence interval for the generation rate and the correlation for the upper confidence interval given by Equation (10.3). The different symbols again show the behavior of the results for different size chambers, with the $10 \mathrm{ft}$ chamber generally being above the correlation result at lower generation rates and the $100 \mathrm{ft}$ extrapolation being generally above the correlation at higher generation rates.

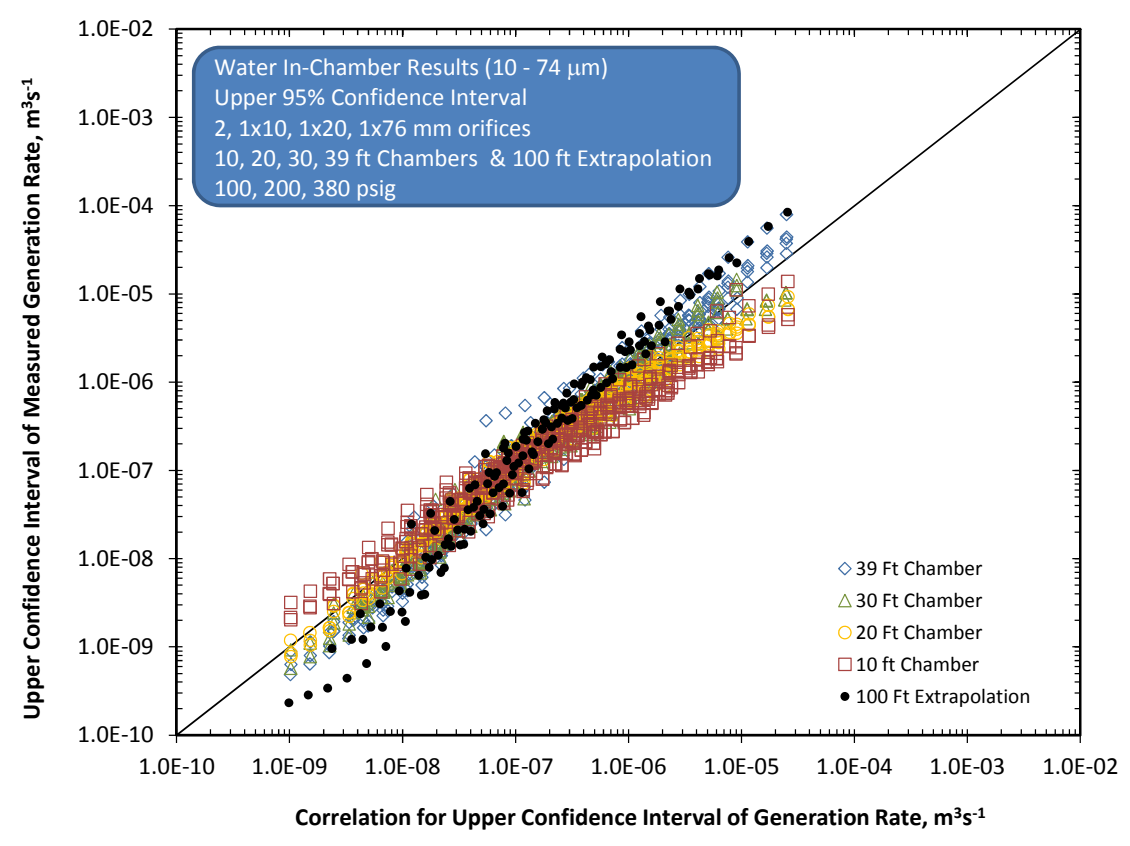

Figure 10.16. Comparison of Measured Upper Confidence Interval Values for Individual Tests with the Correlation for the Upper Confidence Interval Generation Rates

The correlations for the generation rate and the upper confidence interval generation rate have quite similar values for the coefficients for the individual terms. The generation rate estimates from these two correlations give the expected result of the correlation for the upper confidence interval (Equation (10.3)) that exceeds the correlation for the generation rate (Equation (10.2)). Figure 10.17 shows a comparison of the two correlations for the specific test conditions and orifices used for developing the correlations. The correlation for the upper confidence interval (Equation (10.3)) exceeds the correlation for the generation rate data (Equation (10.2)) with the difference being similar for the entire range. Figure 10.18 shows a comparison of the measured values for the upper confidence interval versus the measured generation rate. The diagonal line again represents when the values are identical. The upper confidence interval values tend to be farther above the generation rate data at the higher generation rates and these were some of the most difficult test runs with larger fit uncertainties. 


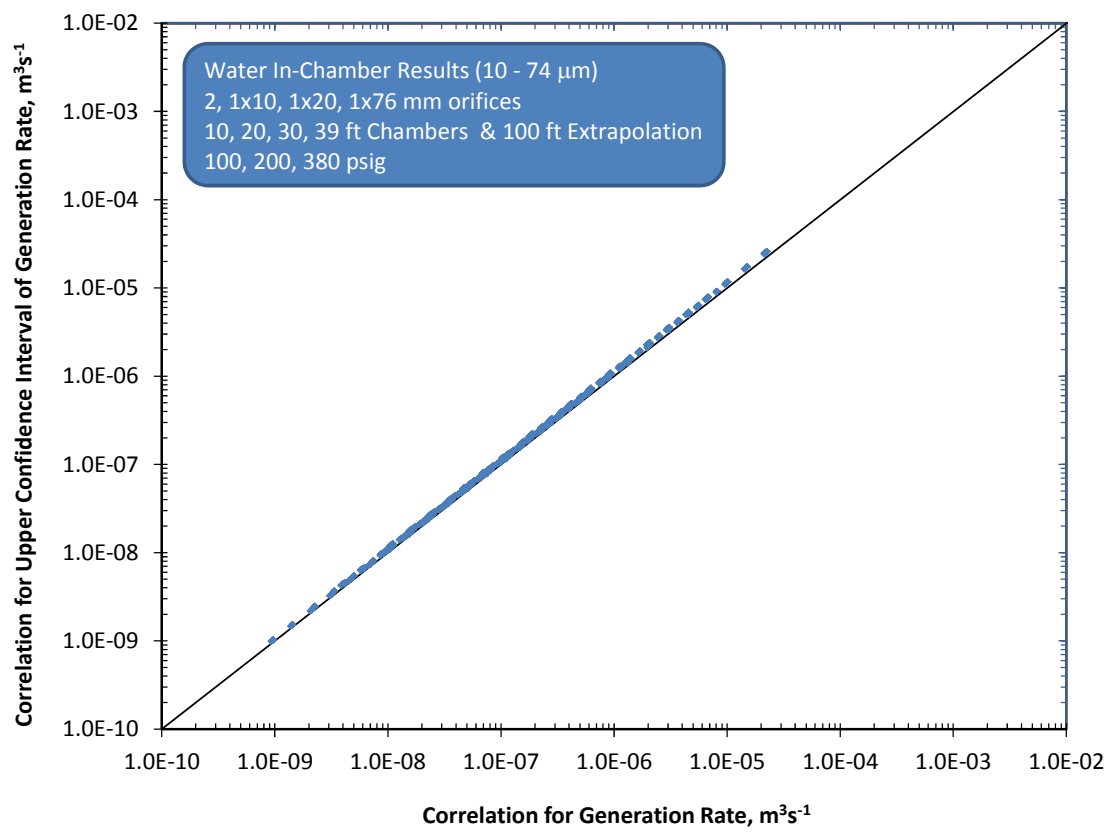

Figure 10.17. Cross Plot Comparison of the Correlations for Generation Rate and Upper Confidence Interval for Generation Rate for the Range of Data Used in Developing the Correlations

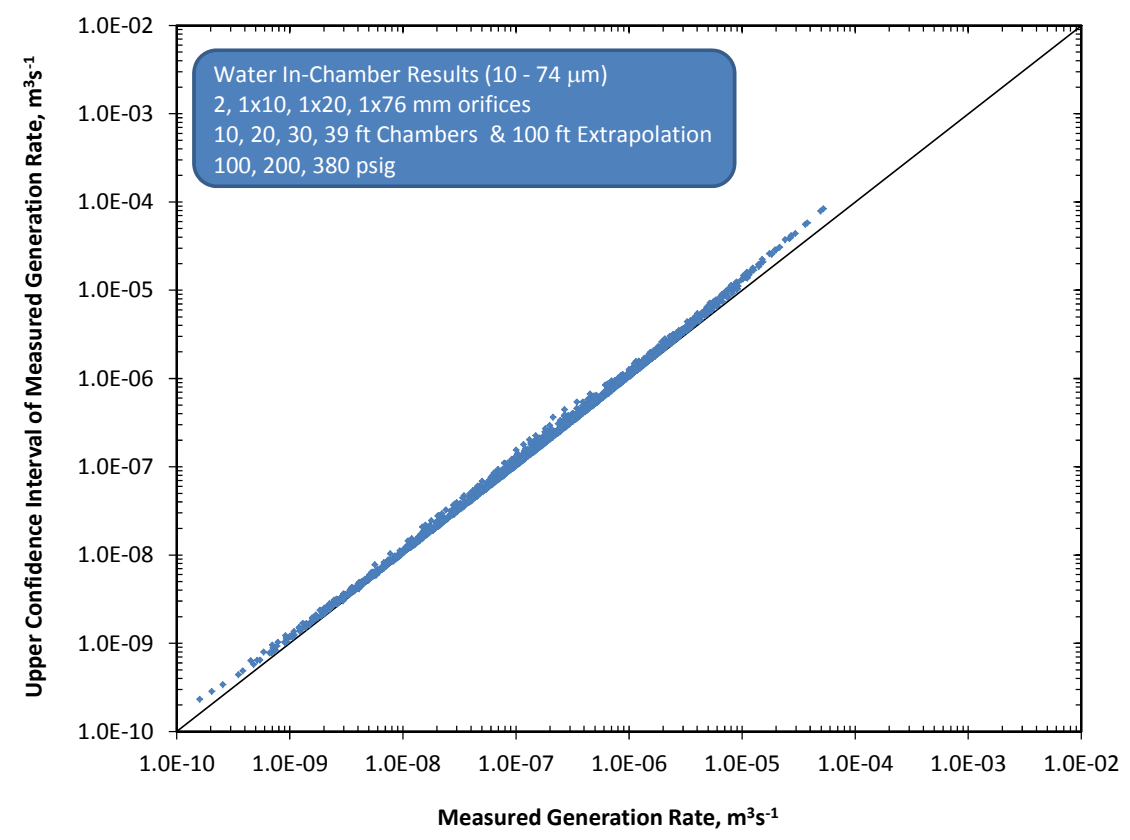

Figure 10.18. Comparison of the Measured Generation Rate and Upper Confidence Interval Values for the Data Used in Developing the Correlations

Because the upper confidence interval correlation and values account for the uncertainty in fitting the chamber concentration data, the correlation for the upper confidence interval will be used in the next step of developing a reasonably conservative correlation. The results in Figure 10.16 show roughly an equal 
number of the measured upper confidence interval values being above or below the correlation for the upper confidence interval, which is expected for a least-squares fit. One approach for obtaining a conservative correlation is to adjust the correlation so that all, or the majority, of the measured values are less than the correlation. The most sensible adjustment is to increase the leading coefficient and not adjust the exponents for the individual parameters of orifice area, spray pressure, and droplet size. The following result, which has a 5.0-fold increase in the leading coefficient, was selected to have nearly all of the upper confidence interval measured values be the same or less than the conservative correlation. This correlation will be referred to as the conservative correlation for generation rate, $G R_{C}{ }^{(1)}$

$$
G R_{C}=3.26 \times 10^{-16}(A)^{0.793}\left(P_{S}\right)^{2.18}\left(d_{p}\right)^{2.40}
$$

Figure 10.19 shows the comparison of the upper confidence interval measured values with the conservative correlation. The results from the individual tests all are at, or below, the conservative correlation, with the exception of a couple individual points. Nearly all the data points closest to, or just exceeding the conservative correlation (near or slightly above the diagonal line), are extrapolated estimates for a $100 \mathrm{ft}$ chamber. Finally, Figure 10.20 shows a comparison of the measured generation rates with the conservative correlation. The measured generation rates are lower than the upper confidence interval values, and this figure shows all of these measured generation rates being farther below the diagonal line, as expected.

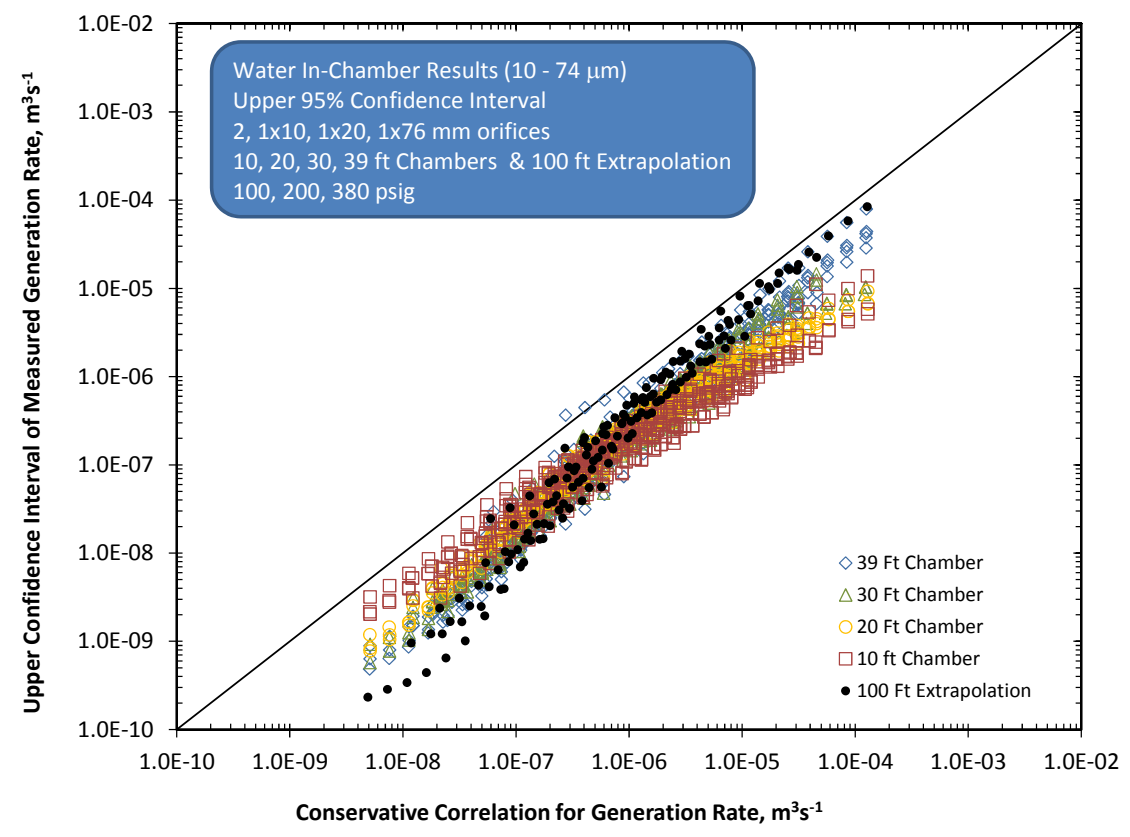

Figure 10.19. Comparison of Measured Upper Confidence Interval of Generation Rate Values for Individual Tests and the $100 \mathrm{ft}$ Extrapolation with the Conservative Correlation for Generation Rate

\footnotetext{
${ }^{1}$ In units of $\mathrm{m}^{3} / \mathrm{s}$ for generation rate, $\mathrm{m}^{2}$ for orifice area, Pa for pressure, and $\mathrm{m}$ for droplet size, the conservative correlation is $G R_{C}=2.01 \times 10^{-5}(A)^{0.793}\left(P_{S}\right)^{2.18}\left(d_{p}\right)^{2.40}$
} 


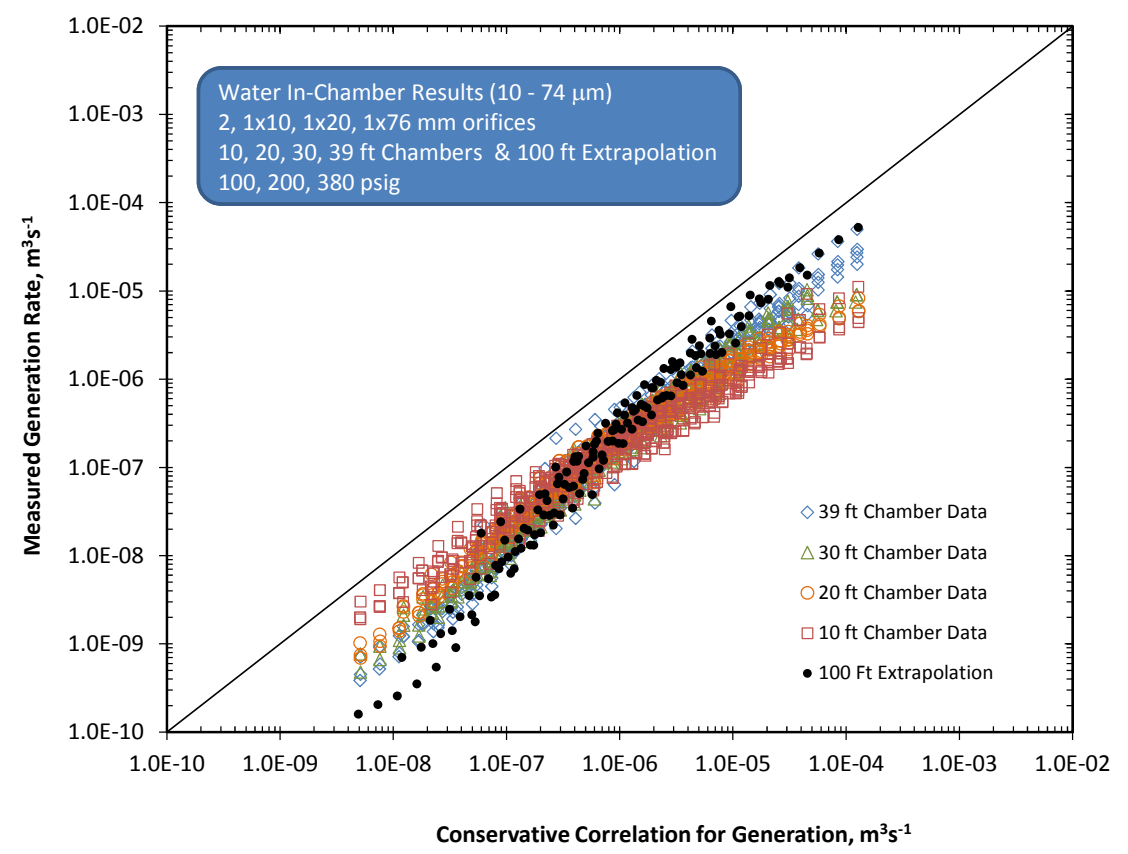

Figure 10.20. Comparison of Measured Generation Rate for Individual Tests and the $100 \mathrm{ft}$ Extrapolations with the Conservative Correlation

\subsection{Comparison of Conservative Generation Rate Correlation to In-Spray Results and Humidity-Corrected (FIO) Generation Rates}

The purpose of this section is to evaluate the conservative correlation to judge if it is reasonably conservative by comparing it to in-spray data for different orifices and spray pressures and also by comparing the conservative correlation to humidity-corrected data from the in-chamber method. The humidity correction is based on humidity measurements obtained from sensors that did not meet the project calibration requirements, so this comparison is FIO and is focused on evaluating if the conservative correlation is sufficient to account for an FIO estimate of the humidity correction for the test data.

In-spray measurements are generally considered to represent an upper limit to aerosol generation from sprays, provided there is no net generation of aerosol droplets by splatter when sprays impact on walls or objects. As summarized in Section 10.2, and discussed more extensively in Chapter 9, the generation rate from a spray decreases as the spray gets closer to the splash wall within a chamber of a specific size. As the spray gets closer to the splash wall, it impacts with a greater velocity and this should increase droplet generation by splatter. The wall also captures droplets when they impact. Net generation is what contributes to aerosol accumulation in the chamber, and the test results with different spray lengths suggest that the net effect of sprays impacting on walls is droplet loss by capture. Accordingly, in-spray measurements represent an upper limit to aerosol generation regardless of the presence of walls or objects. Because the in-spray measurements represent an upper bound, a correlation that is reasonably conservative, but not overly conservative, should not exceed the in-spray results. 
Figure 10.21 through Figure 10.23 show comparisons of the conservative correlation to in-spray results for water for the four orifices tested (i.e., $2 \mathrm{~mm}$ hole, $1 \times 10,1 \times 20,1 \times 76 \mathrm{~mm}$ slots) at the three test pressures $(380,200,100 \mathrm{psig})$. The generation rate values for the in-spray data were obtained by multiplying the in-spray release fraction data with the flow rate measured during the in-spray tests (see Section 1.5). The generation rate from the conservative correlation generally matches the in-spray data, specifically at droplet sizes in the range of 50 to $80 \mu \mathrm{m}$ where there is overlap in the two results. The conservative correlation does over-predict the generation rate in comparison to the in-spray data for the $2 \mathrm{~mm}$ hole, and to a lesser degree for the $1 \times 10$ and $1 \times 20 \mathrm{~mm}$ slots, at 380 psig and the comparison gets better at 200 and 100 psig for all the orifices. The good comparisons in the regions of overlap for different size orifices and different spray pressures confirm that the conservative correlation has orifice area and spray pressure dependences that agree with in-spray data. The slopes of the curves, which show the effect of droplet size on the generation rate, also are very similar for all the orifices and spray pressures, confirming that the conservative correlation has a droplet size dependence that agrees with the in-spray data. Finally, in-spray data are thought to be conservative with a high degree of technical defensibility so the in-chamber results should never be greater than the in-spray results. Because the in-chamber results match the in-spray results, and assuming the in-spray data are accurate, this demonstrates that the conservative correlation has accounted for the potential biases (humidity and method bias) with the in-chamber method without actually quantifying them.

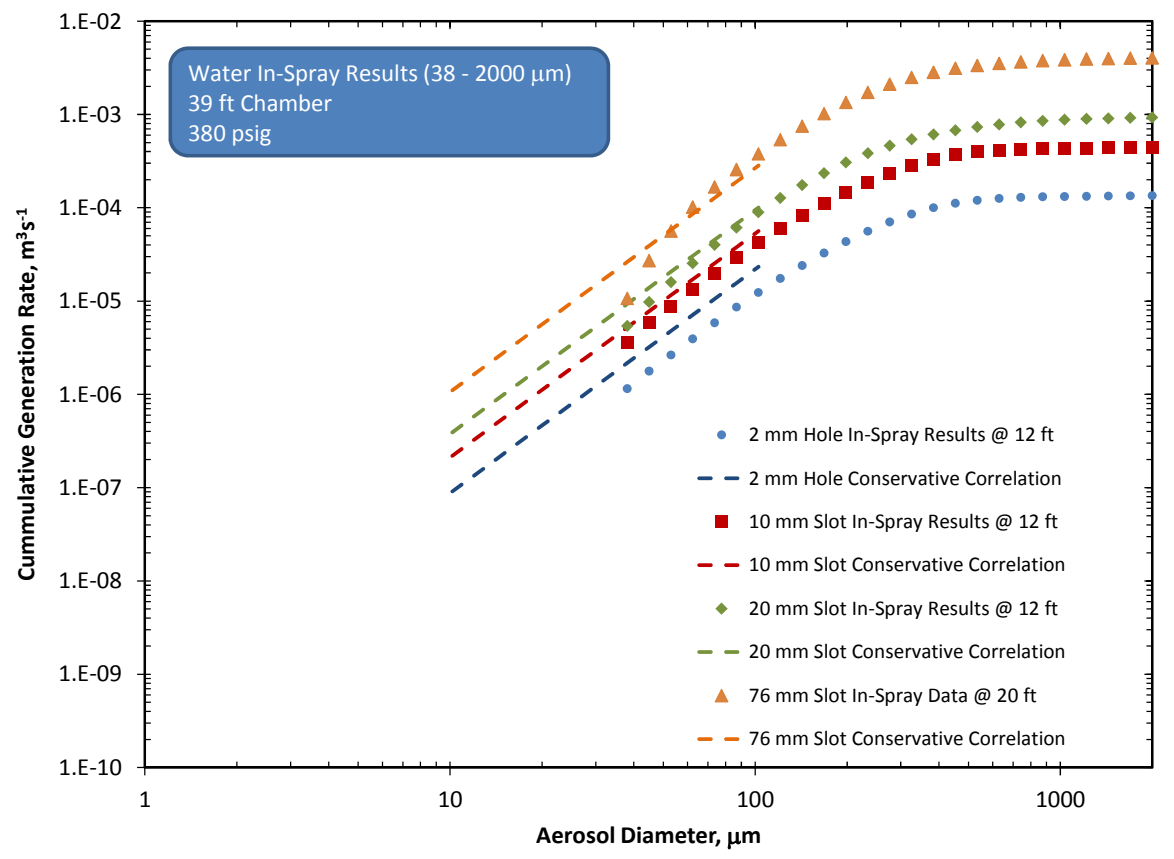

Figure 10.21. Comparison of Conservative Correlation with In-Spray Results for Water Sprays at 380 psig for the Four Orifices Tested. For in-spray data, the measurement distance from the orifice is $12 \mathrm{ft}$ for the $2 \mathrm{~mm}$ hole, $1 \times 10$ and $1 \times 20 \mathrm{~mm}$ slots, and the measurement distance from the orifice is $20 \mathrm{ft}$ for the $1 \times 76 \mathrm{~mm}$ slot. 


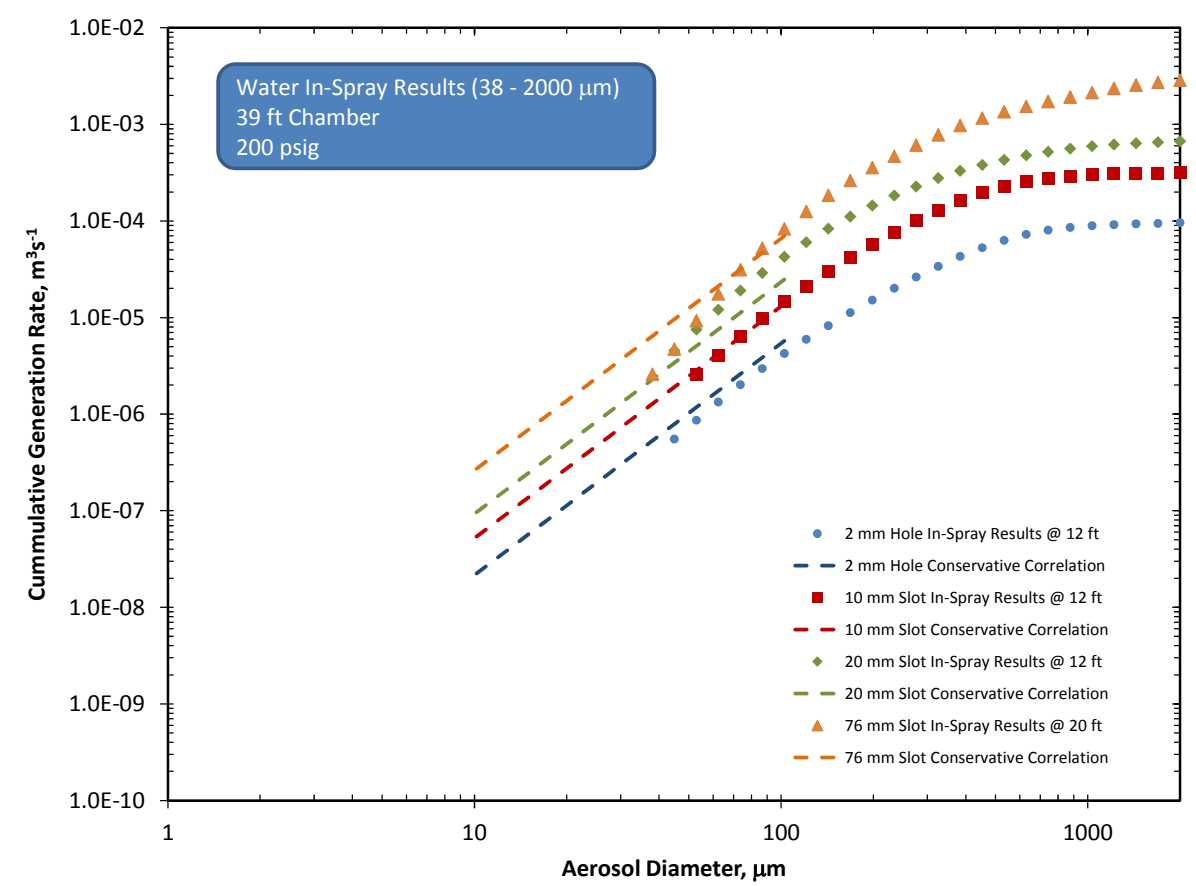

Figure 10.22. Comparison of Conservative Correlation with In-Spray Results for Water Sprays at 200 psig for the Four Orifices Tested. For in-spray data, the measurement distance from the orifice is $12 \mathrm{ft}$ for the $2 \mathrm{~mm}$ hole, $1 \times 10$ and $1 \times 20 \mathrm{~mm}$ slots, and the measurement distance from the orifice is $20 \mathrm{ft}$ for the $1 \times 76 \mathrm{~mm}$ slot.

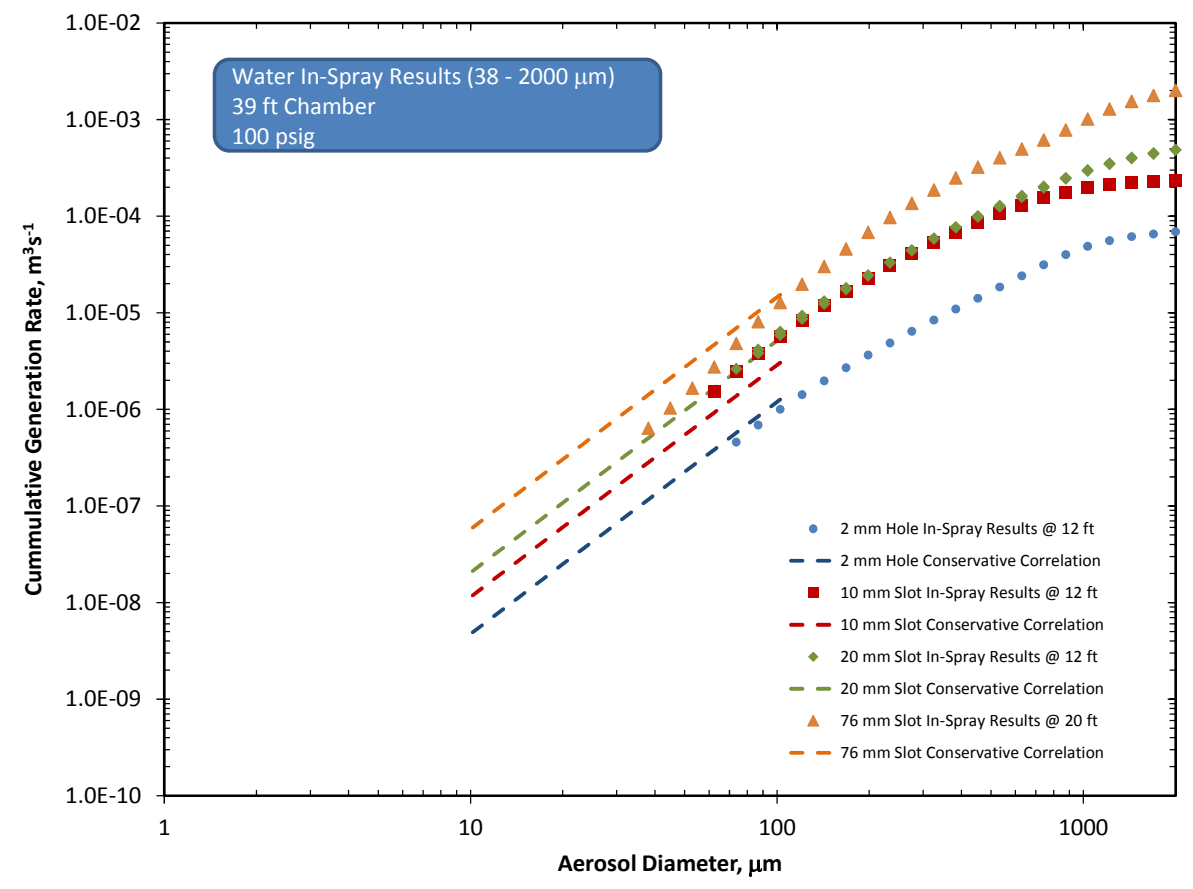

Figure 10.23. Comparison of Conservative Correlation with In-Spray Results for Water Sprays at 100 psig for the Four Orifices Tested. For in-spray data, the measurement distance from the orifice is $12 \mathrm{ft}$ for the $2 \mathrm{~mm}$ hole, $1 \times 10$ and $1 \times 20 \mathrm{~mm}$ slots, and the measurement distance from the orifice is $20 \mathrm{ft}$ for the $1 \times 76 \mathrm{~mm}$ slot. 
The conservative correlation also can be evaluated by comparing it to humidity-corrected, in-chamber results. When the humidity during a test is less than 100 percent $\mathrm{RH}$, evaporation of the droplets causes the reported generation rate values to be biased low. Humidity measurements were collected during each test, but these measurements are FIO because they are based on readings from instruments that do not meet the project calibration requirements. In Appendix A, the test results at different humidity conditions were compared and a humidity correction was estimated for the test results. For water sprays, a maximum correction factor of 1.5 was determined based on the FIO humidity measurements. To evaluate the conservative correlation, the measured values for the generation rate and the upper confidence interval will be increased by the FIO humidity correction of 1.5 and then compared with the conservative correlation. There are no test results to evaluate how humidity affects generation rates for sprays in a $100 \mathrm{ft}$ chamber, so there is additional uncertainty in comparing the conservative correlation with the humidity-corrected test results. Figure 10.24 shows a comparison of the upper confidence interval generation rate values, corrected for humidity based on FIO data, with the conservative correlation. For this comparison, 0.63 percent of the individual data points exceed the conservative correlation. The majority of data points that exceed the correlation correspond to extrapolated $100 \mathrm{ft}$ chamber data. Figure 10.25 shows a comparison of the generation rate data, again corrected for humidity based on FIO data, with the conservative correlation. For this comparison, 0.15 percent of the individual data points exceed the conservative correlation. Both of the comparisons support that the conservative correlation is still reasonably conservative when the test data are corrected for humidity using an estimated maximum correction based on FIO humidity measurements.

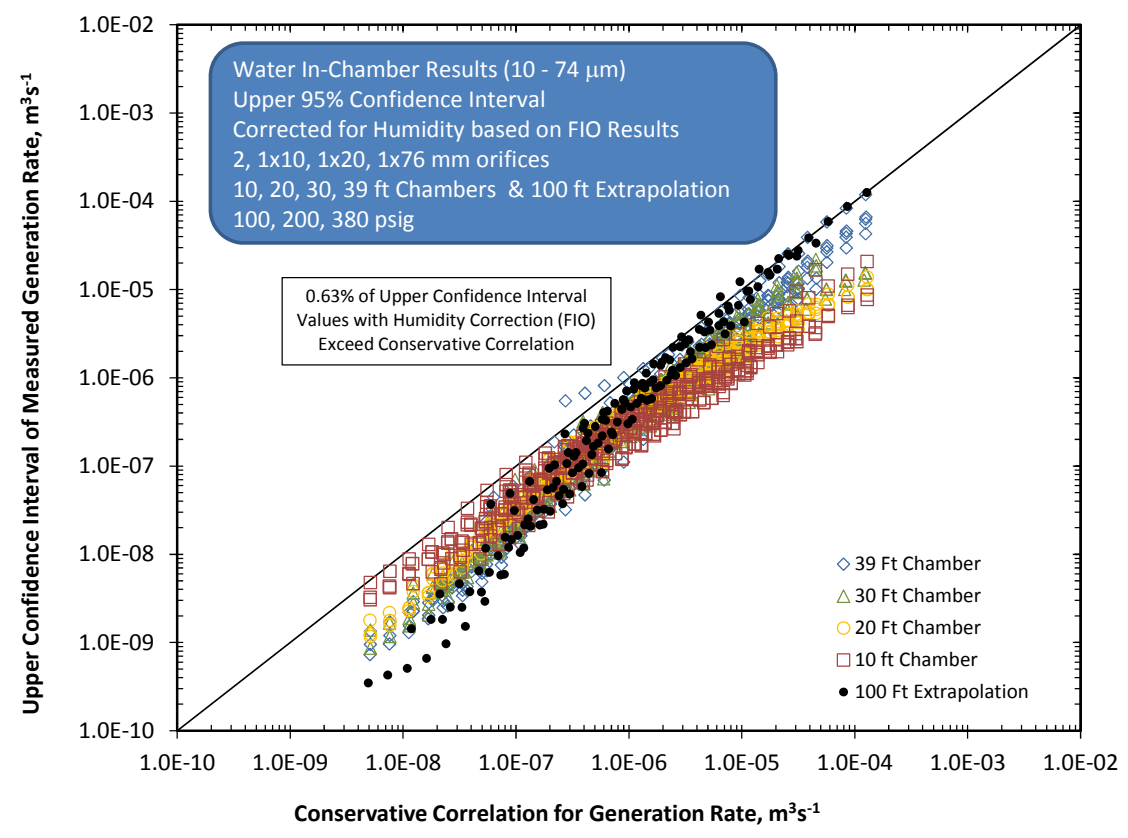

Figure 10.24. Comparison of Upper Confidence Interval Generation Rate Values, Corrected for Humidity Based on FIO Data, Compared to the Conservative Correlation 


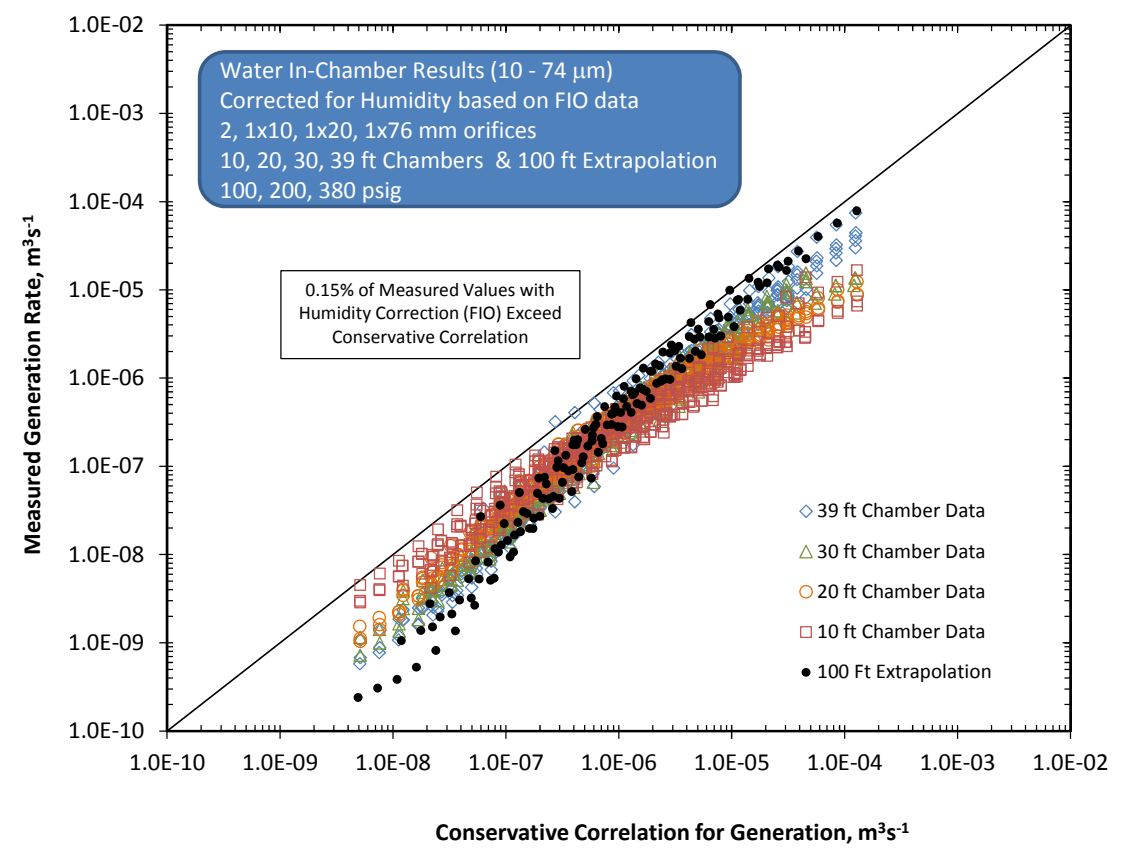

Figure 10.25. Comparison of Measured Generation Rate Data, Corrected for Humidity Based on FIO Data, Compared to the Conservative Correlation

\subsection{Comparison of Conservative Correlation and In-Spray Results to WTP Model and Selected Test Data}

Based on the data collected for the in-chamber and in-spray measurement of aerosol generation, and the comparisons given in this chapter, the conservative correlation appears to fully account for all uncertainties in the in-chamber measurements such as humidity and method bias. Accordingly, this correlation represents reasonably conservative test results that can be compared directly with the WTP model. The conservative correlation also can be used to estimate the generation rate for spray release accident analyses. Because the conservative correlation and in-spray results match and show the same dependence on droplet size, the conservative correlation can be used for droplets in the size range of interest, 10 to $100 \mu \mathrm{m}$, even though the correlation was developed using data for droplets ranging in size from 10 to $74 \mu \mathrm{m}$.

Figure 10.26 through Figure 10.37 show results for each of the orifices tested at each spray pressure. These figures show the conservative correlation, the in-spray results for each condition, and in-chamber generation rate data from the 10 and $39 \mathrm{ft}$ chambers and the $100 \mathrm{ft}$ extrapolations. Figure 10.38 through Figure 10.40 give comparisons of the conservative correlation and the WTP model for three specific orifices and spray pressures (380 psig and $120 \mathrm{~mm}^{2}, 184 \mathrm{psig}$ and $209 \mathrm{~mm}^{2}$, and $540 \mathrm{psig}$ and $290 \mathrm{~mm}^{2}$ ) for process streams that are of interest to the WTP for accidental spray release scenarios. ${ }^{1}$ Comparisons of the conservative correlation results to the WTP model results show three specific observations:

1. The effect of droplet size on generation rate (slope of generation rate versus droplet size lines) is equal for both the conservative correlation and the WTP model. This is equivalent to noting that the

\footnotetext{
${ }^{1}$ Personal communication from A Hassan of BNI to PA Gauglitz of PNNL on March 20, 2013.
} 
exponent of 2.41 for droplet size in Equation (10.4) for the conservative correlation is close to the value of q=2.4 for the Rosin-Rammler distribution used in the WTP model (Schonewill et al. 2012; Mahoney et al. 2013).

2. The WTP model under-represents the increase in generation rate with increasing orifice area when compared to the conservative correlation. For example, Figure 10.26 through Figure 10.29 show a series of comparisons for a $2 \mathrm{~mm}$ hole up to the $1 \times 76 \mathrm{~mm}$ slot for 380 psig sprays. The WTP model overlays the conservative correlation for the $2 \mathrm{~mm}$ hole, but the WTP model under-predicts the generation rate by about a factor of eight for the $1 \times 76 \mathrm{~mm}$ orifice. The orifices of intermediate size show a consistent progression from the small to the large orifice.

3. The WTP model under-represents the increase in generation rate with increasing spray pressure when compared to the conservative correlation. For the $1 \times 76 \mathrm{~mm}$ slot as an example, the WTP model is slightly below the conservative correlation for a 100 psig spray (Figure 10.37), becomes farther below the conservative correlation for a 200 psig spray (Figure 10.33), and is even more below the conservative correlation for a 380 psig spray (Figure 10.29).

Based on these results, the greatest under-estimation in generation rate from the WTP model occurs for the highest postulated pressure and the largest postulated breach, which is consistent with the conclusions from previous testing (Schonewill et al. 2012).

The comparisons in Figure 10.26 through Figure 10.37 also show how selected test data compare to the conservative correlation for specific orifices and spray pressures. While the test data have not been increased to account for the effect of humidity (in Appendix A, the maximum correction based on FIO humidity data was estimated to be 1.5), the comparisons show that the uncorrected test data are below the conservative correlation (but not by a significant margin) for all orifices and test conditions. For example, at the highest test pressure of 380 psig with the $1 \times 76 \mathrm{~mm}$ slot, the results for droplets near $100 \mu \mathrm{m}$ shown in Figure 10.29 are only slightly below the conservative correlation. At the other extreme of testing conditions, which is the lowest test pressure of 100 psig and the smallest orifice ( $2 \mathrm{~mm}$ hole), the results for the $10 \mu \mathrm{m}$ droplets shown in Figure 10.34 are only slightly below the conservative correlation.

The conservative correlation (Equation (10.4)) can be compared to other aerosol generation correlations as well as to the WTP model. Any power-law expression for the Sauter mean diameter (SMD, or $D_{3,2}$ ) of a spray can be converted to a power-law expression for the generation rate of aerosol, if the following two constraints are observed:

- The spray distribution is represented by a Rosin-Rammler size distribution and the value of $q$ describing the spread of the distribution is known

- Generation rates are calculated only for the droplet size range at or below the SMD. For larger sizes the cumulative size distribution flattens as it approaches unity, causing a deviation from the power-law form.

If these constraints are met, then Equation (5.24) can be approximated by using the first term of a Taylor expansion to represent the exponential, giving:

$$
R_{p}^{(\text {model })} \approx\left(\frac{D_{3,2}}{D_{X}} \frac{d_{p}}{D_{3,2}}\right)^{q}
$$


where the factor $\left(D_{3,2} / D_{X}\right)$ depends only on $q$ (Equation (5.25)). The desired power-law expression for $D_{3,2}$ is substituted into the denominator. The cumulative generation rate $G R_{p}^{(\text {model })}$ for any droplet size $d_{p}$ can then be found by multiplying $R_{p}^{(\text {model })}$ by the flow rate predicted by Equation (5.23), using an orifice coefficient that is appropriate for the fluid and the orifice size.

For the purpose of comparing the generation rate predictions for water, the approximation in Equation (10.5) was applied to the WTP model and to three other SMD correlations from the literature. In this comparison, the WTP model for SMD was simplified to its low-viscosity form (accurate to within a few percent for water) by omitting the viscosity terms from Equation (5.19) and Equation (5.20). In another study related to Hanford work, Epstein and Plys (2006) developed a power-law correlation for SMD based on in-spray measurements with a Malvern Spraytec droplet sizer, using water pressures between 180 and $600 \mathrm{psig}$ and a range of orifice sizes from $0.07 \mathrm{~mm}^{2}$ to $12 \mathrm{~mm}^{2}$. They determined that the SMD did not depend on orifice size for most of their orifices (although their largest slot, $1.2 \times 10 \mathrm{~mm}$, showed different behavior) and that a Rosin-Rammler size distribution with $q=2.3$ gave the best fit to their data for all orifices except the largest slot. Merrington and Richardson (1947) conducted experiments with Newtonian liquids with a range of properties (water and glycerine being the ends of the viscosity range), using three nozzle sizes and one sharp-edged orifice (size range $0.5 \mathrm{~mm}^{2}$ to $50 \mathrm{~mm}^{2}$ ), and having jet velocities between 25 and $100 \mathrm{~m} / \mathrm{s}$. Droplet sizes were measured from the marks left where droplets from a vertical downwards jet fell on paper. Merrington and Richardson found a powerlaw expression for the dependence of the mean diameter of the size distribution upon test parameters, and did not find a dependence of mean aerosol diameter on orifice size. Lefebvre (1989) modified the Merrington and Richardson correlation to include a dependence on orifice size. The Lefebvre version is included in the comparison as a correlation likely to be used in general practice, and the original version is included to show the effect of Lefebvre's modification. Following the example of Epstein and Plys, both versions of the Merrington and Richardson correlation are treated as having Rosin-Rammler size distributions with $q=2.3$. This allows the mean diameter to be converted to an SMD, so that power-law expressions can be generated.

Using the same units as in Equation (10.4), the power-law approximations for the correlations from the literature are the following:

- Low-viscosity WTP model:

$$
G R_{p}^{(\text {WTPlow })} \approx 1.59 \times 10^{-13}(A)^{0.2}\left(P_{S}\right)^{1.3}\left(d_{p}\right)^{2.4}
$$

- Epstein and Plys correlation:

$$
G R_{p}^{(E \& P)} \approx 4.35 \times 10^{-13}(A)^{1.0}\left(P_{S}\right)^{1.142}\left(d_{p}\right)^{2.3}
$$

- Merrington and Richardson correlation (assuming Rosin-Rammler distribution with $q=2.3$ ):

$$
G R_{p}^{(M \& R)} \approx 1.27 \times 10^{-15}(A)^{1.0}\left(P_{S}\right)^{1.65}\left(d_{p}\right)^{2.3}
$$

- Merrington and Richardson correlation as modified by Lefebvre (assuming Rosin-Rammler distribution with $q=2.3$ ):

$$
G R_{p}^{(L e f M \& R)} \approx 4.95 \times 10^{-15}(A)^{-0.38}\left(P_{S}\right)^{1.65}\left(d_{p}\right)^{2.3}
$$


The subscript $p$ denotes generation rate is the cumulative rate below the droplet size $d_{p}$.

These expressions, together with the conservative correlation, are plotted in Figure 10.41 to compare the correlations' predictions of the effect of the orifice area on the cumulative generation rate of $<10 \mu \mathrm{m}$ aerosol from a 380 psig water spray. Under these conditions, the conservative correlation consistently predicts higher cumulative generation rates of $<10 \mu \mathrm{m}$ aerosol than the original Merrington and Richardson correlation over the entire depicted range of orifice area, and predicts lower generation rates than the Epstein and Plys correlation. The correlation predicts higher generation rates than the lowviscosity WTP model and the Lefebvre-modified Merrington and Richardson correlation for the larger orifices, but not over the entire orifice size range. The relative behavior of the models and the correlation is much the same for a water spray at 100 psig (not shown).

At 380 psig, as shown in Figure 10.42, the Epstein and Plys correlation predicts $<10 \mu \mathrm{m}$ release fractions that are two to three times more than those predicted by the conservative correlation in the orifice size range $\left(0.07\right.$ to $\left.4.4 \mathrm{~mm}^{2}\right)$ on which the Epstein and Plys correlation was based. This requires some further discussion to establish the extent to which the conservative correlation bounds the spray release fraction. The higher release fractions found by Epstein and Plys can probably be explained by the short downstream distance, less than $1 \mathrm{~m}$, at which Epstein and Plys made measurements. As can be seen in Figure 7.6, for a $2 \mathrm{~mm}$ orifice (which is in the small orifice range of the Epstein and Plys tests) the small droplet release fractions for a 380 psig spray at a downstream measurement distance of $5 \mathrm{ft}$ are about three times those measured at a distance of $12 \mathrm{ft}$. Because the Epstein and Plys measurement distance was usually 0.5 to $1 \mathrm{~m}$ (significantly less than $5 \mathrm{ft}$ ), and the in-spray data from which the conservative correlation were developed were measured at $12 \mathrm{ft}$, the measured release fractions (and generation rates) would be expected to be three or more times higher in Epstein and Plys data than in the conservative correlation, for 380 psig. For completeness, it should be noted that because the conservative correlation has a stronger dependence on pressure than the Epstein and Plys correlation, the difference between the correlations is greater at lower pressures and less at higher pressures.

It is also worth noting that the Epstein and Plys correlation and the conservative correlation show some difference in the generation rate's dependence on orifice area. The present study found a small dependence of release fraction on orifice area (a power-law exponent of about -0.2) whereas Epstein and Plys found no dependence. The difference in dependence is small, and the apparent lack of dependence in the Epstein and Plys tests is probably related to the smaller range of orifice sizes: 0.07 to $4.4 \mathrm{~mm}^{2}$ range for Epstein and Plys, compared to the 3 to $76 \mathrm{~mm}^{2}$ range in the present study. As Figure 9.5 shows, data for larger orifices had to be included before the dependence on orifice size became evident.

The Epstein and Plys correlation ultimately produces larger generation rate predictions than the conservative correlation developed in this chapter, and as such, it could be used to provide "conservative" generation rate predictions for the configurations tested herein. However, based on the Phase II large-scale test data, such use of the Epstein and Plys correlation would provide an overly conservative prediction of aerosol generation rates. The conservative correlation is recommended over the Epstein and Plys correlation for several reasons. As outlined above:

- The conservative correlation is based on a large set of spray release measurements collected with a wide range of orifice size and geometry at and approaching sizes of interest for the WTP. The orifices selected for large-scale testing exceed the range of sizes evaluated by Epstein and Plys by an 
order of magnitude and thereby yield a correlation that is more appropriate for large orifice (e.g., $\sim 200 \mathrm{~mm}^{2}$ ) generation rate estimations or extrapolation.

- The correlation bounds spray lengths relevant to accident scenarios postulated in the WTP and which span 10 to $39 \mathrm{ft}$ (with extrapolation up to $100 \mathrm{ft}$ ). As stated on the previous page, the Epstein and Plys correlation is based on aerosol data for shorter spray lengths $(<10 \mathrm{ft})$.

- The correlation bounds both in-chamber and in-spray test data collected for the simulants tested in this study.

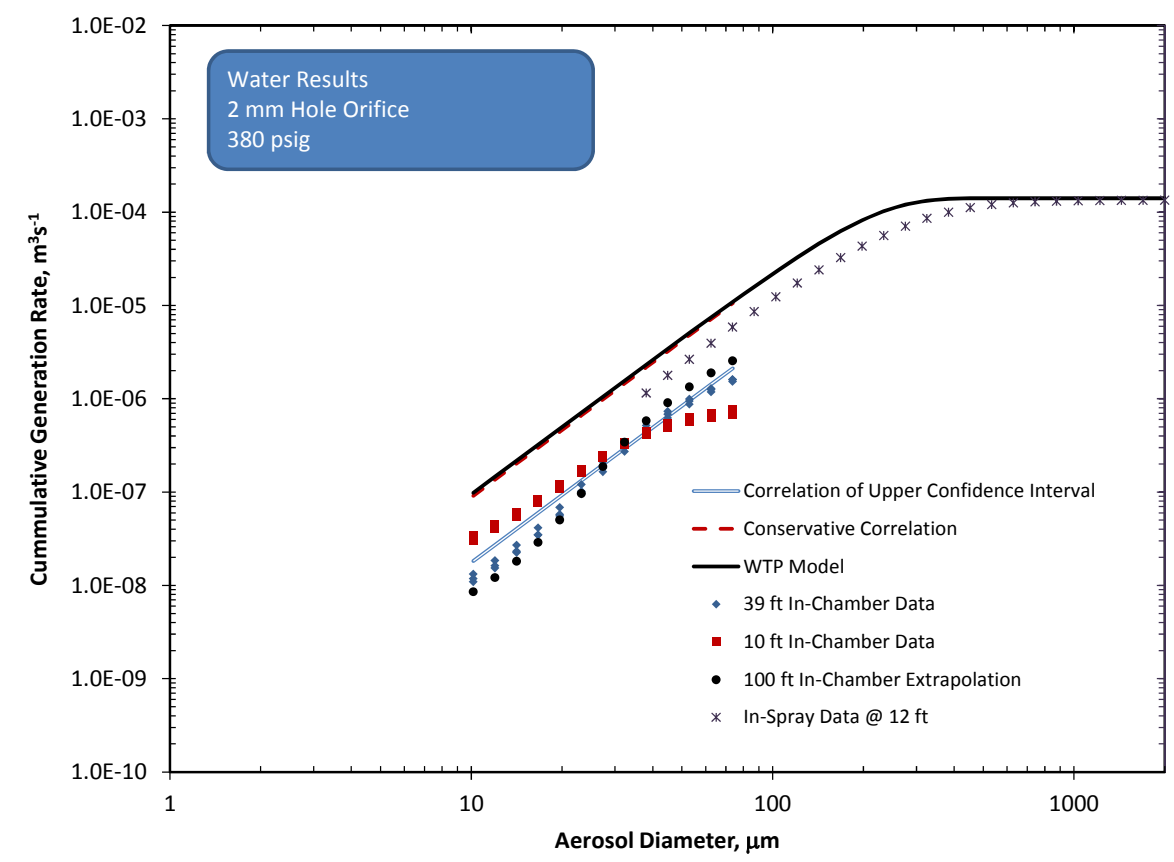

Figure 10.26. Generation Rate Results for a Water Spray from a $2 \mathrm{~mm}$ Hole at 380 psig Showing a Comparison of the Conservative Correlation (hidden by WTP model line), In-Spray Data, and WTP Model. Selected generation rate data (that are biased by humidity and method uncertainties) are shown. 


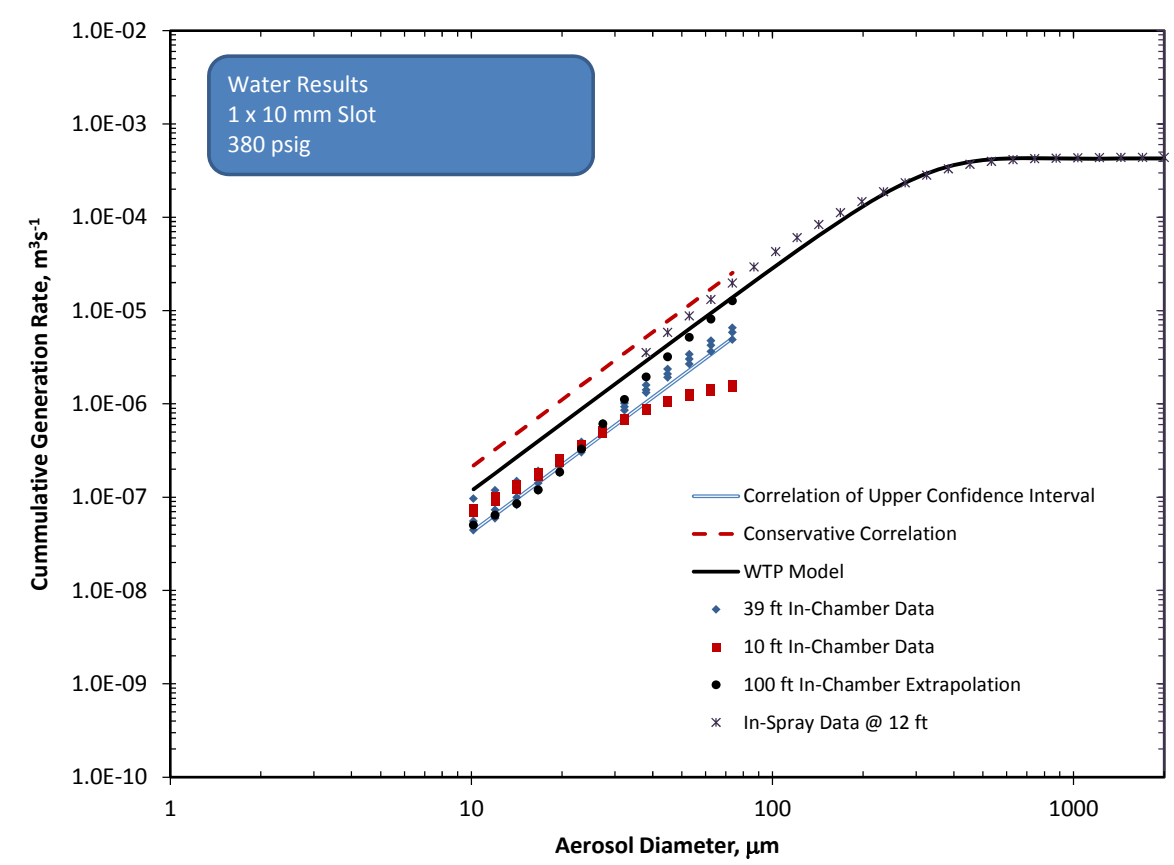

Figure 10.27. Generation Rate Results for a Water Spray from a $1 \times 10 \mathrm{~mm}$ Slot at $380 \mathrm{psig}$ Showing a Comparison of the Conservative Correlation, In-Spray Data, and WTP Model. Selected generation rate data (that are biased by humidity and method uncertainties) are shown.

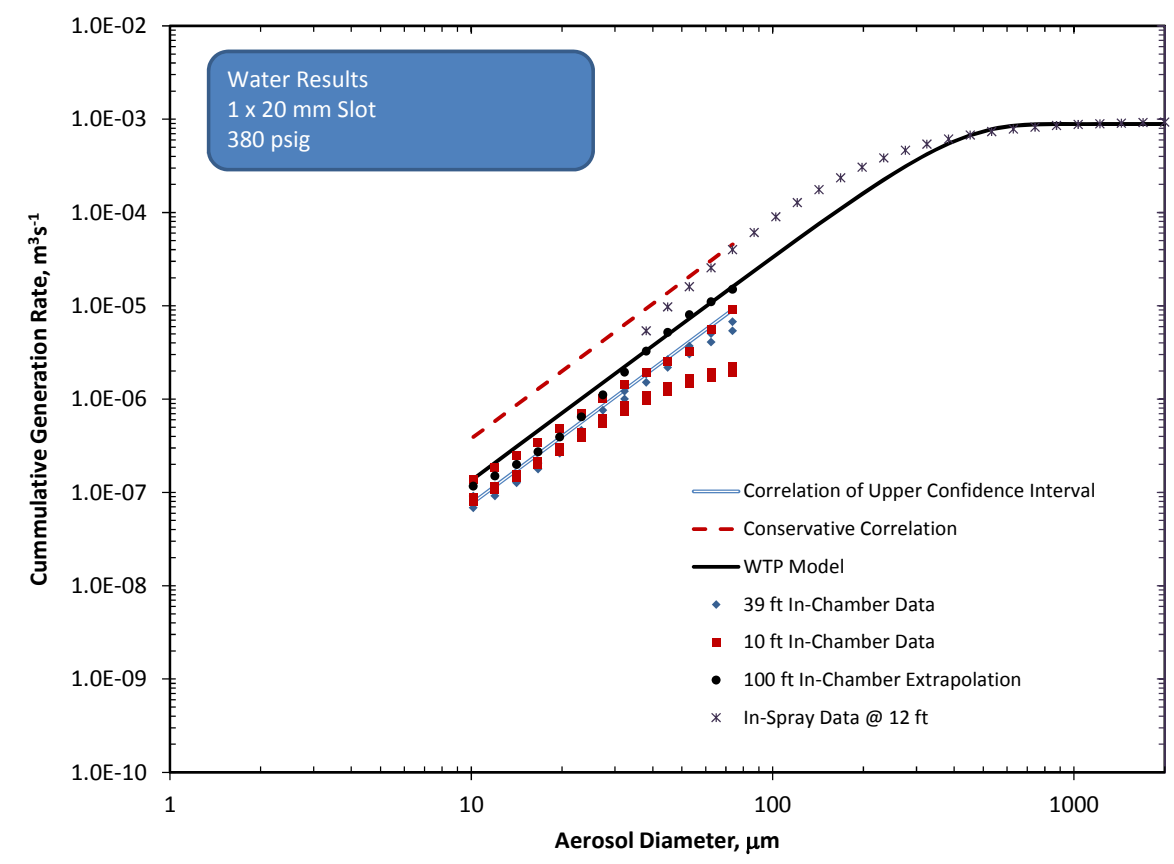

Figure 10.28. Generation Rate Results for a Water Spray from a $1 \times 20 \mathrm{~mm}$ Slot at 380 psig Showing a Comparison of the Conservative Correlation, In-Spray Data, and WTP Model. Selected generation rate data (that are biased by humidity and method uncertainties) are shown. 


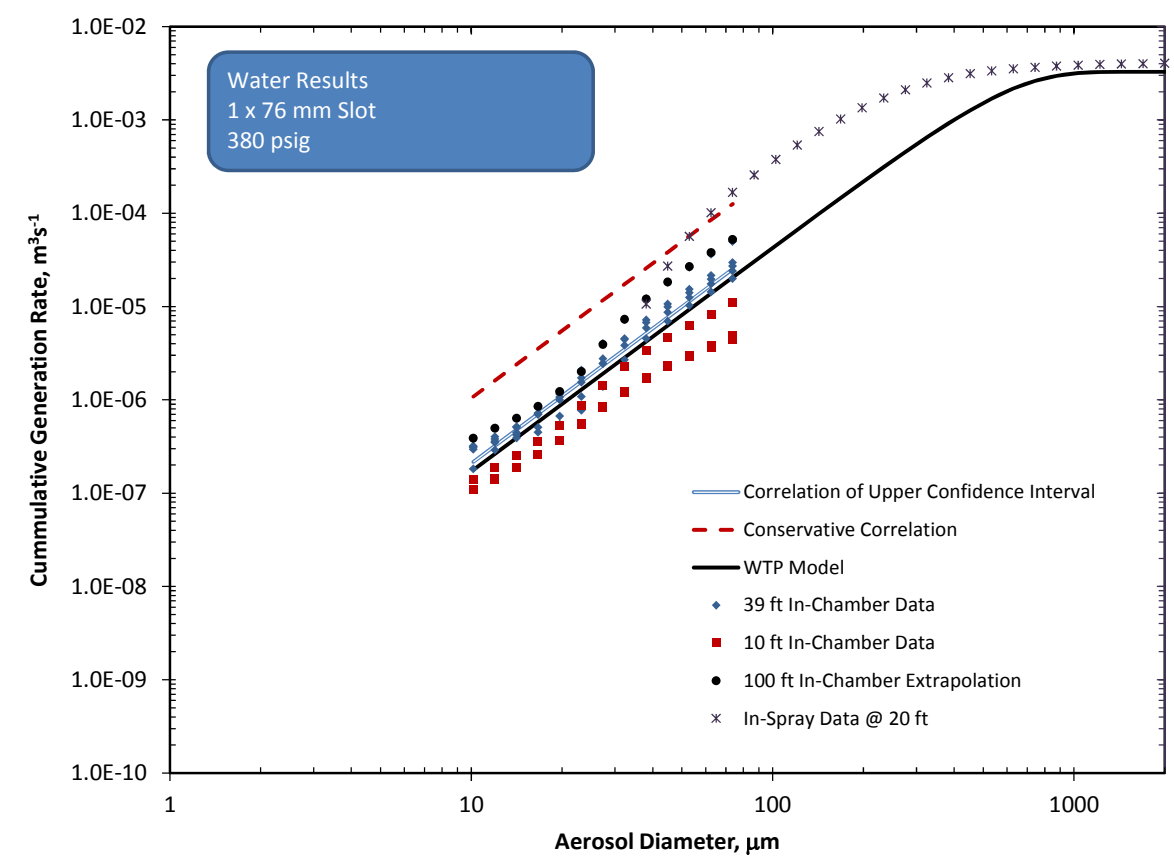

Figure 10.29. Generation Rate Results for a Water Spray from a $1 \times 76 \mathrm{~mm}$ Slot at 380 psig Showing a Comparison of the Conservative Correlation, In-Spray Data, and WTP Model. Selected generation rate data (that are biased by humidity and method uncertainties) are shown.

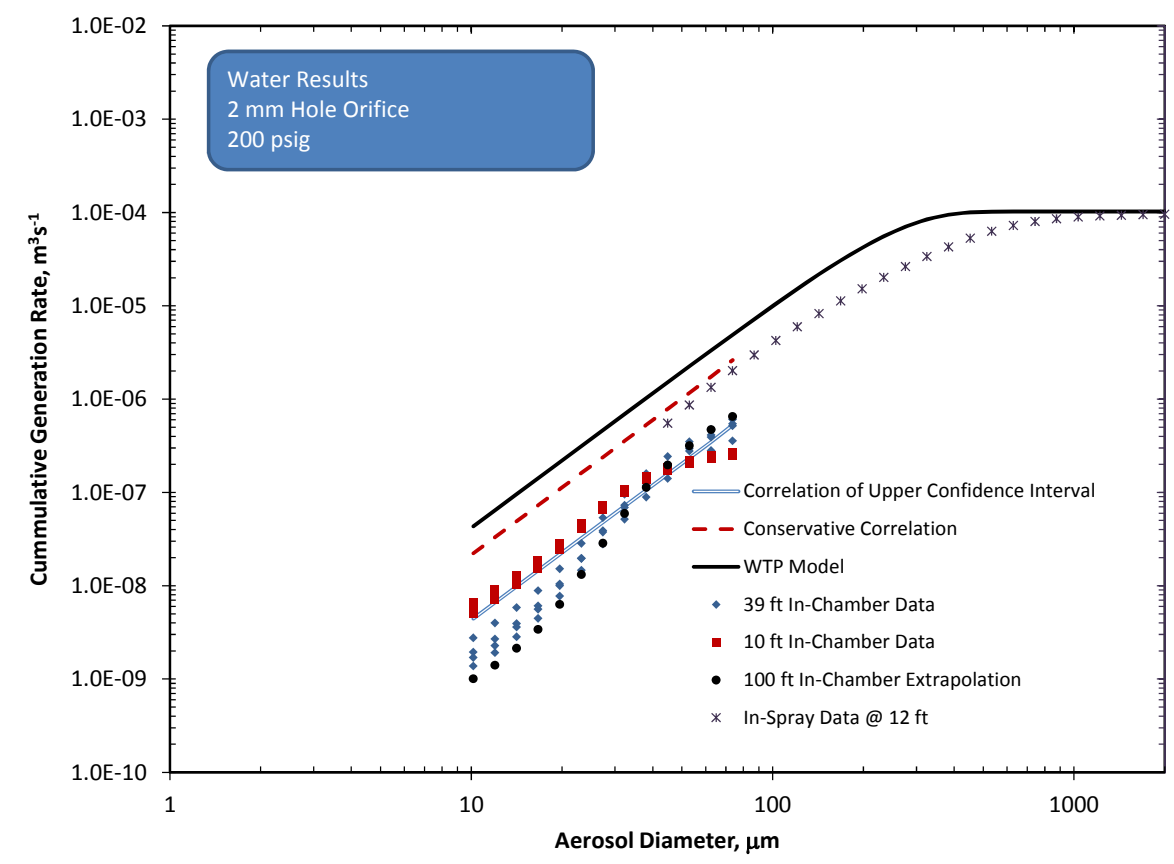

Figure 10.30. Generation Rate Results for a Water Spray from a $2 \mathrm{~mm}$ Hole at 200 psig Showing a Comparison of the Conservative Correlation, In-Spray Data, and WTP Model. Selected generation rate data (that are biased by humidity and method uncertainties) are shown. 


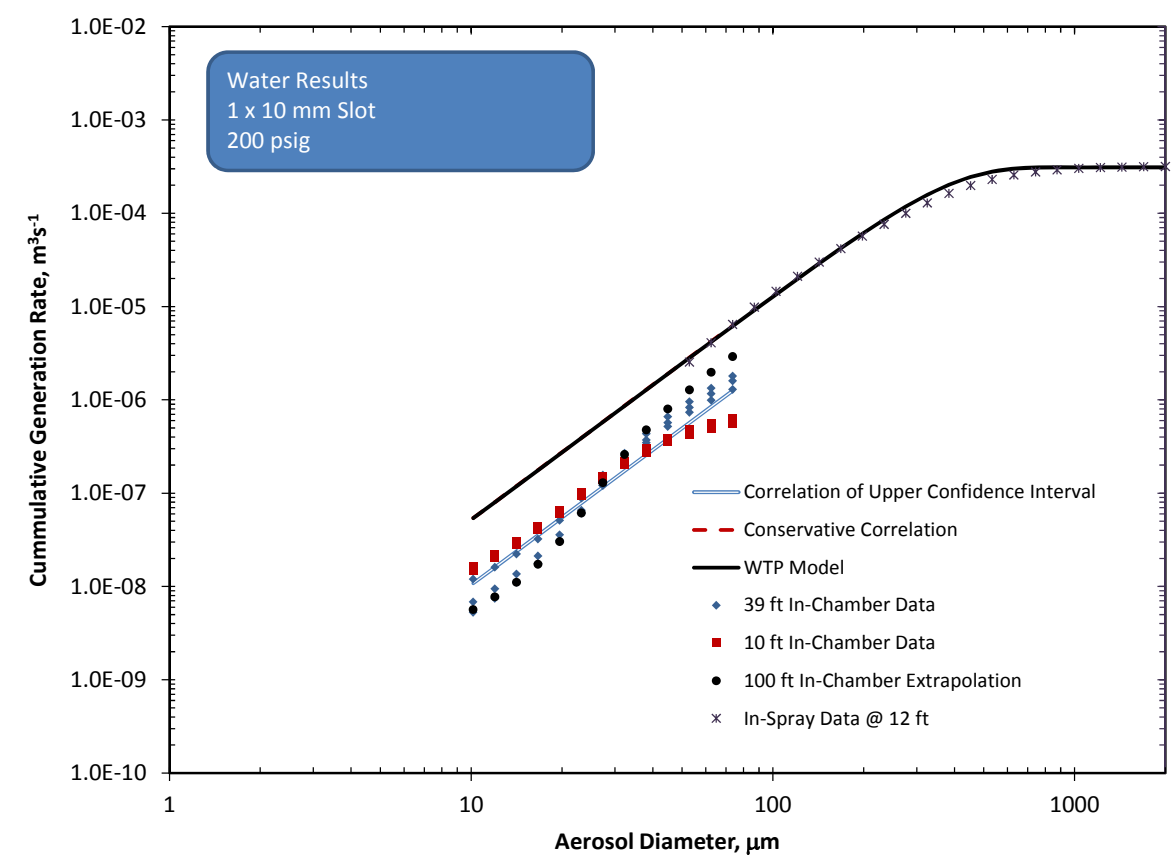

Figure 10.31. Generation Rate Results for a Water Spray from a $1 \times 10 \mathrm{~mm}$ Slot at 200 psig Showing a Comparison of the Conservative Correlation (hidden by WTP model line), In-Spray Data, and WTP Model. Selected generation rate data (that are biased by humidity and method uncertainties) are shown.

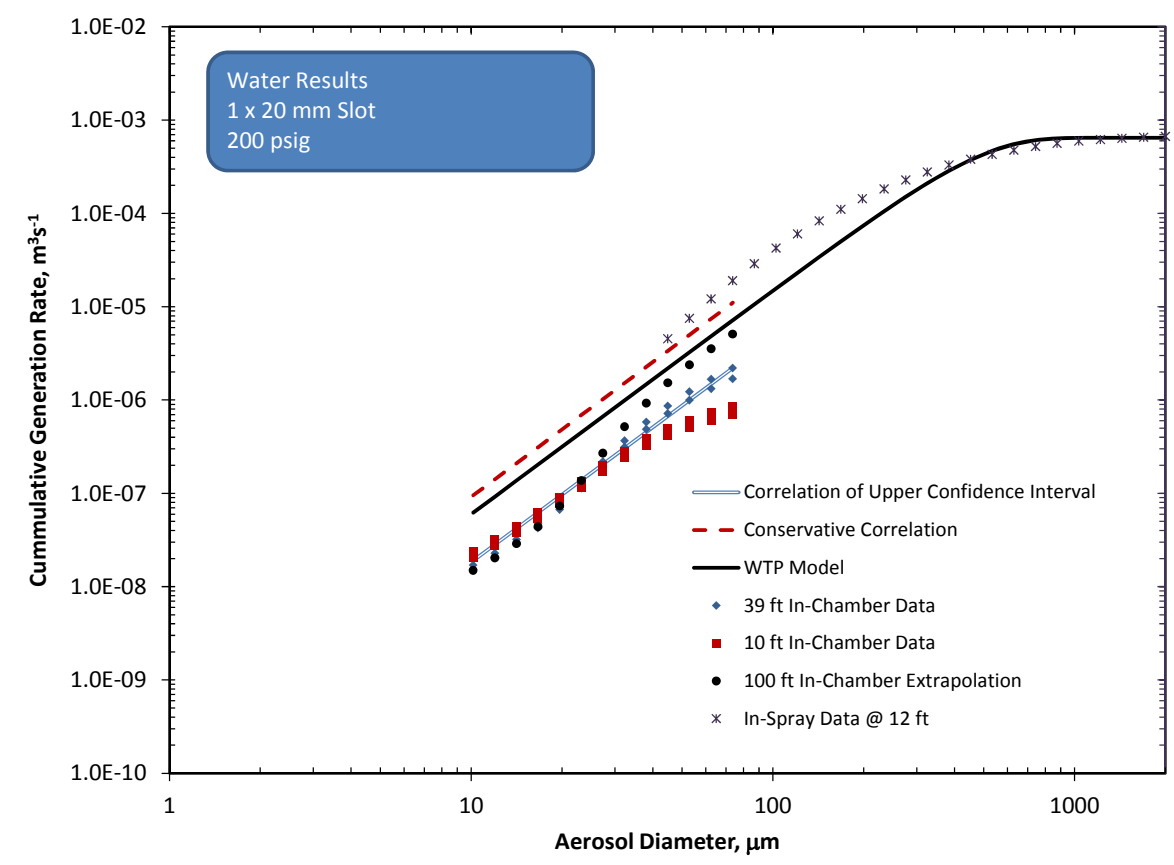

Figure 10.32. Generation Rate Results for a Water Spray from a $1 \times 20 \mathrm{~mm}$ Slot at 200 psig Showing a Comparison of the Conservative Correlation, In-Spray Data, and WTP Model. Selected generation rate data (that are biased by humidity and method uncertainties) are shown. 


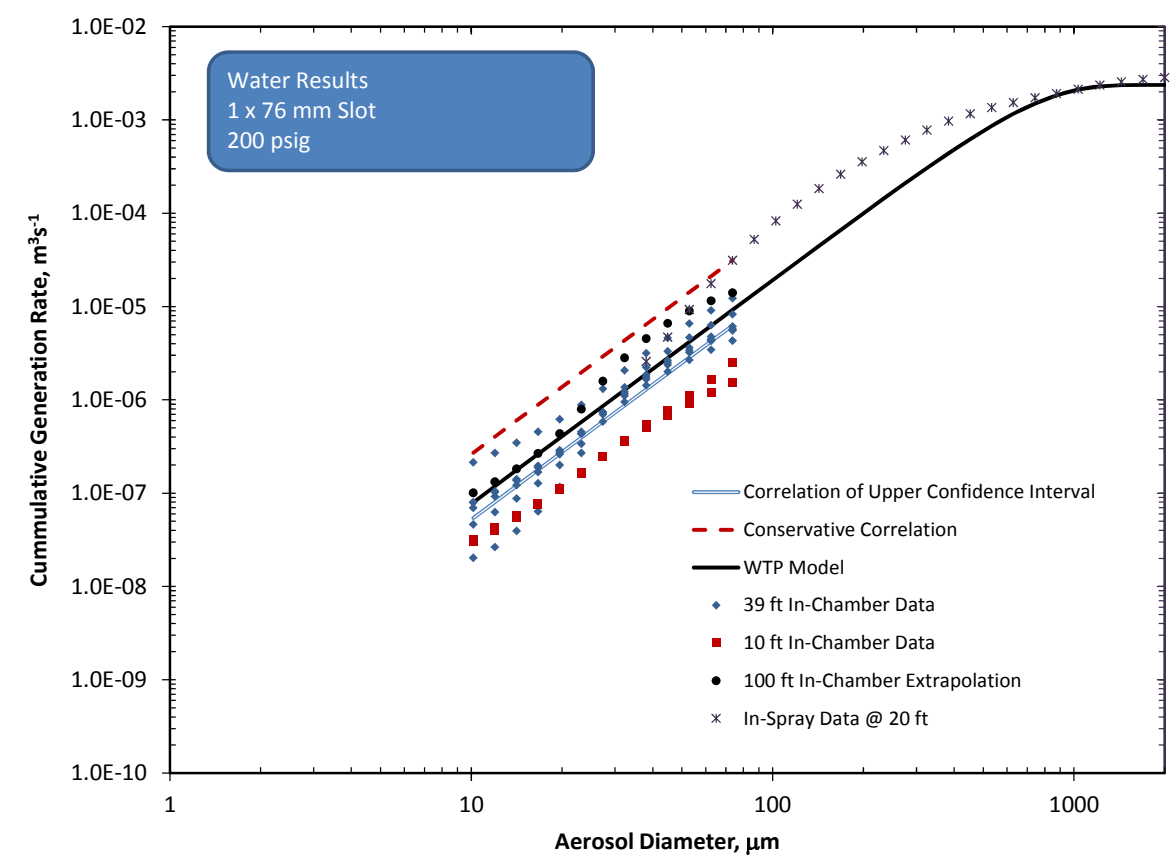

Figure 10.33. Generation Rate Results for a Water Spray from a $1 \times 76 \mathrm{~mm}$ Slot at 200 psig Showing a Comparison of the Conservative Correlation, In-Spray Data, and WTP Model. Selected generation rate data (that are biased by humidity and method uncertainties) are shown.

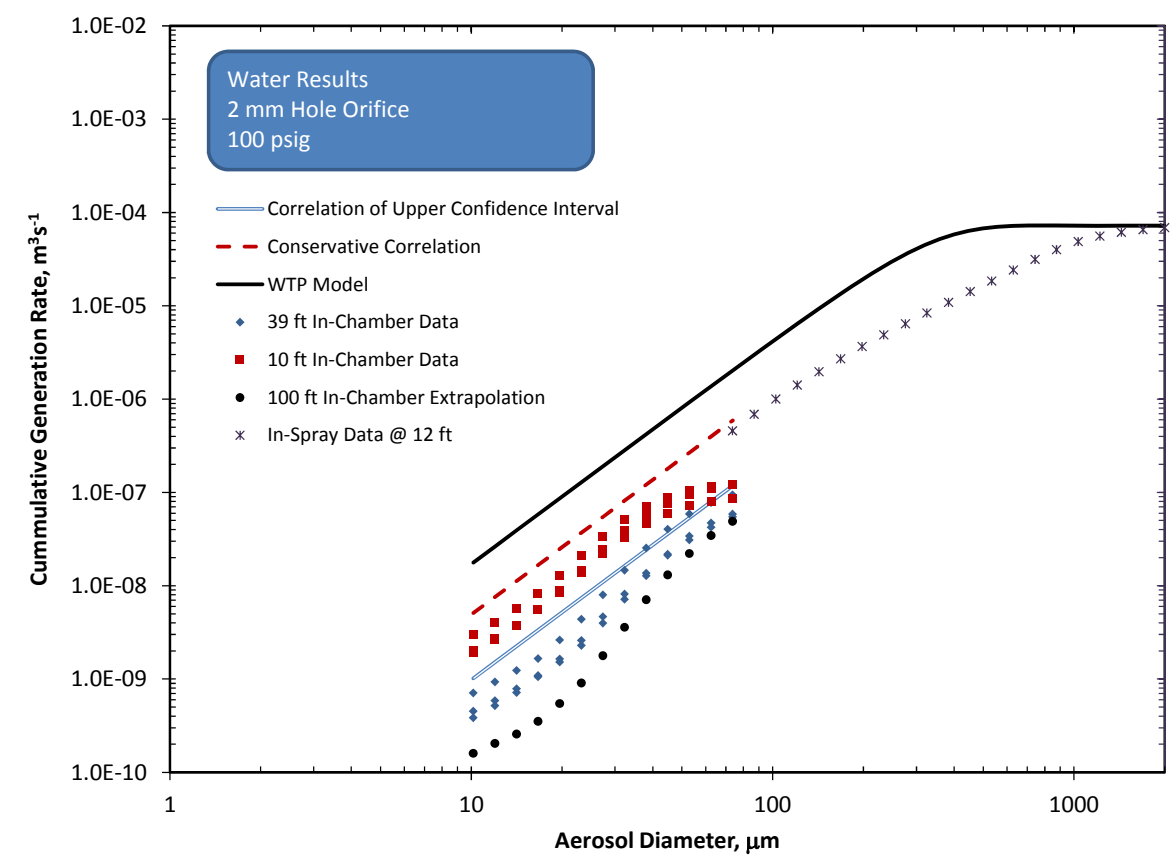

Figure 10.34. Generation Rate Results for a Water Spray from a $2 \mathrm{~mm}$ Hole at 100 psig Showing a Comparison of the Conservative Correlation, In-Spray Data, and WTP Model. Selected generation rate data (that are biased by humidity and method uncertainties) are shown. 


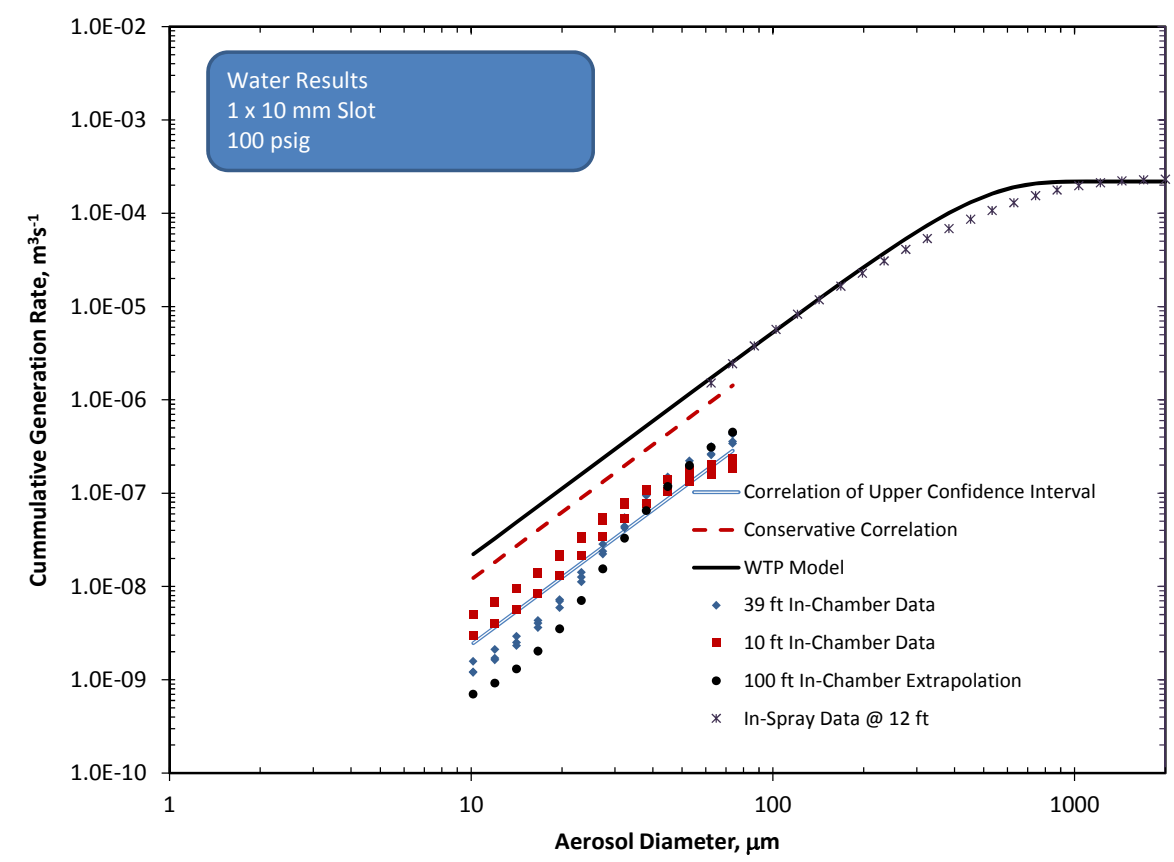

Figure 10.35. Generation Rate Results for a Water Spray from a $1 \times 10 \mathrm{~mm}$ Slot at $100 \mathrm{psig}$ Showing a Comparison of the Conservative Correlation, In-Spray Data, and WTP Model. Selected generation rate data (that are biased by humidity and method uncertainties) are shown.

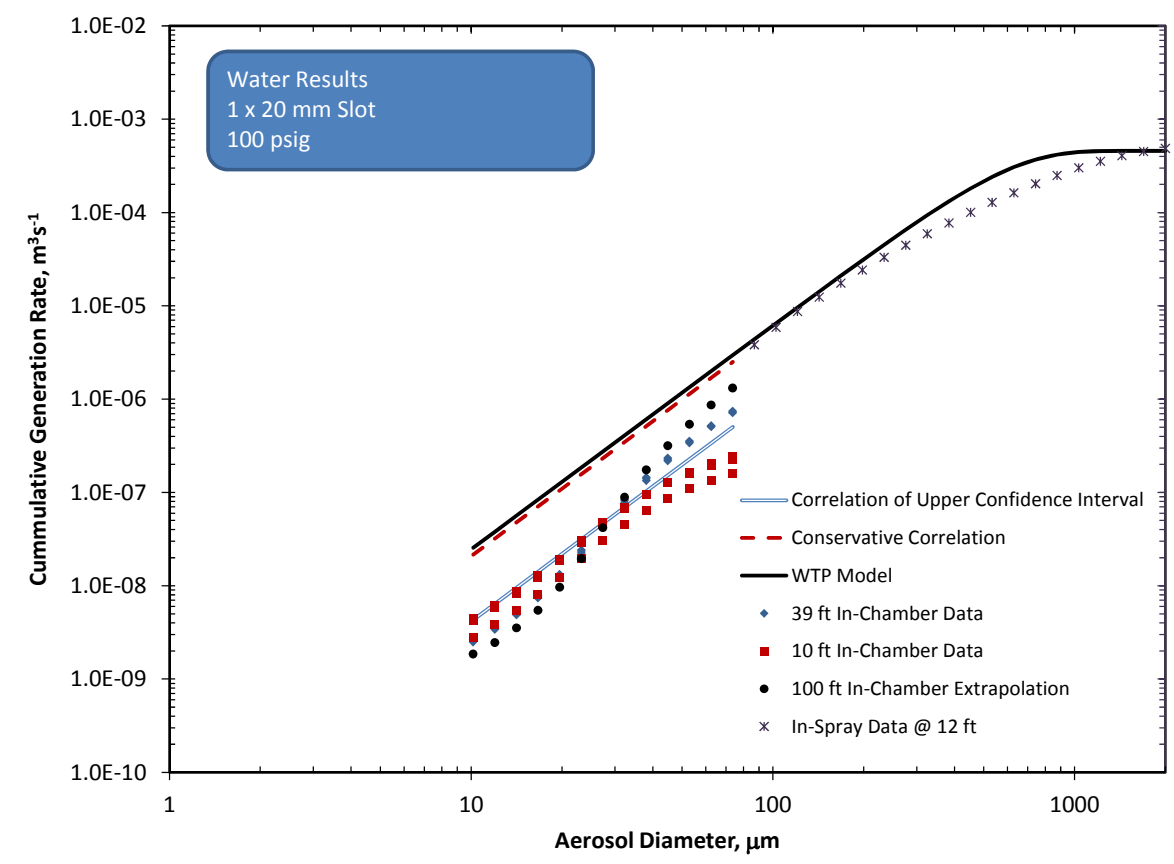

Figure 10.36. Generation Rate Results for a Water Spray from a $1 \times 20 \mathrm{~mm}$ Slot at 100 psig Showing a Comparison of the Conservative Correlation (hidden by WTP model line), In-Spray Data, and WTP Model. Selected generation rate data (that are biased by humidity and method uncertainties) are shown. 


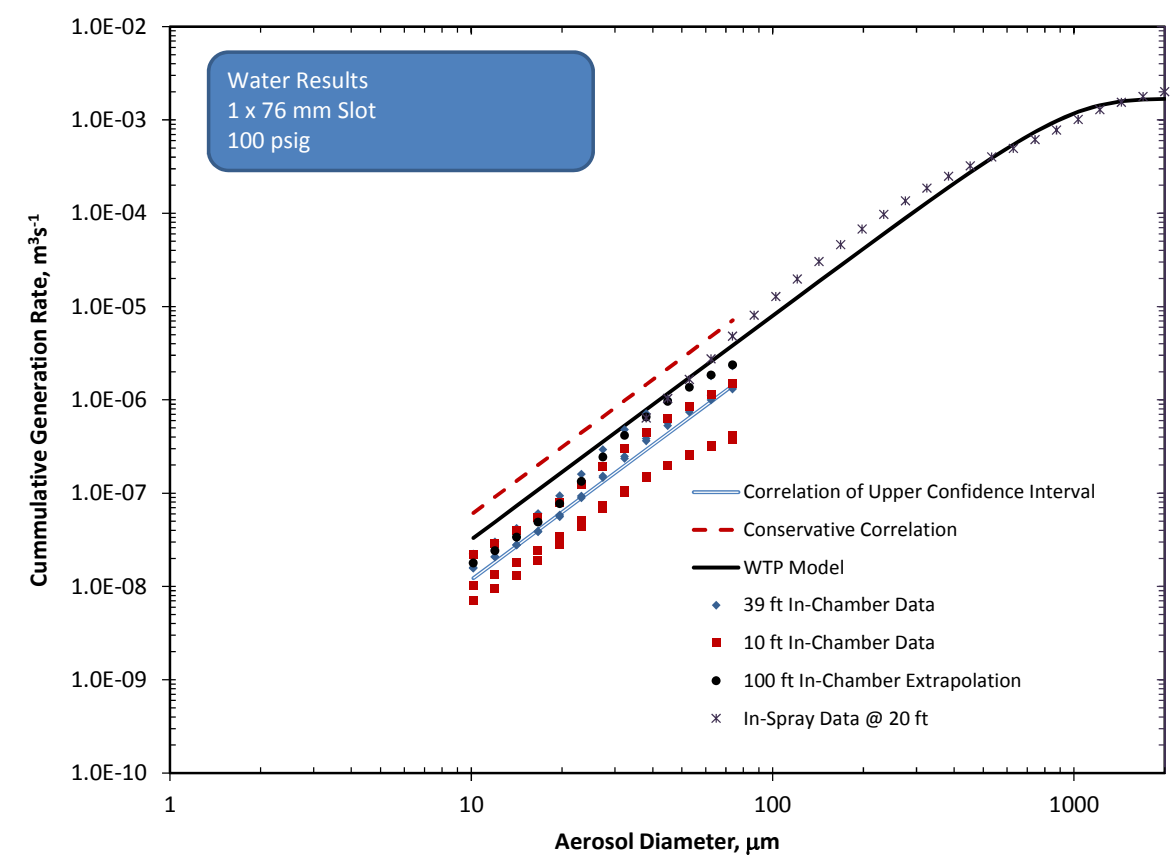

Figure 10.37. Generation Rate Results for a Water Spray from a $1 \times 76 \mathrm{~mm}$ Slot at 100 psig Showing a Comparison of the Conservative Correlation, In-Spray Data, and WTP Model. Selected generation rate data (that are biased by humidity and method uncertainties) are shown.

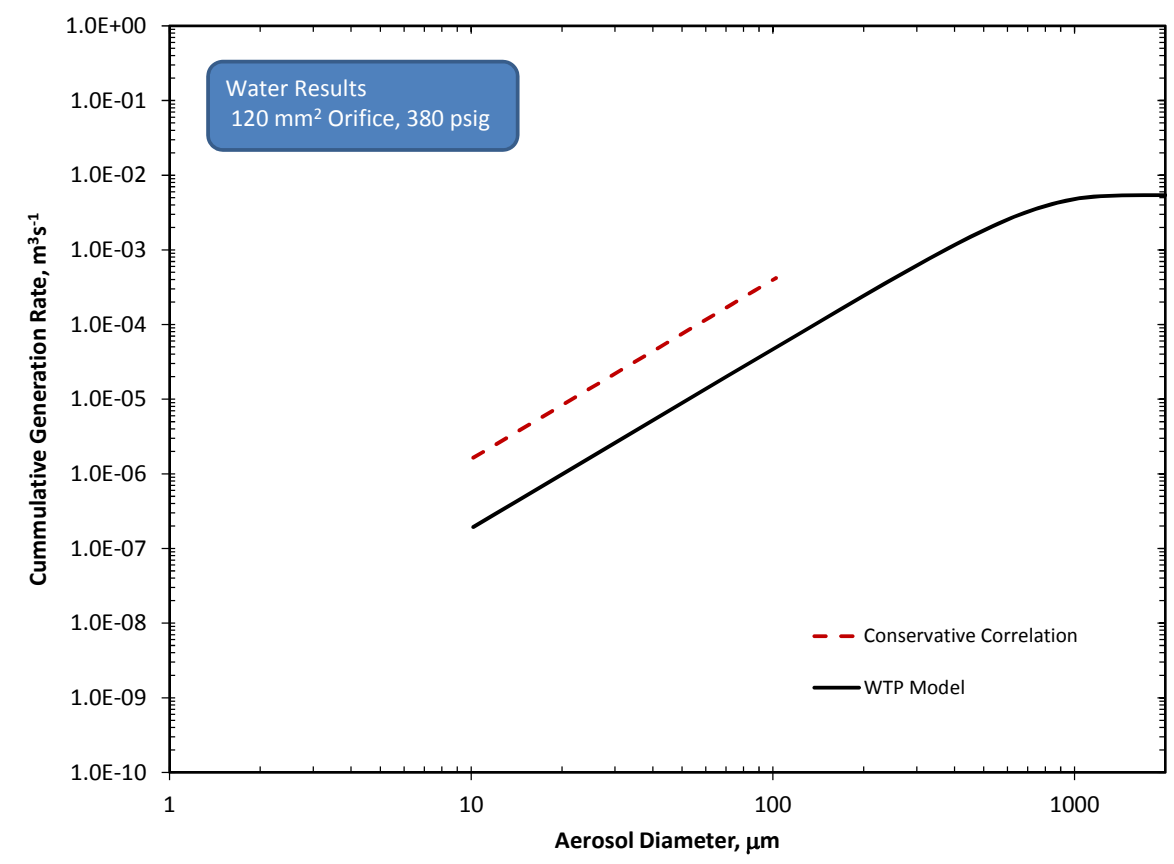

Figure 10.38. Generation Rate Results for a Water Spray from a $120 \mathrm{~mm}^{2}$ Orifice at 380 psig Showing a Comparison of the Conservative Correlation and WTP Model 


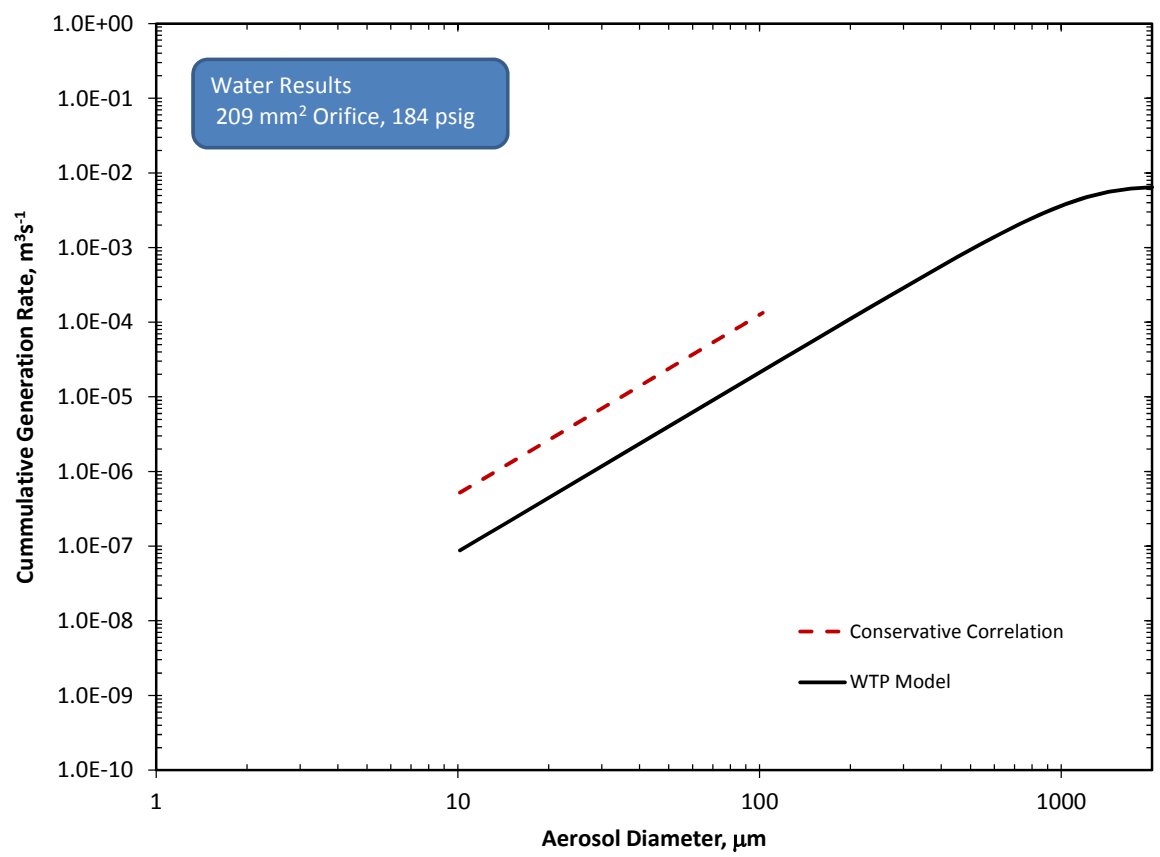

Figure 10.39. Generation Rate Results for a Water Spray from a $209 \mathrm{~mm}^{2}$ Orifice at 184 psig Showing a Comparison of the Conservative Correlation and WTP Model

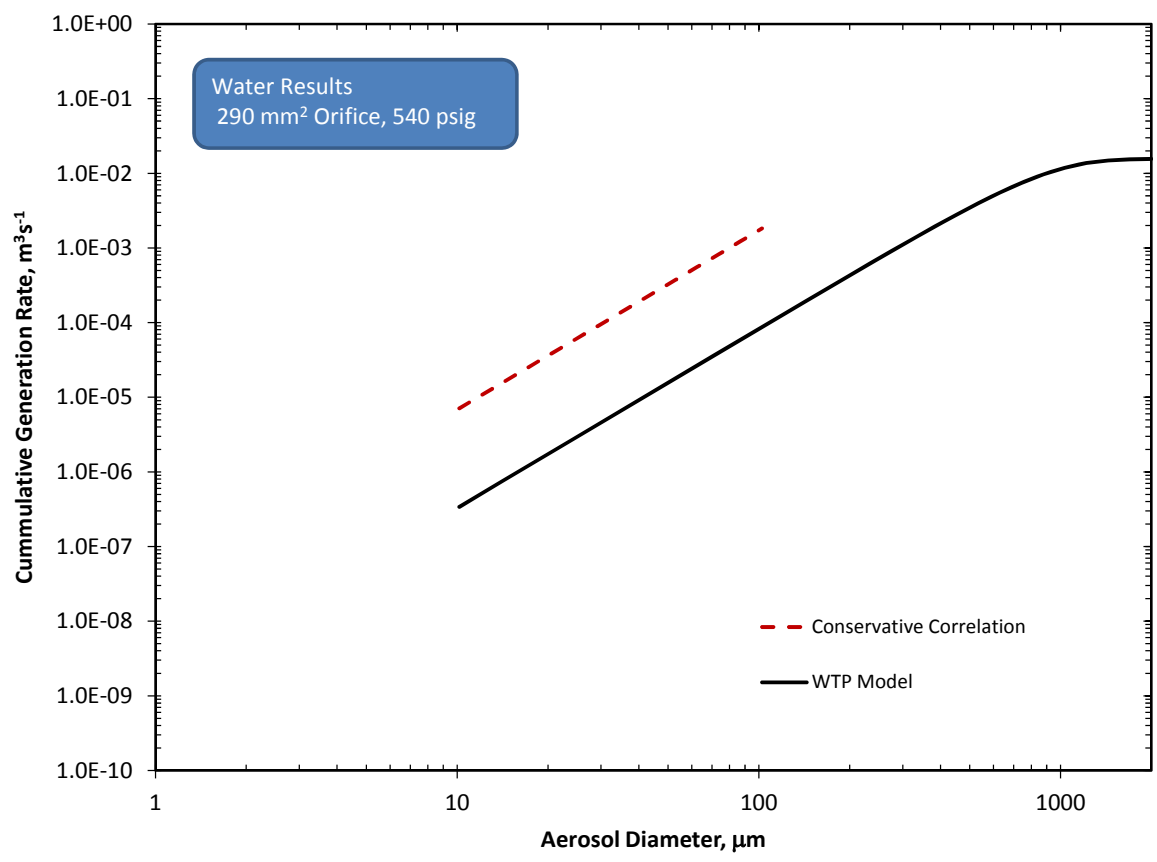

Figure 10.40. Generation Rate Results for a Water Spray from a $290 \mathrm{~mm}^{2}$ Orifice at $540 \mathrm{psig}$ Showing a Comparison of the Conservative Correlation and WTP Model 


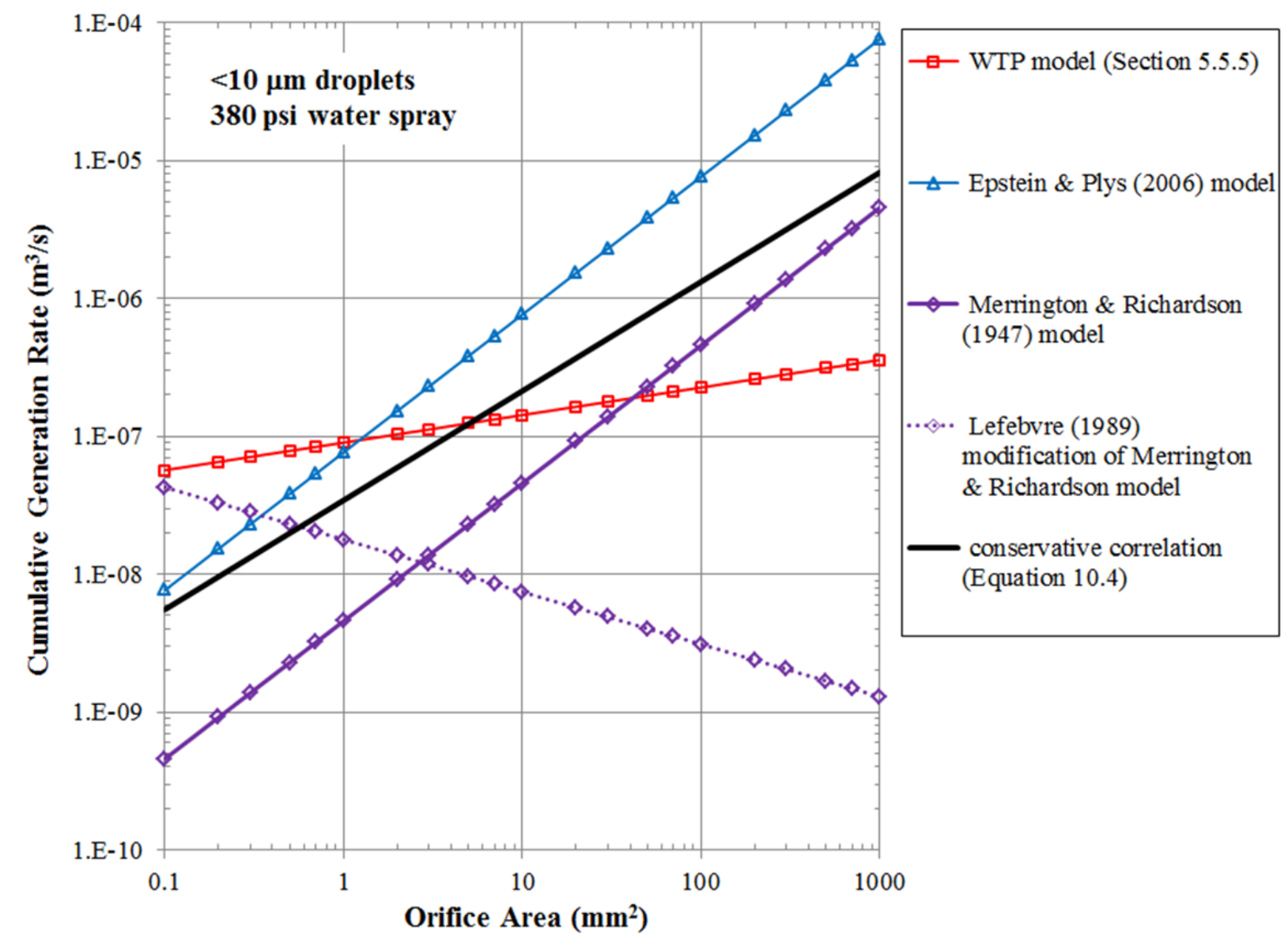

Figure 10.41. Example of the Aerosol Generation Behavior Predicted by the Conservative Correlation and Four Other Models/Correlations

Evaluation of aerosol data in previous sections found that, except for small aerosols $(<20 \mu \mathrm{m})$, release fractions for water were generally larger than those observed for non-Newtonian simulants (6 Pa and $30 \mathrm{~Pa}$ Clay). Furthermore, correction for droplet evaporation caused by testing below 100 percent relative humidity (see Appendix A) will further increase water release fractions relative to clay simulants and may also explain clay having larger-than-water release fractions at small aerosol diameters. These two observations form the basis for selection of water data when developing the conservative generation rate correlation. This chapter deals exclusively with aerosol generation rates rather than release fractions. Because generation rate also depends on the volumetric flow rate of material sprayed from the orifice, it does not immediately follow that, if water release fractions are conservative, water generation rates will also be conservative. Instead, the conservatism of water generation rates relative to that for simulants must also be confirmed by comparing generation rate data for the various simulants test in Phase II.

To accomplish this, the in-chamber generation rate results for the $6 \mathrm{~Pa}$ Clay and $30 \mathrm{~Pa}$ Clay from the $20 \mathrm{ft}$ chamber were compared to $20 \mathrm{ft}$ generation rates for water in Figure 10.42. This figure also includes the conservative correlation developed in this chapter for reference. In all test cases, the measured generation rates from both clay simulants tested are bounded by the conservative correlation and the generation rate data for the $30 \mathrm{~Pa}$ Clay are always less than, and at most equal to, the water, generation rates. However, comparison of water and $6 \mathrm{~Pa}$ Clay generation rates measured in the $20 \mathrm{ft}$ chamber show that the $6 \mathrm{~Pa}$ Clay simulant generation rates are higher than those for water in specific test 
configurations and aerosol diameters that produce generation rates in the lower range of the measured values (on the order of $10^{-9}$ to $10^{-8} \mathrm{~m}^{3} \mathrm{~s}^{-1}$ ). Most of the test configurations where the clay generation rate exceeds the water generation rate are the $2 \mathrm{~mm}$ hole at $100 \mathrm{psig}$ with aerosol diameters $<20 \mu \mathrm{m}$. As discussed in Appendix A, water and clay generation results are susceptible to humidity impacts. For small aerosols $(\sim 10 \mu \mathrm{m})$, low initial test humidity yields a reduction in water generation rates and an increase in clay generation rates. The data shown in Figure 10.42 have not been corrected for humidity effects, and as such, it is likely that the generation rate results at the lower values where the $6 \mathrm{~Pa}$ Clay exceeds water are impacted by low initial test humidity to some extent. Based on the results provided in Appendix A, it is expected that corrections for low test humidity would lessen, but not completely, reverse the difference in water and clay generation rates between $10^{-9}$ and $10^{-8} \mathrm{~m}^{3} \mathrm{~s}^{-1}$.

Another consideration is the impact of chamber size on the conservatism of water generation rates (and release fractions). Currently, chamber size impacts have only been directly evaluated in the large-scale test chamber for water. All non-Newtonian simulant testing employed the $20 \mathrm{ft}$ chamber configuration. As such, the variation of clay release fraction and generation rate with chamber size has not been evaluated, which in turn, introduces uncertainty with respect to the actual conservatism of the generation rate correlation developed herein. If there is little to no variation in the relative difference between water and clay (non-Newtonian simulant) release fractions with chamber length, the current set of water data collected for 10 to $39 \mathrm{ft}$ chambers (and extrapolation to $100 \mathrm{ft}$ chambers) may adequately encompass the range of clay generation rate behavior. Additional testing would be required to address this uncertainty.

While these observations provide motivation for additional study and or evaluation of non-Newtonian simulants, it is important to recognize that the conservative generation rate correlation (shown in Figure 10.42) does adequately bound the non-Newtonian simulant generation rates measured during Phase II testing. 


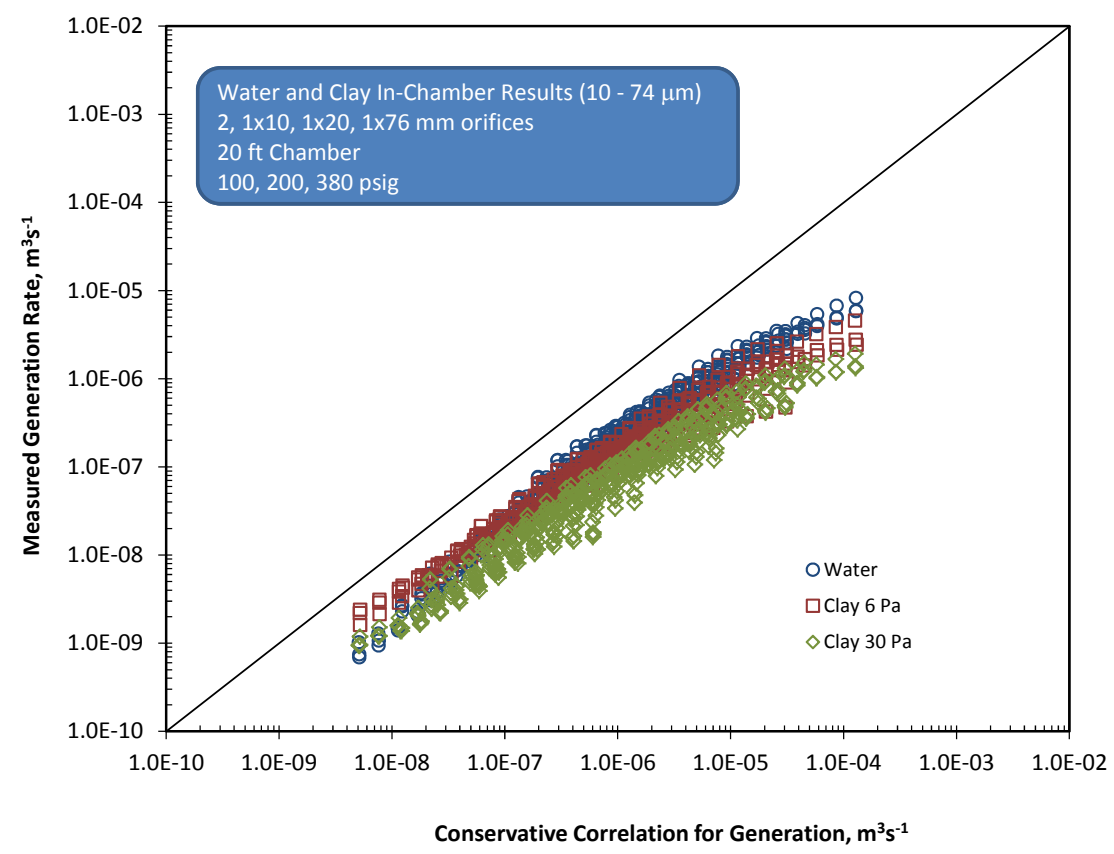

Figure 10.42. Comparison of Measured Generation Rate of Individual Tests Performed in the $20 \mathrm{ft}$ Chamber with Water and Clay Simulants (6 Pa and $30 \mathrm{~Pa}$ Bingham Yield Stress) with the Conservative Correlation Derived from In-Chamber Water Testing

\subsection{Conclusions and Recommendations}

A reasonably conservative correlation was developed based on the in-chamber test data. The correlation was developed for water, but it is appropriate for all the liquids and slurries tested because aerosol generation from the other fluids is overwhelmingly the same or less than water sprays. The primary exception is the results for the non-Newtonian chemical slurry simulants, but these slurries had unusual rheology in comparison to actual waste and the clay simulants (Schonewill et al. 2013), making the applicability of these results questionable. The correlation was compared to in-spray data and was found to match the in-spray data for the range of orifices and spray pressures tested. The good comparisons in the regions of overlap for different size orifices and different spray pressures confirm that the conservative correlation has orifice area, spray pressure, and droplet size dependences that agree with in-spray data.

The in-spray data are thought to be conservative, with a high degree of technical defensibility, so the conservative correlation, to be reasonably but not overly conservative, should never be greater than the in-spray results. Because the conservative correlation matches the in-spray results, this demonstrates that the conservative correlation has accounted for the potential biases (humidity and method bias) with the in-chamber method without actually quantifying them.

A comparison of the WTP model for generation rate to the test results, as represented by the conservative correlation, shows that the WTP model has the correct dependence for droplet size, under-represents the effect of increasing generation rate with orifice area, and under-represents the effect of increasing generation rate with spray pressure. Accordingly, the greatest under-estimation in generation rate from the WTP model occurs for the highest postulated pressure and the largest postulated 
breach, which is consistent with the conclusions from previous testing (Schonewill et al. 2012). For a 540 psig spray from a $290 \mathrm{~mm}^{2}$ breach, the WTP model under-predicts the generation rate compared to the conservative correlation by about a factor of 20 .

\subsubsection{Recommendations for Future Work}

The conservative correlation was developed to represent the in-chamber test results for all chamber sizes, including the extrapolation to $100 \mathrm{ft}$ chambers. A useful result would be an equivalent evaluation of just the $20 \mathrm{ft}$ chamber data to determine how to quantitatively adjust a correlation based on $20 \mathrm{ft}$ chamber data to match the conservative correlation that represented all of the different chamber sizes. With this information, testing results collected in only the $20 \mathrm{ft}$ chamber could be used to quantitatively modify the current conservative correlation. One specific application of this approach would be to determine how the correlation could be adjusted to account for the general decrease in aerosol generation from non-Newtonian slurries with progressively higher Bingham yield stress and consistency. A second application would be to incorporate the Phase I data (Schonewill et al. 2012), which were collected in a $20 \mathrm{ft}$ chamber. This data includes results for a broader range of orifice areas and slot shapes. 


\subsection{Conclusions and Recommendations}

Phase II aerosol generation tests were performed to quantify the release fraction and generation rate for water and a range of non-Newtonian clay slurries that represent expected Waste Treatment Plant (WTP) process stream properties, chamber lengths, spray lengths, and initial chamber humidity. Results were collected from transient concentration measurements in the chambers and from in-spray measurements. We also extrapolated orifice data to larger postulated breaches in 3 and 10 in. process piping and to longer distance sprays. Both the test results and extrapolations were compared with the WTP model predictions. In addition, testing was conducted to evaluate instrument performance, the role of initial chamber humidity, and experimental variation in repeat tests. These results were used to estimate the bias in the in-chamber release fraction measurements and to estimate reasonably conservative aerosol generation rate predictions as a function of orifice area, test pressure, and aerosol diameter. Below is a summary of the key findings.

- Correlation Development for Comparing Test Results to the WTP Model. A reasonably conservative correlation for aerosol generation rate was developed based on the in-chamber test data and extrapolations of the in-chamber data to $100 \mathrm{ft}$ chambers. The correlation was developed for water, but it is appropriate for all the liquids and slurries tested because the aerosol generation from the other fluids is overwhelmingly always the same or less than water sprays. The primary exception is the results for the non-Newtonian chemical slurry simulants, but these slurries had unusual rheology in comparison to actual waste and the clay simulants, making the applicability of these results questionable. The correlation was compared to in-spray data and was found to match the in-spray data for the range of orifices and spray pressures tested. The good comparisons in the regions of overlap for different size orifices and different spray pressures confirm that the conservative correlation has orifice area, spray pressure, and droplet size dependences that agree with in-spray data. Because the conservative correlation matches the in-spray results, this demonstrates that the conservative correlation has accounted for the potential biases (humidity and method bias) with the in-chamber method without actually quantifying them. A comparison of the WTP model for generation rate to the test results, as represented by the conservative correlation, shows that the WTP model has the correct dependence for droplet size, under-represents the effect of increasing generation rate with orifice area, and under-represents the effect of increasing generation rate with spray pressure. Accordingly, the greatest under-estimation in generation rate from the WTP model occurs for the highest postulated pressure and the largest postulated breach, which is consistent with the conclusions from previous testing (Schonewill et al. 2012). One specific process stream of interest for the WTP is a 540-psig spray from a $290 \mathrm{~mm}^{2}$ orifice. For this example, the WTP model under-predicts the generation rate compared to the conservative correlation by about a factor of 20 .

- Performance of Malvern Insitec-S Aerosol Analyzers Using Phase II Chamber and Instrument Measurement Configurations. Functional tests were performed to verify that changes made to both large-scale test chamber and aerosol instruments for Phase II testing did not impact release fraction measurements and prevent direct comparison with Phase I data. The most significant change made to aerosol instruments was installation of the new focusing lens to change the working range of the Malvern Insitec-S from 0.5 to $200 \mu \mathrm{m}$ (100 mm lens, Phase I) to 2.5 to $2500 \mu \mathrm{m}$ (500 mm lens, Phase II). Side-by-side comparison of 100 and $500 \mathrm{~mm}$ lens release fractions measured for water sprayed through a $1 \times 76 \mathrm{~mm}$ orifice at 380 psig indicated that both lenses produced equivalent results at aerosol diameters between 23 and $100 \mu \mathrm{m}$. Instrument sensitivity prevented comparison of 
lens performance at release fractions corresponding to lower aerosol sizes. Additional functional tests were conducted to determine optimal mixing fan settings and instrument purge air rate settings for Phase II analysis and their influence on release fraction measurements. These tests affirmed the adequacy of Phase I mixing fan selections and helped define the range of purge air settings for Phase II analyses.

- Validation of Malvern Insitec-S Aerosol Analyzer Performance. The performance of the Malvern Insitec-S analyzer has been evaluated extensively. Tests examined the accuracy of Malvern Insitec-S concentration and size distribution measurements by testing carefully controlled dilute aqueous slurries of known concentration and PSD and comparing the Malvern Insitec-S result to the known values. Testing included mono- and poly-disperse suspensions and evaluated six Malvern Insitec-S configurations relevant to Phase I and Phase II testing:

- Phase I small-scale configuration: lens - $100 \mathrm{~mm}$; spacer bar length - $150 \mathrm{~mm}$

- Phase II small-scale configuration: lens - $500 \mathrm{~mm}$; spacer bar length -150 mm

- Phase I large-scale configuration: lens - $100 \mathrm{~mm}$; spacer bar length - $500 \mathrm{~mm}$

- Phase II large-scale configuration: lens - $500 \mathrm{~mm}$; spacer bar length - $1000 \mathrm{~mm}$

- Dispersions uniformly distributed across $\sim 400 \mathrm{~mm}$ column analyzed by the $100 \mathrm{~mm}$ lens (Phase I in-chamber configurations)

- Dispersions uniformly distributed across $\sim 900 \mathrm{~mm}$ column analyzed by the $500 \mathrm{~mm}$ lens (Phase II in-chamber configurations).

Testing indicates that under the best measuring circumstances, the Malvern Insitec-S can measure concentration and median dispersion size to approximately 2 percent and 3 percent of list values, respectively. Particle shape was observed to increase the apparent concentration measured by the Malvern Insitec-S (relative to the actual value); however, at the sizes of interest for spray leak analysis (10 to $100 \mu \mathrm{m})$, aerosol droplets are expected to be spherical such that concentration readings will not be impacted. Evaluation of the $500 \mathrm{~mm}$ lens indicates that it is subject to loss of accuracy when used to analyze dispersions that fall between primarily 2.5 and $20 \mu \mathrm{m}$. Aerosols observed in Phase I and Phase II spray leak testing, which always contain significant aerosol fractions greater than $20 \mu \mathrm{m}$ in size, are not expected to be significantly impacted. Spray geometry corrections used to interpret concentration and size measurements for distributed aerosols in Phase I and Phase II have been evaluated and found to provide reasonable and appropriate corrections for laser attenuation. Overall evaluation of the validation results suggests that no bias correction in either aerosol concentration or size distribution measurements is needed for Malvern Insitec-S configurations relevant to Phase I and Phase II testing.

- Assessment of Chamber Aerosol Uniformity. Testing was performed to evaluate chamber uniformity (with respect to aerosol concentration and size distribution) and its impact on accuracy of release fraction measurements and their associated uncertainty. Tests employed the smallest Phase II test orifice (i.e., the $2 \mathrm{~mm}$ hole) to evaluate aerosol uniformity in the $39 \mathrm{ft}$ test chamber under conditions approaching the least turbulent, and therefore least uniform, spray conditions evaluated in Phase II large-scale testing. These measurements indicate that standard in-chamber test locations (which employ aerosol sampling locations $21 \mathrm{in}$. above the orifice) will be subject to loss of large droplets as a result of settling, leading to inaccurate assessment of release fraction and generation rate for droplets $\sim 87 \mu \mathrm{m}$ and greater. Droplet loss is worst above the spray header and becomes less 
severe at downstream positions and closer to the spray. Release fraction estimates for smaller droplets (i.e., 10 to $\sim 80 \mu \mathrm{m}$ ) do not appear to be impacted and are relatively insensitive to measurement location as long as the direct path of the spray is avoided.

- Evaluation of In-Spray Release Fractions as a Function of Measurement Position. In-spray measurements evaluate the release fraction in the direct path (which is defined as the most optically dense region) of the spray. These measurements provide a direct means of assessing the full size distribution of aerosol droplets produced by spray without incurring significant loss of large aerosols from droplet settling or inertial wall capture. Phase II testing evaluated in-spray release fractions for water and two non-Newtonian clay slurries as a function of downstream distance from the orifice. For many test pressure, orifice, and simulant combinations, in-spray release fraction did not change substantially with increasing distance from the orifice. For test cases where significant change did occur, the release fraction contributions were typically shifted to larger aerosol diameters. This increase in overall aerosol size has been attributed to droplet coalescence or to preferential sampling of large aerosols that settle to the bottom of the chamber. Relative to in-spray behavior for the majority of spray configurations and simulants tested, the in-spray trends for water sprays generated by the $1 \times 76 \mathrm{~mm}$ slot were anomalous and indicated that aerosol size decreased with increasing distance from the orifice.

- Assessment of In-Chamber Measurement Basis. In-chamber release fractions are expected to under-estimate true fractional aerosol release because of bias introduced by evaporation (caused by low relative humidity at the start of testing) and aerosol sampling bias introduced by settling of large droplets and mixing limitations. In-spray measurements are not subject to these limitations and are expected to over-estimate (and thus be conservative) actual release fractions in the size range of interest $(10$ to100 $\mu \mathrm{m})$ because of spatial measurement bias. However, measurement of in-spray release fractions in the size range of interest is frequently impossible because of loss of size distribution measurement accuracy at cumulative fractional volumes below $0.5 \times 10^{-2}$. In practical terms, this prevents measurement of size distribution information below $50 \mu \mathrm{m}$. Because in-spray measurements are expected to be conservative (i.e., larger than) the actual release, they can be used as a guide for correcting in-chamber release fractions such that they are "as-conservative" as in-spray measurements. Comparison of in-spray and in-chamber release fraction measurements indicates a multiplicative correction factor of three to in-chamber data will typically render them as conservative as in-spray measurements (see Section 7.4). This "method bias" correction contains size distribution and concentration measurements that impacts both in-spray and in-chamber measurements and also contains, in part, impacts to release fraction for humidity. However, the multiplicative factor of three does not fully account for all humidity effects, and additional correction is necessary.

- Determination of Fractional Aerosol Release from Non-Newtonian Slurries. The effect of non-Newtonian rheology for clay slurry sprays was evaluated within the WTP rheological limits for the Bingham yield stress and consistency of $6 \mathrm{~Pa} / 6 \mathrm{cP}$ and $30 \mathrm{~Pa} / 30 \mathrm{cP}$. Spray tests were conducted for measuring the generation rate and release fraction from aerosol concentration increase in the chamber and directly in-spray. For the in-chamber measurements, the release fraction is the same as water or decreases with increasing Bingham parameters. For in-spray measurements, the release fraction for the clay slurries at $380 \mathrm{psig}$ is the same as water. At 100 and $200 \mathrm{psig}$, the release fraction for the clay slurries decreases with increasing solids content (and increasing rheology as characterized by the Bingham yield stress and consistency). These results show that water results are typically the same or larger than (i.e., conservative relative to) release fraction measured for non-Newtonian clay slurries. 
- Evaluation of the Effects of Spray Length and Chamber Size on Fractional Aerosol Release. The effect of spray length within a chamber was investigated for a range of sprays within a $39 \mathrm{ft}$ chamber. Similar to the Phase I results in a $20 \mathrm{ft}$ chamber, the release fraction always increased with spray length in the $39 \mathrm{ft}$ chamber. Accordingly, for a given size orifice, fluid, spray pressure, and chamber size, the highest release fraction and aerosol generation rate are given by the test results when the spray travels the full length of the chamber. The role of chamber length, when sprays travel the full length of the chamber, was investigated for chambers ranging from 10 to $39 \mathrm{ft}$. For the largest slot tested $(1 \times 76 \mathrm{~mm})$, the release fraction increased with chamber length for all droplet sizes at 380 psig. The $1 \times 76 \mathrm{~mm}$ slot also gave higher generation rates than the smaller orifices, and as a result, it is significant in terms of the worst-case spray for accident analyses. For other orifices and pressures, increasing chamber length typically caused the cumulative release fraction to increase for larger droplets (above roughly $30 \mu \mathrm{m}$ ) and decrease for smaller droplets (below roughly $30 \mu \mathrm{m}$ ). 


\subsection{References}

Bamberger JA, PA Meyer, JR Bontha, CW Enderlin, DA Wilson, AP Poloski, JA Fort, ST Yokuda, HD Smith, F Nigl, MA Friedrich, DE Kurath, GL Smith, JM Bates, and MA Gerber. 2005. Technical Basis for Testing Scaled Pulse Jet Mixing Systems for Non-Newtonian Slurries. PNWD-3551, WTP-RPT-113 Rev 0, Battelle—Pacific Northwest Division, Richland, Washington.

Bartell J, G Ferrazza, and R Schick. 1991. Effects of Spray Nozzle Design and Measurement Techniques on Reported Drop Size Data. Spraying Systems Company, Wheaton, Illinois.

Bohren CF and DR Huffman. 1983. Absorption and Scattering of Light by Small Particles. Wiley, New York, p. 149.

Bruggeman DAG. 1935. "Berechnung verschiedener physikalischer Konstanten von heterogenen Substanzen." Annals of Physics (Leipzig) 24:636-679.

Campbell T, M Parker, A Moon, B Fant, K Clossey, and J Cook. 2010. EFRT Issue M3 PJM Vessel Mixing Assessment, Volume 8-HLP-22. 24590-WTP-RPT-ENG-08-021-08, Rev 1, River Protection Project, Waste Treatment Plant, Richland, Washington.

Deen WM. 1998. Analysis of Transport Phenomena. ISBN 0-19-508494-1. Oxford University Press, New York.

Denn MM. 1980. Process Fluid Mechanics. Prentice-Hall, Inc., Englewood Cliffs, New Jersey.

DOE-3009-94. 2006. "DOE Standard Preparation Guide for U.S. Department of Energy Non-Reactor Nuclear Facility Documented Safety Analysis, DOE-3009-94.” Change Notice No. 3, U.S. Department of Energy, Washington, D.C.

Dombrowski N and WR Johns. 1963. "The Aerodynamic Instability and Disintegration of Viscous Liquid Sheets.” Chemical Engineering Science 18:203-414.

Garnett JCM. 1904. "Colours in Metal Glasses and in Metallic Films." Philosophical Transactions of the Royal Society of London 203:385-420.

Jillavenkatesa A, SJ Dapkunas, and LSH Lum. 2001. NIST Recommended Practice Guide: Particle Size Characterization. Special Publication 960-1, National Institute of Standard and Technology, U.S. Government Printing Office, Washington, D.C.

Larson AR and BT Allen. 2010. WTP Methodology for Spray Leak Scenarios. 24590-WTP-RPT-ENS 10-001, Rev 1, River Protection Project, Waste Treatment Plant, Richland, Washington.

Looyenga H. 1965. "Dielectric Constants of Heterogeneous Mixtures.” Physica 31:401-406.

Mahoney LA, PA Gauglitz, J Blanchard, ML Kimura, and DE Kurath. 2012. Small-Scale Spray Releases: Orifice Plugging Test Results. PNNL-21361, WTP-RPT-219 Rev 0, Pacific Northwest National Laboratory, Richland, Washington. 
Mahoney LA, PA Gauglitz, ML Kimura, GN Brown, DE Kurath, J Blanchard, C Song, RC Daniel, BE Wells, D Tran, WC Buchmiller, CA Burns, and DM Smith. 2013. Small-Scale Spray Releases: Initial Aerosol Test Results. PNNL-21367, WTP-RPT-216 Rev1, Pacific Northwest National Laboratory, Richland, Washington.

Malvern Instruments, Ltd. 2010. RTSizer and Insitec Analyser User Manual. MAN0467 Issue 1.0, Malvern Instruments Ltd., Malvern, Worcestershire, United Kingdom.

McAllister J. 2010. Severity Level Calculations for the Pretreatment Facility Based on Updated MAR. Calculation No. 24590-PTF-Z0C-W14T-00036, Rev B, River Protection Project, Waste Treatment Plant, Bechtel National, Inc., Richland, Washington.

Monecke J. 1994. "Bergman Spectral Representation of a Simple Expression for the Dielectric Response of a Symmetric Two-Component Composite." Journal of Physics: Condensed Matter 6:907-912.

Poloski AP, PA Meyer, LK Jagoda, and PR Hrma. 2004. Non-Newtonian Slurry Simulant Development and Selection for Pulse Jet Mixer Testing. PNWD-3495, WTP-RPT-111 Rev 0, Battelle-Pacific Northwest Division, Richland, Washington.

Rosin P and E Rammler. 1933. "The Laws Governing the Fineness of Powdered Coal." Journal of the Institute of Fuel 7:29-36.

Russell RL, CW Stewart, SD Rassat, CD Johnson, ST Arm, PA Meyer, MS Fountain, CE Guzman-Leong, and BK Hatchell. 2005. Final Report: Gas Retention and Release in Hybrid Pulse Jet Mixed Tanks Containing Non-Newtonian Waste Simulants. PNWD-3552, WTP-RPT-114 Rev 1, Battelle-Pacific Northwest Division, Richland, Washington.

Schonewill PP, LA Mahoney, PA Gauglitz, DN Tran, ML Kimura, CA Burns, GN Brown, and DE Kurath. 2013. Small-Scale Spray Releases: Additional Aerosol Test Results. PNNL-22402, WTP-RPT-222 Rev 0, Pacific Northwest National Laboratory, Richland, Washington.

Schonewill PP, PA Gauglitz, JR Bontha, RC Daniel, DE Kurath, HE Adkins, Jr., JM Billing, CA Burns, JM Davis, CW Enderlin, CM Fischer, JJ Jenks, CD Lukins, PJ MacFarlan, JI Shutthanandam, and DM Smith. 2012. Large-Scale Spray Releases: Initial Aerosol Test Results. PNNL-21333, WTP-RPT-217 Rev 0, Pacific Northwest National Laboratory, Richland, Washington.

van de Hulst HC. 1981. Light Scattering by Small Particles. Dover Publications, New York.

Wells BE, DE Kurath, LA Mahoney, Y Onishi, JL Huckaby, SK Cooley, CA Burns, EC Buck, JM Tingey, RC Daniel, and KK Anderson. 2011. Hanford Waste Physical and Rheological Properties: Data and Gaps. PNNL-20646, Pacific Northwest National Laboratory, Richland, Washington. 
Appendix A

Functional Tests 



\section{Appendix A}

\section{Functional Tests}

Generation of aerosol in a fixed volume chamber is governed by complex physical phenomena occurring at the free jet surface/air interface in a turbulent flow regime. To establish that test data were collected at conditions permitting meaningful analysis of aerosol behavior in spite of the complexity, functional testing was conducted to assess the quality and reproducibility of the aerosol data as a function of chamber/test configuration.

In Phase I of the project, a series of tests were conducted to identify the best equipment configuration and operating test conditions. For more details, Chapter 8 of the Phase I large-scale spray release testing report (Schonewill et al. 2012), presents a thorough discussion of Phase I functional testing.

In Phase II, functional testing with water also was conducted prior to aerosol release fraction testing (i.e., matrix tests, see Section 5.4) to verify and/or establish several system parameters such as chamber mixing fan configuration, Malvern Insitec-S vertical and horizontal positioning, Malvern Insitec-S detector lens purge rate, Malvern Insitec-S lens sizes, and chamber relative humidity (RH). Although several of these parameters were established during the Phase I testing, they were confirmed and/or modified for the expanded $39 \mathrm{ft}$ chamber and new Malvern Insitec-S instrument configurations (e.g., $500 \mathrm{~mm}$ lens and $1000 \mathrm{~mm}$ spacer bars). In the sections that follow, Phase II functional testing is discussed. As part of the discussion of $\mathrm{RH}$, release fraction bias correction factors for low starting $\mathrm{RH}$ are proposed. In the final section, an overall bias correction for in-chamber measurements is presented that includes the in-spray to in-chamber release fraction bias correction factor and the low starting RH bias correction factor.

\section{A.1 Objectives}

The general objective of the Phase II functional tests was to improve reliability and repeatability of the data collected. Specifically, the Phase II functional test objectives were to:

- Assess the influence of enhanced chamber mixing with bilge fans at various fan speeds and fan configurations

- Assess chamber aerosol homogeneity with Malvern Insitec-S instruments at varying vertical and horizontal positions within the chamber

- Assess the impact of Malvern Insitec-S detector lenses on measured release fractions when using a $500 \mathrm{~mm}$ lens during Phase II and a $100 \mathrm{~mm}$ lens during Phase I

- Determine the influence of Malvern Insitec-S lens purge rates for a range of test conditions (orifice, pressure, Malvern Insitec-S position) that are sufficient to prevent aerosol contamination of the windows without impacting the aerosol concentration in the measurement volume

- Determine the influence of starting chamber RH on measured release fractions during spray release tests. 


\section{A.2 Fan Configuration and Speed}

Phase II mixing fan functional tests were performed to assess how different bilge fan ${ }^{1}$ configurations and speeds affected the aerosol concentration and, ultimately, the release fraction calculated from the concentration data. Figure A.1 illustrates the fan array and standard Malvern Insitec-S instrument positions in the $39 \mathrm{ft}$ chamber. MAL1852 (position \#3) is shown slightly offset from the actual standard position (10 ft) so the fan array position could be clearly depicted. The array of four "shrouded" bilge fans was installed $\sim 10 \mathrm{ft}$ downstream of the spray header and just above the collection tray divider. The bilge fans were installed with flexible ducting that moved the air intake closer to the bottom of the chamber and spray header to preclude any influence on the jet.

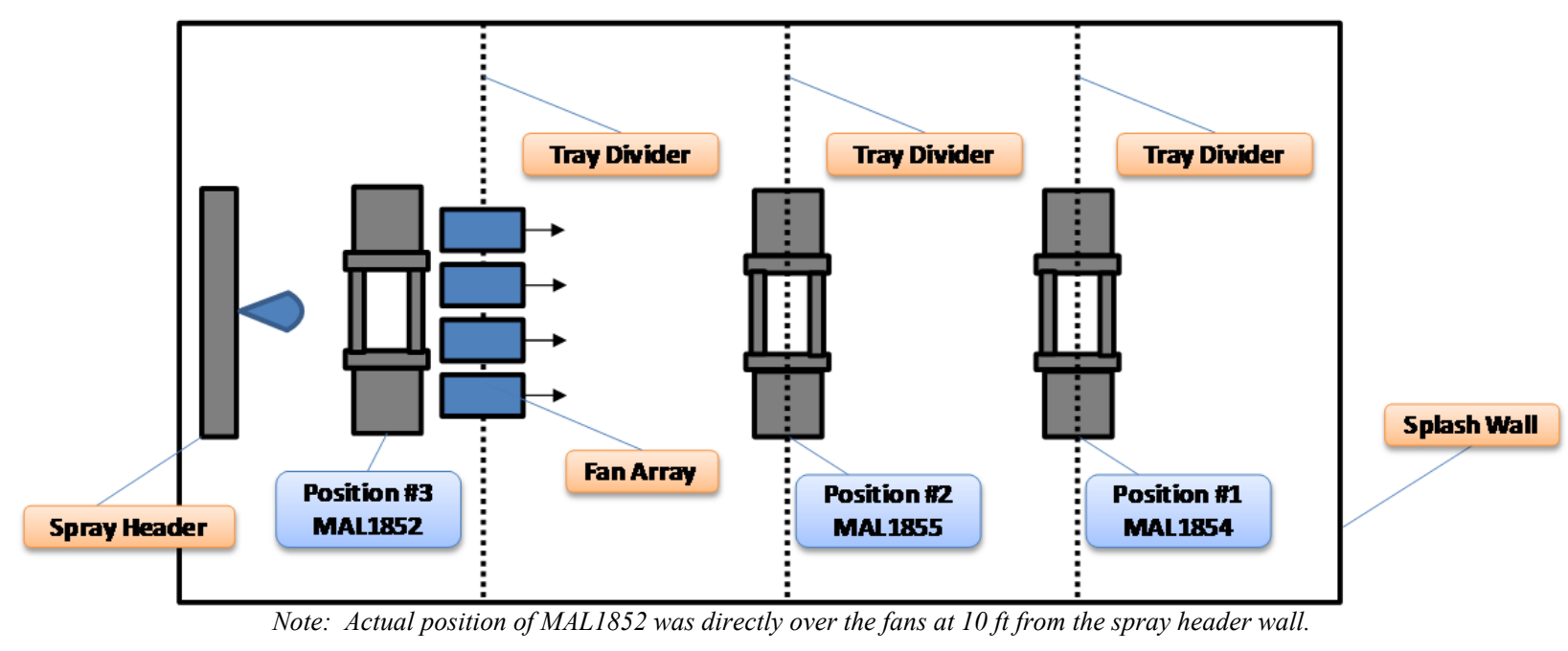

Figure A.1. Top View of Malvern Insitec-S Instruments and Mixing Fan Arrangement

Table A.1 summarizes the various mixing fan configurations and speeds used during Phase II functional tests. More extensive mixing fan testing was conducted during Phase I work (discussed in Section 8 of Schonewill et al. 2012) with the $20 \mathrm{ft}$ chamber using a nominal $2 \mathrm{~mm}$ hole. Phase I functional testing determined that the use of the two center bilge fans, operating at $12 \mathrm{~V}$, resulted in a higher measured release fraction and improved spatial distribution of aerosol in the chamber. During Phase II testing, these mixing fan tests were repeated with the $2 \mathrm{~mm}$ hole to verify that changes to the chamber configuration (namely the increase in chamber length from 20 to $39 \mathrm{ft}$ ) did not require increased mixing power or use of additional fans. The nominal $2 \mathrm{~mm}$ hole represents the smallest orifice tested in Phase II and the smallest spray volume. Because of this, the effect of mixing fan configuration on chamber homogeneity should be the most pronounced for the $2 \mathrm{~mm}$ hole. The smallest spray for the $2 \mathrm{~mm}$ hole would occur at $100 \mathrm{psig}$, but this pressure was not tested because of concerns related to sensitivity of measurement (i.e., release fraction resolution). Instead, the 380 psig spray was tested to provide increased confidence with respect to trends in the release fraction result.

\footnotetext{
${ }^{1}$ Model \#751RC, 4 in. diameter in-line blower, capable of $240 \mathrm{ft}^{3} / \mathrm{min}$ at 13.6V, DetMar Corporation, Detroit, Michigan.
} 
Table A.1. Actual Functional Tests to Establish Effect of Mixing Fans

\begin{tabular}{|c|c|c|c|c|c|c|c|}
\hline \multirow[b]{2}{*}{ Simulant } & \multirow[b]{2}{*}{$\begin{array}{c}\text { Chamber } \\
\text { Configuration }^{(\mathrm{a})}\end{array}$} & \multicolumn{2}{|c|}{$\begin{array}{l}\text { Malvern Insitec-S } \\
\text { Configuration }^{(\mathrm{b})}\end{array}$} & \multirow[b]{2}{*}{$\begin{array}{l}\text { Mixing Fan } \\
\text { Conditions. }\end{array}$} & \multirow{2}{*}{$\begin{array}{l}\text { Test Fluid } \\
\text { Pressure } \\
\text { (psig) }\end{array}$} & \multirow{2}{*}{$\begin{array}{l}\text { Nominal } \\
\text { Orifice } \\
(\mathrm{mm})\end{array}$} & \multirow[b]{2}{*}{$\begin{array}{c}\text { Accepted Functional } \\
\text { Tests }^{(\mathrm{d})}\end{array}$} \\
\hline & & $\begin{array}{l}\text { Purge Rate } \\
\text { (SCFH) }\end{array}$ & Locations $^{(\mathrm{c})}$ & & & & \\
\hline Water & $39 \mathrm{~S} 3 \mathrm{~A}$ & 25 & Standard & No Fans & 380 & Circular, 2.0 & $\begin{array}{l}\text { W273, W274, W277, } \\
\text { W282 }\end{array}$ \\
\hline Water & $39 \mathrm{~S} 3 \mathrm{~A}$ & 25 & Standard & $\begin{array}{l}12 \mathrm{~V}, \text { two } \\
\text { center fans }\end{array}$ & 380 & Circular, 2.0 & W283, W284, W285 \\
\hline Water & $39 \mathrm{~S} 3 \mathrm{~A}$ & 25 & Standard & $\begin{array}{l}6 \mathrm{~V}, \text { two } \\
\text { center fans }\end{array}$ & 380 & Circular, 2.0 & W286, W287, W288 \\
\hline Water & $39 \mathrm{~S} 3 \mathrm{~A}$ & 25 & Standard & $\begin{array}{l}12 \mathrm{~V} \text {, four } \\
\text { fans }\end{array}$ & 380 & Circular, 2.0 & W289, W290, W291 \\
\hline
\end{tabular}

(a) The five-character chamber configuration code (e.g., 39S3A) represents: $39=$ total chamber length in feet, $\mathrm{S}=$ spacer bars (in-chamber testing), 3 = Malvern Insitec-S analyzers used, and the last character represents lengths of spray header extensions where $\mathrm{A}=$ no spray header extension, $\mathrm{B}=10 \mathrm{ft}, \mathrm{C}=19 \mathrm{ft}$, and $\mathrm{D}=34.3 \mathrm{ft}$ spray header extension.

(b) The Malvern Insitec-S instruments were configured with $500 \mathrm{~mm}$ lens and $1000 \mathrm{~mm}$ spacer bars for all mixing fan functional tests.

(c) Standard Malvern Insitec-S Locations: (Position 1) A $=75 \%$ of chamber length (360 in.), $\mathrm{B} \& \mathrm{C}=28$ in., $\mathrm{D} \& \mathrm{E}=28$ in.; (Position 2) $\mathrm{A}=50 \%$ of chamber length (240 in.), $\mathrm{B} \& \mathrm{C}=28$ in., $\mathrm{D} \& \mathrm{E}=28$ in.; (Position 3 ) $\mathrm{A}=25 \%$ of chamber length (120 in.), $\mathrm{B} \& \mathrm{C}=28$ in., $\mathrm{D} \& \mathrm{E}=28$ in. Dimensions A, B, C, D, and $\mathrm{E}$ are defined and illustrated in Figure 4.9.

(d) Detector 31 of Malvern Insitec-S analyzer MAL1854 malfunctioned during the mixing fan functional tests. NCR OTS-1270 documented the discovery and the technical assessment concluded the relative effects of chamber configuration assessed during functional tests should not be impacted.

Figure A.2 illustrates the relative impact on the release fraction using the nominal $2 \mathrm{~mm}$ hole, 380 psig, and Malvern Insitec-S instruments in standard locations with no fans, two center fans at $6 \mathrm{~V}$, two center fans at $12 \mathrm{~V}$, and four fans at $12 \mathrm{~V}$. No significant influence of the number of fans and fan speeds on the release fraction was observed.

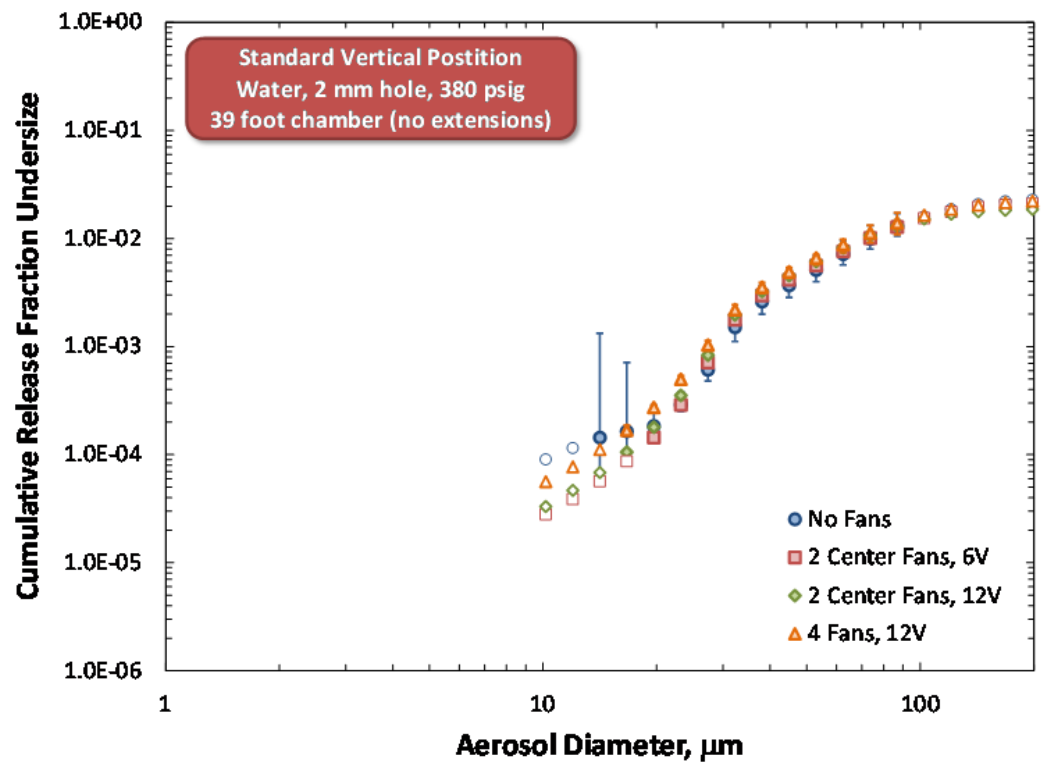

Note: All fan combinations and fan speeds at the standard vertical Malvern Insitec-S instrument position of 28 in. from the chamber ceiling.

Figure A.2. Results from Fan Configuration and Speed Testing 
The comparison results reported here were impacted by a malfunction of detector 31 on the Malvern Insitec-S instrument identified as MAL1854 (position 1) and were the subject of the nonconformance report (NCR) OTS-1270. The conclusion of the NCR was that the detector malfunction did not impact repeatability of the MAL1854 instrument, thus the relative effects of chamber configuration assessed during functional tests should not be impacted.

The Phase II mixing fan functional tests demonstrated that the chamber fans employed promoted mixing and did not lead to inhomogeneity in the chamber aerosol concentrations. Furthermore, functional tests also confirmed that the fan configuration and operational parameters identified in Phase I were adequate for tests conducted in the $39 \mathrm{ft}$ chamber. As such, no changes were made to fan configuration or operations for Phase II and all tests were conducted using two center fans operating at $12 \mathrm{~V}$.

\section{A.3 Chamber Homogeneity}

The mixing fans, as discussed in the previous section, were employed to enhance convective mixing provided by the turbulent jet and promote homogeneity in the aerosol chamber. A homogeneous chamber has the following two connotations: 1) the total volume concentration of aerosol is the same everywhere in the chamber and 2) the size distribution of aerosol is the same everywhere in the chamber. Therefore, in a well-mixed chamber, the measurement of aerosol concentration will not depend on location and the evaluation of representative release fractions are made possible. Therefore, the uniformity of the aerosol concentration throughout the chamber was assessed at several measurement locations. A set of tests, summarized in Table A.2, were conducted to assess whether the vertical and lateral position of the Malvern Insitec-S instruments had a significant effect on the measured aerosol concentration. These heights, as measured from the top ceiling of the aerosol chamber to the bottom edge of the Malvern Insitec-S mounting end plate edge, are 28, 36, and 54 in. ${ }^{1}$ The results of chamber uniformity/homogeneity testing are described in detail in Section 7.2 of this report, and as such, will not be reported in this appendix beyond listing the group of functional tests corresponding to discussion and analysis of chamber homogeneity testing.

\footnotetext{
${ }^{1}$ These vertical distances are approximate and varied \pm 0.5 in. between the various Malvern Insitec-S instruments.
} 
Table A.2. Actual Functional Tests to Establish Effect of Malvern Insitec-S Instrument Location

\begin{tabular}{|c|c|c|c|c|c|c|c|}
\hline \multirow[b]{2}{*}{ Simulant } & \multirow[b]{2}{*}{$\begin{array}{c}\text { Chamber } \\
\text { Configuration }^{(a)}\end{array}$} & \multicolumn{2}{|c|}{ Malvern Insitec-S Configuration $^{(b)}$} & \multirow[b]{2}{*}{$\begin{array}{l}\text { Mixing Fan } \\
\text { Conditions }\end{array}$} & \multirow{2}{*}{$\begin{array}{l}\text { Test Fluid } \\
\text { Pressure } \\
\text { (psig) }\end{array}$} & \multirow{2}{*}{$\begin{array}{c}\text { Nominal } \\
\text { Orifice } \\
(\mathrm{mm})\end{array}$} & \multirow[b]{2}{*}{$\begin{array}{c}\text { Accepted } \\
\text { Functional Tests }\end{array}$} \\
\hline & & $\begin{array}{l}\text { Purge Rate } \\
\text { (SCFH) }\end{array}$ & Locations $^{(\mathrm{c})}$ & & & & \\
\hline Water & $39 \mathrm{~S} 3 \mathrm{~A}$ & 1 & Standard & $\begin{array}{l}12 \mathrm{~V}, \text { two } \\
\text { center fans }\end{array}$ & 380 & Circular, 2.0 & $\begin{array}{l}\text { W533,W537, } \\
\text { W540 }\end{array}$ \\
\hline Water & $39 \mathrm{~S} 3 \mathrm{~A}$ & 1 & $\begin{array}{l}\text { Standard except } \\
\mathrm{D} \& \mathrm{E}=36 "\end{array}$ & $\begin{array}{l}12 \mathrm{~V}, \text { two } \\
\text { center fans }\end{array}$ & 380 & Circular, 2.0 & W569, W570 \\
\hline Water & $39 \mathrm{~S} 3 \mathrm{~A}$ & $50^{(\mathrm{d})}$ & $\begin{array}{l}\text { Standard except } \\
\mathrm{D} \& \mathrm{E}=54 "\end{array}$ & $\begin{array}{l}12 \mathrm{~V}, \text { two } \\
\text { center fans }\end{array}$ & 380 & Circular, 2.0 & W571, W572 \\
\hline Water & $39 \mathrm{~S} 3 \mathrm{~A}$ & $50^{(\mathrm{d})}$ & $\begin{array}{l}A=(15,25,35 \mathrm{ft}) \\
D \& E=54 "\end{array}$ & $\begin{array}{l}12 \mathrm{~V}, \text { two } \\
\text { center fans }\end{array}$ & 380 & Circular, 2.0 & W573 \\
\hline Water & $39 \mathrm{~S} 3 \mathrm{~A}$ & 1 & $\begin{array}{l}A=(15,25,35 \mathrm{ft}) \\
D \& E=36 "\end{array}$ & $\begin{array}{l}12 \mathrm{~V}, \text { two } \\
\text { center fans }\end{array}$ & 380 & Circular, 2.0 & W574 \\
\hline Water & $39 \mathrm{~S} 3 \mathrm{~A}$ & 1 & $\begin{array}{l}A=(15,25,35 \mathrm{ft}) \\
D \& E=28 "\end{array}$ & $\begin{array}{l}12 \mathrm{~V}, \text { two } \\
\text { center fans }\end{array}$ & 380 & Circular, 2.0 & $\begin{array}{l}\text { W575, W576, } \\
\text { W577 }\end{array}$ \\
\hline
\end{tabular}

(a) The five-character chamber configuration code (e.g., 39S3A) represents: $39=$ total chamber length in feet, $\mathrm{S}=$ spacer bars (in-chamber testing), 3 = Malvern Insitec-S analyzers used, and the last character represents lengths of spray header extensions where $\mathrm{A}=$ no spray header extension, $\mathrm{B}=10 \mathrm{ft}, \mathrm{C}=19 \mathrm{ft}$, and $\mathrm{D}=34.3 \mathrm{ft}$ spray header extension.

(b) The Malvern Insitec-S instruments were configured with $500 \mathrm{~mm}$ lens and $1000 \mathrm{~mm}$ spacer bars for all Malvern Insitec-S instrument location functional tests.

(c) Standard Malvern Insitec-S Locations: (Position 1) A = 75\% of chamber length (360 in.), B\&C $=28$ in., D\&E $=28$ in.; (Position 2) $\mathrm{A}=50 \%$ of chamber length (240 in.), $\mathrm{B} \& \mathrm{C}=28$ in., $\mathrm{D} \& \mathrm{E}=28$ in.; (Position 3 ) $\mathrm{A}=25 \%$ of chamber length (120 in.), $\mathrm{B} \& \mathrm{C}=28$ in., $\mathrm{D} \& \mathrm{E}=28$ in. Dimensions $\mathrm{A}, \mathrm{B}, \mathrm{C}, \mathrm{D}$, and $\mathrm{E}$ are defined and illustrated in Figure 4.9.

(d) The Malvern Insitec-S purge air rate was increased to $50 \mathrm{SCFH}$ to minimize aerosol droplet contamination on the Malvern Insitec-S optical windows while the instruments were in close proximity to the spray jet (i.e., D\&E $=54$ in.). The functional test comparisons presented here are not affected by this purge rate change. A more detailed discussion of the effect of Malvern Insitec-S purge rate is provided in Section A.5 below.

\section{A.4 Malvern Insitec-S Lens Tests}

As discussed in Section 4.5.3, the Malvern Insitec-S lens is a part of the detection unit and is responsible for translating (focusing) fixed angles of scattered light to a fixed light displacement on the photodetector rings. The focal length of the lens determines the range and resolution of light scattering angles that can be interpreted by the photodetector and determines the range of aerosol sizes accessible to the Malvern Insitec-S analyzer. In Phase II, the three aerosol analyzers were modified from using $100 \mathrm{~mm}$ (Phase I) to $500 \mathrm{~mm}$ (Phase II) lenses because of concerns that aerosols larger than $200 \mu \mathrm{m} \mathrm{had}$ not been adequately measured during Phase I (100 mm lens) tests. The $500 \mathrm{~mm}$ lens has a nominal working range of 2.5 to $2500 \mu \mathrm{m}$, whereas the $100 \mathrm{~mm}$ lens has a working range of 0.5 to $200 \mu \mathrm{m}$.

Comparative functional testing of the $100 \mathrm{~mm}$ and $500 \mathrm{~mm}$ lenses was performed to evaluate the impact of lenses on measured release fractions. Table A.3 summarizes the individual test conditions for functional testing of the Malvern Insitec-S lenses in Phase II. The testing was performed in the $39 \mathrm{ft}$ large-scale chamber configuration (with no spray header extensions) using a nominal $1 \times 76 \mathrm{~mm}$ slot orifice with spray release tests conducted at $380 \mathrm{psig}$, a purge air rate of $50 \mathrm{SCFH}$, and two center chamber mixing fans operated at $12 \mathrm{~V}$ fans.

The cumulative release fraction results comparing the 100 and $500 \mathrm{~mm}$ lens are shown in Figure A.3. Similar to mixing fan functional tests, detector 31 of Malvern Insitec-S analyzer MAL1854 malfunctioned 
during the lens functional tests. NCR OTS-1270 documented the discovery of the malfunction and the conclusion that while the malfunction did impact the accuracy of the PSD measurement, it did not impact the conclusions drawn from studies involving comparison of data sets collected using the malfunctioning MAL1854 instrument. As such, the relative effects of chamber configuration assessed during functional tests should not be impacted.

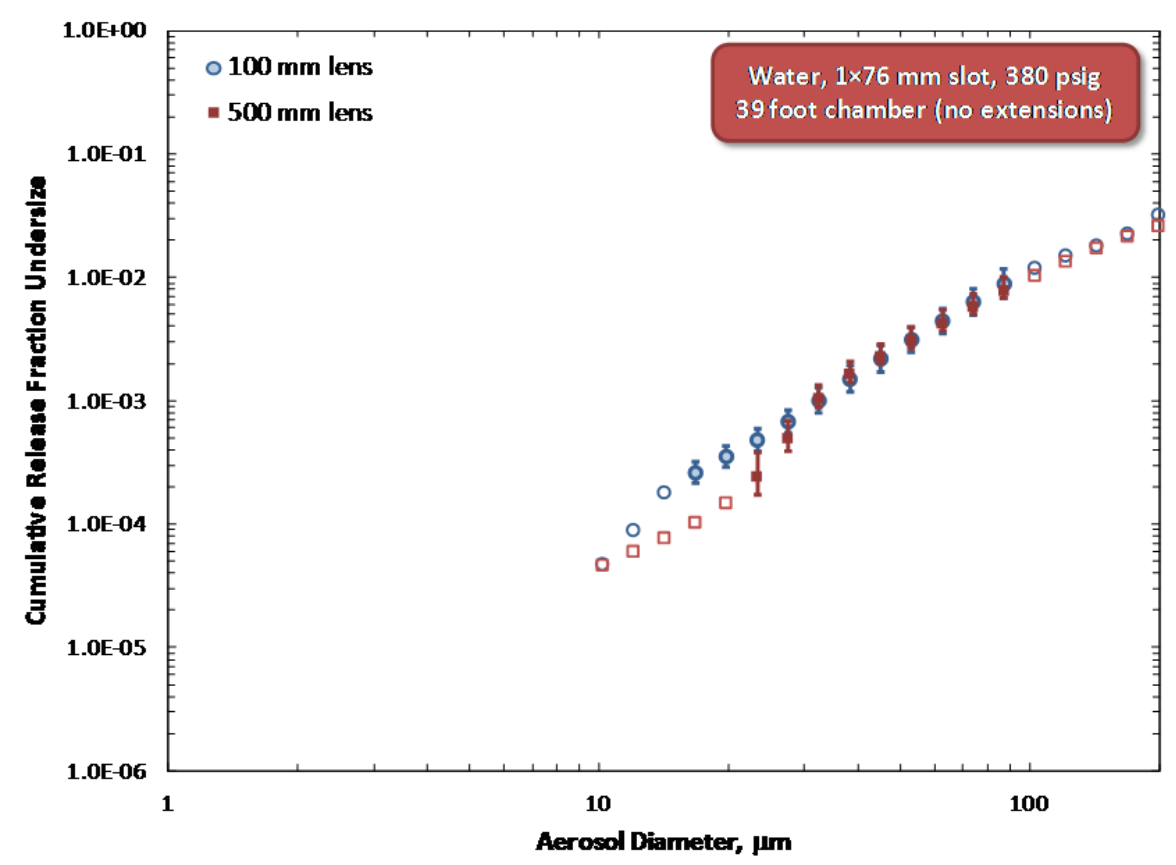

Figure A.3. Comparison of Release Fraction Measurements Made Using the $100 \mathrm{~mm}$ Lens (blue circles) and the $500 \mathrm{~mm}$ Lens (red squares). Data correspond to release fractions measured for water spray through a nominal $1 \times 76 \mathrm{~mm}$ orifice at $380 \mathrm{psig}$. Each measurement curve represents the average of at least two repeat tests; error bars correspond to the maximum and minimum release fractions measured in individual tests. Open circles represent points at aerosol diameters that fall outside the nominal Malvern Insitec-S measuring range for in-chamber measurements and, as a result, are subject to increased uncertainty. Criteria for determining suspect points for in-chamber measurements are described in Section 8.1 of this report.

Comparison of the release fraction results indicated that both lenses gave equivalent results at droplet diameters above $\sim 25 \mu \mathrm{m}$ (based on expected uncertainty and reproducibility of release fractions determined in Phase I testing). Increased measurement uncertainty below $25 \mu \mathrm{m}$ prevents assessment of lens performance at lower aerosol diameters. Because use of the $500 \mathrm{~mm}$ lens did not appear to affect the ability to measure release fraction in the size range of interest (10 to $100 \mu \mathrm{m}$ ), it was selected for use in all Phase II water and clay matrix tests. Exclusive use of the $500 \mathrm{~mm}$ lens avoided the need for frequent switch-out of Malvern Insitec-S optical lens (which requires realignment and potentially can damage the instrument) and allowed measurement of sizes above $200 \mu \mathrm{m}$ for the bulk of Phase II testing, eliminating speculation regarding the existence of larger aerosols in both in-chamber and in-spray measurements. 
Table A.3. Actual Functional Tests to Establish the Effect of Malvern Insitec-S Lens Size on Performance

\begin{tabular}{|c|c|c|c|c|c|c|c|c|}
\hline \multirow[b]{2}{*}{ Simulant } & \multirow[b]{2}{*}{$\begin{array}{c}\text { Chamber } \\
\text { Configuration }^{(a, b)}\end{array}$} & \multicolumn{4}{|c|}{ Malvern Insitec-S Configuration } & \multirow{2}{*}{$\begin{array}{c}\text { Test Fluid } \\
\text { Pressure } \\
\text { (psig) }\end{array}$} & \multirow[b]{2}{*}{$\begin{array}{c}\text { Nominal } \\
\text { Orifice (mm) }\end{array}$} & \multirow{2}{*}{$\begin{array}{l}\text { Accepted } \\
\text { Functional } \\
\text { Tests }^{(d)}\end{array}$} \\
\hline & & $\begin{array}{l}\text { Lens } \\
(\mathrm{mm})\end{array}$ & $\begin{array}{l}\text { Spacers } \\
(\mathrm{mm})\end{array}$ & $\begin{array}{c}\text { Purge Rate } \\
(\mathrm{SCFH})\end{array}$ & Locations $^{(\mathrm{c})}$ & & & \\
\hline Water & $39 \mathrm{~S} 3 \mathrm{~A}$ & 100 & 1000 & 50 & Standard & 380 & $1.0 \times 76.2$ slot & $\begin{array}{c}\text { W315, W316, } \\
\text { W317 }\end{array}$ \\
\hline Water & $39 \mathrm{~S} 3 \mathrm{~A}$ & 500 & 1000 & 50 & Standard & 380 & $1.0 \times 76.2$ slot & W318, W320 \\
\hline
\end{tabular}

(a) The five-character chamber configuration code (e.g., 39S3A) represents: $39=$ total chamber length in feet, $\mathrm{S}=$ spacer bars (in-chamber testing), 3 = Malvern Insitec-S analyzers used, and the last character represents lengths of spray header extensions where $\mathrm{A}=$ no spray header extension, $\mathrm{B}=10 \mathrm{ft}, \mathrm{C}=19 \mathrm{ft}$, and $\mathrm{D}=34.3 \mathrm{ft}$ spray header extension.

(b) Chamber mixing fans were operated at $12 \mathrm{~V}$, two center fans only.

(c) Standard Malvern Insitec-S Locations: (Position 1) A = 75\% of chamber length (360 in.), B\&C = 28 in., D\&E $=28$ in.; (Position 2) $\mathrm{A}=50 \%$ of chamber length (240 in.), B\&C = 28 in., D\&E = 28 in.; (Position 3) A = 25\% of chamber length (120 in.), $B \& C=28$ in., $D \& E=28$ in. Dimensions A, B, C, D, and E are defined and illustrated in Figure 4.9.

(d) Detector 31 of Malvern Insitec-S analyzer MAL1854 malfunctioned during the lens functional tests. NCR OTS-1270 documented the discovery and the technical assessment concluded the relative effects of chamber configuration assessed during functional tests should not be impacted.

\section{A.5 Malvern Insitec-S Purge Rate Tests}

The electronic components of the Malvern Insitec-S aerosol analyzers are protected from direct contact with the aerosol through specialized enclosures for the laser and detector. However, the laser and scattered light pass through optical windows where deposition of aerosol or the presence of contaminants will impact the quality of the measurement. Any minor surface contamination or surface imperfections (such as scratches) can be accounted for by measuring the "background" scattering intensity generated by the laser prior to aerosol generation (i.e., when the measurement volume is substantially free of aerosol). However, contamination of the window during aerosol generation is of significant concern, as particles and aerosol that attach to the window will yield a static scattering signal that will remain through the measurement. To prevent contamination of the windows by direct spray impact, all Malvern Insitec-S instruments were equipped with a $40 \mathrm{~mm}$ cylindrical spray shroud. The spray shroud protects the optics from direct impact, but does not provide protection against diffusive or convective transport of aerosol particles. As such, the Malvern Insitec-S instruments were also equipped with an air purge system that blows air across the optical windows and pushes aerosol out of the volume enclosed by the spray shrouds. Both the laser and detector windows had shrouds and purge ports and received equal purges rates (e.g., $1 \mathrm{SCFH} /$ window equals $2 \mathrm{SCFH} /$ instrument). Functional tests to determine the influence of purge air flow rate were conducted prior to matrix tests and are summarized in Table A.4. 
Table A.4. Actual Functional Tests to Establish the Effect of Malvern Insitec-S Purge Air Rates

\begin{tabular}{|c|c|c|c|c|c|c|c|c|}
\hline \multirow[b]{2}{*}{ Simulant } & \multirow[b]{2}{*}{$\begin{array}{c}\text { Chamber } \\
\text { Configuration }^{(\mathrm{a}, \mathrm{b})}\end{array}$} & \multicolumn{4}{|c|}{ Malvern Insitec-S Configuration } & \multirow{2}{*}{$\begin{array}{l}\text { Test Fluid } \\
\text { Pressure } \\
\text { (psig) }\end{array}$} & \multirow{2}{*}{$\begin{array}{c}\text { Nominal } \\
\text { Orifice } \\
(\mathrm{mm})\end{array}$} & \multirow{2}{*}{$\begin{array}{c}\text { Accepted } \\
\text { Functional } \\
\text { Tests }^{(\mathrm{d})}\end{array}$} \\
\hline & & $\begin{array}{l}\text { Lens } \\
(\mathrm{mm})\end{array}$ & $\begin{array}{c}\text { Spacers } \\
(\mathrm{mm})\end{array}$ & $\begin{array}{l}\text { Purge Rate } \\
\text { (SCFH) }\end{array}$ & Locations $^{(\mathrm{c})}$ & & & \\
\hline Water & 39S3A & 100 & 1000 & 1 & Standard & 380 & Circular, 2.0 & $\begin{array}{l}\text { W321, W322, } \\
\text { W323 }\end{array}$ \\
\hline & & & & & & & $1.0 \times 20$ slot & W324 \\
\hline Water & $39 \mathrm{~S} 3 \mathrm{~A}$ & 100 & 1000 & 3 & Standard & 380 & $\begin{array}{l}\text { Circular, } 2.0 \\
1.0 \times 20 \text { slot }\end{array}$ & $\begin{array}{l}\text { W327 } \\
\text { W325, W326 }\end{array}$ \\
\hline Water & 39S3A & 100 & 1000 & 25 & Standard & 380 & $1.0 \times 20$ slot & W328 \\
\hline
\end{tabular}

(a) The 5-character chamber configuration code (e.g., 39S3A) represents: $39=$ total chamber length in feet, $\mathrm{S}=$ spacer bars (in-chamber testing), 3 = Malvern Insitec-S analyzers used, and the last character represents lengths of spray header extensions where $\mathrm{A}=$ no spray header extension, $\mathrm{B}=10 \mathrm{ft}, \mathrm{C}=19 \mathrm{ft}$, and $\mathrm{D}=34.3 \mathrm{ft}$ spray header extension.

(b) Chamber mixing fans were operated at $12 \mathrm{~V}$, two center fans only.

(c) Standard Malvern Insitec-S Locations: (Position 1) A $=75 \%$ of chamber length (360in.), $\mathrm{B} \& \mathrm{C}=28 \mathrm{in}$., $\mathrm{D} \& \mathrm{E}=28 \mathrm{in}$.; (Position 2) $\mathrm{A}=50 \%$ of chamber length (240 in.), $\mathrm{B} \& \mathrm{C}=28$ in., $\mathrm{D} \& \mathrm{E}=28$ in.; (Position 3 ) $\mathrm{A}=25 \%$ of chamber length (120 in.), B\&C $=28$ in., D\&E = 28 in. Dimensions A, B, C, D, and E are defined and illustrated in Figure 4.9.

(d) Detector 31 of Malvern Insitec-S analyzer MAL1854 malfunctioned during the purge air functional tests. NCR OTS-1270 documented the discovery and the technical assessment concluded the relative effects of chamber configuration assessed during functional tests should not be impacted.

Similar to lens size functional tests, detector 31 of Malvern Insitec-S analyzer MAL1854 malfunctioned during the Malvern Insitec-S purge air rate functional tests. NCR OTS-1270 documented the discovery of the malfunction and the conclusion that the malfunction did not impact repeatability of the MAL1854 instrument, and as such, the relative effects of chamber configuration assessed during functional tests should not be impacted.

Using the nominal $2 \mathrm{~mm}$ circular orifice, water spray release tests were completed at 1 and $3 \mathrm{SCFH}$, and the release fraction results of these tests are presented in Figure A.4. The release fractions are comparable, but indicate slight, but significant relative to measurement repeatability, increase in release fraction measurements with increasing purge rate. Additional functional tests were conducted using the nominal $1 \times 20 \mathrm{~mm}$ slot orifice at 1,3 , and $25 \mathrm{SCFH}$ purge air rates, and the results are illustrated in Figure A.5. Similar to the $2 \mathrm{~mm}$ orifice, a slight increase in release fraction with increasing purge air rate was observed. Based on these observations, purge rate selection does impact release fraction measurements. During Phase II testing, selection of the purge rate needed to balance the impact of increased purge on the release fraction against rejection of test data as a result of lens contamination by simulant droplets. During Phase II testing, the operational approach adopted was to generally use a $1 \mathrm{SCFH}$ air purge rate for the nominal $2 \mathrm{~mm}$ and $1 \times 10 \mathrm{~mm}$ orifice and at least a $25 \mathrm{SCFH}$ rate for the nominal $1 \times 20 \mathrm{~mm}$ and $1 \times 76 \mathrm{~mm}$ orifices with a secondary objective to maintain consistent purge rate conditions throughout water and clay matrix testing. When window contamination was highly likely (i.e., in-spray testing) or could not be controlled at the minimal and typical air purge rate, larger purge rate rates (e.g., $100 \mathrm{SCFH})$ were employed. 


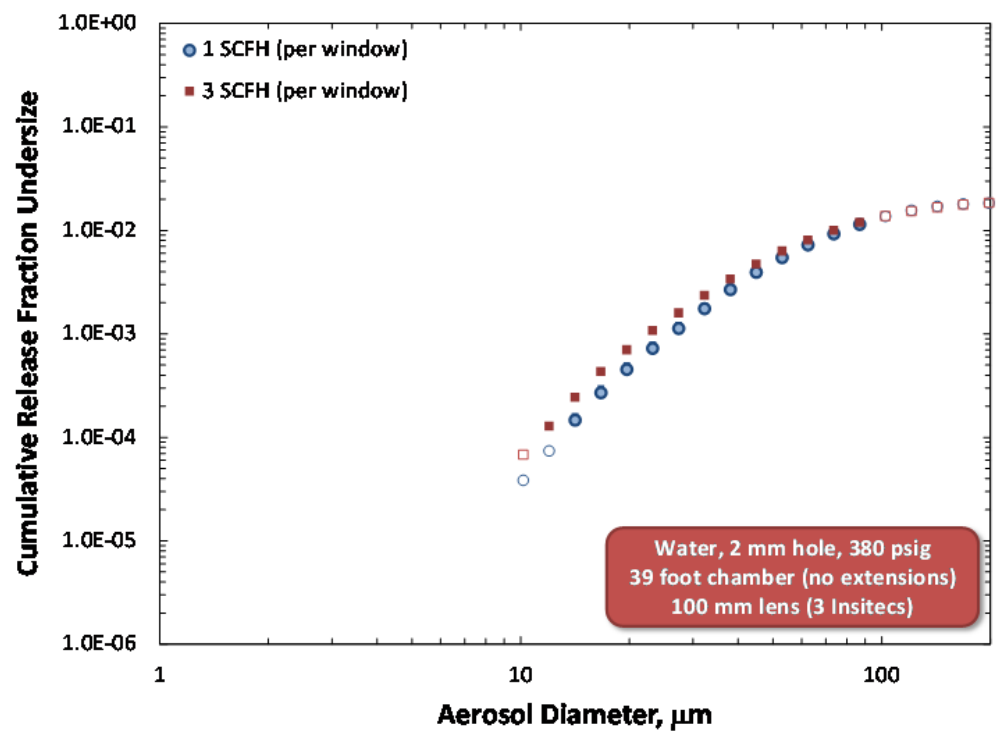

Figure A.4. Influence of Purge Air Rate Using a Nominal $2 \mathrm{~mm}$ Circular Orifice at $380 \mathrm{psig}$

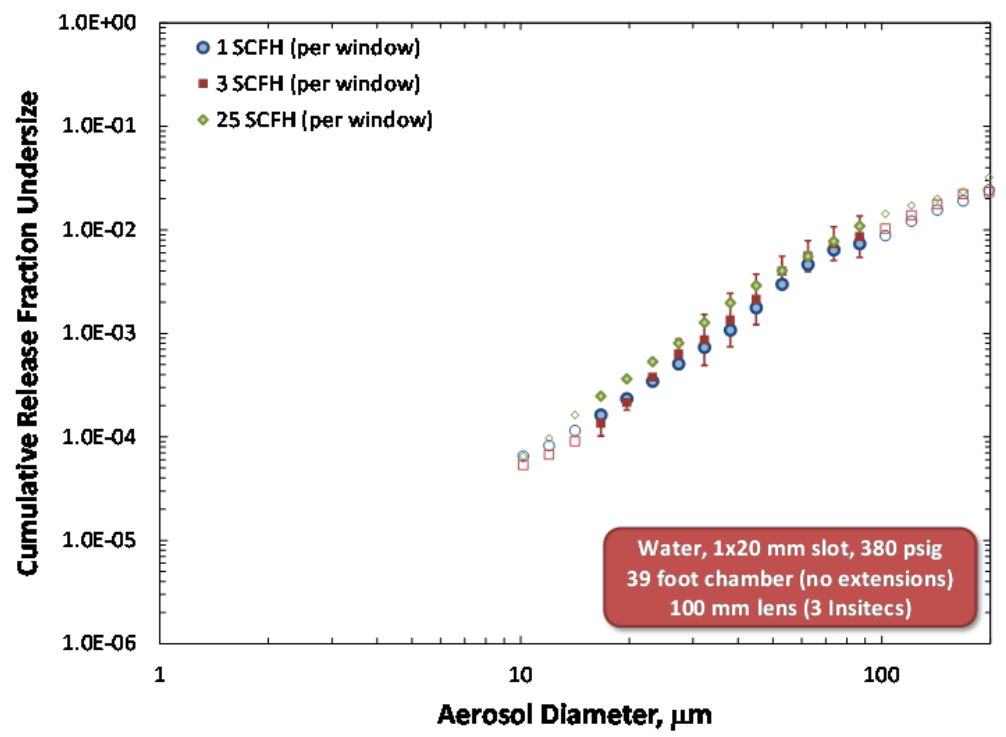

Figure A.5. Influence of Purge Air Rate Using a Nominal $1 \times 20 \mathrm{~mm}$ Slot Orifice at $380 \mathrm{psig}$

\section{A.6 Evaluation of Impact of Humidity on Release Fraction}

Relative humidity directly impacts apparent release fraction measurements made using the in-chamber test approach through evaporation of droplets, a phenomenon not accounted for in the droplet material balance presented in earlier sections of this report. Functionally, apparent in-chamber release fraction should depend on the chamber RH as long as the evaporation process is not mass transfer limited. Spray of liquid or slurry into the chamber is expected to increase RH during the test; however, because release fraction is calculated by the slope of the concentration increase at the start of spray, the $\mathrm{RH}$ at the start of testing is expected to be the controlling variable (and not the instantaneous value of release fraction measured during spray). A sequence of water and clay simulant large-scale test chamber 
aerosolization studies was performed to evaluate the impact of humidity on release fraction measurements. For this these studies, initial chamber RH was varied from 40 to $\sim 99$ percent (as measured by FIO instruments). Here, release fraction is measured using the standard in-chamber test setup and methodology.

The goal of RH testing is to evaluate the magnitude of bias in release fraction measurements caused by low initial starting humidity. All release fraction results presented in Chapters 1 through 10 of this report are presented under the implicit assumption that droplet evaporation does not influence the magnitude of release fraction. The standard test protocol employed in large-scale testing dictated that chamber RH be above 75 percent before initiating spray, and while this protocol limited the impact of initial humidity on the measured release fraction, the vast majority of Phase II results described in this report include some measurement bias caused by low humidity as the number of tests with starting humidity at or near 100 percent was limited. At the start of testing, it was believed that an initial RH less than 100 percent would cause droplets to evaporate before reaching the measurement zone, resulting in an under-estimation (i.e., bias low) of in-chamber release fractions. However, the magnitude of bias introduced was not known and is the subject of the investigation presented herein.

Before presenting the humidity test results, it should be stressed that neither of the two humidity sensors used in large-scale Phase I and Phase II testing were calibrated per the project requirements. However, the humidity sensors were procured with a factory calibration. Additionally, a performance check of the sensors toward the end of large-scale testing (and before the final humidity tests - $20 \mathrm{ft}$ water testing) indicated that the humidity sensors were providing reasonably accurate ${ }^{1}$ measurement of chamber humidity. As such, while the release fraction measurements presented herein are qualified, the value of initial RH associated with that result is "For Information Only." For this reason, all of humidity test results presented in this section and any conclusions derived from these results were not qualified (in terms of PNNL's NQA-1 program requirements as described in Section 2) and should be considered "For Information Only."

Testing of RH impact on water sprays was conducted in the 20,30, and $39 \mathrm{ft}$ chambers. Humidity tests with the clay simulant employed the $20 \mathrm{ft}$ chamber and $6 \mathrm{~Pa}$ Clay simulant. Tests using simulant were performed primarily to evaluate the role of dry particulate, which represent the lower limit of evaporation in clay systems, on the humidity bias. Testing of a single simulant system was deemed sufficient to meet this test objective, and for this reason, no humidity tests were performed with the $30 \mathrm{~Pa}$ Clay simulant. Table A.5 lists the sequence of tests conducted at each chamber length and simulant material along with the starting (initial) RH for each test. All RH tests were performed using a nominal $2 \mathrm{~mm}$ hole and a 380 psig spray. In terms of test orifice size, the $2 \mathrm{~mm}$ hole represents the smallest Phase II orifice tested and produces the smallest spray rate. Because of this, the $2 \mathrm{~mm}$ hole should be most strongly impacted by evaporation out of the four Phase II orifices tested. Likewise, the smallest $2 \mathrm{~mm}$ hole spray would correspond to a test pressure of $100 \mathrm{psig}$. This pressure was not tested because the concentration of spray produced with this combination of test orifice and pressure is near the detection limits of the Malvern Insitec-S aerosol analyzers. As such, there was concern that variation in release fractions with humidity would not be resolvable. Instead, the 380 psig spray was tested. At this pressure,

\footnotetext{
${ }^{1}$ All large-scale chamber humidity sensor readings were within $5 \%$ of the true value and were typically lower than the true value, as measured by a calibrated Vaisala MI70 humidity sensor (PNNL IS\&T, M\&TE System ID: HIVA3-0001).
} 
the $2 \mathrm{~mm}$ hole provides easily resolvable aerosol concentrations in the large-scale chamber, which provide increased confidence that trends in the release fraction result could be observed and quantified accurately.

Table A.5. List of Humidity Test Measurements for Water and $6 \mathrm{~Pa}$ Clay Simulant (RH data are not qualified and should be used "For Information Only")

\begin{tabular}{|c|c|c|c|c|c|c|c|}
\hline \multirow[b]{2}{*}{ Simulant } & \multirow[b]{2}{*}{$\begin{array}{c}\text { Chamber } \\
\text { Configuration }^{(a)}\end{array}$} & \multicolumn{2}{|c|}{$\begin{array}{l}\text { Malvern Insitec-S } \\
\text { Configuration }^{(\mathrm{b})}\end{array}$} & \multirow[b]{2}{*}{$\begin{array}{l}\text { Mixing Fan } \\
\text { Conditions }\end{array}$} & \multirow{2}{*}{$\begin{array}{l}\text { Test } \\
\text { Fluid } \\
\text { Pressure } \\
\text { (psig) }\end{array}$} & \multirow{2}{*}{$\begin{array}{l}\text { Nominal } \\
\text { Orifice } \\
(\mathrm{mm})\end{array}$} & \multirow[b]{2}{*}{$\begin{array}{l}\text { Accepted Functional } \\
\text { Tests }^{(\mathrm{d})}\end{array}$} \\
\hline & & $\begin{array}{l}\text { Purge Rate } \\
\text { (SCFH) }\end{array}$ & Locations $^{(\mathrm{c})}$ & & & & \\
\hline Water & $20 \mathrm{~S} 3 \mathrm{~A}$ & 1 & Standard & $\begin{array}{l}12 \mathrm{~V} \text {, two } \\
\text { center fans }\end{array}$ & 380 & Circular2.0 & $\begin{array}{l}\text { W859 (94\%), W860 (96\%), } \\
\text { W861 (97\%), W863 (50\%), } \\
\text { W864 (52\%), W865 (87\%), } \\
\text { W866 (65\%), W867 (75\%) }\end{array}$ \\
\hline Water & 30S3A & 1 & Standard & $\begin{array}{l}12 \mathrm{~V} \text {, two } \\
\text { center fans }\end{array}$ & 380 & Circular2.0 & $\begin{array}{l}\text { W659 (45\%), W660 (44\%), } \\
\text { W661 (48\%), W655 (98\%), } \\
\text { W656 (98\%), W657 (98\%), } \\
\text { W658 (98\%) }\end{array}$ \\
\hline Water & 39S3A & 1 & Standard & $\begin{array}{l}12 \mathrm{~V} \text {, two } \\
\text { center fans }\end{array}$ & 380 & Circular2.0 & $\begin{array}{l}\text { W563 }(45 \%), \text { W564 (48\%), } \\
\text { W565 (41\%), W578 (55\%), } \\
\text { W579 (42\%), W580 (70\%), } \\
\text { W566 (81\%), W567 (87\%) }\end{array}$ \\
\hline 6 Pa Clay & 20S3A & 1 & Standard & $\begin{array}{l}12 \mathrm{~V}, \text { two } \\
\text { center fans }\end{array}$ & 380 & Circular2.0 & $\begin{array}{l}\text { S } 851(57 \%), S 852(43 \%), \\
\text { S853 (49\%), S854 (67\%), } \\
\text { S855 (77\%), S856 (87\%), } \\
\text { S857 (95\%), S858 (96\%) }\end{array}$ \\
\hline \multicolumn{8}{|c|}{$\begin{array}{l}\text { (a) The five-character chamber configuration code (e.g., } 39 \mathrm{~S} 3 \mathrm{~A} \text { ) represents: } 39=\text { total chamber length in feet, } \mathrm{S}=\text { spacer bars } \\
\text { (in-chamber testing), } 3=\text { Malvern Insitec-S analyzers used, and the last character represents lengths of spray header extension } \\
\text { where } \mathrm{A}=\text { no spray header extension, } \mathrm{B}=10 \mathrm{ft}, \mathrm{C}=19 \mathrm{ft} \text {, and } \mathrm{D}=34.3 \mathrm{ft} \text { spray header extension. } \\
\text { (b) The Malvern Insitec-S instruments were configured with } 500 \mathrm{~mm} \text { lens and } 1000 \mathrm{~mm} \text { spacers for all humidity functional tests. } \\
\text { (c) Standard Malvern Insitec-S Locations: (Position } 1) \mathrm{A}=75 \% \text { of chamber length ( } 360 \mathrm{in} \text {.), } \mathrm{B} \& \mathrm{C}=28 \mathrm{in} \text {., } \mathrm{D} \& \mathrm{E}=28 \mathrm{in} \text {.; } \\
\text { (Position } 2) \mathrm{A}=50 \% \text { of chamber length }(240 \mathrm{in} \text {.), } \mathrm{B} \& \mathrm{C}=28 \mathrm{in} \text {., } \mathrm{D} \& \mathrm{E}=28 \text { in.; (Position } 3) \mathrm{A}=25 \% \text { of chamber length } \\
(120 \text { in.), } \mathrm{B} \& \mathrm{C}=28 \mathrm{in} ., \mathrm{D} \& \mathrm{E}=28 \mathrm{in} \text {. Dimensions } \mathrm{A}, \mathrm{B}, \mathrm{C}, \mathrm{D} \text {, and } \mathrm{E} \text { are defined and illustrated in Figure } 4.9 \text {. } \\
\text { (d) The starting (initial) } \mathrm{RH} \text { associated with each test is listed next to the test identification number in parentheses. }\end{array}$} \\
\hline
\end{tabular}

Figure A.6 shows the impact of RH on release fraction measurements of water. The results indicate that, regardless of chamber size, release fraction is reduced at low humidity across all droplet sizes. While release fraction measurements for water appear to be impacted more strongly at smaller droplet sizes $(\leq 20 \mu \mathrm{m})$, the difference in the reduction for small and large droplets does not appear to be great. In other words, the impact of humidity on droplet size is significant for both large and small aerosols. The degree of reduction appears to scale proportionally to the difference between the test humidity and 100 percent, and any divergence from this behavior appears to derive from measurement uncertainty rather than a phenomenological mechanism. 


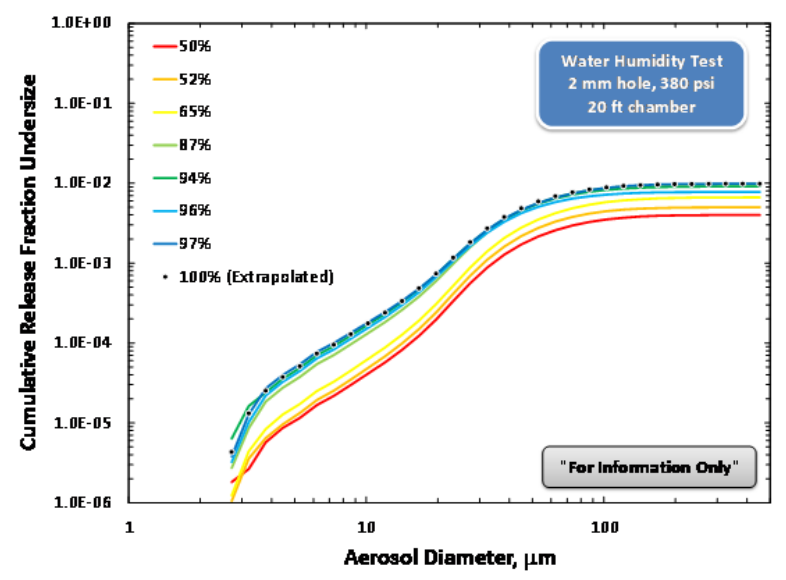

(a) - Water, $20 \mathrm{ft}$ chamber

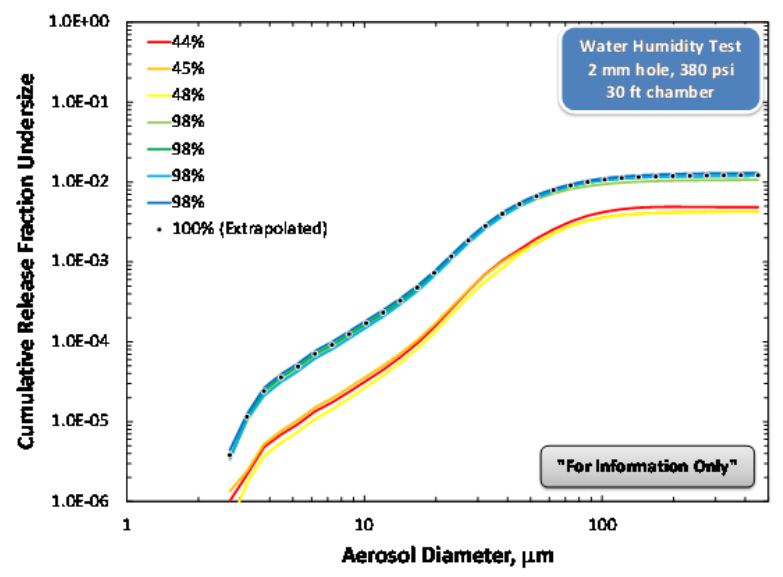

(b) - Water, $30 \mathrm{ft}$ chamber

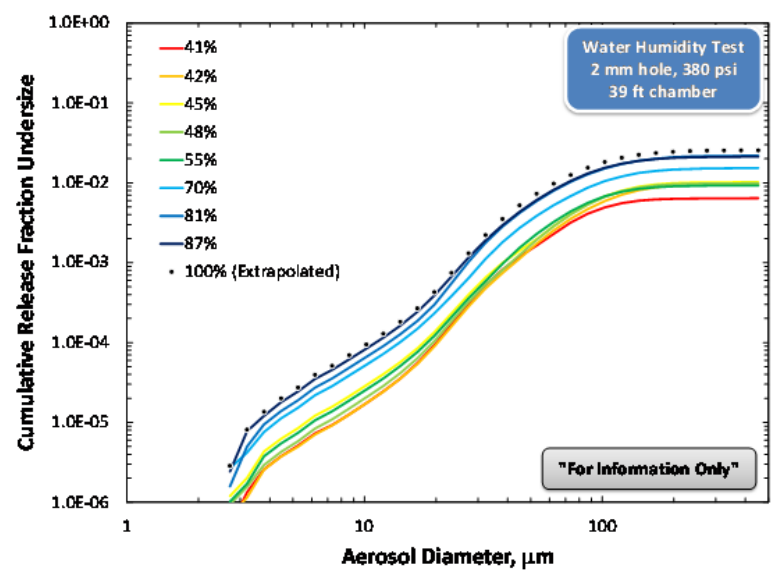

(c) - Water, $39 \mathrm{ft}$ chamber

Figure A.6. Impact of Starting RH on Water Release Fraction Results for Tests in (a) the $20 \mathrm{ft}$ Chamber, (b) the $30 \mathrm{ft}$ Chamber, and (c) $39 \mathrm{ft}$ Chamber. Actual measurements are shown using solid lines. An extrapolation, based on actual measurements, to the release fraction corresponding to 100 percent $\mathrm{RH}$ is shown (black dots). While release fraction measurements are qualified, their corresponding humidity measurements are not and should be considered "For Information Only." 
Figure A.7 shows the impact of humidity on release fraction measurements for $6 \mathrm{~Pa}$ Clay in the $20 \mathrm{ft}$ chamber. As expected, the presence of particles in the clay slurry alters how RH impacts release fraction. At large droplet sizes $(>50 \mu \mathrm{m})$, which are expected to correspond to droplets whose composition is similar to that of the bulk slurry, lowered RH yields a decrease in the apparent release fraction. This behavior is consistent with evaporative loss of droplets and matches the water behavior. In contrast, the small clay droplets $(<20 \mu \mathrm{m})$ show the opposite behavior, and exhibit an increase in release fraction with decreasing humidity. At these sizes, the aerosol diameter is similar to the dry clay particulate that makes up the slurry. As such, the increase in release fraction with lowered humidity at these sizes likely derives from the increased production of dry clay particulate by evaporative drying at low RH. This dry particulate is not subject to further size reduction by evaporation, unlike water droplets produced in this size range, which will continue to evaporate until they disappear or until air is saturated with water.

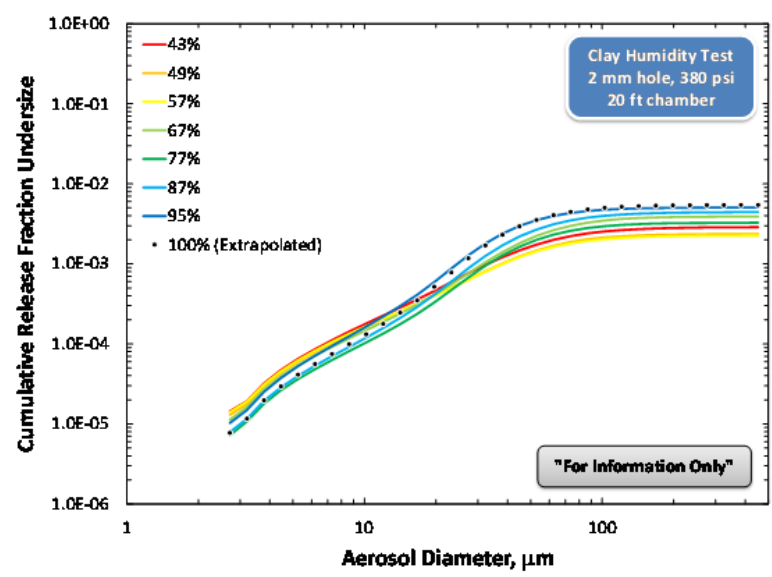

Figure A.7. Impact of Starting (Initial) RH on 6 Pa Clay Release Fraction Results for a Test in the $20 \mathrm{ft}$ Chamber. Actual measurements are shown using solid lines. An extrapolation, based on actual measurements, to the release fraction corresponding to 100 percent $\mathrm{RH}$ is shown (black dots). While release fraction measurements are qualified, their corresponding humidity measurements are not and should be considered "For Information Only."

The results presented in Figure A.6 and Figure A.7 show a strong dependence of measured release fraction on starting (initial) RH. In an attempt to quantify how release fraction depends on RH, release fraction data at measured particle diameters $(2.5$ to $450 \mu \mathrm{m})$ were fit to the following linear correlation using least-squares analysis:

$$
R_{k}=m_{k} H_{o}+b_{k}
$$

Here, $R_{k}$ is the cumulative release fraction of aerosols below diameter $d_{p}$. The terms $\mathrm{m}_{\mathrm{k}}$ and $\mathrm{b}_{\mathrm{k}}$ represent the slope and intercept of the linear fit, and are determined by linear least-square analysis of release fraction, $R_{k}$, as a function of starting $\mathrm{RH}, H_{o}$. Two example fits of the data are shown in Figure A.8 for the 30 and $39 \mathrm{ft}$ chambers. In general, the fits of release fraction data against Equation (A.1) are of reasonable quality. Correlation coefficients (i.e., $R^{2}$ ) for water data generally range from 0.8 to 0.98 , and indicate reasonable correlation between the release fraction and humidity data with respect to Equation (A.1). Because of the trend reversal observed in $6 \mathrm{~Pa}$ Clay release fractions with humidity at large and small aerosol diameters, there is a size region in which the clay release fraction does not depend strongly on humidity. This leads to poor correlation coefficients (i.e., $R^{2}<0.5$ ) at intermediate 
(i.e., 5 to $20 \mu \mathrm{m}$ ) aerosol size ranges for clay. Outside of this size range, correlation coefficients for clay are reasonable and can range from 0.50 to 0.82 .

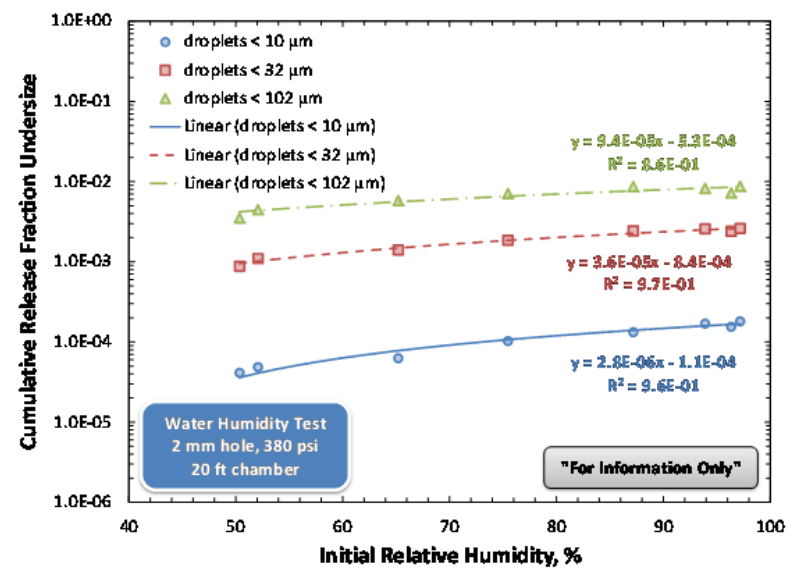

(a) - Water, $20 \mathrm{ft}$ chamber

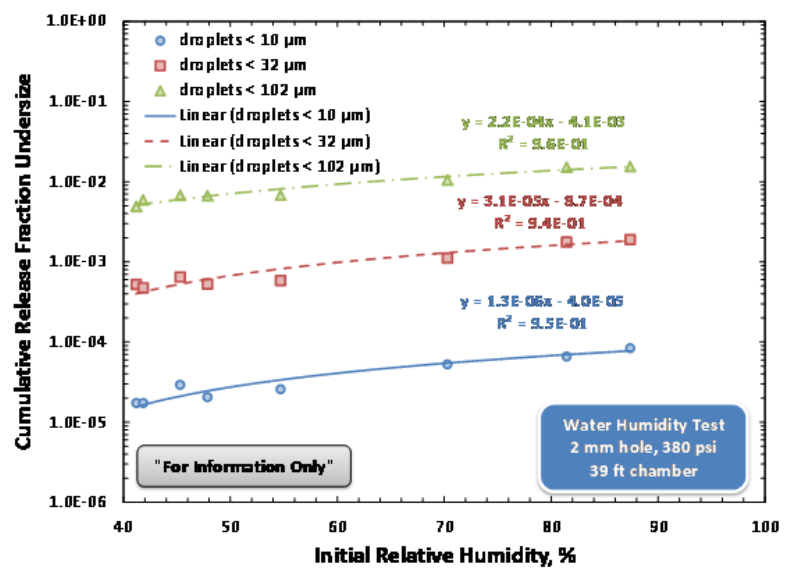

(b) - Water, $39 \mathrm{ft}$ chamber

Figure A.8. Two Examples of Linear Least-Square Analysis of Release Fraction as a Function of Starting (Initial) RH. Fits of 10, 32, and $102 \mu \mathrm{m}$ aerosol droplet release fractions are shown for (a) the $20 \mathrm{ft}$ chamber and (b) the $39 \mathrm{ft}$ chamber. Data correspond to a water spray at 380 psig through a $2 \mathrm{~mm}$ hole. Actual release fraction measurements are shown using symbols (blue circles $-10 \mu \mathrm{m}$, red squares $-32 \mu \mathrm{m}$, and green triangles $-102 \mu \mathrm{m}$ ). The corresponding linear fits to the data are shown using solid and dashed lines. The equations included in the charts represent the linear fit of the data to Equation (A.1) along with the $R^{2}$ value of the fit. While release fraction measurements are qualified, their corresponding humidity measurements are not and should be considered "For Information Only."

Linear regression provides best fit values for $m_{k}$ and $b_{k}$ for each aerosol size considered. From these values, it is possible to interpolate or extrapolate measured release fraction values to RH ranges not tested. Indeed, several examples of extrapolation to saturation (i.e., 100 percent RH) are shown in Figure A.6 and Figure A.7. Likewise, the bias incurred as a result of testing at an RH below saturation can be assessed by evaluating the ratio of the saturated release fraction (i.e., that at 100 percent $\mathrm{RH}$, denoted by $R_{k}^{(S A T)}$ and determined by extrapolation) to the current test release fraction (denoted by $R_{k}^{(R E F)}$ and determined by the best fit correlation to a reference test humidity, $H_{R E F}$ ). To this end, a relative release fraction humidity ratio for each aerosol size (denoted as $\eta_{k}$ ) can be defined from Equation (A.2):

$$
\eta_{k}=\frac{R_{k}^{(S A T)}}{R_{k}^{(R E F)}}=\frac{m_{k} H_{S A T}+b_{k}}{m_{k} H_{R E F}+b_{k}}
$$

where $H_{S A T}=100$ percent.

Note that $\eta_{k}$ represents a multiplicative correction factor that can be applied to release fraction data (measured at the specified aerosol diameter and under a specific test configuration [e.g., $2 \mathrm{~mm}$ hole, $380 \mathrm{psig}$, and $39 \mathrm{ft}$ chamber length]) to correct under-estimation of release fractions resulting from low initial test humidity. Universal application of correction for humidity bias is not possible for either Phase I or Phase II large-scale test chamber data for two reasons. First, humidity correction correlations (as defined by a unique set of $m_{k}$ and $b_{k}$ coefficients) are not available for each unique test combination of 
orifice size, test pressure, and chamber configuration. Furthermore, the humidity measurements on which these correlations would be based are not qualified, and as a result, any corrected result derived therefrom would be unqualified as well. Despite this limitation, the existing humidity correction data derived from humidity testing with the $2 \mathrm{~mm}$ hole at 380 psig can provide an FIO overall estimate of humidity bias and its impact on the main release fraction test results for water and clay presented in the body of this report. To do this, the overall range of starting (initial) RH observed in testing is considered first. Figure A.9 shows the distribution (i.e., frequency of occurrence) of starting (initial) RH in water (a) and clay (b) matrix testing. Water testing shows a broad range of initial relative test humidity, ranging from 74 to 102 percent. Clay simulant testing shows a slightly smaller range of relative test humidity, ranging from 81 to 98 percent.

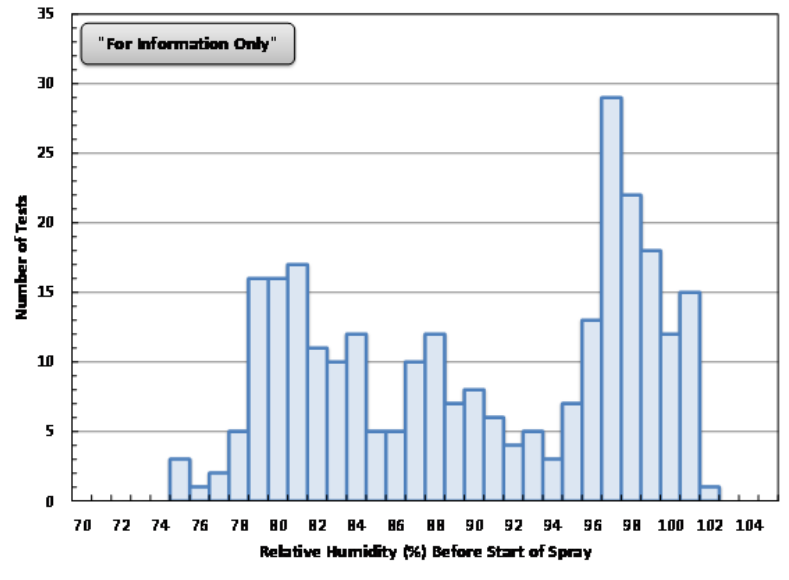

(a) - water matrix testing

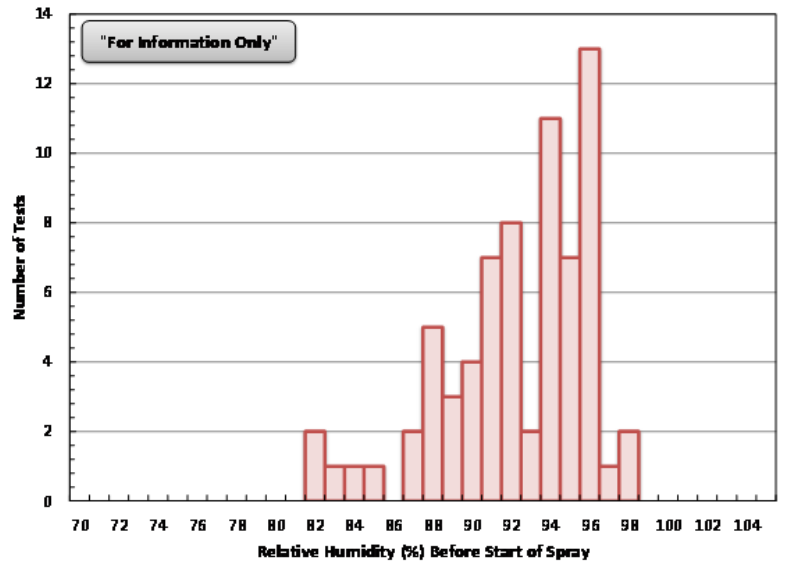

(b) - clay simulant matrix testing

Figure A.9. Frequency and Distribution of Starting (Initial) RH in (a) Water Matrix Testing and (b) Clay Simulant Matrix Testing. Humidity data presented here are not qualified and should be considered "For Information Only."

The humidity frequency results shown in Figure A.9 indicate that application of a single correction factor can possibility over-estimate or under-estimate releases for a significant portion of the test population. Specifically, if low reference humidity is used to determine the correction factor for all tests, then a large portion of the corrected release fractions may over-estimate their actual saturation value. Likewise, if high reference humidity is used, there will be a portion of tests for which release fraction is under-estimated. For the purpose of estimating a correction factor, two reference humidity values shall consider 1) the minimum test humidity and 2) the median test humidity observed for all test cases. Humidity corrections for water and clay are considered separately because of the difference in each simulant's aerosol behavior with decreasing humidity. Furthermore, analysis of humidity bias will be restricted to the size range of interest for the current aerosol studies (i.e., 10 to $100 \mu \mathrm{m}$ ).

Table A.6 provides the minimum and median humidity for water and clay test matrices as well as humidity bias correction factors $\eta_{k}$ for 10,32 , and $102 \mu \mathrm{m}$ droplets for the various test configurations examined during humidity testing. For water testing, the minimum starting (initial) RH measured during an accepted test was 74.2 percent. The bias introduced to measurements of cumulative release fraction for water by testing at this low initial RH can be corrected by application of 1.4 and 1.7 multiplicative factors for large $(102 \mu \mathrm{m})$ and small $(10 \mu \mathrm{m})$ droplets, respectively. As expected, a larger correction 
factor is needed for smaller droplets as they are more strongly impacted by evaporation because of their higher surface area to droplet volume ratio.

Table A.6. Humidity Bias Correction Factors Based on the Minimum and Median Initial RHs Measured in Phase II Large-Scale Water and Clay Matrix Testing. Correction factors are listed for the various test configurations evaluated. Representative humidity readings and listed correction factors are provided on a "For Information Only" basis.

\begin{tabular}{|c|c|c|c|c|}
\hline Property & $\begin{array}{c}\text { Water } \\
20 \mathrm{ft} \text { Chamber }\end{array}$ & $\begin{array}{c}\text { Water } \\
30 \mathrm{ft} \text { Chamber }\end{array}$ & $\begin{array}{c}\text { Water } \\
39 \mathrm{ft} \text { Chamber }\end{array}$ & $\begin{array}{c}6 \text { Pa Clay } \\
20 \mathrm{ft} \text { Chamber }\end{array}$ \\
\hline$H_{o}$ (minimum) $^{(\mathrm{a})}$ & $74 \%$ & $74 \%$ & $74 \%$ & $81 \%$ \\
\hline$H_{o}(\text { median })^{(\mathrm{b})}$ & $90 \%$ & $90 \%$ & $90 \%$ & $92 \%$ \\
\hline \multicolumn{5}{|c|}{ Median Humidity Bias Correction $\eta_{k}$ (based on median test matrix humidity) } \\
\hline $10 \mu \mathrm{m}$ & 1.20 & 1.18 & 1.17 & 0.97 \\
\hline $32 \mu \mathrm{m}$ & 1.16 & 1.17 & 1.17 & 1.08 \\
\hline $102 \mu \mathrm{m}$ & 1.12 & 1.14 & 1.15 & 1.09 \\
\hline \multicolumn{5}{|c|}{ Maximum Humidity Bias Correction $\eta_{k}$ (based on minimum test matrix humidity) } \\
\hline $10 \mu \mathrm{m}$ & 1.71 & 1.63 & 1.58 & 0.92 \\
\hline $32 \mu \mathrm{m}$ & 1.51 & 1.57 & 1.56 & 1.24 \\
\hline $102 \mu \mathrm{m}$ & 1.38 & 1.44 & 1.46 & 1.26 \\
\hline
\end{tabular}

(a) Minimum starting (initial) RH recorded during execution of Phase II large-scale water or clay test matrix.

(b) Median starting (initial) RH recorded during execution of Phase II large-scale water or clay test matrix.

The impact of humidity appears to be a stronger function of droplet size in small test chambers; however, the overall uncertainty in the humidity bias correction factor generally appears to be \pm 0.2 (as evaluated by statistical analysis of uncertainty in $m_{k}$ and $b_{k}$ and propagation of those uncertainties through Equation (A.2)). As such, changes in overall correction factor with size, much less with chamber length, appear to be statistically insignificant. The consequence of the latter finding is that humidity correction factors, based on the minimum test humidity, are similar and that a $1.5 \pm 0.2$ correction factor can be universally applied to all water release fraction to correct for humidity bias. It should be noted that application of this single correction factor will over-estimate release fraction for the majority of water tests. Median humidity correction factors show similar behavior and indicate a multiplicative correction factor of $1.2 \pm 0.2$ will correct (or over-correct) for humidity bias in half of the water release fraction measurements.

As discussed above, low RH impacts clay release fraction differently than it does water release fraction. Therefore, it is not surprising to observe different humidity correction factors for the $6 \mathrm{~Pa}$ Clay tested. The difference derives both from different median and minimum RH values for 6 Pa Clay, which are 92 percent and 81 percent, respectively, and from the presence of particulate that limits the role of evaporation with respect to aerosol size below aerosol diameters of $\sim 50 \mu \mathrm{m}$. The presence of non-volatile clay solids also greatly reduces the impact of humidity on release fraction and reduces the overall magnitude of the worst-case humidity correction from $\sim 1.7$ for water to $\sim 1.3$ for clay.

In our evaluation of the humidity corrections for the $6 \mathrm{~Pa}$ Clay listed in Table A.6, we found that almost no correction was needed for $10 \mu \mathrm{m}$ particles. Any correction serves to lower the release fraction 
by $\sim 10$ percent in the worst-case humidity correction. For the 32 and $102 \mu \mathrm{m}$ particles, the overall multiplicative humidity bias correction fraction is $\sim 1.2$ for the worst-case correction and $\sim 1.1$ for the median correction. Uncertainty in the humidity correction factor for clay is reduced relative to that of water, and generally yields a \pm 0.1 variation in the correction factor. As such, a universal humidity bias correction factor of $1.2 \pm 0.1$ seems appropriate when correcting release fraction measurements of clay systems measured during Phase II large-scale testing. This factor allows correction (or over-correction) of the humidity bias in clay release fraction data, such that measurements are conservative with respect to the actual saturation (i.e., 100 percent $\mathrm{RH}$ ) release fraction. Correction of release fraction for the median $\mathrm{RH}$ yields a multiplier of $1.1 \pm 0.1$ that yields a release fraction data set in which 50 percent of the measurements are conservative (with respect to the expected release fraction at 100 percent $\mathrm{RH}$ ).

Overall, evaluations of the impact of RH on in-chamber release fraction measurements indicates that low humidity can have a significant impact on the measured release fraction results, even when humidity was maintained with the control range identified for testing ( $>75$ percent). The range of starting (initial) RHs observed during testing is large and spans the lower limit of the control range $\sim 75$ percent up to saturation (100 percent), and as such, release fraction measurements are subject to bias from low RH to a varying extent. Two approaches for recommending a single correction factor for low humidity bias were taken. The first approach is based on the minimum RH, while the second represents the median RH observed in all the water and clay matrix testing. For the first and second approaches, the correction factors for water are $1.5 \pm 0.2$ and $1.2 \pm 0.2$, respectively. For clay, these same correction factors are $1.2 \pm 0.1$ and $1.1 \pm 0.1$, respectively. It should be noted that these correction factors are relatively small compared to the suspected magnitude of the method bias and the experimental variability noted between different experimental conditions.

\section{A.7 Overall Correction of Release Fraction Data}

In overall terms, humidity bias is not the only source of uncertainty for in-chamber measurements. Another significant source of uncertainty is termed method bias and is discussed in detail in Chapter 7 . Method bias, as defined in this report, concerns observed differences in relative magnitude of in-chamber and in-spray release fraction measurements and include bias in the in-spray methods that derive from spatial measurement of aerosol size distribution and bias in the in-chamber methods that derive from incomplete mixing and gravitational settling of aerosol. Because in-spray bias is expected to yield higher-than-actual release fraction measurements, in-spray release fractions are considered conservative measurements of aerosol release. However, in-spray release fractions are not generally accurate below $50 \mu \mathrm{m}$, and as a result, determination of release fraction typically relies on available in-chamber measurements. In-chamber method bias is expected to lower measured release fractions relative to the true value, which makes use of in-chamber results without correction non-conservative. From the available data, correction of either in-spray or in-chamber measurements for method bias is not possible. However, comparison of in-chamber measurements and in-spray measurements at sizes where the two methods overlap can indicate the degree of correction needed to make in-chamber measurements "as conservative" as in-spray measurements. For simplicity, this correction will simply be referred to as "a method bias" correction for in-chamber even though the correction does yields a "conservative" estimate of actual release (rather than the true value).

Comparison of in-spray and in-chamber measurements indicate that for the 10 to $100 \mu \mathrm{m}$ particles, the method bias yields a factor of three decrease in in-chamber measurements relative to in-spray 
measurements (which are considered conservative). Because the in-chamber data employed to determine this factor are impacted to varying extents by humidity bias, the factor of three also includes a portion of the humidity bias correct as well, but does not correct for all humidity bias. For the purpose of evaluating an overall correction factor, no credit is taken for the humidity correction intrinsic in the proposed method bias correction factor of three.

Overall correction of the data for both humidity and method bias is determined by the combination of multiplicative correction factors such that:

$$
R_{k}^{(c)}=\hat{\eta} \hat{\kappa} R_{k}
$$

where an individual cumulative release fraction undersize $d_{k}$ (denoted by $R_{k}$ ) is corrected by a universal multiplicative correction factor for humidity bias, $\hat{\eta}$, and method bias, $\hat{\kappa}$, to yield a corrected release fraction, $R_{k}{ }^{(c)}$. Let the overall data correction by defined as:

$$
R_{k}^{(c)}=\widehat{\varphi} R_{k}
$$

with $\widehat{\varphi}=\hat{\eta} \hat{\kappa}$. As indicated above, $\widehat{\varphi}$ does not properly correct for measurement biases impacting in-chamber release fractions. Instead, it attempts to make in-chamber release fractions as-conservative as in-spray measurements. Using estimates of humidity bias from the preceding section (Section A.6) and estimates of method bias from Chapter 7 of this report, values for $\widehat{\varphi}$ can be proposed. Table A.7 presents overall in-chamber release fraction correction factors, $\widehat{\varphi}$, for water and clay test simulants. It should be noted that the method bias correction does not accommodate loss of aerosol from settling (which limits the upper size of aerosols measured by in-chamber methods). In general, this means that the overall correction is valid only over size ranges not impacted by in-chamber droplet loss (10 to $80 \mu \mathrm{m})$. The range of $\widehat{\varphi}$ presented in Table A.7 represents the best estimates of Phase II in-chamber measurement uncertainty; however, this estimate is subject to the assumption that RH values measured in testing are accurate. Because of data quality concerns with respect to humidity measurements, the overall correction factors listed in Table A.7 should be used "For Information Only." Under these limitations, application of this correction factor to data should yield an in-chamber result that is conservative. The overall data correction factor indicates that in-chamber data for water are at most a factor of three to five too low (from the combined effects of humidity and method bias); for the clay system, data are a factor of approximately 3 to 4 times too low.

Table A.7. Estimated Correction Factors for Method Bias and Humidity Bias and the Combined Overall Correction Factor (see Equation (A.4)) for In-Chamber Measurements. Correction factors are provided on a "For Information Only" basis.

\begin{tabular}{lccc}
\hline System & $\begin{array}{c}\text { Method Bias } \\
\text { Correction, } \hat{\eta}\end{array}$ & $\begin{array}{c}\text { Humidity Bias } \\
\text { Correction, } \hat{\kappa}\end{array}$ & $\begin{array}{c}\text { Overall } \\
\text { Correction, } \widehat{\varphi}\end{array}$ \\
\hline \multicolumn{4}{c}{ Maximum Correction } \\
\hline Water & 3 & $1.5 \pm 0.2$ & $4.5 \pm 0.6$ \\
Clay & 3 & $1.2 \pm 0.1$ & $3.6 \pm 0.3$ \\
\hline \multicolumn{4}{c}{ Median Correction } \\
\hline Water & 3 & $1.2 \pm 0.2$ & $3.6 \pm 0.6$ \\
Clay & 3 & $1.1 \pm 0.1$ & $3.3 \pm 0.3$ \\
\hline
\end{tabular}


Appendix B

\section{Phase II Large-Scale Test Run Log}





\section{Appendix B}

\section{Phase II Large-Scale Test Run Log}

The tables and charts in this appendix present information and data from the parametric test matrices that were performed in the large-scale spray release system. This excludes the functional tests presented in Appendix A and Malvern Insitec-S validation testing described in Chapter 6 of the report. Table B.1 contains the parameters established during functional testing that apply to all matrix tests. Exceptions to these parameters are footnoted in the succeeding tables as appropriate. Table B.2 contains the nominal water matrix test conditions and corresponding individual Test Identification (TID) numbers. Table B.3 contains the clay (6 Pa and $30 \mathrm{~Pa}$ ) matrix test conditions and TID numbers. In-spray matrix testing with TID numbers can be found in Table B.4.

Selected PLC data for all accepted tests are presented sequentially by TID number in Table B.5. PLC data presented include average test pressure, the feed tank temperature before the start of spray, the spray leak flow rate $(Q)$ as determined by tank mass change and by differential Coriolis meter flow rate, and the discharge coefficient $\left(C_{D}\right)$ determined by tank mass and Coriolis flow rate, and the chamber relative humidity before the start of spray $\left(H_{o}\right)$. Both $Q$ and $C_{D}$ as determined by differential Coriolis meter flow rate were calculated using a Coriolis mass flow meter that was for information only (FIO) at the time of testing; thus these results can only be considered FIO and its inclusion in the table is for completeness but does not confer a pedigree equal to $Q$ and $C_{D}$ values determined from change in feed tank mass. Release fraction calculations presented in previous sections of this report are solely based on $Q$ and $C_{D}$ calculated from change in feed tank mass. Relative humidity readings $\left(H_{o}\right)$ also are derived from uncalibrated instruments and can only be considered FIO. Select PLC data are not reported for tests that were rejected during the data review cycle. Tests could be rejected for one or more reasons, typically for operational issues noted by the test crew, PLC data collection errors, poor Malvern Insitec-S performance, or Malvern Insitec-S data collection errors. Histograms summarizing $C_{D}$ by mass and flow for accepted in-chamber matrix tests using water and clay simulant (6 Pa and $30 \mathrm{~Pa}$ ) are provided in Figure B.1 and Figure B.2, respectively. The accompanying table of statistics is summarized and provided in Table B.6.

Actual test operating conditions including pressure, temperature, flow rate, humidity, and tank masses were within the tolerances provided by the governing test plan, project plan, and test instructions. The test fluid pressure for accepted in-chamber water tests at 100, 200, and 380 psig target pressures is summarized in histograms presented in Figure B.3. Similarly, in-chamber clay test pressure histograms are provided in Figure B.4. The accompanying table of statistics for both water and clay in-chamber test pressures is provided in Table B.7.

Following the tables and figures, charts of the release fractions estimated for the individual tests are presented in the order set forth in Table B.2 to Table B.4. Each figure has the release fraction calculated by the cumulative and differential methods, the corresponding 95 percent confidence intervals of the fit algorithm, and the WTP model prediction for the experimental conditions of the test. The release fractions calculated by the algorithm that were rejected based on the adjusted coefficient of determination being less than 0.5 are also shown (as " $\times$ ") without any confidence intervals. Confidence intervals that are not connected to the release fractions represent instances where the lower bound could not be estimated. Information about the test conditions is shown in the title of each figure. Note that the figures 
are not captioned and thus can only be referenced via the unique test number and other information provided in the title.

Table B.1. Typical Configurations for Matrix Tests Using Large-Scale Spray Release System

\begin{tabular}{|c|c|c|c|}
\hline & $\begin{array}{c}\text { Table B.2 } \\
\text { Water Matrix Testing }\end{array}$ & $\begin{array}{c}\text { Table B.3 } \\
\text { Clay Matrix Testing }\end{array}$ & $\begin{array}{c}\text { Table B.4 } \\
\text { In-Spray Testing }\end{array}$ \\
\hline Simulant (s) & Richland City Water & $\begin{array}{l}\text { Kaolin-Bentonite Clay } \\
(6 \mathrm{~Pa} \text { and } 30 \mathrm{~Pa})\end{array}$ & $\begin{array}{l}\text { Richland City Water and } \\
\text { Kaolin-Bentonite Clay } \\
(6 \mathrm{~Pa} \text { and } 30 \mathrm{~Pa})\end{array}$ \\
\hline $\begin{array}{l}\text { Simulant Temperature } \\
\text { Range }\left({ }^{\circ} \mathrm{F}\right)\end{array}$ & $65-85^{\circ} \mathrm{F}$ & $65-85^{\circ} \mathrm{F}$ & $65-85^{\circ} \mathrm{F}$ \\
\hline Relative Humidity (\%) & $>75 \%(\mathrm{FIO}$, varies by test $)$ & $>80 \%$ (FIO, varies by test $)$ & $>80 \%$ (FIO, varies by test $)$ \\
\hline Mixing Fan Configuration & 2 center fans, $12 \mathrm{~V}$ & 2 center fans, $12 \mathrm{~V}$ & No fans used \\
\hline Lens Size & $500 \mathrm{~mm}$ & $500 \mathrm{~mm}$ & $500 \mathrm{~mm}$ \\
\hline $\begin{array}{l}\text { Malvern Insitec-S } \\
\text { Spacing }\end{array}$ & $1000 \mathrm{~mm}$ (spacer bars) & $1000 \mathrm{~mm}$ (spacer bars) & $1000 \mathrm{~mm}$ (frames) \\
\hline $\begin{array}{l}\text { Malvern Insitec-S } \\
\text { Window Purge Rate }\end{array}$ & $\begin{array}{l}1,50, \text { or } 100 \mathrm{SCFH} \\
\text { depending on orifice and } \\
\text { pressure }\end{array}$ & $\begin{array}{l}1,50 \text {, or } 100 \mathrm{SCFH} \\
\text { depending on orifice and } \\
\text { pressure }\end{array}$ & $\begin{array}{l}50 \text { to } 150 \mathrm{SCFH} \\
\text { depending on orifice, } \\
\text { pressure, and Malvern } \\
\text { Insitec-S position }\end{array}$ \\
\hline $\begin{array}{l}\text { Malvern Insitec-S } \\
\text { Chamber Positions (see } \\
\text { Figure } 4.9 \text { and Figure } 4.12 \\
\text { for an explanation of key } \\
\text { dimensions [i.e., A, B, C, } \\
\text { D, and E] and position } \\
\text { identification [i.e., 1, 2, } \\
\text { and 3], respectively). }\end{array}$ & $\begin{array}{l}\text { A - Positions } 1,2 \text { and } 3 \text { at } \\
75,50 \text { and } 25 \% \text { of } \\
\text { chamber length from spray } \\
\text { header wall } \\
\text { B\&C }-28 \text { in. } \\
\text { D\&E }-28 \text { in. }\end{array}$ & $\begin{array}{l}\text { A - Positions } 1,2 \text { and } 3 \text { at } \\
15,10 \text {, and } 5 \mathrm{ft} \text { from spray } \\
\text { header wall } \\
\text { B\&C }-28 \text { in. } \\
\text { D\&E }-28 \text { in. }\end{array}$ & $\begin{array}{l}\text { A - Positions } 1,2 \text { and } 3 \text { at } \\
12,8.5 \text {, and } 5 \mathrm{ft} \text { from } \\
\text { spray header wall } \\
\text { Dimensions B, C, D, and } \\
\text { E - adjusted as necessary } \\
\text { to center the core of spray } \\
\text { in the Malvern Insitec-S } \\
\text { measurement laser beam }\end{array}$ \\
\hline
\end{tabular}


Table B.2. In-Chamber Matrix Tests with Water

\begin{tabular}{|c|c|c|c|c|c|}
\hline Simulant & $\begin{array}{l}\text { Chamber } \\
\text { Length }(\mathrm{ft})\end{array}$ & $\begin{array}{c}\text { Spray } \\
\text { Orifice } \\
\text { Distance } \\
\text { from Splash } \\
\text { Wall (ft) }\end{array}$ & $\begin{array}{l}\text { Orifice } \\
(\mathrm{mm})\end{array}$ & $\begin{array}{l}\text { Target Test } \\
\text { Fluid } \\
\text { Pressure } \\
\text { (psig) }\end{array}$ & Accepted Tests \\
\hline Water & 39 & 38 & 2 & $\begin{array}{l}100 \\
200 \\
380\end{array}$ & $\begin{array}{l}\text { W531, W534, W538 } \\
\text { W532, W535, W536, W539 } \\
\text { W533, W537, W540 }\end{array}$ \\
\hline Water & 39 & 38 & $1 \times 10$ & $\begin{array}{l}100 \\
200 \\
380\end{array}$ & $\begin{array}{l}\text { W541, W546, W547 } \\
\text { W548, W549, W550 } \\
\text { W551, W552, W553 }\end{array}$ \\
\hline Water & 39 & 38 & $1 \times 20$ & $\begin{array}{l}100 \\
200 \\
380\end{array}$ & $\begin{array}{l}\text { W554, W557, W560 } \\
\text { W558, W561 } \\
\text { W556, W562 }\end{array}$ \\
\hline Water & 39 & 38 & $1 \times 76$ & $\begin{array}{l}100 \\
200 \\
380\end{array}$ & $\begin{array}{l}\text { W581, W584, W587 } \\
\text { W585, W588, W589, W593, W594, W595 } \\
\text { W583, W586, W590, W591, W592 }\end{array}$ \\
\hline Water & 39 & 28 & 2 & $\begin{array}{l}100 \\
200 \\
380\end{array}$ & $\begin{array}{l}\text { W385 } \\
\text { W380, W383, W386 } \\
\text { W381, W384, W387 }\end{array}$ \\
\hline Water & 39 & 28 & $1 \times 10$ & $\begin{array}{l}100 \\
200 \\
380\end{array}$ & $\begin{array}{l}\text { W388, W391, W393 } \\
\text { W389, W392, W394 } \\
\text { W390, W395, W396, W397, W398 }\end{array}$ \\
\hline Water & 39 & 28 & $1 \times 20$ & $\begin{array}{l}100 \\
200 \\
380\end{array}$ & $\begin{array}{l}\text { W403, W405, W407, W409 } \\
\text { W404, W406, W408, W410 } \\
\text { W401, W402 }\end{array}$ \\
\hline Water & 39 & 28 & $1 \times 76$ & $\begin{array}{l}100 \\
200 \\
380\end{array}$ & $\begin{array}{l}\text { W412, W413, W414 } \\
\text { W415, W416, W417, W419 } \\
\text { W420, W421, W422 }\end{array}$ \\
\hline Water & 39 & 19 & $1 \times 76$ & $\begin{array}{l}100 \\
200 \\
380\end{array}$ & $\begin{array}{l}\text { W441, W446, W450 } \\
\text { W439, W440, W442, W447, W448, W451 } \\
\text { W443, W444, W445, W449, W452, W454 }\end{array}$ \\
\hline Water & 39 & 19 & 2 & $\begin{array}{l}100 \\
200 \\
380\end{array}$ & $\begin{array}{l}\text { W455, W458, W461 } \\
\text { W456, W459, W462 } \\
\text { W457, W460, W463 }\end{array}$ \\
\hline Water & 39 & 19 & $1 \times 10$ & $\begin{array}{l}100 \\
200 \\
380\end{array}$ & $\begin{array}{l}\text { W464, W467, W470 } \\
\text { W465, W468, W471 } \\
\text { W466, W469, W472, W528, W529, W530 }\end{array}$ \\
\hline Water & 39 & 19 & $1 \times 20$ & $\begin{array}{l}100 \\
200 \\
380\end{array}$ & $\begin{array}{l}\text { W473, W474, W475, W479, W482, W485 } \\
\text { W476, W480, W483, W486 } \\
\text { W478, W481, W484, W488 }\end{array}$ \\
\hline Water & 39 & 3.5 & 2 & $\begin{array}{l}100 \\
200 \\
380\end{array}$ & $\begin{array}{l}\text { W489, W492, W495 } \\
\text { W490, W493, W496 } \\
\text { W494, W497 }\end{array}$ \\
\hline Water & 39 & 3.5 & $1 \times 10$ & $\begin{array}{l}100 \\
200 \\
380\end{array}$ & $\begin{array}{l}\text { W498, W501, W504 } \\
\text { W499, W502, W505 } \\
\text { W500, W503, W506 }\end{array}$ \\
\hline
\end{tabular}


Table B.2. (contd)

\begin{tabular}{|c|c|c|c|c|c|}
\hline Simulant & $\begin{array}{l}\text { Chamber } \\
\text { Length (ft) }\end{array}$ & $\begin{array}{c}\text { Spray } \\
\text { Orifice } \\
\text { Distance } \\
\text { from Splash } \\
\text { Wall (ft) }\end{array}$ & $\begin{array}{c}\text { Nominal } \\
\text { Orifice } \\
(\mathrm{mm})\end{array}$ & $\begin{array}{l}\text { Target Test } \\
\text { Fluid } \\
\text { Pressure } \\
\text { (psig) }\end{array}$ & Accepted Tests \\
\hline \multirow{3}{*}{ Water } & \multirow{3}{*}{39} & \multirow{3}{*}{3.5} & \multirow{3}{*}{$1 \times 20$} & 100 & W507, W511, W514 \\
\hline & & & & 200 & W509, W512, W515, W517 \\
\hline & & & & 380 & W510, W513, W516, W518 \\
\hline \multirow{3}{*}{ Water } & \multirow{3}{*}{39} & \multirow{3}{*}{3.5} & \multirow{3}{*}{$1 \times 76$} & 100 & W519, W522, W525 \\
\hline & & & & 200 & W520, W523, W526 \\
\hline & & & & 380 & W521, W524, W527 \\
\hline \multirow{3}{*}{ Water } & \multirow{3}{*}{30} & \multirow{3}{*}{29} & \multirow{3}{*}{2} & 100 & W620, W623 \\
\hline & & & & 200 & W618, W621, W624 \\
\hline & & & & 380 & W619, W622, W625 \\
\hline \multirow{3}{*}{ Water } & \multirow{3}{*}{30} & \multirow{3}{*}{29} & \multirow{3}{*}{$1 \times 10$} & 100 & W626, W629, W632 \\
\hline & & & & 200 & W627, W630, W633 \\
\hline & & & & 380 & W631, W634 \\
\hline \multirow{3}{*}{ Water } & \multirow{3}{*}{30} & \multirow{3}{*}{29} & \multirow{3}{*}{$1 \times 20$} & 100 & W635, W638, W641 \\
\hline & & & & 200 & W636, W639, W642 \\
\hline & & & & 380 & W637, W640, W643 \\
\hline \multirow{3}{*}{ Water } & \multirow{3}{*}{30} & \multirow{3}{*}{29} & \multirow{3}{*}{$1 \times 76$} & 100 & W644, W647, W650 \\
\hline & & & & 200 & W645, W648, W651 \\
\hline & & & & 380 & W649, W653, W654 \\
\hline \multirow{3}{*}{ Water } & & & & 100 & W662, W665, W668 \\
\hline & 20 & 19 & 2 & 200 & W663, W666, W669 \\
\hline & & & & 380 & W664, W667, W670 \\
\hline & & & & 100 & W671, W674, W677 \\
\hline Water & 20 & 19 & $1 \times 10$ & 200 & W672, W675, W678 \\
\hline & & & & 380 & W673, W676, W679 \\
\hline & & & & 100 & W680, W683, W686 \\
\hline Water & 20 & 19 & $1 \times 20$ & 200 & W681, W684, W687 \\
\hline & & & & 380 & W682, W685, W688, W689 \\
\hline & & & & 100 & W699, W703, W704 \\
\hline Water & 20 & 19 & $1 \times 76$ & 200 & W700, W701, W705 \\
\hline & & & & 380 & W702, W706, W707 \\
\hline & & & & 100 & W717, W720, W723 \\
\hline Water & 10 & 9.0 & 2 & 200 & W718, W721, W724 \\
\hline & & & & 380 & W719, W722, W725 \\
\hline & & & & 100 & W726, W727, W728 \\
\hline Water & 10 & 9.0 & $1 \times 10$ & 200 & W729, W731, W733 \\
\hline & & & & 380 & W730, W732, W734 \\
\hline & & & & 100 & W735, W741, W743 \\
\hline Water & 10 & 9.0 & $1 \times 20$ & 200 & W736, W742, W744 \\
\hline & & & & 380 & W737, W738, W740, W745 \\
\hline & & & & 100 & W708, W711, W714 \\
\hline Water & 10 & 9.0 & $1 \times 76$ & 200 & W709, W712, W715 \\
\hline & & & & 380 & W710, W713, W716 \\
\hline
\end{tabular}


Table B.3. In-Chamber Matrix Tests with $6 \mathrm{~Pa}$ and $30 \mathrm{~Pa}$ Clay Simulant

\begin{tabular}{|c|c|c|c|c|}
\hline Simulant & $\begin{array}{l}\text { Chamber } \\
\text { Length (ft) }\end{array}$ & $\begin{array}{l}\text { Nominal } \\
\text { Orifice } \\
(\mathrm{mm})\end{array}$ & $\begin{array}{l}\text { Target Test } \\
\text { Fluid } \\
\text { Pressure } \\
\text { (psig) }\end{array}$ & Accepted Tests \\
\hline \multirow{3}{*}{ Clay (6 Pa) } & \multirow{3}{*}{20} & \multirow{3}{*}{2} & 100 & S810, S811, S812 \\
\hline & & & 200 & S814, S815 \\
\hline & & & 380 & S816, S817, S818 \\
\hline \multirow{3}{*}{ Clay (6 Pa) } & \multirow{3}{*}{20} & \multirow{3}{*}{$1 \times 10$} & 100 & S819, S824, S826 \\
\hline & & & 200 & S820, S825, S827 \\
\hline & & & 380 & $\mathrm{~S} 821, \mathrm{~S} 822, \mathrm{~S} 823$ \\
\hline \multirow{3}{*}{ Clay (6 Pa) } & \multirow{3}{*}{20} & \multirow{3}{*}{$1 \times 20$} & 100 & $\mathrm{~S} 829, \mathrm{~S} 830$ \\
\hline & & & 200 & S831, S832, S833 \\
\hline & & & 380 & S835, S836, S837 \\
\hline \multirow{3}{*}{ Clay (6 Pa) } & \multirow{3}{*}{20} & \multirow{3}{*}{$1 \times 76$} & 100 & S838, S839, S842, S843 \\
\hline & & & 200 & $\mathrm{~S} 844, \mathrm{~S} 845, \mathrm{~S} 846$ \\
\hline & & & 380 & $\mathrm{~S} 848, \mathrm{~S} 849, \mathrm{~S} 850$ \\
\hline \multirow{3}{*}{ Clay (30 Pa) } & \multirow{3}{*}{20} & \multirow{3}{*}{2} & 100 & S746, S747, S748, S749 \\
\hline & & & 200 & S757, S758, S759 \\
\hline & & & 380 & S753, S760, S763 \\
\hline \multirow{3}{*}{ Clay (30 Pa) } & \multirow{3}{*}{20} & \multirow{3}{*}{$1 \times 10$} & 100 & S754, S755, S756 \\
\hline & & & 200 & $\mathrm{~S} 750, \mathrm{~S} 751, \mathrm{~S} 752$ \\
\hline & & & 380 & S764, S765 \\
\hline \multirow{3}{*}{ Clay (30 Pa) } & \multirow{3}{*}{20} & \multirow{3}{*}{$1 \times 20$} & 100 & S761, S762, S766 \\
\hline & & & 200 & S767, S769, S770 \\
\hline & & & 380 & S768, S771, S772 \\
\hline \multirow{3}{*}{ Clay (30 Pa) } & \multirow{3}{*}{20} & \multirow{3}{*}{$1 \times 76$} & 100 & S773, S774, S775 \\
\hline & & & 200 & S776, S777, S778 \\
\hline & & & 380 & $\mathrm{~S} 780, \mathrm{~S} 781$ \\
\hline
\end{tabular}


Table B.4. In-Spray Matrix Tests with Water and Clay (6 Pa, 30 Pa) Simulants

\begin{tabular}{|c|c|c|c|c|}
\hline Simulant & $\begin{array}{c}\text { Chamber } \\
\text { Length }(\mathrm{ft})\end{array}$ & $\begin{array}{c}\text { Nominal } \\
\text { Orifice } \\
(\mathrm{mm})\end{array}$ & $\begin{array}{l}\text { Target Test } \\
\text { Fluid } \\
\text { Pressure } \\
\text { (psig) }\end{array}$ & Accepted Tests \\
\hline \multirow{3}{*}{ Water } & \multirow{3}{*}{39} & \multirow{3}{*}{2} & 100 & W606, W607, W608 \\
\hline & & & 200 & W603, W604, W605 \\
\hline & & & 380 & W601, W602 \\
\hline \multirow{3}{*}{ Water } & \multirow{3}{*}{39} & \multirow{3}{*}{$1 \times 10$} & 100 & W609 \\
\hline & & & 200 & W610 \\
\hline & & & 380 & W611 \\
\hline \multirow{3}{*}{ Water } & \multirow{3}{*}{39} & \multirow{3}{*}{$1 \times 20$} & 100 & W612, W614 \\
\hline & & & 200 & W613 \\
\hline & & & 380 & W615, W616 \\
\hline \multirow{3}{*}{ Water } & \multirow{3}{*}{39} & \multirow{3}{*}{$1 \times 76^{(\mathrm{a})}$} & 100 & W596 \\
\hline & & & 200 & W597, W598 \\
\hline & & & 380 & W599 \\
\hline \multirow{3}{*}{ Clay $(6 \mathrm{~Pa})$} & \multirow{3}{*}{20} & \multirow{3}{*}{2} & 100 & $\mathrm{~S} 801$ \\
\hline & & & 200 & $\mathrm{~S} 802$ \\
\hline & & & 380 & $\mathrm{~S} 803$ \\
\hline \multirow{3}{*}{ Clay (6 Pa) } & \multirow{3}{*}{20} & \multirow{3}{*}{$1 \times 10$} & 100 & $\mathrm{~S} 804$ \\
\hline & & & 200 & $\mathrm{~S} 805$ \\
\hline & & & 380 & S806 \\
\hline \multirow{3}{*}{ Clay (6 Pa) } & \multirow{3}{*}{20} & \multirow{3}{*}{$1 \times 20$} & 100 & S807 \\
\hline & & & 200 & S808 \\
\hline & & & 380 & S809 \\
\hline \multirow{3}{*}{ Clay (6 Pa) } & \multirow{3}{*}{20} & \multirow{3}{*}{$1 \times 76$} & 100 & $\mathrm{~S} 800$ \\
\hline & & & 200 & S799 \\
\hline & & & 380 & S798 \\
\hline \multirow{3}{*}{ Clay (30 Pa) } & \multirow{3}{*}{20} & \multirow{3}{*}{2} & 100 & S783 \\
\hline & & & 200 & S785 \\
\hline & & & 380 & S786 \\
\hline \multirow{3}{*}{ Clay (30 Pa) } & & & 100 & S788 \\
\hline & 20 & $1 \times 10$ & 200 & S789 \\
\hline & & & 380 & S791 \\
\hline & & & 100 & S790 \\
\hline Clay (30 Pa) & 20 & $1 \times 20$ & 200 & S792 \\
\hline & & & 380 & S793 \\
\hline & & & 100 & S794 \\
\hline Clay (30 Pa) & 20 & $1 \times 76$ & 200 & S795 \\
\hline & & & 380 & S796, S797 \\
\hline
\end{tabular}

(a) Malvern Insitec-S positions were different for water in-spray testing with the nominal $1 \times 76.2 \mathrm{~mm}$ slot orifice. For these tests, dimension A was 20, 12.5 and $5 \mathrm{ft}$ for positions 1, 2 and 3, respectively, with dimensions B, C, D, and E adjusted as necessary to center the core of the spray in the Malvern Insitec-S measurement laser beam (see Table B.1). 
Table B.5. Selected PLC Data for Accepted Large-Scale In-Chamber, In-Spray, and Functional Tests

\begin{tabular}{|c|c|c|c|c|c|c|c|c|c|c|c|c|c|}
\hline Test ID & $\begin{array}{c}\text { Test } \\
\text { Category }\end{array}$ & Simulant & $\begin{array}{c}\text { Chamber } \\
\text { ID }\end{array}$ & $\begin{array}{l}\text { Orifice } \\
\text { ID }\end{array}$ & $\begin{array}{c}\text { Average } \\
\text { Pressure } \\
\text { [psig] }\end{array}$ & $\begin{array}{c}\text { Test Temp. } \\
{\left[{ }^{\circ} \mathrm{F}\right]}\end{array}$ & $\begin{array}{c}Q \text { (mass) } \\
{[\mathrm{gpm}]}\end{array}$ & $\begin{array}{c}Q \times 10^{3} \\
(\mathrm{mass}) \\
{\left[\mathrm{m}^{3} \mathrm{~s}^{-1}\right]}\end{array}$ & $\begin{array}{c}Q \\
\text { (flow) }^{(\mathrm{a})} \\
{[\mathrm{gpm}]}\end{array}$ & $\begin{array}{c}Q \times 10^{3} \\
(\text { flow) } \\
(\mathrm{a}) \\
{\left[\mathrm{m}^{3} \mathrm{~s}^{-1}\right]}\end{array}$ & $\begin{array}{c}C_{D} \\
\text { (mass) }\end{array}$ & $\begin{array}{c}C_{D} \\
\text { (flow) }^{\text {(a) }}\end{array}$ & $\begin{array}{c}H_{o}^{(\mathrm{a})} \\
{[\%]}\end{array}$ \\
\hline W79 & Functional & Water & $20 \mathrm{~S} 3 \mathrm{~A}$ & S1D & 102.2 & 67.8 & 1.3 & 0.082 & 1.3 & 0.085 & 0.623 & 0.646 & $--^{(b)}$ \\
\hline W80 & Functional & Water & $20 \mathrm{~S} 3 \mathrm{~A}$ & S1D & 199.4 & 67.3 & 1.8 & 0.114 & 1.7 & 0.109 & 0.620 & 0.591 & $--^{(b)}$ \\
\hline W81 & Functional & Water & $20 \mathrm{~S} 3 \mathrm{~A}$ & S1D & 379.3 & 68.7 & 2.5 & 0.161 & 2.5 & 0.160 & 0.635 & 0.630 & $--^{(b)}$ \\
\hline W86 & Functional & Water & $20 \mathrm{~S} 3 \mathrm{~A}$ & S1D & 99.7 & 68.1 & 1.3 & 0.084 & 1.3 & 0.083 & 0.649 & 0.638 & $--^{(b)}$ \\
\hline W87 & Functional & Water & $20 \mathrm{~S} 3 \mathrm{~A}$ & S1D & 200.1 & 68.2 & 1.8 & 0.115 & 1.2 & 0.077 & 0.625 & 0.417 & $--^{(b)}$ \\
\hline W88 & Functional & Water & $20 \mathrm{~S} 3 \mathrm{~A}$ & S1D & 376.6 & 70.0 & 2.5 & 0.158 & - - $^{(c)}$ & $--^{(c)}$ & 0.626 & - -( $^{(c)}$ & $--^{(b)}$ \\
\hline W267 & Functional & Water & $39 \mathrm{~S} 3 \mathrm{~A}$ & S8A & 379.5 & 73.4 & 2.0 & 0.127 & 2.5 & 0.157 & 0.559 & 0.690 & 100.9 \\
\hline W268 & Functional & Water & $39 \mathrm{~S} 3 \mathrm{~A}$ & S8A & 379.7 & 74.5 & 2.1 & 0.131 & 1.8 & 0.114 & 0.578 & 0.500 & 101.2 \\
\hline W270 & Functional & Water & $39 \mathrm{~S} 3 \mathrm{~A}$ & $\mathrm{~S} 8 \mathrm{C}$ & 375.7 & 75.2 & 15.5 & 0.981 & 15.0 & 0.953 & 0.687 & 0.667 & 102.0 \\
\hline W271 & Functional & Water & 39S3A & $\mathrm{S} 8 \mathrm{C}$ & 374.6 & 67.6 & 14.4 & 0.912 & 13.6 & 0.859 & 0.640 & 0.602 & 88.6 \\
\hline W273 & Functional & Water & $39 \mathrm{~S} 3 \mathrm{~A}$ & S8A & 377.8 & 70.0 & 2.0 & 0.129 & 2.3 & 0.145 & 0.570 & 0.638 & 86.5 \\
\hline W274 & Functional & Water & $39 \mathrm{~S} 3 \mathrm{~A}$ & S8A & 377.7 & 71.9 & 2.2 & 0.137 & 2.1 & 0.135 & 0.606 & 0.595 & 85.6 \\
\hline W275 & Functional & Water & 39S3A & $\mathrm{S} 8 \mathrm{C}$ & 374.5 & 73.6 & 15.2 & 0.961 & 14.2 & 0.897 & 0.674 & 0.629 & 85.1 \\
\hline W276 & Functional & Water & $39 \mathrm{~S} 3 \mathrm{~A}$ & $\mathrm{~S} 8 \mathrm{C}$ & 374.7 & 75.1 & 15.0 & 0.950 & 14.9 & 0.946 & 0.666 & 0.663 & 96.3 \\
\hline W277 & Functional & Water & $39 \mathrm{~S} 3 \mathrm{~A}$ & S8A & 378.5 & 70.1 & 2.1 & 0.132 & 2.3 & 0.145 & 0.581 & 0.638 & 70.9 \\
\hline W282 & Functional & Water & 39S3A & S8A & 377.7 & 69.1 & 2.0 & 0.127 & 2.7 & 0.173 & 0.559 & 0.763 & 67.3 \\
\hline W283 & Functional & Water & 39S3A & S8A & 377.8 & 70.6 & 2.0 & 0.130 & 1.7 & 0.110 & 0.572 & 0.485 & 75.2 \\
\hline W284 & Functional & Water & 39S3A & S8A & 377.7 & 72.4 & 2.0 & 0.129 & 2.4 & 0.151 & 0.570 & 0.666 & 77.6 \\
\hline W285 & Functional & Water & $39 \mathrm{~S} 3 \mathrm{~A}$ & S8A & 377.7 & 73.8 & 2.0 & 0.126 & 2.6 & 0.168 & 0.557 & 0.739 & 75.3 \\
\hline W286 & Functional & Water & 39S3A & S8A & 377.3 & 74.9 & 2.0 & 0.127 & 2.7 & 0.173 & 0.561 & 0.761 & 75.6 \\
\hline W287 & Functional & Water & 39S3A & S8A & 377.0 & 75.9 & 2.1 & 0.132 & 2.5 & 0.158 & 0.583 & 0.695 & 72.6 \\
\hline W288 & Functional & Water & 39S3A & S8A & 377.2 & 77.0 & 2.1 & 0.132 & 2.2 & 0.137 & 0.581 & 0.604 & 72.0 \\
\hline W289 & Functional & Water & 39S3A & S8A & 376.1 & 75.4 & 2.1 & 0.132 & 2.5 & 0.158 & 0.585 & 0.700 & 76.1 \\
\hline W290 & Functional & Water & 39S3A & S8A & 377.9 & 75.2 & 2.0 & 0.127 & 2.6 & 0.162 & 0.559 & 0.712 & 82.9 \\
\hline W291 & Functional & Water & 39S3A & S8A & 378.3 & 76.2 & 2.1 & 0.131 & 2.0 & 0.129 & 0.576 & 0.568 & 77.2 \\
\hline W292 & Functional & Water & 39S3A & S8A & 378.3 & 63.6 & 2.1 & 0.130 & 3.1 & 0.198 & 0.574 & 0.873 & 53.6 \\
\hline W293 & Functional & Water & 39S3A & S8A & 378.2 & 65.6 & 2.1 & 0.130 & 3.7 & 0.236 & 0.575 & 1.040 & 74.1 \\
\hline W294 & Functional & Water & 39S3A & S8A & 378.1 & 70.1 & 2.0 & 0.129 & 2.9 & 0.182 & 0.567 & 0.802 & 90.2 \\
\hline W295 & Functional & Water & 39S3A & S8A & 377.4 & 70.0 & 2.0 & 0.129 & 2.0 & 0.126 & 0.570 & 0.554 & 93.4 \\
\hline W296 & Functional & Water & 39S3A & S8A & 377.3 & 71.1 & 2.0 & 0.129 & 2.8 & 0.179 & 0.570 & 0.789 & 91.8 \\
\hline
\end{tabular}

(a) -Instrument(s) upon which measurements are based were not calibrated. Result is "For-Information Only"; (b) - Humidity data collected but not reported for W79-W81 and W86-W88; (c) - Coriolis meter instrument reading/sensitivity issue yielded anomalous result. 
Table B.5. (contd)

\begin{tabular}{|c|c|c|c|c|c|c|c|c|c|c|c|c|c|}
\hline Test ID & $\begin{array}{c}\text { Test } \\
\text { Category }\end{array}$ & Simulant & $\begin{array}{c}\text { Chamber } \\
\text { ID }\end{array}$ & $\begin{array}{c}\text { Orifice } \\
\text { ID }\end{array}$ & $\begin{array}{c}\text { Average } \\
\text { Pressure } \\
\text { [psig] }\end{array}$ & $\begin{array}{c}\text { Test Temp. } \\
{\left[{ }^{\circ} \mathrm{F}\right]}\end{array}$ & $\begin{array}{c}Q \text { (mass) } \\
{[\mathrm{gpm}]}\end{array}$ & $\begin{array}{c}Q \times 10^{3} \\
(\mathrm{mass}) \\
{\left[\mathrm{m}^{3} \mathrm{~s}^{-1}\right]}\end{array}$ & $\begin{array}{c}Q \\
(\text { flow) } \\
{[\text { (a) }}\end{array}$ & $\begin{array}{l}Q \times 10^{3} \\
(\text { flow) } \\
(\mathrm{a}) \\
{\left[\mathrm{m}^{3} \mathrm{~s}^{-1}\right]}\end{array}$ & $\begin{array}{c}C_{D} \\
\text { (mass) }\end{array}$ & $\begin{array}{c}C_{D} \\
\text { (flow) }^{\text {(a) }}\end{array}$ & $\begin{array}{c}H_{o}^{(\mathrm{a})} \\
{[\%]}\end{array}$ \\
\hline W297 & Functional & Water & 39S3A & S8A & 376.3 & 71.9 & 2.1 & 0.130 & 2.3 & 0.148 & 0.575 & 0.656 & 91.2 \\
\hline W298 & Functional & Water & 39S3A & S8A & 375.9 & 73.0 & 2.0 & 0.130 & 2.3 & 0.143 & 0.572 & 0.631 & 90.1 \\
\hline W299 & Functional & Water & 39S3A & S8A & 376.4 & 73.7 & 2.0 & 0.130 & 2.2 & 0.142 & 0.573 & 0.629 & 89.1 \\
\hline W300 & Functional & Water & $39 \mathrm{~S} 3 \mathrm{~A}$ & S8A & 375.9 & 71.0 & 2.1 & 0.130 & 1.7 & 0.110 & 0.575 & 0.487 & 71.7 \\
\hline W301 & Functional & Water & 39S3A & S8A & 375.8 & 71.2 & 2.0 & 0.127 & 2.9 & 0.182 & 0.564 & 0.805 & 80.8 \\
\hline W302 & Functional & Water & 39S3A & S8A & 375.2 & 71.2 & 2.0 & 0.129 & 2.1 & 0.132 & 0.573 & 0.586 & 79.8 \\
\hline W303 & Functional & Water & 39S3A & S8A & 375.9 & 71.6 & 2.0 & 0.128 & 1.7 & 0.106 & 0.567 & 0.467 & 79.0 \\
\hline W304 & Functional & Water & $39 \mathrm{~S} 3 \mathrm{~A}$ & S8A & 374.8 & 72.5 & 2.0 & 0.127 & 2.6 & 0.166 & 0.563 & 0.736 & 78.2 \\
\hline W305 & Functional & Water & 39S3A & S8A & 375.1 & 72.9 & 2.0 & 0.126 & 3.1 & 0.198 & 0.560 & 0.876 & 76.7 \\
\hline W315 & Functional & Water & 39S3A & S4A & 370.9 & 67.9 & 63.3 & 4.010 & 58.2 & 3.683 & 0.766 & 0.704 & 83.5 \\
\hline W316 & Functional & Water & 39S3A & S4A & 372.2 & 69.8 & 63.5 & 4.025 & 57.7 & 3.653 & 0.767 & 0.697 & 93.2 \\
\hline W317 & Functional & Water & 39S3A & S4A & 357.5 & 69.4 & 64.4 & 4.077 & 56.7 & 3.592 & 0.793 & 0.699 & 95.4 \\
\hline W318 & Functional & Water & 39S3A & S4A & 378.2 & 77.0 & 63.1 & 3.995 & 57.8 & 3.663 & 0.755 & 0.692 & 91.9 \\
\hline W320 & Functional & Water & 39S3A & S4A & 378.2 & 75.5 & 63.2 & 4.001 & 58.6 & 3.713 & 0.756 & 0.702 & 98.1 \\
\hline W321 & Functional & Water & 39S3A & S8A & 380.0 & 71.7 & 2.5 & 0.161 & 1.9 & 0.121 & 0.708 & 0.531 & 85.1 \\
\hline W322 & Functional & Water & 39S3A & S8A & 379.9 & 72.3 & 2.6 & 0.164 & 1.8 & 0.112 & 0.720 & 0.492 & 84.8 \\
\hline W323 & Functional & Water & 39S3A & S8A & 379.7 & 73.2 & 2.6 & 0.163 & 1.8 & 0.113 & 0.719 & 0.498 & 82.1 \\
\hline W324 & Functional & Water & 39S3A & $\mathrm{S} 8 \mathrm{C}$ & 379.1 & 73.8 & 14.7 & 0.931 & 13.6 & 0.862 & 0.649 & 0.600 & 80.4 \\
\hline W325 & Functional & Water & 39S3A & $\mathrm{S} 8 \mathrm{C}$ & 379.0 & 74.1 & 14.7 & 0.933 & 13.8 & 0.872 & 0.650 & 0.607 & 87.9 \\
\hline W326 & Functional & Water & 39S3A & $\mathrm{S} 8 \mathrm{C}$ & 379.0 & 74.7 & 14.7 & 0.929 & 13.5 & 0.857 & 0.647 & 0.597 & 91.5 \\
\hline W327 & Functional & Water & 39S3A & S8A & 379.3 & 76.2 & 2.9 & 0.185 & 2.2 & 0.137 & 0.813 & 0.603 & 91.8 \\
\hline W328 & Functional & Water & 39S3A & $\mathrm{S} 8 \mathrm{C}$ & 378.6 & 76.4 & 14.6 & 0.924 & 13.7 & 0.869 & 0.644 & 0.606 & 83.8 \\
\hline W380 & In-chamber & Water & 39S3B & S8A & 201.8 & 74.1 & 1.2 & 0.074 & 1.6 & 0.104 & 0.449 & 0.625 & 78.2 \\
\hline W381 & In-chamber & Water & 39S3B & S8A & 382.4 & 75.9 & 2.0 & 0.128 & 2.1 & 0.130 & 0.561 & 0.570 & 75.2 \\
\hline W383 & In-chamber & Water & 39S3B & S8A & 201.7 & 73.3 & 1.0 & 0.061 & 1.5 & 0.096 & 0.371 & 0.578 & 77.4 \\
\hline W384 & In-chamber & Water & 39S3B & S8A & 382.0 & 74.6 & 2.1 & 0.135 & 1.9 & 0.121 & 0.590 & 0.528 & 79.1 \\
\hline W385 & In-chamber & Water & 39S3B & S8A & 104.3 & 73.3 & 0.2 & 0.014 & 1.2 & 0.075 & 0.120 & 0.628 & 79.6 \\
\hline W386 & In-chamber & Water & 39S3B & S8A & 201.6 & 73.3 & 1.5 & 0.096 & 1.4 & 0.089 & 0.582 & 0.539 & 80.5 \\
\hline W387 & In-chamber & Water & 39S3B & S8A & 382.1 & 74.9 & 2.2 & 0.140 & 1.9 & 0.119 & 0.613 & 0.522 & 80.0 \\
\hline W388 & In-chamber & Water & 39S3B & S8B & 104.1 & 69.8 & 3.6 & 0.226 & 4.0 & 0.255 & 0.624 & 0.705 & 83.2 \\
\hline
\end{tabular}

(a) -Instrument(s) upon which measurements are based were not calibrated. Result is "For-Information Only"; (b) - Humidity data collected but not reported for W79-W81 and W86-W88; (c) - Coriolis meter instrument reading/sensitivity issue yielded anomalous result. 
Table B.5. (contd)

\begin{tabular}{|c|c|c|c|c|c|c|c|c|c|c|c|c|c|}
\hline Test ID & $\begin{array}{c}\text { Test } \\
\text { Category }\end{array}$ & Simulant & $\begin{array}{c}\text { Chamber } \\
\text { ID }\end{array}$ & $\begin{array}{c}\text { Orifice } \\
\text { ID }\end{array}$ & $\begin{array}{c}\text { Average } \\
\text { Pressure } \\
\text { [psig] }\end{array}$ & $\begin{array}{c}\text { Test Temp. } \\
{\left[{ }^{\circ} \mathrm{F}\right]}\end{array}$ & $\begin{array}{c}Q \text { (mass) } \\
{[\mathrm{gpm}]}\end{array}$ & $\begin{array}{c}Q \times 10^{3} \\
(\mathrm{mass}) \\
{\left[\mathrm{m}^{3} \mathrm{~s}^{-1}\right]}\end{array}$ & $\begin{array}{c}Q \\
(\text { flow) } \\
{[\text { (a) }}\end{array}$ & $\begin{array}{l}Q \times 10^{3} \\
(\text { flow) } \\
(\mathrm{a}) \\
{\left[\mathrm{m}^{3} \mathrm{~s}^{-1}\right]}\end{array}$ & $\begin{array}{c}C_{D} \\
\text { (mass) }\end{array}$ & $\begin{array}{c}C_{D} \\
\text { (flow) }^{\text {(a) }}\end{array}$ & $\begin{array}{c}H_{o}^{(\mathrm{a})} \\
{[\%]}\end{array}$ \\
\hline W389 & In-chamber & Water & 39S3B & S8B & 201.1 & 70.3 & 5.2 & 0.332 & 5.0 & 0.319 & 0.661 & 0.635 & 81.5 \\
\hline W390 & In-chamber & Water & 39S3B & S8B & 380.8 & 72.2 & 7.2 & 0.459 & 6.7 & 0.422 & 0.663 & 0.610 & 79.5 \\
\hline W391 & In-chamber & Water & 39S3B & S8B & 103.9 & 71.7 & 3.1 & 0.199 & 3.6 & 0.225 & 0.550 & 0.623 & 82.0 \\
\hline W392 & In-chamber & Water & 39S3B & S8B & 200.9 & 72.2 & 4.9 & 0.310 & 4.8 & 0.306 & 0.617 & 0.610 & 80.2 \\
\hline W393 & In-chamber & Water & 39S3B & S8B & 104.3 & 71.8 & 3.2 & 0.202 & 3.6 & 0.230 & 0.558 & 0.636 & 80.5 \\
\hline W394 & In-chamber & Water & 39S3B & S8B & 200.8 & 71.6 & 5.3 & 0.333 & 4.9 & 0.312 & 0.664 & 0.622 & 79.5 \\
\hline W395 & In-chamber & Water & 39S3B & S8B & 380.6 & 73.4 & 7.3 & 0.459 & 6.8 & 0.432 & 0.664 & 0.625 & 83.3 \\
\hline W396 & In-chamber & Water & 39S3B & S8B & 380.6 & 75.6 & 7.3 & 0.460 & 7.2 & 0.459 & 0.665 & 0.664 & 80.7 \\
\hline W397 & In-chamber & Water & 39S3B & S8B & 381.0 & 70.5 & 6.4 & 0.405 & 6.8 & 0.429 & 0.586 & 0.620 & 90.5 \\
\hline W398 & In-chamber & Water & 39S3B & S8B & 380.8 & 70.6 & 6.3 & 0.401 & 6.6 & 0.416 & 0.580 & 0.602 & 89.1 \\
\hline W401 & In-chamber & Water & 39S3B & $\mathrm{S} 8 \mathrm{C}$ & 379.3 & 68.5 & 14.7 & 0.934 & 15.0 & 0.948 & 0.651 & 0.660 & 84.4 \\
\hline W402 & In-chamber & Water & 39S3B & $\mathrm{S} 8 \mathrm{C}$ & 379.2 & 69.6 & 14.8 & 0.935 & 14.7 & 0.931 & 0.651 & 0.649 & 82.5 \\
\hline W403 & In-chamber & Water & 39S3B & $\mathrm{S} 8 \mathrm{C}$ & 103.3 & 67.7 & 7.8 & 0.494 & 7.7 & 0.490 & 0.660 & 0.654 & 82.4 \\
\hline W404 & In-chamber & Water & 39S3B & $\mathrm{S} 8 \mathrm{C}$ & 199.9 & 67.3 & 10.1 & 0.640 & 10.7 & 0.678 & 0.614 & 0.651 & 78.6 \\
\hline W405 & In-chamber & Water & 39S3B & $\mathrm{S} 8 \mathrm{C}$ & 103.1 & 66.3 & 7.7 & 0.490 & 7.6 & 0.482 & 0.654 & 0.643 & 79.1 \\
\hline W406 & In-chamber & Water & 39S3B & $\mathrm{S} 8 \mathrm{C}$ & 199.8 & 66.2 & 9.8 & 0.622 & 10.1 & 0.643 & 0.597 & 0.617 & 80.8 \\
\hline W407 & In-chamber & Water & 39S3B & $\mathrm{S} 8 \mathrm{C}$ & 103.3 & 66.3 & 7.0 & 0.444 & 7.1 & 0.451 & 0.593 & 0.602 & 78.8 \\
\hline W408 & In-chamber & Water & 39S3B & $\mathrm{S} 8 \mathrm{C}$ & 199.9 & 66.3 & 9.3 & 0.591 & 9.9 & 0.628 & 0.567 & 0.603 & 79.2 \\
\hline W409 & In-chamber & Water & 39S3B & $\mathrm{S} 8 \mathrm{C}$ & 103.1 & 65.5 & 6.3 & 0.402 & 7.0 & 0.446 & 0.537 & 0.596 & 80.4 \\
\hline W410 & In-chamber & Water & 39S3B & $\mathrm{S} 8 \mathrm{C}$ & 199.8 & 65.6 & 9.6 & 0.609 & 9.9 & 0.628 & 0.585 & 0.603 & 79.5 \\
\hline W412 & In-chamber & Water & 39S3B & S4A & 101.3 & 65.4 & 30.5 & 1.932 & 29.4 & 1.865 & 0.706 & 0.682 & 79.2 \\
\hline W413 & In-chamber & Water & 39S3B & S4A & 101.1 & 66.0 & 31.2 & 1.976 & 29.5 & 1.865 & 0.723 & 0.683 & 78.6 \\
\hline W414 & In-chamber & Water & 39S3B & S4A & 100.8 & 66.7 & 30.5 & 1.930 & 29.2 & 1.850 & 0.707 & 0.678 & 77.9 \\
\hline W415 & In-chamber & Water & 39S3B & S4A & 200.2 & 70.1 & 44.0 & 2.788 & 41.8 & 2.647 & 0.725 & 0.688 & 76.4 \\
\hline W416 & In-chamber & Water & 39S3B & S4A & 200.4 & 71.4 & 44.8 & 2.840 & 41.7 & 2.644 & 0.738 & 0.687 & 81.8 \\
\hline W417 & In-chamber & Water & 39S3B & S4A & 200.4 & 72.5 & 44.3 & 2.808 & 42.1 & 2.669 & 0.730 & 0.693 & 83.4 \\
\hline W419 & In-chamber & Water & 39S3B & S4A & 199.8 & 66.6 & 44.4 & 2.812 & 41.8 & 2.649 & 0.732 & 0.689 & 79.3 \\
\hline W420 & In-chamber & Water & 39S3B & S4A & 376.9 & 70.9 & 62.6 & 3.966 & 59.4 & 3.761 & 0.751 & 0.713 & 79.1 \\
\hline W421 & In-chamber & Water & 39S3B & S4A & 376.1 & 73.9 & 62.7 & 3.973 & 59.4 & 3.763 & 0.753 & 0.714 & 84.9 \\
\hline W422 & In-chamber & Water & 39S3B & S4A & 375.4 & 73.3 & 62.1 & 3.930 & 58.5 & 3.706 & 0.746 & 0.703 & 86.2 \\
\hline
\end{tabular}

(a) -Instrument(s) upon which measurements are based were not calibrated. Result is "For-Information Only"; (b) - Humidity data collected but not reported for W79-W81 and W86-W88; (c) - Coriolis meter instrument reading/sensitivity issue yielded anomalous result. 
Table B.5. (contd)

\begin{tabular}{|c|c|c|c|c|c|c|c|c|c|c|c|c|c|}
\hline Test ID & $\begin{array}{c}\text { Test } \\
\text { Category }\end{array}$ & Simulant & $\begin{array}{c}\text { Chamber } \\
\text { ID }\end{array}$ & $\begin{array}{c}\text { Orifice } \\
\text { ID }\end{array}$ & $\begin{array}{c}\text { Average } \\
\text { Pressure } \\
\text { [psig] }\end{array}$ & $\begin{array}{c}\text { Test Temp. } \\
{\left[{ }^{\circ} \mathrm{F}\right]}\end{array}$ & $\begin{array}{c}Q \text { (mass) } \\
{[\mathrm{gpm}]}\end{array}$ & $\begin{array}{c}Q \times 10^{3} \\
(\mathrm{mass}) \\
{\left[\mathrm{m}^{3} \mathrm{~s}^{-1}\right]}\end{array}$ & $\begin{array}{c}Q \\
(\text { flow) } \\
\text { (a) } \\
{[\mathrm{gpm}]}\end{array}$ & $\begin{array}{l}Q \times 10^{3} \\
(\text { flow) } \\
{\left[\mathrm{m}^{3} \mathrm{~s}^{-1}\right]}\end{array}$ & $\begin{array}{c}C_{D} \\
\text { (mass) }\end{array}$ & $\begin{array}{c}C_{D} \\
\text { (flow) }^{\text {(a) }}\end{array}$ & $\begin{array}{l}H_{o}^{(\mathrm{a})} \\
{[\%]}\end{array}$ \\
\hline W439 & In-chamber & Water & 39S3C & S4A & 199.2 & 67.0 & 44.1 & 2.795 & 42.2 & 2.670 & 0.729 & 0.696 & 76.4 \\
\hline W440 & In-chamber & Water & 39S3C & S4A & 199.1 & 68.5 & 44.4 & 2.813 & 42.0 & 2.662 & 0.733 & 0.694 & 78.0 \\
\hline W441 & In-chamber & Water & $39 \mathrm{~S} 3 \mathrm{C}$ & S4A & 101.7 & 72.1 & 31.2 & 1.975 & 29.9 & 1.893 & 0.720 & 0.691 & 86.7 \\
\hline W442 & In-chamber & Water & $39 \mathrm{~S} 3 \mathrm{C}$ & S4A & 199.0 & 71.1 & 44.4 & 2.809 & 42.2 & 2.674 & 0.732 & 0.697 & 91.3 \\
\hline W443 & In-chamber & Water & $39 \mathrm{~S} 3 \mathrm{C}$ & S4A & 372.5 & 71.6 & 63.8 & 4.044 & 59.2 & 3.749 & 0.771 & 0.714 & 88.3 \\
\hline W444 & In-chamber & Water & 39S3C & S4A & 375.4 & 69.1 & 64.2 & 4.069 & 59.8 & 3.787 & 0.773 & 0.719 & 83.8 \\
\hline W445 & In-chamber & Water & $39 \mathrm{~S} 3 \mathrm{C}$ & S4A & 374.4 & 70.1 & 64.4 & 4.076 & 59.6 & 3.774 & 0.775 & 0.717 & 83.9 \\
\hline W446 & In-chamber & Water & $39 \mathrm{~S} 3 \mathrm{C}$ & S4A & 102.2 & 67.9 & 31.3 & 1.983 & 29.9 & 1.891 & 0.721 & 0.688 & 81.8 \\
\hline W447 & In-chamber & Water & 39S3C & S4A & 199.1 & 70.0 & 44.7 & 2.829 & 42.2 & 2.674 & 0.737 & 0.697 & 78.5 \\
\hline W448 & In-chamber & Water & 39S3C & S4A & 199.2 & 69.5 & 44.9 & 2.846 & 42.1 & 2.666 & 0.742 & 0.695 & 81.6 \\
\hline W449 & In-chamber & Water & $39 \mathrm{~S} 3 \mathrm{C}$ & S4A & 374.8 & 71.5 & 64.3 & 4.070 & 59.0 & 3.735 & 0.773 & 0.710 & 80.6 \\
\hline W450 & In-chamber & Water & 39S3C & S4A & 101.8 & 69.7 & 31.5 & 1.996 & 29.8 & 1.888 & 0.728 & 0.688 & 80.2 \\
\hline W451 & In-chamber & Water & 39S3C & S4A & 199.1 & 70.5 & 44.6 & 2.826 & 42.3 & 2.676 & 0.737 & 0.698 & 80.3 \\
\hline W452 & In-chamber & Water & 39S3C & S4A & 374.7 & 72.6 & 65.5 & 4.147 & 59.7 & 3.783 & 0.788 & 0.719 & 80.8 \\
\hline W454 & In-chamber & Water & $39 \mathrm{~S} 3 \mathrm{C}$ & S4A & 376.0 & 71.5 & 64.2 & 4.067 & 59.7 & 3.784 & 0.771 & 0.718 & 81.3 \\
\hline W455 & In-chamber & Water & 39S3C & S8A & 103.2 & 72.1 & 1.0 & 0.063 & 1.0 & 0.064 & 0.535 & 0.542 & 80.6 \\
\hline W456 & In-chamber & Water & $39 \mathrm{~S} 3 \mathrm{C}$ & S8A & 200.9 & 70.8 & 1.5 & 0.095 & 1.6 & 0.102 & 0.576 & 0.620 & 77.1 \\
\hline W457 & In-chamber & Water & $39 \mathrm{~S} 3 \mathrm{C}$ & S8A & 378.8 & 71.9 & 2.2 & 0.138 & 2.1 & 0.133 & 0.608 & 0.587 & 81.8 \\
\hline W458 & In-chamber & Water & $39 \mathrm{~S} 3 \mathrm{C}$ & S8A & 103.0 & 70.5 & 1.0 & 0.064 & 1.0 & 0.065 & 0.541 & 0.551 & 79.5 \\
\hline W459 & In-chamber & Water & 39S3C & S8A & 200.7 & 70.0 & 1.5 & 0.096 & 1.7 & 0.105 & 0.584 & 0.637 & 84.2 \\
\hline W460 & In-chamber & Water & 39S3C & S8A & 378.8 & 71.0 & 2.2 & 0.138 & 2.2 & 0.142 & 0.608 & 0.627 & 85.9 \\
\hline W461 & In-chamber & Water & 39S3C & S8A & 103.3 & 69.2 & 1.0 & 0.062 & 1.1 & 0.067 & 0.526 & 0.563 & 83.5 \\
\hline W462 & In-chamber & Water & $39 \mathrm{~S} 3 \mathrm{C}$ & S8A & 200.3 & 68.9 & 1.5 & 0.098 & 1.5 & 0.093 & 0.594 & 0.565 & 85.9 \\
\hline W463 & In-chamber & Water & $39 \mathrm{~S} 3 \mathrm{C}$ & S8A & 378.4 & 70.2 & 2.1 & 0.135 & 2.0 & 0.128 & 0.597 & 0.564 & 85.8 \\
\hline W464 & In-chamber & Water & $39 \mathrm{~S} 3 \mathrm{C}$ & S8B & 102.6 & 70.2 & 3.7 & 0.233 & 3.6 & 0.231 & 0.650 & 0.643 & 88.2 \\
\hline W465 & In-chamber & Water & 39S3C & S8B & 200.5 & 69.8 & 5.2 & 0.332 & 4.9 & 0.313 & 0.662 & 0.623 & 87.1 \\
\hline W466 & In-chamber & Water & 39S3C & S8B & 379.0 & 71.2 & 7.3 & 0.461 & 6.8 & 0.431 & 0.668 & 0.625 & 87.4 \\
\hline W467 & In-chamber & Water & 39S3C & S8B & 102.8 & 70.2 & 3.7 & 0.231 & 3.6 & 0.231 & 0.644 & 0.643 & 88.6 \\
\hline W468 & In-chamber & Water & 39S3C & S8B & 200.7 & 69.9 & 5.2 & 0.329 & 5.0 & 0.315 & 0.656 & 0.627 & 87.7 \\
\hline W469 & In-chamber & Water & $39 \mathrm{~S} 3 \mathrm{C}$ & S8B & 379.1 & 70.3 & 7.3 & 0.460 & 6.9 & 0.437 & 0.666 & 0.633 & 86.5 \\
\hline
\end{tabular}

(a) -Instrument(s) upon which measurements are based were not calibrated. Result is "For-Information Only"; (b) - Humidity data collected but not reported for W79-W81 and W86-W88; (c) - Coriolis meter instrument reading/sensitivity issue yielded anomalous result. 
Table B.5. (contd)

\begin{tabular}{|c|c|c|c|c|c|c|c|c|c|c|c|c|c|}
\hline Test ID & $\begin{array}{c}\text { Test } \\
\text { Category }\end{array}$ & Simulant & $\begin{array}{c}\text { Chamber } \\
\text { ID }\end{array}$ & $\begin{array}{c}\text { Orifice } \\
\text { ID }\end{array}$ & $\begin{array}{c}\text { Average } \\
\text { Pressure } \\
\text { [psig] }\end{array}$ & $\begin{array}{c}\text { Test Temp. } \\
{\left[{ }^{\circ} \mathrm{F}\right]}\end{array}$ & $\begin{array}{c}Q \text { (mass) } \\
{[\mathrm{gpm}]}\end{array}$ & $\begin{array}{c}Q \times 10^{3} \\
(\mathrm{mass}) \\
{\left[\mathrm{m}^{3} \mathrm{~s}^{-1}\right]}\end{array}$ & $\begin{array}{c}Q \\
(\text { flow) } \\
{[\text { (a) }}\end{array}$ & $\begin{array}{l}Q \times 10^{3} \\
(\text { flow) } \\
(\mathrm{a}) \\
{\left[\mathrm{m}^{3} \mathrm{~s}^{-1}\right]}\end{array}$ & $\begin{array}{c}C_{D} \\
\text { (mass) }\end{array}$ & $\begin{array}{c}C_{D} \\
\text { (flow) }^{(\text {a) }}\end{array}$ & $\begin{array}{l}H_{o}^{(\mathrm{a})} \\
{[\%]}\end{array}$ \\
\hline W470 & In-chamber & Water & 39S3C & S8B & 102.8 & 69.6 & 3.7 & 0.232 & 3.6 & 0.229 & 0.647 & 0.637 & 87.6 \\
\hline W471 & In-chamber & Water & $39 \mathrm{~S} 3 \mathrm{C}$ & S8B & 201.0 & 69.4 & 5.3 & 0.338 & 5.2 & 0.330 & 0.673 & 0.657 & 86.5 \\
\hline W472 & In-chamber & Water & $39 \mathrm{~S} 3 \mathrm{C}$ & S8B & 378.9 & 70.6 & 7.3 & 0.461 & 6.6 & 0.416 & 0.669 & 0.603 & 86.2 \\
\hline W473 & In-chamber & Water & $39 \mathrm{~S} 3 \mathrm{C}$ & $\mathrm{S} 8 \mathrm{C}$ & 102.5 & 69.4 & 7.3 & 0.462 & 7.2 & 0.455 & 0.619 & 0.610 & 85.2 \\
\hline W474 & In-chamber & Water & $39 \mathrm{~S} 3 \mathrm{C}$ & $\mathrm{S} 8 \mathrm{C}$ & 102.1 & 69.0 & 7.4 & 0.466 & 7.2 & 0.455 & 0.626 & 0.612 & 86.5 \\
\hline W475 & In-chamber & Water & $39 \mathrm{~S} 3 \mathrm{C}$ & $\mathrm{S} 8 \mathrm{C}$ & 102.0 & 66.4 & 7.2 & 0.459 & 7.2 & 0.457 & 0.616 & 0.614 & 87.3 \\
\hline W476 & In-chamber & Water & $39 \mathrm{~S} 3 \mathrm{C}$ & $\mathrm{S} 8 \mathrm{C}$ & 199.6 & 67.5 & 10.4 & 0.661 & 9.8 & 0.621 & 0.635 & 0.597 & 88.0 \\
\hline W478 & In-chamber & Water & $39 \mathrm{~S} 3 \mathrm{C}$ & $\mathrm{S} 8 \mathrm{C}$ & 379.5 & 77.2 & 15.6 & 0.988 & 14.6 & 0.925 & 0.688 & 0.644 & 86.1 \\
\hline W479 & In-chamber & Water & $39 \mathrm{~S} 3 \mathrm{C}$ & $\mathrm{S} 8 \mathrm{C}$ & 102.4 & 74.7 & 8.0 & 0.506 & 7.6 & 0.483 & 0.678 & 0.648 & 86.5 \\
\hline W480 & In-chamber & Water & 39S3C & $\mathrm{S} 8 \mathrm{C}$ & 200.0 & 72.9 & 11.1 & 0.702 & 10.6 & 0.674 & 0.674 & 0.647 & 82.4 \\
\hline W481 & In-chamber & Water & $39 \mathrm{~S} 3 \mathrm{C}$ & $\mathrm{S} 8 \mathrm{C}$ & 378.7 & 73.1 & 15.5 & 0.981 & 14.3 & 0.904 & 0.684 & 0.630 & 84.2 \\
\hline W482 & In-chamber & Water & $39 \mathrm{~S} 3 \mathrm{C}$ & $\mathrm{S} 8 \mathrm{C}$ & 102.3 & 71.2 & 8.0 & 0.504 & 7.5 & 0.475 & 0.676 & 0.637 & 84.2 \\
\hline W483 & In-chamber & Water & $39 \mathrm{~S} 3 \mathrm{C}$ & $\mathrm{S} 8 \mathrm{C}$ & 199.8 & 70.2 & 11.2 & 0.711 & 10.7 & 0.676 & 0.682 & 0.649 & 80.6 \\
\hline W484 & In-chamber & Water & $39 \mathrm{~S} 3 \mathrm{C}$ & $\mathrm{S} 8 \mathrm{C}$ & 378.3 & 71.8 & 15.4 & 0.973 & 14.5 & 0.921 & 0.679 & 0.643 & 78.3 \\
\hline W485 & In-chamber & Water & $39 \mathrm{~S} 3 \mathrm{C}$ & $\mathrm{S} 8 \mathrm{C}$ & 102.0 & 69.7 & 7.8 & 0.495 & 7.7 & 0.487 & 0.665 & 0.654 & 82.3 \\
\hline W486 & In-chamber & Water & 39S3C & $\mathrm{S} 8 \mathrm{C}$ & 199.7 & 69.5 & 11.2 & 0.712 & 10.6 & 0.670 & 0.684 & 0.644 & 74.9 \\
\hline W488 & In-chamber & Water & $39 \mathrm{~S} 3 \mathrm{C}$ & $\mathrm{S} 8 \mathrm{C}$ & 378.6 & 72.1 & 15.6 & 0.986 & 14.4 & 0.912 & 0.688 & 0.636 & 74.2 \\
\hline W489 & In-chamber & Water & 39S3D & S8A & 102.1 & 64.7 & 1.0 & 0.062 & 0.9 & 0.057 & 0.525 & 0.484 & 78.8 \\
\hline W490 & In-chamber & Water & 39S3D & S8A & 200.1 & 65.6 & 1.4 & 0.090 & 1.7 & 0.106 & 0.548 & 0.643 & 78.9 \\
\hline W492 & In-chamber & Water & 39S3D & S8A & 101.8 & 68.4 & 1.0 & 0.062 & 1.0 & 0.066 & 0.525 & 0.564 & 81.5 \\
\hline W493 & In-chamber & Water & 39S3D & S8A & 200.2 & 68.4 & 1.5 & 0.093 & 1.6 & 0.103 & 0.566 & 0.623 & 82.2 \\
\hline W494 & In-chamber & Water & 39S3D & S8A & 380.9 & 69.8 & 2.1 & 0.133 & 2.0 & 0.130 & 0.586 & 0.569 & 82.3 \\
\hline W495 & In-chamber & Water & 39S3D & S8A & 102.5 & 69.4 & 1.0 & 0.061 & 1.1 & 0.070 & 0.513 & 0.593 & 83.7 \\
\hline W496 & In-chamber & Water & 39S3D & S8A & 200.0 & 69.5 & 1.5 & 0.094 & 1.6 & 0.100 & 0.570 & 0.604 & 83.3 \\
\hline W497 & In-chamber & Water & 39S3D & S8A & 380.8 & 70.6 & 2.1 & 0.132 & 1.9 & 0.123 & 0.582 & 0.541 & 82.1 \\
\hline W498 & In-chamber & Water & 39S3D & S8B & 101.4 & 68.0 & 3.6 & 0.229 & 3.5 & 0.224 & 0.641 & 0.629 & 89.1 \\
\hline W499 & In-chamber & Water & 39S3D & S8B & 199.0 & 69.1 & 5.1 & 0.325 & 5.1 & 0.323 & 0.651 & 0.646 & 88.8 \\
\hline W500 & In-chamber & Water & 39S3D & S8B & 379.6 & 71.7 & 7.3 & 0.461 & 6.6 & 0.419 & 0.667 & 0.606 & 88.6 \\
\hline W501 & In-chamber & Water & 39S3D & S8B & 101.2 & 70.5 & 3.7 & 0.231 & 3.6 & 0.226 & 0.649 & 0.633 & 90.6 \\
\hline W502 & In-chamber & Water & 39S3D & S8B & 198.9 & 70.0 & 5.0 & 0.316 & 4.9 & 0.313 & 0.633 & 0.627 & 89.7 \\
\hline
\end{tabular}

(a) -Instrument(s) upon which measurements are based were not calibrated. Result is "For-Information Only"; (b) - Humidity data collected but not reported for W79-W81 and W86-W88; (c) - Coriolis meter instrument reading/sensitivity issue yielded anomalous result. 
Table B.5. (contd)

\begin{tabular}{|c|c|c|c|c|c|c|c|c|c|c|c|c|c|}
\hline Test ID & $\begin{array}{c}\text { Test } \\
\text { Category }\end{array}$ & Simulant & $\begin{array}{c}\text { Chamber } \\
\text { ID }\end{array}$ & $\begin{array}{c}\text { Orifice } \\
\text { ID }\end{array}$ & $\begin{array}{c}\text { Average } \\
\text { Pressure } \\
\text { [psig] }\end{array}$ & $\begin{array}{c}\text { Test Temp. } \\
{\left[{ }^{\circ} \mathrm{F}\right]}\end{array}$ & $\begin{array}{c}Q \text { (mass) } \\
{[\mathrm{gpm}]}\end{array}$ & $\begin{array}{c}Q \times 10^{3} \\
(\mathrm{mass}) \\
{\left[\mathrm{m}^{3} \mathrm{~s}^{-1}\right]}\end{array}$ & $\begin{array}{c}Q \\
\text { (flow) }^{(a)} \\
{[\mathrm{gpm}]}\end{array}$ & $\begin{array}{c}Q \times 10^{3} \\
(\text { flow) } \\
(\mathrm{a}) \\
{\left[\mathrm{m}^{3} \mathrm{~s}^{-1}\right]}\end{array}$ & $\begin{array}{c}C_{D} \\
\text { (mass) }\end{array}$ & $\begin{array}{c}C_{D} \\
\text { (flow) }^{(\text {a) }}\end{array}$ & $\begin{array}{l}H_{o}^{(\mathrm{a})} \\
{[\%]}\end{array}$ \\
\hline W503 & In-chamber & Water & 39S3D & S8B & 379.4 & 70.7 & 7.2 & 0.455 & 6.9 & 0.439 & 0.659 & 0.636 & 88.3 \\
\hline W504 & In-chamber & Water & 39S3D & S8B & 101.1 & 69.8 & 3.6 & 0.228 & 3.7 & 0.231 & 0.640 & 0.649 & 88.2 \\
\hline W505 & In-chamber & Water & 39S3D & S8B & 198.6 & 69.2 & 5.2 & 0.329 & 5.0 & 0.315 & 0.659 & 0.631 & 87.2 \\
\hline W506 & In-chamber & Water & 39S3D & S8B & 379.2 & 70.5 & 7.2 & 0.457 & 6.6 & 0.419 & 0.663 & 0.608 & 86.5 \\
\hline W507 & In-chamber & Water & 39S3D & $\mathrm{S} 8 \mathrm{C}$ & 101.0 & 67.5 & 7.4 & 0.466 & 7.6 & 0.482 & 0.629 & 0.651 & 82.0 \\
\hline W509 & In-chamber & Water & 39S3D & $\mathrm{S} 8 \mathrm{C}$ & 198.7 & 69.3 & 11.1 & 0.702 & 10.5 & 0.662 & 0.675 & 0.638 & 80.0 \\
\hline W510 & In-chamber & Water & 39S3D & $\mathrm{S} 8 \mathrm{C}$ & 380.0 & 71.7 & 15.4 & 0.974 & 14.6 & 0.928 & 0.678 & 0.646 & 80.1 \\
\hline W511 & In-chamber & Water & 39S3D & $\mathrm{S} 8 \mathrm{C}$ & 100.7 & 70.6 & 7.8 & 0.494 & 7.6 & 0.484 & 0.668 & 0.654 & 83.9 \\
\hline W512 & In-chamber & Water & 39S3D & $\mathrm{S} 8 \mathrm{C}$ & 198.5 & 70.2 & 11.1 & 0.700 & 10.5 & 0.668 & 0.674 & 0.643 & 82.7 \\
\hline W513 & In-chamber & Water & 39S3D & $\mathrm{S} 8 \mathrm{C}$ & 380.0 & 71.1 & 15.3 & 0.970 & 14.5 & 0.920 & 0.675 & 0.640 & 81.9 \\
\hline W514 & In-chamber & Water & 39S3D & S8C & 100.6 & 69.4 & 7.8 & 0.493 & 7.5 & 0.475 & 0.666 & 0.643 & 81.4 \\
\hline W515 & In-chamber & Water & 39S3D & $\mathrm{S} 8 \mathrm{C}$ & 198.4 & 70.1 & 11.1 & 0.703 & 10.5 & 0.664 & 0.677 & 0.640 & 80.0 \\
\hline W516 & In-chamber & Water & 39S3D & $\mathrm{S} 8 \mathrm{C}$ & 379.9 & 70.3 & 15.5 & 0.981 & 14.4 & 0.911 & 0.683 & 0.634 & 80.2 \\
\hline W517 & In-chamber & Water & 39S3D & $\mathrm{S} 8 \mathrm{C}$ & 198.6 & 69.9 & 11.1 & 0.701 & 10.7 & 0.675 & 0.675 & 0.650 & 79.7 \\
\hline W518 & In-chamber & Water & 39S3D & S8C & 379.3 & 70.4 & 15.5 & 0.982 & 14.5 & 0.916 & 0.684 & 0.638 & 78.6 \\
\hline W519 & In-chamber & Water & 39S3D & S4A & 99.4 & 68.2 & 30.7 & 1.944 & 29.6 & 1.877 & 0.717 & 0.693 & 79.0 \\
\hline W520 & In-chamber & Water & 39S3D & S4A & 195.0 & 68.1 & 43.0 & 2.724 & 41.4 & 2.622 & 0.718 & 0.691 & 78.6 \\
\hline W521 & In-chamber & Water & 39S3D & S4A & 376.4 & 69.5 & 62.9 & 3.982 & 59.8 & 3.790 & 0.755 & 0.719 & 78.1 \\
\hline W522 & In-chamber & Water & 39S3D & $\mathrm{S} 4 \mathrm{~A}$ & 99.5 & 68.5 & 30.8 & 1.953 & 29.6 & 1.875 & 0.720 & 0.692 & 80.6 \\
\hline W523 & In-chamber & Water & 39S3D & $\mathrm{S} 4 \mathrm{~A}$ & 196.8 & 68.4 & 43.9 & 2.778 & 42.0 & 2.658 & 0.729 & 0.697 & 78.5 \\
\hline W524 & In-chamber & Water & 39S3D & S4A & 376.1 & 69.6 & 62.9 & 3.985 & 59.8 & 3.785 & 0.756 & 0.718 & 78.7 \\
\hline W525 & In-chamber & Water & 39S3D & S4A & 99.4 & 68.6 & 30.6 & 1.936 & 29.6 & 1.874 & 0.714 & 0.691 & 80.0 \\
\hline W526 & In-chamber & Water & 39S3D & S4A & 196.6 & 68.5 & 44.1 & 2.790 & 41.9 & 2.655 & 0.732 & 0.697 & 78.3 \\
\hline W527 & In-chamber & Water & 39S3D & $\mathrm{S} 4 \mathrm{~A}$ & 376.6 & 69.3 & 62.5 & 3.958 & 59.3 & 3.758 & 0.750 & 0.712 & 77.9 \\
\hline W528 & In-chamber & Water & $39 \mathrm{~S} 3 \mathrm{C}$ & S8B & 378.6 & 69.0 & 7.3 & 0.461 & 6.9 & 0.438 & 0.668 & 0.635 & 83.6 \\
\hline W529 & In-chamber & Water & 39S3C & S8B & 378.3 & 71.3 & 7.4 & 0.466 & 6.9 & 0.439 & 0.676 & 0.637 & 87.5 \\
\hline W530 & In-chamber & Water & $39 \mathrm{~S} 3 \mathrm{C}$ & S8B & 377.9 & 71.9 & 7.3 & 0.465 & 6.9 & 0.434 & 0.675 & 0.630 & 89.6 \\
\hline W531 & In-chamber & Water & 39S3A & S8A & 101.5 & 67.1 & 0.9 & 0.058 & 1.1 & 0.069 & 0.498 & 0.588 & 78.3 \\
\hline W532 & In-chamber & Water & 39S3A & S8A & 199.4 & 67.3 & 1.4 & 0.089 & 1.6 & 0.100 & 0.541 & 0.606 & 82.4 \\
\hline W533 & In-chamber & Water & 39S3A & S8A & 380.9 & 68.8 & 2.1 & 0.133 & 2.4 & 0.149 & 0.582 & 0.655 & 83.3 \\
\hline
\end{tabular}

(a) -Instrument(s) upon which measurements are based were not calibrated. Result is "For-Information Only"; (b) - Humidity data collected but not reported for W79-W81 and W86-W88; (c) - Coriolis meter instrument reading/sensitivity issue yielded anomalous result. 
Table B.5. (contd)

\begin{tabular}{|c|c|c|c|c|c|c|c|c|c|c|c|c|c|}
\hline Test ID & $\begin{array}{c}\text { Test } \\
\text { Category }\end{array}$ & Simulant & $\begin{array}{c}\text { Chamber } \\
\text { ID }\end{array}$ & $\begin{array}{c}\text { Orifice } \\
\text { ID }\end{array}$ & $\begin{array}{c}\text { Average } \\
\text { Pressure } \\
\text { [psig] }\end{array}$ & $\begin{array}{c}\text { Test Temp. } \\
{\left[{ }^{\circ} \mathrm{F}\right]}\end{array}$ & $\begin{array}{c}Q \text { (mass) } \\
{[\mathrm{gpm}]}\end{array}$ & $\begin{array}{c}Q \times 10^{3} \\
(\mathrm{mass}) \\
{\left[\mathrm{m}^{3} \mathrm{~s}^{-1}\right]}\end{array}$ & $\begin{array}{c}Q \\
\text { (flow) }^{(a)} \\
{[\mathrm{gpm}]}\end{array}$ & $\begin{array}{c}Q \times 10^{3} \\
(\text { flow) } \\
(\mathrm{a}) \\
{\left[\mathrm{m}^{3} \mathrm{~s}^{-1}\right]}\end{array}$ & $\begin{array}{c}C_{D} \\
\text { (mass) }\end{array}$ & $\begin{array}{c}C_{D} \\
\text { (flow) }^{(\text {a) }}\end{array}$ & $\begin{array}{l}H_{o}^{(\mathrm{a})} \\
{[\%]}\end{array}$ \\
\hline W534 & In-chamber & Water & 39S3A & S8A & 102.0 & 69.1 & 1.0 & 0.065 & 1.1 & 0.070 & 0.552 & 0.595 & 79.6 \\
\hline W535 & In-chamber & Water & 39S3A & S8A & 201.1 & 69.0 & 1.5 & 0.092 & 1.5 & 0.098 & 0.559 & 0.590 & 81.8 \\
\hline W536 & In-chamber & Water & 39S3A & S8A & 201.0 & 67.9 & 1.5 & 0.093 & 1.6 & 0.099 & 0.564 & 0.596 & 77.8 \\
\hline W537 & In-chamber & Water & 39S3A & S8A & 380.7 & 70.5 & 2.1 & 0.131 & 2.1 & 0.131 & 0.576 & 0.575 & 83.5 \\
\hline W538 & In-chamber & Water & 39S3A & S8A & 102.0 & 69.8 & 1.0 & 0.066 & 1.1 & 0.067 & 0.561 & 0.568 & 87.4 \\
\hline W539 & In-chamber & Water & 39S3A & S8A & 200.8 & 69.8 & 1.4 & 0.092 & 1.4 & 0.087 & 0.554 & 0.525 & 87.1 \\
\hline W540 & In-chamber & Water & 39S3A & S8A & 380.4 & 71.1 & 2.0 & 0.129 & 2.0 & 0.126 & 0.565 & 0.555 & 87.8 \\
\hline W541 & In-chamber & Water & 39S3A & S8B & 101.6 & 71.3 & 3.5 & 0.225 & 3.5 & 0.224 & 0.629 & 0.627 & 86.6 \\
\hline W546 & In-chamber & Water & 39S3A & S8B & 101.5 & 68.1 & 3.6 & 0.225 & 3.6 & 0.230 & 0.630 & 0.644 & 90.9 \\
\hline W547 & In-chamber & Water & 39S3A & S8B & 101.5 & 67.5 & 3.6 & 0.227 & 3.6 & 0.226 & 0.636 & 0.634 & 90.1 \\
\hline W548 & In-chamber & Water & 39S3A & S8B & 200.0 & 67.1 & 5.0 & 0.320 & 5.0 & 0.315 & 0.638 & 0.629 & 89.4 \\
\hline W549 & In-chamber & Water & 39S3A & S8B & 200.0 & 67.2 & 5.0 & 0.318 & 4.9 & 0.312 & 0.634 & 0.622 & 89.5 \\
\hline W550 & In-chamber & Water & 39S3A & S8B & 199.9 & 67.5 & 5.1 & 0.322 & 5.2 & 0.328 & 0.643 & 0.655 & 89.3 \\
\hline W551 & In-chamber & Water & 39S3A & S8B & 379.3 & 68.0 & 6.9 & 0.437 & 7.1 & 0.450 & 0.633 & 0.652 & 87.8 \\
\hline W552 & In-chamber & Water & $39 \mathrm{~S} 3 \mathrm{~A}$ & S8B & 379.0 & 70.3 & 6.9 & 0.440 & 6.8 & 0.432 & 0.637 & 0.625 & 91.4 \\
\hline W553 & In-chamber & Water & $39 \mathrm{~S} 3 \mathrm{~A}$ & S8B & 378.8 & 72.0 & 6.9 & 0.435 & 6.9 & 0.436 & 0.631 & 0.632 & 92.7 \\
\hline W554 & In-chamber & Water & 39S3A & $\mathrm{S} 8 \mathrm{C}$ & 100.8 & 69.6 & 7.7 & 0.487 & 7.6 & 0.481 & 0.658 & 0.650 & 95.4 \\
\hline W556 & In-chamber & Water & 39S3A & $\mathrm{S} 8 \mathrm{C}$ & 379.9 & 70.4 & 15.0 & 0.952 & 14.3 & 0.907 & 0.663 & 0.631 & 96.2 \\
\hline W557 & In-chamber & Water & 39S3A & $\mathrm{S} 8 \mathrm{C}$ & 100.5 & 68.5 & 7.8 & 0.492 & 7.5 & 0.478 & 0.666 & 0.647 & 98.0 \\
\hline W558 & In-chamber & Water & 39S3A & $\mathrm{S} 8 \mathrm{C}$ & 198.7 & 68.3 & 10.9 & 0.689 & 10.7 & 0.680 & 0.663 & 0.655 & 97.8 \\
\hline W560 & In-chamber & Water & 39S3A & $\mathrm{S} 8 \mathrm{C}$ & 100.4 & 68.5 & 7.9 & 0.499 & 7.4 & 0.467 & 0.675 & 0.633 & 98.9 \\
\hline W561 & In-chamber & Water & 39S3A & $\mathrm{S} 8 \mathrm{C}$ & 198.8 & 68.5 & 11.0 & 0.696 & 10.6 & 0.673 & 0.670 & 0.648 & 98.7 \\
\hline W562 & In-chamber & Water & $39 \mathrm{~S} 3 \mathrm{~A}$ & $\mathrm{~S} 8 \mathrm{C}$ & 381.4 & 69.9 & 14.9 & 0.945 & 15.6 & 0.989 & 0.656 & 0.687 & 98.6 \\
\hline W563 & Humidity & Water & 39S3A & S8A & 380.7 & 69.8 & 2.2 & 0.137 & 1.9 & 0.120 & 0.602 & 0.525 & 45.3 \\
\hline W564 & Humidity & Water & 39S3A & S8A & 379.9 & 66.5 & 2.6 & 0.167 & 2.1 & 0.133 & 0.733 & 0.586 & 47.8 \\
\hline W565 & Humidity & Water & 39S3A & S8A & 379.8 & 70.0 & 2.2 & 0.139 & 2.1 & 0.131 & 0.613 & 0.577 & 41.2 \\
\hline W566 & Humidity & Water & 39S3A & S8A & 379.8 & 72.8 & 2.2 & 0.139 & 2.1 & 0.135 & 0.611 & 0.594 & 81.4 \\
\hline W567 & Humidity & Water & 39S3A & S8A & 379.8 & 75.6 & 2.2 & 0.139 & 1.8 & 0.117 & 0.611 & 0.513 & 87.4 \\
\hline W568 & Humidity & Water & 39S3A & S8A & 379.5 & 69.2 & 2.1 & 0.133 & 2.7 & 0.170 & 0.583 & 0.748 & 31.2 \\
\hline W569 & Functional & Water & 39S3A & S8A & 379.6 & 73.3 & 2.2 & 0.137 & 1.6 & 0.101 & 0.603 & 0.446 & 84.7 \\
\hline
\end{tabular}

(a) -Instrument(s) upon which measurements are based were not calibrated. Result is "For-Information Only"; (b) - Humidity data collected but not reported for W79-W81 and W86-W88; (c) - Coriolis meter instrument reading/sensitivity issue yielded anomalous result. 
Table B.5. (contd)

\begin{tabular}{|c|c|c|c|c|c|c|c|c|c|c|c|c|c|}
\hline Test ID & $\begin{array}{c}\text { Test } \\
\text { Category }\end{array}$ & Simulant & $\begin{array}{c}\text { Chamber } \\
\text { ID }\end{array}$ & $\begin{array}{c}\text { Orifice } \\
\text { ID }\end{array}$ & $\begin{array}{c}\text { Average } \\
\text { Pressure } \\
\text { [psig] }\end{array}$ & $\begin{array}{c}\text { Test Temp. } \\
{\left[{ }^{\circ} \mathrm{F}\right]}\end{array}$ & $\begin{array}{c}Q \text { (mass) } \\
{[\mathrm{gpm}]}\end{array}$ & $\begin{array}{c}Q \times 10^{3} \\
(\mathrm{mass}) \\
{\left[\mathrm{m}^{3} \mathrm{~s}^{-1}\right]}\end{array}$ & $\begin{array}{c}Q \\
(\text { flow) } \\
{[\text { (a) }}\end{array}$ & $\begin{array}{l}Q \times 10^{3} \\
(\text { flow) } \\
(\mathrm{a}) \\
{\left[\mathrm{m}^{3} \mathrm{~s}^{-1}\right]}\end{array}$ & $\begin{array}{c}C_{D} \\
\text { (mass) }\end{array}$ & $\begin{array}{c}C_{D} \\
\text { (flow) }^{\text {(a) }}\end{array}$ & $\begin{array}{c}H_{o}^{(\mathrm{a})} \\
{[\%]}\end{array}$ \\
\hline W570 & Functional & Water & 39S3A & S8A & 379.4 & 72.7 & 2.2 & 0.139 & 1.9 & 0.120 & 0.610 & 0.529 & 88.2 \\
\hline W571 & Functional & Water & 39S3A & S8A & 379.3 & 69.8 & 2.2 & 0.137 & 1.5 & 0.098 & 0.604 & 0.430 & 81.3 \\
\hline W572 & Functional & Water & 39S3A & S8A & 379.0 & 70.5 & 2.2 & 0.139 & 1.5 & 0.095 & 0.614 & 0.417 & 87.2 \\
\hline W573 & Functional & Water & $39 \mathrm{~S} 3 \mathrm{~A}$ & S8A & 380.5 & 69.3 & 2.1 & 0.136 & 1.8 & 0.117 & 0.598 & 0.514 & 79.6 \\
\hline W574 & Functional & Water & 39S3A & S8A & 380.0 & 68.3 & 2.6 & 0.164 & 1.2 & 0.079 & 0.719 & 0.346 & 83.7 \\
\hline W575 & Functional & Water & 39S3A & S8A & 381.2 & 72.0 & 2.7 & 0.172 & 0.9 & 0.056 & 0.756 & 0.244 & 81.6 \\
\hline W576 & Functional & Water & 39S3A & S8A & 380.0 & 69.9 & 2.6 & 0.163 & 1.7 & 0.105 & 0.718 & 0.460 & 85.6 \\
\hline W577 & Functional & Water & $39 \mathrm{~S} 3 \mathrm{~A}$ & S8A & 379.9 & 71.6 & 2.8 & 0.174 & 1.0 & 0.064 & 0.767 & 0.280 & 92.3 \\
\hline W578 & Humidity & Water & 39S3A & S8A & 379.6 & 68.8 & 2.6 & 0.167 & 0.5 & 0.032 & 0.736 & 0.141 & 54.7 \\
\hline W579 & Humidity & Water & 39S3A & S8A & 379.9 & 71.7 & 2.6 & 0.163 & 0.4 & 0.026 & 0.717 & 0.113 & 41.8 \\
\hline W580 & Humidity & Water & 39S3A & S8A & 379.8 & 72.7 & 2.7 & 0.172 & 0.1 & 0.009 & 0.755 & 0.040 & 70.3 \\
\hline W581 & In-chamber & Water & 39S3A & S4A & 101.2 & 71.3 & 31.2 & 1.974 & 29.8 & 1.887 & 0.722 & 0.690 & 91.2 \\
\hline W583 & In-chamber & Water & 39S3A & S4A & 376.9 & 71.0 & 63.1 & 3.999 & 60.0 & 3.800 & 0.758 & 0.720 & 92.4 \\
\hline W584 & In-chamber & Water & 39S3A & S4A & 102.1 & 68.9 & 31.3 & 1.982 & 30.1 & 1.909 & 0.722 & 0.695 & 97.8 \\
\hline W585 & In-chamber & Water & 39S3A & S4A & 199.8 & 68.3 & 44.6 & 2.828 & 42.2 & 2.671 & 0.736 & 0.695 & 98.1 \\
\hline W586 & In-chamber & Water & 39S3A & S4A & 375.8 & 69.3 & 63.0 & 3.992 & 59.2 & 3.751 & 0.758 & 0.712 & 97.5 \\
\hline W587 & In-chamber & Water & 39S3A & S4A & 102.7 & 69.0 & 30.8 & 1.949 & 30.0 & 1.900 & 0.708 & 0.690 & 98.4 \\
\hline W588 & In-chamber & Water & 39S3A & S4A & 200.9 & 68.5 & 44.4 & 2.815 & 42.6 & 2.697 & 0.731 & 0.700 & 97.1 \\
\hline W589 & In-chamber & Water & 39S3A & S4A & 200.9 & 68.2 & 44.4 & 2.815 & 42.5 & 2.689 & 0.731 & 0.698 & 95.6 \\
\hline W590 & In-chamber & Water & 39S3A & S4A & 375.5 & 69.4 & 62.4 & 3.949 & 59.5 & 3.769 & 0.750 & 0.715 & 94.5 \\
\hline W591 & In-chamber & Water & 39S3A & S4A & 375.4 & 69.8 & 63.2 & 4.006 & 59.5 & 3.767 & 0.760 & 0.715 & 98.5 \\
\hline W592 & In-chamber & Water & 39S3A & S4A & 376.0 & 69.8 & 63.4 & 4.017 & 59.9 & 3.791 & 0.762 & 0.719 & 99.5 \\
\hline W593 & In-chamber & Water & 39S3A & S4A & 200.1 & 68.9 & 44.4 & 2.815 & 42.1 & 2.666 & 0.732 & 0.693 & 99.8 \\
\hline W594 & In-chamber & Water & 39S3A & S4A & 200.0 & 68.5 & 45.0 & 2.851 & 42.4 & 2.688 & 0.741 & 0.699 & 99.3 \\
\hline W595 & In-chamber & Water & $39 \mathrm{~S} 3 \mathrm{~A}$ & S4A & 200.3 & 67.7 & 44.5 & 2.820 & 42.5 & 2.690 & 0.733 & 0.699 & 96.9 \\
\hline W596 & In-spray & Water & 39F3A & S4A & 101.8 & 65.4 & 31.6 & 2.002 & 29.9 & 1.896 & 0.730 & 0.691 & 79.3 \\
\hline W597 & In-spray & Water & 39F3A & S4A & 199.4 & 67.0 & 44.8 & 2.839 & 42.4 & 2.682 & 0.740 & 0.699 & 82.8 \\
\hline W598 & In-spray & Water & 39F3A & S4A & 199.4 & 68.7 & 45.1 & 2.856 & 42.3 & 2.681 & 0.744 & 0.698 & 87.2 \\
\hline W599 & In-spray & Water & 39F3A & S4A & 375.0 & 72.7 & 63.7 & 4.034 & 59.4 & 3.762 & 0.766 & 0.715 & 90.2 \\
\hline W601 & In-spray & Water & 39F3A & S8A & 380.3 & 79.4 & 2.1 & 0.135 & 2.1 & 0.136 & 0.592 & 0.595 & 91.3 \\
\hline
\end{tabular}

(a) -Instrument(s) upon which measurements are based were not calibrated. Result is "For-Information Only"; (b) - Humidity data collected but not reported for W79-W81 and W86-W88; (c) - Coriolis meter instrument reading/sensitivity issue yielded anomalous result. 
Table B.5. (contd)

\begin{tabular}{|c|c|c|c|c|c|c|c|c|c|c|c|c|c|}
\hline Test ID & $\begin{array}{c}\text { Test } \\
\text { Category }\end{array}$ & Simulant & $\begin{array}{c}\text { Chamber } \\
\text { ID }\end{array}$ & $\begin{array}{c}\text { Orifice } \\
\text { ID }\end{array}$ & $\begin{array}{c}\text { Average } \\
\text { Pressure } \\
\text { [psig] }\end{array}$ & $\begin{array}{c}\text { Test Temp. } \\
{\left[{ }^{\circ} \mathrm{F}\right]}\end{array}$ & $\begin{array}{c}Q \text { (mass) } \\
{[\mathrm{gpm}]}\end{array}$ & $\begin{array}{c}Q \times 10^{3} \\
(\mathrm{mass}) \\
{\left[\mathrm{m}^{3} \mathrm{~s}^{-1}\right]}\end{array}$ & $\begin{array}{c}Q \\
(\text { flow) } \\
{[\text { (a) }}\end{array}$ & $\begin{array}{l}Q \times 10^{3} \\
(\text { flow) } \\
(\mathrm{a}) \\
{\left[\mathrm{m}^{3} \mathrm{~s}^{-1}\right]}\end{array}$ & $\begin{array}{c}C_{D} \\
\text { (mass) }\end{array}$ & $\begin{array}{c}C_{D} \\
\text { (flow) }^{(\text {a) }}\end{array}$ & $\begin{array}{l}H_{o}^{(\mathrm{a})} \\
{[\%]}\end{array}$ \\
\hline W602 & In-spray & Water & 39F3A & S8A & 380.3 & 79.7 & 2.1 & 0.134 & 2.0 & 0.126 & 0.589 & 0.555 & 91.8 \\
\hline W603 & In-spray & Water & 39F3A & S8A & 201.2 & 78.3 & 1.5 & 0.095 & 1.5 & 0.096 & 0.574 & 0.579 & 87.4 \\
\hline W604 & In-spray & Water & 39F3A & S8A & 201.2 & 77.2 & 1.5 & 0.096 & 1.4 & 0.089 & 0.583 & 0.536 & 87.9 \\
\hline W605 & In-spray & Water & 39F3A & S8A & 201.2 & 76.0 & 1.5 & 0.095 & 1.5 & 0.096 & 0.575 & 0.580 & 88.2 \\
\hline W606 & In-spray & Water & 39F3A & S8A & 104.0 & 71.4 & 1.1 & 0.070 & 1.1 & 0.070 & 0.590 & 0.588 & 83.1 \\
\hline W607 & In-spray & Water & 39F3A & S8A & 103.9 & 69.6 & 1.1 & 0.068 & 1.1 & 0.072 & 0.571 & 0.609 & 84.0 \\
\hline W608 & In-spray & Water & 39F3A & S8A & 103.9 & 68.8 & 1.1 & 0.068 & 1.0 & 0.066 & 0.576 & 0.553 & 85.0 \\
\hline W609 & In-spray & Water & 39F3A & S8B & 102.4 & 73.8 & 3.7 & 0.231 & 3.6 & 0.228 & 0.645 & 0.637 & 94.9 \\
\hline W610 & In-spray & Water & 39F3A & S8B & 201.1 & 73.3 & 5.0 & 0.317 & 5.0 & 0.320 & 0.630 & 0.636 & 95.5 \\
\hline W611 & In-spray & Water & $39 \mathrm{~F} 3 \mathrm{~A}$ & S8B & 381.5 & 74.4 & 6.9 & 0.439 & 7.0 & 0.442 & 0.634 & 0.638 & 93.8 \\
\hline W612 & In-spray & Water & 39F3A & $\mathrm{S} 8 \mathrm{C}$ & 102.9 & 74.4 & 7.7 & 0.485 & 7.7 & 0.490 & 0.648 & 0.655 & 98.5 \\
\hline W613 & In-spray & Water & 39F3A & $\mathrm{S} 8 \mathrm{C}$ & 198.9 & 73.8 & 10.6 & 0.668 & 10.5 & 0.666 & 0.643 & 0.641 & 98.9 \\
\hline W614 & In-spray & Water & 39F3A & $\mathrm{S} 8 \mathrm{C}$ & 103.1 & 72.6 & 7.7 & 0.485 & 7.6 & 0.482 & 0.648 & 0.644 & 99.3 \\
\hline W615 & In-spray & Water & 39F3A & $\mathrm{S} 8 \mathrm{C}$ & 377.6 & 73.0 & 14.6 & 0.926 & 14.6 & 0.926 & 0.646 & 0.647 & 99.4 \\
\hline W616 & In-spray & Water & 39F3A & $\mathrm{S} 8 \mathrm{C}$ & 377.5 & 74.4 & 14.7 & 0.929 & 14.7 & 0.929 & 0.649 & 0.648 & 99.7 \\
\hline W618 & In-chamber & Water & 30S3A & S8A & 200.4 & 71.8 & 1.5 & 0.095 & 1.5 & 0.092 & 0.574 & 0.557 & 96.9 \\
\hline W619 & In-chamber & Water & $30 \mathrm{~S} 3 \mathrm{~A}$ & S8A & 381.2 & 73.7 & 2.0 & 0.126 & 2.0 & 0.128 & 0.553 & 0.560 & 96.9 \\
\hline W620 & In-chamber & Water & $30 \mathrm{~S} 3 \mathrm{~A}$ & S8A & 101.9 & 72.4 & 1.1 & 0.067 & 1.1 & 0.067 & 0.570 & 0.568 & 96.8 \\
\hline W621 & In-chamber & Water & $30 \mathrm{~S} 3 \mathrm{~A}$ & S8A & 202.1 & 72.3 & 1.5 & 0.098 & 1.5 & 0.095 & 0.591 & 0.575 & 96.3 \\
\hline W622 & In-chamber & Water & $30 \mathrm{~S} 3 \mathrm{~A}$ & S8A & 380.1 & 73.1 & 2.0 & 0.126 & 2.0 & 0.125 & 0.554 & 0.550 & 95.9 \\
\hline W623 & In-chamber & Water & $30 \mathrm{~S} 3 \mathrm{~A}$ & S8A & 102.0 & 71.5 & 1.0 & 0.066 & 1.1 & 0.068 & 0.562 & 0.574 & 96.3 \\
\hline W624 & In-chamber & Water & $30 \mathrm{~S} 3 \mathrm{~A}$ & S8A & 202.1 & 69.4 & 1.4 & 0.091 & 1.4 & 0.089 & 0.546 & 0.536 & 94.0 \\
\hline W625 & In-chamber & Water & $30 \mathrm{~S} 3 \mathrm{~A}$ & S8A & 380.0 & 70.5 & 2.0 & 0.126 & 2.3 & 0.144 & 0.556 & 0.635 & 95.2 \\
\hline W626 & In-chamber & Water & $30 \mathrm{~S} 3 \mathrm{~A}$ & S8B & 101.6 & 69.9 & 3.6 & 0.227 & 3.6 & 0.226 & 0.636 & 0.633 & 96.1 \\
\hline W627 & In-chamber & Water & $30 \mathrm{~S} 3 \mathrm{~A}$ & S8B & 202.0 & 69.7 & 5.2 & 0.332 & 5.1 & 0.322 & 0.659 & 0.640 & 96.0 \\
\hline W629 & In-chamber & Water & $30 \mathrm{~S} 3 \mathrm{~A}$ & S8B & 101.8 & 70.4 & 3.6 & 0.230 & 3.5 & 0.220 & 0.642 & 0.614 & 97.3 \\
\hline W630 & In-chamber & Water & $30 \mathrm{~S} 3 \mathrm{~A}$ & S8B & 202.0 & 69.5 & 5.1 & 0.323 & 5.0 & 0.314 & 0.641 & 0.623 & 96.7 \\
\hline W631 & In-chamber & Water & $30 \mathrm{~S} 3 \mathrm{~A}$ & S8B & 380.3 & 70.7 & 7.2 & 0.459 & 7.0 & 0.445 & 0.664 & 0.644 & 96.6 \\
\hline W632 & In-chamber & Water & $30 \mathrm{~S} 3 \mathrm{~A}$ & S8B & 101.8 & 70.0 & 3.6 & 0.225 & 3.6 & 0.228 & 0.629 & 0.638 & 97.5 \\
\hline W633 & In-chamber & Water & $30 \mathrm{~S} 3 \mathrm{~A}$ & S8B & 202.4 & 69.8 & 5.1 & 0.321 & 5.0 & 0.319 & 0.637 & 0.632 & 96.7 \\
\hline
\end{tabular}

(a) -Instrument(s) upon which measurements are based were not calibrated. Result is "For-Information Only"; (b) - Humidity data collected but not reported for W79-W81 and W86-W88; (c) - Coriolis meter instrument reading/sensitivity issue yielded anomalous result. 
Table B.5. (contd)

\begin{tabular}{|c|c|c|c|c|c|c|c|c|c|c|c|c|c|}
\hline Test ID & $\begin{array}{c}\text { Test } \\
\text { Category }\end{array}$ & Simulant & $\begin{array}{c}\text { Chamber } \\
\text { ID }\end{array}$ & $\begin{array}{c}\text { Orifice } \\
\text { ID }\end{array}$ & $\begin{array}{c}\text { Average } \\
\text { Pressure } \\
\text { [psig] }\end{array}$ & $\begin{array}{c}\text { Test Temp. } \\
{\left[{ }^{\circ} \mathrm{F}\right]}\end{array}$ & $\begin{array}{c}Q \text { (mass) } \\
{[\mathrm{gpm}]}\end{array}$ & $\begin{array}{c}Q \times 10^{3} \\
(\mathrm{mass}) \\
{\left[\mathrm{m}^{3} \mathrm{~s}^{-1}\right]}\end{array}$ & $\begin{array}{c}Q \\
\text { (flow) }^{(\mathrm{a})} \\
{[\mathrm{gpm}]}\end{array}$ & $\begin{array}{l}Q \times 10^{3} \\
(\text { flow) } \\
(\mathrm{a}) \\
{\left[\mathrm{m}^{3} \mathrm{~s}^{-1}\right]}\end{array}$ & $\begin{array}{c}C_{D} \\
\text { (mass) }\end{array}$ & $\begin{array}{c}C_{D} \\
\text { (flow) }^{(\text {a) }}\end{array}$ & $\begin{array}{l}H_{o}^{(a)} \\
{[\%]}\end{array}$ \\
\hline W634 & In-chamber & Water & 30S3A & S8B & 381.1 & 70.8 & 7.0 & 0.440 & 6.8 & 0.429 & 0.636 & 0.620 & 96.6 \\
\hline W635 & In-chamber & Water & 30S3A & $\mathrm{S} 8 \mathrm{C}$ & 101.6 & 70.3 & 7.6 & 0.478 & 7.6 & 0.483 & 0.644 & 0.650 & 97.6 \\
\hline W636 & In-chamber & Water & $30 \mathrm{~S} 3 \mathrm{~A}$ & $\mathrm{~S} 8 \mathrm{C}$ & 201.4 & 70.2 & 10.8 & 0.687 & 10.7 & 0.679 & 0.657 & 0.649 & 96.5 \\
\hline W637 & In-chamber & Water & $30 \mathrm{~S} 3 \mathrm{~A}$ & $\mathrm{~S} 8 \mathrm{C}$ & 379.4 & 71.2 & 14.6 & 0.925 & 14.6 & 0.926 & 0.644 & 0.645 & 96.7 \\
\hline W638 & In-chamber & Water & $30 \mathrm{~S} 3 \mathrm{~A}$ & $\mathrm{~S} 8 \mathrm{C}$ & 101.5 & 69.1 & 7.7 & 0.488 & 7.7 & 0.486 & 0.657 & 0.654 & 98.8 \\
\hline W639 & In-chamber & Water & $30 \mathrm{~S} 3 \mathrm{~A}$ & $\mathrm{~S} 8 \mathrm{C}$ & 201.4 & 69.1 & 10.7 & 0.678 & 10.7 & 0.677 & 0.648 & 0.647 & 97.2 \\
\hline W640 & In-chamber & Water & 30S3A & $\mathrm{S} 8 \mathrm{C}$ & 380.3 & 70.1 & 14.4 & 0.915 & 14.3 & 0.903 & 0.636 & 0.628 & 96.4 \\
\hline W641 & In-chamber & Water & 30S3A & $\mathrm{S} 8 \mathrm{C}$ & 101.5 & 69.6 & 7.6 & 0.483 & 7.6 & 0.479 & 0.651 & 0.645 & 98.8 \\
\hline W642 & In-chamber & Water & 30S3A & $\mathrm{S} 8 \mathrm{C}$ & 201.3 & 69.5 & 10.6 & 0.669 & 10.8 & 0.682 & 0.640 & 0.652 & 98.6 \\
\hline W643 & In-chamber & Water & 30S3A & $\mathrm{S} 8 \mathrm{C}$ & 379.2 & 70.6 & 14.5 & 0.919 & 14.9 & 0.941 & 0.641 & 0.655 & 97.6 \\
\hline W644 & In-chamber & Water & $30 \mathrm{~S} 3 \mathrm{~A}$ & S4A & 101.2 & 64.5 & 31.1 & 1.972 & 29.9 & 1.891 & 0.721 & 0.692 & 91.7 \\
\hline W645 & In-chamber & Water & 30S3A & S4A & 199.2 & 66.0 & 44.9 & 2.844 & 42.4 & 2.685 & 0.741 & 0.700 & 92.2 \\
\hline W647 & In-chamber & Water & $30 \mathrm{~S} 3 \mathrm{~A}$ & S4A & 101.2 & 69.0 & 31.3 & 1.981 & 29.9 & 1.891 & 0.724 & 0.691 & 96.6 \\
\hline W648 & In-chamber & Water & 30S3A & S4A & 199.7 & 69.8 & 44.7 & 2.833 & 42.4 & 2.688 & 0.737 & 0.700 & 95.9 \\
\hline W649 & In-chamber & Water & $30 \mathrm{~S} 3 \mathrm{~A}$ & S4A & 374.2 & 72.4 & 63.0 & 3.992 & 59.2 & 3.748 & 0.759 & 0.713 & 96.1 \\
\hline W650 & In-chamber & Water & $30 \mathrm{~S} 3 \mathrm{~A}$ & S4A & 101.0 & 69.6 & 31.2 & 1.979 & 29.8 & 1.888 & 0.724 & 0.691 & 98.6 \\
\hline W651 & In-chamber & Water & $30 \mathrm{~S} 3 \mathrm{~A}$ & S4A & 198.3 & 69.4 & 43.9 & 2.779 & 42.2 & 2.670 & 0.726 & 0.697 & 97.0 \\
\hline W653 & In-chamber & Water & $30 \mathrm{~S} 3 \mathrm{~A}$ & S4A & 370.5 & 70.4 & 62.6 & 3.965 & 58.8 & 3.721 & 0.758 & 0.711 & 97.0 \\
\hline W654 & In-chamber & Water & $30 \mathrm{~S} 3 \mathrm{~A}$ & S4A & 374.7 & 70.4 & 62.7 & 3.972 & 59.9 & 3.791 & 0.755 & 0.720 & 96.6 \\
\hline W655 & Humidity & Water & $30 \mathrm{~S} 3 \mathrm{~A}$ & S8A & 382.6 & 79.1 & 2.0 & 0.128 & 1.8 & 0.116 & 0.559 & 0.509 & 98.2 \\
\hline W656 & Humidity & Water & $30 \mathrm{~S} 3 \mathrm{~A}$ & S8A & 382.5 & 76.4 & 2.1 & 0.130 & 1.5 & 0.097 & 0.572 & 0.425 & 97.9 \\
\hline W657 & Humidity & Water & $30 \mathrm{~S} 3 \mathrm{~A}$ & S8A & 382.3 & 74.5 & 2.0 & 0.128 & 2.0 & 0.126 & 0.561 & 0.553 & 98.0 \\
\hline W658 & Humidity & Water & $30 \mathrm{~S} 3 \mathrm{~A}$ & S8A & 382.4 & 74.8 & 2.1 & 0.130 & 1.6 & 0.103 & 0.570 & 0.453 & 97.9 \\
\hline W659 & Humidity & Water & 30S3A & S8A & 382.1 & 70.7 & 2.0 & 0.127 & 2.1 & 0.135 & 0.558 & 0.592 & 45.1 \\
\hline W660 & Humidity & Water & $30 \mathrm{~S} 3 \mathrm{~A}$ & S8A & 382.1 & 68.5 & 2.0 & 0.126 & 1.7 & 0.105 & 0.552 & 0.460 & 44.3 \\
\hline W661 & Humidity & Water & $30 \mathrm{~S} 3 \mathrm{~A}$ & S8A & 382.0 & 70.9 & 2.1 & 0.130 & 1.5 & 0.096 & 0.570 & 0.422 & 47.9 \\
\hline W662 & In-chamber & Water & $20 \mathrm{~S} 3 \mathrm{~A}$ & S8A & 101.6 & 63.8 & 1.0 & 0.065 & 1.1 & 0.070 & 0.555 & 0.598 & 93.9 \\
\hline W663 & In-chamber & Water & $20 \mathrm{~S} 3 \mathrm{~A}$ & S8A & 200.1 & 66.2 & 1.4 & 0.091 & 1.3 & 0.081 & 0.550 & 0.492 & 94.5 \\
\hline W664 & In-chamber & Water & $20 \mathrm{~S} 3 \mathrm{~A}$ & S8A & 381.6 & 69.2 & 2.2 & 0.139 & 1.5 & 0.094 & 0.610 & 0.412 & 95.3 \\
\hline W665 & In-chamber & Water & $20 \mathrm{~S} 3 \mathrm{~A}$ & S8A & 102.0 & 69.4 & 1.1 & 0.069 & 1.0 & 0.064 & 0.587 & 0.539 & 94.8 \\
\hline
\end{tabular}

(a) -Instrument(s) upon which measurements are based were not calibrated. Result is "For-Information Only"; (b) - Humidity data collected but not reported for W79-W81 and W86-W88; (c) - Coriolis meter instrument reading/sensitivity issue yielded anomalous result. 
Table B.5. (contd)

\begin{tabular}{|c|c|c|c|c|c|c|c|c|c|c|c|c|c|}
\hline Test ID & $\begin{array}{c}\text { Test } \\
\text { Category }\end{array}$ & Simulant & $\begin{array}{c}\text { Chamber } \\
\text { ID }\end{array}$ & $\begin{array}{c}\text { Orifice } \\
\text { ID }\end{array}$ & $\begin{array}{c}\text { Average } \\
\text { Pressure } \\
\text { [psig] }\end{array}$ & $\begin{array}{c}\text { Test Temp. } \\
{\left[{ }^{\circ} \mathrm{F}\right]}\end{array}$ & $\begin{array}{c}Q \text { (mass) } \\
{[\mathrm{gpm}]}\end{array}$ & $\begin{array}{c}Q \times 10^{3} \\
(\mathrm{mass}) \\
{\left[\mathrm{m}^{3} \mathrm{~s}^{-1}\right]}\end{array}$ & $\begin{array}{c}Q \\
\text { (flow) }^{(\mathrm{a})} \\
{[\mathrm{gpm}]}\end{array}$ & $\begin{array}{l}Q \times 10^{3} \\
(\text { flow) } \\
(\mathrm{a}) \\
{\left[\mathrm{m}^{3} \mathrm{~s}^{-1}\right]}\end{array}$ & $\begin{array}{c}C_{D} \\
\text { (mass) }\end{array}$ & $\begin{array}{c}C_{D} \\
\text { (flow) }^{(\text {a) }}\end{array}$ & $\begin{array}{l}H_{o}^{(a)} \\
{[\%]}\end{array}$ \\
\hline W666 & In-chamber & Water & $20 \mathrm{~S} 3 \mathrm{~A}$ & S8A & 200.4 & 69.6 & 1.5 & 0.092 & 1.6 & 0.100 & 0.560 & 0.606 & 94.9 \\
\hline W667 & In-chamber & Water & $20 \mathrm{~S} 3 \mathrm{~A}$ & S8A & 381.7 & 71.1 & 2.1 & 0.134 & 1.8 & 0.116 & 0.589 & 0.507 & 95.4 \\
\hline W668 & In-chamber & Water & $20 \mathrm{~S} 3 \mathrm{~A}$ & S8A & 102.0 & 70.2 & 1.0 & 0.066 & 1.1 & 0.071 & 0.556 & 0.601 & 96.5 \\
\hline W669 & In-chamber & Water & $20 \mathrm{~S} 3 \mathrm{~A}$ & S8A & 200.4 & 70.4 & 1.4 & 0.091 & 1.5 & 0.096 & 0.552 & 0.582 & 96.2 \\
\hline W670 & In-chamber & Water & $20 \mathrm{~S} 3 \mathrm{~A}$ & S8A & 381.7 & 71.7 & 2.1 & 0.136 & 1.8 & 0.114 & 0.596 & 0.500 & 96.2 \\
\hline W671 & In-chamber & Water & $20 \mathrm{~S} 3 \mathrm{~A}$ & S8B & 101.7 & 70.6 & 3.6 & 0.225 & 3.6 & 0.231 & 0.630 & 0.646 & 96.7 \\
\hline W672 & In-chamber & Water & $20 \mathrm{~S} 3 \mathrm{~A}$ & S8B & 199.9 & 70.7 & 5.0 & 0.320 & 5.1 & 0.322 & 0.638 & 0.643 & 96.5 \\
\hline W673 & In-chamber & Water & $20 \mathrm{~S} 3 \mathrm{~A}$ & S8B & 380.5 & 71.9 & 7.0 & 0.446 & 6.7 & 0.424 & 0.645 & 0.613 & 96.8 \\
\hline W674 & In-chamber & Water & $20 \mathrm{~S} 3 \mathrm{~A}$ & S8B & 101.7 & 70.7 & 3.6 & 0.227 & 3.6 & 0.226 & 0.636 & 0.632 & 97.6 \\
\hline W675 & In-chamber & Water & $20 \mathrm{~S} 3 \mathrm{~A}$ & S8B & 199.8 & 70.2 & 5.0 & 0.317 & 5.1 & 0.322 & 0.632 & 0.643 & 97.3 \\
\hline W676 & In-chamber & Water & $20 \mathrm{~S} 3 \mathrm{~A}$ & S8B & 380.5 & 71.6 & 7.0 & 0.446 & 6.8 & 0.433 & 0.645 & 0.626 & 97.1 \\
\hline W677 & In-chamber & Water & $20 \mathrm{~S} 3 \mathrm{~A}$ & S8B & 101.6 & 70.4 & 3.5 & 0.224 & 3.6 & 0.229 & 0.626 & 0.640 & 97.6 \\
\hline W678 & In-chamber & Water & $20 \mathrm{~S} 3 \mathrm{~A}$ & S8B & 199.7 & 70.3 & 5.0 & 0.320 & 5.0 & 0.315 & 0.638 & 0.629 & 97.3 \\
\hline W679 & In-chamber & Water & $20 \mathrm{~S} 3 \mathrm{~A}$ & S8B & 380.3 & 71.9 & 7.0 & 0.444 & 7.0 & 0.443 & 0.643 & 0.641 & 97.1 \\
\hline W680 & In-chamber & Water & $20 \mathrm{~S} 3 \mathrm{~A}$ & $\mathrm{~S} 8 \mathrm{C}$ & 102.4 & 69.9 & 7.5 & 0.476 & 7.6 & 0.484 & 0.638 & 0.650 & 89.7 \\
\hline W681 & In-chamber & Water & $20 \mathrm{~S} 3 \mathrm{~A}$ & $\mathrm{~S} 8 \mathrm{C}$ & 201.6 & 71.1 & 10.9 & 0.687 & 10.7 & 0.679 & 0.657 & 0.649 & 94.1 \\
\hline W682 & In-chamber & Water & $20 \mathrm{~S} 3 \mathrm{~A}$ & $\mathrm{~S} 8 \mathrm{C}$ & 380.2 & 73.8 & 15.3 & 0.969 & 14.7 & 0.928 & 0.674 & 0.646 & 96.2 \\
\hline W683 & In-chamber & Water & $20 \mathrm{~S} 3 \mathrm{~A}$ & $\mathrm{~S} 8 \mathrm{C}$ & 102.7 & 65.4 & 7.6 & 0.482 & 7.7 & 0.487 & 0.646 & 0.652 & 94.9 \\
\hline W684 & In-chamber & Water & $20 \mathrm{~S} 3 \mathrm{~A}$ & $\mathrm{~S} 8 \mathrm{C}$ & 201.7 & 66.7 & 11.0 & 0.696 & 10.8 & 0.683 & 0.665 & 0.653 & 95.9 \\
\hline W685 & In-chamber & Water & $20 \mathrm{~S} 3 \mathrm{~A}$ & $\mathrm{~S} 8 \mathrm{C}$ & 380.0 & 69.6 & 15.3 & 0.970 & 14.5 & 0.921 & 0.675 & 0.641 & 96.9 \\
\hline W686 & In-chamber & Water & $20 \mathrm{~S} 3 \mathrm{~A}$ & $\mathrm{~S} 8 \mathrm{C}$ & 101.1 & 70.1 & 7.6 & 0.483 & 7.6 & 0.484 & 0.651 & 0.653 & 98.4 \\
\hline W687 & In-chamber & Water & $20 \mathrm{~S} 3 \mathrm{~A}$ & $\mathrm{~S} 8 \mathrm{C}$ & 201.8 & 71.2 & 10.9 & 0.688 & 10.7 & 0.679 & 0.657 & 0.648 & 98.8 \\
\hline W688 & In-chamber & Water & $20 \mathrm{~S} 3 \mathrm{~A}$ & $\mathrm{~S} 8 \mathrm{C}$ & 380.1 & 73.8 & 15.4 & 0.976 & 14.7 & 0.929 & 0.679 & 0.646 & 99.0 \\
\hline W689 & In-chamber & Water & 20S3A & $\mathrm{S} 8 \mathrm{C}$ & 380.1 & 74.8 & 14.6 & 0.927 & 14.9 & 0.945 & 0.645 & 0.657 & 99.6 \\
\hline W690 & Functional & Water & $20 \mathrm{~S} 3 \mathrm{~A}$ & S8A & 101.3 & 73.6 & 1.1 & 0.069 & 1.1 & 0.072 & 0.587 & 0.612 & 94.6 \\
\hline W691 & Functional & Water & $20 \mathrm{~S} 3 \mathrm{~A}$ & S8A & 202.2 & 73.4 & 1.5 & 0.096 & 1.5 & 0.097 & 0.577 & 0.587 & 95.4 \\
\hline W692 & Functional & Water & $20 \mathrm{~S} 3 \mathrm{~A}$ & S8A & 381.4 & 74.1 & 2.1 & 0.134 & 1.8 & 0.116 & 0.590 & 0.511 & 96.0 \\
\hline W693 & Functional & Water & $20 \mathrm{~S} 3 \mathrm{~A}$ & S8A & 102.0 & 72.9 & 1.1 & 0.067 & 1.0 & 0.064 & 0.566 & 0.546 & 97.3 \\
\hline W694 & Functional & Water & $20 \mathrm{~S} 3 \mathrm{~A}$ & S8A & 202.3 & 73.0 & 1.5 & 0.096 & 1.6 & 0.101 & 0.578 & 0.606 & 97.2 \\
\hline W695 & Functional & Water & $20 \mathrm{~S} 3 \mathrm{~A}$ & S8A & 381.5 & 74.0 & 2.2 & 0.136 & 2.0 & 0.127 & 0.599 & 0.558 & 97.0 \\
\hline
\end{tabular}

(a) -Instrument(s) upon which measurements are based were not calibrated. Result is "For-Information Only"; (b) - Humidity data collected but not reported for W79-W81 and W86-W88; (c) - Coriolis meter instrument reading/sensitivity issue yielded anomalous result. 
Table B.5. (contd)

\begin{tabular}{|c|c|c|c|c|c|c|c|c|c|c|c|c|c|}
\hline Test ID & $\begin{array}{c}\text { Test } \\
\text { Category }\end{array}$ & Simulant & $\begin{array}{c}\text { Chamber } \\
\text { ID }\end{array}$ & $\begin{array}{c}\text { Orifice } \\
\text { ID }\end{array}$ & $\begin{array}{c}\text { Average } \\
\text { Pressure } \\
\text { [psig] }\end{array}$ & $\begin{array}{c}\text { Test Temp. } \\
{\left[{ }^{\circ} \mathrm{F}\right]}\end{array}$ & $\begin{array}{c}Q \text { (mass) } \\
{[\mathrm{gpm}]}\end{array}$ & $\begin{array}{c}Q \times 10^{3} \\
\text { (mass) } \\
{\left[\mathrm{m}^{3} \mathrm{~s}^{-1}\right]}\end{array}$ & $\begin{array}{c}Q \\
\text { (flow) }^{(a)} \\
{[\mathrm{gpm}]}\end{array}$ & $\begin{array}{c}Q \times 10^{3} \\
(\text { flow) } \\
(\mathrm{a}) \\
{\left[\mathrm{m}^{3} \mathrm{~s}^{-1}\right]}\end{array}$ & $\begin{array}{c}C_{D} \\
\text { (mass) }\end{array}$ & $\begin{array}{c}C_{D} \\
\text { (flow) }^{\text {(a) }}\end{array}$ & $\begin{array}{c}H_{o}^{(\mathrm{a})} \\
{[\%]}\end{array}$ \\
\hline W696 & Functional & Water & $20 \mathrm{~S} 3 \mathrm{~A}$ & S8A & 102.0 & 72.9 & 1.1 & 0.070 & 0.9 & 0.060 & 0.592 & 0.505 & 97.5 \\
\hline W697 & Functional & Water & $20 \mathrm{~S} 3 \mathrm{~A}$ & S8A & 202.3 & 72.9 & 1.5 & 0.096 & 1.8 & 0.113 & 0.579 & 0.683 & 97.1 \\
\hline W698 & Functional & Water & $20 \mathrm{~S} 3 \mathrm{~A}$ & S8A & 381.5 & 74.9 & 2.2 & 0.138 & 2.2 & 0.139 & 0.604 & 0.609 & 97.0 \\
\hline W699 & In-chamber & Water & $20 \mathrm{~S} 3 \mathrm{~A}$ & S4A & 100.5 & 68.3 & 31.4 & 1.991 & 29.8 & 1.884 & 0.731 & 0.691 & 93.2 \\
\hline W700 & In-chamber & Water & $20 \mathrm{~S} 3 \mathrm{~A}$ & S4A & 199.6 & 69.3 & 44.2 & 2.802 & 42.4 & 2.682 & 0.729 & 0.698 & 95.4 \\
\hline W701 & In-chamber & Water & $20 \mathrm{~S} 3 \mathrm{~A}$ & S4A & 199.9 & 68.8 & 44.9 & 2.842 & 42.3 & 2.681 & 0.739 & 0.698 & 97.1 \\
\hline W702 & In-chamber & Water & $20 \mathrm{~S} 3 \mathrm{~A}$ & S4A & 381.4 & 71.1 & 63.9 & 4.046 & 60.2 & 3.816 & 0.762 & 0.719 & 97.7 \\
\hline W703 & In-chamber & Water & $20 \mathrm{~S} 3 \mathrm{~A}$ & S4A & 100.4 & 69.1 & 31.1 & 1.970 & 29.6 & 1.874 & 0.723 & 0.688 & 98.9 \\
\hline W704 & In-chamber & Water & $20 \mathrm{~S} 3 \mathrm{~A}$ & S4A & 100.4 & 69.2 & 31.5 & 1.994 & 29.8 & 1.889 & 0.732 & 0.693 & 99.2 \\
\hline W705 & In-chamber & Water & $20 \mathrm{~S} 3 \mathrm{~A}$ & S4A & 199.0 & 70.1 & 44.5 & 2.816 & 42.2 & 2.671 & 0.734 & 0.696 & 99.5 \\
\hline W706 & In-chamber & Water & $20 \mathrm{~S} 3 \mathrm{~A}$ & S4A & 381.1 & 70.7 & 63.9 & 4.044 & 59.8 & 3.785 & 0.762 & 0.713 & 99.8 \\
\hline W707 & In-chamber & Water & $20 \mathrm{~S} 3 \mathrm{~A}$ & S4A & 381.8 & 70.8 & 63.8 & 4.038 & 60.1 & 3.809 & 0.760 & 0.717 & 100.3 \\
\hline W708 & In-chamber & Water & $10 \mathrm{~S} 2 \mathrm{~A}$ & S4A & 99.2 & 66.8 & 31.2 & 1.975 & 29.5 & 1.870 & 0.730 & 0.690 & 94.4 \\
\hline W710 & In-chamber & Water & $10 \mathrm{~S} 2 \mathrm{~A}$ & S4A & 380.6 & 71.3 & 64.5 & 4.084 & 60.3 & 3.818 & 0.770 & 0.720 & 98.3 \\
\hline W711 & In-chamber & Water & $10 \mathrm{~S} 2 \mathrm{~A}$ & S4A & 99.6 & 68.8 & 31.0 & 1.964 & 29.6 & 1.875 & 0.724 & 0.691 & 100.2 \\
\hline W712 & In-chamber & Water & $10 \mathrm{~S} 2 \mathrm{~A}$ & S4A & 197.5 & 69.5 & 44.5 & 2.817 & 42.0 & 2.659 & 0.737 & 0.696 & 100.3 \\
\hline W713 & In-chamber & Water & $10 \mathrm{~S} 2 \mathrm{~A}$ & S4A & 381.4 & 71.0 & 62.6 & 3.966 & 60.4 & 3.824 & 0.747 & 0.720 & 100.5 \\
\hline W714 & In-chamber & Water & $10 \mathrm{~S} 2 \mathrm{~A}$ & S4A & 99.6 & 68.2 & 30.7 & 1.944 & 29.6 & 1.875 & 0.717 & 0.691 & 100.7 \\
\hline W715 & In-chamber & Water & $10 \mathrm{~S} 2 \mathrm{~A}$ & S4A & 197.7 & 69.1 & 43.8 & 2.773 & 42.2 & 2.672 & 0.725 & 0.699 & 100.9 \\
\hline W716 & In-chamber & Water & $10 \mathrm{~S} 2 \mathrm{~A}$ & S4A & 381.4 & 71.0 & 64.3 & 4.070 & 60.5 & 3.833 & 0.767 & 0.722 & 101.1 \\
\hline W717 & In-chamber & Water & $10 \mathrm{~S} 2 \mathrm{~A}$ & S8A & 101.6 & 67.3 & 0.9 & 0.059 & 1.1 & 0.068 & 0.501 & 0.581 & 90.4 \\
\hline W718 & In-chamber & Water & $10 \mathrm{~S} 2 \mathrm{~A}$ & S8A & 202.0 & 68.7 & 1.5 & 0.094 & 1.5 & 0.096 & 0.569 & 0.579 & 92.9 \\
\hline W719 & In-chamber & Water & $10 \mathrm{~S} 2 \mathrm{~A}$ & S8A & 381.0 & 71.6 & 2.0 & 0.128 & 1.8 & 0.113 & 0.562 & 0.498 & 95.1 \\
\hline W720 & In-chamber & Water & $10 \mathrm{~S} 2 \mathrm{~A}$ & S8A & 101.8 & 72.4 & 0.9 & 0.059 & 1.0 & 0.066 & 0.501 & 0.558 & 97.7 \\
\hline W721 & In-chamber & Water & $10 \mathrm{~S} 2 \mathrm{~A}$ & S8A & 202.1 & 73.2 & 1.5 & 0.092 & 1.5 & 0.096 & 0.557 & 0.580 & 98.3 \\
\hline W722 & In-chamber & Water & $10 \mathrm{~S} 2 \mathrm{~A}$ & S8A & 381.3 & 73.9 & 2.0 & 0.128 & 2.0 & 0.124 & 0.561 & 0.543 & 98.9 \\
\hline W723 & In-chamber & Water & $10 \mathrm{~S} 2 \mathrm{~A}$ & S8A & 102.0 & 71.6 & 1.0 & 0.061 & 1.0 & 0.065 & 0.519 & 0.551 & 99.6 \\
\hline W724 & In-chamber & Water & $10 \mathrm{~S} 2 \mathrm{~A}$ & S8A & 200.1 & 70.6 & 1.4 & 0.090 & 1.3 & 0.085 & 0.548 & 0.512 & 99.7 \\
\hline W725 & In-chamber & Water & $10 \mathrm{~S} 2 \mathrm{~A}$ & S8A & 381.1 & 72.2 & 2.1 & 0.130 & 1.9 & 0.121 & 0.572 & 0.530 & 99.7 \\
\hline W726 & In-chamber & Water & $10 \mathrm{~S} 2 \mathrm{~A}$ & S8B & 101.3 & 69.5 & 3.6 & 0.230 & 3.6 & 0.229 & 0.645 & 0.642 & 90.2 \\
\hline
\end{tabular}

(a) -Instrument(s) upon which measurements are based were not calibrated. Result is "For-Information Only"; (b) - Humidity data collected but not reported for W79-W81 and W86-W88; (c) - Coriolis meter instrument reading/sensitivity issue yielded anomalous result. 
Table B.5. (contd)

\begin{tabular}{|c|c|c|c|c|c|c|c|c|c|c|c|c|c|}
\hline Test ID & $\begin{array}{c}\text { Test } \\
\text { Category }\end{array}$ & Simulant & $\begin{array}{c}\text { Chamber } \\
\text { ID }\end{array}$ & $\begin{array}{c}\text { Orifice } \\
\text { ID }\end{array}$ & $\begin{array}{c}\text { Average } \\
\text { Pressure } \\
\text { [psig] }\end{array}$ & $\begin{array}{c}\text { Test Temp. } \\
{\left[{ }^{\circ} \mathrm{F}\right]}\end{array}$ & $\begin{array}{c}Q \text { (mass) } \\
{[\mathrm{gpm}]}\end{array}$ & $\begin{array}{c}Q \times 10^{3} \\
(\mathrm{mass}) \\
{\left[\mathrm{m}^{3} \mathrm{~s}^{-1}\right]}\end{array}$ & $\begin{array}{c}Q \\
(\text { flow) } \\
{[\text { (a) }}\end{array}$ & $\begin{array}{c}Q \times 10^{3} \\
(\text { flow) } \\
(\mathrm{a}) \\
{\left[\mathrm{m}^{3} \mathrm{~s}^{-1}\right]}\end{array}$ & $\begin{array}{c}C_{D} \\
\text { (mass) }\end{array}$ & $\begin{array}{c}C_{D} \\
\text { (flow) }^{(\text {a) }}\end{array}$ & $\begin{array}{c}H_{o}^{(\mathrm{a})} \\
{[\%]}\end{array}$ \\
\hline W727 & In-chamber & Water & $10 \mathrm{~S} 2 \mathrm{~A}$ & S8B & 101.0 & 69.8 & 3.5 & 0.223 & 3.6 & 0.229 & 0.627 & 0.643 & 95.3 \\
\hline W728 & In-chamber & Water & $10 \mathrm{~S} 2 \mathrm{~A}$ & S8B & 101.0 & 70.2 & 3.5 & 0.224 & 3.6 & 0.229 & 0.630 & 0.642 & 97.1 \\
\hline W729 & In-chamber & Water & $10 \mathrm{~S} 2 \mathrm{~A}$ & S8B & 199.6 & 71.2 & 4.9 & 0.313 & 5.0 & 0.315 & 0.625 & 0.629 & 97.7 \\
\hline W730 & In-chamber & Water & $10 \mathrm{~S} 2 \mathrm{~A}$ & S8B & 379.8 & 73.6 & 6.9 & 0.439 & 6.6 & 0.418 & 0.636 & 0.604 & 98.8 \\
\hline W731 & In-chamber & Water & $10 \mathrm{~S} 2 \mathrm{~A}$ & S8B & 199.5 & 71.9 & 5.0 & 0.314 & 5.1 & 0.321 & 0.627 & 0.640 & 99.6 \\
\hline W732 & In-chamber & Water & $10 \mathrm{~S} 2 \mathrm{~A}$ & S8B & 379.7 & 72.3 & 6.9 & 0.435 & 7.1 & 0.447 & 0.631 & 0.647 & 100.1 \\
\hline W733 & In-chamber & Water & $10 \mathrm{~S} 2 \mathrm{~A}$ & S8B & 200.0 & 71.3 & 5.0 & 0.315 & 4.9 & 0.313 & 0.629 & 0.625 & 100.3 \\
\hline W734 & In-chamber & Water & $10 \mathrm{~S} 2 \mathrm{~A}$ & S8B & 379.6 & 72.5 & 6.9 & 0.436 & 7.0 & 0.446 & 0.631 & 0.646 & 100.6 \\
\hline W735 & In-chamber & Water & $10 \mathrm{~S} 2 \mathrm{~A}$ & $\mathrm{~S} 8 \mathrm{C}$ & 100.7 & 71.4 & 7.5 & 0.477 & 7.6 & 0.478 & 0.644 & 0.646 & 92.1 \\
\hline W736 & In-chamber & Water & $10 \mathrm{~S} 2 \mathrm{~A}$ & $\mathrm{~S} 8 \mathrm{C}$ & 199.9 & 72.4 & 10.6 & 0.672 & 10.6 & 0.670 & 0.645 & 0.643 & 95.9 \\
\hline W737 & In-chamber & Water & $10 \mathrm{~S} 2 \mathrm{~A}$ & $\mathrm{~S} 8 \mathrm{C}$ & 376.8 & 72.7 & 14.5 & 0.915 & 14.7 & 0.929 & 0.640 & 0.649 & 97.7 \\
\hline W738 & In-chamber & Water & $10 \mathrm{~S} 2 \mathrm{~A}$ & $\mathrm{~S} 8 \mathrm{C}$ & 377.8 & 73.9 & 14.6 & 0.928 & 14.7 & 0.929 & 0.647 & 0.648 & 98.9 \\
\hline W740 & In-chamber & Water & $10 \mathrm{~S} 2 \mathrm{~A}$ & $\mathrm{~S} 8 \mathrm{C}$ & 378.1 & 75.3 & 14.5 & 0.920 & 14.5 & 0.918 & 0.642 & 0.641 & 100.3 \\
\hline W741 & In-chamber & Water & $10 \mathrm{~S} 2 \mathrm{~A}$ & $\mathrm{~S} 8 \mathrm{C}$ & 100.8 & 70.1 & 7.7 & 0.485 & 7.5 & 0.476 & 0.655 & 0.644 & 100.6 \\
\hline W742 & In-chamber & Water & $10 \mathrm{~S} 2 \mathrm{~A}$ & $\mathrm{~S} 8 \mathrm{C}$ & 199.8 & 70.0 & 10.7 & 0.675 & 10.5 & 0.664 & 0.647 & 0.637 & 100.7 \\
\hline W743 & In-chamber & Water & $10 \mathrm{~S} 2 \mathrm{~A}$ & $\mathrm{~S} 8 \mathrm{C}$ & 100.5 & 68.8 & 7.5 & 0.472 & 7.6 & 0.480 & 0.639 & 0.650 & 100.7 \\
\hline W744 & In-chamber & Water & $10 \mathrm{~S} 2 \mathrm{~A}$ & $\mathrm{~S} 8 \mathrm{C}$ & 199.7 & 68.7 & 10.6 & 0.674 & 10.6 & 0.671 & 0.648 & 0.644 & 100.7 \\
\hline W745 & In-chamber & Water & $10 \mathrm{~S} 2 \mathrm{~A}$ & $\mathrm{~S} 8 \mathrm{C}$ & 377.4 & 70.0 & 14.5 & 0.917 & 14.6 & 0.928 & 0.641 & 0.648 & 100.8 \\
\hline S746 & In-chamber & $30 \mathrm{~Pa}$ Clay & $20 \mathrm{~S} 3 \mathrm{~A}$ & S8A & 101.4 & 72.2 & 0.8 & 0.049 & 1.0 & 0.062 & 0.465 & 0.592 & 81.5 \\
\hline S747 & In-chamber & $30 \mathrm{~Pa}$ Clay & $20 \mathrm{~S} 3 \mathrm{~A}$ & S8A & 102.6 & 72.5 & 0.9 & 0.055 & 1.0 & 0.062 & 0.516 & 0.585 & 87.9 \\
\hline S748 & In-chamber & $30 \mathrm{~Pa}$ Clay & $20 \mathrm{~S} 3 \mathrm{~A}$ & S8A & 102.5 & 73.1 & 0.9 & 0.059 & 1.0 & 0.065 & 0.556 & 0.613 & 87.4 \\
\hline S749 & In-chamber & $30 \mathrm{~Pa}$ Clay & $20 \mathrm{~S} 3 \mathrm{~A}$ & S8A & 102.1 & 73.5 & 0.9 & 0.060 & 1.1 & 0.072 & 0.566 & 0.687 & 90.4 \\
\hline $\mathrm{S} 750$ & In-chamber & $30 \mathrm{~Pa}$ Clay & $20 \mathrm{~S} 3 \mathrm{~A}$ & S8B & 202.0 & 75.6 & 2.3 & 0.148 & 2.3 & 0.144 & 0.329 & 0.319 & 91.8 \\
\hline S751 & In-chamber & $30 \mathrm{~Pa}$ Clay & $20 \mathrm{~S} 3 \mathrm{~A}$ & S8B & 202.5 & 76.8 & 2.4 & 0.151 & 2.5 & 0.158 & 0.334 & 0.351 & 94.2 \\
\hline S752 & In-chamber & $30 \mathrm{~Pa}$ Clay & $20 \mathrm{~S} 3 \mathrm{~A}$ & S8B & 202.5 & 77.9 & 2.3 & 0.145 & 2.4 & 0.153 & 0.322 & 0.339 & 95.1 \\
\hline $\mathrm{S} 753$ & In-chamber & $30 \mathrm{~Pa}$ Clay & $20 \mathrm{~S} 3 \mathrm{~A}$ & S8A & 380.3 & 81.4 & 2.1 & 0.130 & 2.2 & 0.138 & 0.640 & 0.680 & 95.9 \\
\hline S754 & In-chamber & $30 \mathrm{~Pa}$ Clay & $20 \mathrm{~S} 3 \mathrm{~A}$ & S8B & 101.0 & 73.8 & 1.5 & 0.096 & 1.7 & 0.109 & 0.300 & 0.342 & 87.0 \\
\hline S755 & In-chamber & 30 Pa Clay & $20 \mathrm{~S} 3 \mathrm{~A}$ & S8B & 100.1 & 74.4 & 1.2 & 0.077 & 1.5 & 0.093 & 0.243 & 0.292 & 90.4 \\
\hline S756 & In-chamber & $30 \mathrm{~Pa}$ Clay & $20 \mathrm{~S} 3 \mathrm{~A}$ & S8B & 101.3 & 75.0 & 1.6 & 0.100 & 1.8 & 0.113 & 0.313 & 0.354 & 92.0 \\
\hline $\mathrm{S} 757$ & In-chamber & $30 \mathrm{~Pa}$ Clay & $20 \mathrm{~S} 3 \mathrm{~A}$ & S8A & 200.4 & 76.7 & 1.4 & 0.090 & 1.4 & 0.086 & 0.609 & 0.585 & 93.2 \\
\hline
\end{tabular}

(a) -Instrument(s) upon which measurements are based were not calibrated. Result is "For-Information Only"; (b) - Humidity data collected but not reported for W79-W81 and W86-W88; (c) - Coriolis meter instrument reading/sensitivity issue yielded anomalous result. 
Table B.5. (contd)

\begin{tabular}{|c|c|c|c|c|c|c|c|c|c|c|c|c|c|}
\hline Test ID & $\begin{array}{c}\text { Test } \\
\text { Category }\end{array}$ & Simulant & $\begin{array}{c}\text { Chamber } \\
\text { ID }\end{array}$ & $\begin{array}{c}\text { Orifice } \\
\text { ID }\end{array}$ & $\begin{array}{c}\text { Average } \\
\text { Pressure } \\
\text { [psig] }\end{array}$ & $\begin{array}{c}\text { Test Temp. } \\
{\left[{ }^{\circ} \mathrm{F}\right]}\end{array}$ & $\begin{array}{c}Q \text { (mass) } \\
{[\mathrm{gpm}]}\end{array}$ & $\begin{array}{c}Q \times 10^{3} \\
(\mathrm{mass}) \\
{\left[\mathrm{m}^{3} \mathrm{~s}^{-1}\right]}\end{array}$ & $\begin{array}{c}Q \\
(\text { flow) } \\
{[\text { (a) }}\end{array}$ & $\begin{array}{l}Q \times 10^{3} \\
(\text { flow) } \\
(\mathrm{a}) \\
{\left[\mathrm{m}^{3} \mathrm{~s}^{-1}\right]}\end{array}$ & $\begin{array}{c}C_{D} \\
\text { (mass) }\end{array}$ & $\begin{array}{c}C_{D} \\
\text { (flow) }^{\text {(a) }}\end{array}$ & $\begin{array}{c}H_{o}^{(\mathrm{a})} \\
{[\%]}\end{array}$ \\
\hline S758 & In-chamber & $30 \mathrm{~Pa}$ Clay & $20 \mathrm{~S} 3 \mathrm{~A}$ & S8A & 200.4 & 78.1 & 1.5 & 0.092 & 1.6 & 0.102 & 0.626 & 0.687 & 94.3 \\
\hline S759 & In-chamber & 30 Pa Clay & $20 \mathrm{~S} 3 \mathrm{~A}$ & S8A & 199.7 & 79.3 & 1.4 & 0.086 & 1.7 & 0.108 & 0.580 & 0.735 & 95.1 \\
\hline S760 & In-chamber & 30 Pa Clay & $20 \mathrm{~S} 3 \mathrm{~A}$ & S8A & 379.3 & 82.6 & 2.1 & 0.136 & 2.1 & 0.132 & 0.668 & 0.651 & 95.7 \\
\hline S761 & In-chamber & $30 \mathrm{~Pa}$ Clay & $20 \mathrm{~S} 3 \mathrm{~A}$ & $\mathrm{~S} 8 \mathrm{C}$ & 100.7 & 82.3 & 6.8 & 0.429 & 6.9 & 0.437 & 0.648 & 0.661 & 94.7 \\
\hline S762 & In-chamber & 30 Pa Clay & $20 \mathrm{~S} 3 \mathrm{~A}$ & $\mathrm{~S} 8 \mathrm{C}$ & 101.4 & 82.2 & 6.6 & 0.417 & 6.6 & 0.415 & 0.628 & 0.625 & 95.4 \\
\hline S763 & In-chamber & 30 Pa Clay & $20 \mathrm{~S} 3 \mathrm{~A}$ & S8A & 379.5 & 85.3 & 2.2 & 0.141 & 2.2 & 0.138 & 0.693 & 0.678 & 95.9 \\
\hline S764 & In-chamber & 30 Pa Clay & $20 \mathrm{~S} 3 \mathrm{~A}$ & S8B & 377.5 & 73.4 & 6.3 & 0.397 & 6.7 & 0.426 & 0.645 & 0.692 & 88.3 \\
\hline S765 & In-chamber & 30 Pa Clay & $20 \mathrm{~S} 3 \mathrm{~A}$ & S8B & 377.2 & 79.1 & 6.8 & 0.428 & 6.8 & 0.430 & 0.695 & 0.699 & 93.6 \\
\hline S766 & In-chamber & 30 Pa Clay & $20 \mathrm{~S} 3 \mathrm{~A}$ & $\mathrm{~S} 8 \mathrm{C}$ & 101.2 & 80.1 & 6.9 & 0.439 & 7.1 & 0.449 & 0.661 & 0.678 & 93.2 \\
\hline S767 & In-chamber & 30 Pa Clay & $20 \mathrm{~S} 3 \mathrm{~A}$ & $\mathrm{~S} 8 \mathrm{C}$ & 199.2 & 81.5 & 9.5 & 0.600 & 9.5 & 0.599 & 0.645 & 0.644 & 93.1 \\
\hline S768 & In-chamber & 30 Pa Clay & $20 \mathrm{~S} 3 \mathrm{~A}$ & $\mathrm{~S} 8 \mathrm{C}$ & 374.6 & 86.1 & 14.3 & 0.909 & 14.0 & 0.884 & 0.712 & 0.692 & 95.3 \\
\hline S769 & In-chamber & 30 Pa Clay & $20 \mathrm{~S} 3 \mathrm{~A}$ & $\mathrm{~S} 8 \mathrm{C}$ & 202.6 & 77.9 & 10.0 & 0.636 & 10.4 & 0.656 & 0.678 & 0.699 & 81.5 \\
\hline $\mathrm{S} 770$ & In-chamber & 30 Pa Clay & $20 \mathrm{~S} 3 \mathrm{~A}$ & $\mathrm{~S} 8 \mathrm{C}$ & 202.1 & 78.7 & 10.4 & 0.656 & 10.4 & 0.658 & 0.700 & 0.703 & 89.0 \\
\hline S771 & In-chamber & 30 Pa Clay & $20 \mathrm{~S} 3 \mathrm{~A}$ & $\mathrm{~S} 8 \mathrm{C}$ & 377.6 & 81.8 & 14.1 & 0.894 & 14.2 & 0.899 & 0.698 & 0.702 & 90.7 \\
\hline S772 & In-chamber & 30 Pa Clay & $20 \mathrm{~S} 3 \mathrm{~A}$ & $\mathrm{~S} 8 \mathrm{C}$ & 376.8 & 84.1 & 14.2 & 0.901 & 14.2 & 0.901 & 0.703 & 0.704 & 93.7 \\
\hline S773 & In-chamber & 30 Pa Clay & $20 \mathrm{~S} 3 \mathrm{~A}$ & S4A & 101.3 & 74.2 & 28.4 & 1.802 & 27.3 & 1.729 & 0.736 & 0.706 & 82.3 \\
\hline S774 & In-chamber & 30 Pa Clay & $20 \mathrm{~S} 3 \mathrm{~A}$ & S4A & 102.8 & 74.5 & 29.1 & 1.841 & 27.6 & 1.749 & 0.747 & 0.710 & 88.0 \\
\hline S775 & In-chamber & 30 Pa Clay & $20 \mathrm{~S} 3 \mathrm{~A}$ & S4A & 103.3 & 74.7 & 29.1 & 1.846 & 27.9 & 1.769 & 0.747 & 0.716 & 89.4 \\
\hline S776 & In-chamber & $30 \mathrm{~Pa}$ Clay & $20 \mathrm{~S} 3 \mathrm{~A}$ & S4A & 196.5 & 77.6 & 41.1 & 2.604 & 38.8 & 2.456 & 0.764 & 0.721 & 91.3 \\
\hline S777 & In-chamber & 30 Pa Clay & $20 \mathrm{~S} 3 \mathrm{~A}$ & S4A & 199.0 & 77.3 & 38.6 & 2.444 & 37.0 & 2.345 & 0.713 & 0.684 & 91.7 \\
\hline S778 & In-chamber & 30 Pa Clay & $20 \mathrm{~S} 3 \mathrm{~A}$ & S4A & 196.1 & 75.5 & 41.3 & 2.613 & 38.0 & 2.407 & 0.768 & 0.707 & 87.8 \\
\hline S780 & In-chamber & 30 Pa Clay & $20 \mathrm{~S} 3 \mathrm{~A}$ & S4A & 379.0 & 74.3 & 57.8 & 3.662 & 54.8 & 3.474 & 0.774 & 0.734 & 84.6 \\
\hline S781 & In-chamber & 30 Pa Clay & $20 \mathrm{~S} 3 \mathrm{~A}$ & S4A & 377.9 & 77.4 & 53.3 & 3.377 & 48.6 & 3.077 & 0.715 & 0.651 & 94.8 \\
\hline S783 & In-spray & 30 Pa Clay & $20 \mathrm{~F} 3 \mathrm{~A}$ & S8A & 102.3 & 77.5 & 0.9 & 0.059 & 1.3 & 0.080 & 0.564 & 0.756 & 91.4 \\
\hline S785 & In-spray & $30 \mathrm{~Pa}$ Clay & $20 \mathrm{~F} 3 \mathrm{~A}$ & S8A & 201.7 & 81.5 & 1.6 & 0.104 & 1.5 & 0.094 & 0.700 & 0.635 & 94.3 \\
\hline S786 & In-spray & 30 Pa Clay & $20 \mathrm{~F} 3 \mathrm{~A}$ & S8A & 378.0 & 85.2 & 2.2 & 0.141 & 2.3 & 0.147 & 0.694 & 0.725 & 90.1 \\
\hline S788 & In-spray & 30 Pa Clay & $20 \mathrm{~F} 3 \mathrm{~A}$ & S8B & 102.0 & 74.2 & 2.7 & 0.169 & 2.9 & 0.186 & 0.528 & 0.583 & 90.7 \\
\hline S789 & In-spray & 30 Pa Clay & $20 \mathrm{~F} 3 \mathrm{~A}$ & S8B & 204.0 & 76.9 & 3.0 & 0.190 & 3.3 & 0.208 & 0.421 & 0.459 & 90.8 \\
\hline S790 & In-spray & 30 Pa Clay & $20 \mathrm{~F} 3 \mathrm{~A}$ & $\mathrm{~S} 8 \mathrm{C}$ & 103.6 & 76.7 & 5.4 & 0.340 & 5.8 & 0.369 & 0.506 & 0.550 & 91.7 \\
\hline S791 & In-spray & 30 Pa Clay & $20 \mathrm{~F} 3 \mathrm{~A}$ & S8B & 380.0 & 80.9 & 4.2 & 0.269 & 4.4 & 0.277 & 0.436 & 0.448 & 94.1 \\
\hline
\end{tabular}

(a) -Instrument(s) upon which measurements are based were not calibrated. Result is "For-Information Only"; (b) - Humidity data collected but not reported for W79-W81 and W86-W88; (c) - Coriolis meter instrument reading/sensitivity issue yielded anomalous result. 
Table B.5. (contd)

\begin{tabular}{|c|c|c|c|c|c|c|c|c|c|c|c|c|c|}
\hline Test ID & $\begin{array}{c}\text { Test } \\
\text { Category }\end{array}$ & Simulant & $\begin{array}{c}\text { Chamber } \\
\text { ID }\end{array}$ & $\begin{array}{c}\text { Orifice } \\
\text { ID }\end{array}$ & $\begin{array}{c}\text { Average } \\
\text { Pressure } \\
\text { [psig] }\end{array}$ & $\begin{array}{c}\text { Test Temp. } \\
{\left[{ }^{\circ} \mathrm{F}\right]}\end{array}$ & $\begin{array}{c}Q \text { (mass) } \\
{[\mathrm{gpm}]}\end{array}$ & $\begin{array}{c}Q \times 10^{3} \\
(\mathrm{mass}) \\
{\left[\mathrm{m}^{3} \mathrm{~s}^{-1}\right]}\end{array}$ & $\begin{array}{c}Q \\
\text { (flow) }^{(\mathrm{a})} \\
{[\mathrm{gpm}]}\end{array}$ & $\begin{array}{c}Q \times 10^{3} \\
(\text { flow) } \\
(\mathrm{a}) \\
{\left[\mathrm{m}^{3} \mathrm{~s}^{-1}\right]}\end{array}$ & $\begin{array}{c}C_{D} \\
\text { (mass) }\end{array}$ & $\begin{array}{c}C_{D} \\
\text { (flow) }^{(\text {a) }}\end{array}$ & $\begin{array}{c}H_{o}^{(\mathrm{a})} \\
{[\%]}\end{array}$ \\
\hline S792 & In-spray & $30 \mathrm{~Pa}$ Clay & $20 \mathrm{~F} 3 \mathrm{~A}$ & $\mathrm{~S} 8 \mathrm{C}$ & 202.0 & 82.7 & 9.3 & 0.587 & 9.7 & 0.614 & 0.626 & 0.655 & 91.4 \\
\hline S793 & In-spray & $30 \mathrm{~Pa}$ Clay & $20 \mathrm{~F} 3 \mathrm{~A}$ & $\mathrm{~S} 8 \mathrm{C}$ & 380.1 & 86.4 & 11.1 & 0.704 & 11.3 & 0.717 & 0.547 & 0.557 & 96.0 \\
\hline S794 & In-spray & $30 \mathrm{~Pa}$ Clay & $20 \mathrm{~F} 3 \mathrm{~A}$ & S4A & 100.1 & 72.7 & 30.2 & 1.911 & 26.7 & 1.690 & 0.786 & 0.695 & 91.3 \\
\hline S795 & In-spray & $30 \mathrm{~Pa}$ Clay & $20 \mathrm{~F} 3 \mathrm{~A}$ & S4A & 198.9 & 75.3 & 40.2 & 2.546 & 35.0 & 2.214 & 0.743 & 0.646 & 90.2 \\
\hline S796 & In-spray & 30 Pa Clay & $20 \mathrm{~F} 3 \mathrm{~A}$ & S4A & 377.1 & 79.3 & 58.2 & 3.683 & 51.7 & 3.274 & 0.780 & 0.693 & 96.6 \\
\hline S797 & In-spray & 30 Pa Clay & $20 \mathrm{~F} 3 \mathrm{~A}$ & S4A & 378.5 & 79.6 & 56.7 & 3.588 & 49.1 & 3.111 & 0.759 & 0.658 & 94.6 \\
\hline S798 & In-spray & 6 Pa Clay & $20 \mathrm{~F} 3 \mathrm{~A}$ & S4A & 383.9 & 70.6 & 56.2 & 3.560 & 52.5 & 3.325 & 0.733 & 0.685 & 94.8 \\
\hline S799 & In-spray & 6 Pa Clay & $20 \mathrm{~F} 3 \mathrm{~A}$ & S4A & 198.4 & 67.6 & 45.5 & 2.879 & 41.5 & 2.631 & 0.825 & 0.754 & 94.7 \\
\hline S800 & In-spray & 6 Pa Clay & $20 \mathrm{~F} 3 \mathrm{~A}$ & S4A & 101.1 & 66.9 & 26.5 & 1.678 & 23.7 & 1.501 & 0.674 & 0.603 & 89.3 \\
\hline S801 & In-spray & 6 Pa Clay & $20 \mathrm{~F} 3 \mathrm{~A}$ & S8A & 101.4 & 65.6 & 1.0 & 0.066 & 1.1 & 0.069 & 0.618 & 0.648 & 90.1 \\
\hline S802 & In-spray & 6 Pa Clay & $20 \mathrm{~F} 3 \mathrm{~A}$ & S8A & 201.0 & 68.6 & 1.6 & 0.100 & 1.8 & 0.113 & 0.663 & 0.750 & 89.7 \\
\hline S803 & In-spray & 6 Pa Clay & $20 \mathrm{~F} 3 \mathrm{~A}$ & S8A & 379.7 & 75.0 & 2.3 & 0.149 & 2.3 & 0.148 & 0.717 & 0.716 & 91.5 \\
\hline S804 & In-spray & 6 Pa Clay & $20 \mathrm{~F} 3 \mathrm{~A}$ & S8B & 101.6 & 66.1 & 3.6 & 0.226 & 3.7 & 0.236 & 0.694 & 0.724 & 88.2 \\
\hline S805 & In-spray & 6 Pa Clay & $20 \mathrm{~F} 3 \mathrm{~A}$ & S8B & 199.4 & 69.4 & 5.1 & 0.322 & 5.1 & 0.320 & 0.707 & 0.702 & 90.8 \\
\hline S806 & In-spray & 6 Pa Clay & $20 \mathrm{~F} 3 \mathrm{~A}$ & S8B & 378.8 & 76.5 & 7.1 & 0.451 & 7.0 & 0.443 & 0.717 & 0.704 & 94.6 \\
\hline S807 & In-spray & 6 Pa Clay & $20 \mathrm{~F} 3 \mathrm{~A}$ & $\mathrm{~S} 8 \mathrm{C}$ & 101.0 & 69.4 & 7.5 & 0.475 & 7.4 & 0.467 & 0.703 & 0.691 & 91.2 \\
\hline S808 & In-spray & 6 Pa Clay & $20 \mathrm{~F} 3 \mathrm{~A}$ & $\mathrm{~S} 8 \mathrm{C}$ & 200.4 & 72.2 & 10.6 & 0.673 & 10.8 & 0.681 & 0.708 & 0.716 & 91.0 \\
\hline S809 & In-spray & 6 Pa Clay & $20 \mathrm{~F} 3 \mathrm{~A}$ & $\mathrm{~S} 8 \mathrm{C}$ & 378.8 & 75.5 & 14.4 & 0.910 & 14.9 & 0.941 & 0.695 & 0.720 & 95.5 \\
\hline $\mathrm{S} 810$ & In-chamber & 6 Pa Clay & $20 \mathrm{~S} 3 \mathrm{~A}$ & S8A & 102.7 & 66.1 & 1.1 & 0.067 & 1.1 & 0.067 & 0.626 & 0.620 & 83.4 \\
\hline S811 & In-chamber & 6 Pa Clay & $20 \mathrm{~S} 3 \mathrm{~A}$ & S8A & 102.8 & 66.6 & 1.1 & 0.067 & 1.1 & 0.072 & 0.618 & 0.667 & 86.6 \\
\hline S812 & In-chamber & 6 Pa Clay & $20 \mathrm{~S} 3 \mathrm{~A}$ & S8A & 102.8 & 67.0 & 1.1 & 0.071 & 1.3 & 0.081 & 0.659 & 0.748 & 88.2 \\
\hline S814 & In-chamber & 6 Pa Clay & $20 \mathrm{~S} 3 \mathrm{~A}$ & S8A & 201.1 & 71.8 & 1.7 & 0.106 & 1.7 & 0.106 & 0.704 & 0.706 & 89.0 \\
\hline S815 & In-chamber & 6 Pa Clay & $20 \mathrm{~S} 3 \mathrm{~A}$ & S8A & 201.5 & 72.8 & 1.6 & 0.103 & 1.8 & 0.112 & 0.679 & 0.745 & 91.6 \\
\hline S816 & In-chamber & 6 Pa Clay & $20 \mathrm{~S} 3 \mathrm{~A}$ & S8A & 379.8 & 74.5 & 2.3 & 0.144 & 2.3 & 0.147 & 0.696 & 0.711 & 93.0 \\
\hline S817 & In-chamber & 6 Pa Clay & $20 \mathrm{~S} 3 \mathrm{~A}$ & S8A & 379.9 & 74.3 & 2.0 & 0.126 & 2.4 & 0.154 & 0.609 & 0.744 & 90.8 \\
\hline S818 & In-chamber & 6 Pa Clay & $20 \mathrm{~S} 3 \mathrm{~A}$ & S8A & 380.0 & 74.9 & 2.7 & 0.173 & 2.2 & 0.138 & 0.836 & 0.668 & 90.9 \\
\hline S819 & In-chamber & 6 Pa Clay & $20 \mathrm{~S} 3 \mathrm{~A}$ & S8B & 102.0 & 66.4 & 3.6 & 0.226 & 3.7 & 0.233 & 0.693 & 0.716 & 90.6 \\
\hline S820 & In-chamber & 6 Pa Clay & $20 \mathrm{~S} 3 \mathrm{~A}$ & S8B & 200.3 & 68.4 & 5.0 & 0.317 & 5.2 & 0.329 & 0.693 & 0.719 & 92.0 \\
\hline S821 & In-chamber & 6 Pa Clay & $20 \mathrm{~S} 3 \mathrm{~A}$ & S8B & 379.9 & 72.8 & 7.1 & 0.452 & 7.0 & 0.443 & 0.718 & 0.703 & 93.5 \\
\hline S822 & In-chamber & 6 Pa Clay & $20 \mathrm{~S} 3 \mathrm{~A}$ & S8B & 379.2 & 72.1 & 7.1 & 0.451 & 7.2 & 0.459 & 0.717 & 0.729 & 91.9 \\
\hline
\end{tabular}

(a) -Instrument(s) upon which measurements are based were not calibrated. Result is "For-Information Only"; (b) - Humidity data collected but not reported for W79-W81 and W86-W88; (c) - Coriolis meter instrument reading/sensitivity issue yielded anomalous result. 
Table B.5. (contd)

\begin{tabular}{|c|c|c|c|c|c|c|c|c|c|c|c|c|c|}
\hline Test ID & $\begin{array}{c}\text { Test } \\
\text { Category }\end{array}$ & Simulant & $\begin{array}{c}\text { Chamber } \\
\text { ID }\end{array}$ & $\begin{array}{c}\text { Orifice } \\
\text { ID }\end{array}$ & $\begin{array}{c}\text { Average } \\
\text { Pressure } \\
\text { [psig] }\end{array}$ & $\begin{array}{c}\text { Test Temp. } \\
{\left[{ }^{\circ} \mathrm{F}\right]}\end{array}$ & $\begin{array}{c}Q \text { (mass) } \\
{[\mathrm{gpm}]}\end{array}$ & $\begin{array}{c}Q \times 10^{3} \\
\text { (mass) } \\
{\left[\mathrm{m}^{3} \mathrm{~s}^{-1}\right]}\end{array}$ & $\begin{array}{c}Q \\
(\text { flow) } \\
\text { (a) } \\
\text { gpm }]\end{array}$ & $\begin{array}{c}Q \times 10^{3} \\
(\text { flow) } \\
(\mathrm{a}) \\
{\left[\mathrm{m}^{3} \mathrm{~s}^{-1}\right]}\end{array}$ & $\begin{array}{c}C_{D} \\
\text { (mass) }\end{array}$ & $\begin{array}{c}C_{D} \\
\text { (flow) }^{(\mathrm{a})}\end{array}$ & $\begin{array}{l}H_{o}^{(\mathrm{a})} \\
{[\%]}\end{array}$ \\
\hline S823 & In-chamber & 6 Pa Clay & $20 \mathrm{~S} 3 \mathrm{~A}$ & S8B & 381.5 & 73.9 & 7.0 & 0.441 & 5.8 & 0.368 & 0.699 & 0.582 & 93.5 \\
\hline S824 & In-chamber & 6 Pa Clay & $20 \mathrm{~S} 3 \mathrm{~A}$ & S8B & 102.4 & 71.0 & 3.5 & 0.222 & 3.7 & 0.236 & 0.679 & 0.723 & 94.5 \\
\hline S825 & In-chamber & 6 Pa Clay & $20 \mathrm{~S} 3 \mathrm{~A}$ & S8B & 198.9 & 71.0 & 5.2 & 0.329 & 5.2 & 0.330 & 0.723 & 0.724 & 95.2 \\
\hline S826 & In-chamber & 6 Pa Clay & $20 \mathrm{~S} 3 \mathrm{~A}$ & S8B & 101.6 & 69.6 & 3.6 & 0.227 & 3.8 & 0.238 & 0.696 & 0.731 & 95.8 \\
\hline S827 & In-chamber & 6 Pa Clay & $20 \mathrm{~S} 3 \mathrm{~A}$ & S8B & 199.7 & 70.1 & 5.0 & 0.319 & 5.2 & 0.329 & 0.698 & 0.721 & 95.8 \\
\hline S829 & In-chamber & 6 Pa Clay & $20 \mathrm{~S} 3 \mathrm{~A}$ & $\mathrm{~S} 8 \mathrm{C}$ & 98.4 & 70.1 & 5.5 & 0.346 & 5.5 & 0.350 & 0.520 & 0.525 & 93.0 \\
\hline $\mathrm{S} 830$ & In-chamber & 6 Pa Clay & $20 \mathrm{~S} 3 \mathrm{~A}$ & $\mathrm{~S} 8 \mathrm{C}$ & 100.3 & 68.6 & 5.2 & 0.328 & 5.6 & 0.355 & 0.487 & 0.527 & 94.2 \\
\hline S831 & In-chamber & 6 Pa Clay & $20 \mathrm{~S} 3 \mathrm{~A}$ & $\mathrm{~S} 8 \mathrm{C}$ & 200.1 & 68.9 & 7.1 & 0.452 & 7.0 & 0.446 & 0.476 & 0.469 & 94.5 \\
\hline S832 & In-chamber & 6 Pa Clay & $20 \mathrm{~S} 3 \mathrm{~A}$ & $\mathrm{~S} 8 \mathrm{C}$ & 200.9 & 68.6 & 7.2 & 0.455 & 7.4 & 0.472 & 0.477 & 0.495 & 95.1 \\
\hline S833 & In-chamber & 6 Pa Clay & $20 \mathrm{~S} 3 \mathrm{~A}$ & $\mathrm{~S} 8 \mathrm{C}$ & 200.9 & 68.2 & 6.9 & 0.438 & 7.1 & 0.451 & 0.460 & 0.473 & 95.5 \\
\hline $\mathrm{S} 835$ & In-chamber & 6 Pa Clay & $20 \mathrm{~S} 3 \mathrm{~A}$ & $\mathrm{~S} 8 \mathrm{C}$ & 379.7 & 73.4 & 13.9 & 0.883 & 14.0 & 0.887 & 0.674 & 0.677 & 93.4 \\
\hline S836 & In-chamber & 6 Pa Clay & $20 \mathrm{~S} 3 \mathrm{~A}$ & $\mathrm{~S} 8 \mathrm{C}$ & 380.4 & 76.1 & 13.7 & 0.865 & 13.7 & 0.868 & 0.660 & 0.662 & 95.0 \\
\hline S837 & In-chamber & 6 Pa Clay & $20 \mathrm{~S} 3 \mathrm{~A}$ & $\mathrm{~S} 8 \mathrm{C}$ & 379.4 & 76.3 & 14.1 & 0.896 & 13.9 & 0.881 & 0.684 & 0.673 & 96.7 \\
\hline S838 & In-chamber & 6 Pa Clay & $20 \mathrm{~S} 3 \mathrm{~A}$ & S4A & 100.7 & 77.9 & 32.9 & 2.083 & 30.6 & 1.939 & 0.837 & 0.779 & 93.7 \\
\hline S839 & In-chamber & 6 Pa Clay & $20 \mathrm{~S} 3 \mathrm{~A}$ & S4A & 101.5 & 73.2 & 32.0 & 2.027 & 30.0 & 1.903 & 0.812 & 0.762 & 97.4 \\
\hline $\mathrm{S} 842$ & In-chamber & 6 Pa Clay & $20 \mathrm{~S} 3 \mathrm{~A}$ & S4A & 101.3 & 75.6 & 32.5 & 2.057 & 30.5 & 1.934 & 0.825 & 0.775 & 91.1 \\
\hline S843 & In-chamber & 6 Pa Clay & $20 \mathrm{~S} 3 \mathrm{~A}$ & S4A & 100.5 & 72.6 & 31.8 & 2.014 & 30.5 & 1.934 & 0.811 & 0.778 & 91.9 \\
\hline S844 & In-chamber & 6 Pa Clay & $20 \mathrm{~S} 3 \mathrm{~A}$ & S4A & 197.3 & 68.7 & 44.4 & 2.810 & 43.1 & 2.727 & 0.807 & 0.783 & 89.6 \\
\hline S845 & In-chamber & 6 Pa Clay & $20 \mathrm{~S} 3 \mathrm{~A}$ & S4A & 199.2 & 70.3 & 47.7 & 3.019 & 43.4 & 2.751 & 0.863 & 0.787 & 87.0 \\
\hline S846 & In-chamber & 6 Pa Clay & $20 \mathrm{~S} 3 \mathrm{~A}$ & S4A & 199.9 & 70.8 & 47.2 & 2.992 & 43.4 & 2.747 & 0.854 & 0.784 & 88.6 \\
\hline S848 & In-chamber & 6 Pa Clay & $20 \mathrm{~S} 3 \mathrm{~A}$ & S4A & 383.0 & 73.6 & 68.9 & 4.365 & 61.6 & 3.899 & 0.900 & 0.804 & 90.5 \\
\hline S849 & In-chamber & 6 Pa Clay & $20 \mathrm{~S} 3 \mathrm{~A}$ & S4A & 380.7 & 75.7 & 64.2 & 4.069 & 58.2 & 3.686 & 0.841 & 0.762 & 93.2 \\
\hline $\mathrm{S} 850$ & In-chamber & 6 Pa Clay & $20 \mathrm{~S} 3 \mathrm{~A}$ & S4A & 378.3 & 78.2 & 63.4 & 4.018 & 57.2 & 3.623 & 0.833 & 0.751 & 97.7 \\
\hline S851 & Humidity & 6 Pa Clay & $20 \mathrm{~S} 3 \mathrm{~A}$ & S8A & 379.0 & 79.0 & 2.2 & 0.141 & 2.2 & 0.141 & 0.682 & 0.679 & 56.8 \\
\hline S852 & Humidity & 6 Pa Clay & $20 \mathrm{~S} 3 \mathrm{~A}$ & S8A & 378.6 & 78.4 & 2.3 & 0.145 & 2.4 & 0.153 & 0.700 & 0.740 & 43.2 \\
\hline S853 & Humidity & 6 Pa Clay & $20 \mathrm{~S} 3 \mathrm{~A}$ & S8A & 379.8 & 79.1 & 2.3 & 0.146 & 2.1 & 0.133 & 0.702 & 0.642 & 49.5 \\
\hline S854 & Humidity & 6 Pa Clay & $20 \mathrm{~S} 3 \mathrm{~A}$ & S8A & 379.9 & 78.1 & 2.3 & 0.143 & 2.5 & 0.160 & 0.688 & 0.772 & 67.4 \\
\hline $\mathrm{S} 855$ & Humidity & 6 Pa Clay & $20 \mathrm{~S} 3 \mathrm{~A}$ & S8A & 379.1 & 77.5 & 2.4 & 0.150 & - -c $^{(c)}$ & $--^{(c)}$ & 0.723 & - - $^{(c)}$ & 76.7 \\
\hline S856 & Humidity & 6 Pa Clay & $20 \mathrm{~S} 3 \mathrm{~A}$ & S8A & 378.6 & 76.8 & 2.3 & 0.147 & 3.0 & 0.192 & 0.711 & 0.927 & 87.2 \\
\hline S857 & Humidity & 6 Pa Clay & $20 \mathrm{~S} 3 \mathrm{~A}$ & S8A & 378.3 & 74.2 & 2.3 & 0.147 & 3.0 & 0.190 & 0.709 & 0.919 & 94.7 \\
\hline
\end{tabular}

(a) -Instrument(s) upon which measurements are based were not calibrated. Result is "For-Information Only"; (b) - Humidity data collected but not reported for W79-W81 and W86-W88; (c) - Coriolis meter instrument reading/sensitivity issue yielded anomalous result. 
Table B.5. (contd)

\begin{tabular}{|c|c|c|c|c|c|c|c|c|c|c|c|c|c|}
\hline Test ID & $\begin{array}{c}\text { Test } \\
\text { Category }\end{array}$ & Simulant & $\begin{array}{c}\text { Chamber } \\
\text { ID }\end{array}$ & $\begin{array}{l}\text { Orifice } \\
\text { ID }\end{array}$ & $\begin{array}{c}\text { Average } \\
\text { Pressure } \\
\text { [psig] }\end{array}$ & $\begin{array}{c}\text { Test Temp. } \\
{\left[{ }^{\circ} \mathrm{F}\right]}\end{array}$ & $\begin{array}{c}Q \text { (mass) } \\
{[\text { gpm] }}\end{array}$ & $\begin{array}{c}Q \times 10^{3} \\
(\text { mass) } \\
{\left[\mathrm{m}^{3} \mathrm{~s}^{-1}\right]}\end{array}$ & $\begin{array}{c}Q \\
\text { (flow) }^{(a)} \\
{[\mathrm{gpm}]}\end{array}$ & $\begin{array}{c}Q \times 10^{3} \\
(\text { flow) } \\
{\left[\mathrm{m}^{3} \mathrm{~s}^{-1}\right]}\end{array}$ & $\begin{array}{c}C_{D} \\
\text { (mass) }\end{array}$ & $\begin{array}{c}C_{D} \\
\text { (flow) }^{\text {(a) }}\end{array}$ & $\begin{array}{c}H_{o}^{(a)} \\
{[\%]}\end{array}$ \\
\hline S858 & Humidity & 6 Pa Clay & $20 \mathrm{~S} 3 \mathrm{~A}$ & S8A & 378.0 & 74.5 & 2.4 & 0.152 & 2.6 & 0.164 & 0.737 & 0.795 & 95.7 \\
\hline W859 & Humidity & Water & $20 \mathrm{~S} 3 \mathrm{~A}$ & S8A & 379.3 & 77.8 & 2.5 & 0.156 & 3.0 & 0.188 & 0.686 & 0.827 & 93.9 \\
\hline W860 & Humidity & Water & $20 \mathrm{~S} 3 \mathrm{~A}$ & S8A & 379.5 & 76.5 & 2.5 & 0.156 & -- (c) & --(c) & 0.684 & -- (c) & 96.3 \\
\hline W861 & Humidity & Water & $20 \mathrm{~S} 3 \mathrm{~A}$ & S8A & 379.2 & 78.3 & 2.5 & 0.157 & -- (c) & --. (c) & 0.693 & -- (c) & 97.2 \\
\hline W863 & Humidity & Water & $20 \mathrm{~S} 3 \mathrm{~A}$ & S8A & 378.4 & 76.2 & 2.5 & 0.157 & 3.6 & 0.225 & 0.692 & 0.991 & 50.3 \\
\hline W864 & Humidity & Water & $20 \mathrm{~S} 3 \mathrm{~A}$ & S8A & 378.6 & 75.8 & 2.4 & 0.150 & - - $^{(c)}$ & - -(c) $^{(c)}$ & 0.660 & - -(c) $^{(c)}$ & 52.1 \\
\hline W865 & Humidity & Water & $20 \mathrm{~S} 3 \mathrm{~A}$ & S8A & 378.3 & 75.1 & 2.4 & 0.153 & 0.3 & 0.022 & 0.675 & 0.095 & 87.2 \\
\hline W866 & Humidity & Water & $20 \mathrm{~S} 3 \mathrm{~A}$ & S8A & 378.4 & 74.4 & 2.5 & 0.161 & $--^{(c)}$ & $--^{(c)}$ & 0.707 & --(c) & 65.2 \\
\hline W867 & Humidity & Water & $20 \mathrm{~S} 3 \mathrm{~A}$ & S8A & 378.2 & 74.3 & 2.4 & 0.150 & 0.3 & 0.017 & 0.663 & 0.076 & 75.5 \\
\hline
\end{tabular}

(a) -Instrument(s) upon which measurements are based were not calibrated. Result is "For-Information Only"; (b) - Humidity data collected but not reported for W79-W81 and W86-W88; (c) - Coriolis meter instrument reading/sensitivity issue yielded anomalous result. 


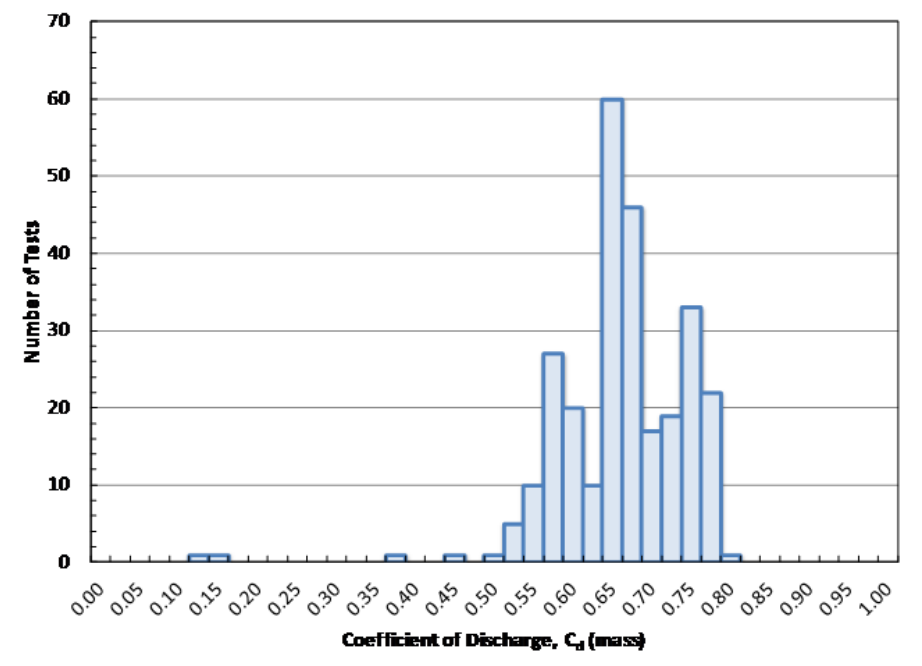

(A)

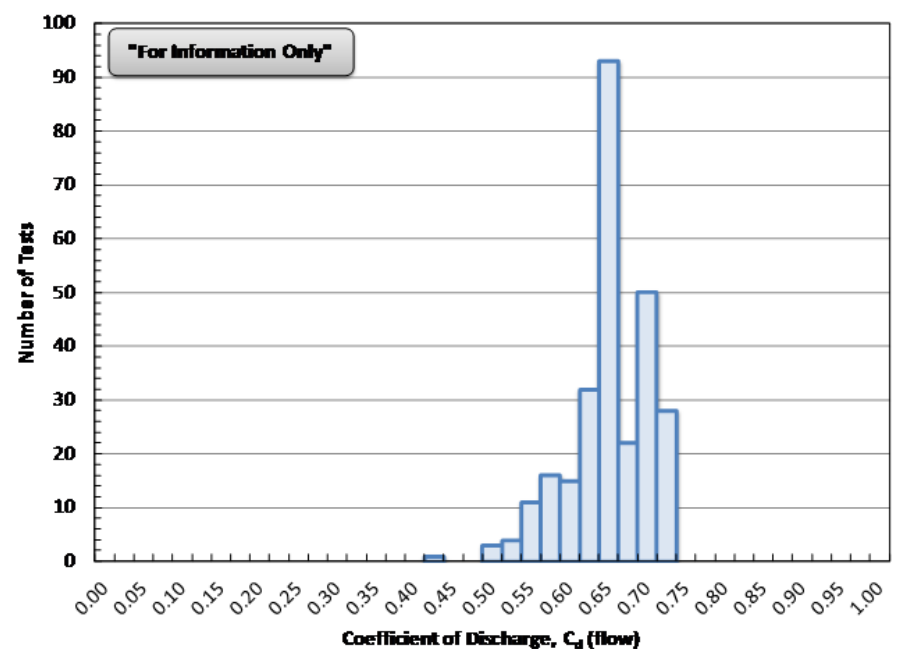

(B)

Figure B.1. Histogram of (A) $C_{D \text {, mass }}$ and (B) $C_{D \text {, flow }}$ for Accepted In-Chamber Matrix Tests Using Water

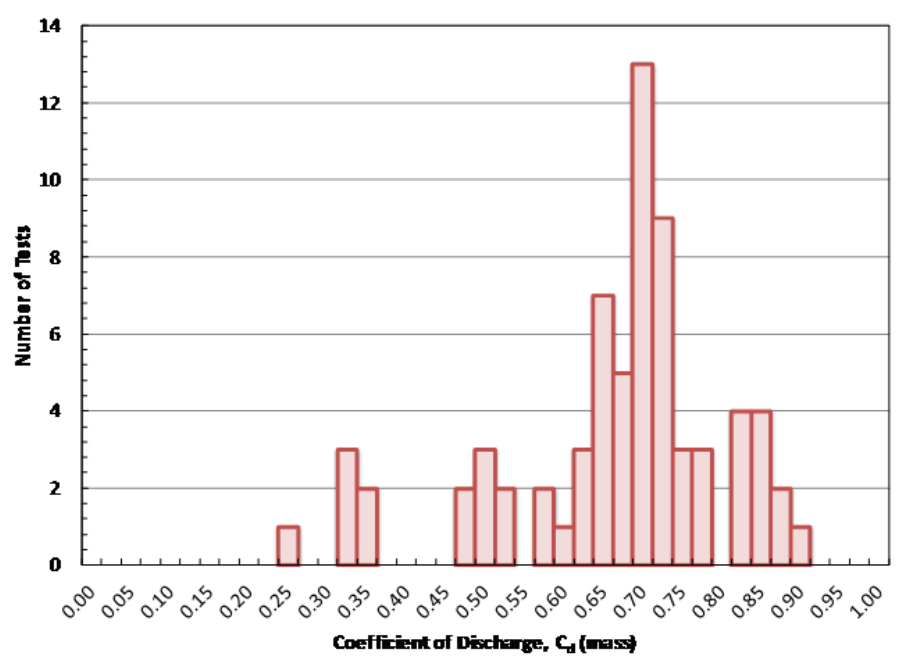

(A)

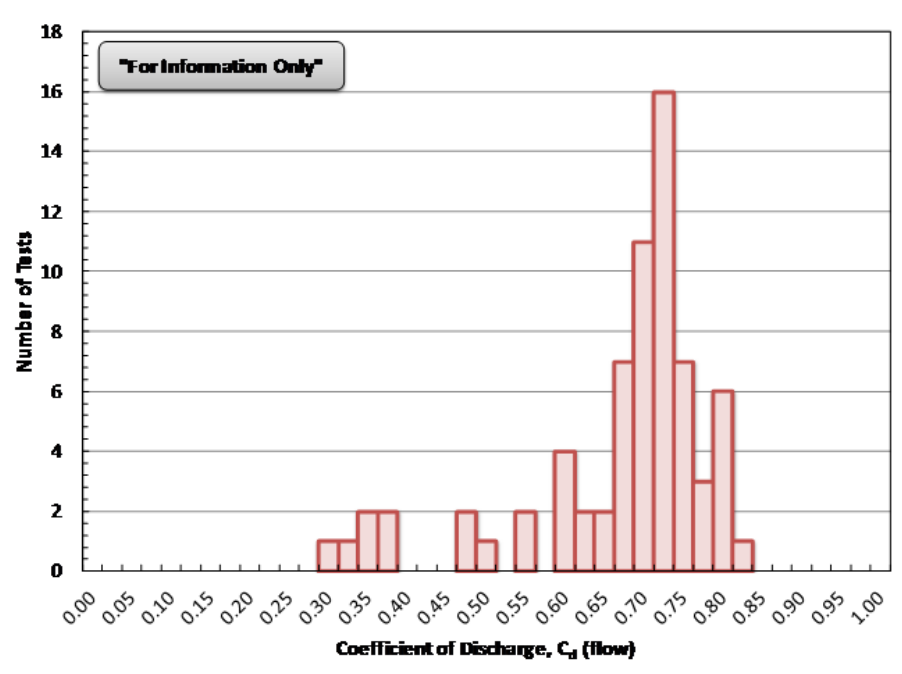

(B)

Figure B.2. Histogram of (A) $C_{D \text {, mass }}$ and (B) $C_{D}$, flow for Accepted In-Chamber Matrix Tests Using 6 Pa Clay and 30 Pa Clay 
Table B.6. Discharge Coefficient Statistics for Accepted In-Chamber Matrix Test Using Water and Clay (6 $\mathrm{Pa}$ and $30 \mathrm{~Pa}$ ) Simulants

\begin{tabular}{ccccc}
\hline & \multirow{2}{*}{$\begin{array}{c}\text { Discharge } \\
\text { Coefficient }\end{array}$} & \multicolumn{3}{c}{ Discharge Coefficient } \\
\cline { 3 - 5 } Simulant & Basis & Average & Median & $\begin{array}{c}\text { Standard } \\
\text { Deviation }\end{array}$ \\
\hline \multirow{2}{*}{ Water } & Mass & 0.649 & 0.651 & 0.083 \\
& Flow & 0.639 & 0.643 & 0.053 \\
\multirow{2}{*}{ Clay (6 Pa and 30 Pa) } & Mass & 0.653 & 0.688 & 0.145 \\
& Flow & 0.655 & 0.696 & 0.123 \\
\hline
\end{tabular}

Table B.7. Test Fluid Pressure Statistics for Accepted In-Chamber Matrix Test Using Water and Clay (6 $\mathrm{Pa}$ and $30 \mathrm{~Pa}$ ) Simulants

\begin{tabular}{ccccc}
\hline & Target & \multicolumn{3}{c}{ Test Fluid Pressure } \\
\cline { 3 - 5 } Simulant & Pressure & & & Standard \\
\cline { 3 - 5 } Conditions & Average & Median & Deviation \\
\hline \multirow{2}{*}{ Water } & 100 & 101.7 & 101.7 & 1.1 \\
& 200 & 200.0 & 200.0 & 1.3 \\
& 380 & 379.0 & 379.5 & 2.3 \\
Clay (6 Pa and 30 Pa) & 100 & 101.6 & 101.4 & 1.1 \\
& 200 & 200.1 & 200.3 & 1.8 \\
& 380 & 379.2 & 379.5 & 1.8 \\
\hline
\end{tabular}


(A)

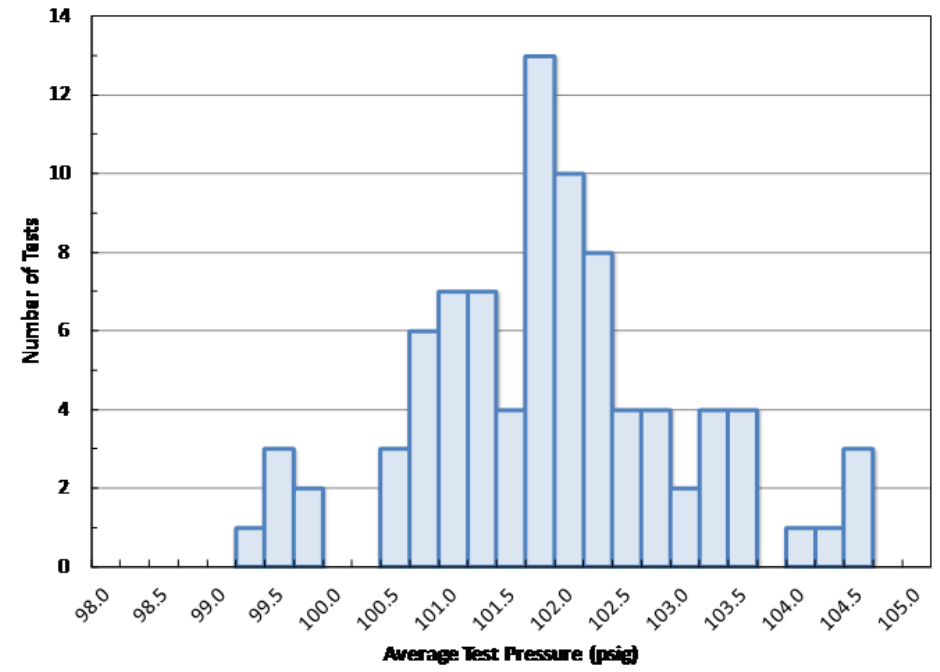

(B)
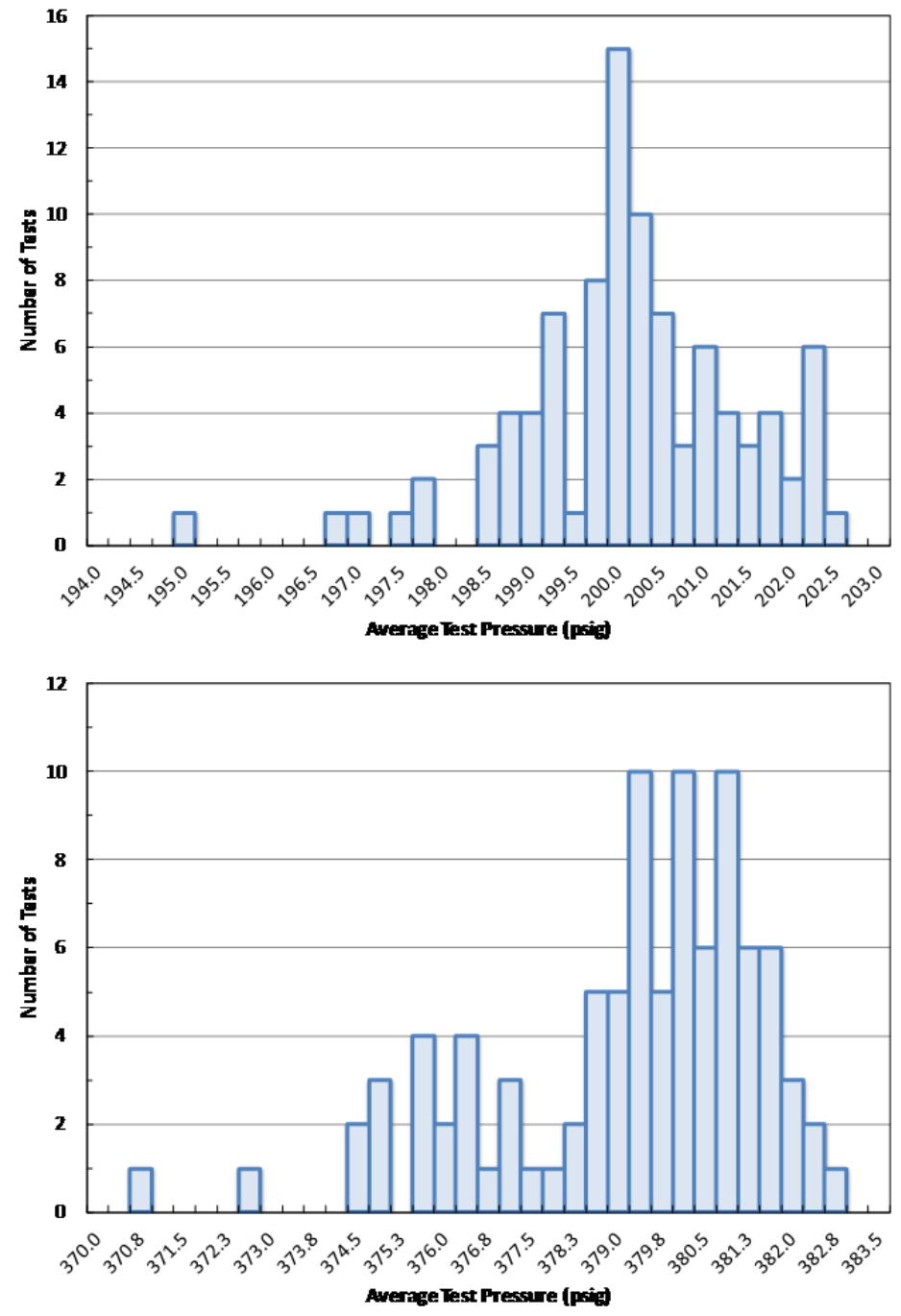

Figure B.3. Histogram of Test Fluid Pressure Using Water for (A) 100 psig, (B) 200, and (C) 380 psig Target Pressure Conditions 
(A)

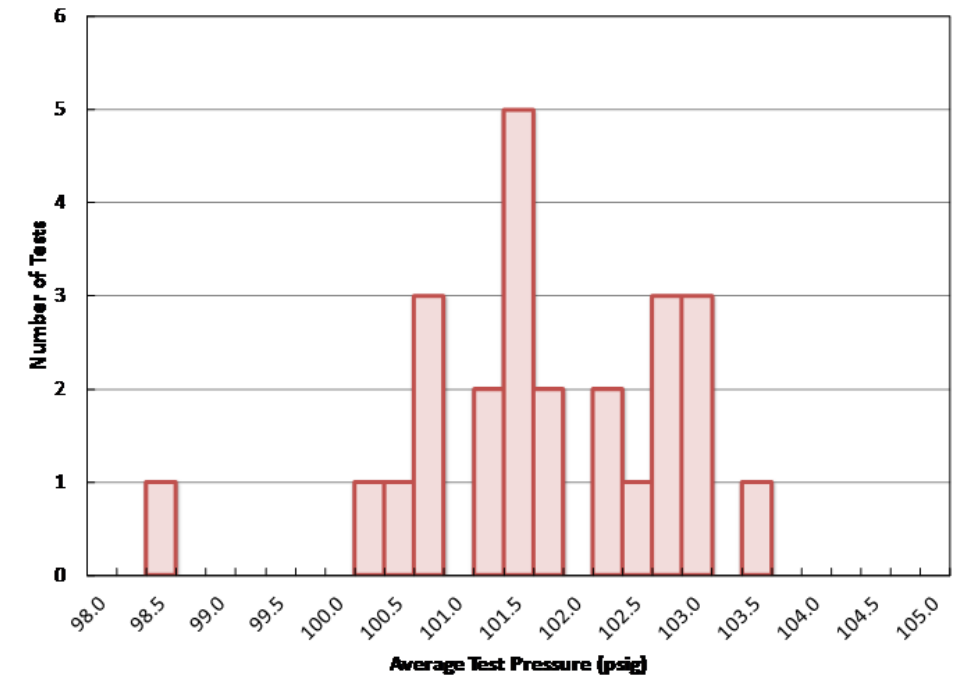

(B)
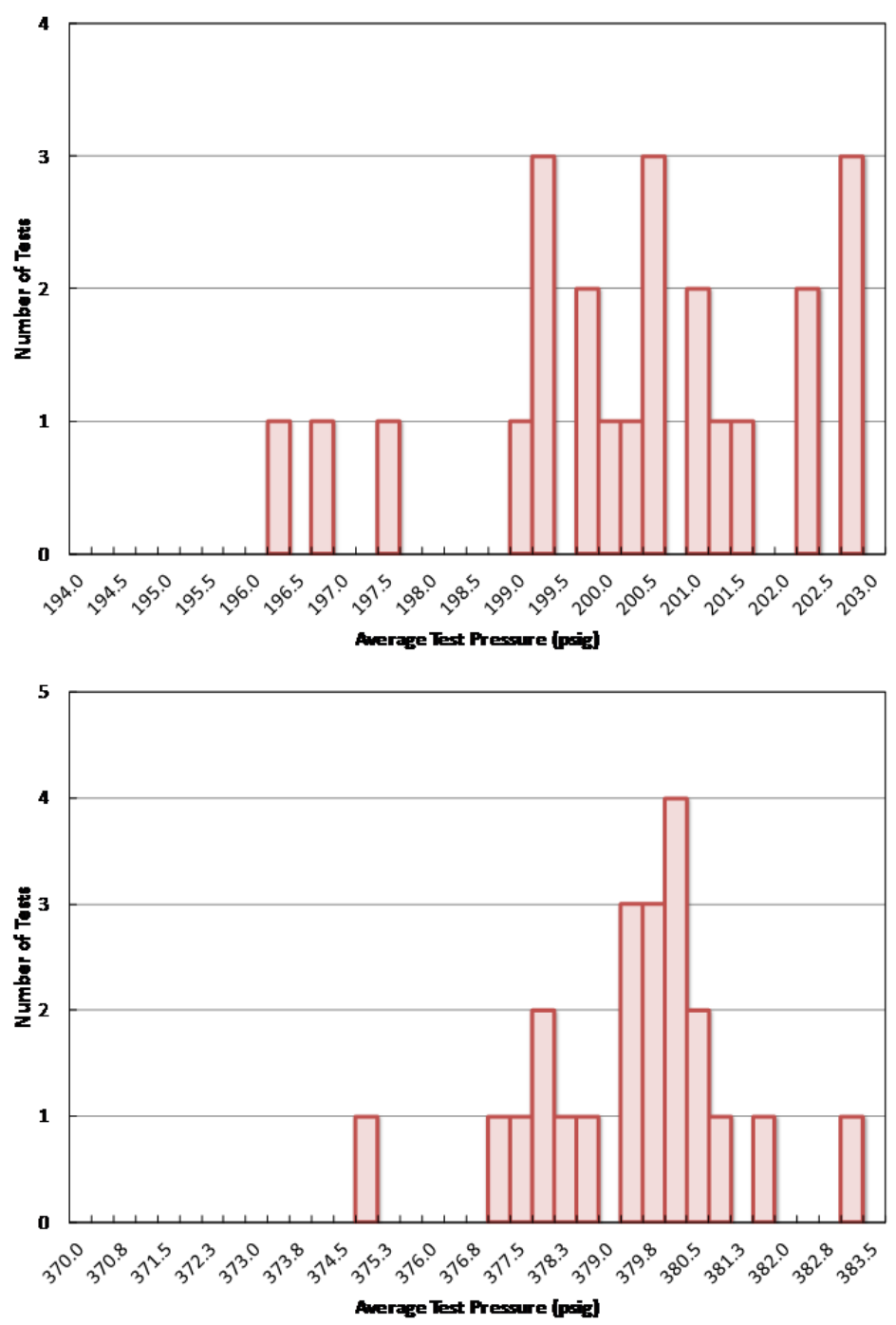

Figure B.4. Histogram of Test Fluid Pressure Using Clay for (A) 100 psig, (B) 200, and (C) 380 psig Target Pressure Conditions 


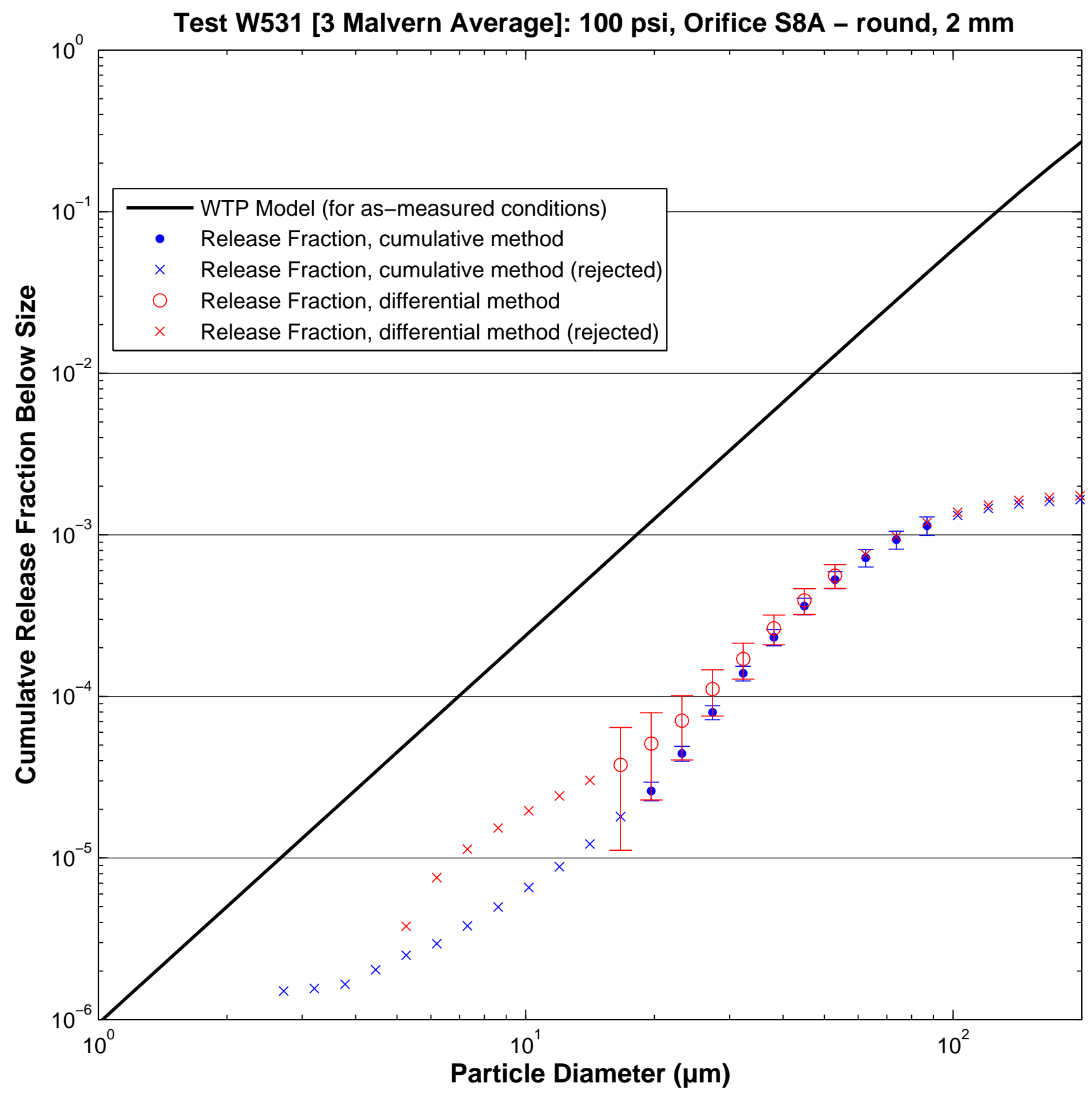




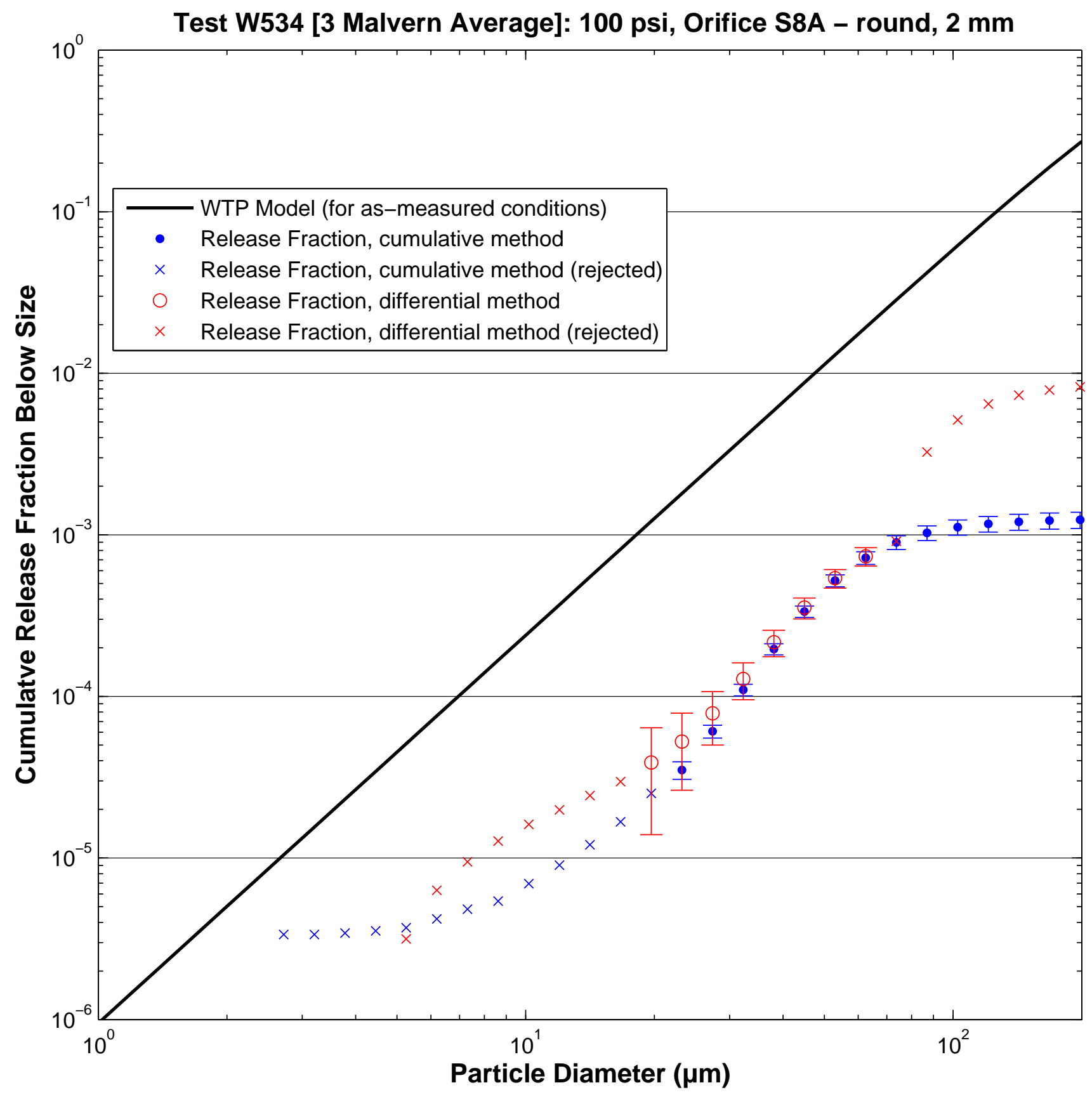




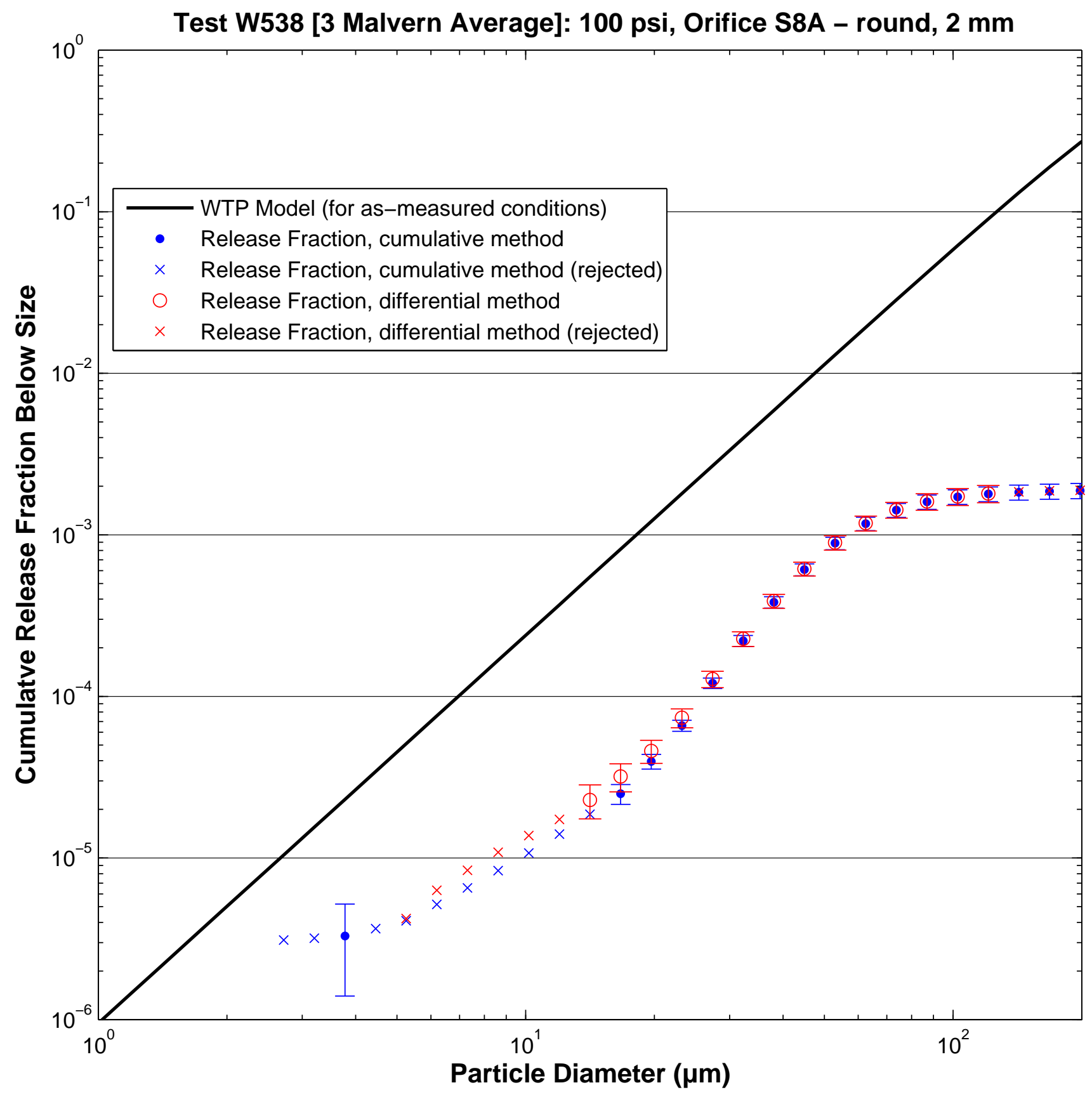




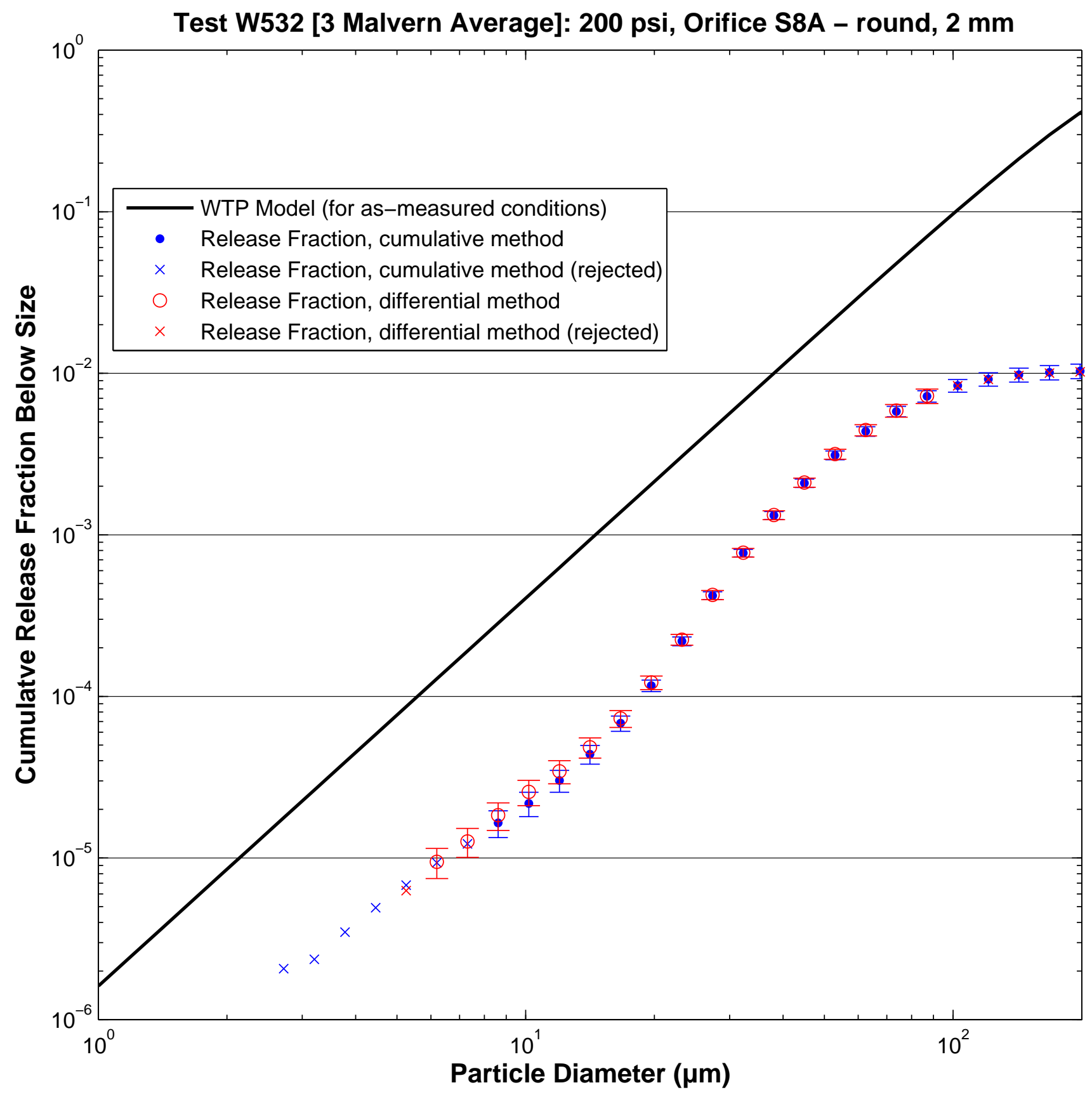




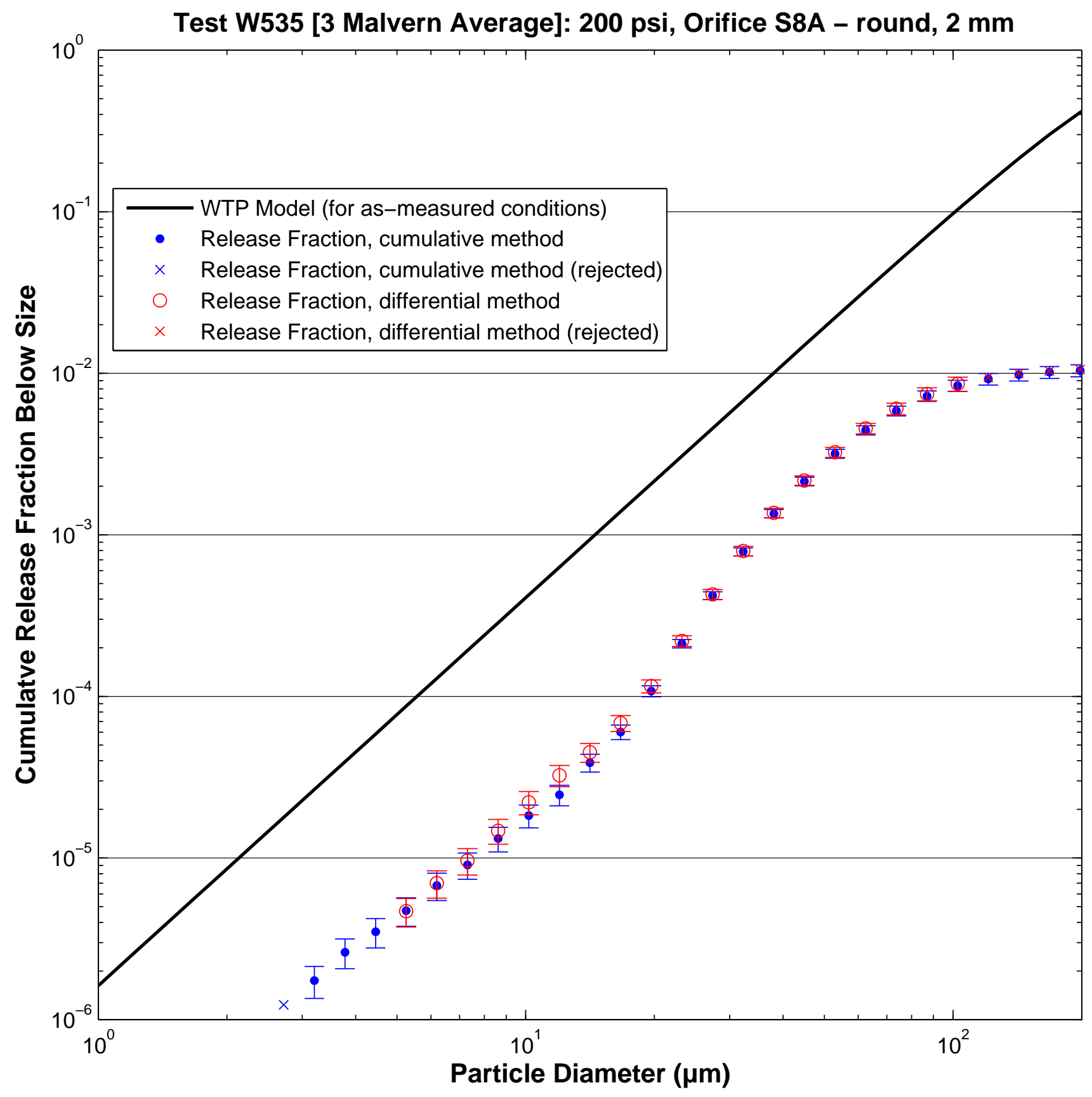




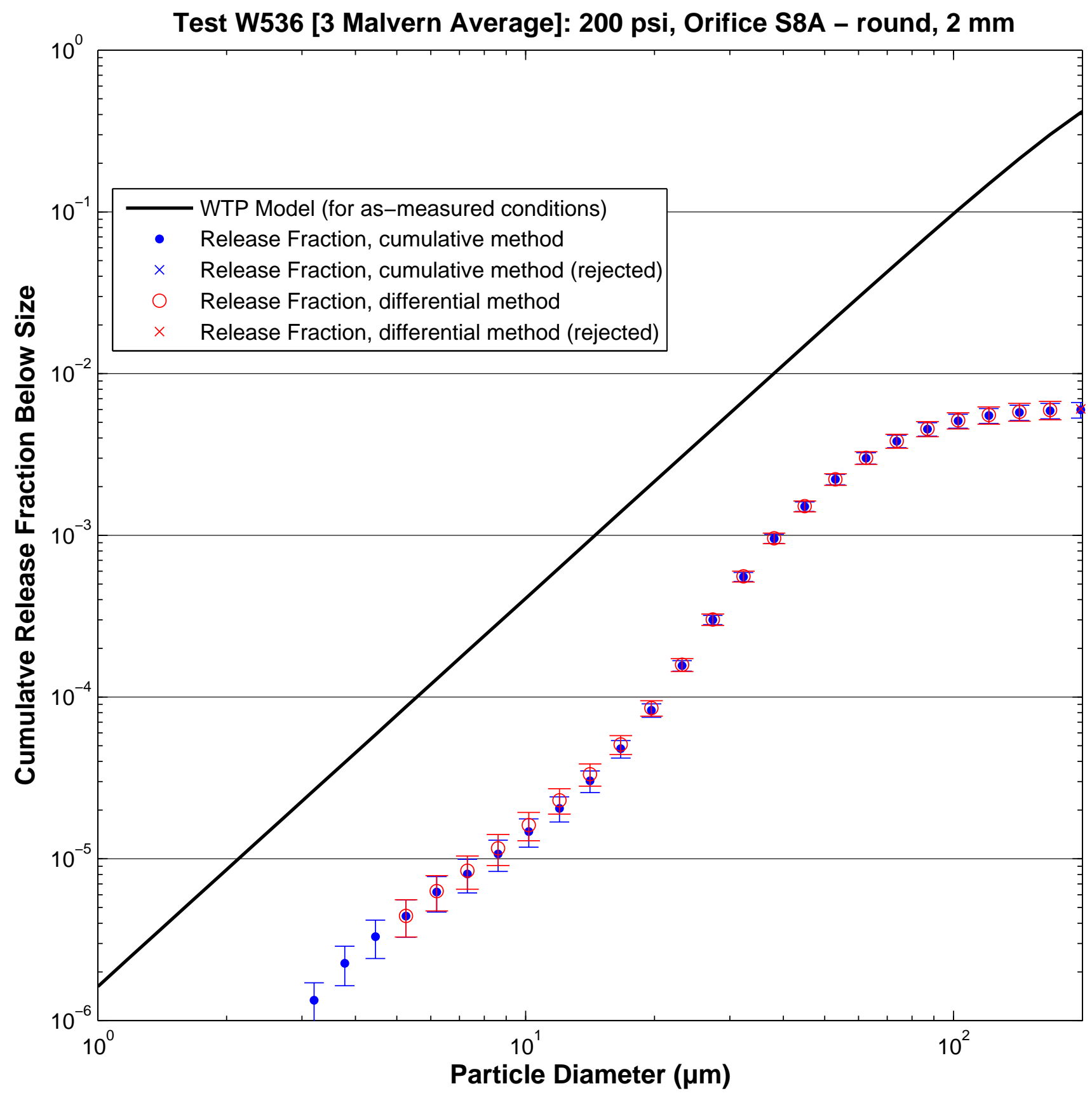




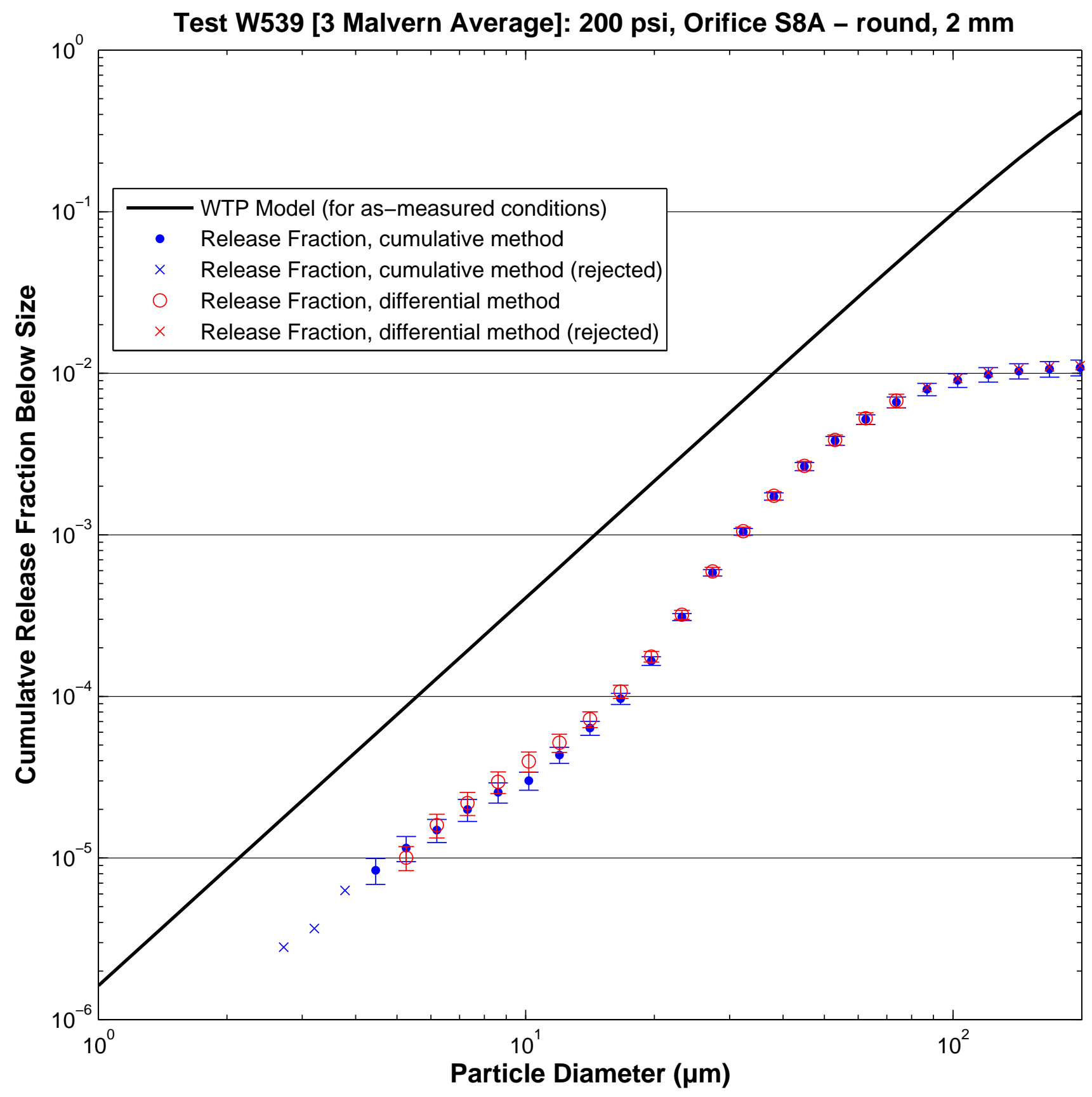




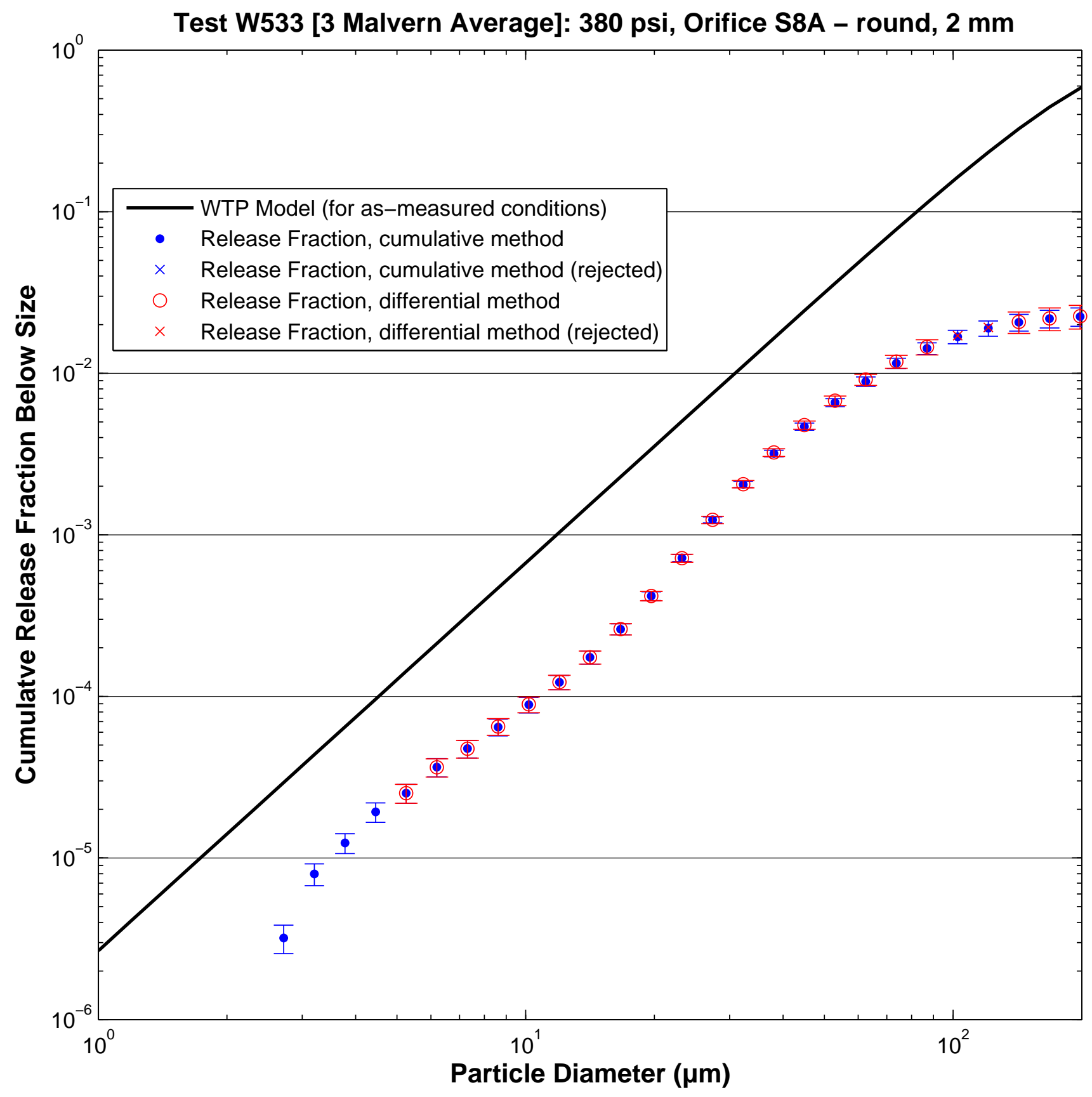




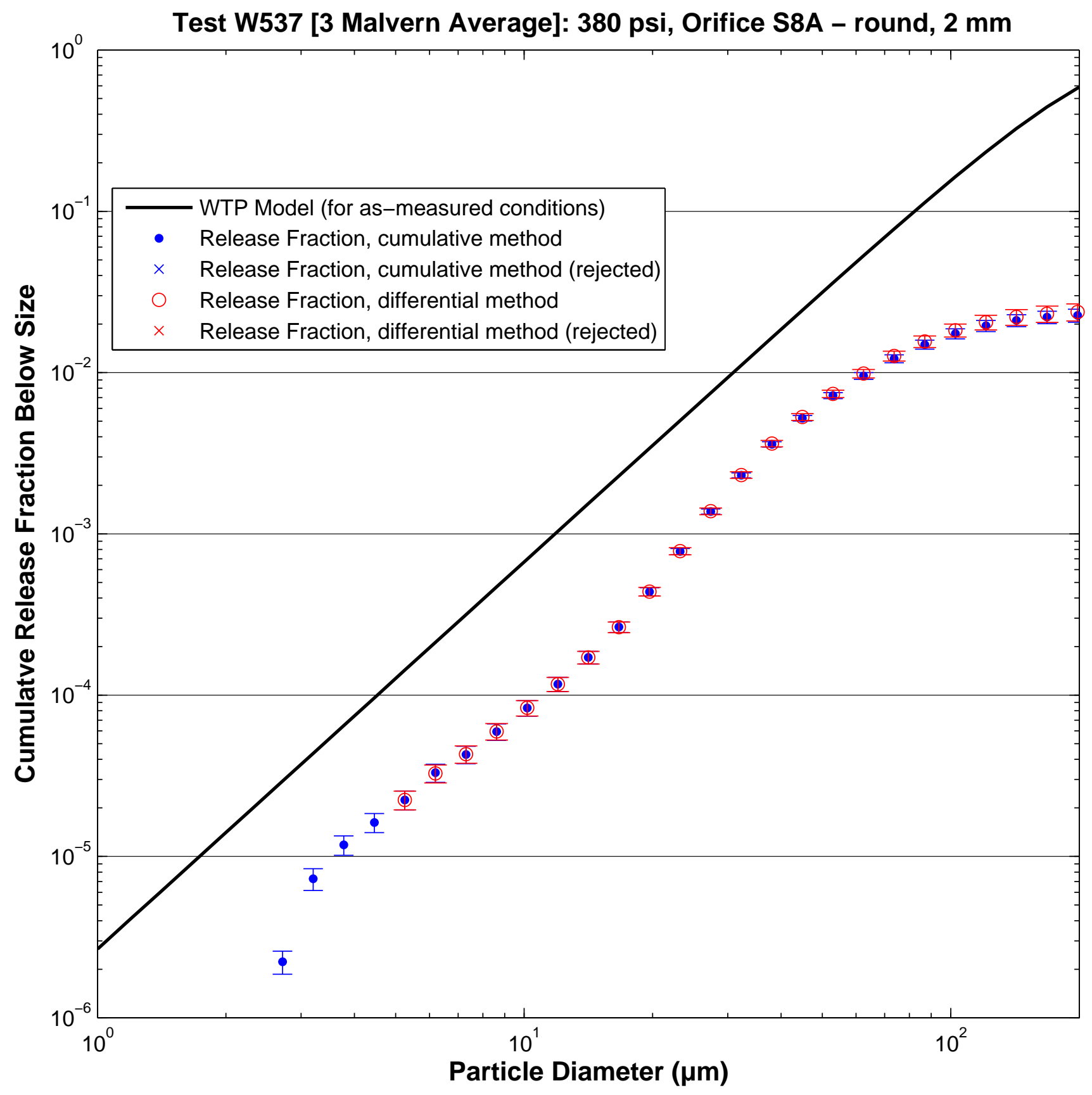




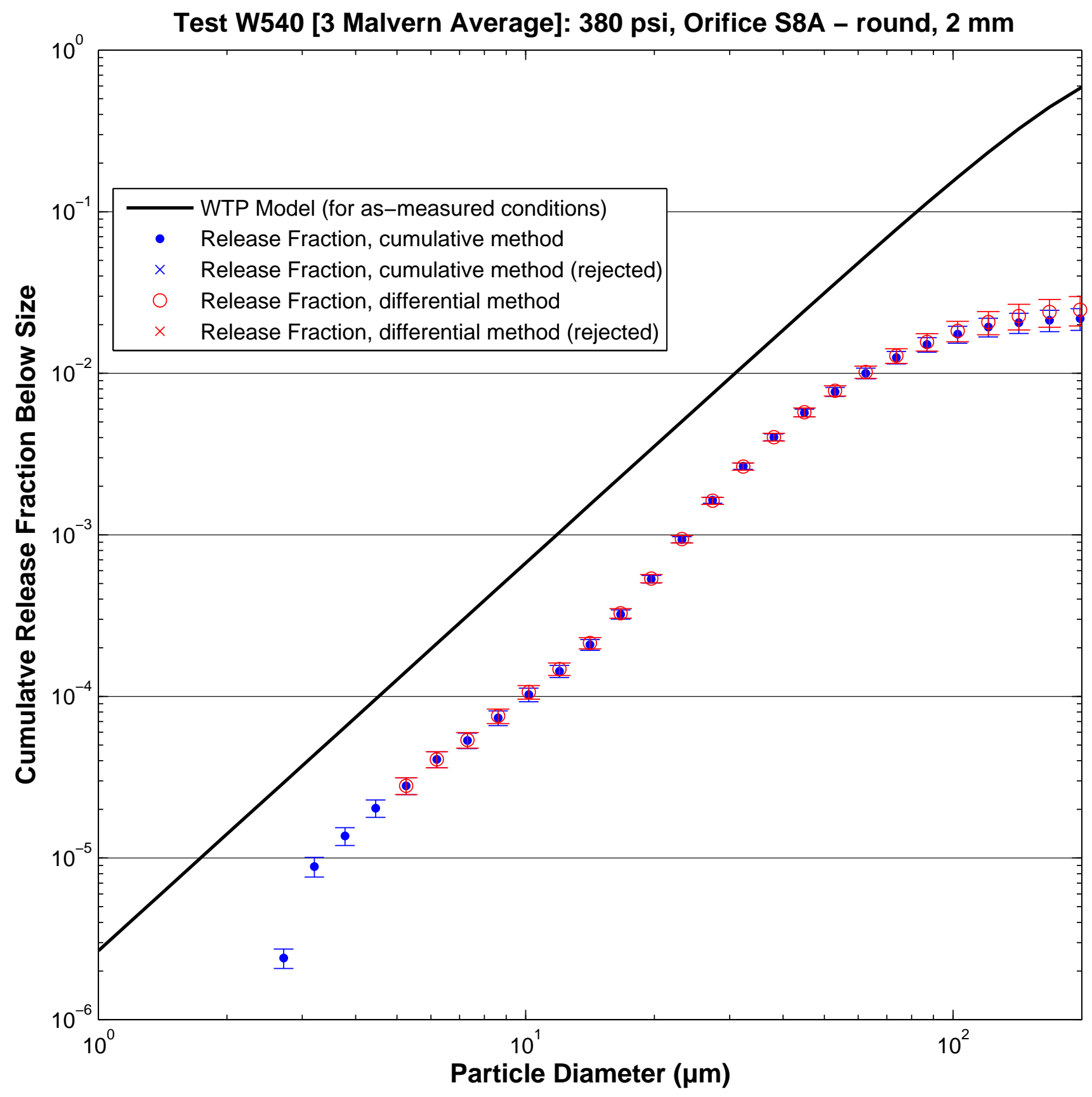




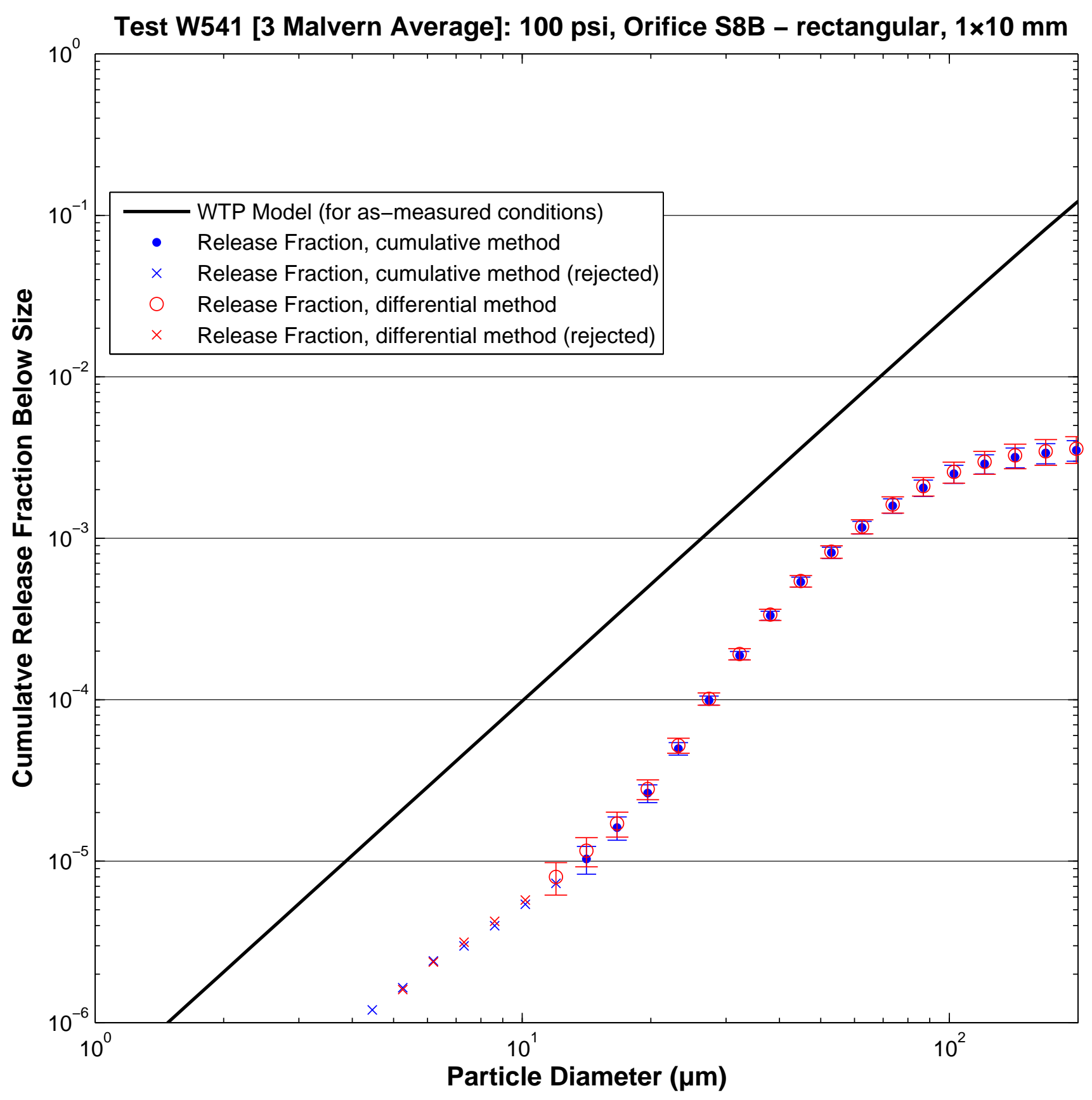




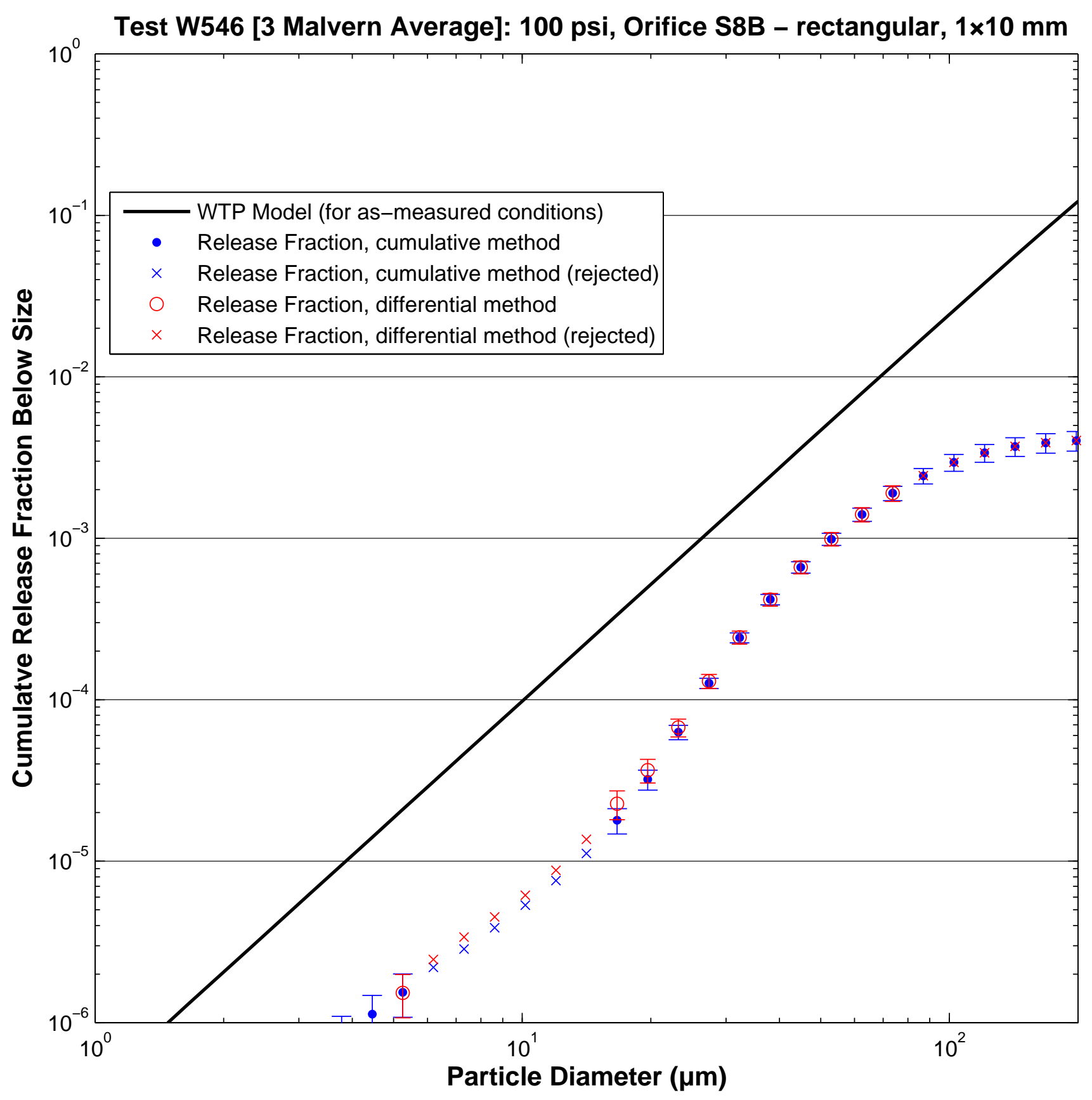




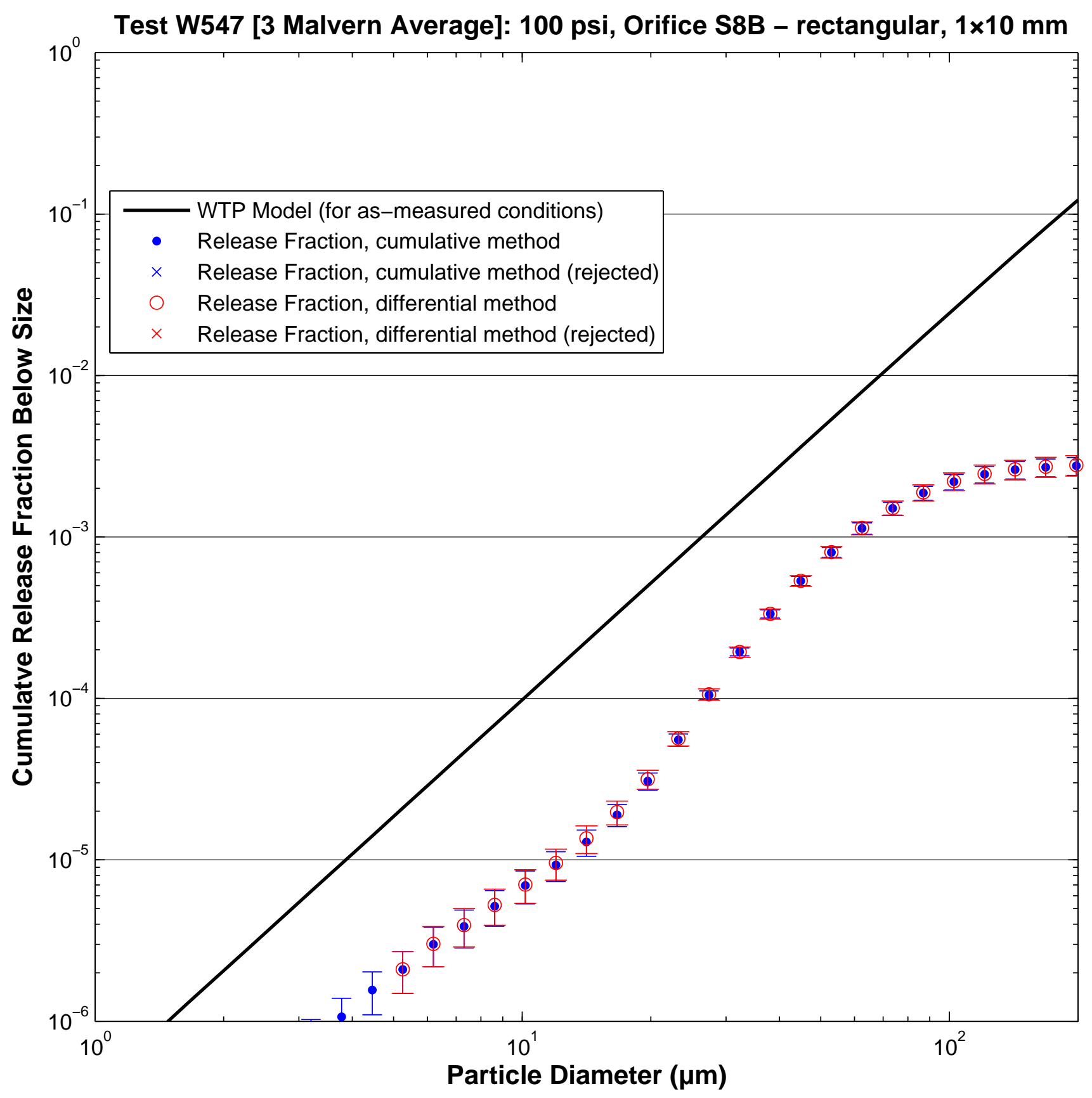




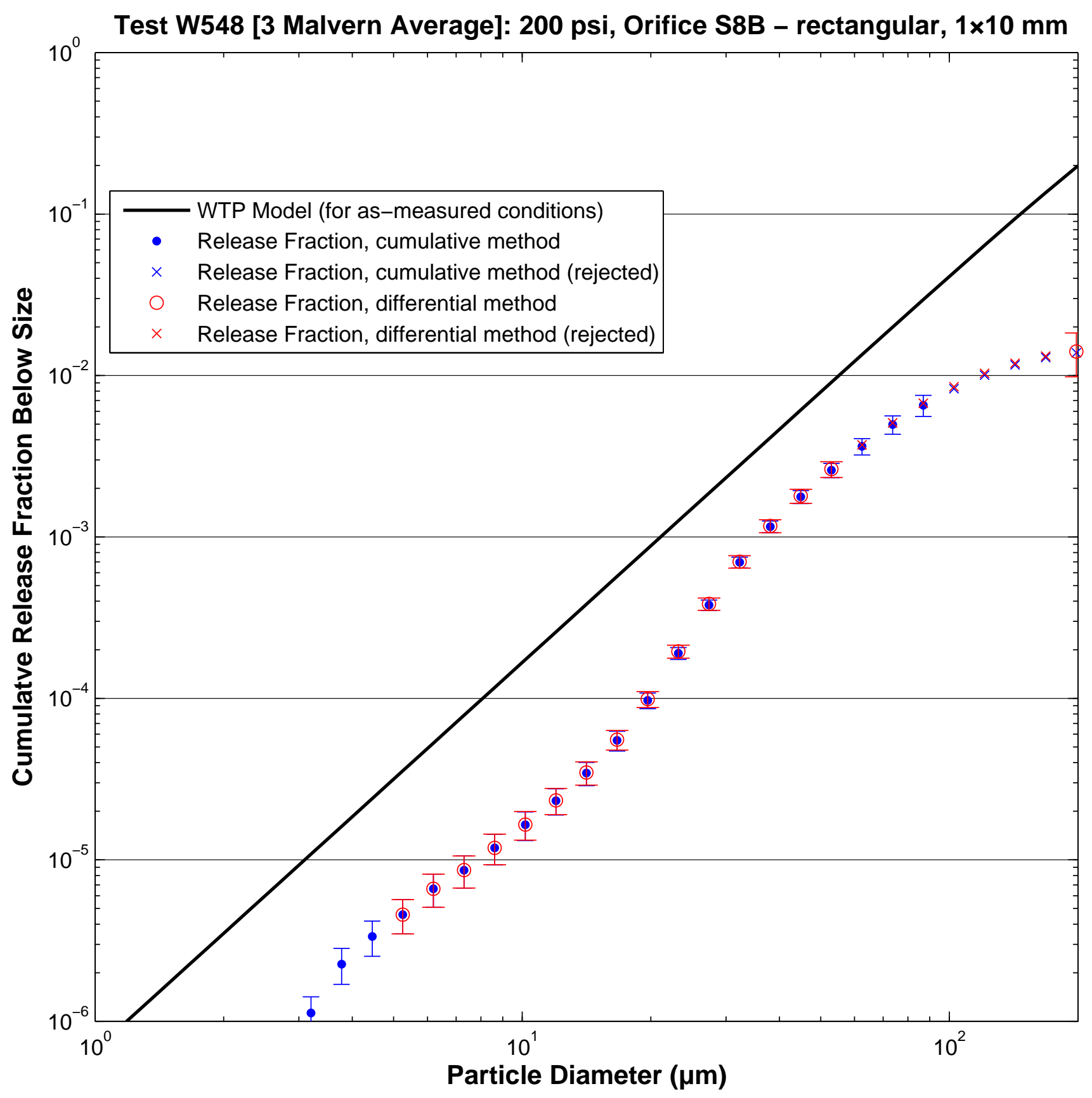




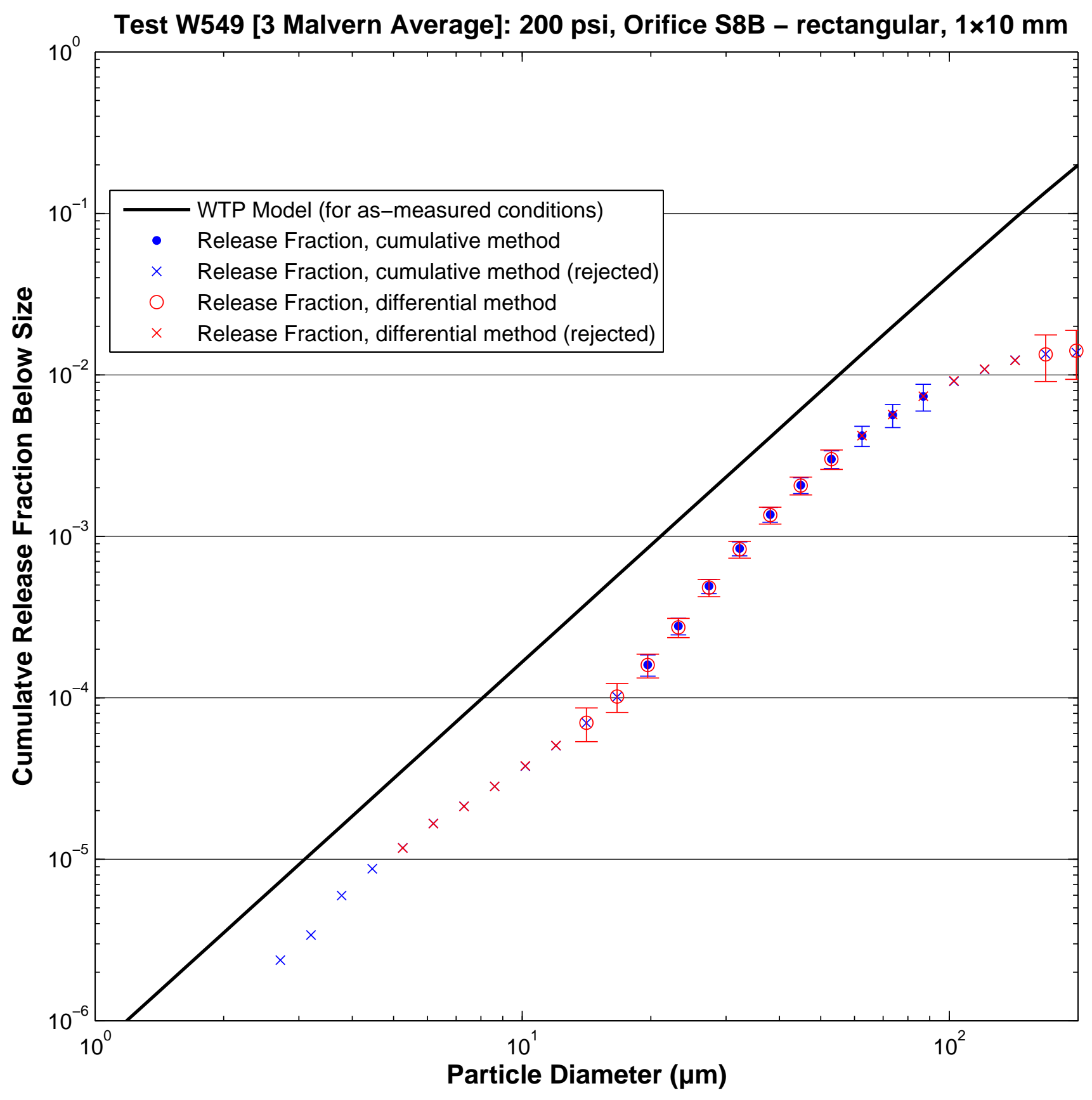




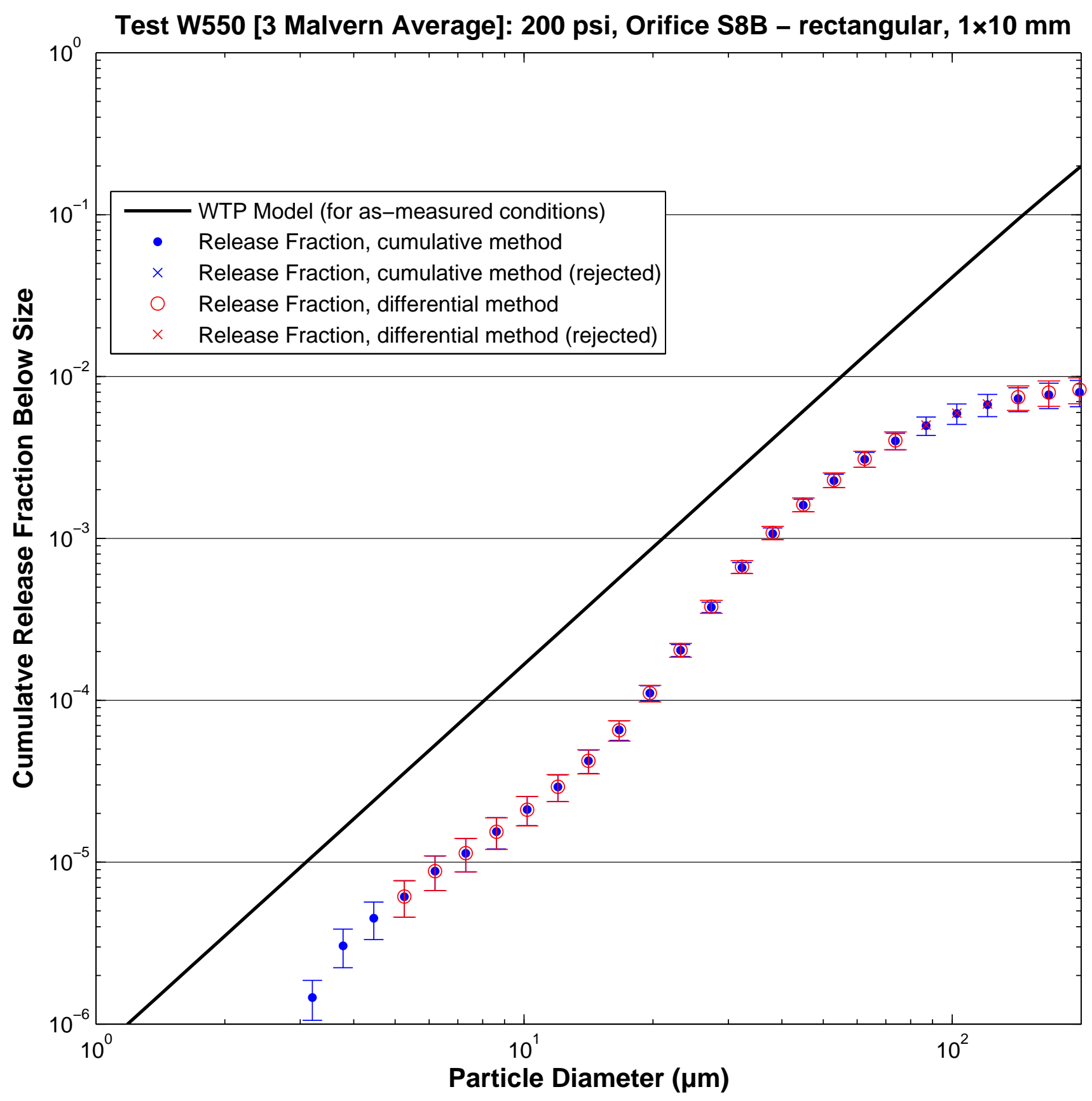




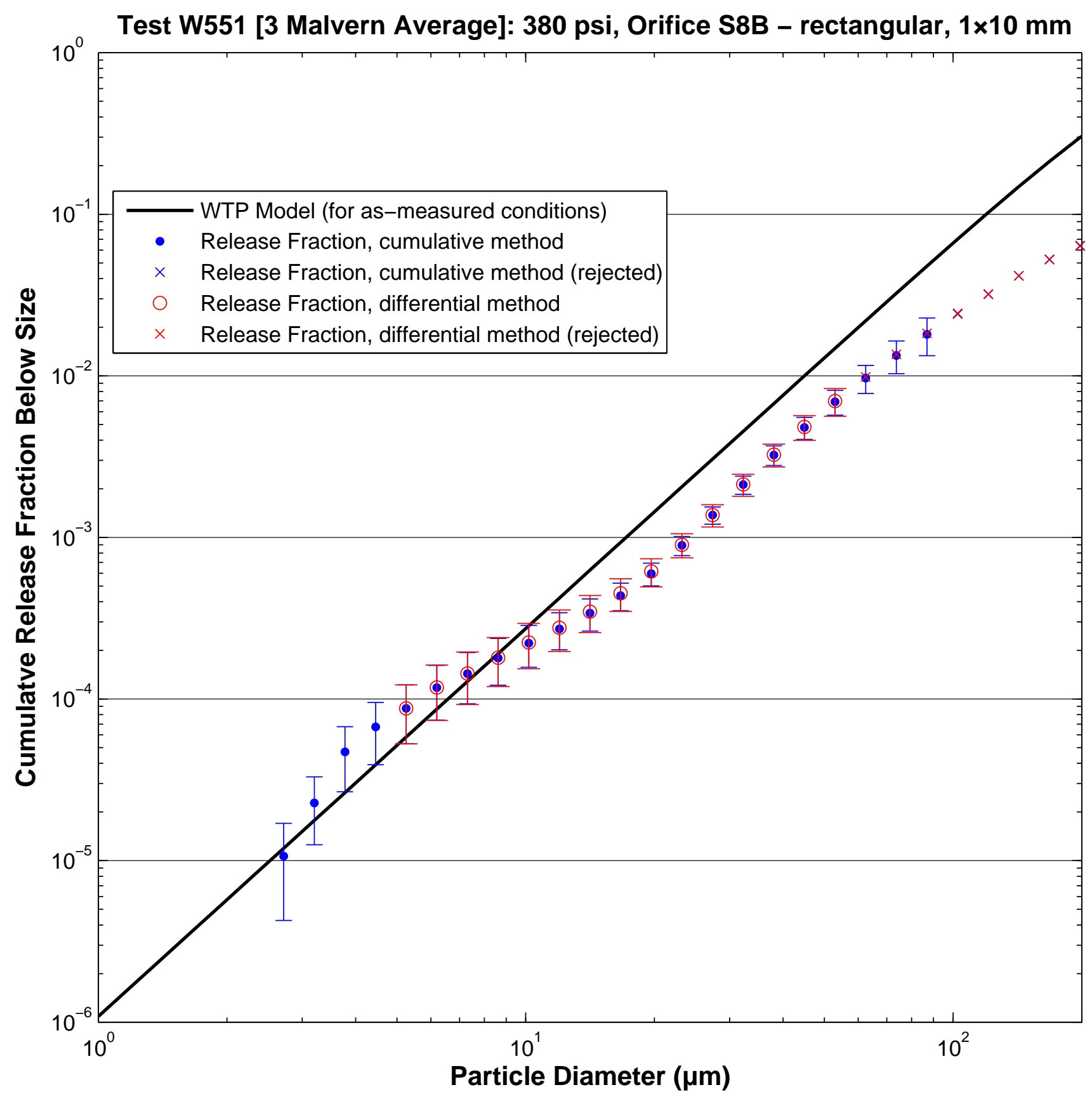




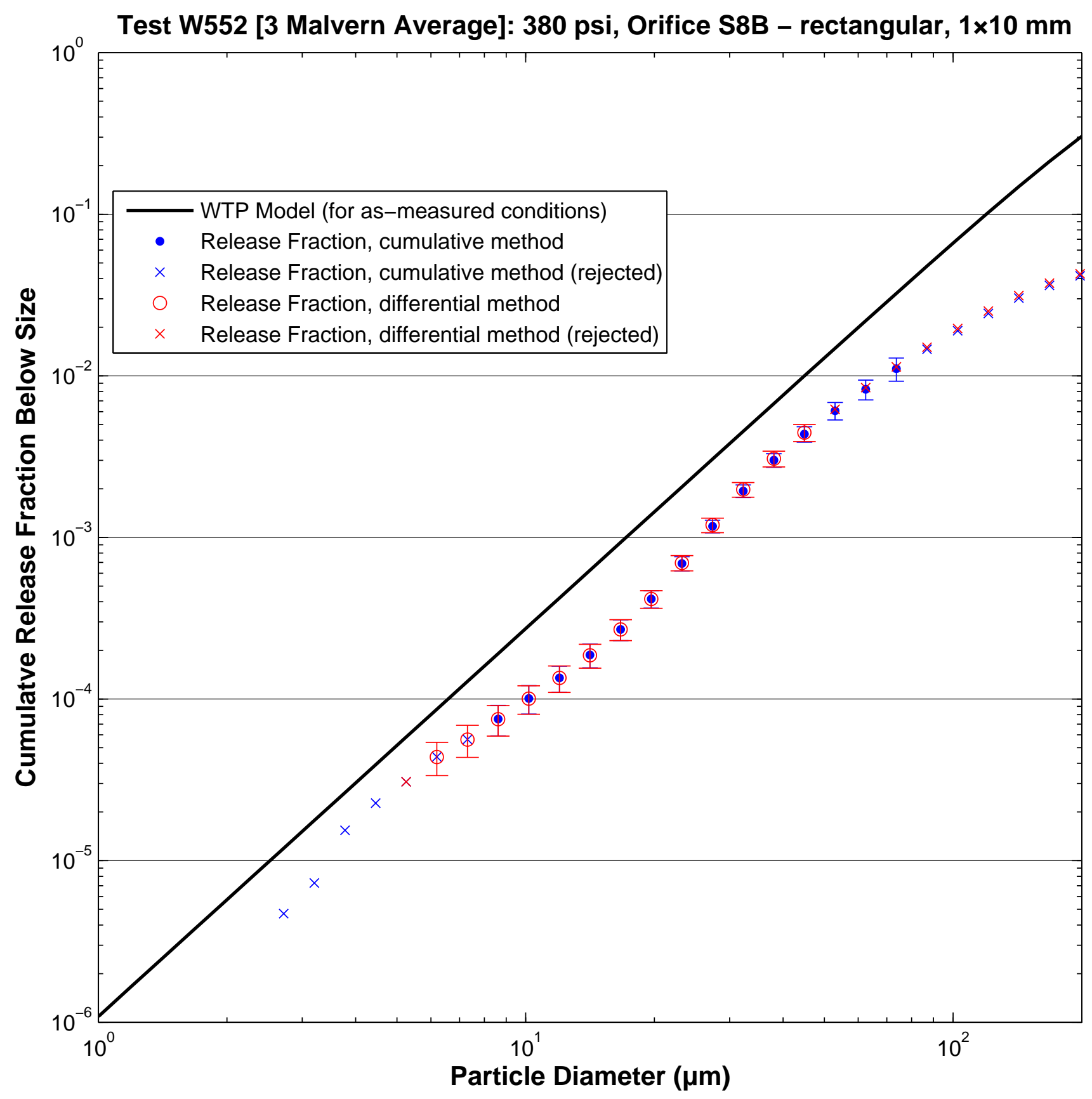




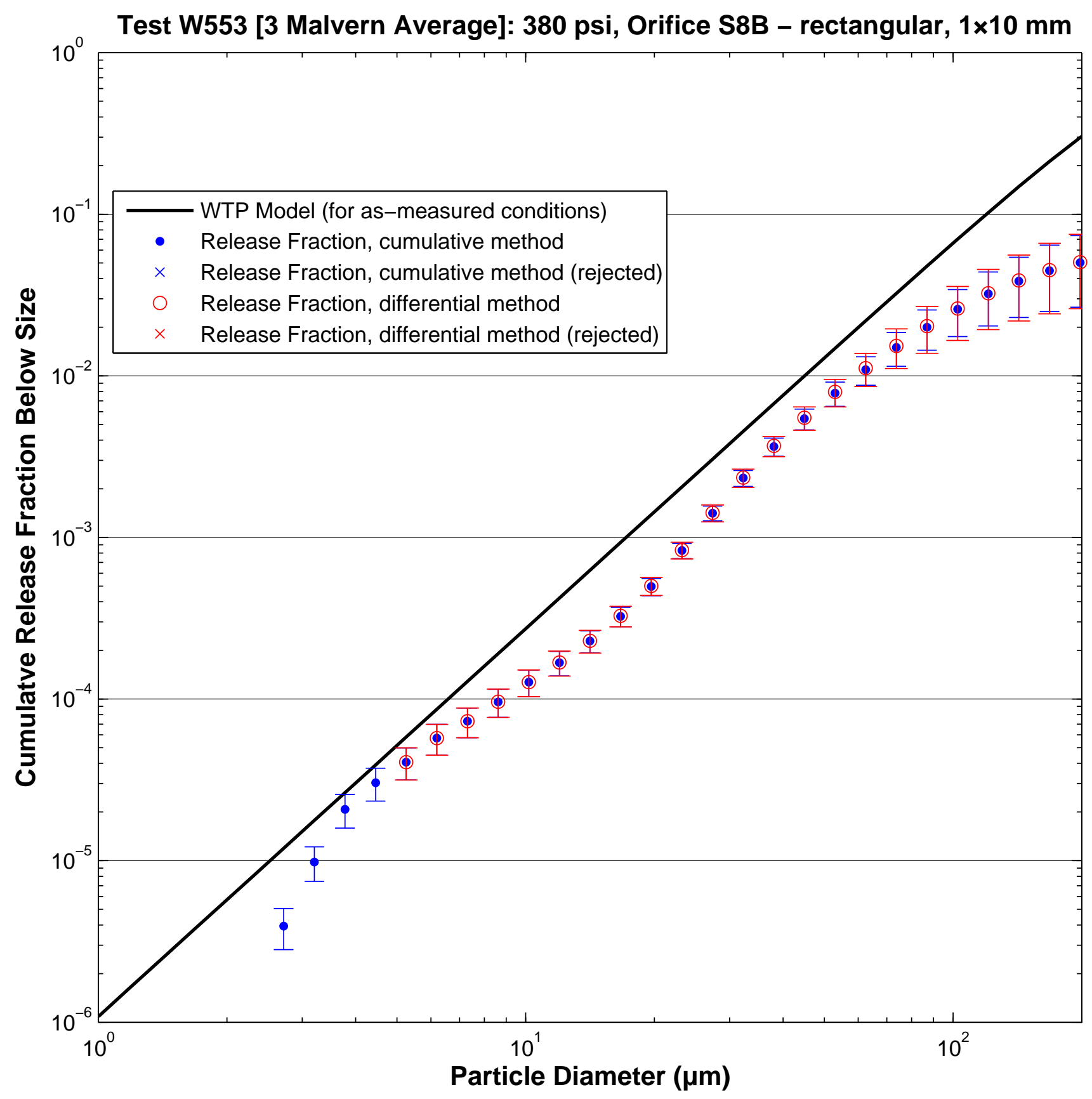




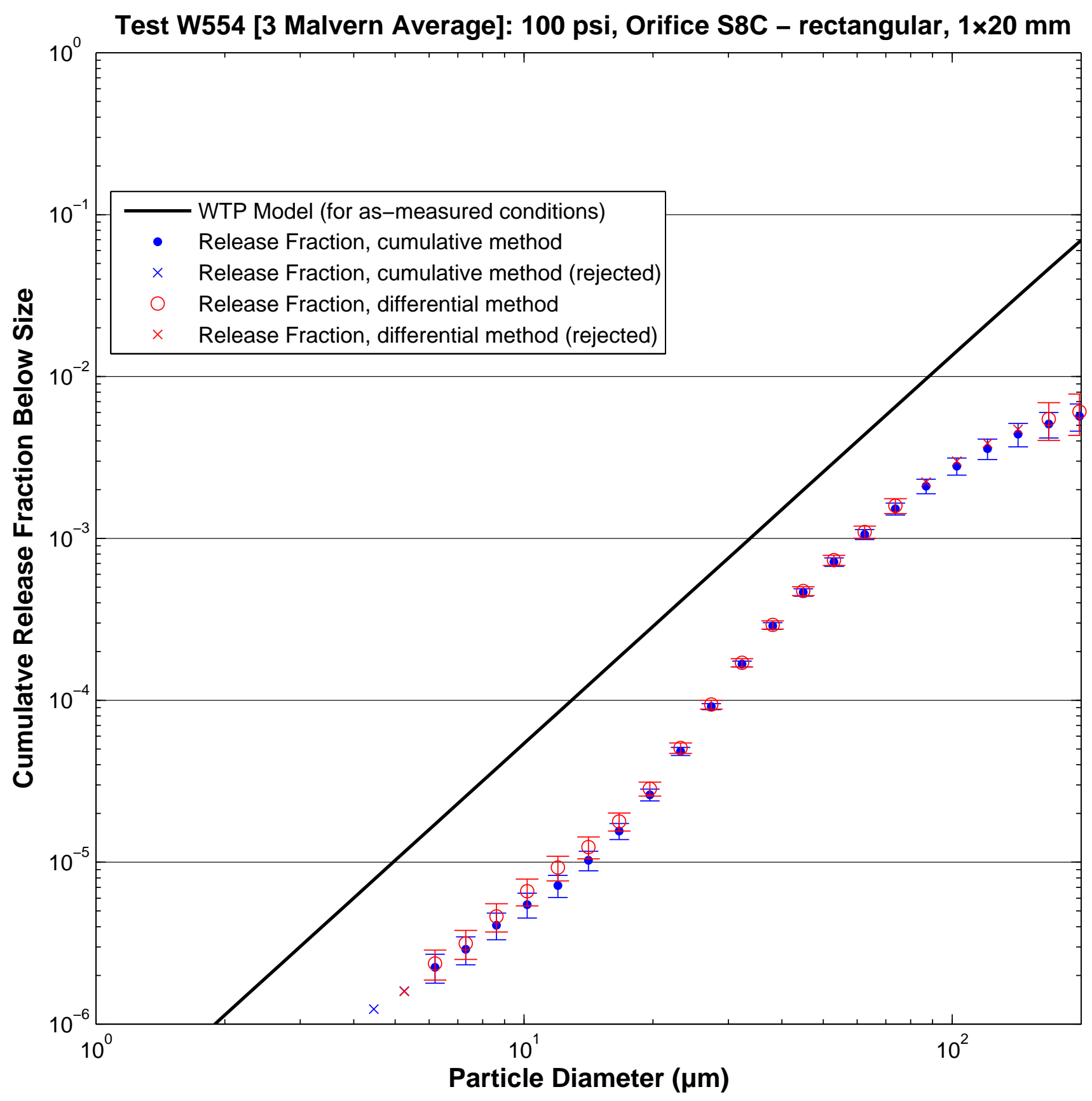




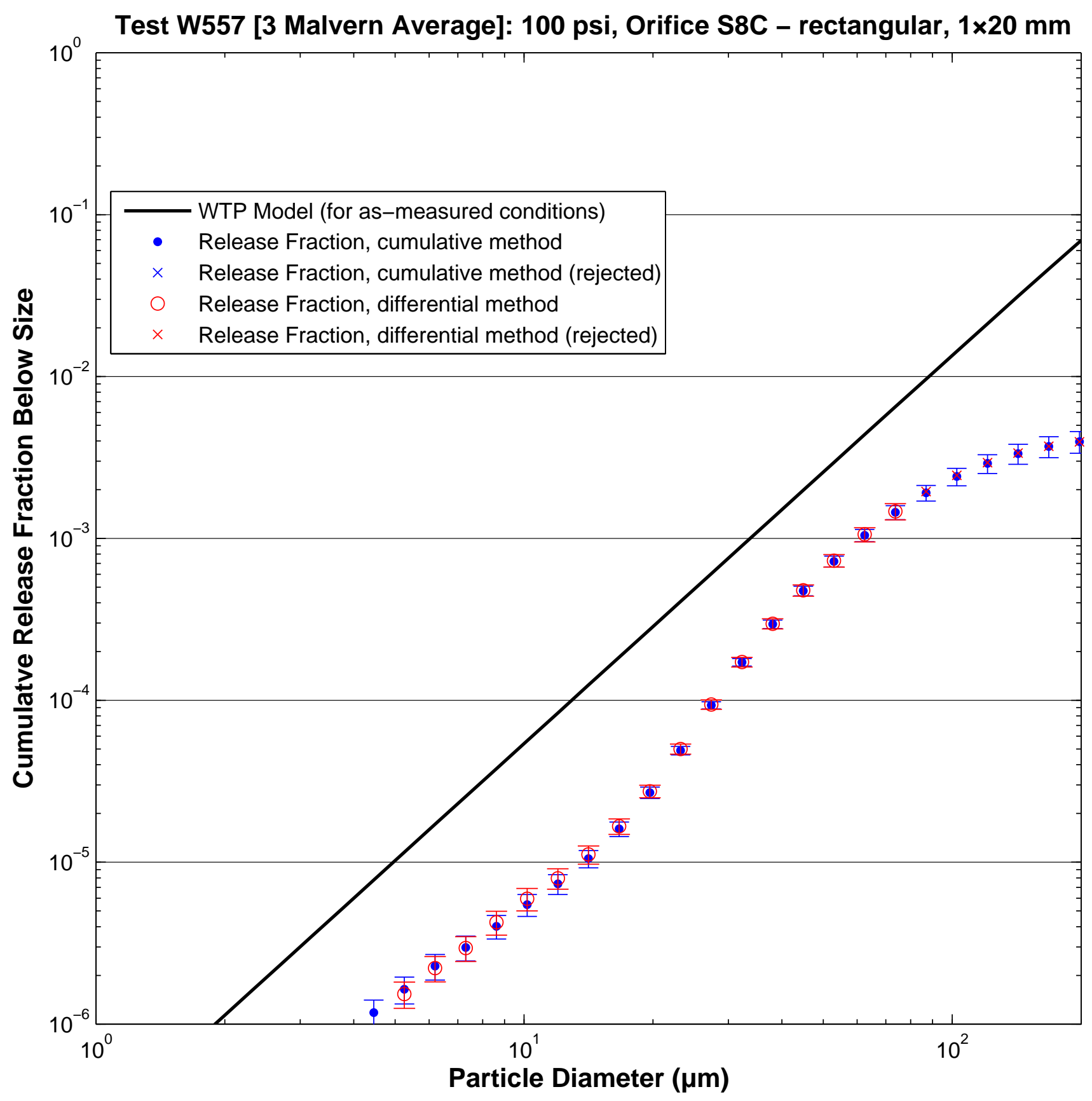




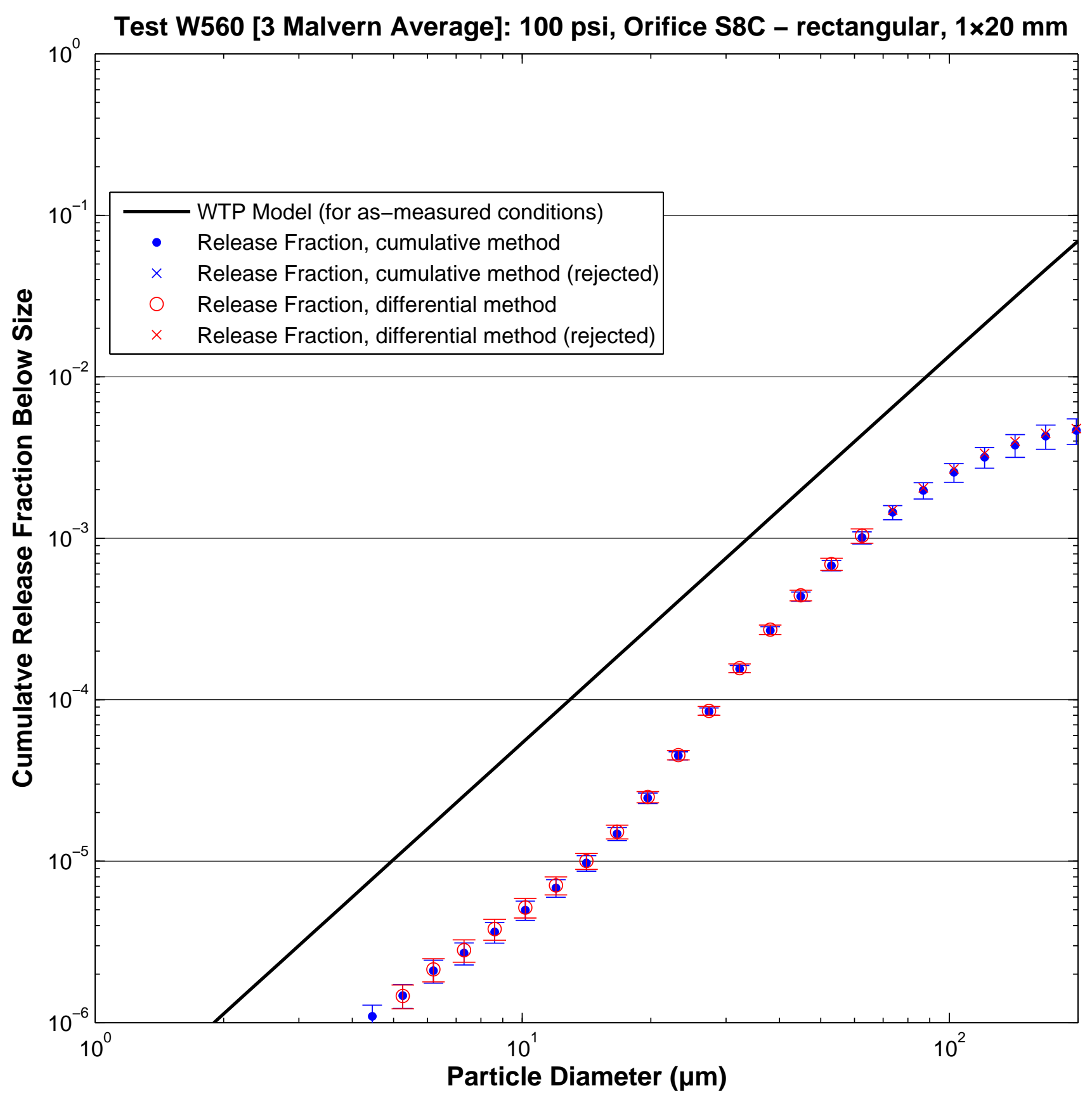




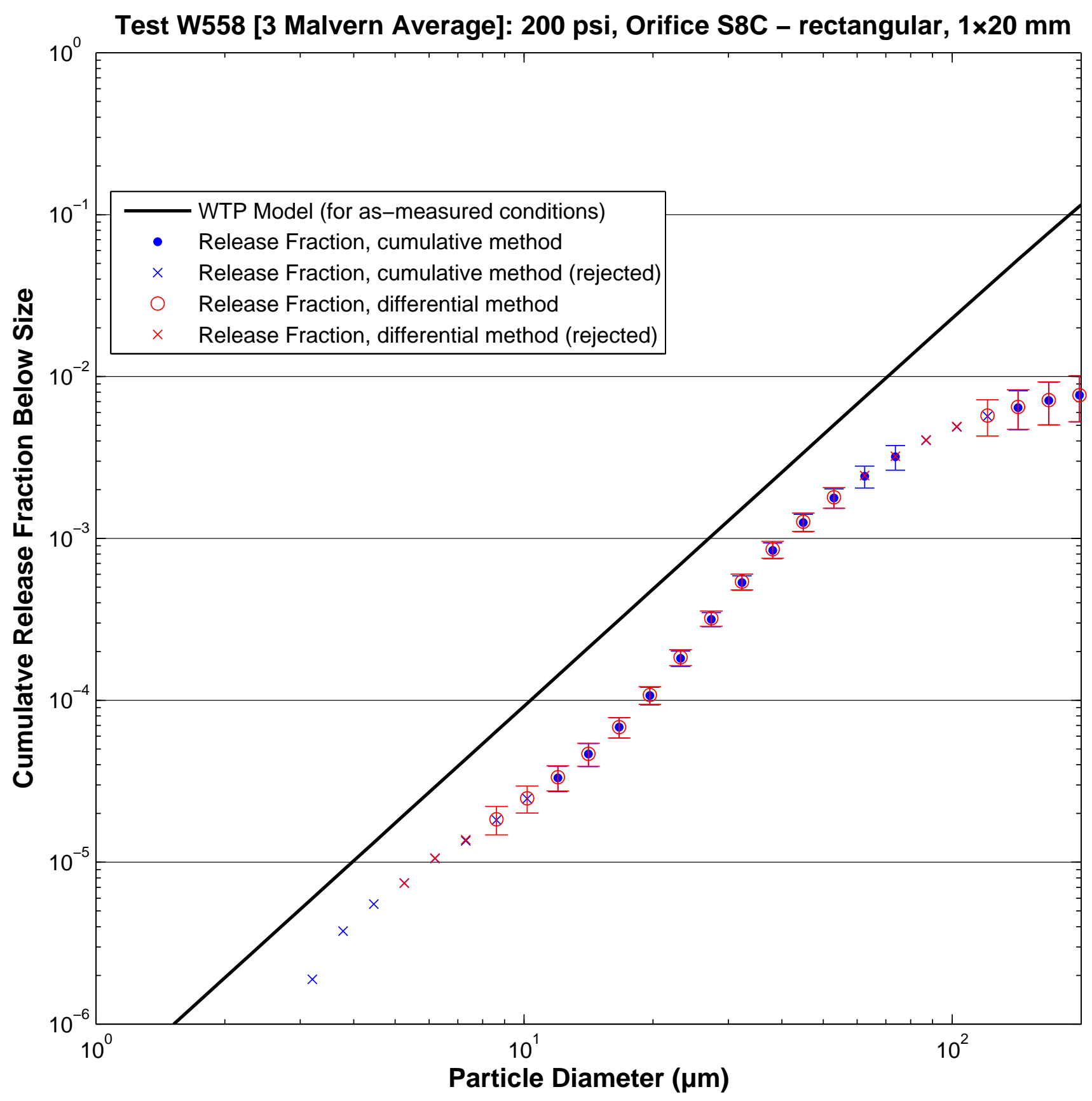




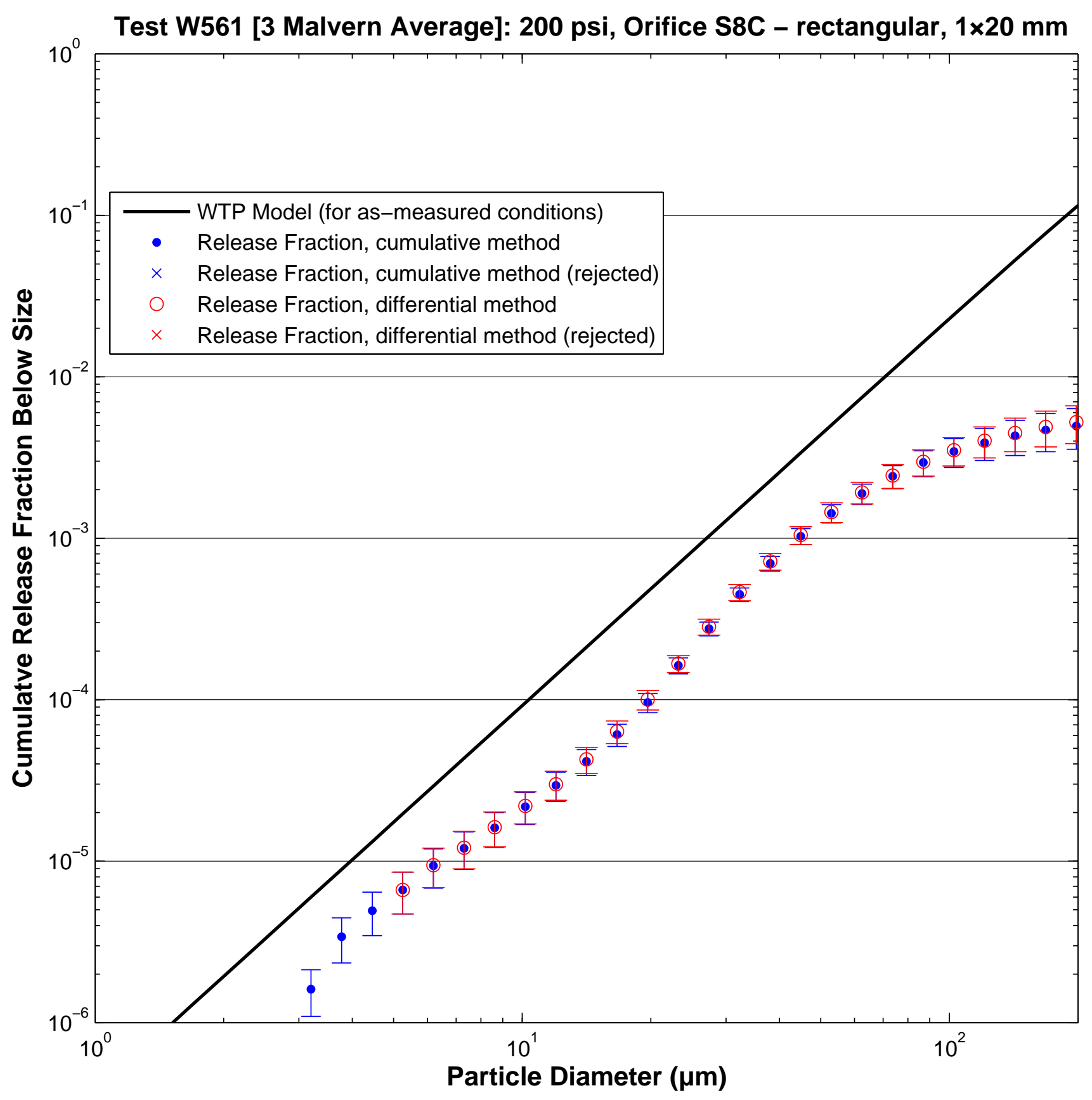




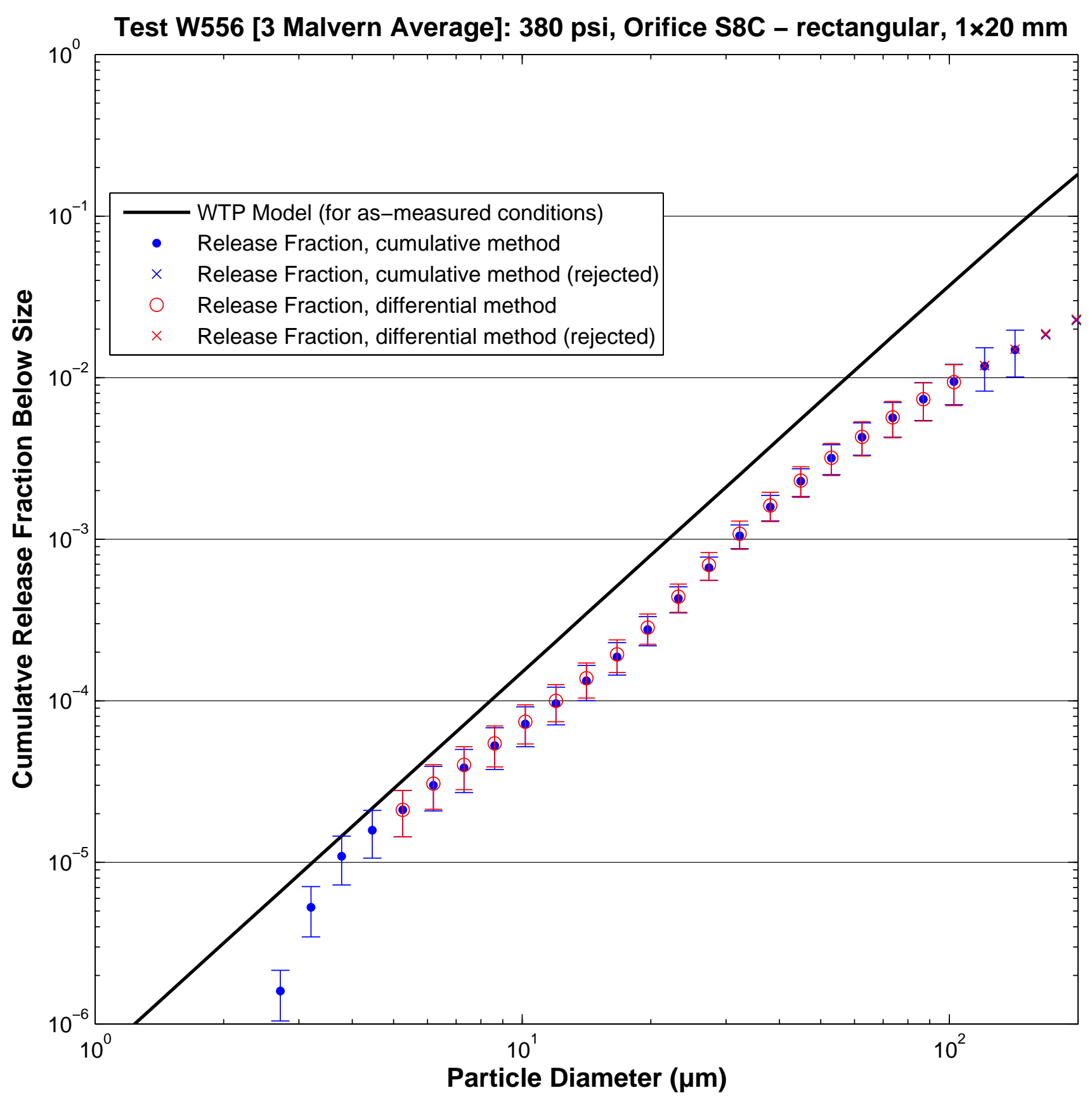




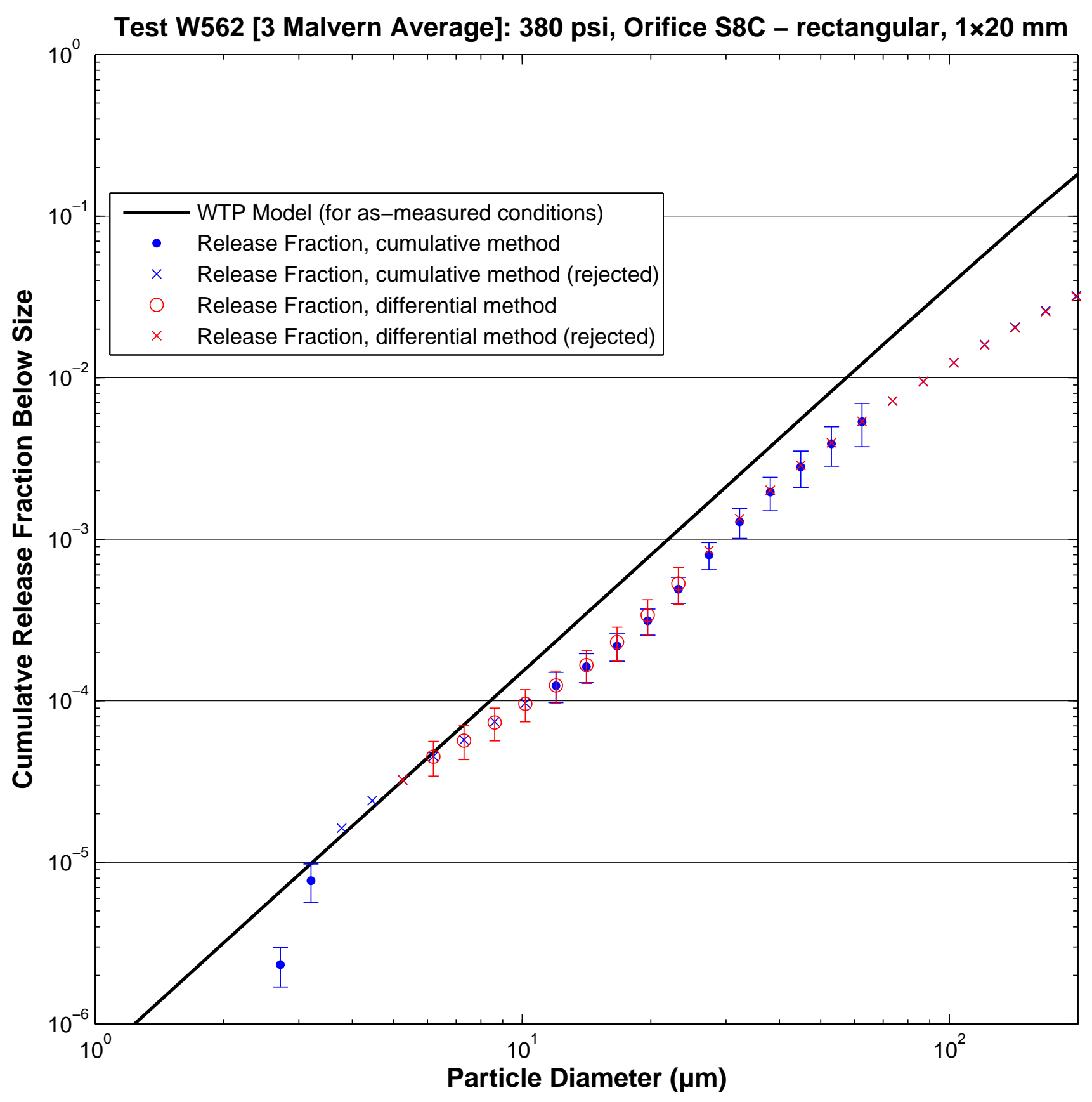




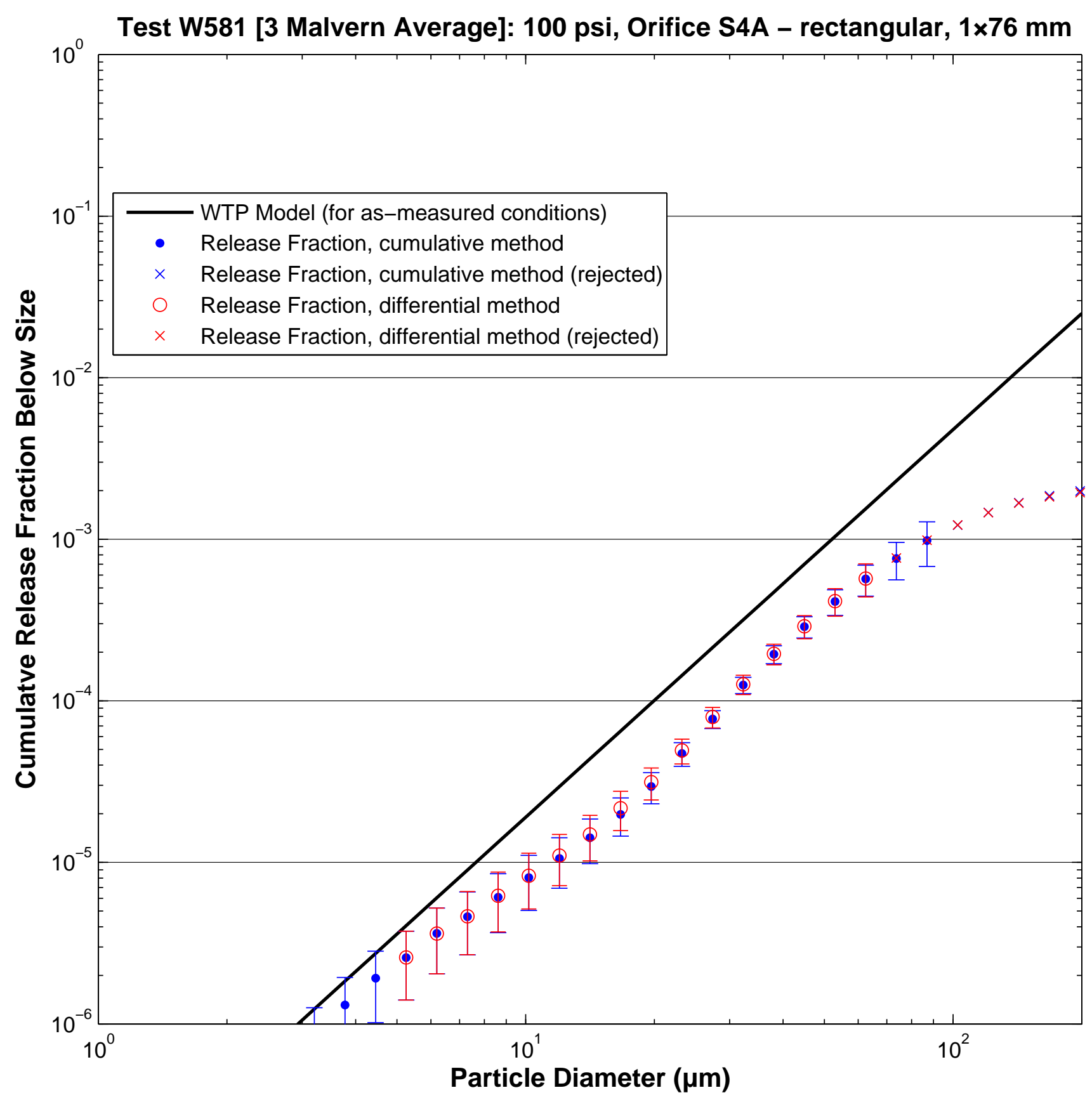




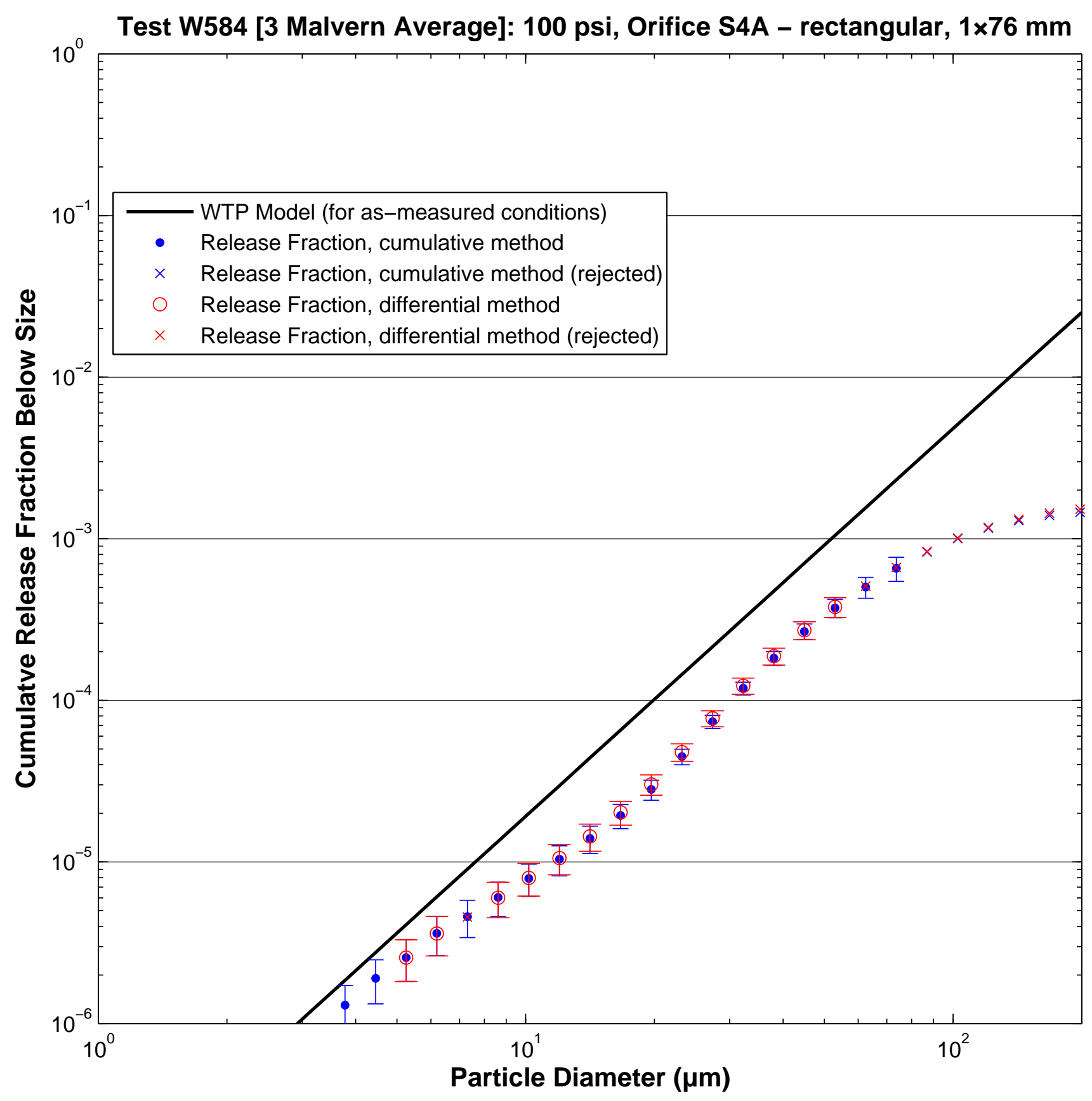




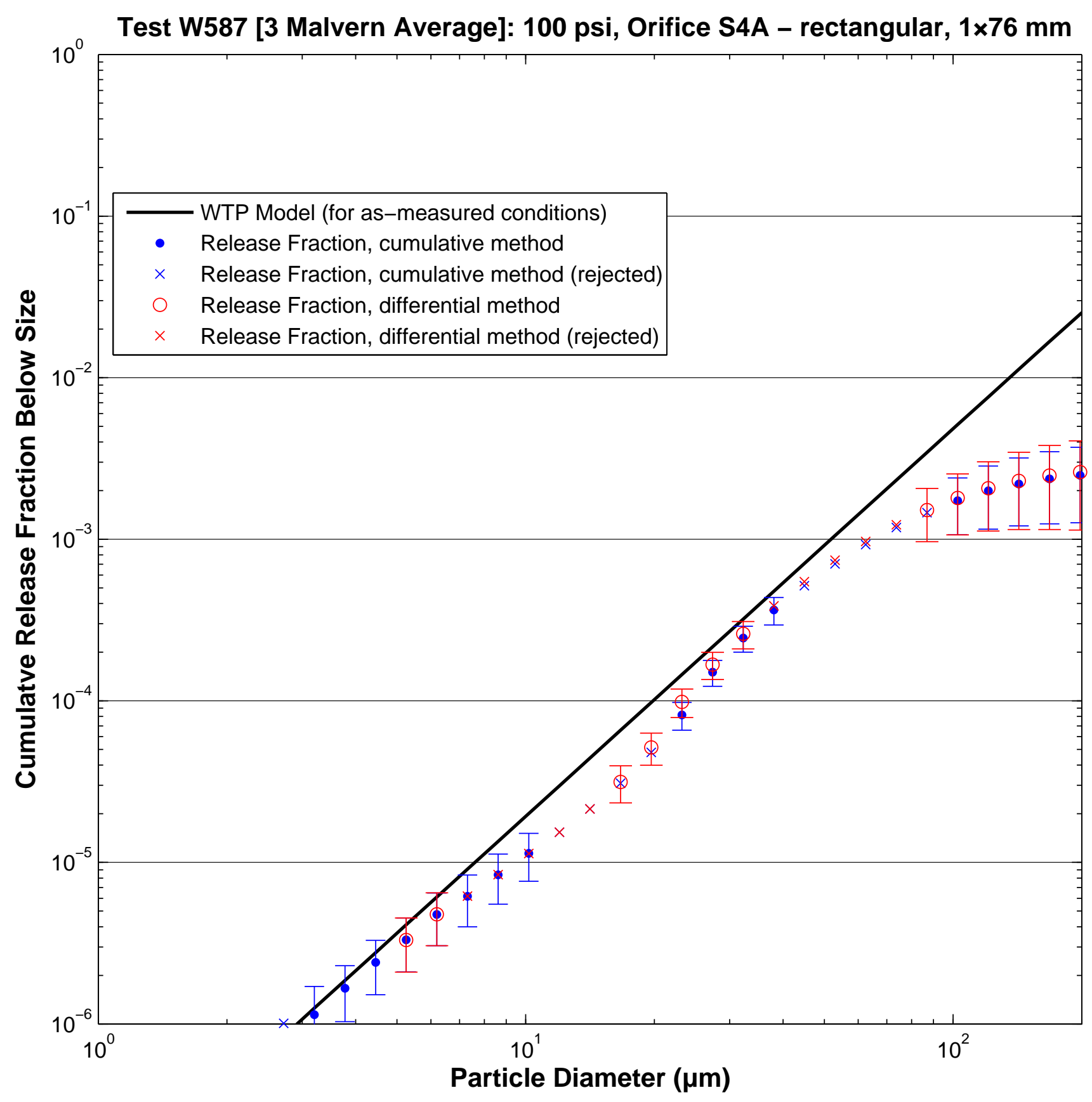




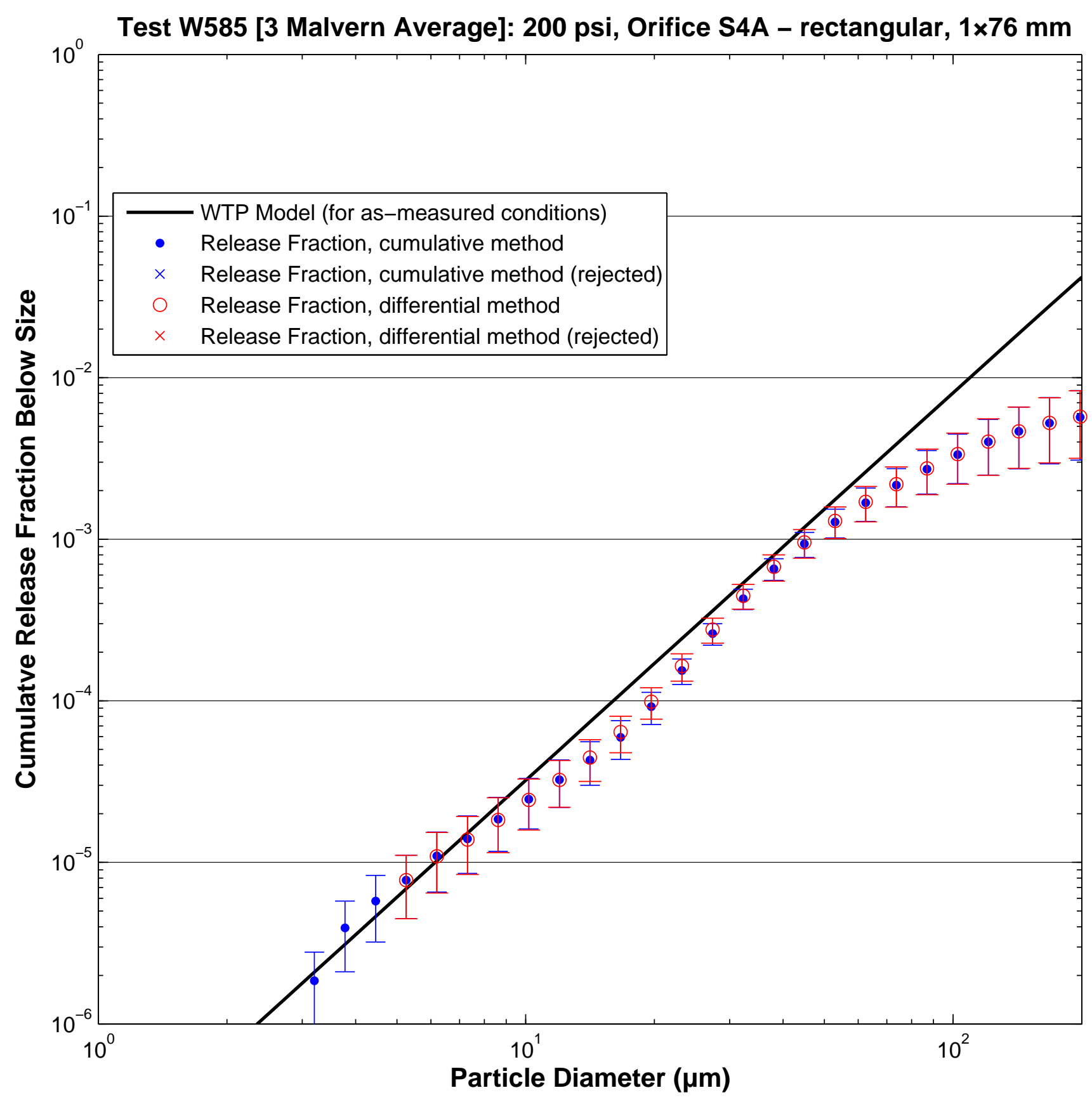




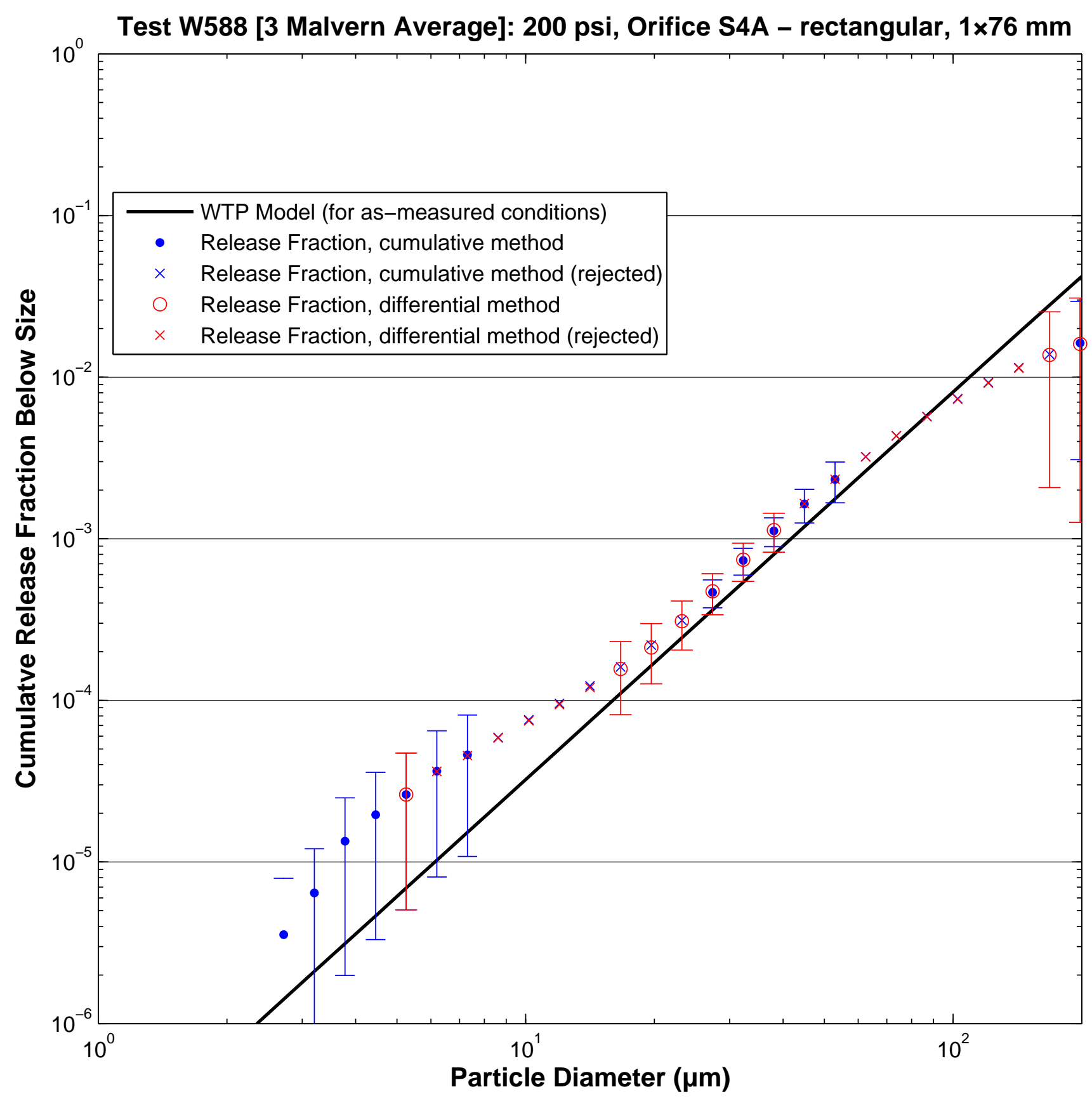




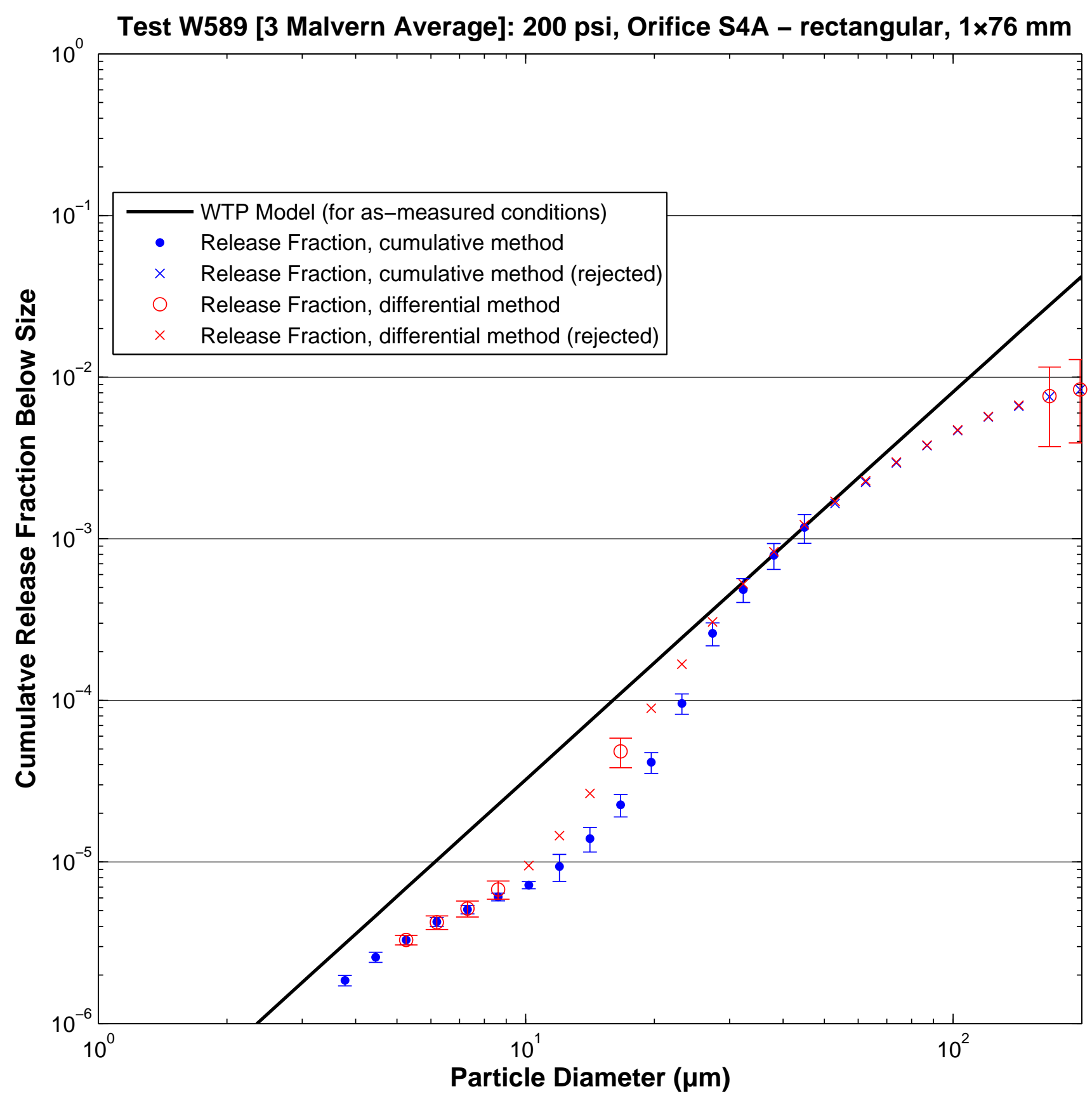




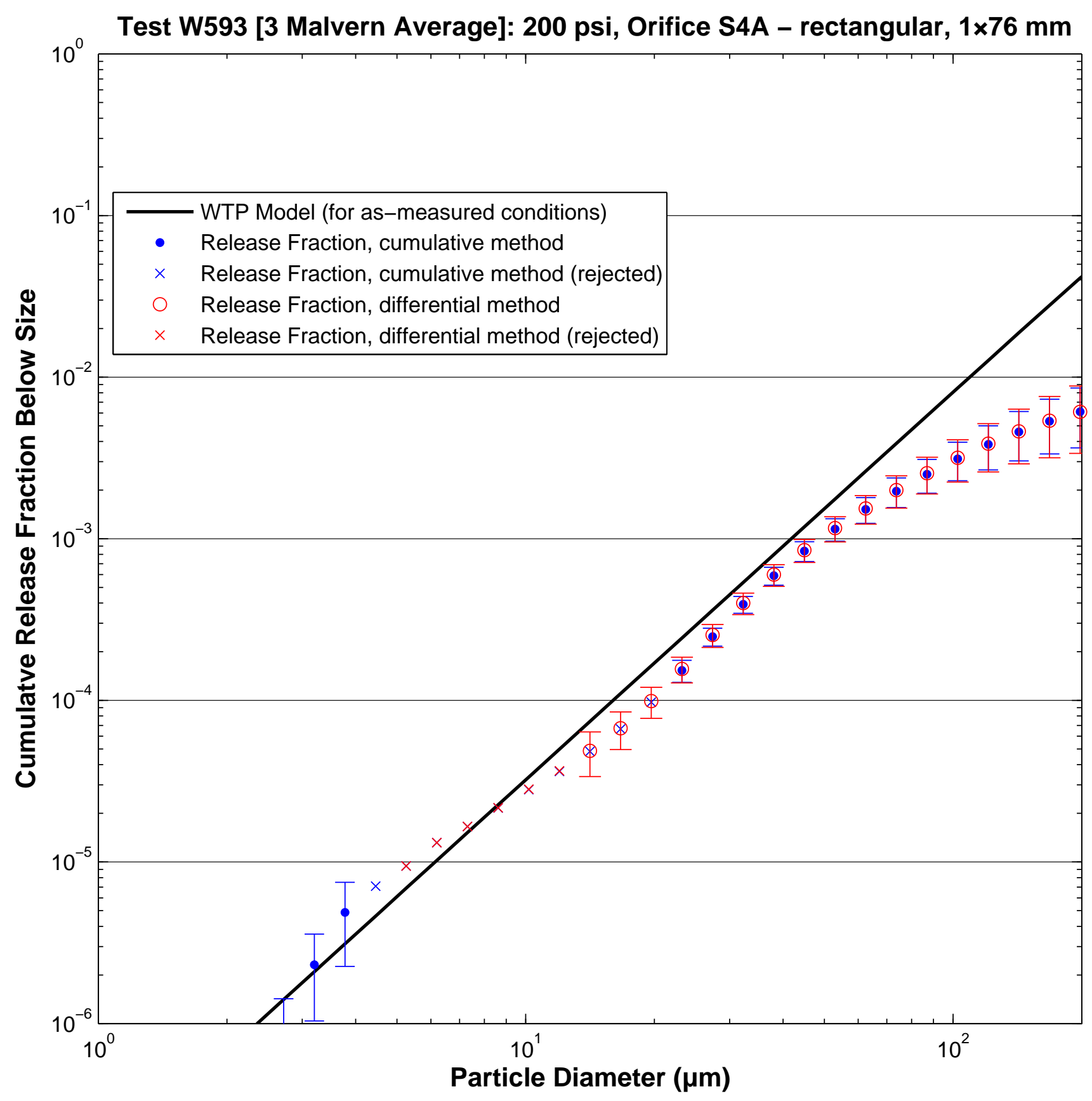




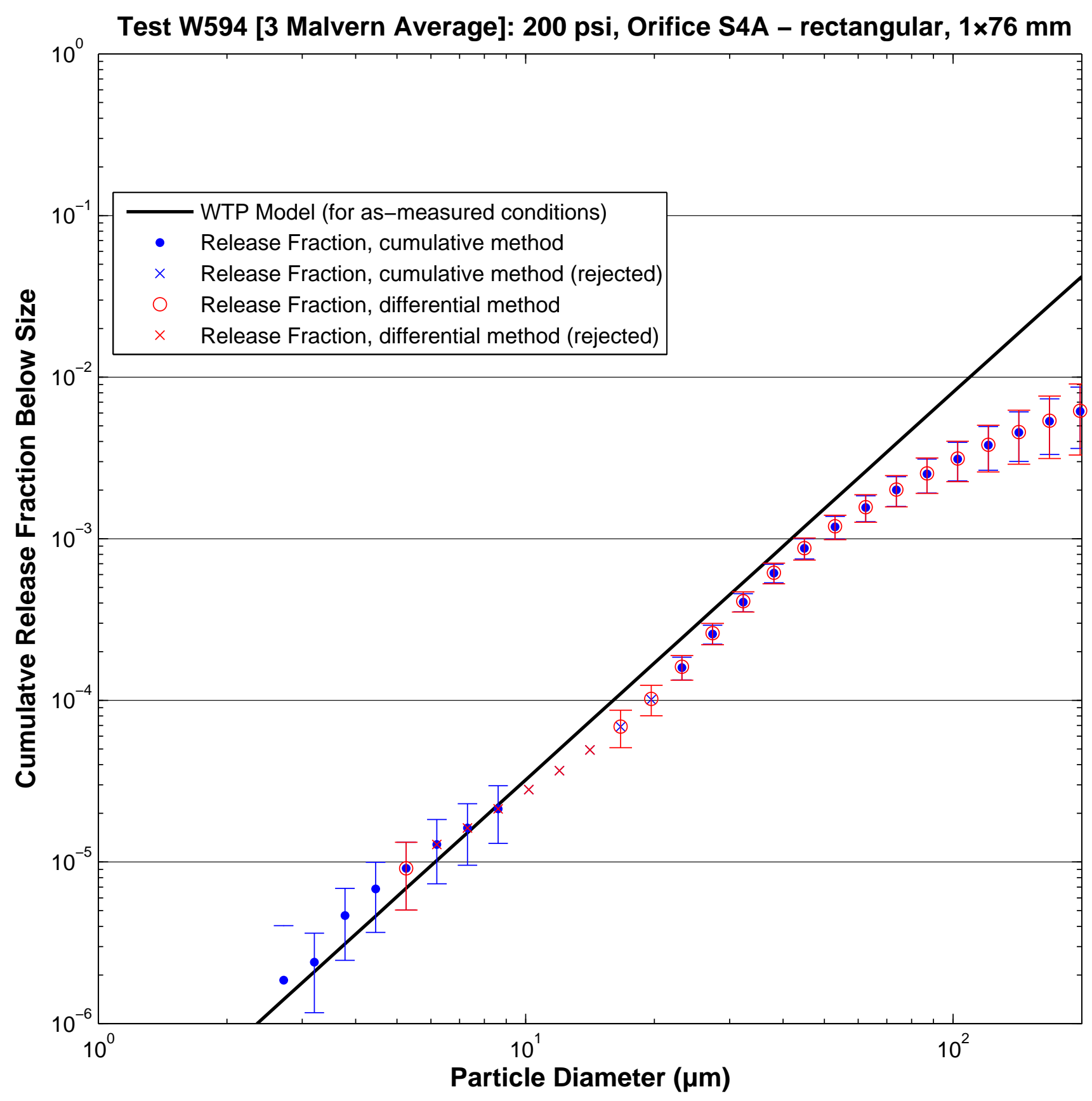




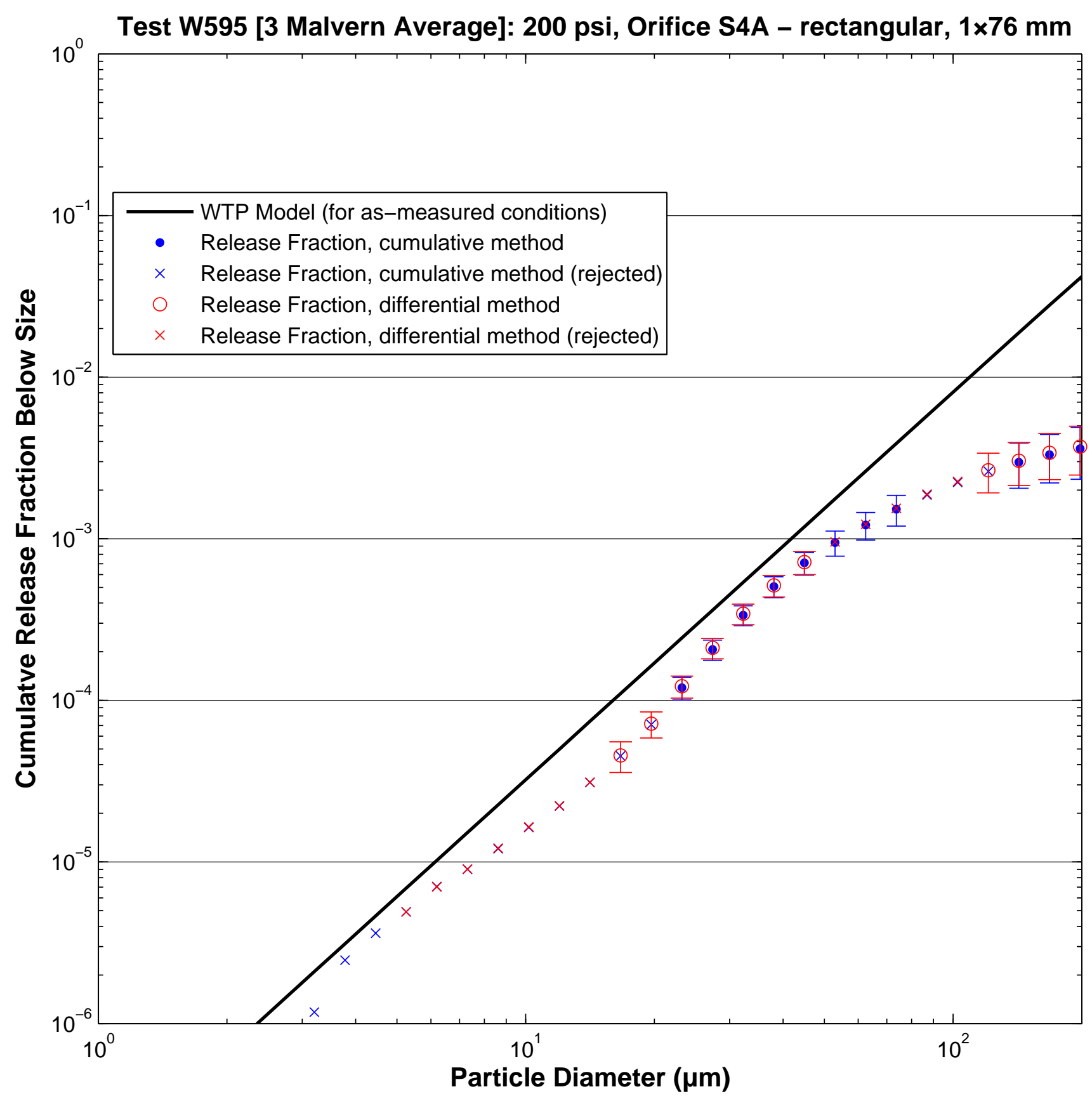




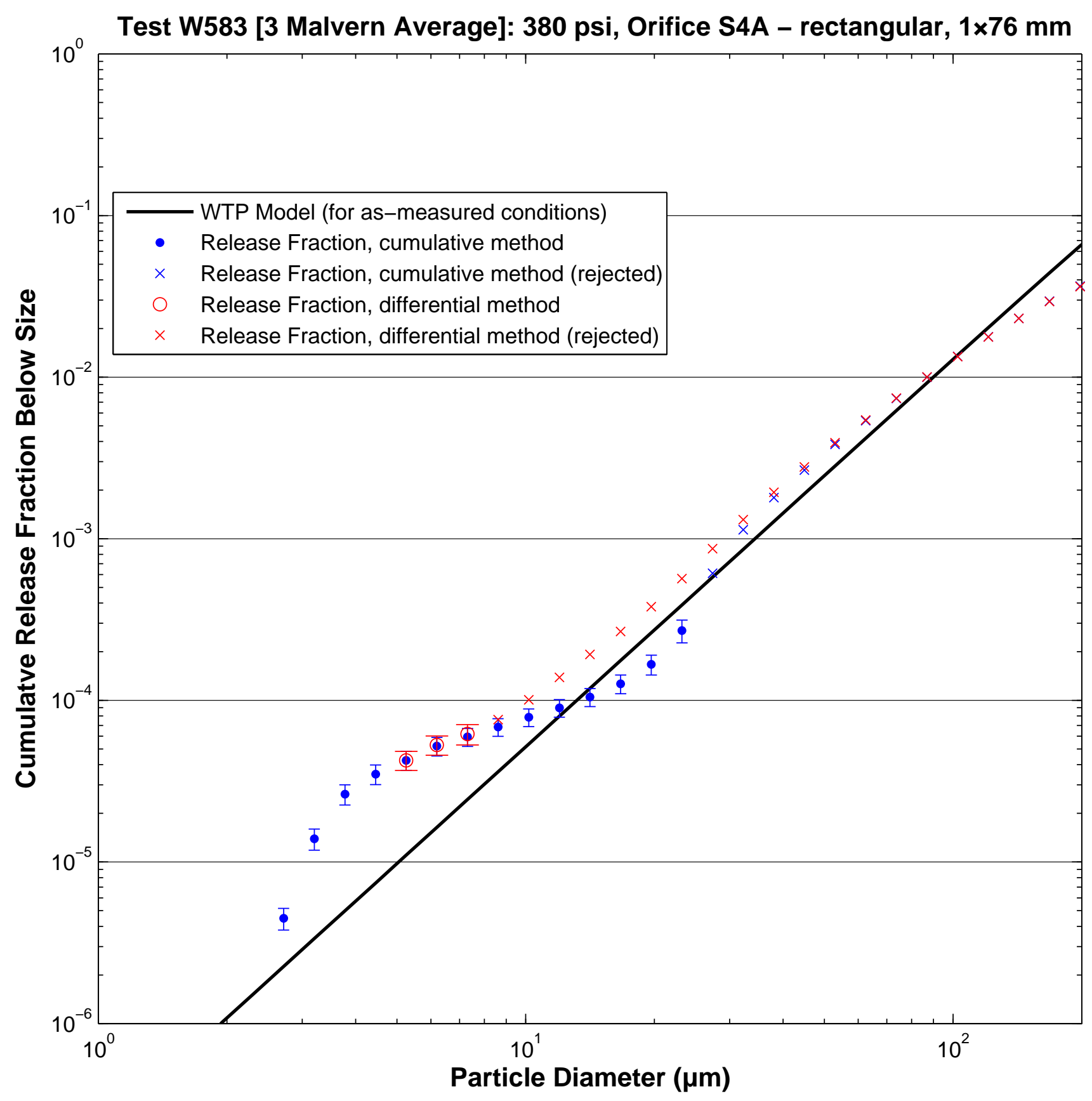




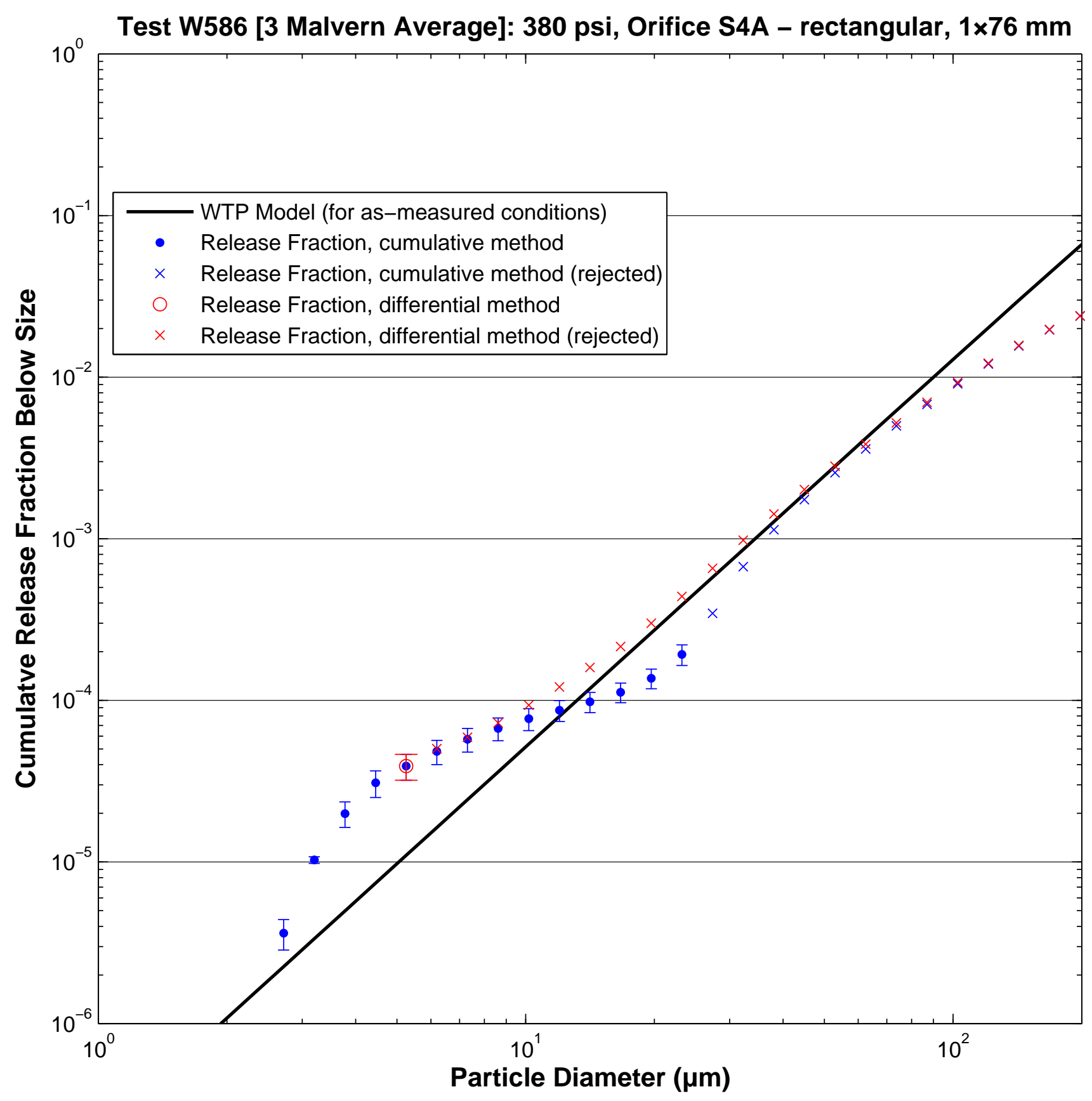




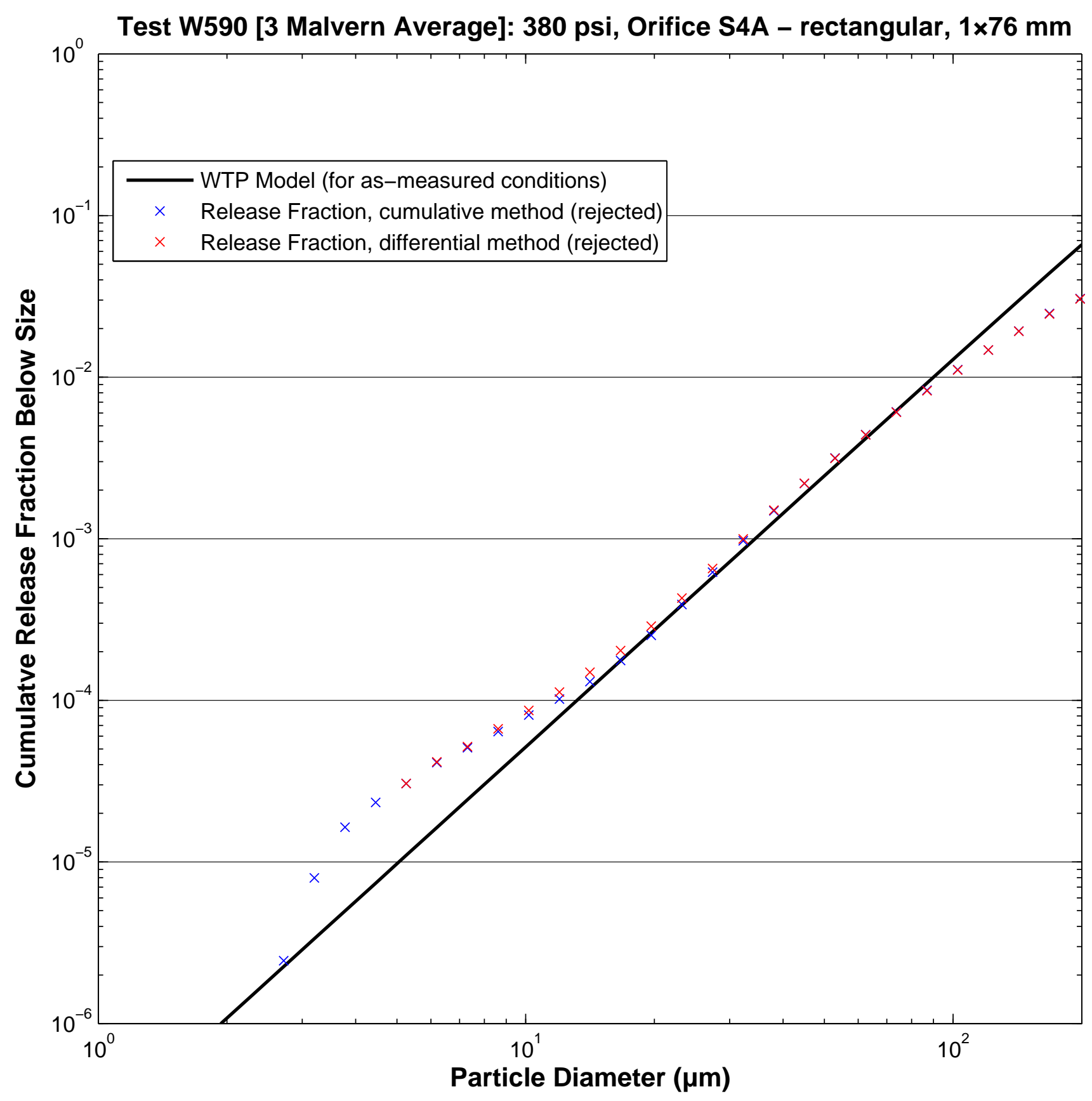




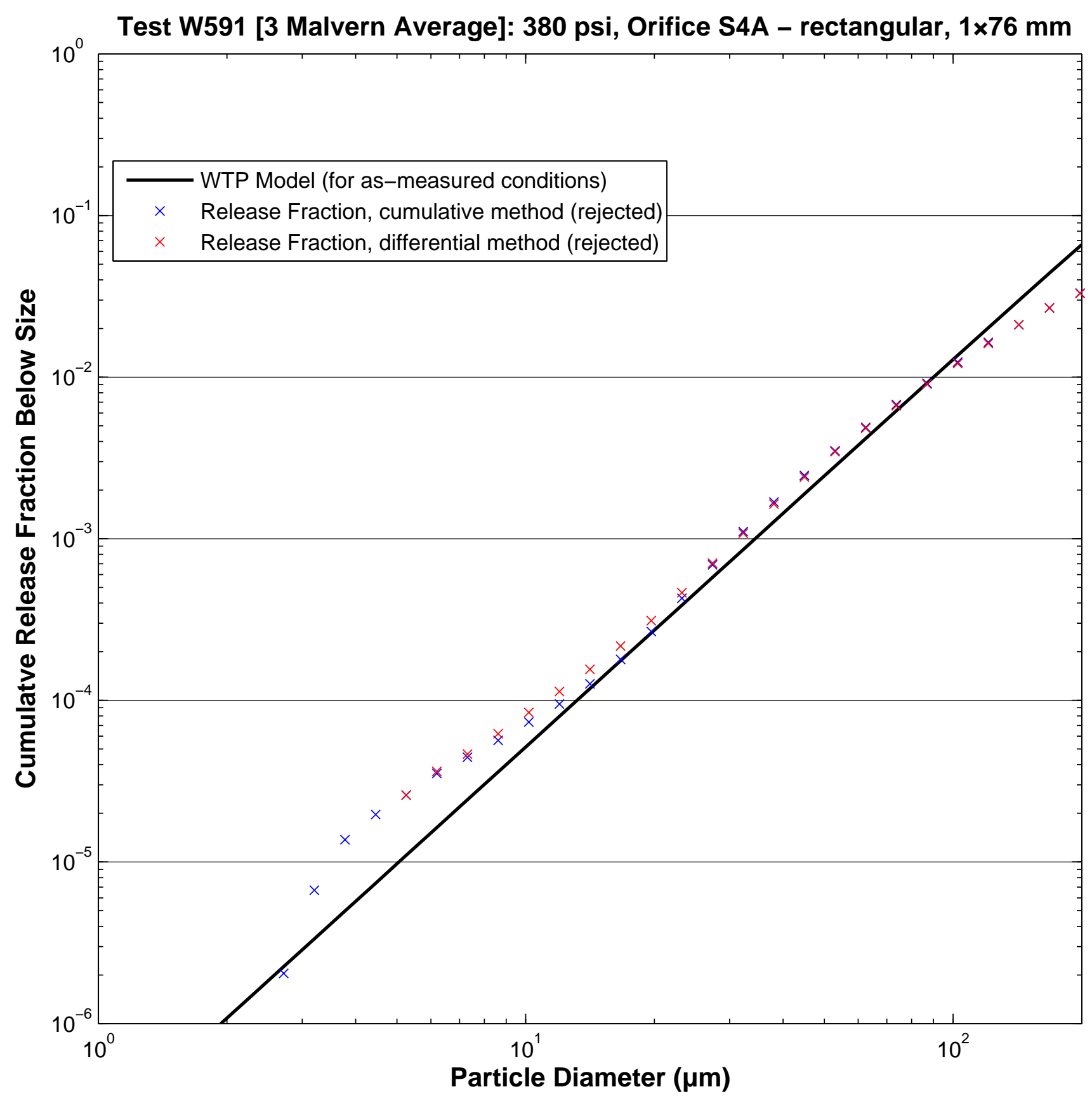




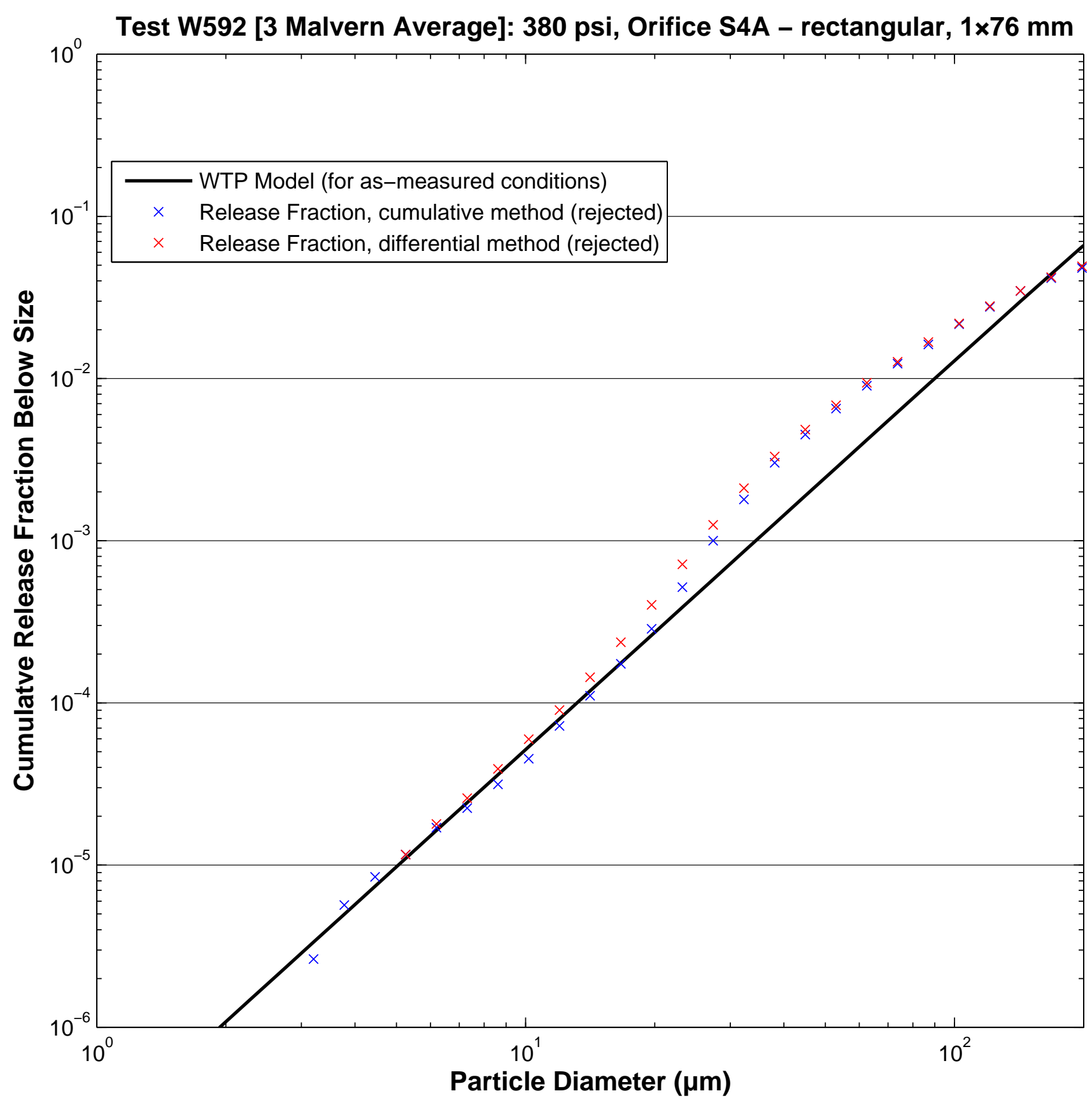




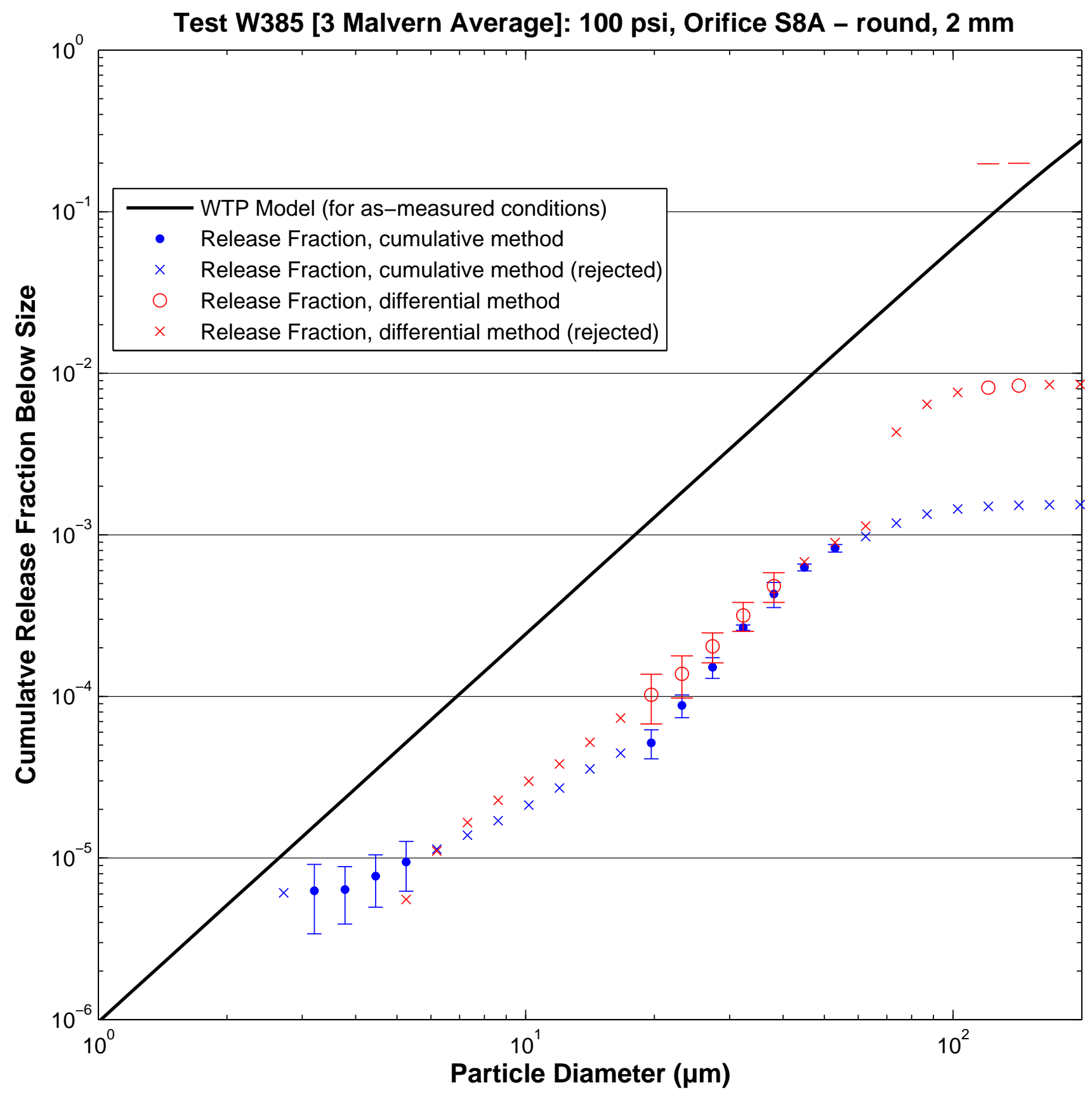




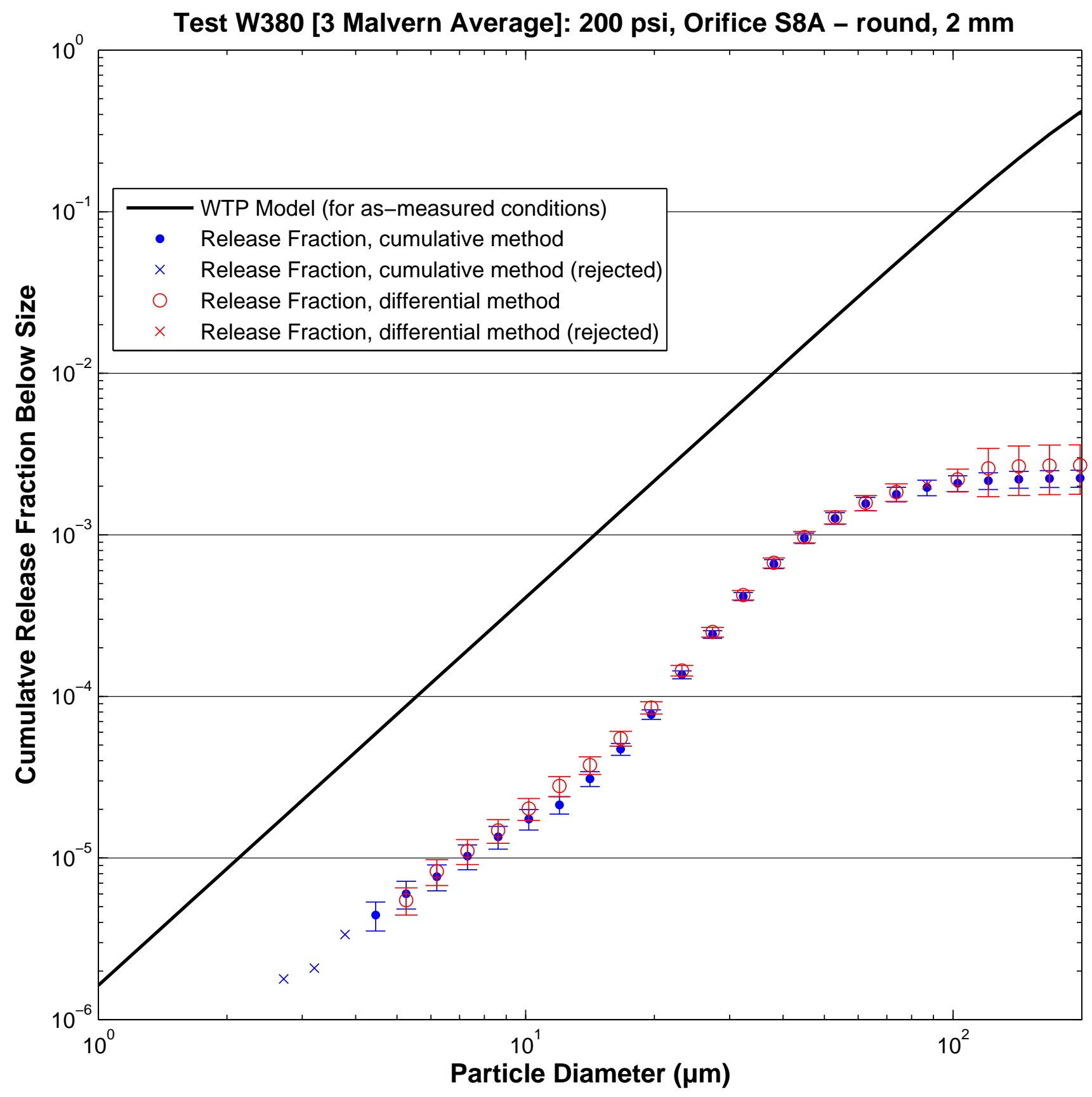




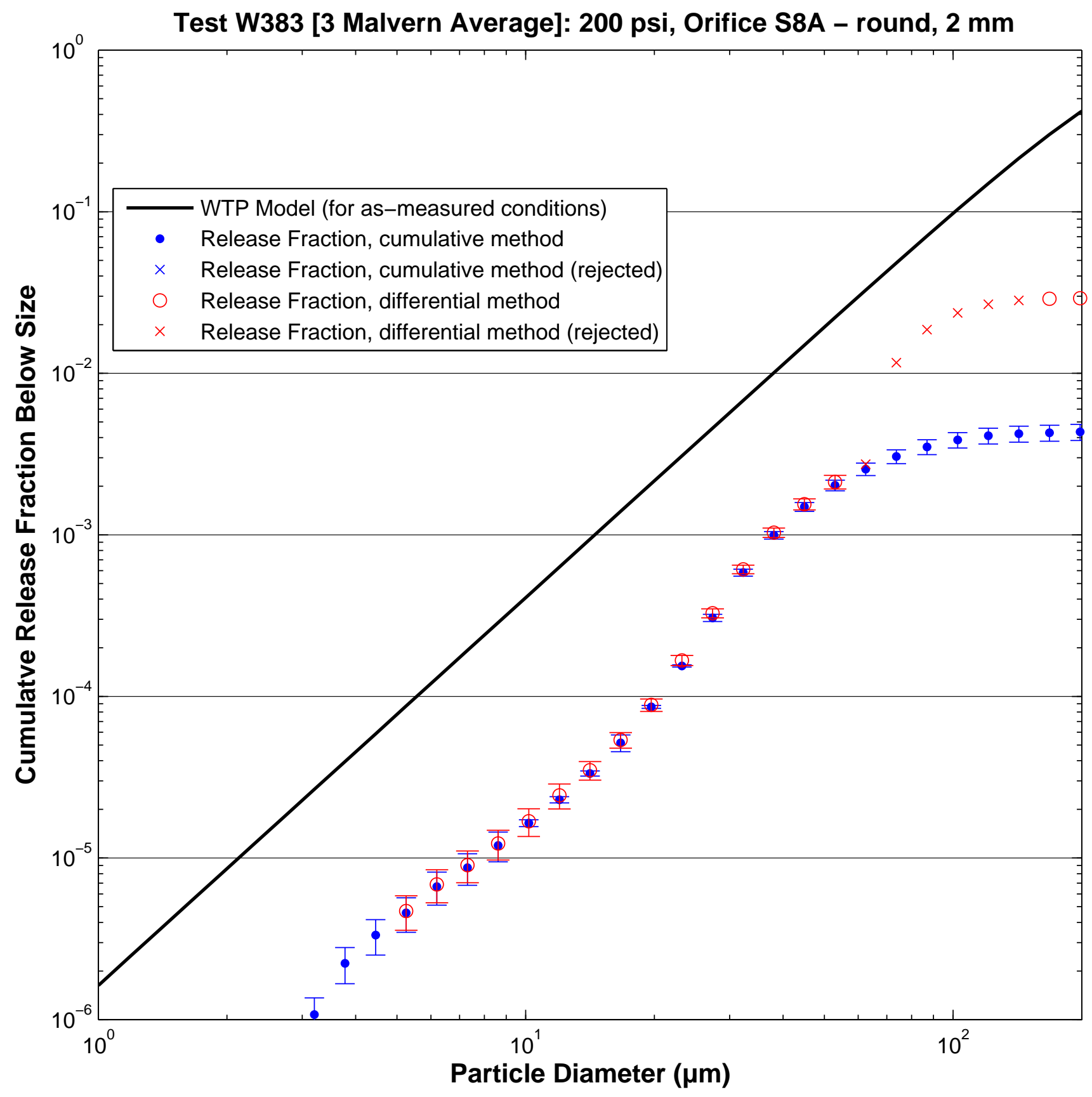




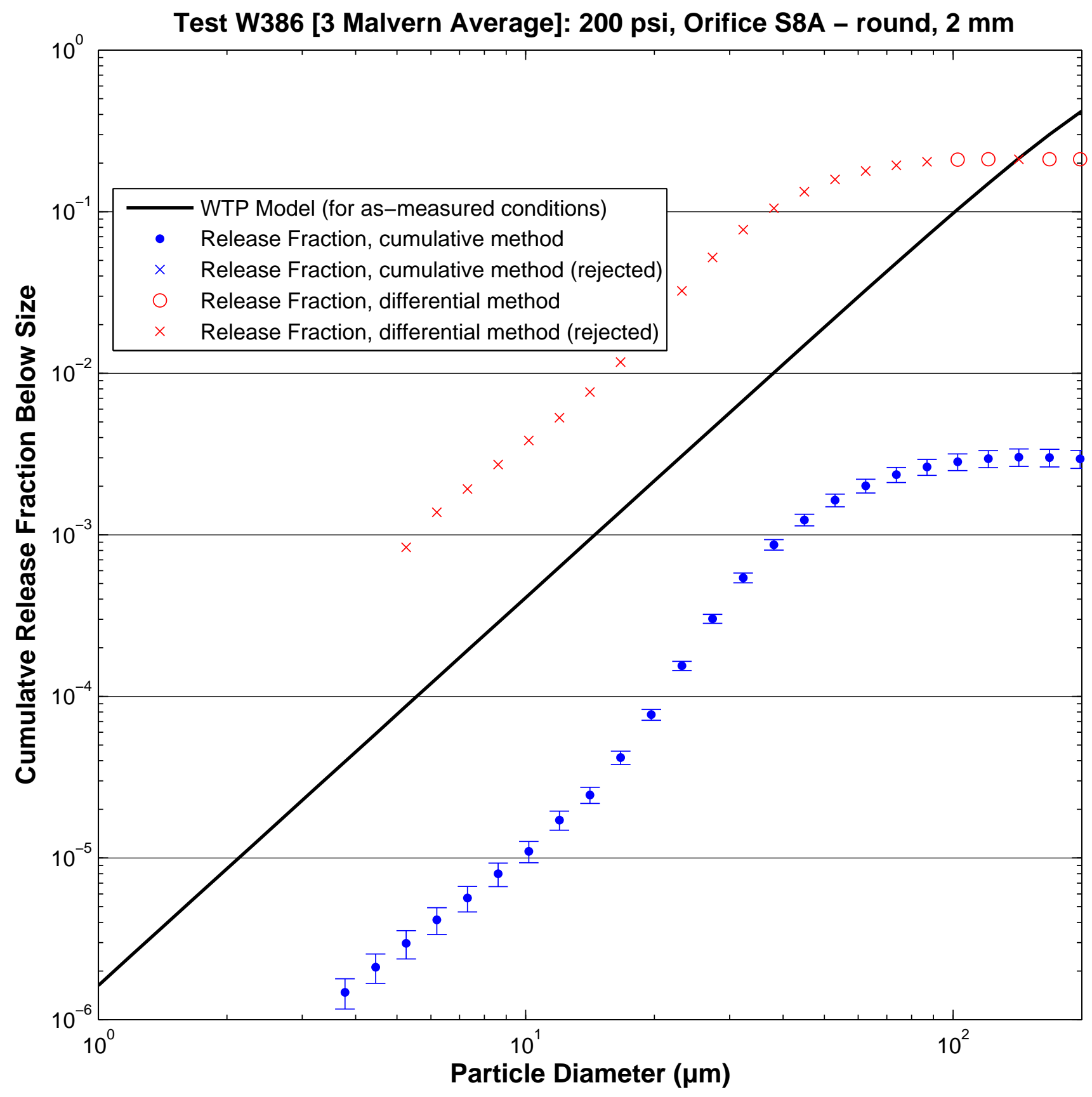




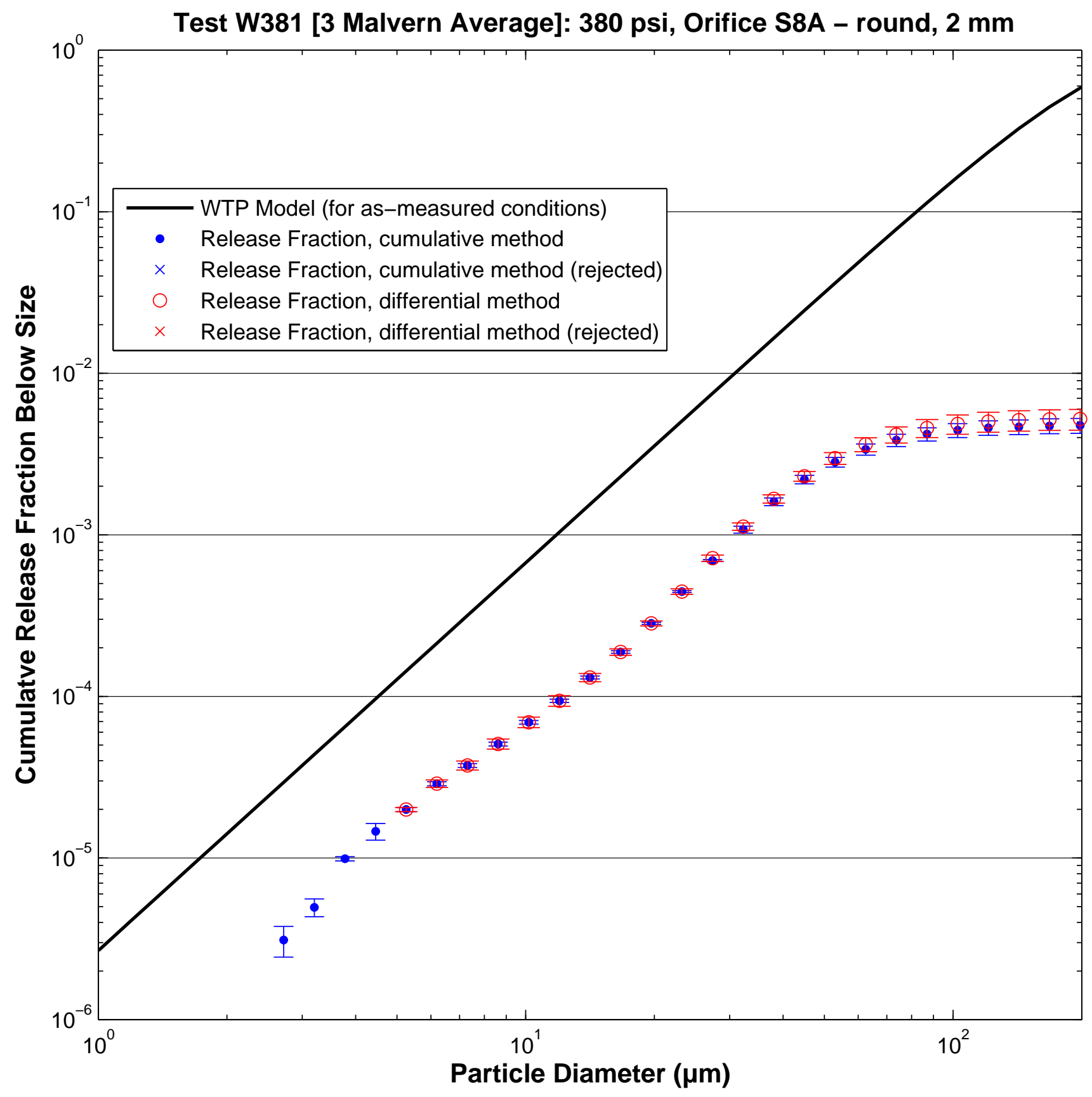




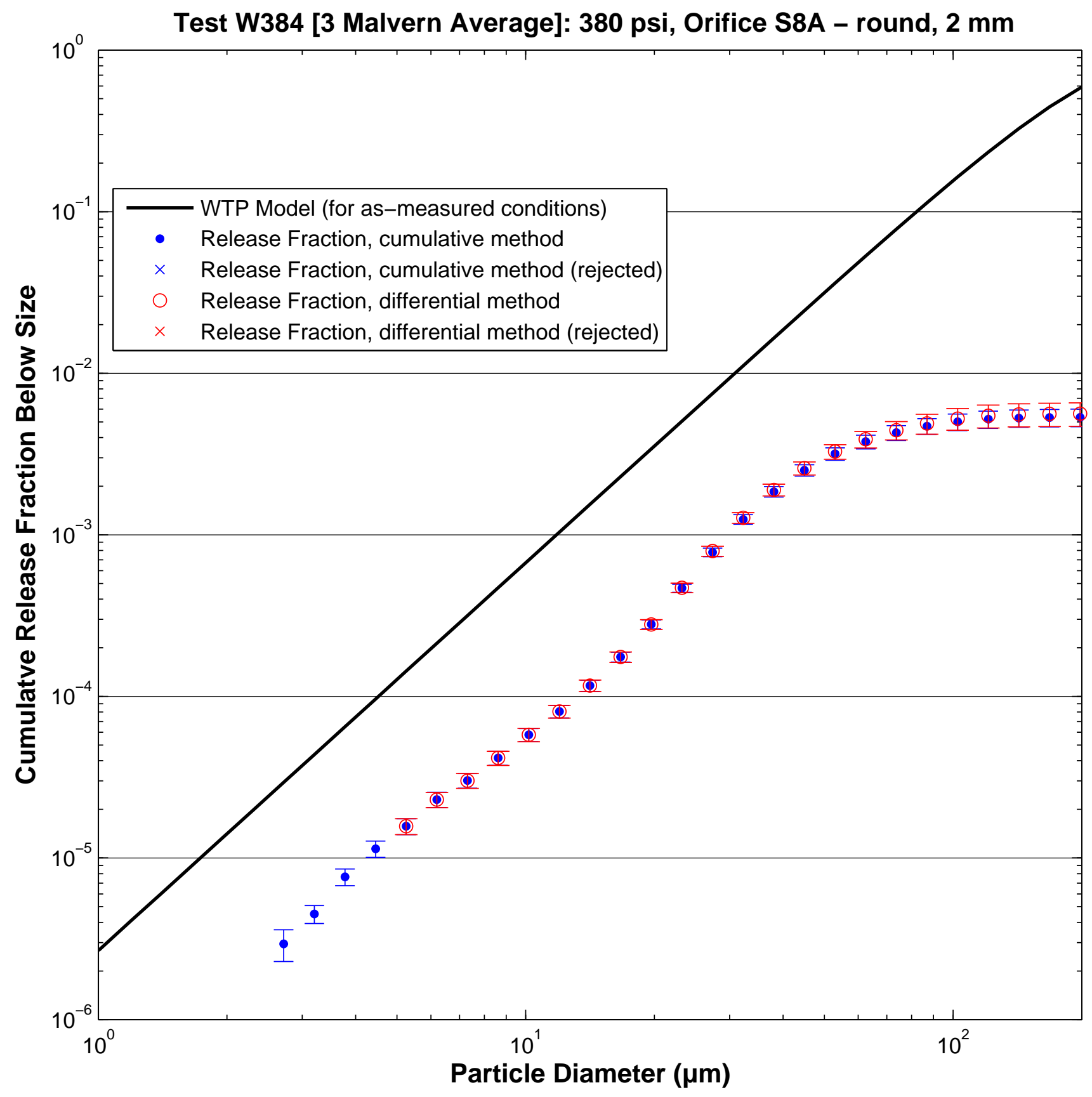




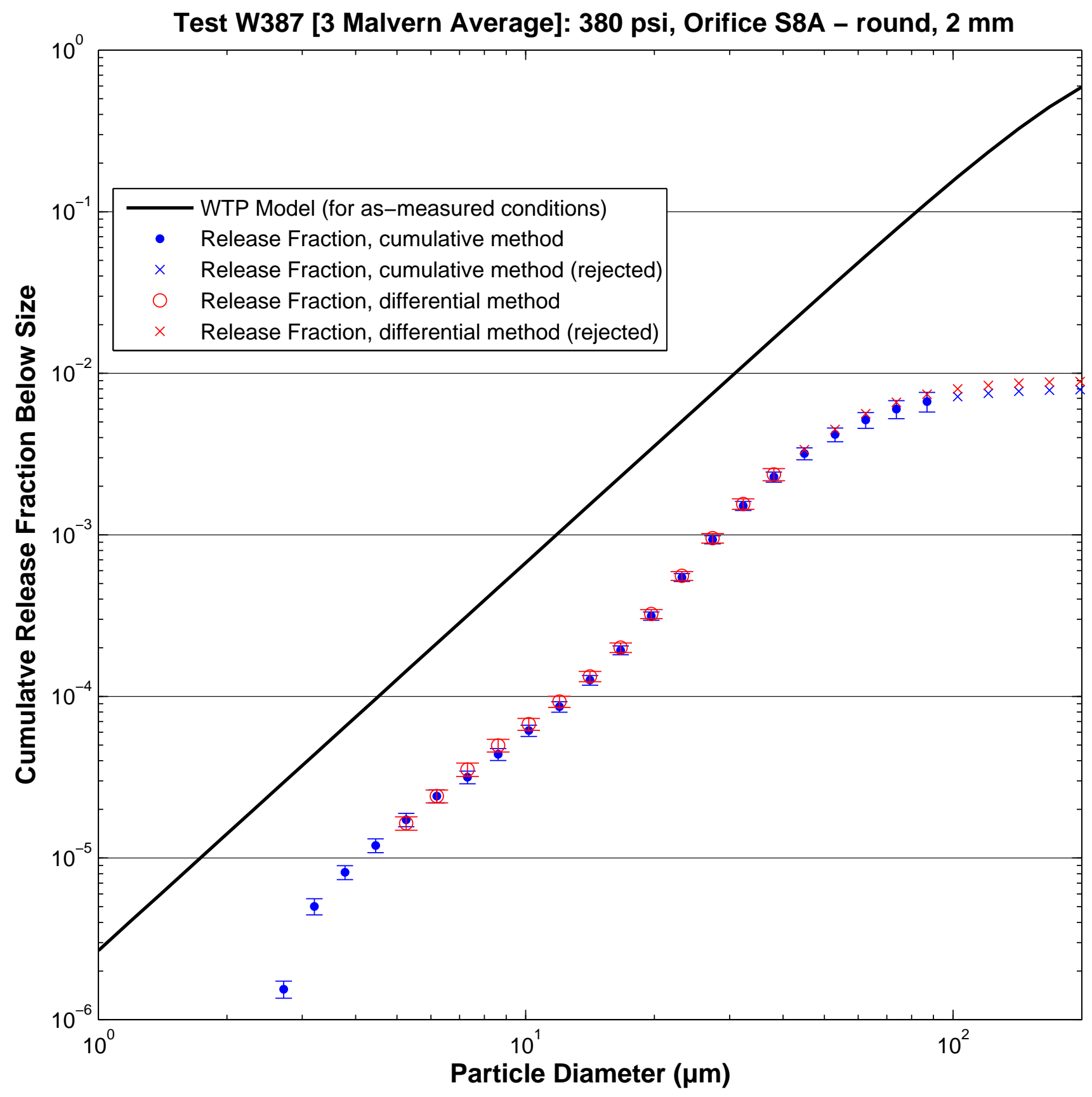




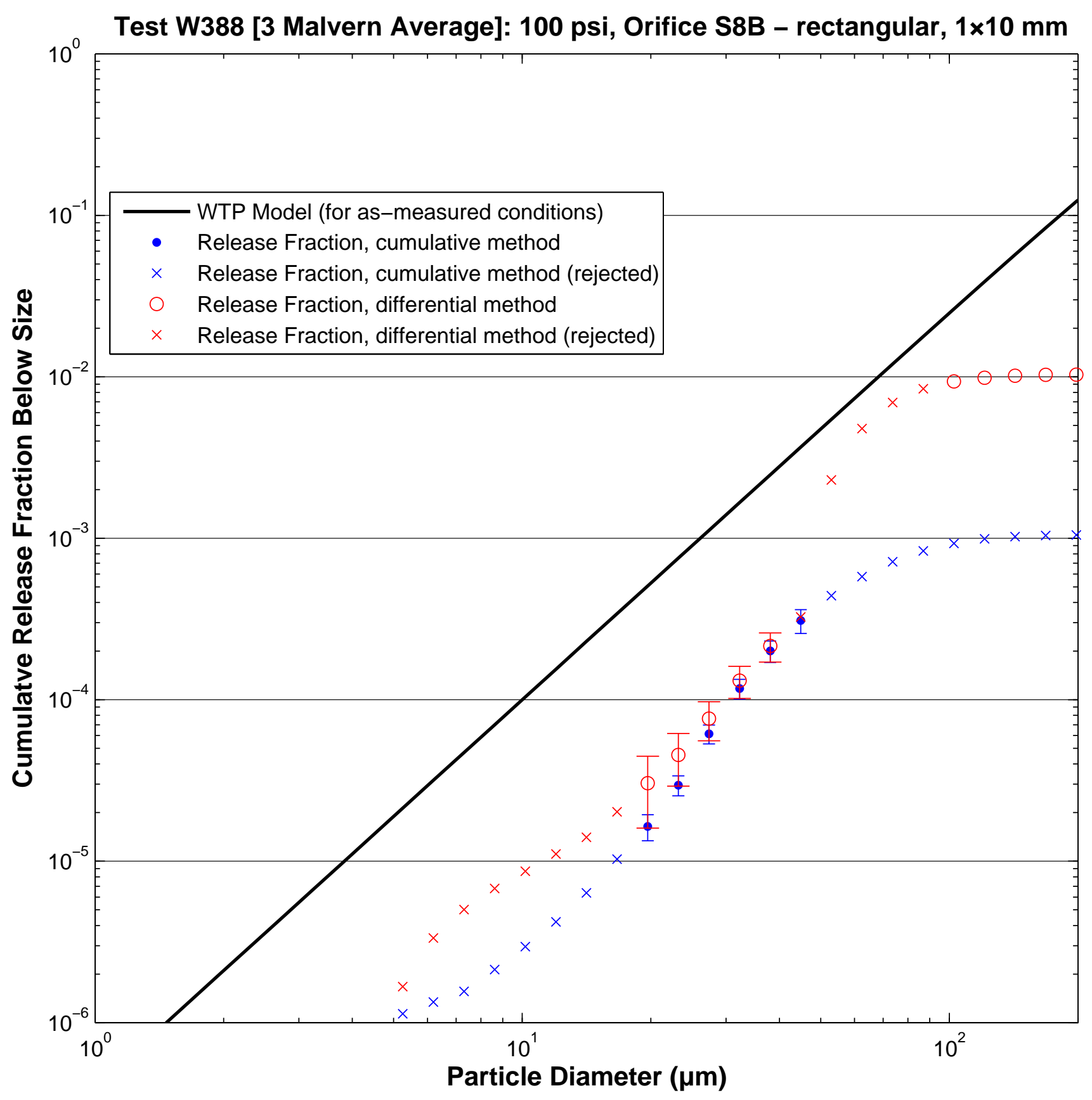




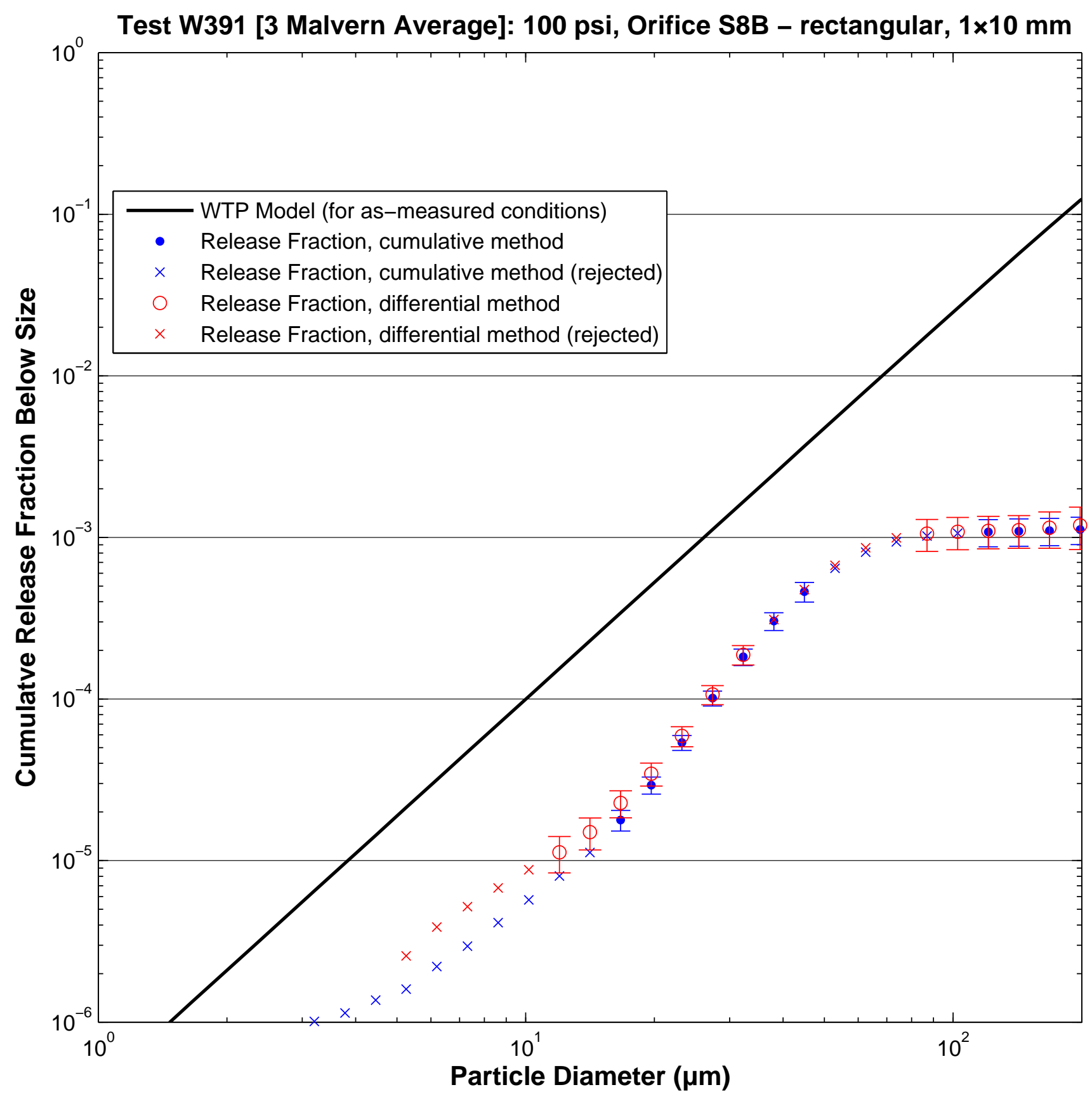




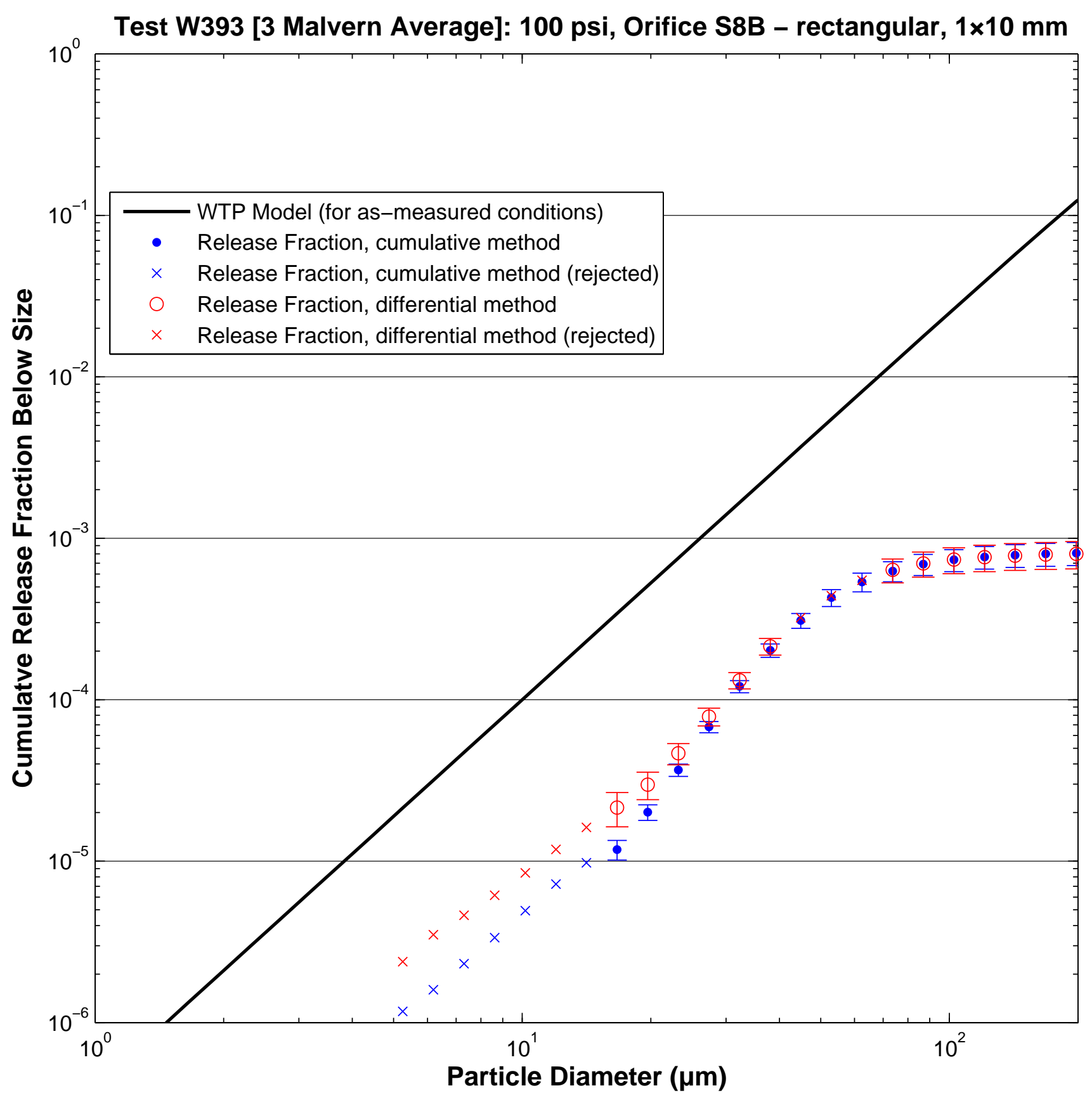




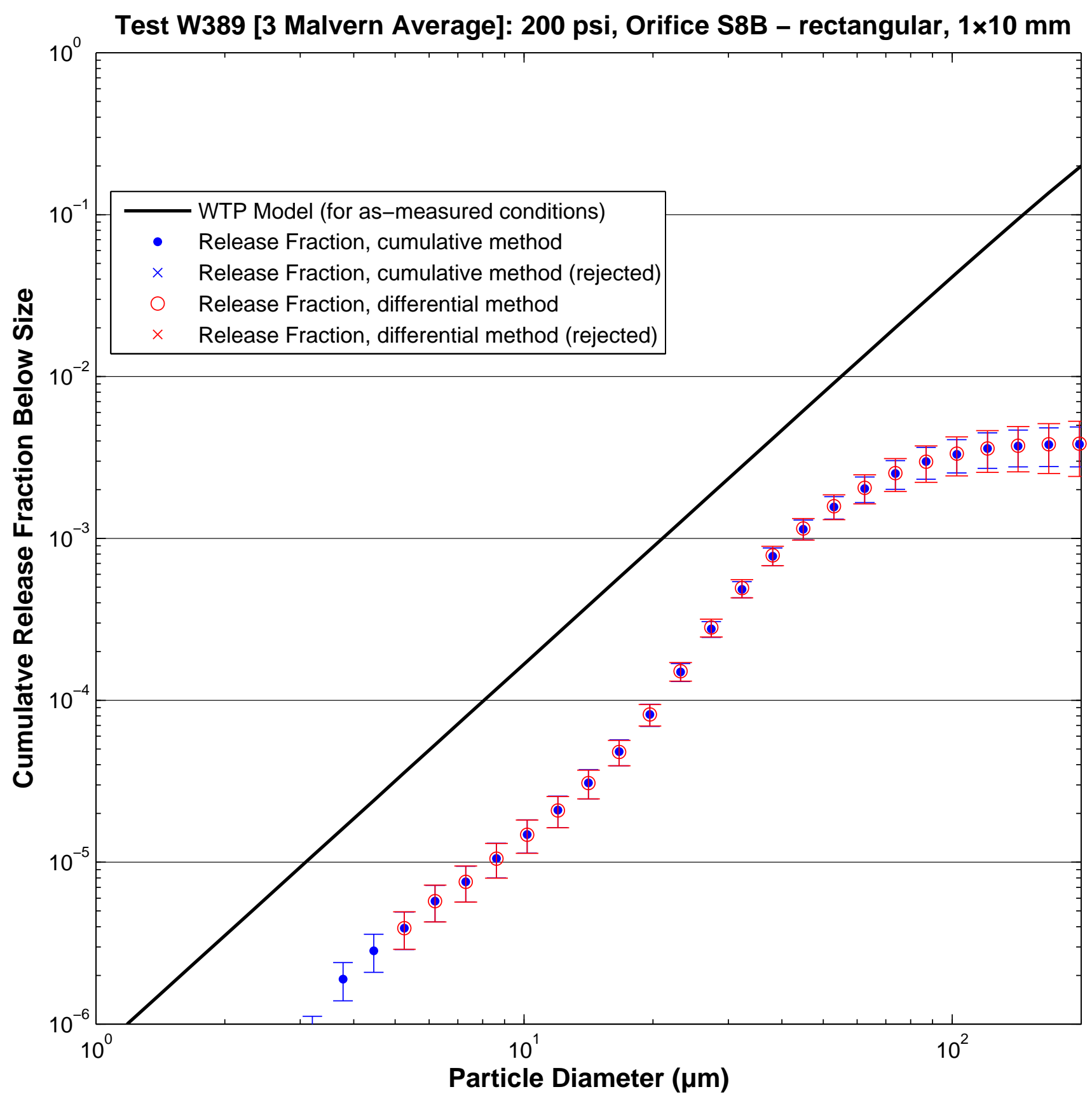




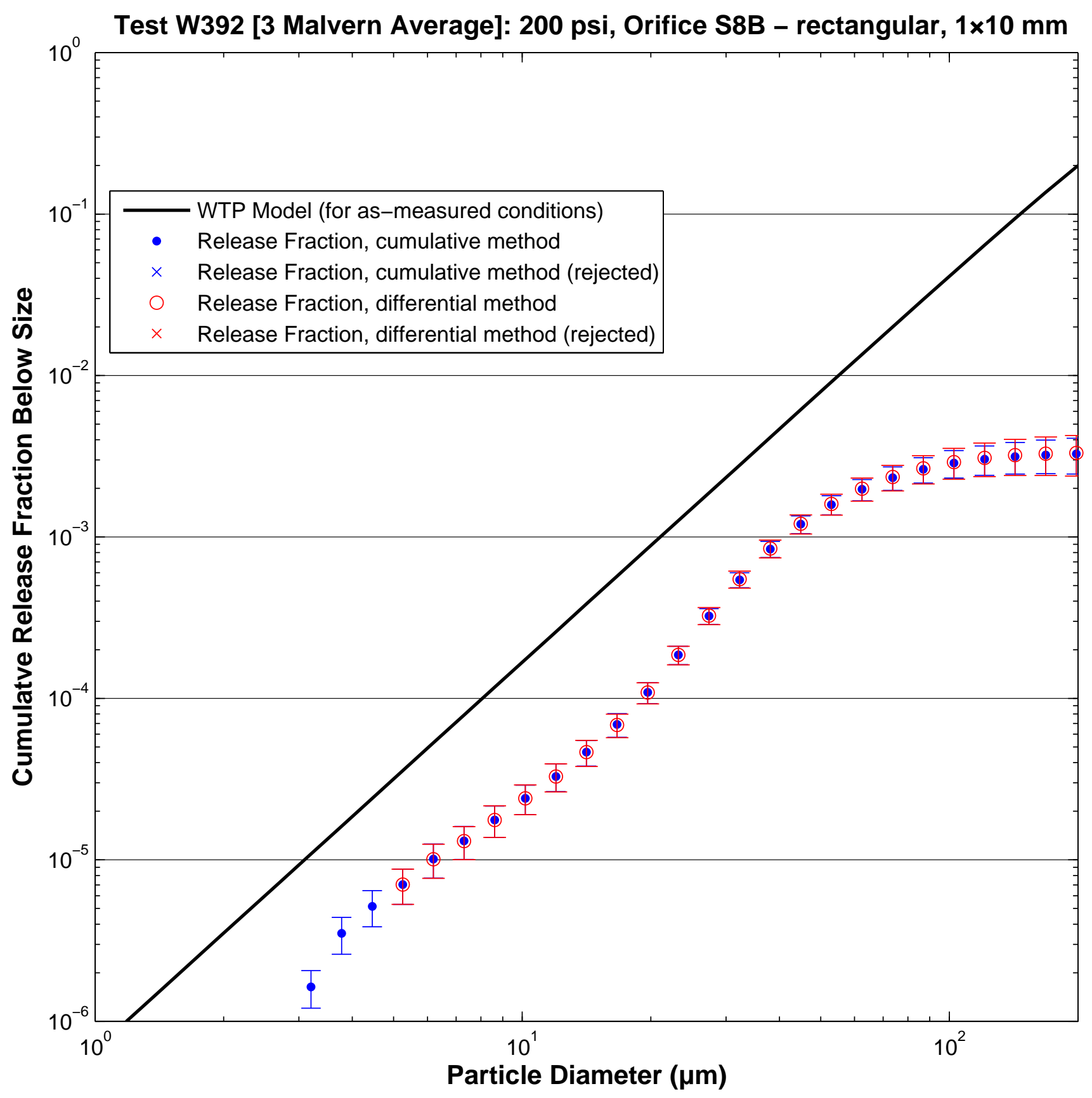




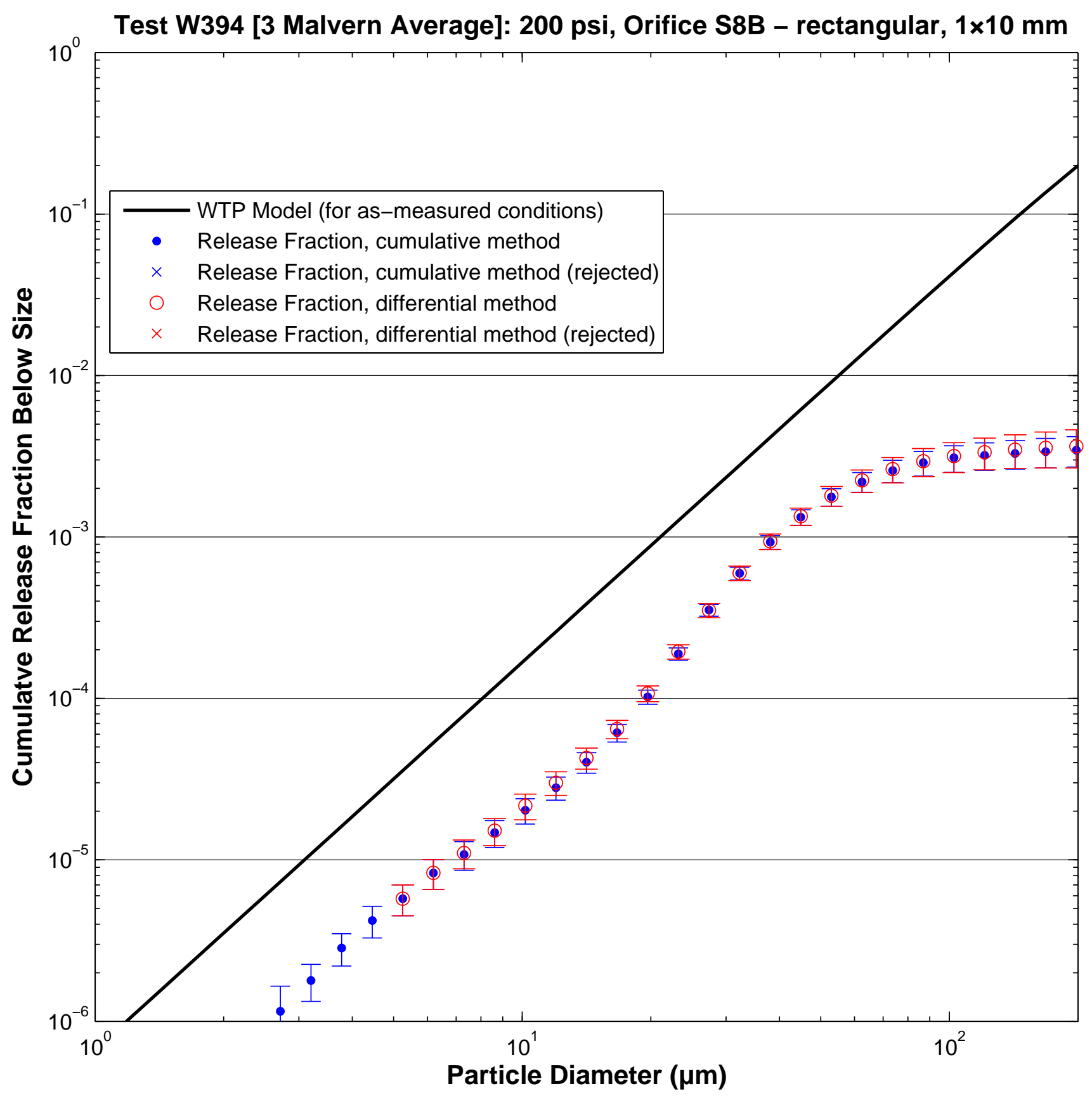




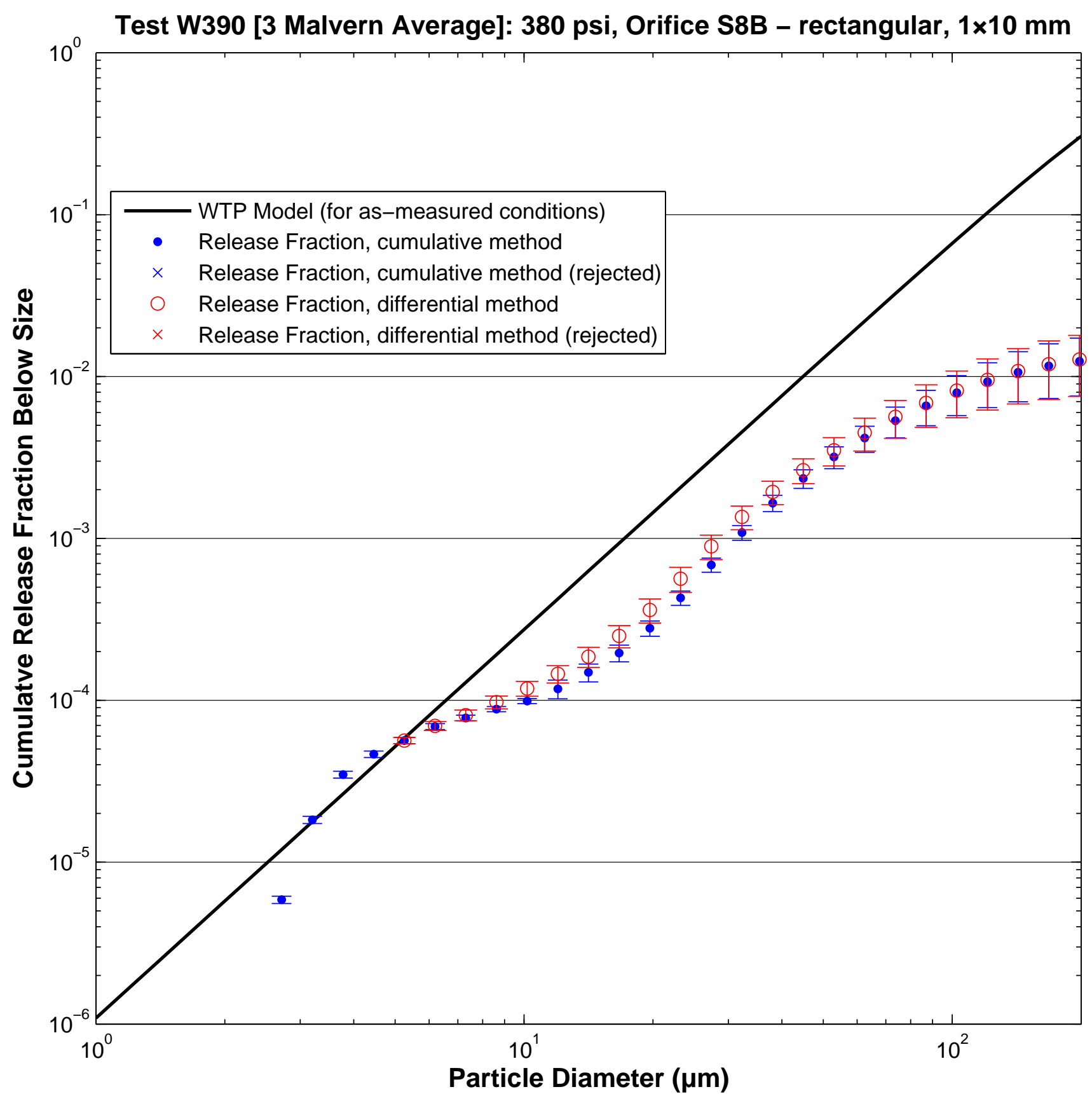




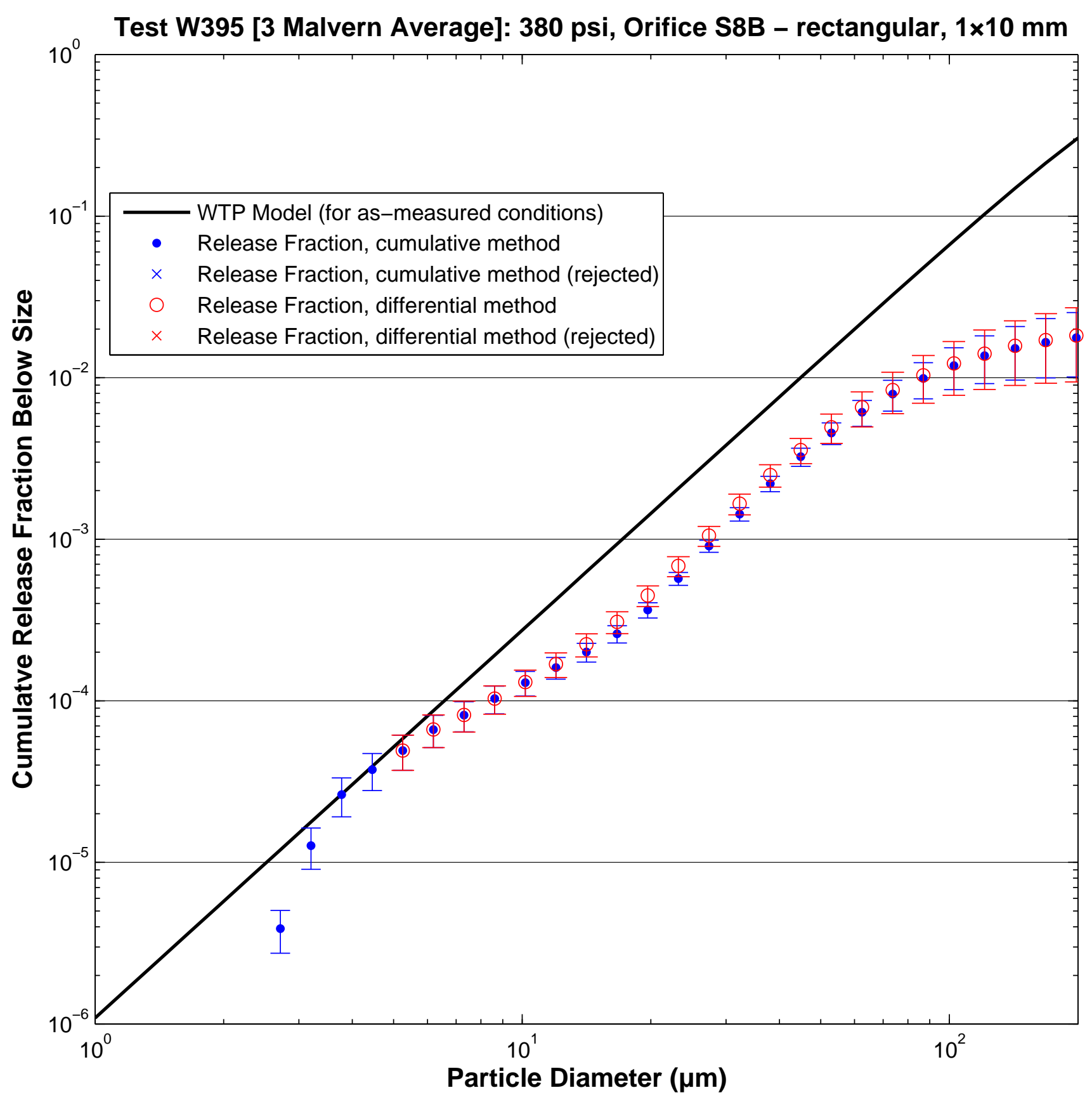




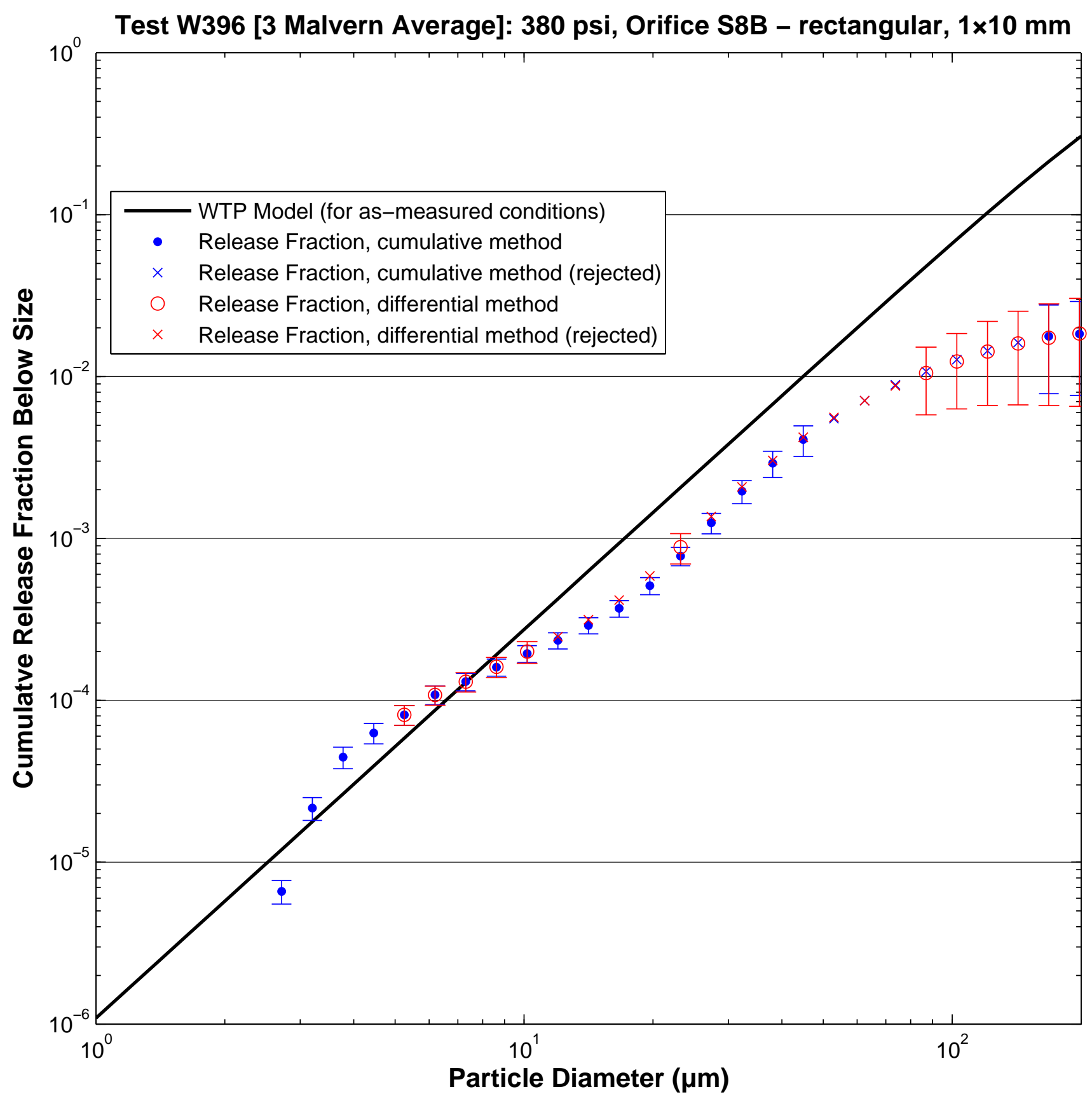




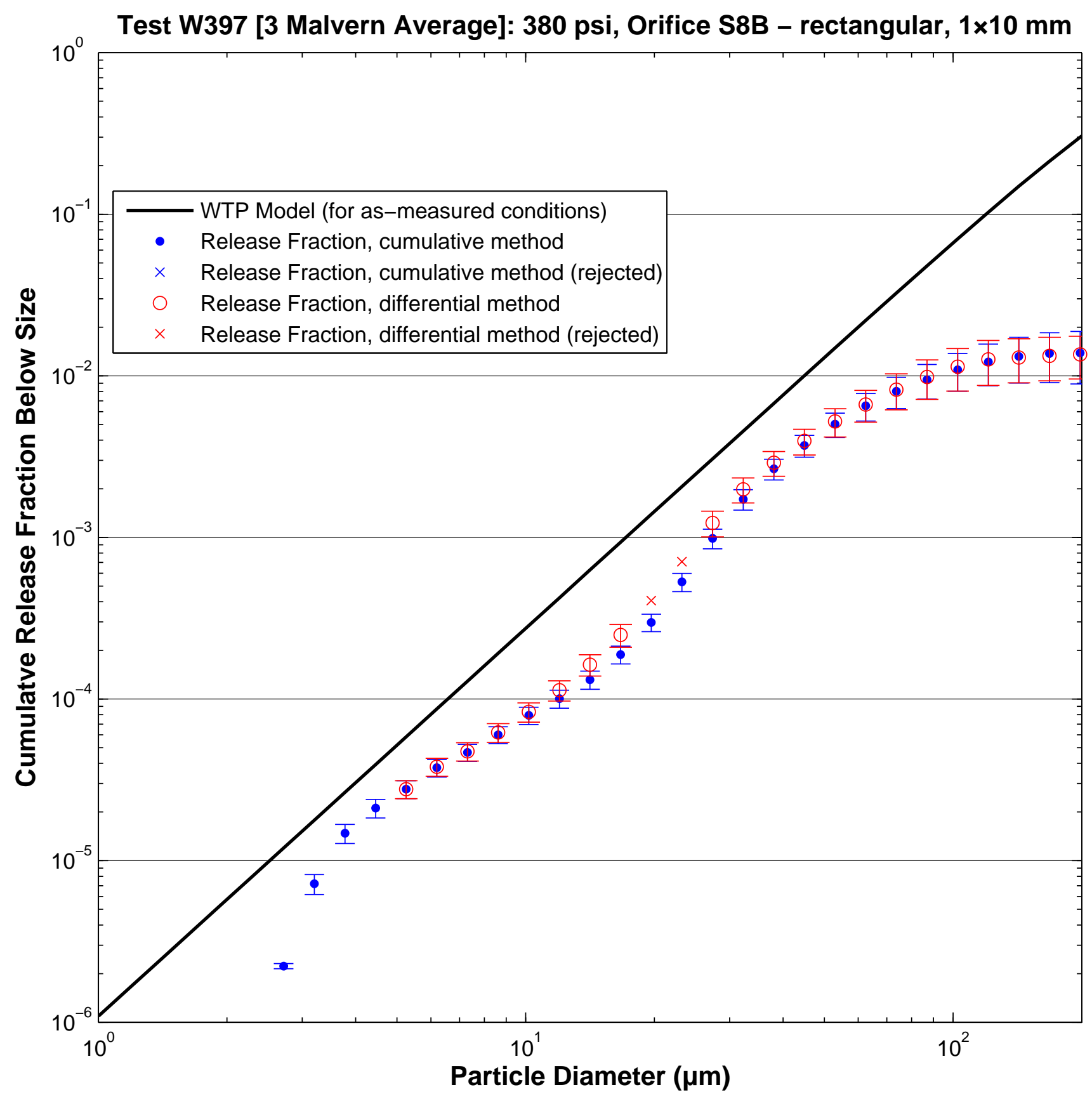




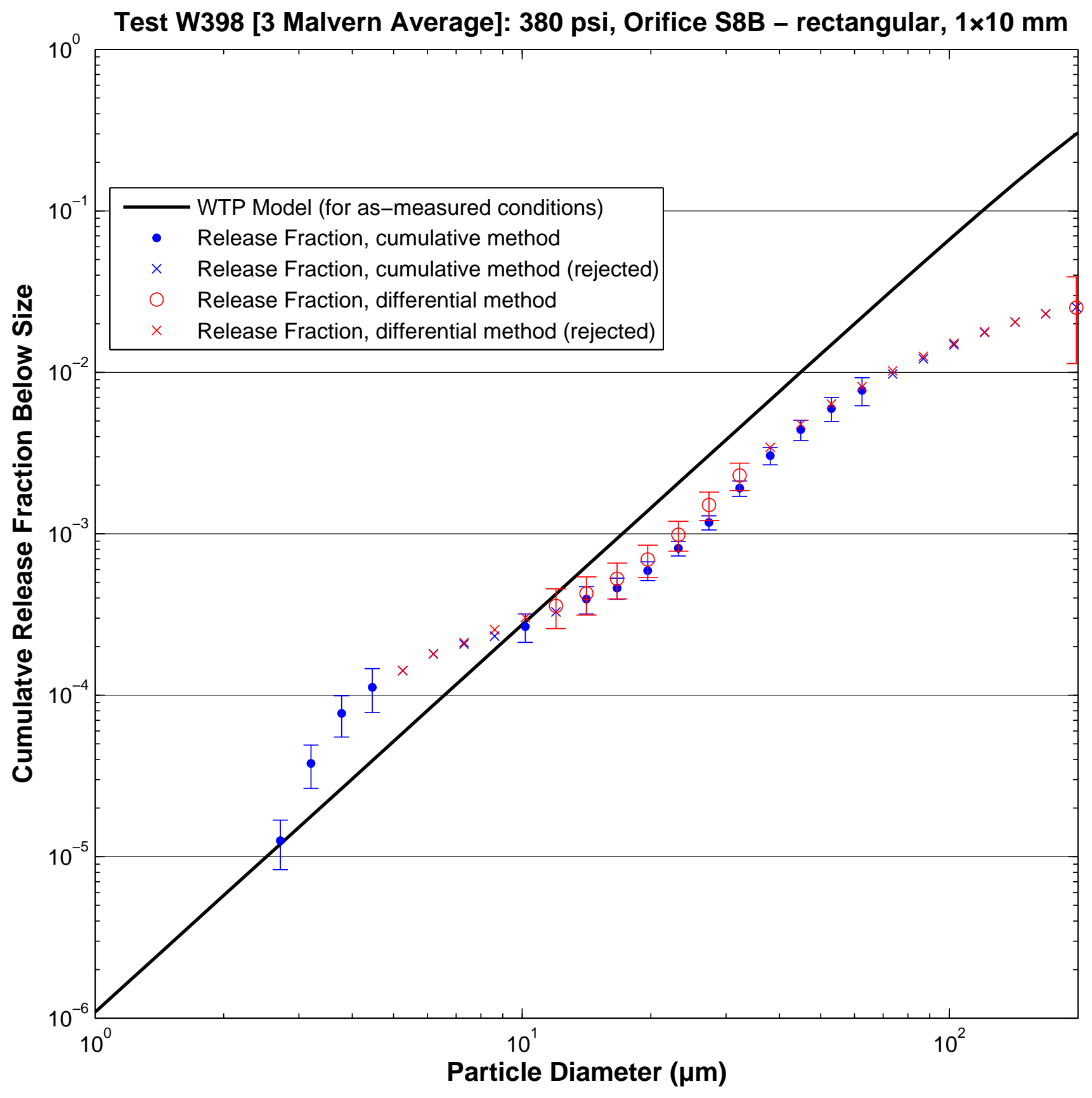




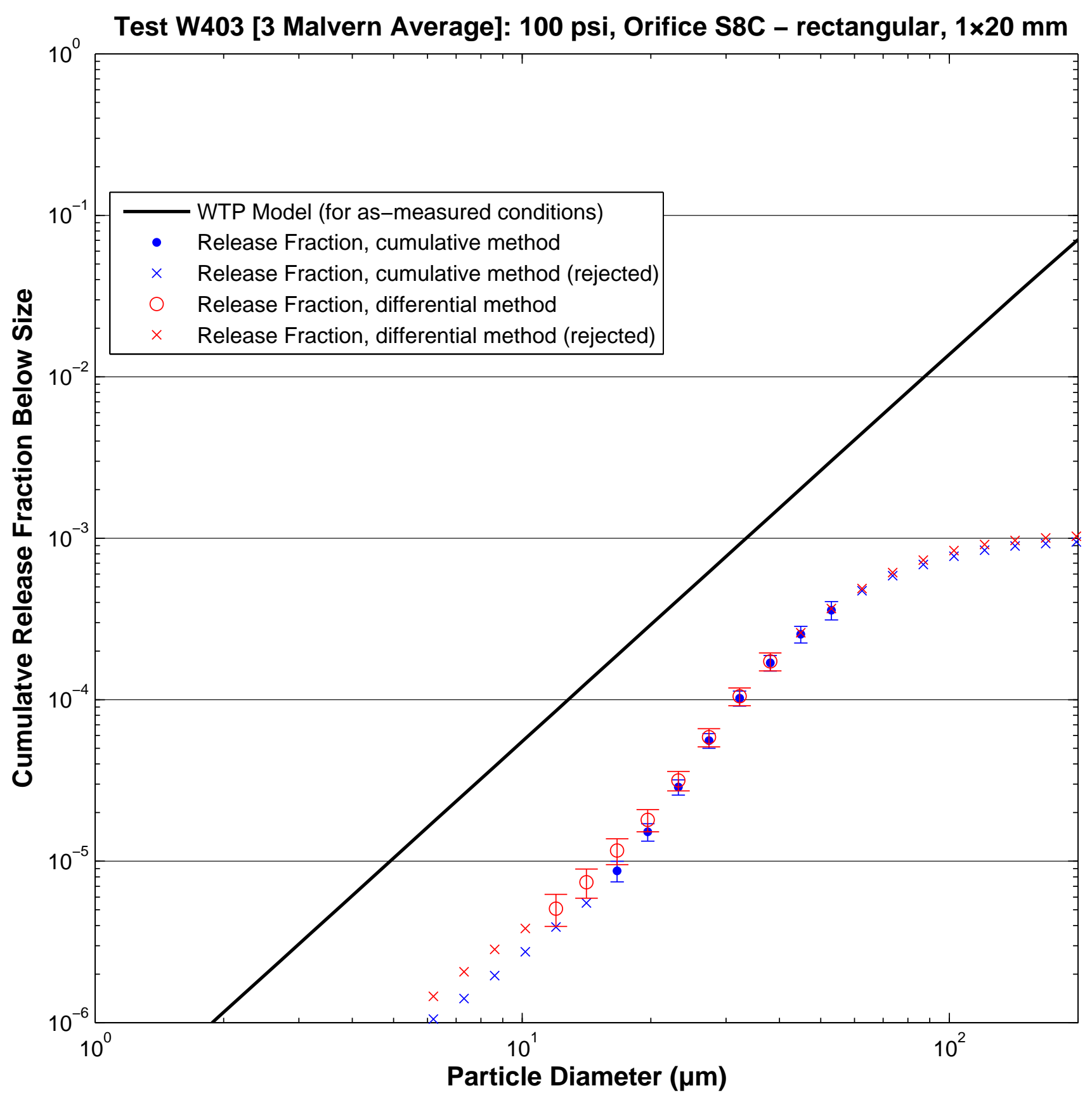




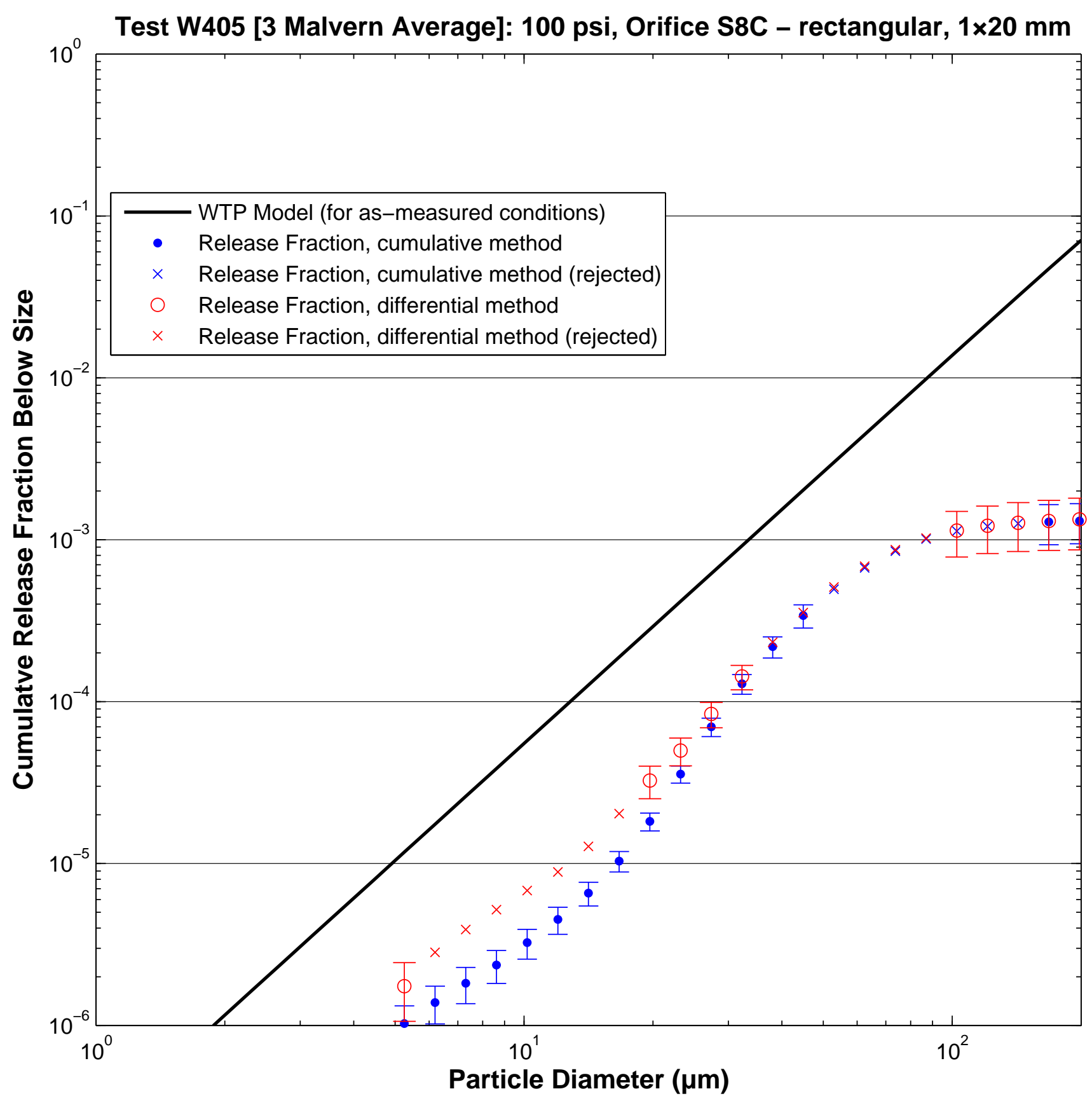




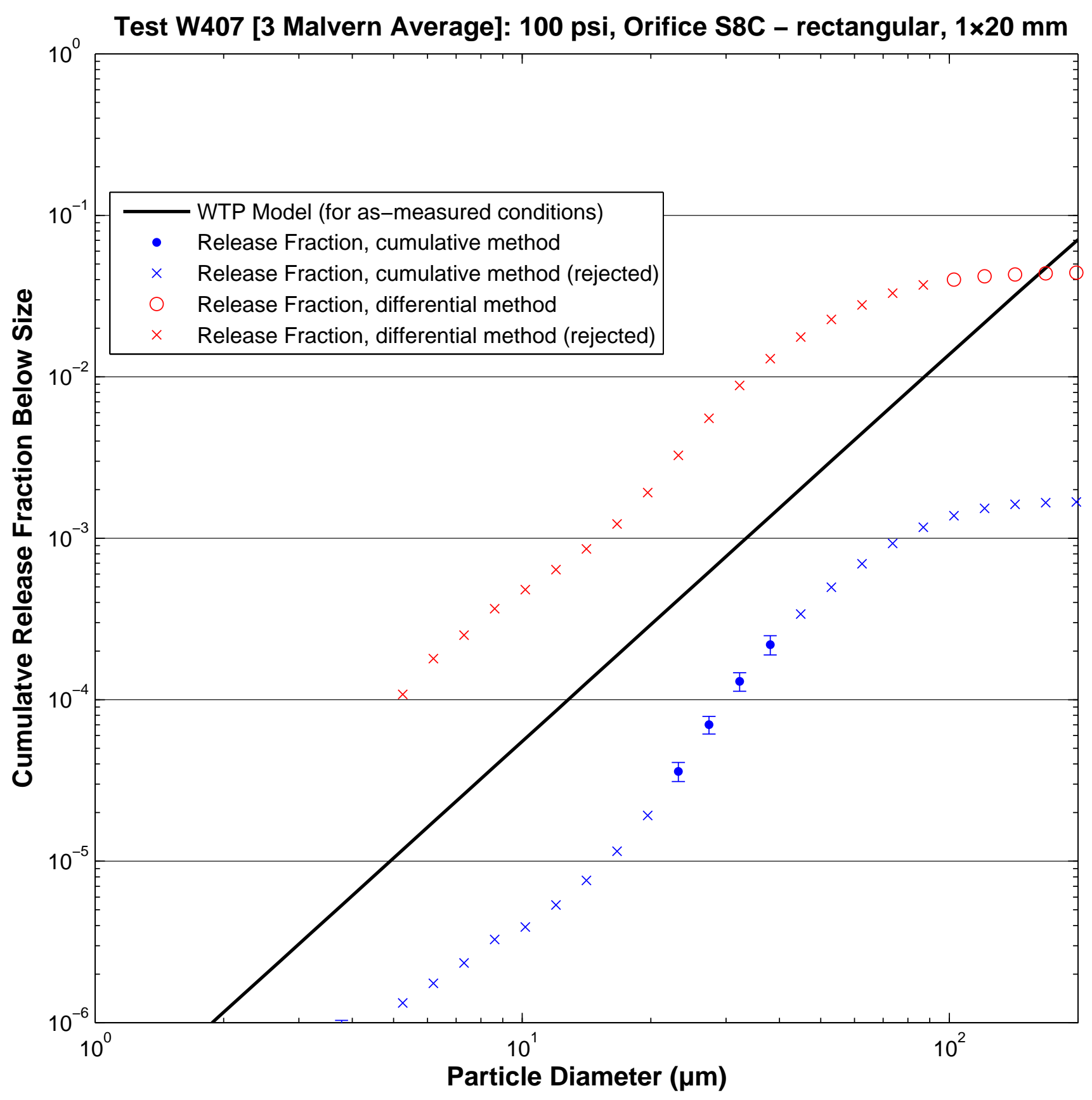




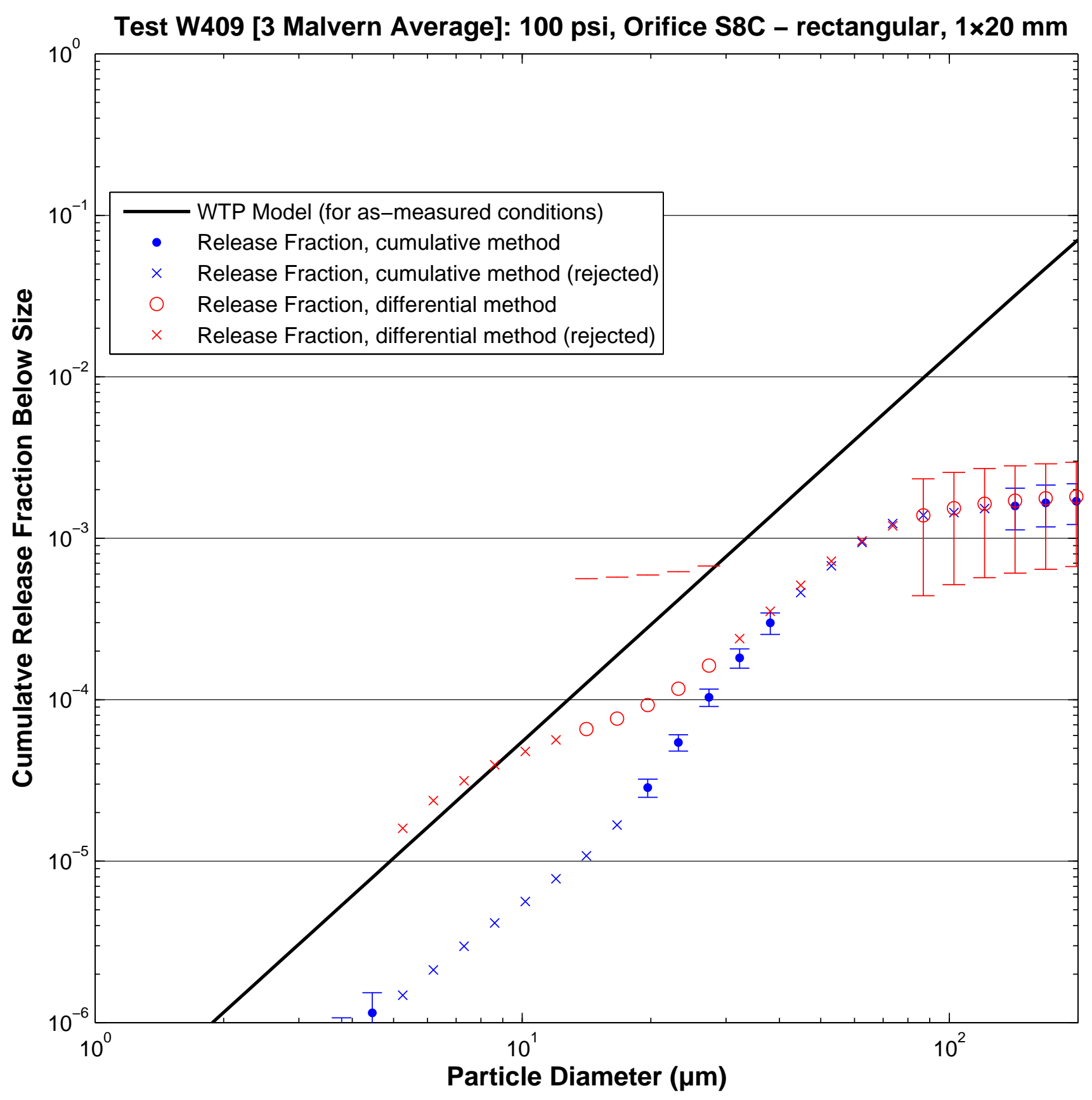




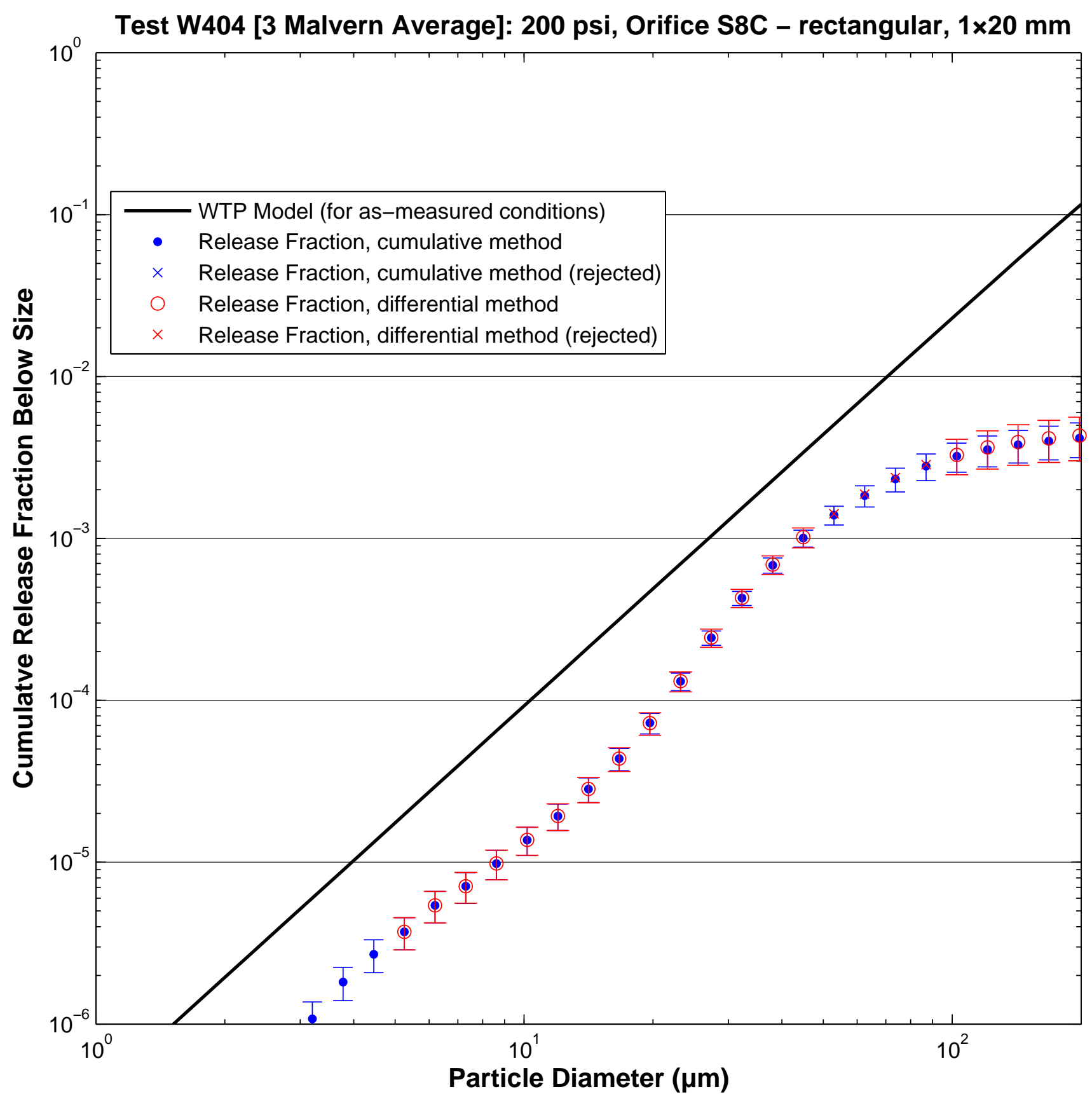




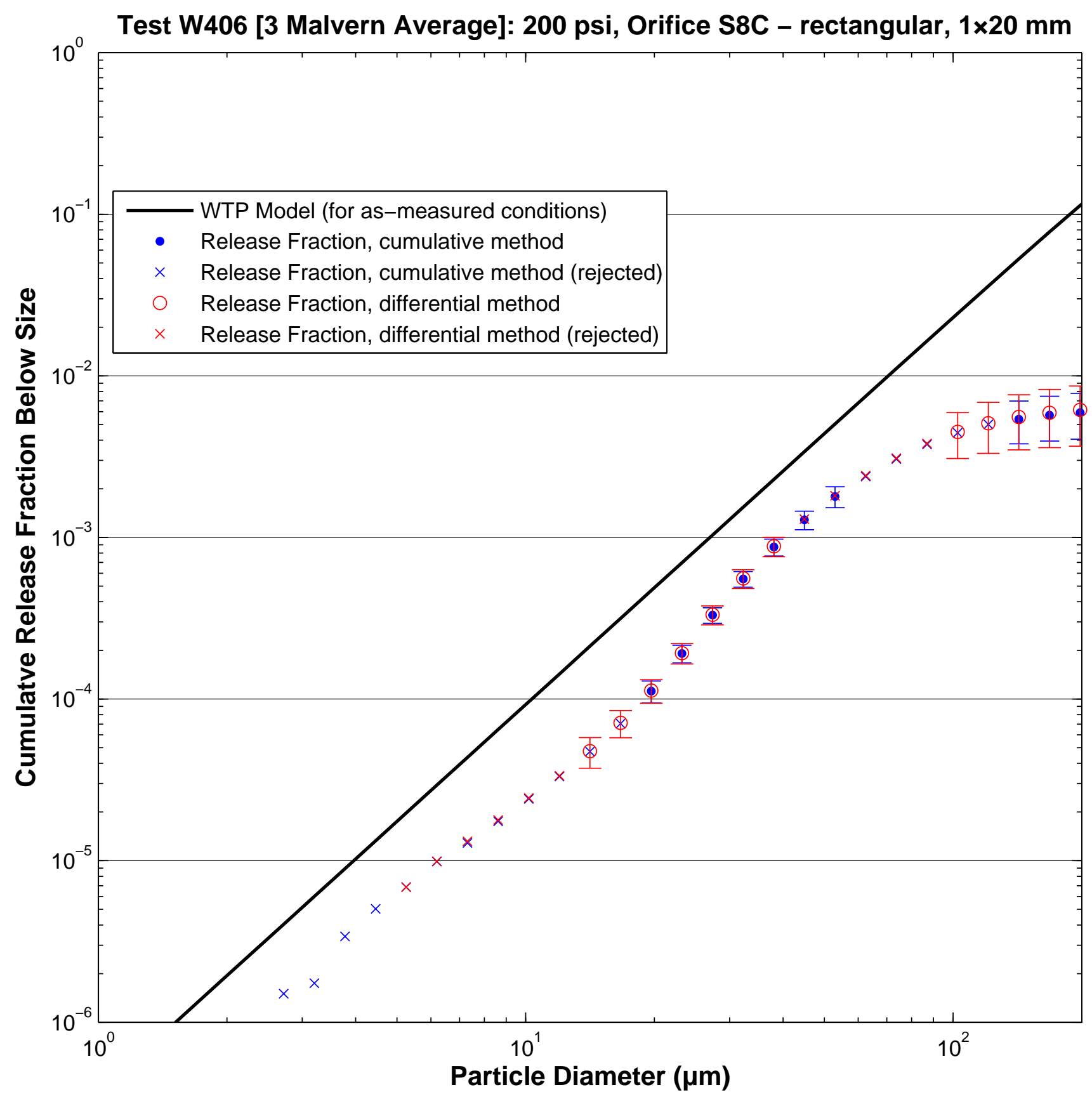




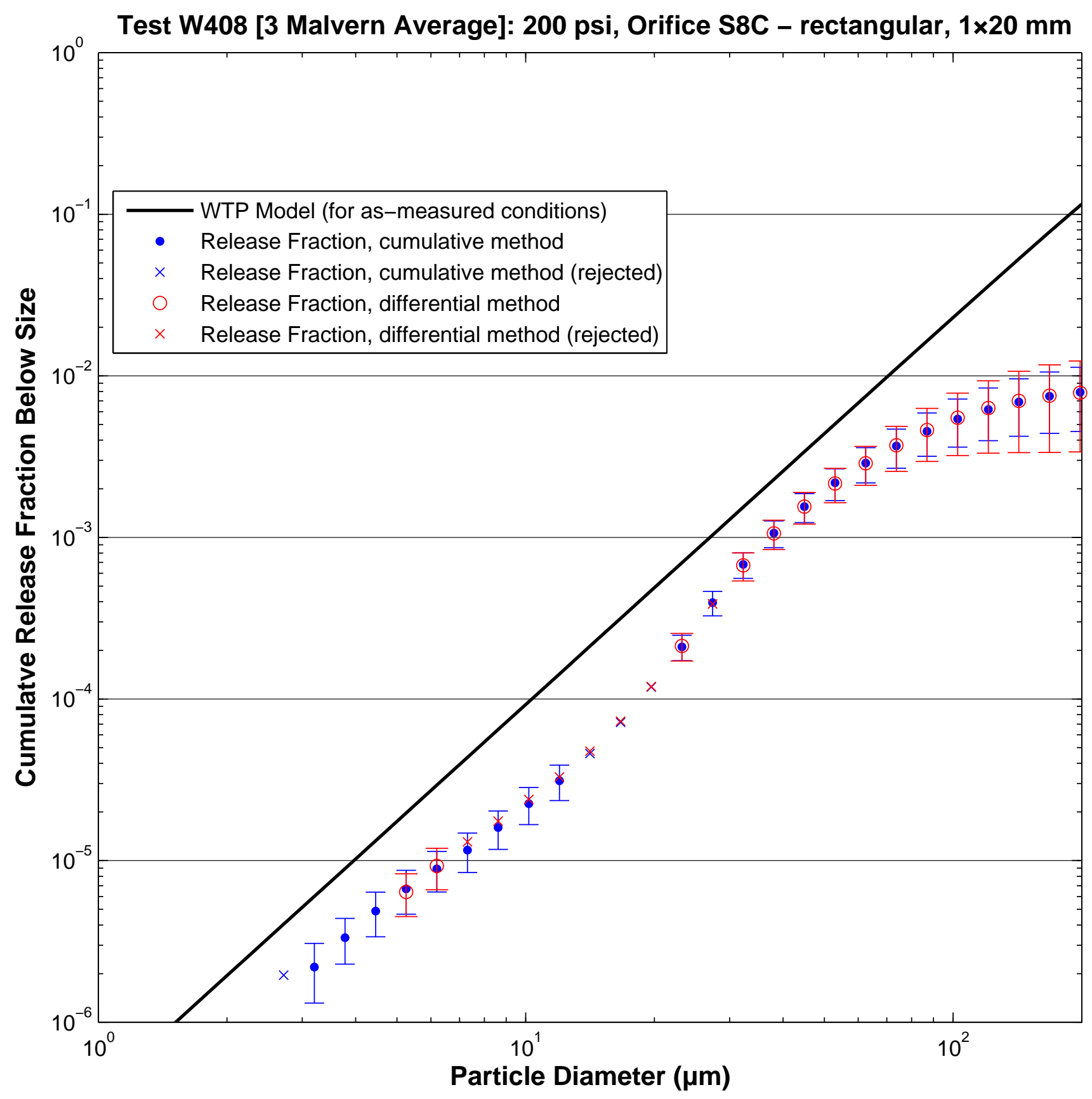




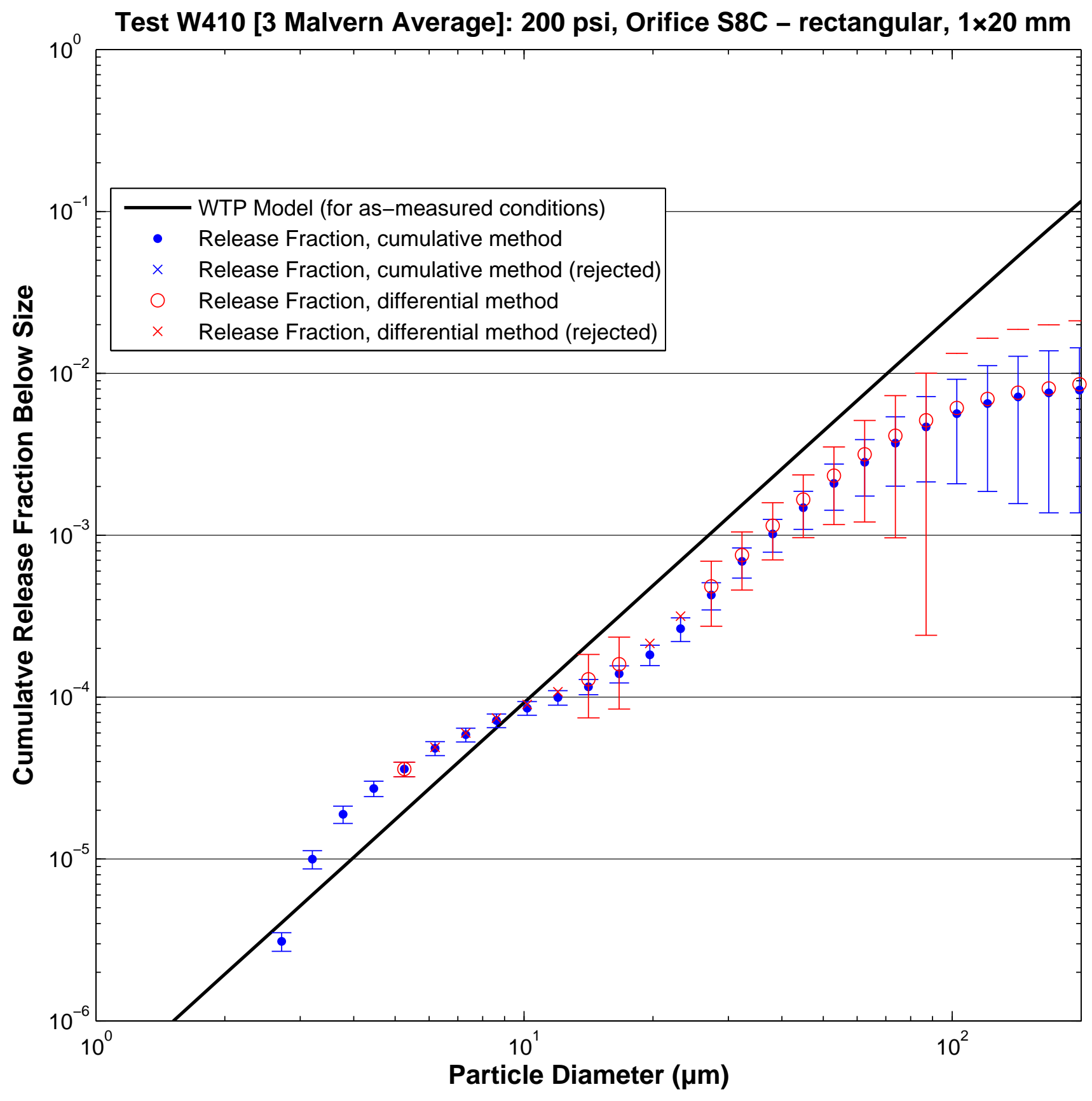




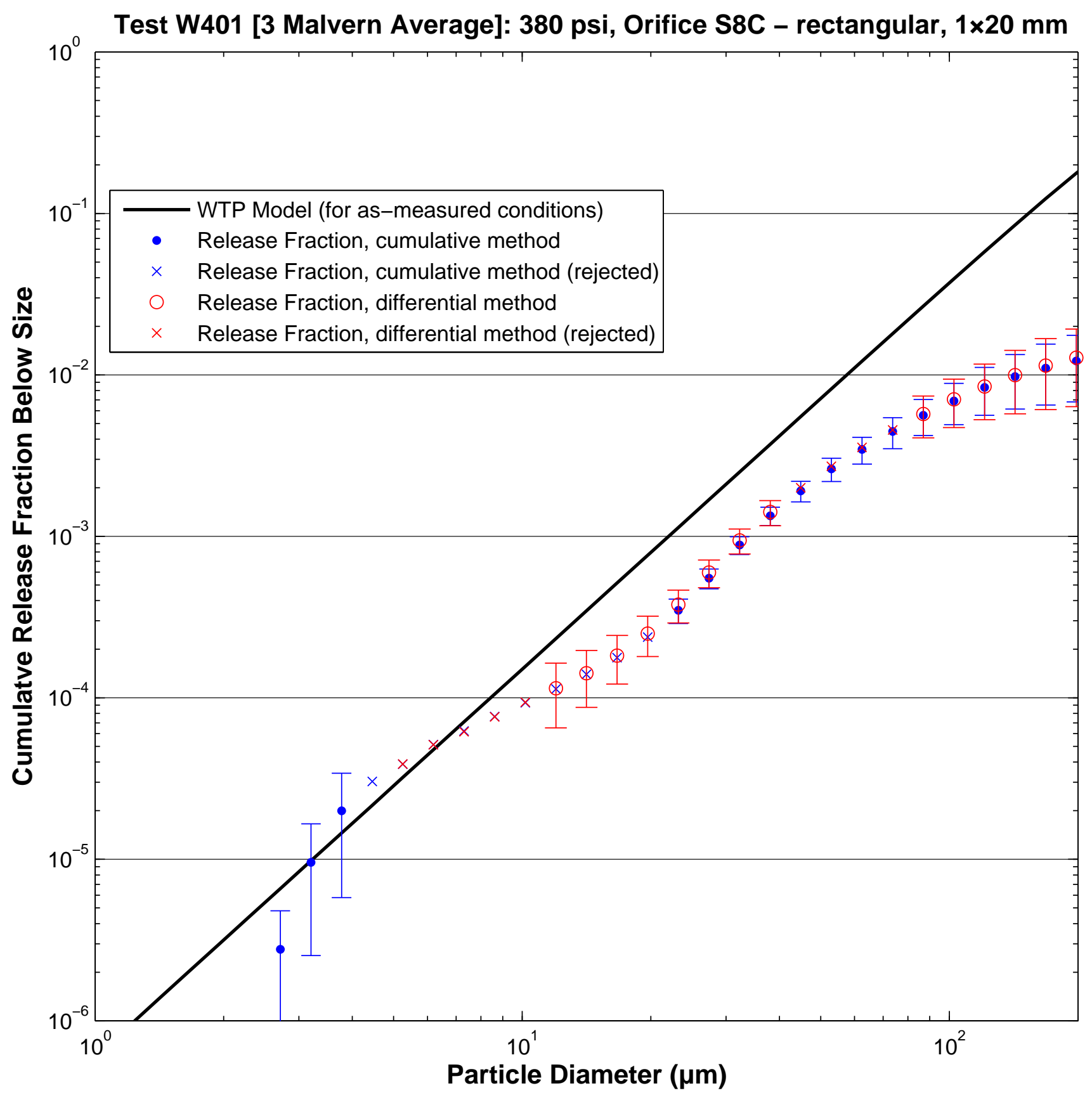




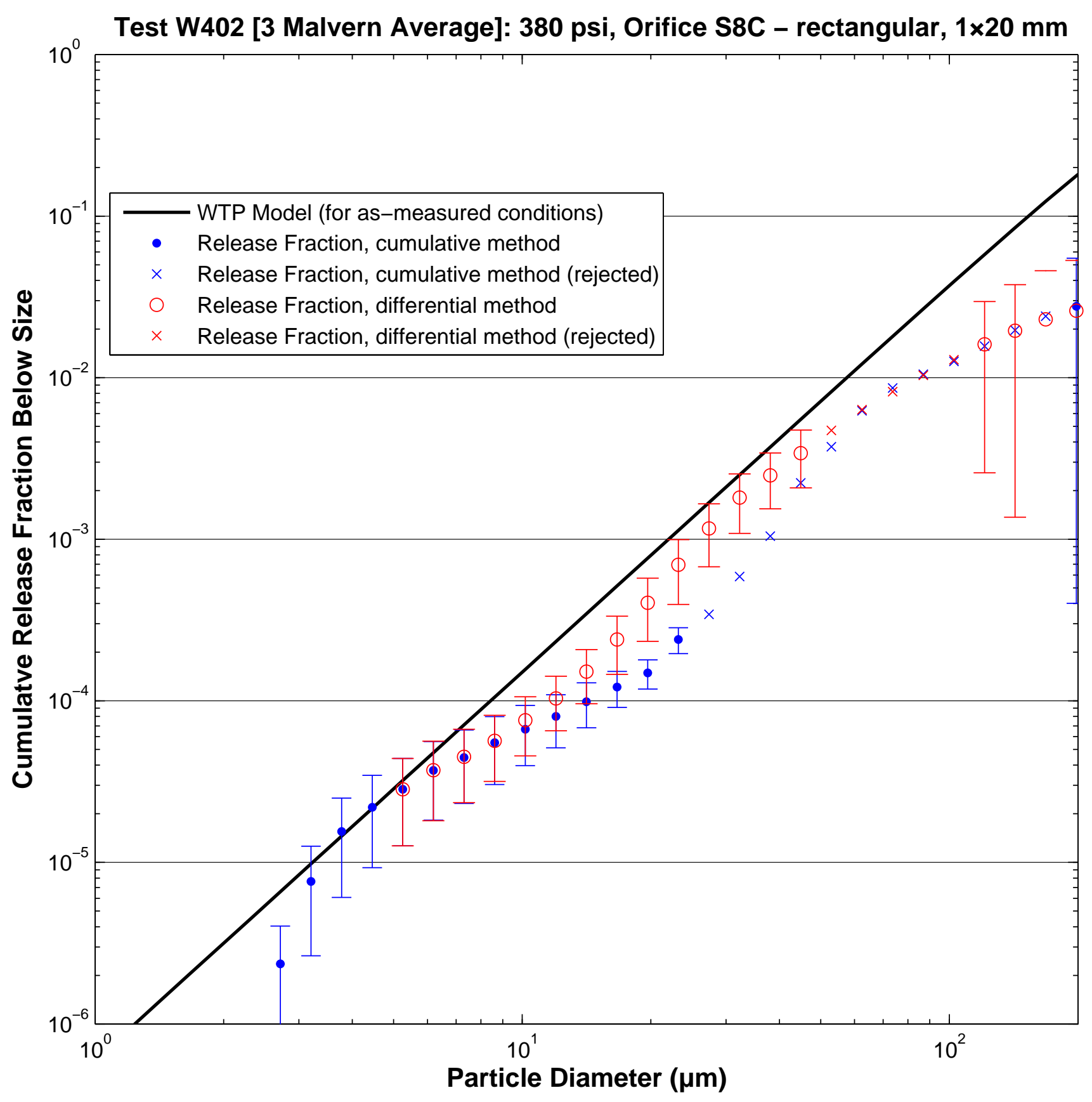




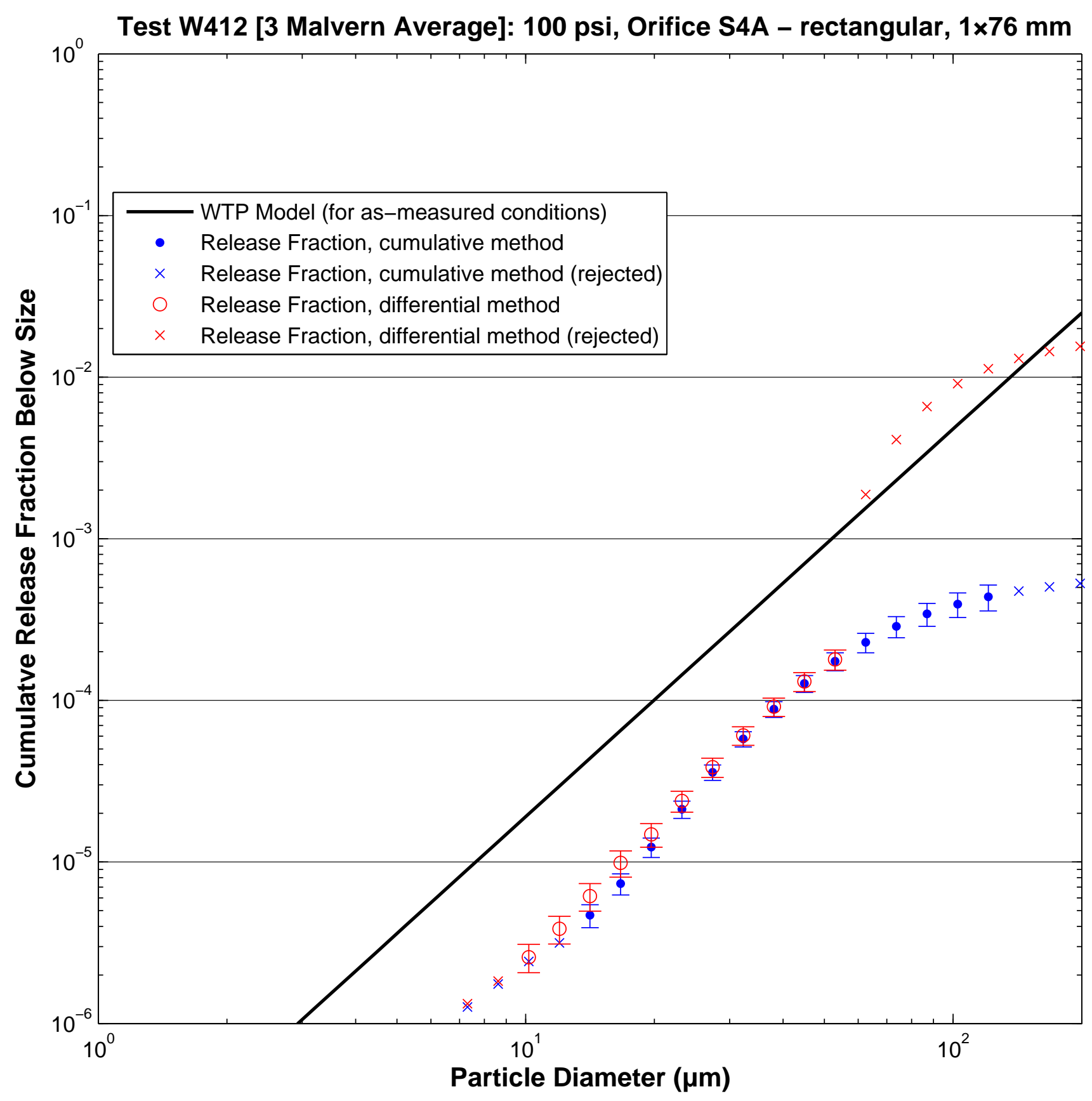




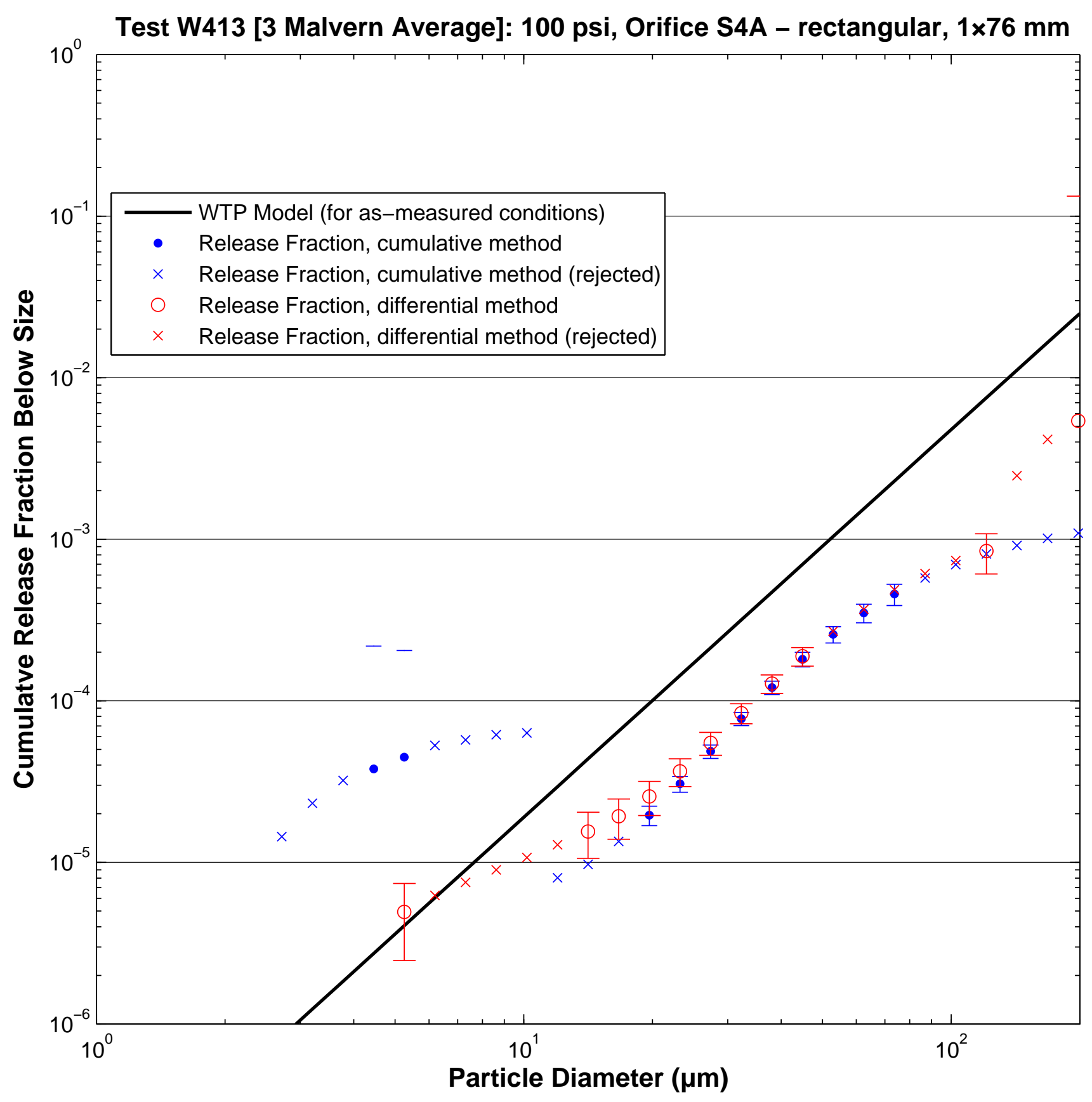




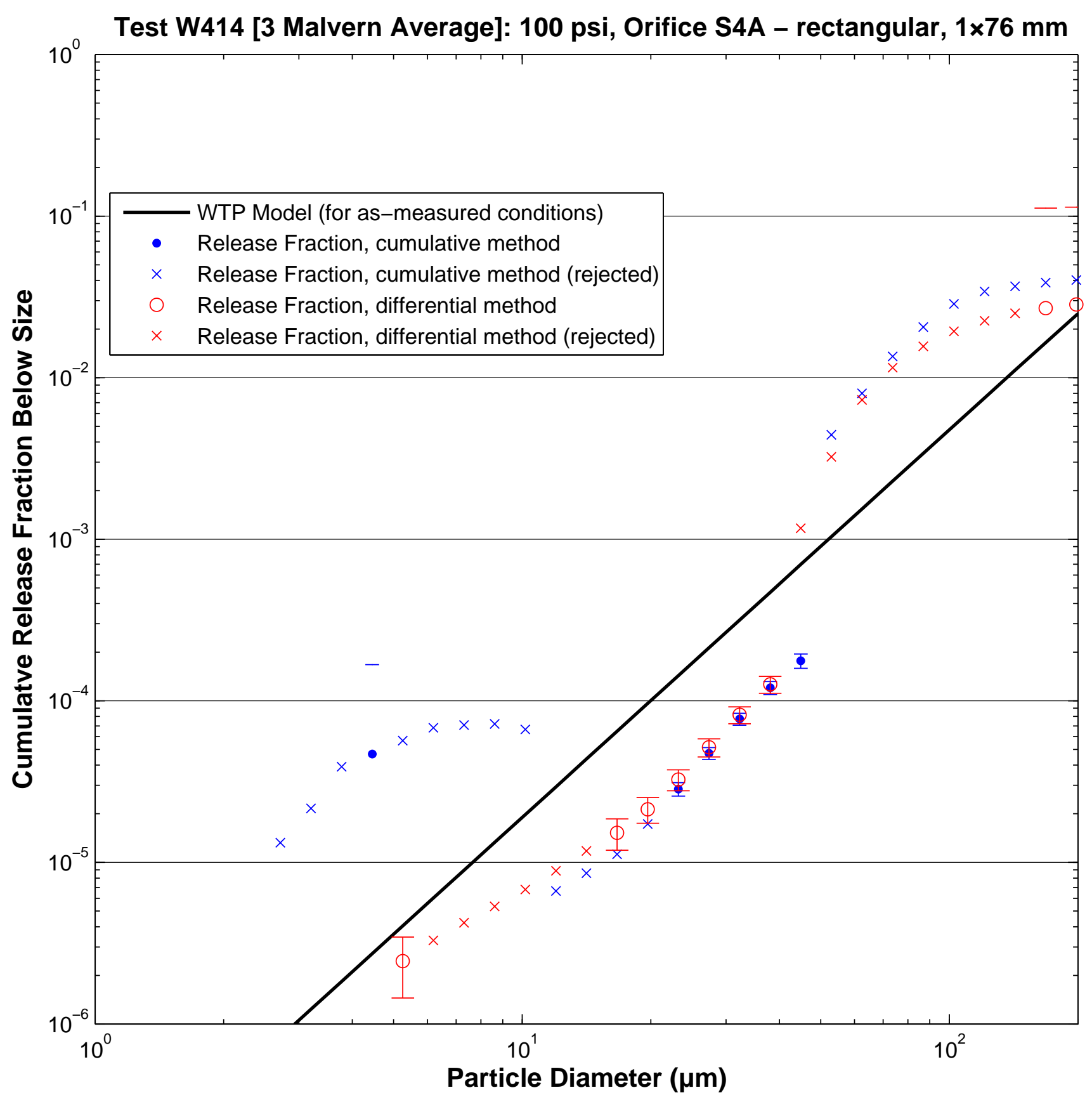




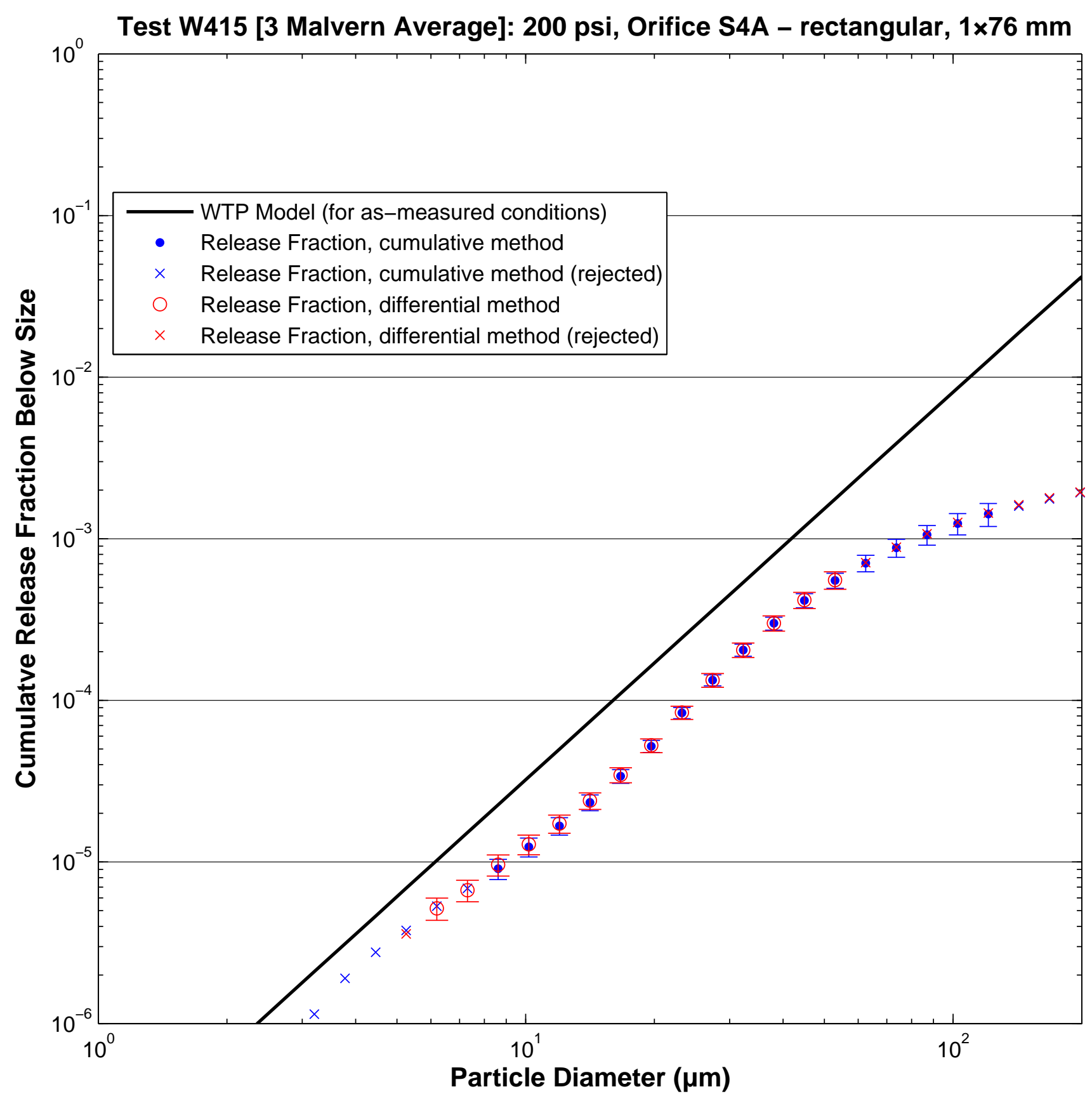




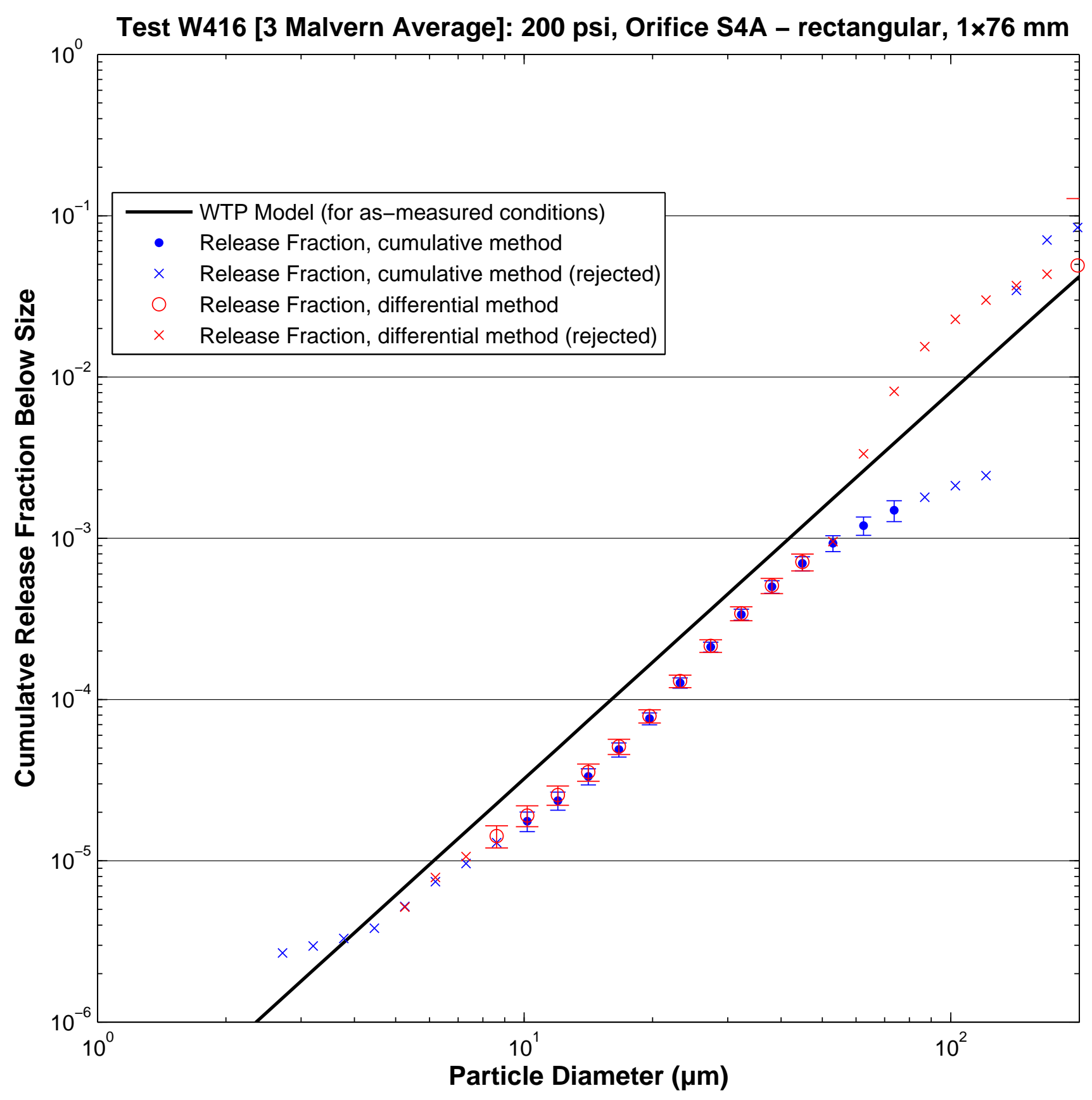

B. 100 


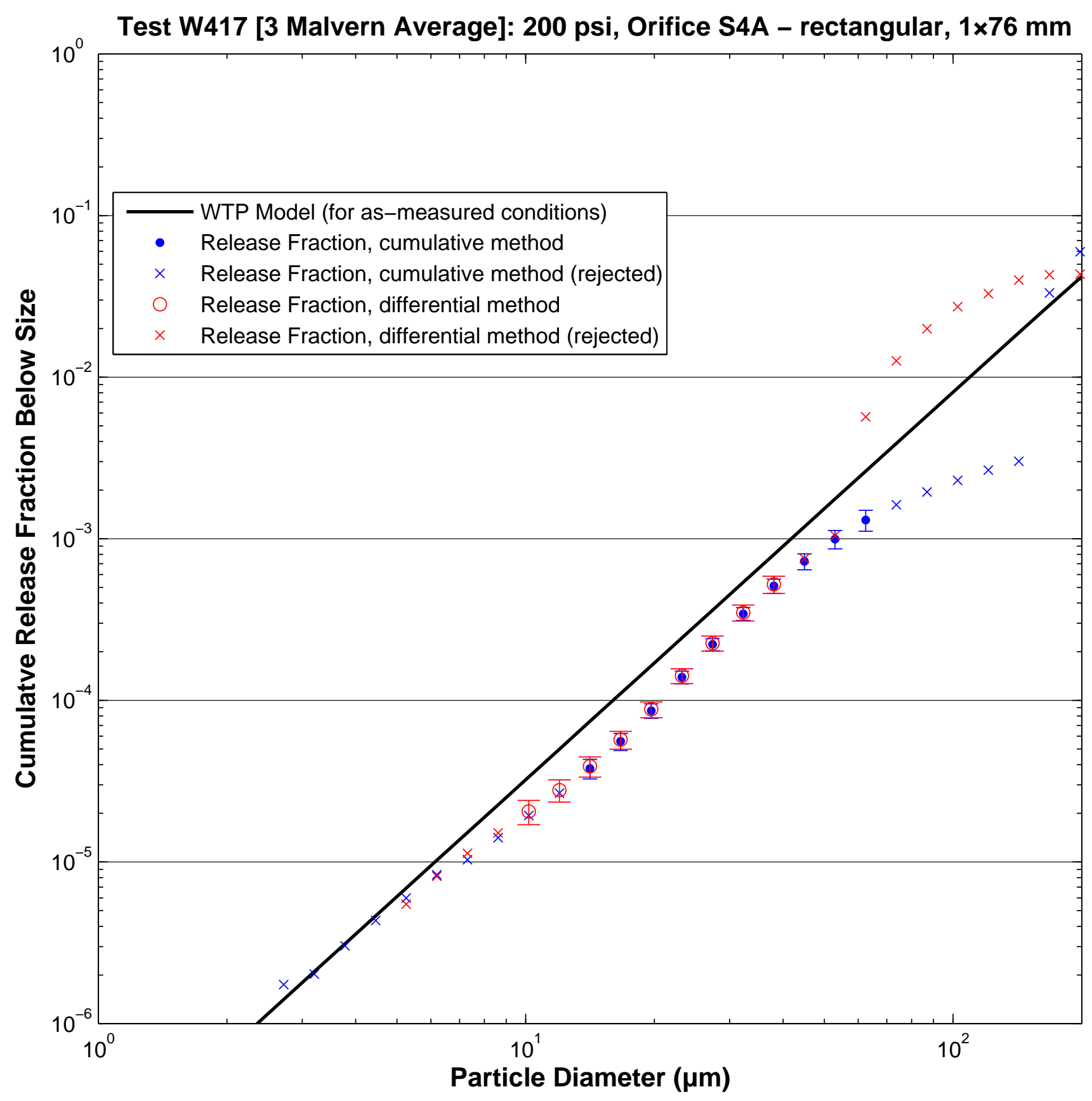




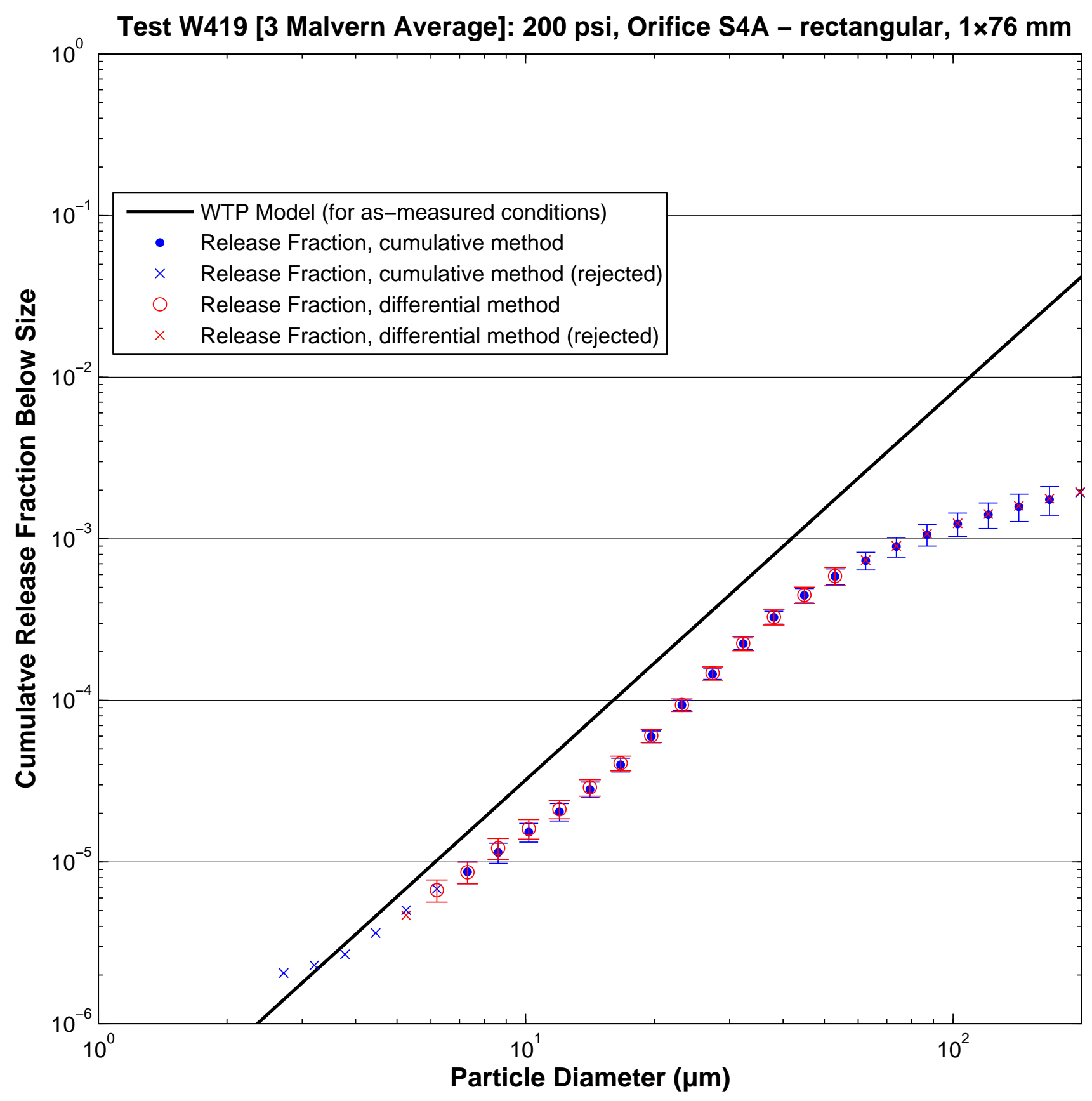

B. 102 


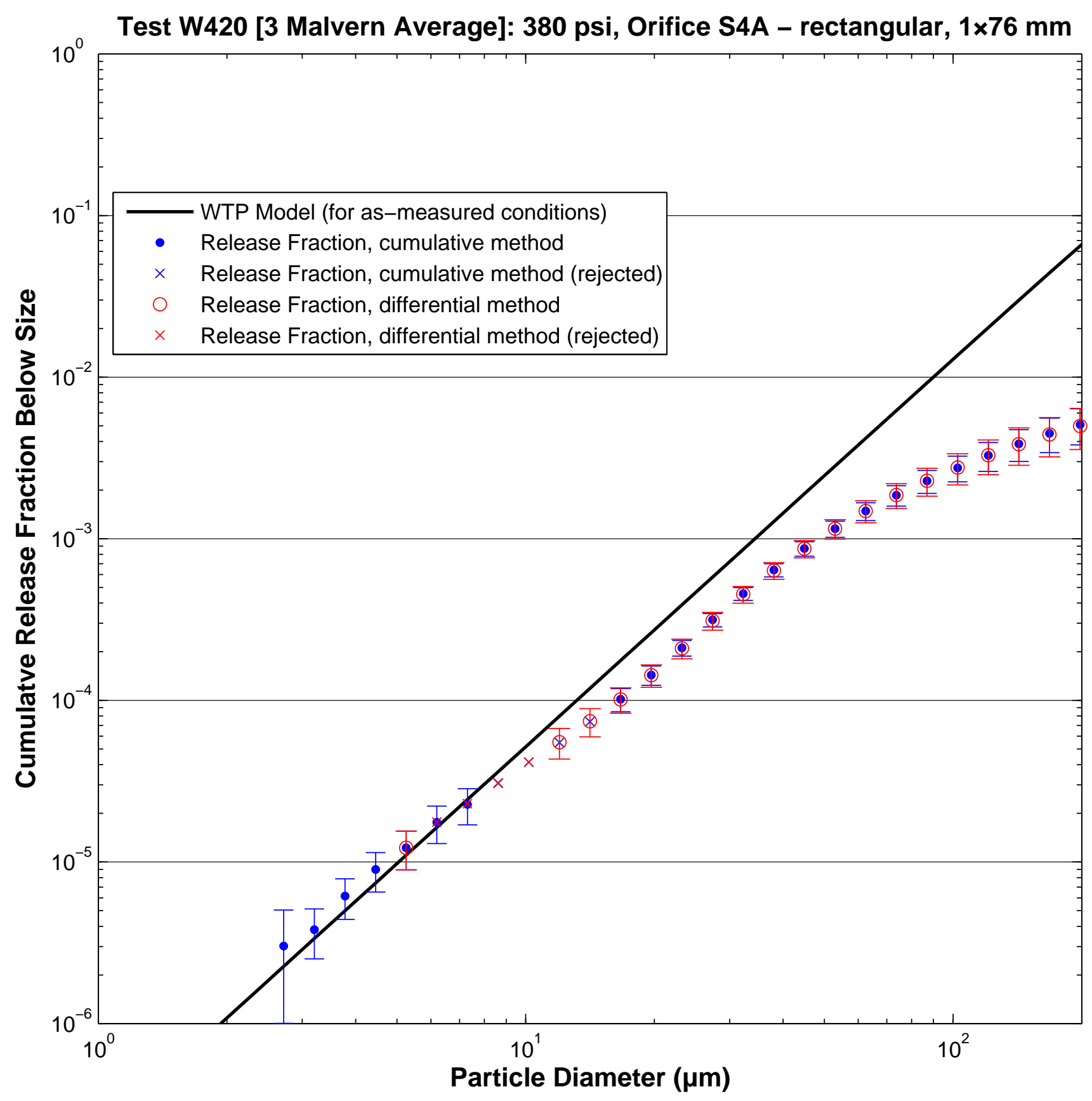

B. 103 


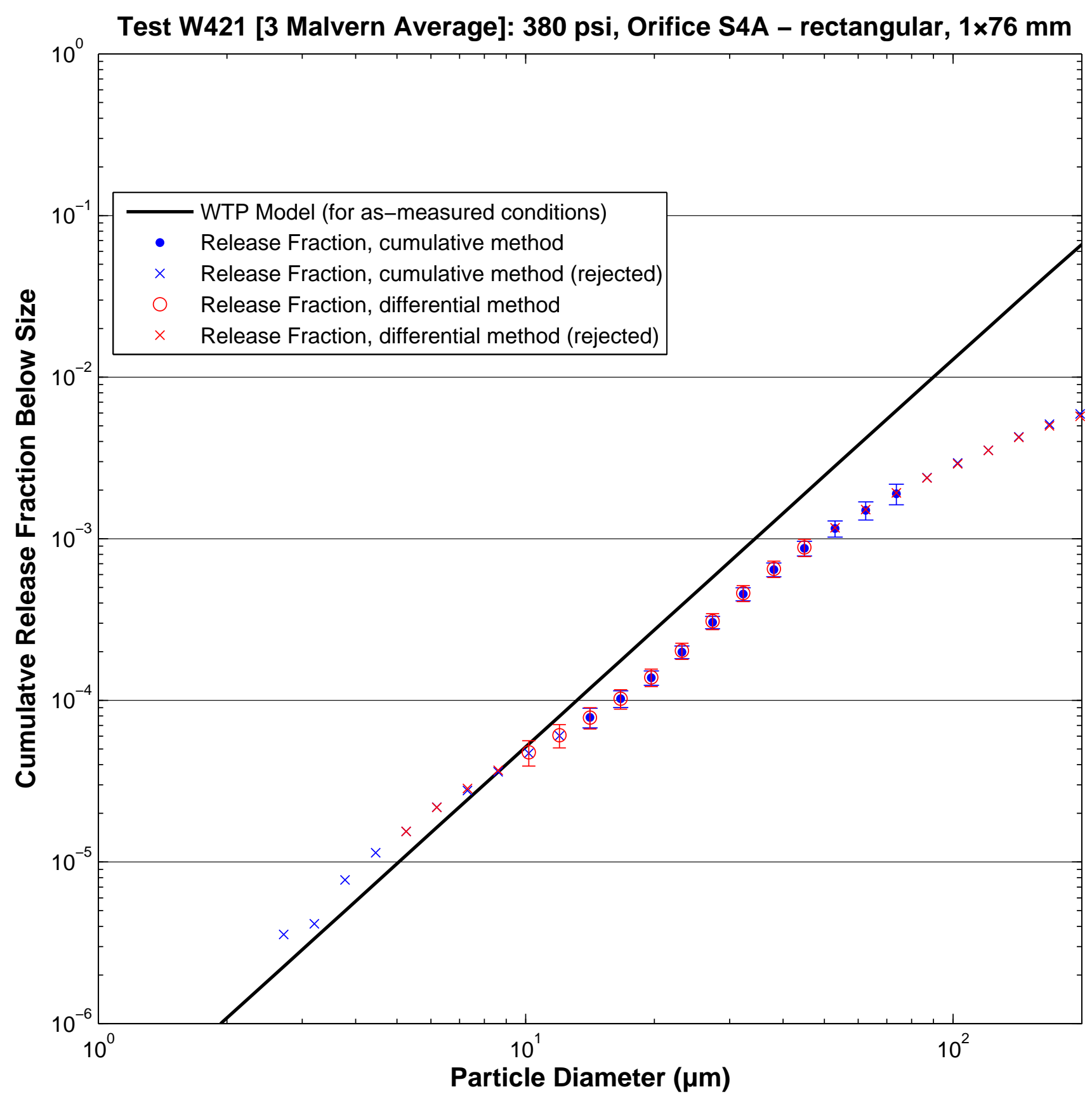

B. 104 


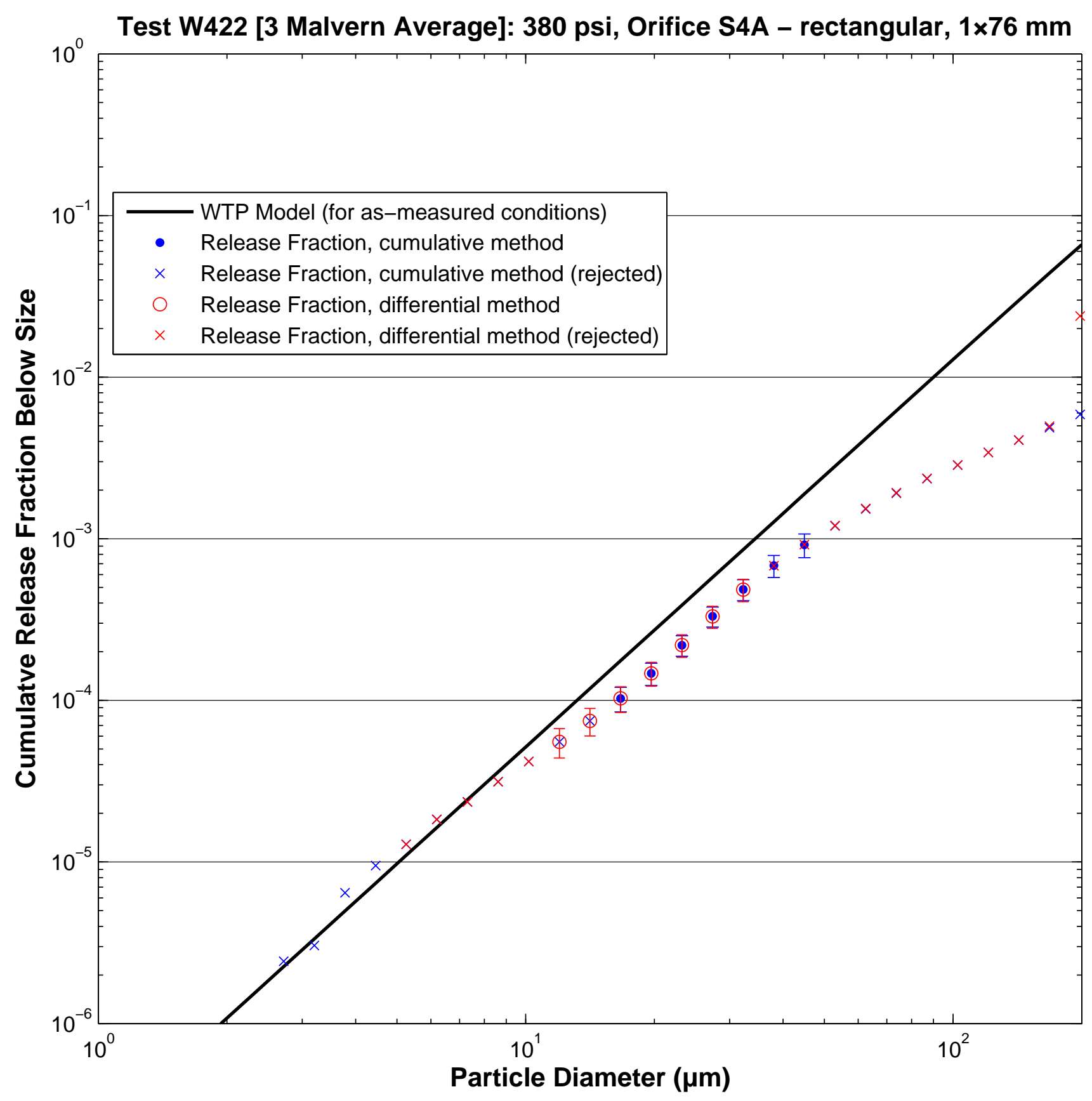

B. 105 


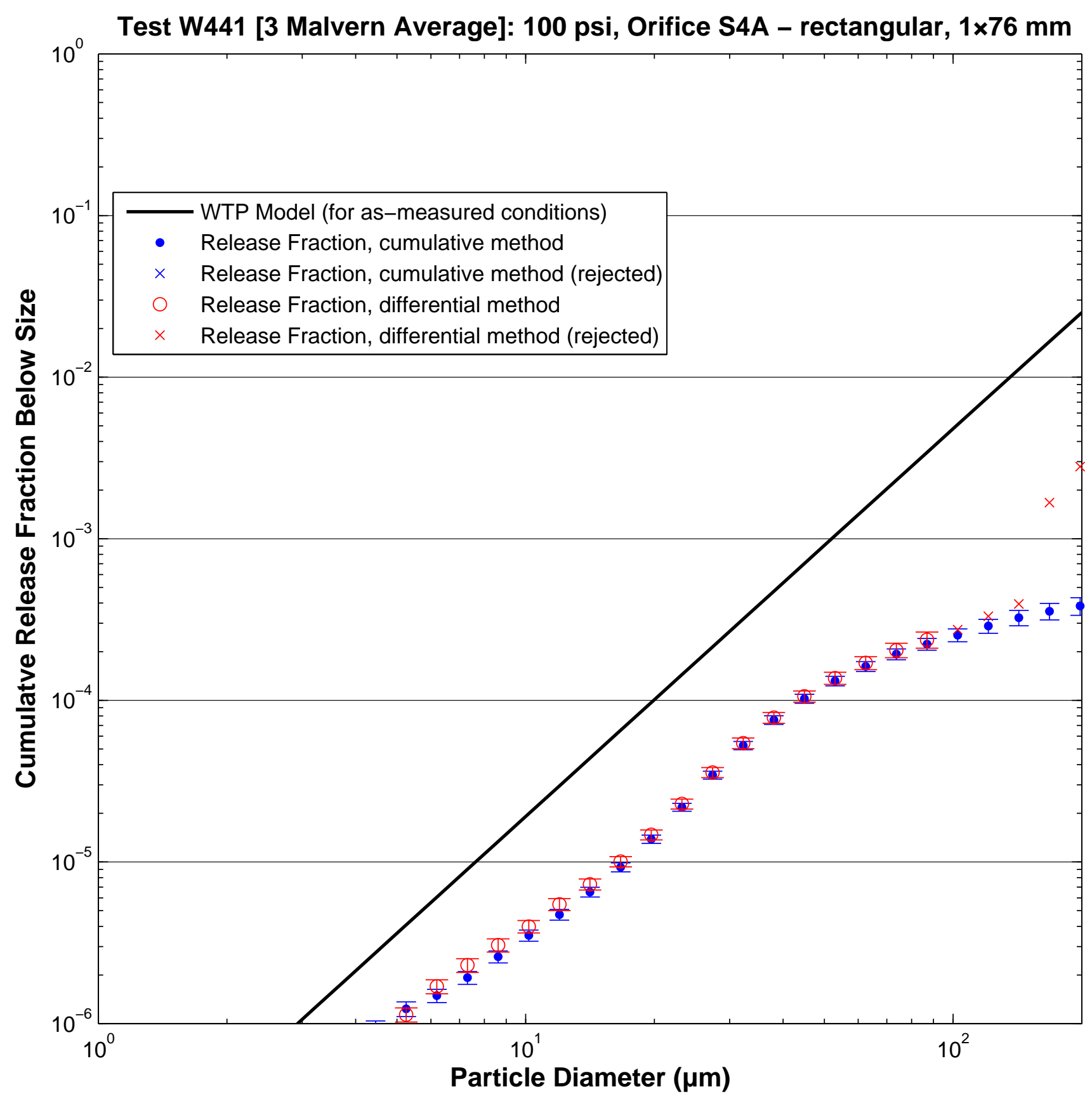

B. 106 


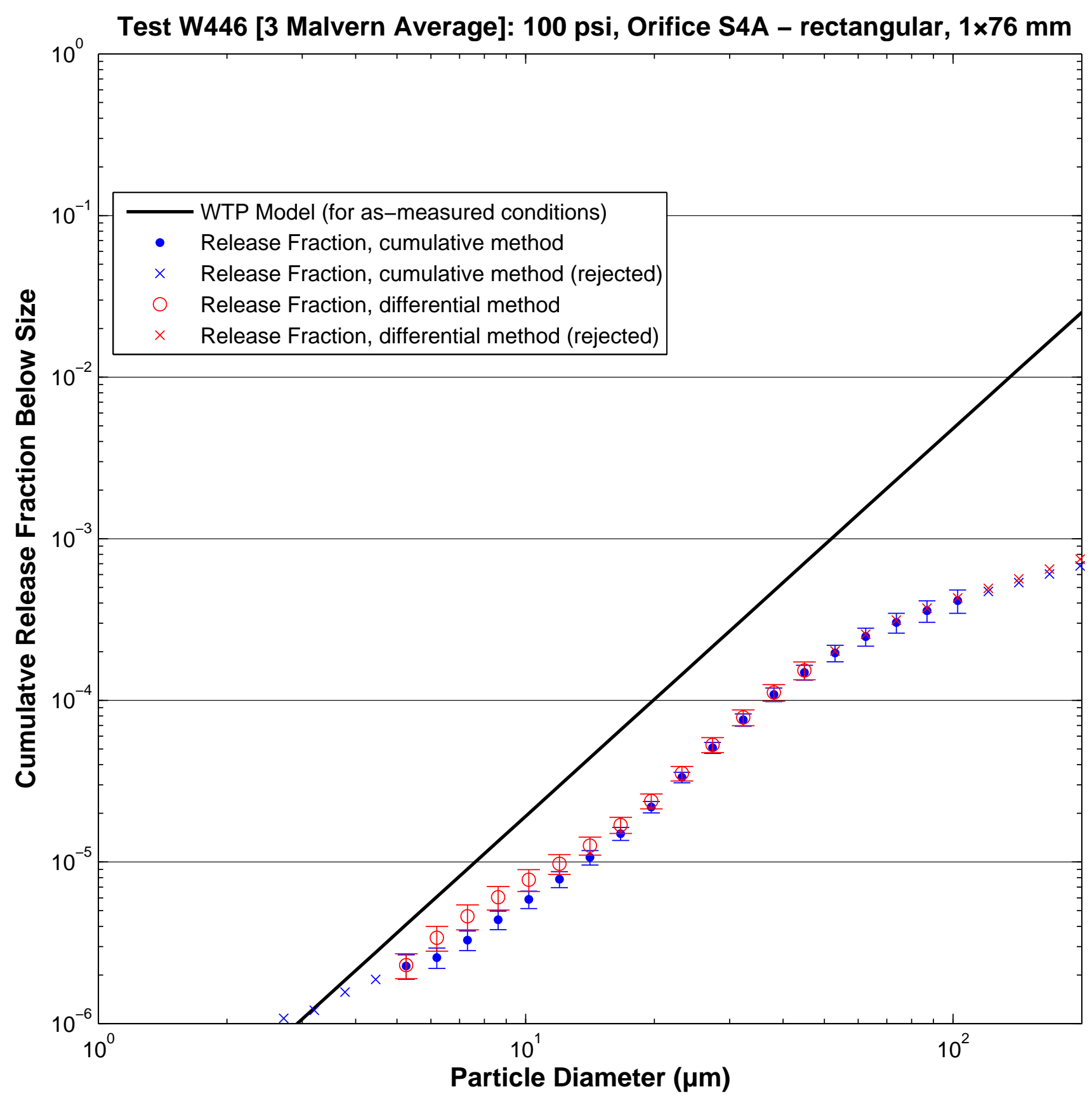




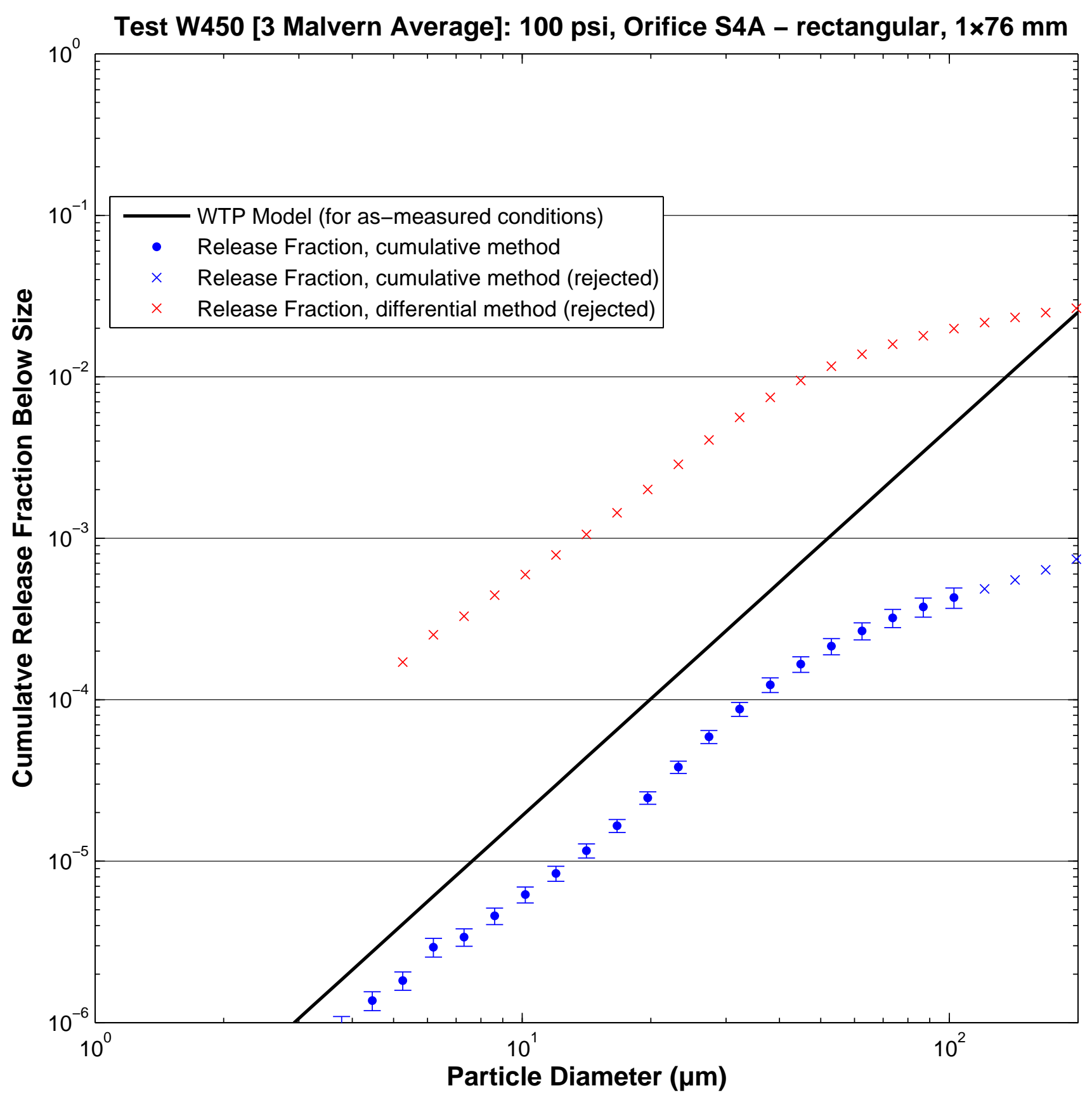

B. 108 


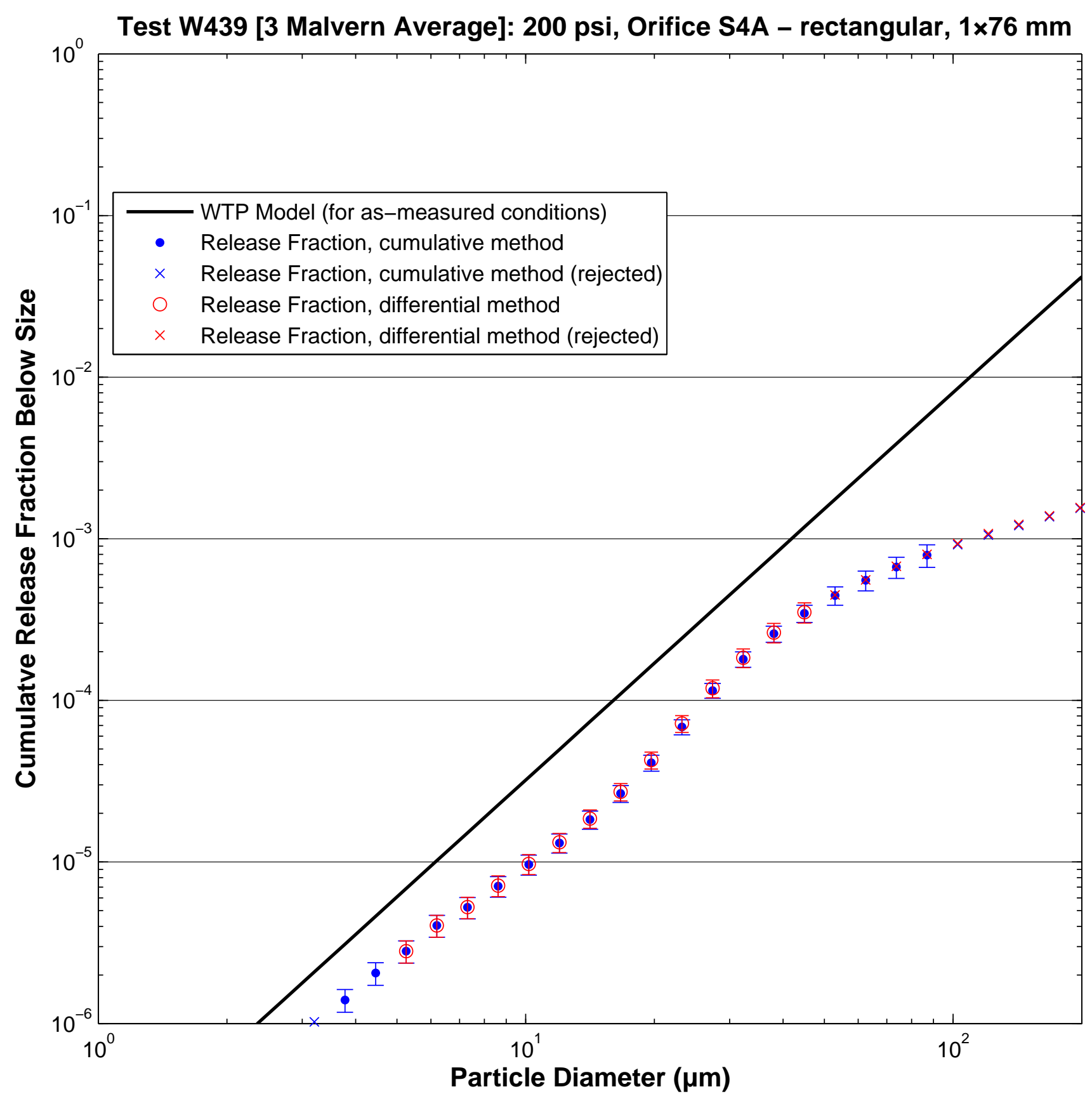

B. 109 


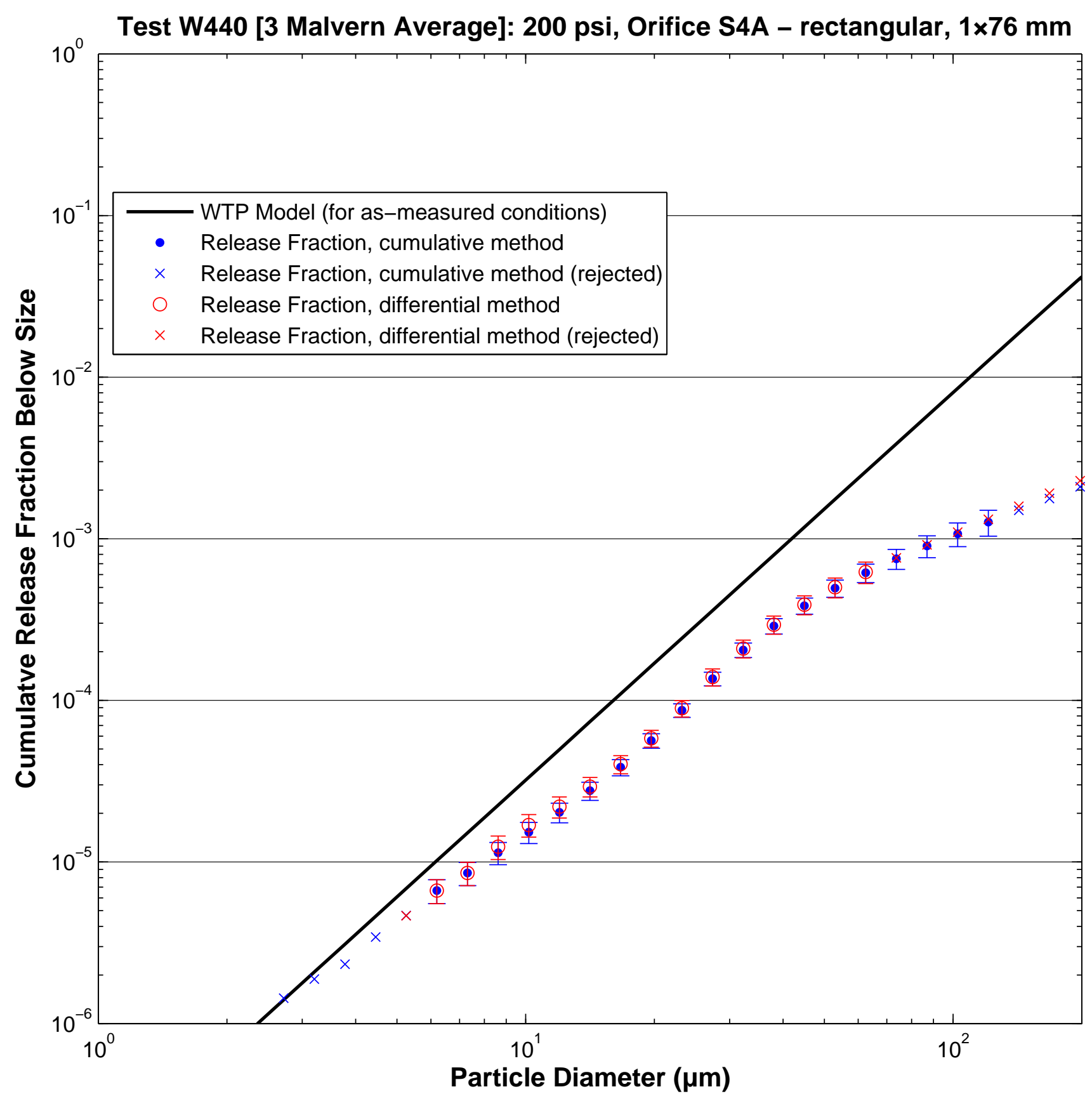

B. 110 


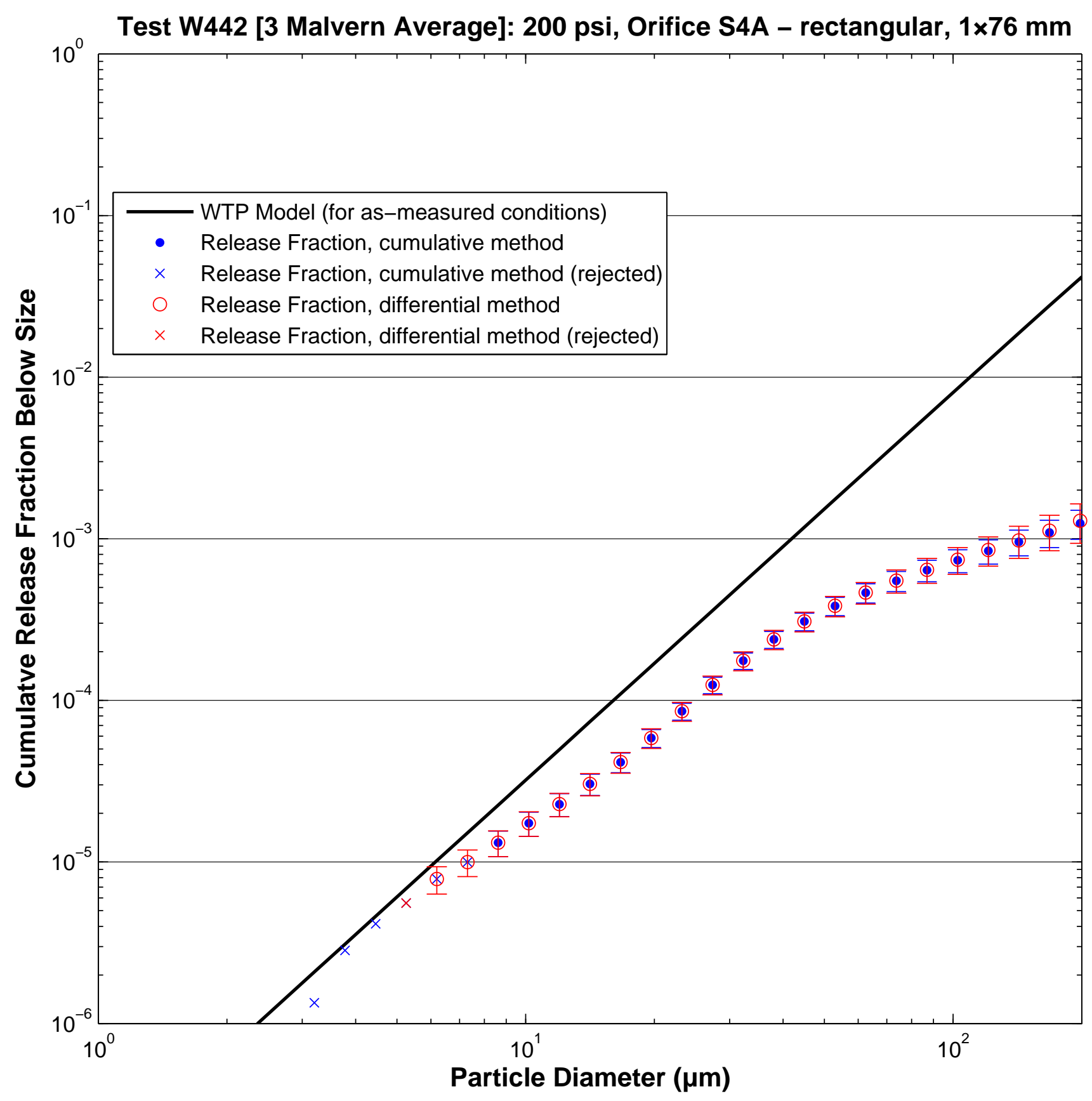




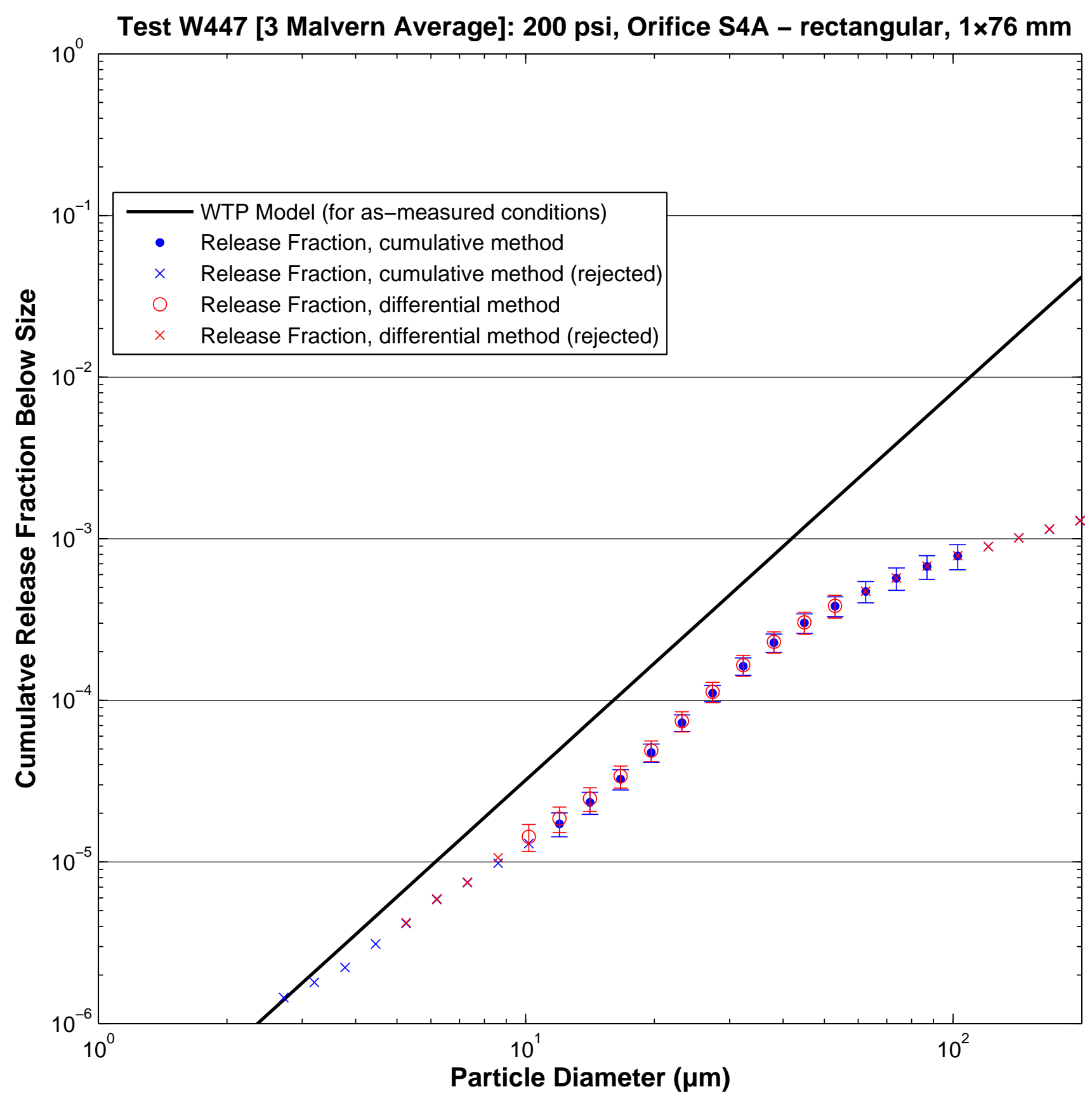




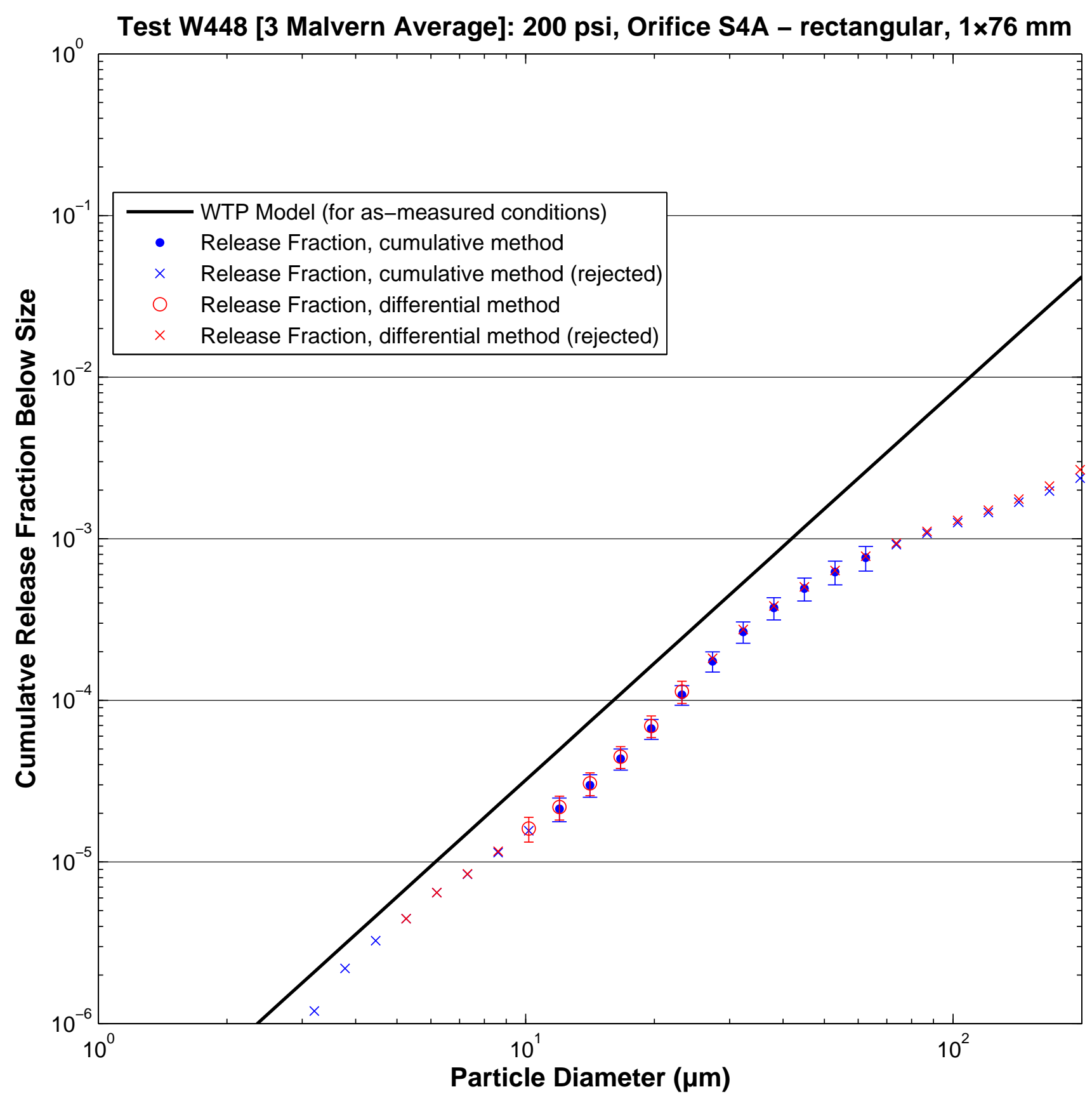




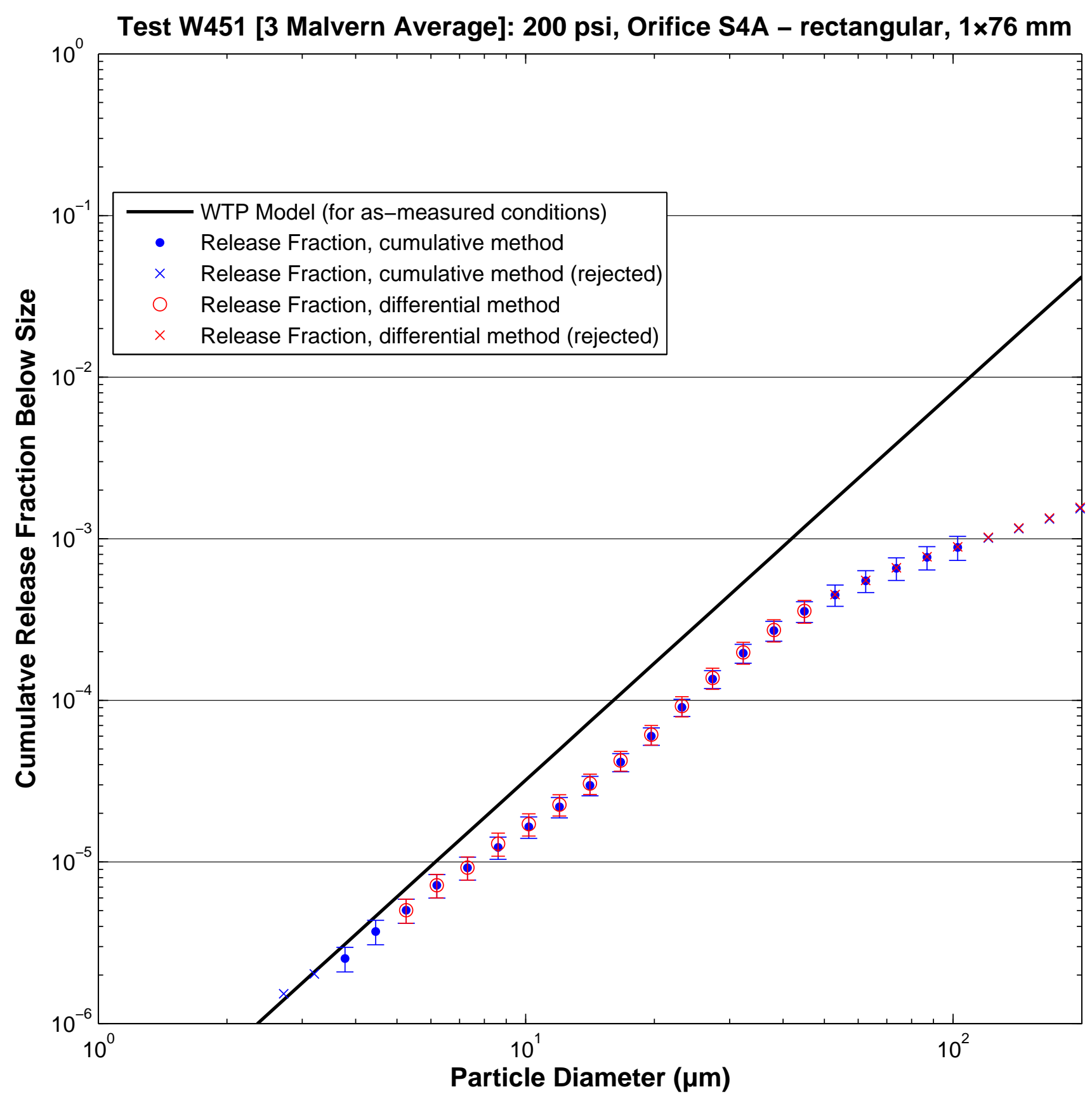

B. 114 


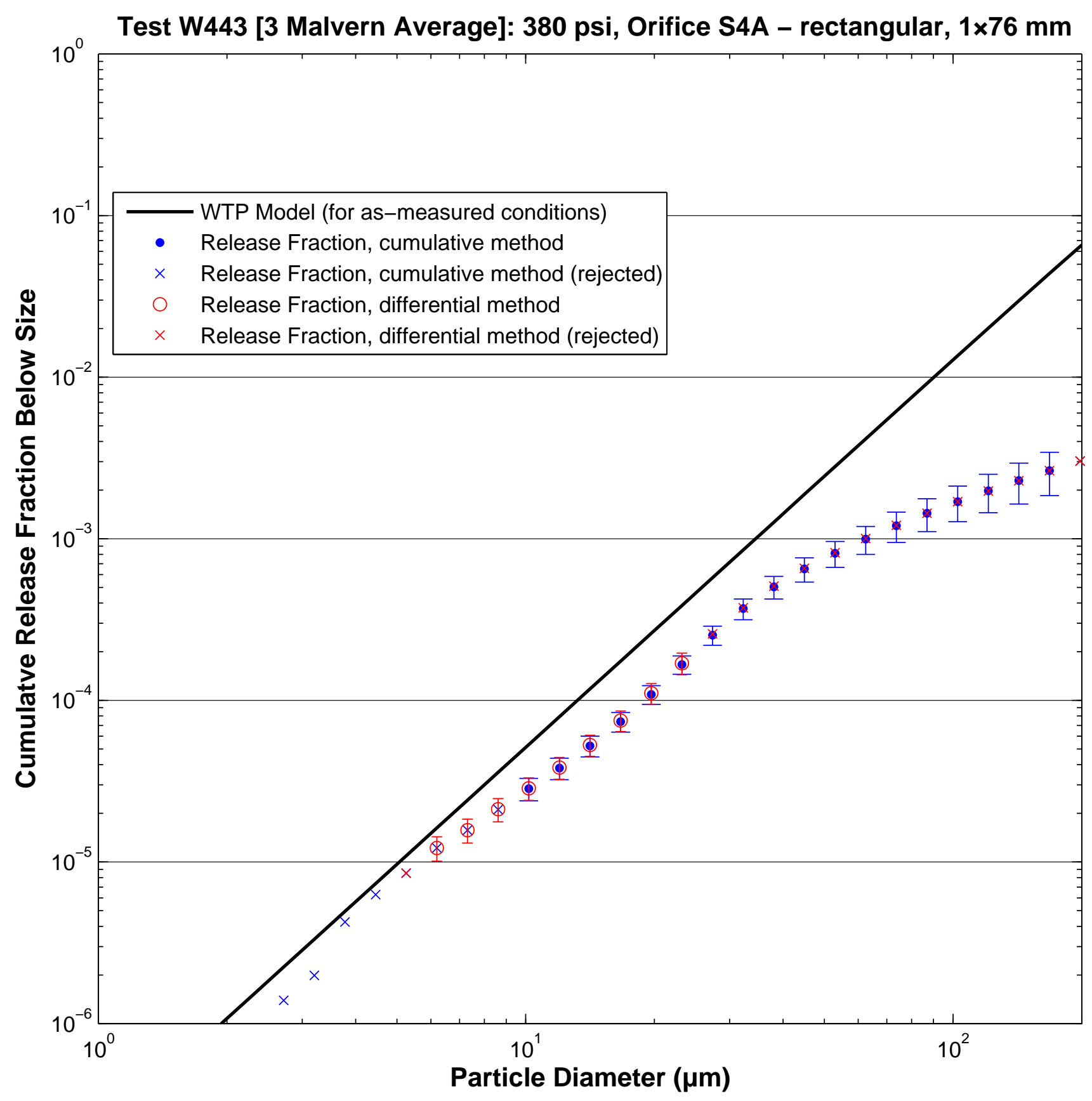




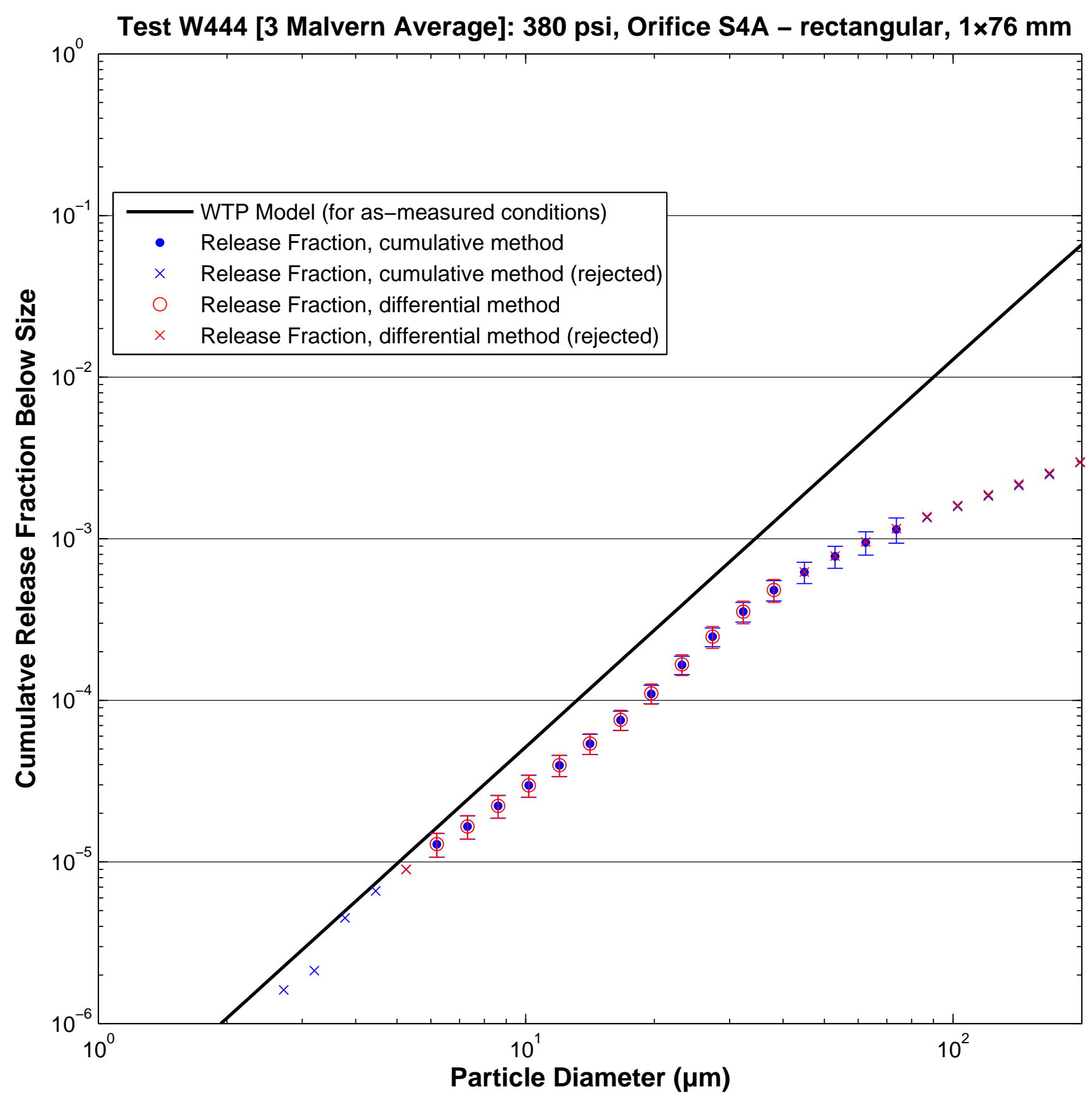

B. 116 


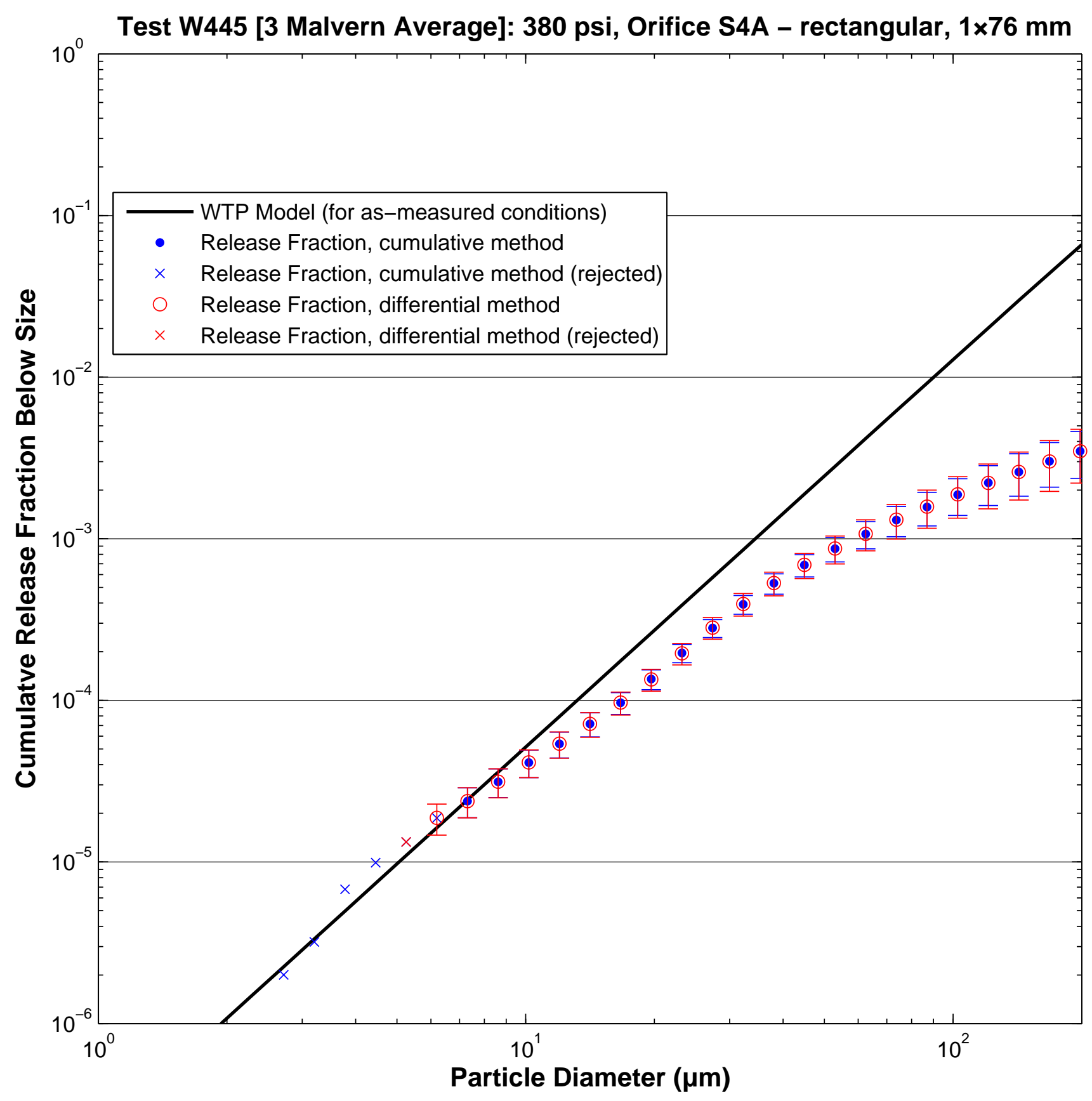




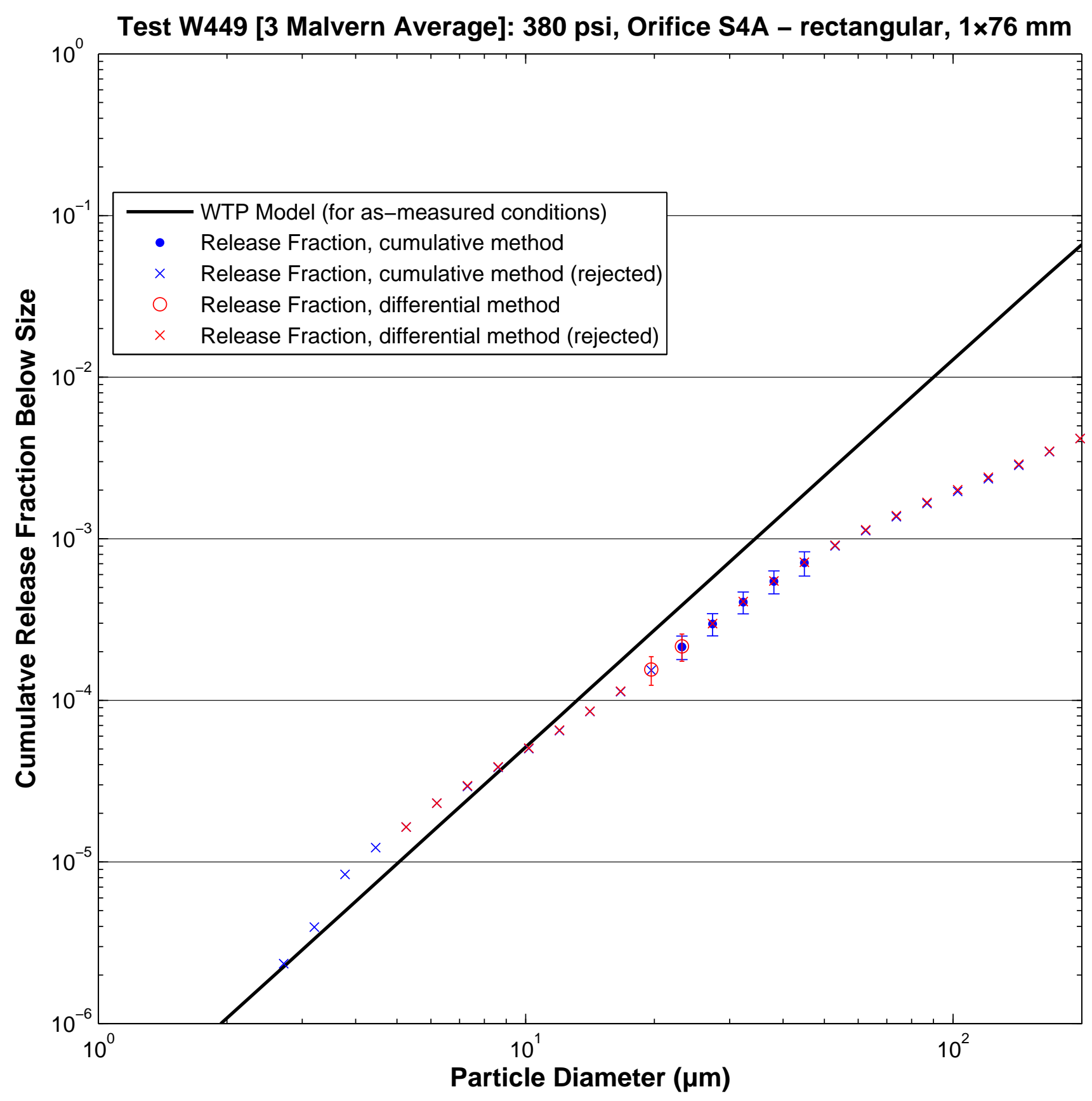

B. 118 


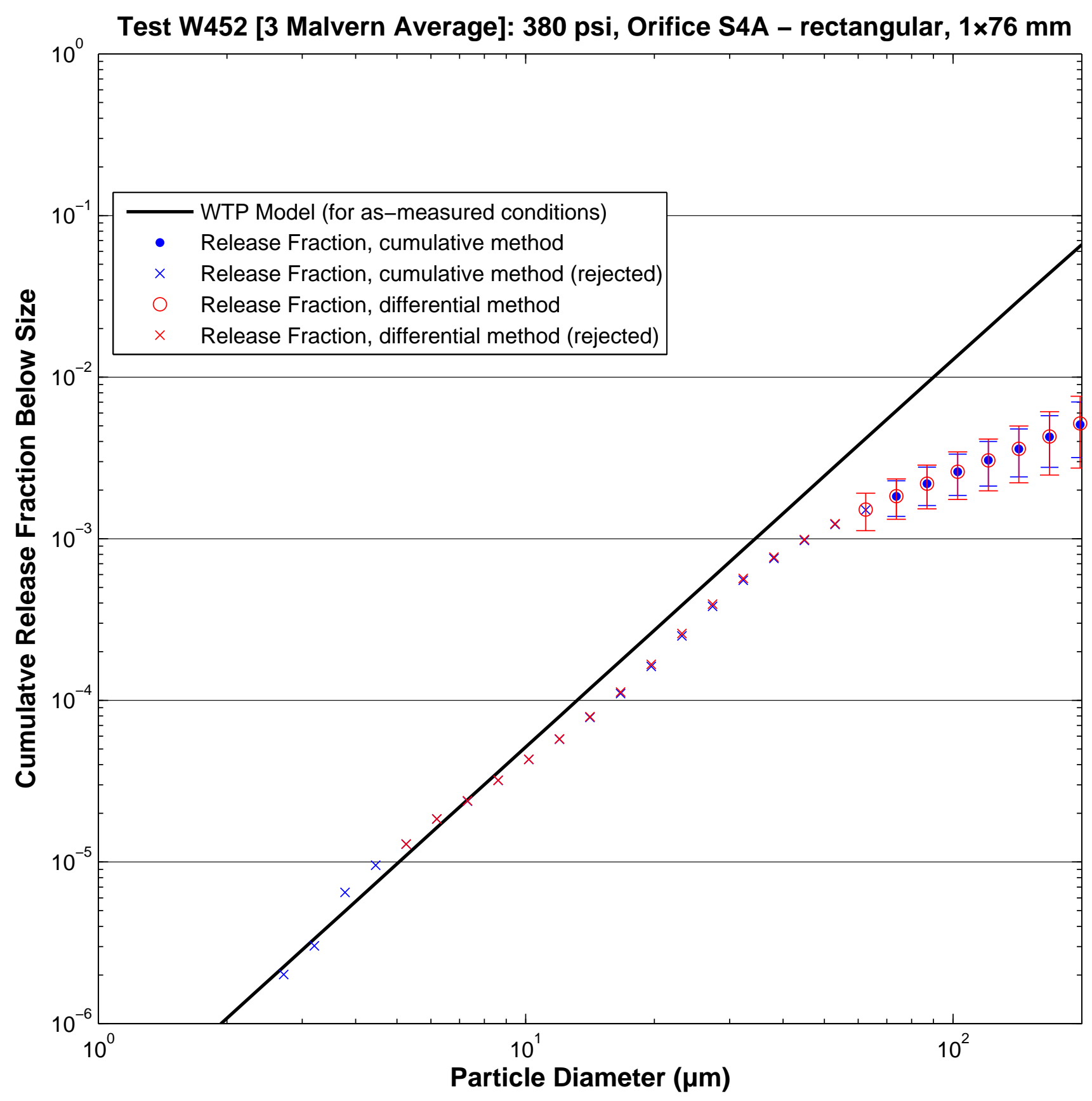

B. 119 


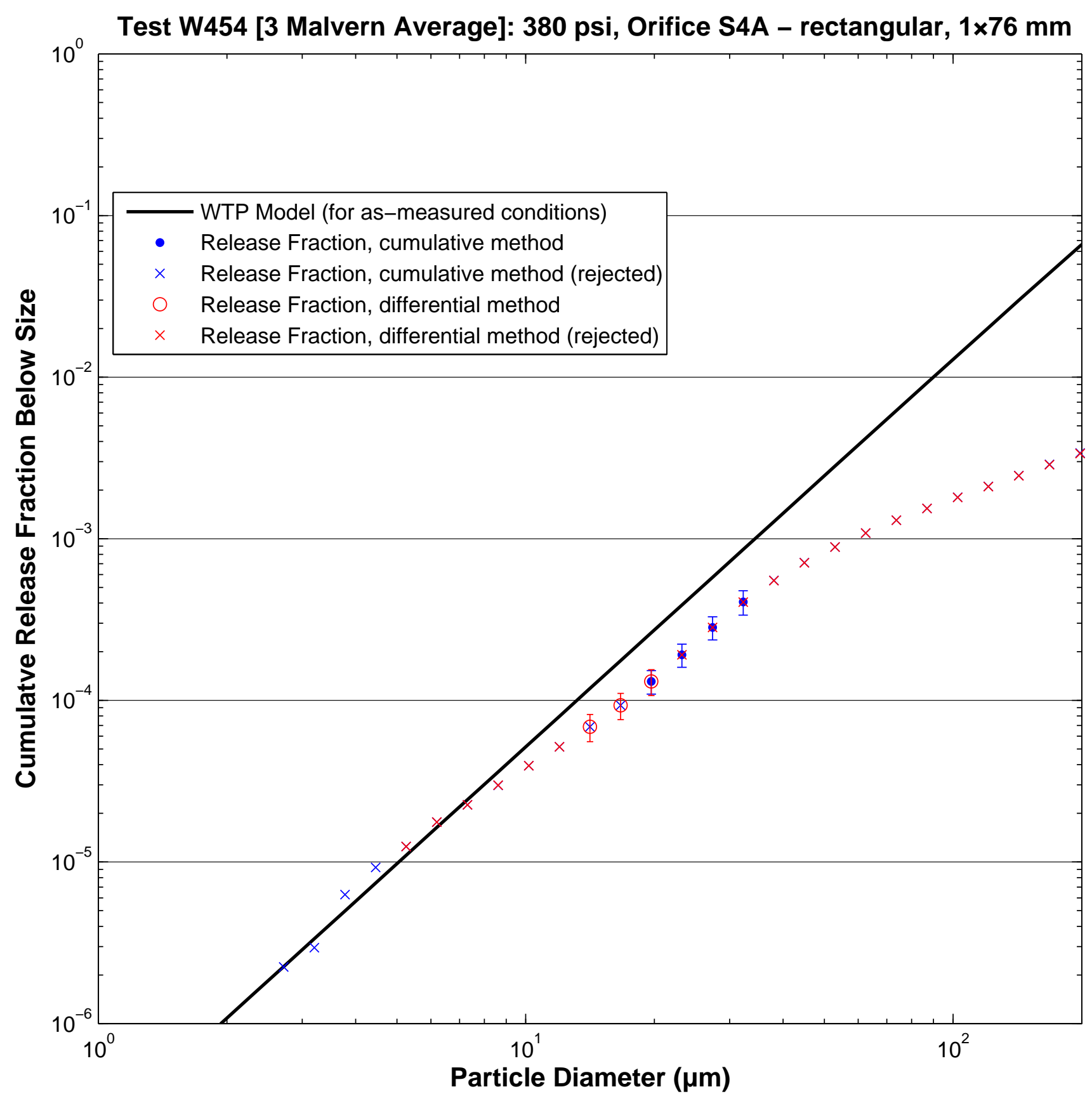

B. 120 


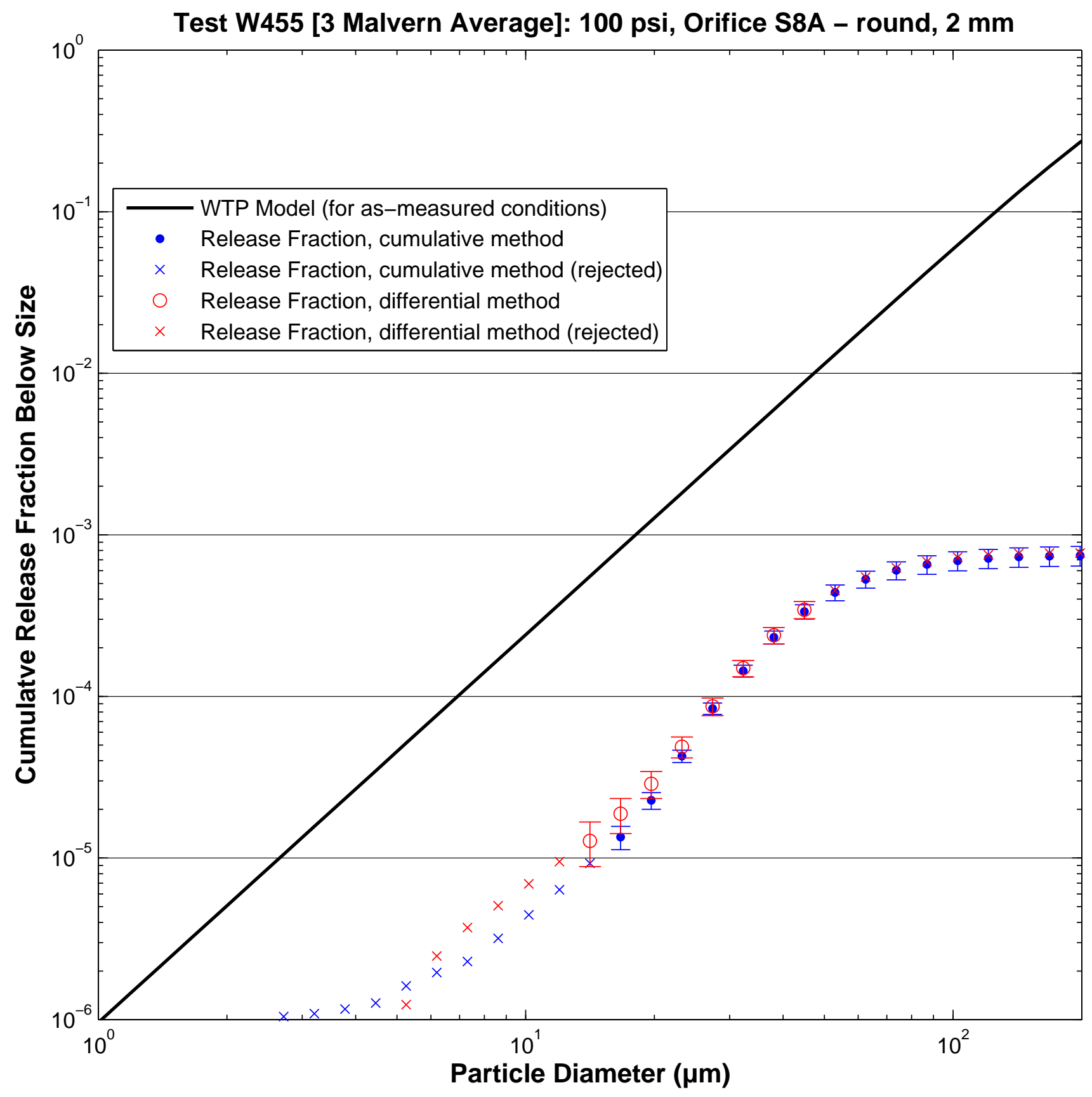




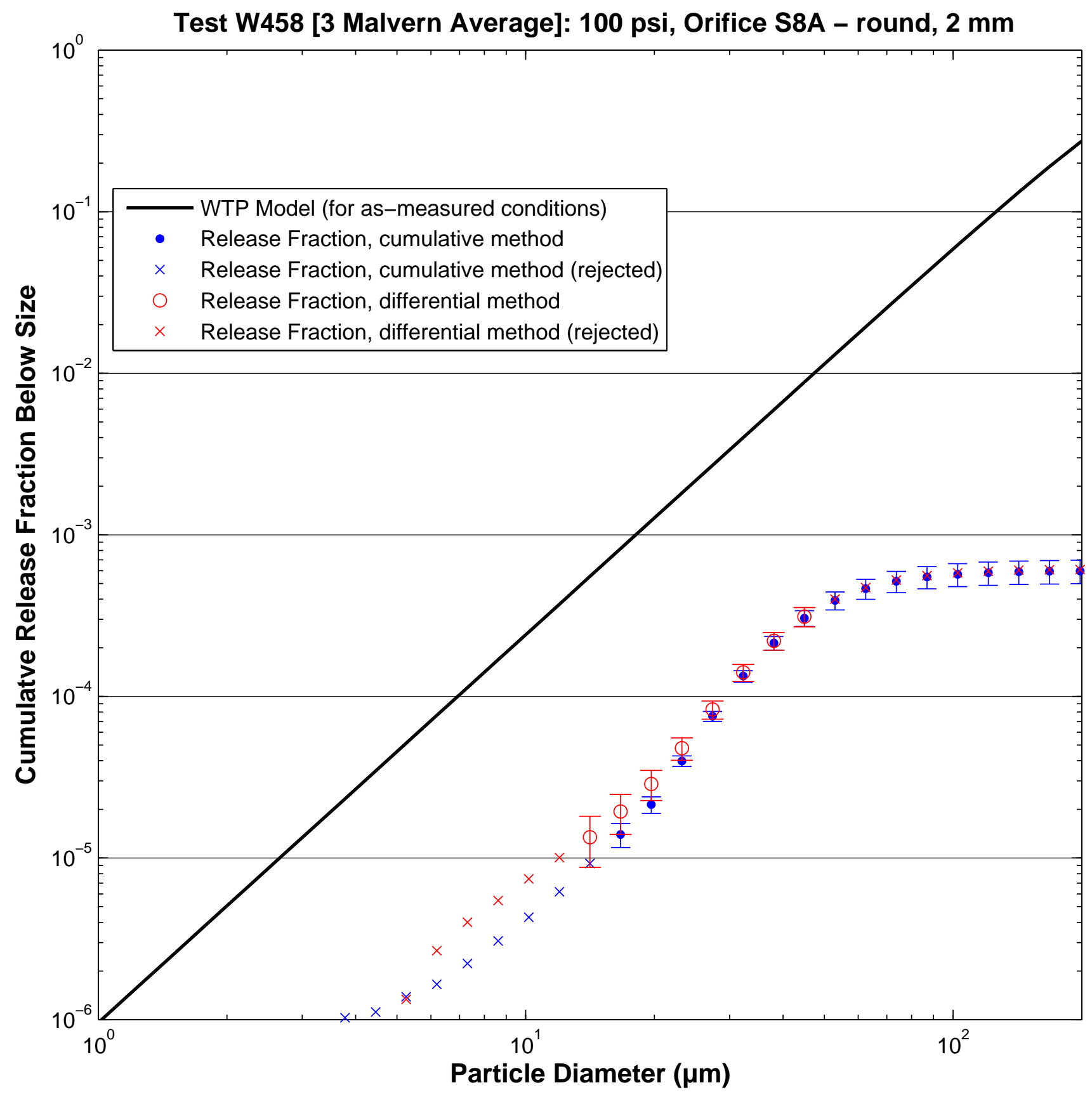

B. 122 


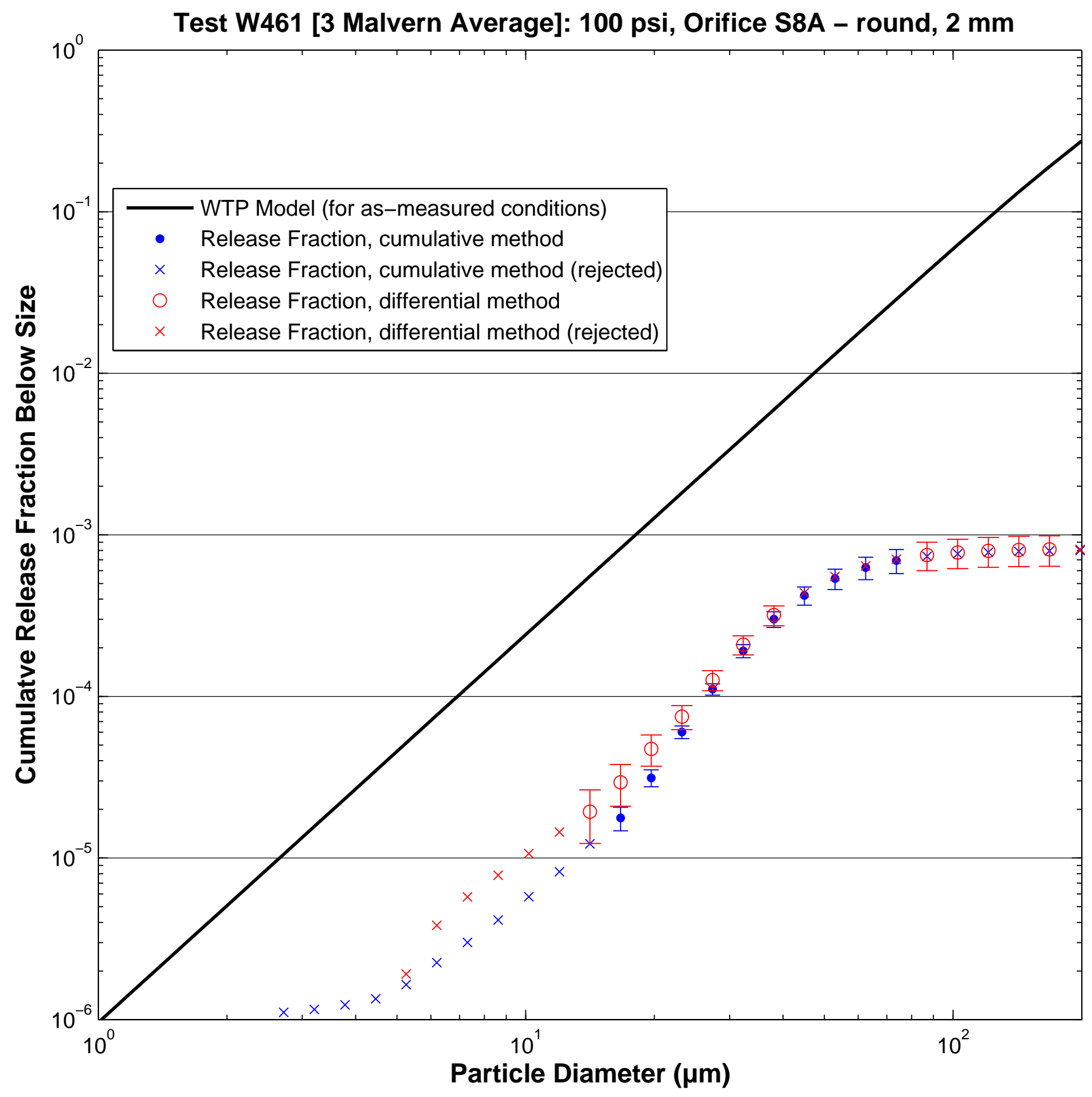

B. 123 


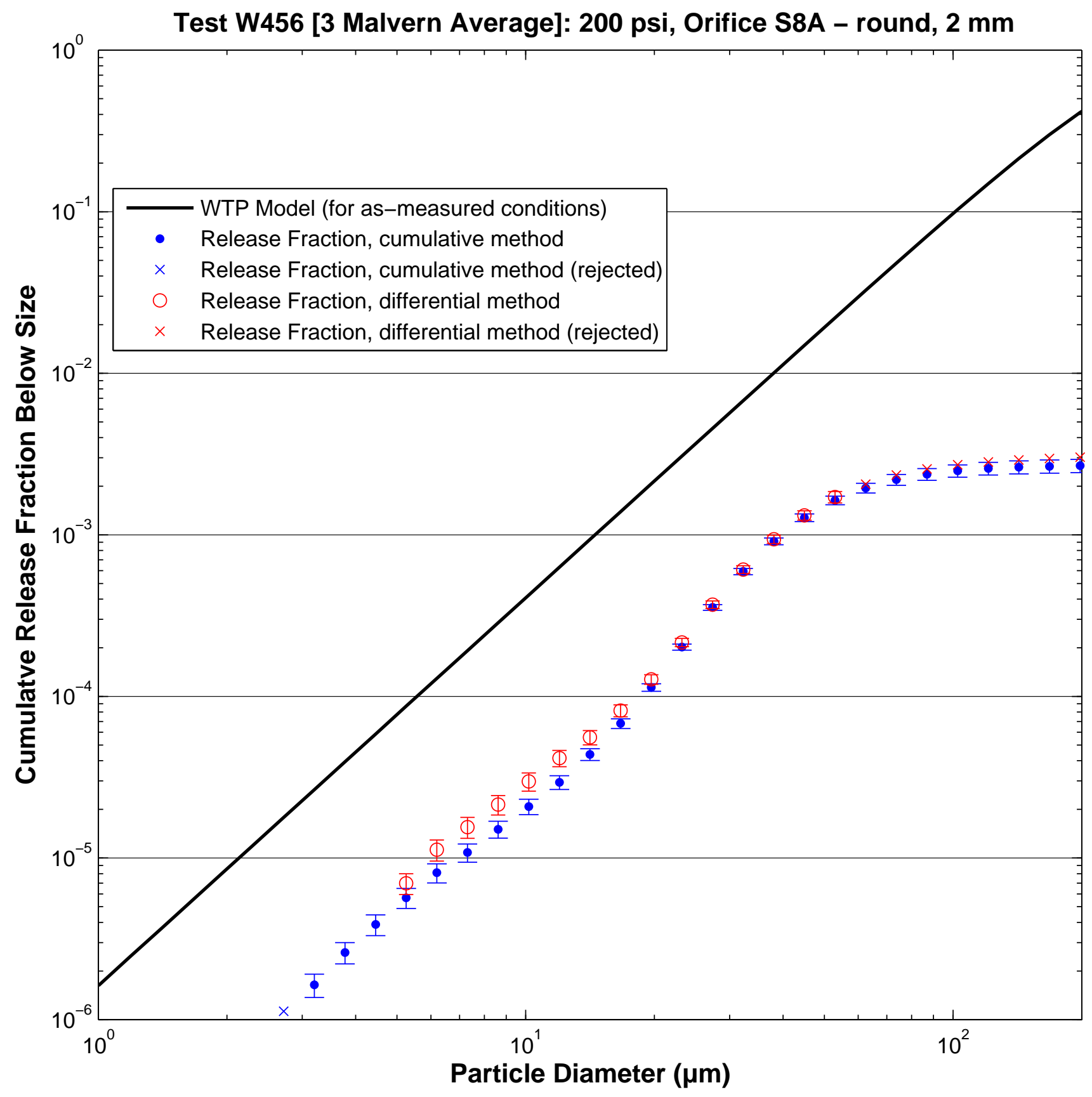

B. 124 


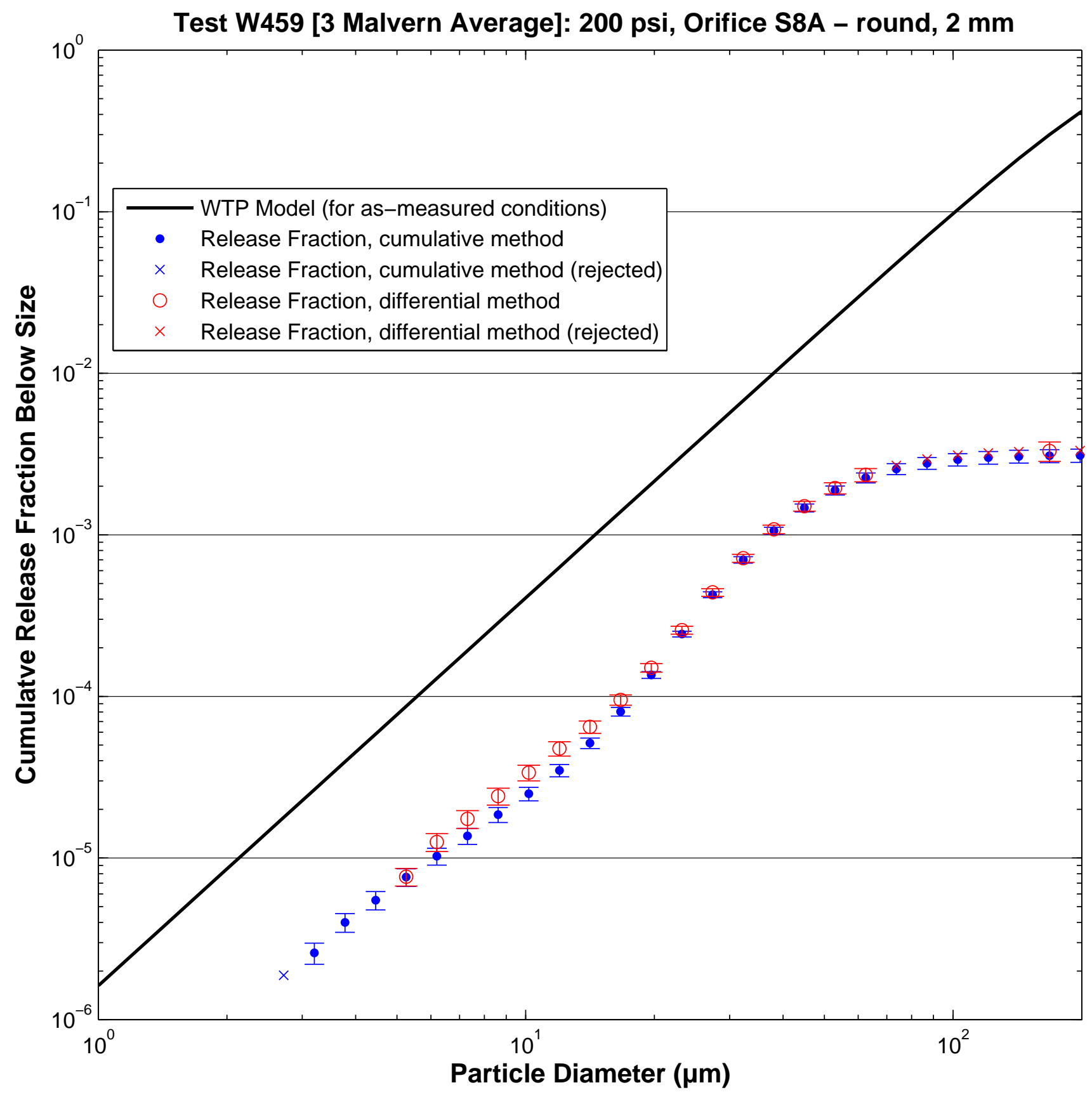

B. 125 


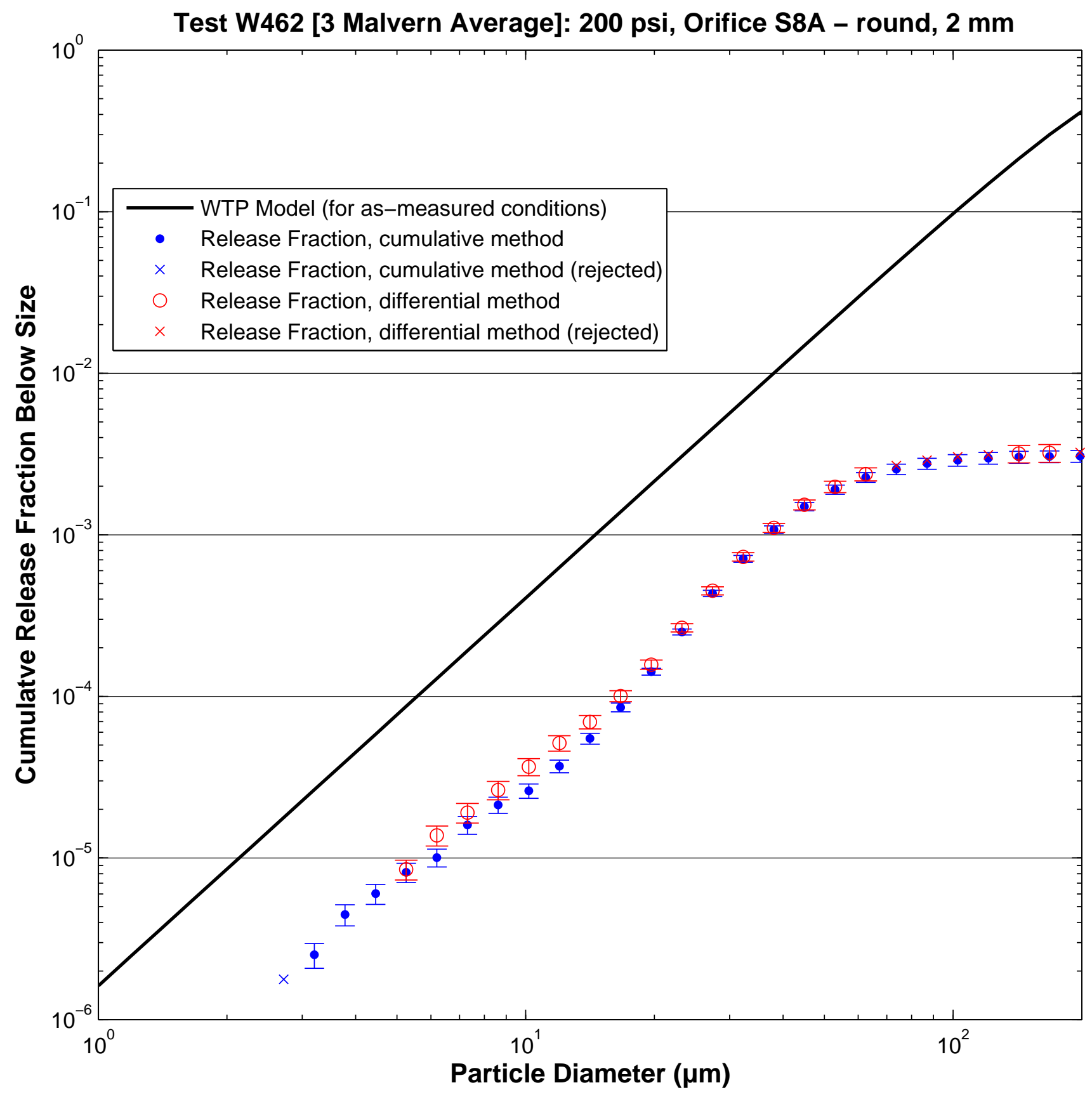

B. 126 


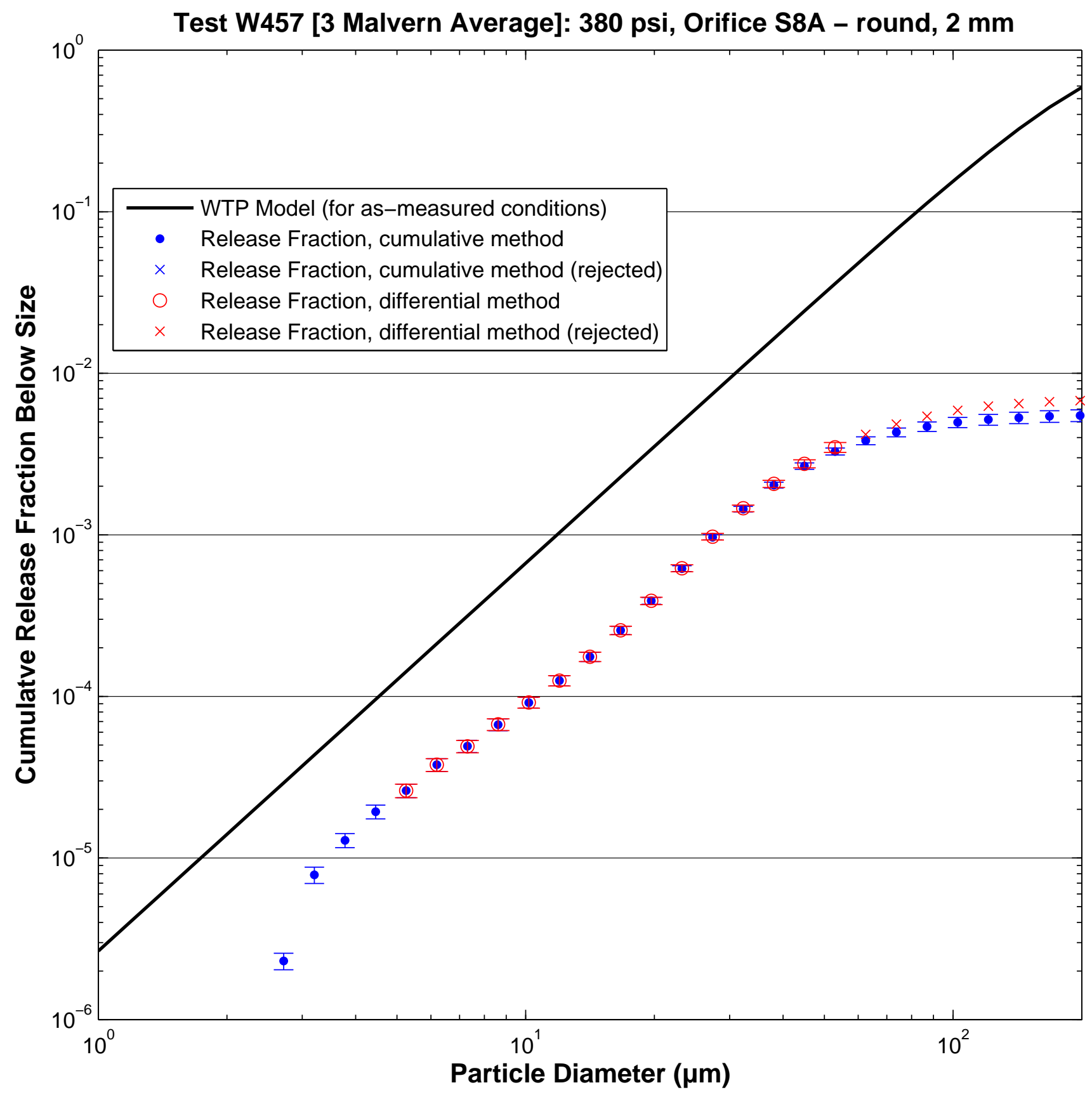




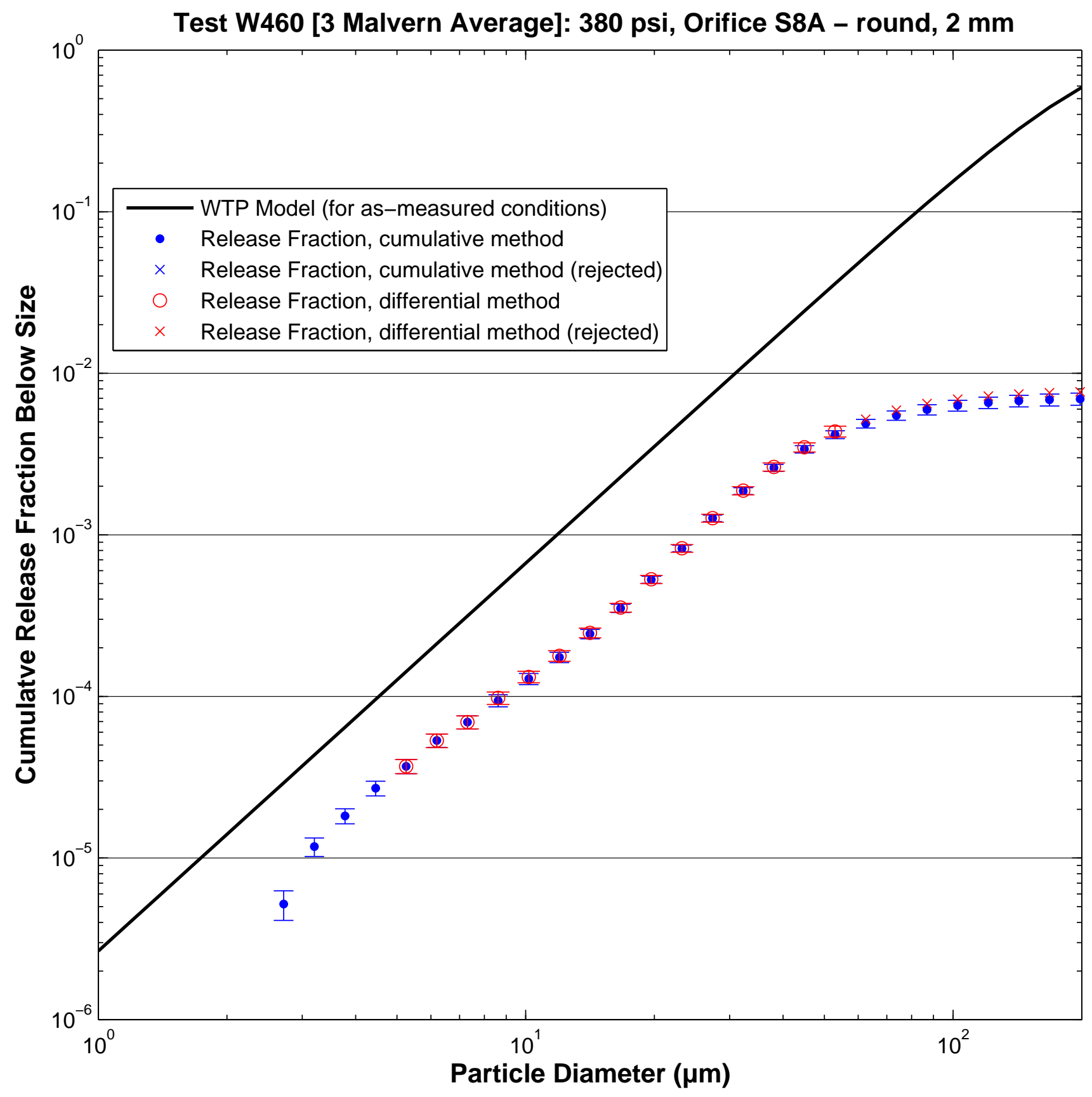

B. 128 


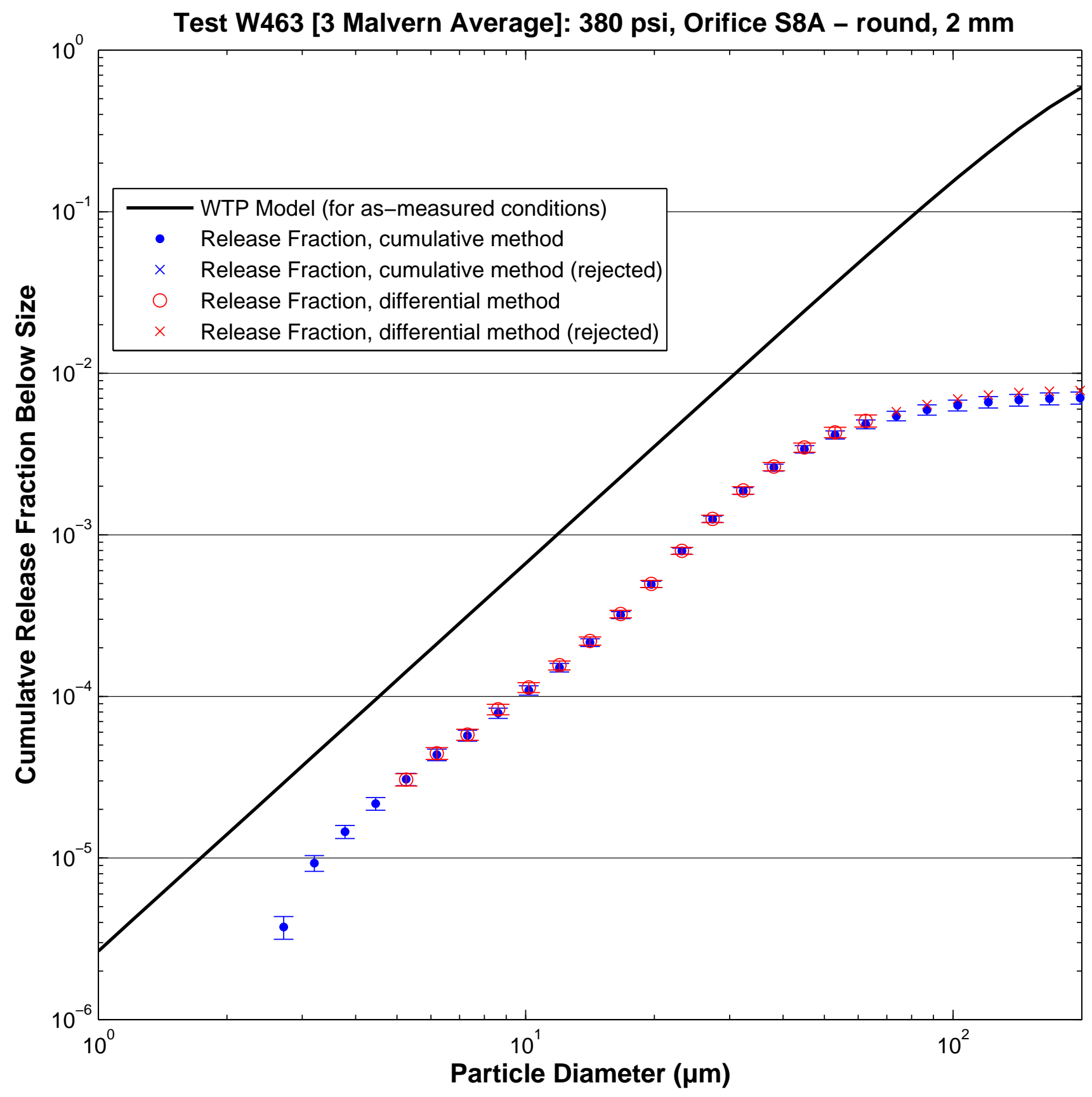




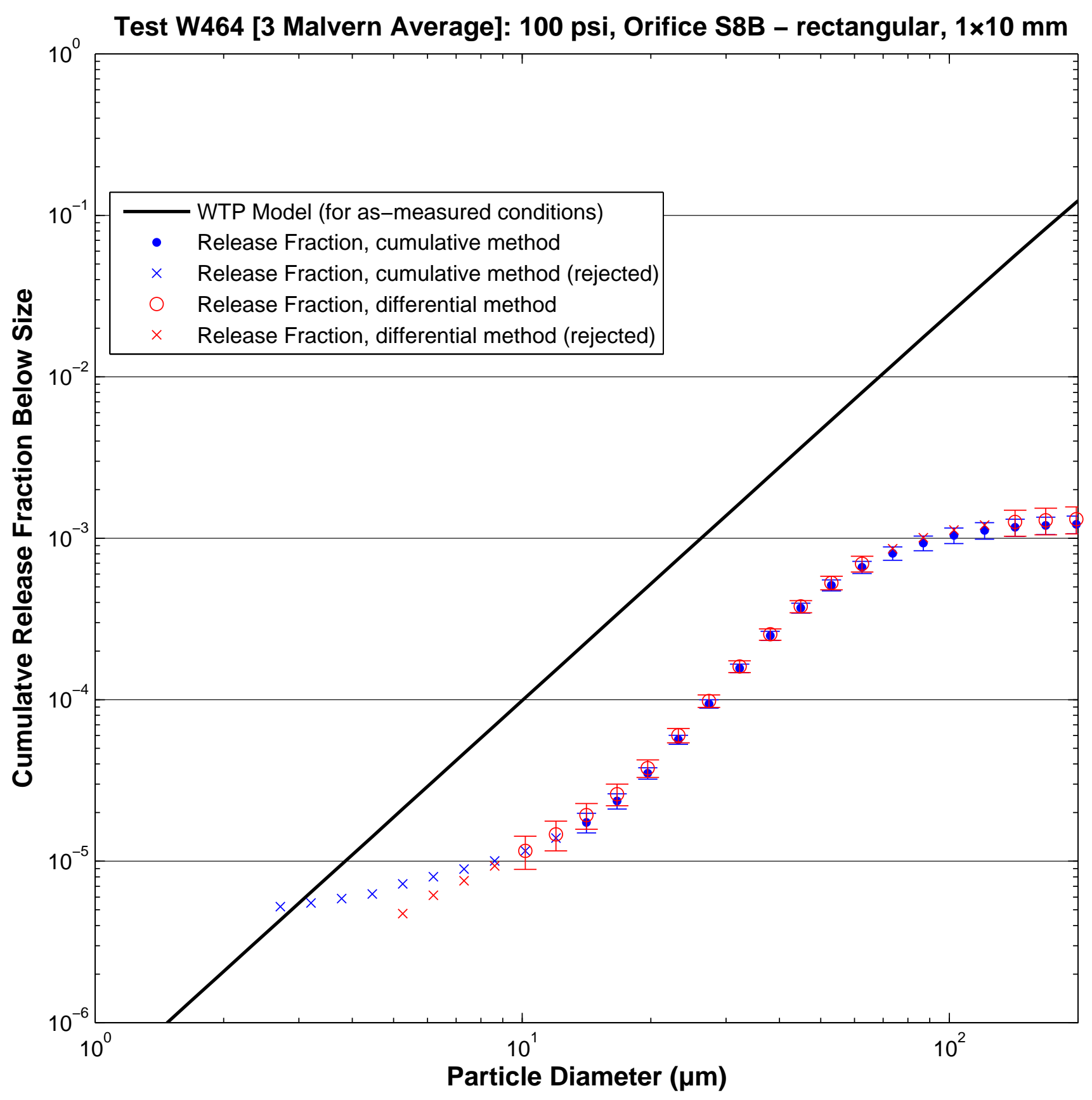

B. 130 


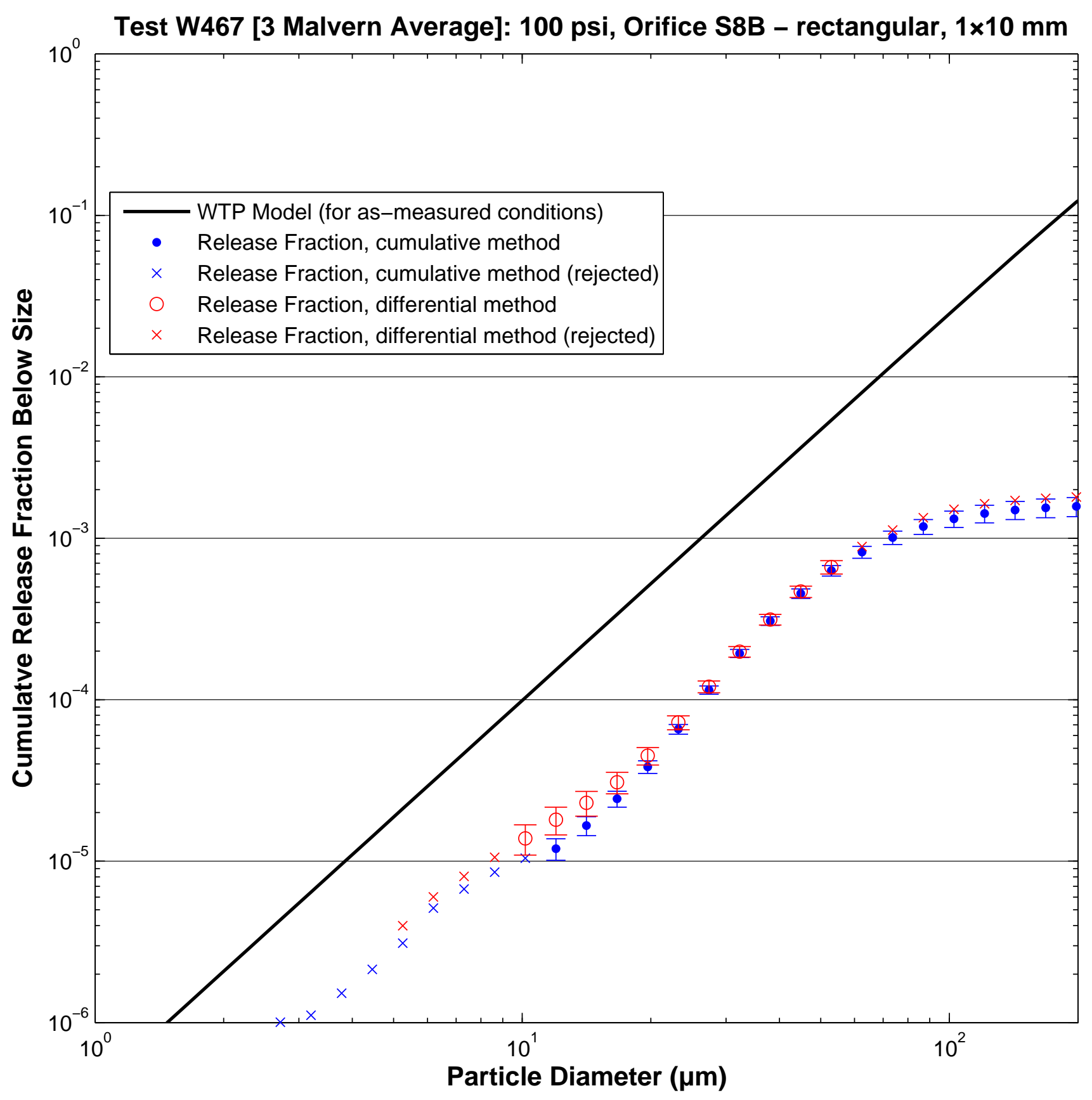




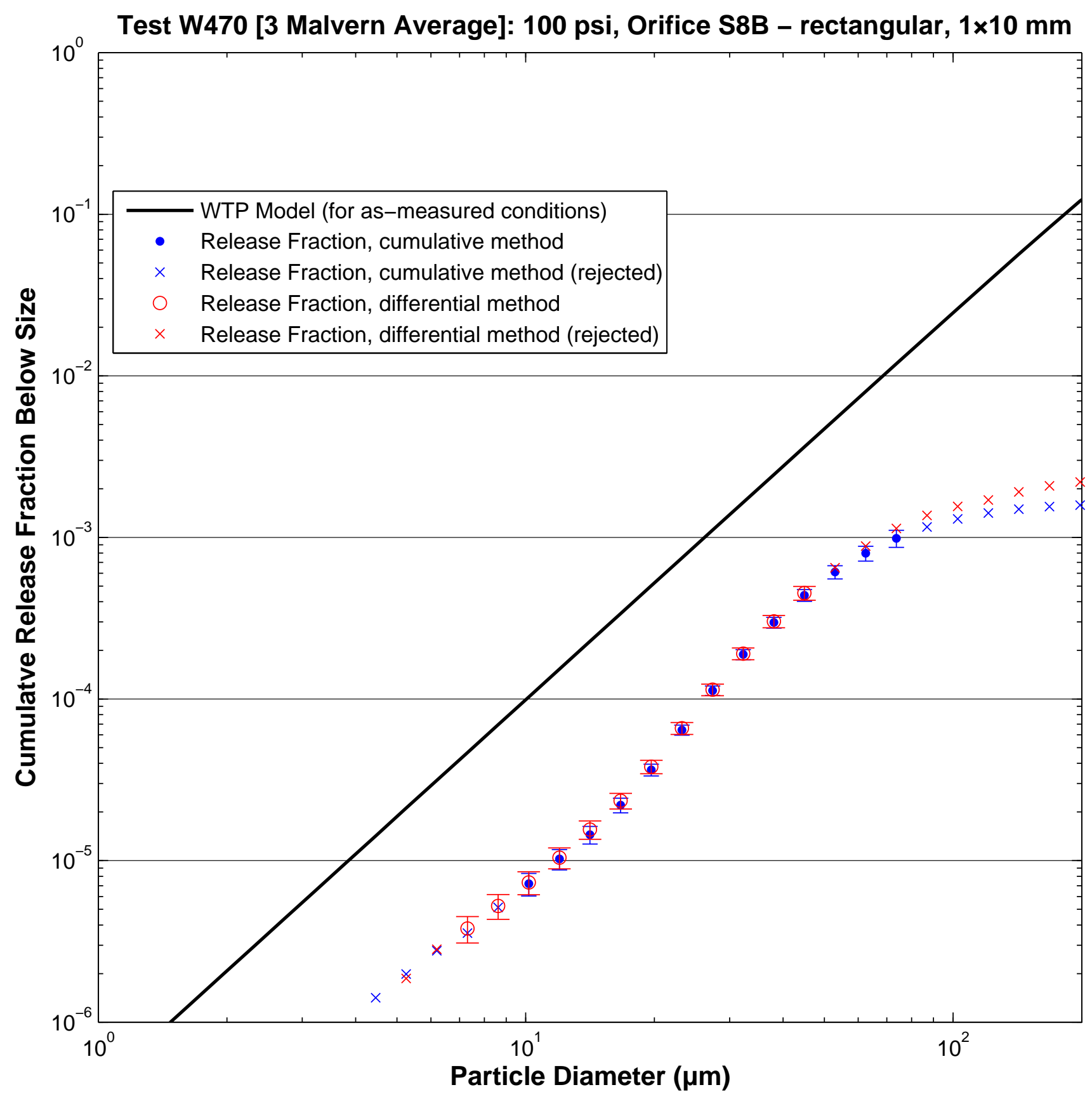

B. 132 


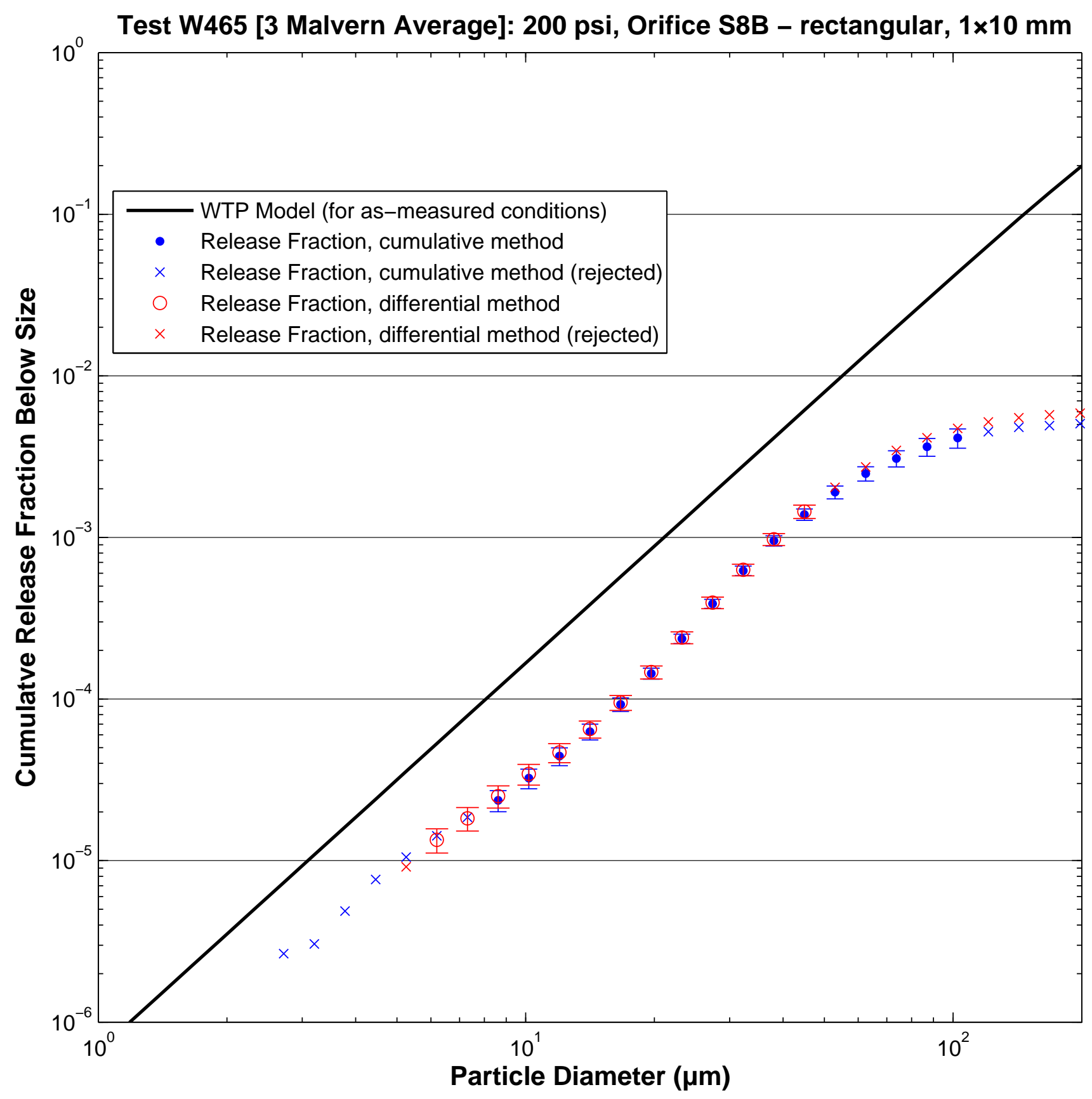

B. 133 


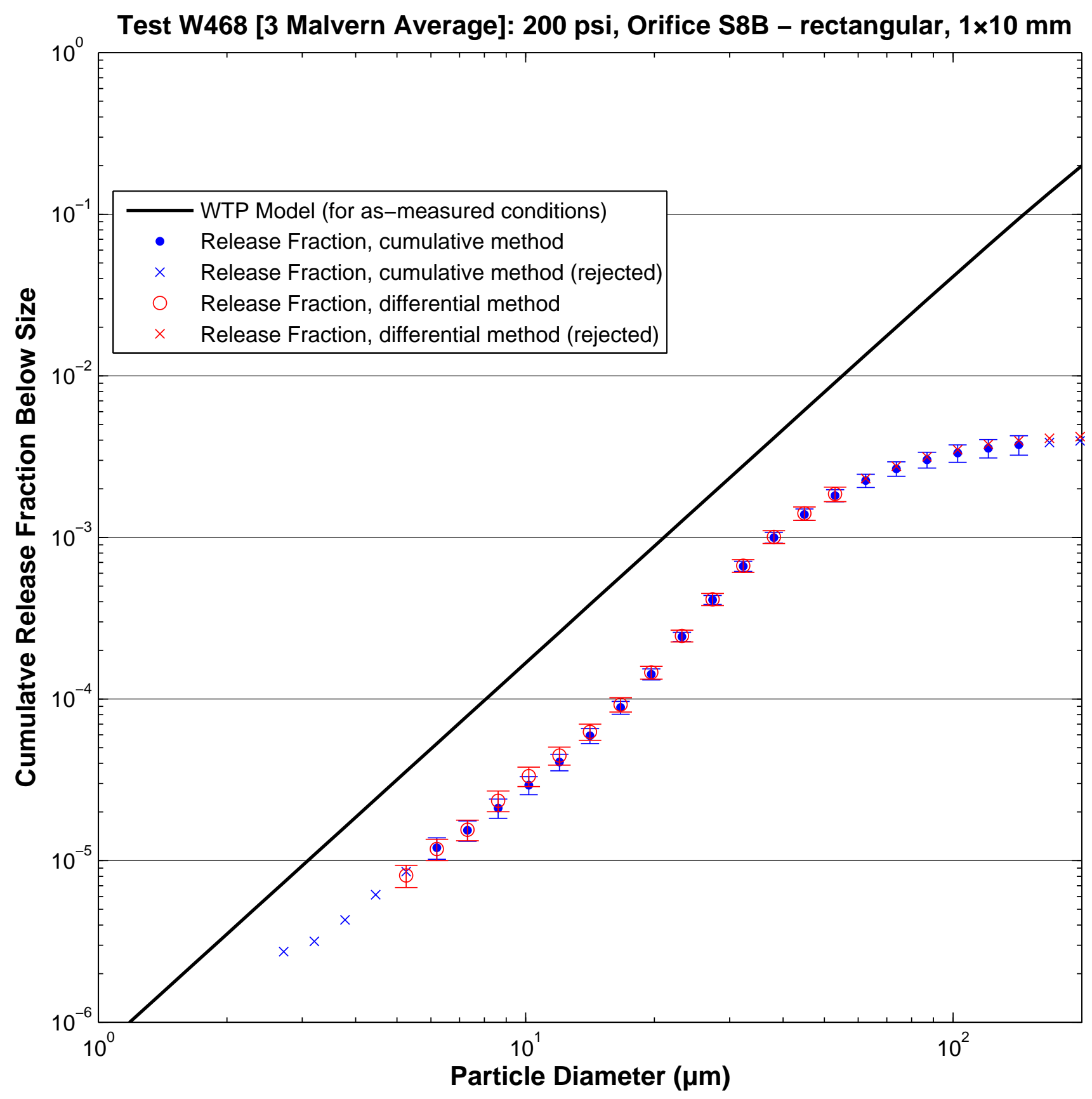

B. 134 


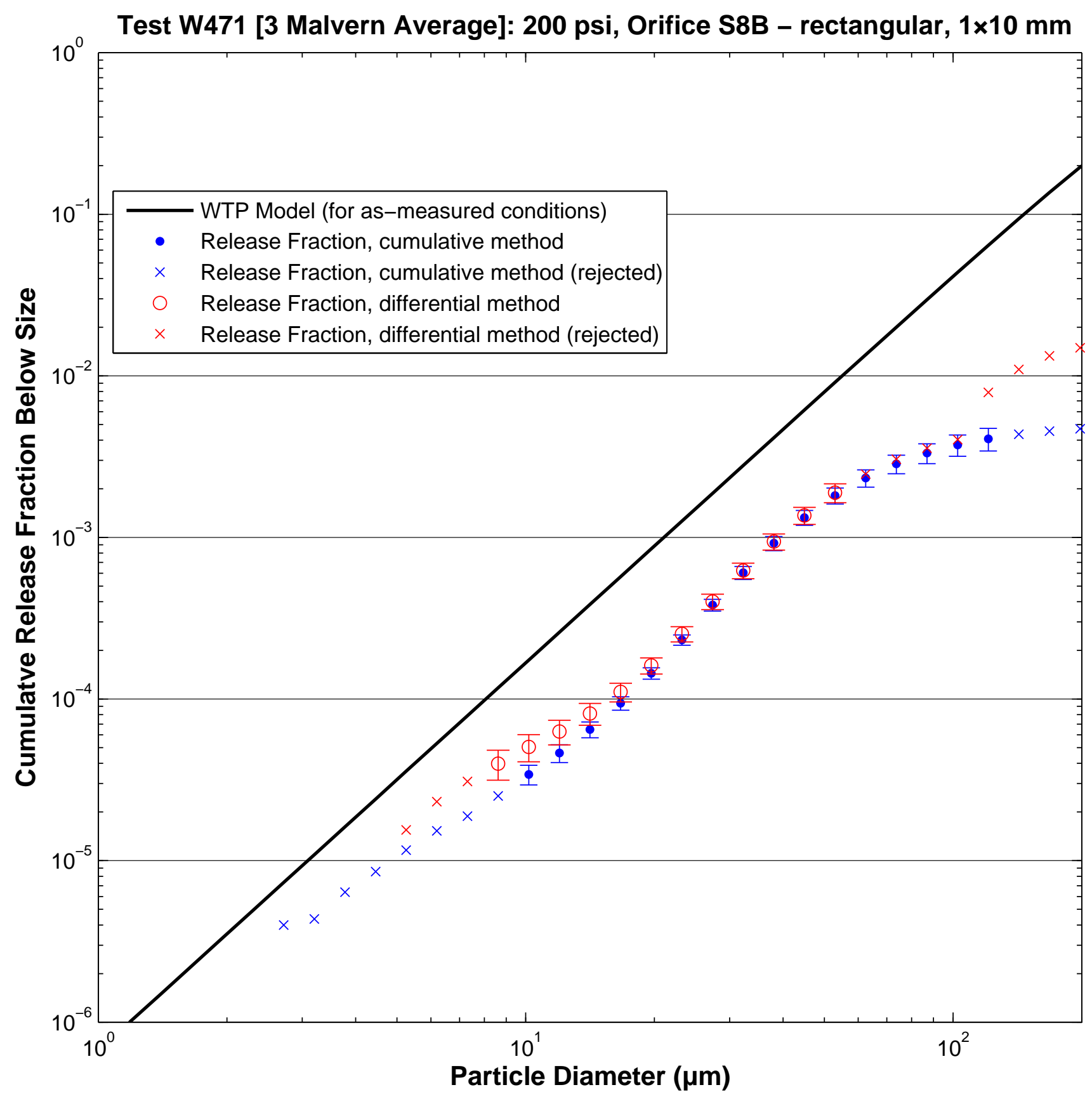

B. 135 


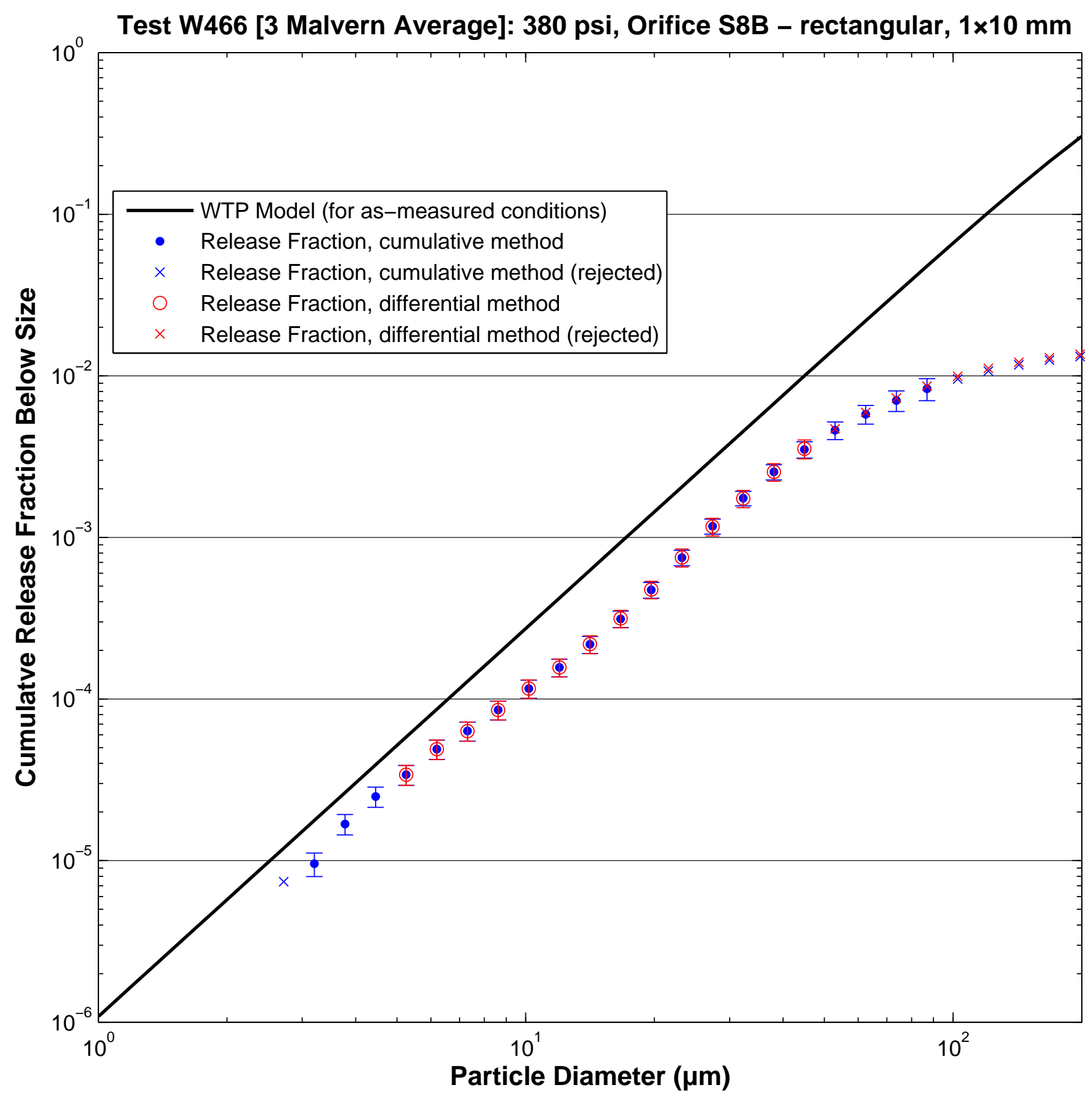

B. 136 


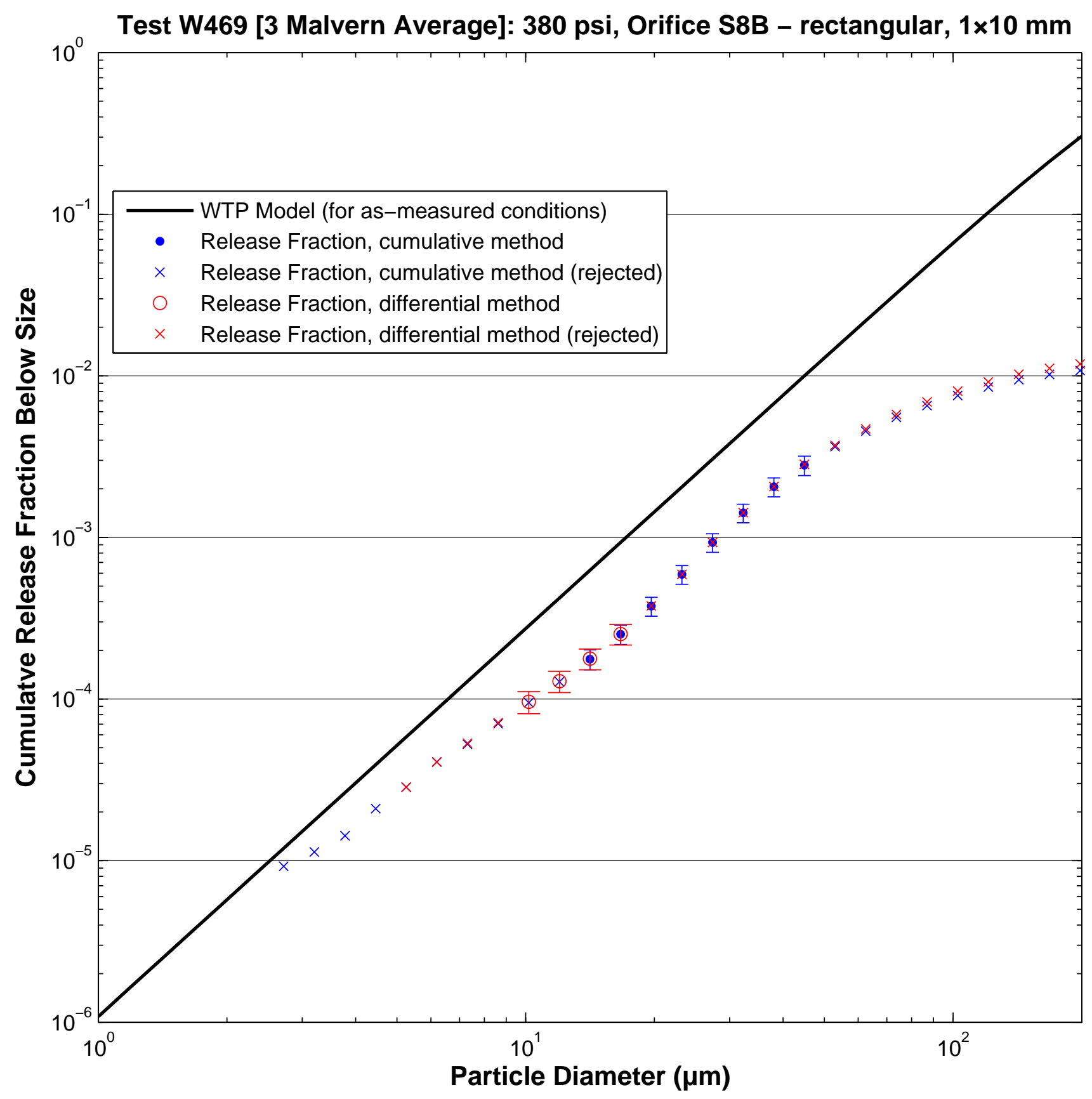




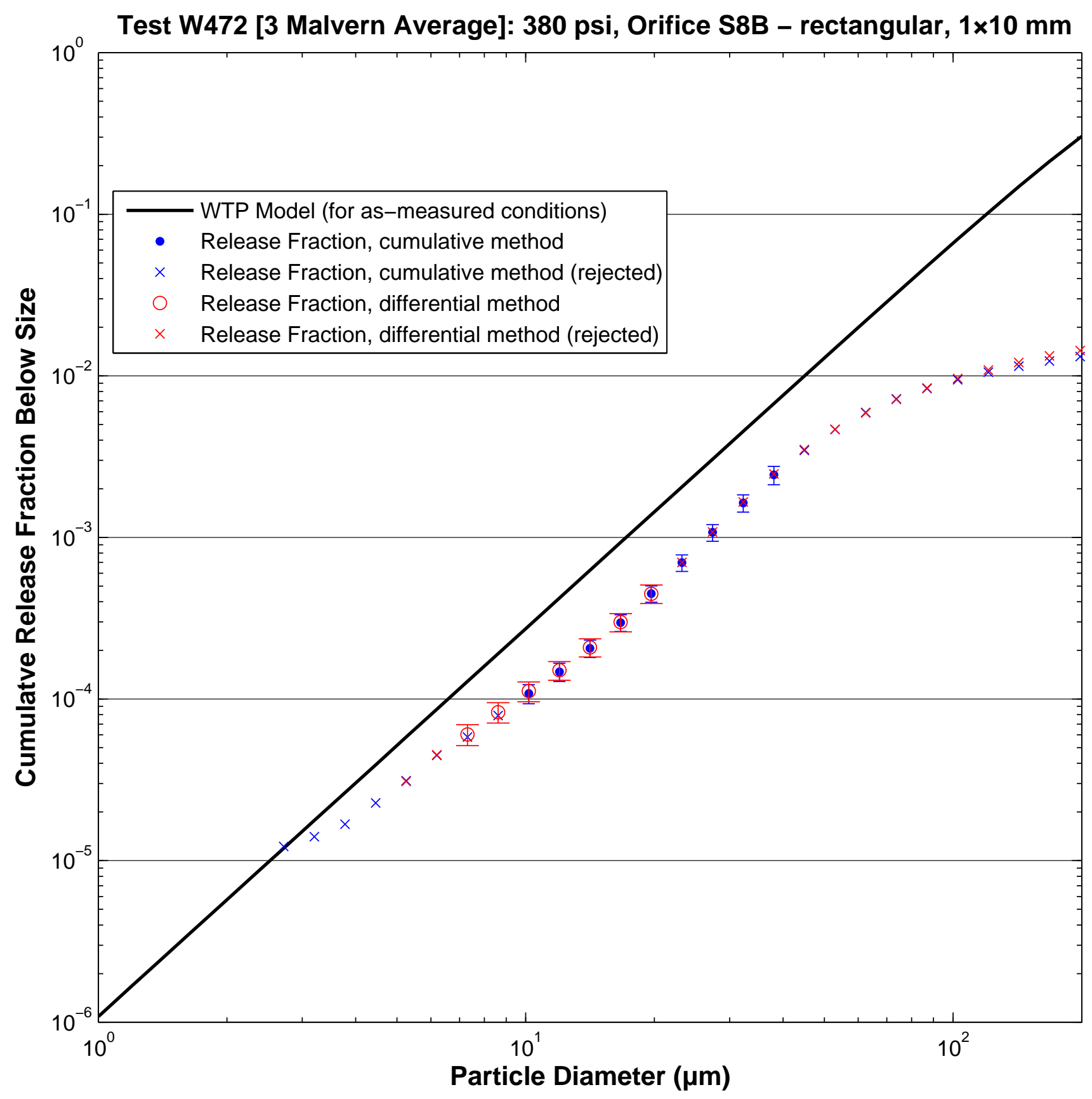

B. 138 


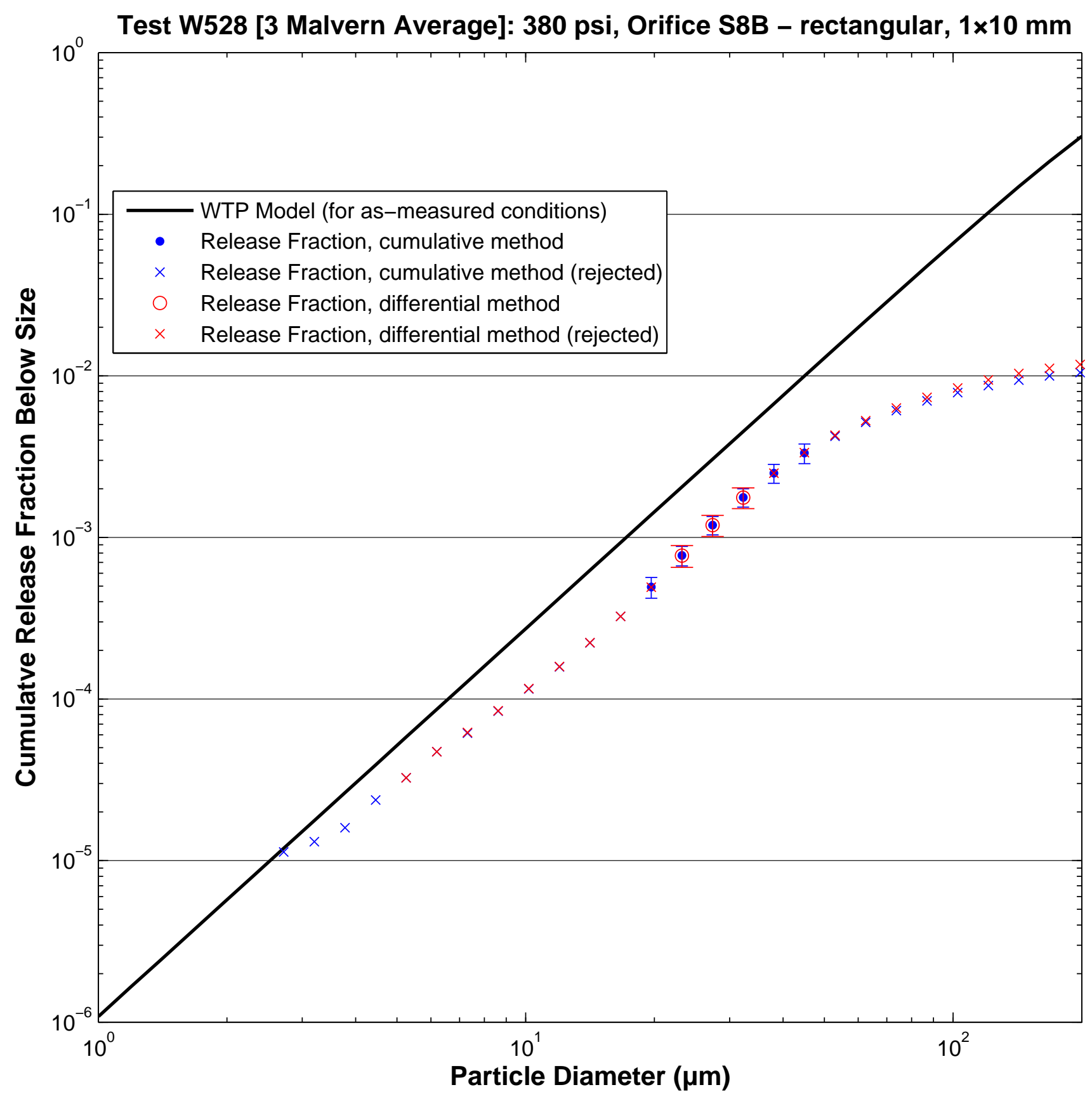

B. 139 


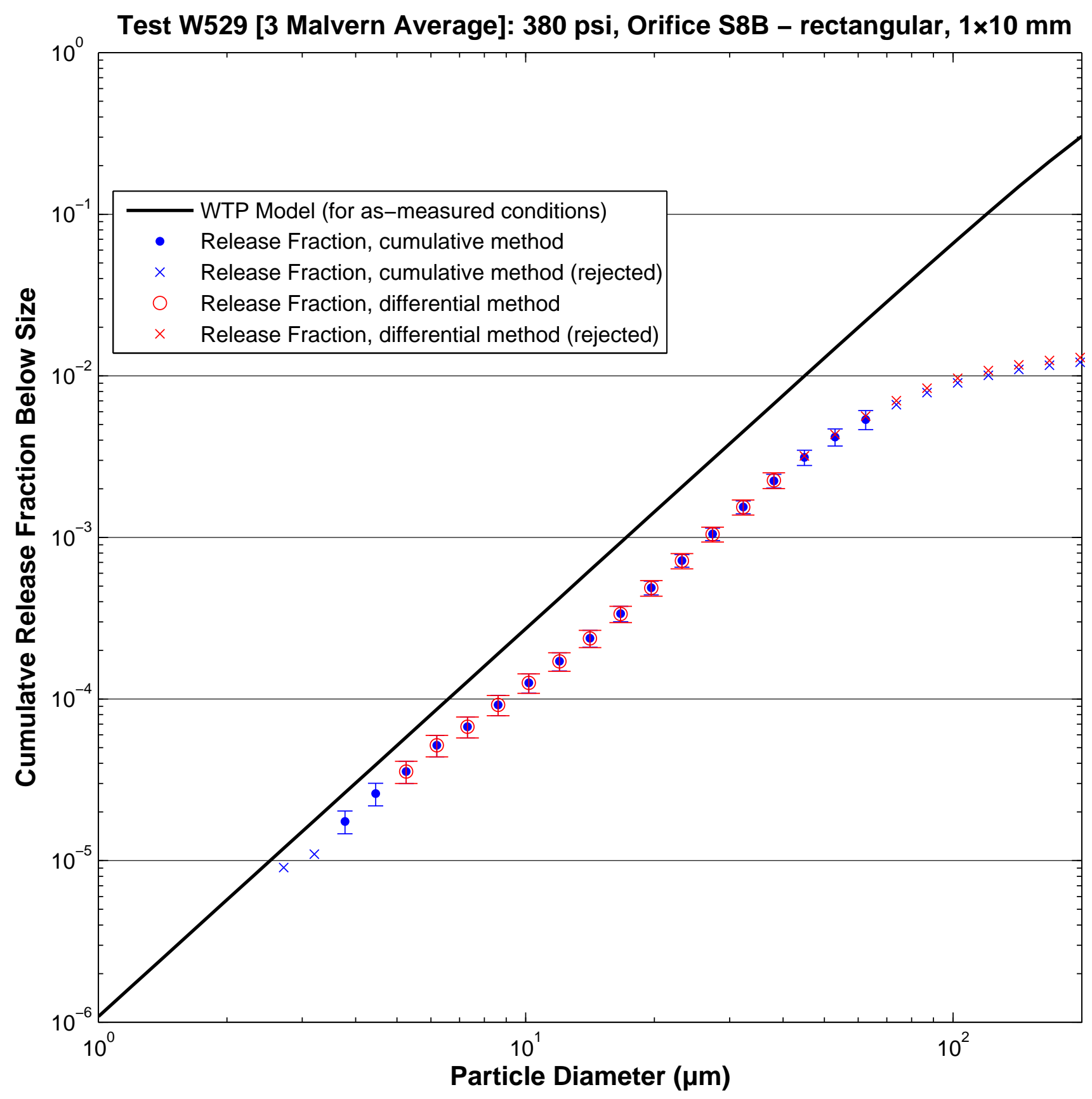

B. 140 


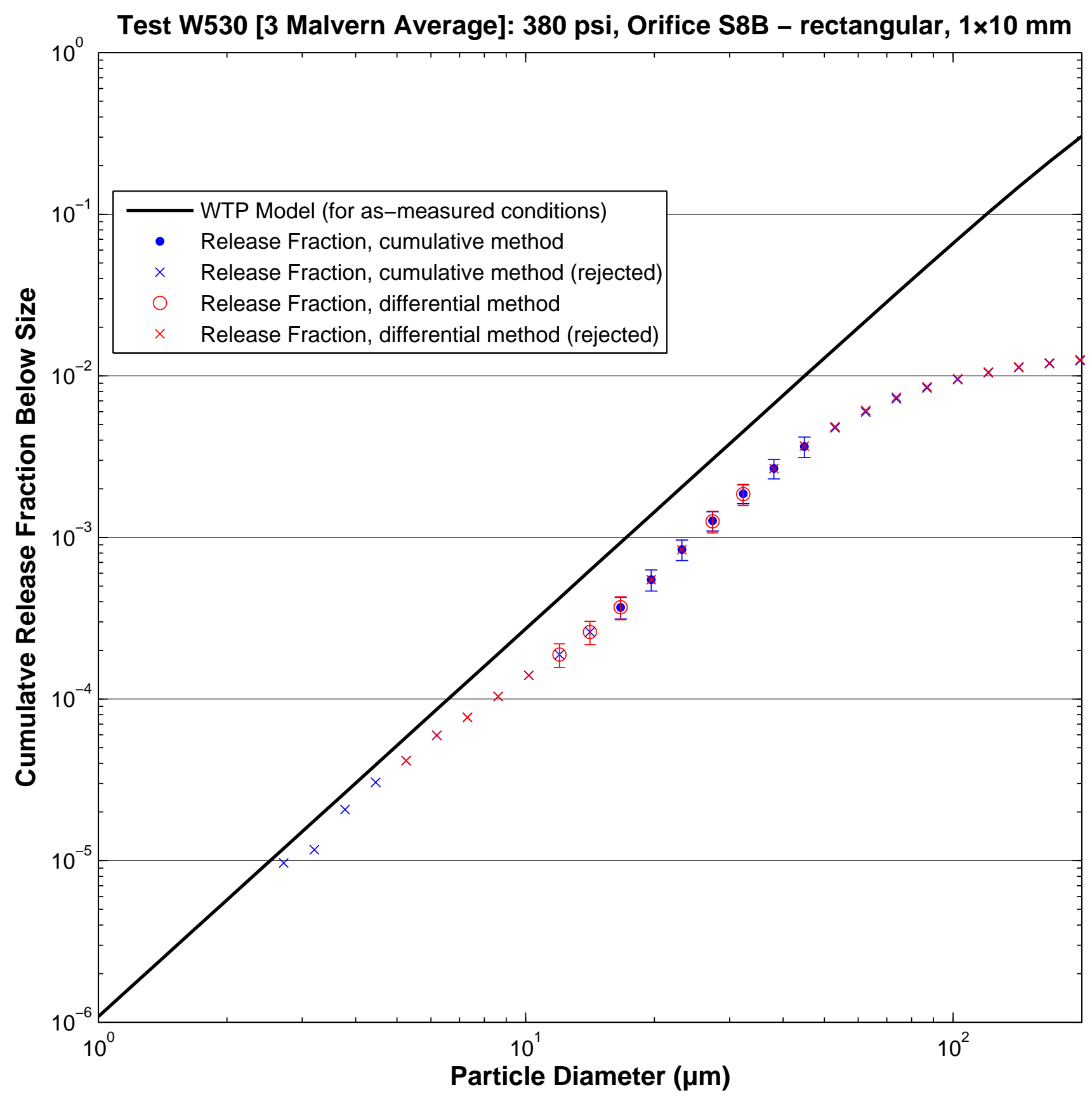




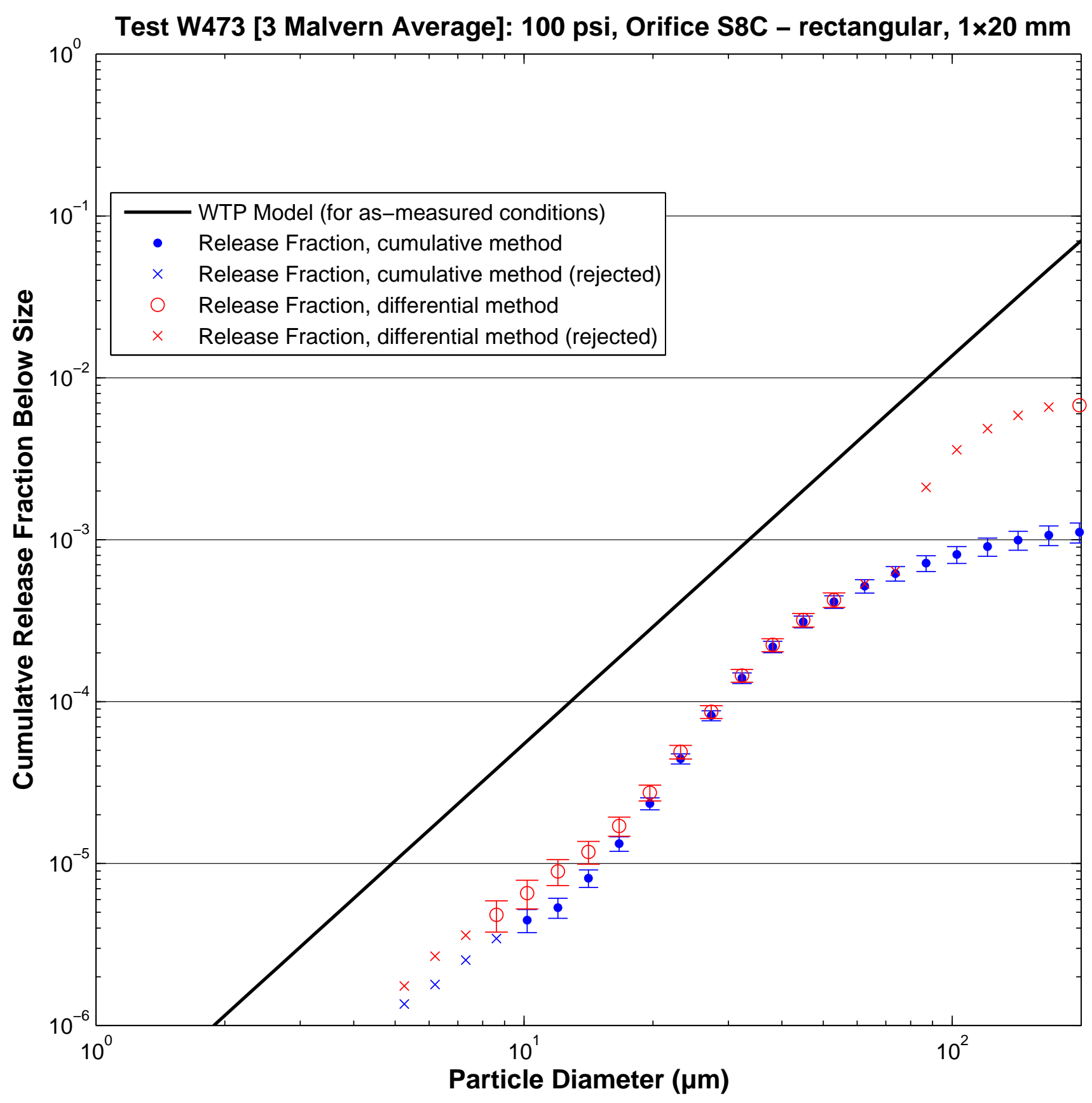

B. 142 


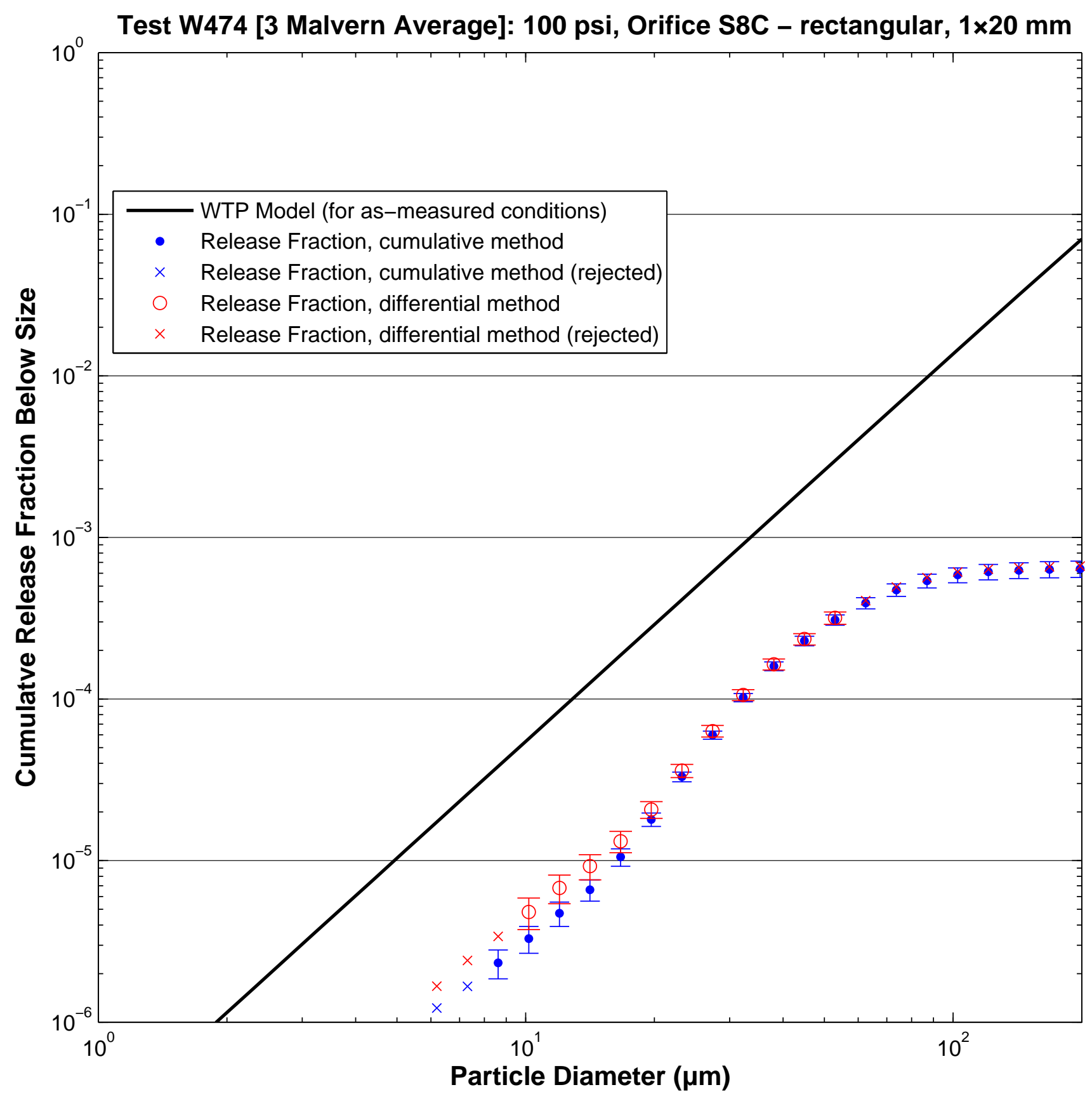




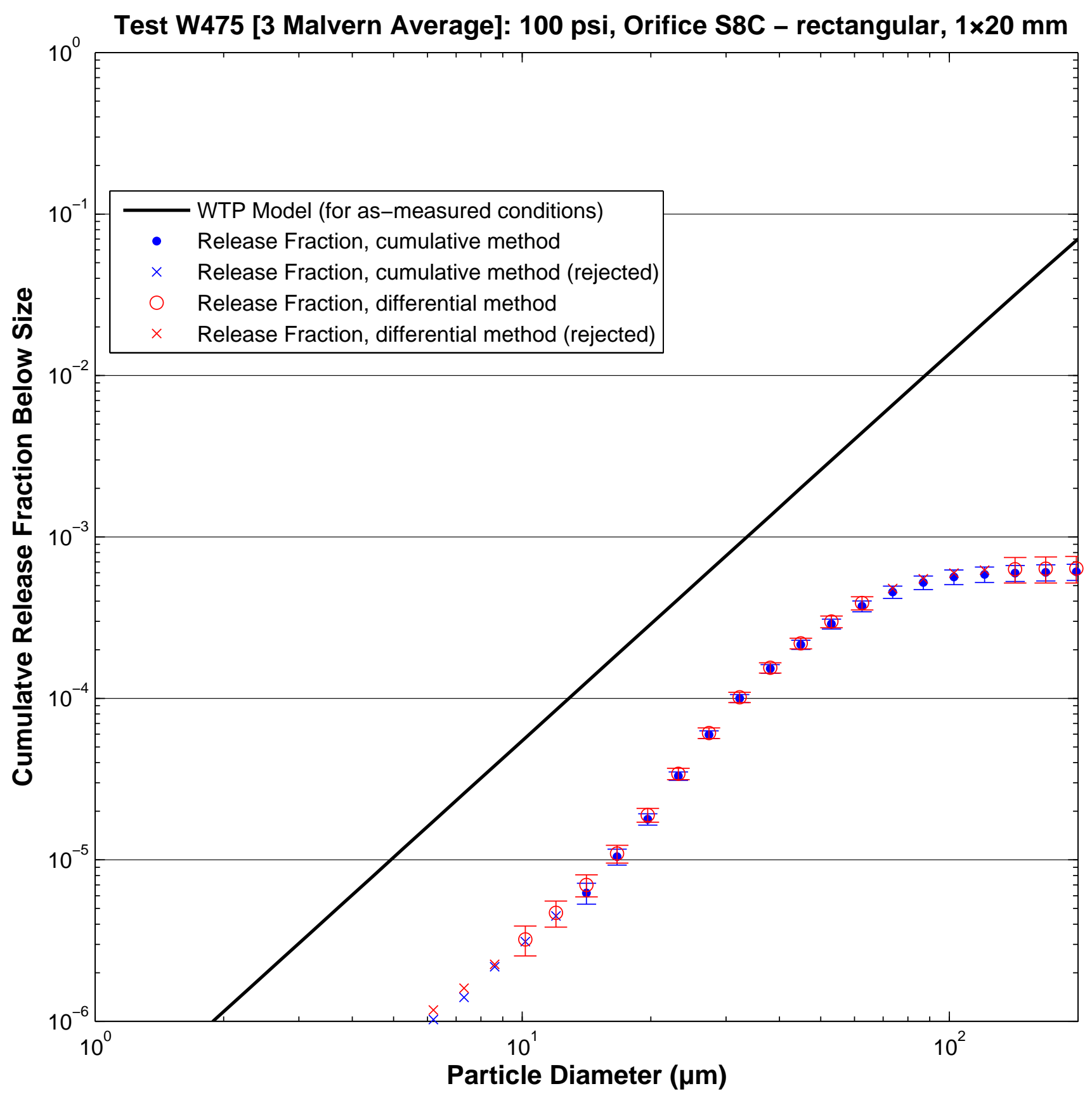




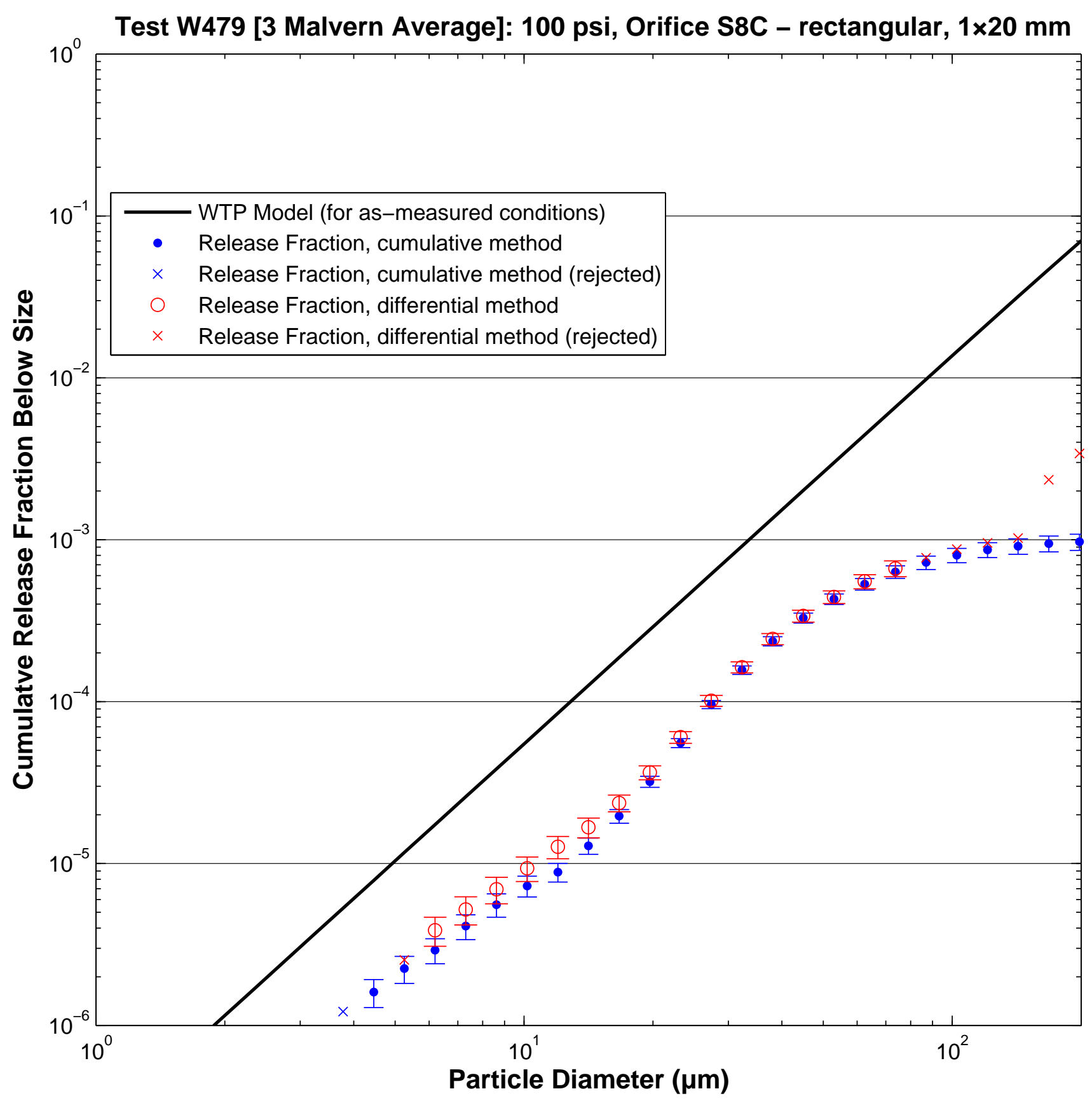




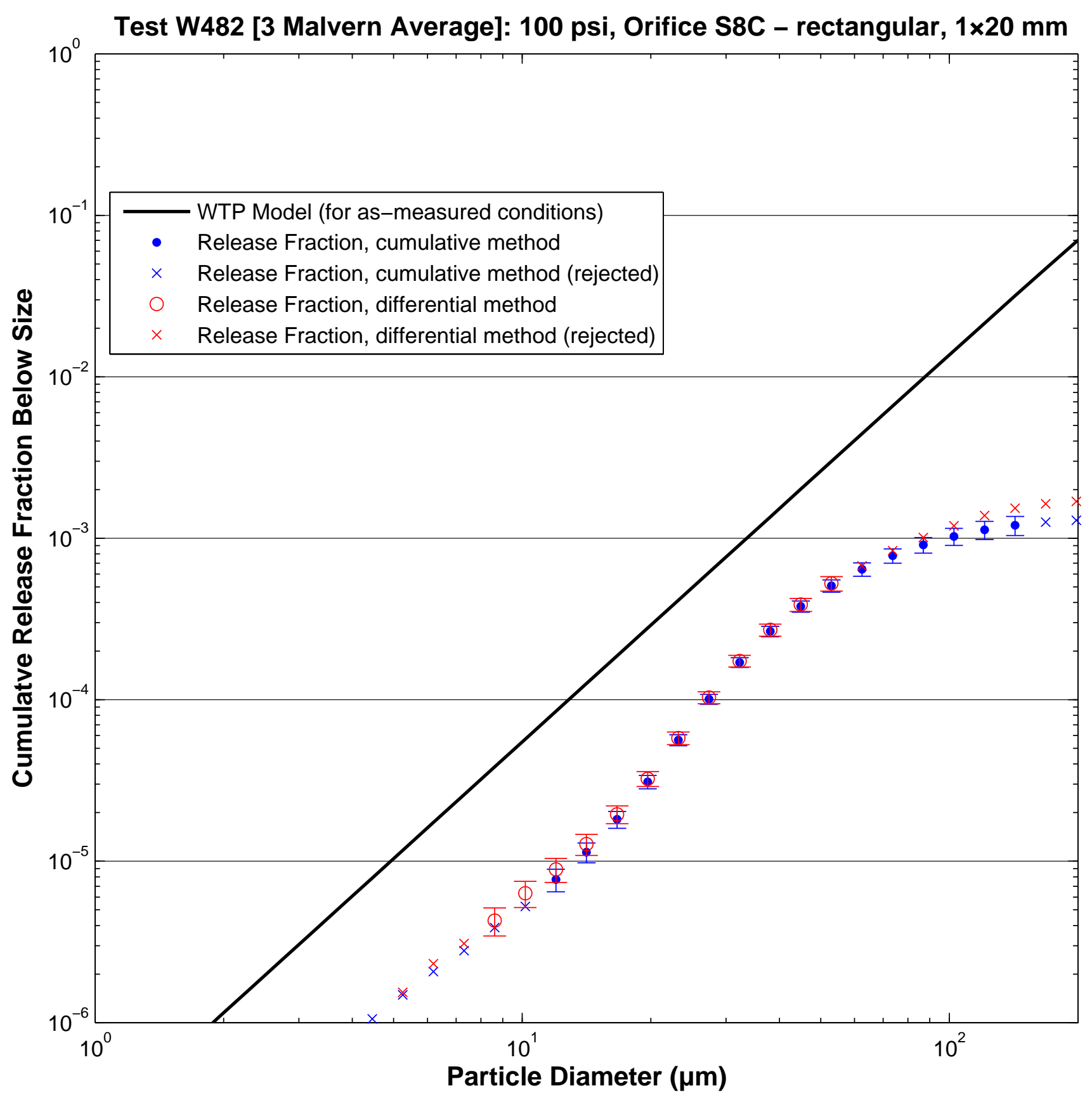




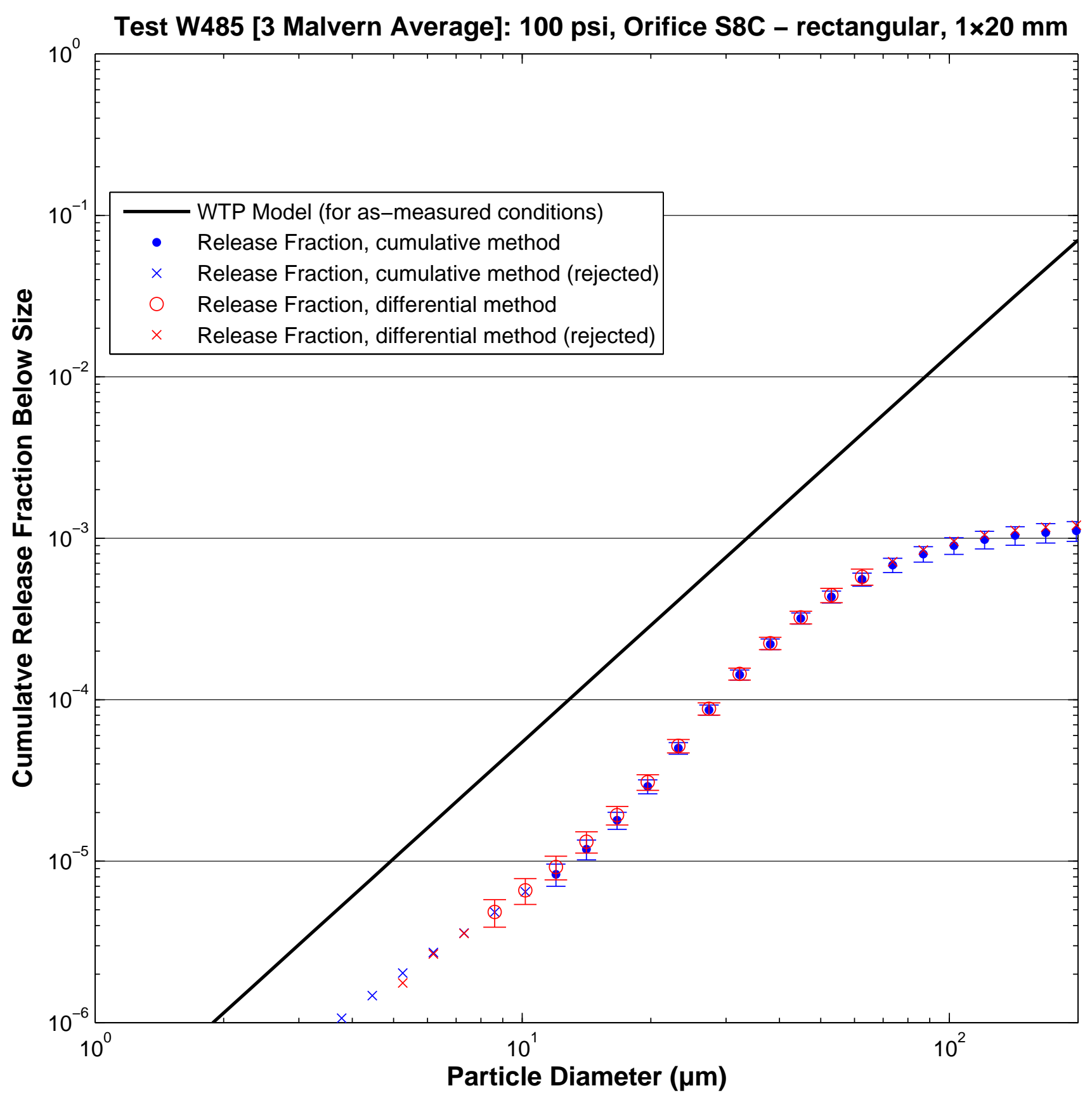




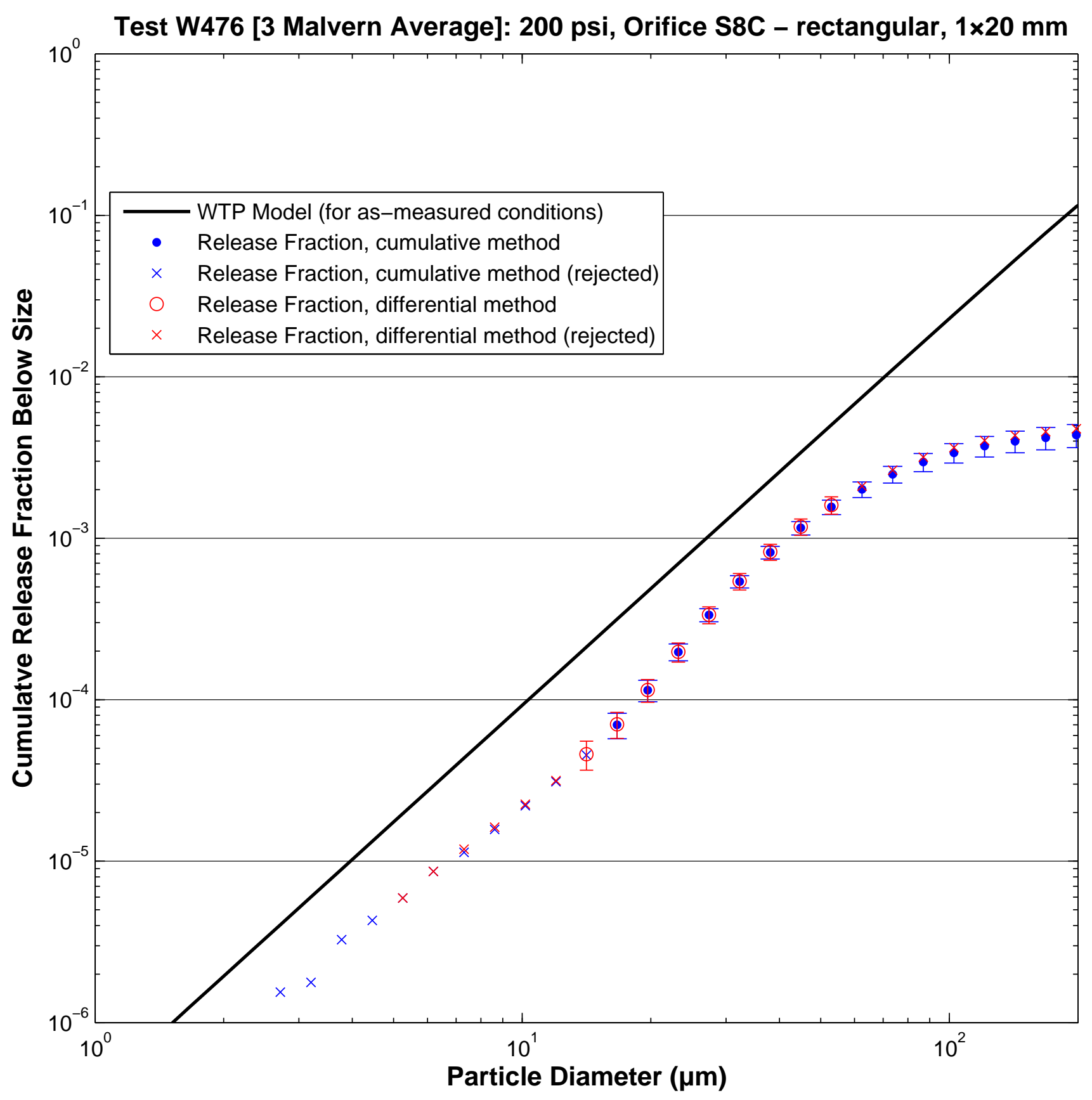

B. 148 


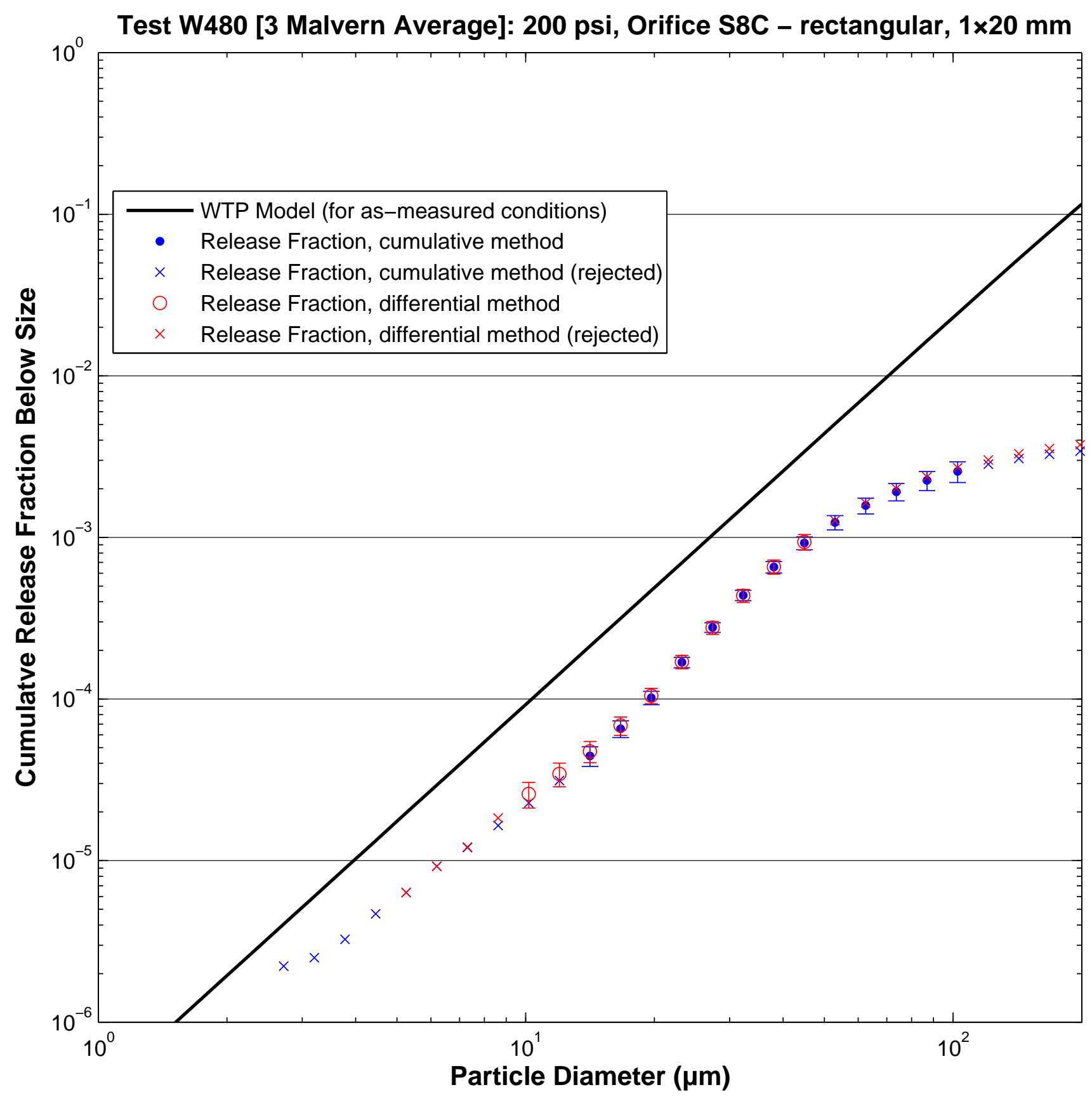

B. 149 


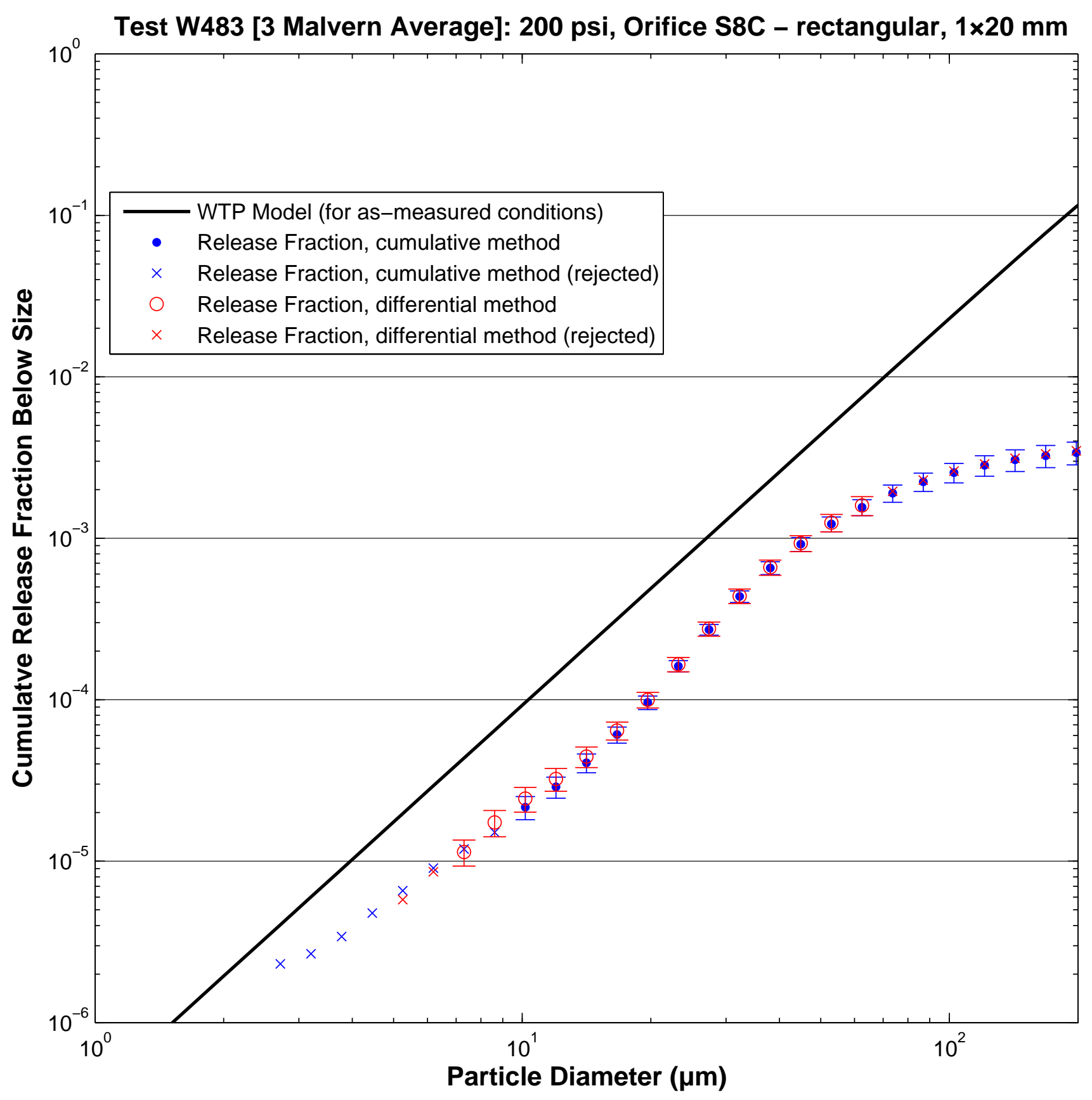

B. 150 


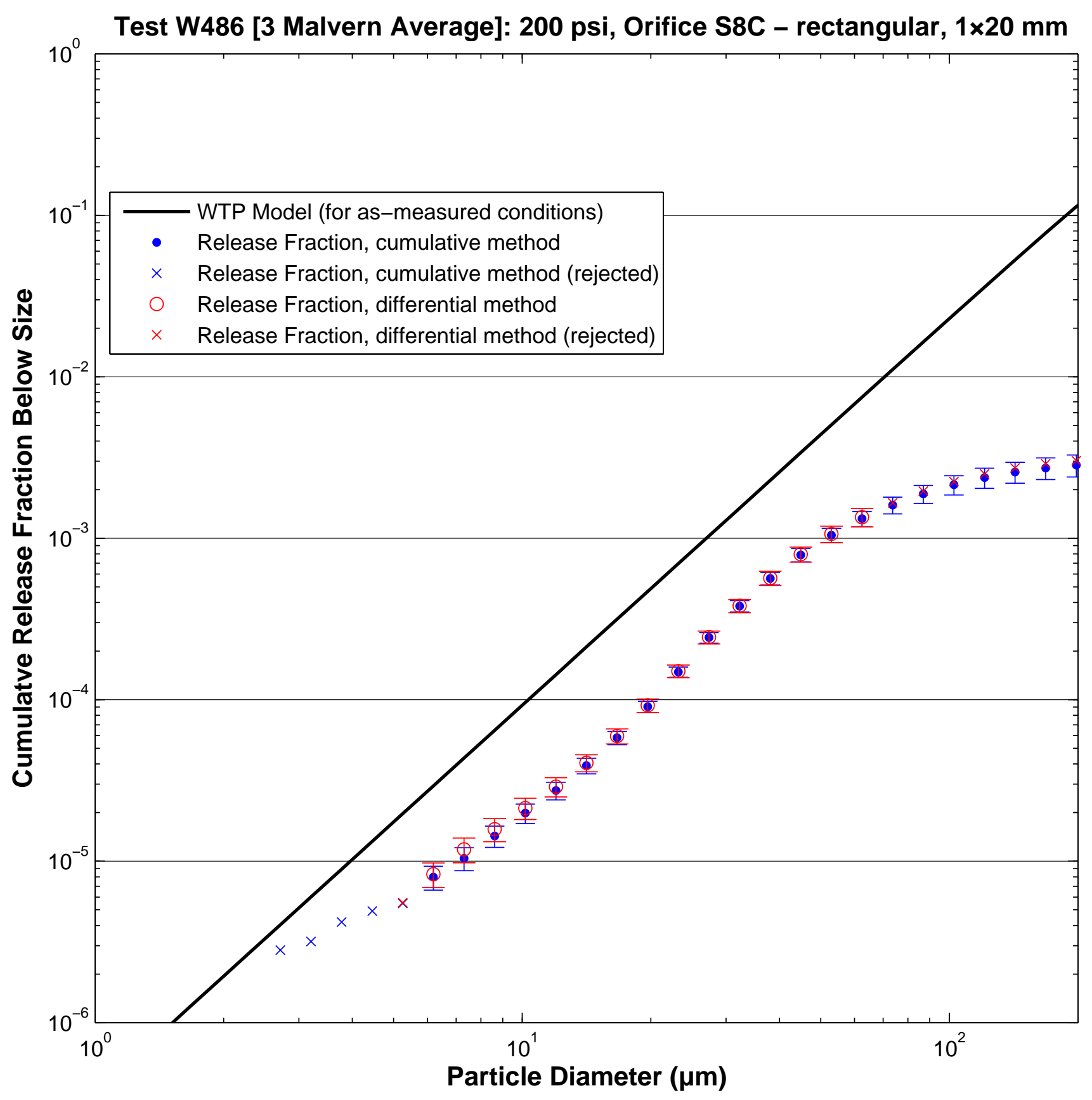




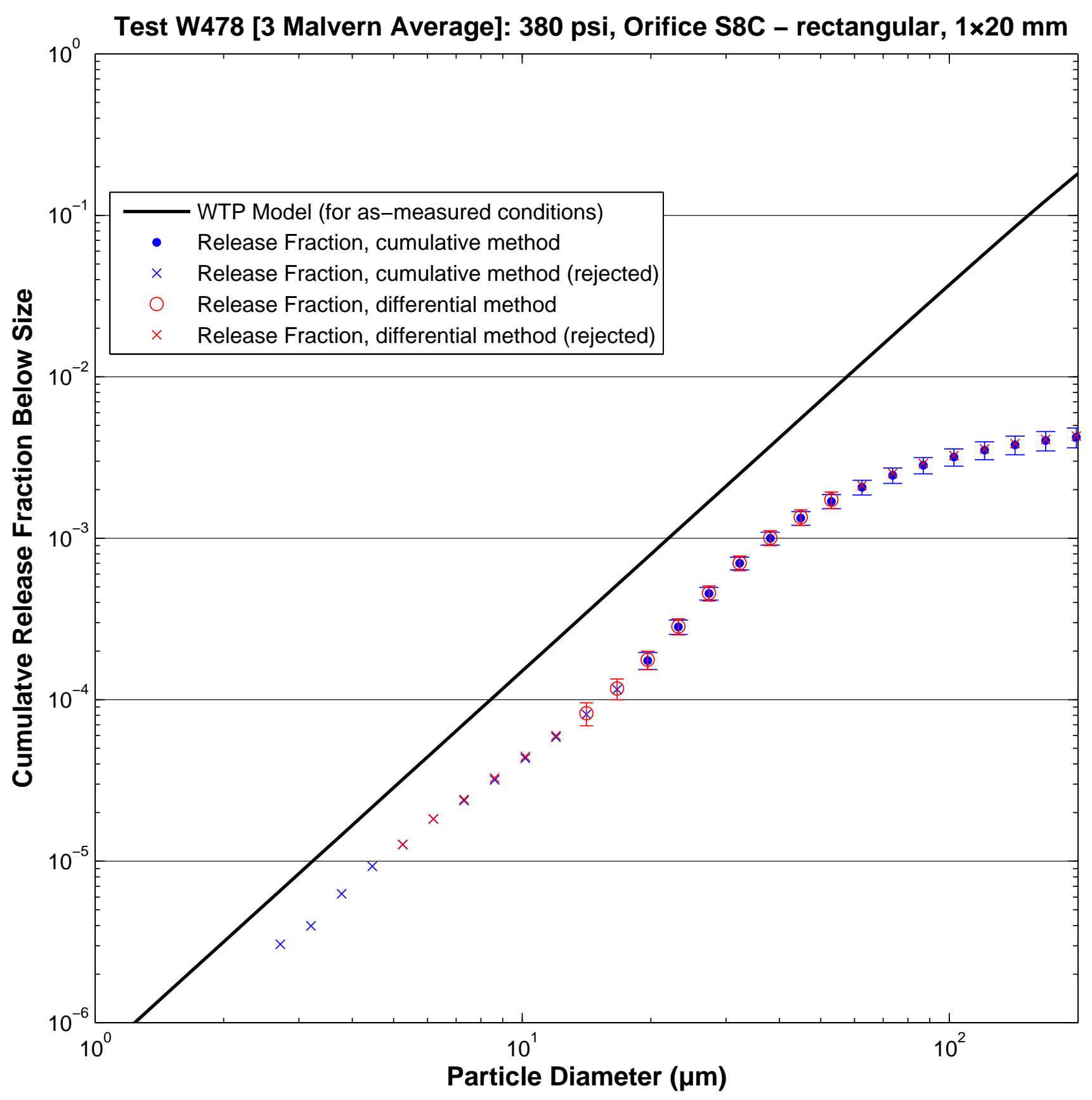

B. 152 


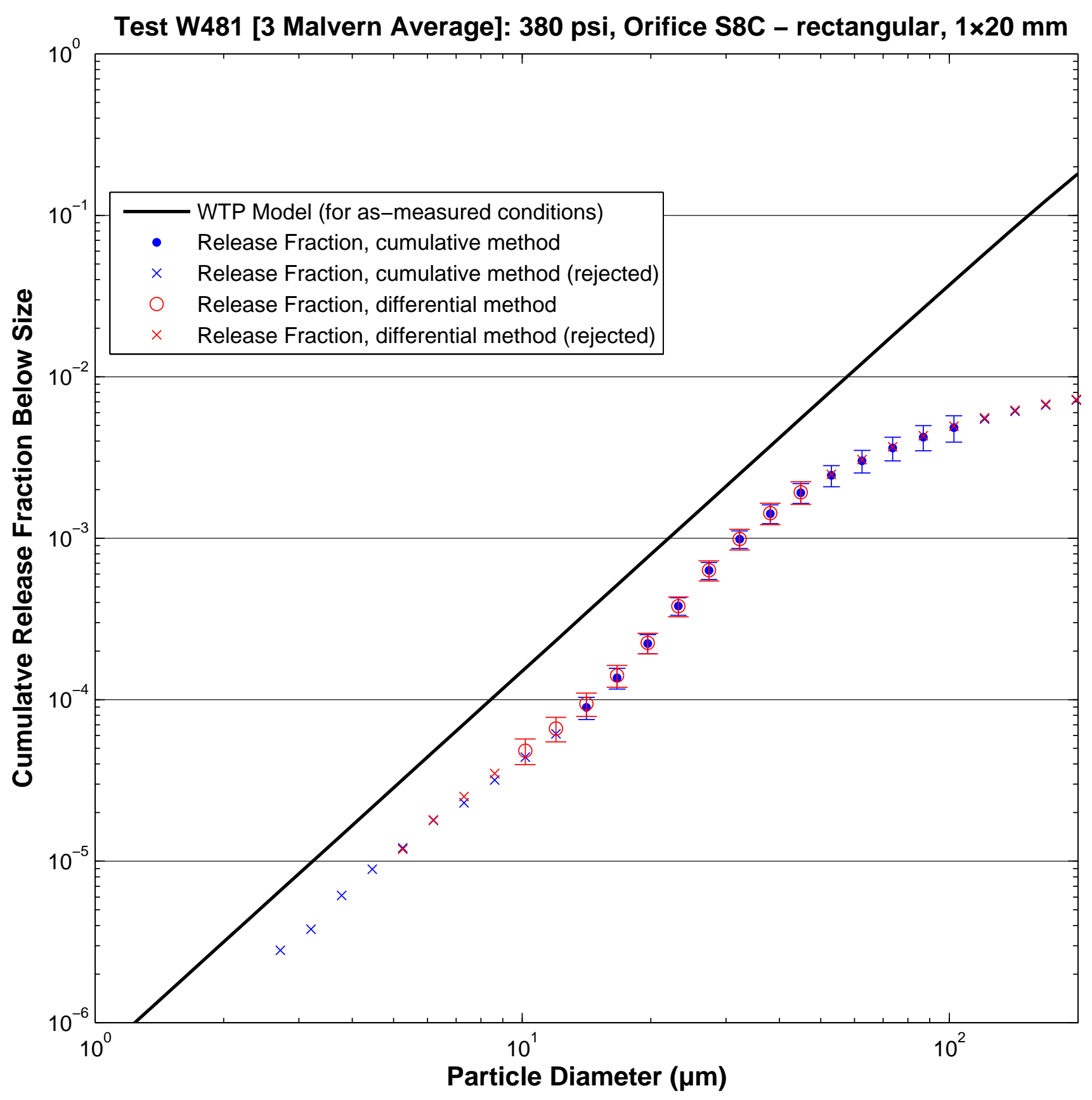




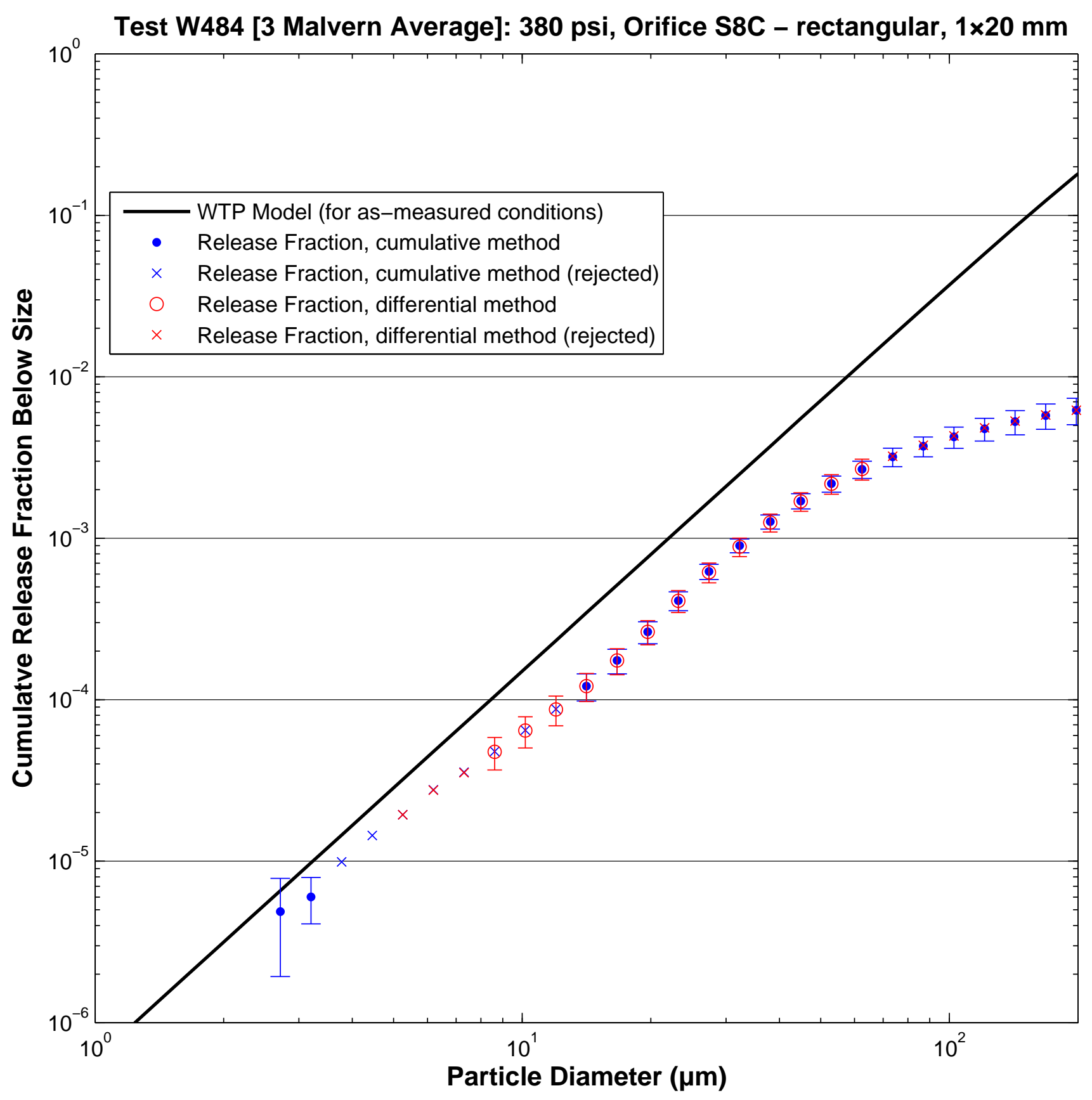

B. 154 


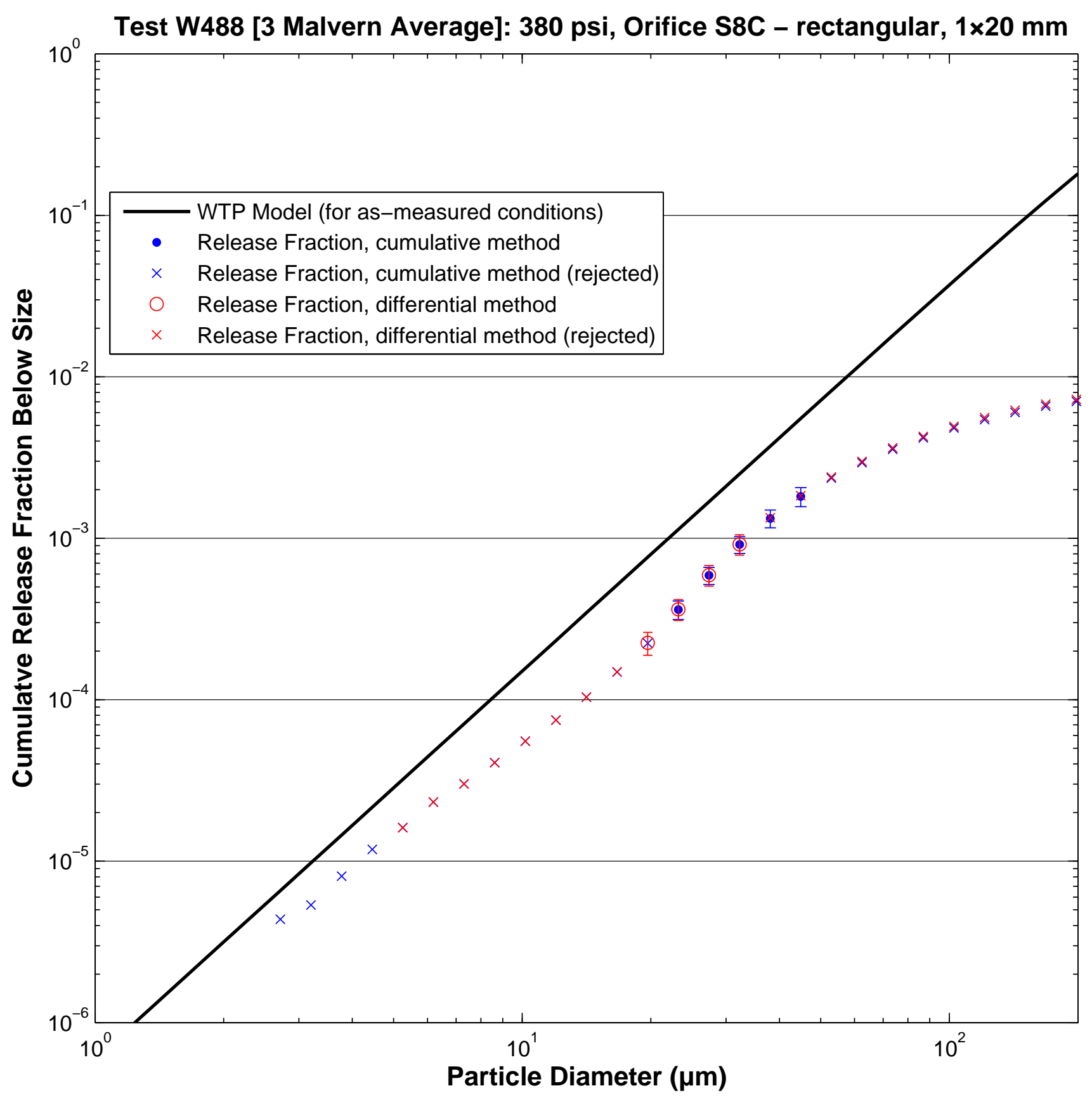

B. 155 


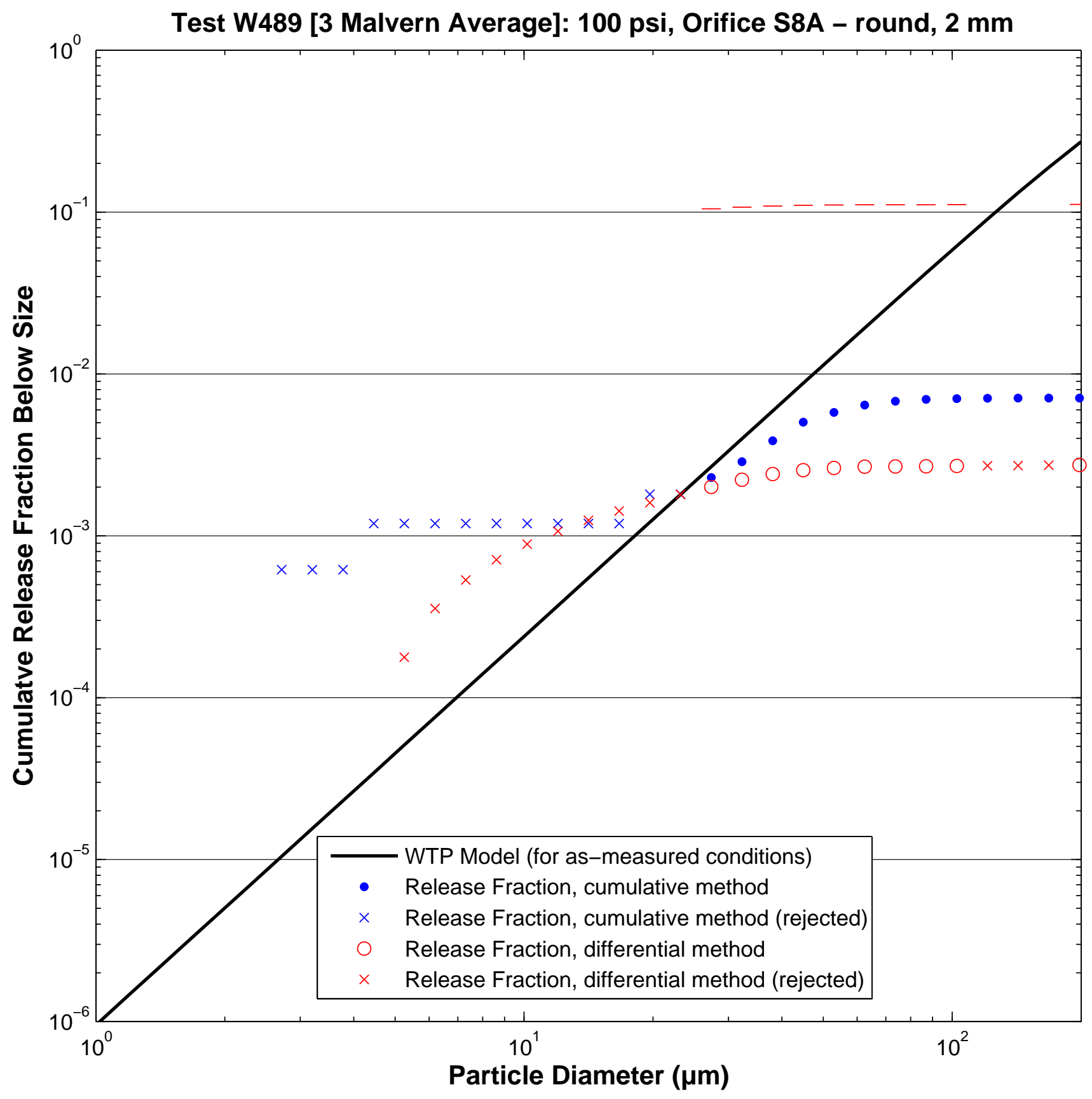

B. 156 


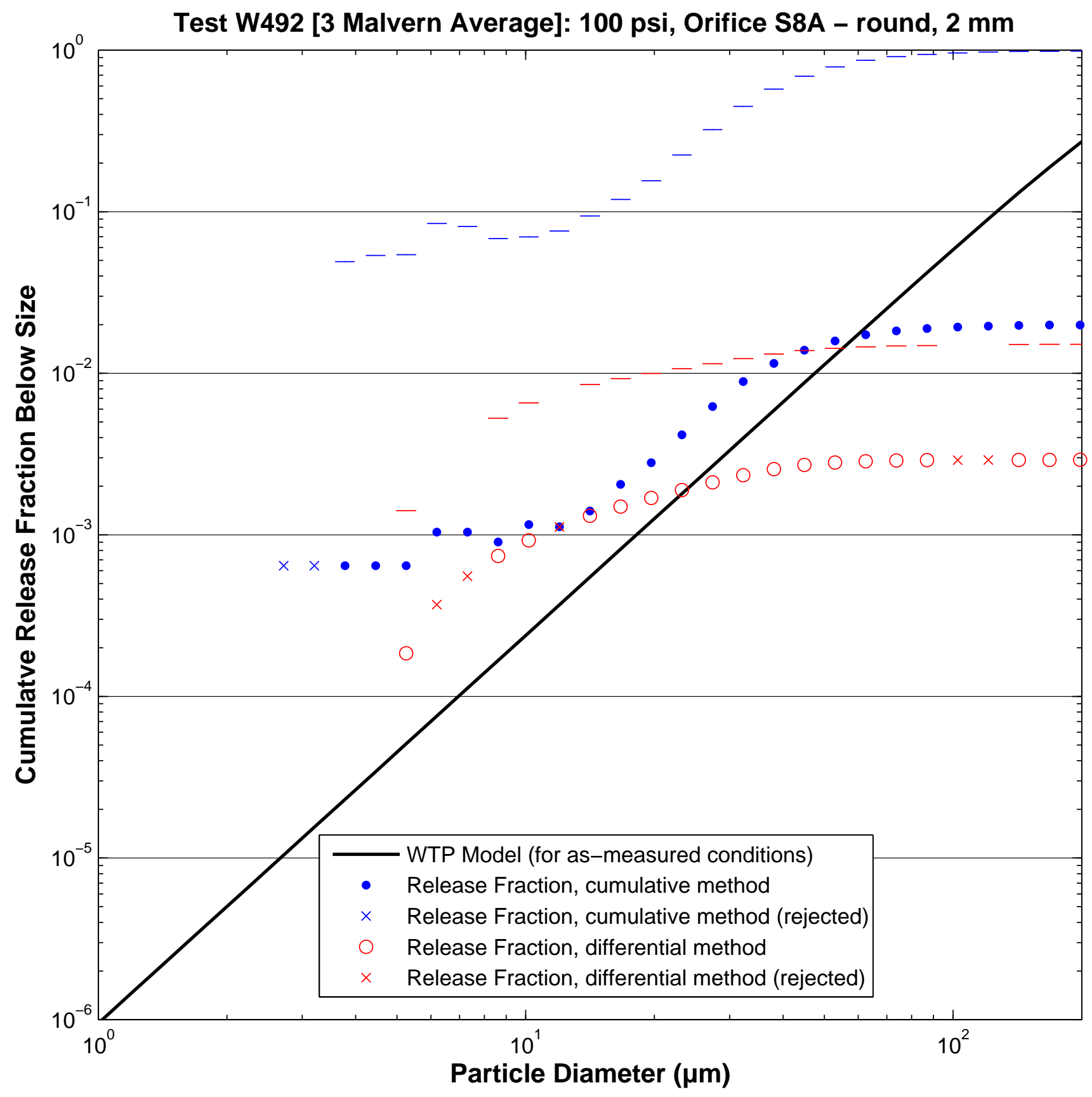




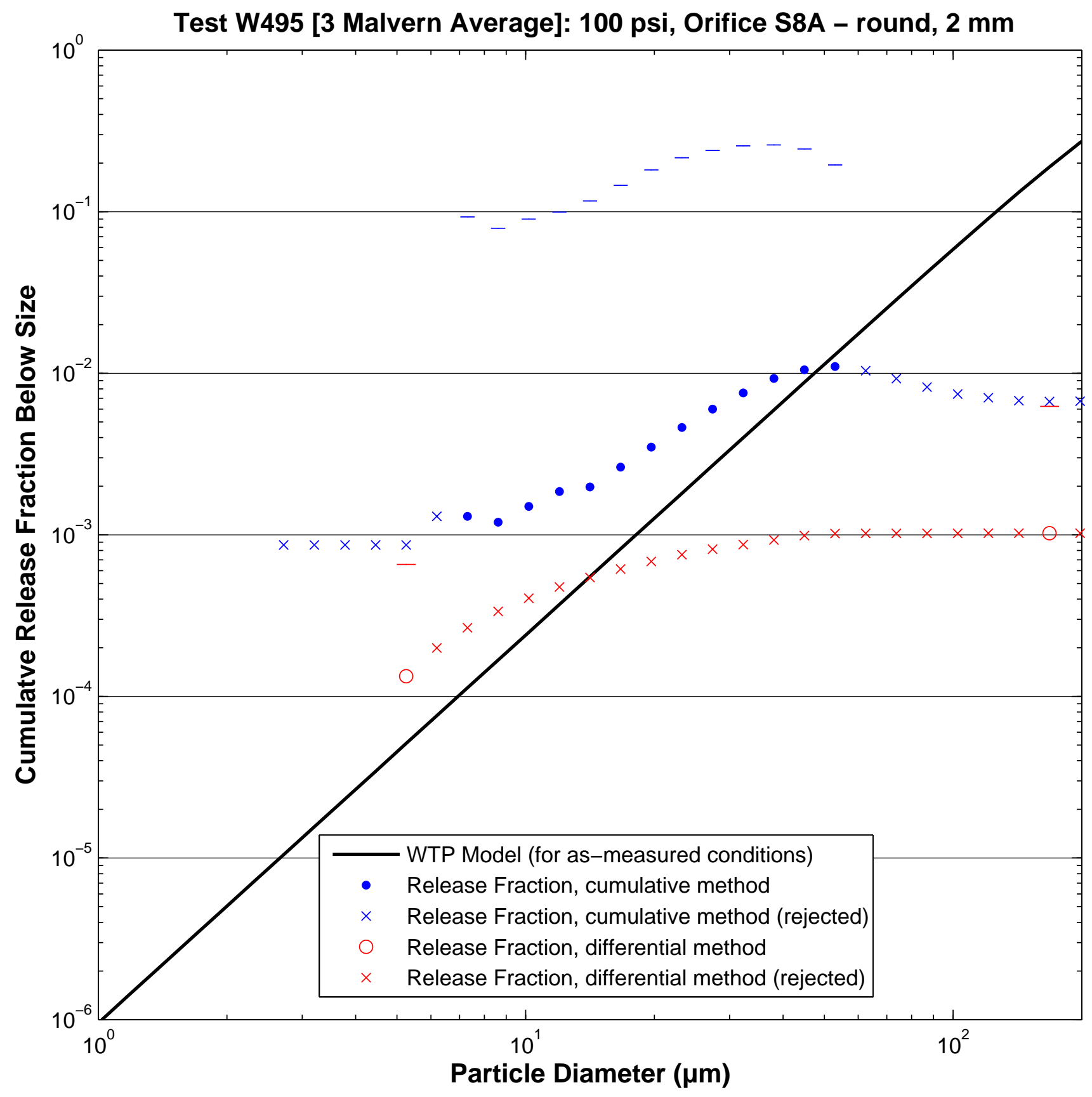

B. 158 


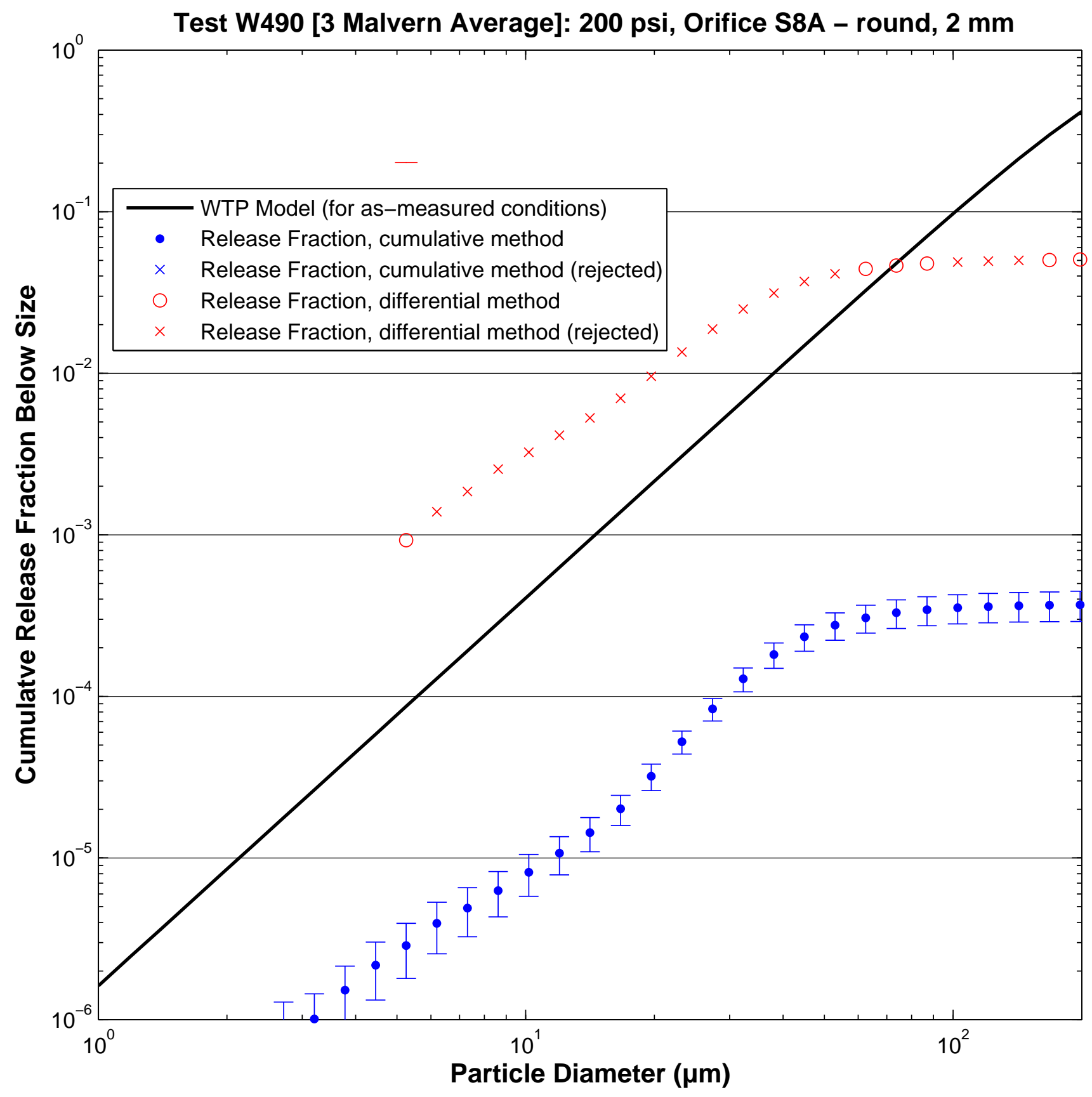

B. 159 


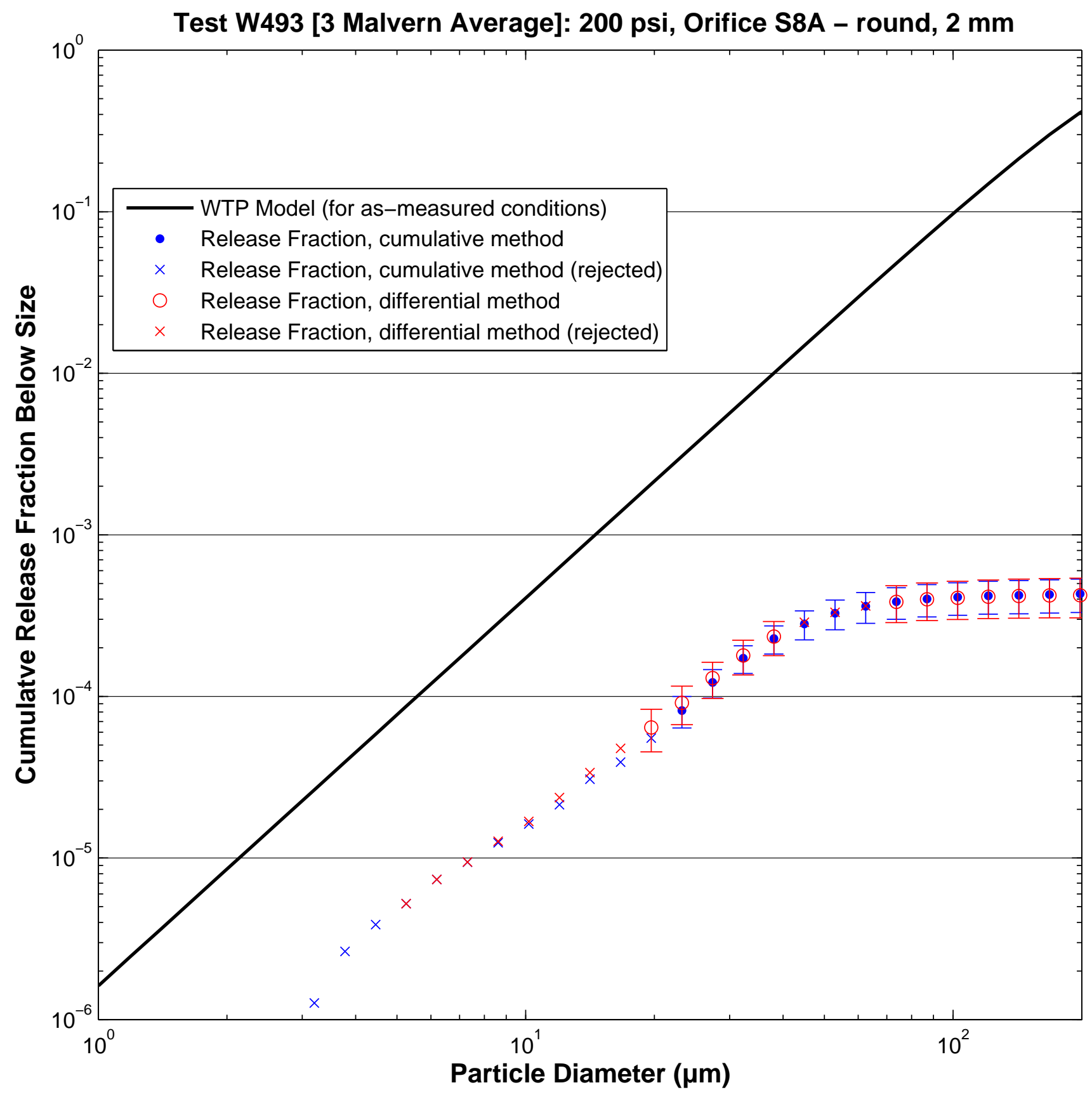

B. 160 


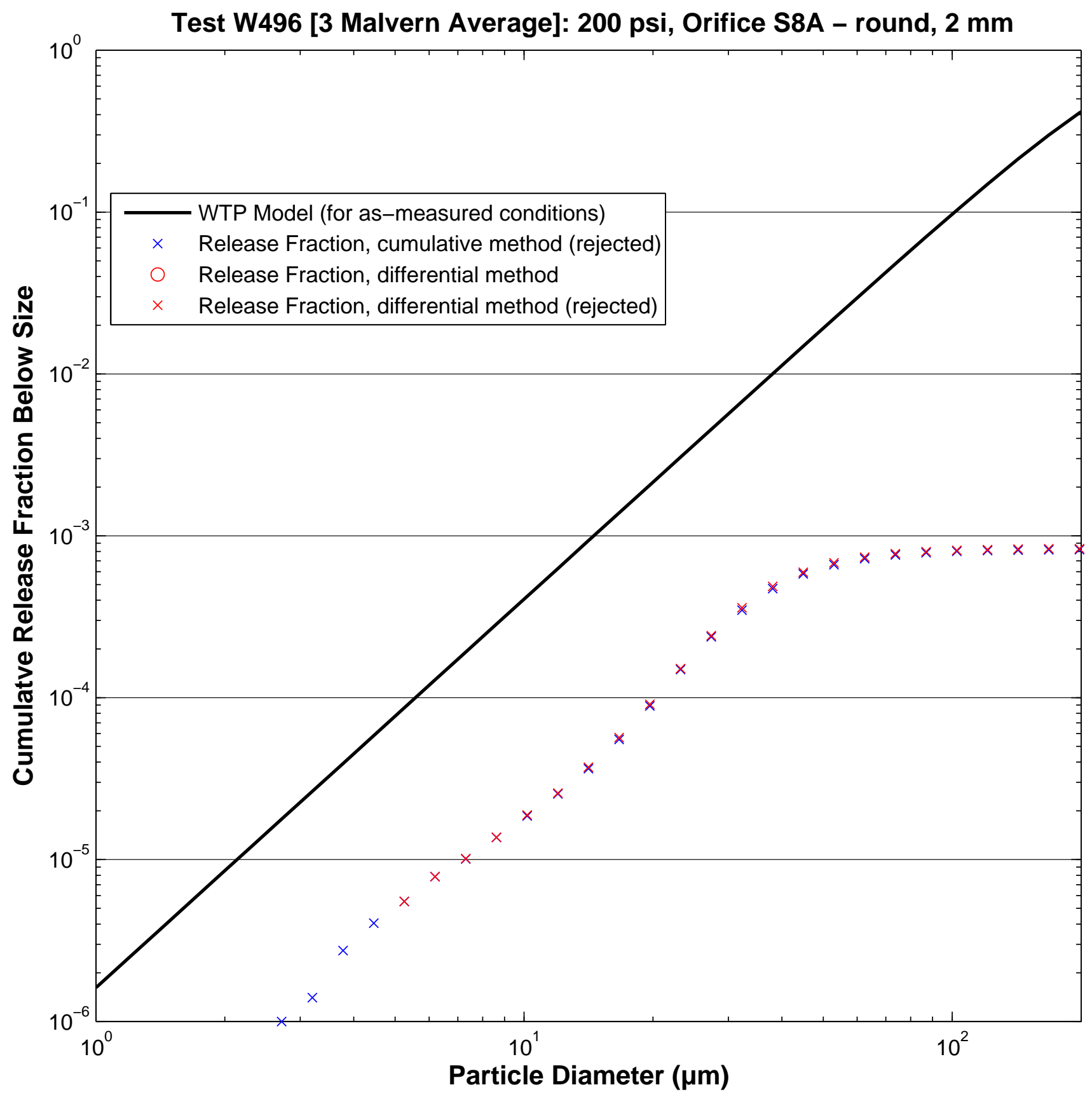




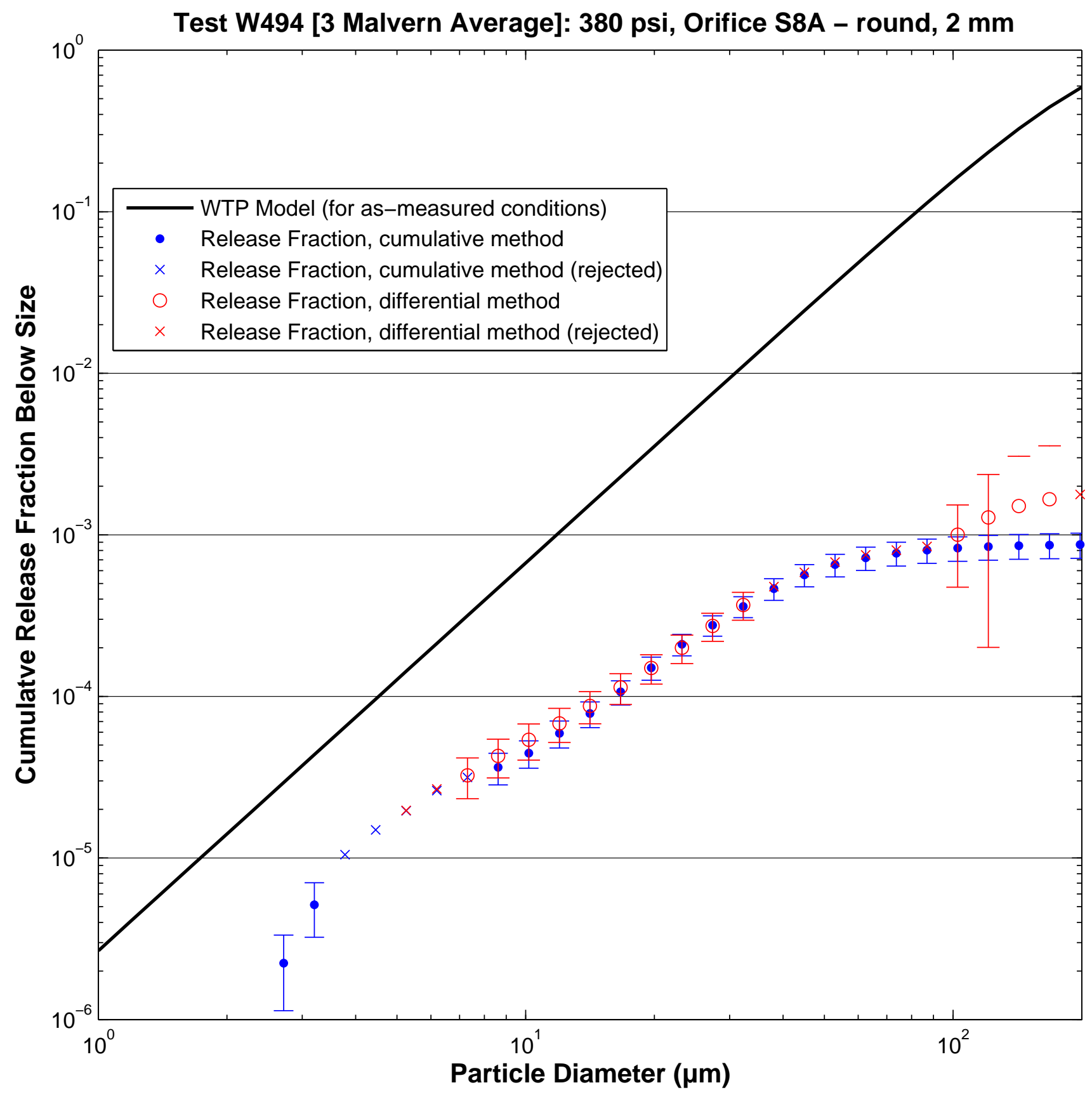




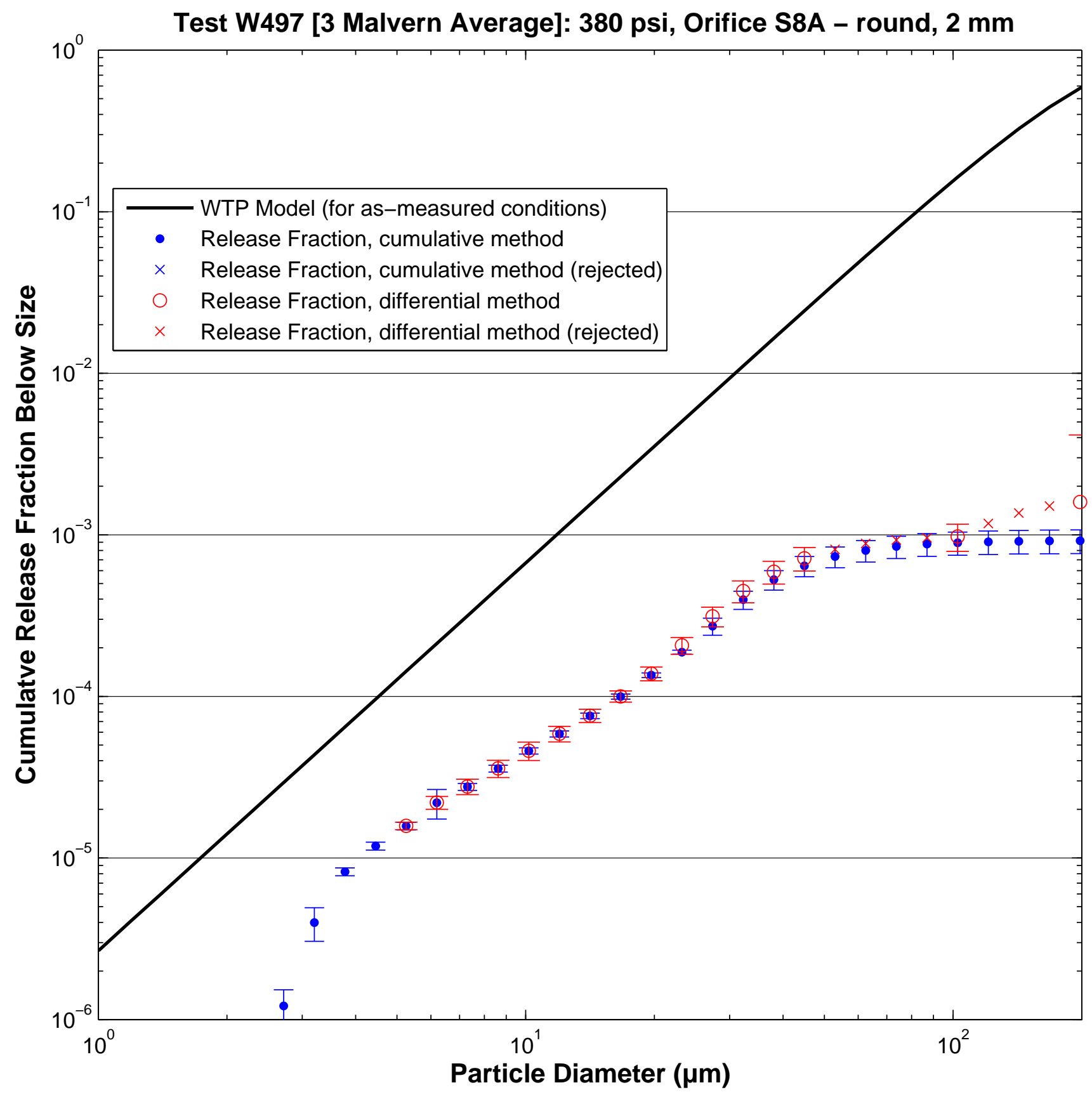




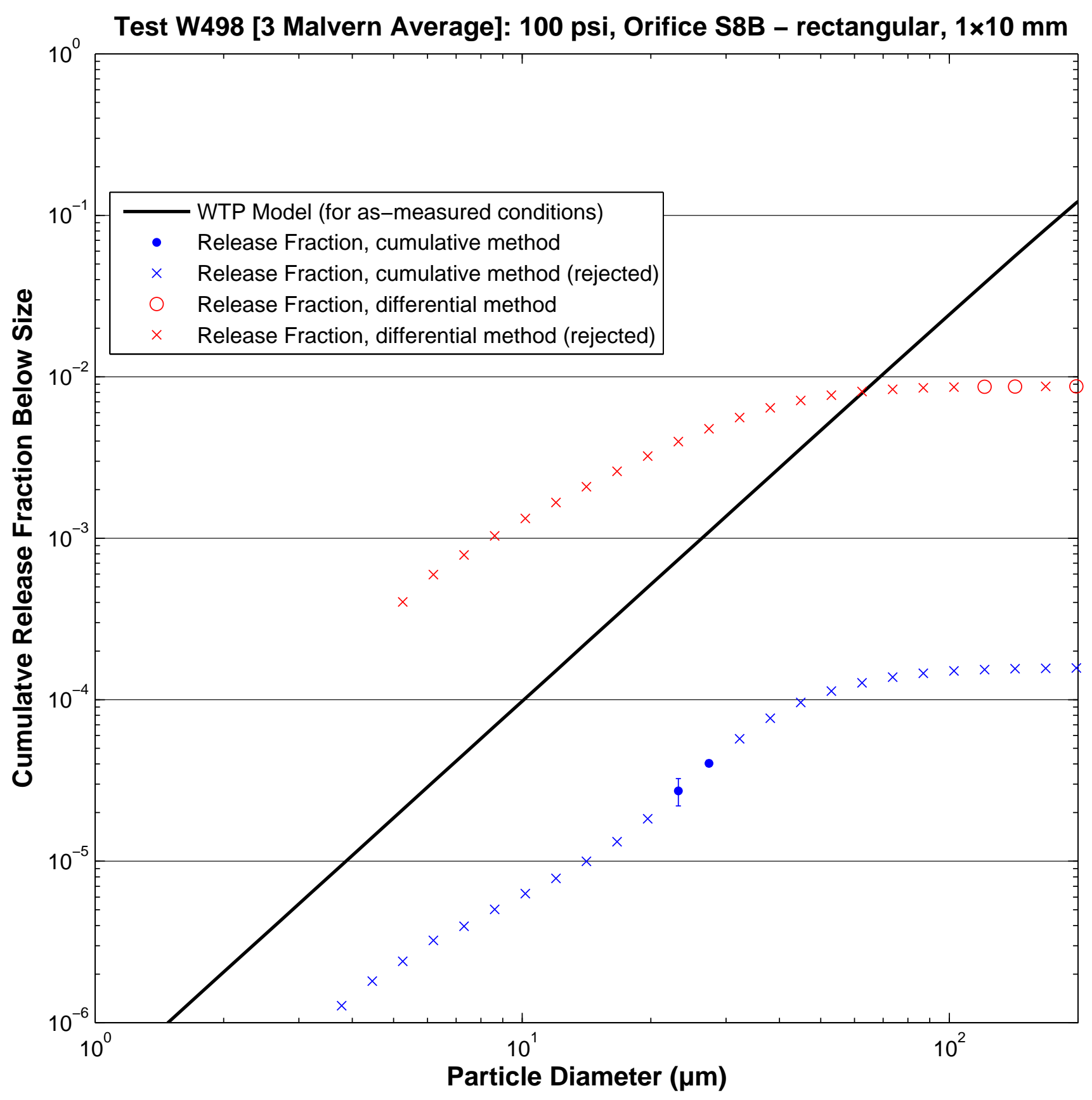

B. 164 


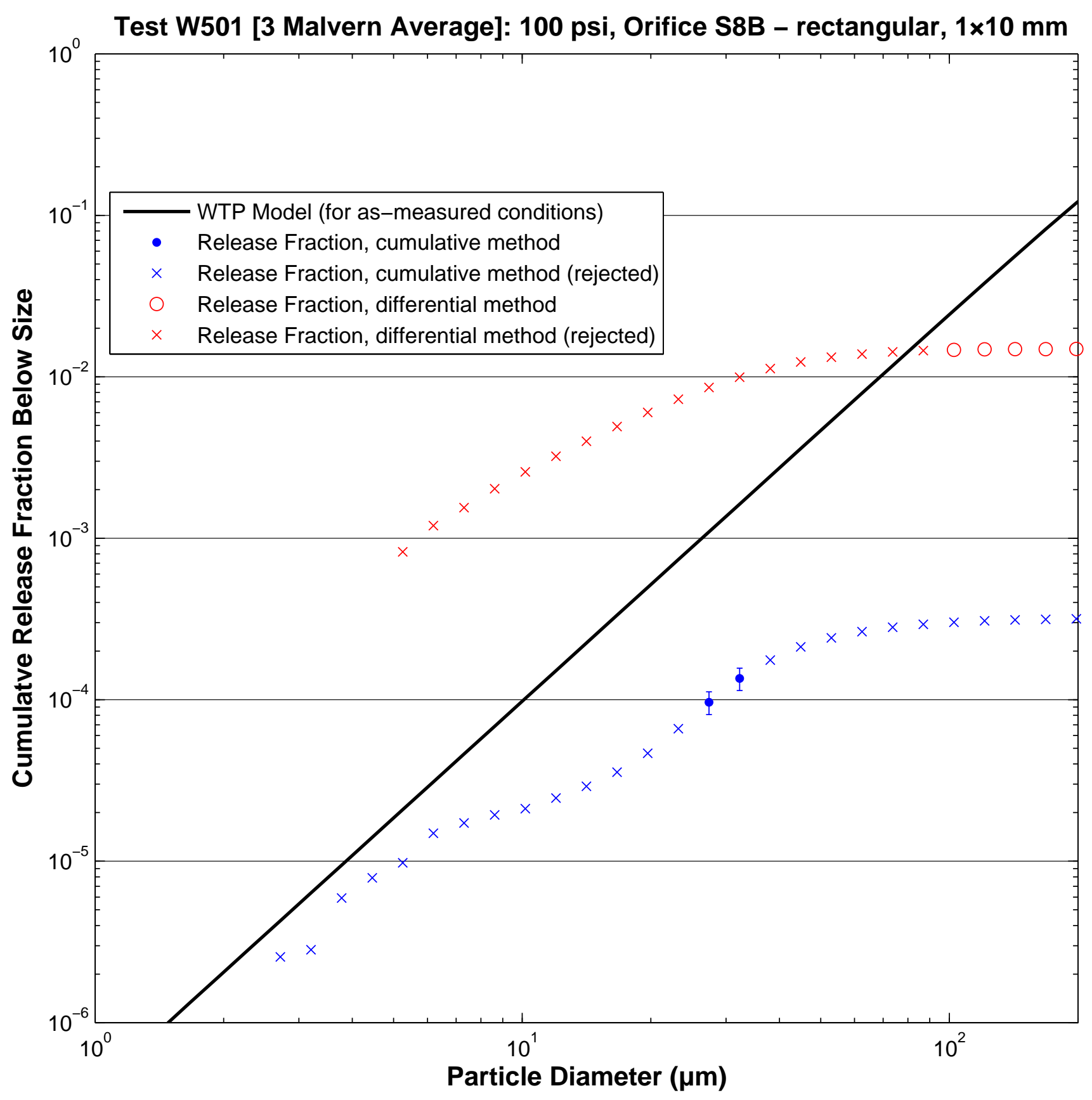

B. 165 


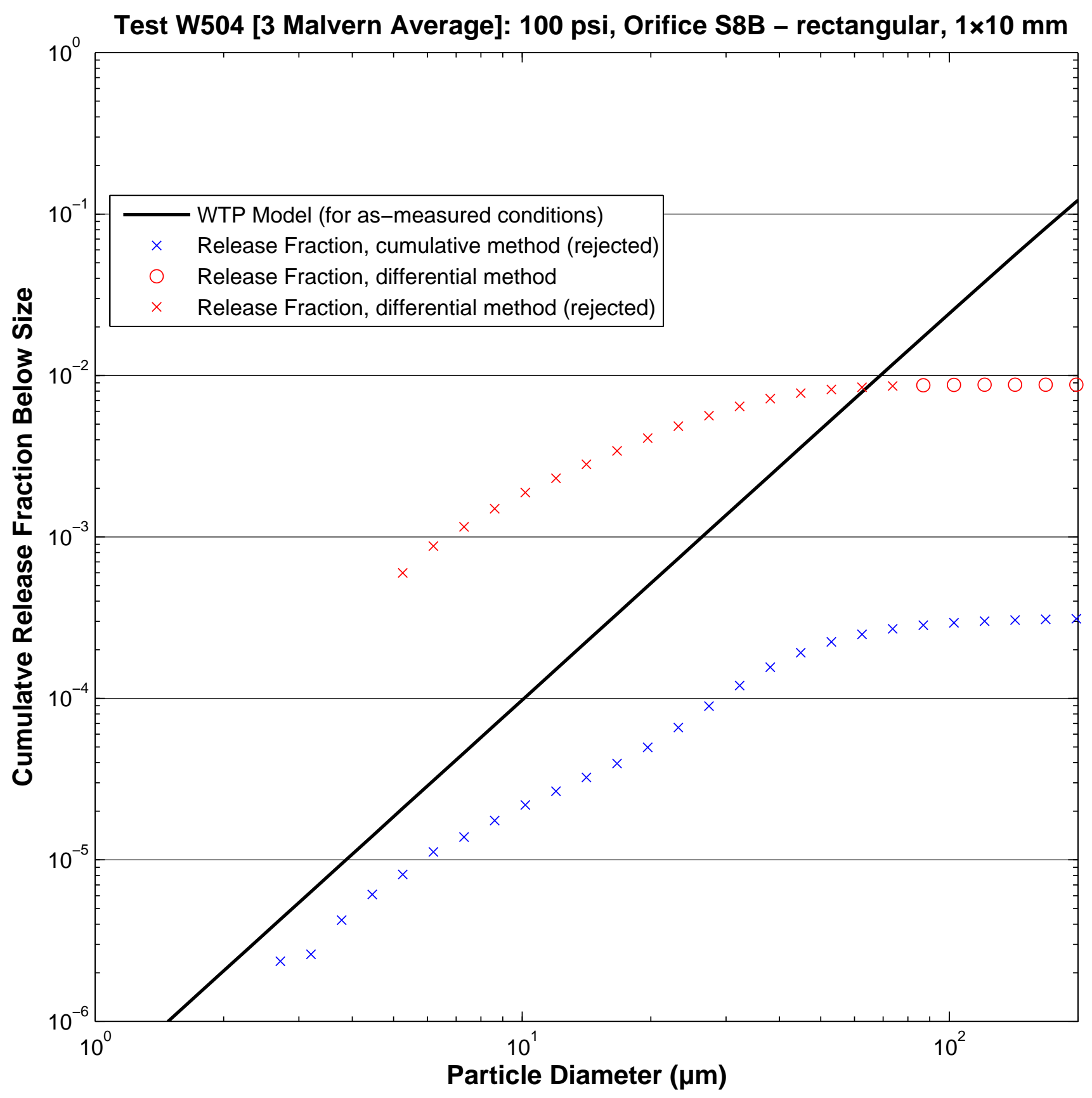




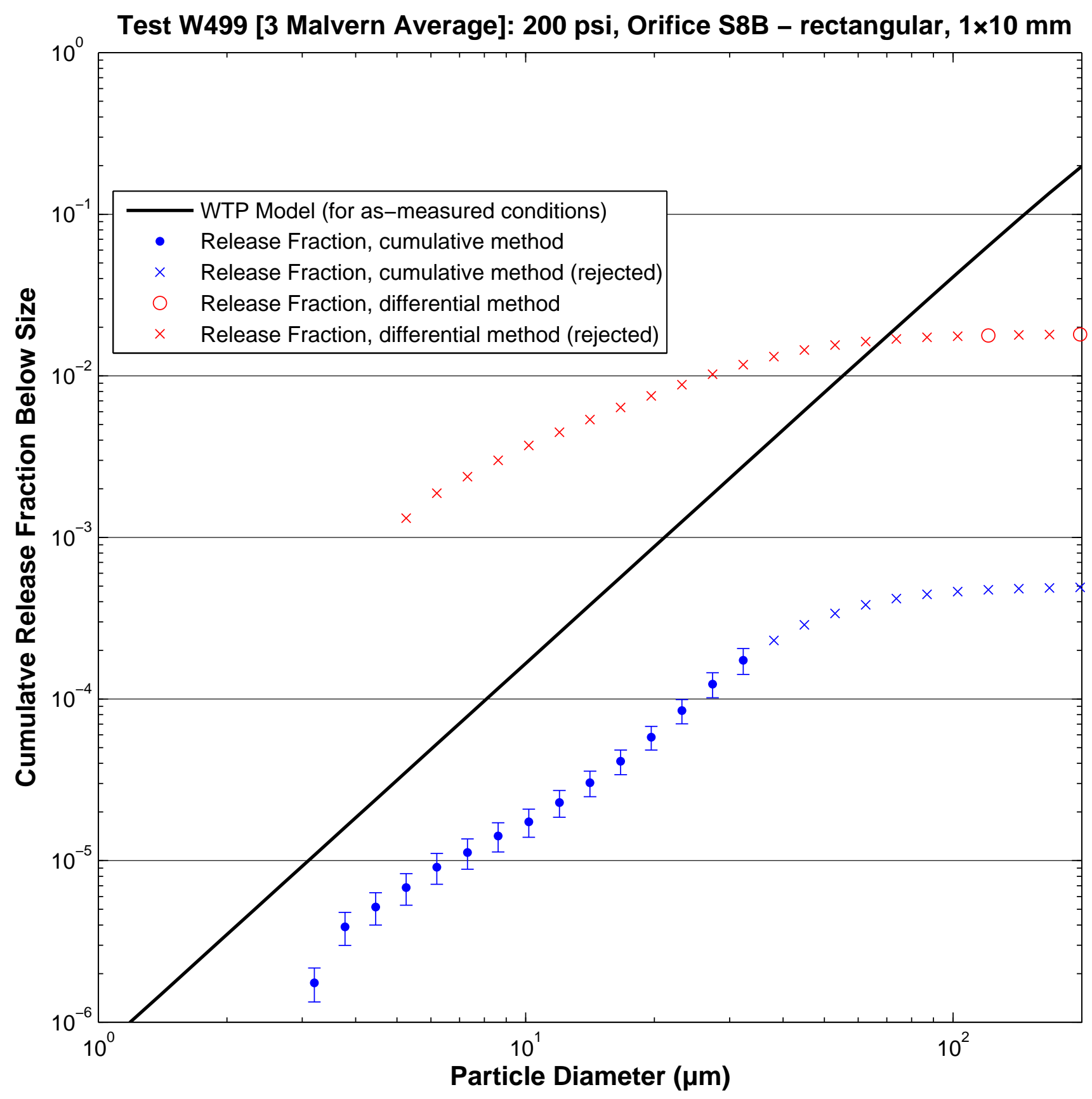




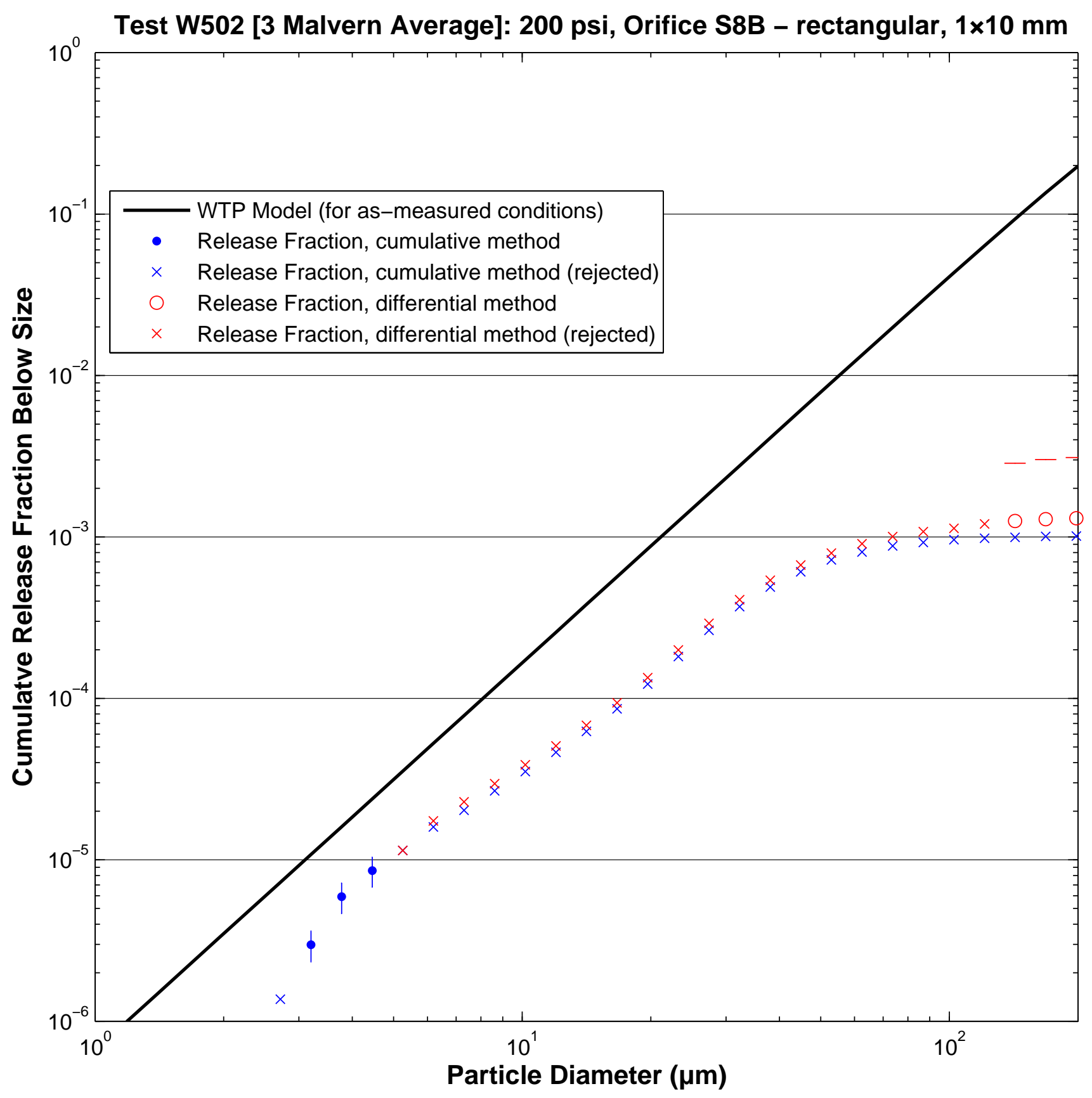




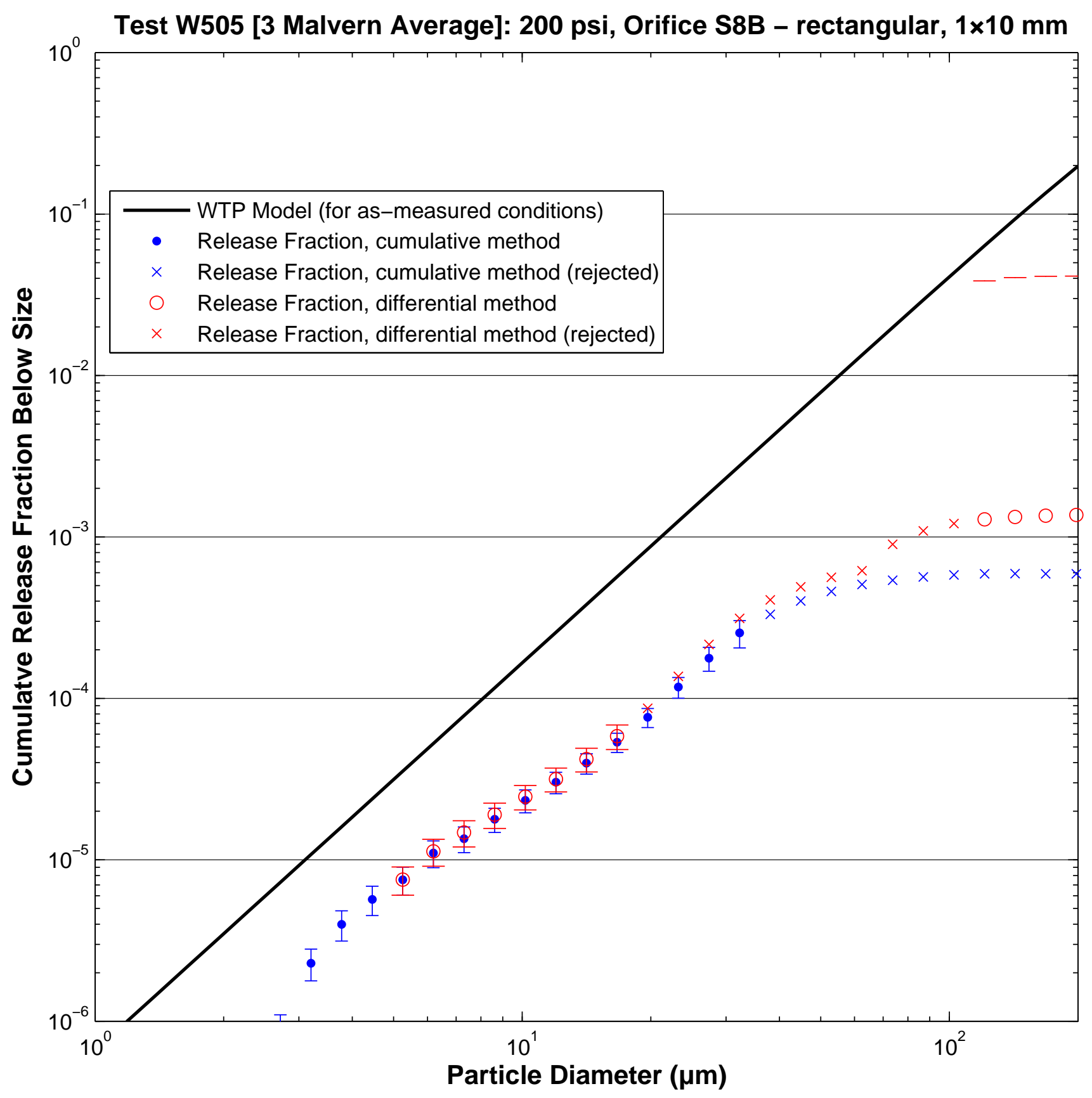

B. 169 


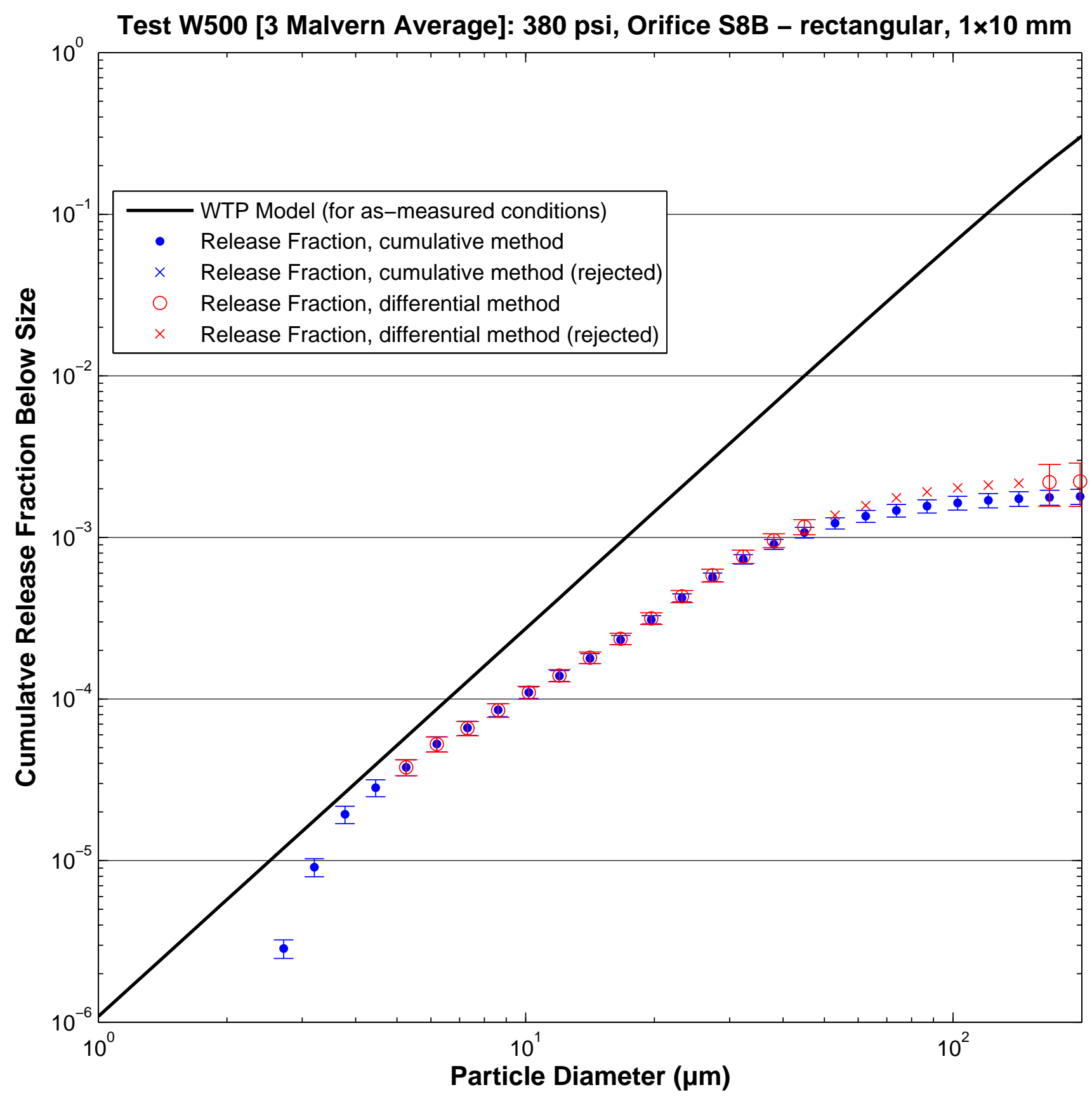

B. 170 


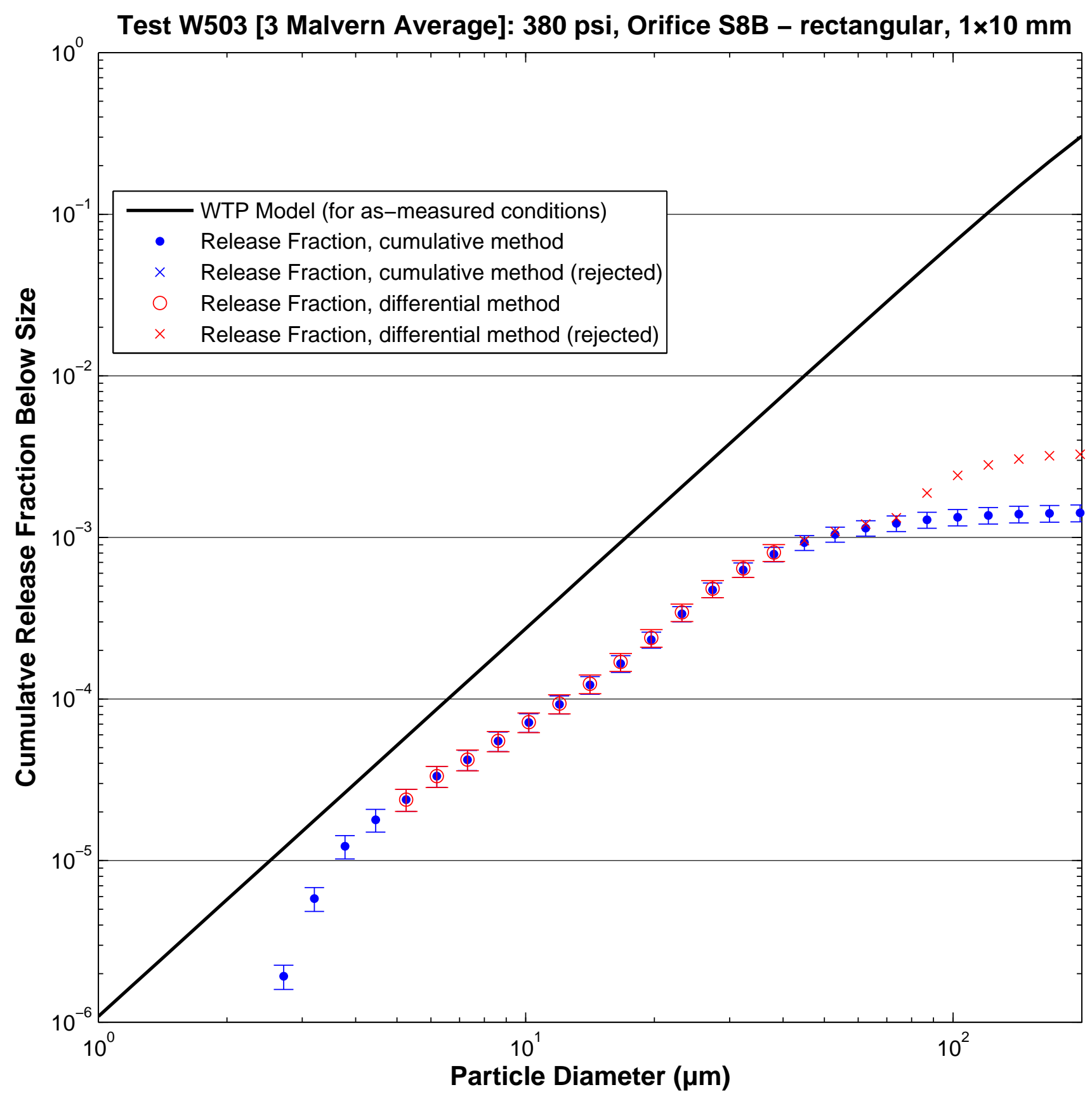




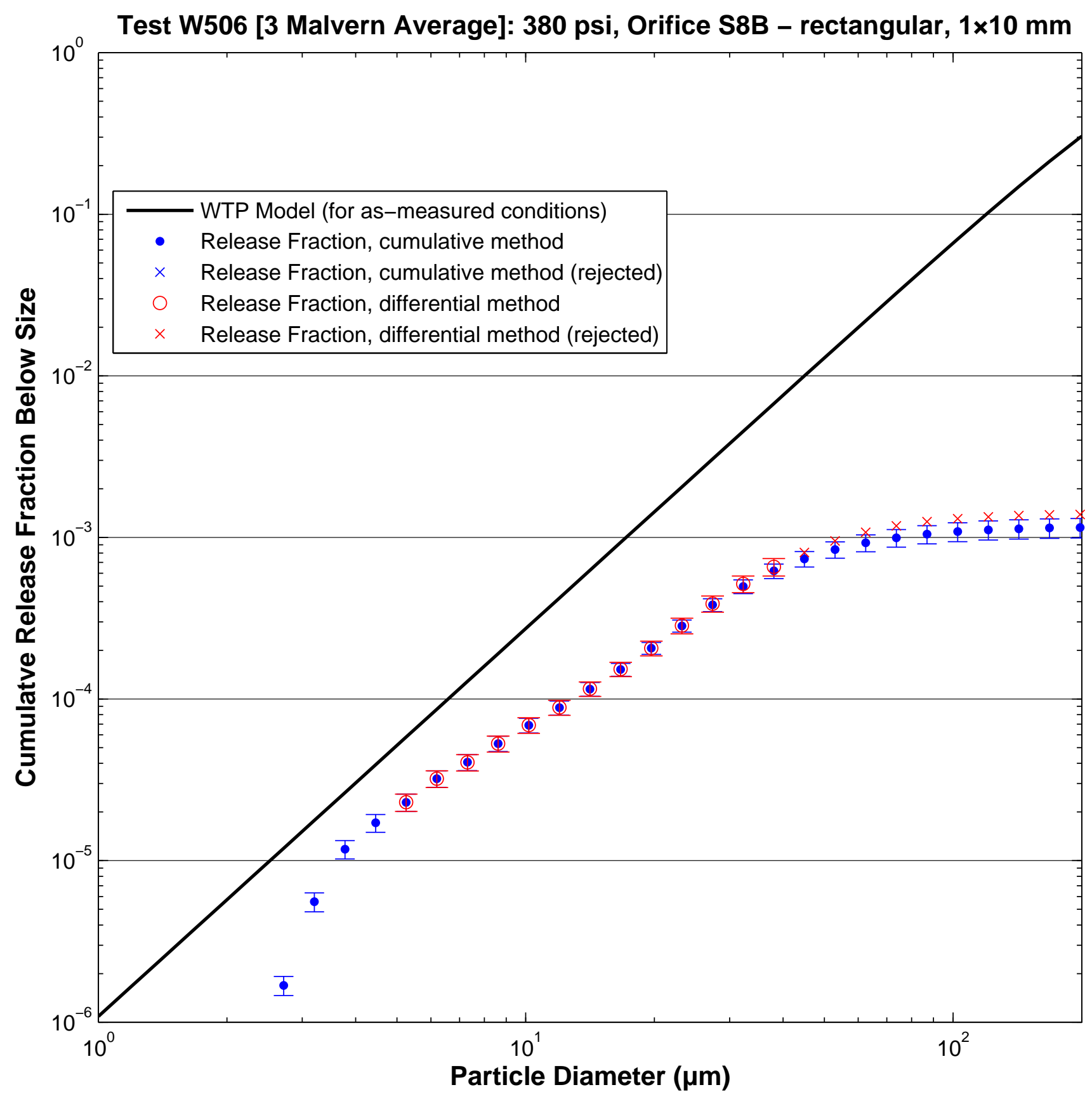




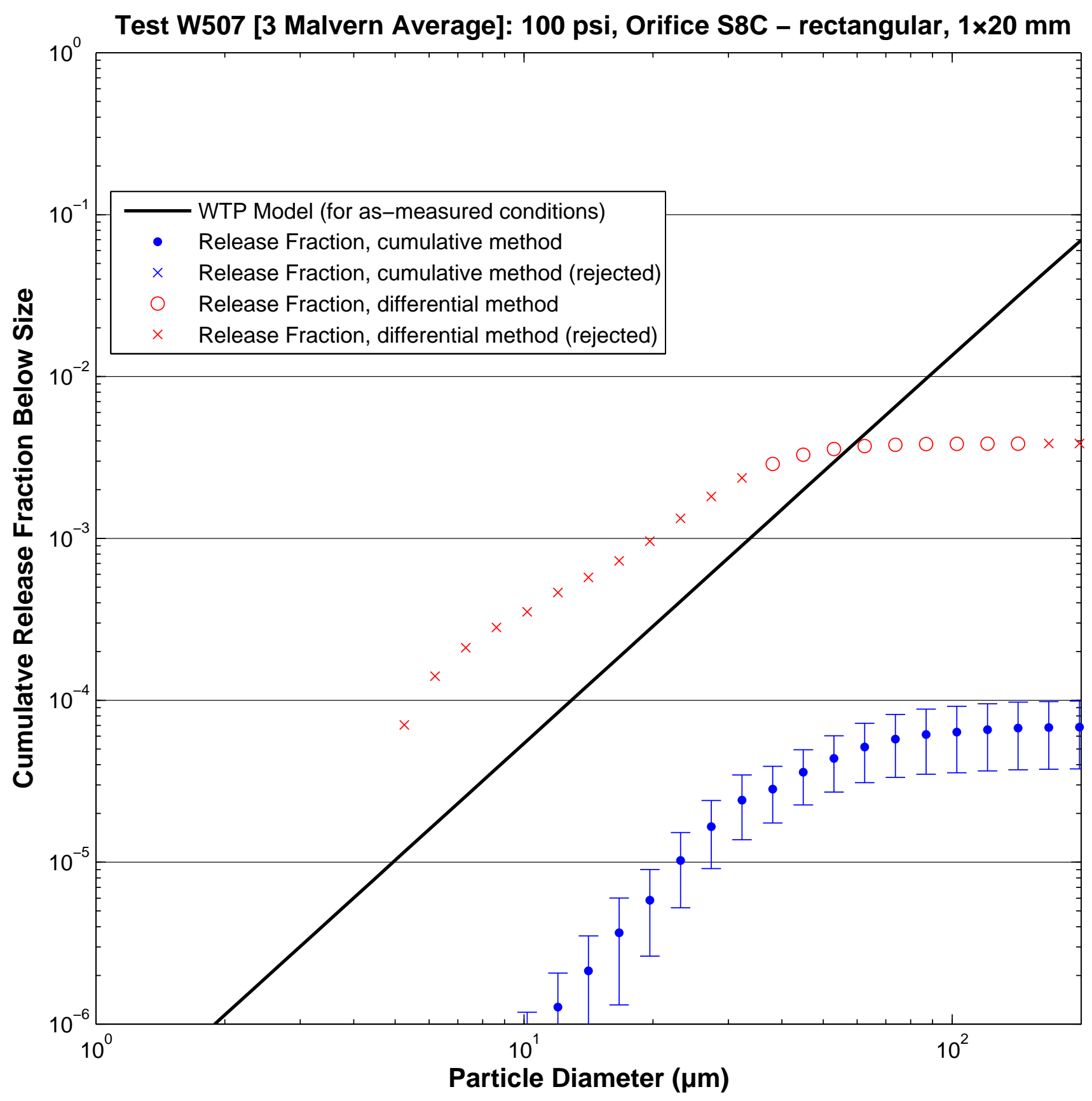




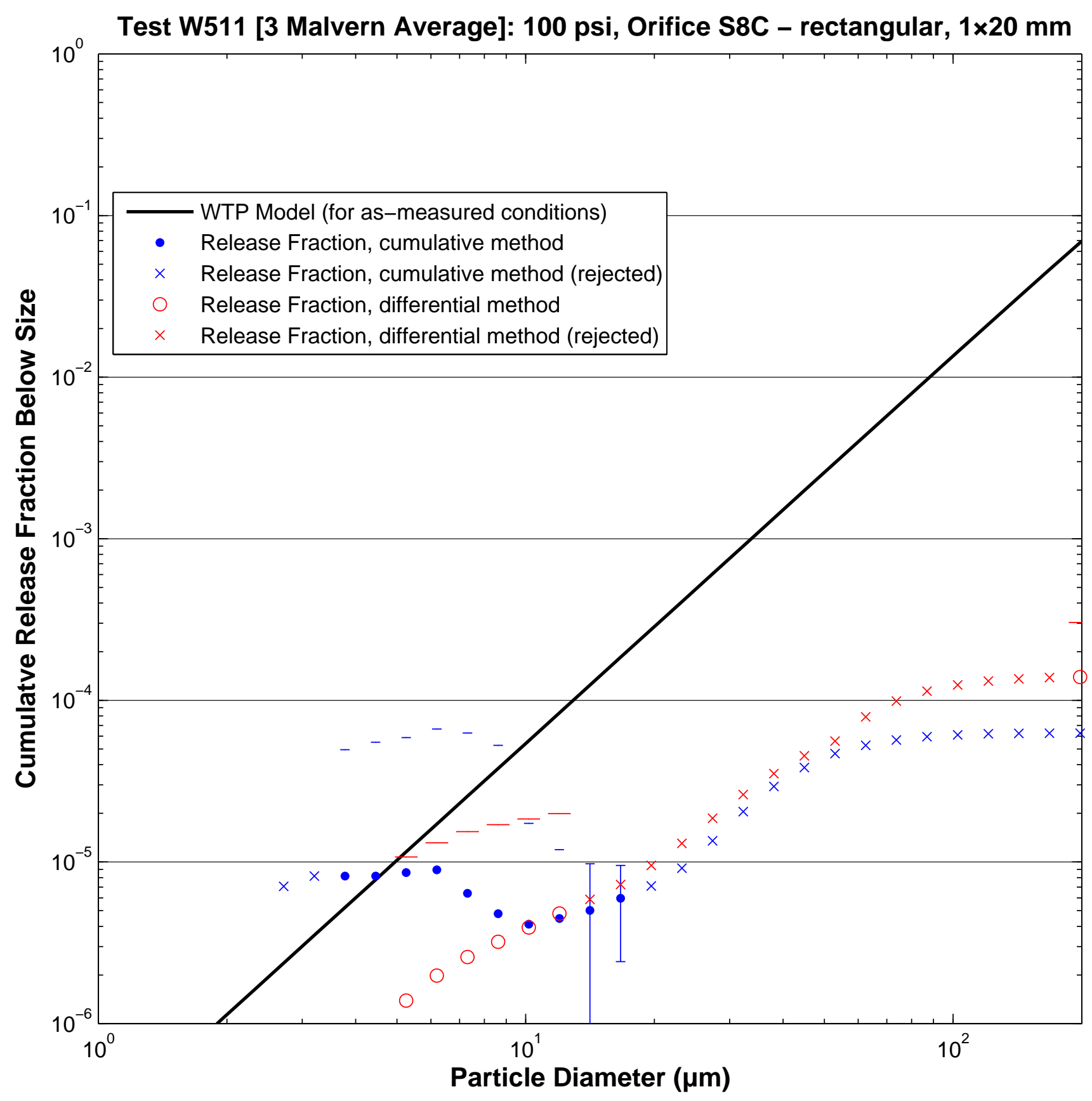




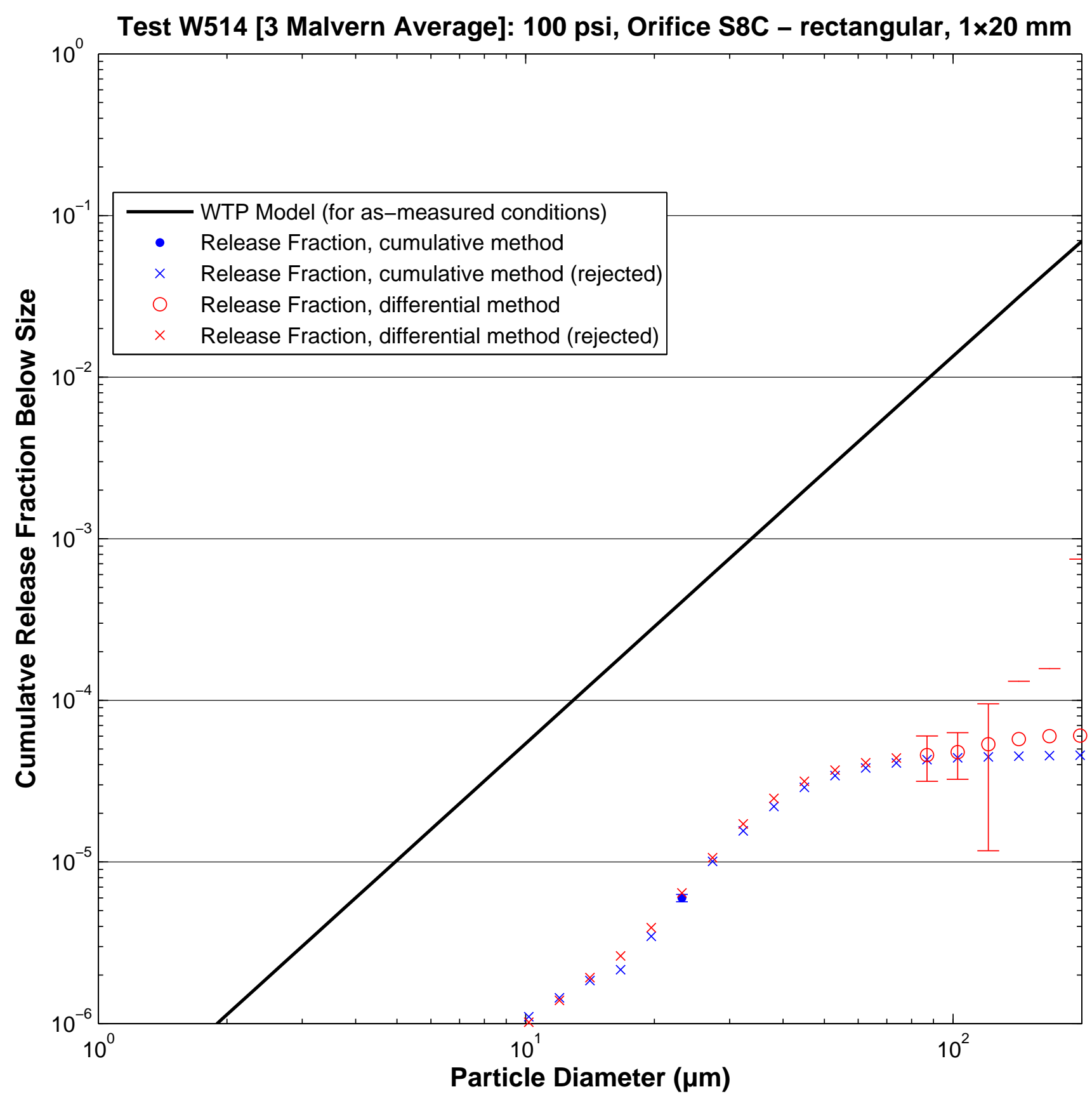




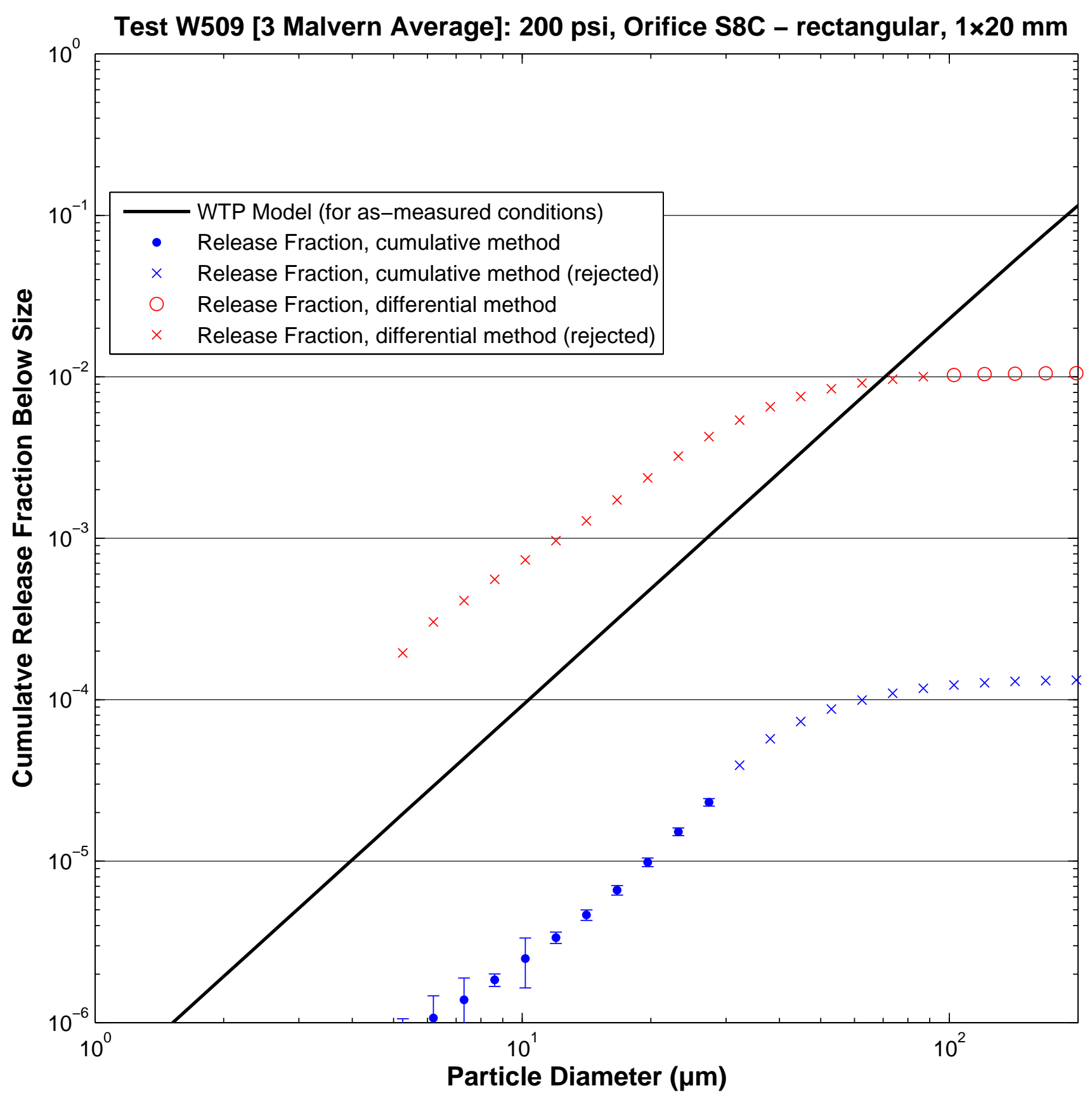

B. 176 


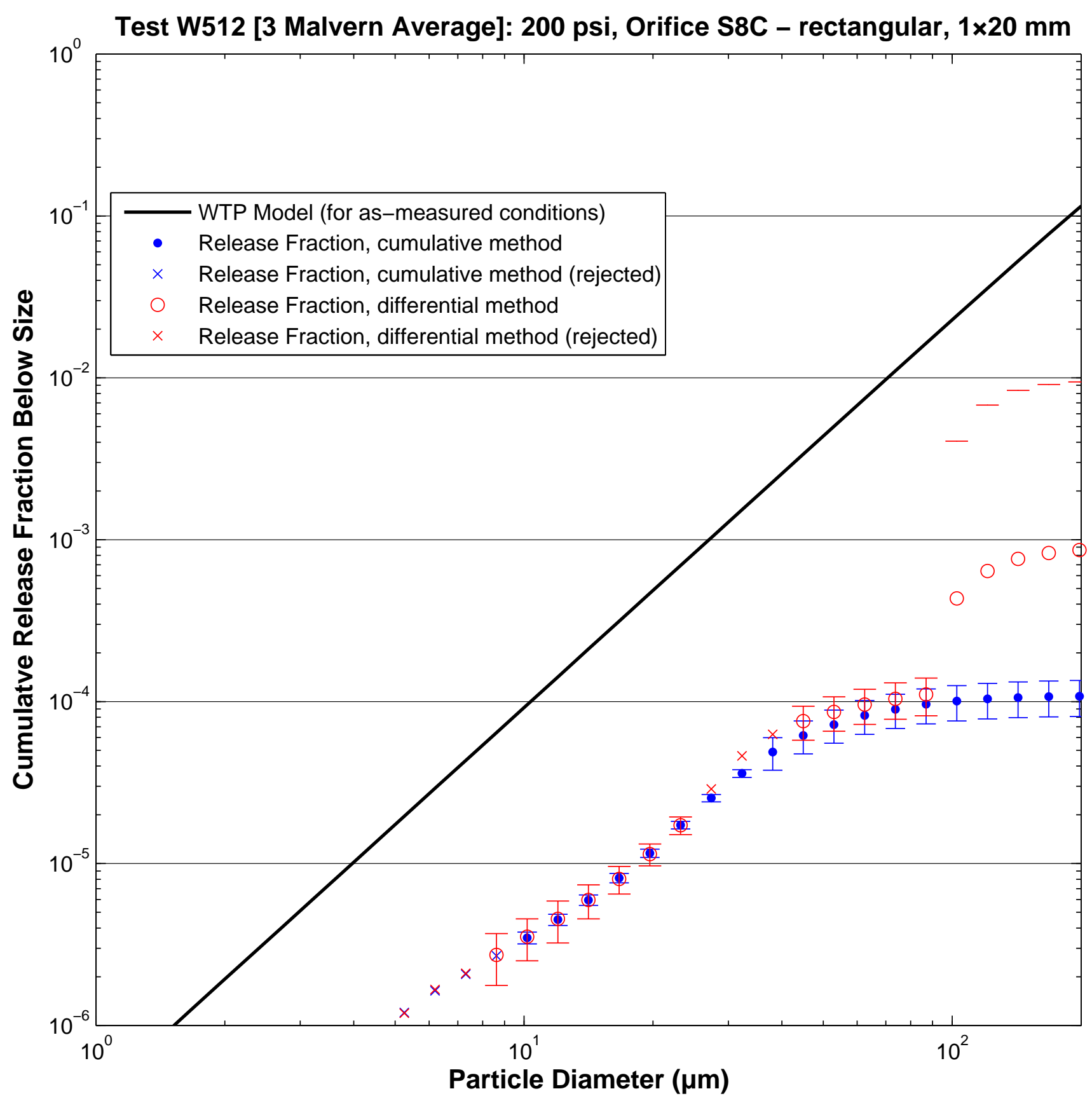




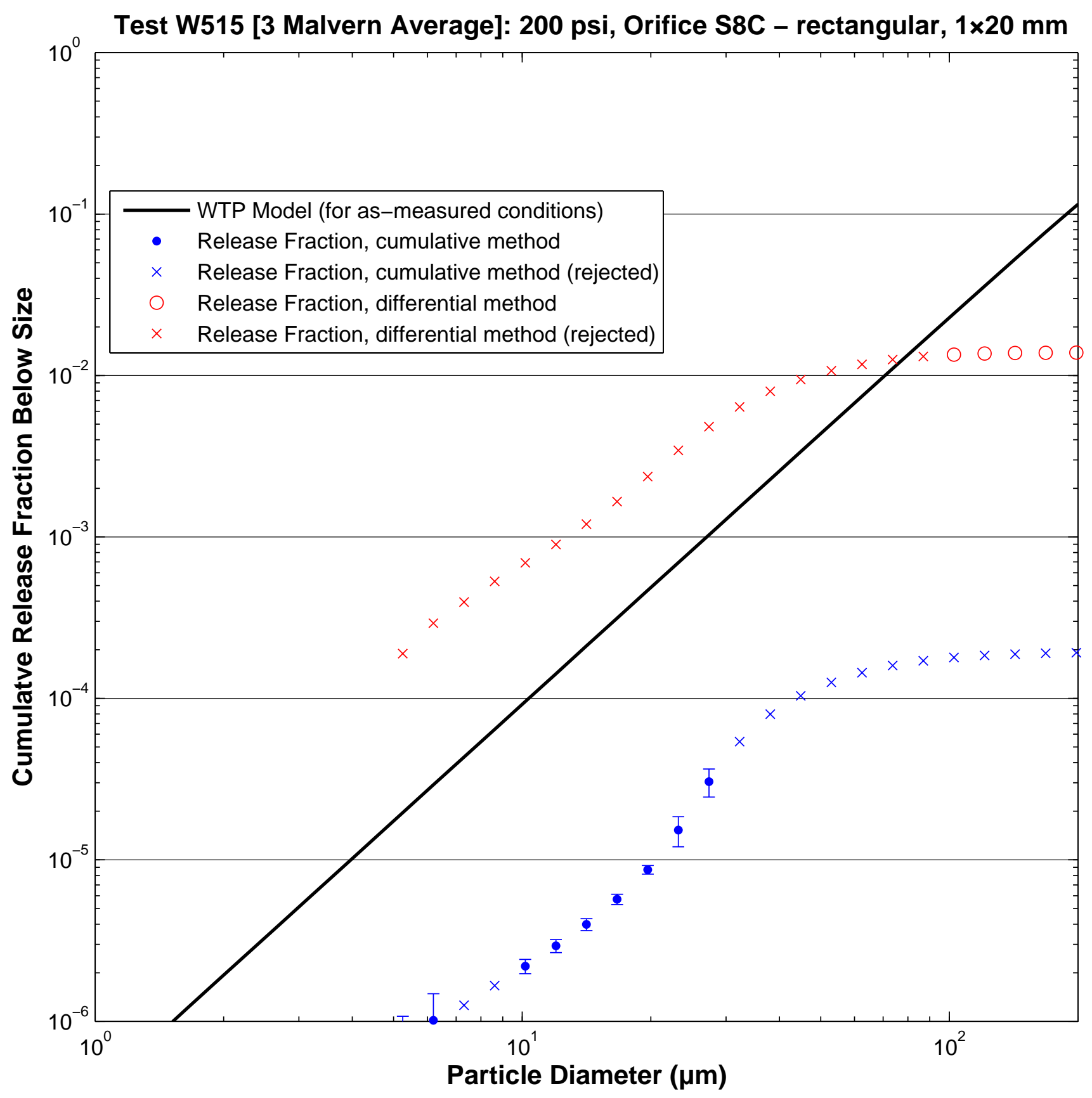

B. 178 


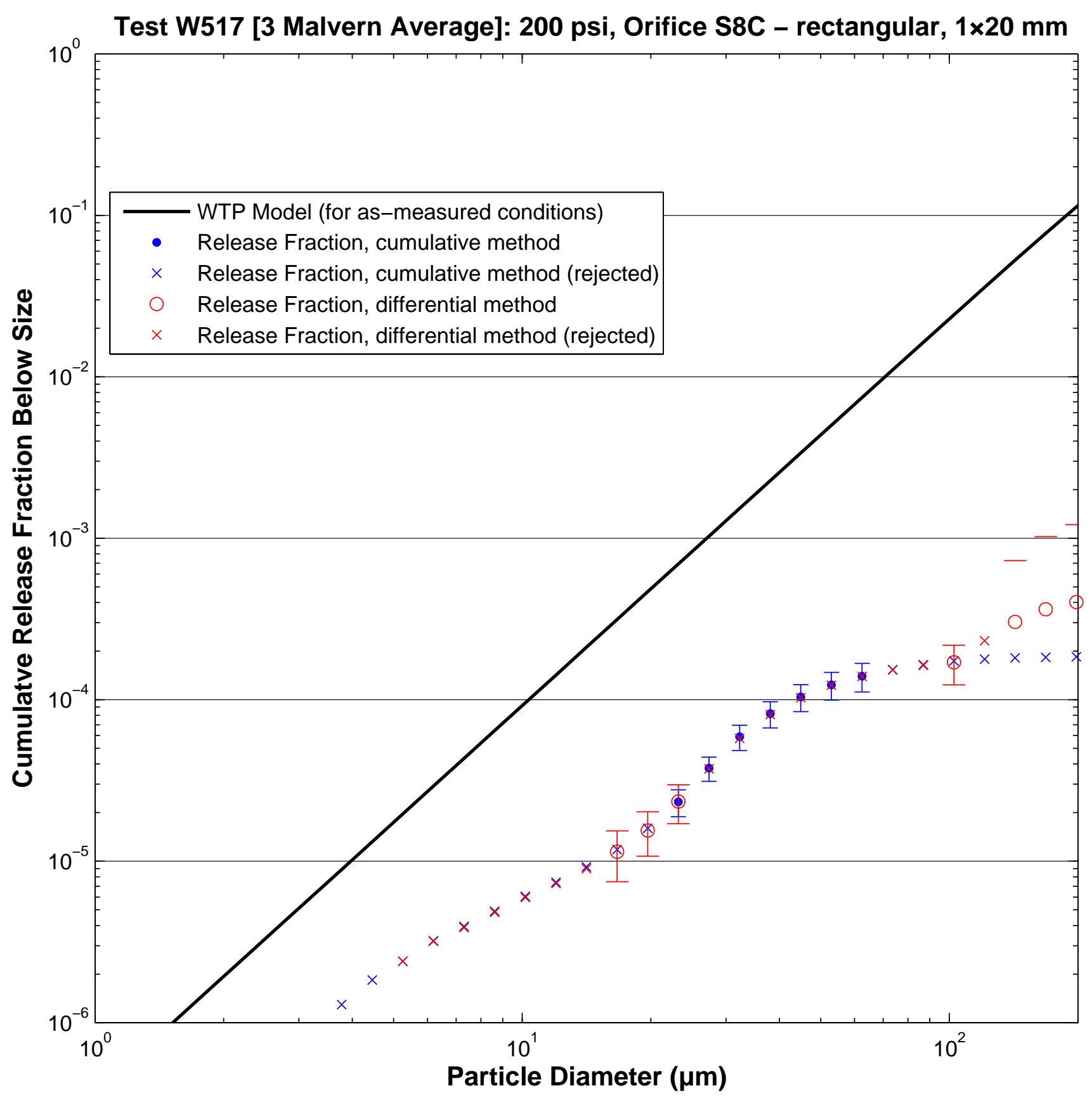




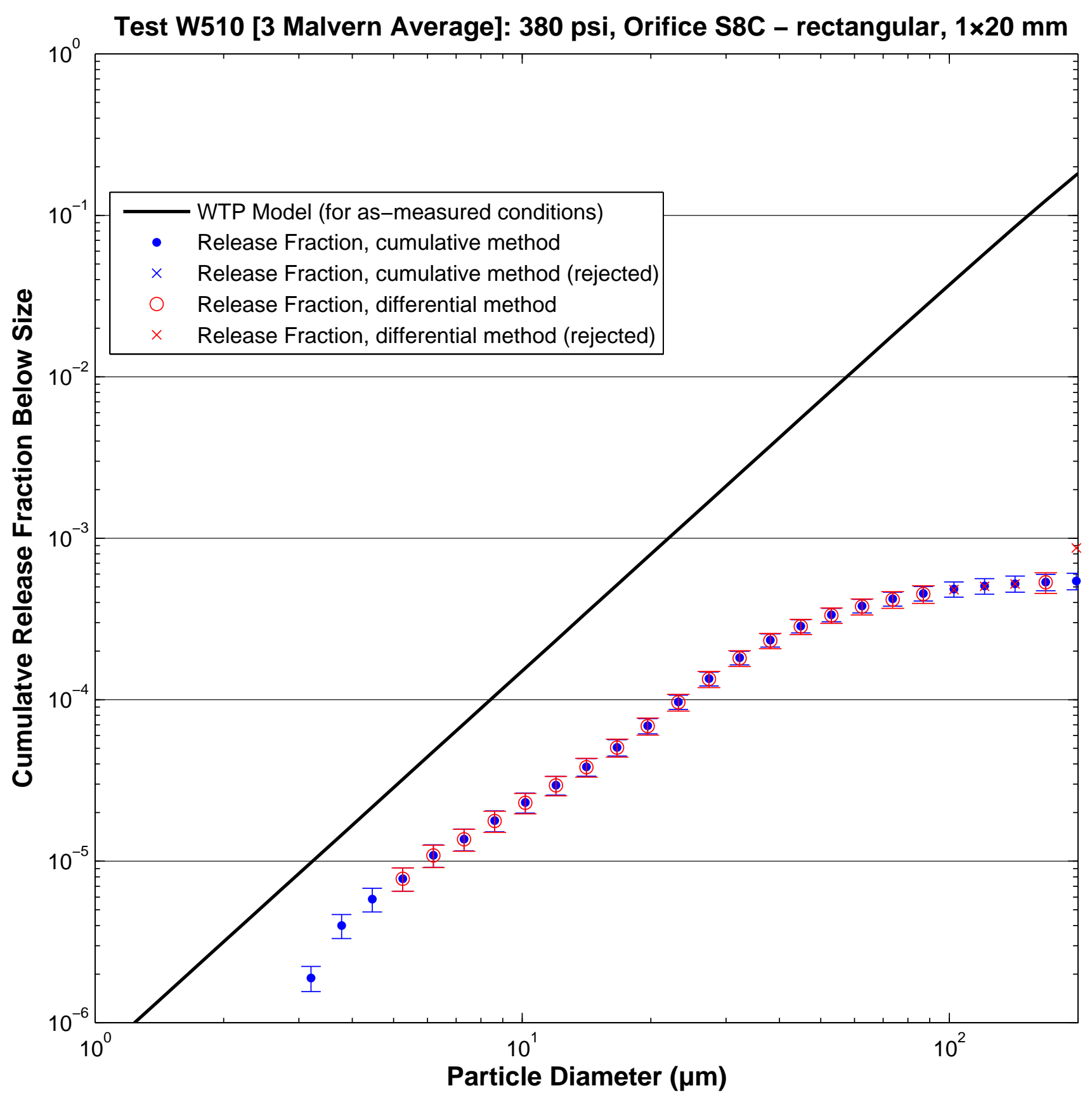

B. 180 


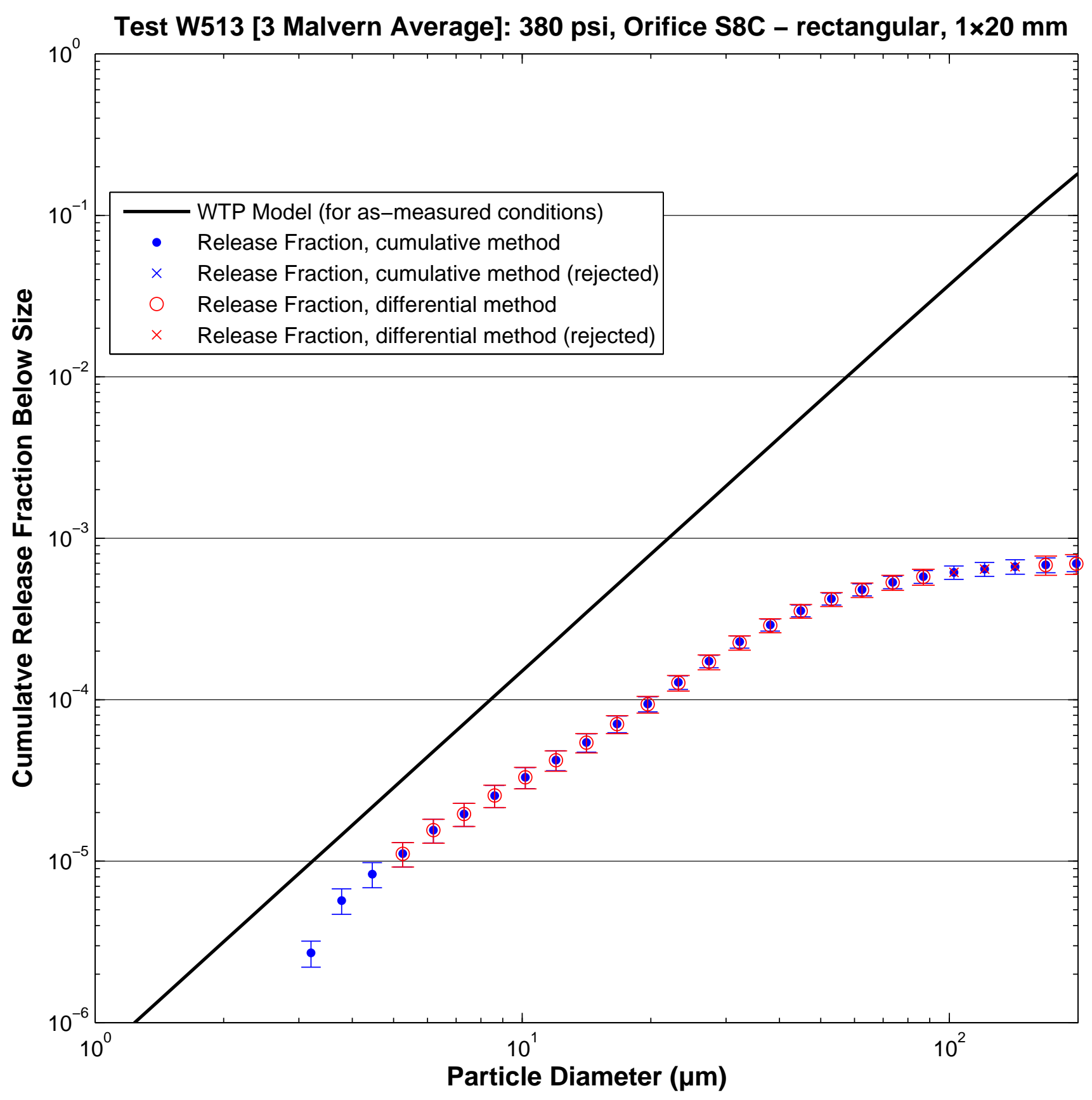




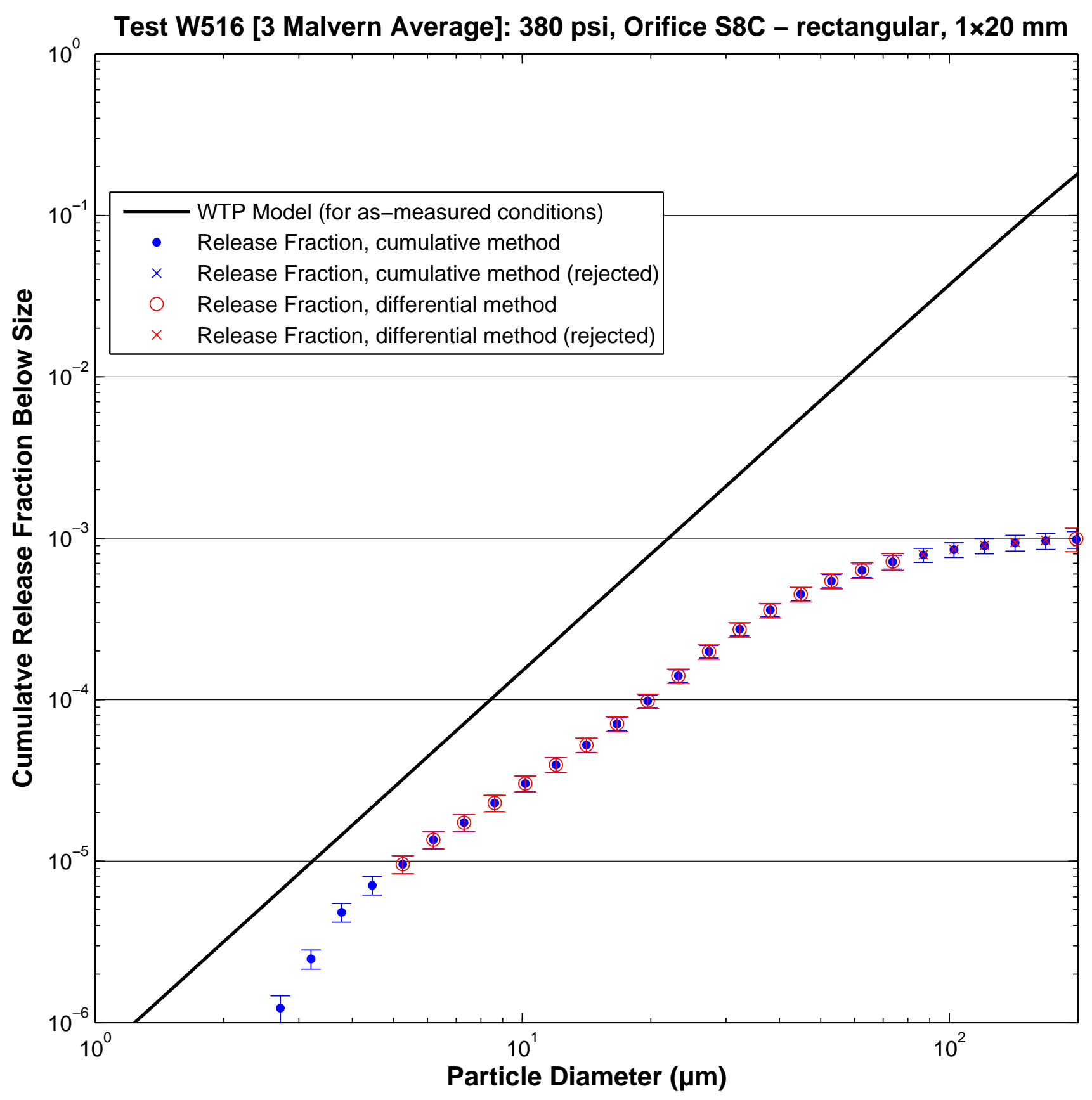

B. 182 


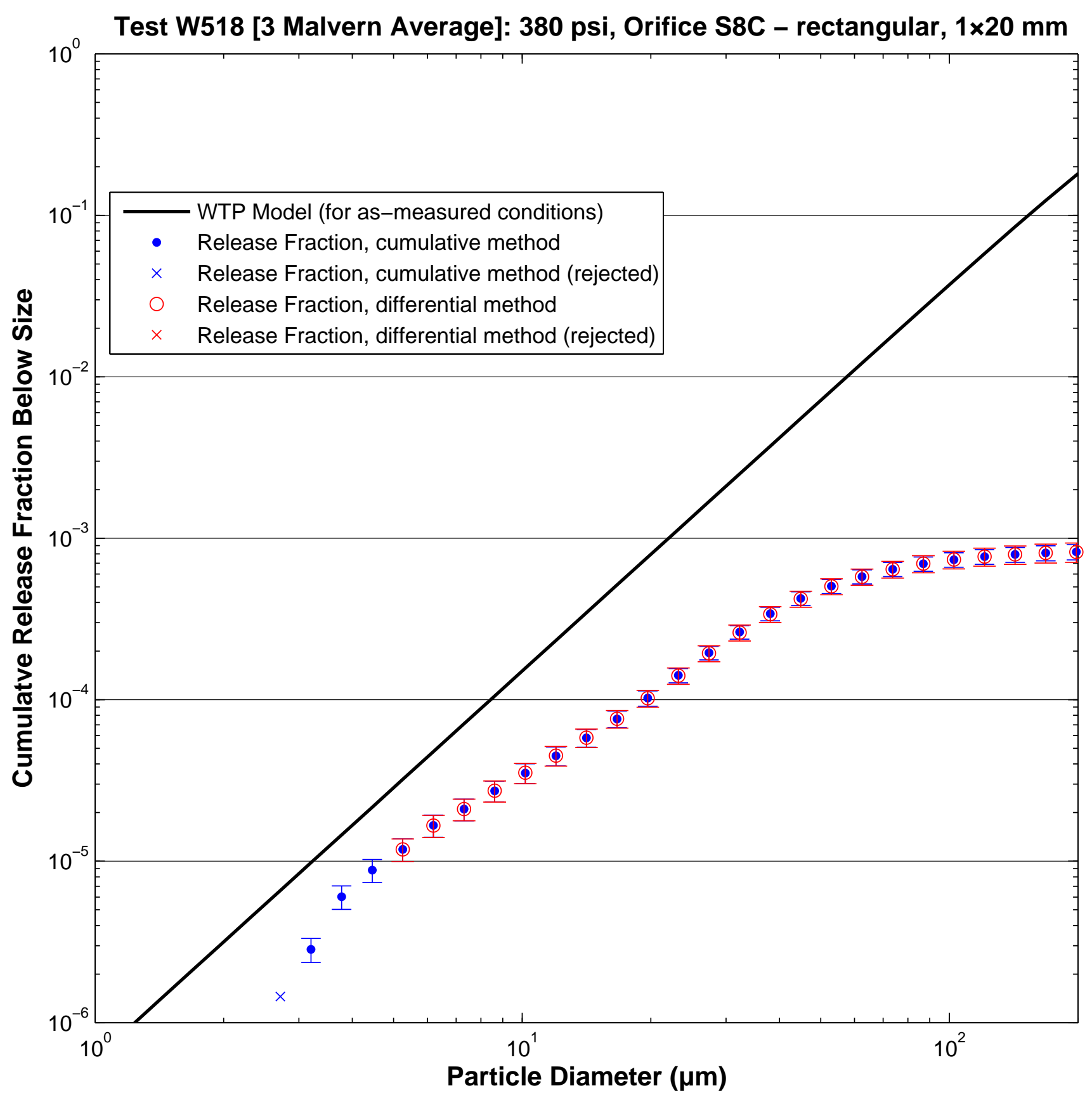




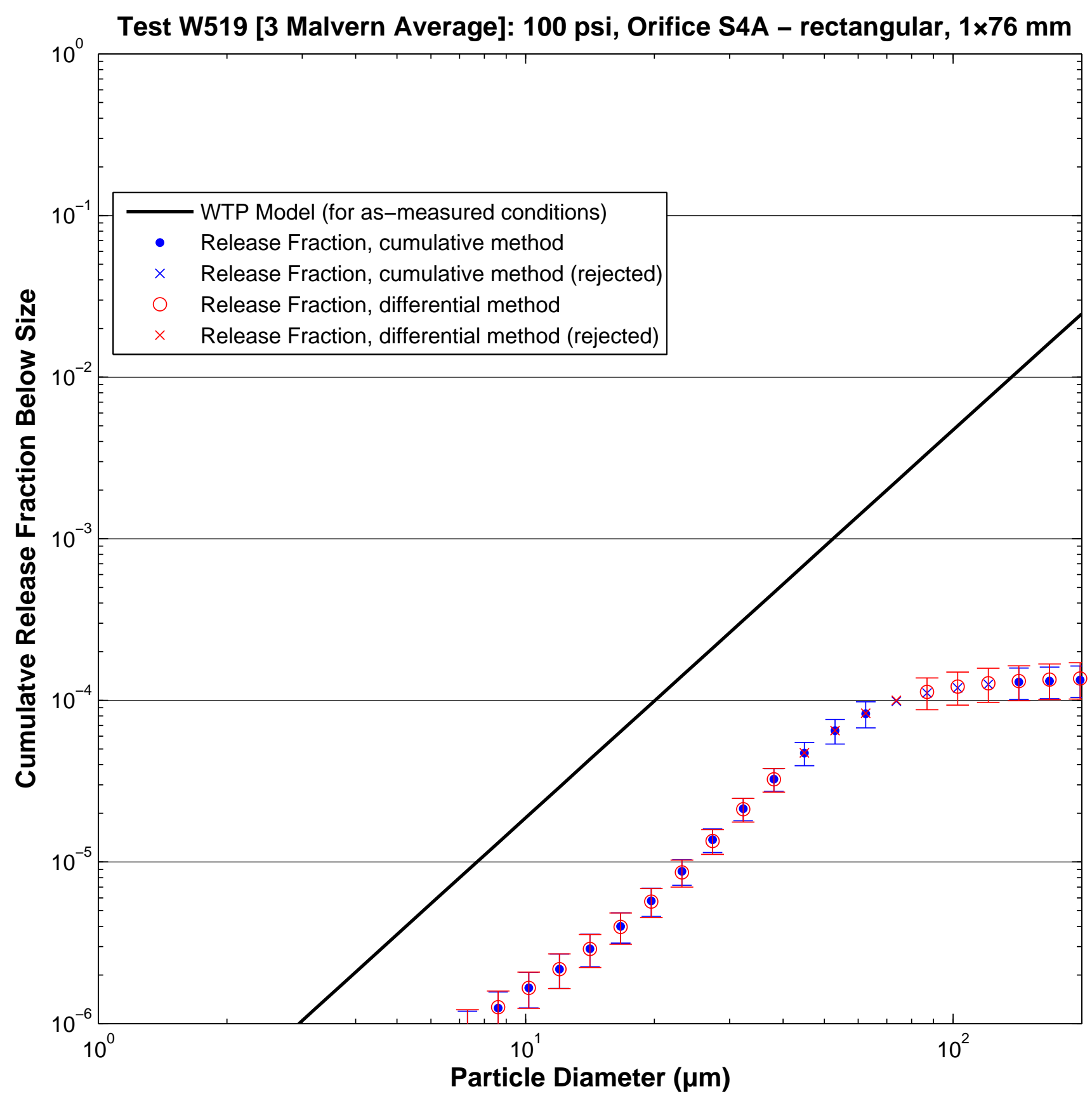

B. 184 


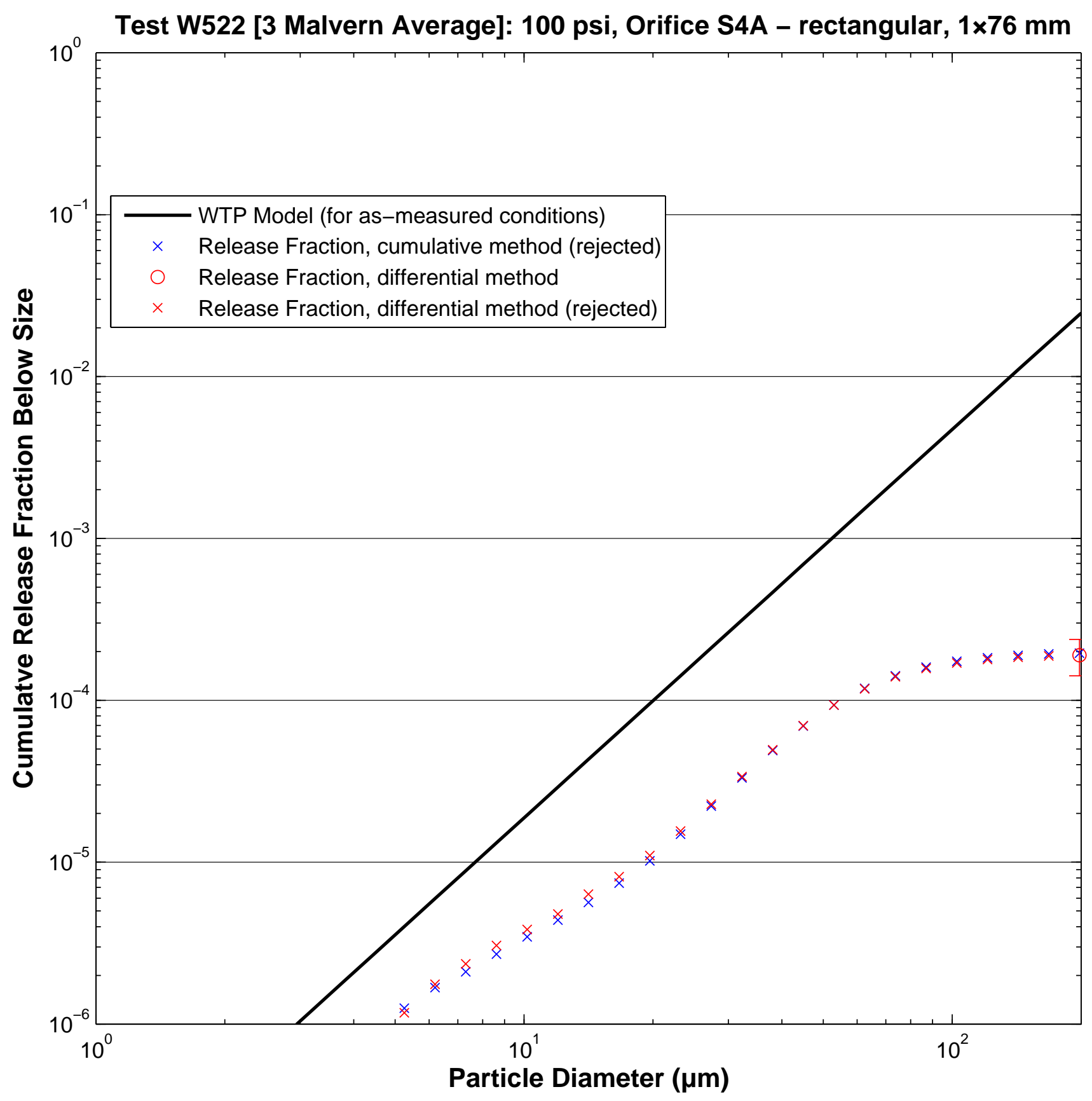

B. 185 


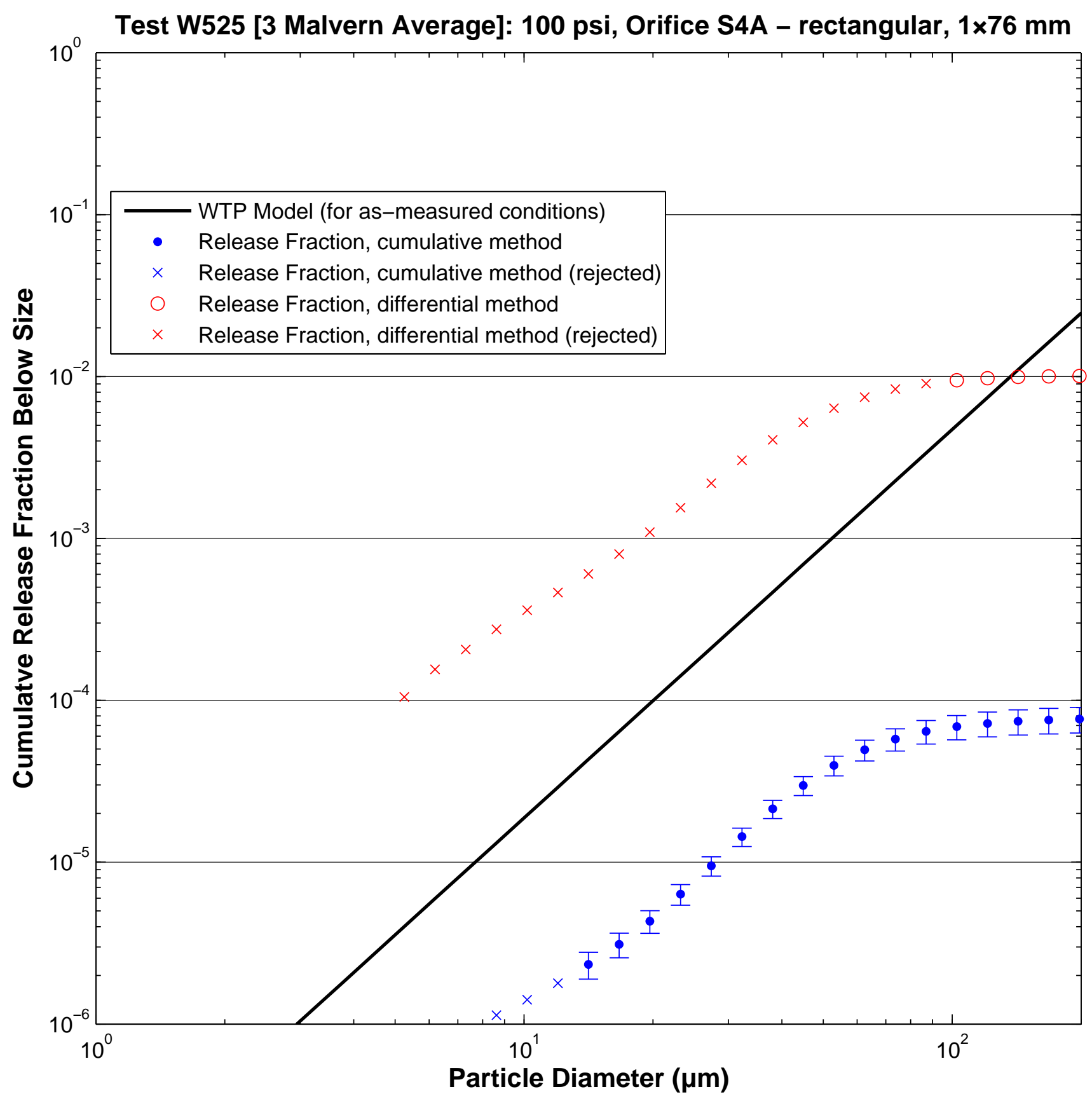




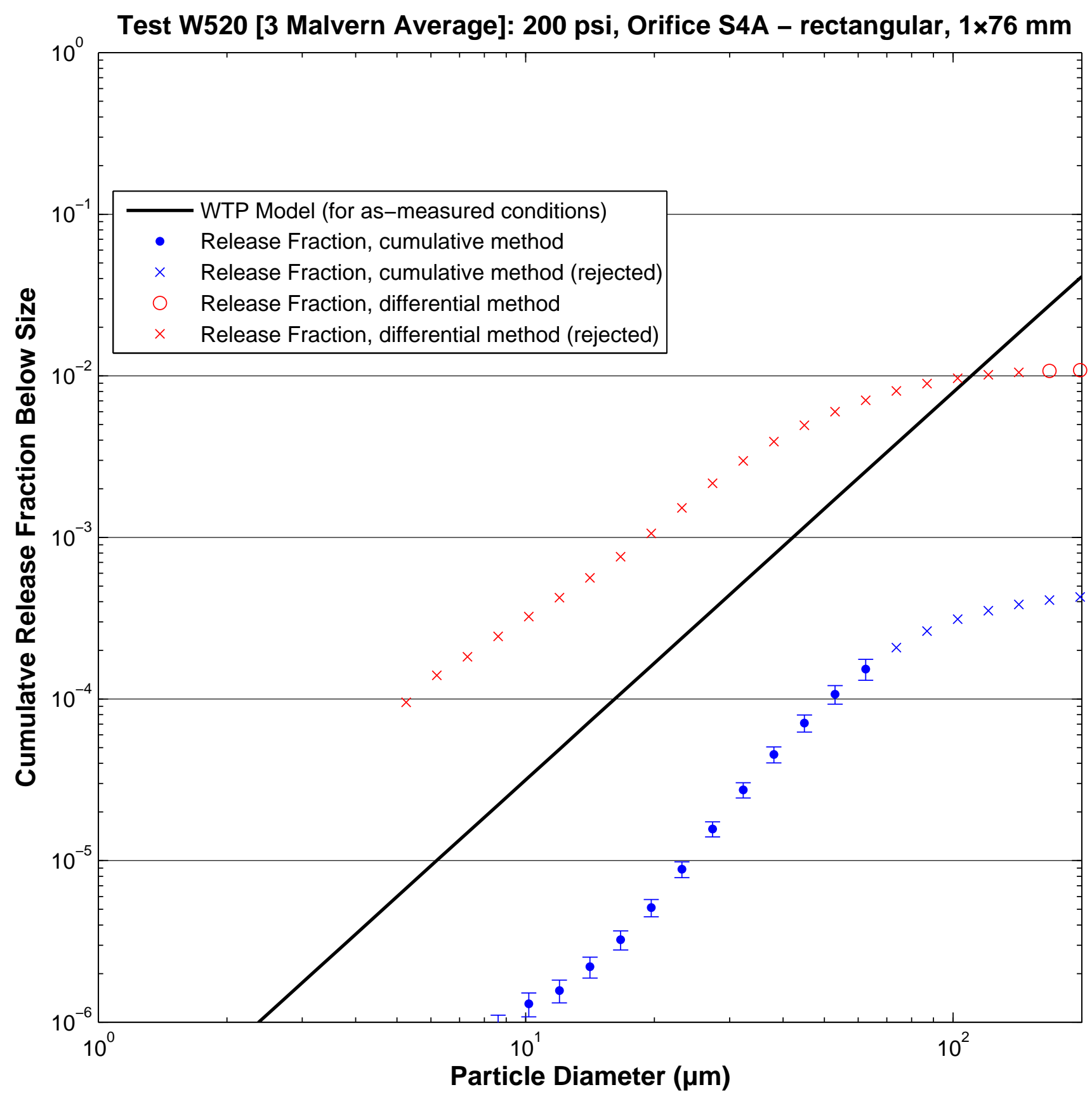

B. 187 


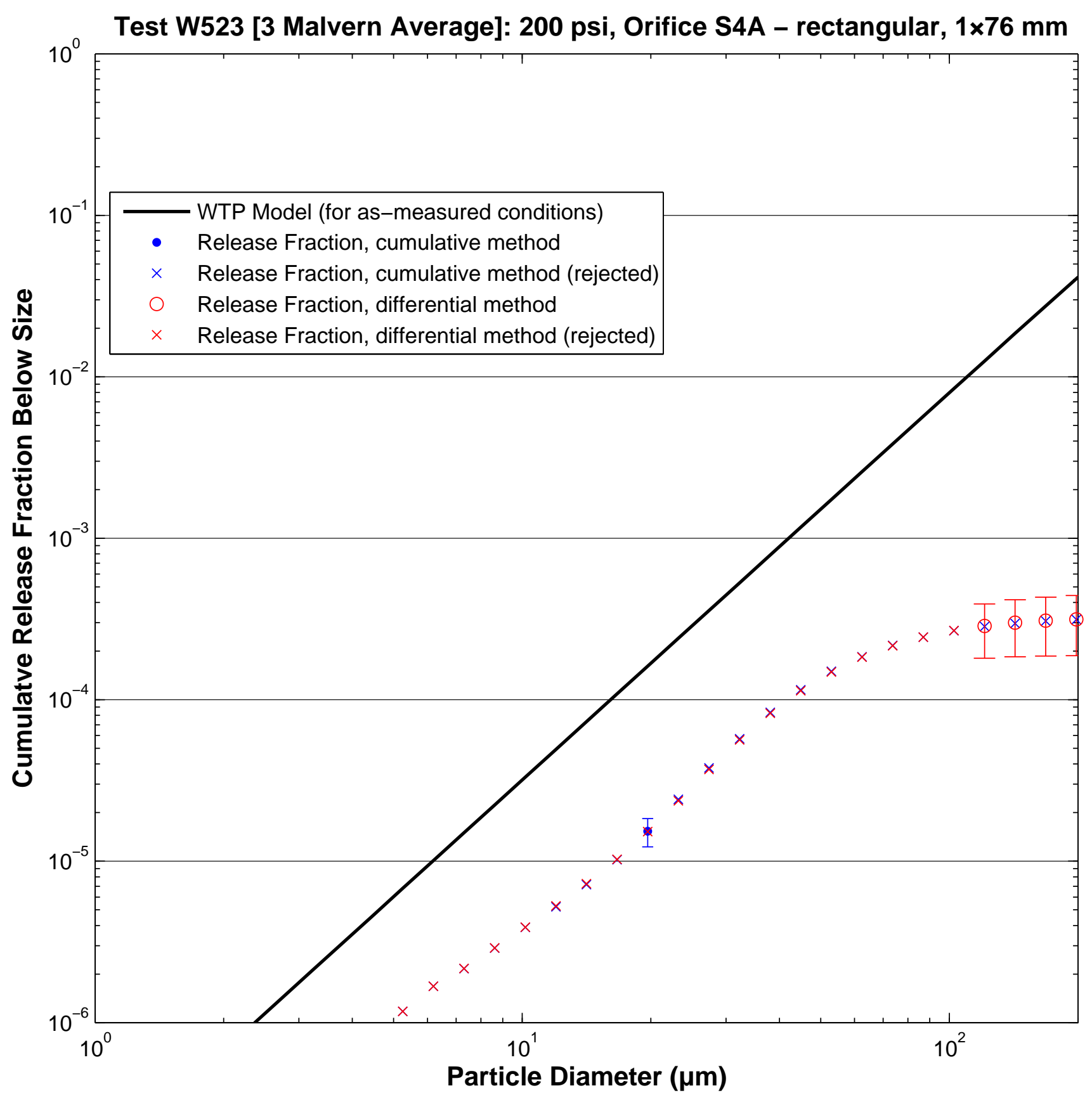

B. 188 


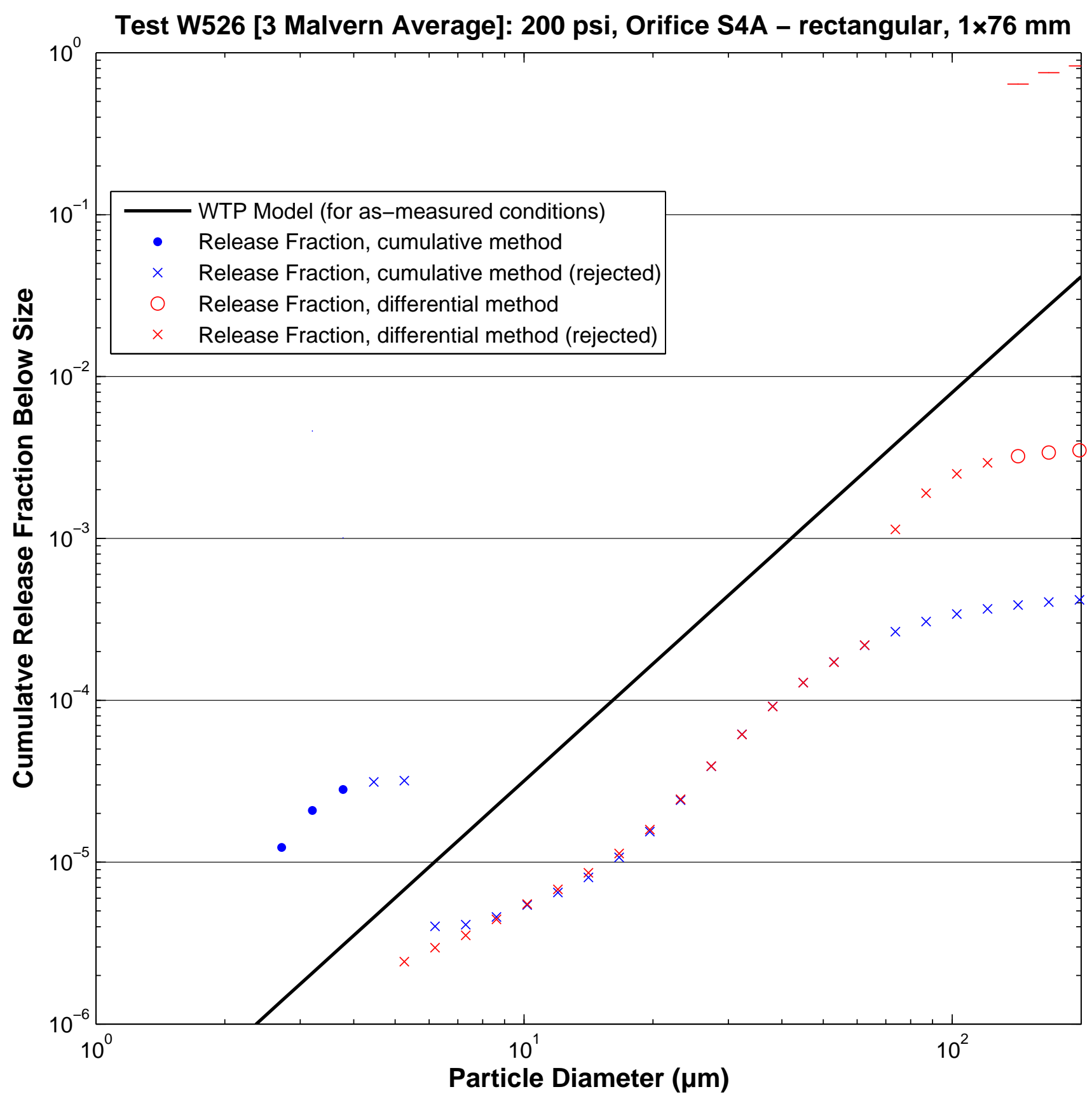




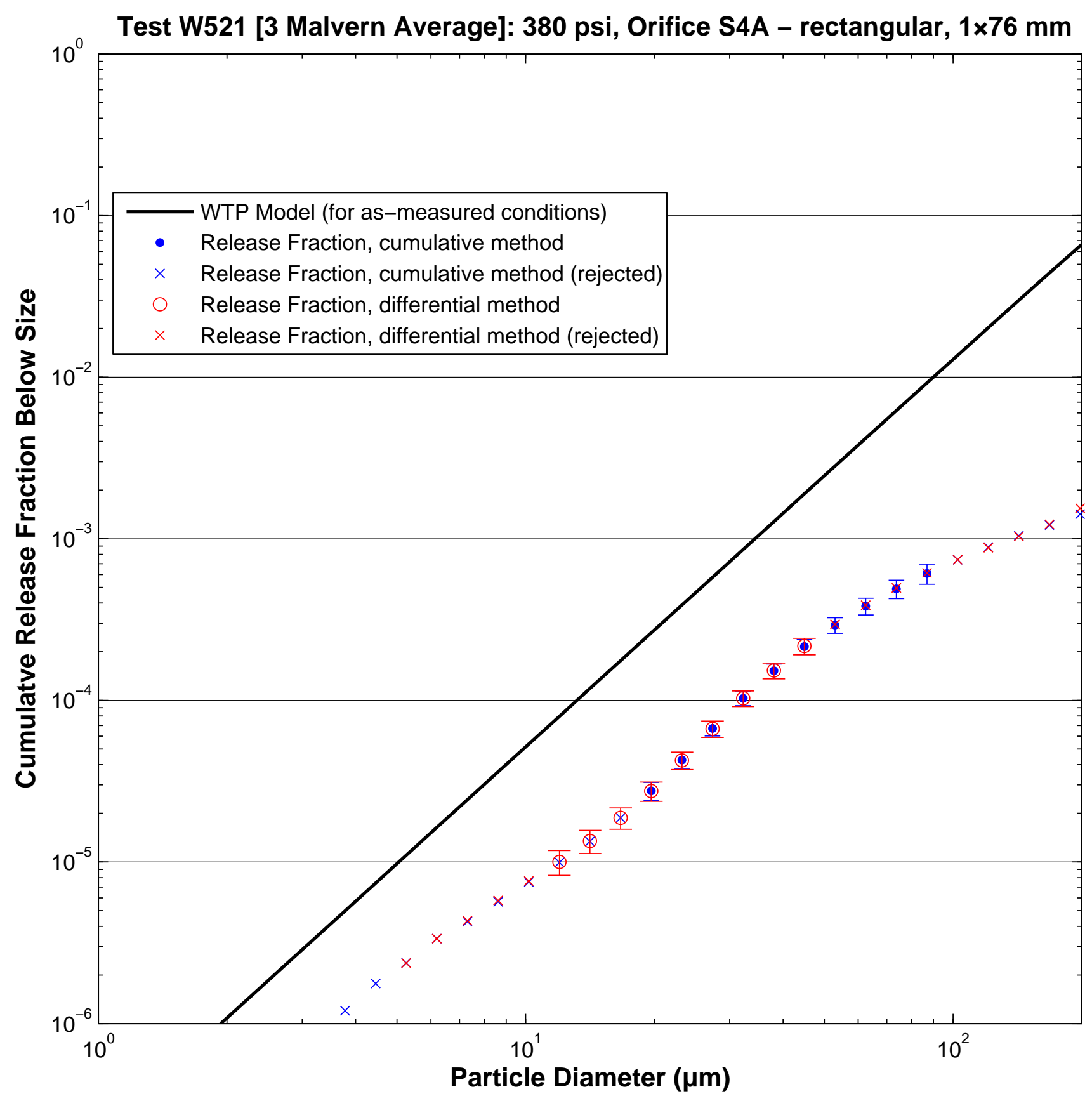

B. 190 


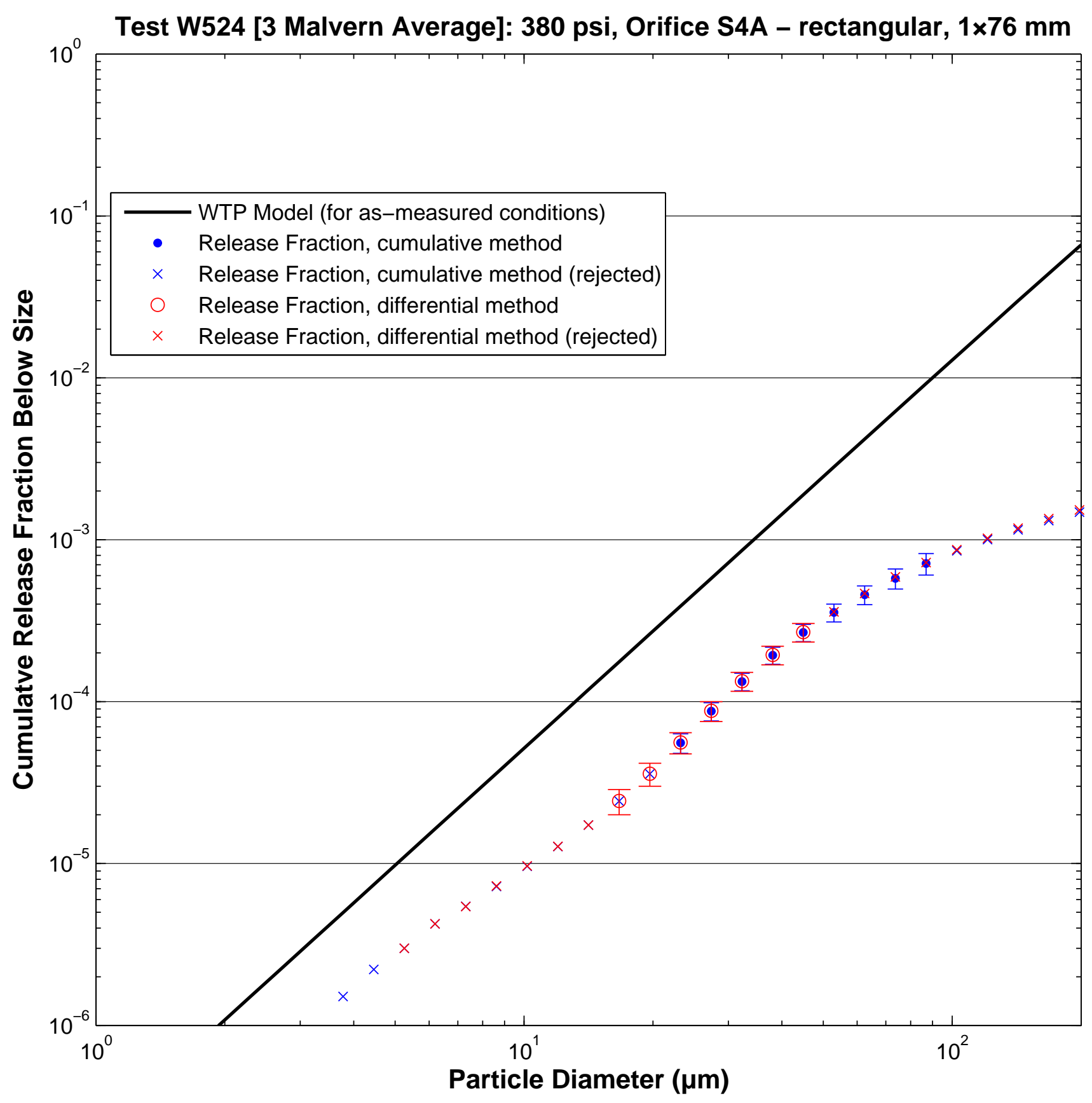




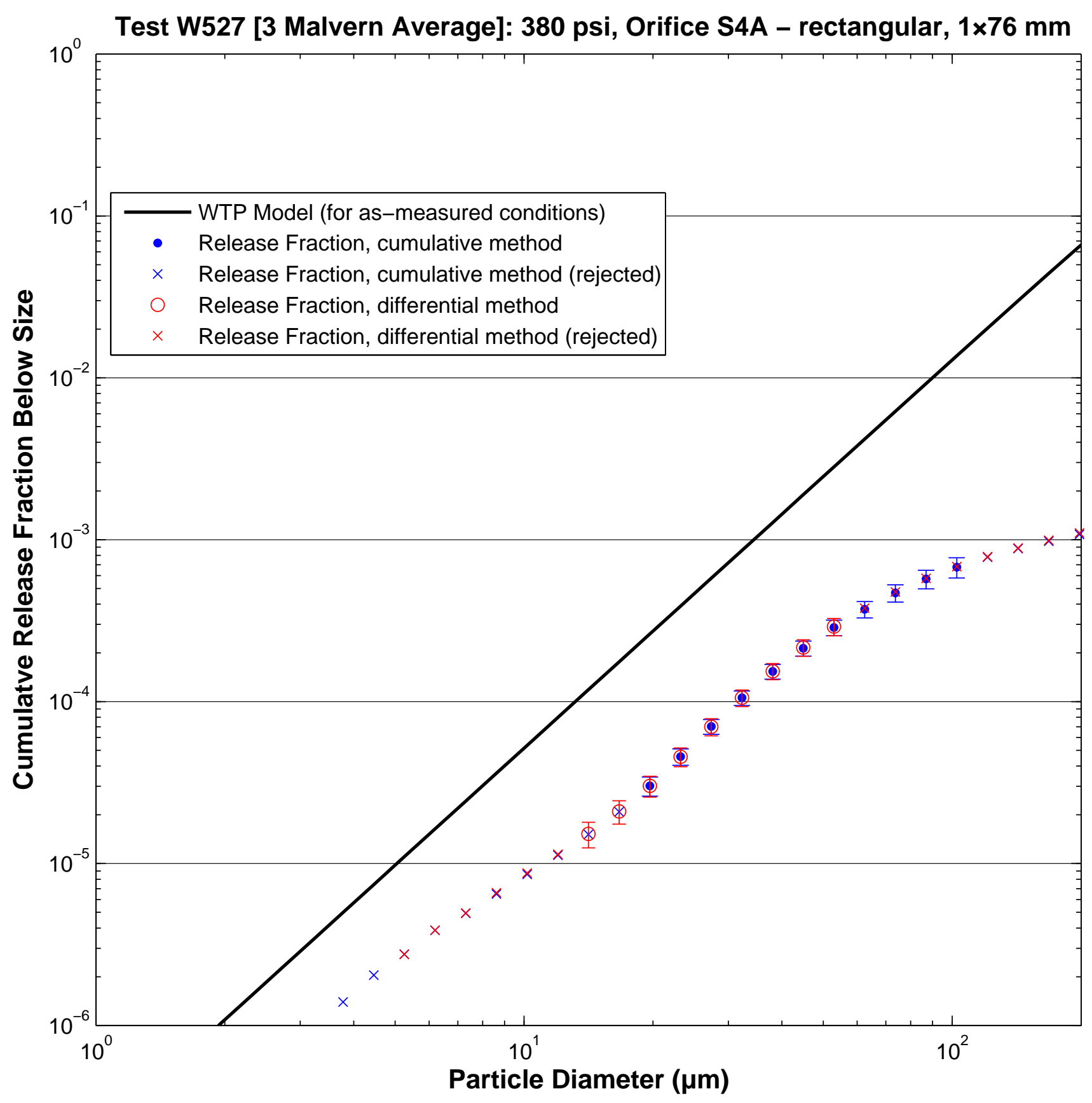




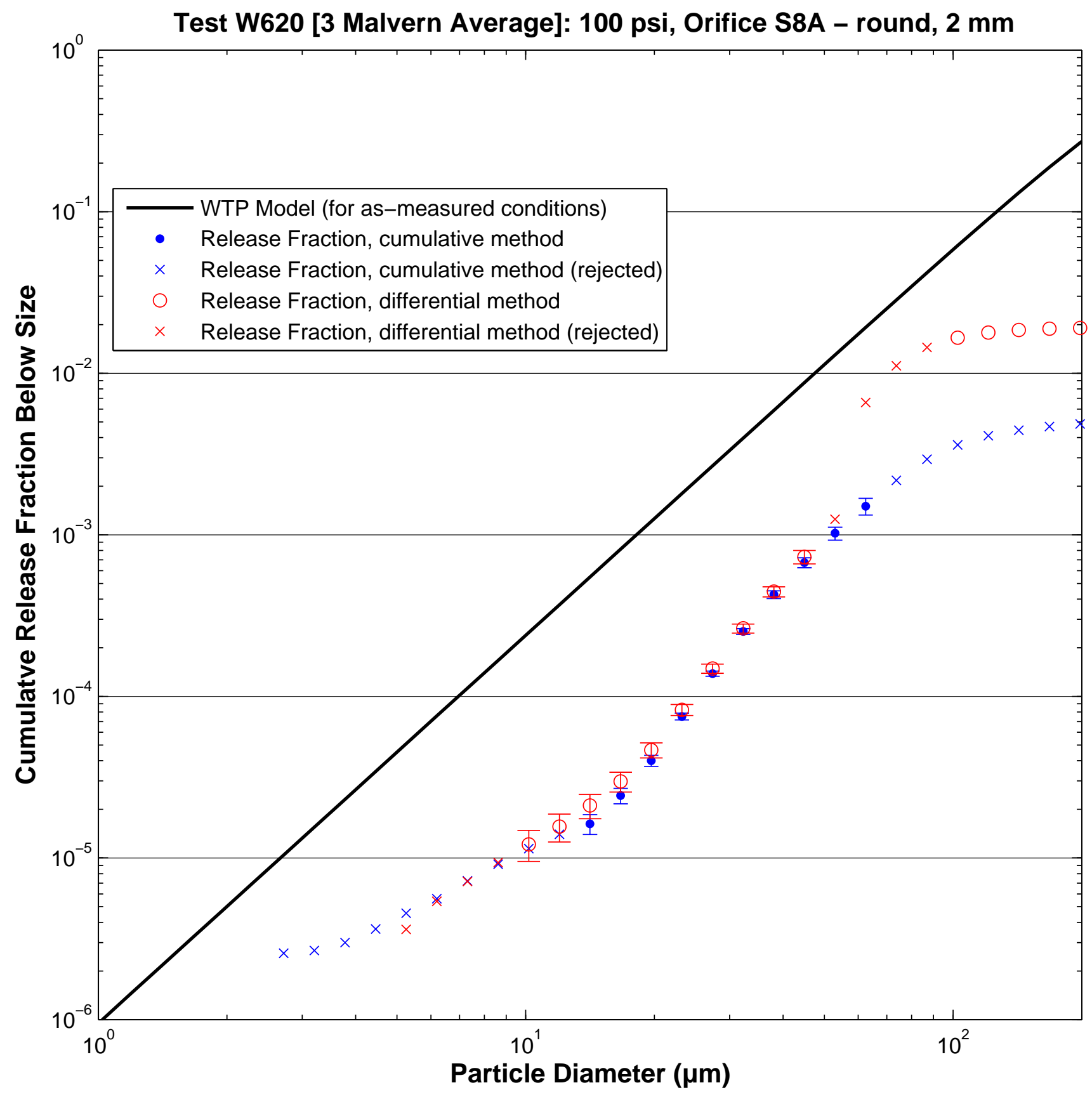




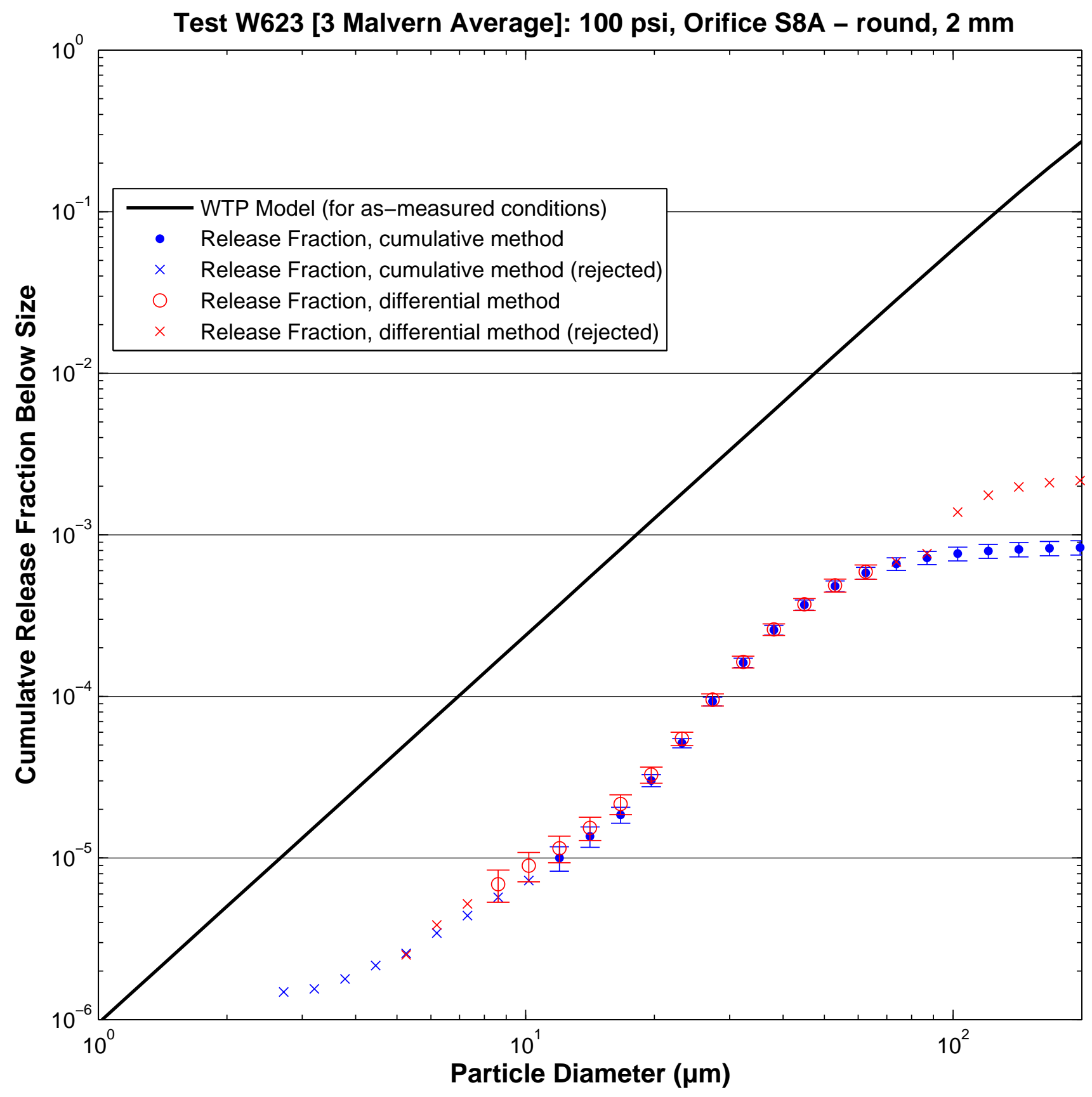




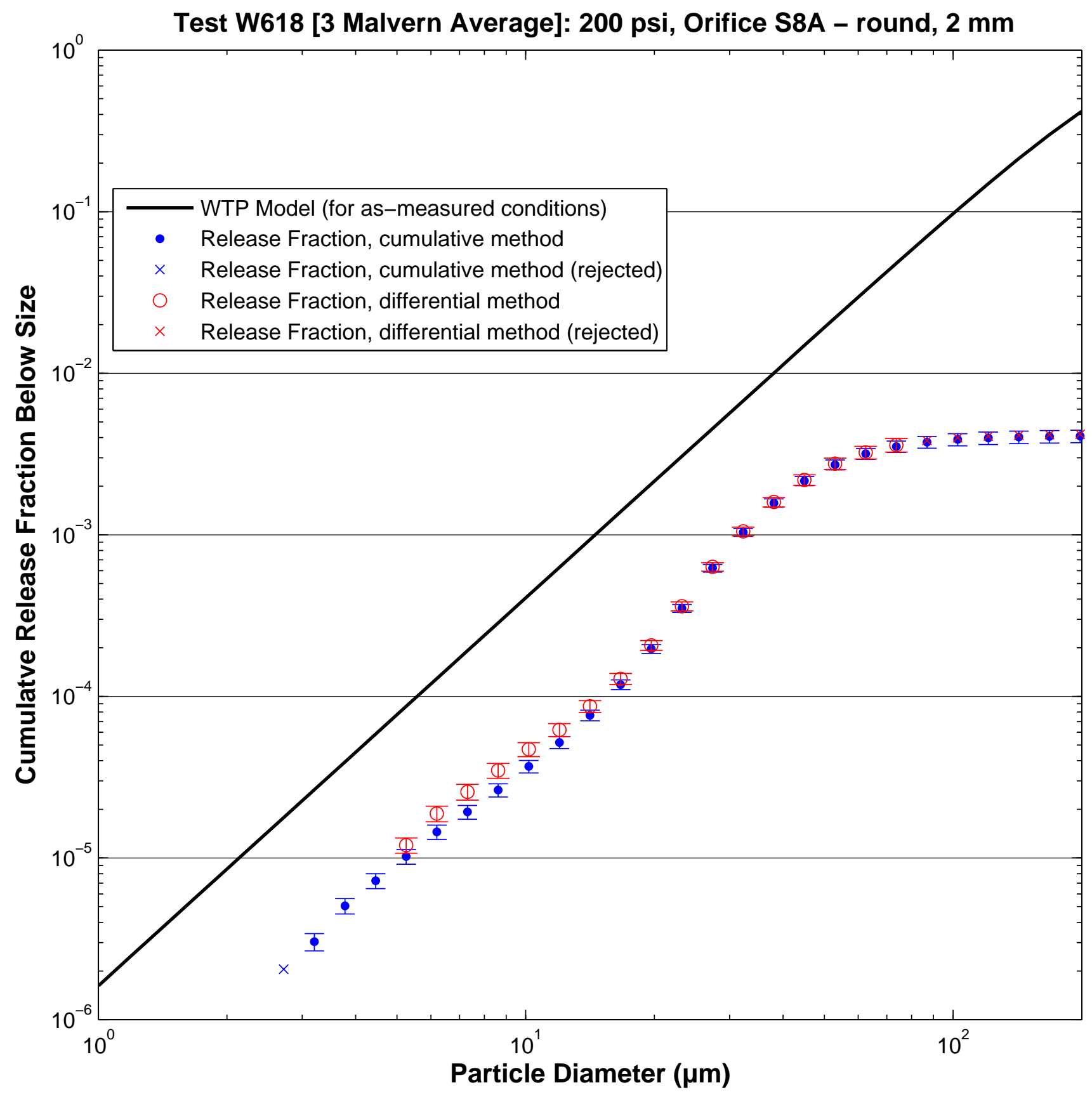

B. 195 


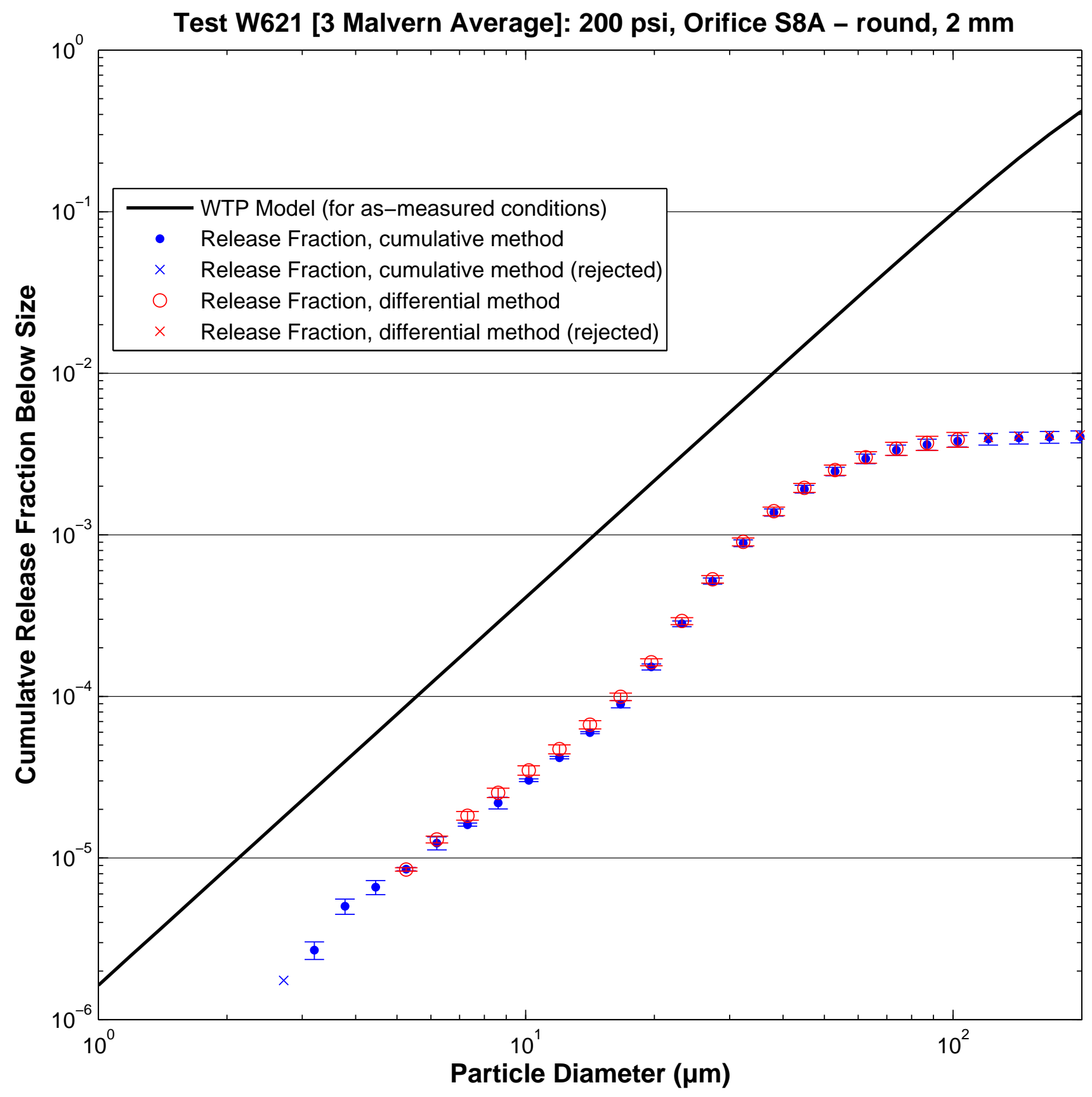




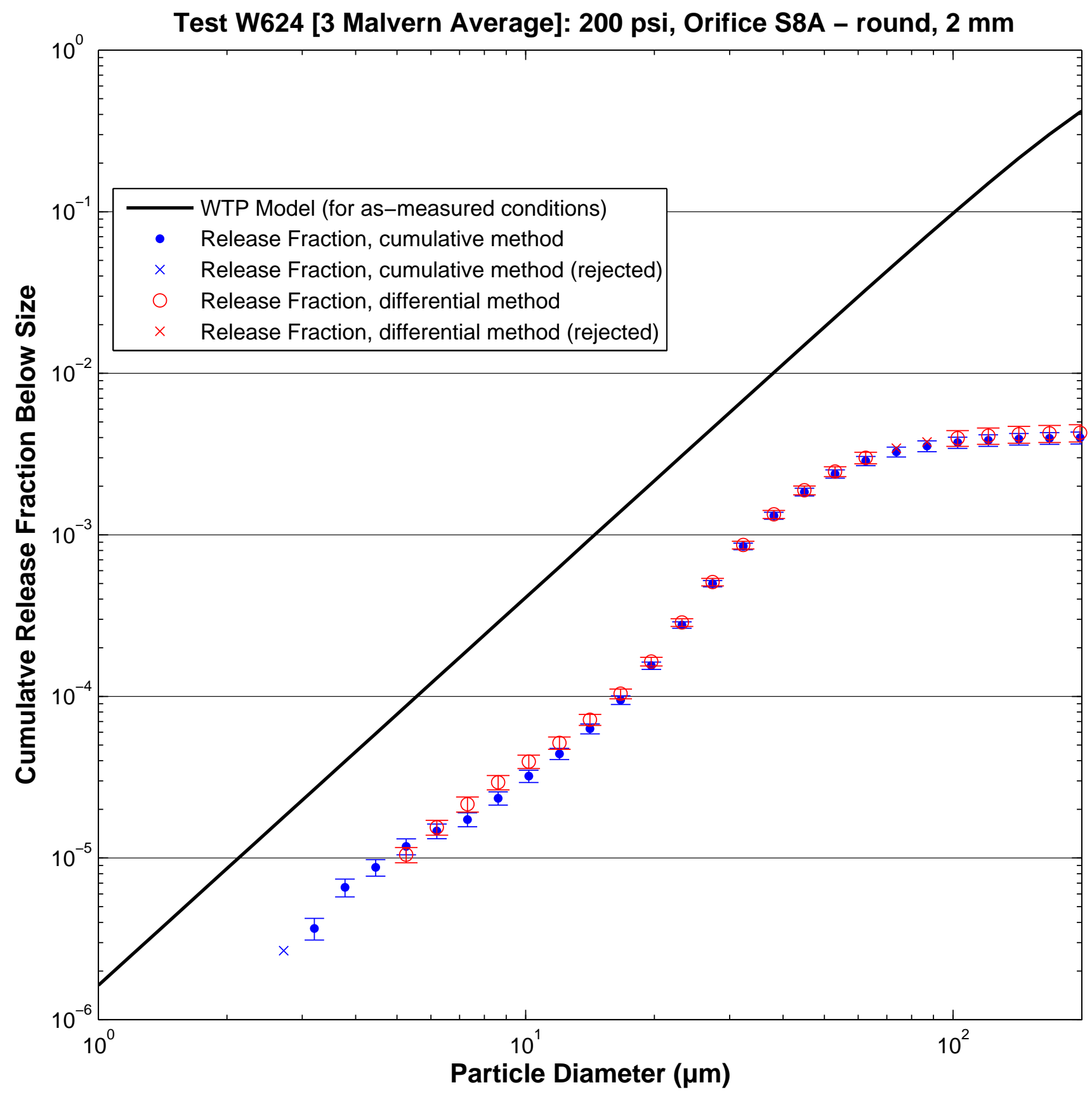




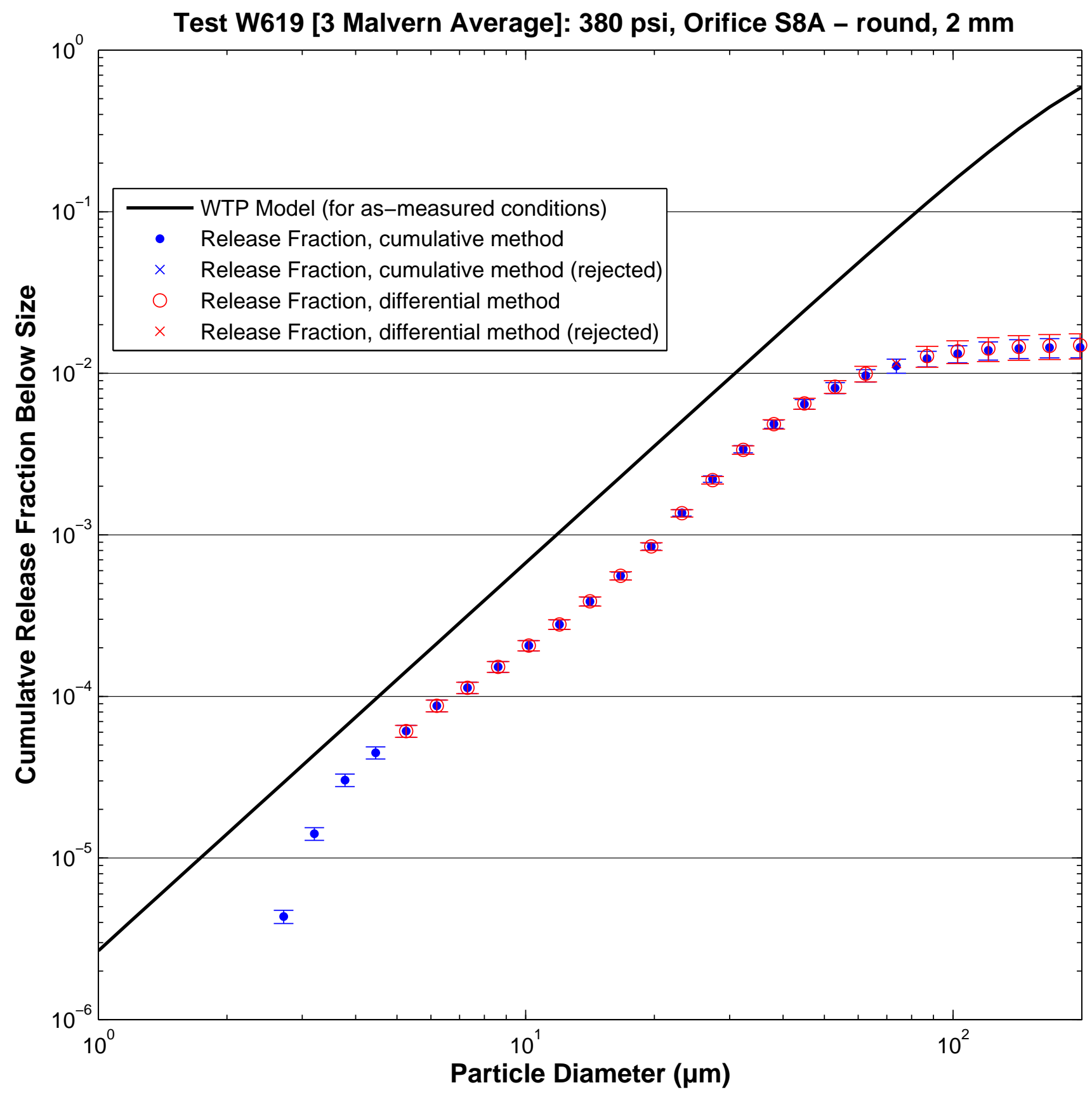




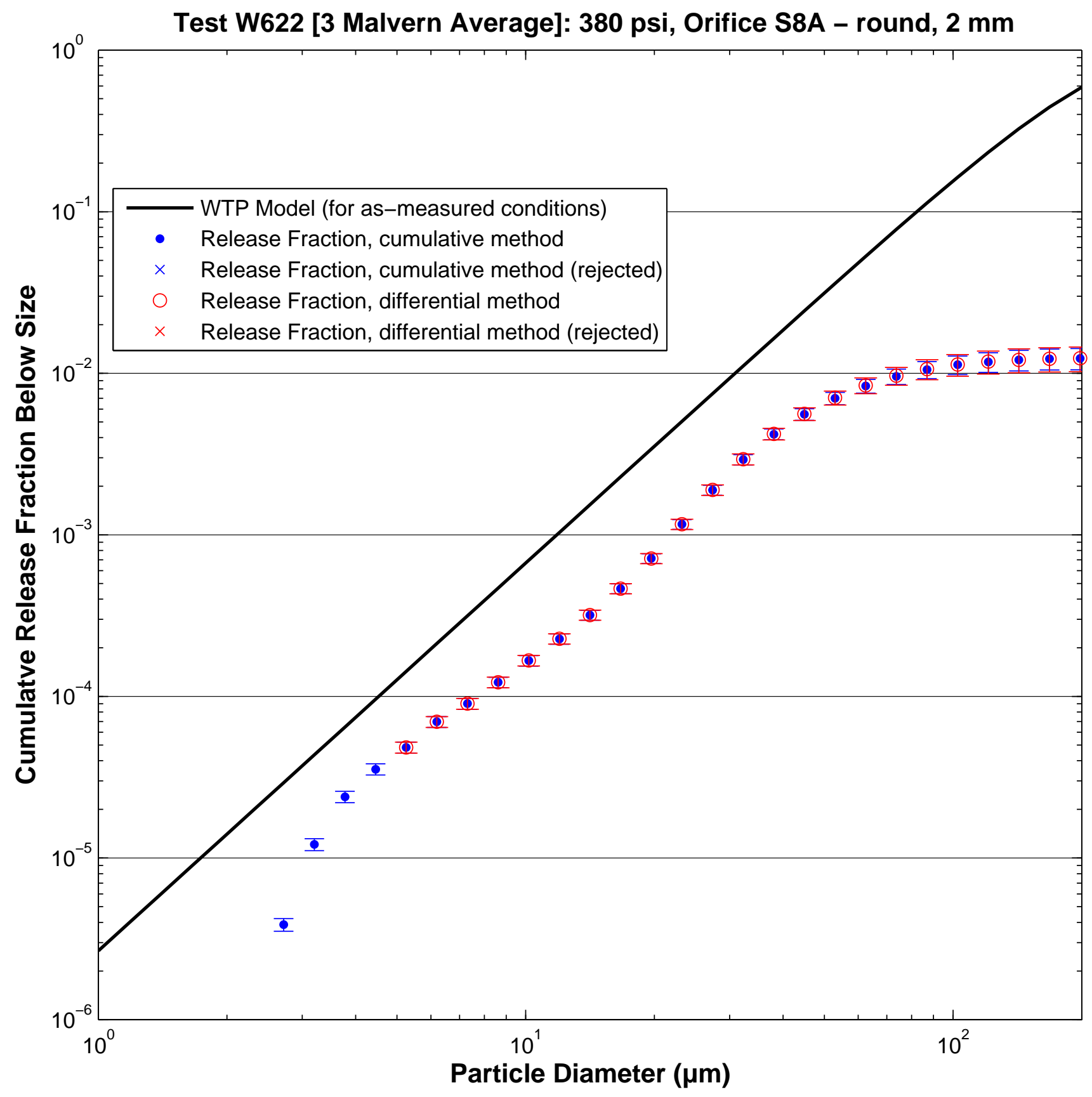

B.199 


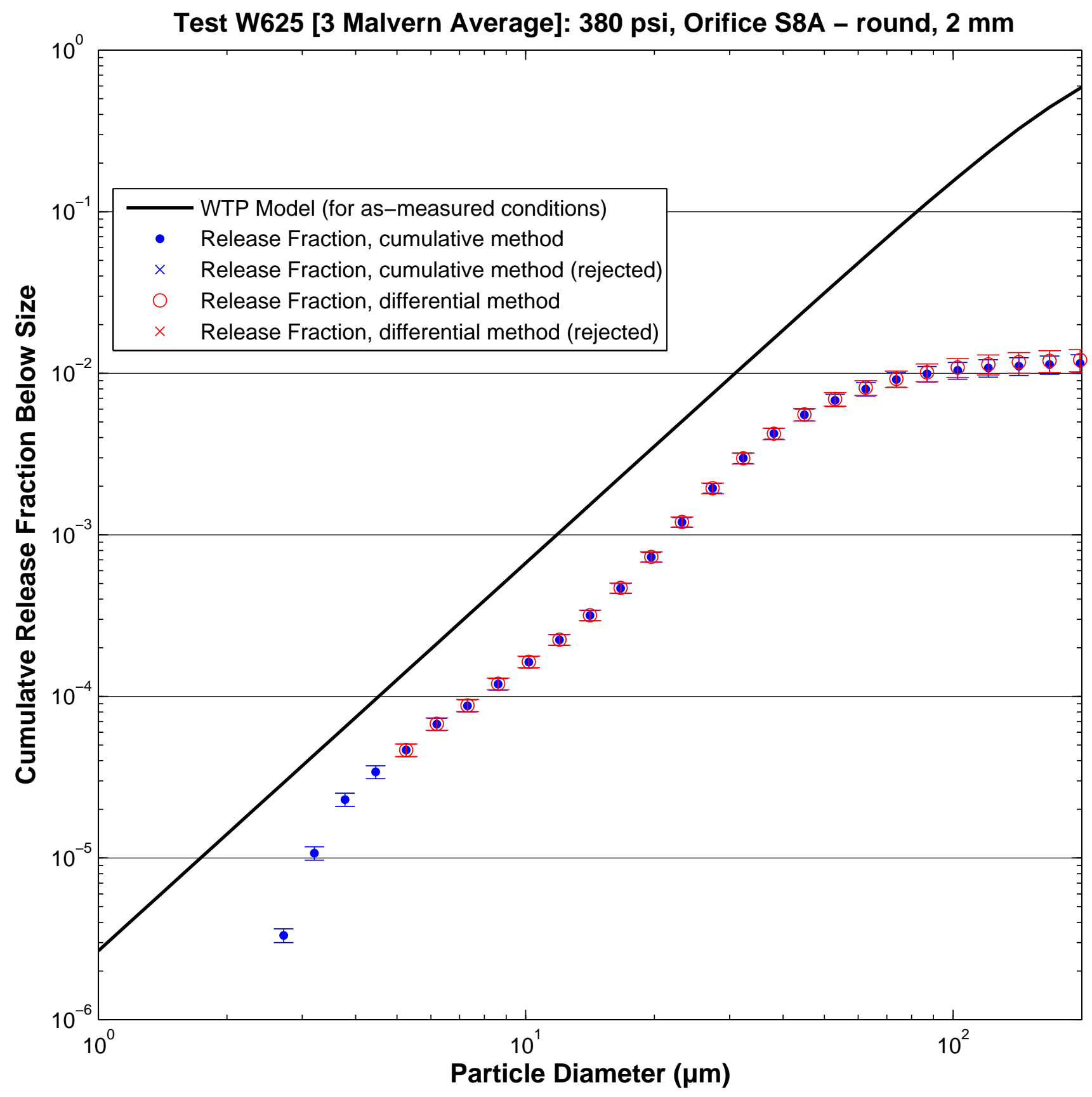




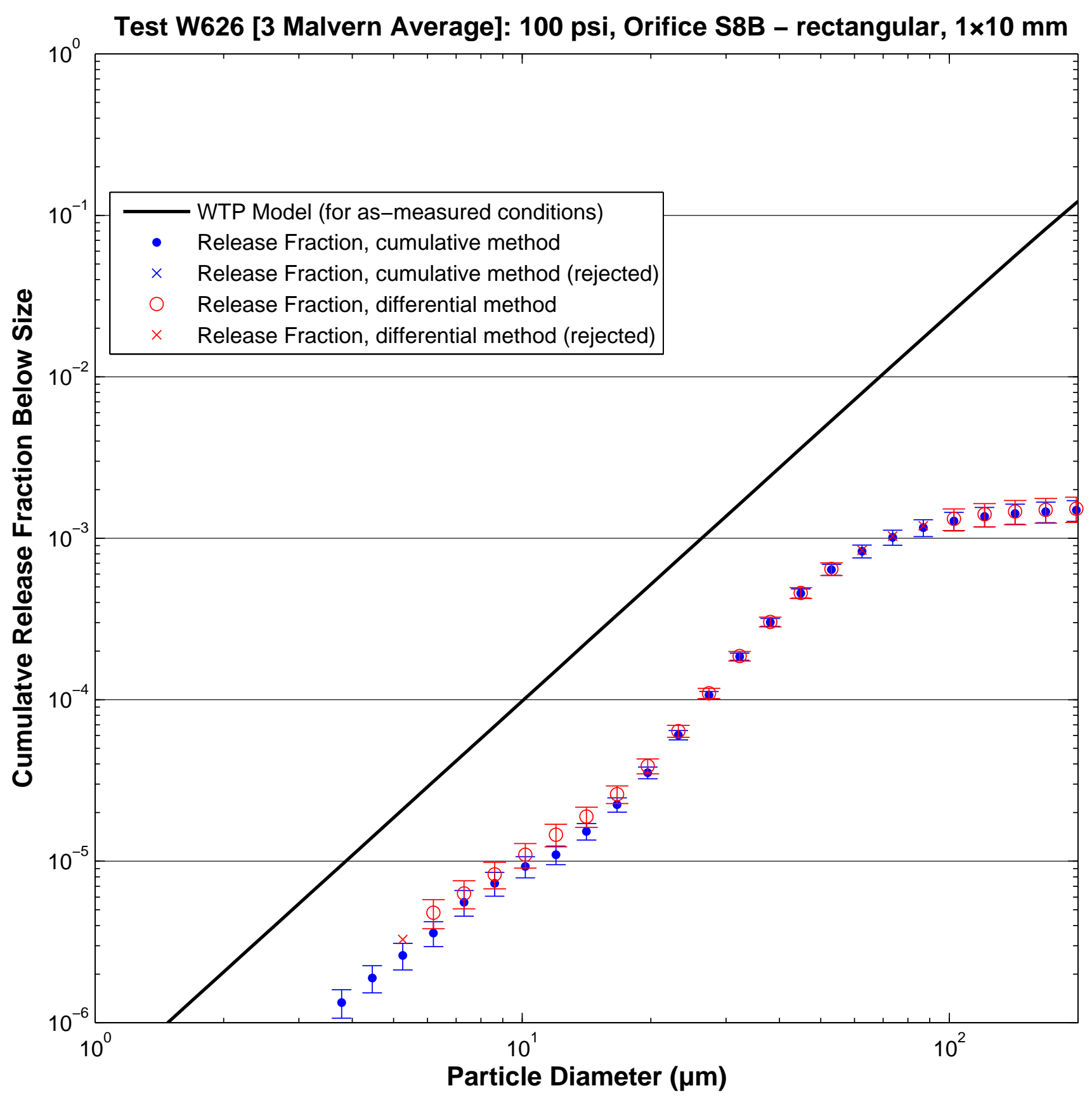




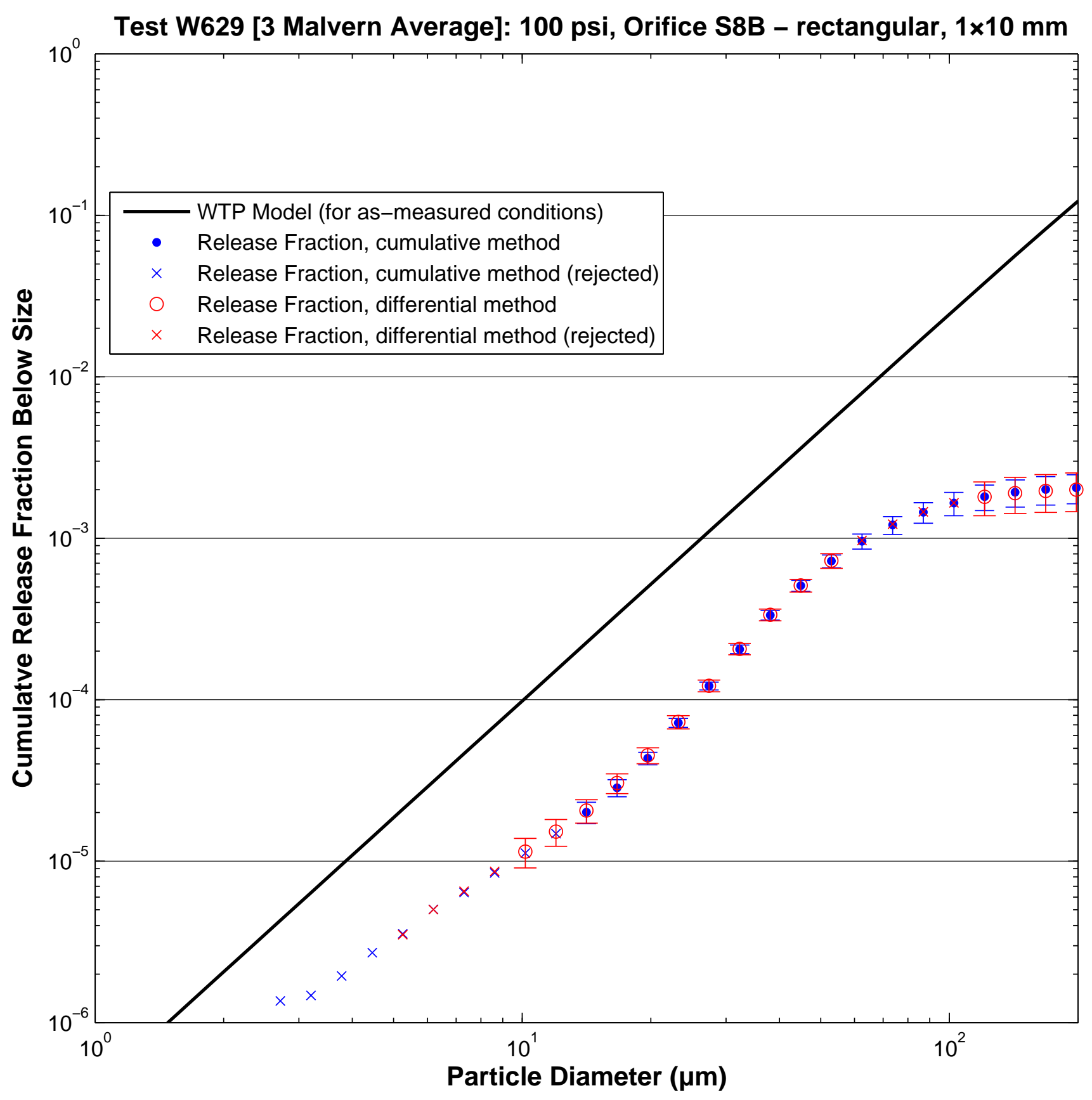




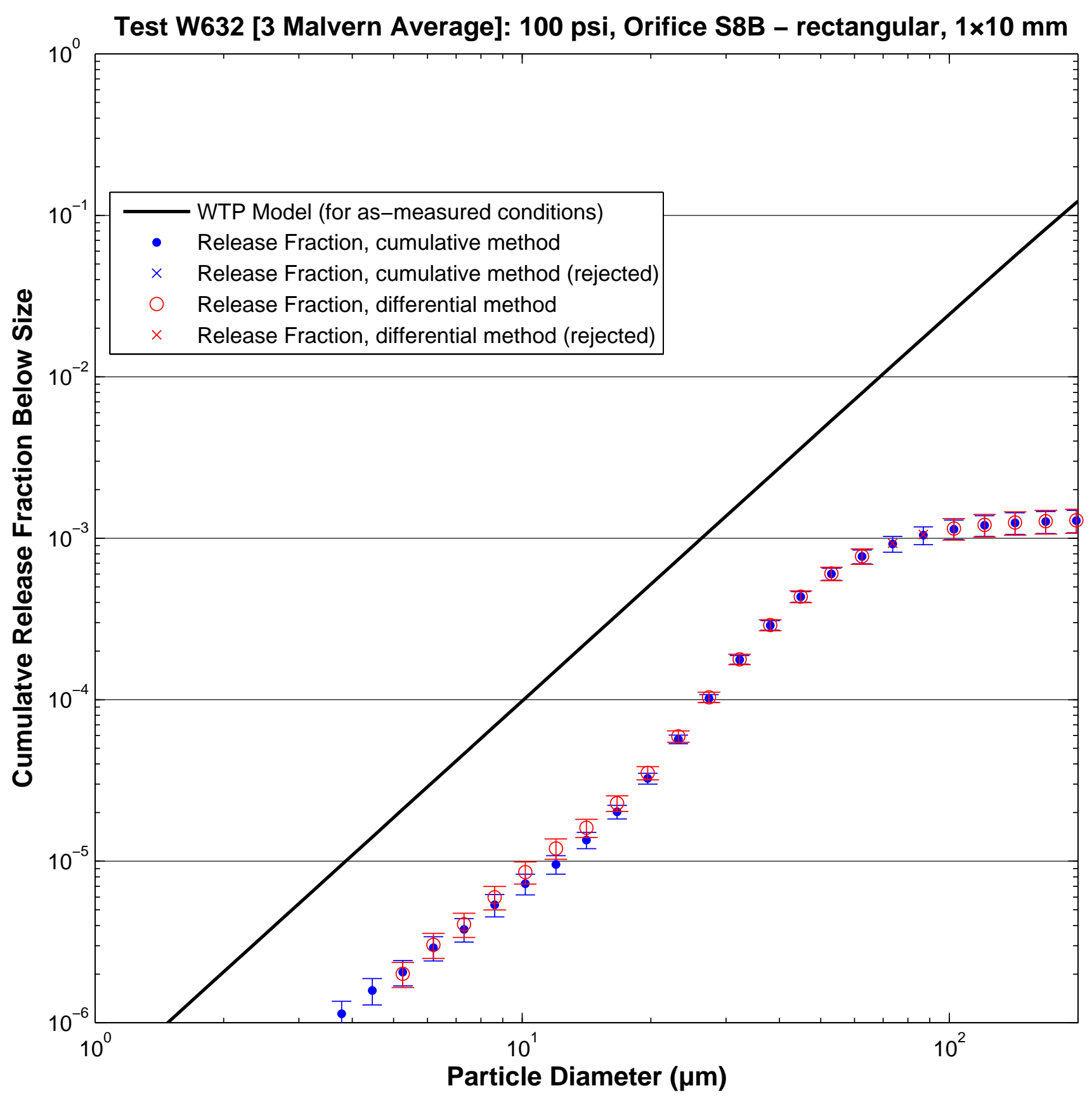




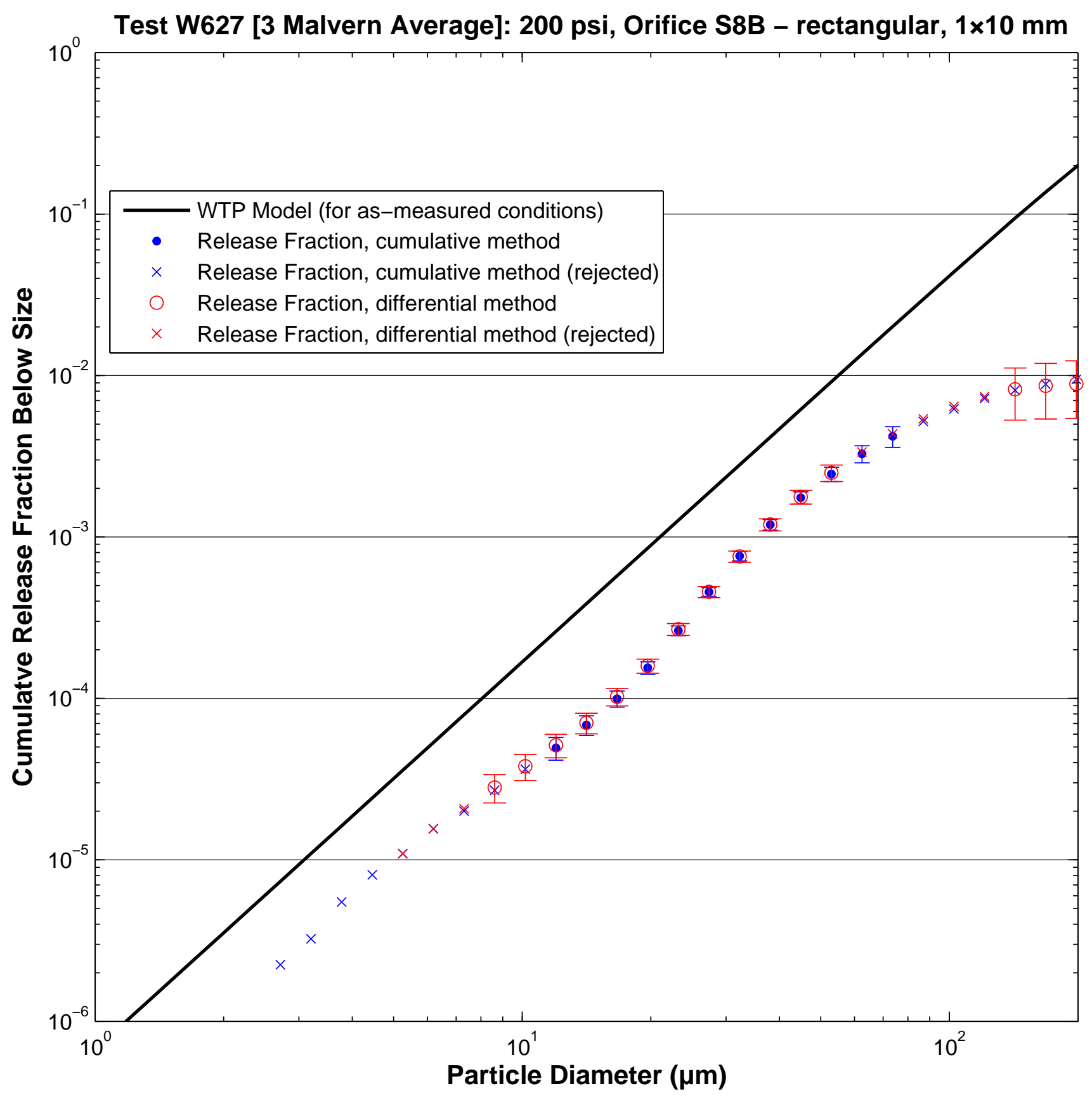




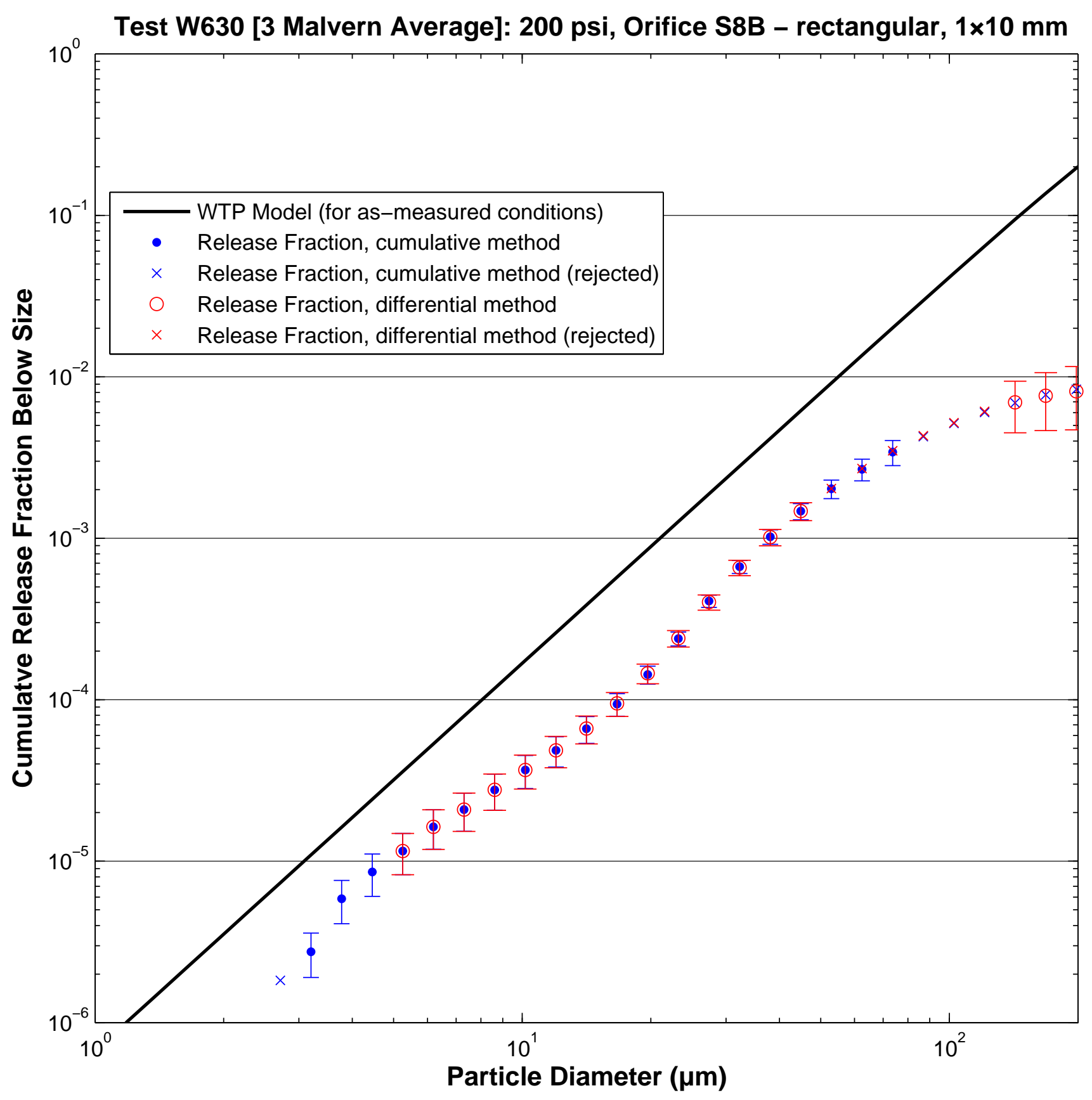




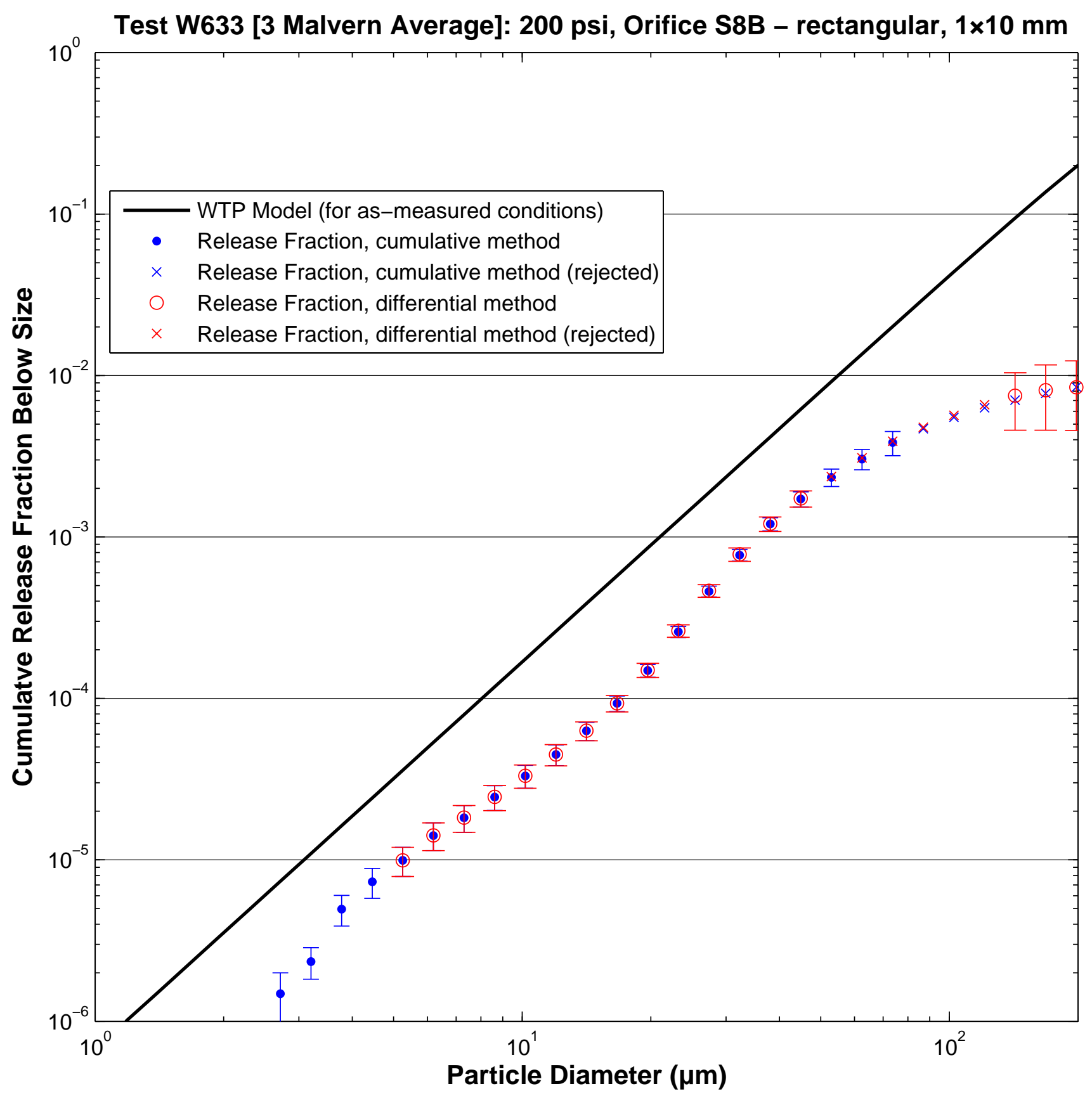




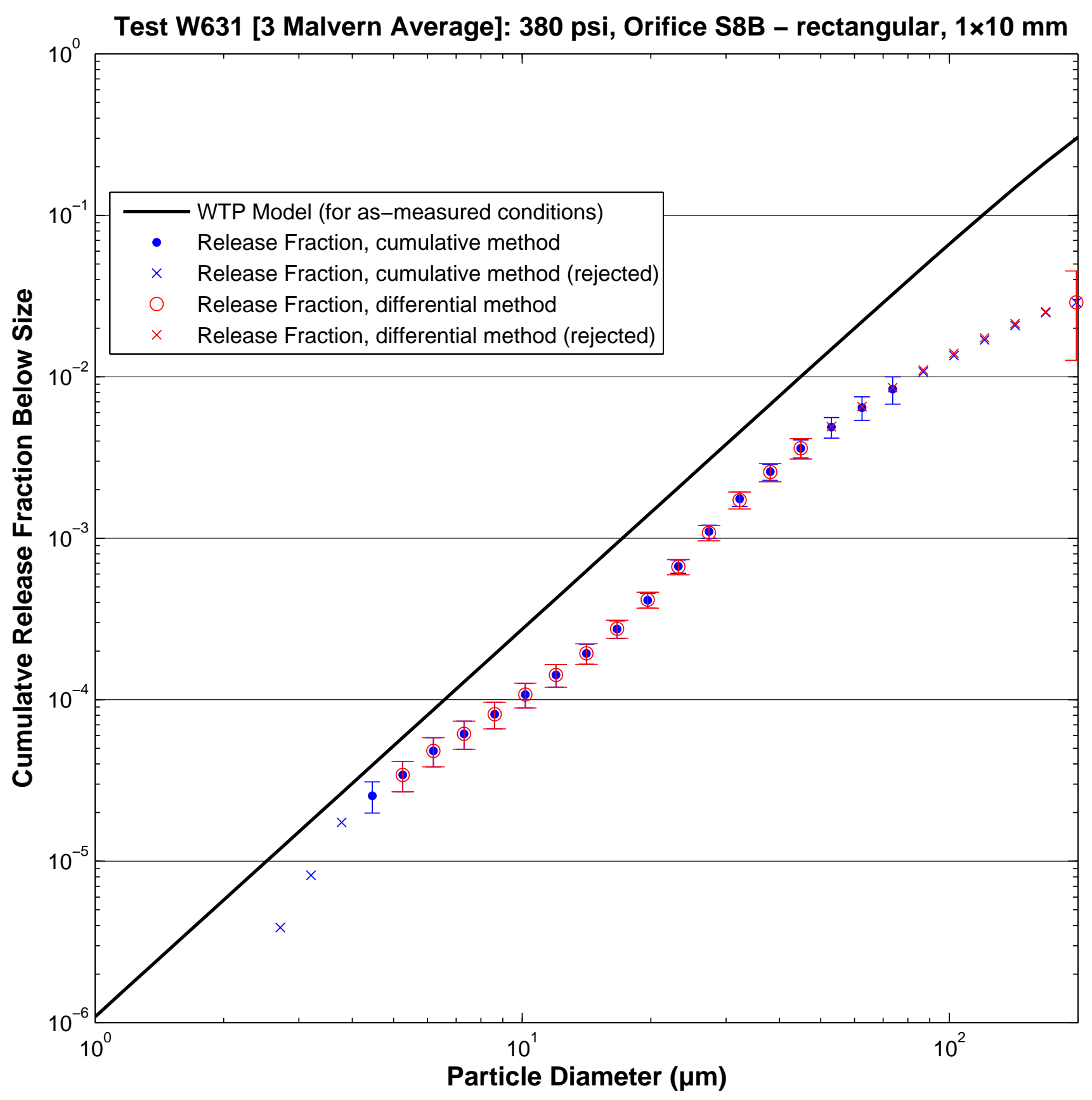




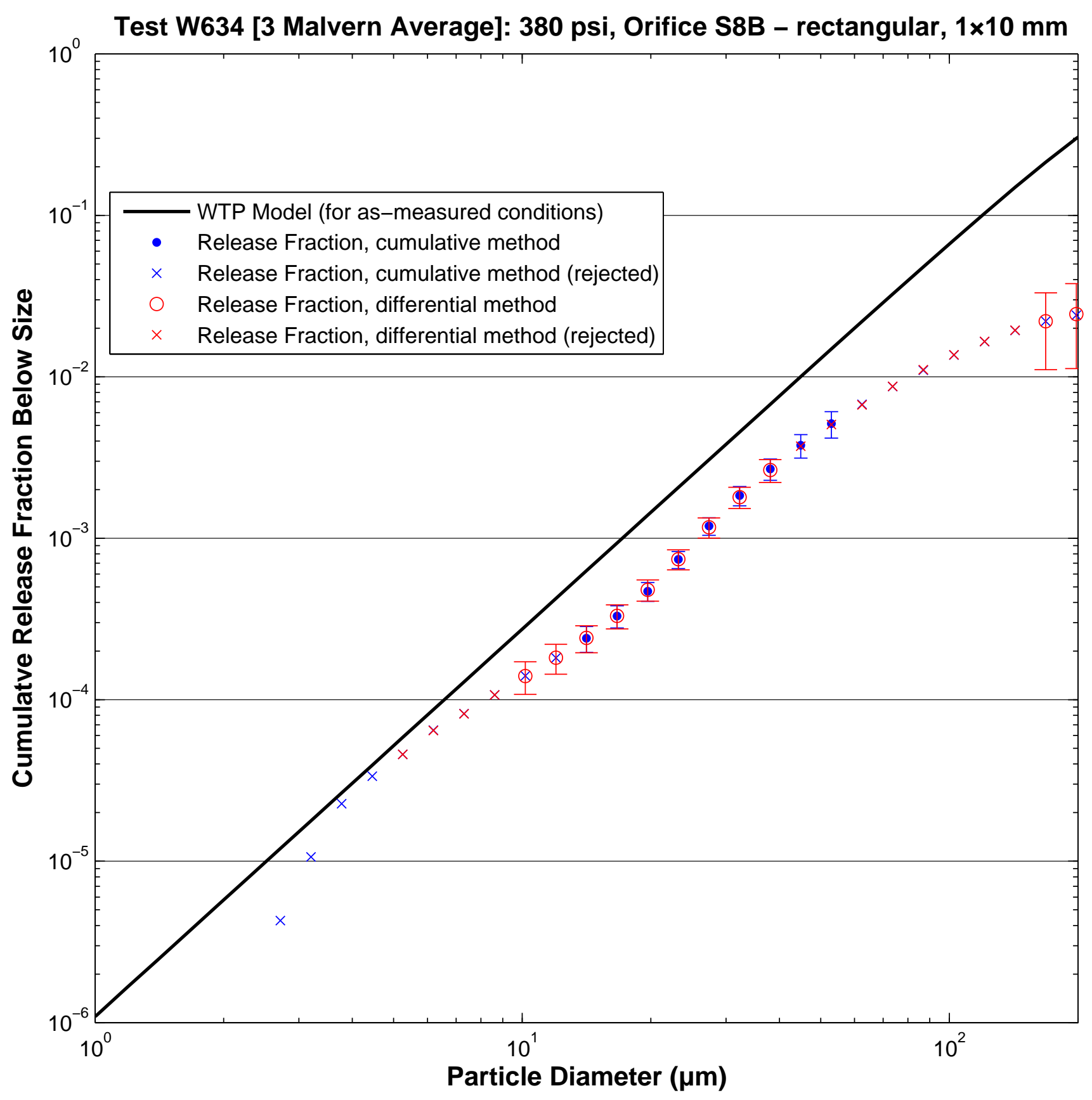




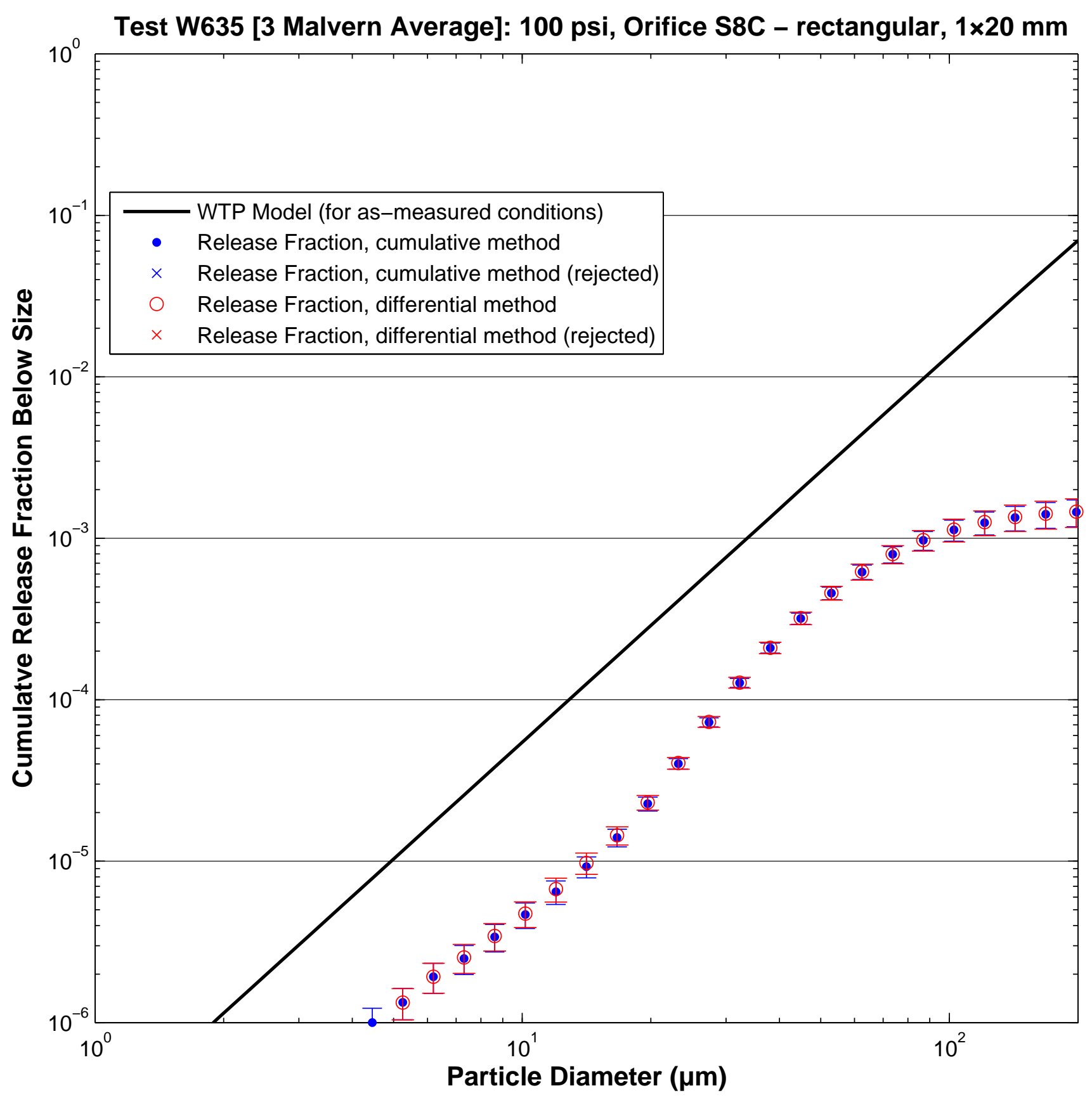




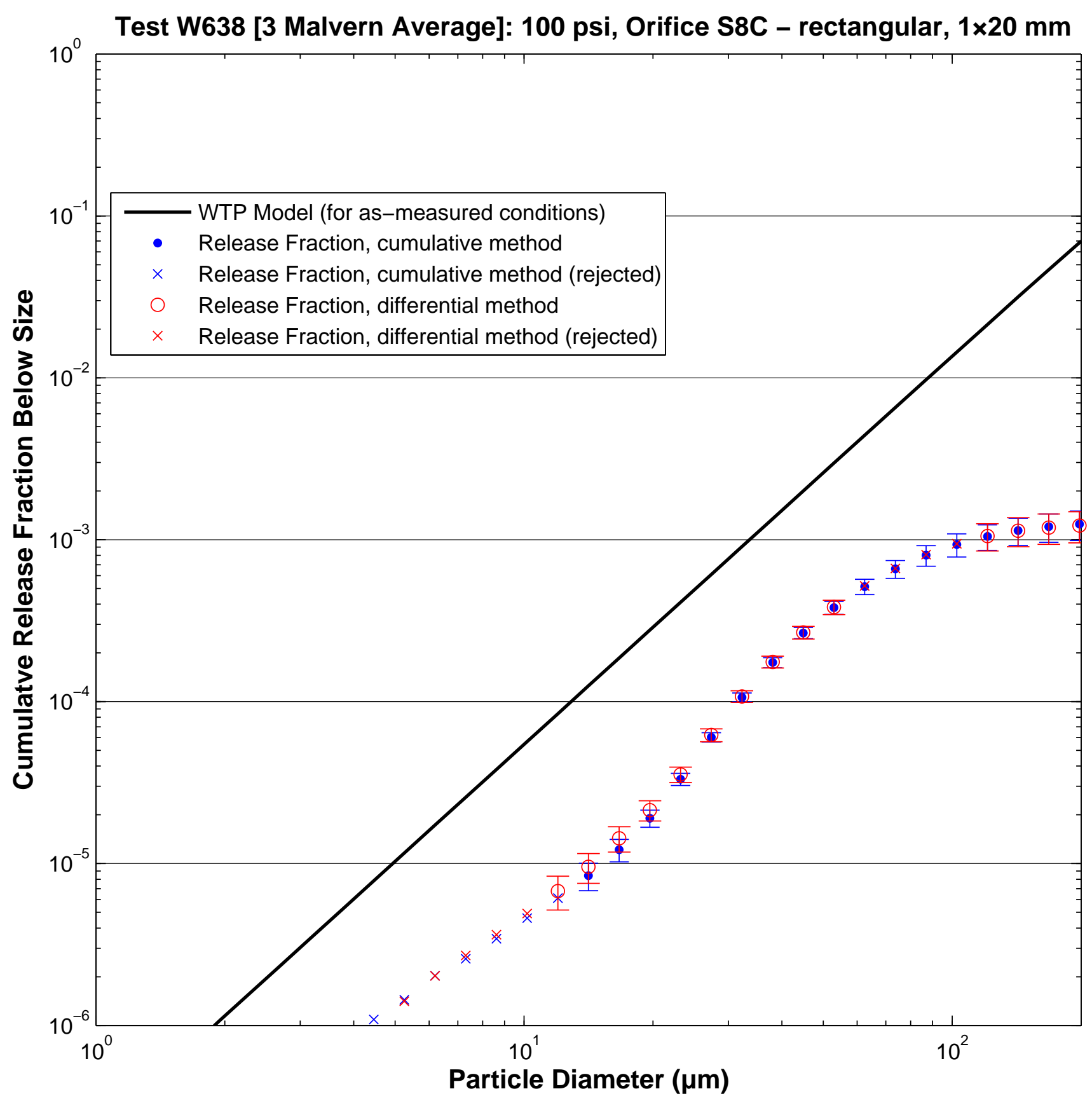




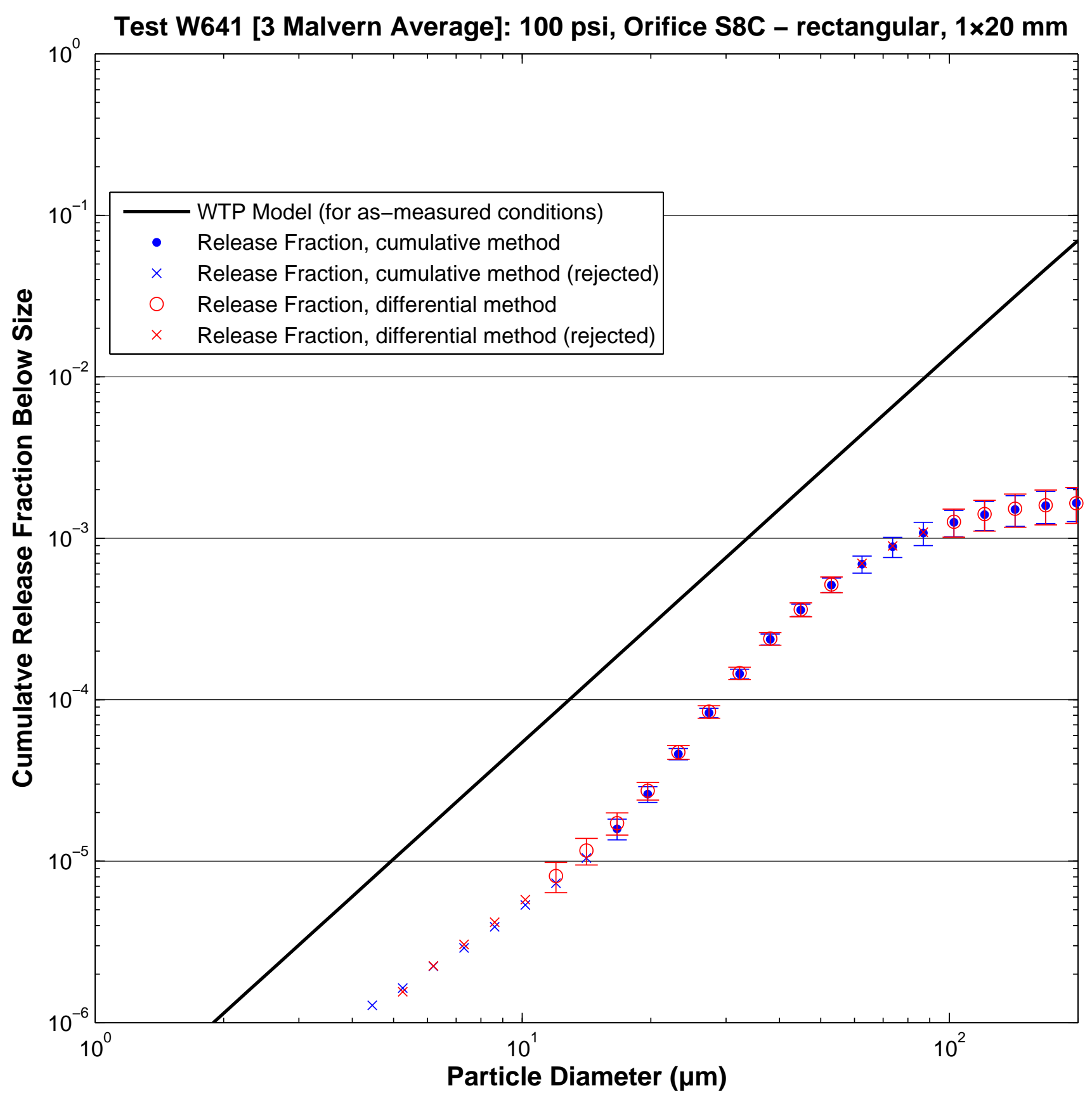




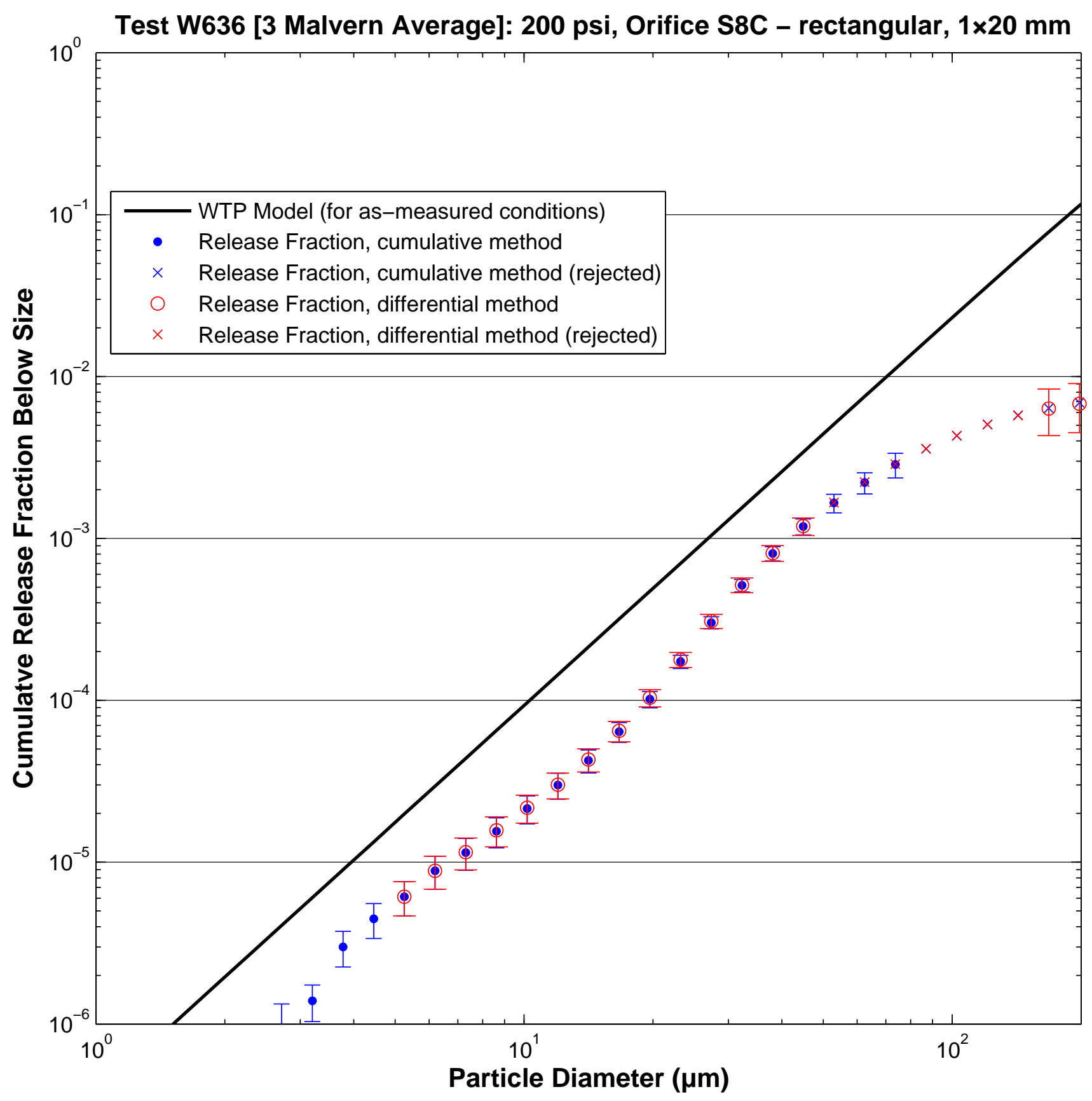




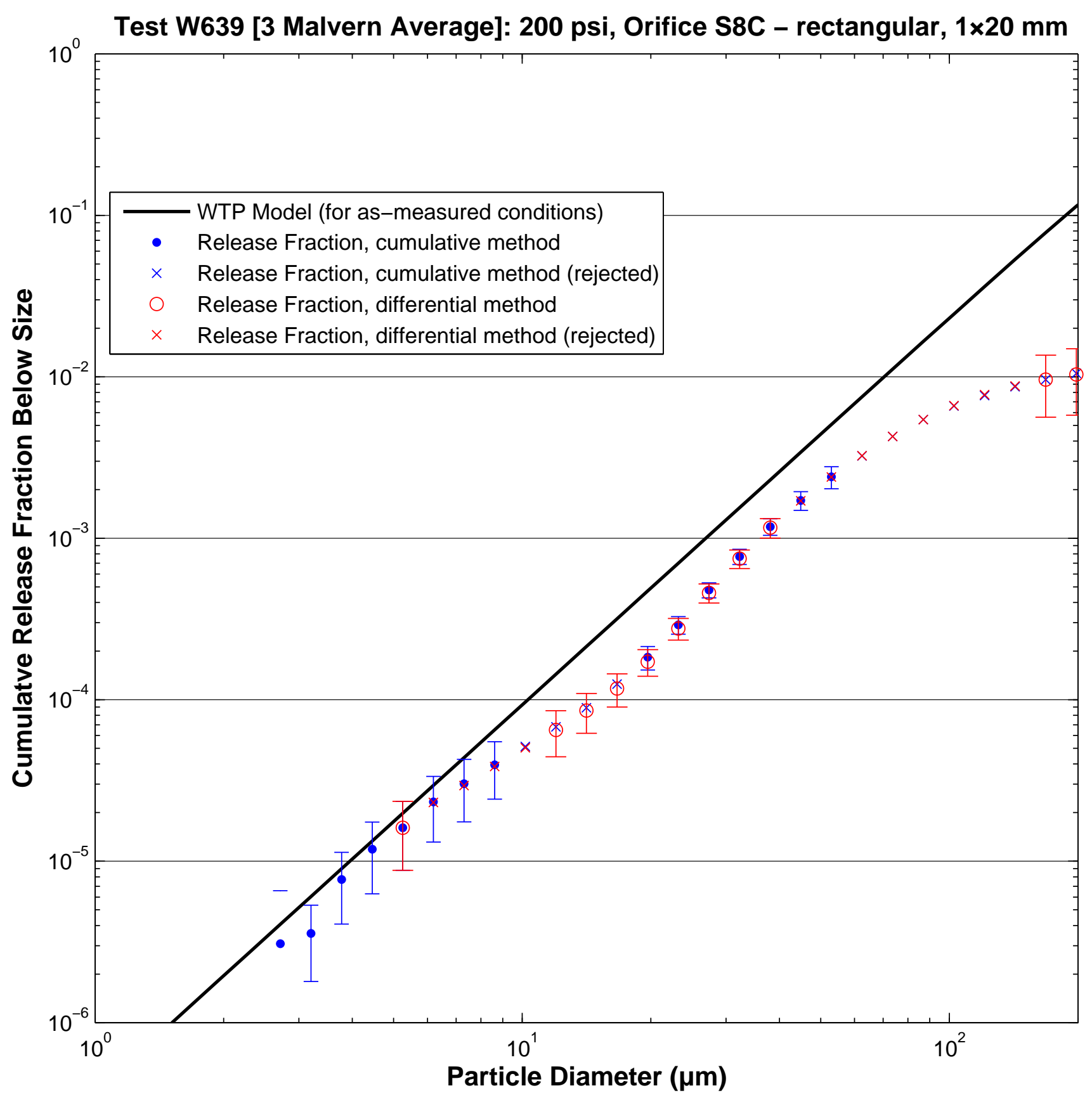




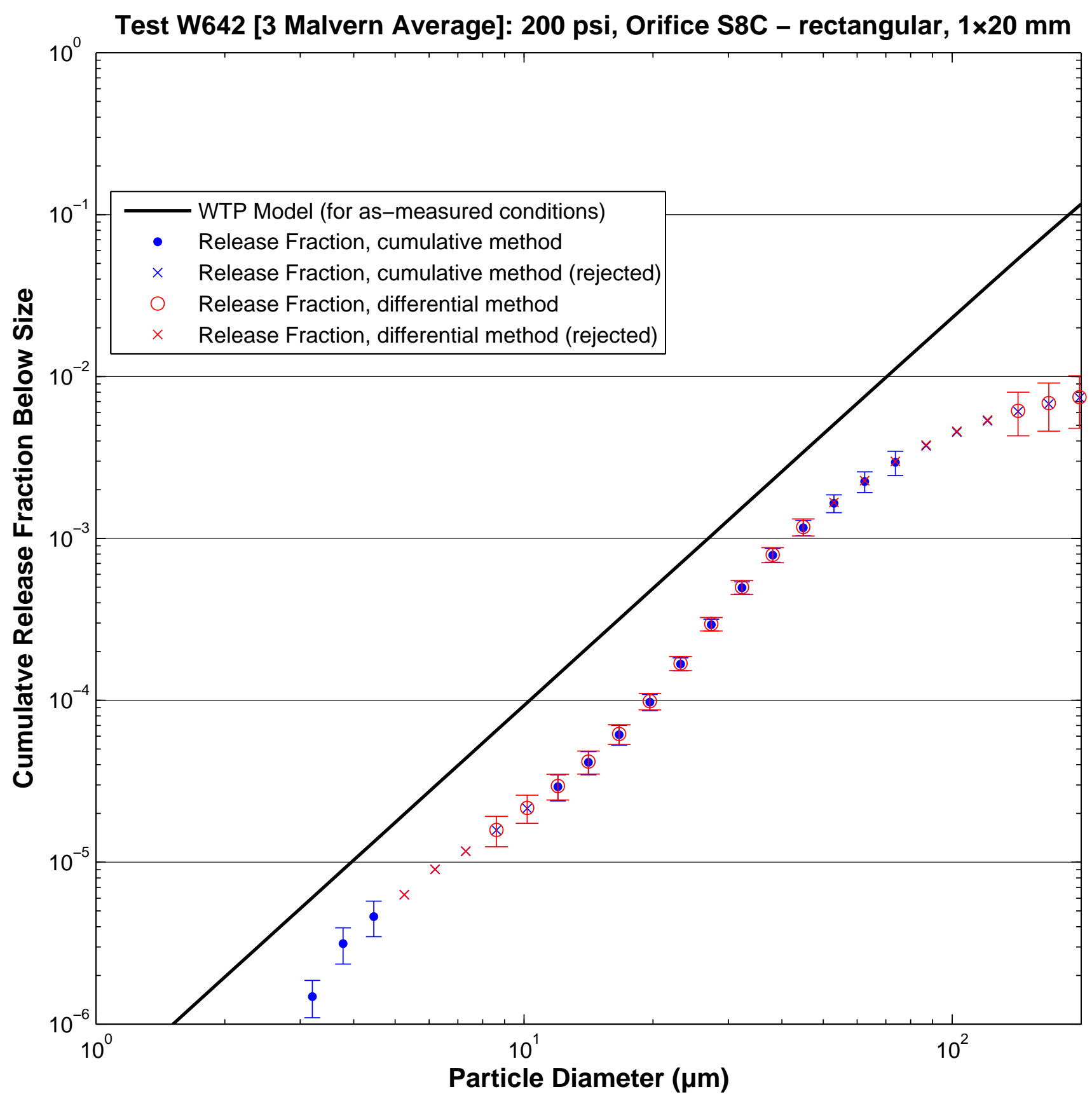




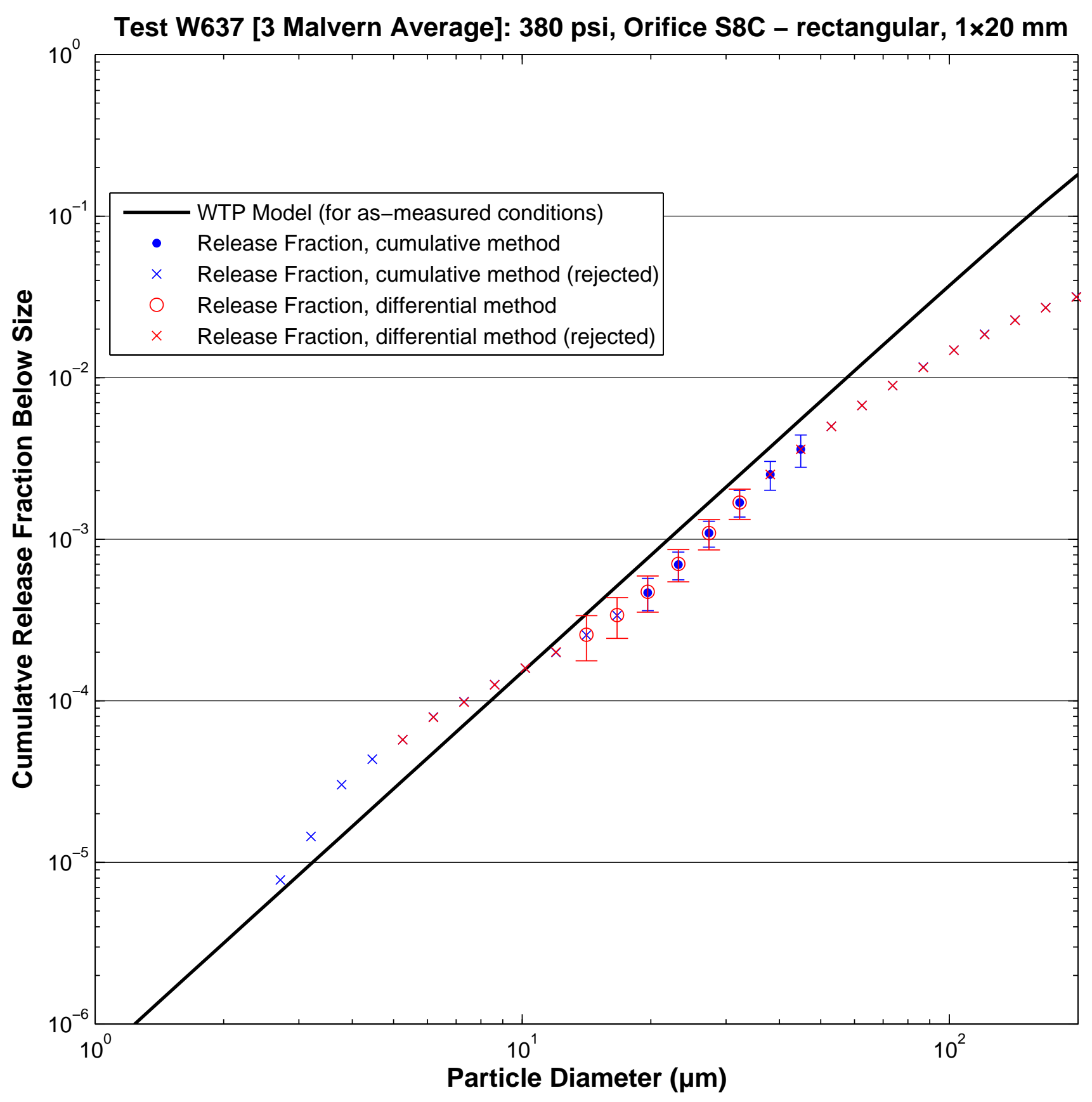




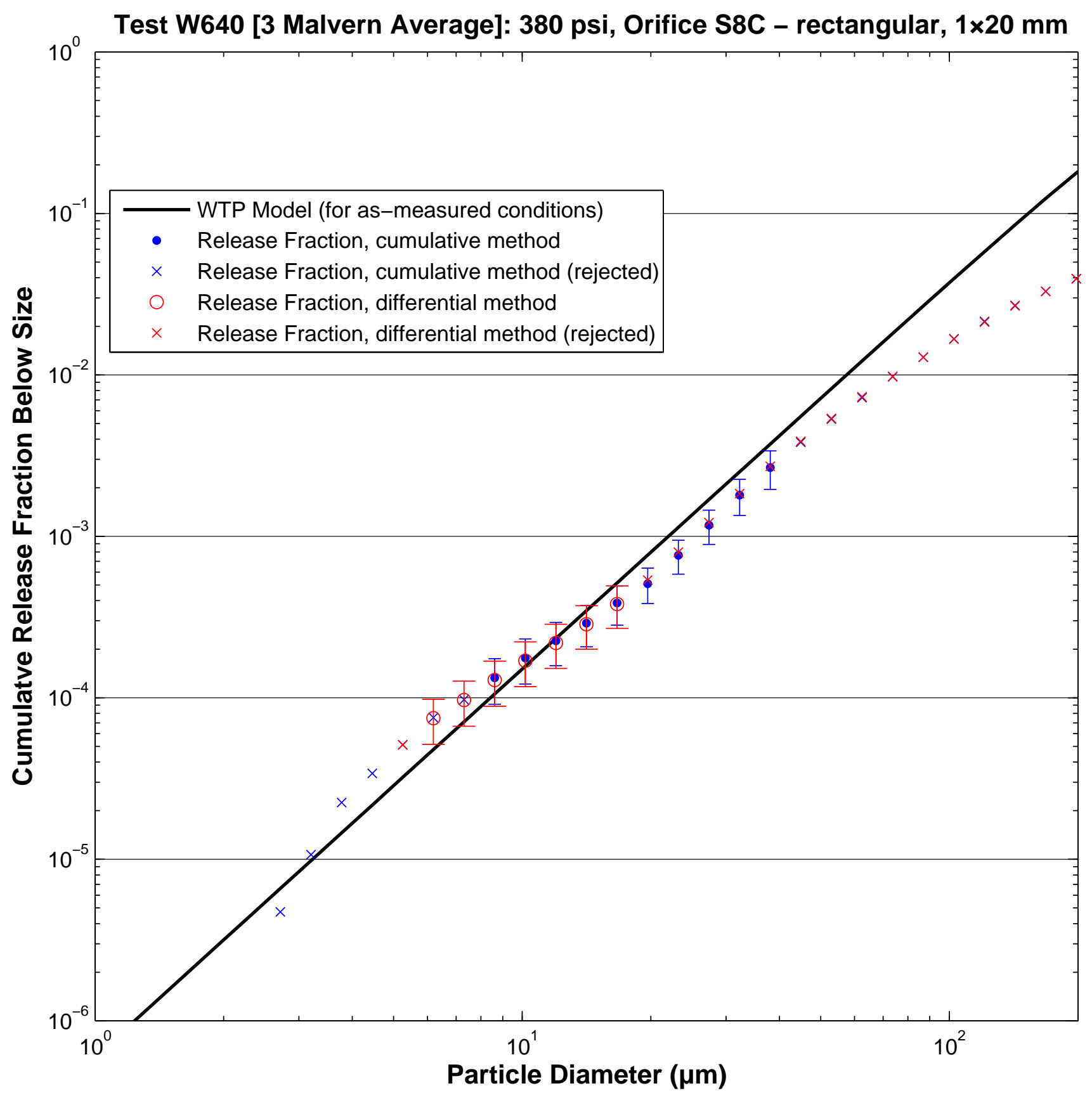




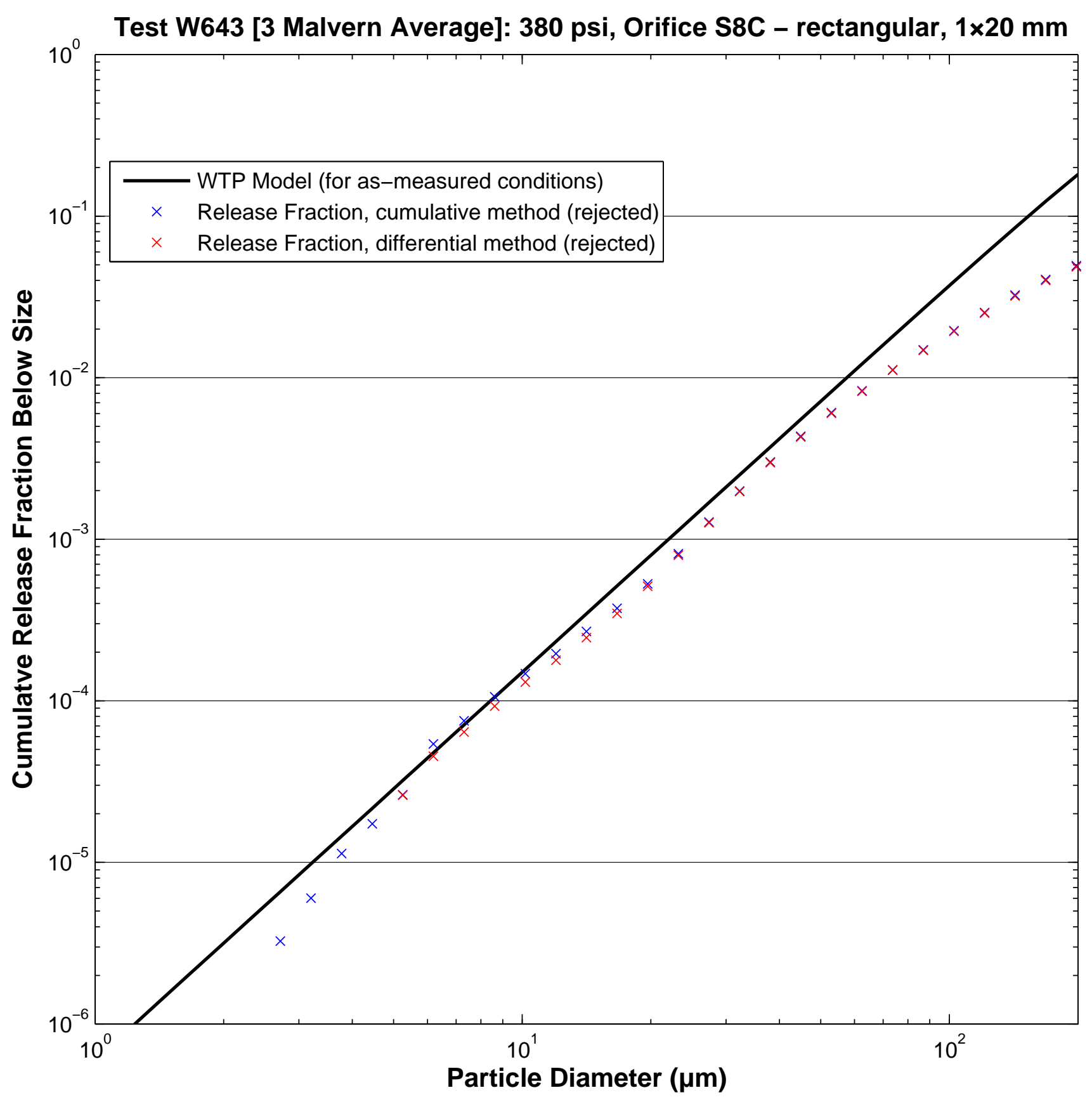




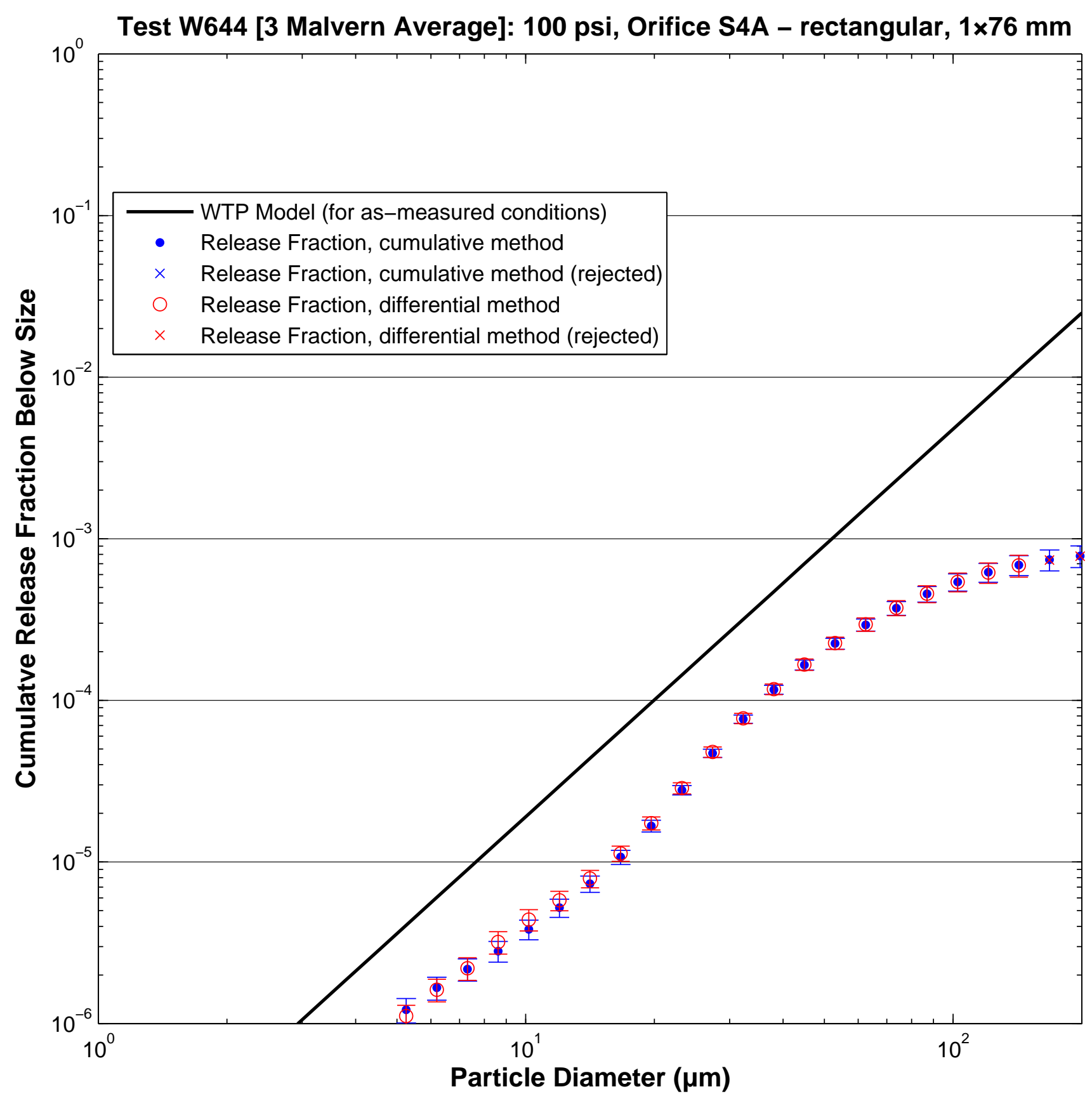




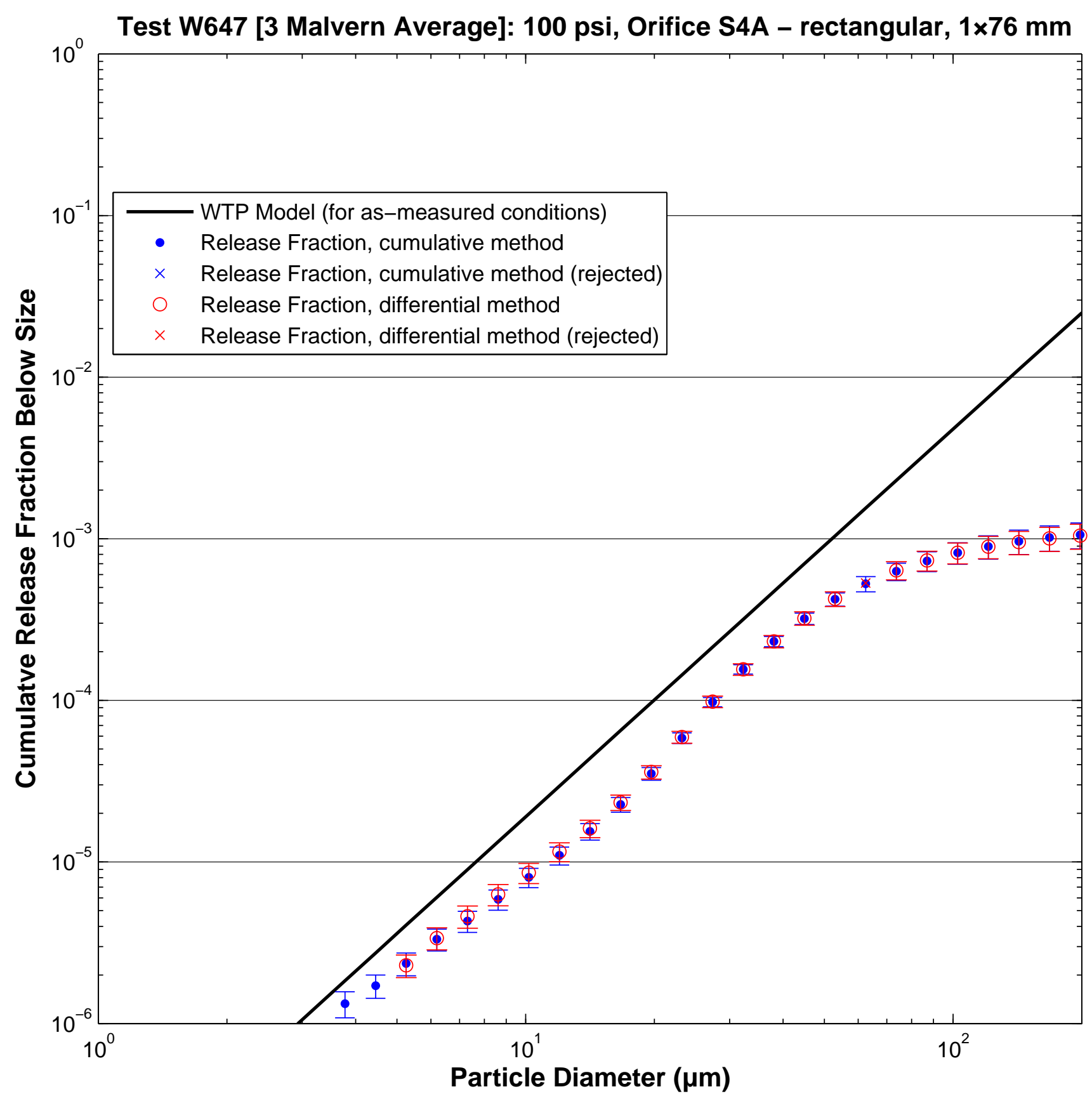




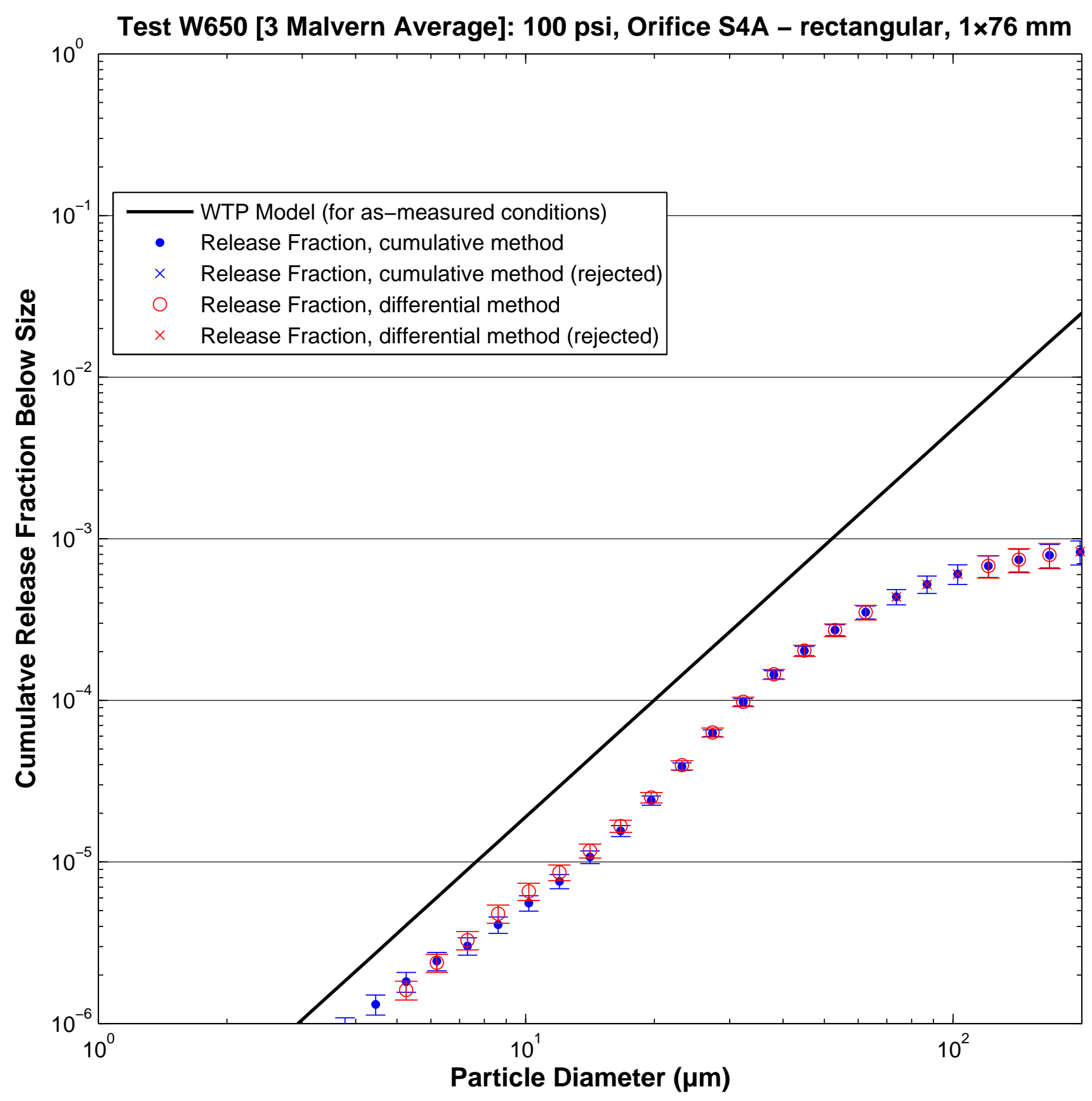




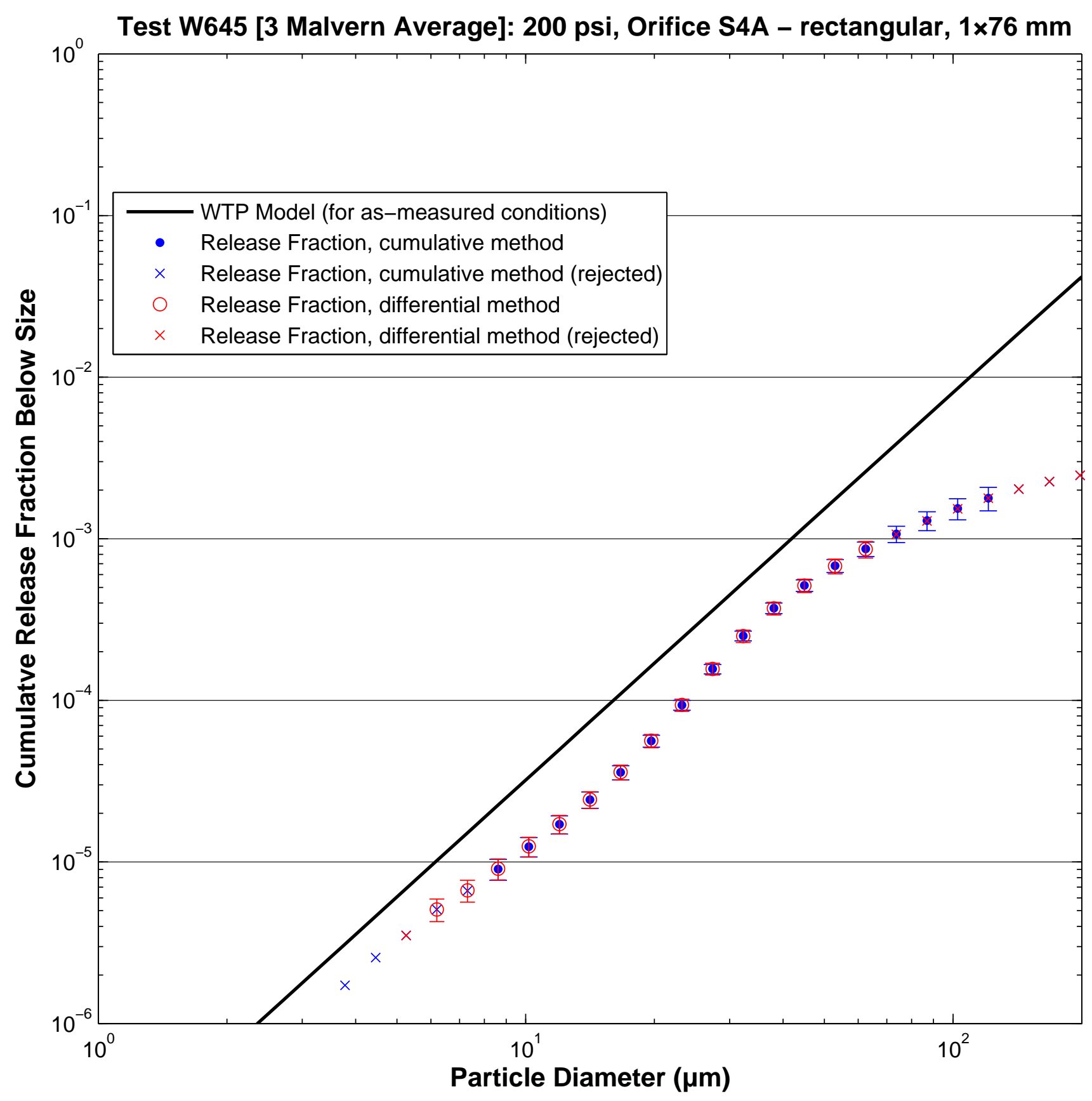




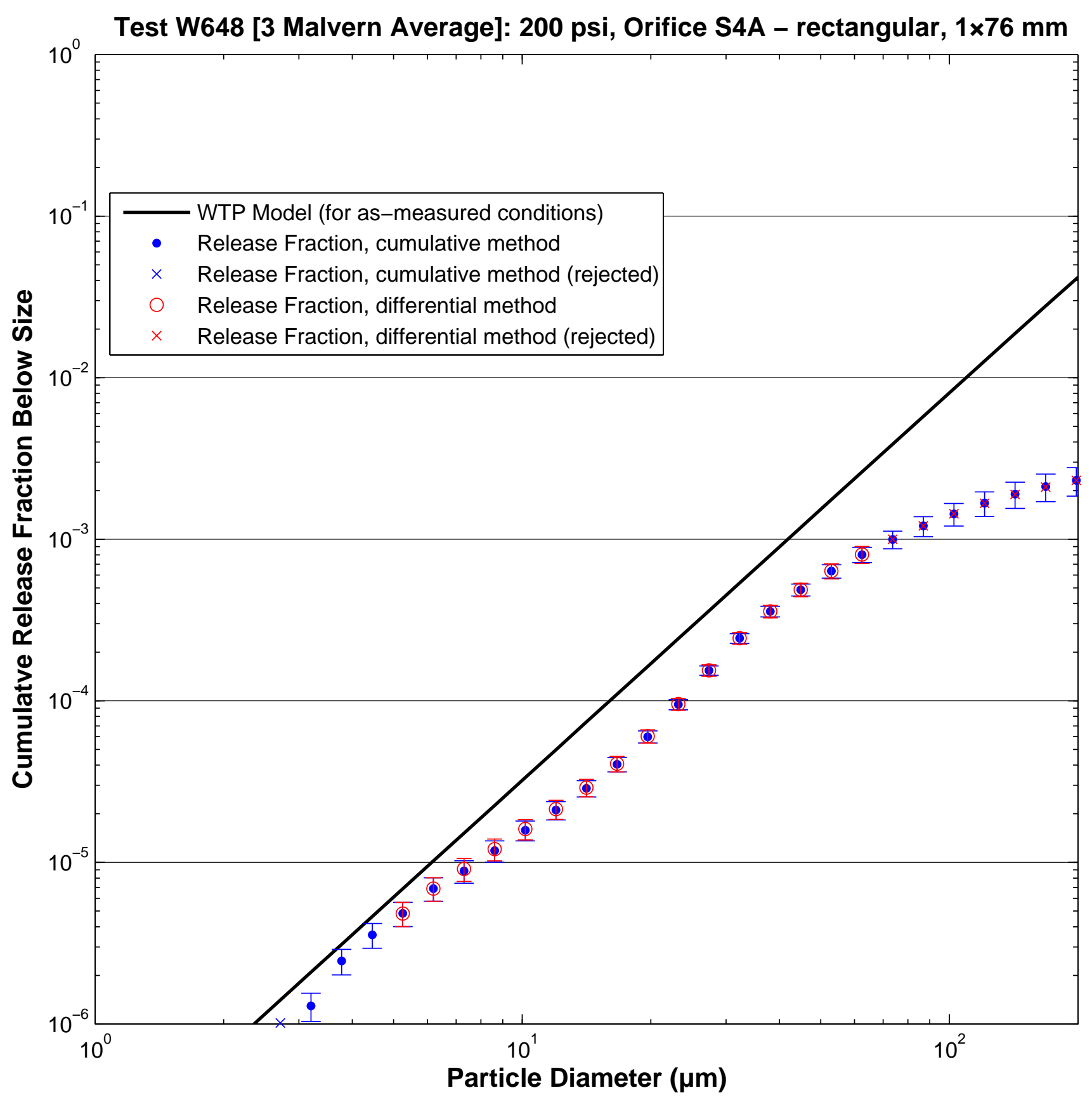




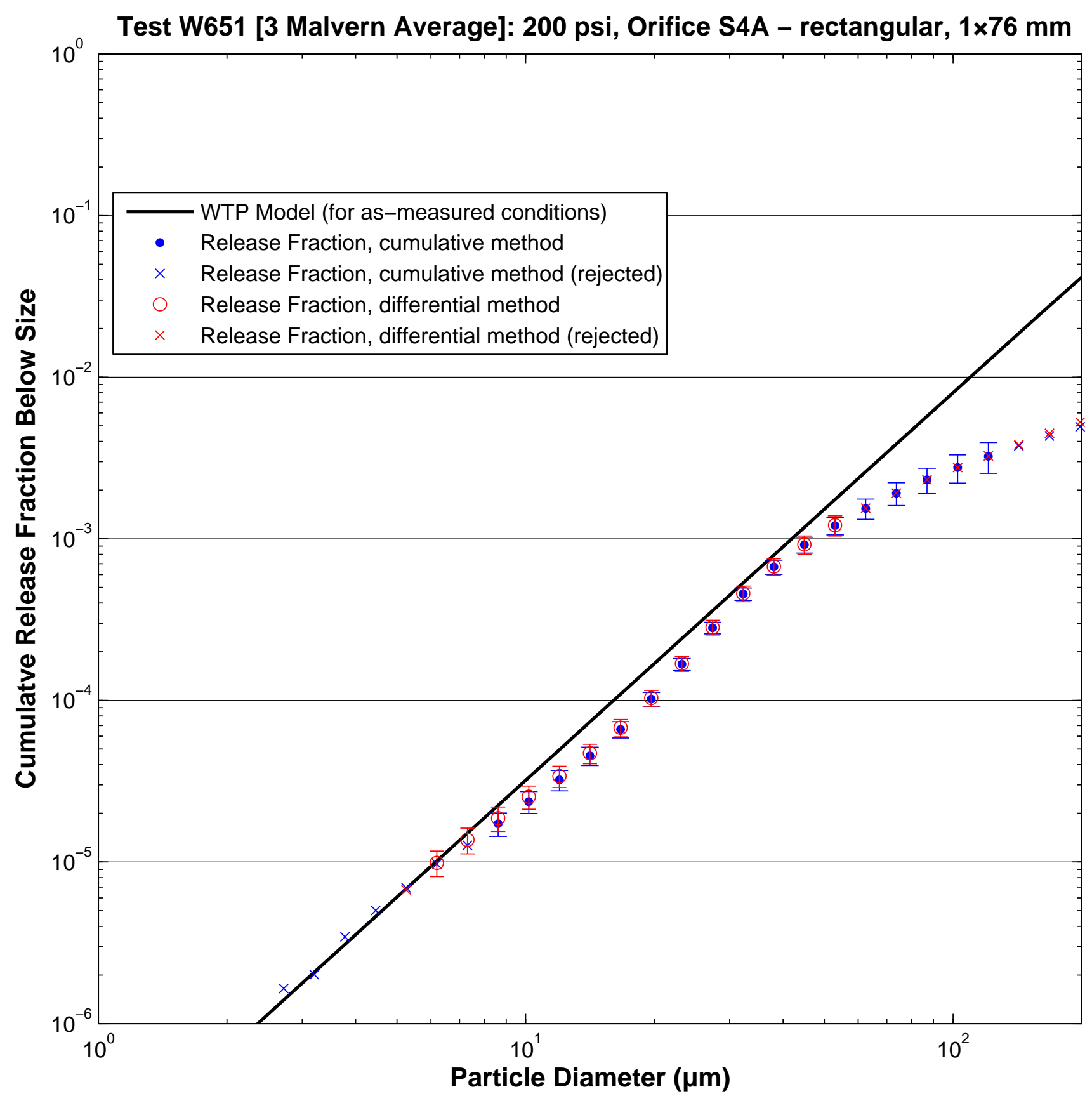




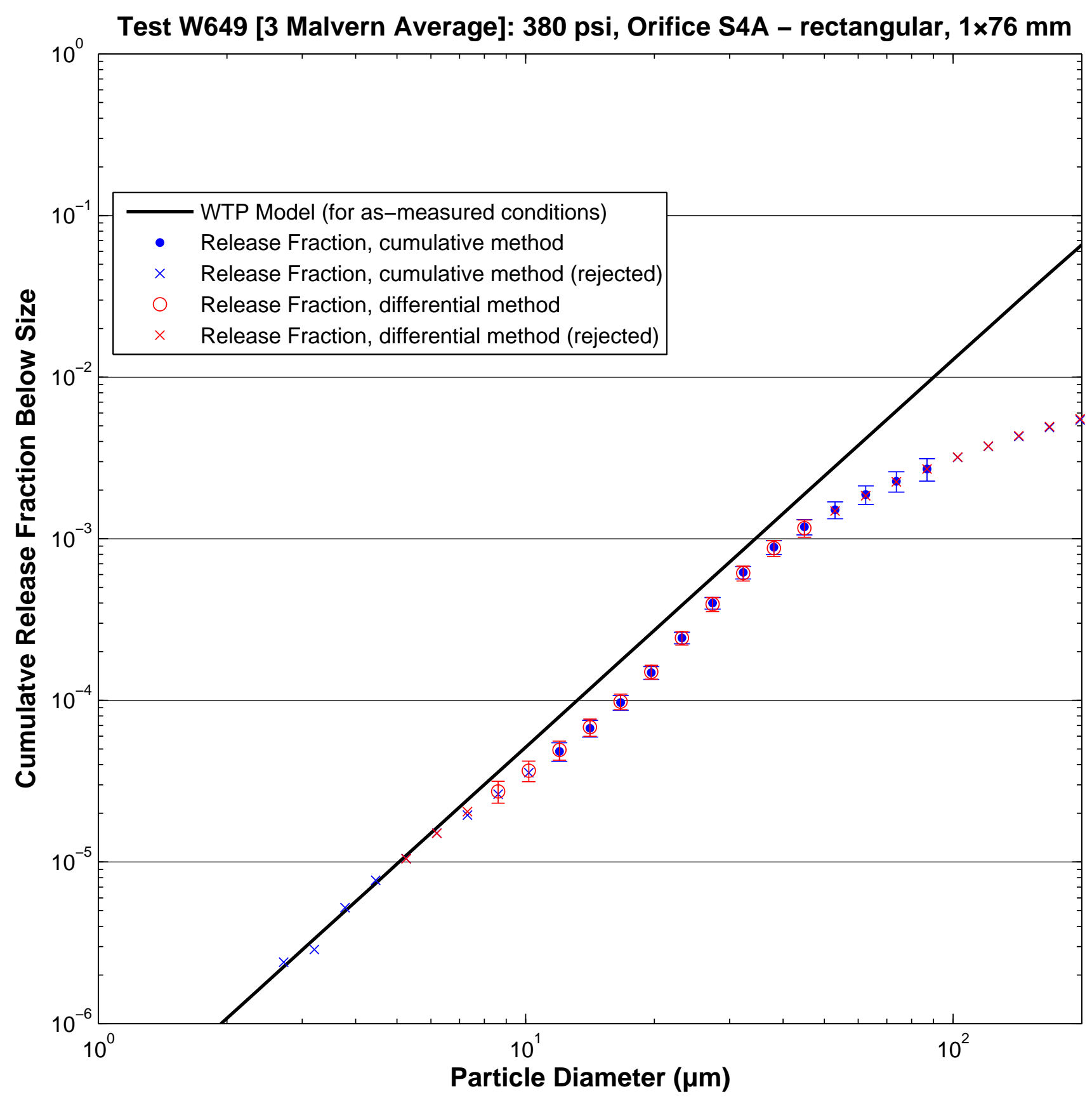




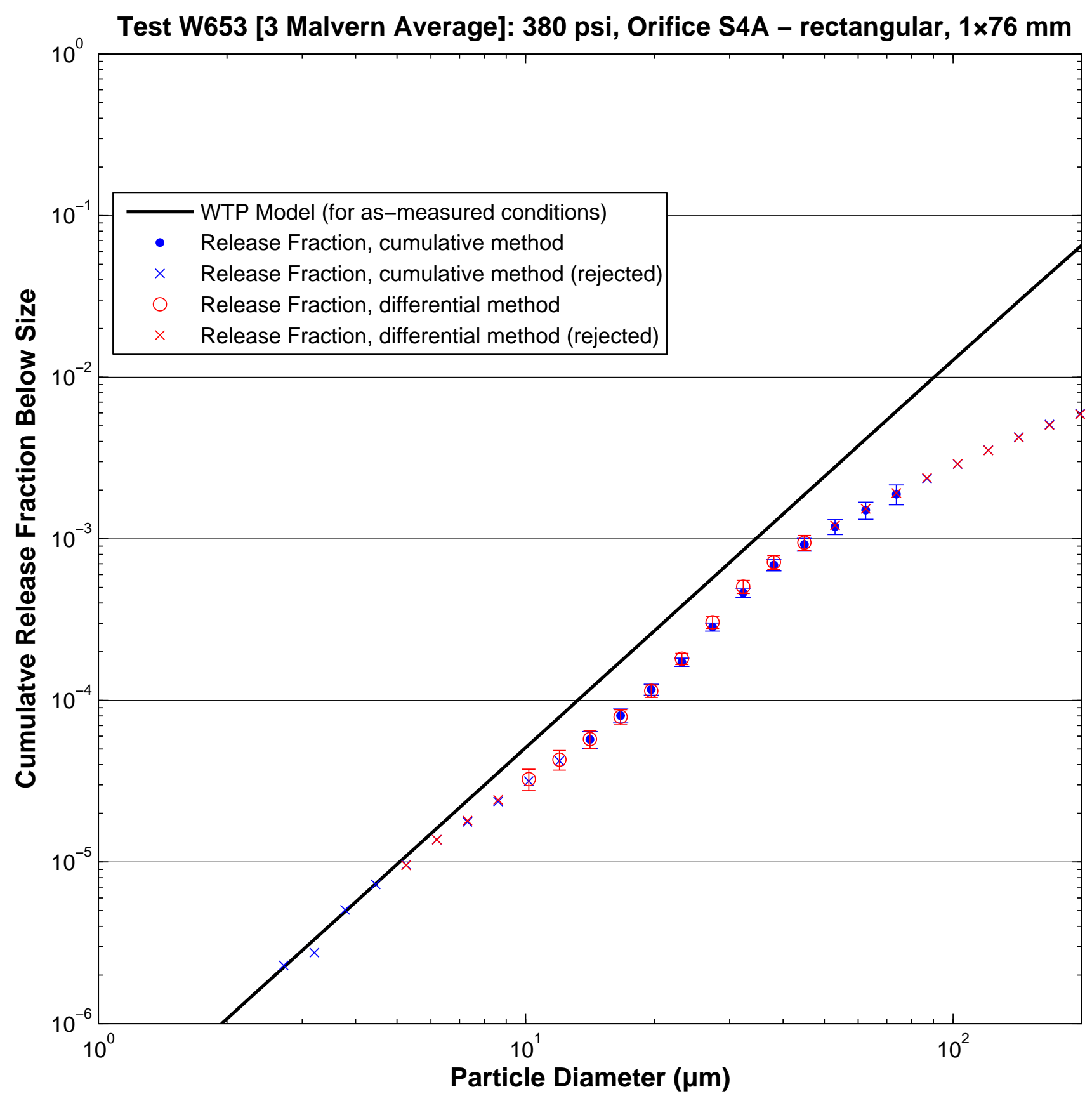




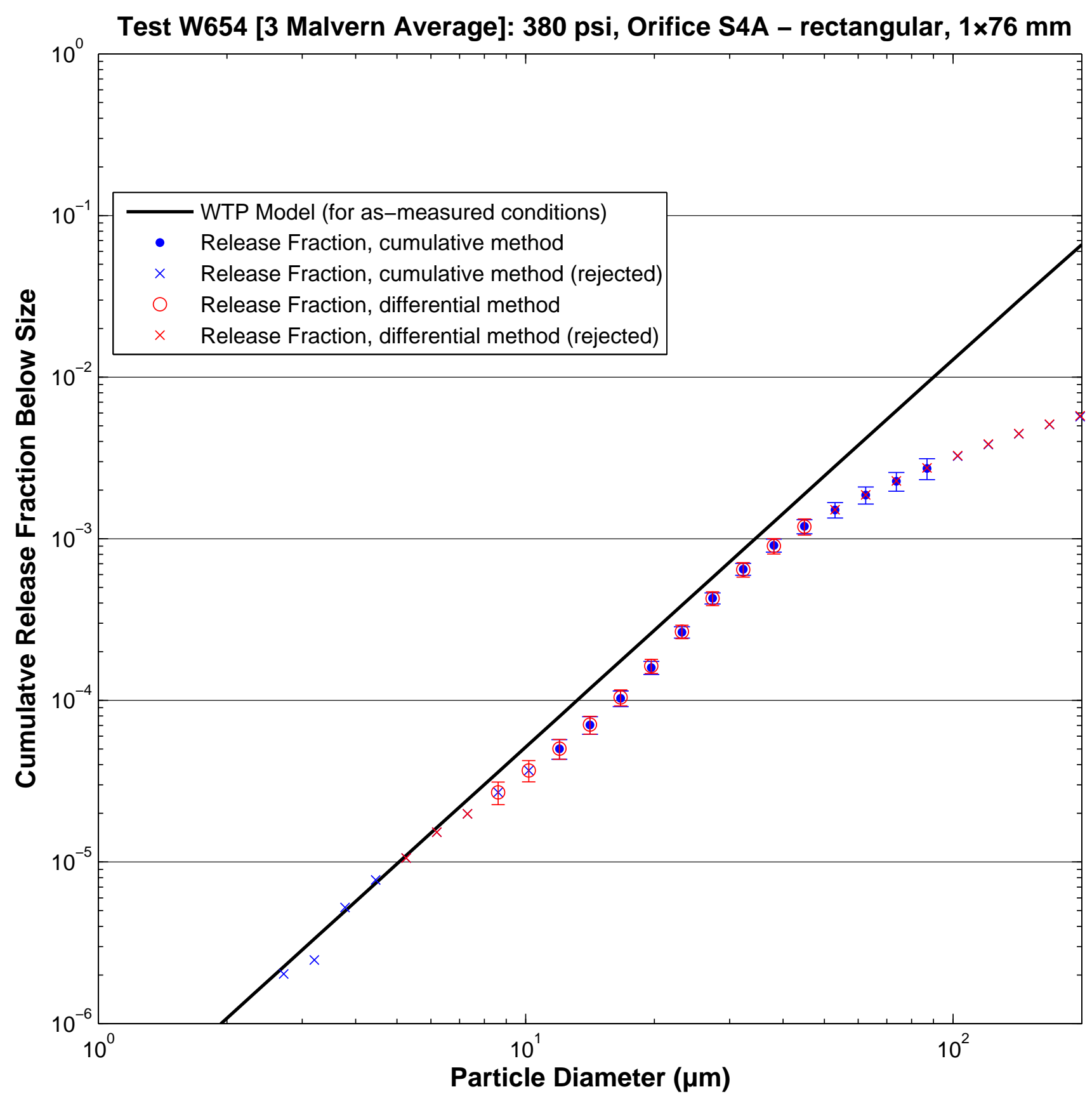




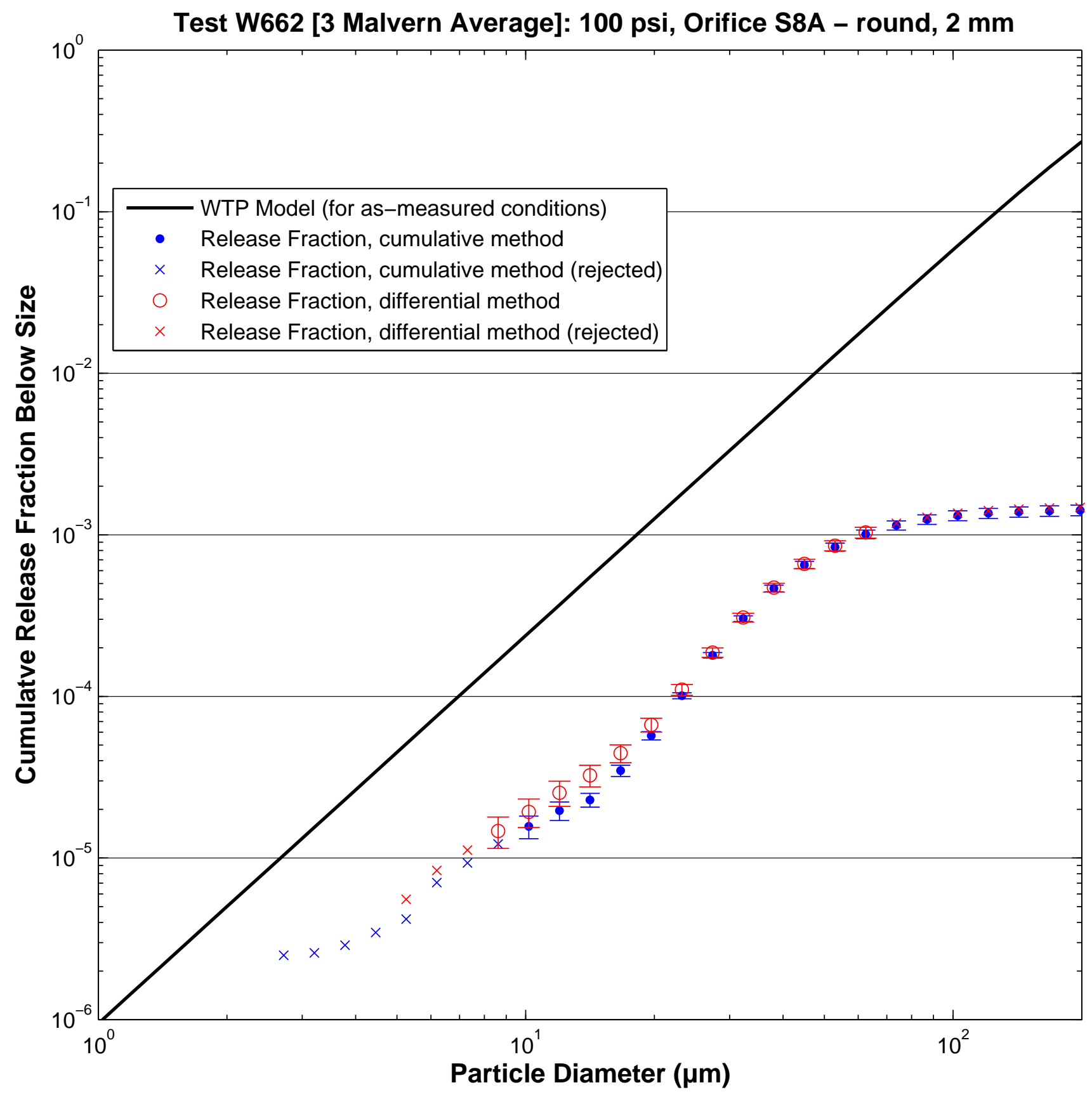




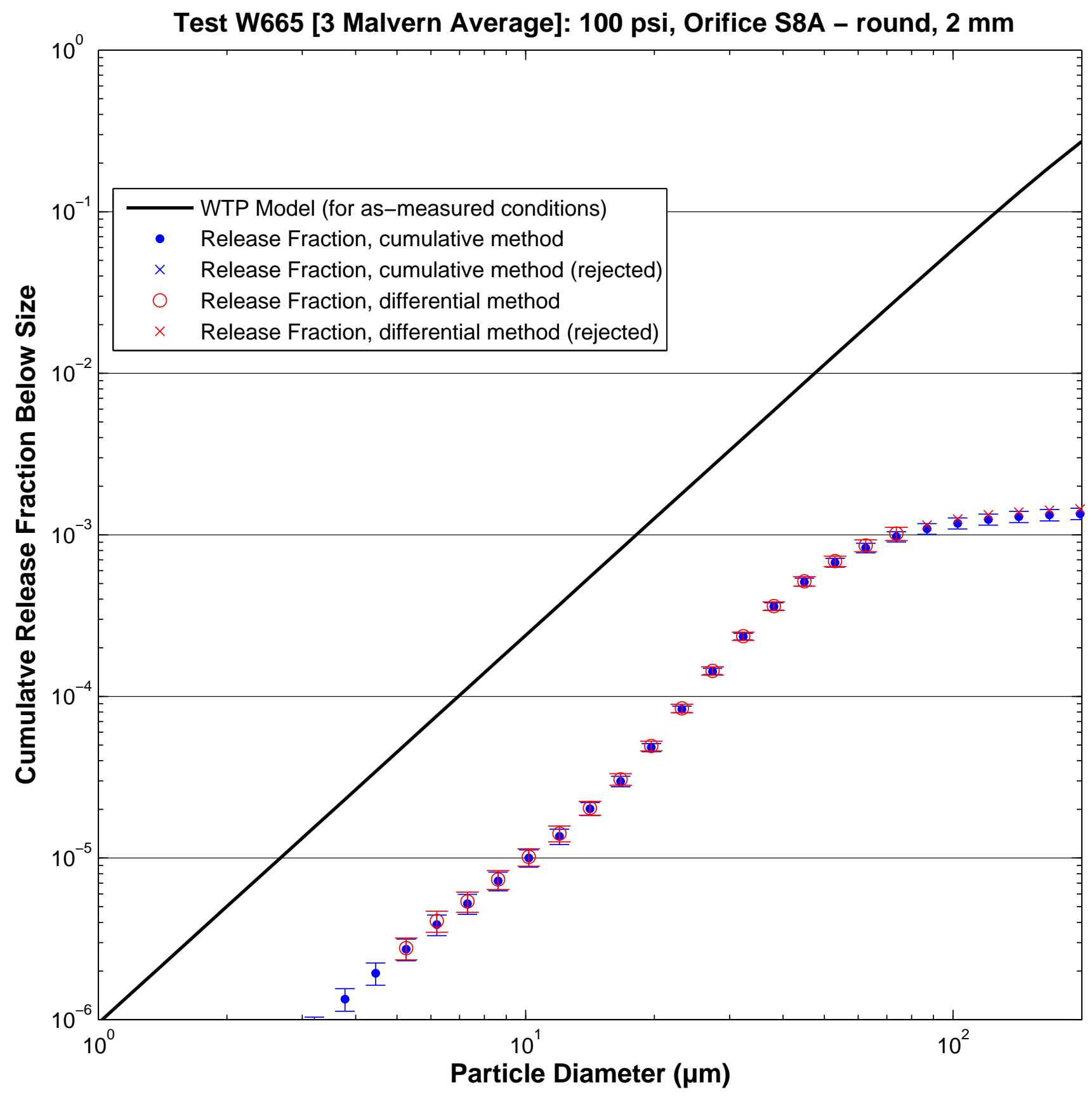




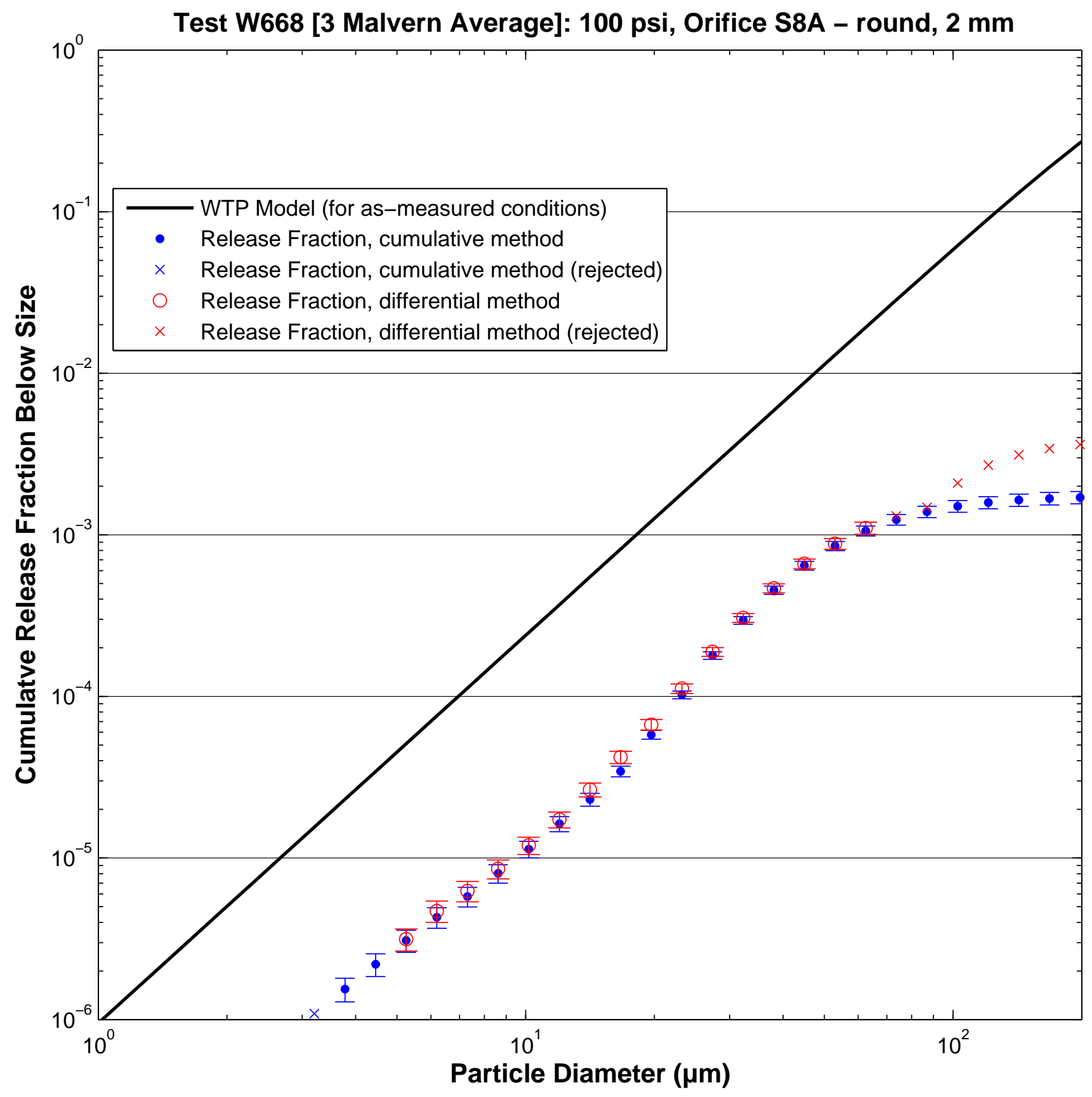




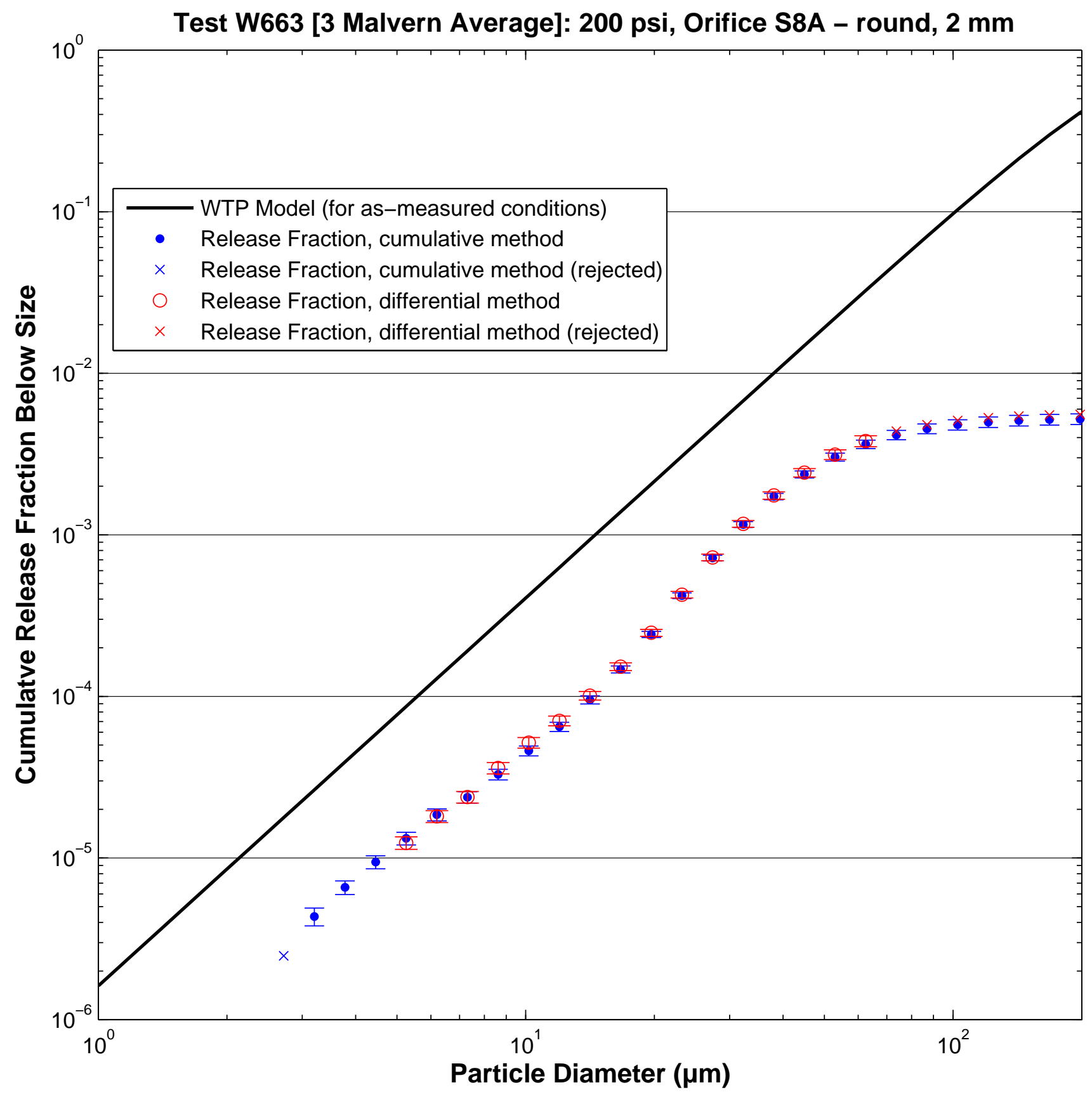




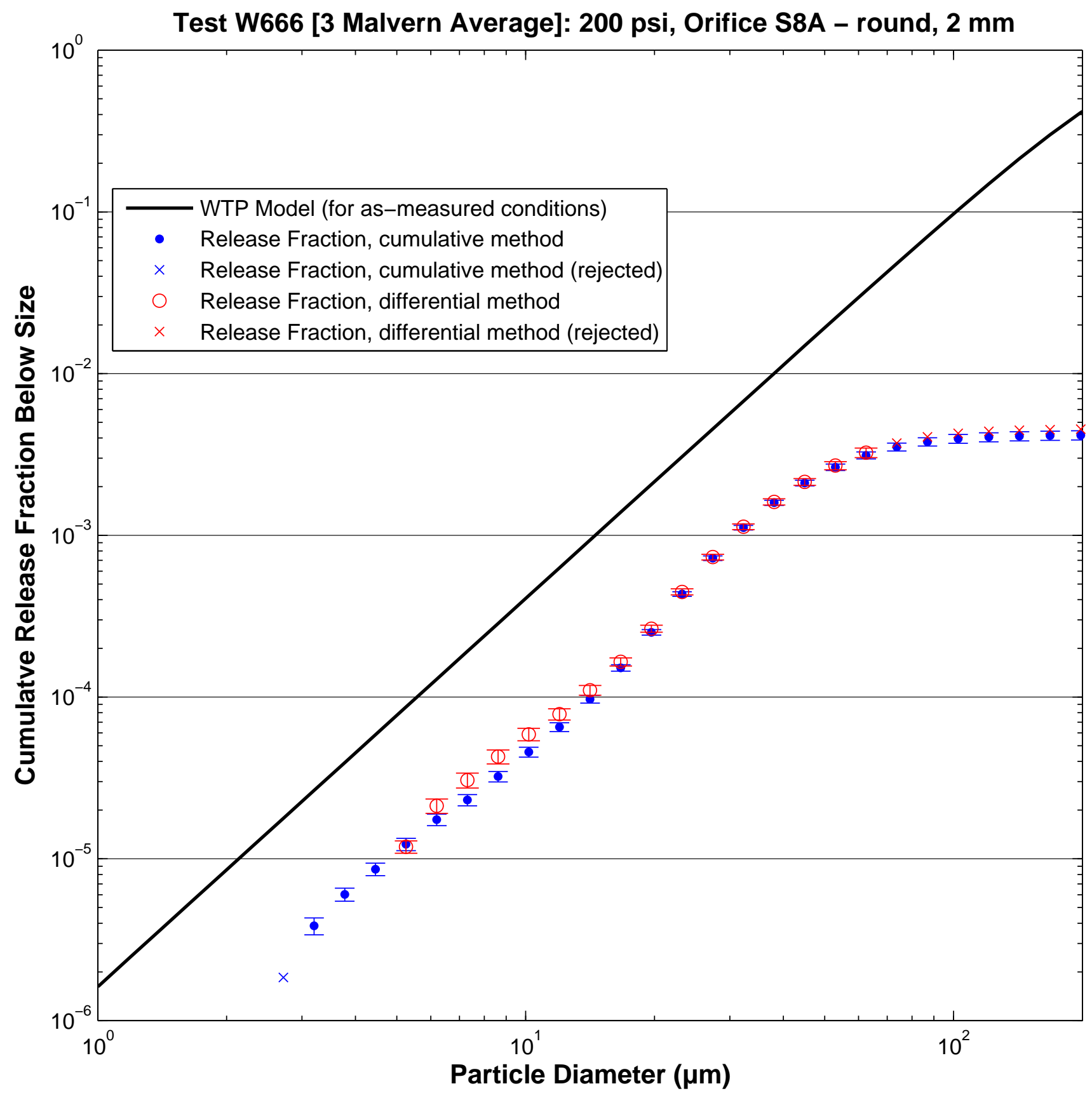




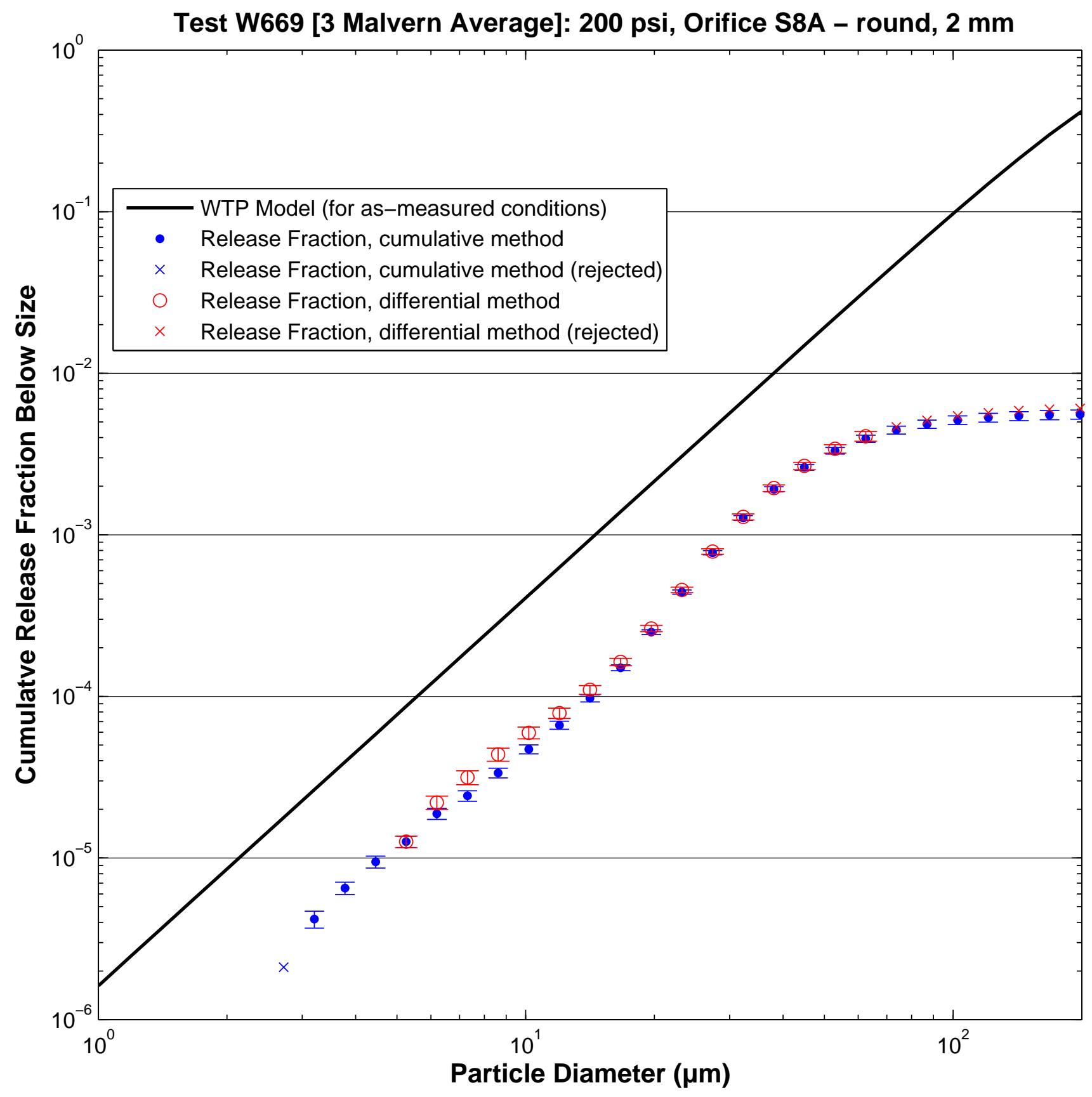




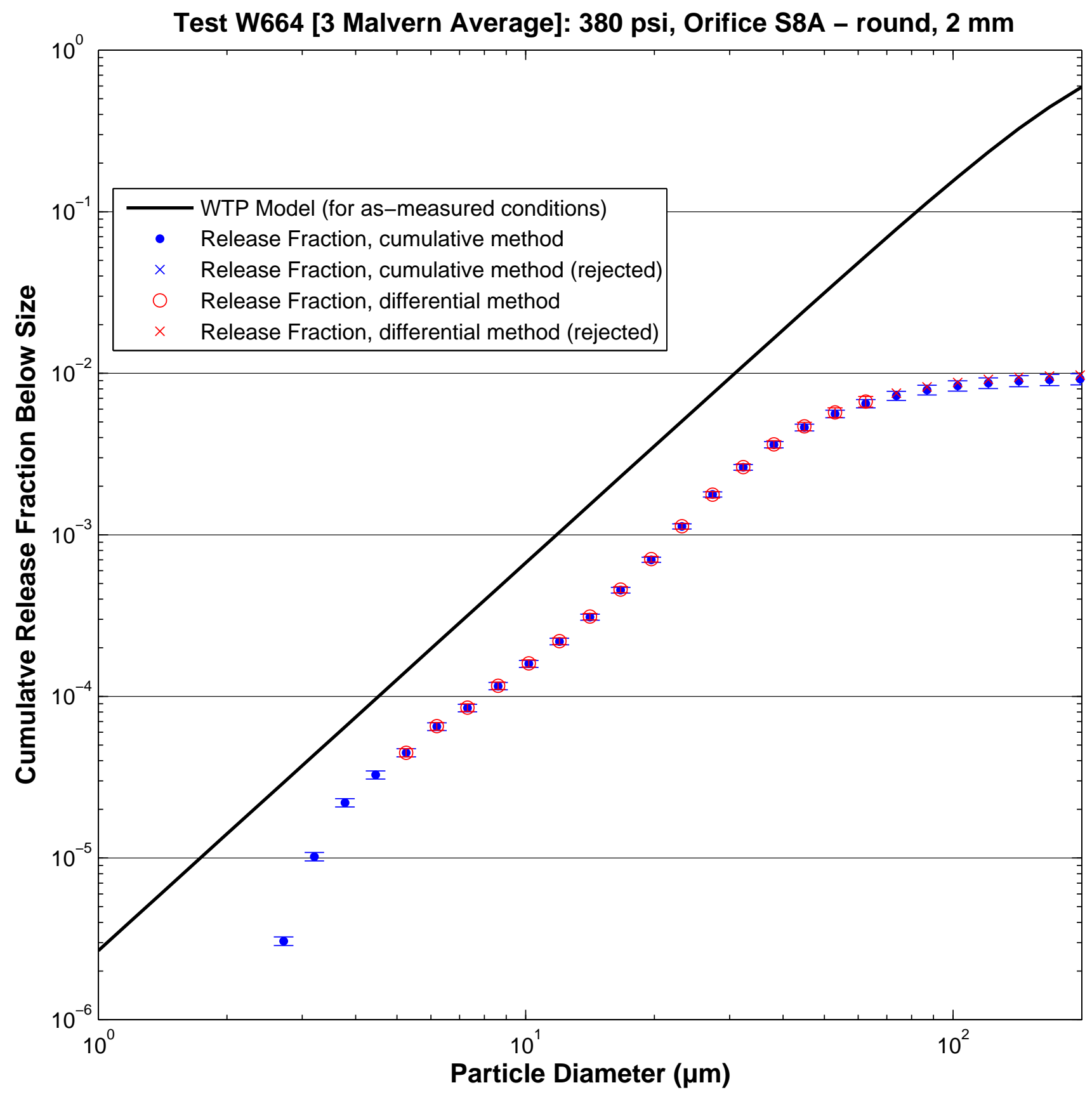




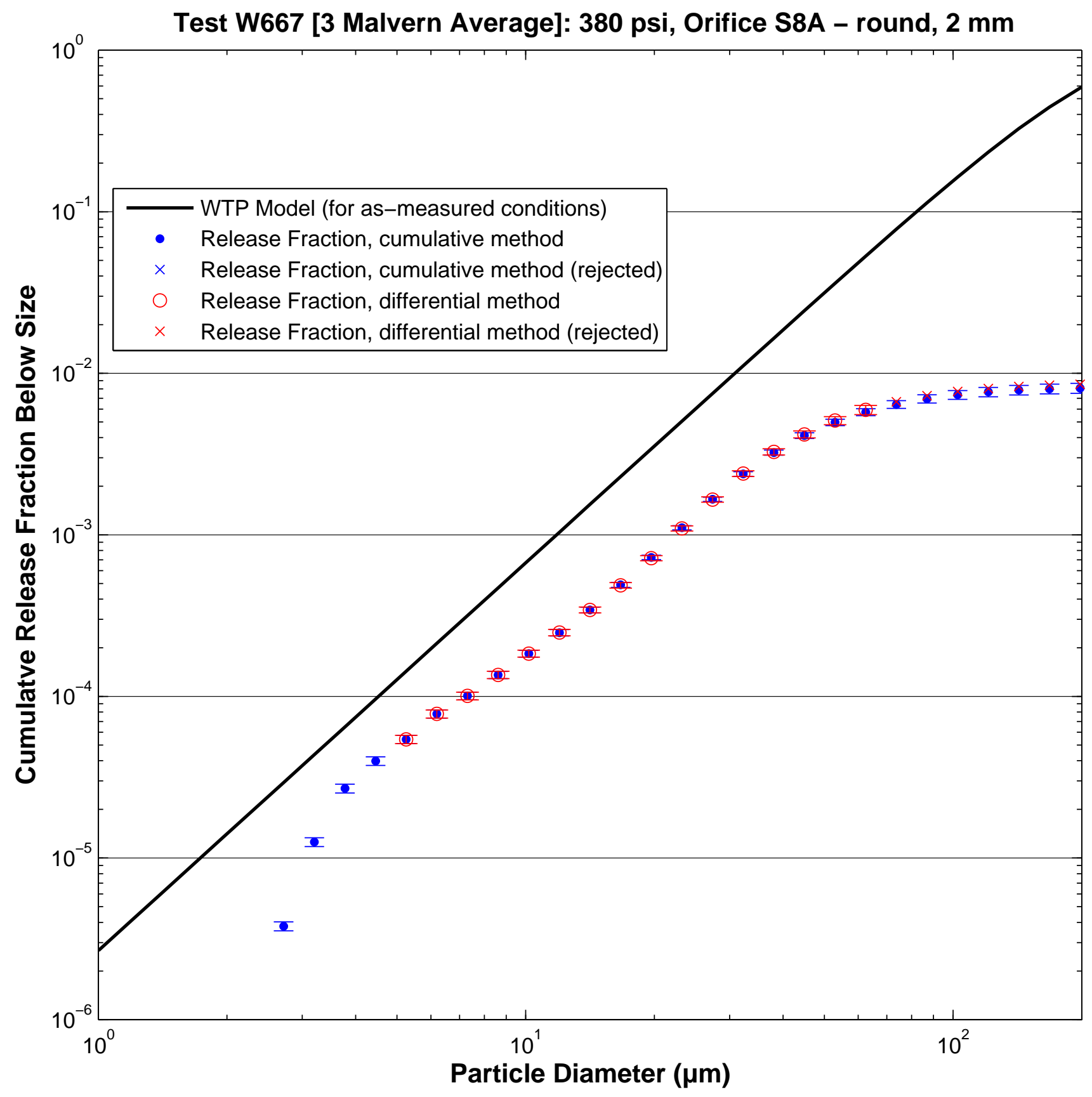




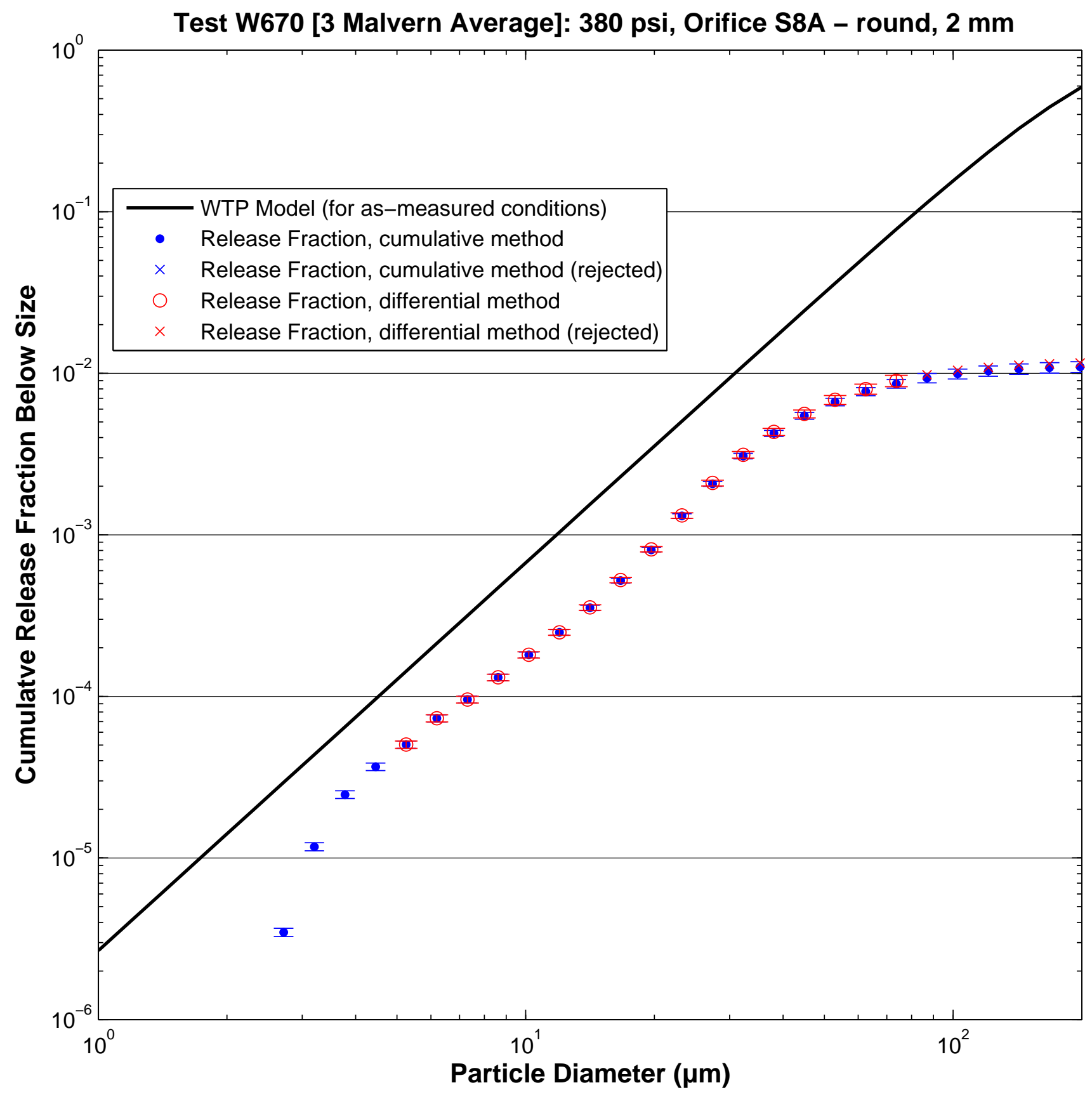




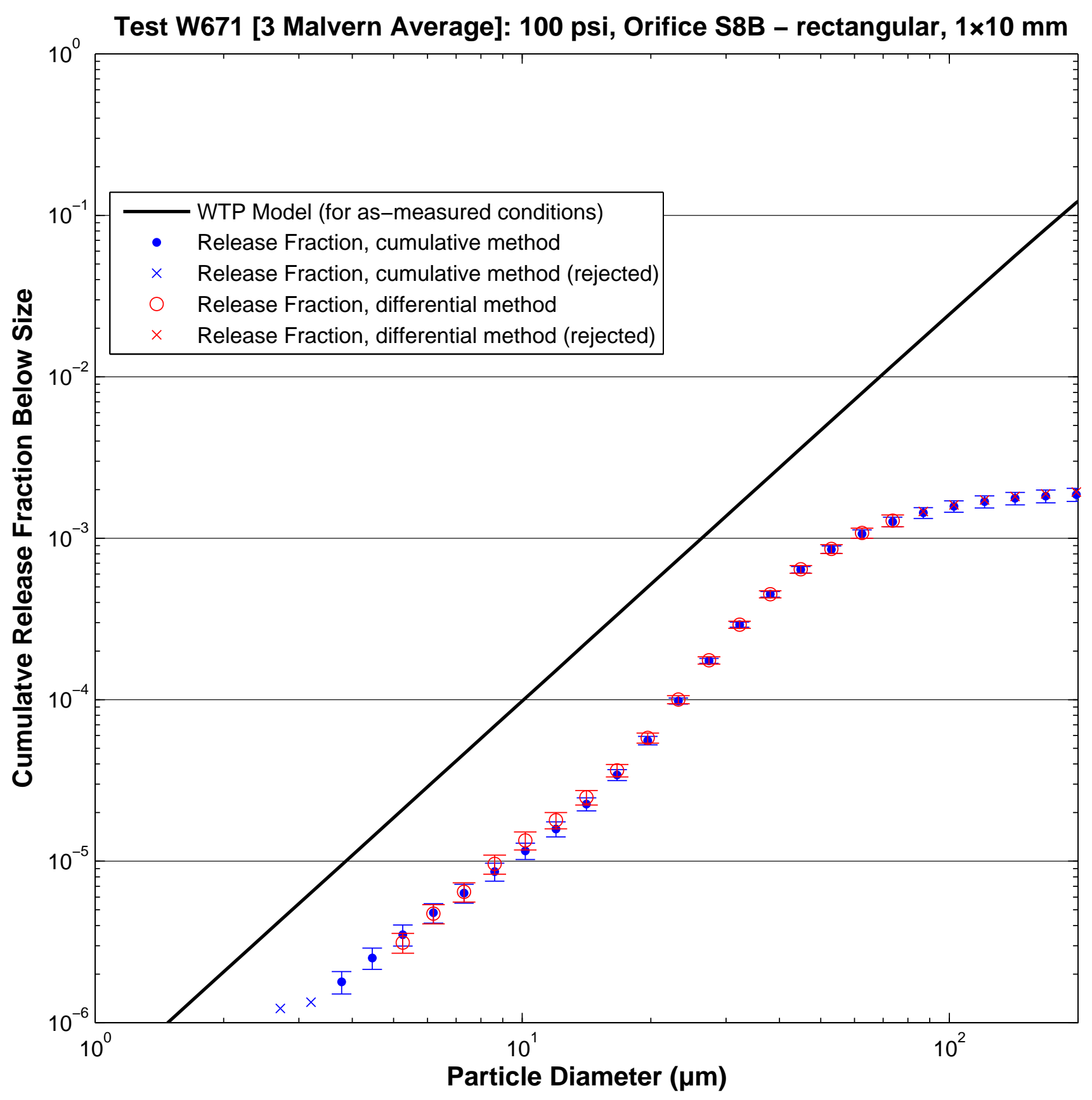




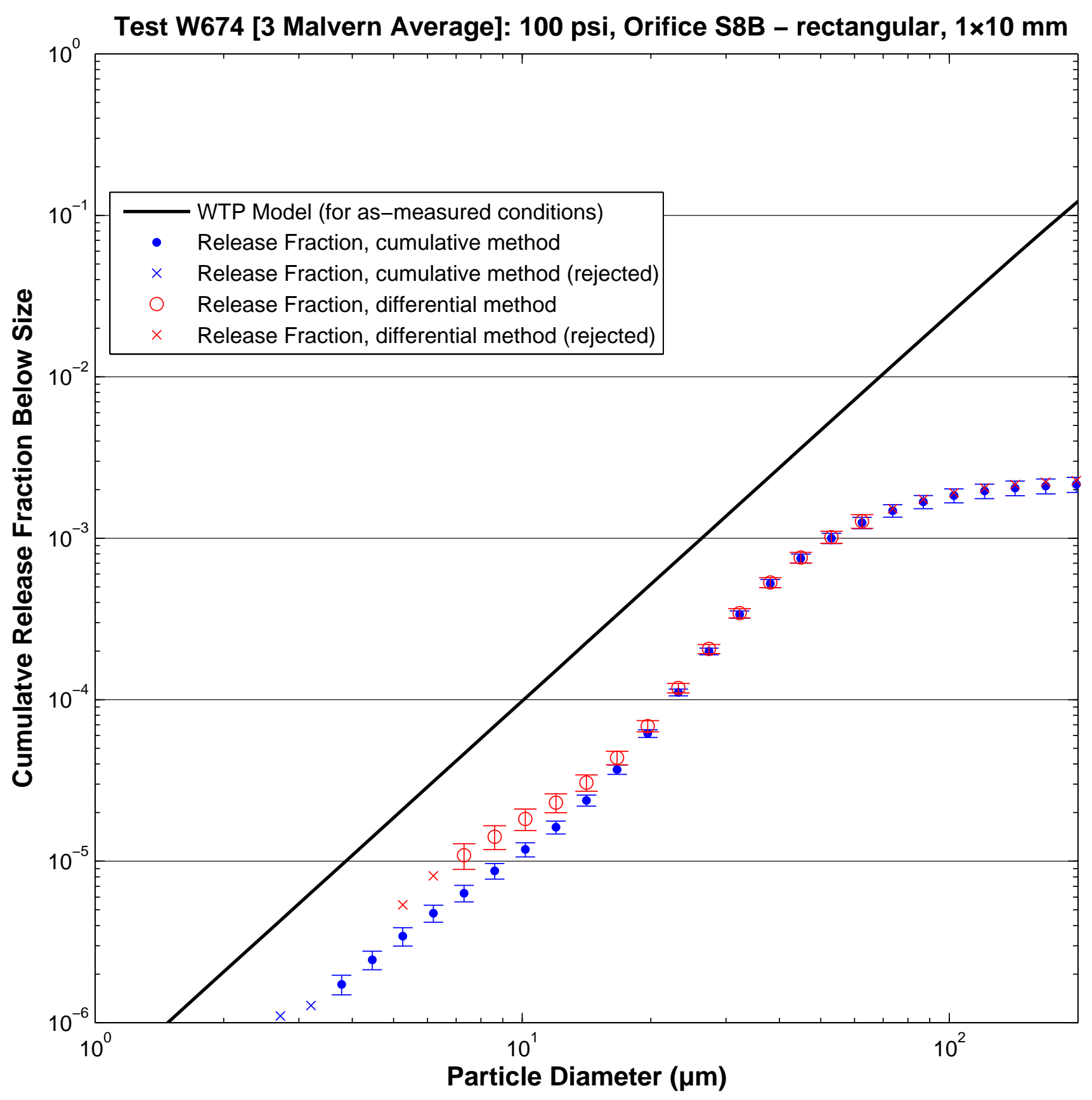




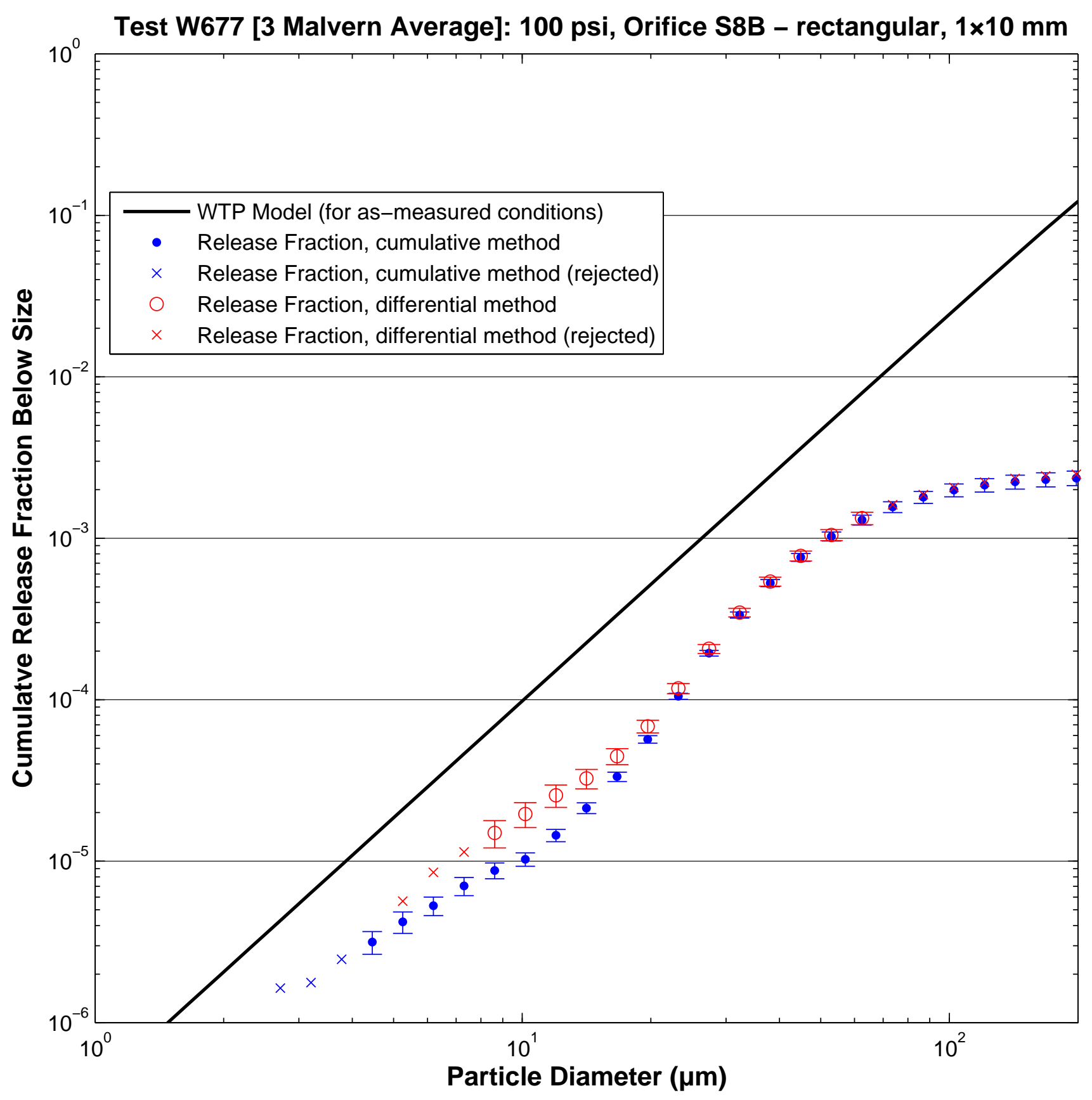




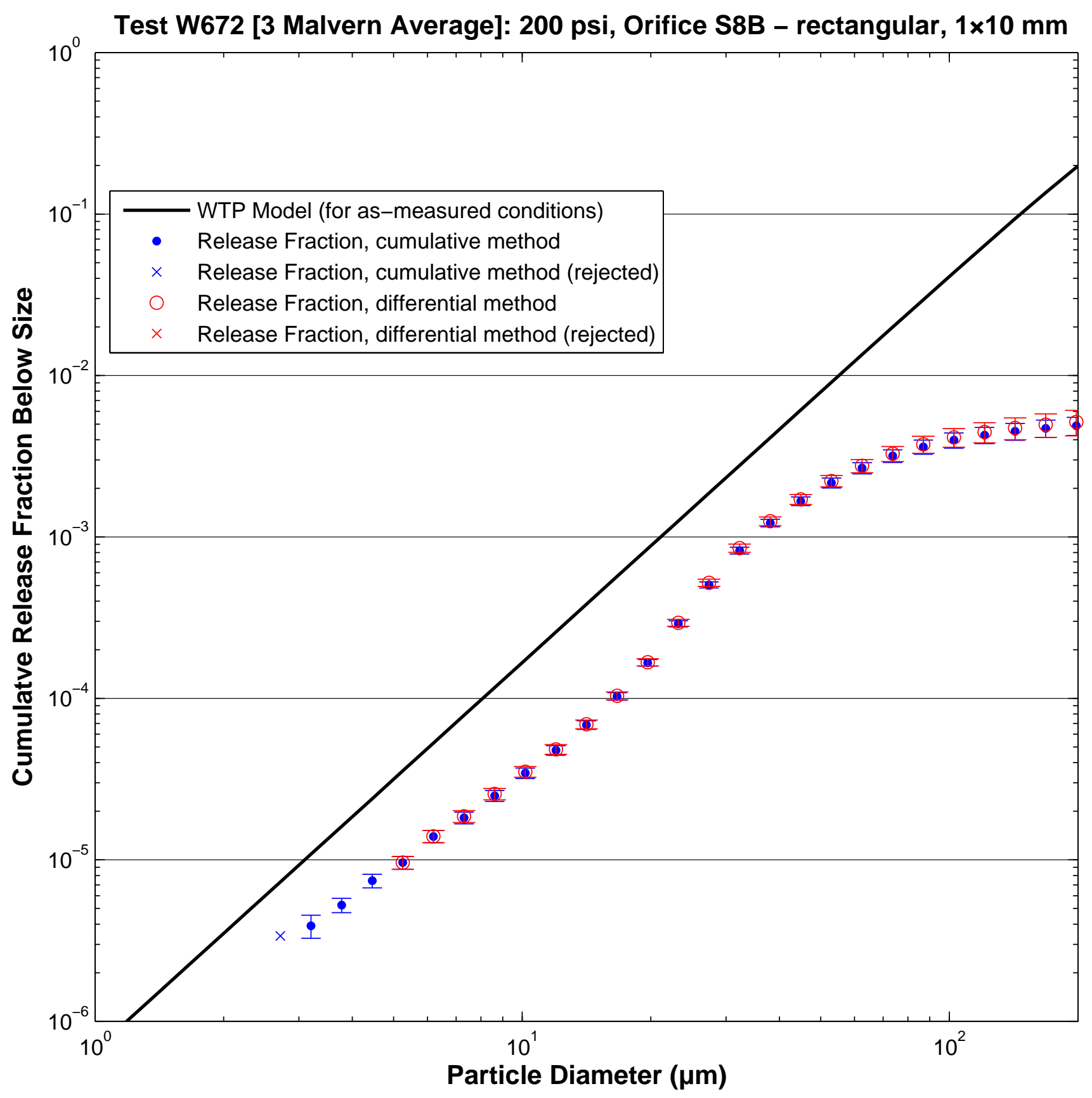




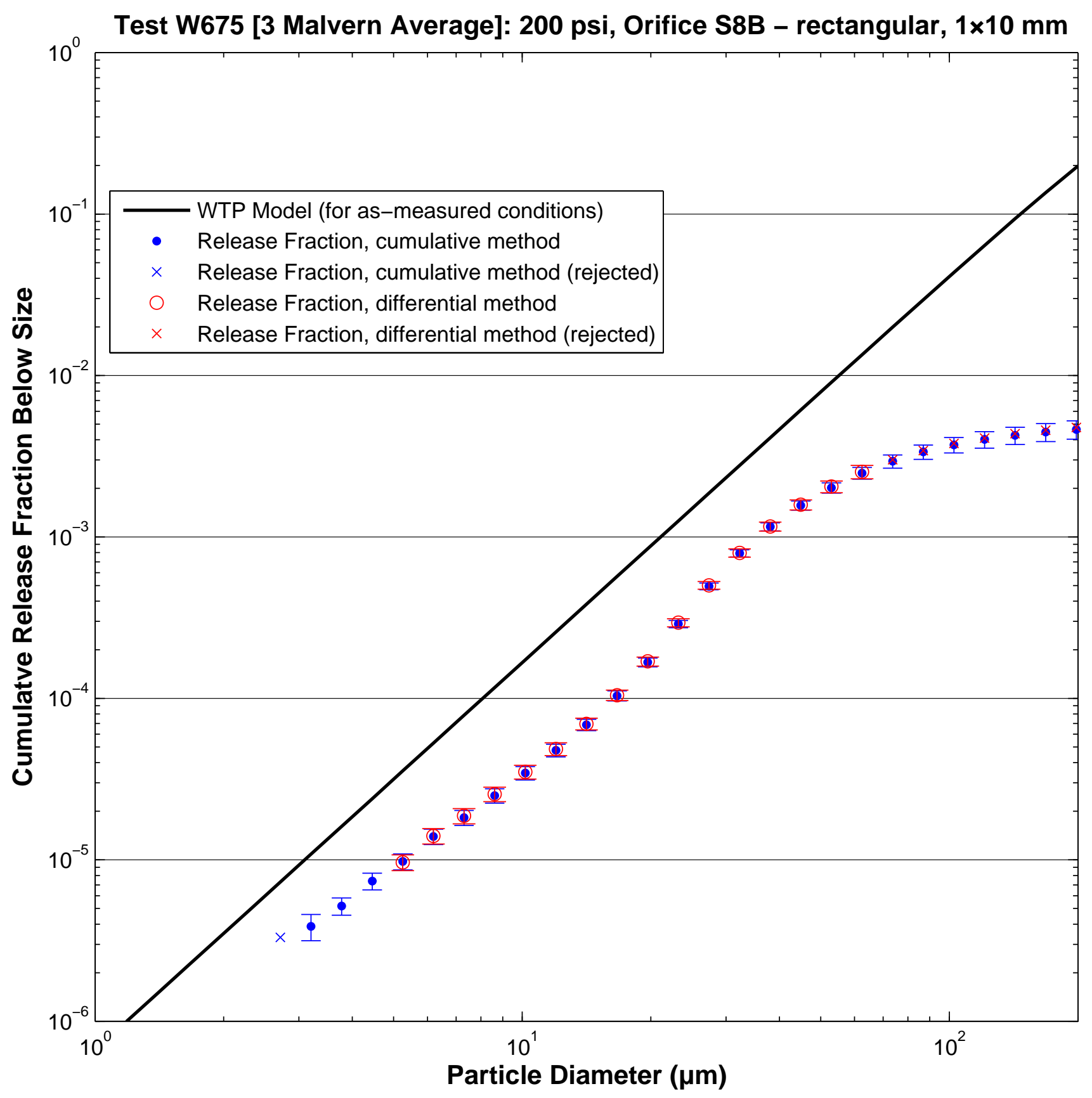




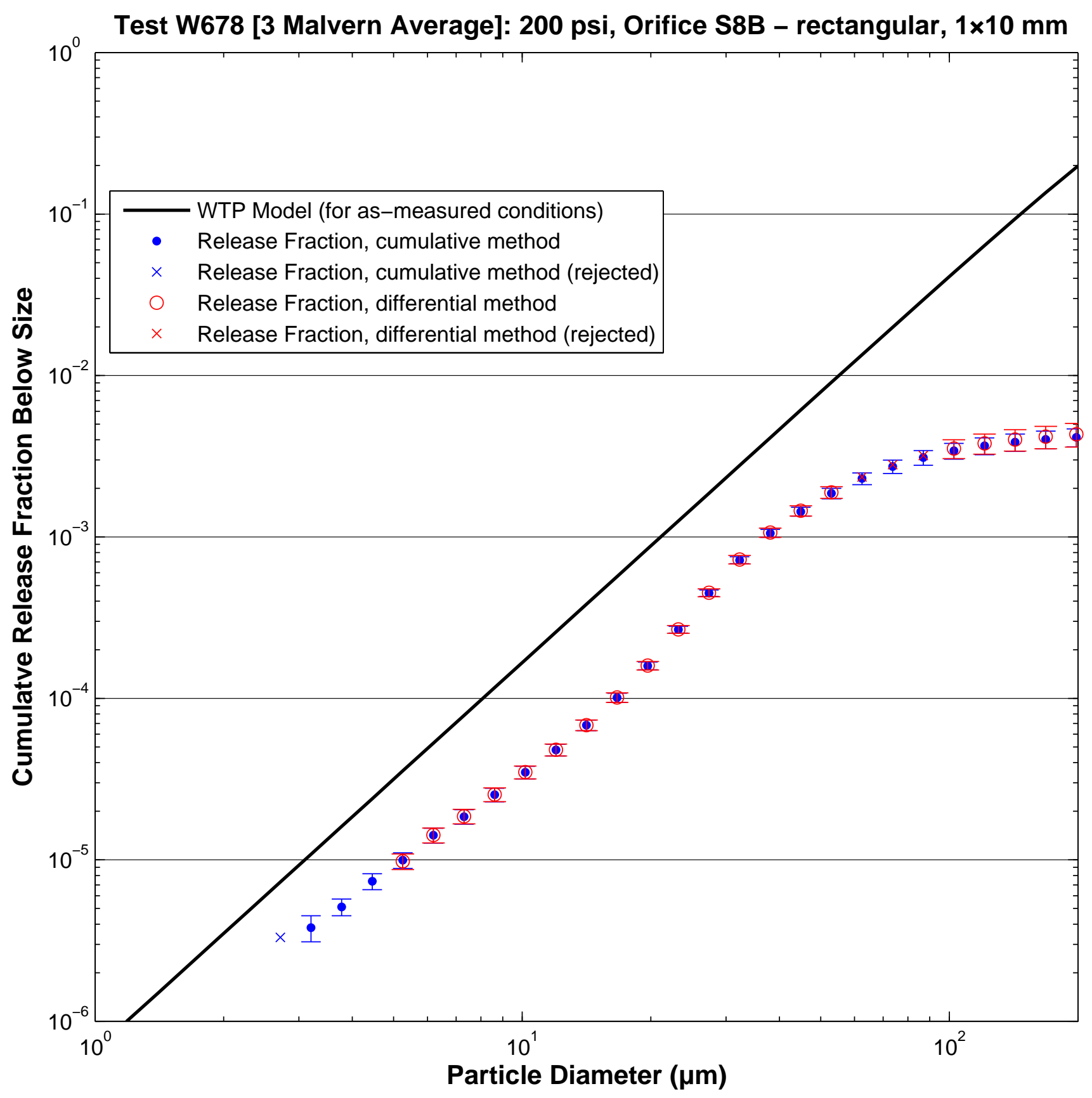




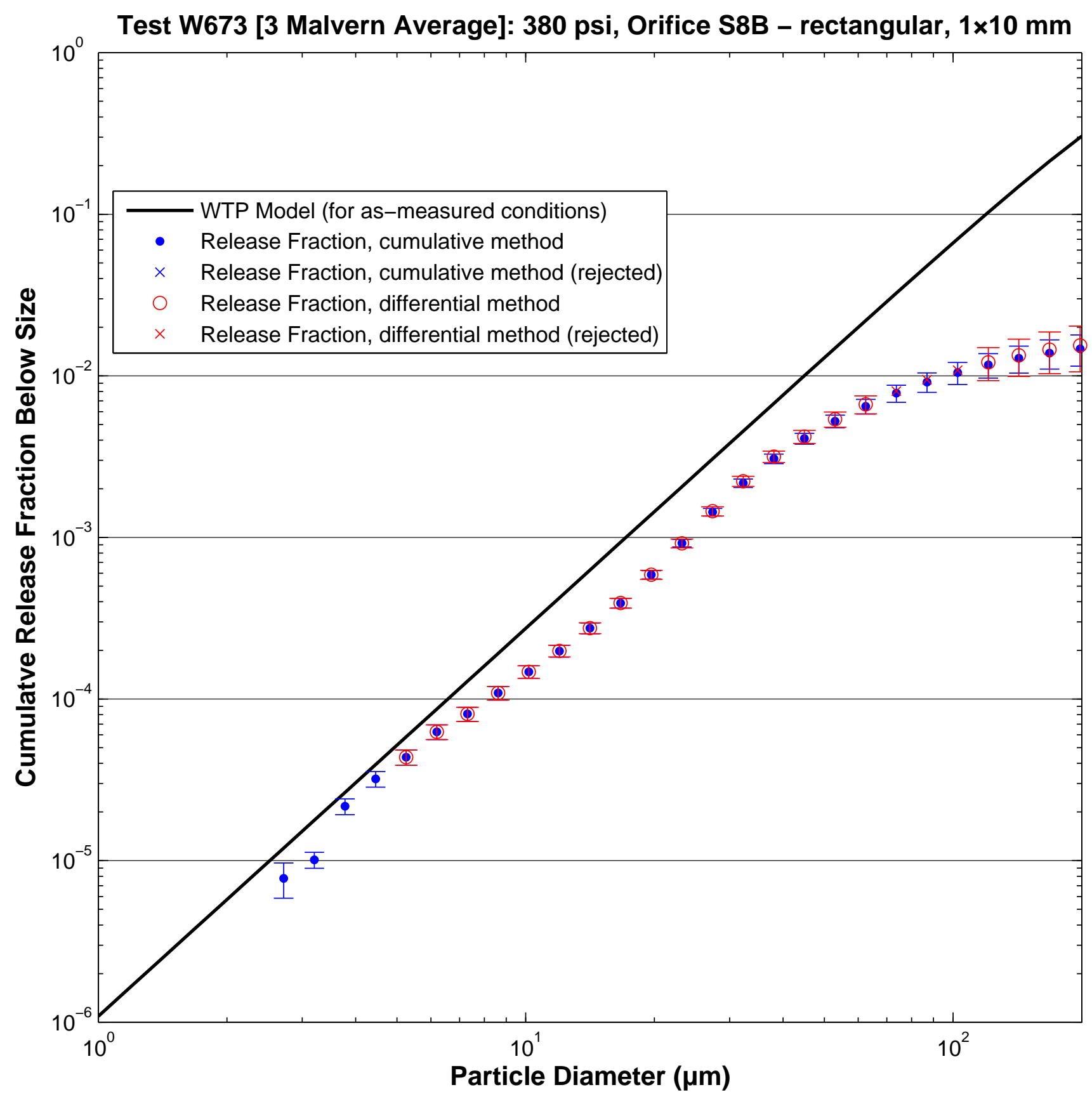




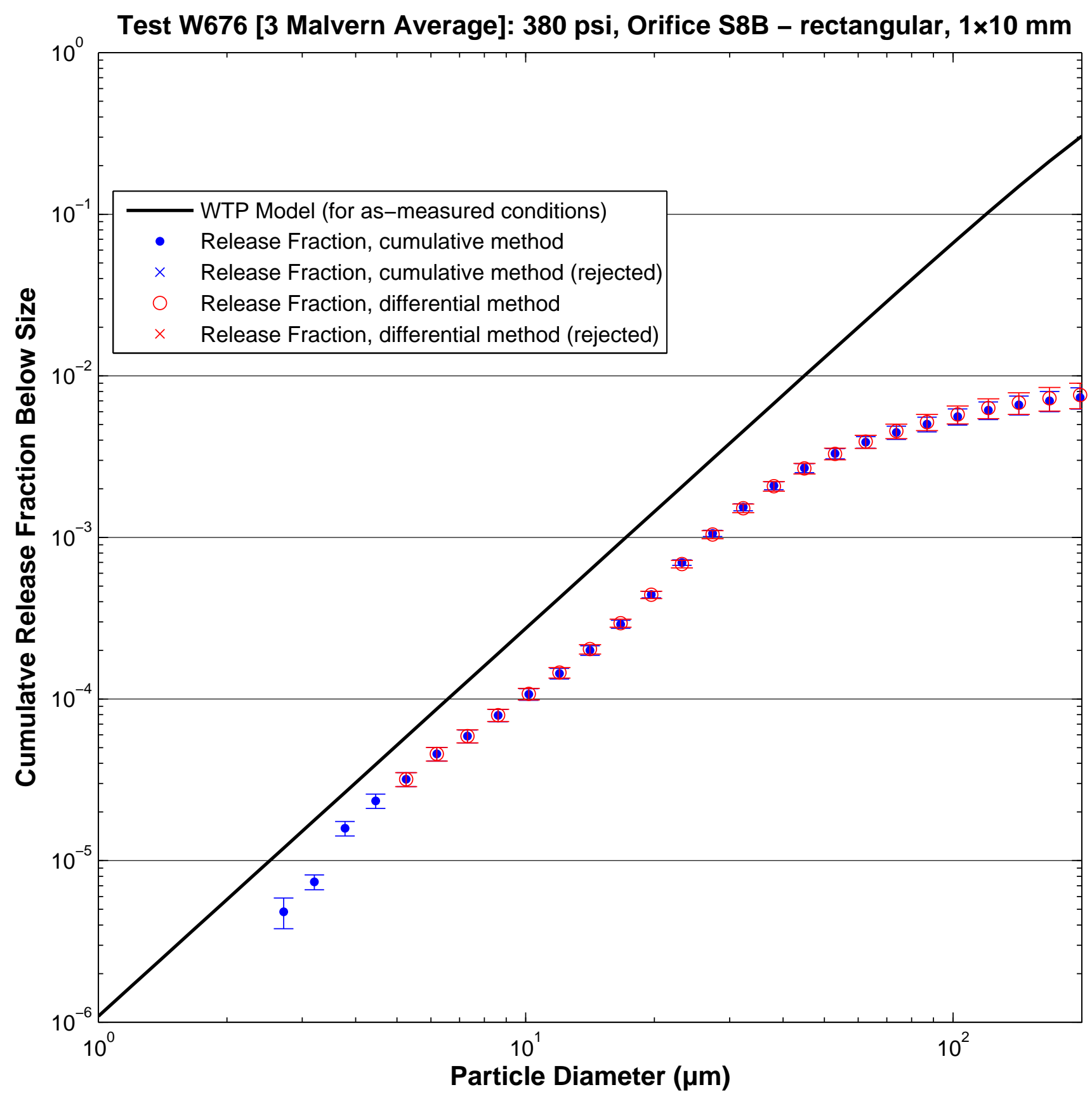




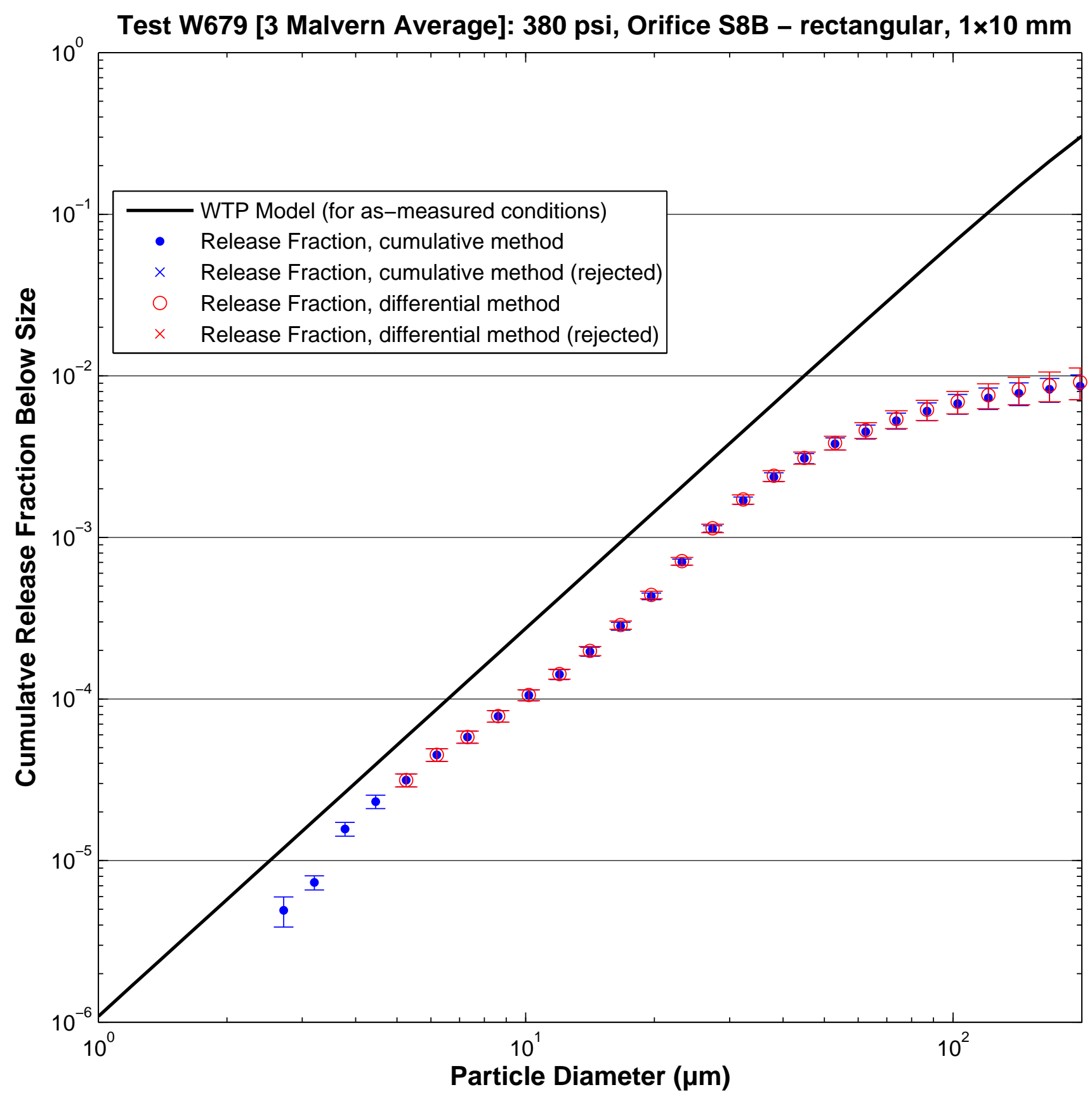




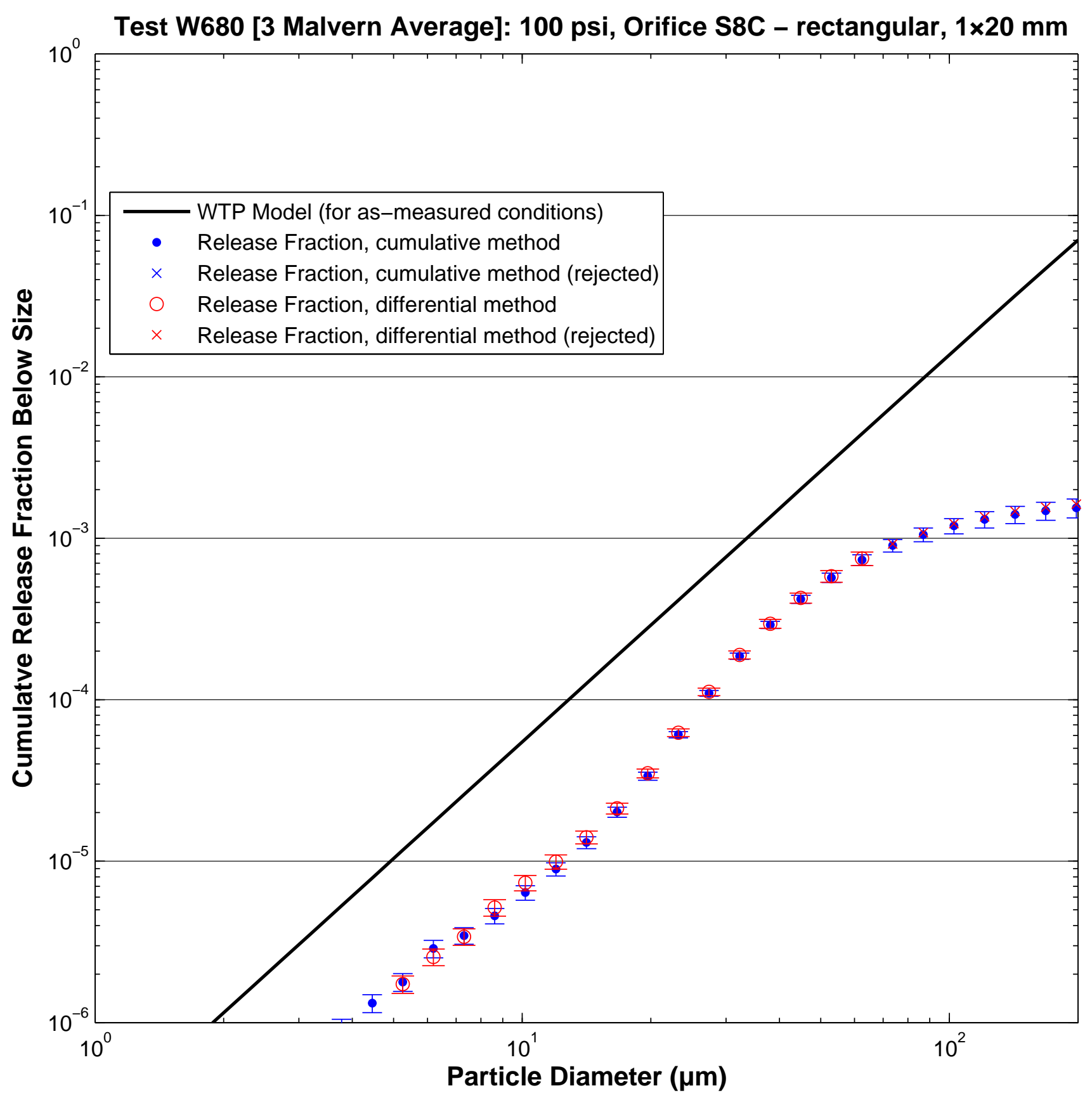




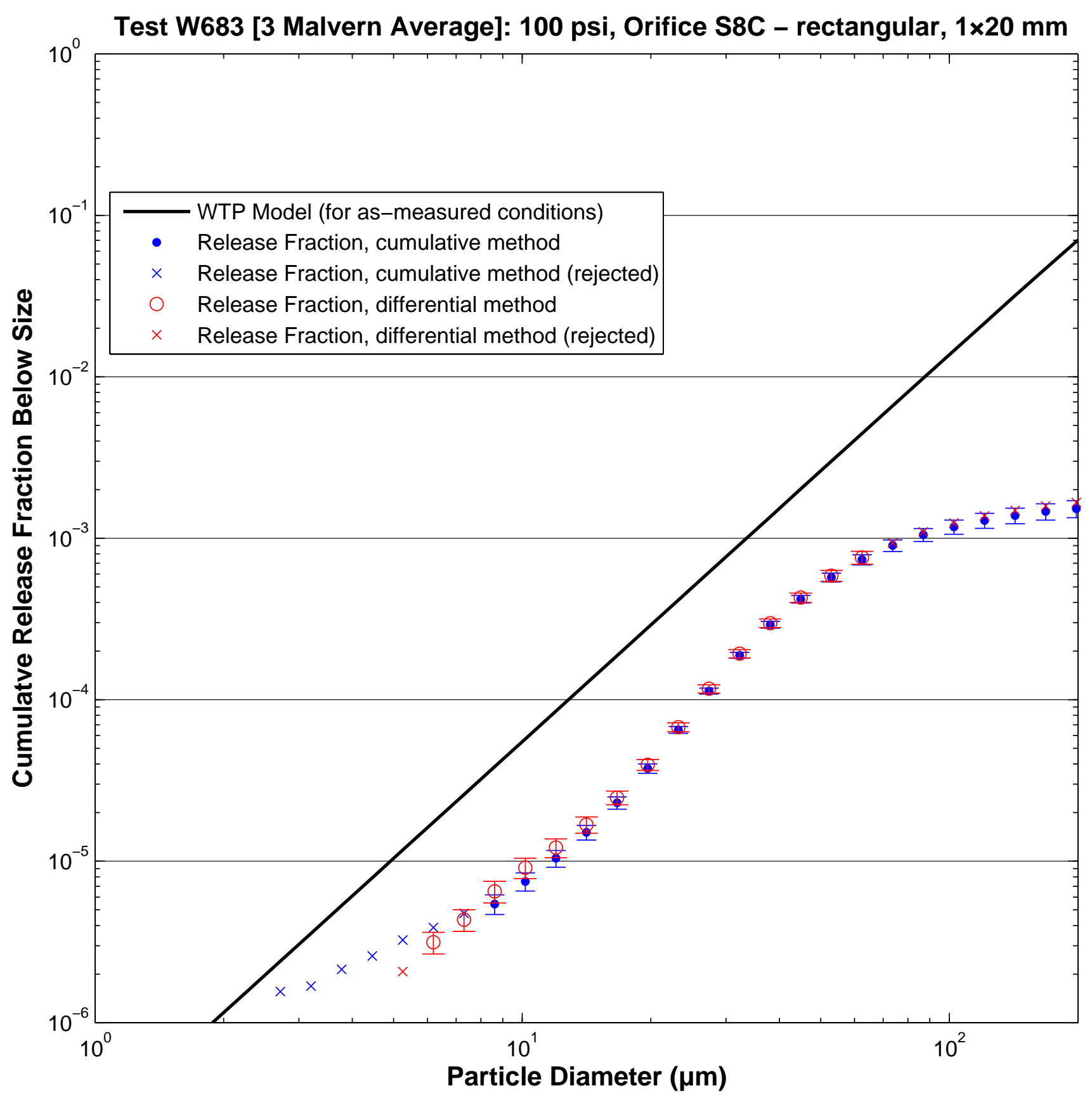




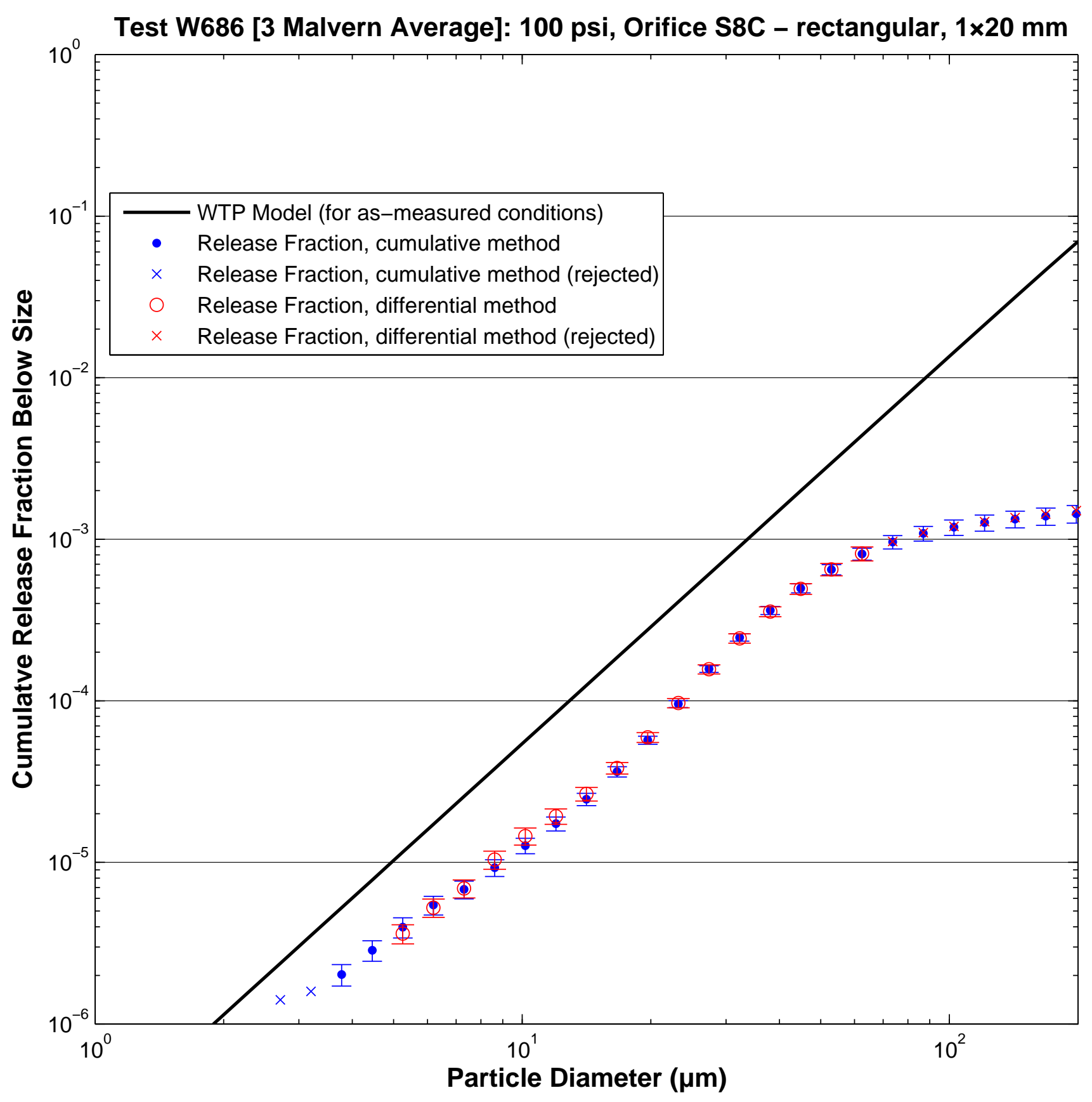




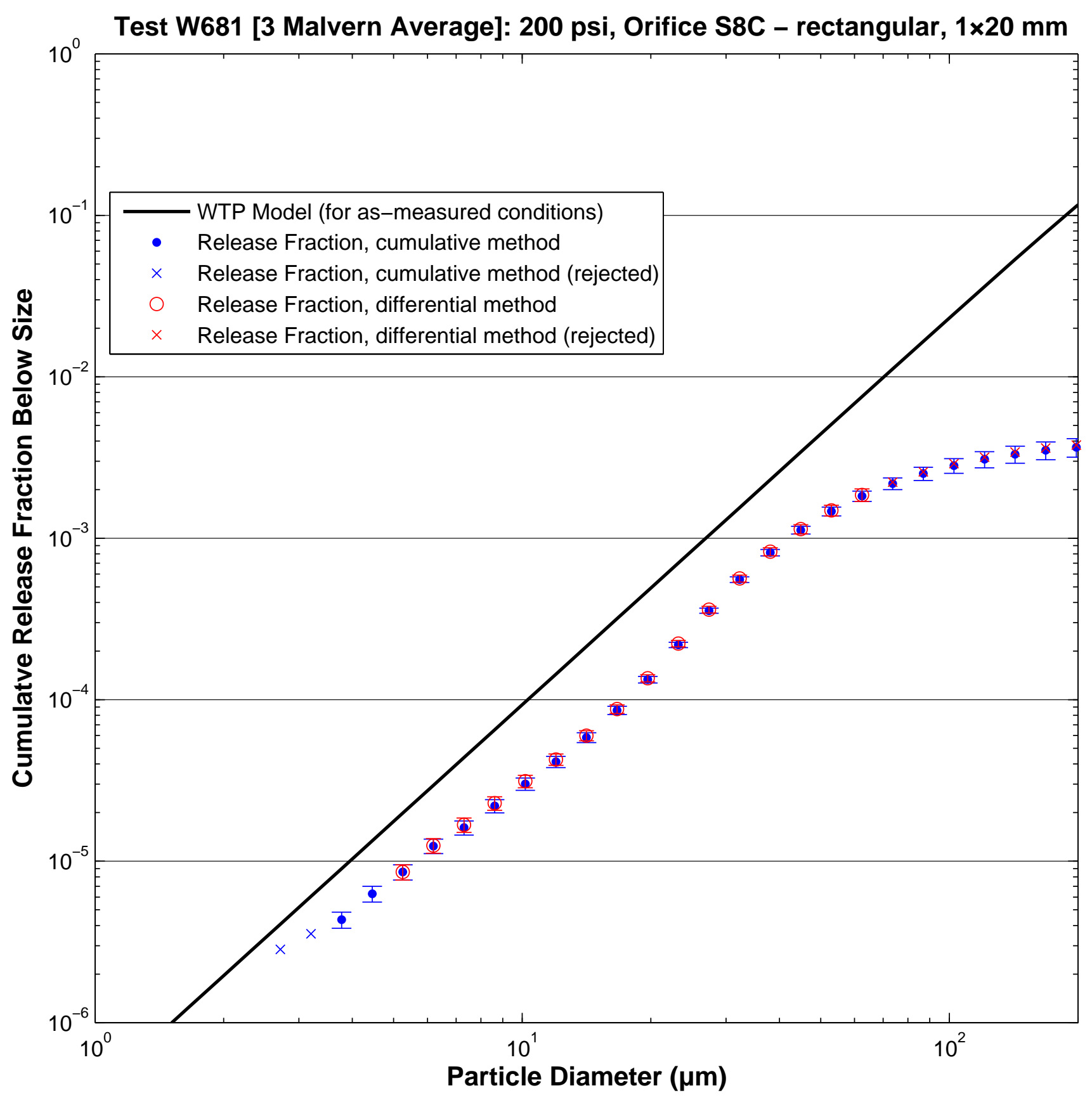




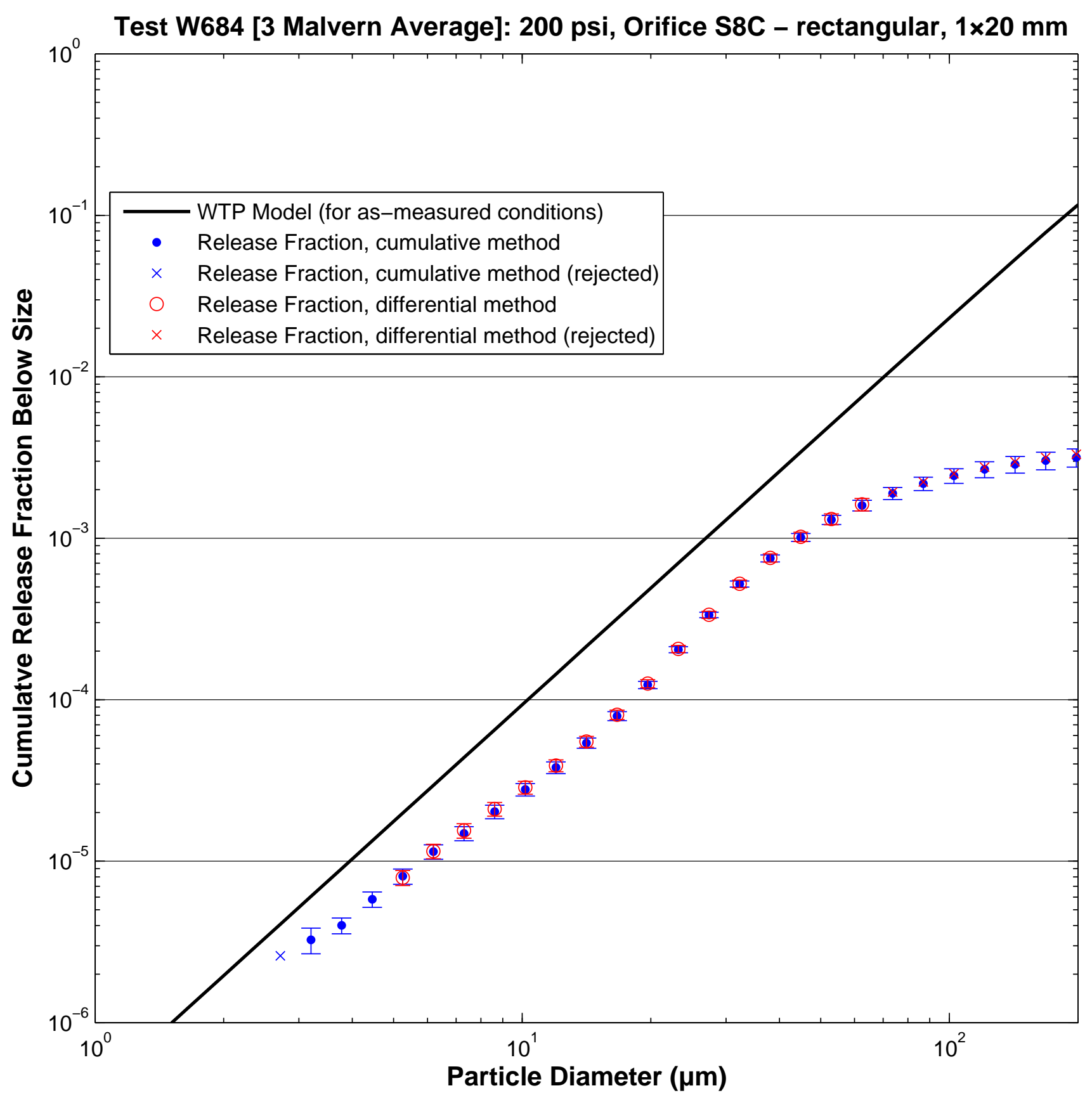




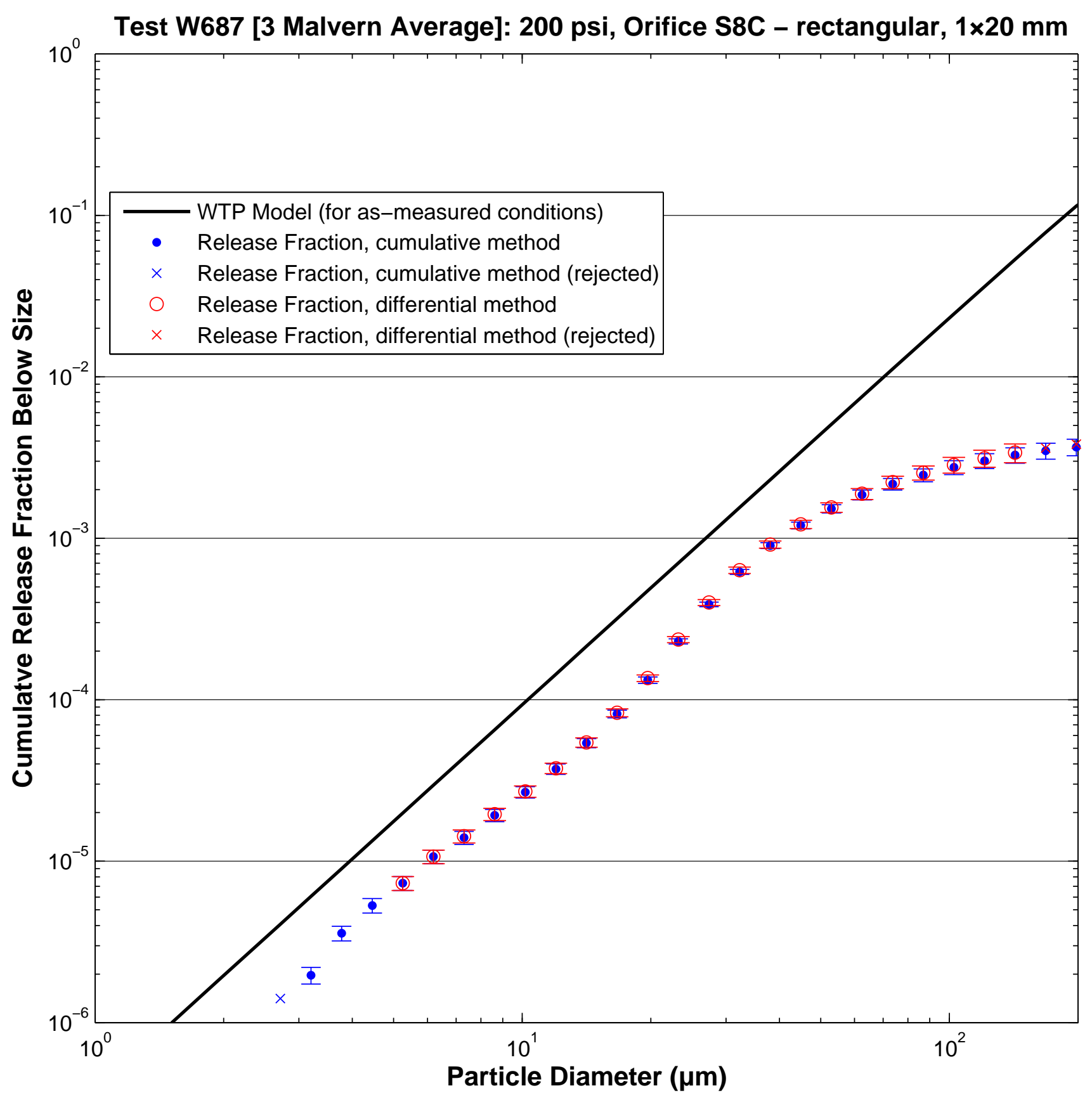




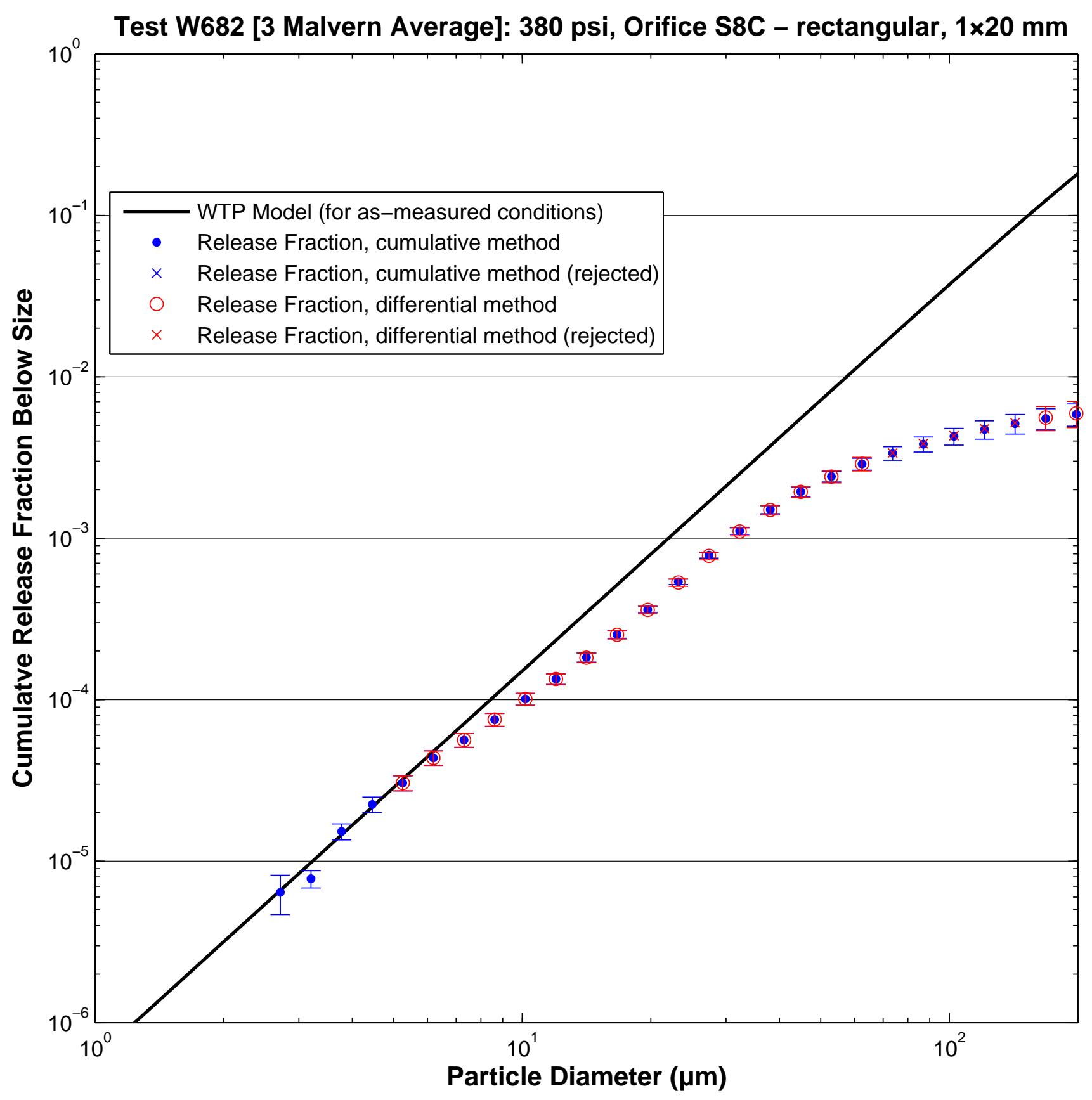




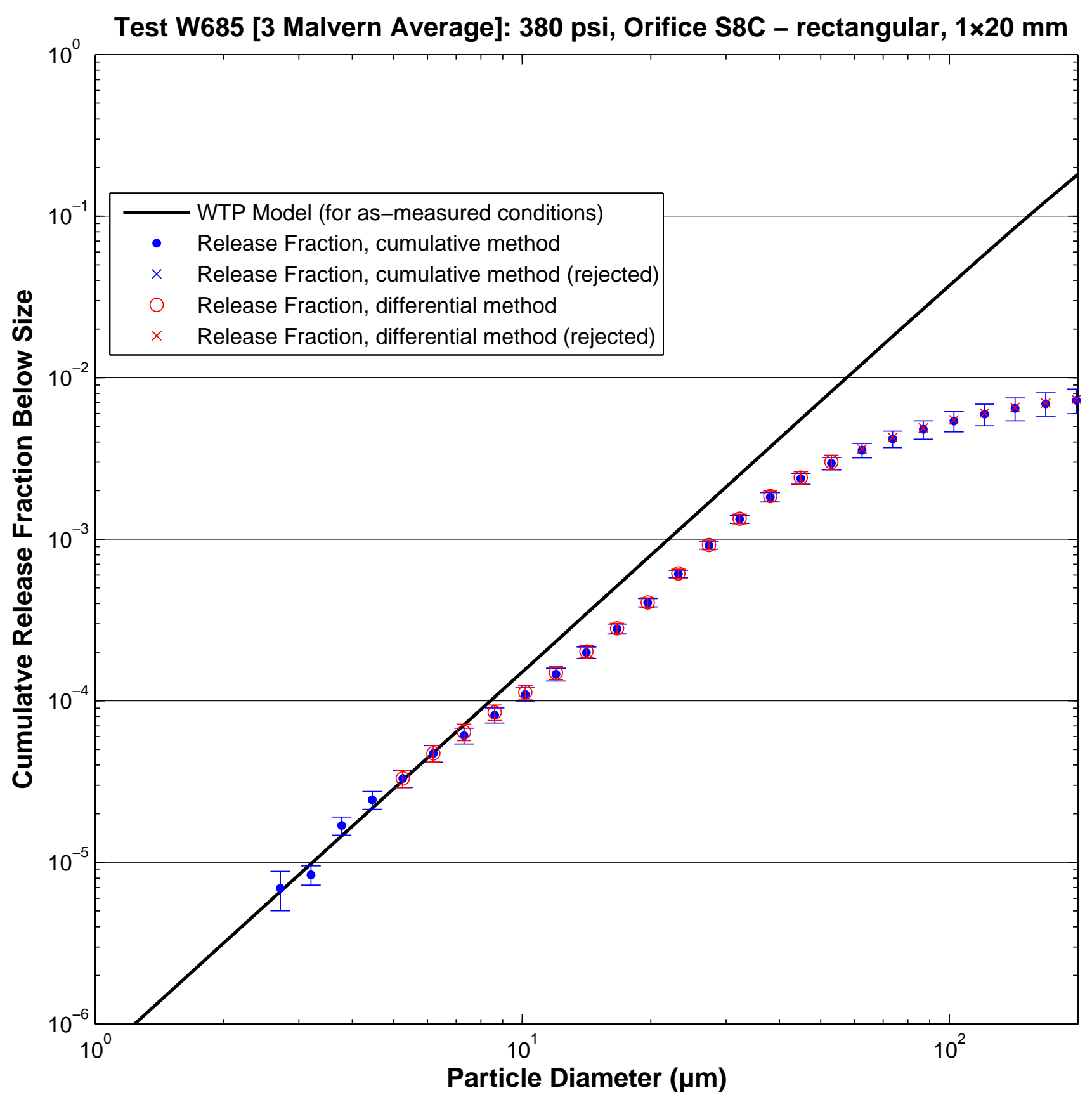




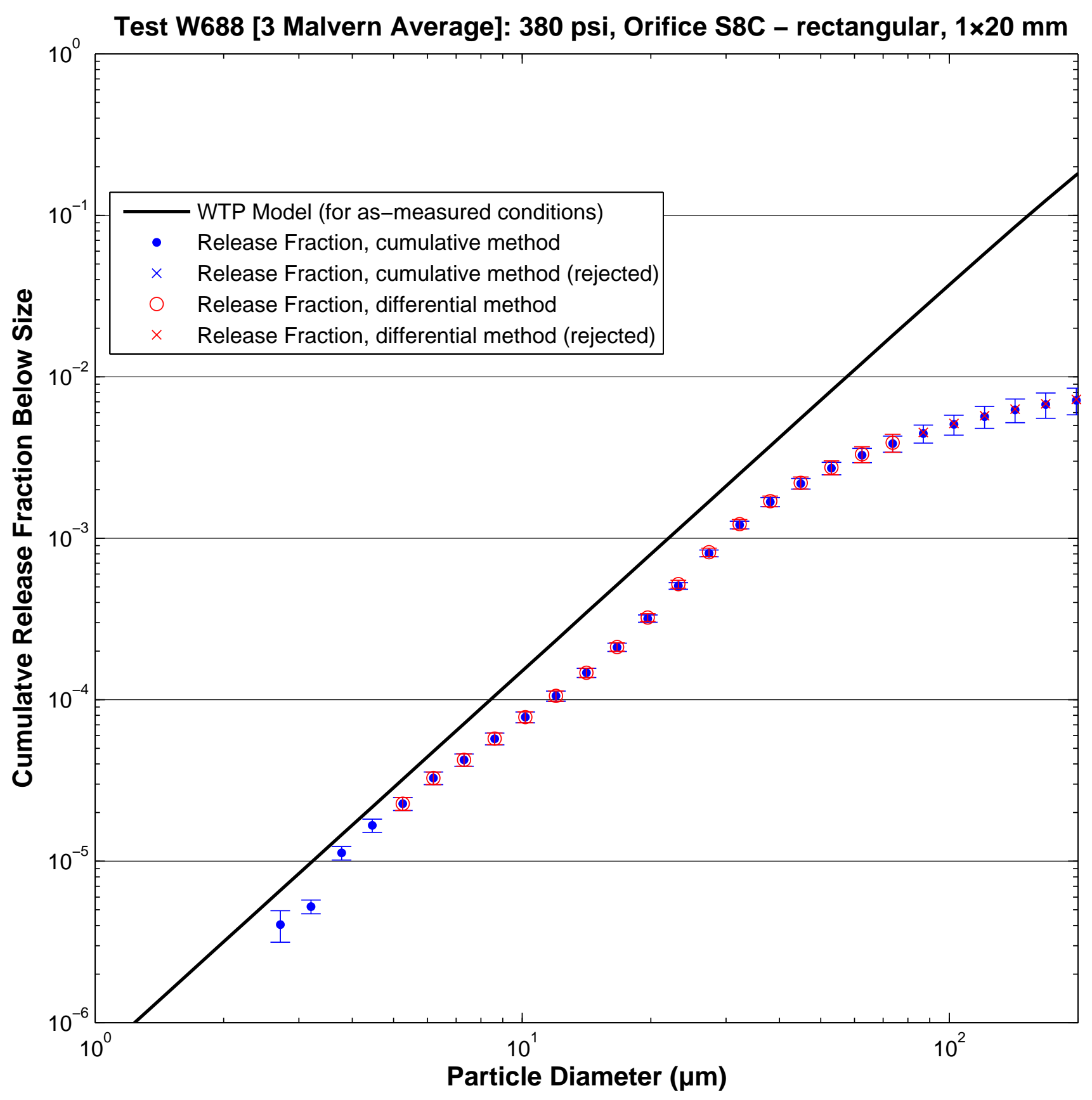




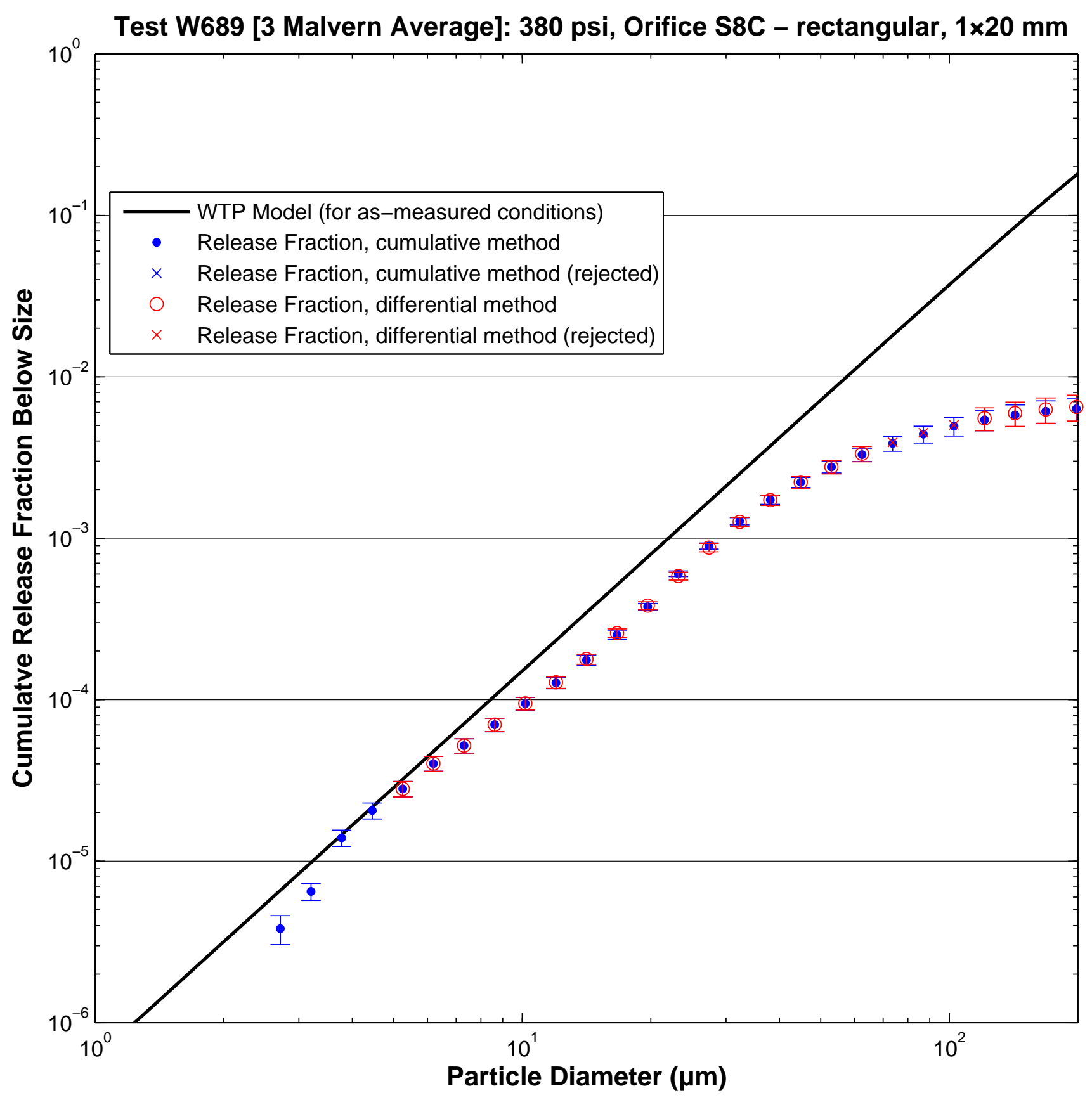




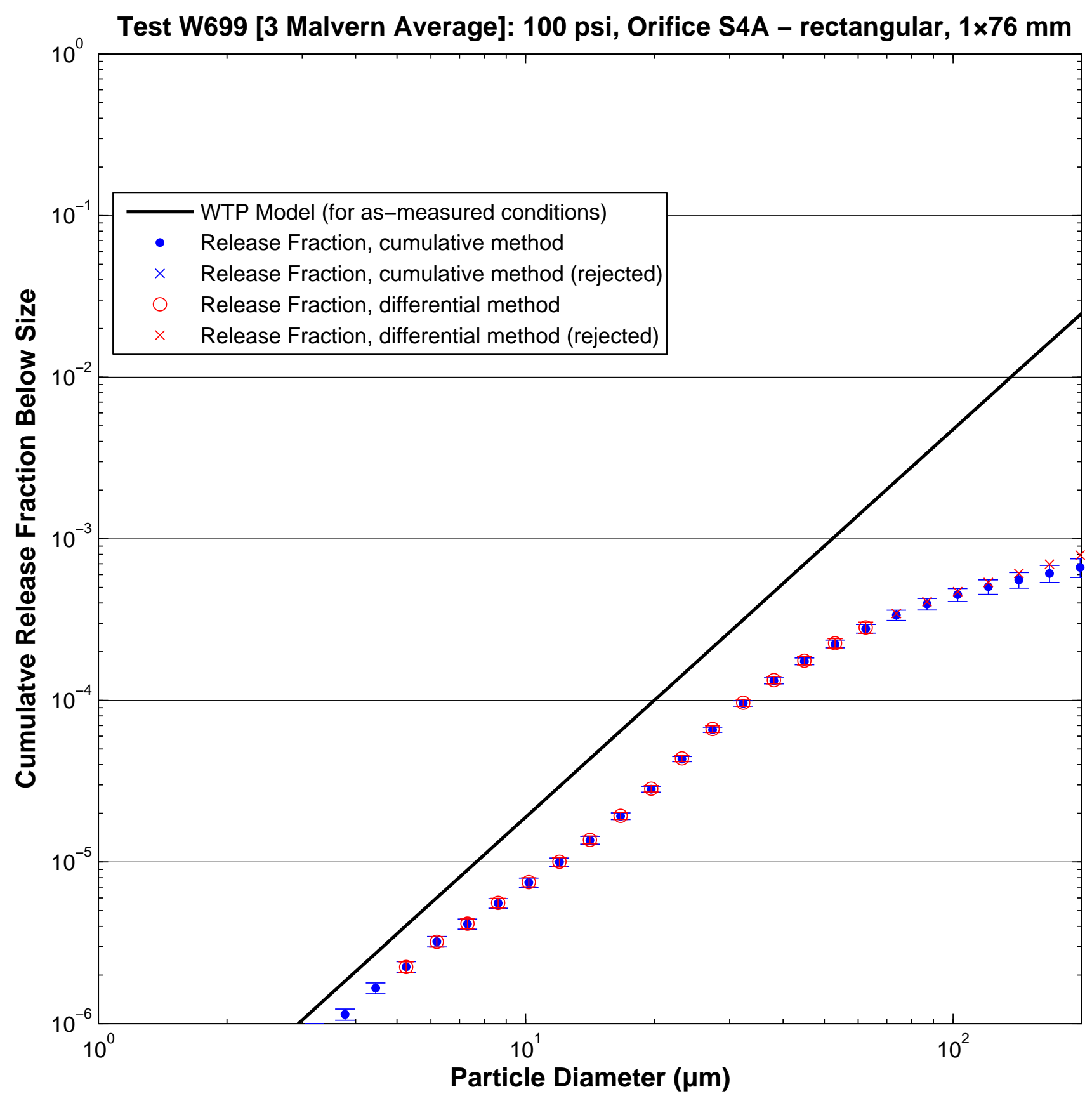




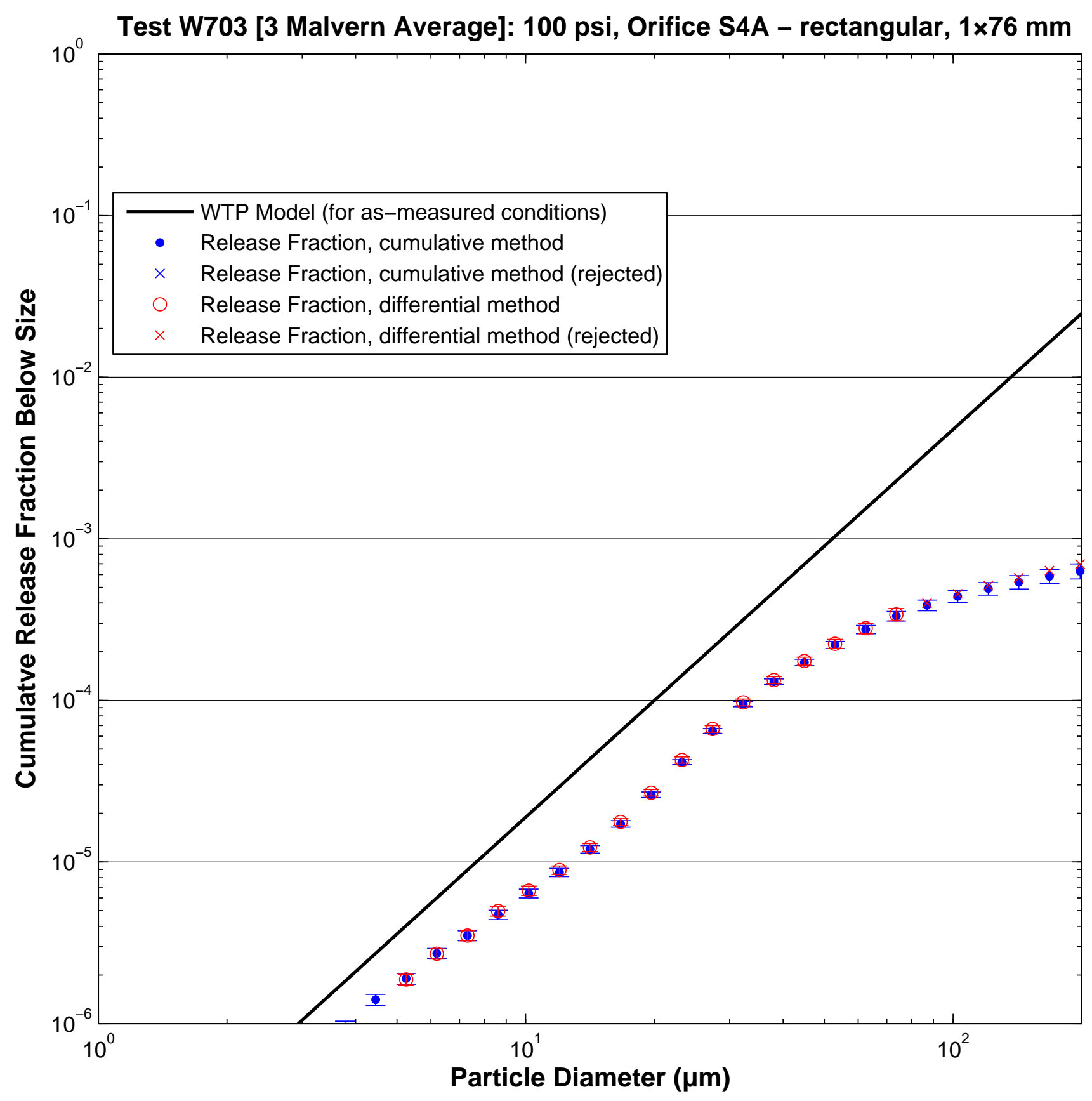




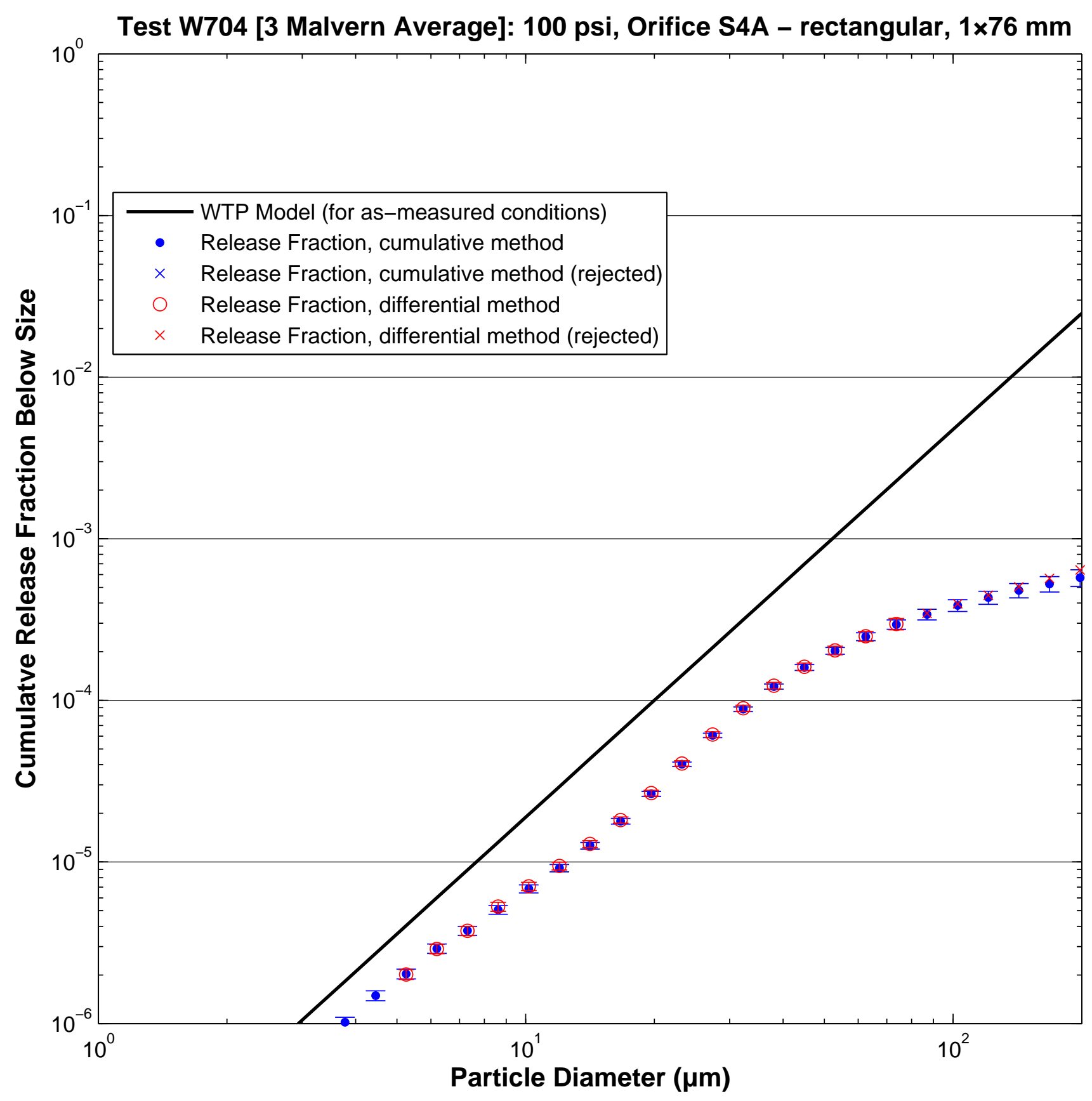




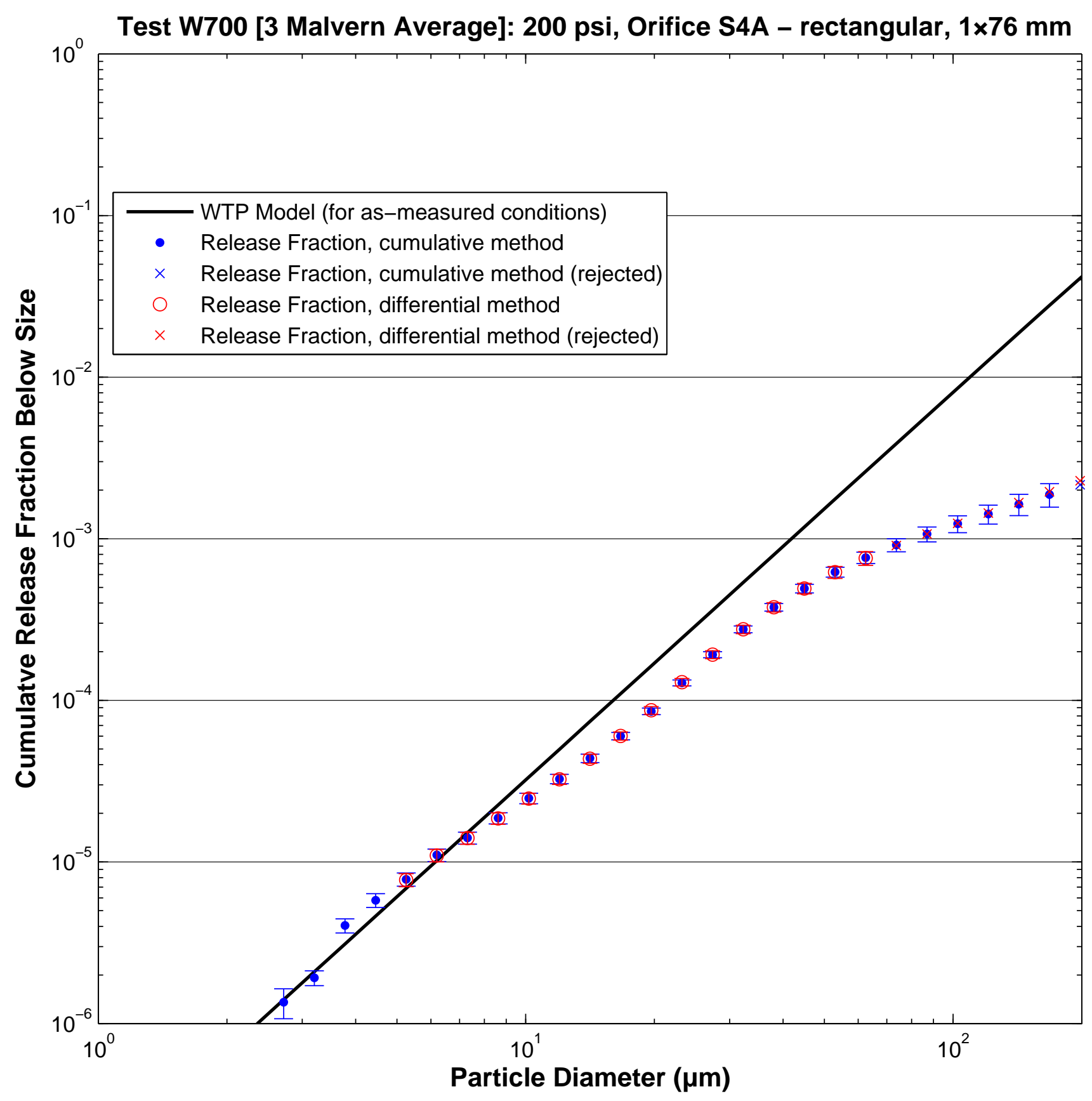




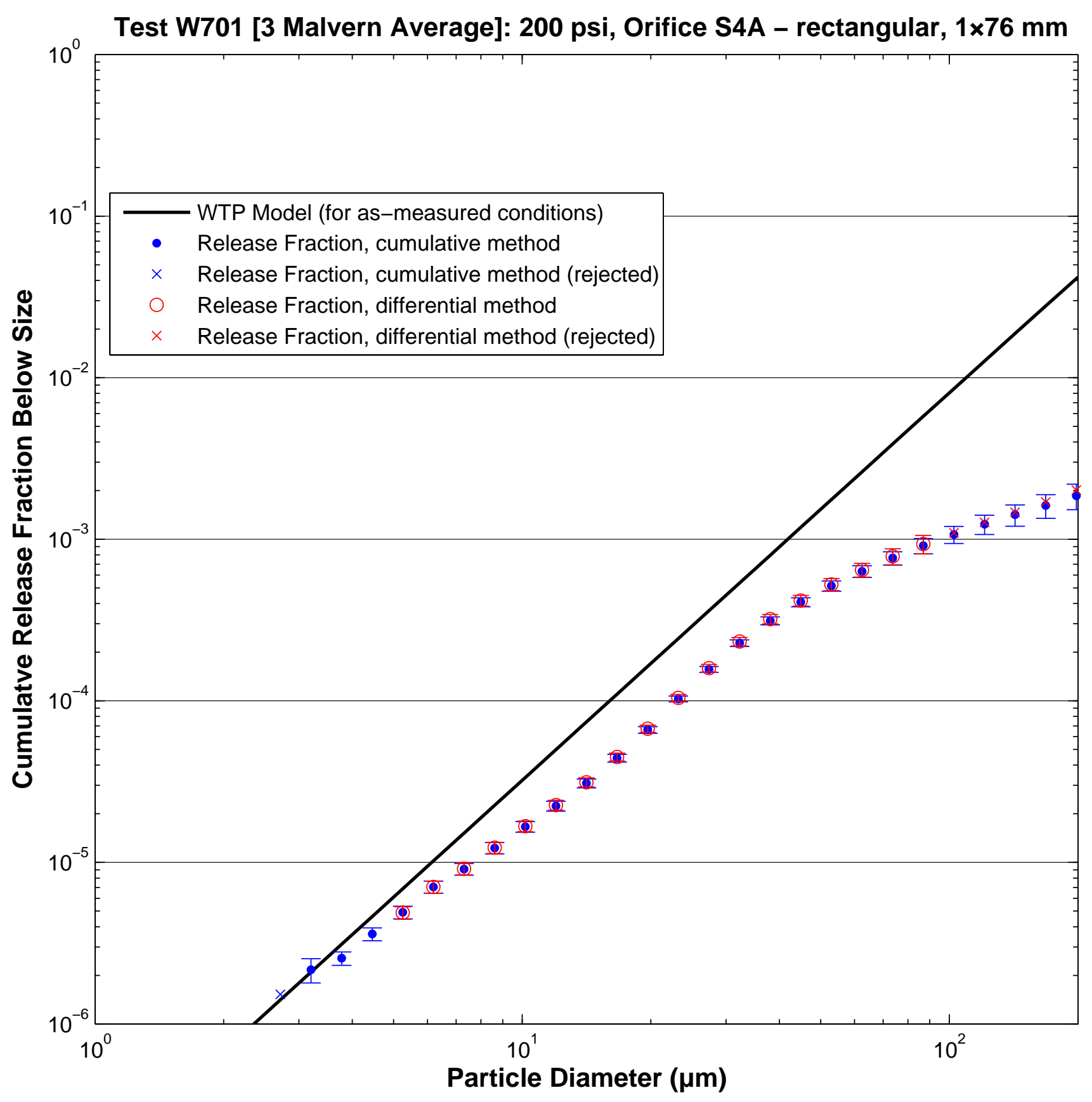




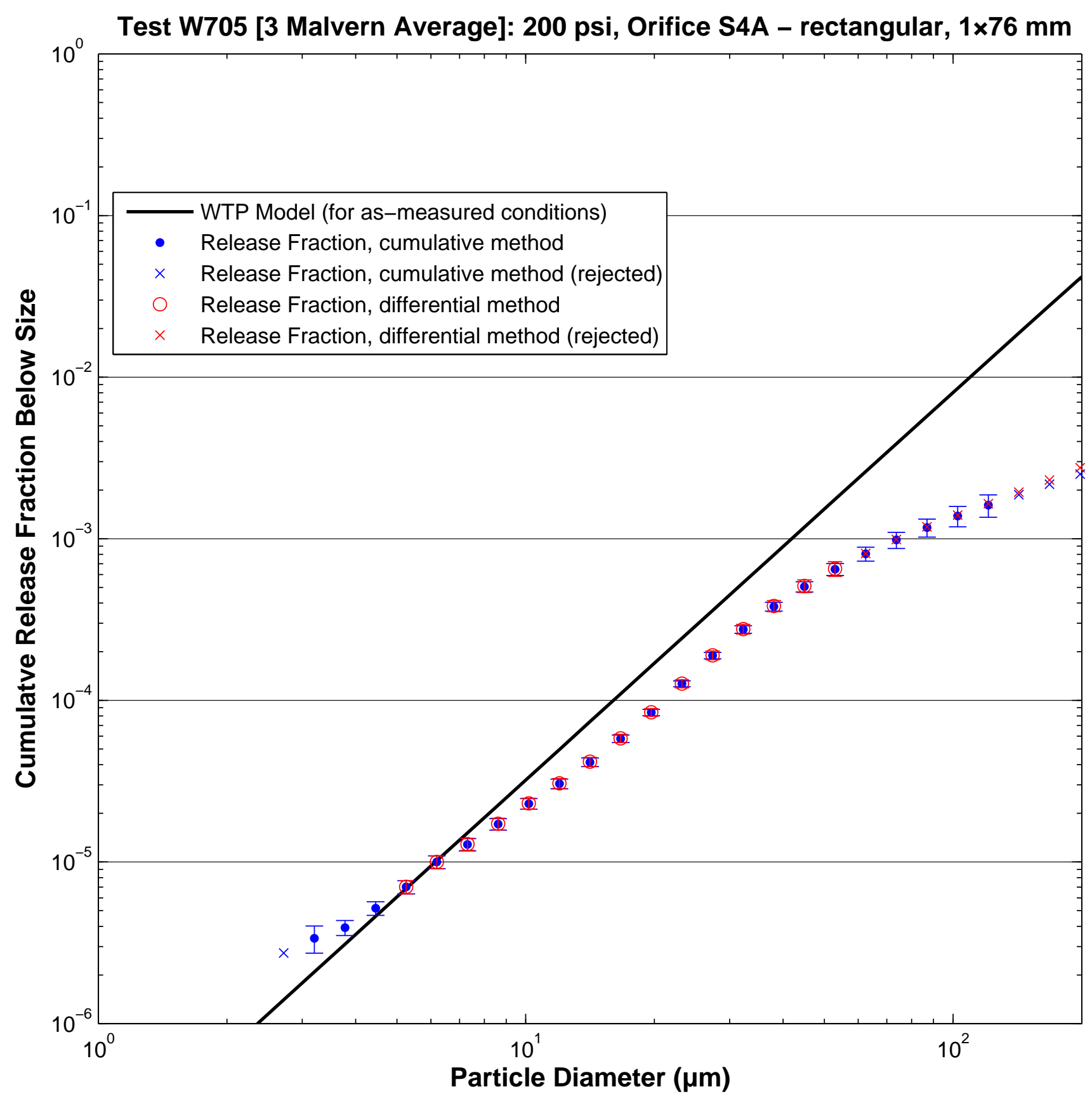




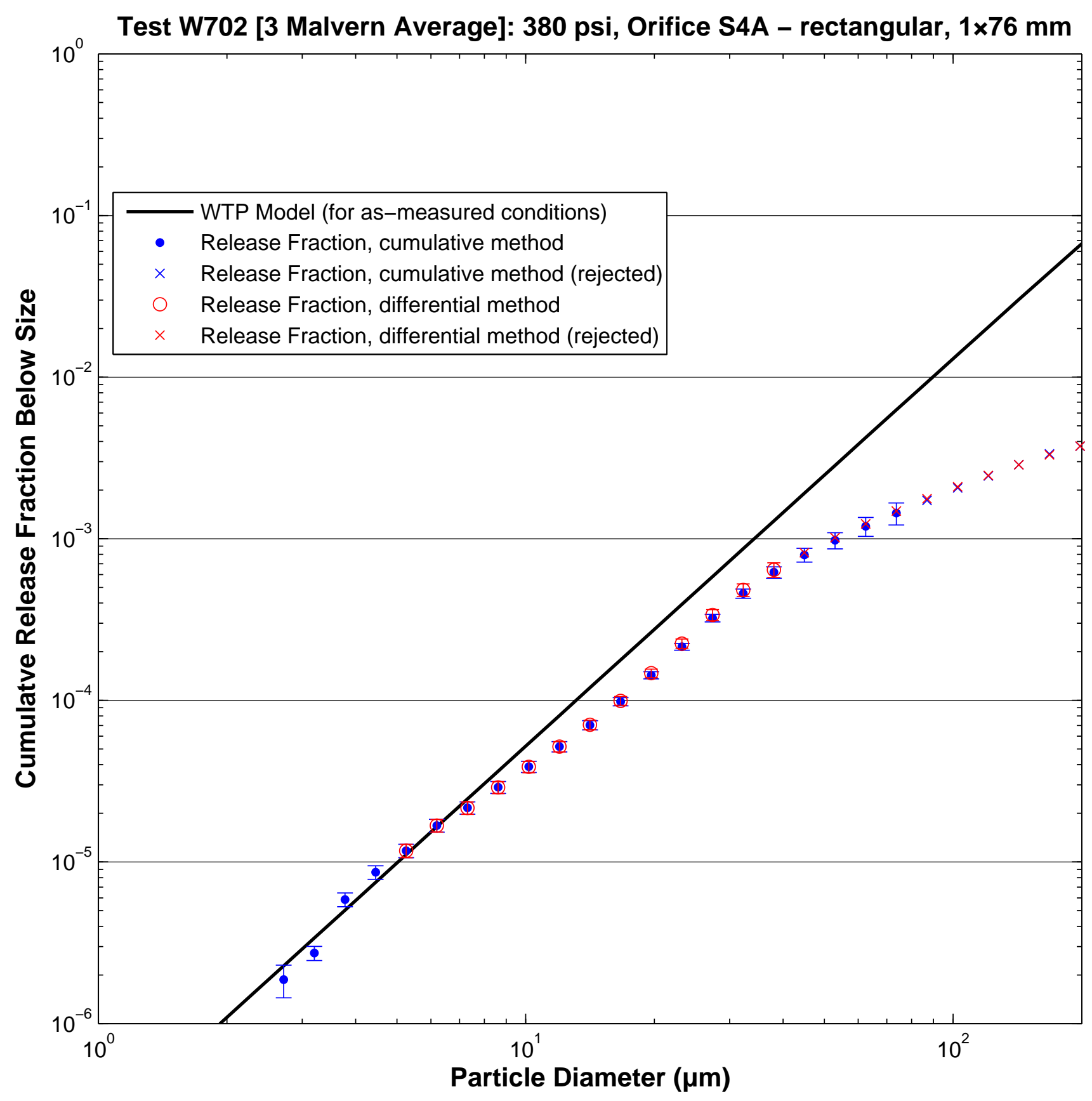




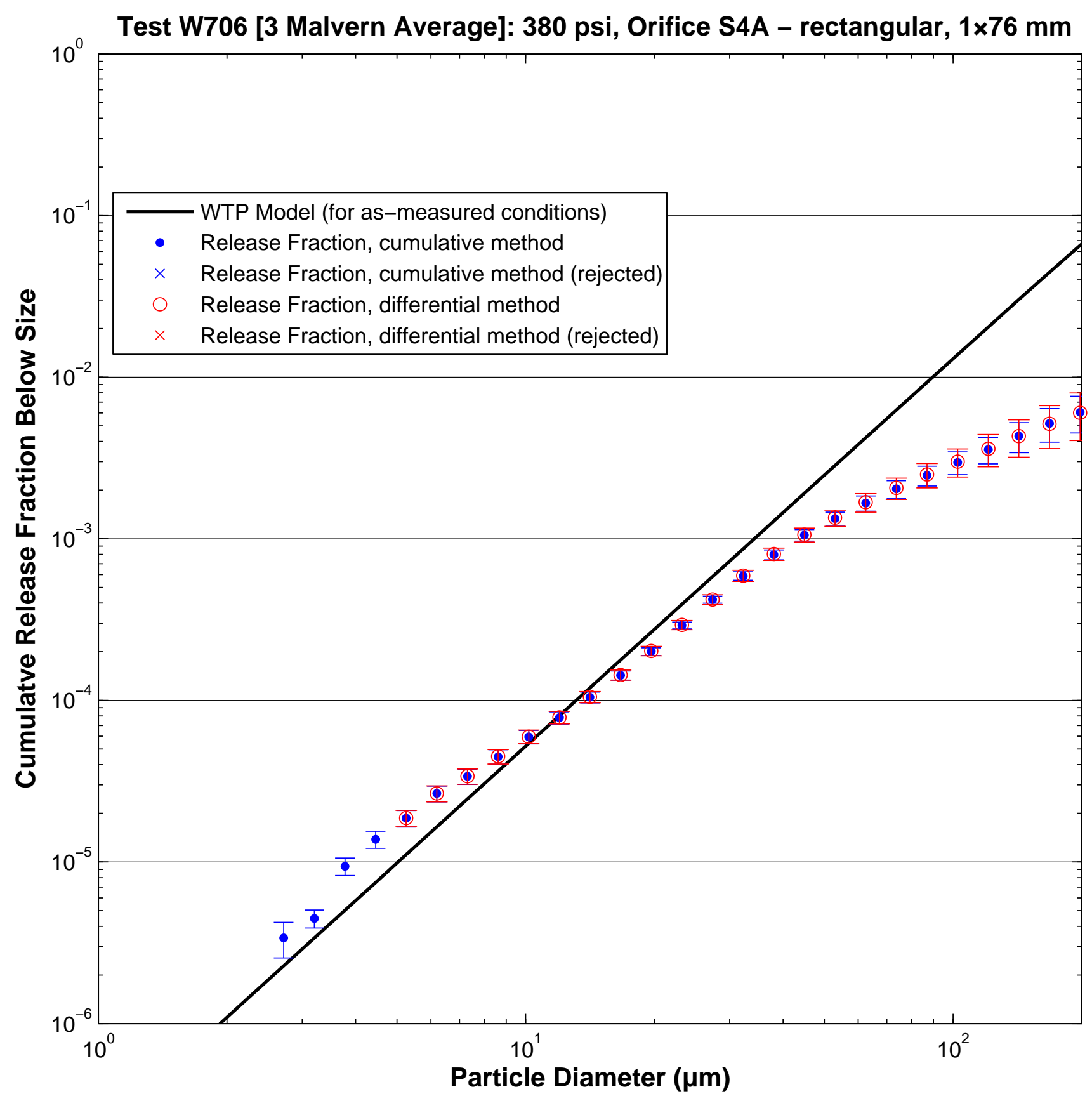




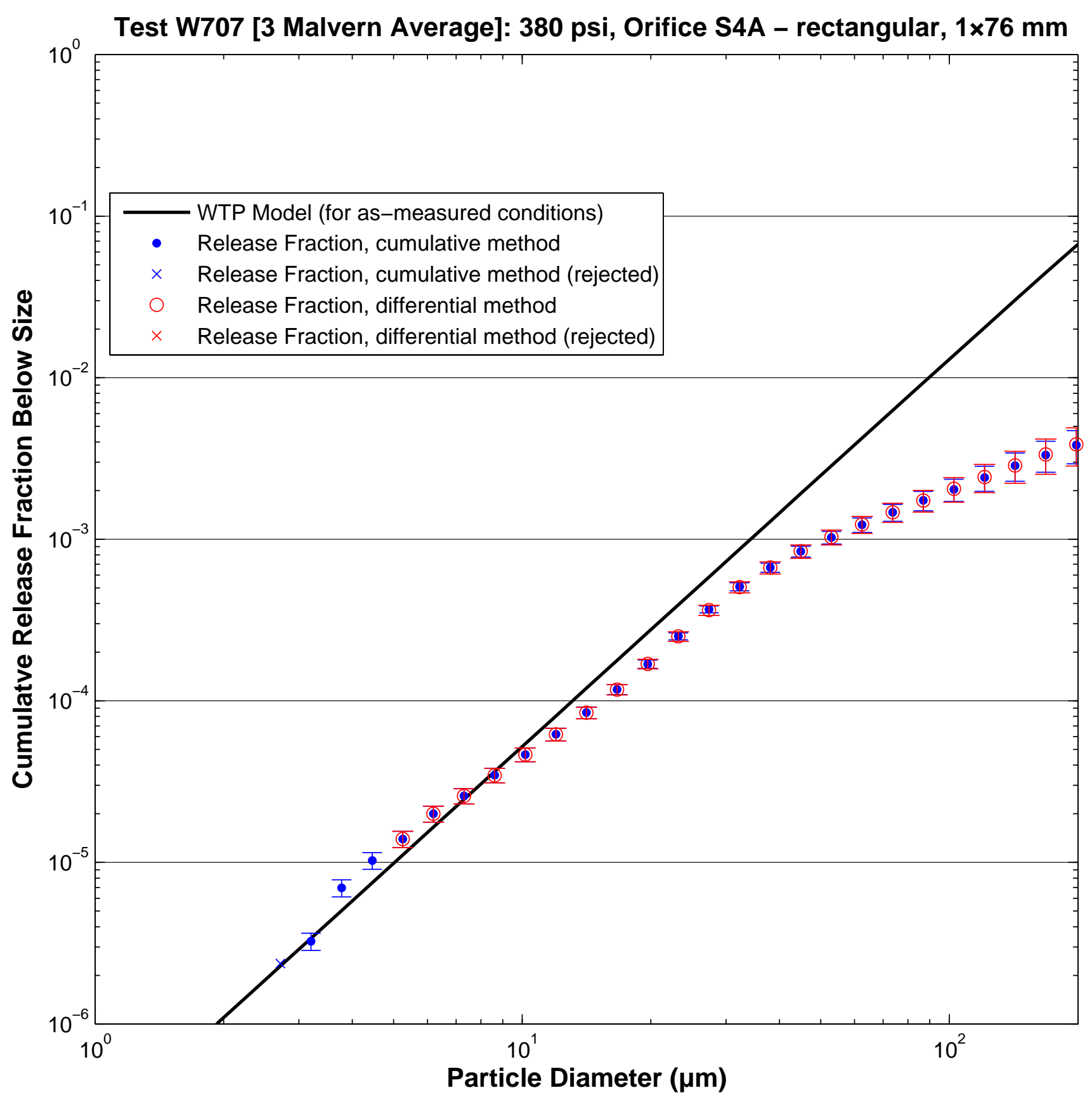




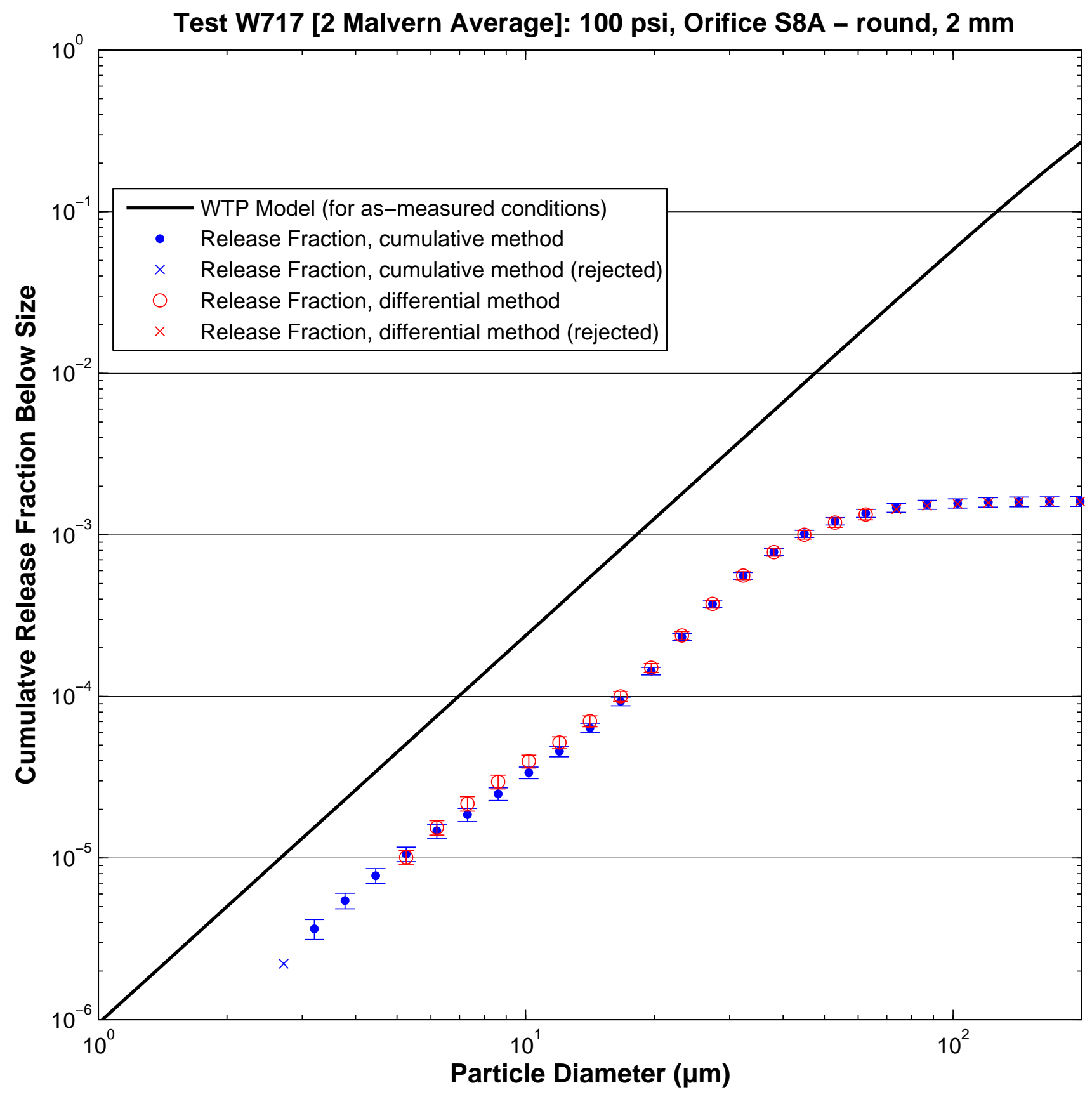




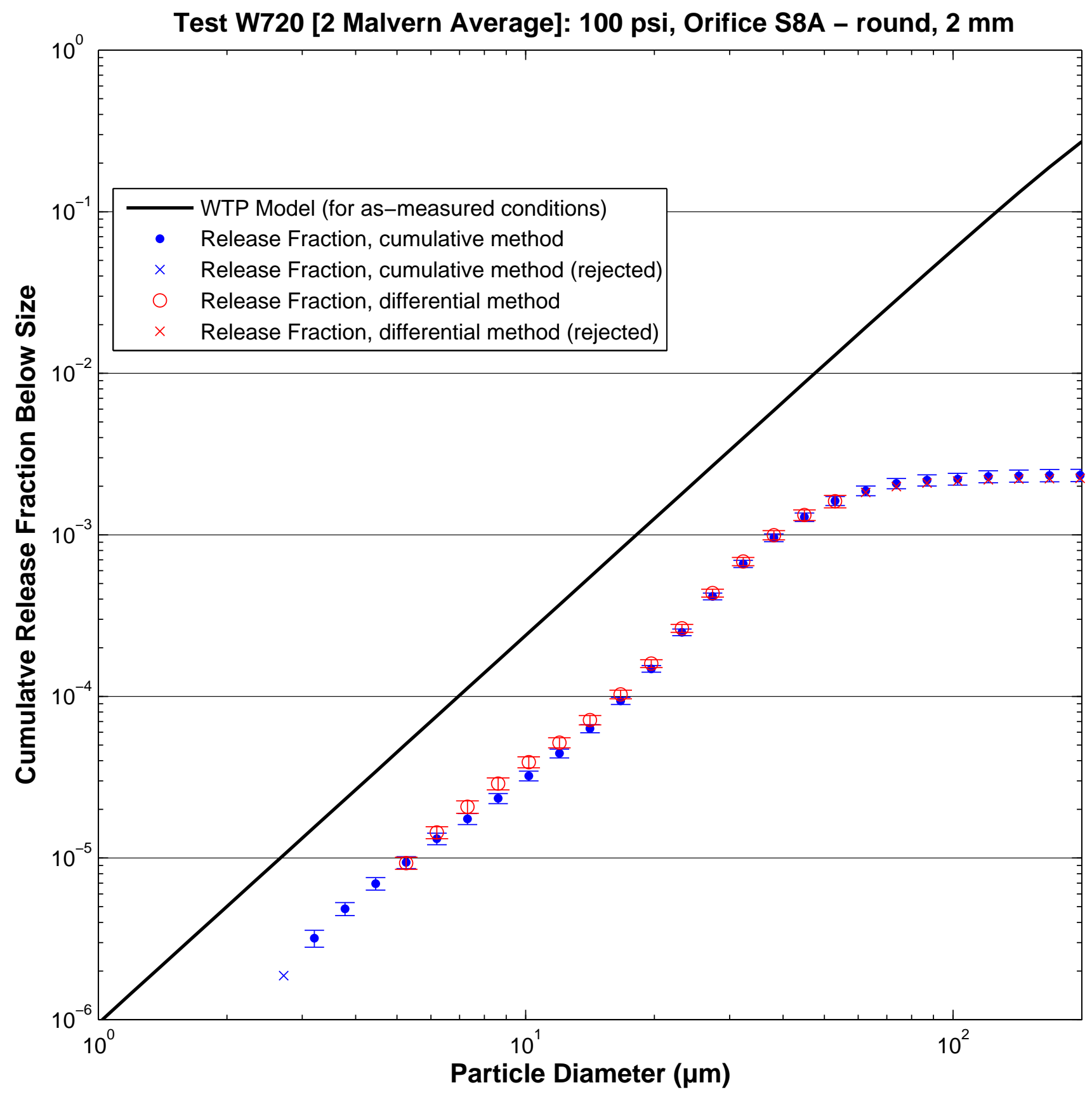




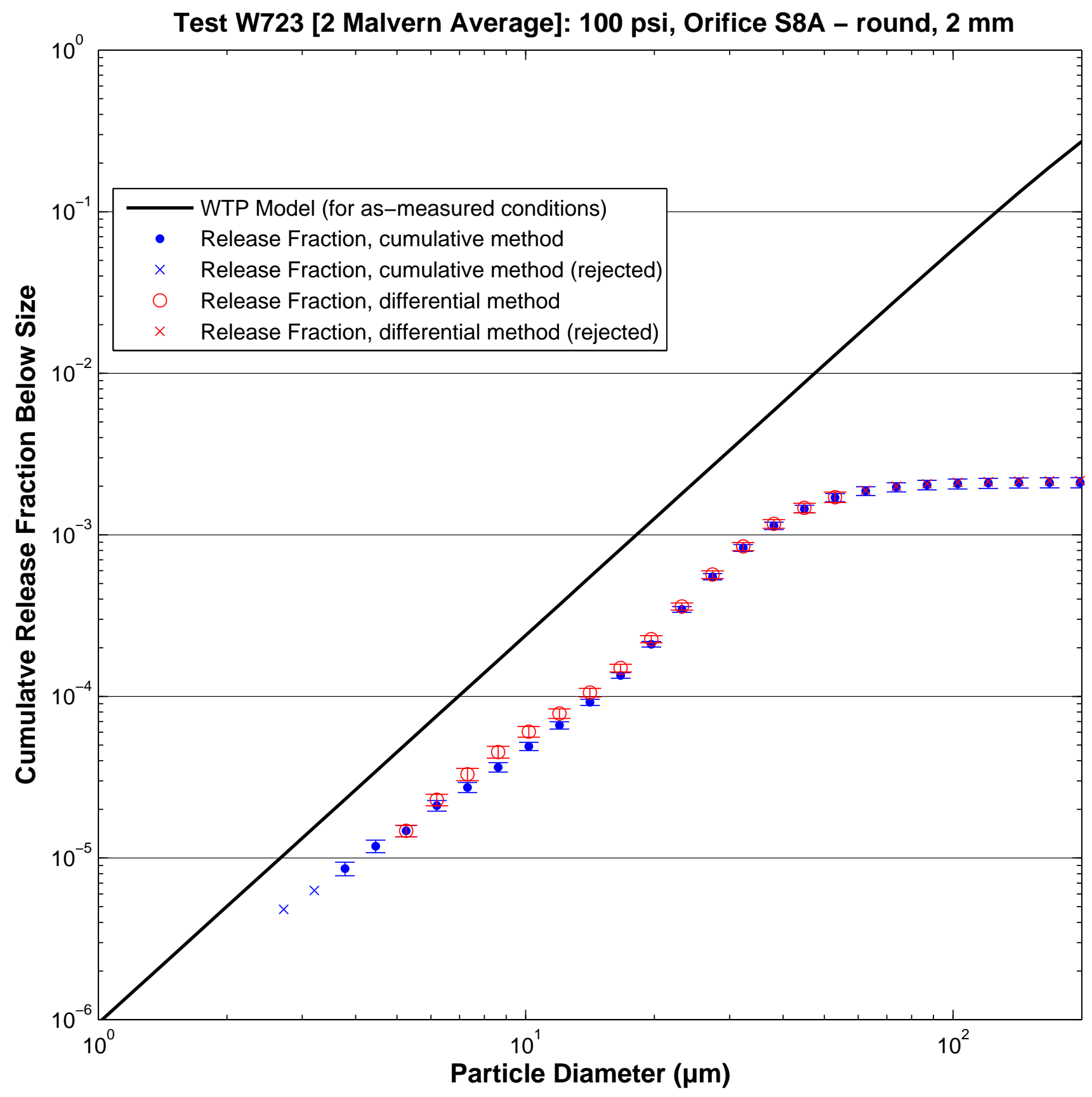




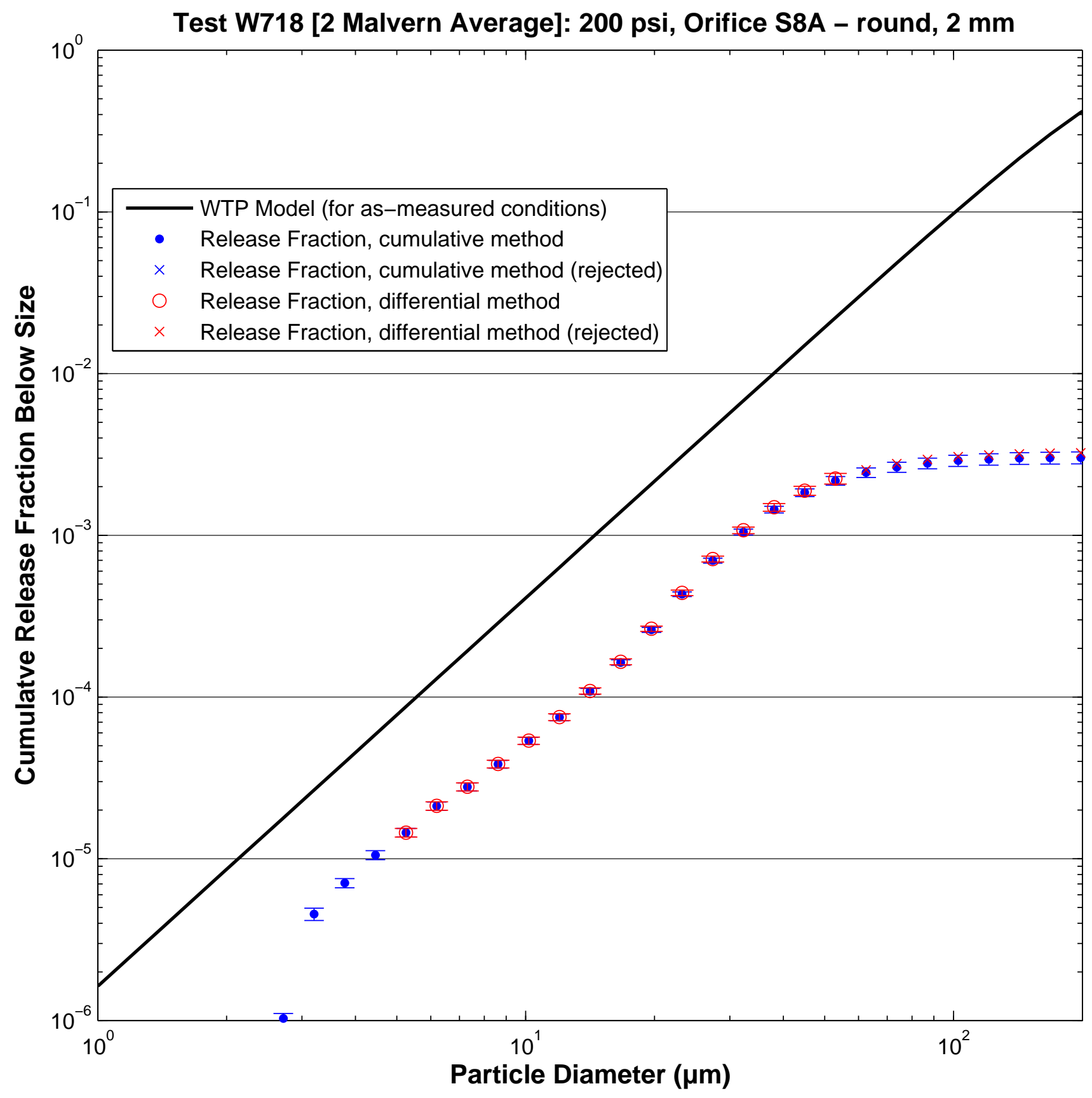




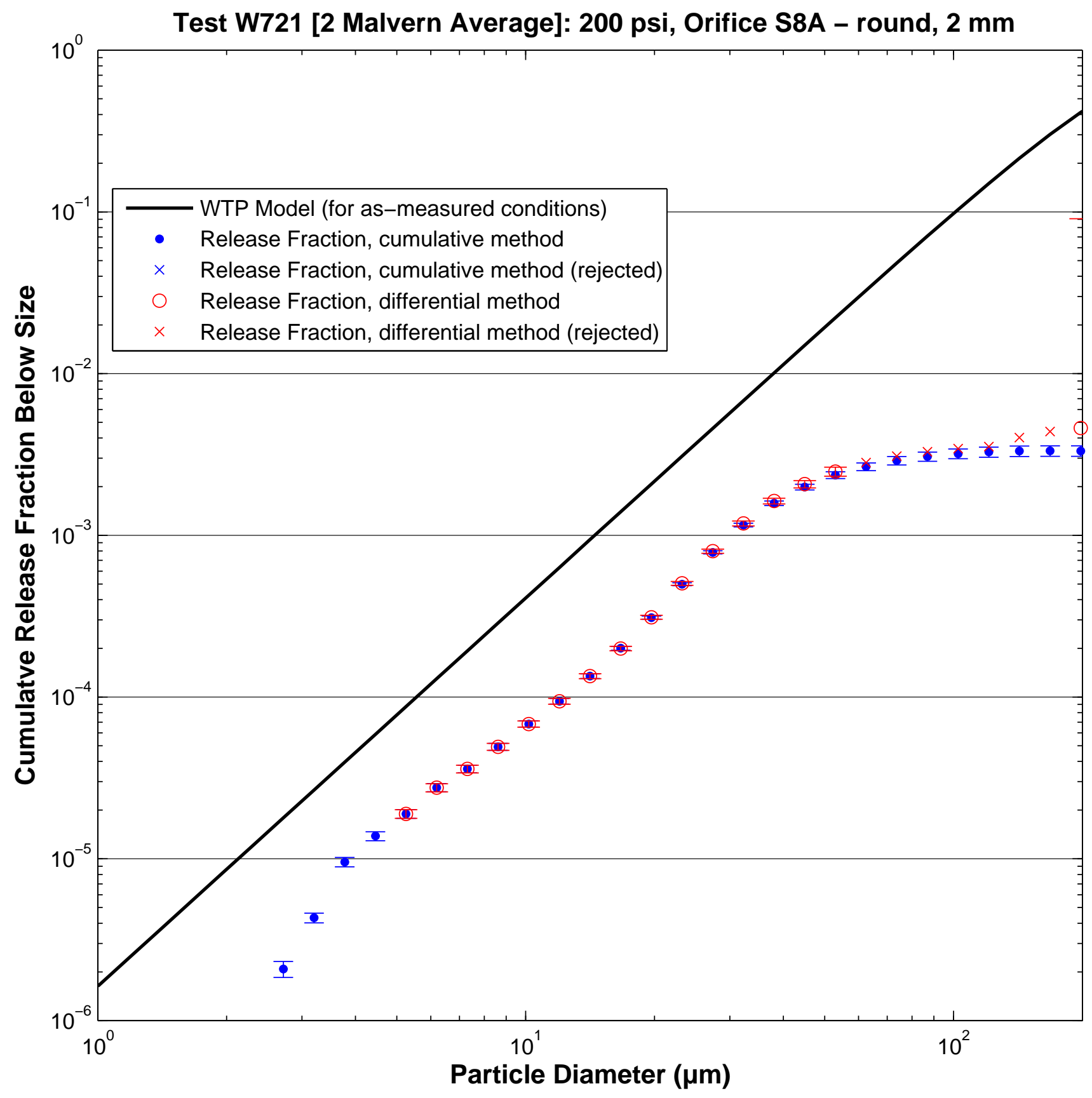




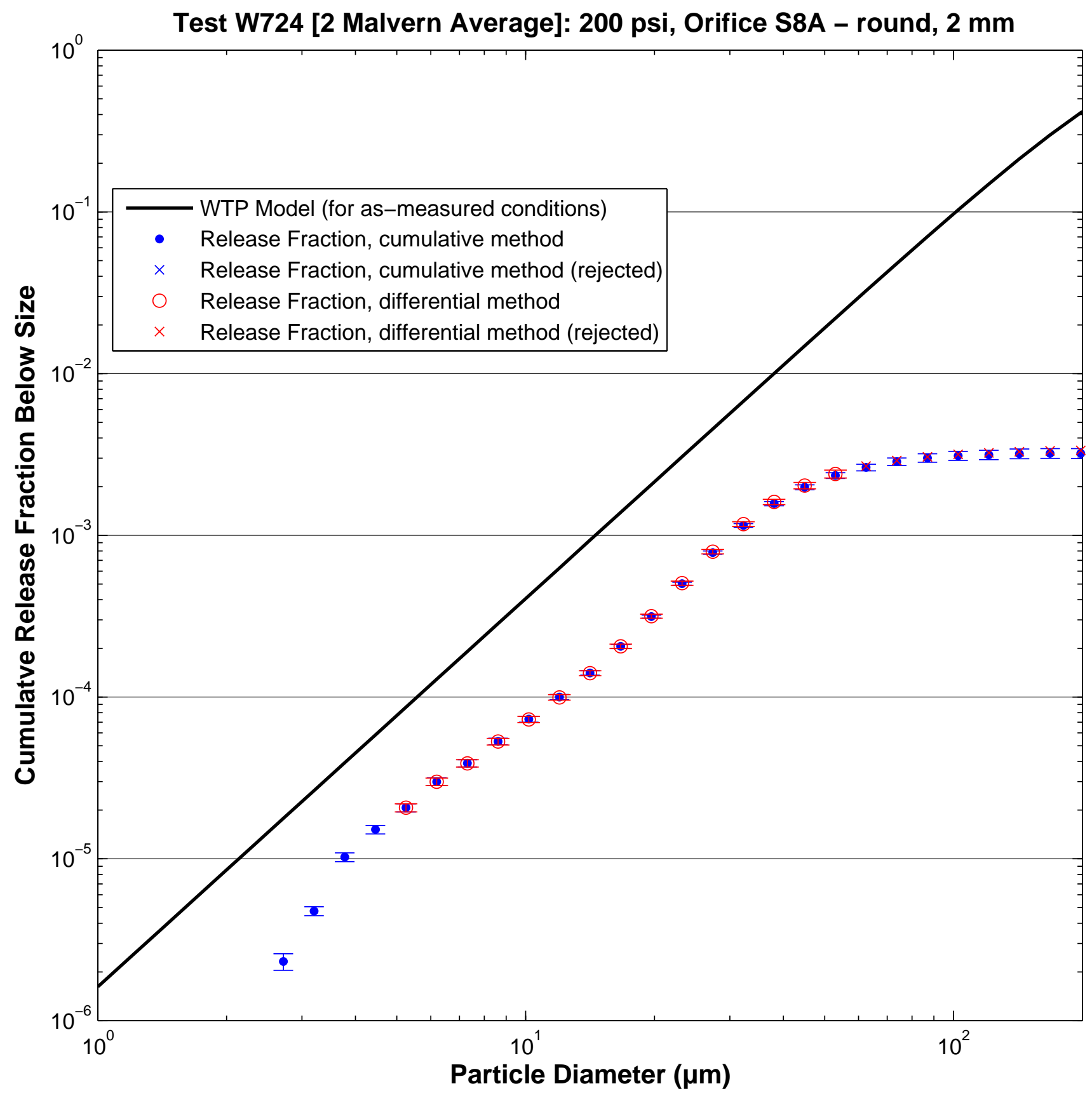




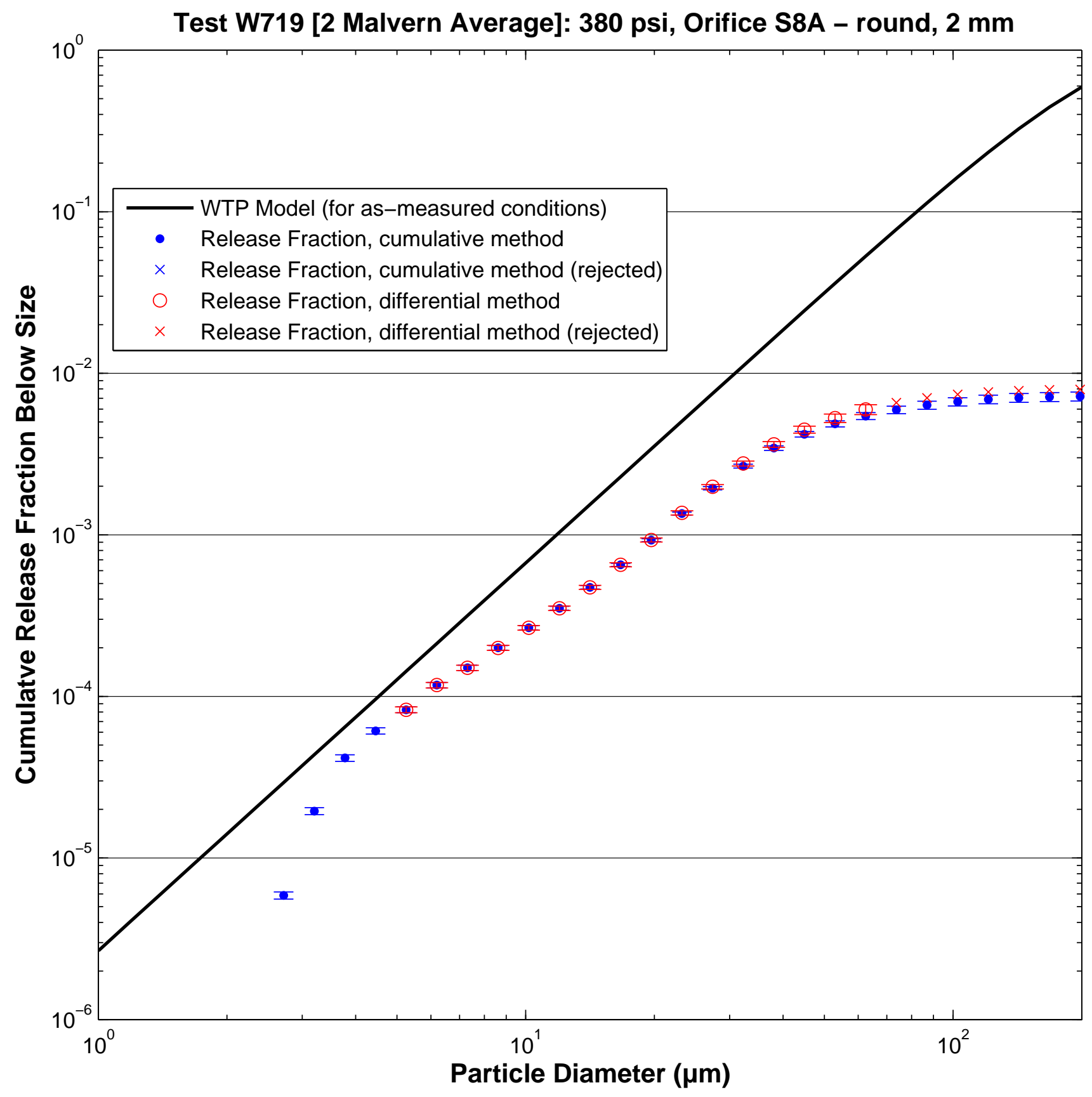




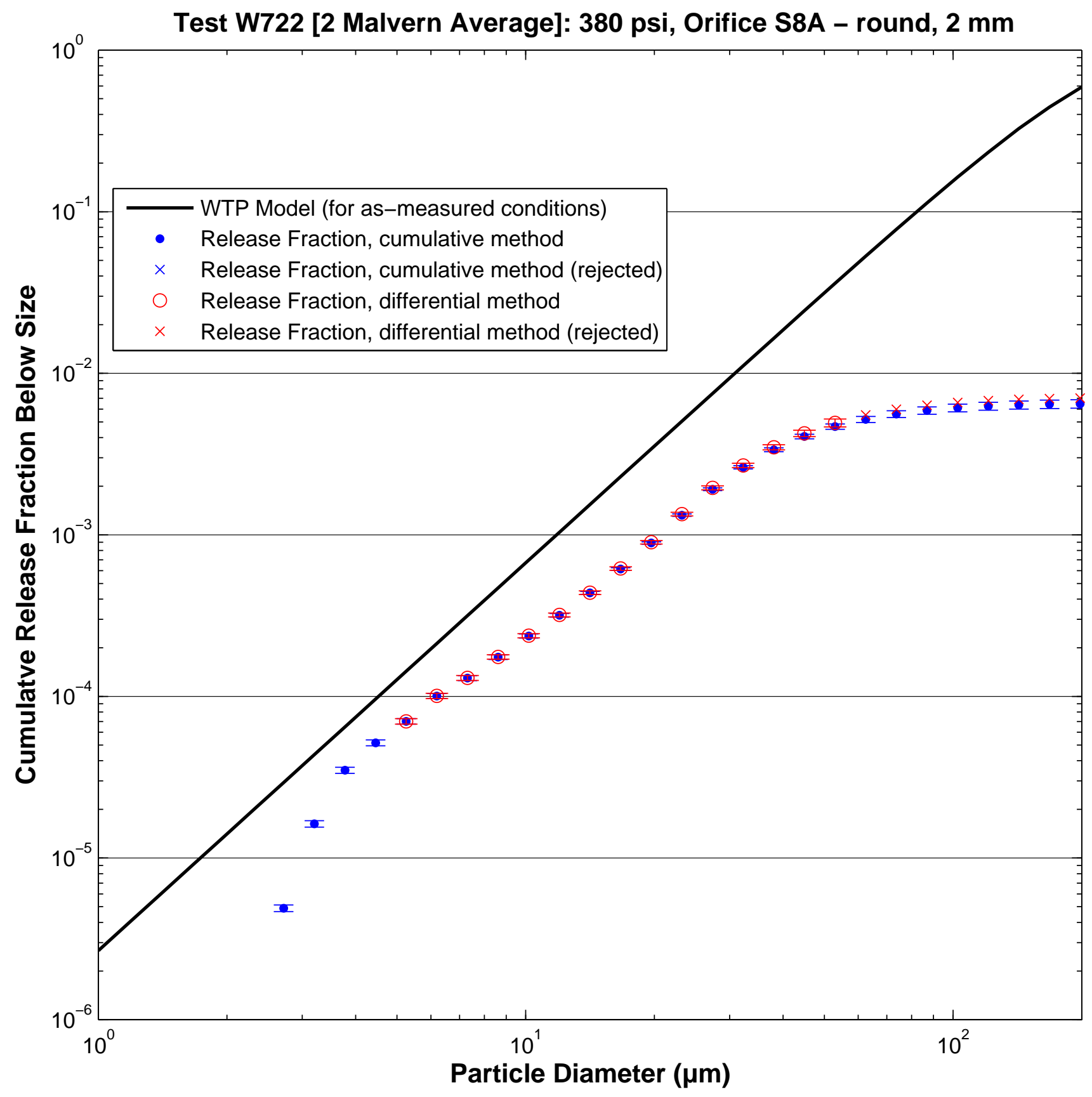




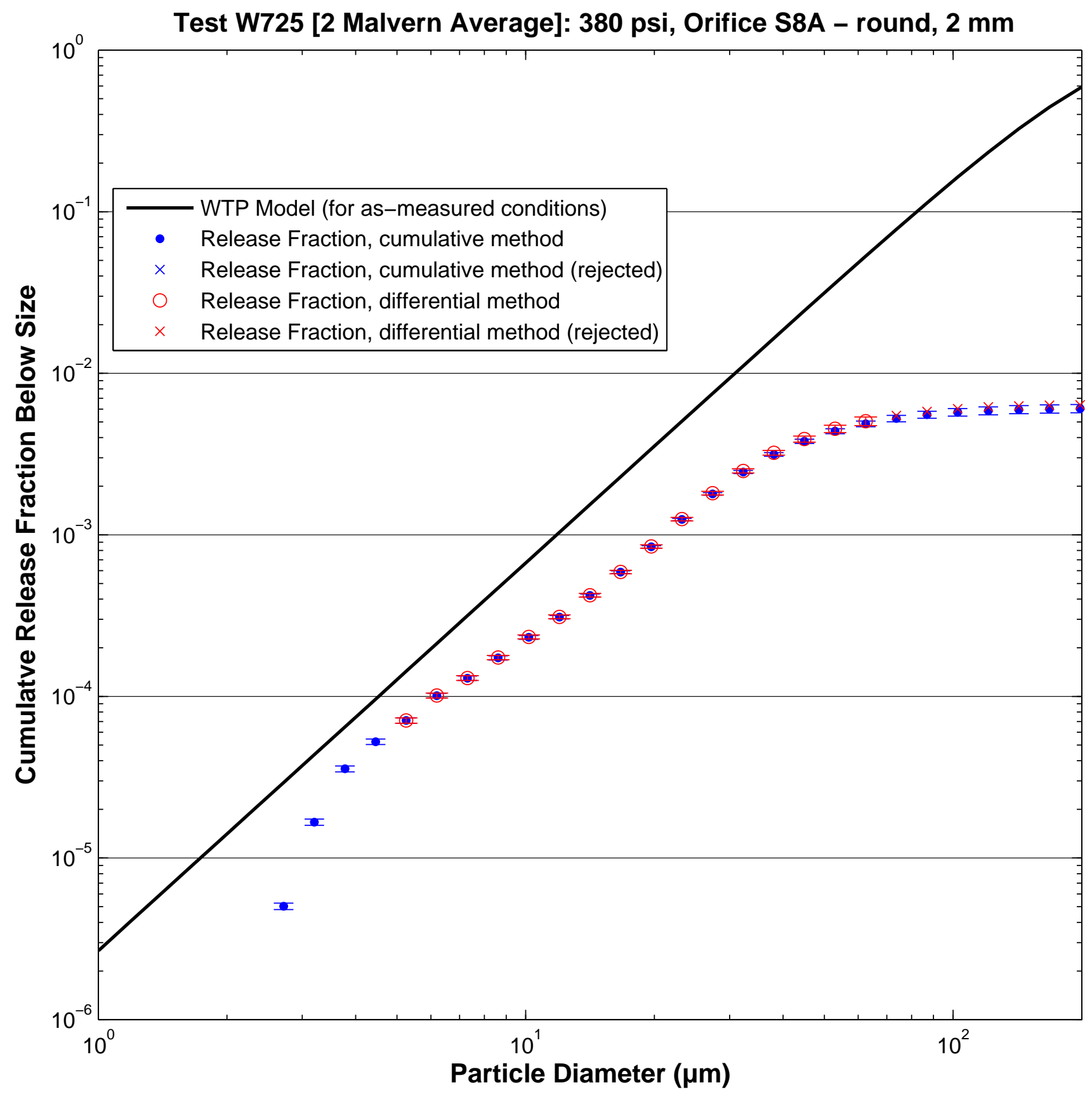




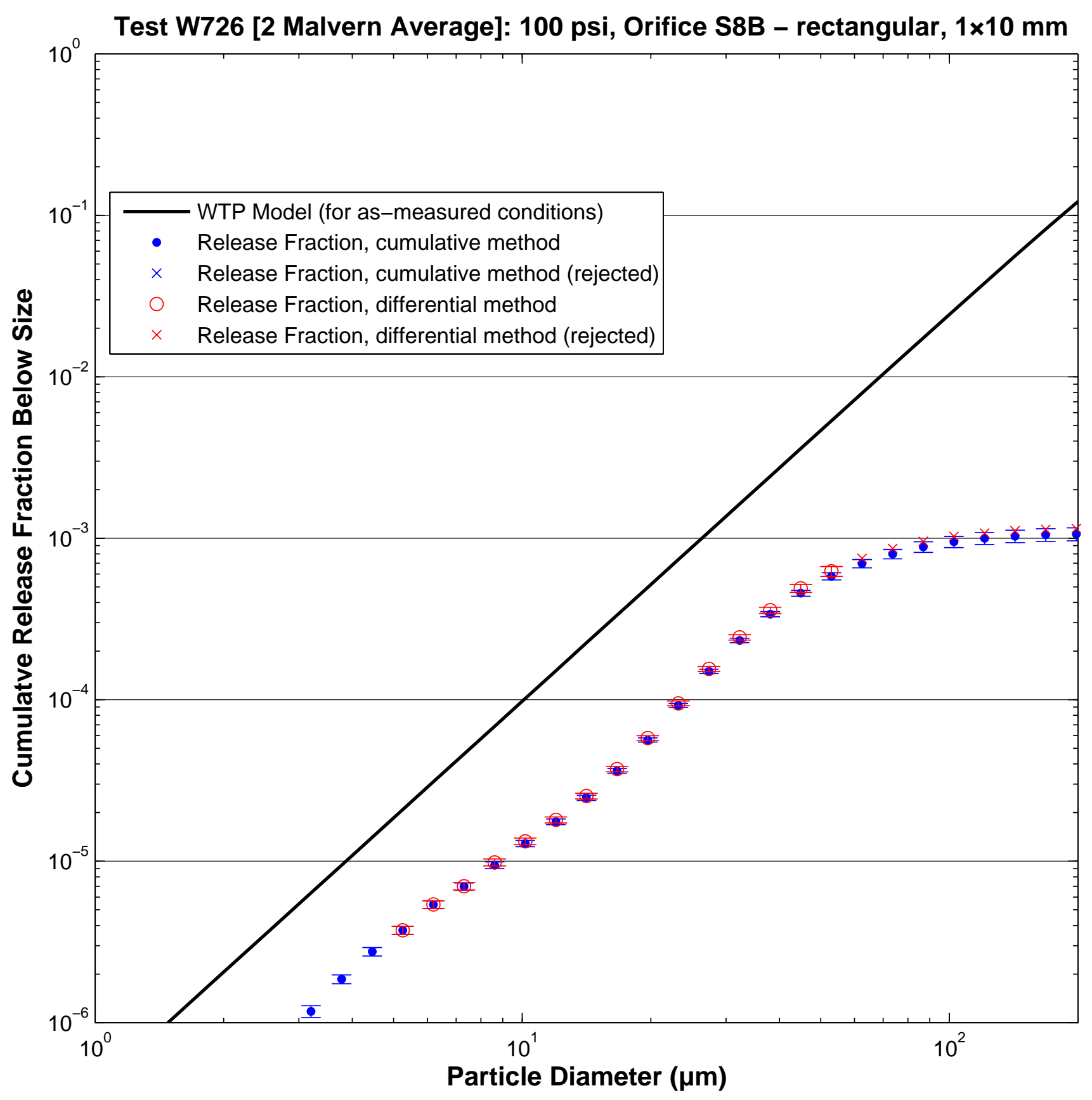




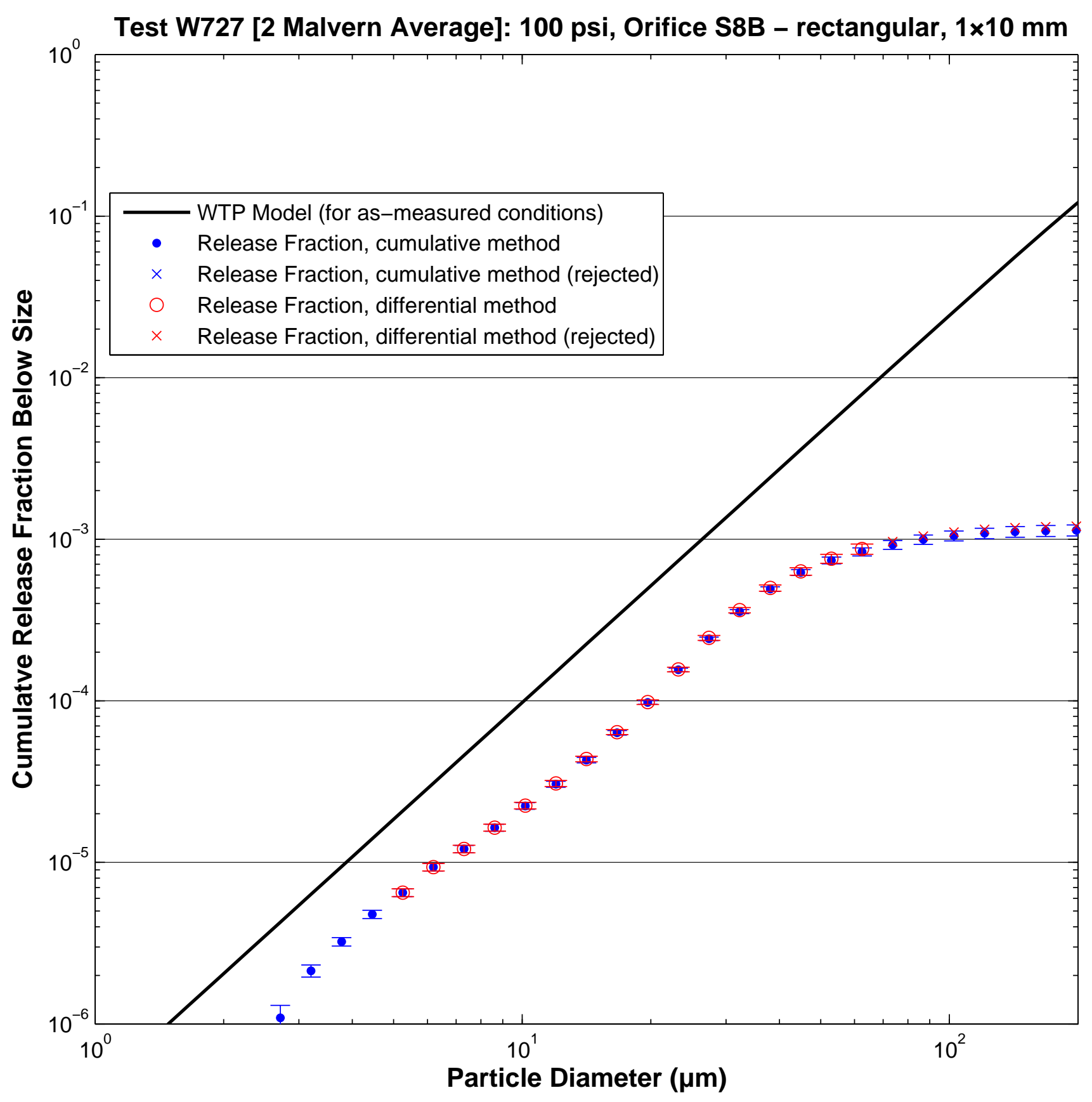




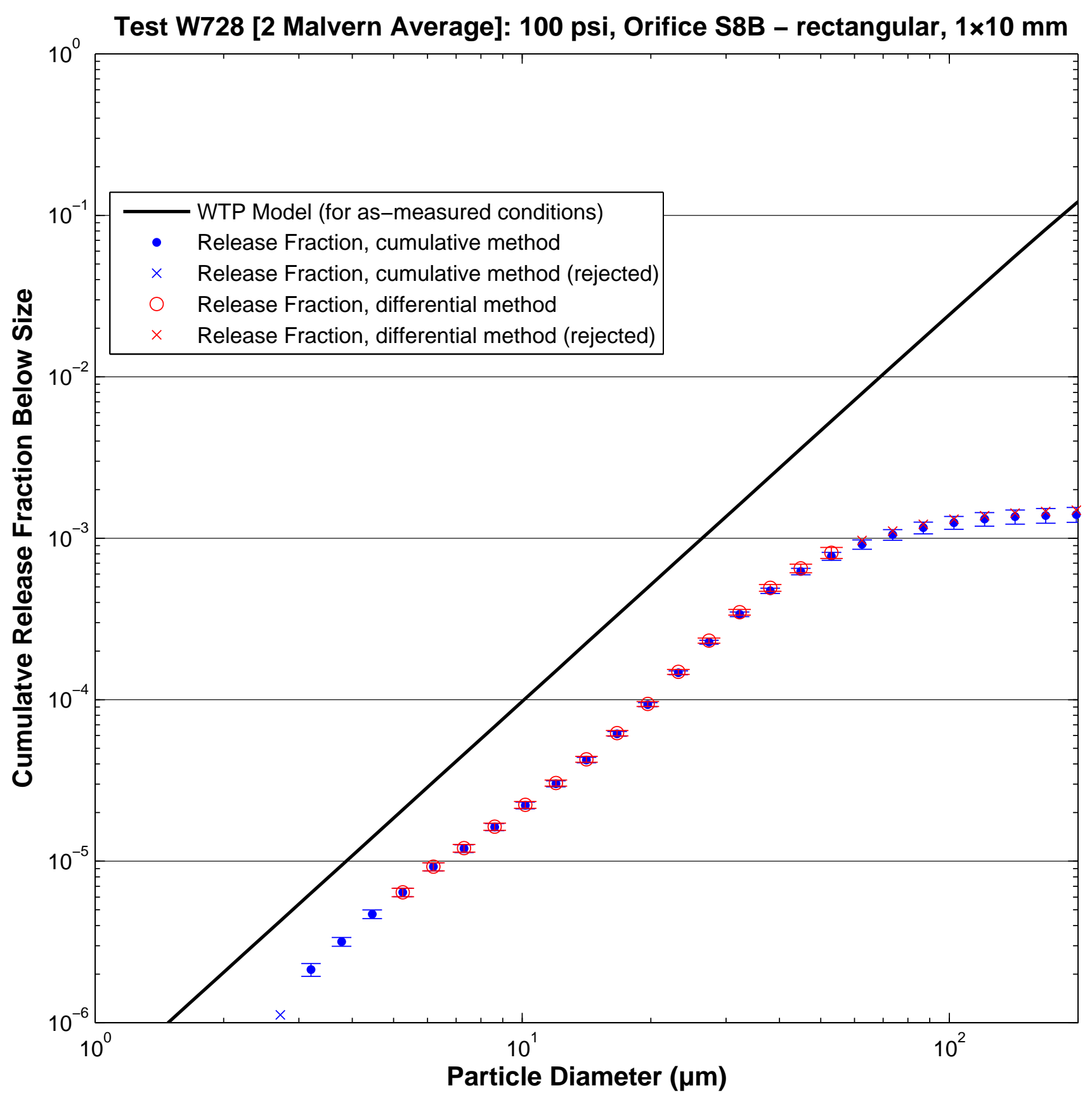




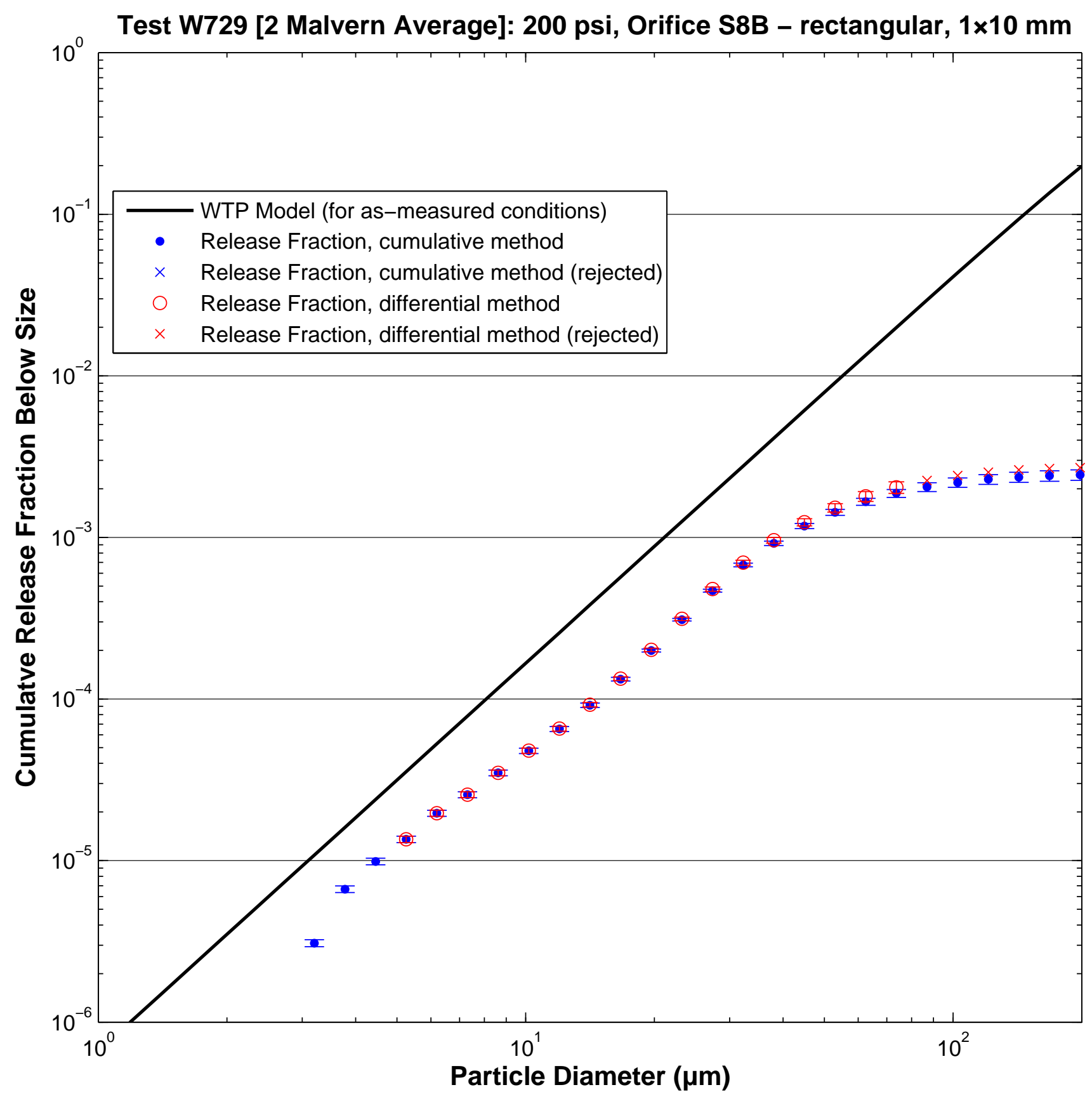




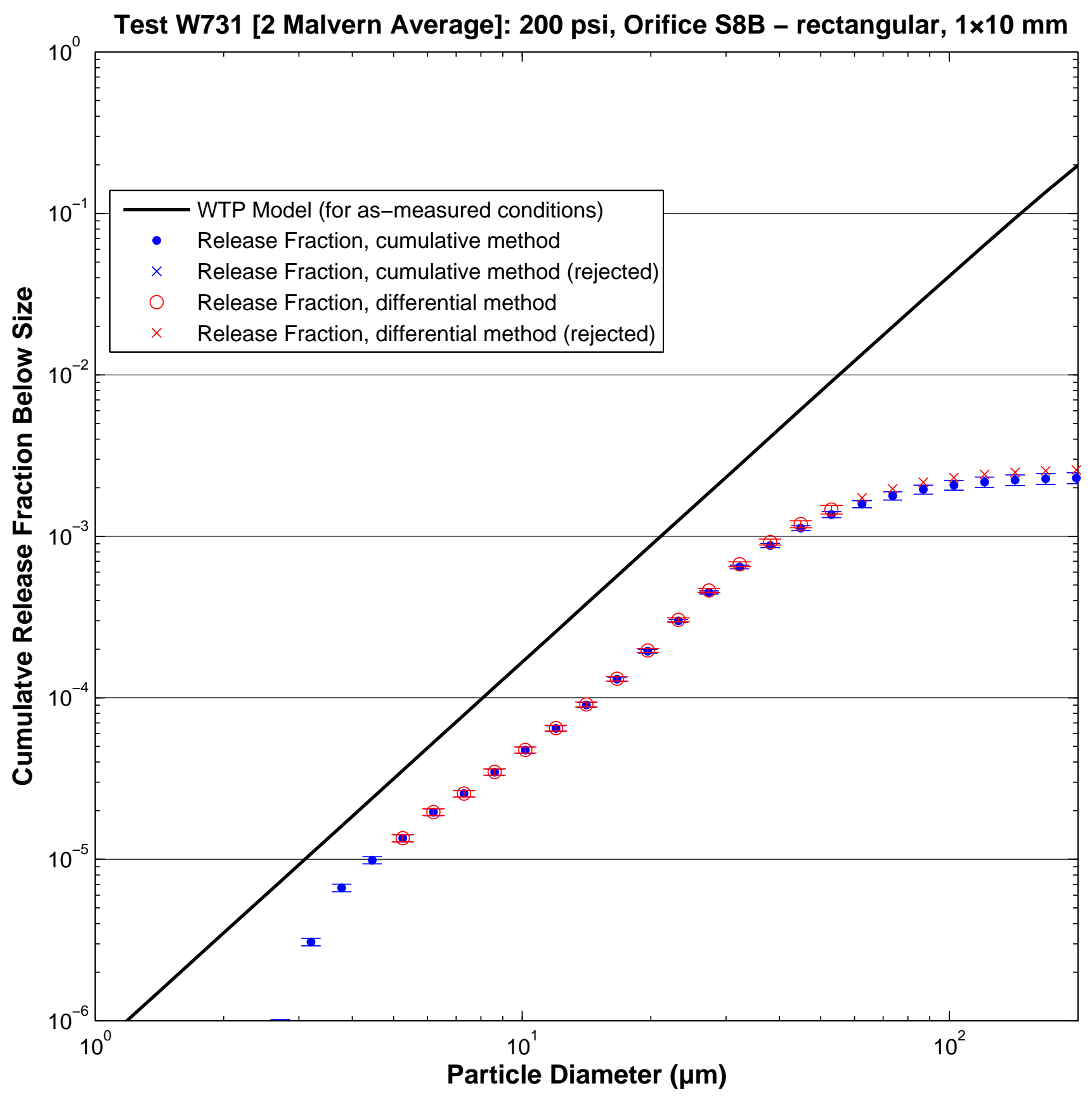




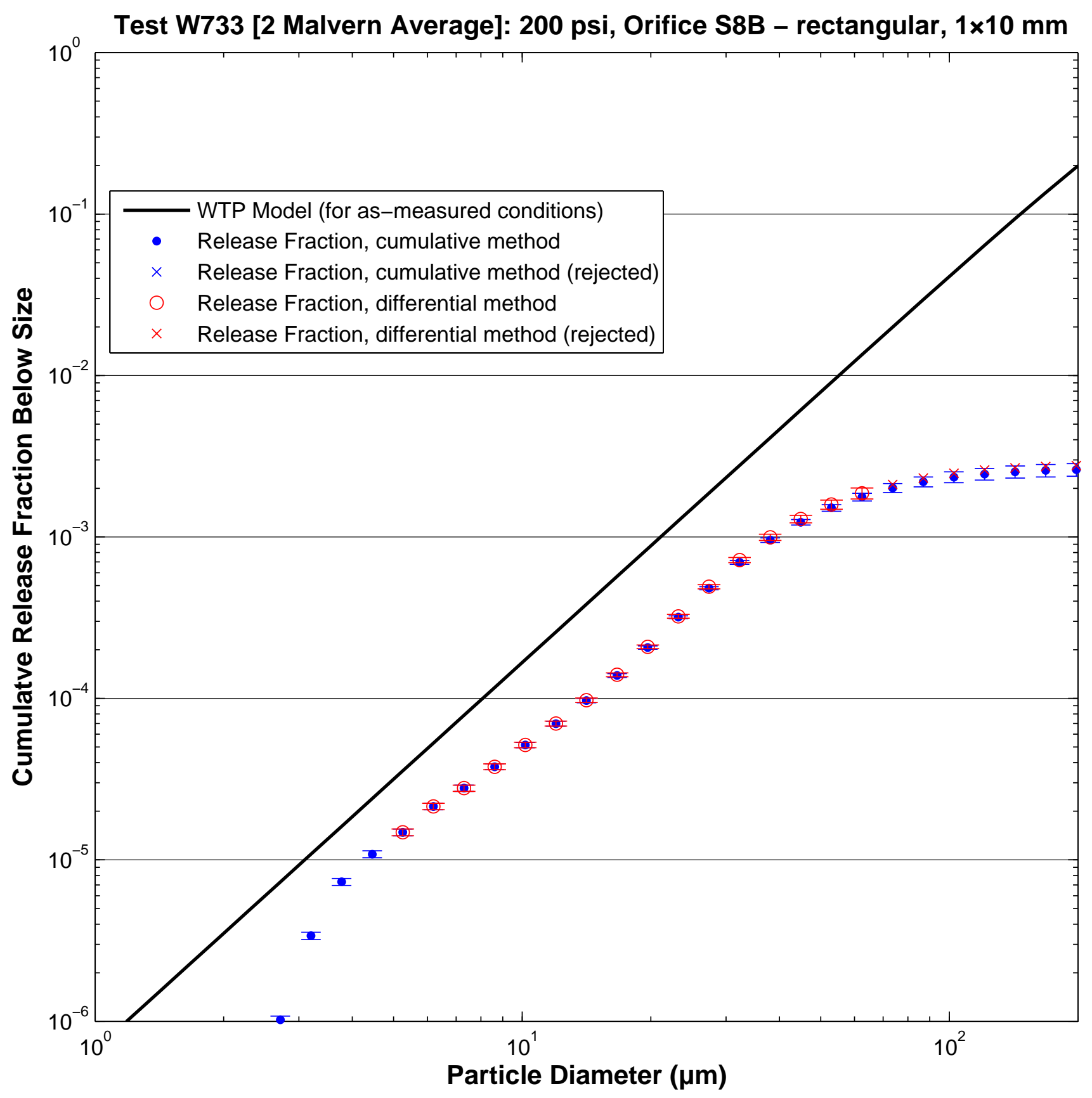




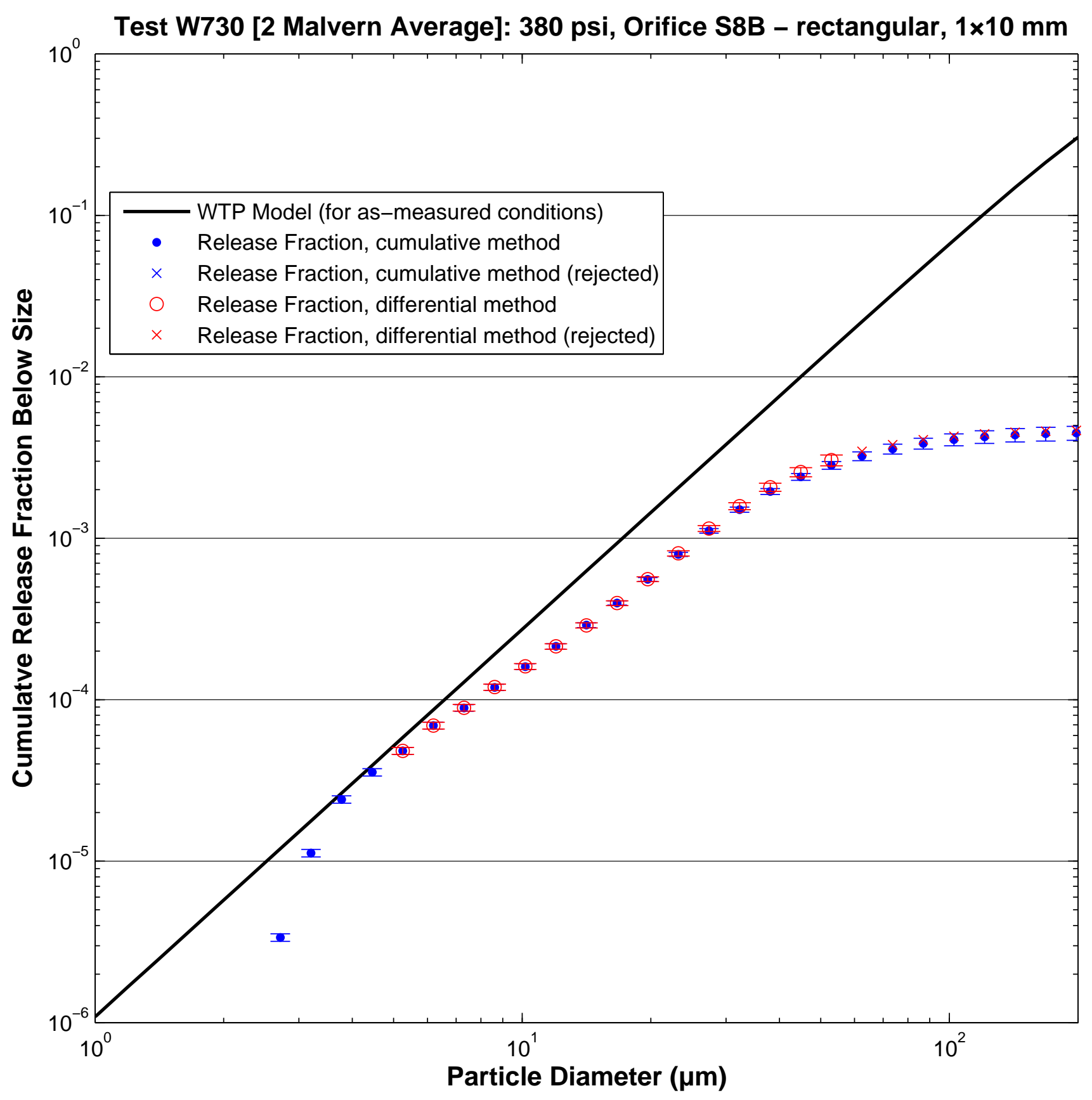




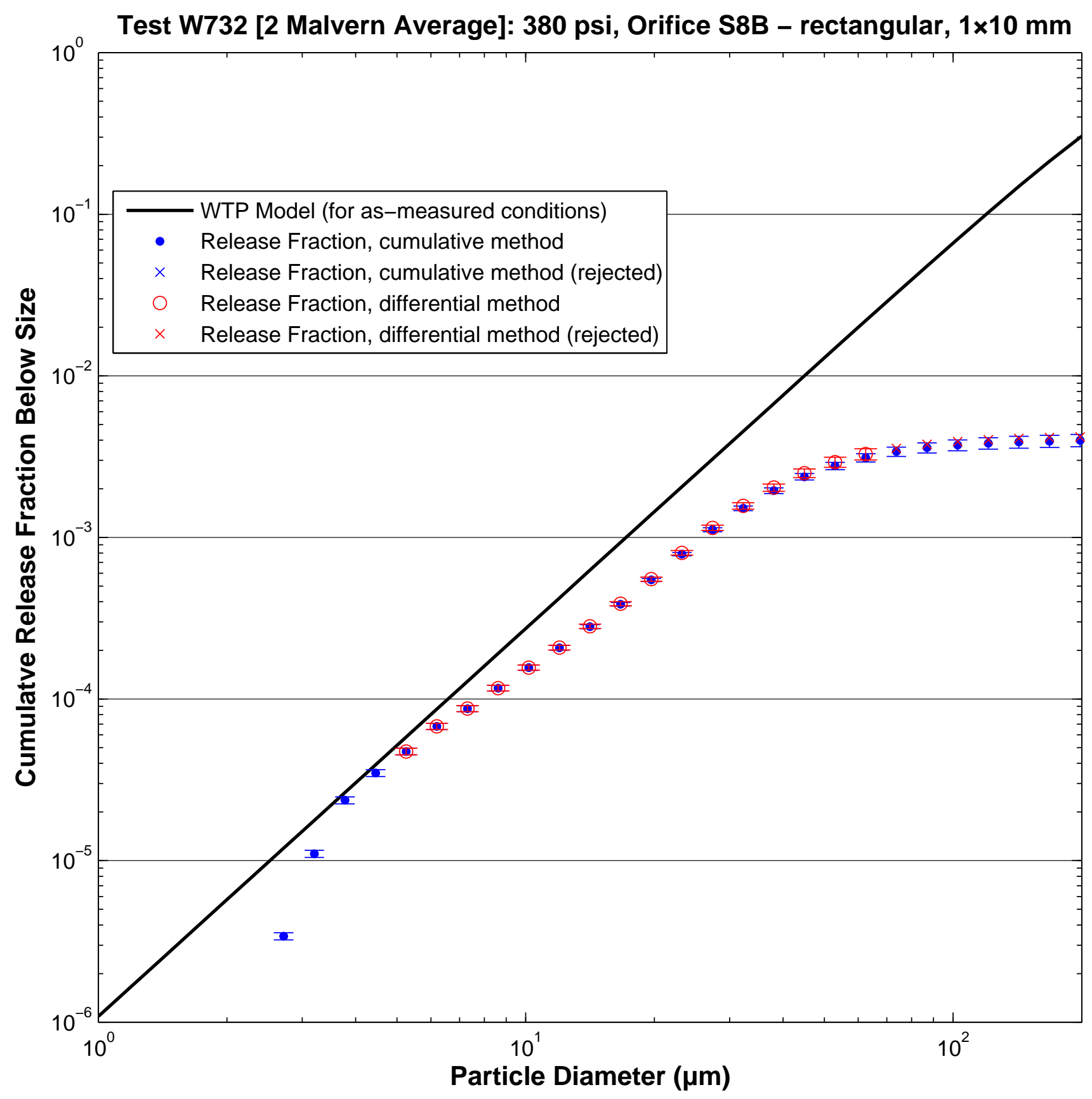




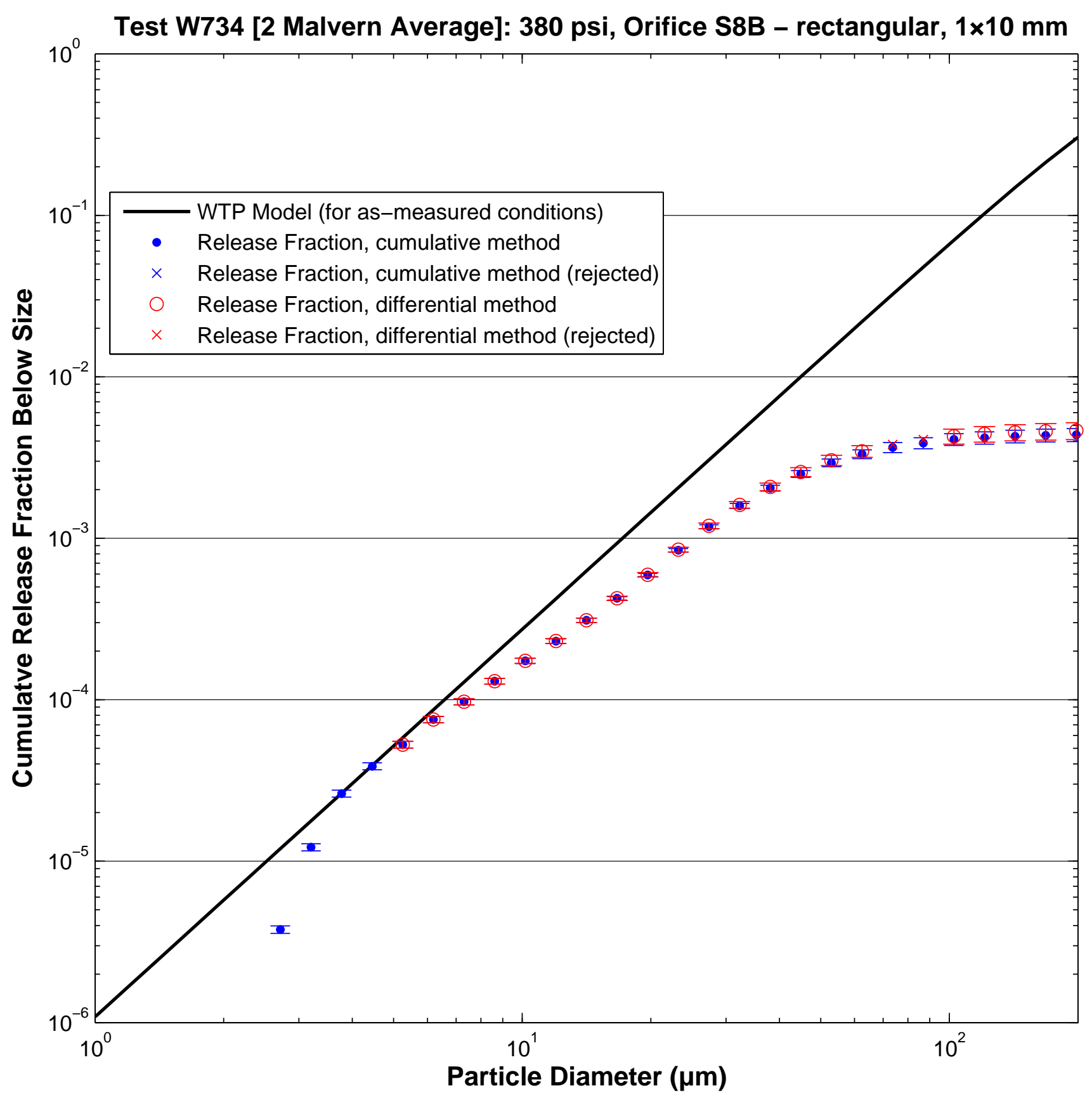




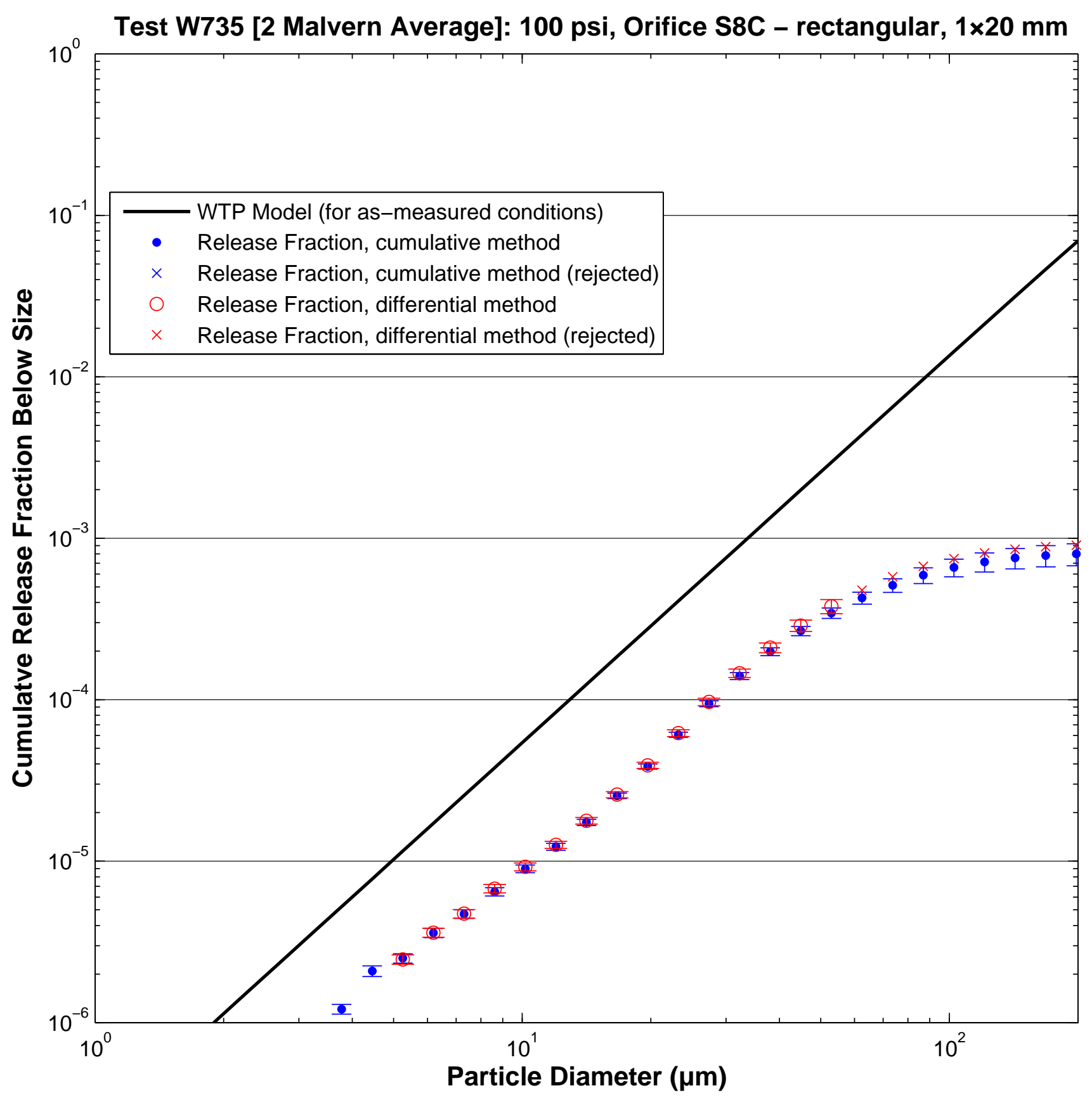




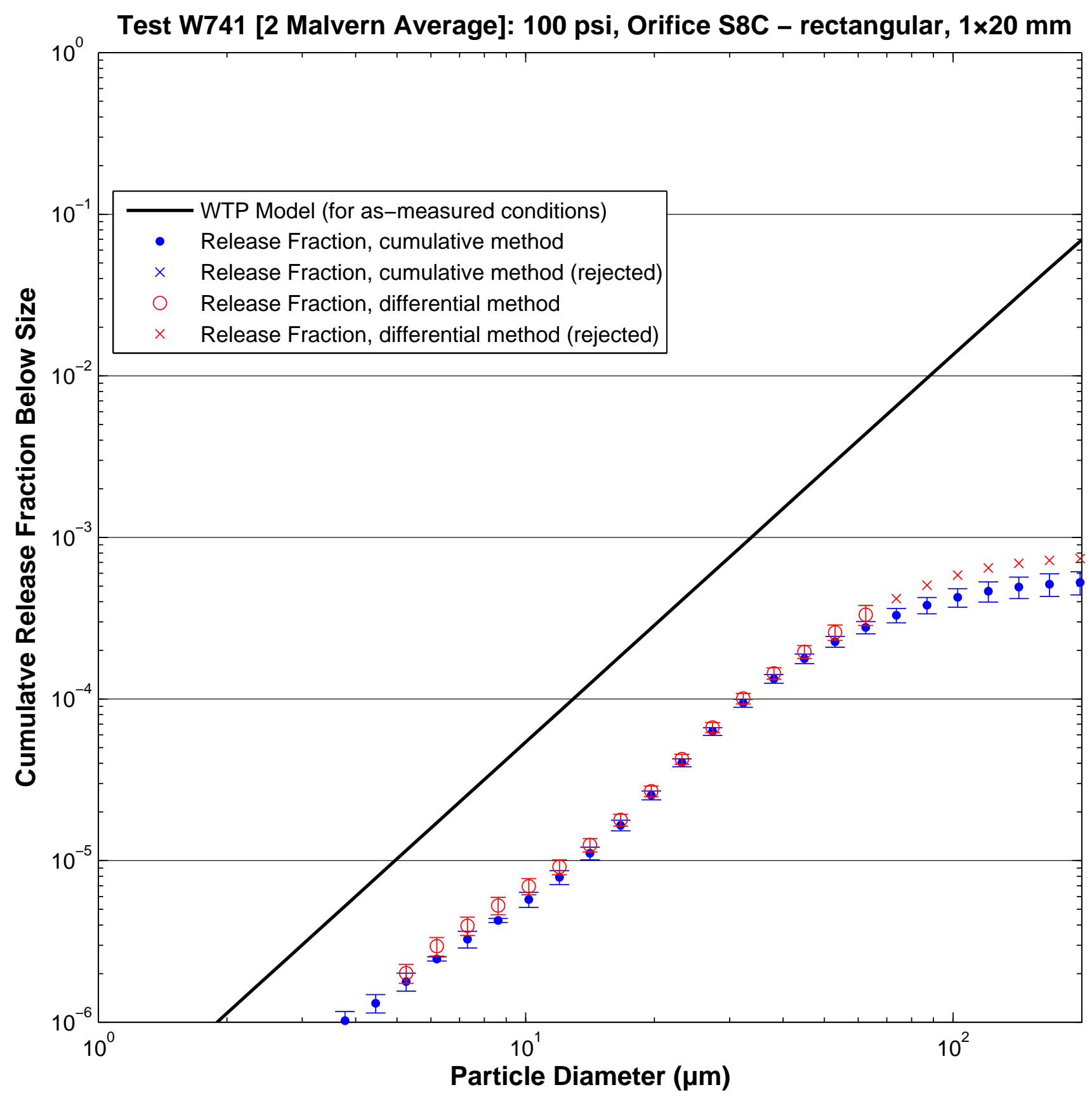




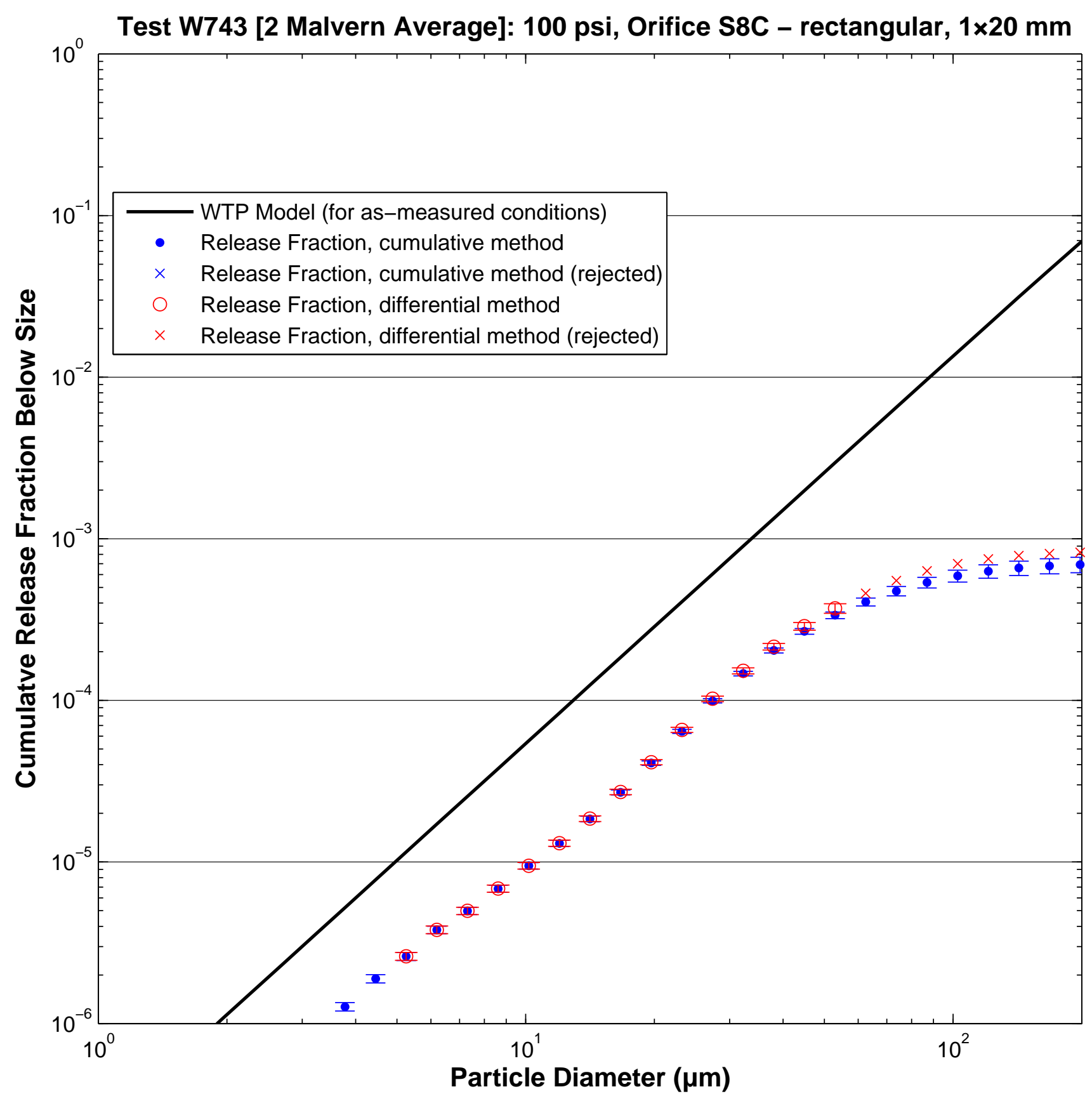




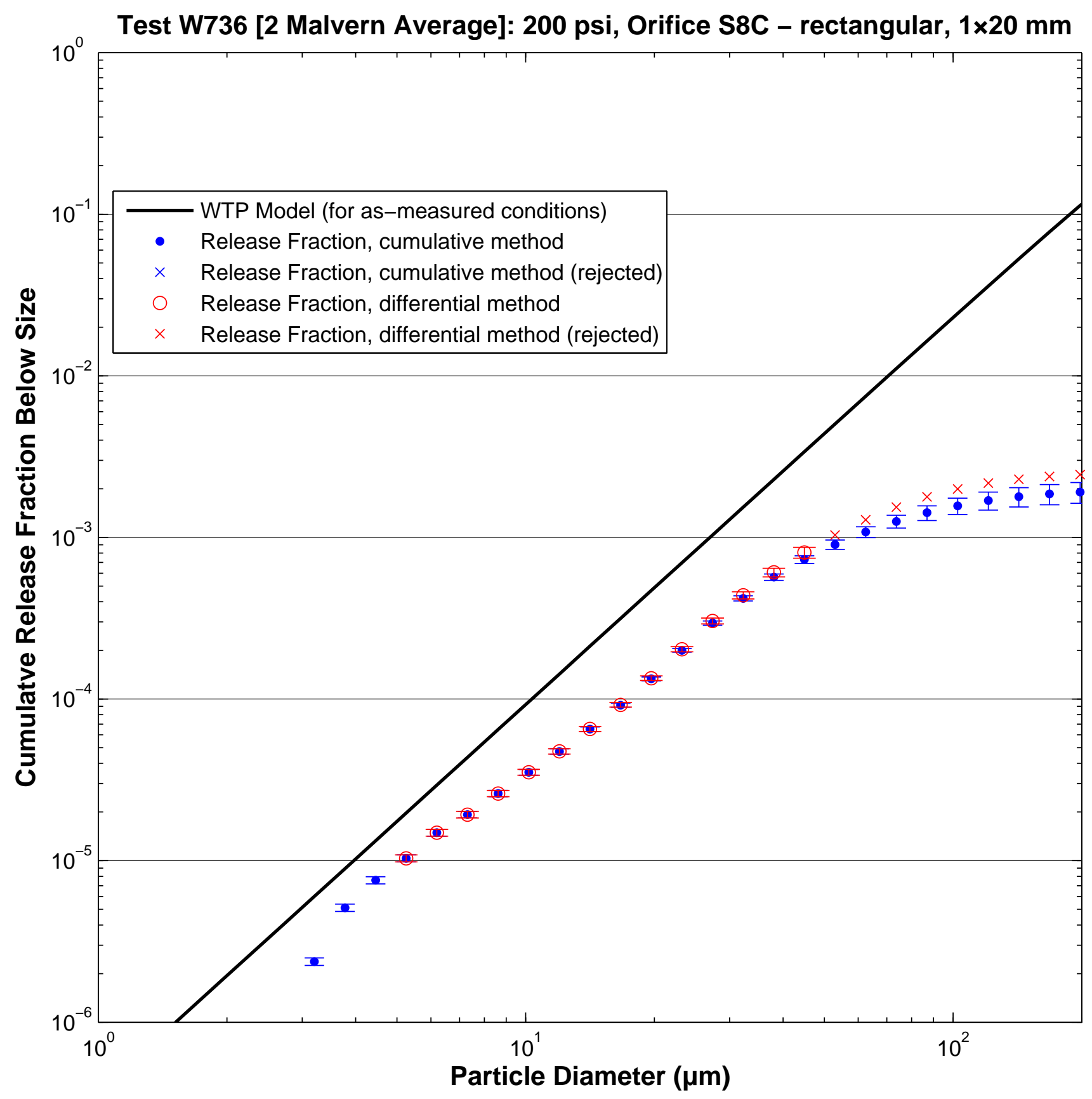




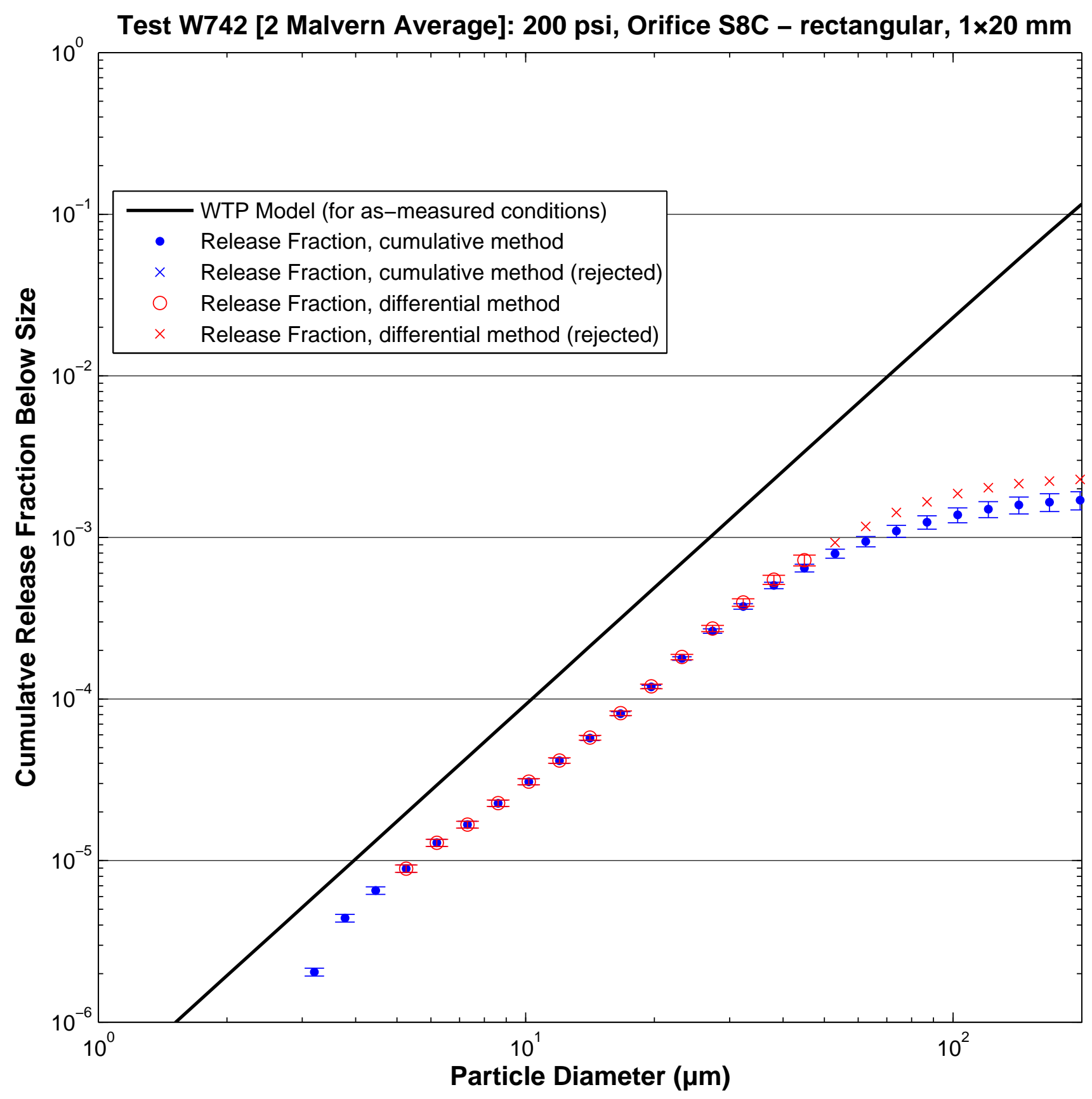




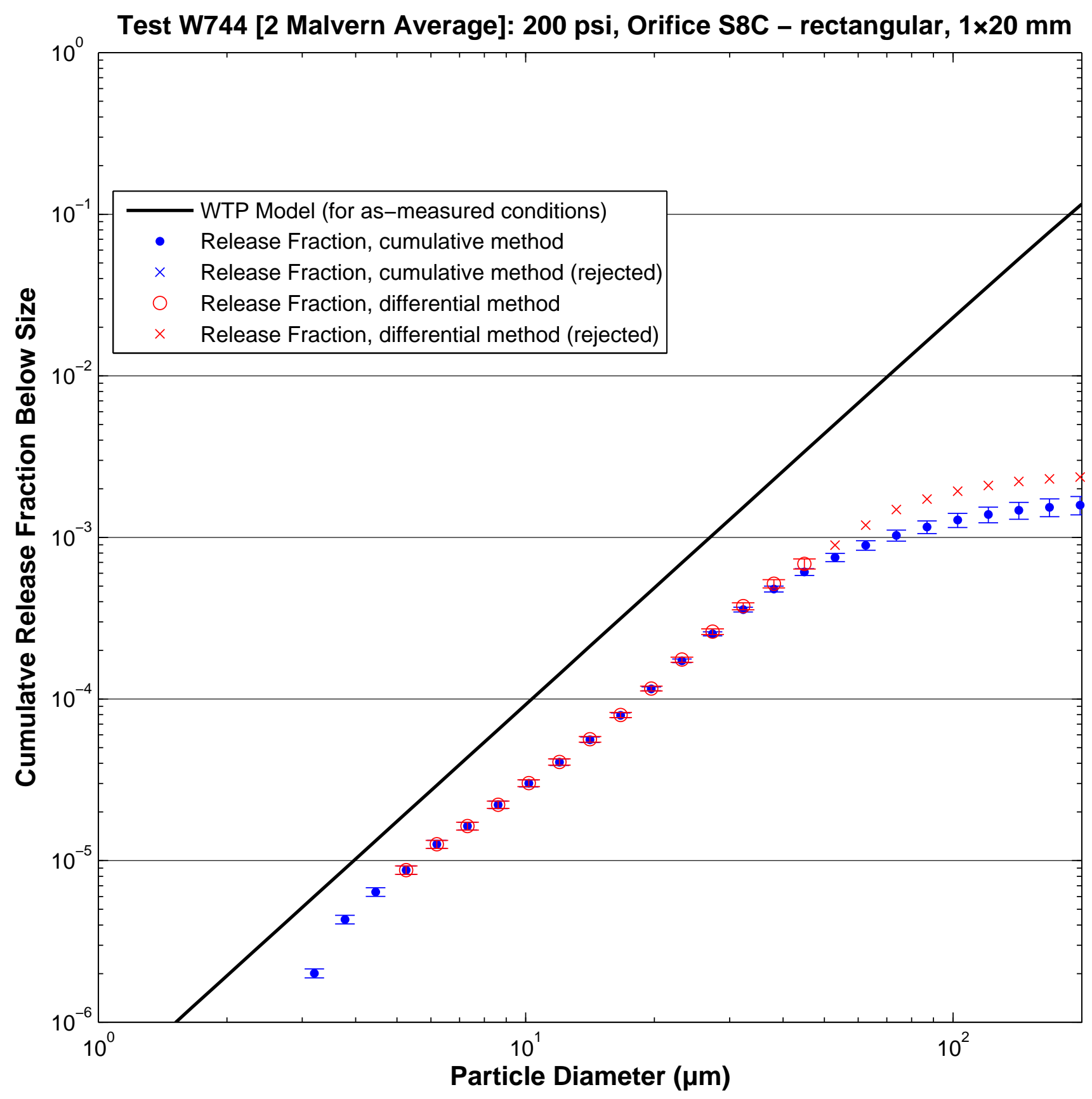




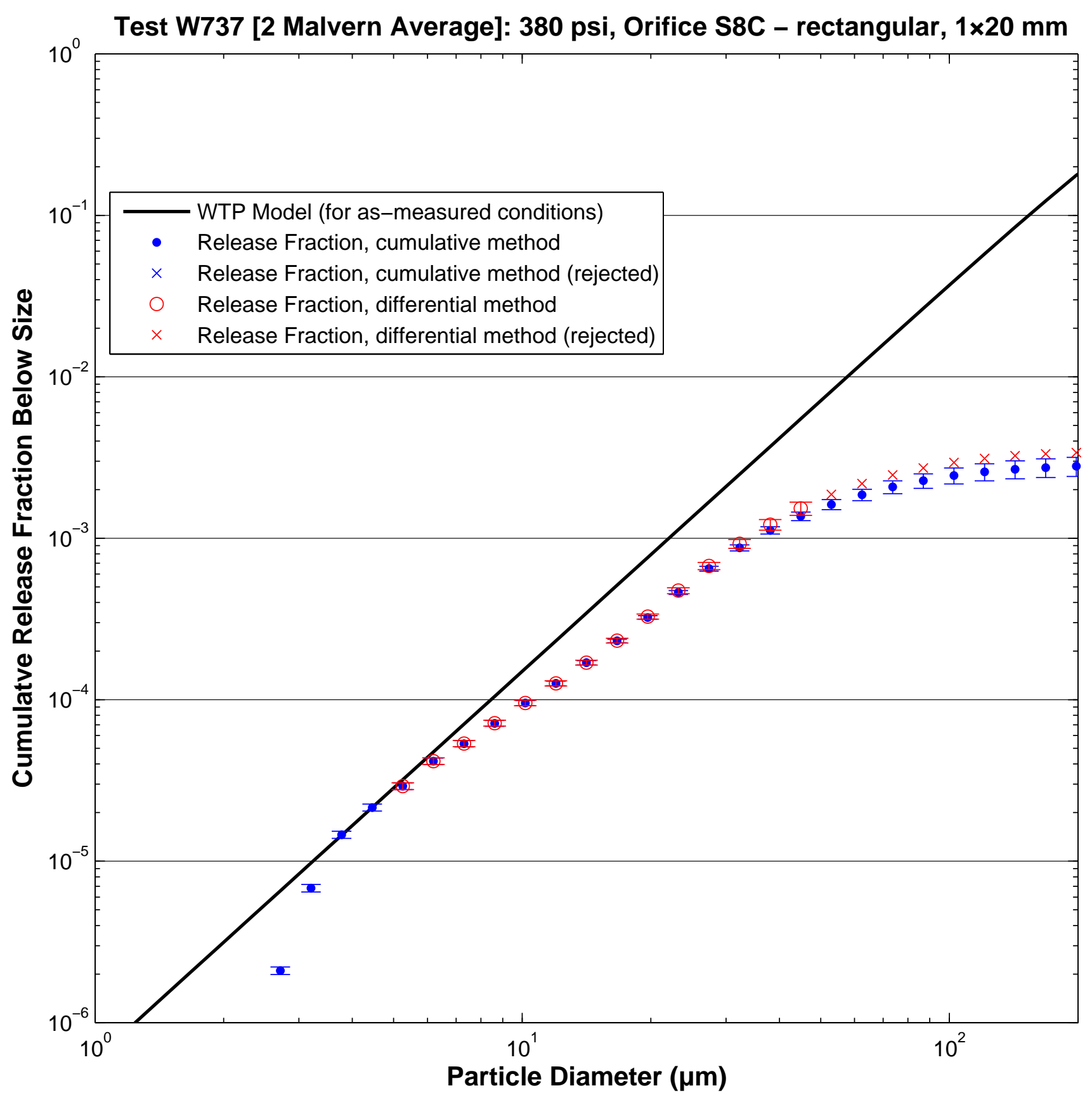




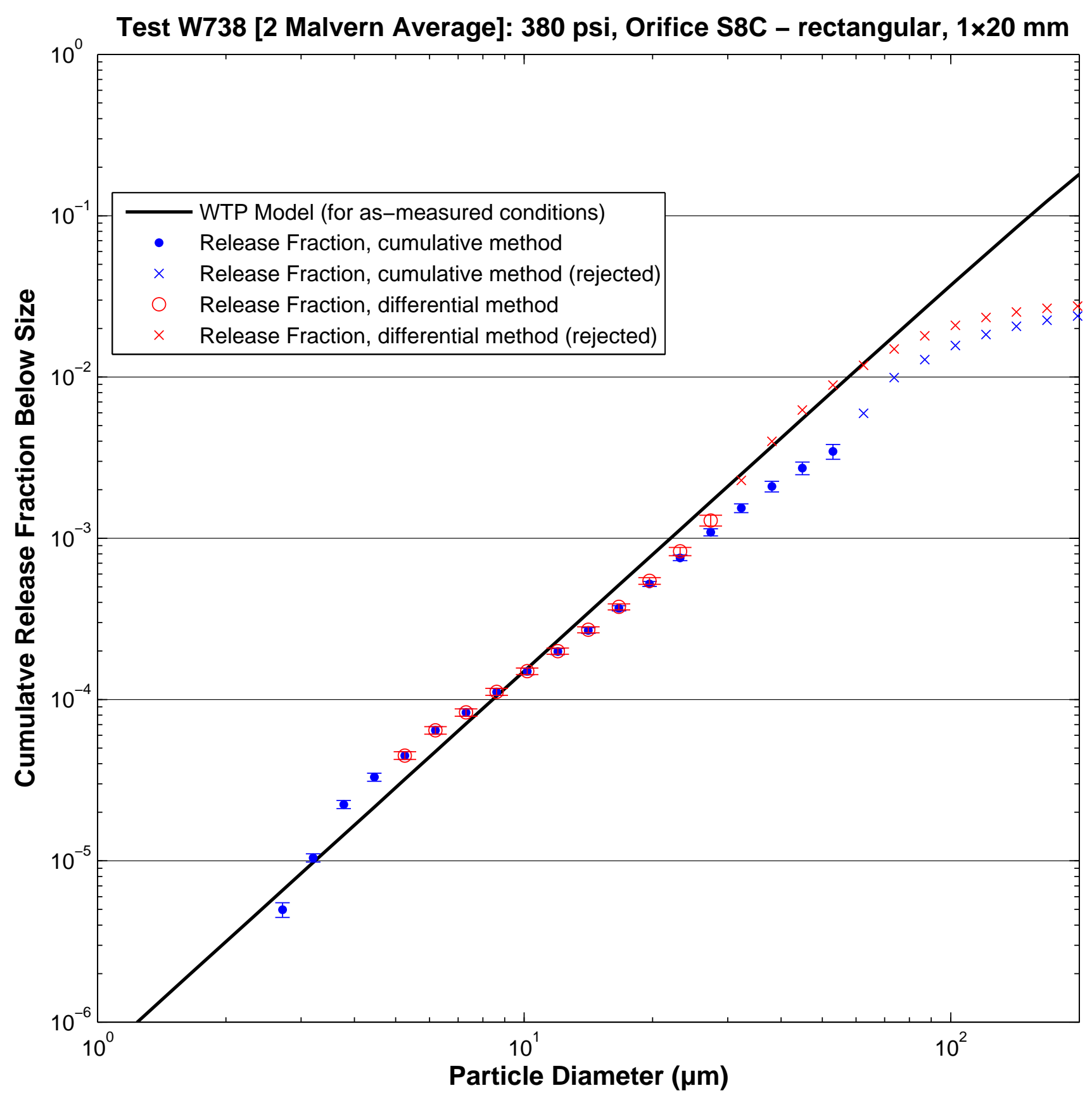




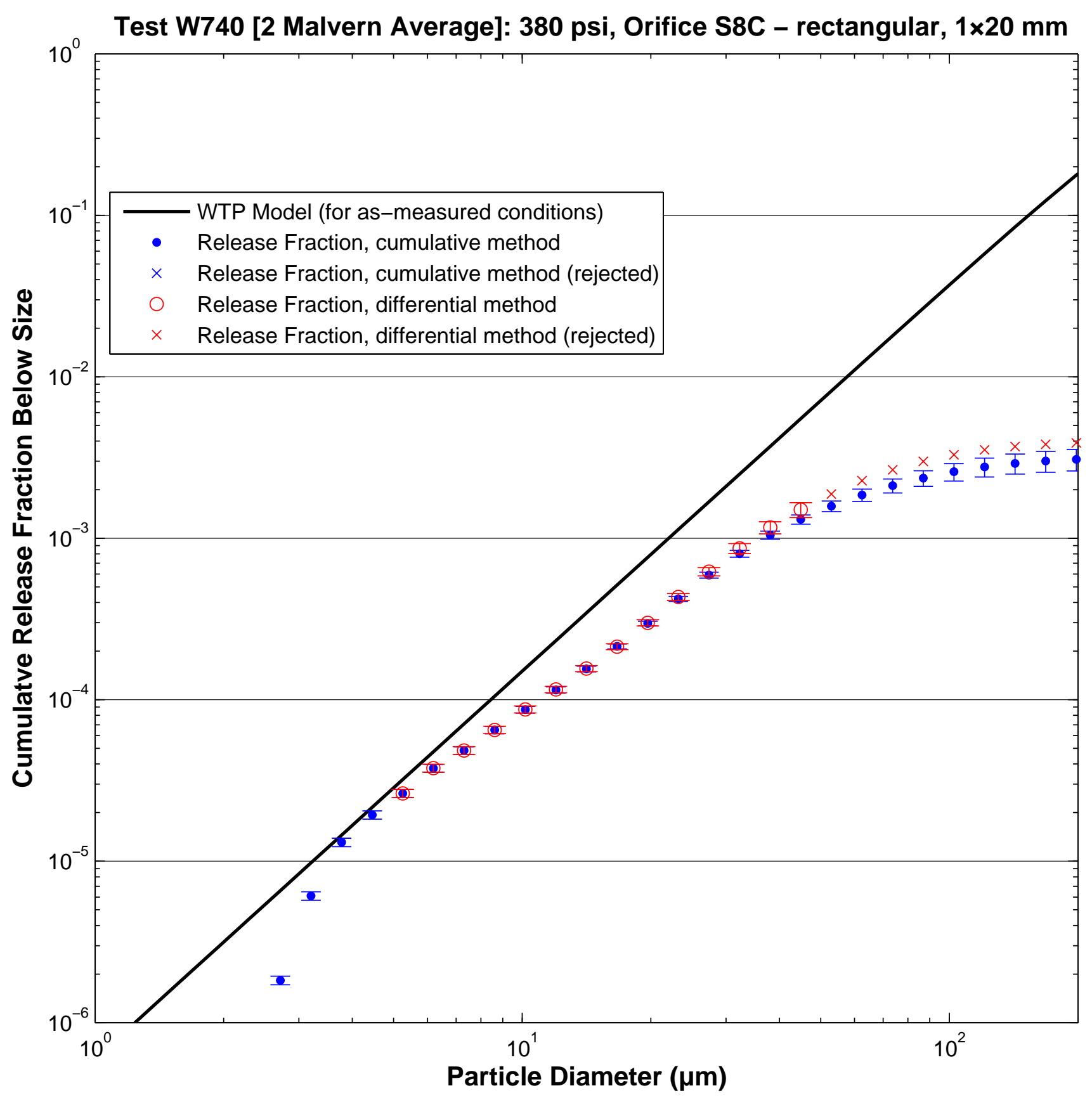




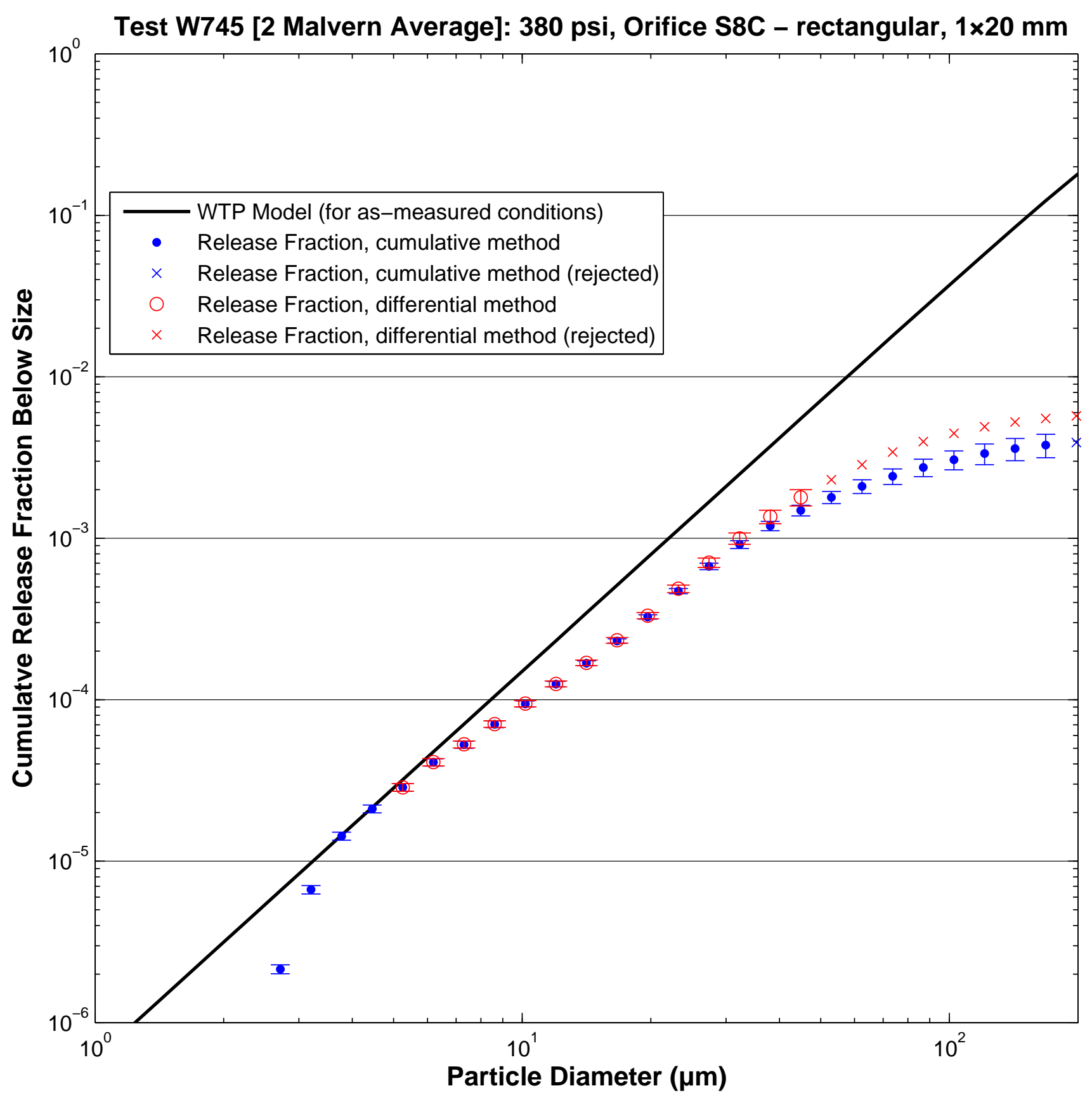




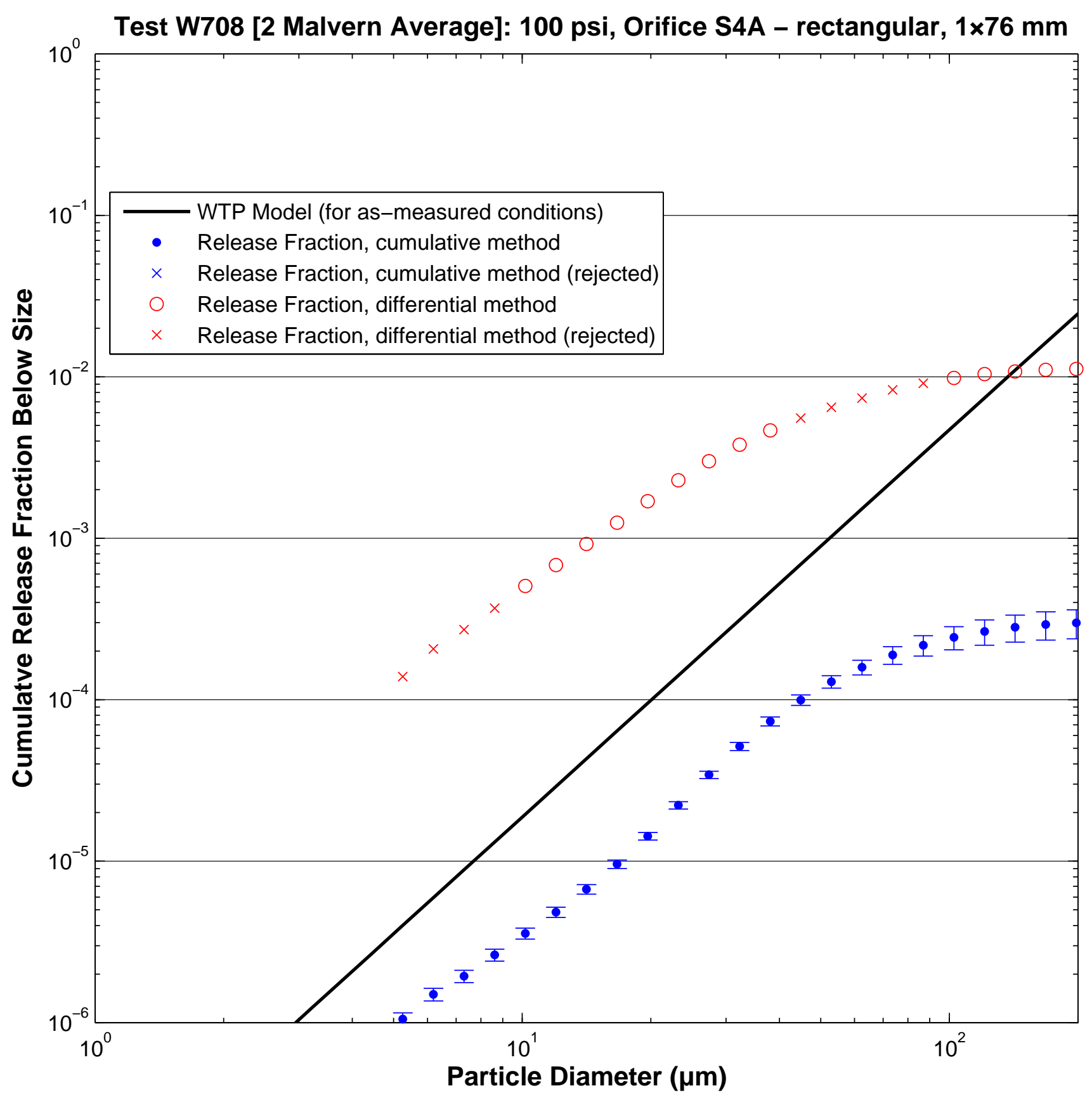




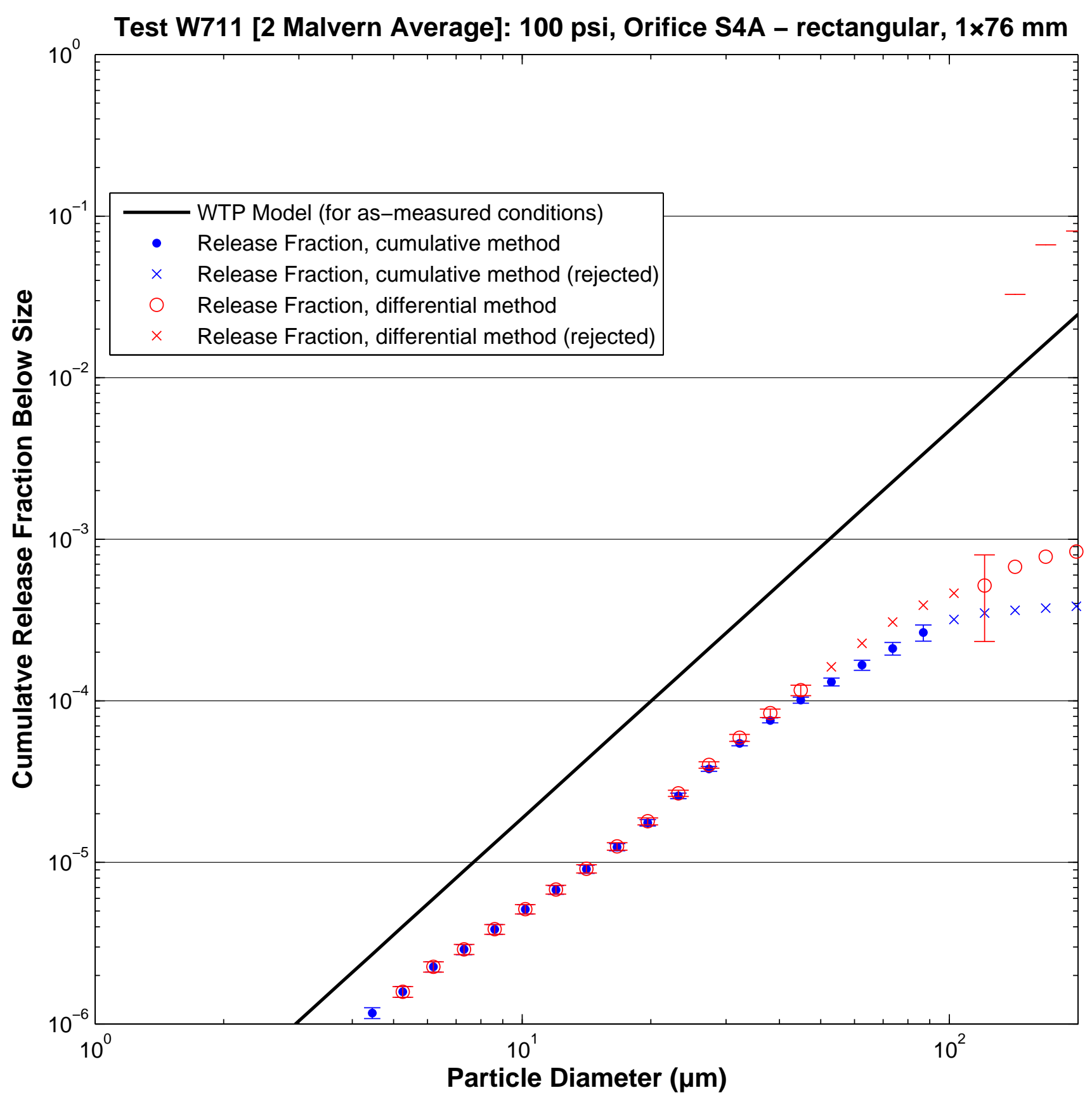




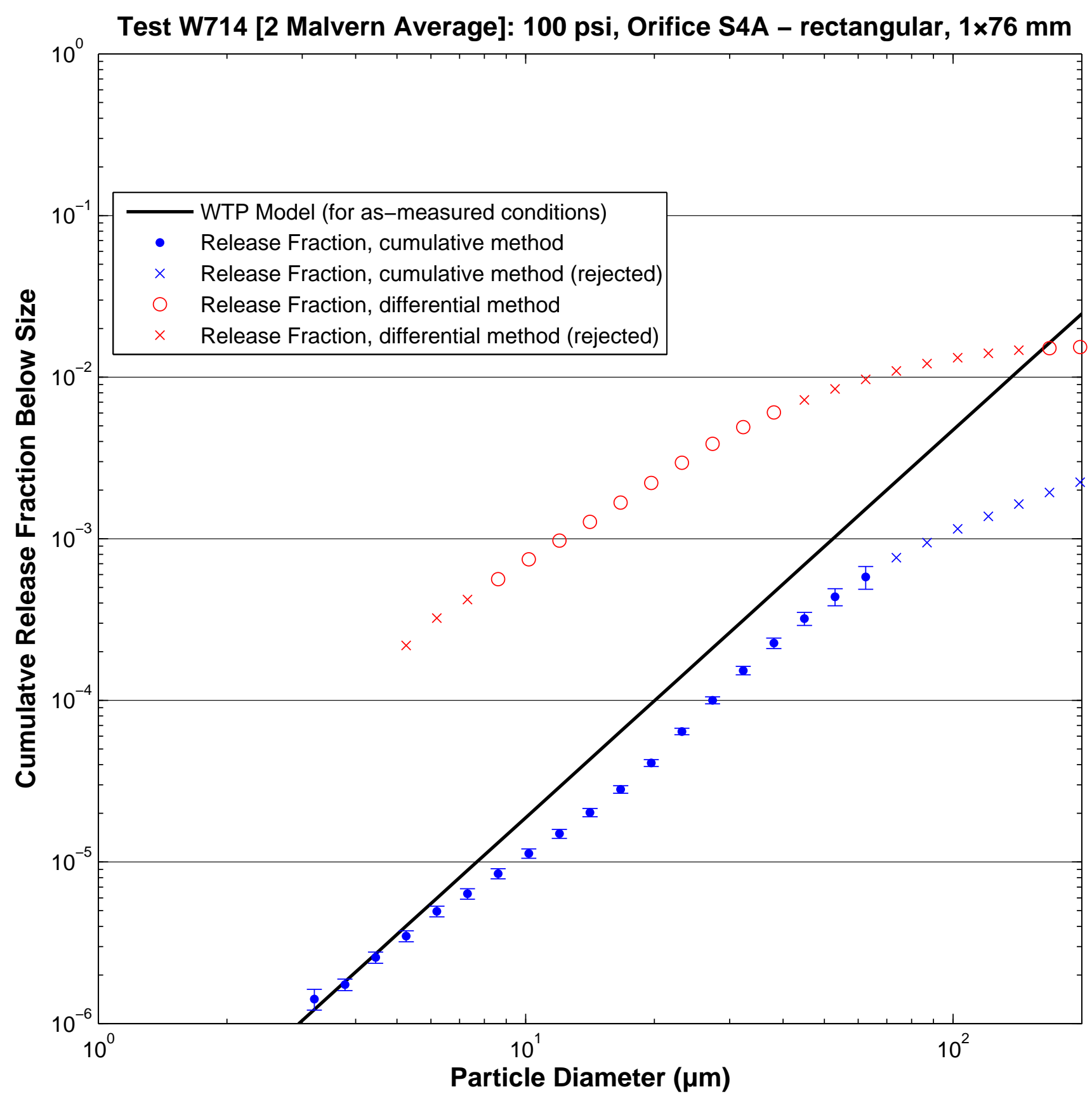




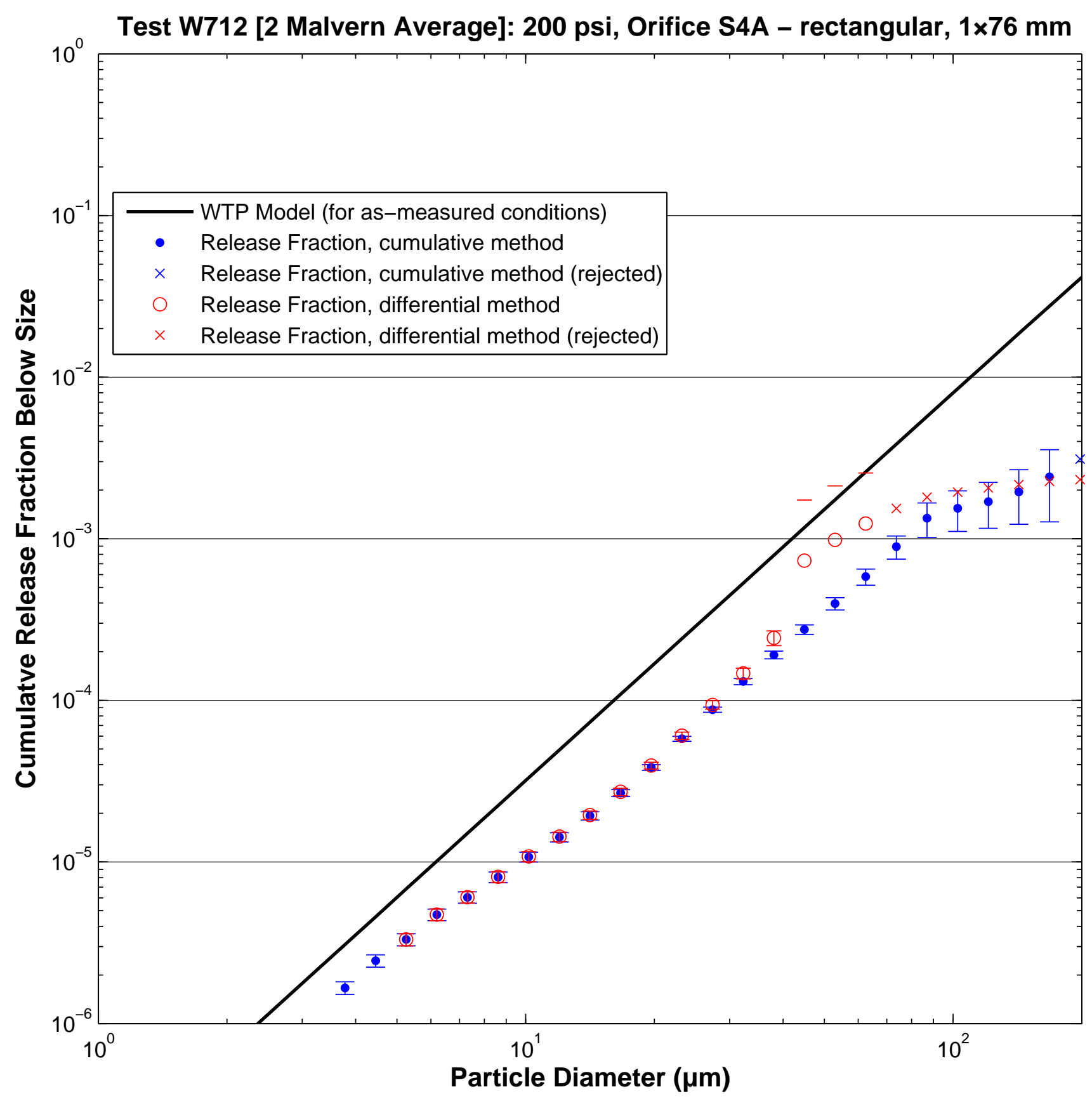




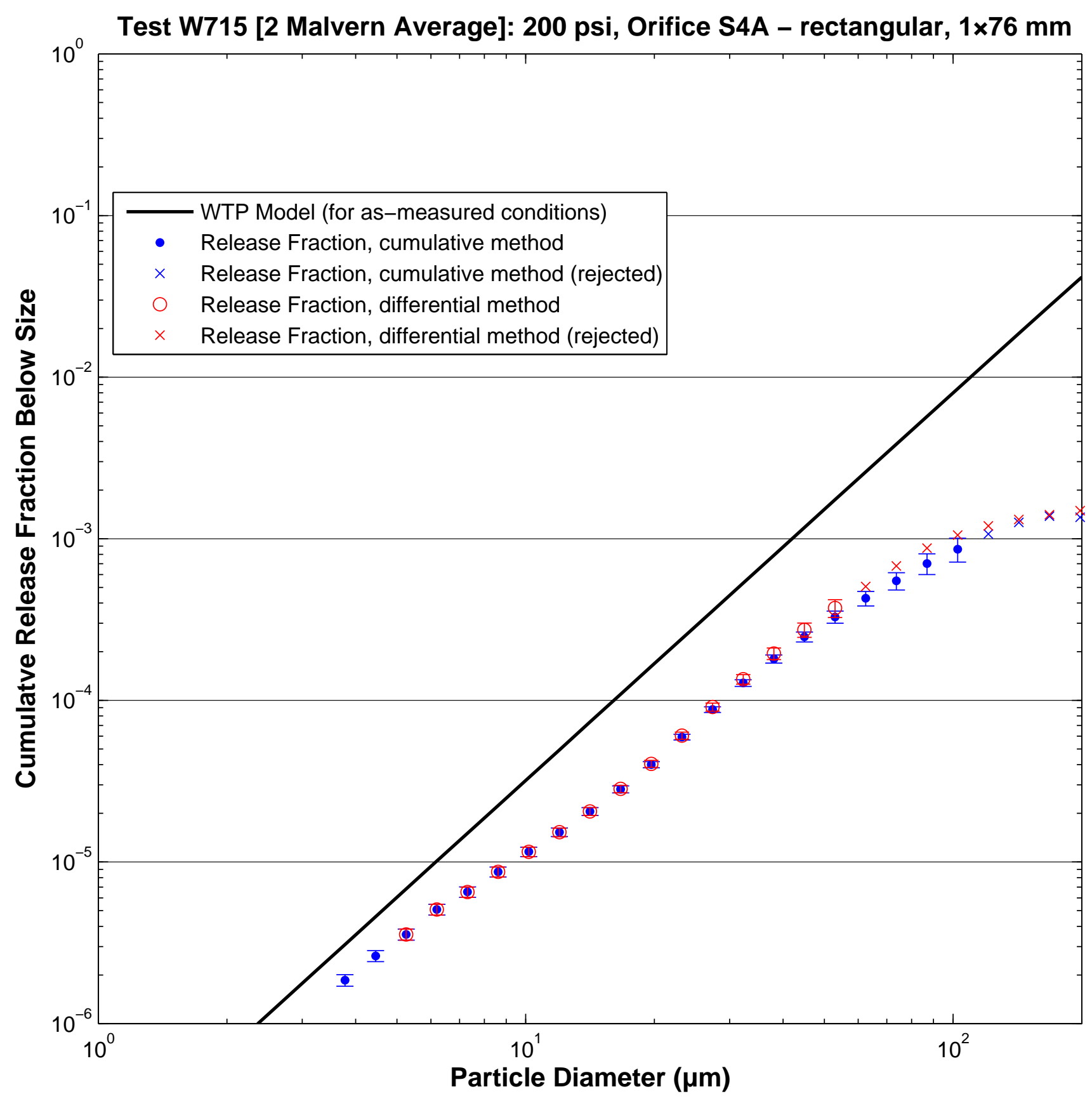




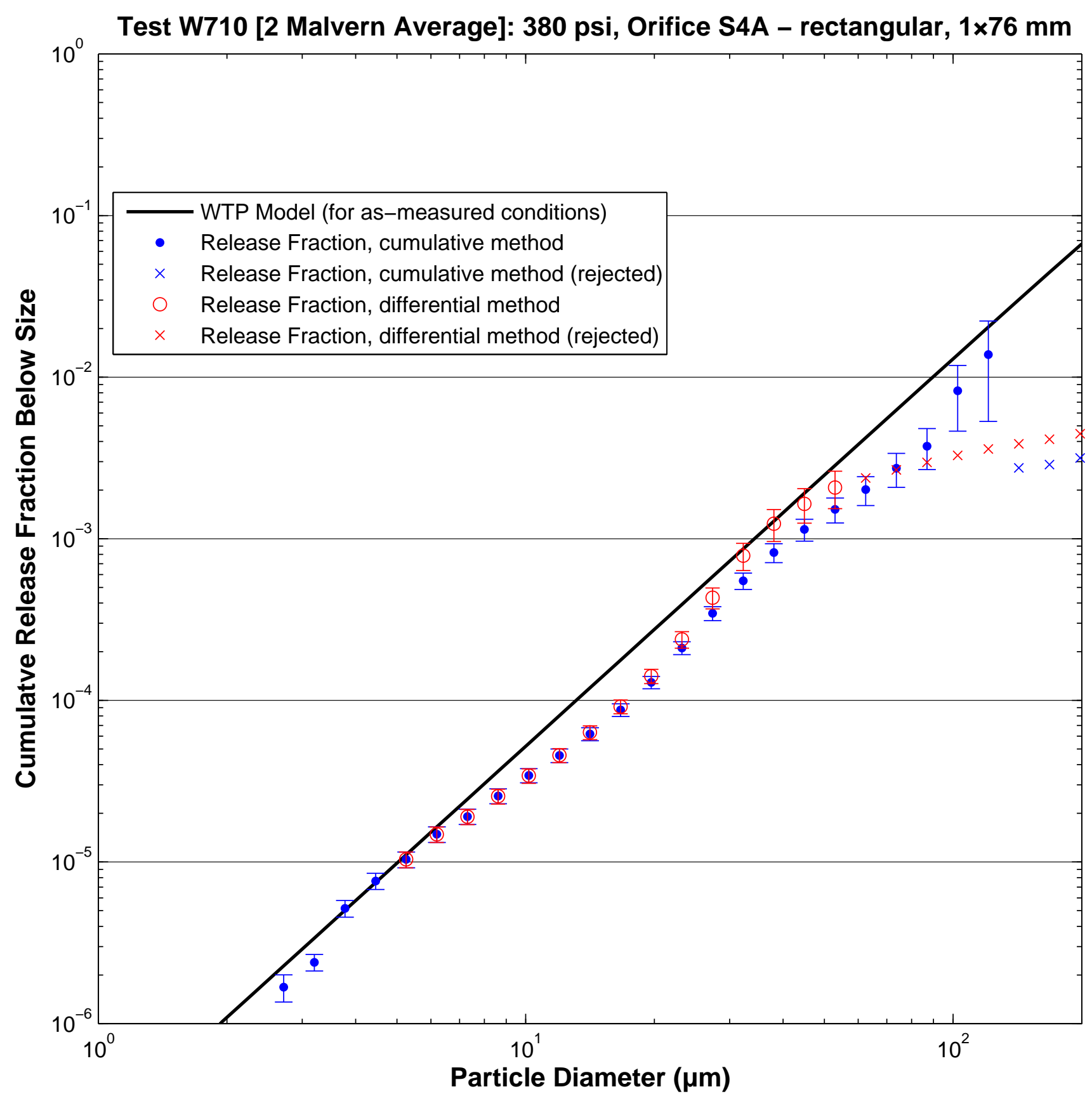




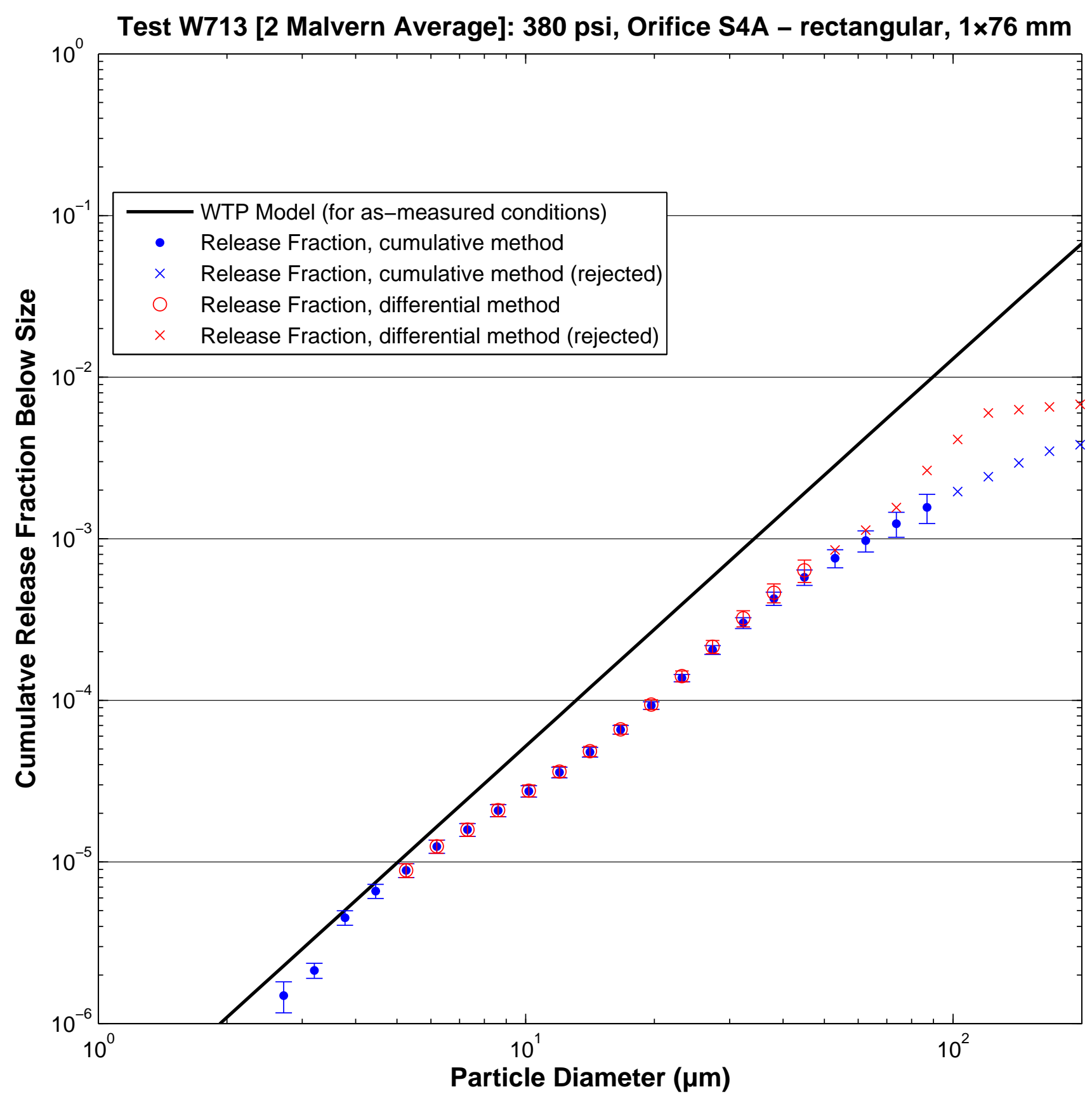




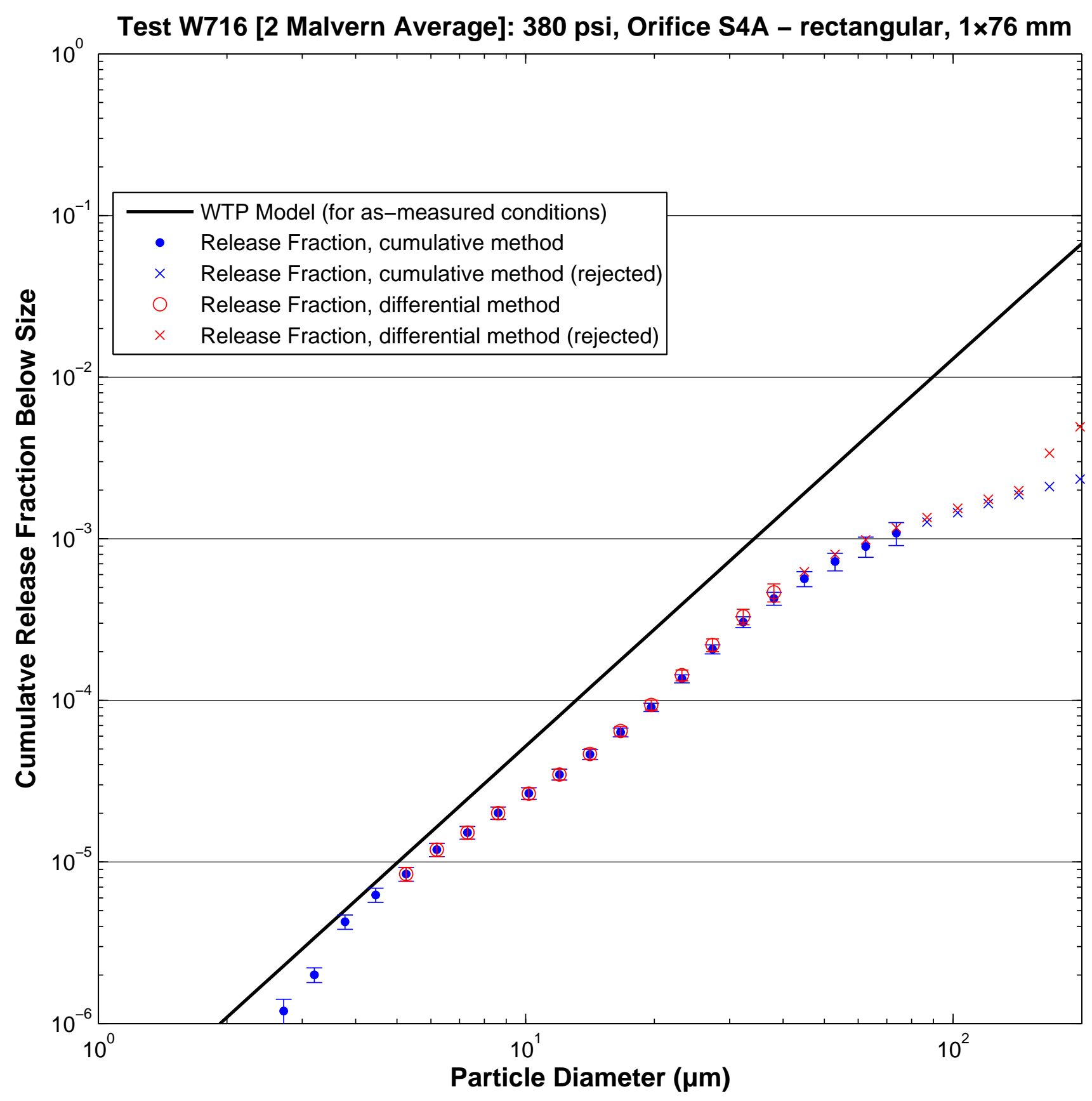




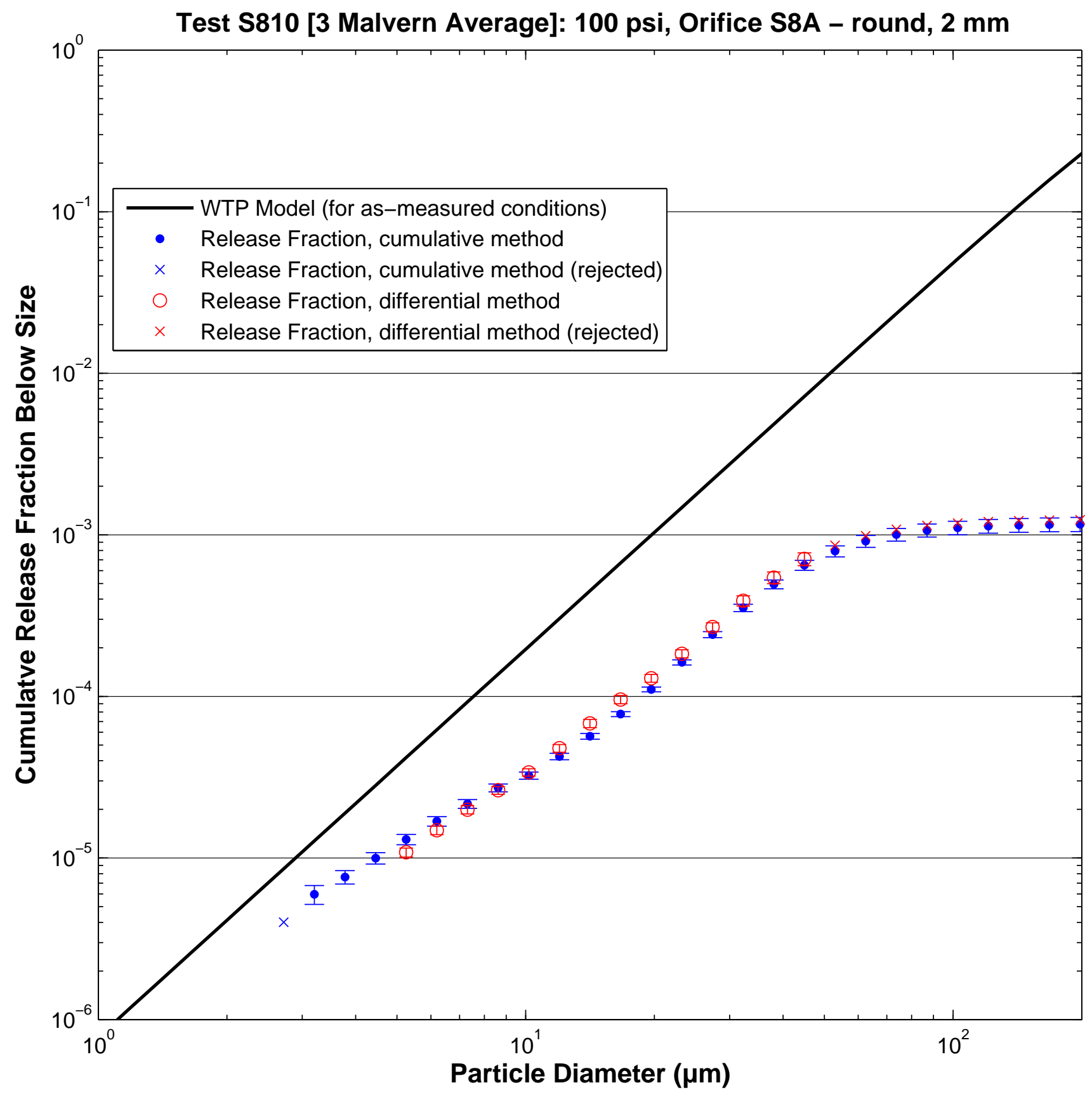




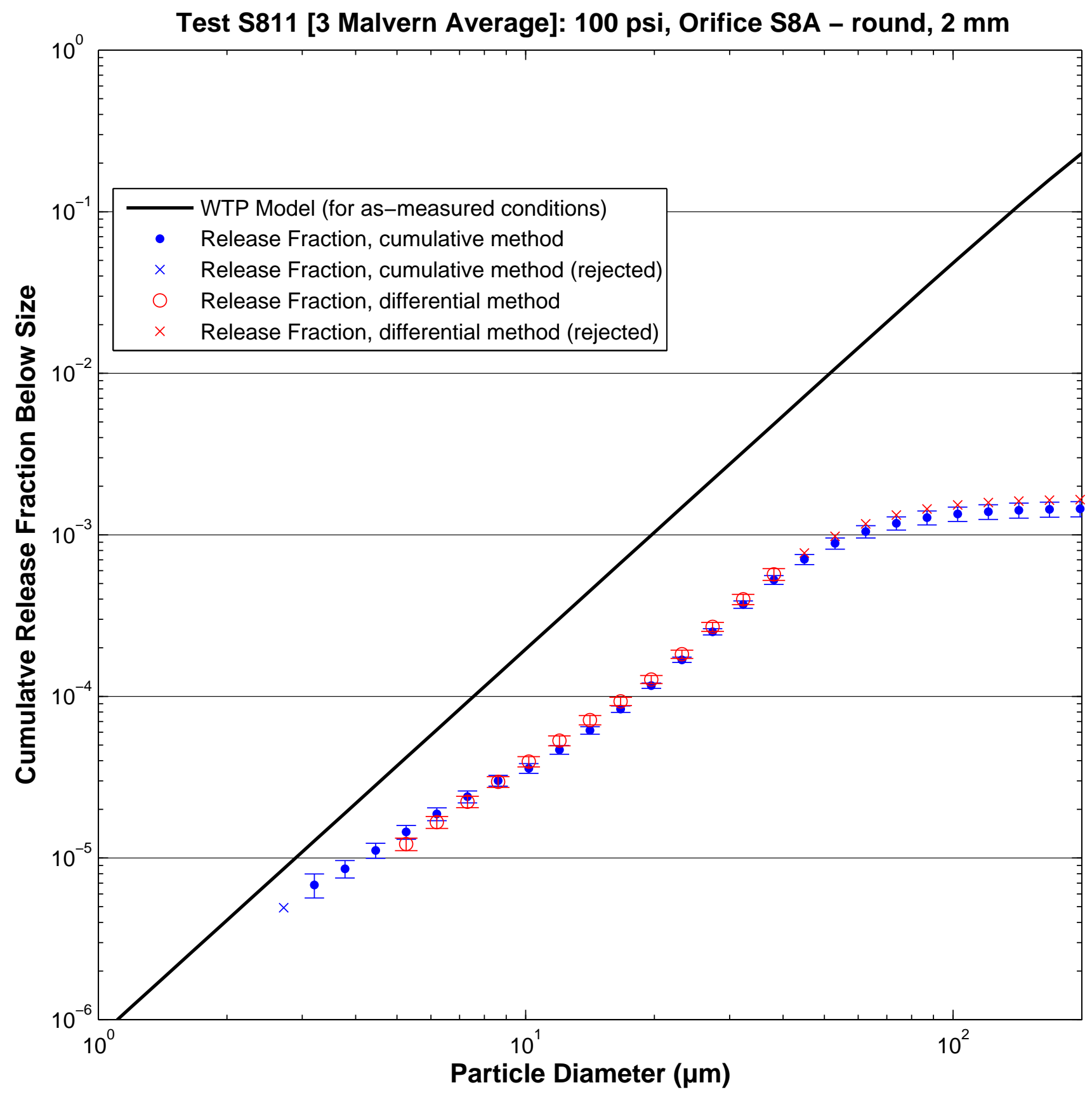




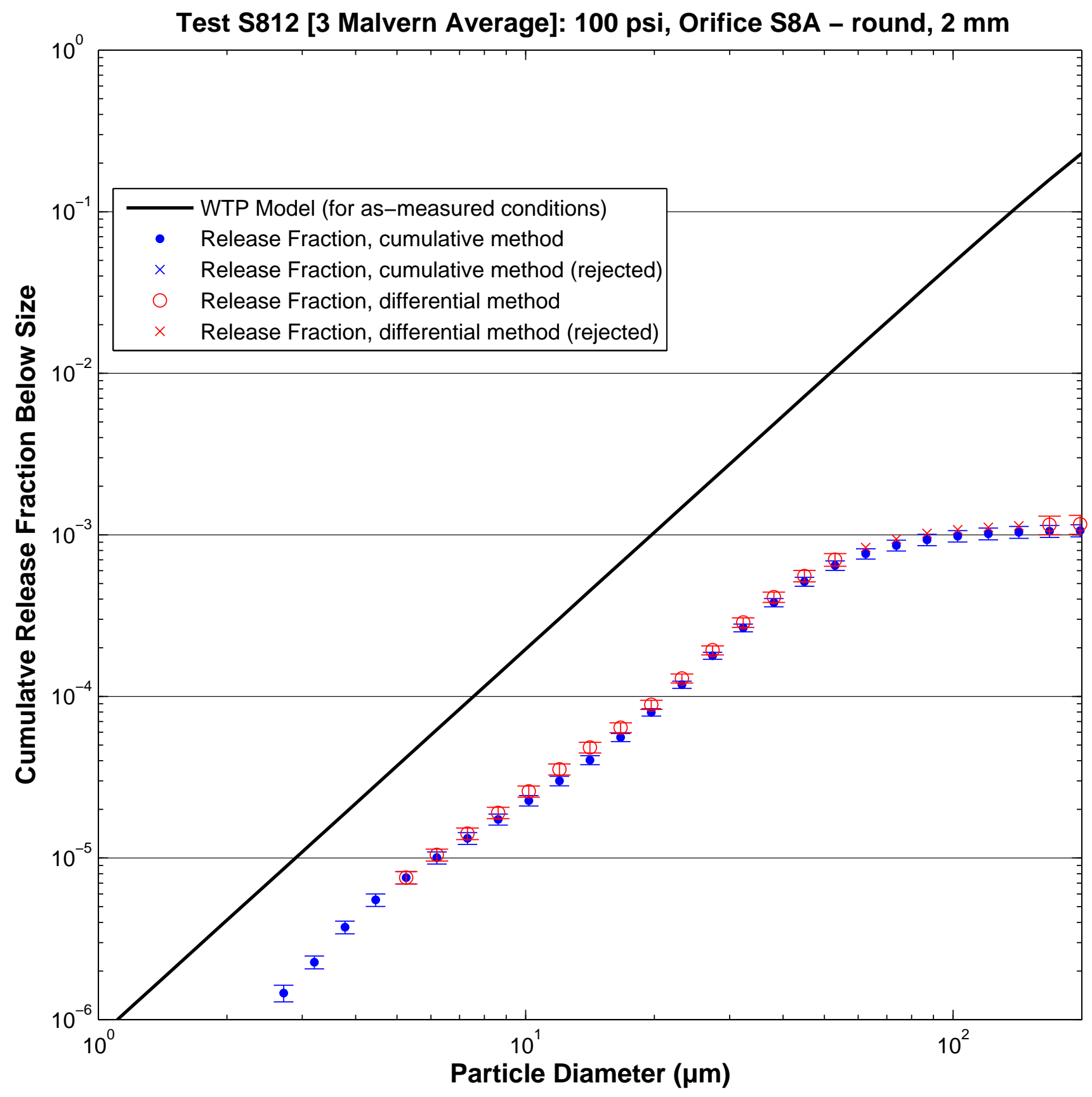




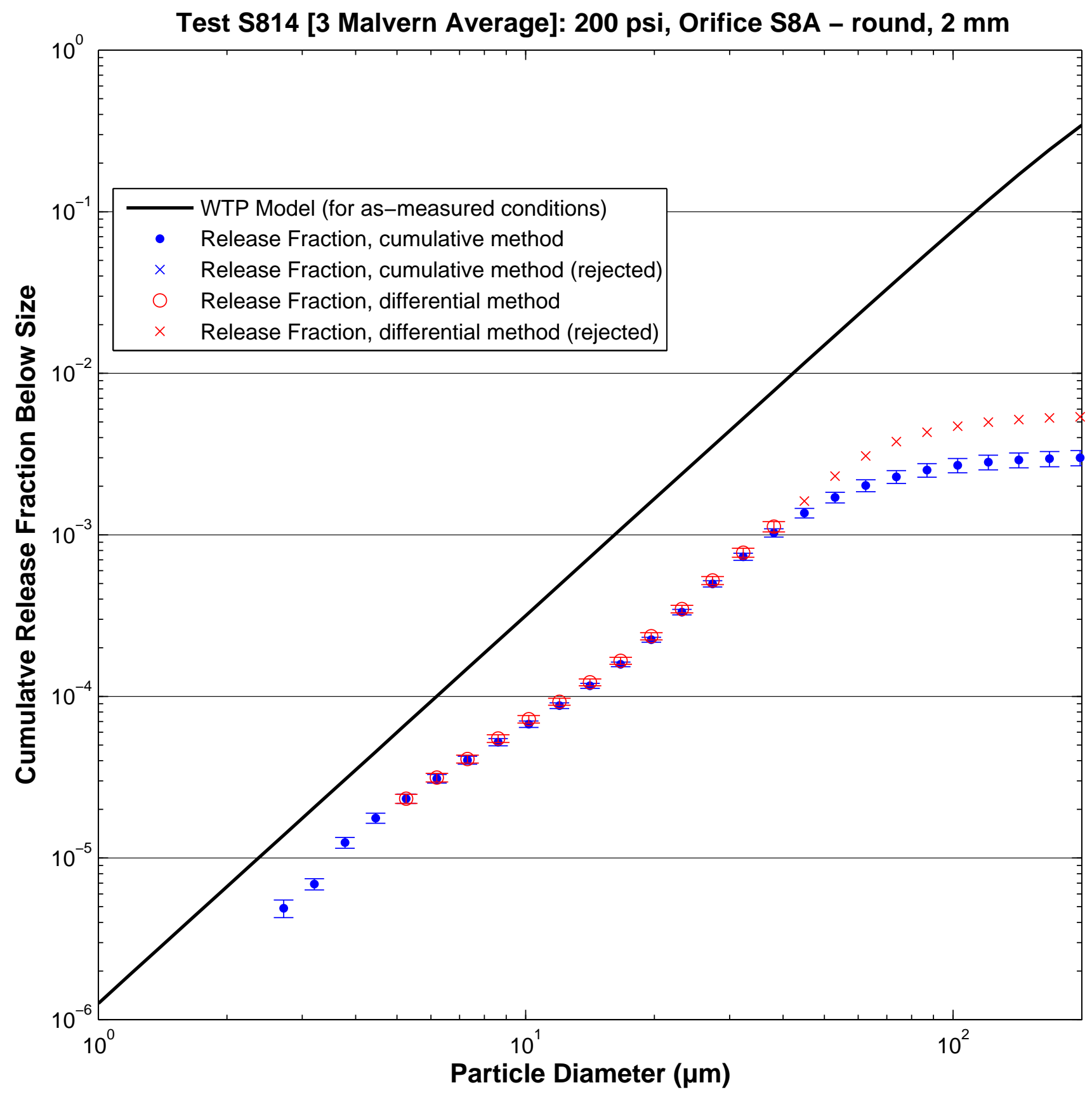




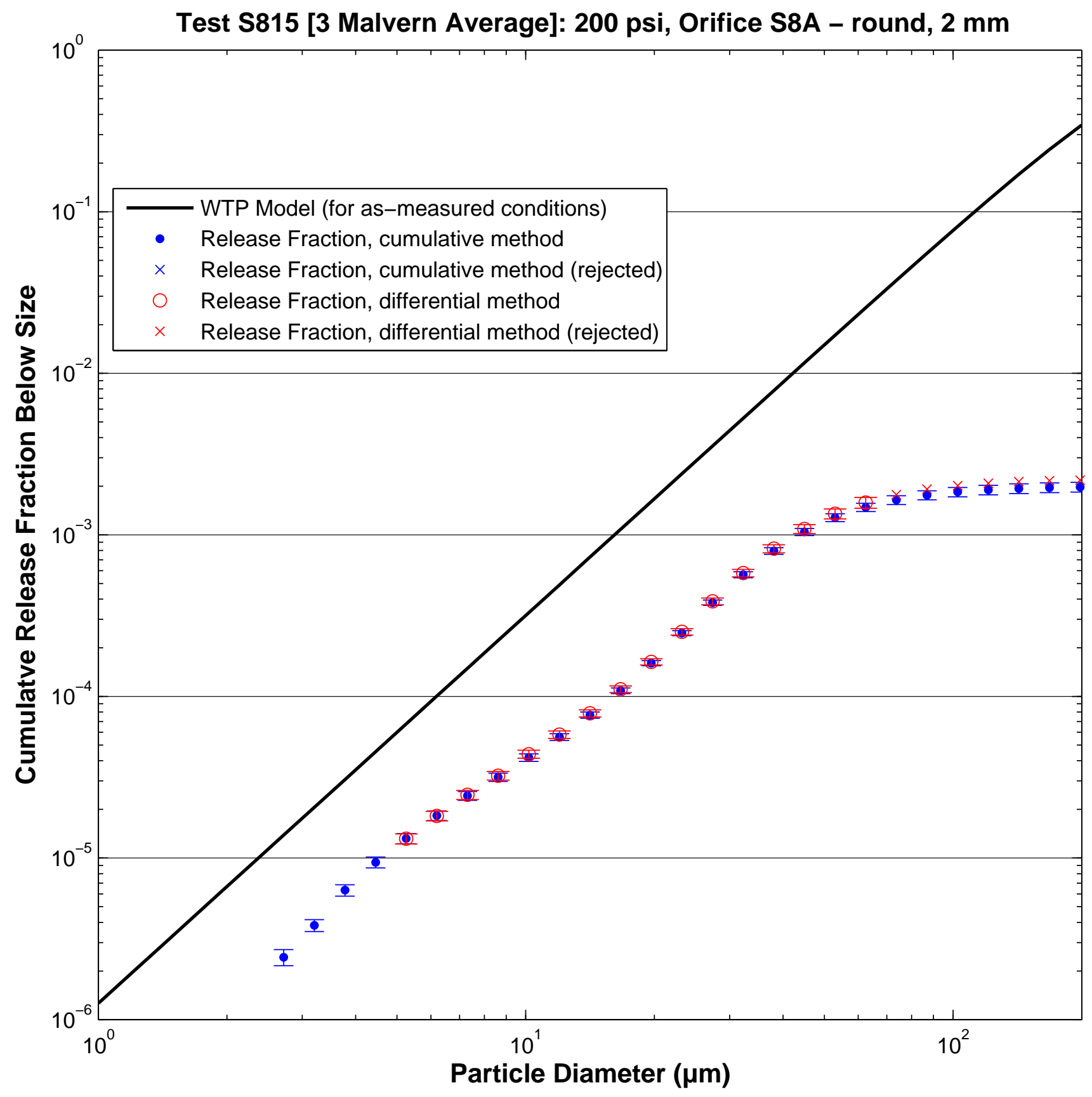




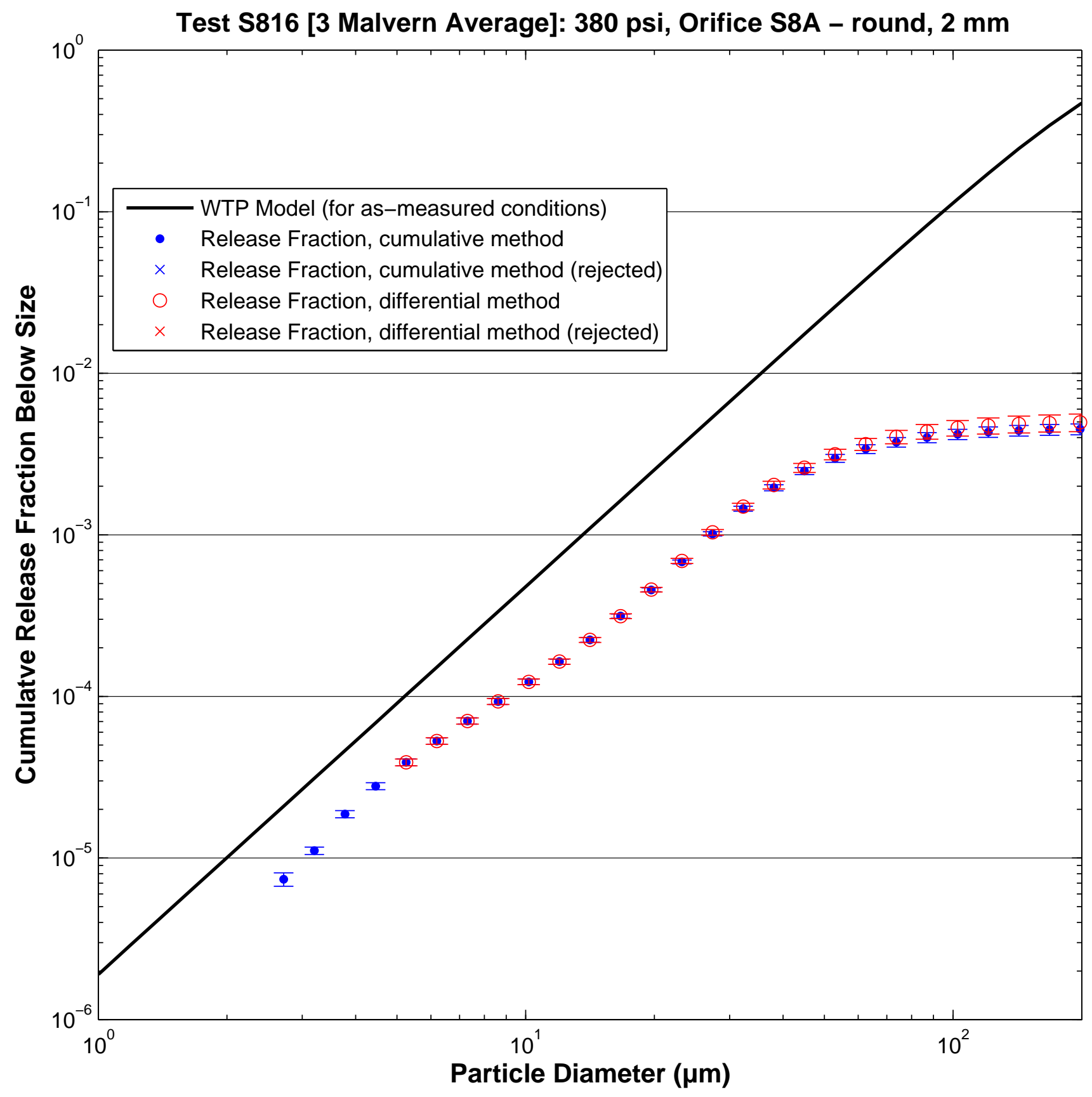




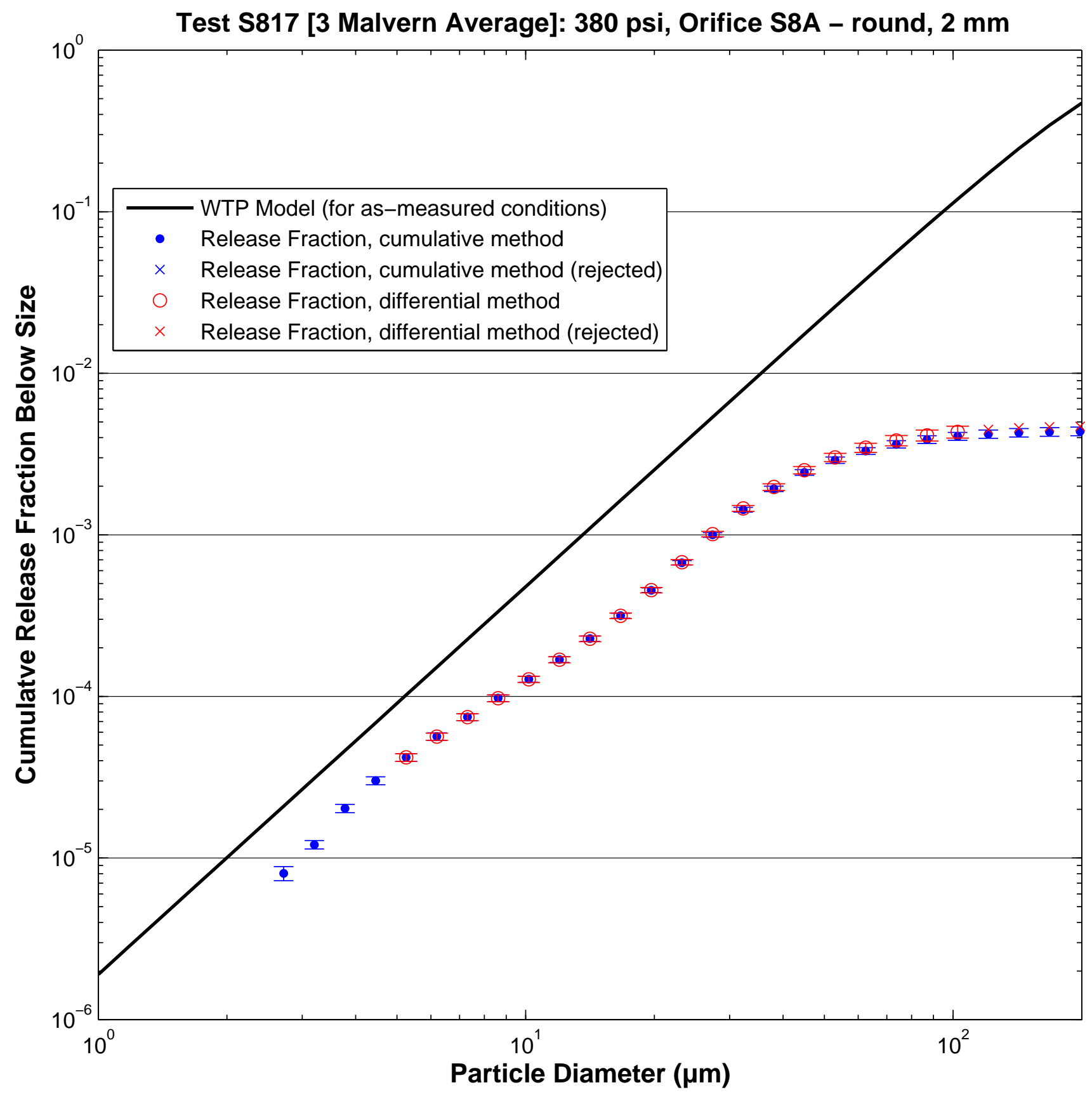




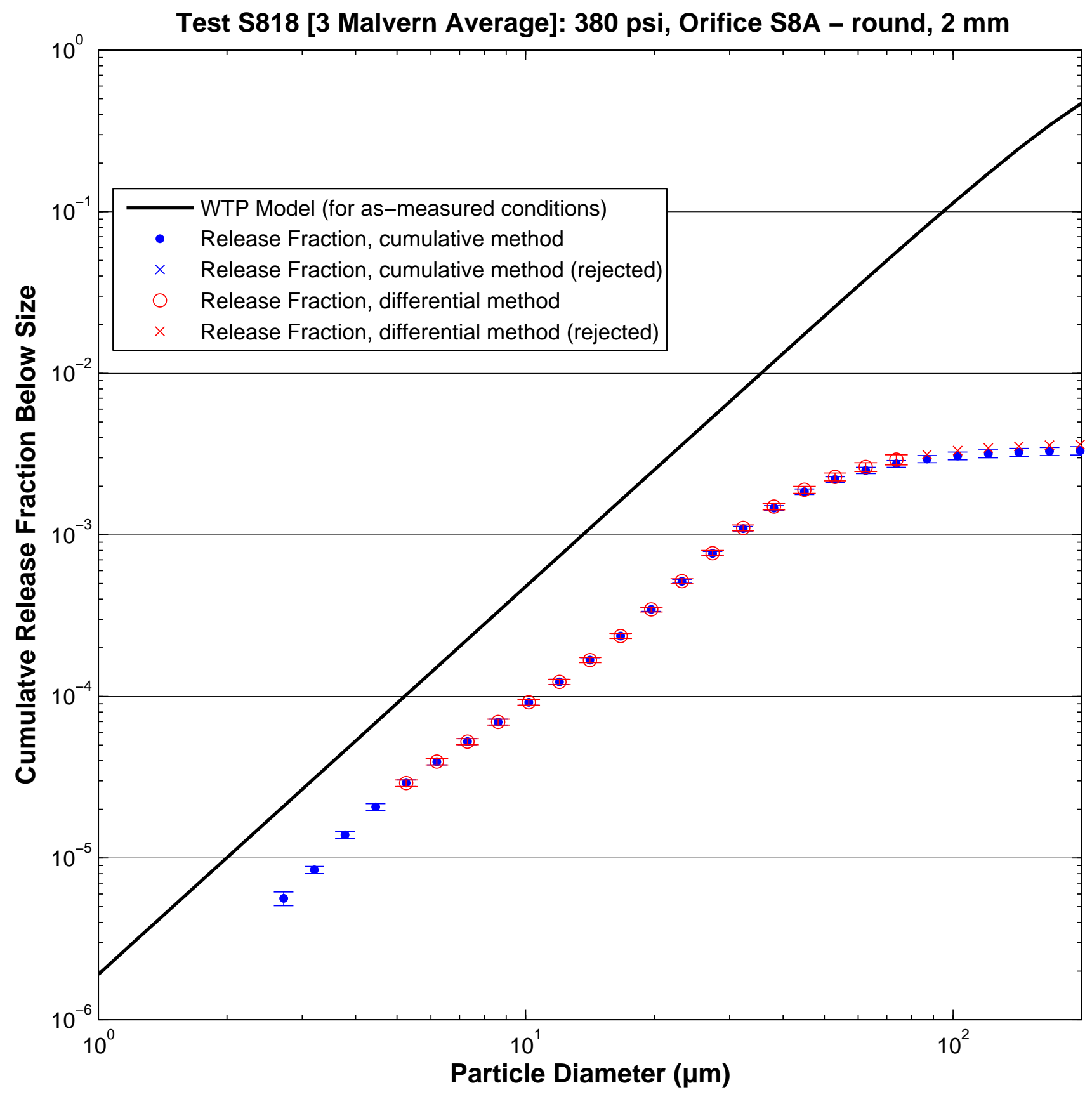




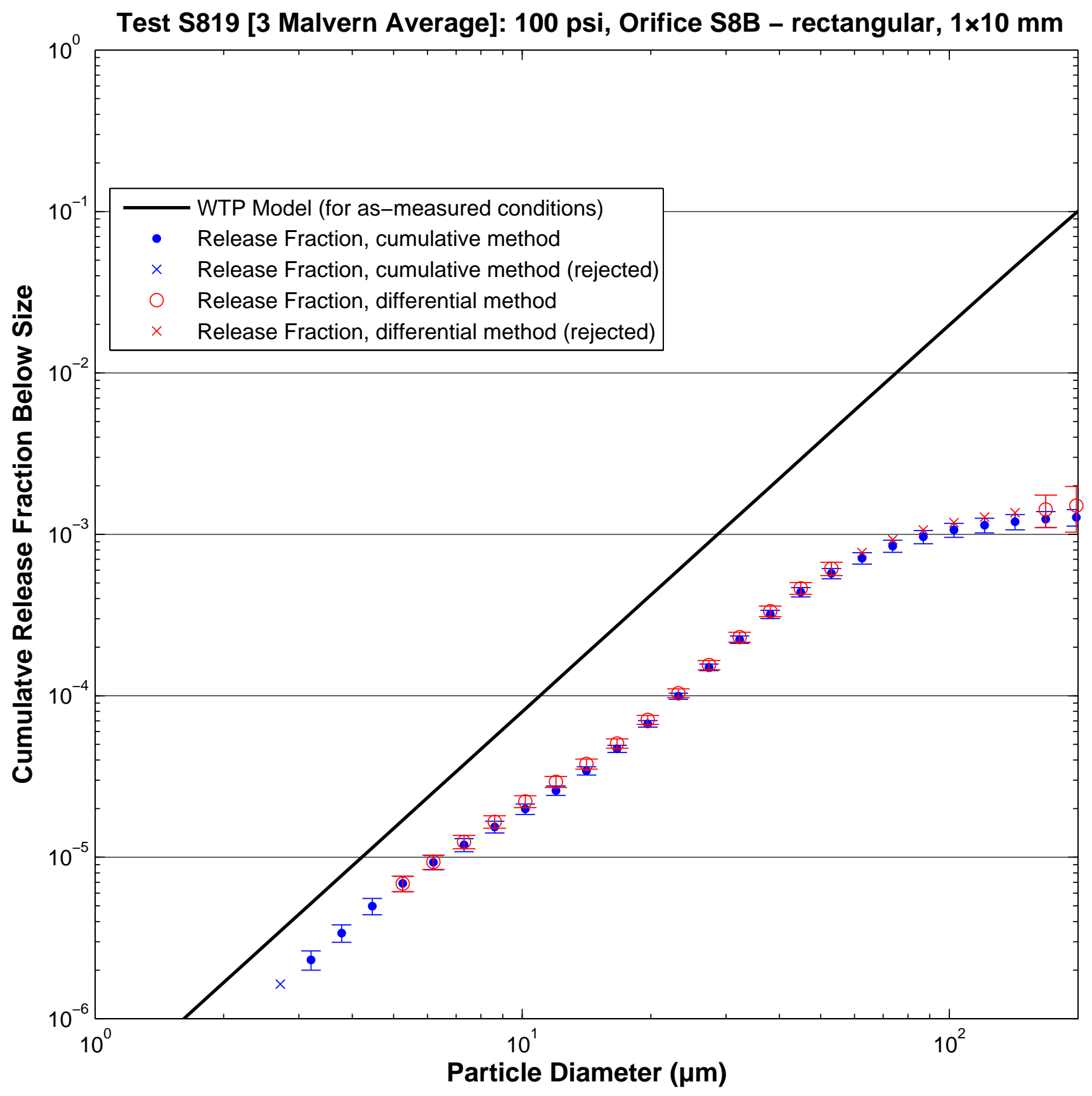




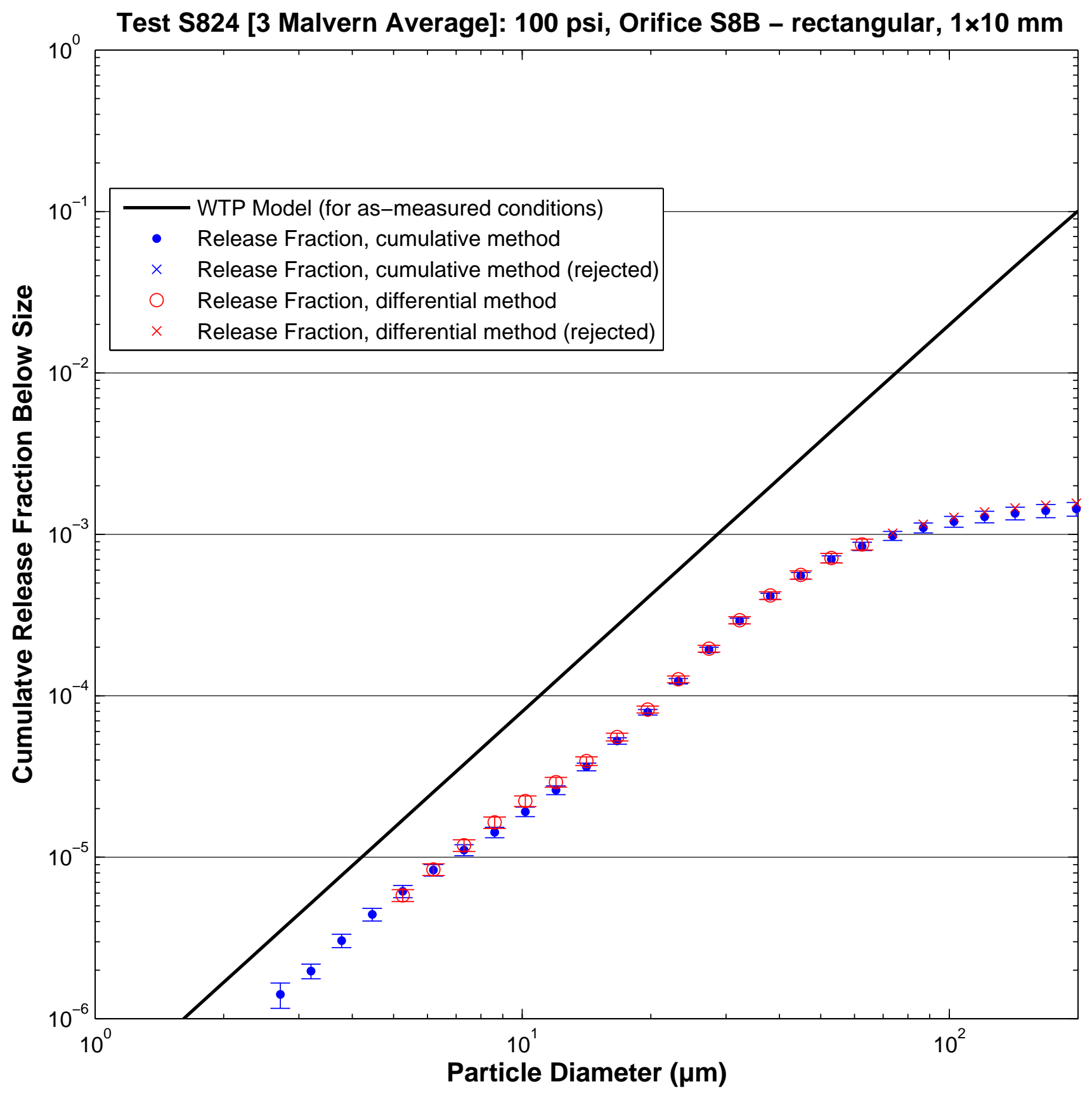




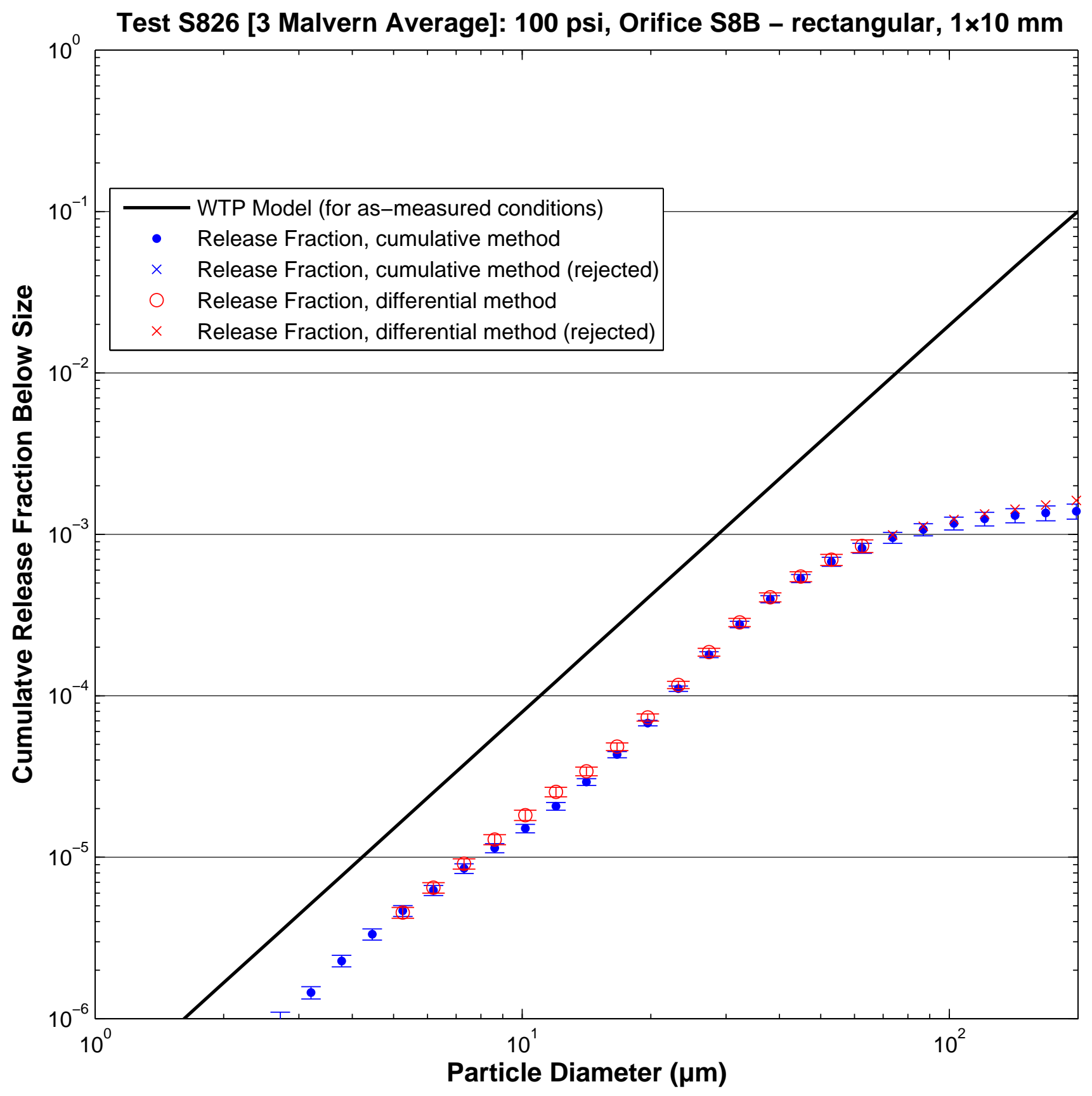




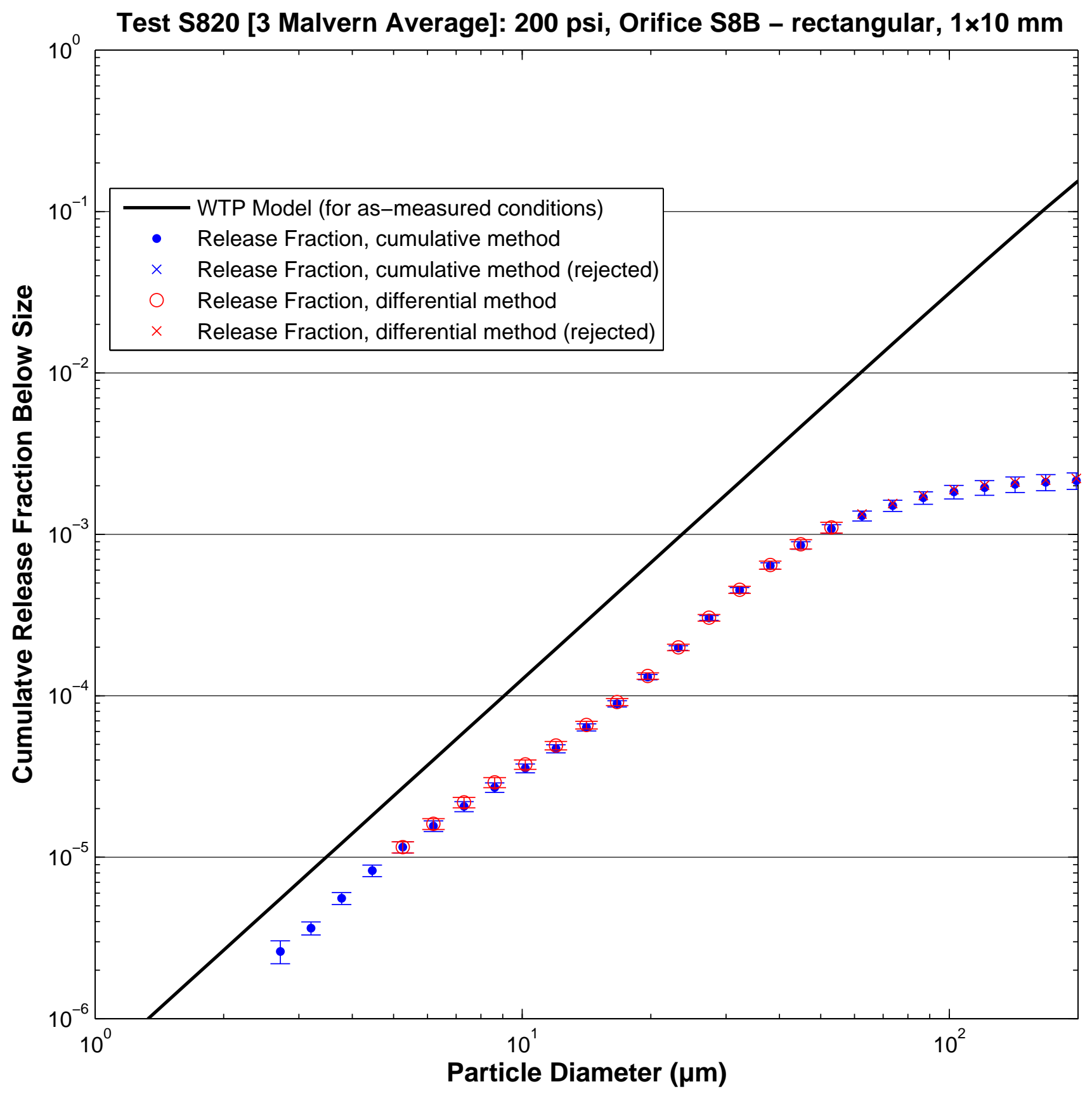




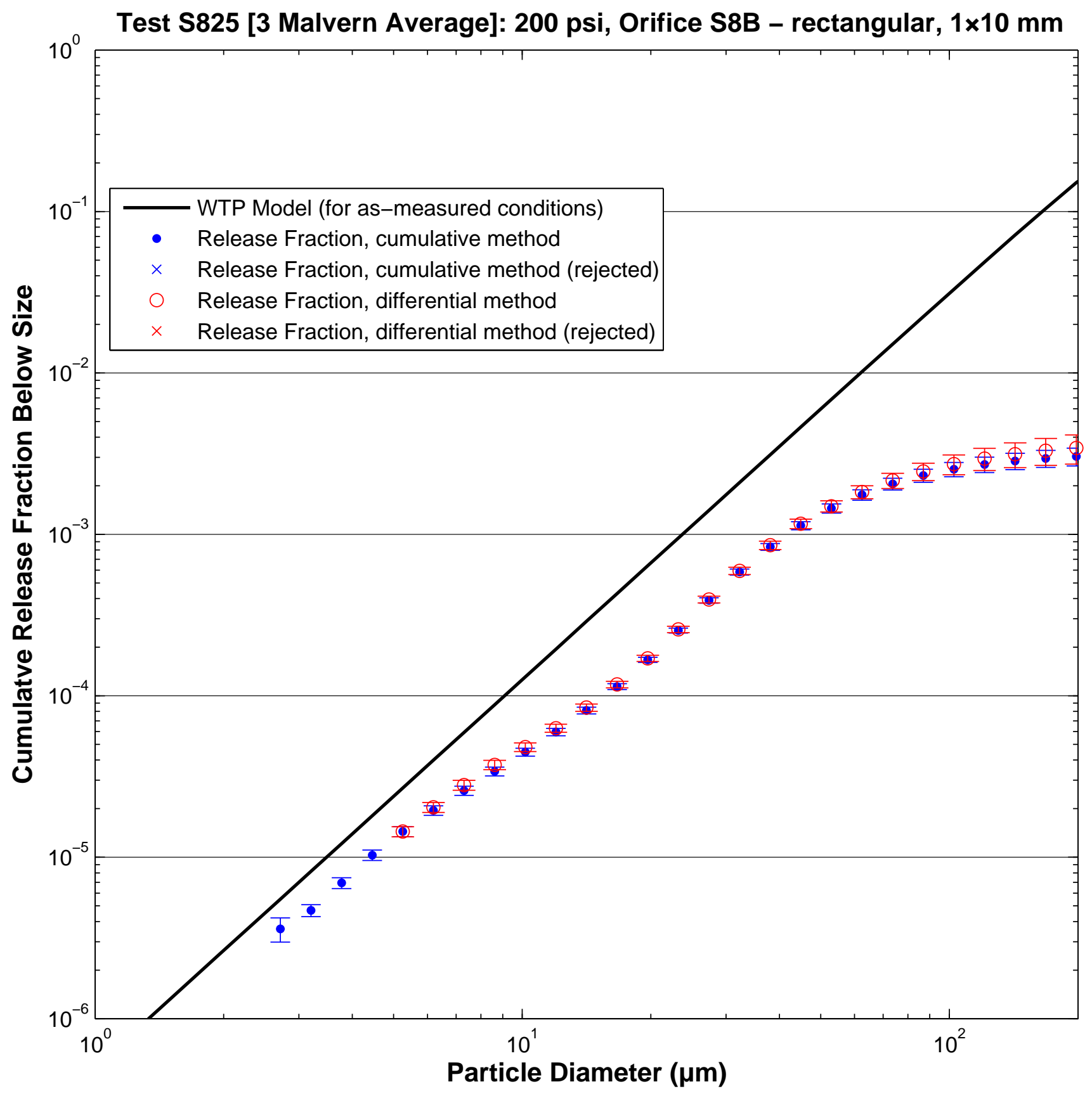




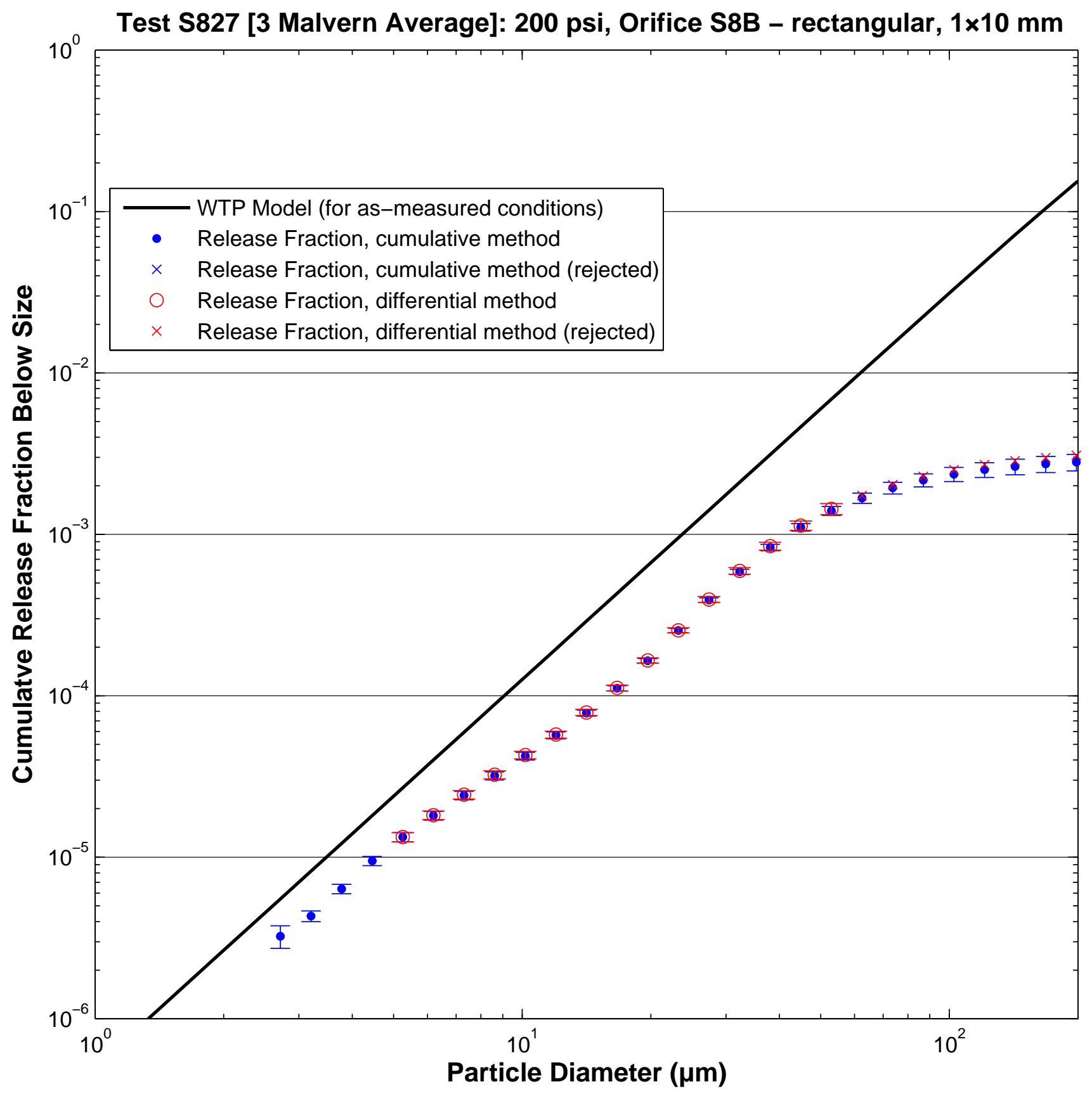




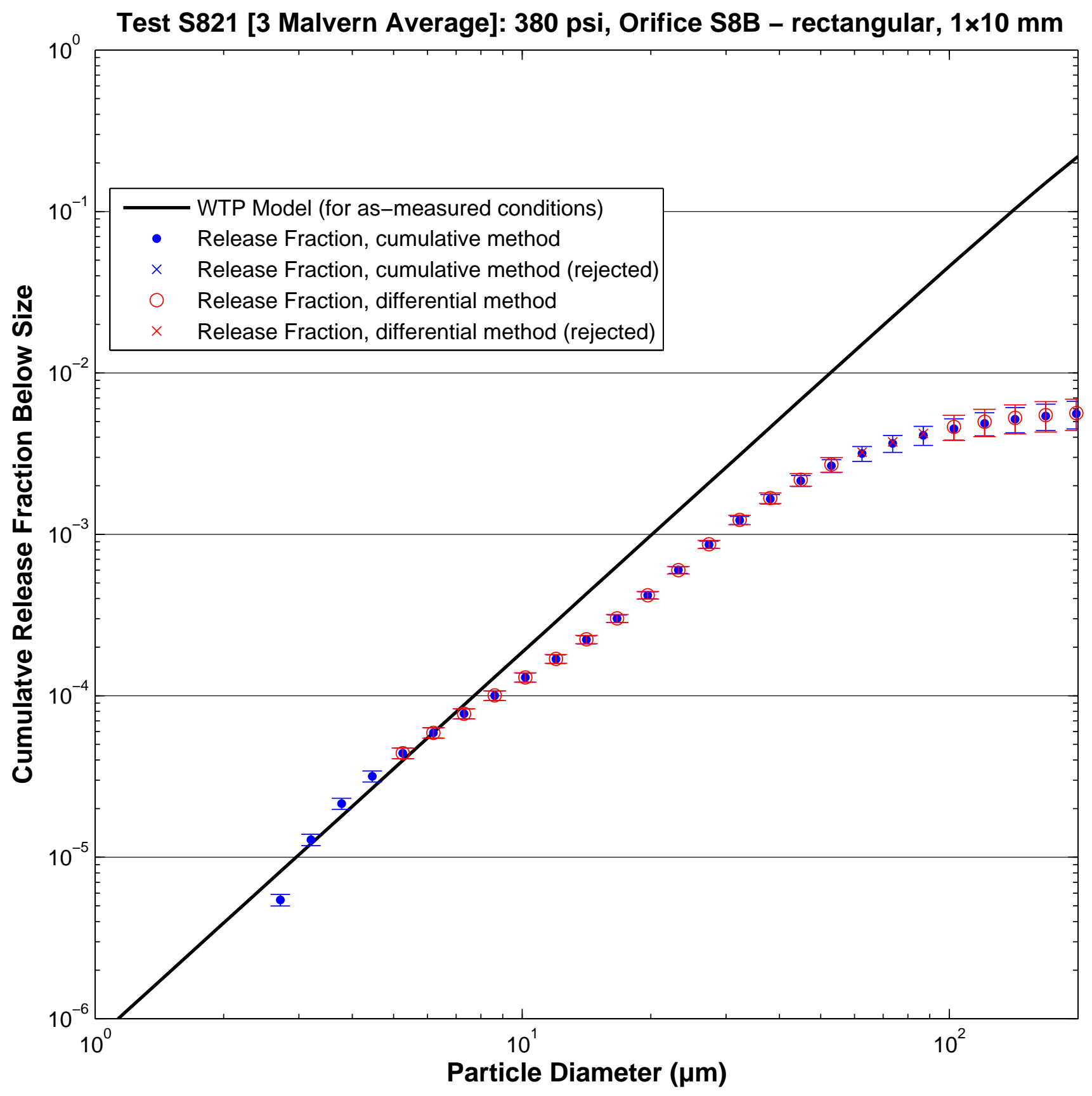




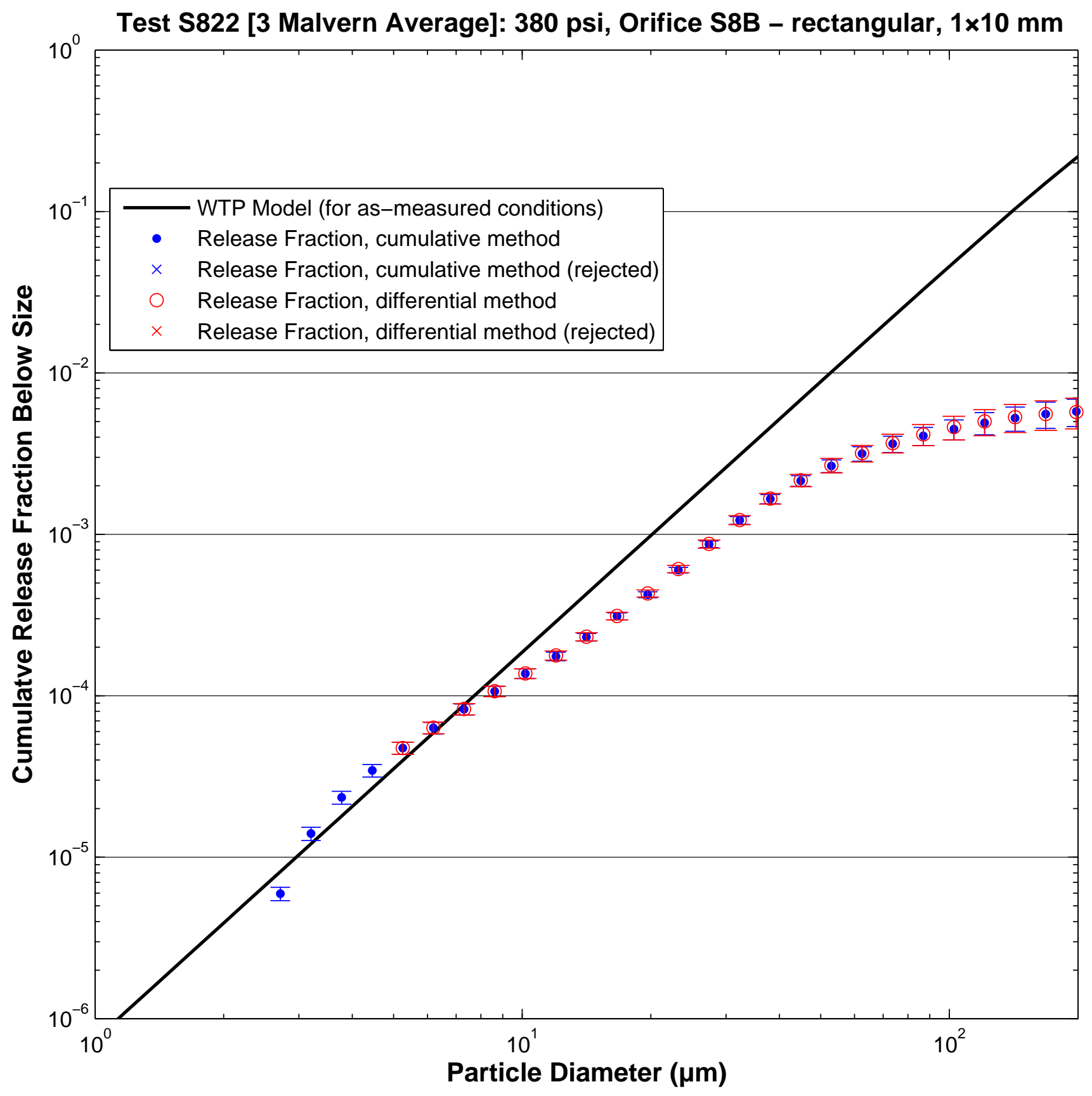




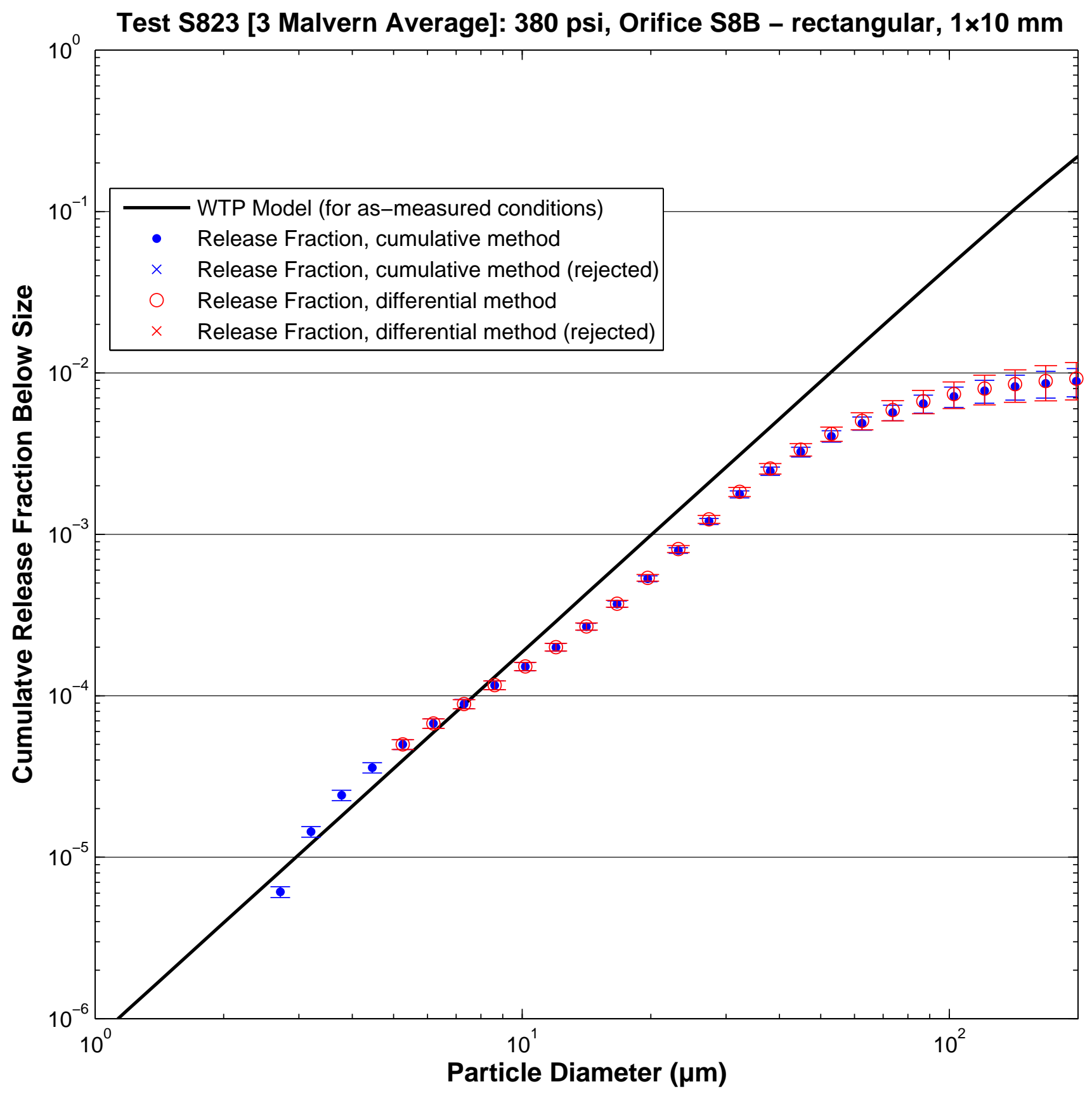




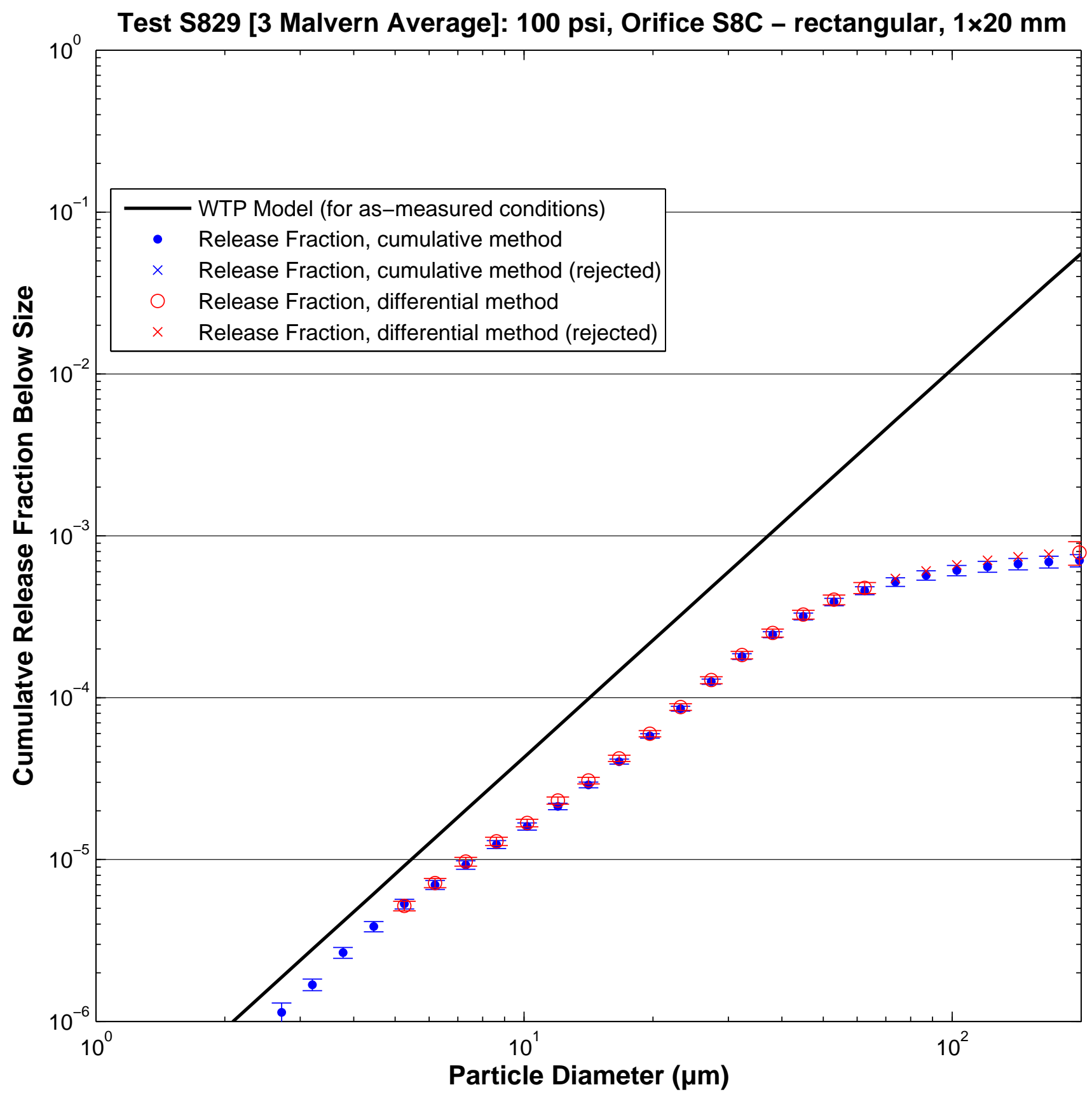




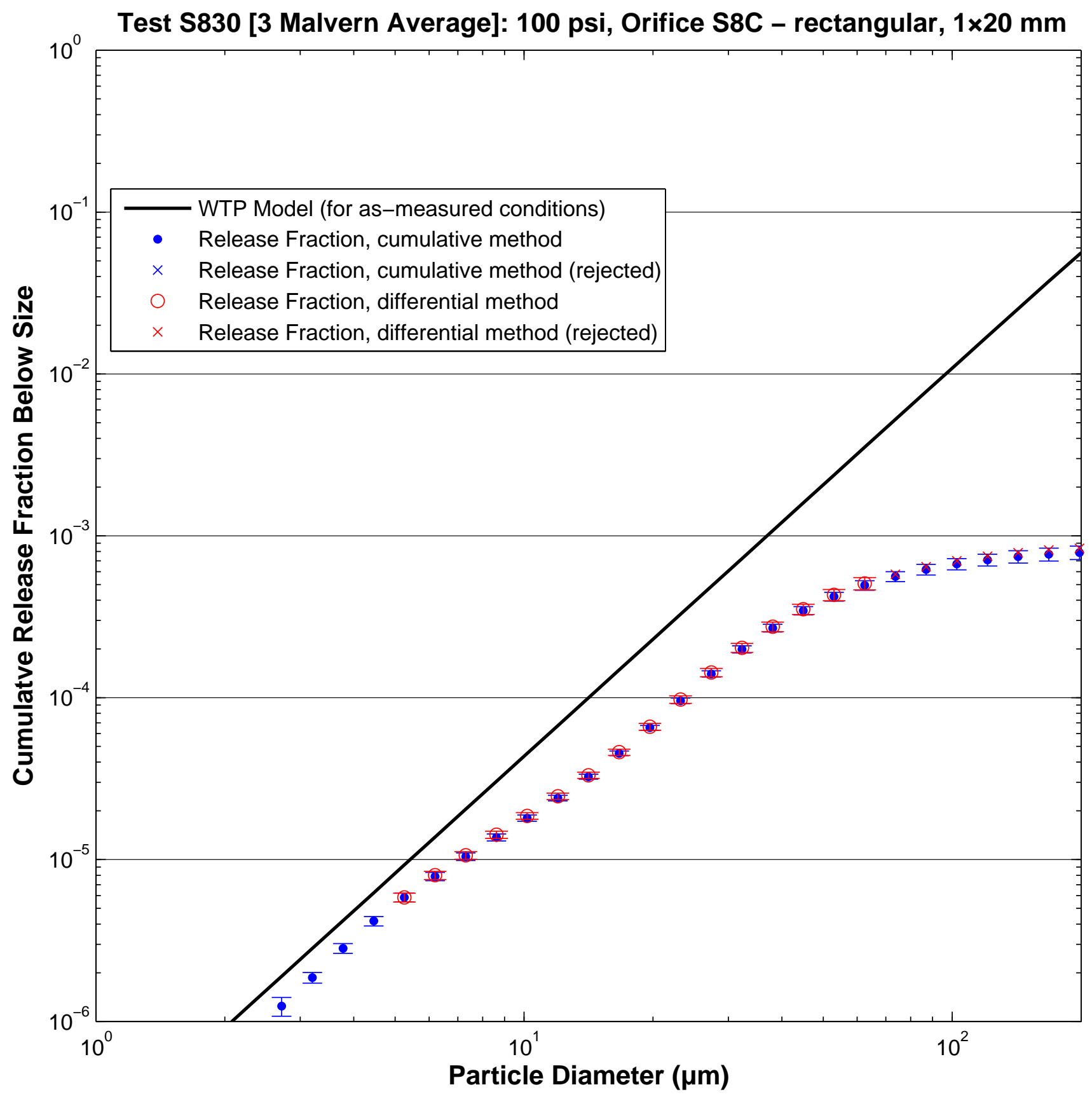




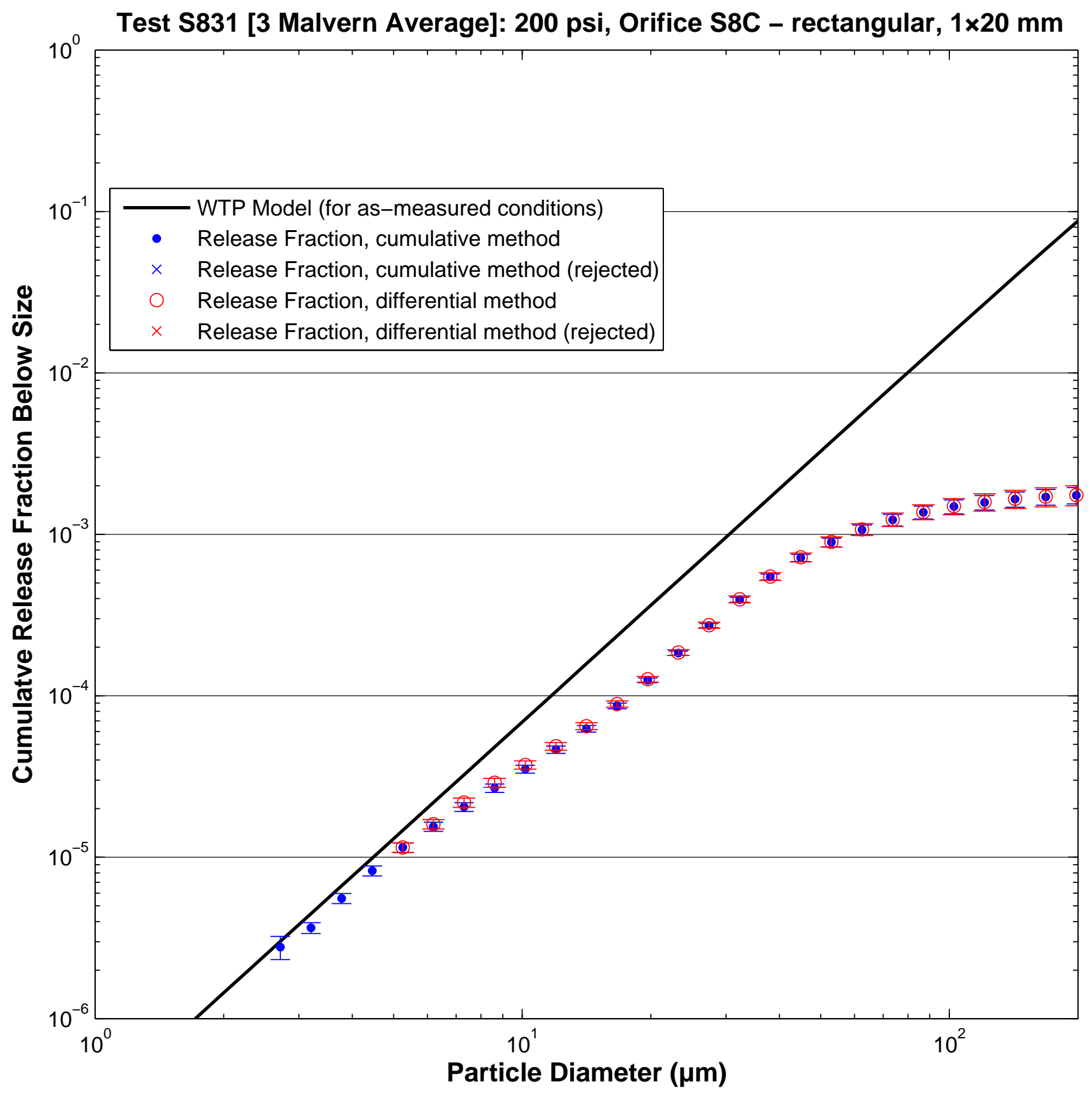




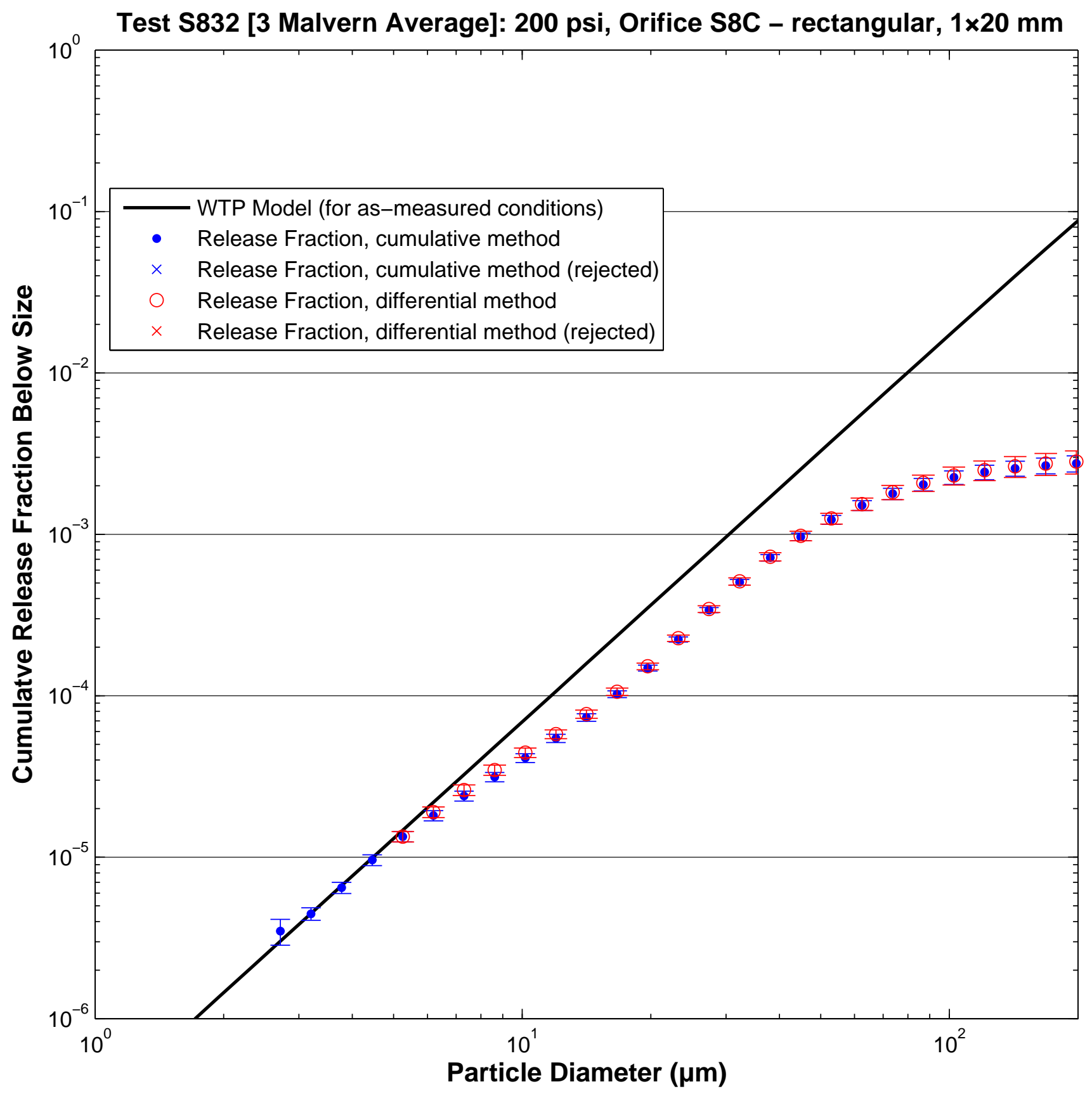




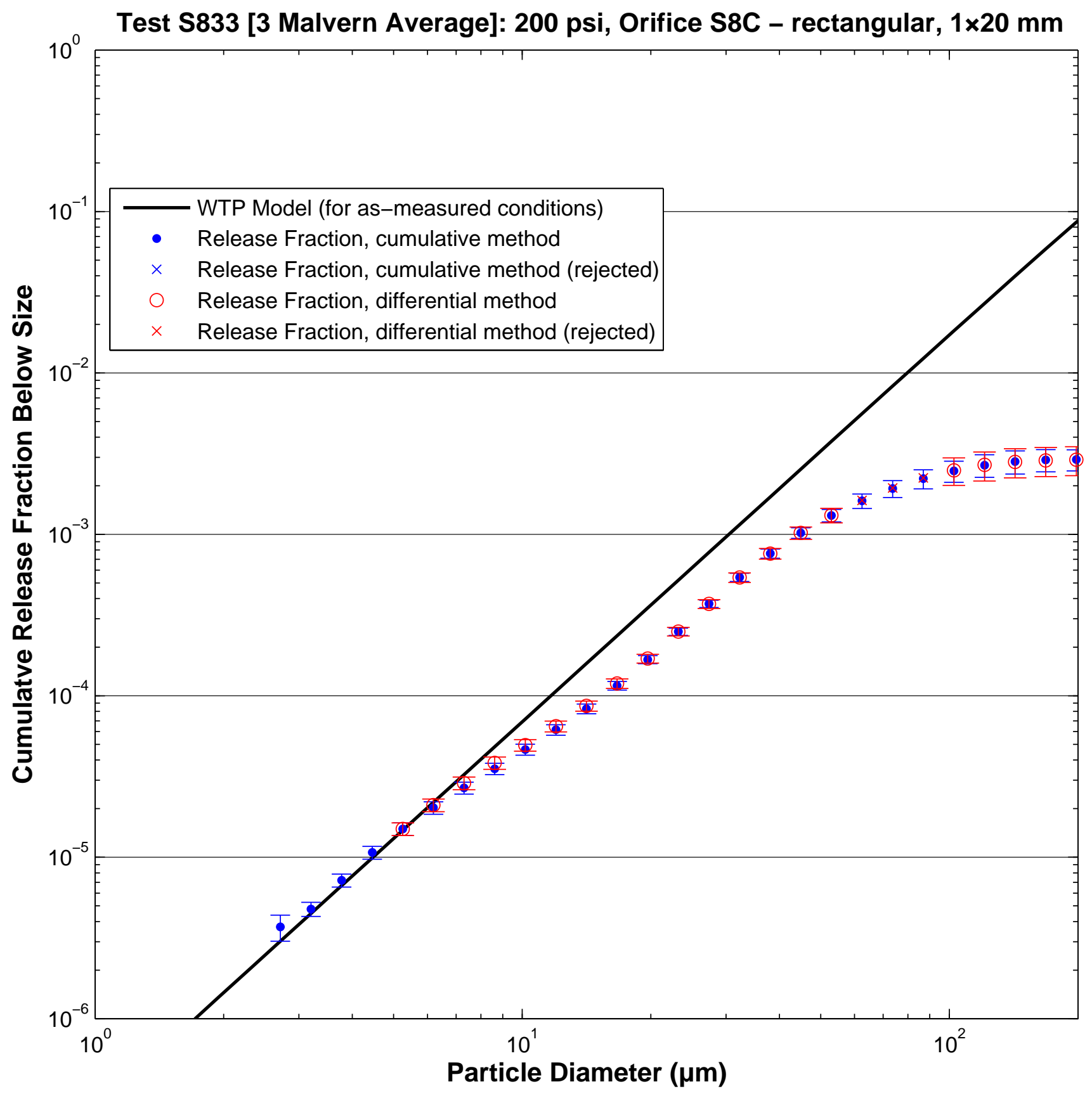




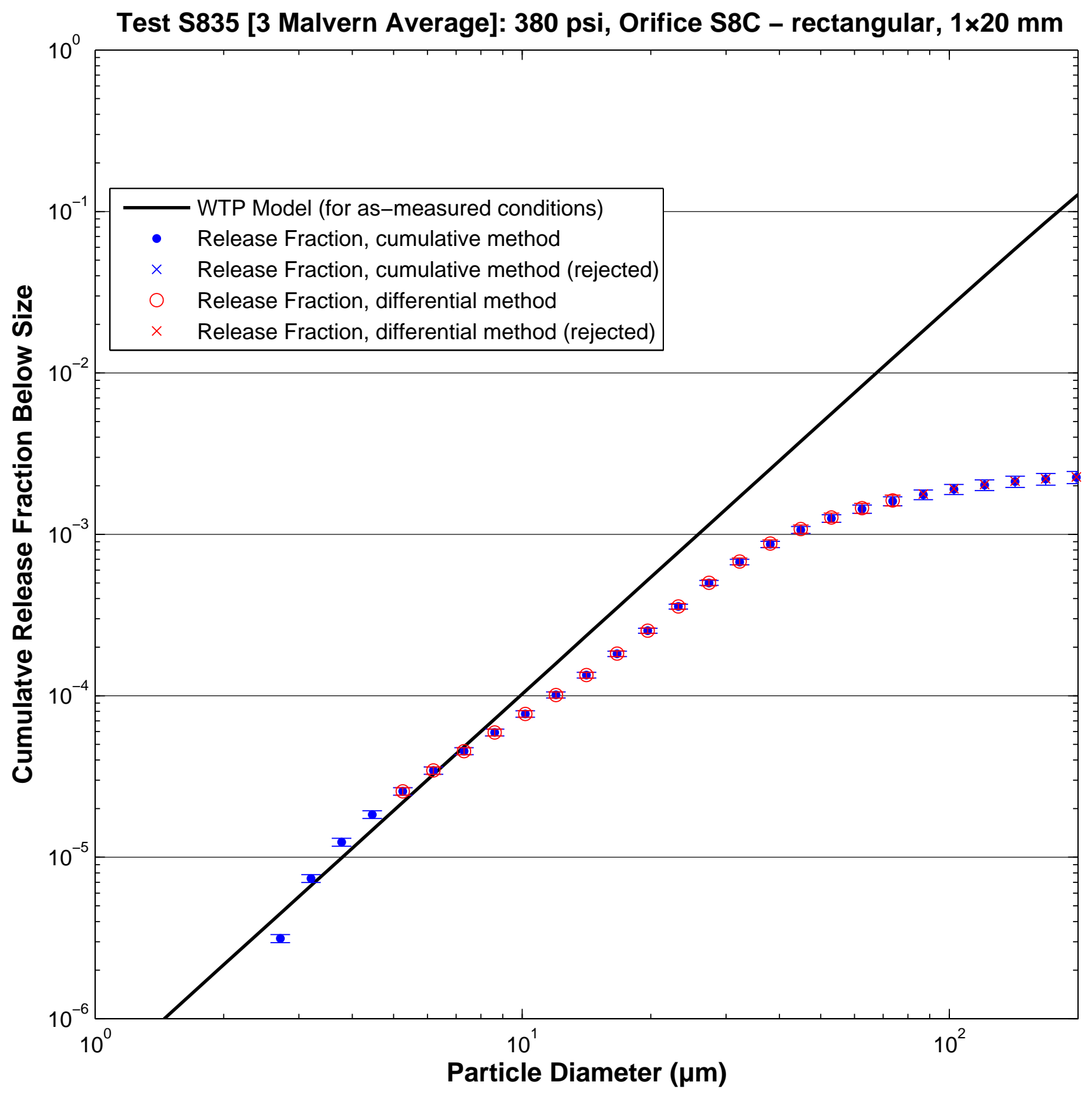




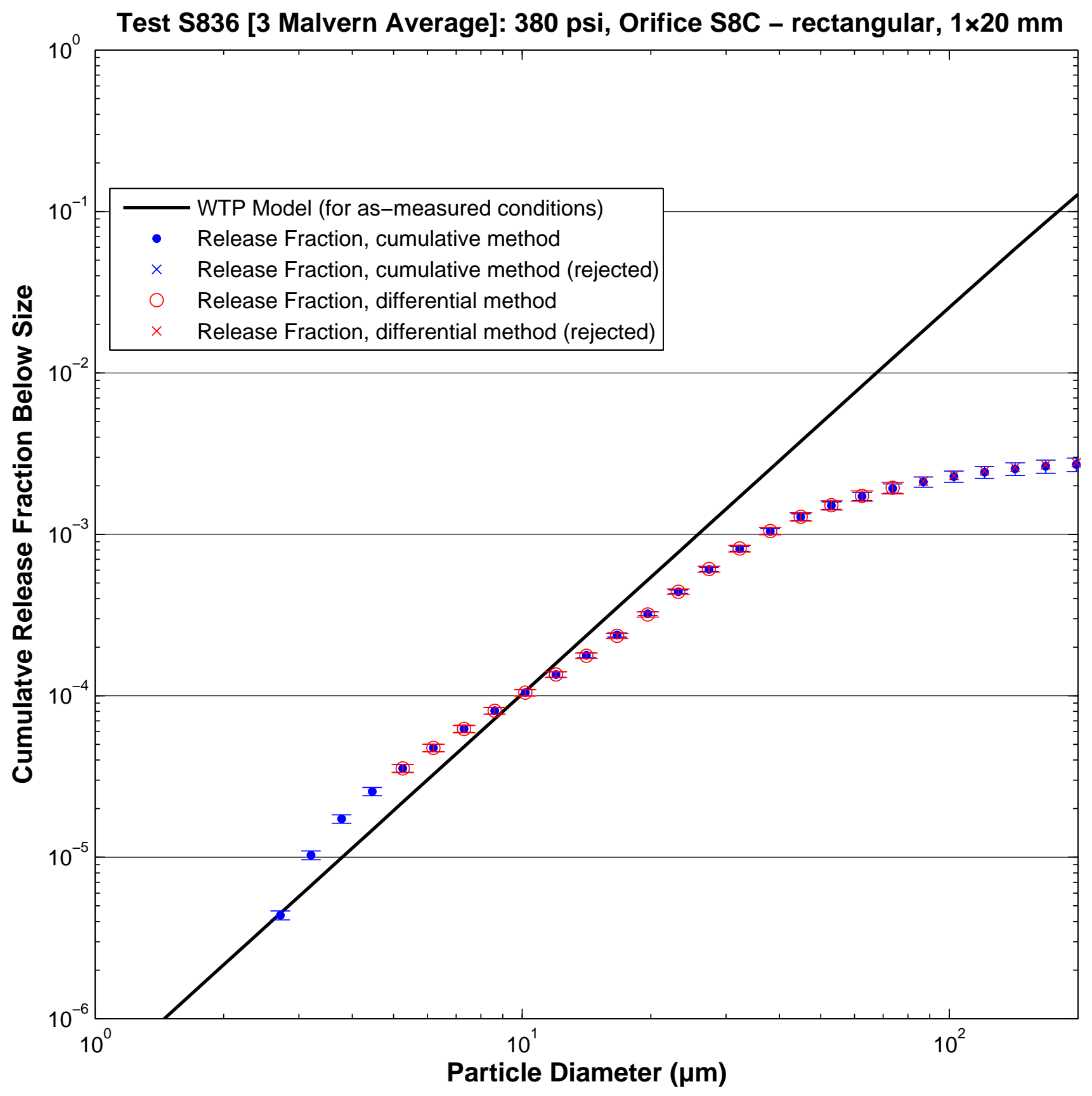




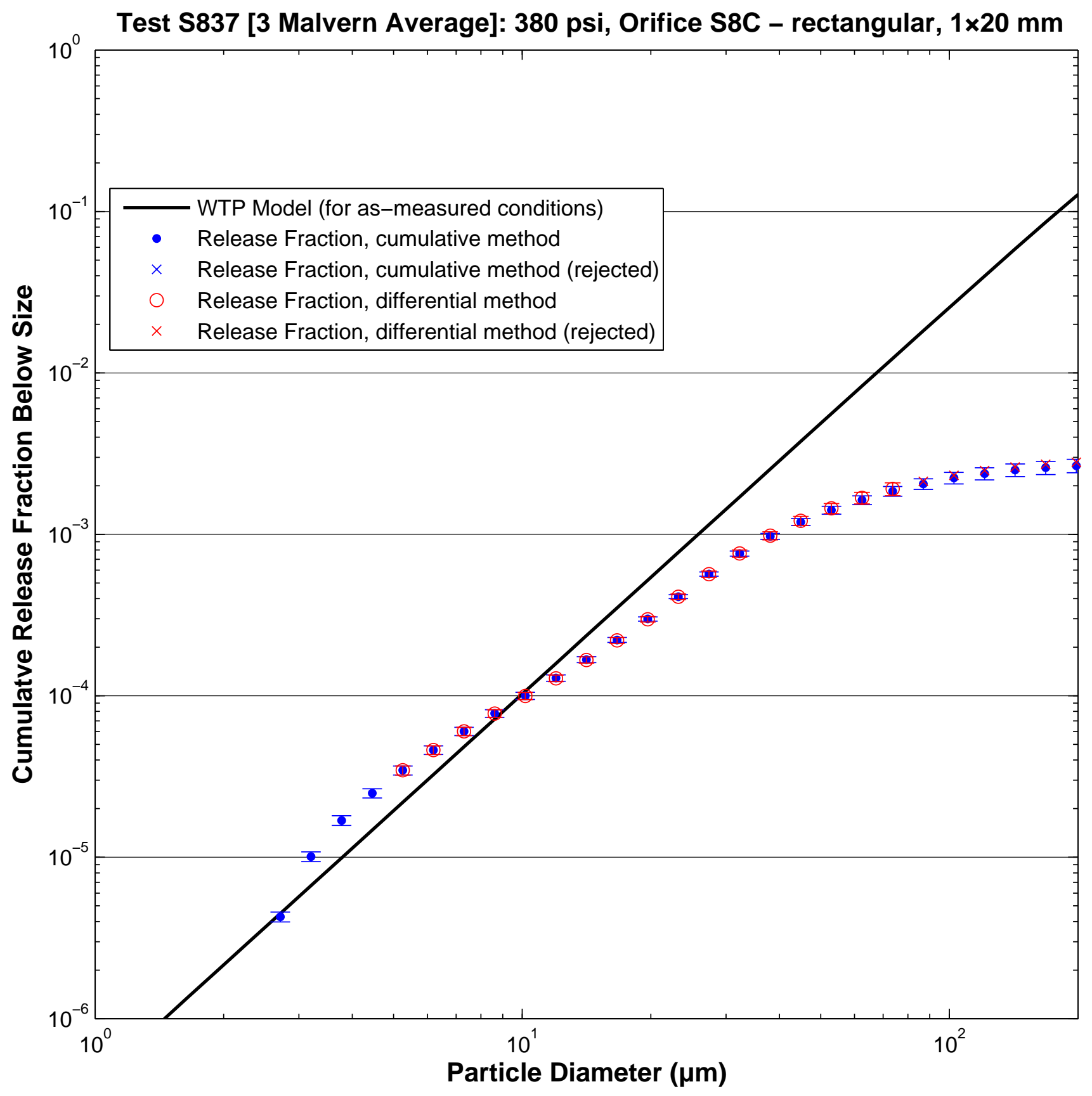




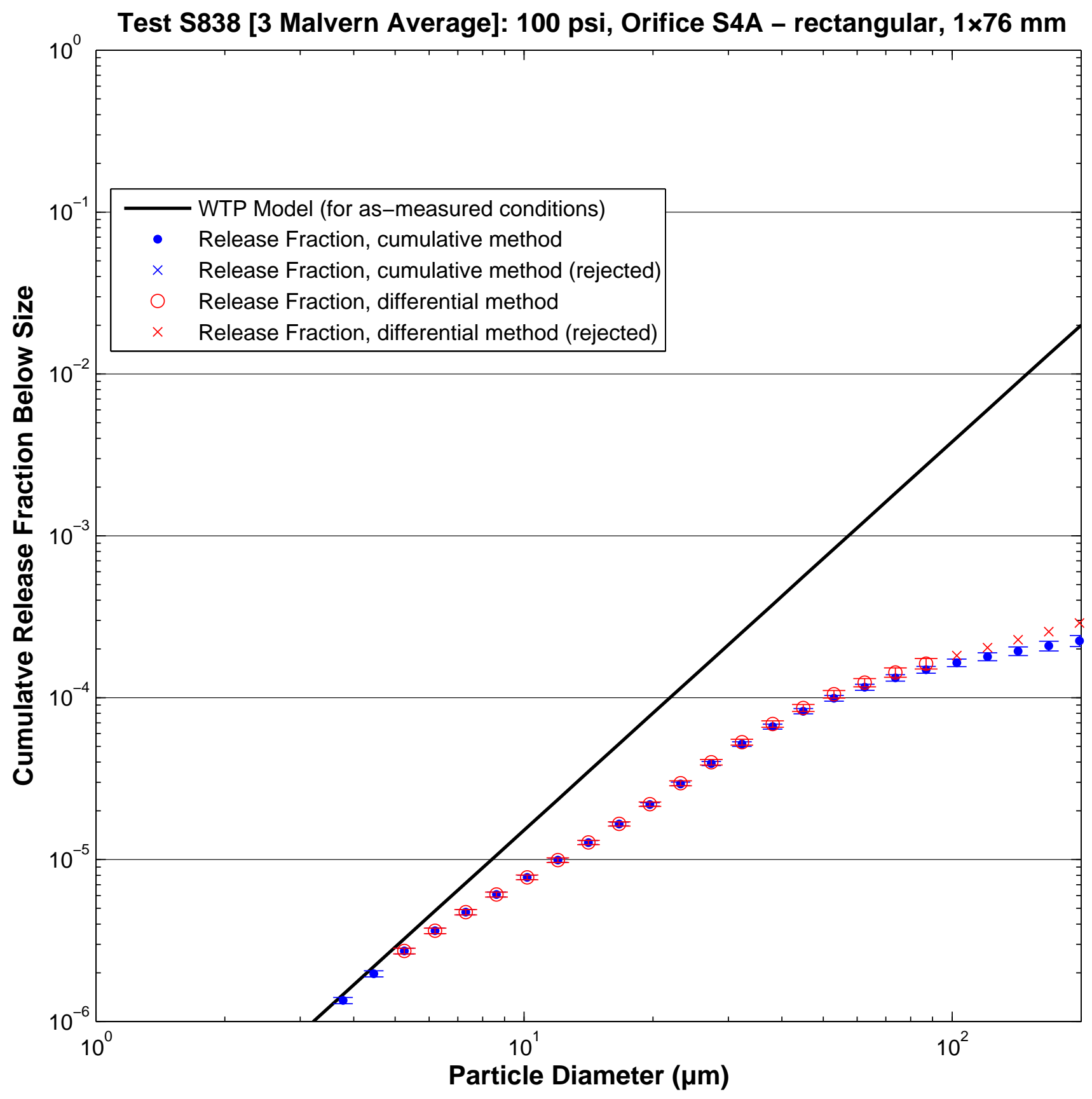




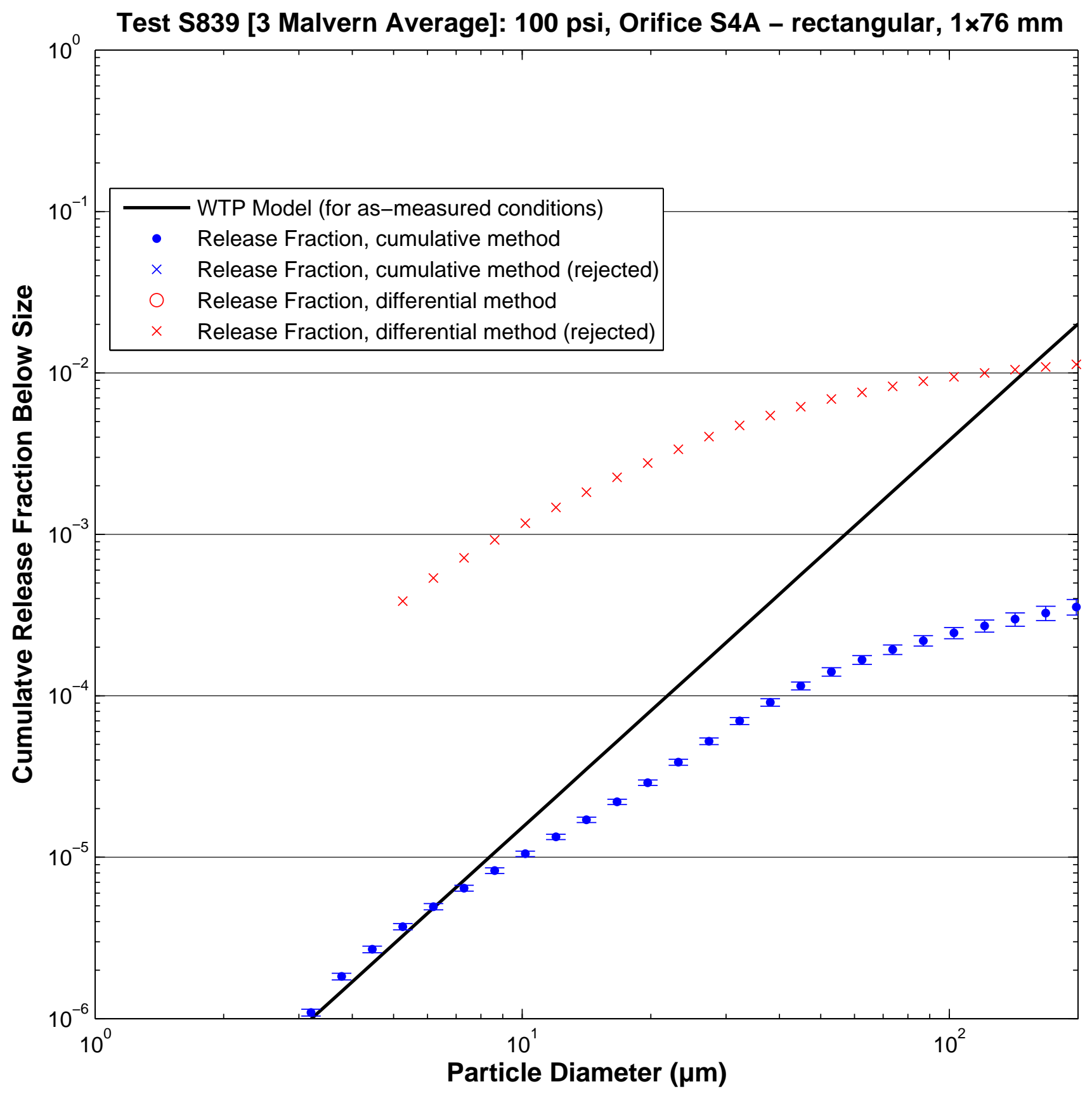




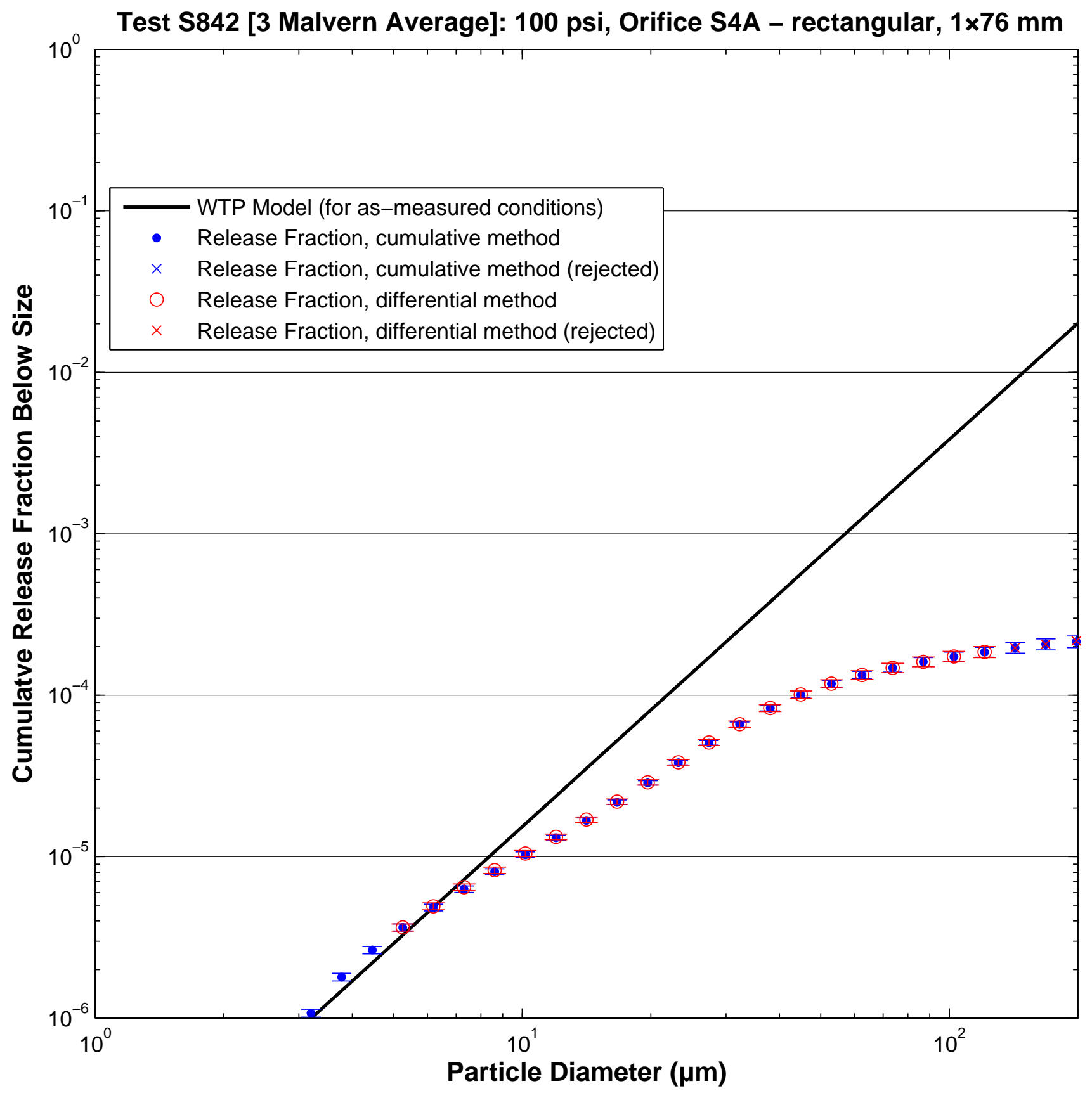




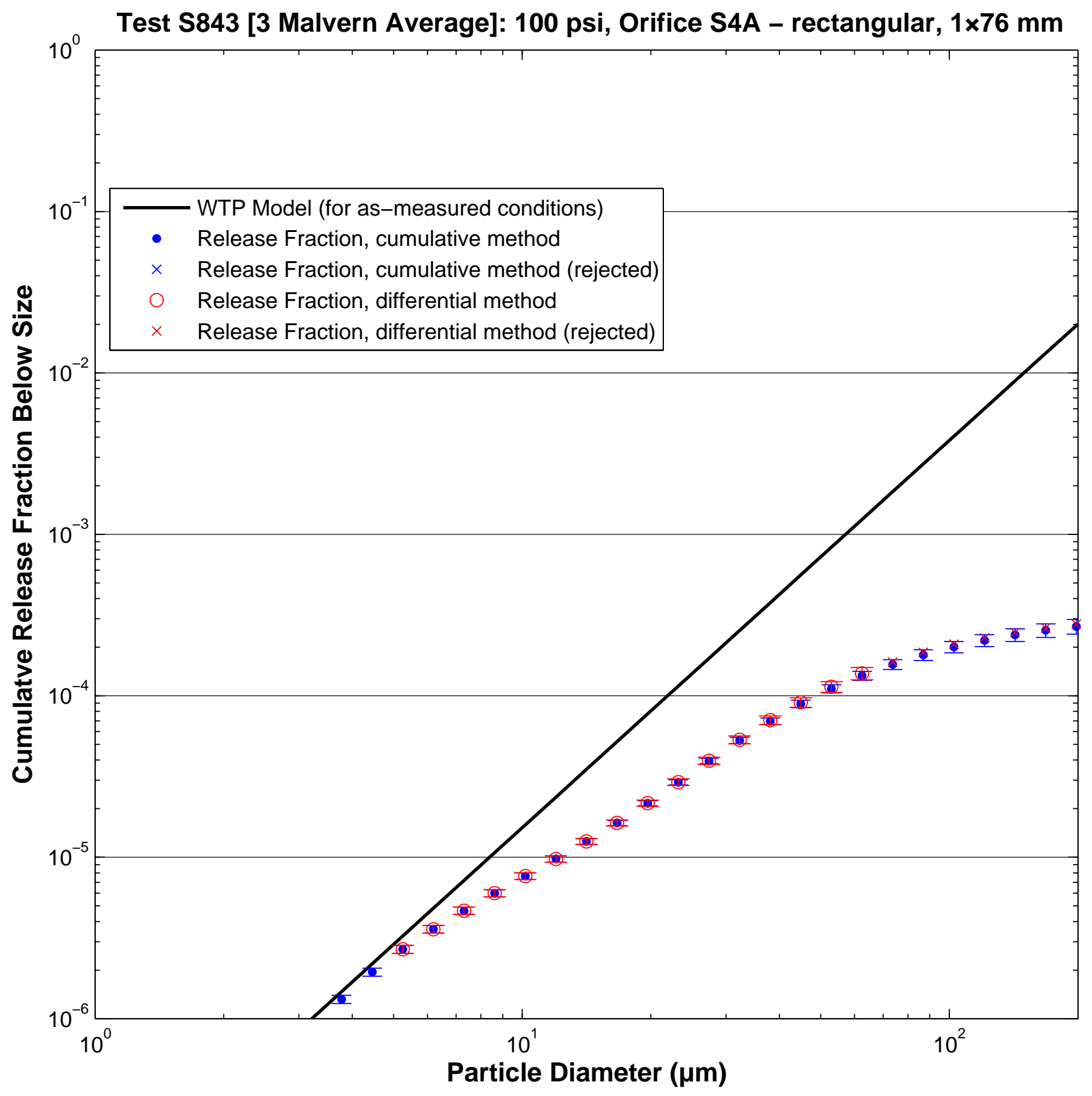




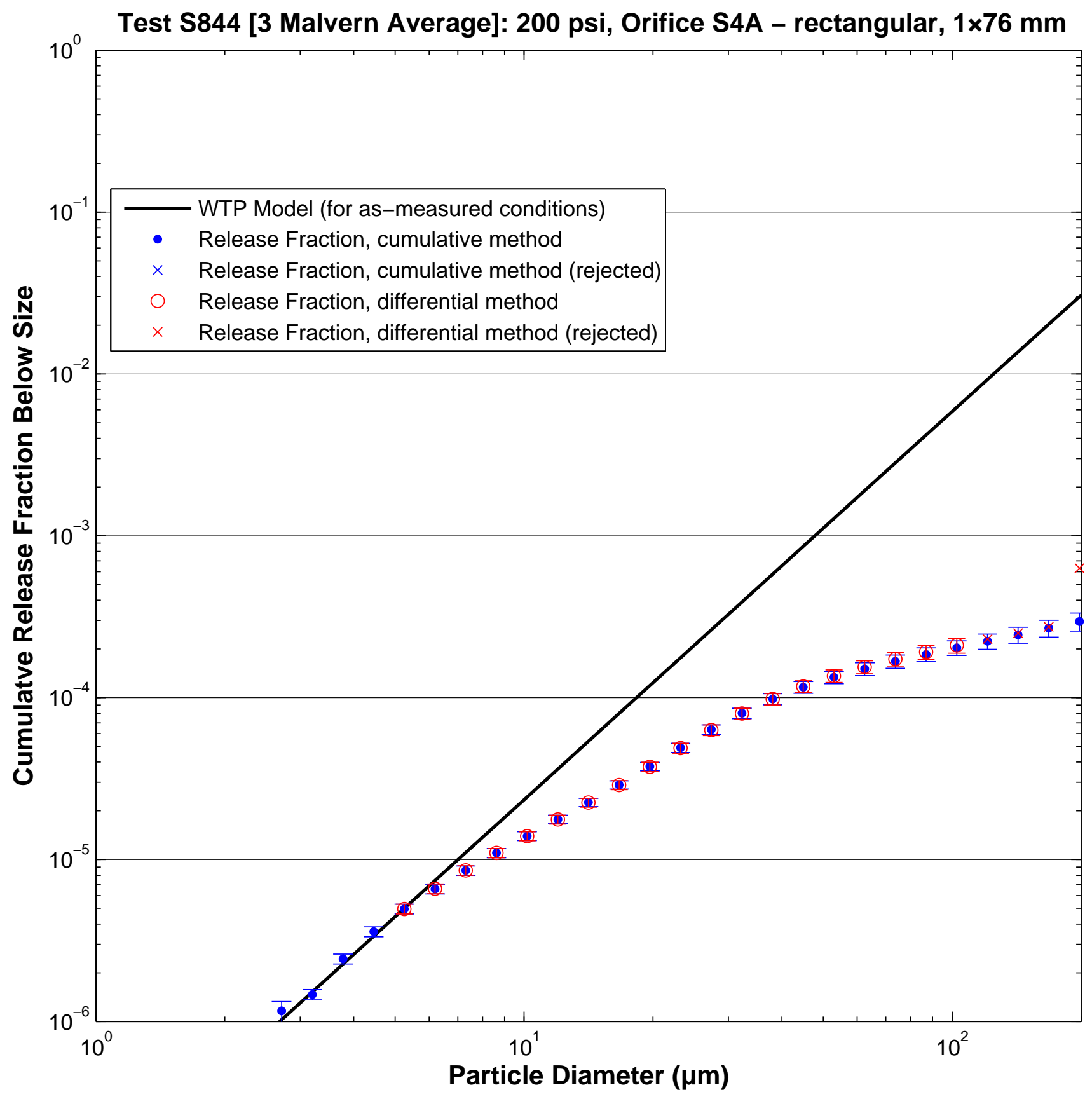




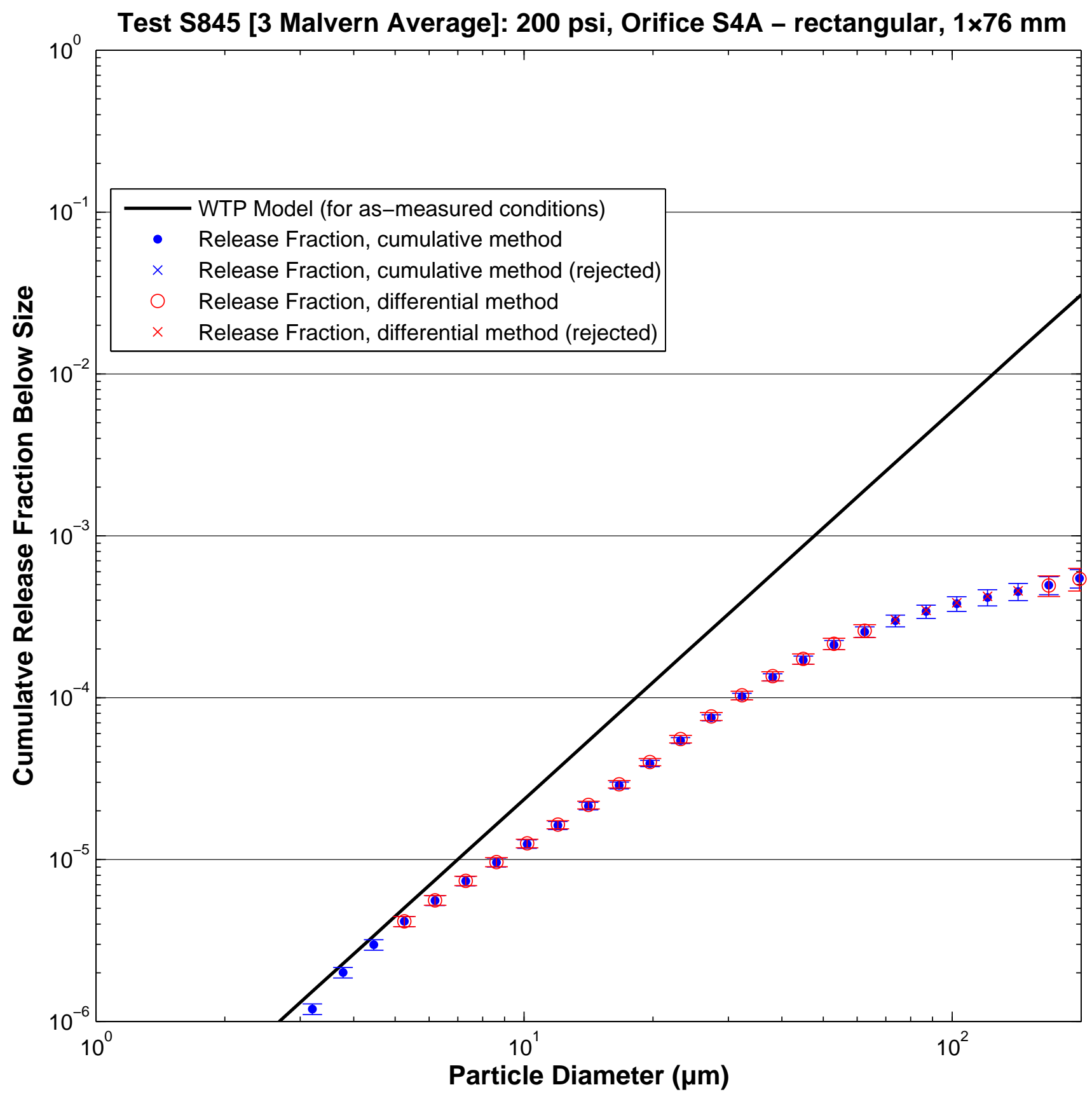




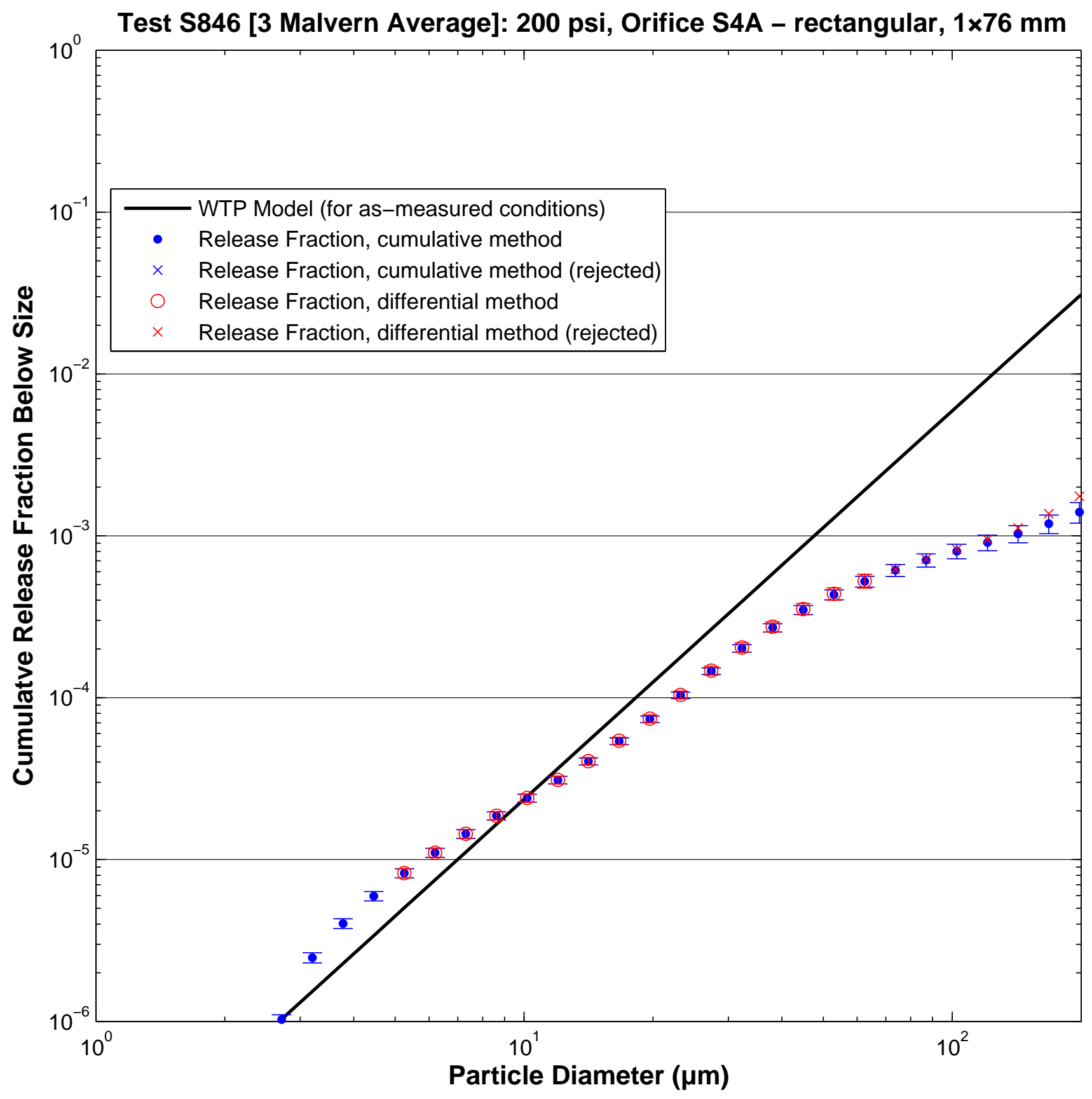




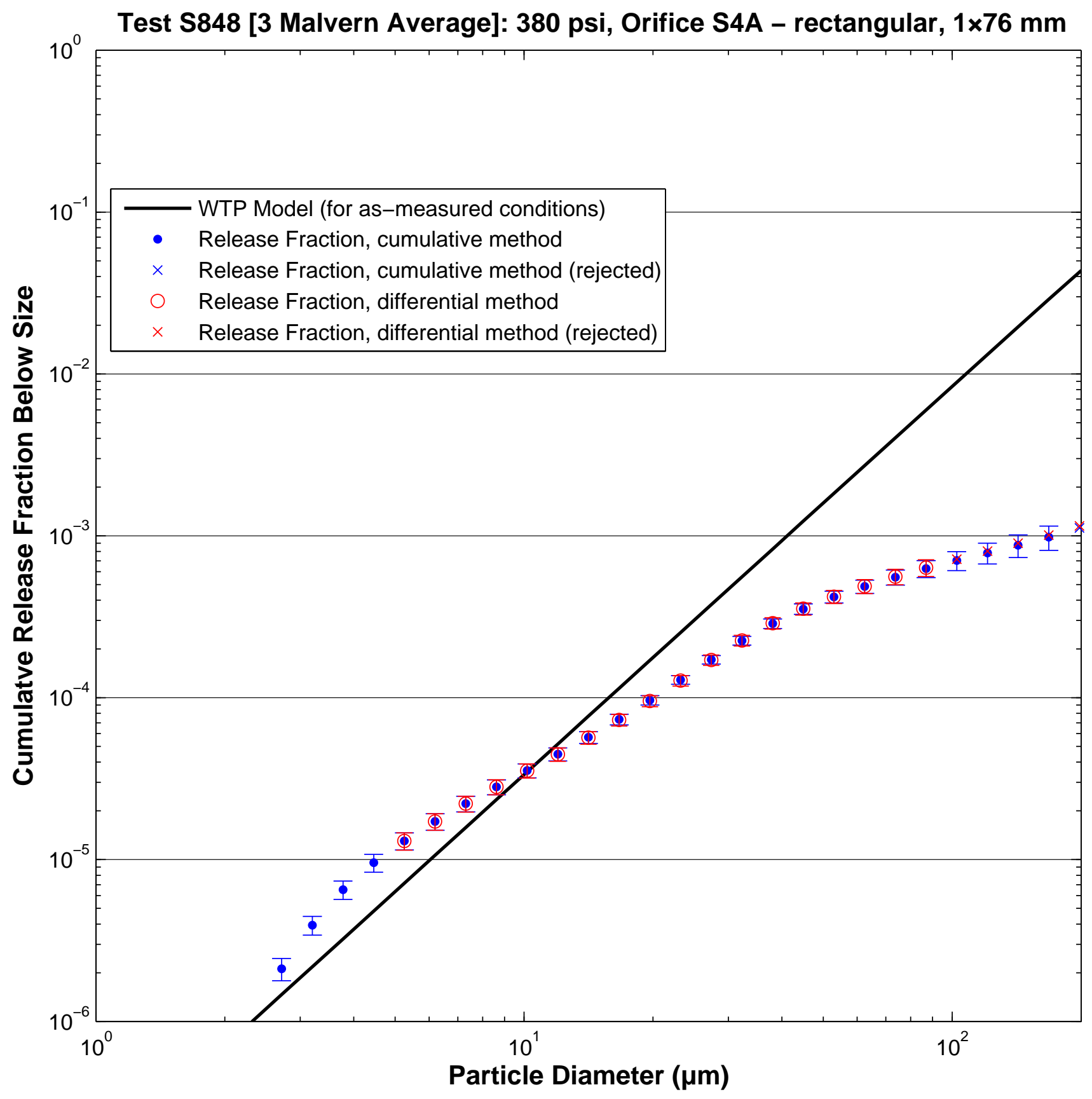




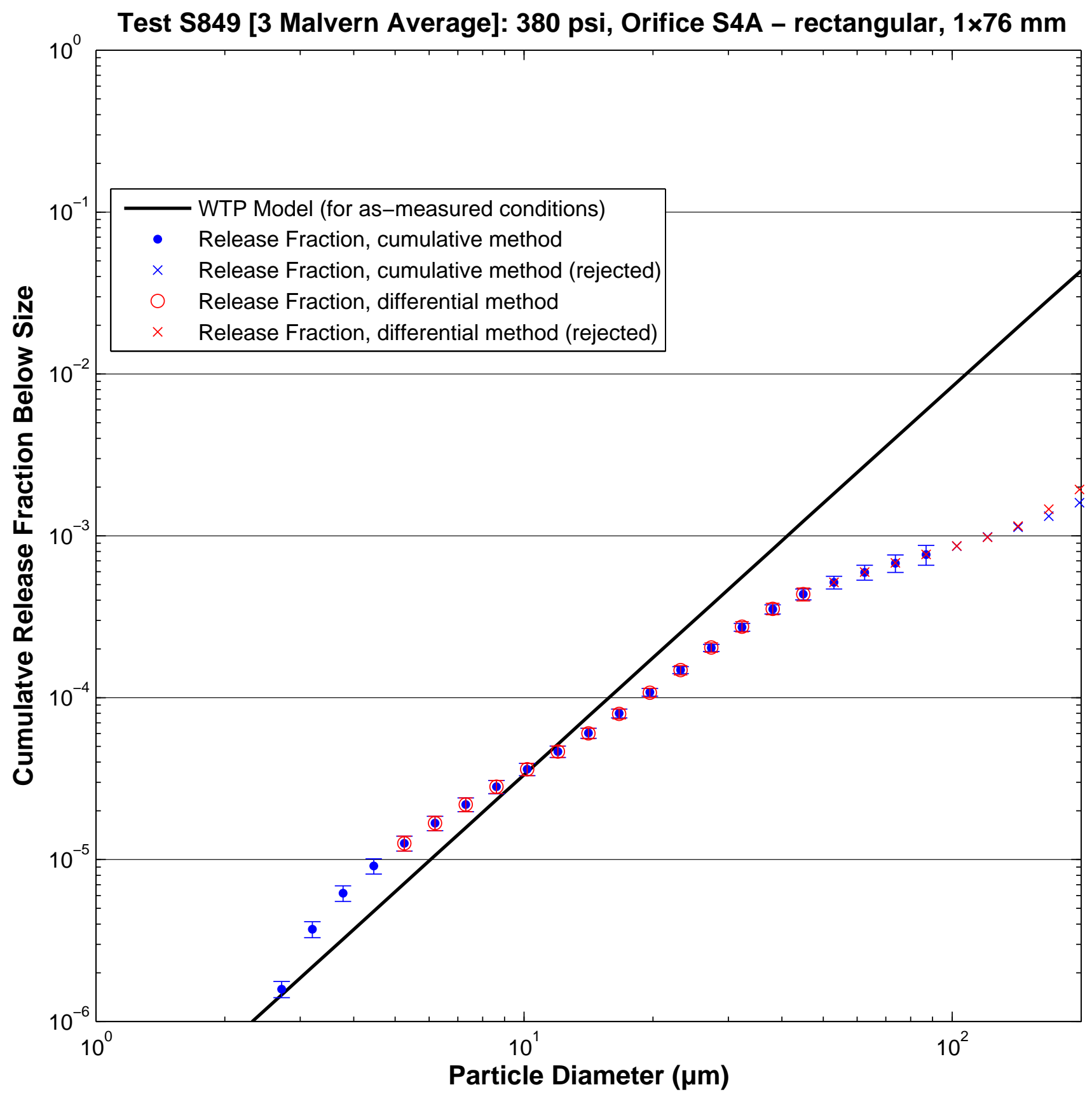




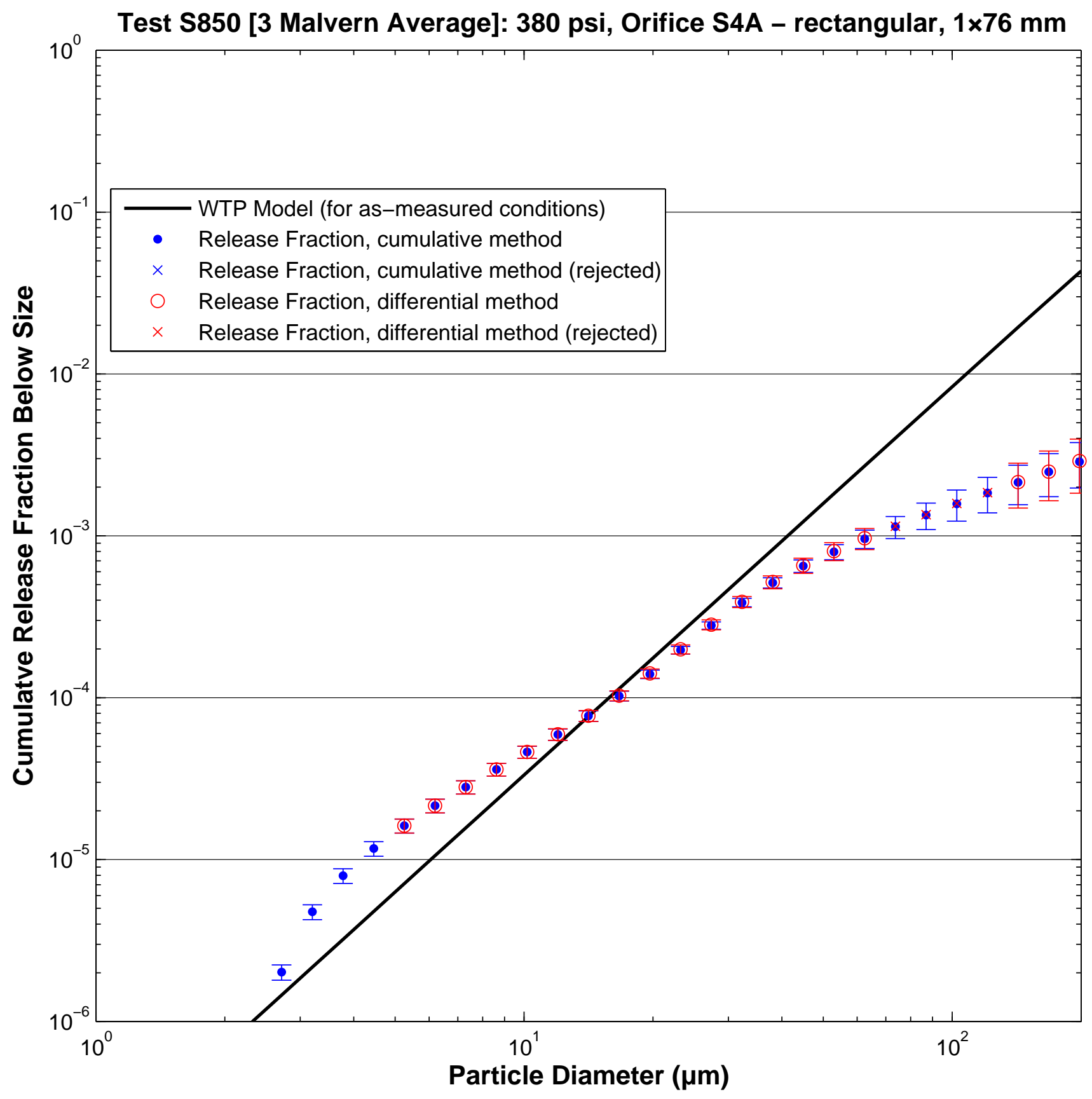




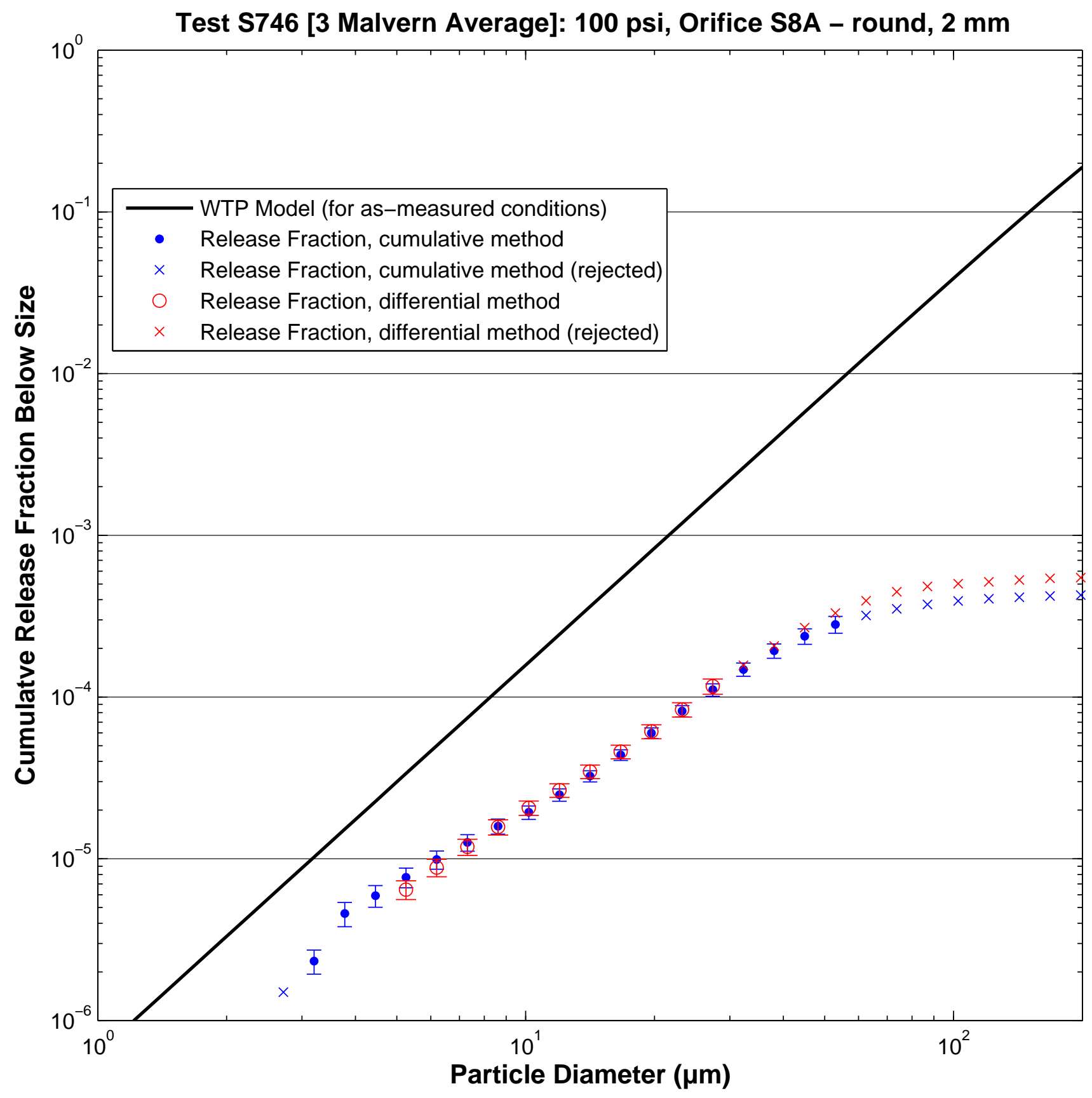




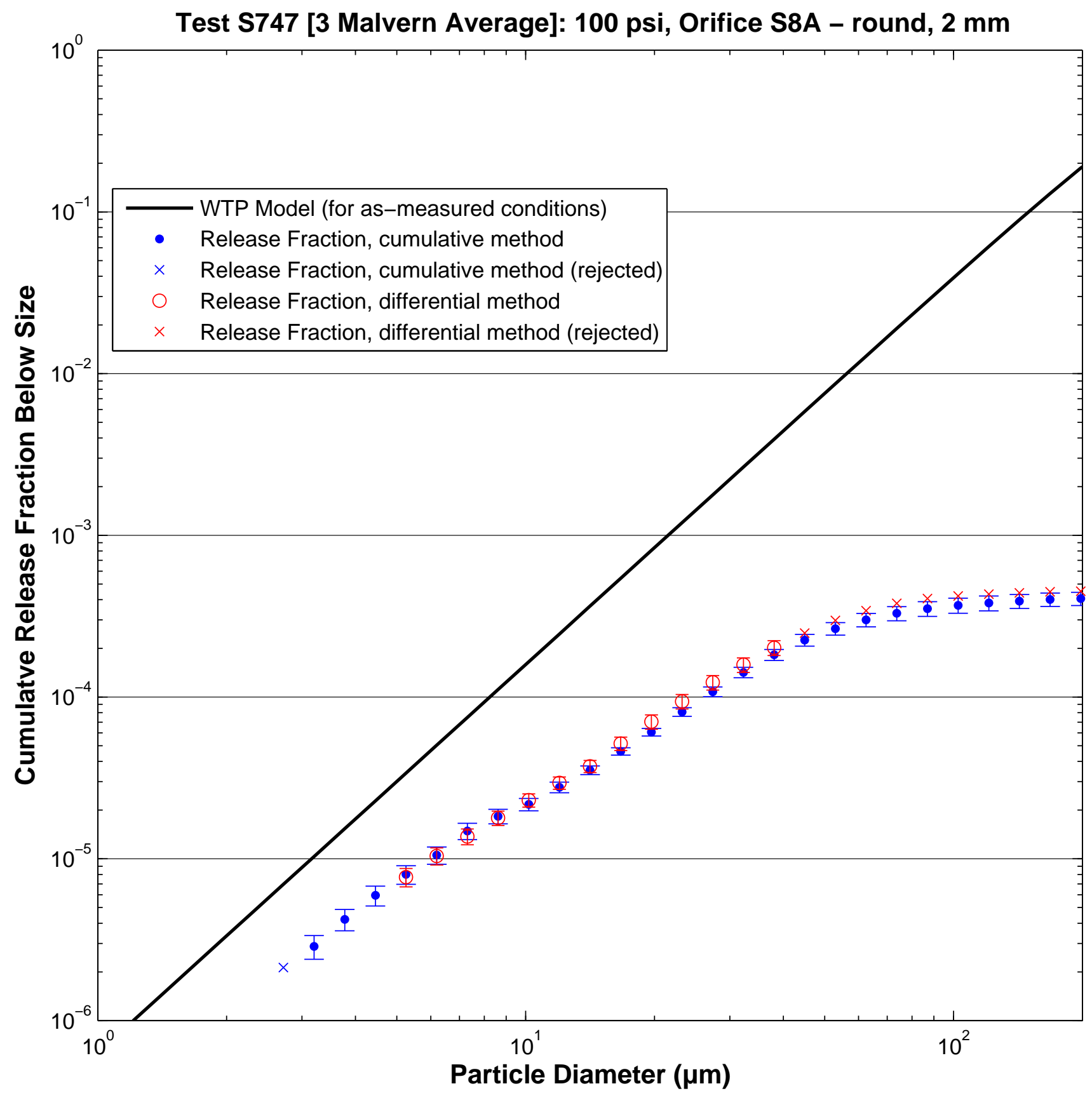




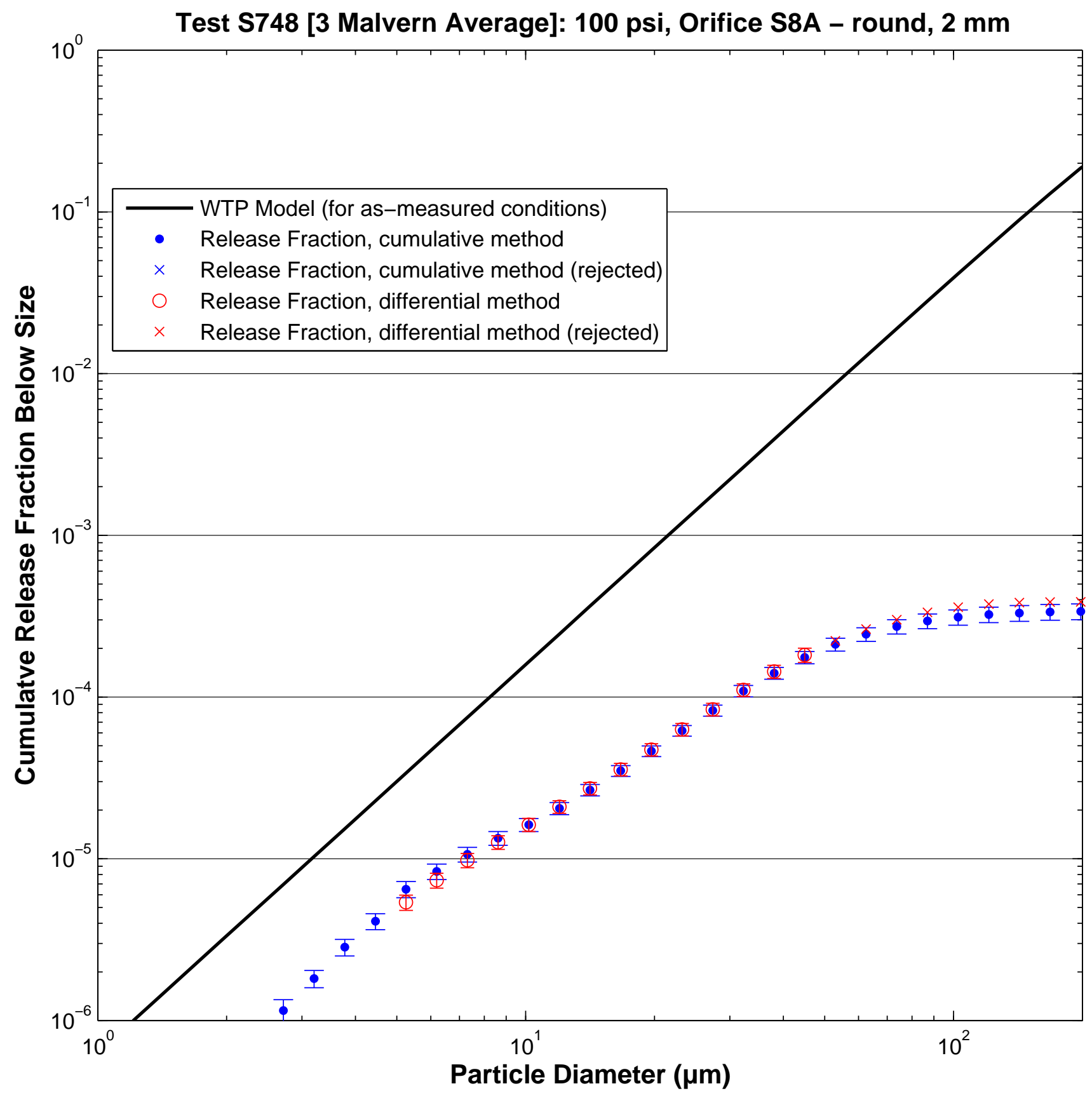




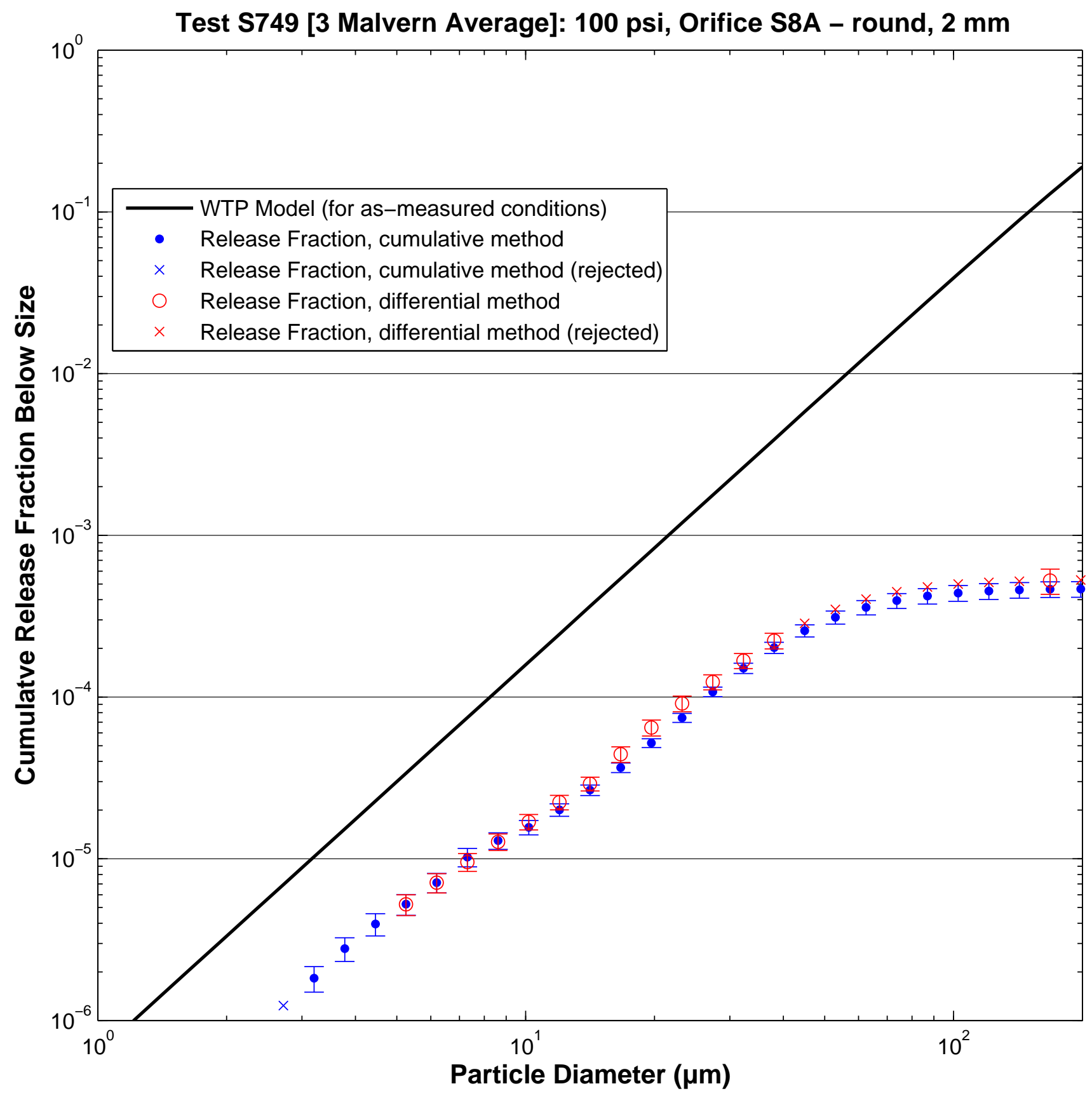




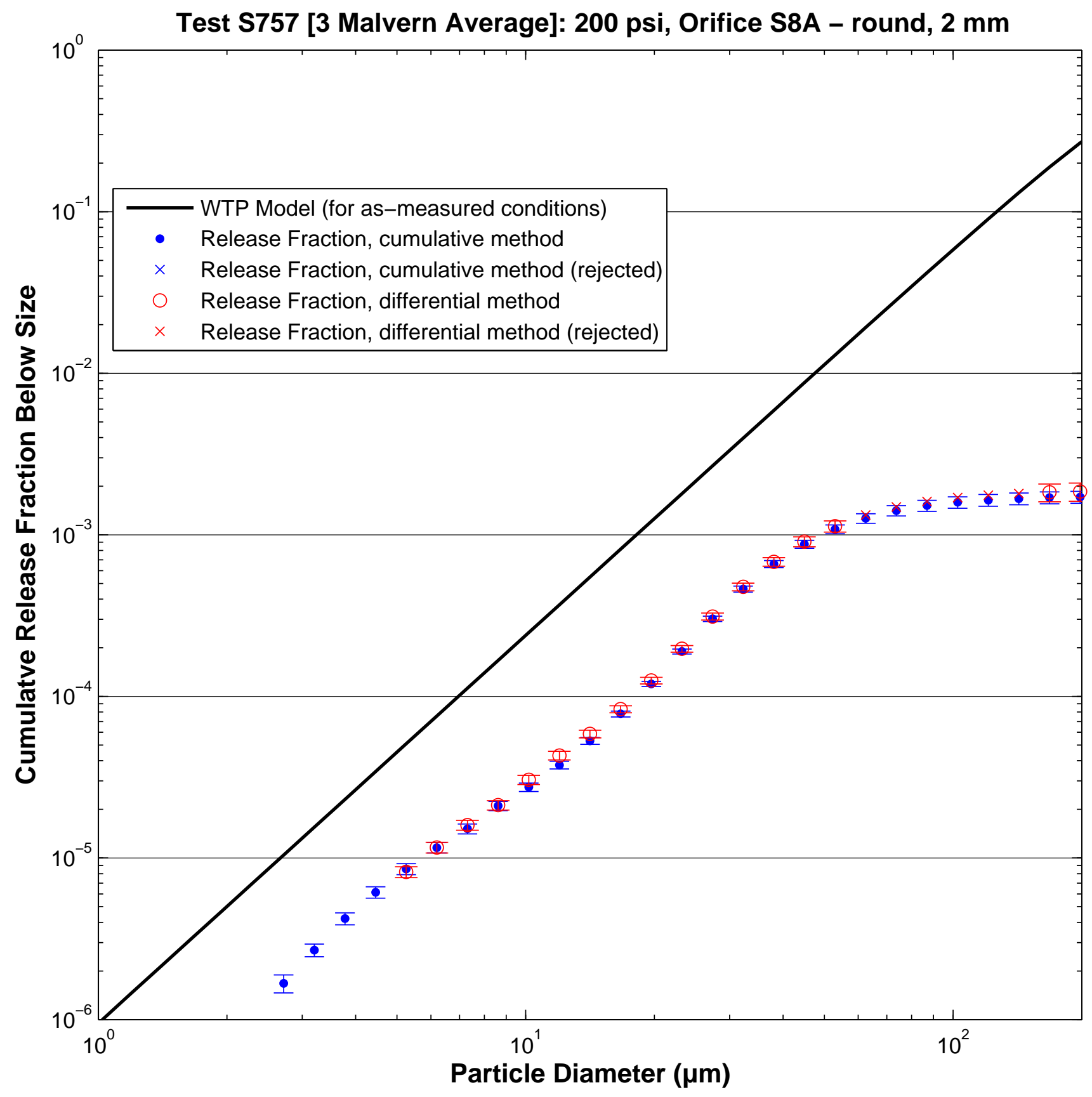




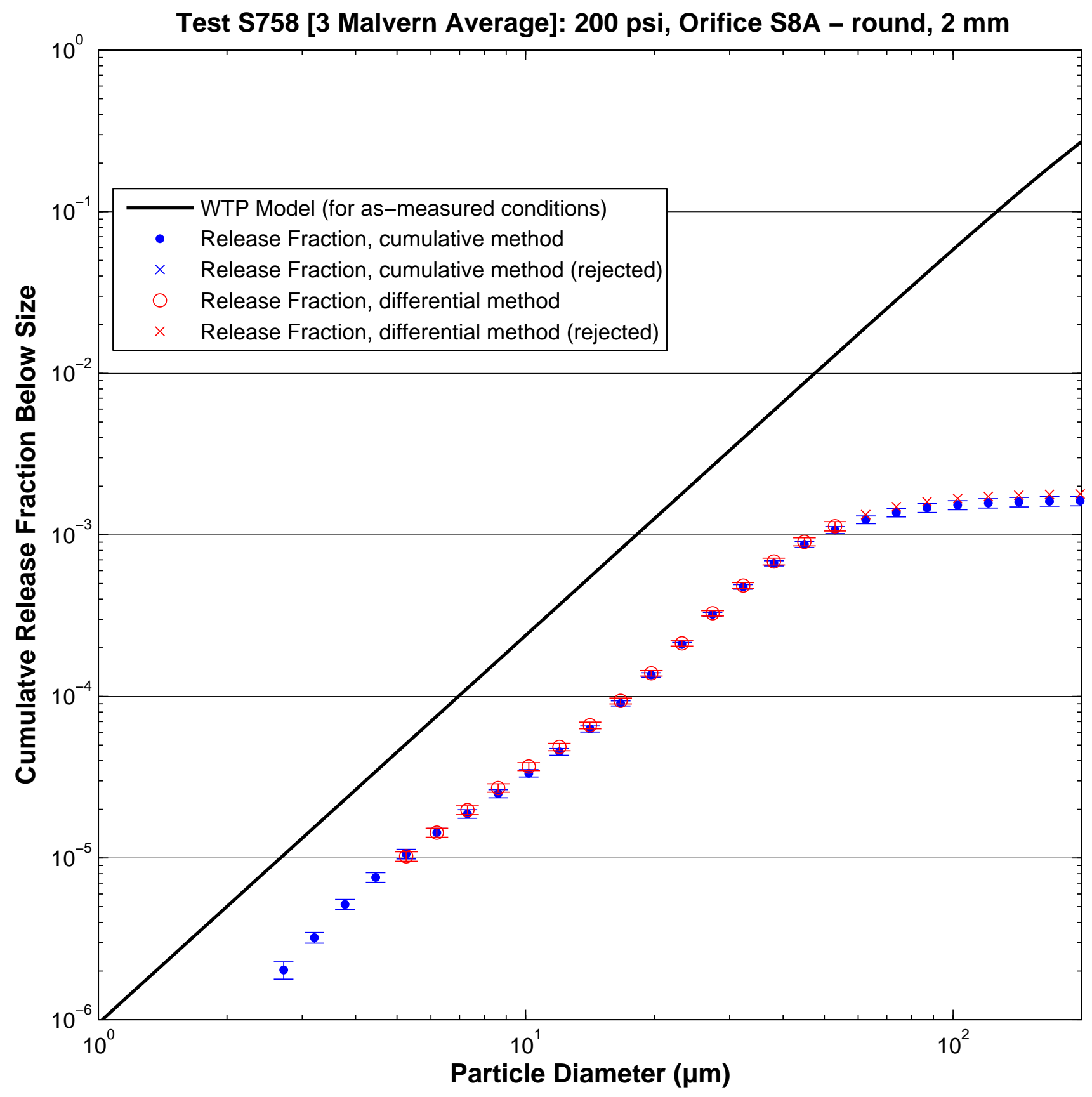




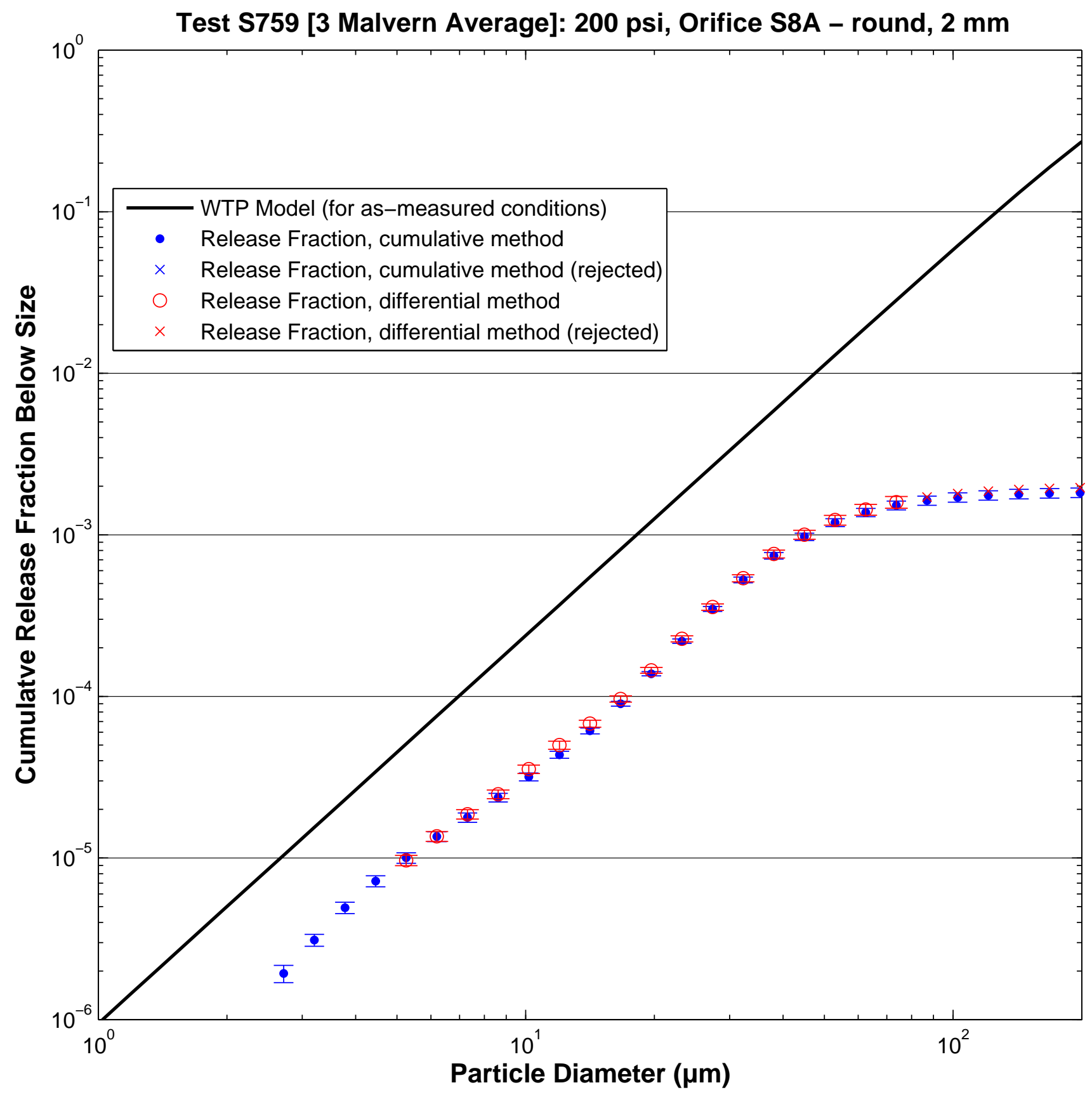




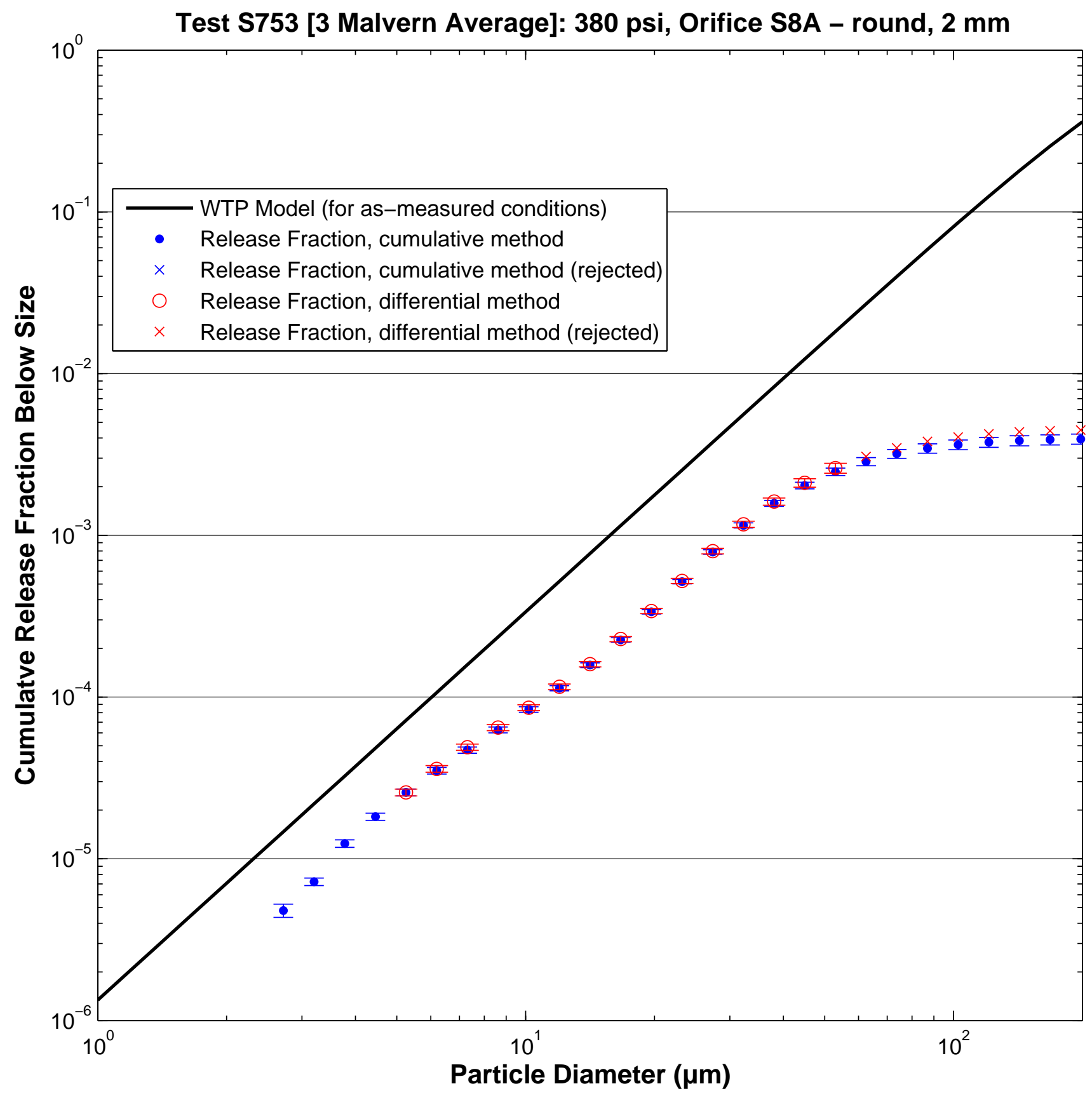




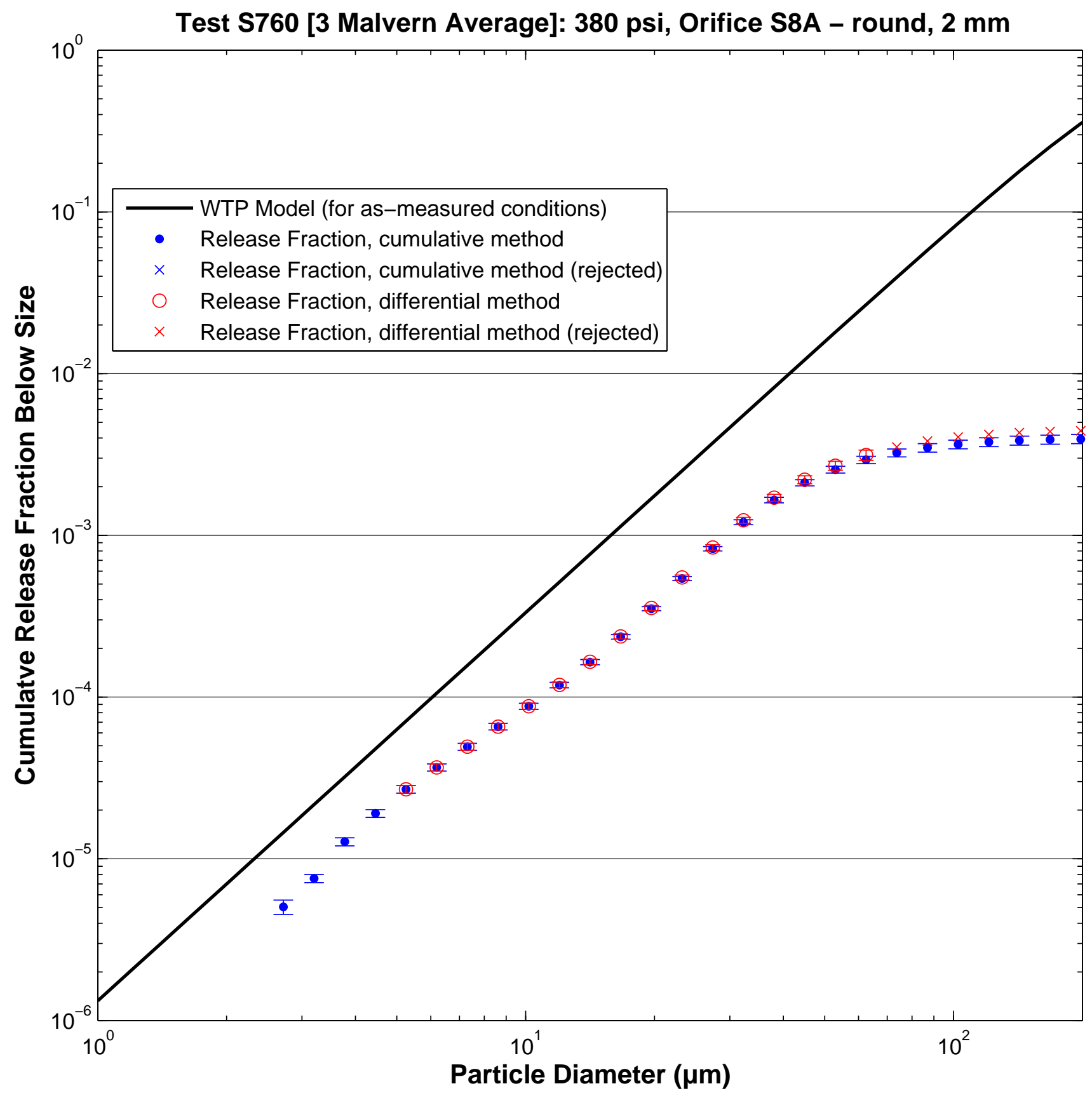




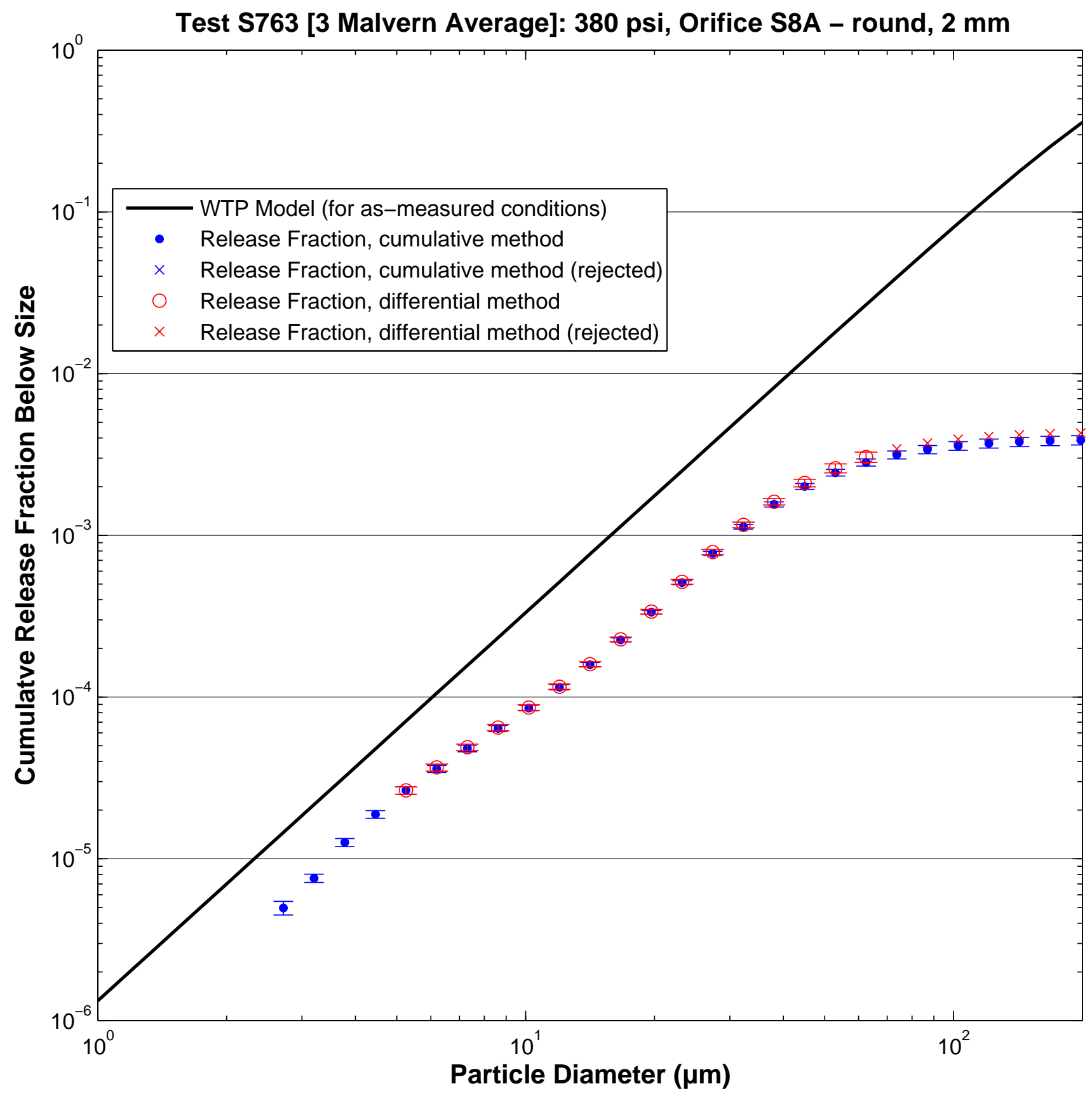




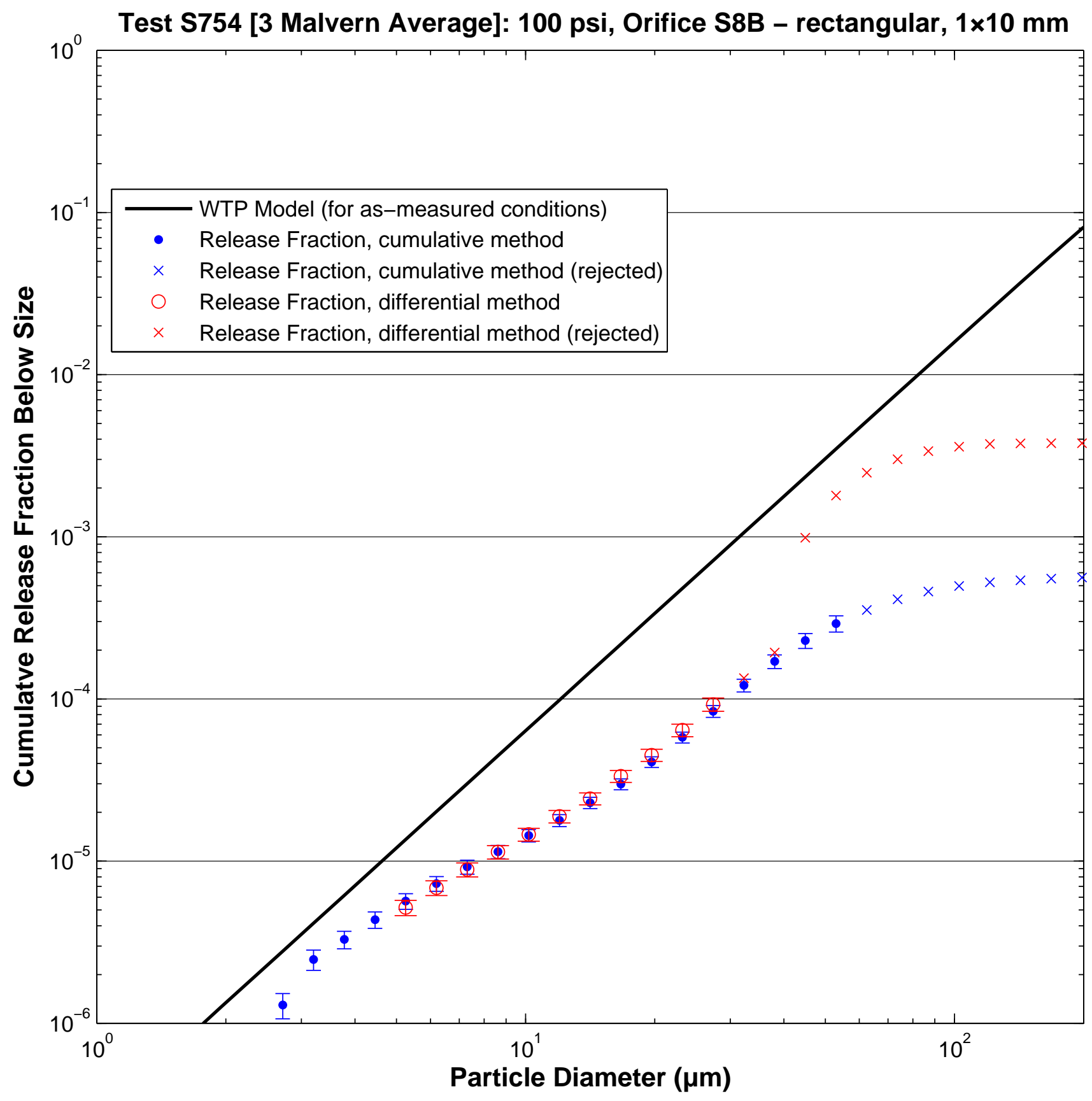




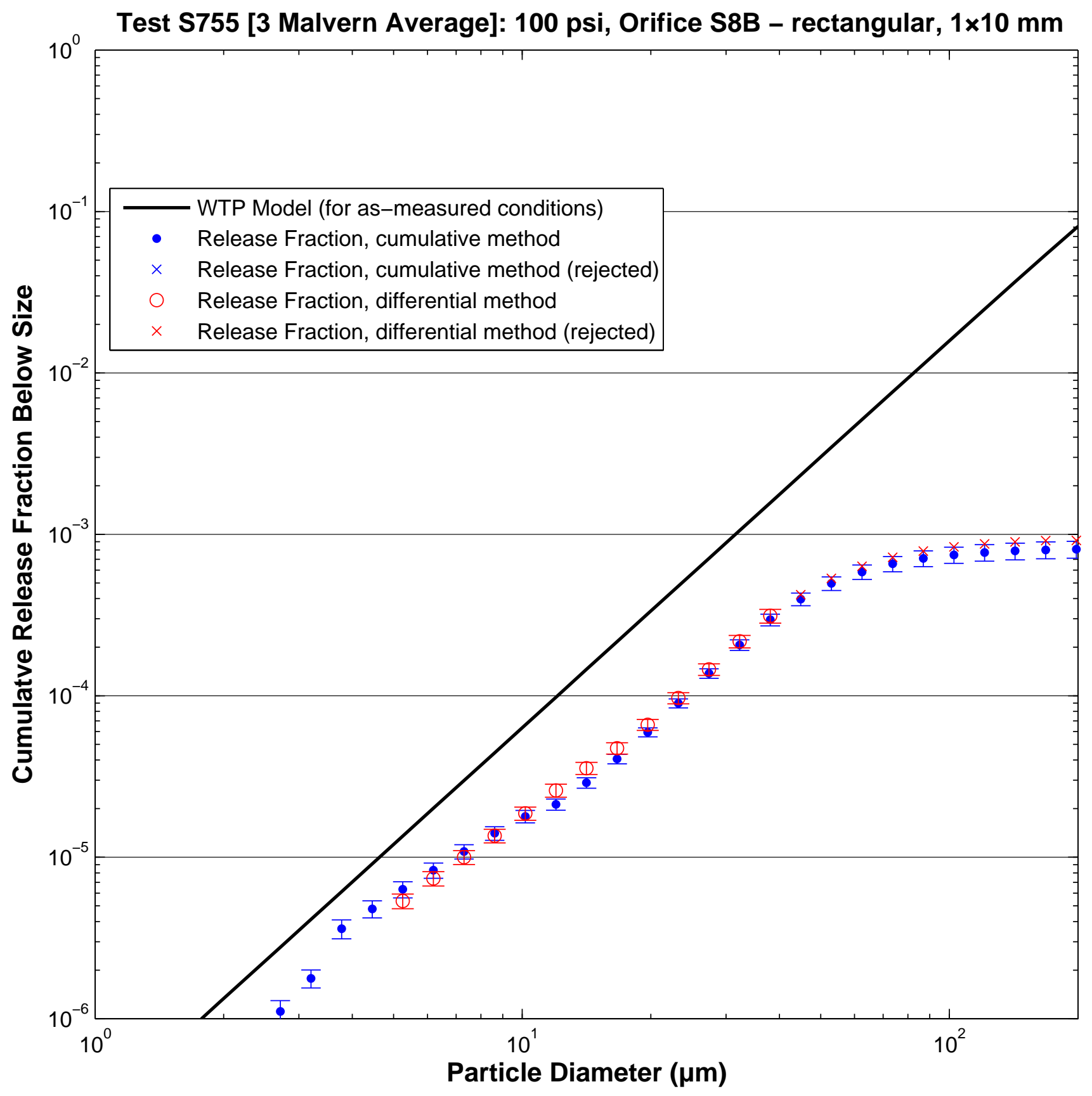




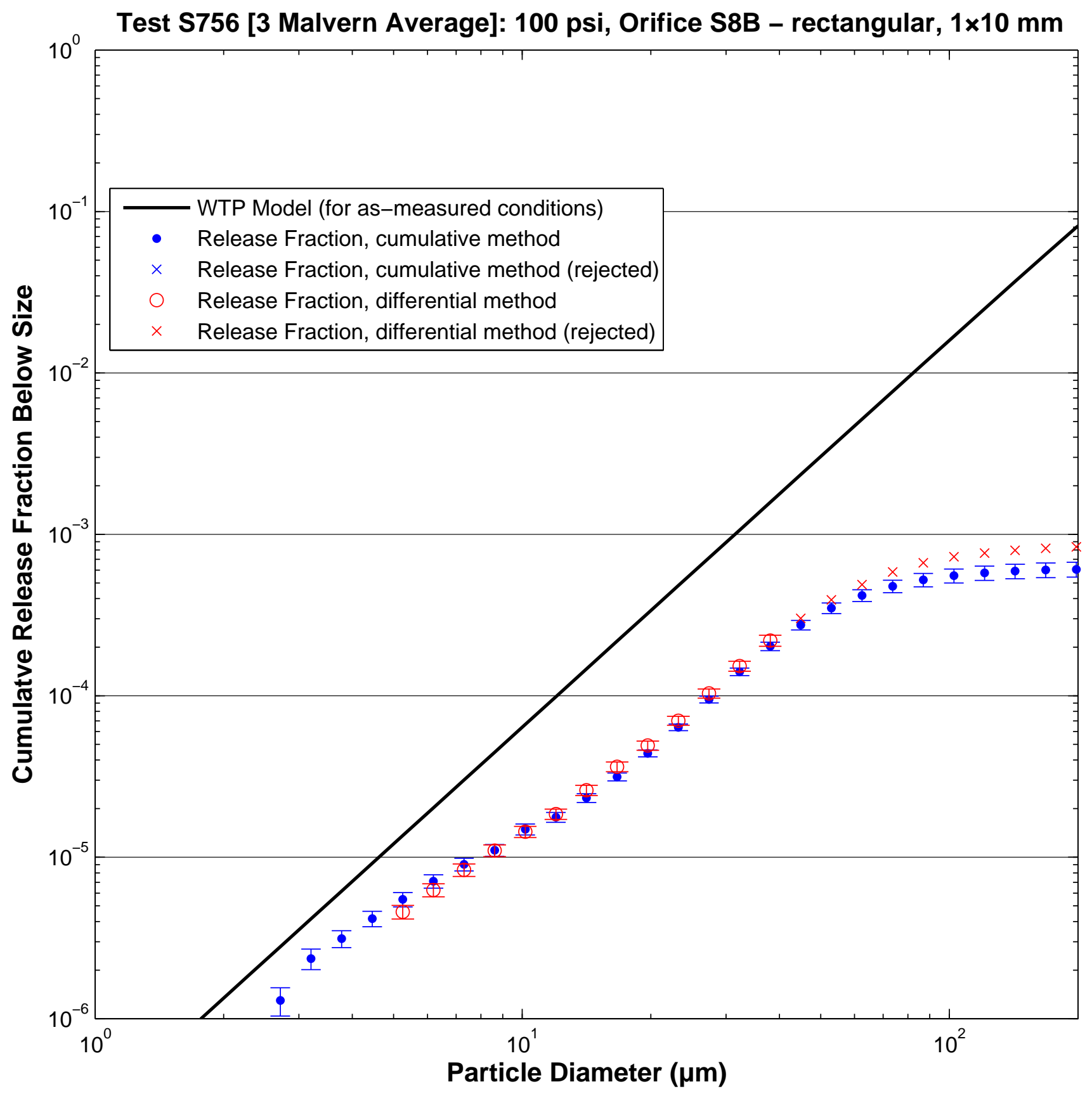




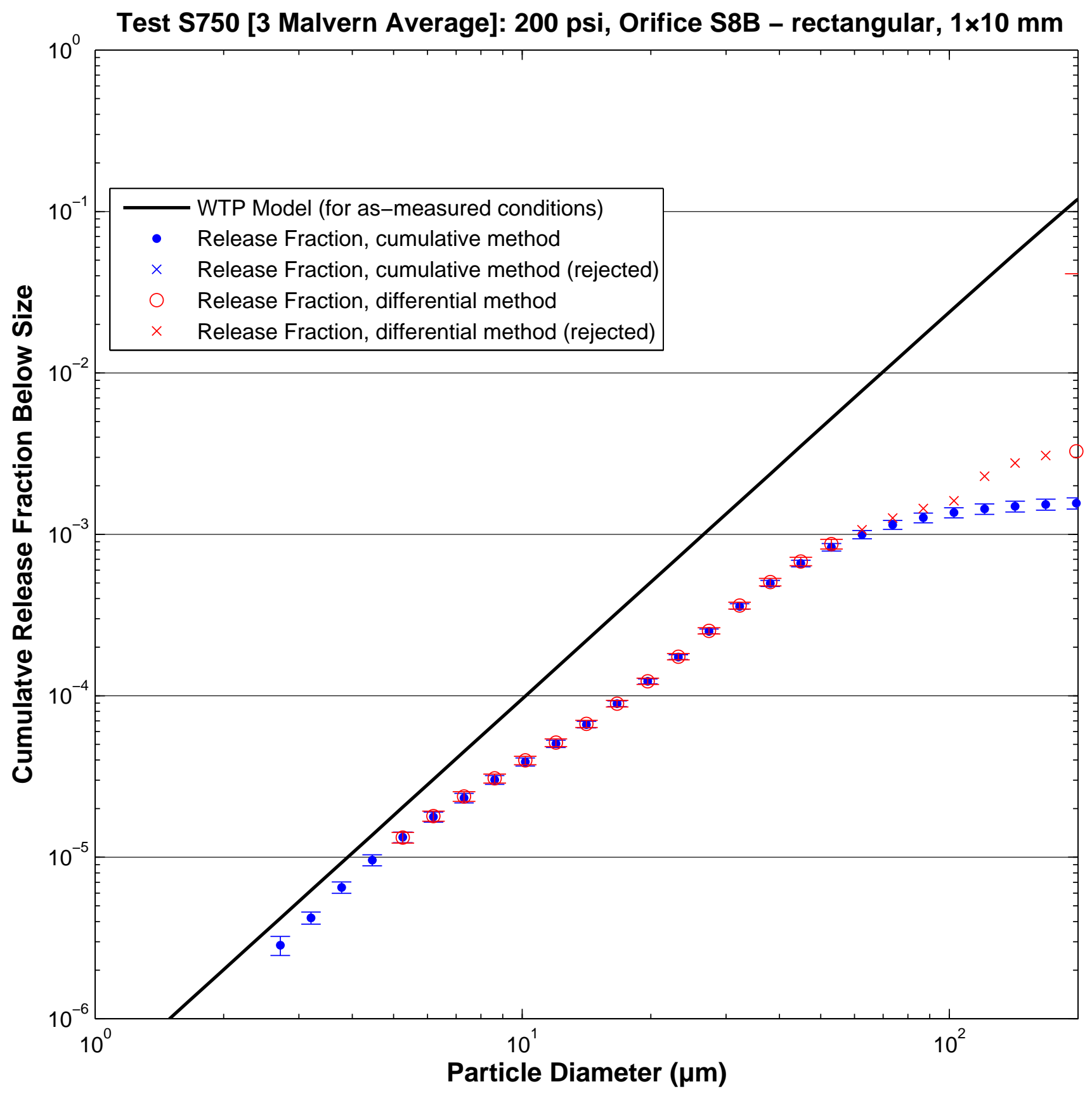




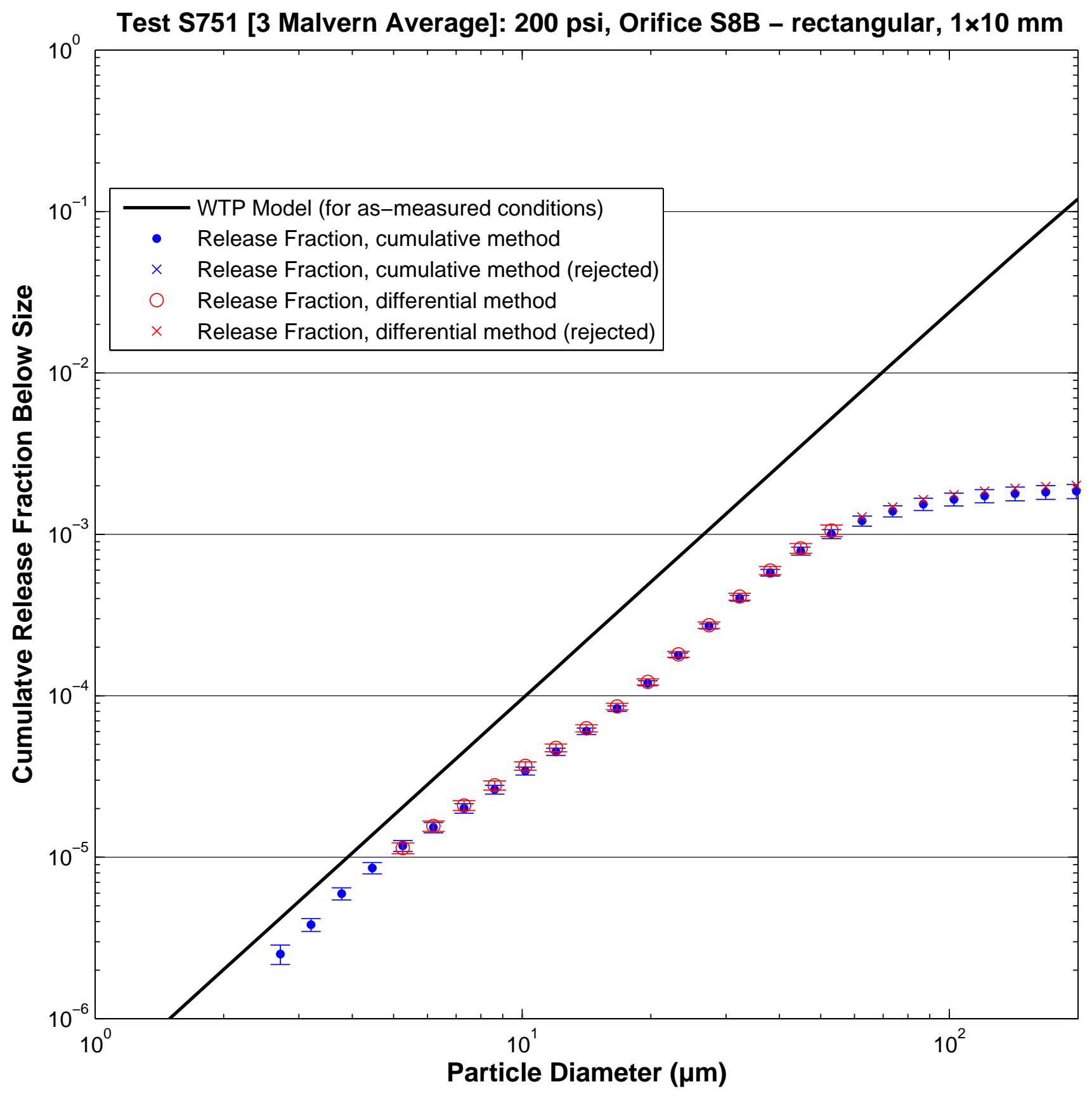




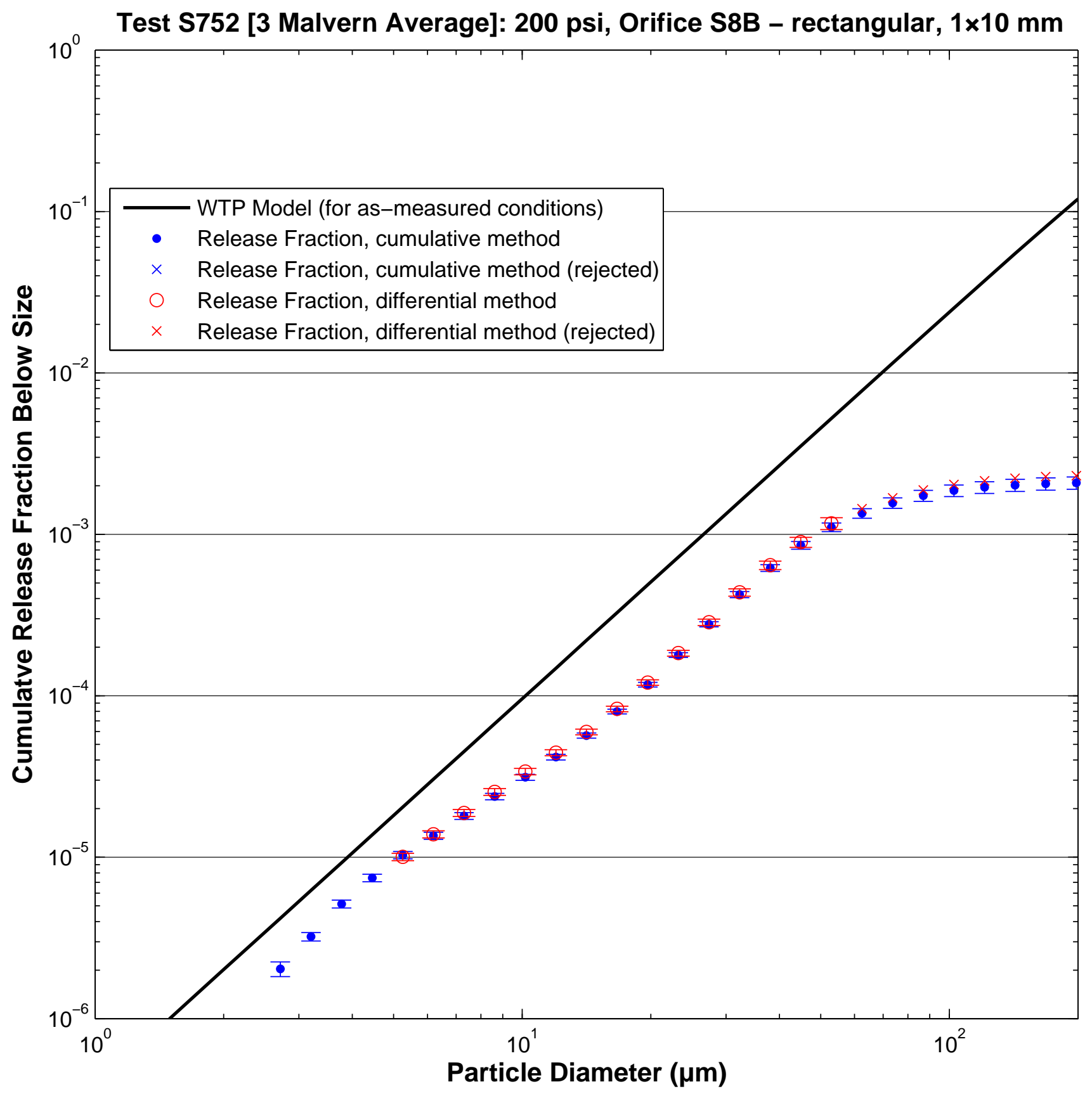




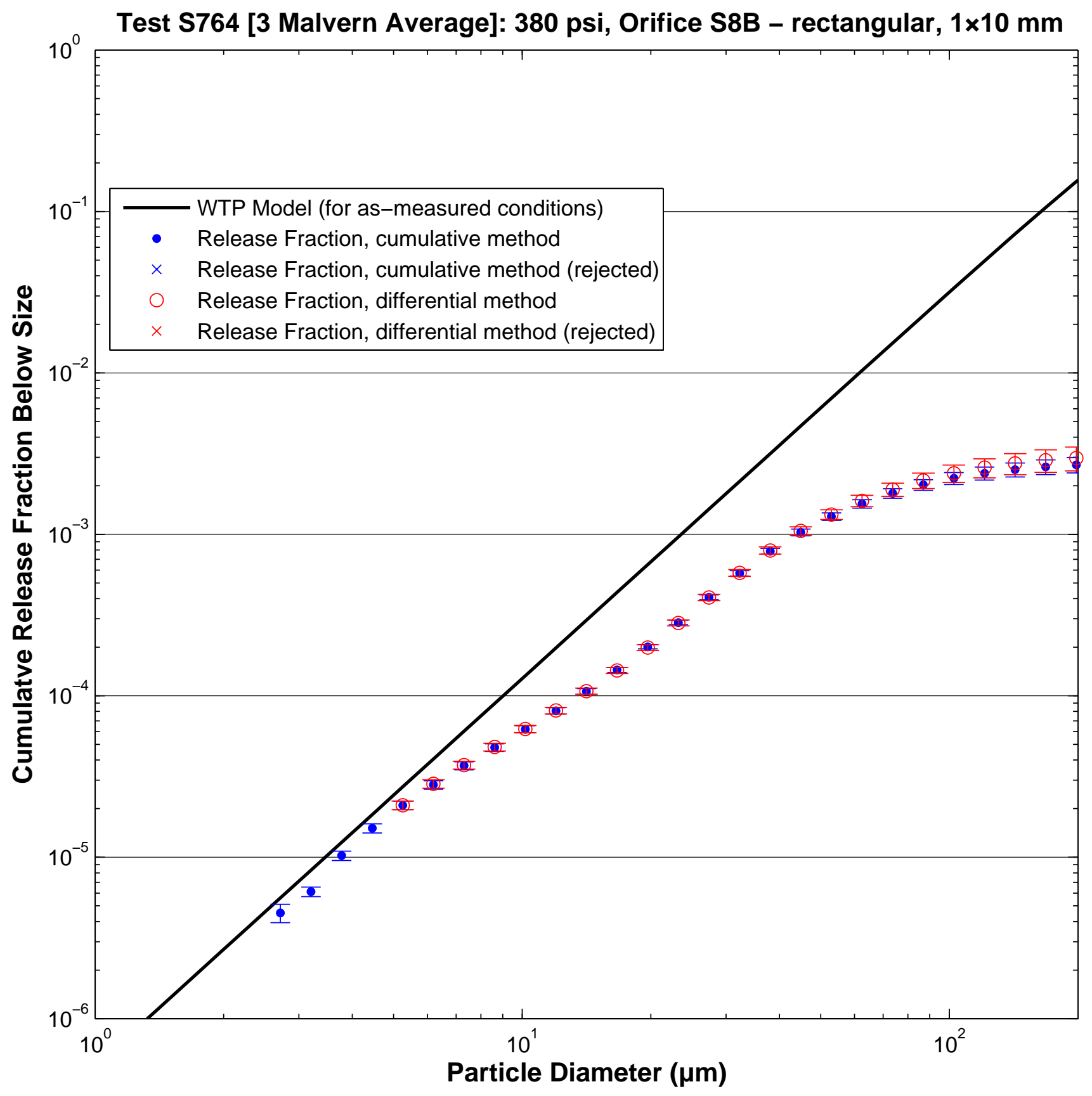




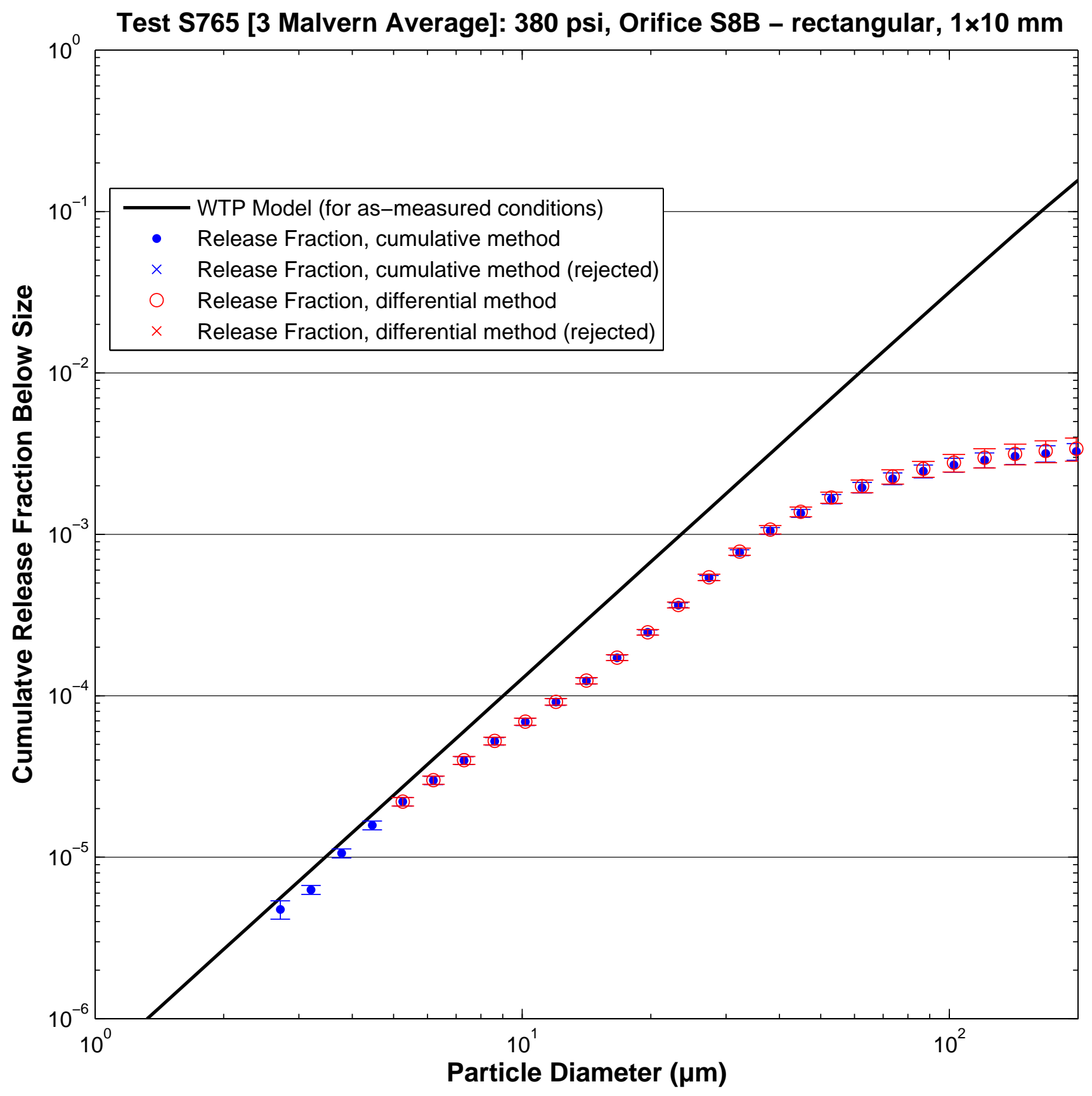




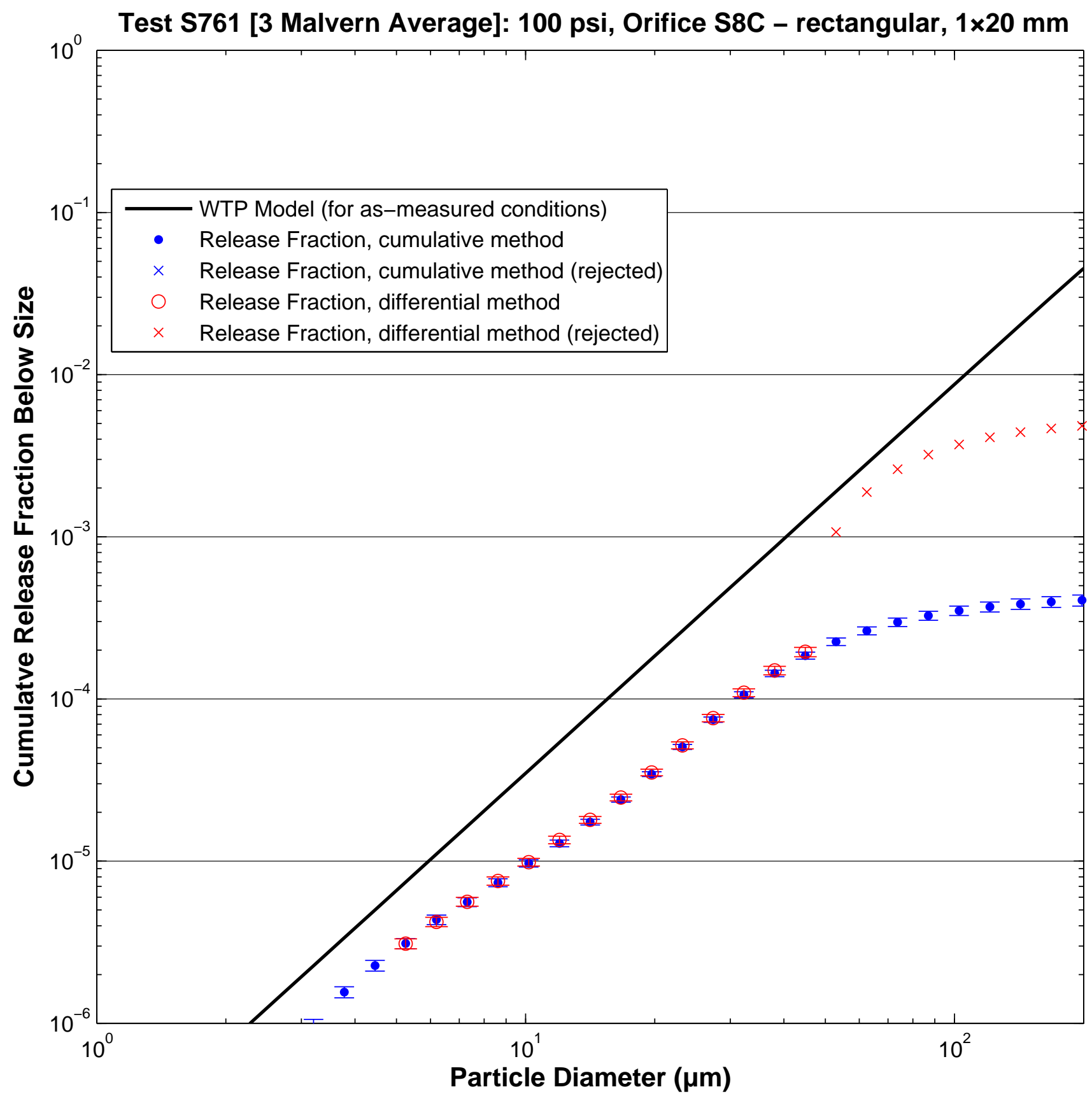




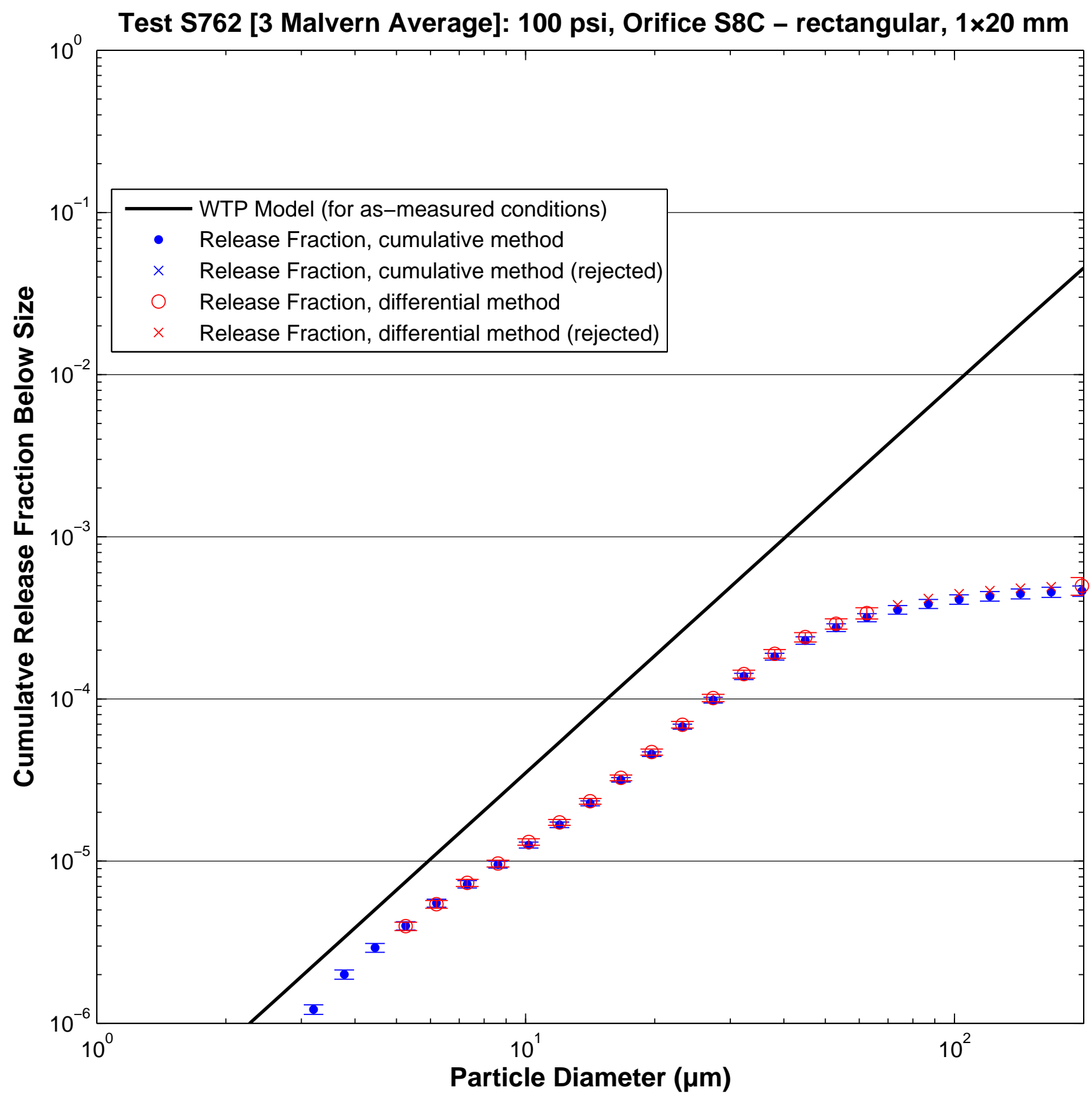




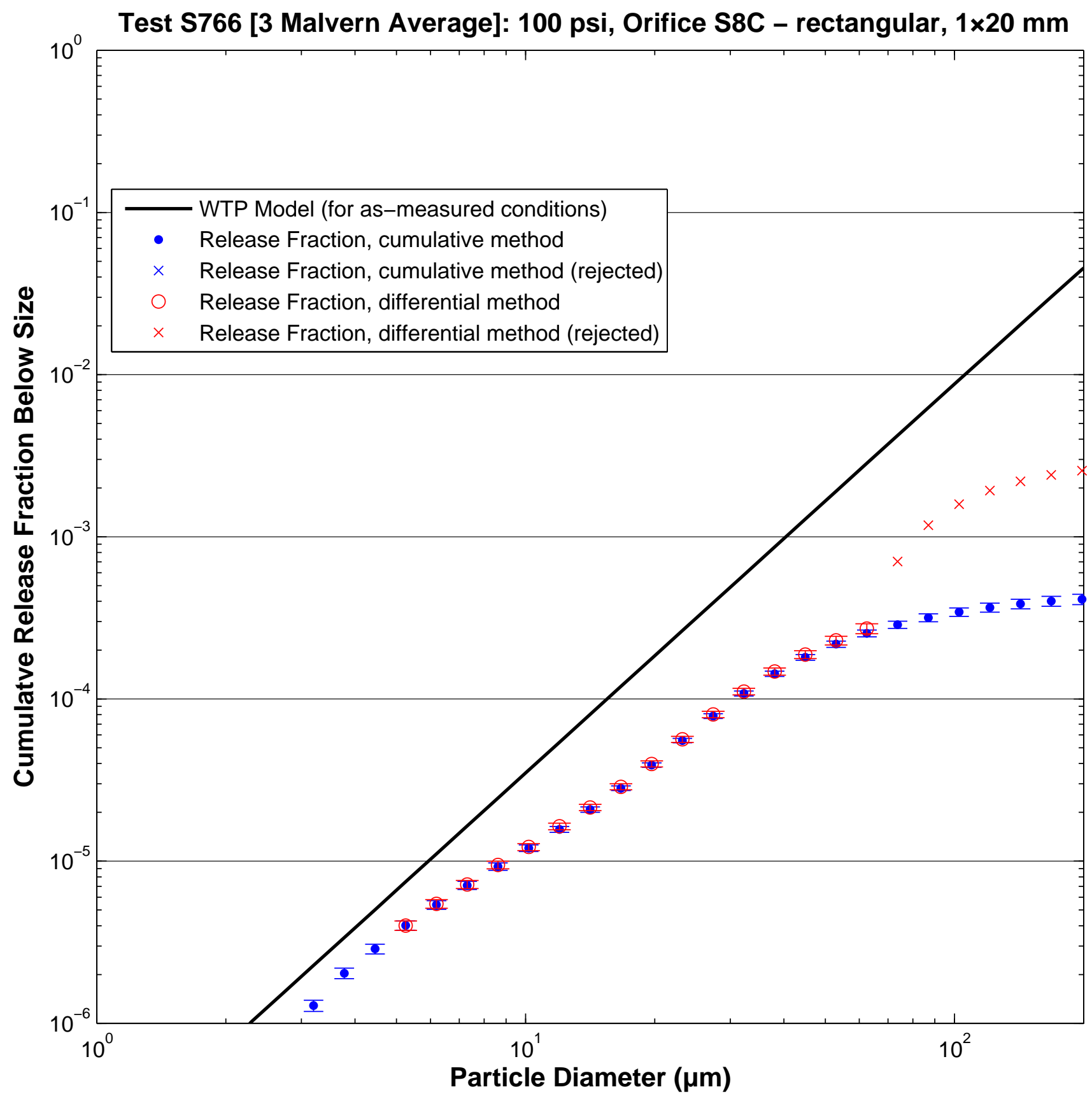




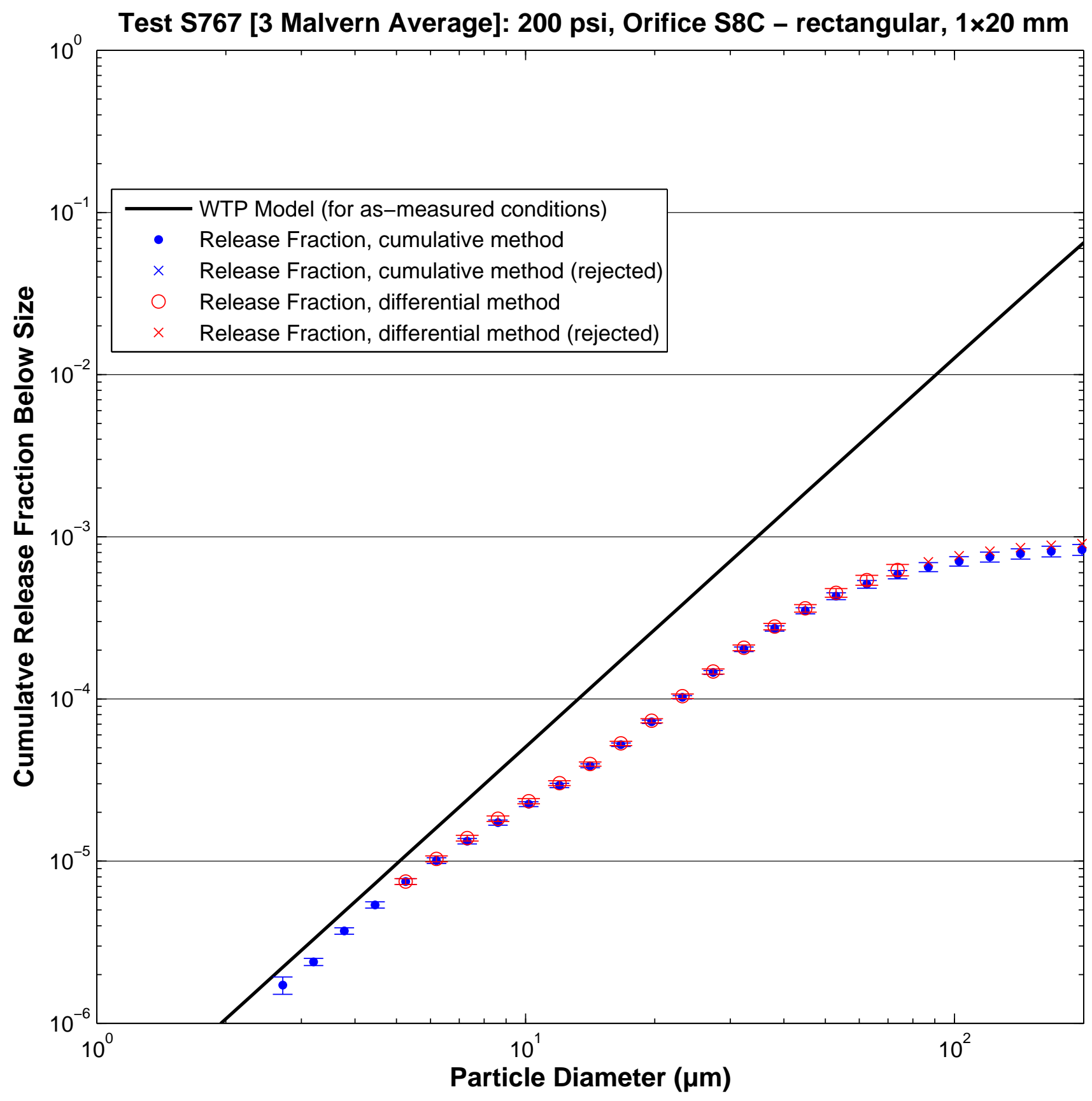




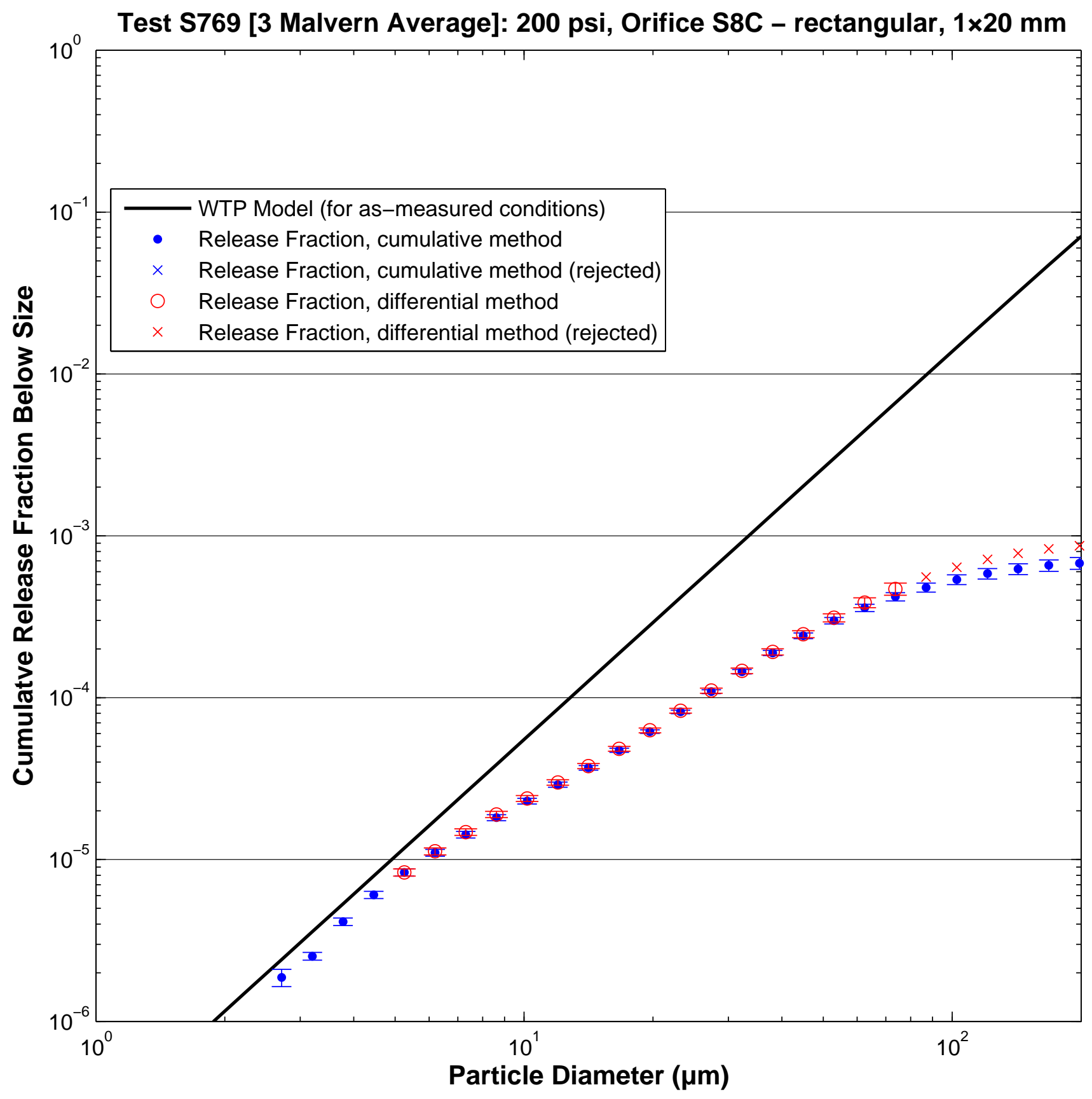




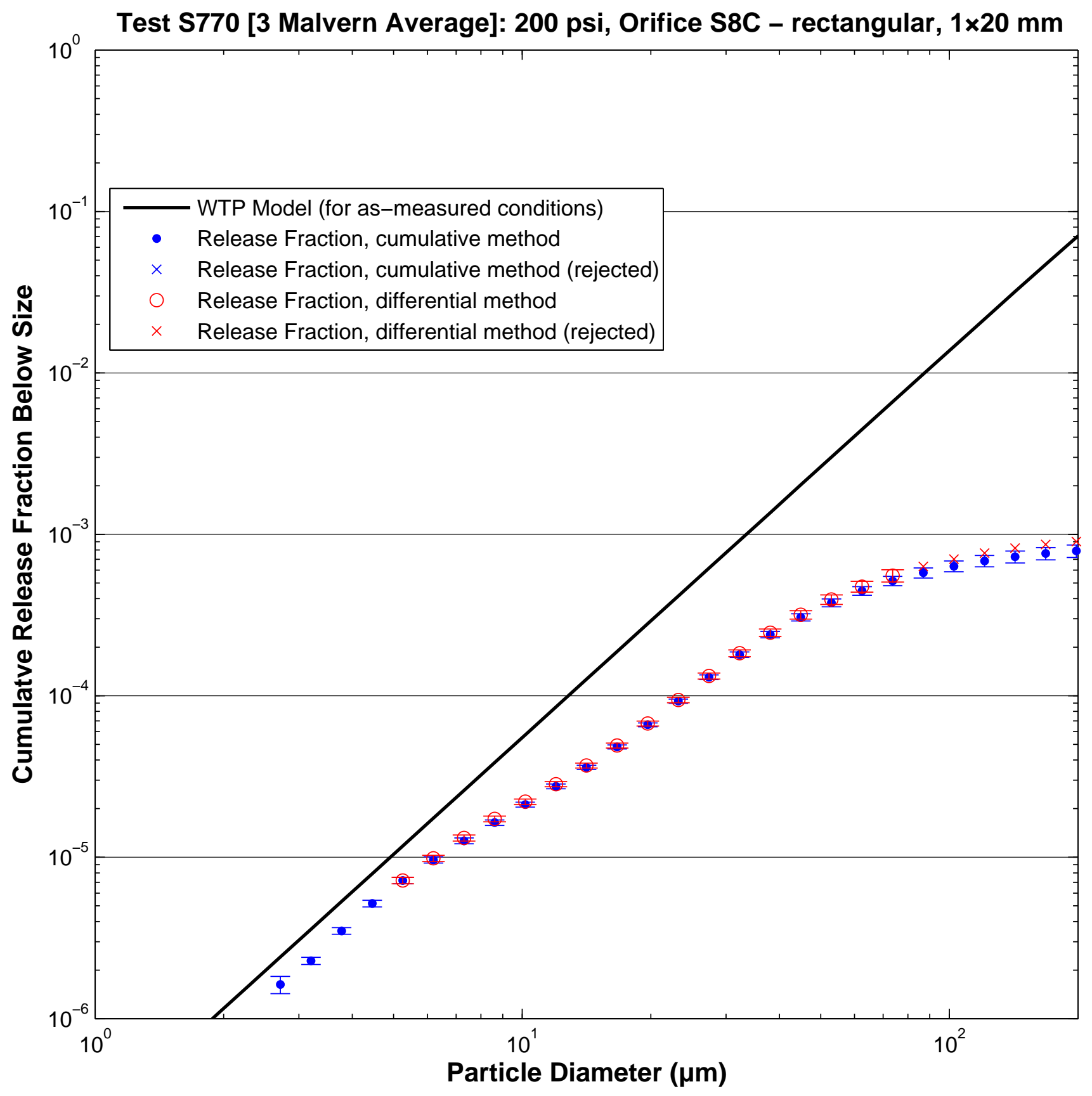




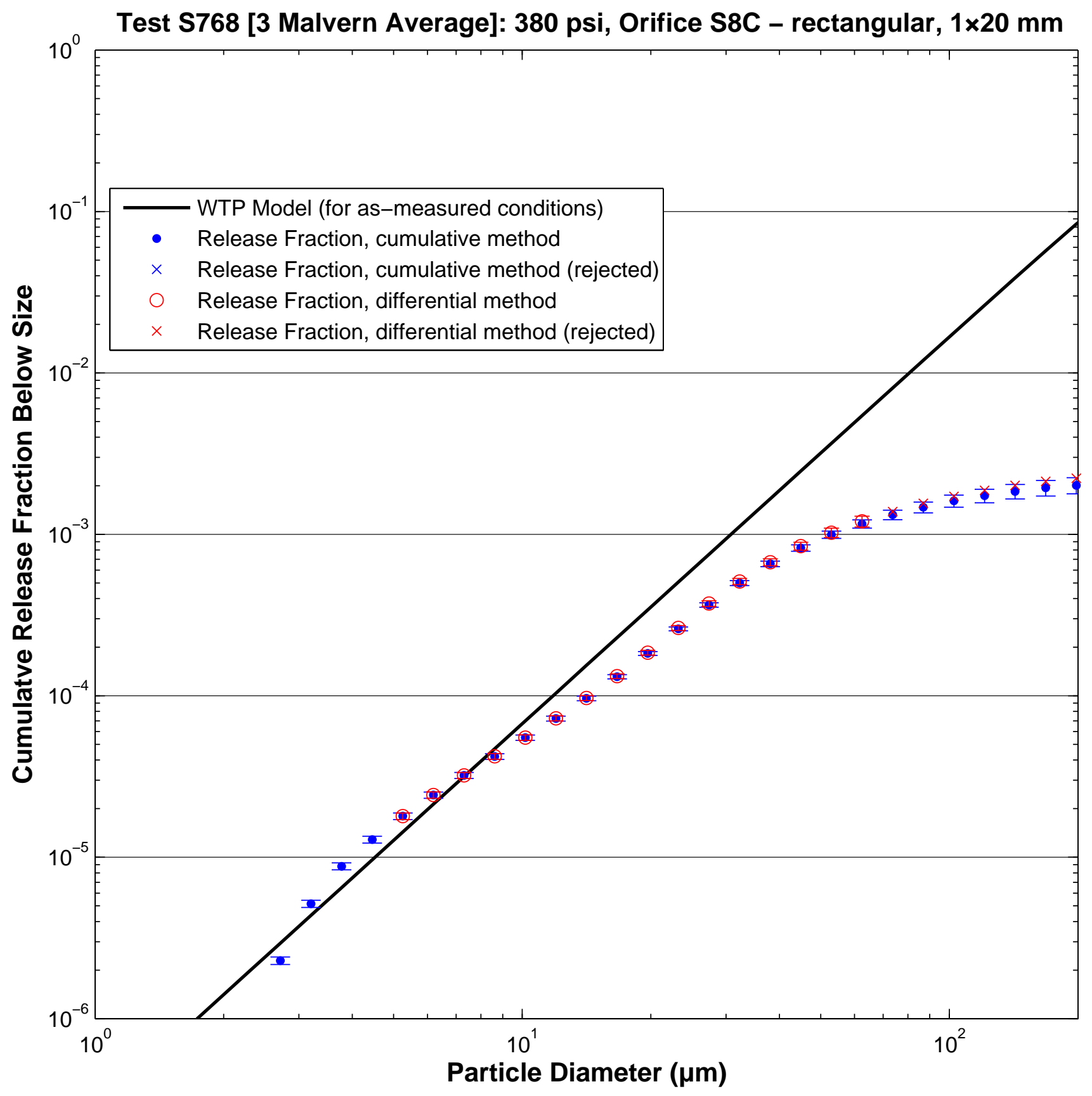




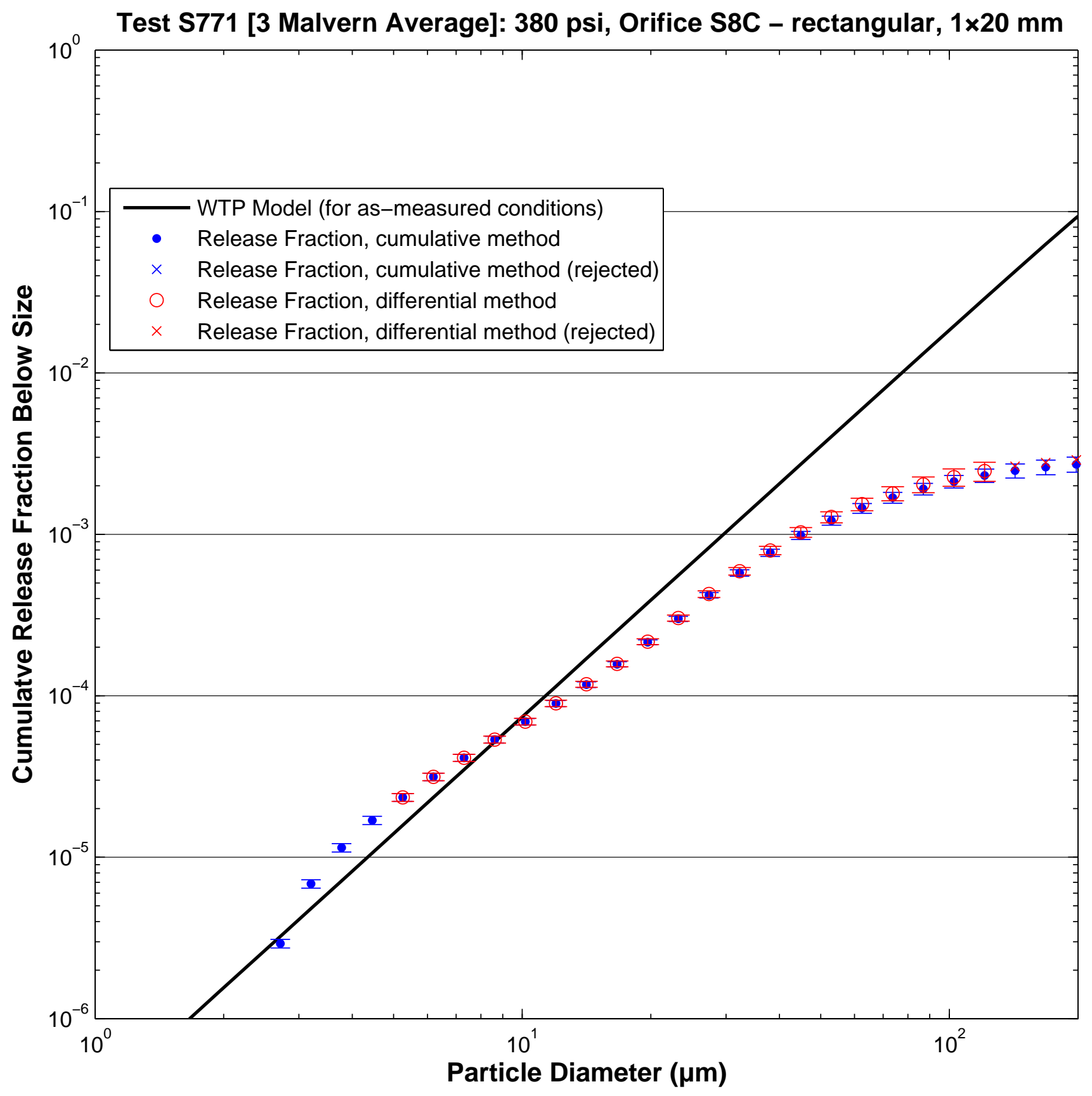




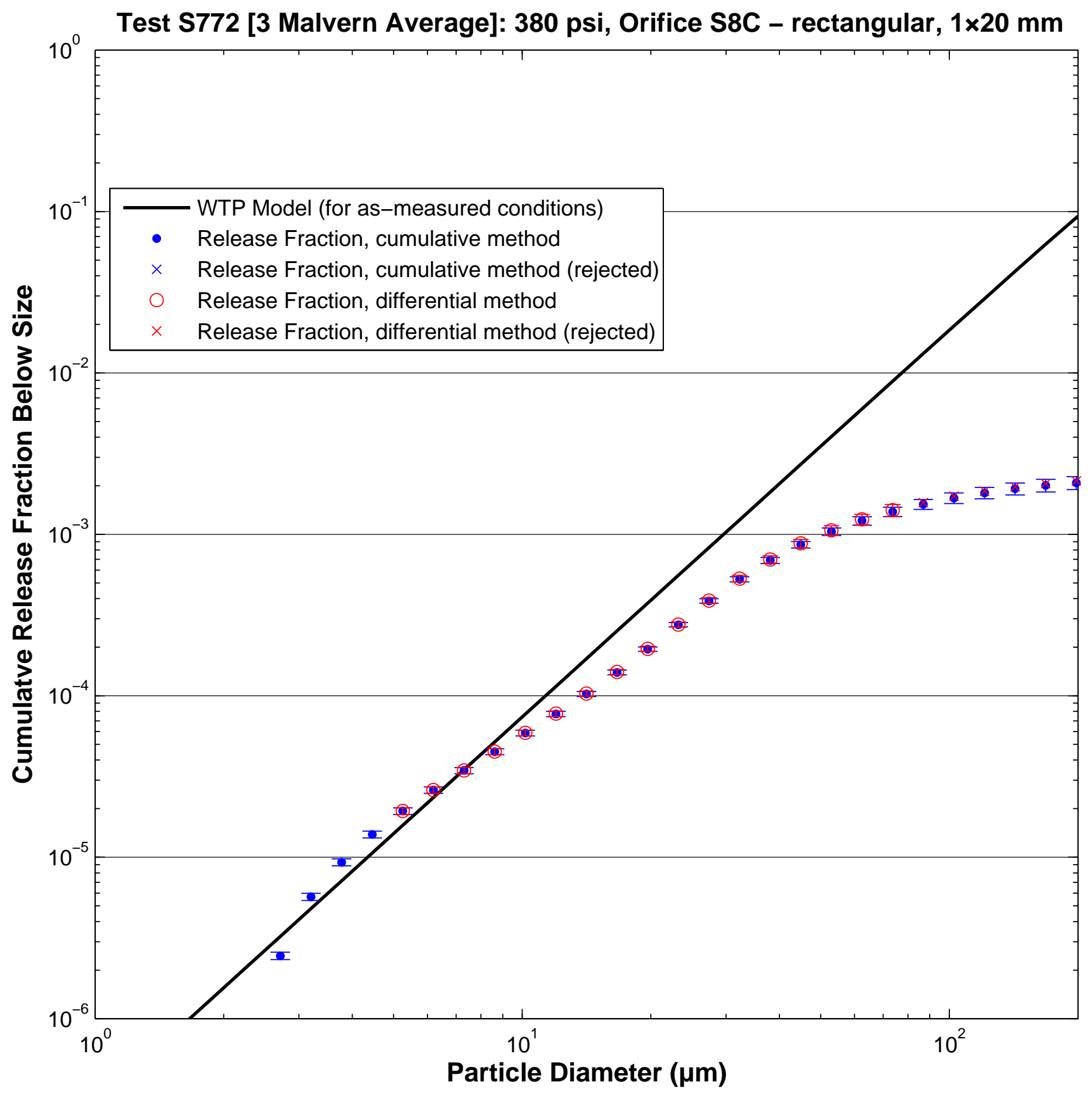




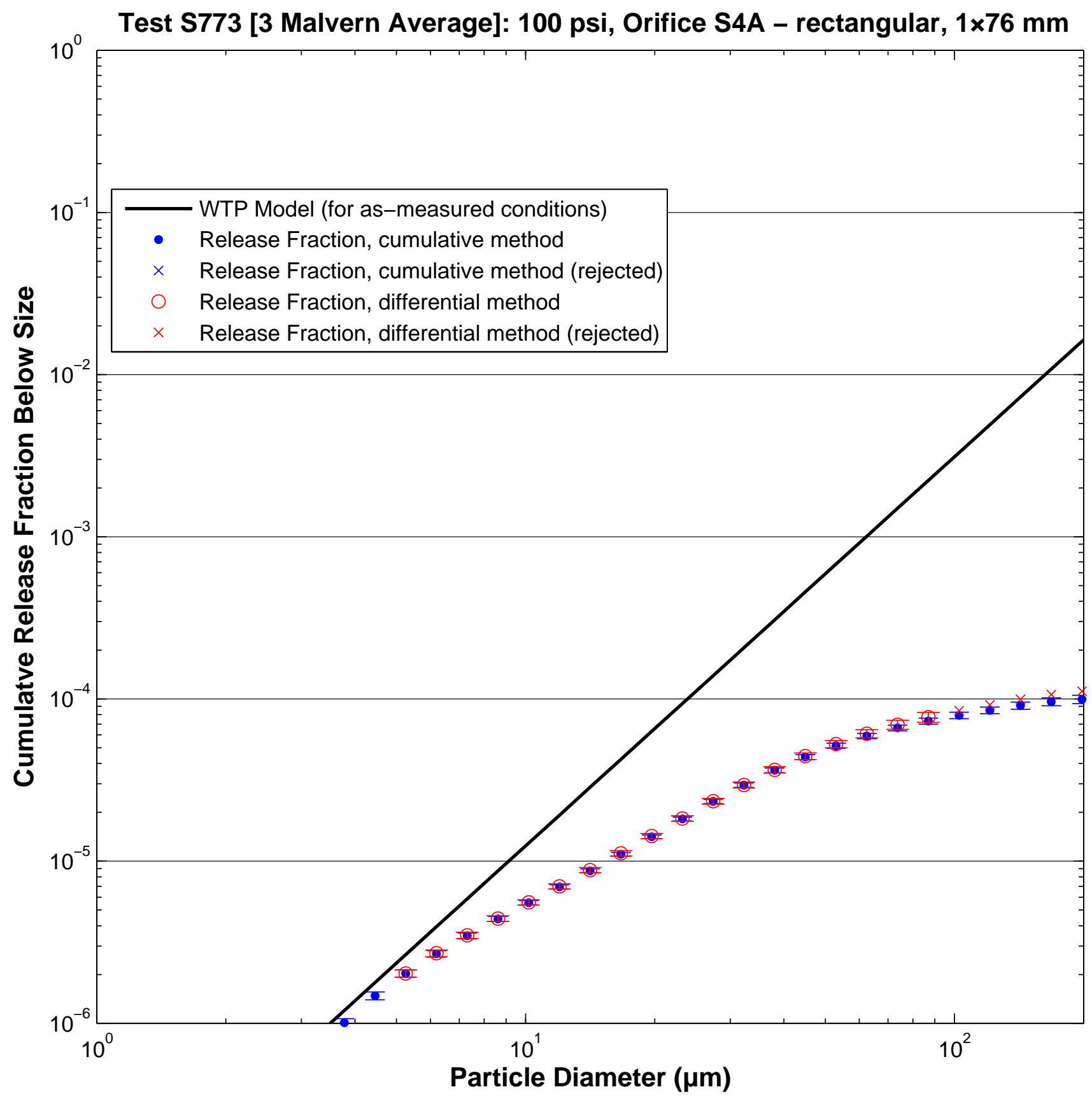




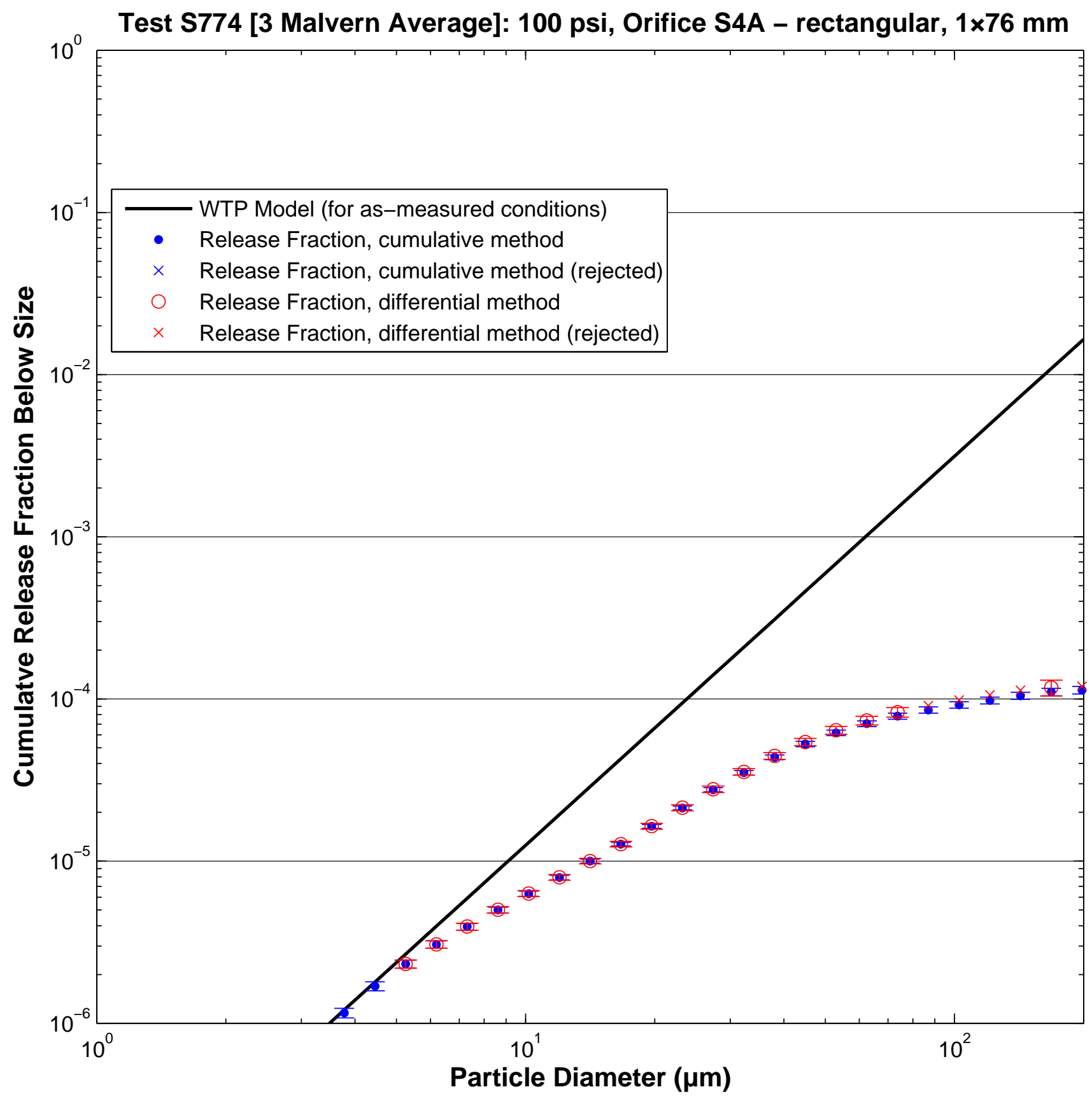




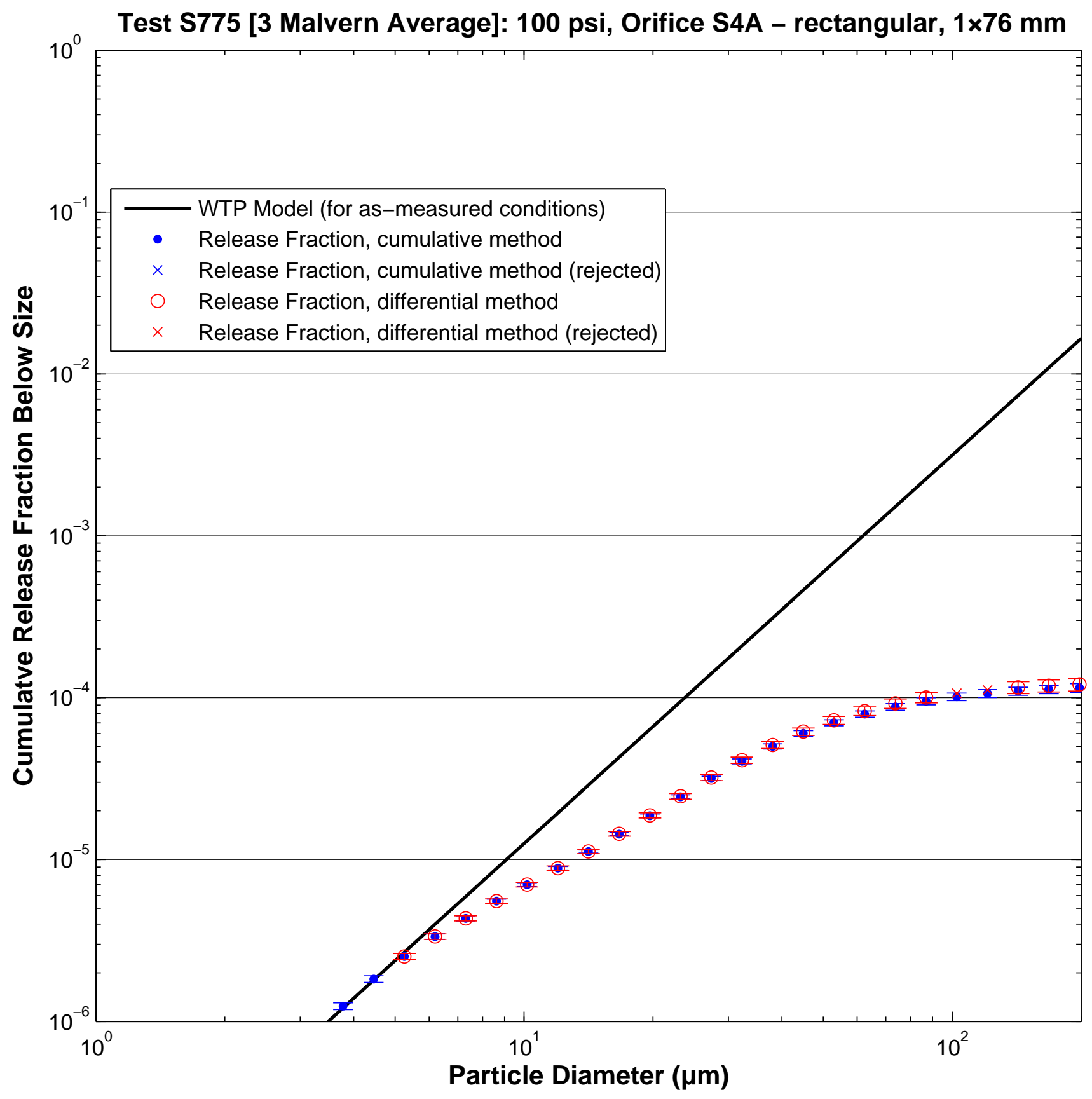




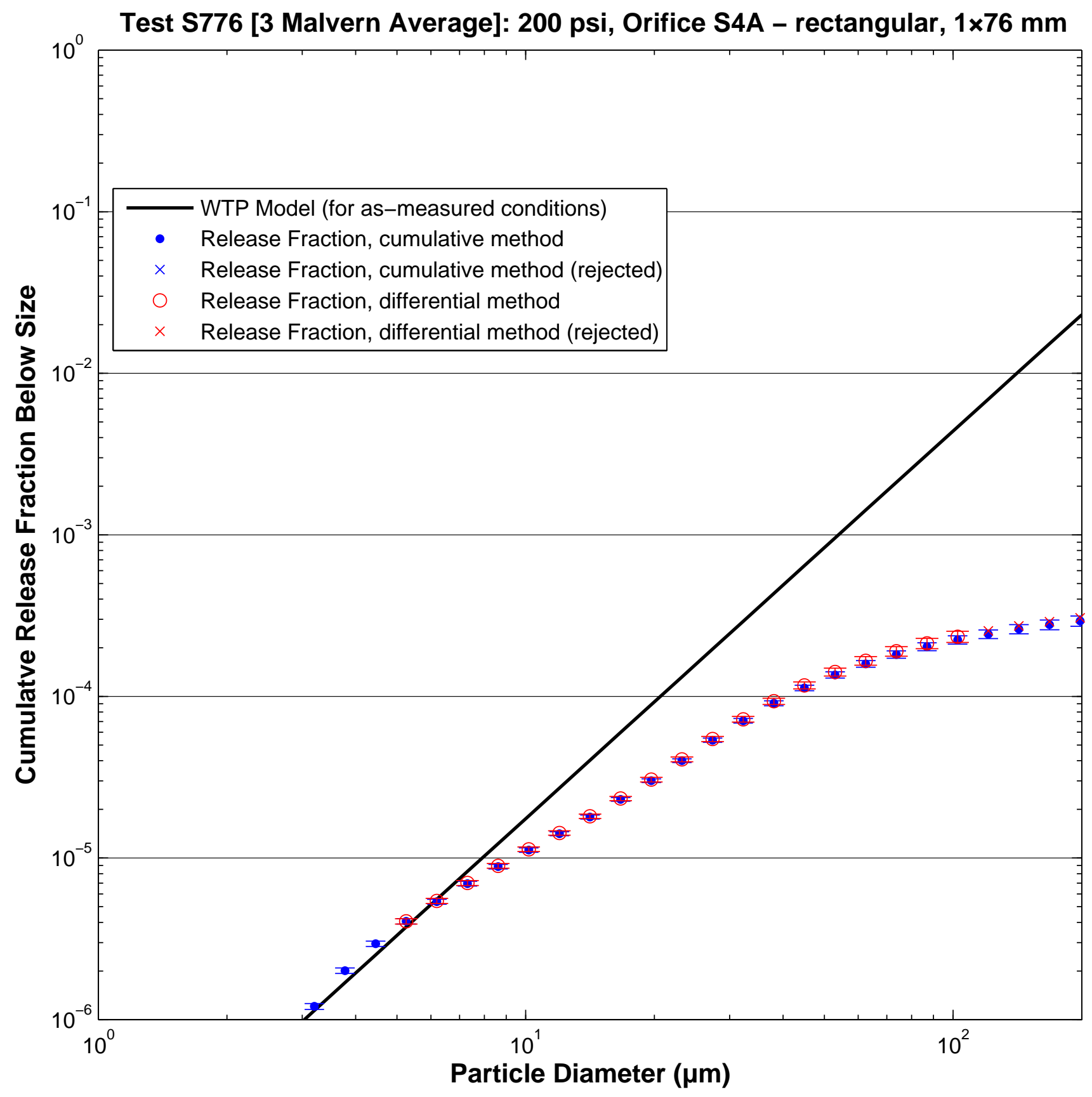




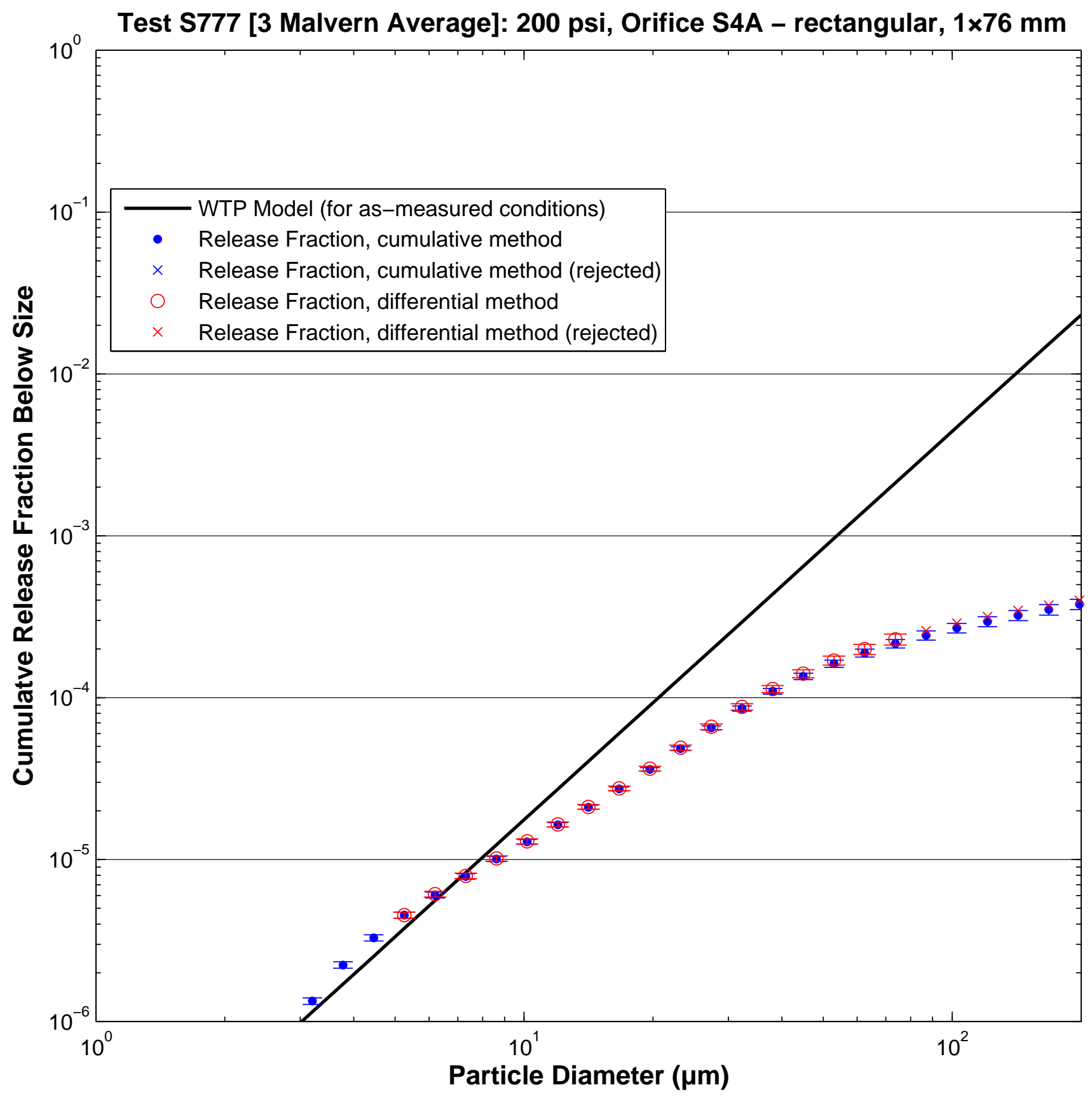




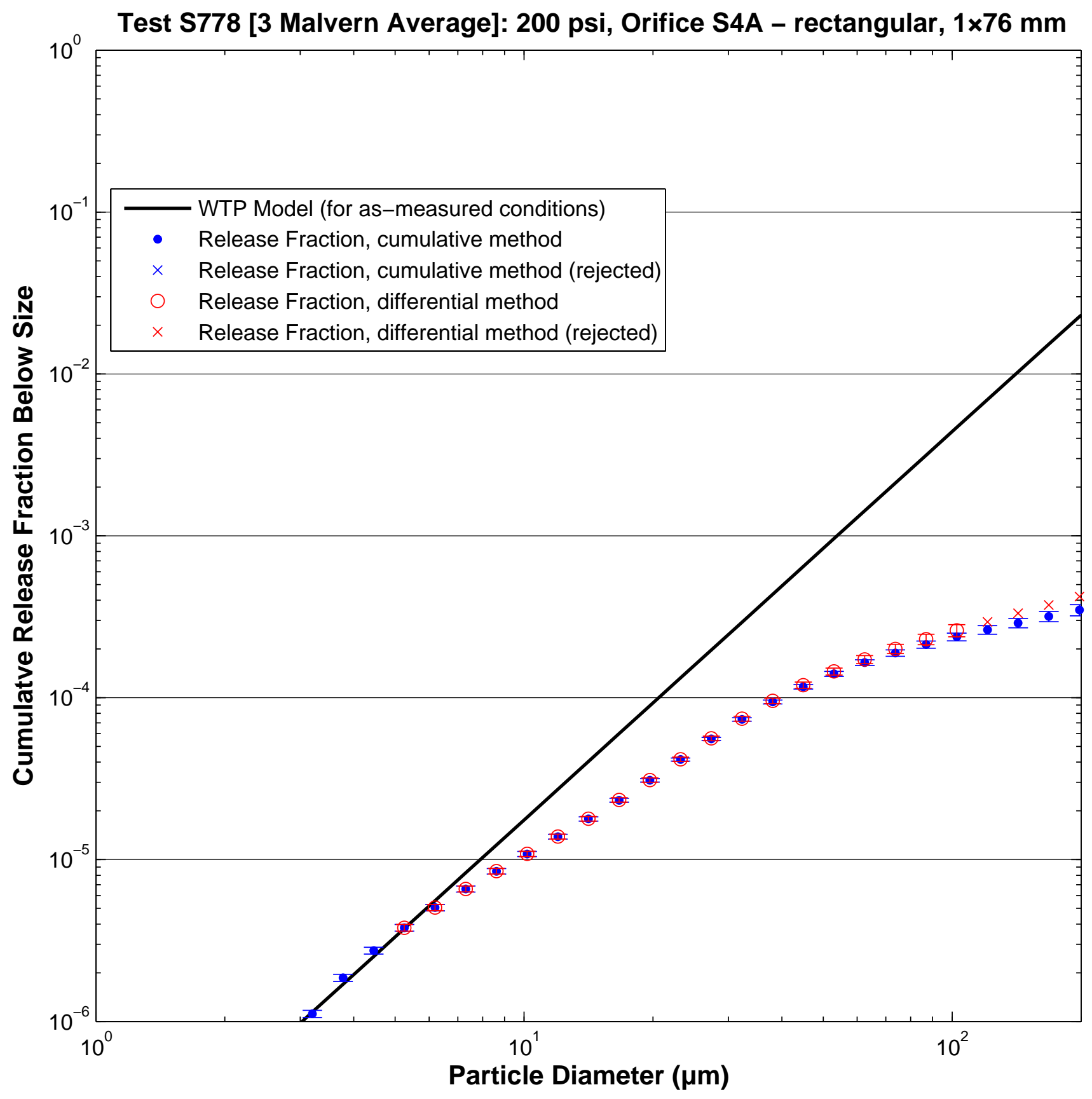




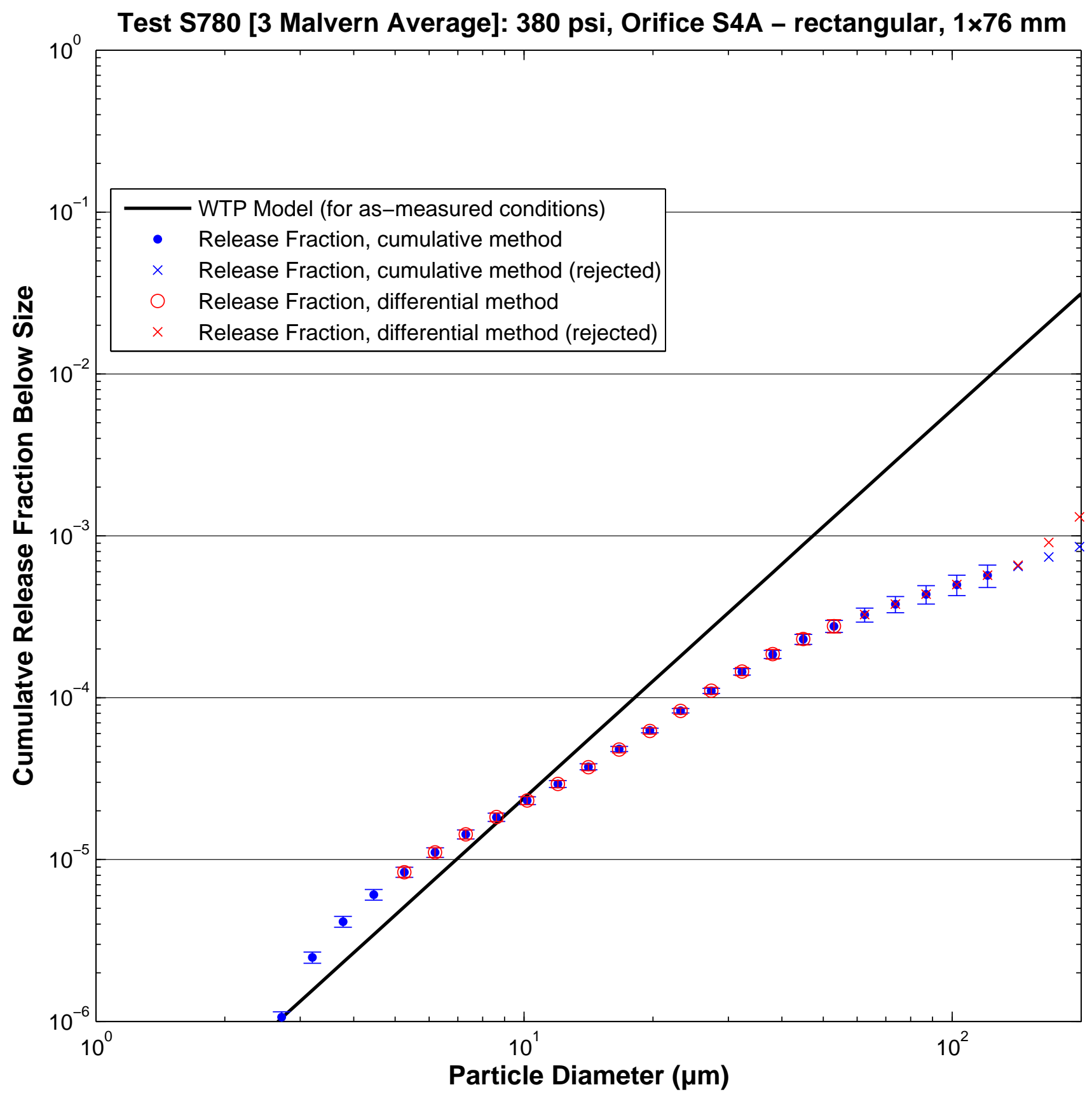




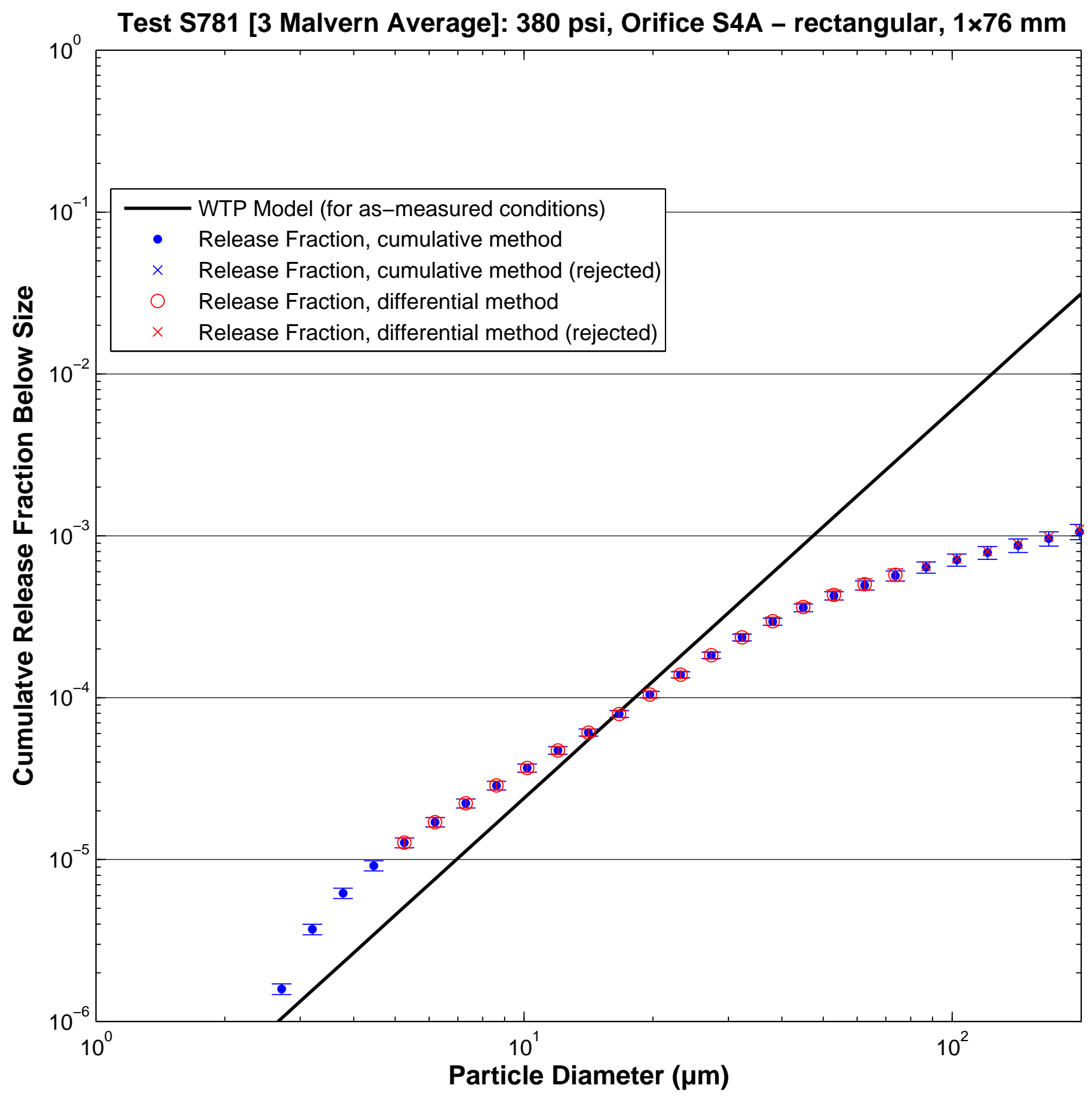




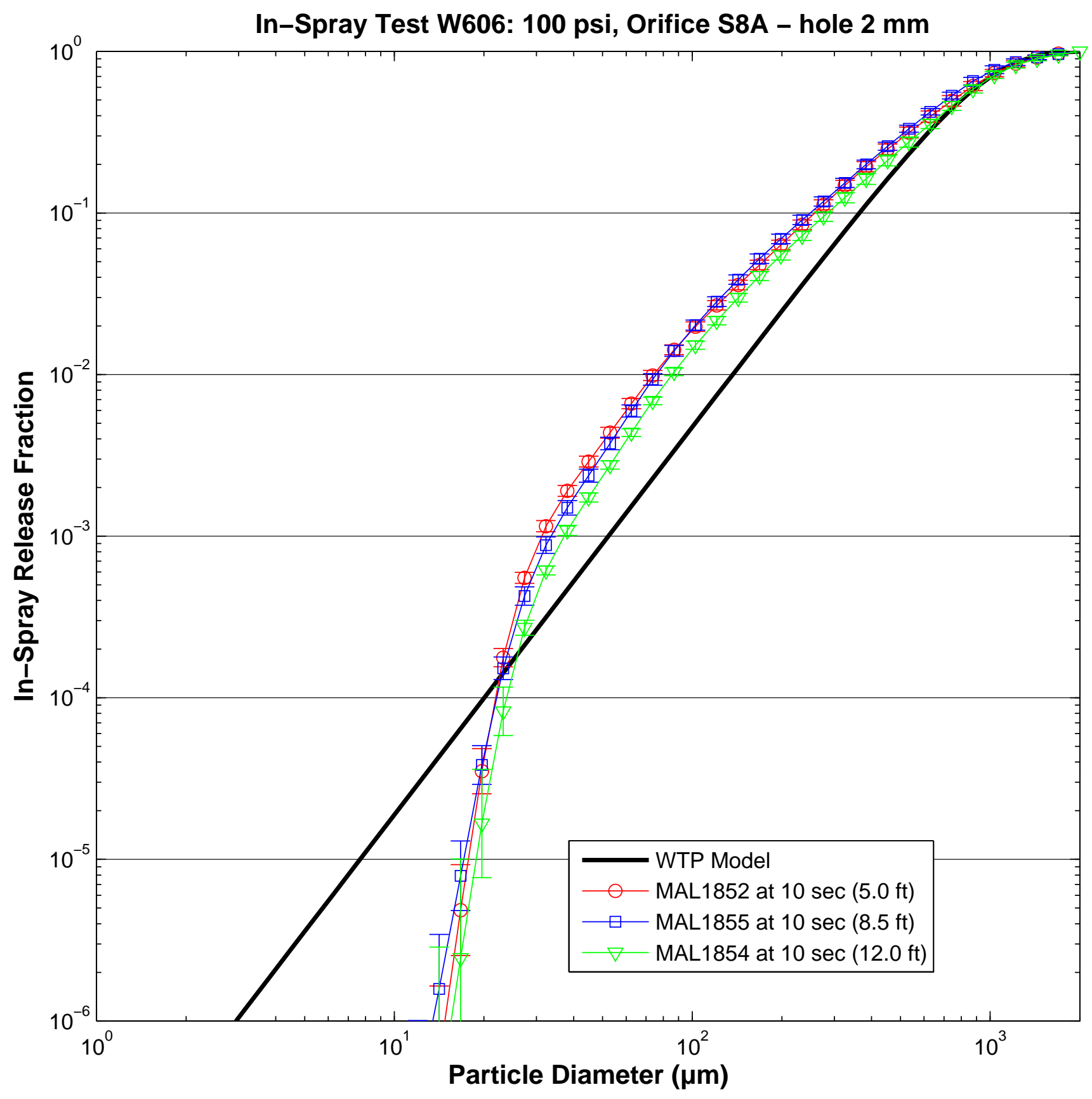




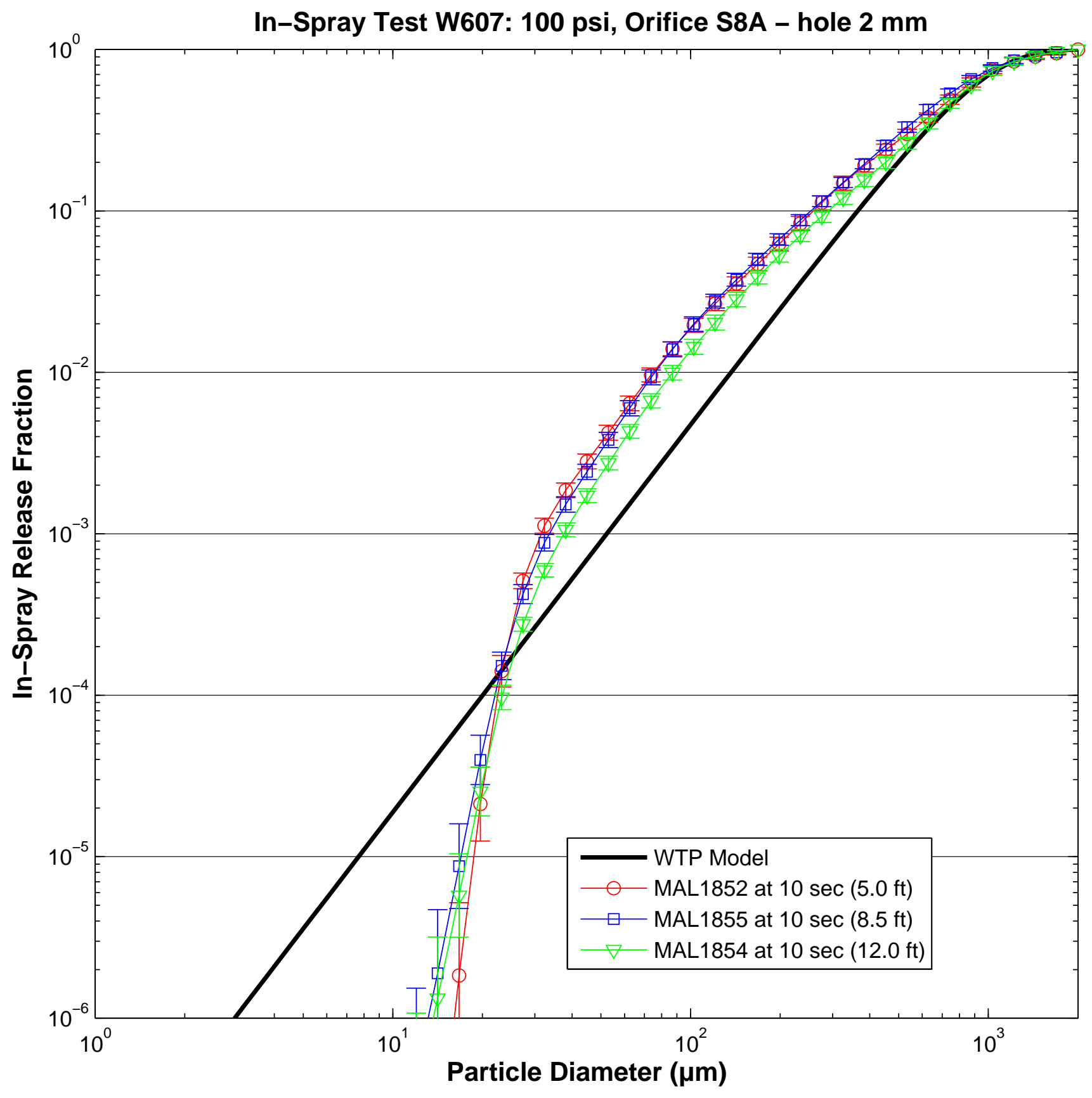




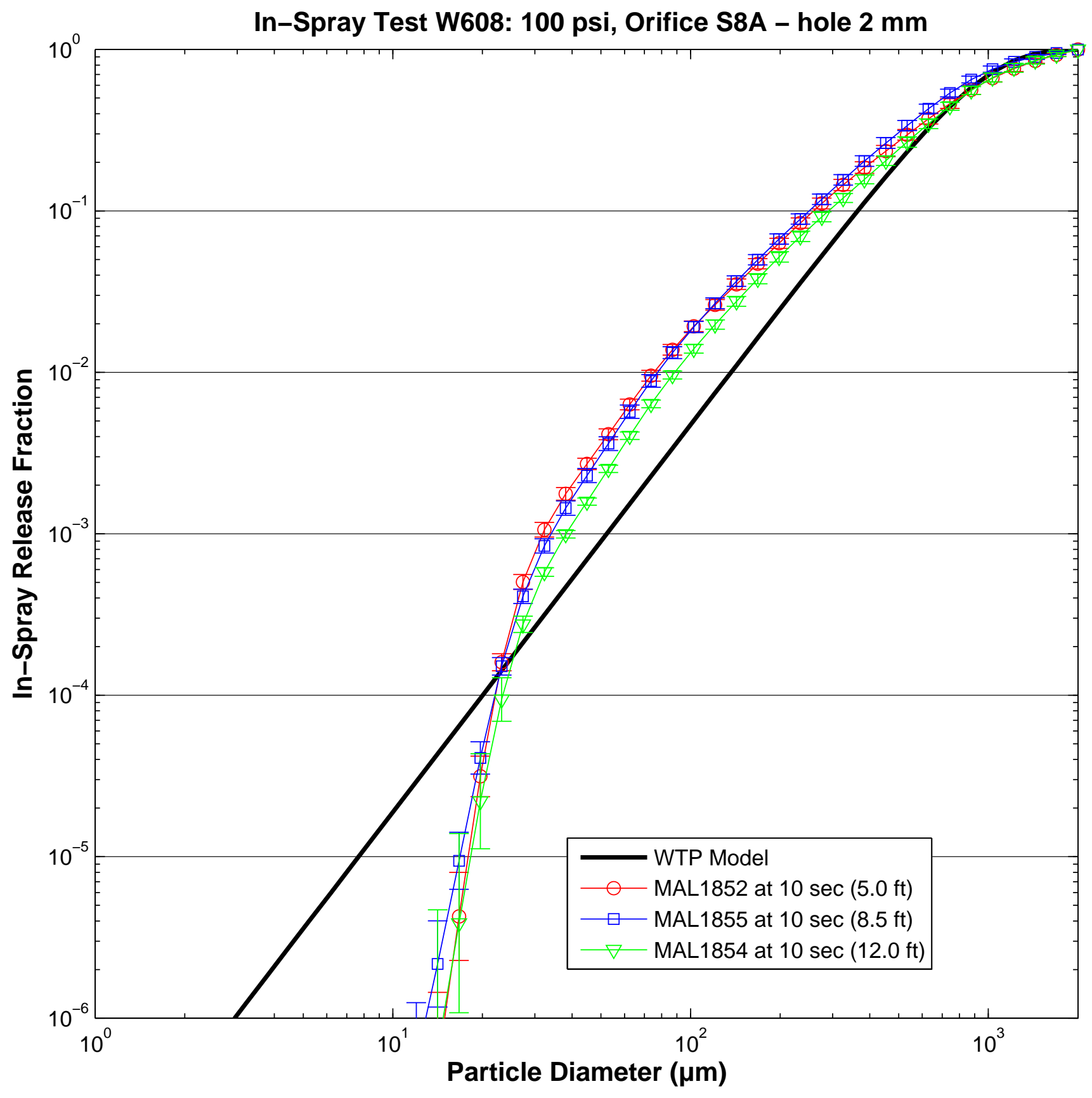




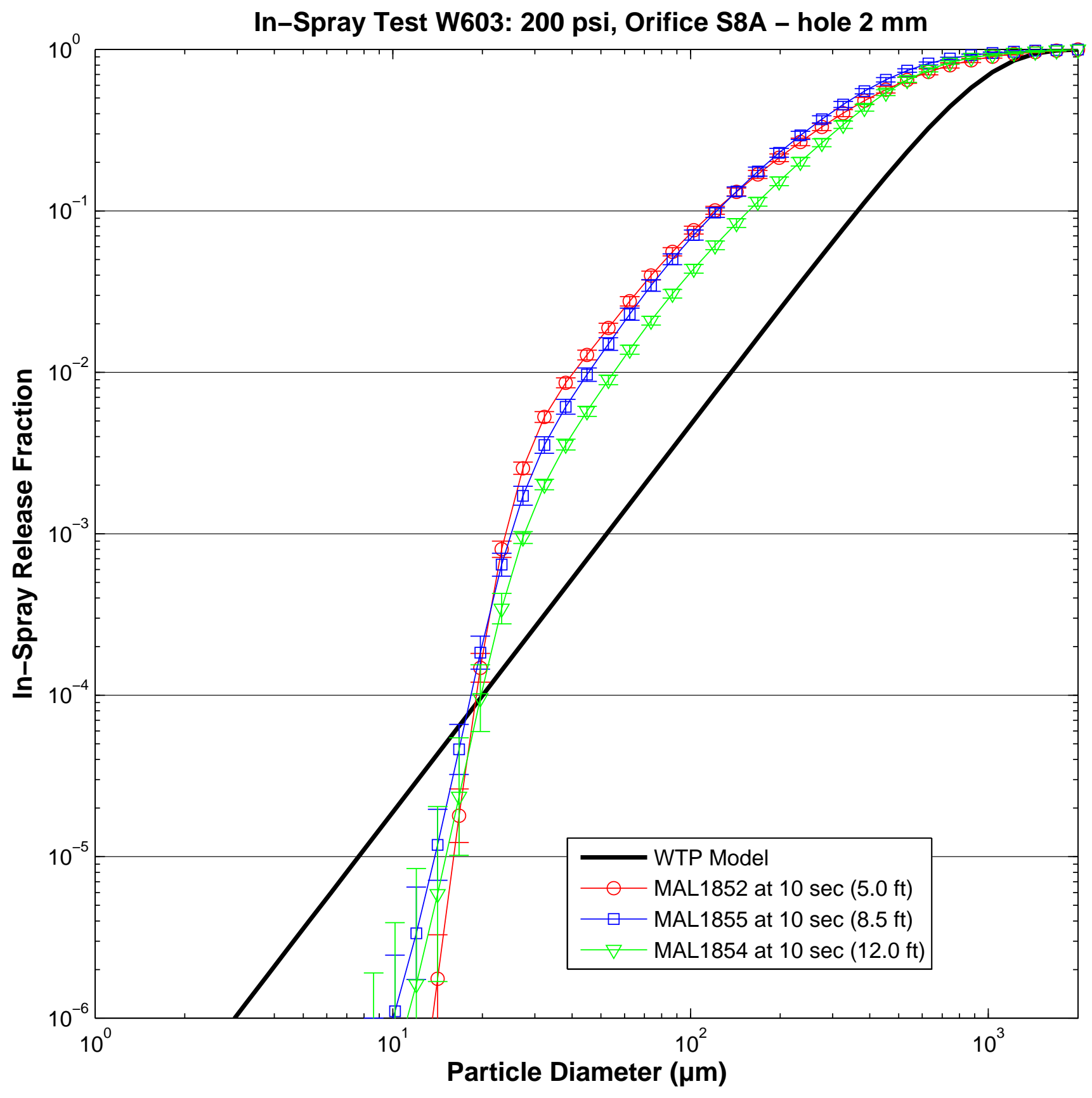




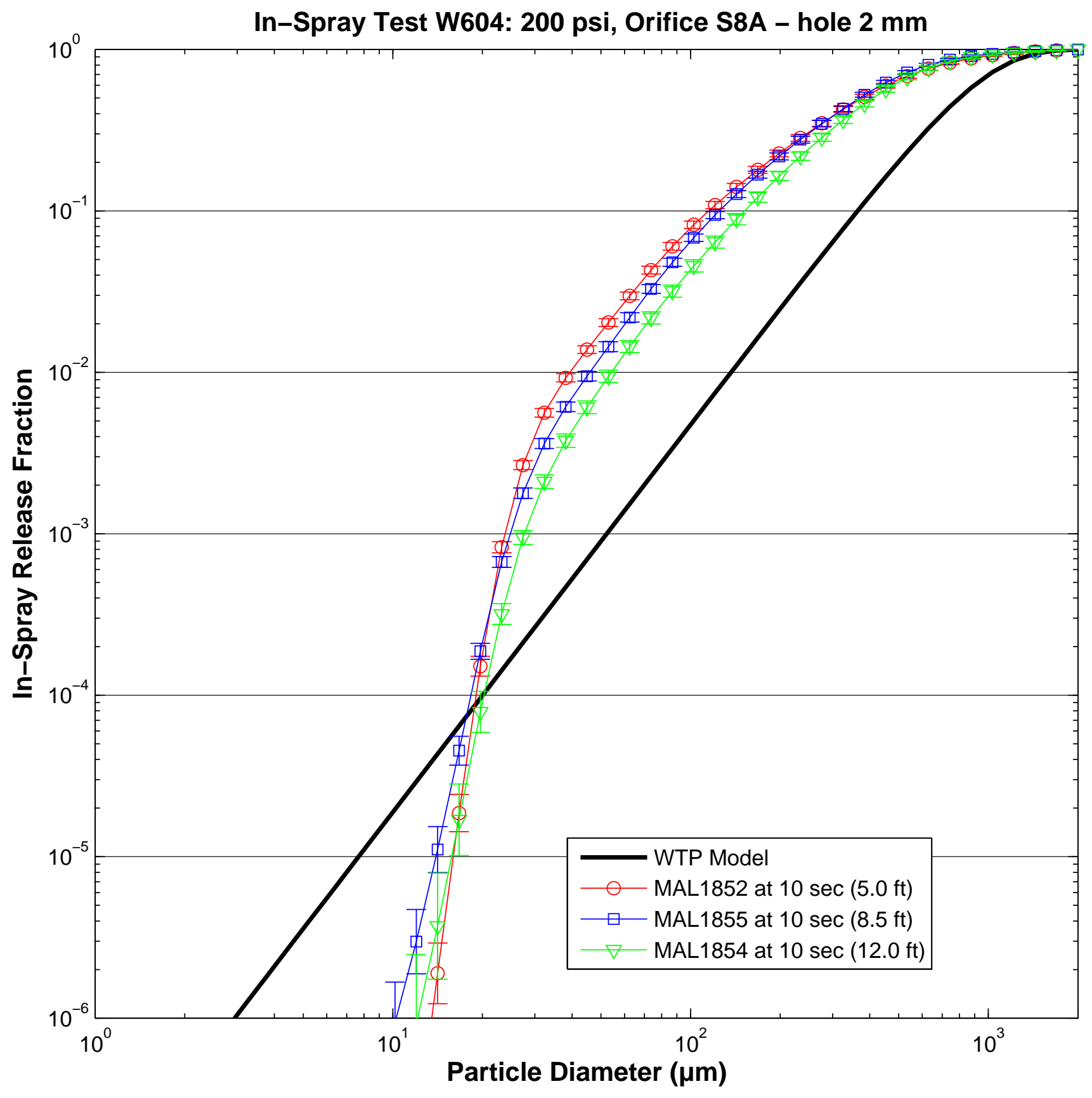




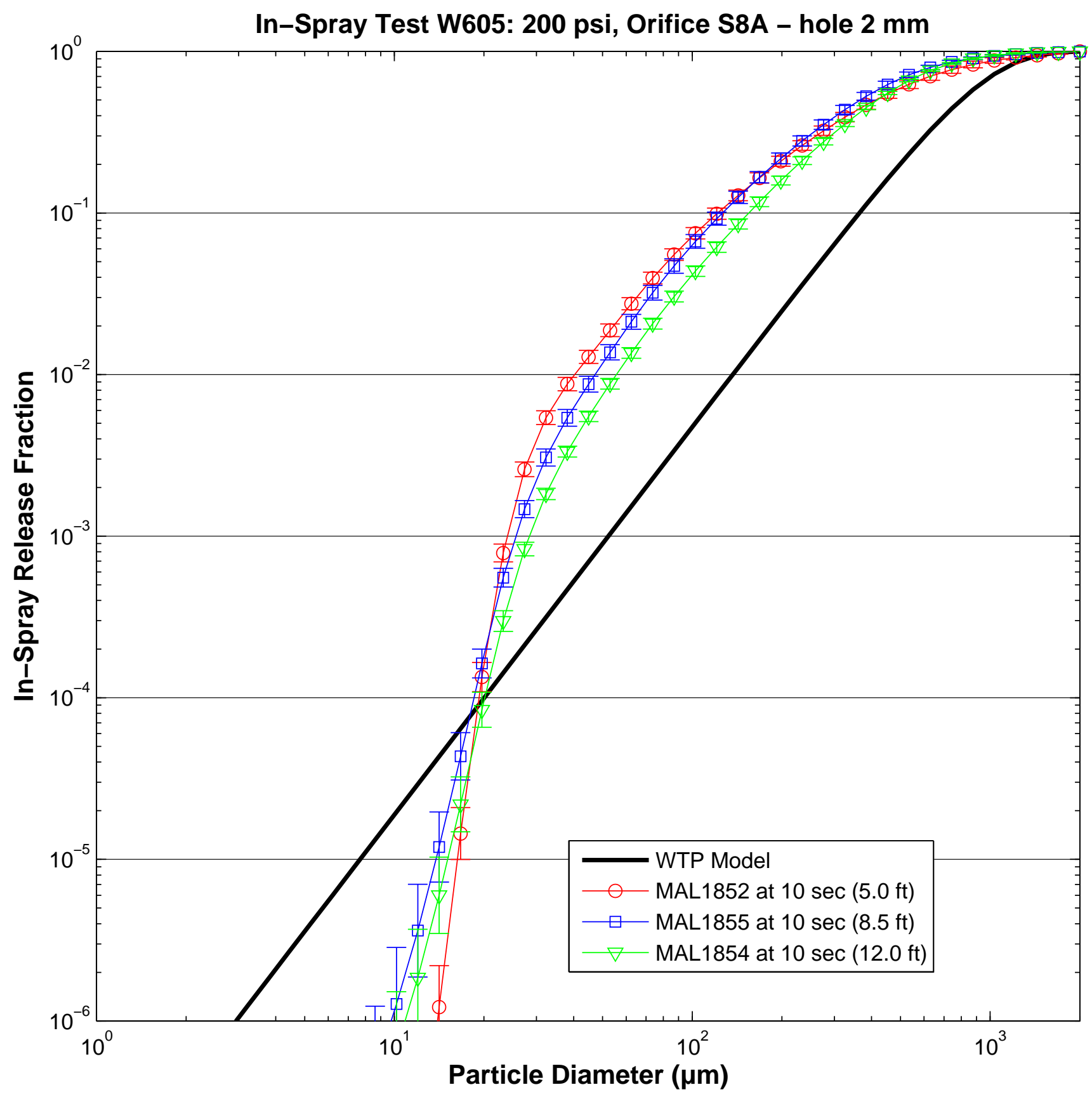




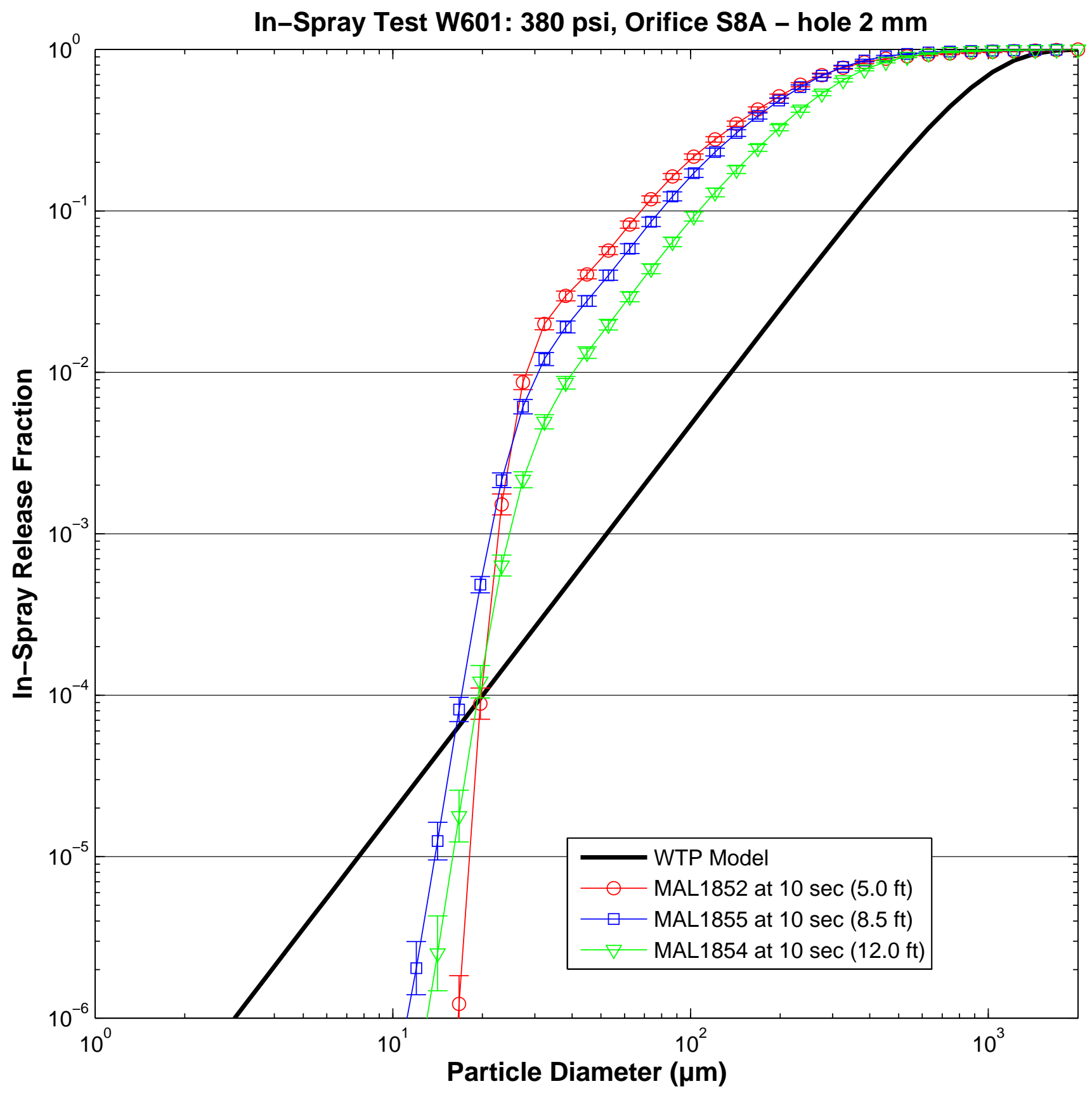




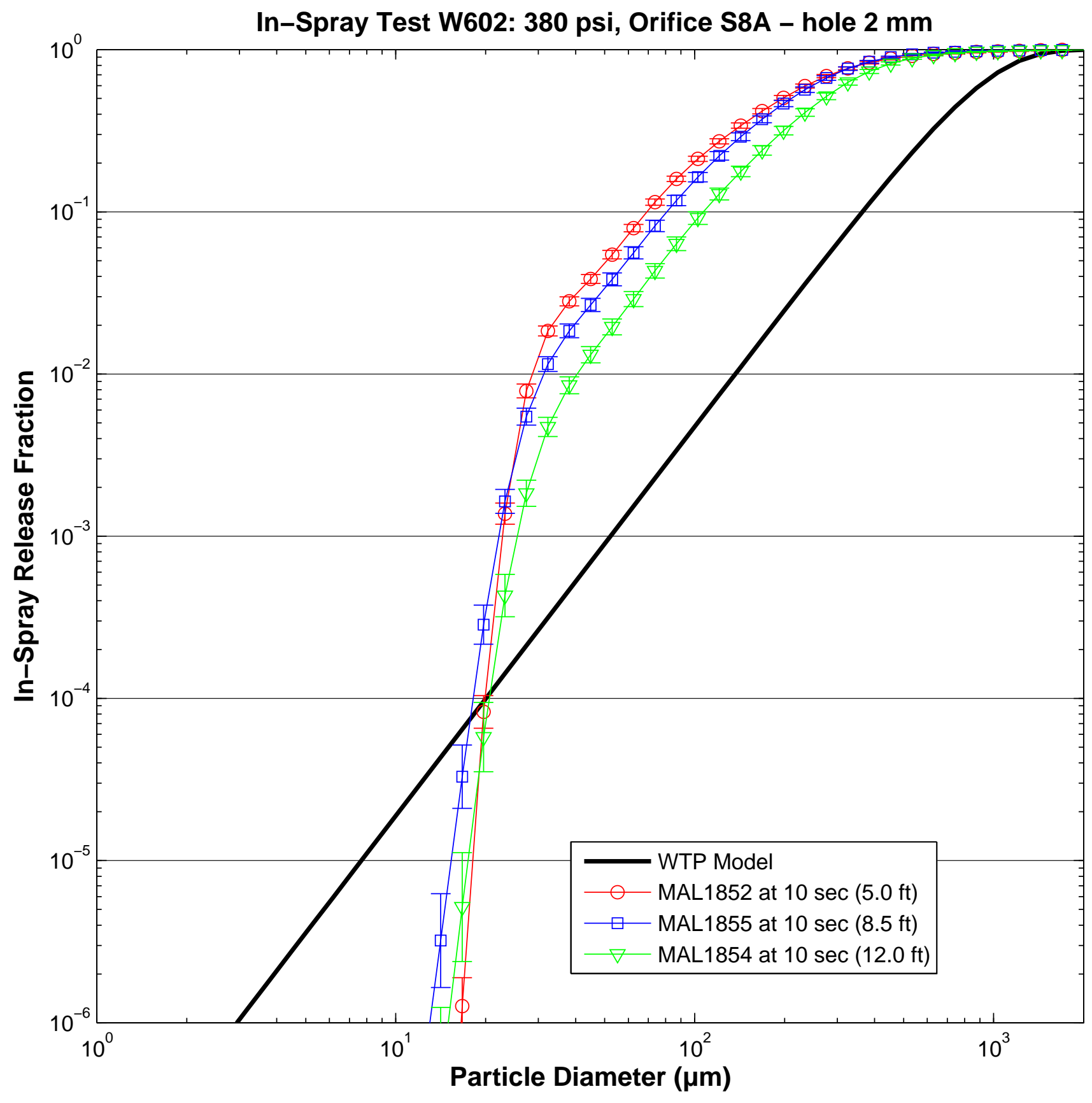




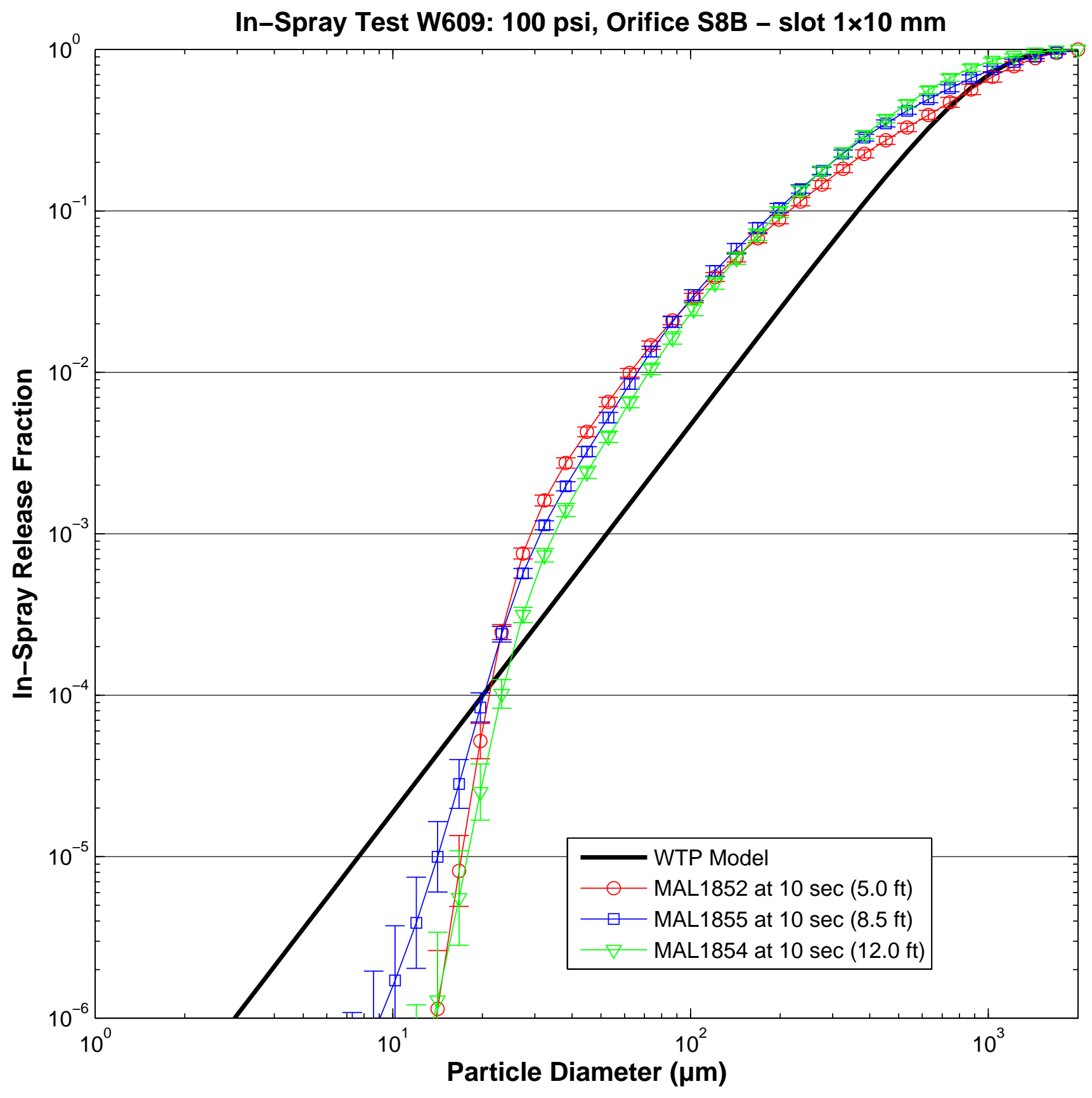




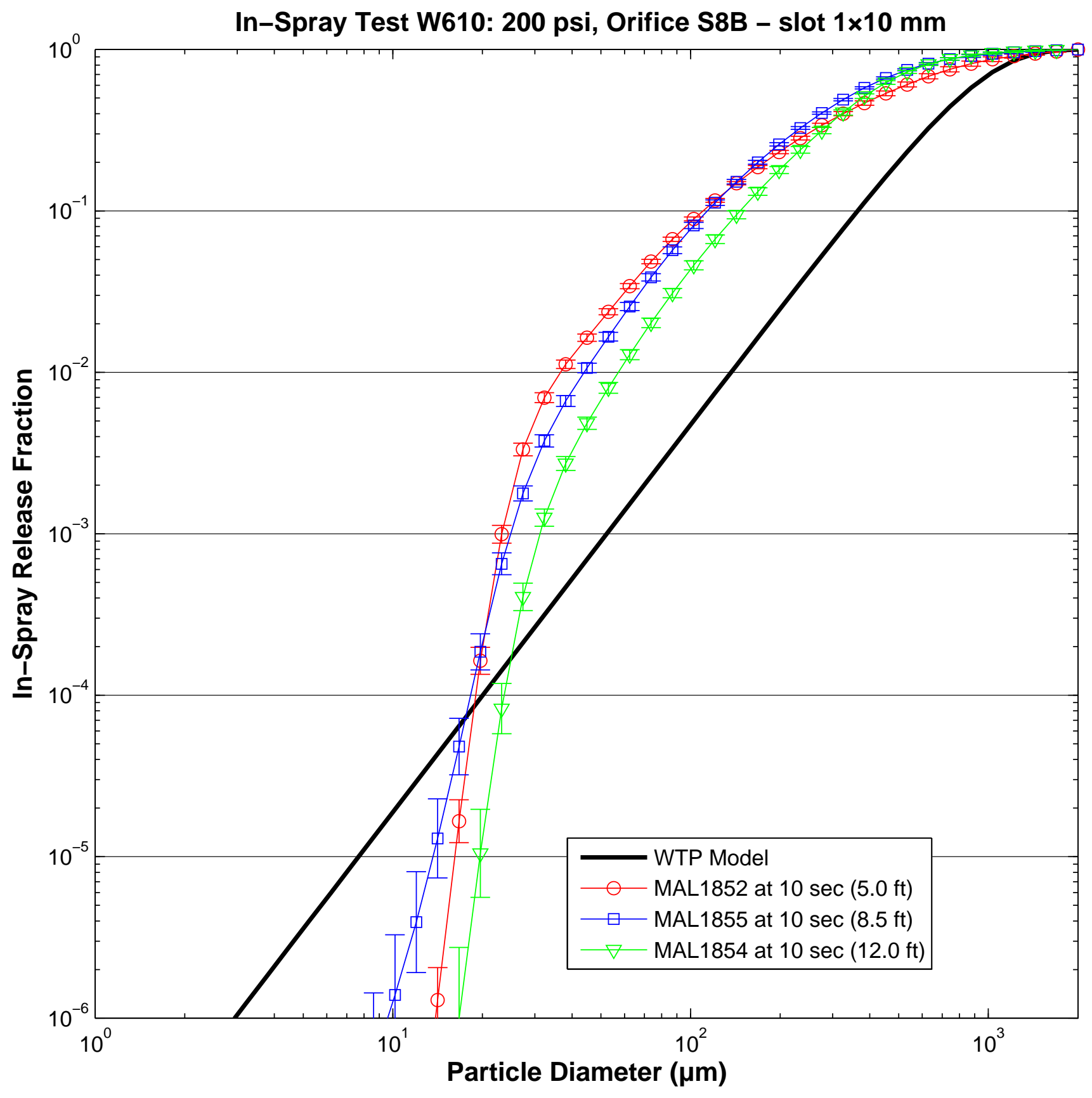




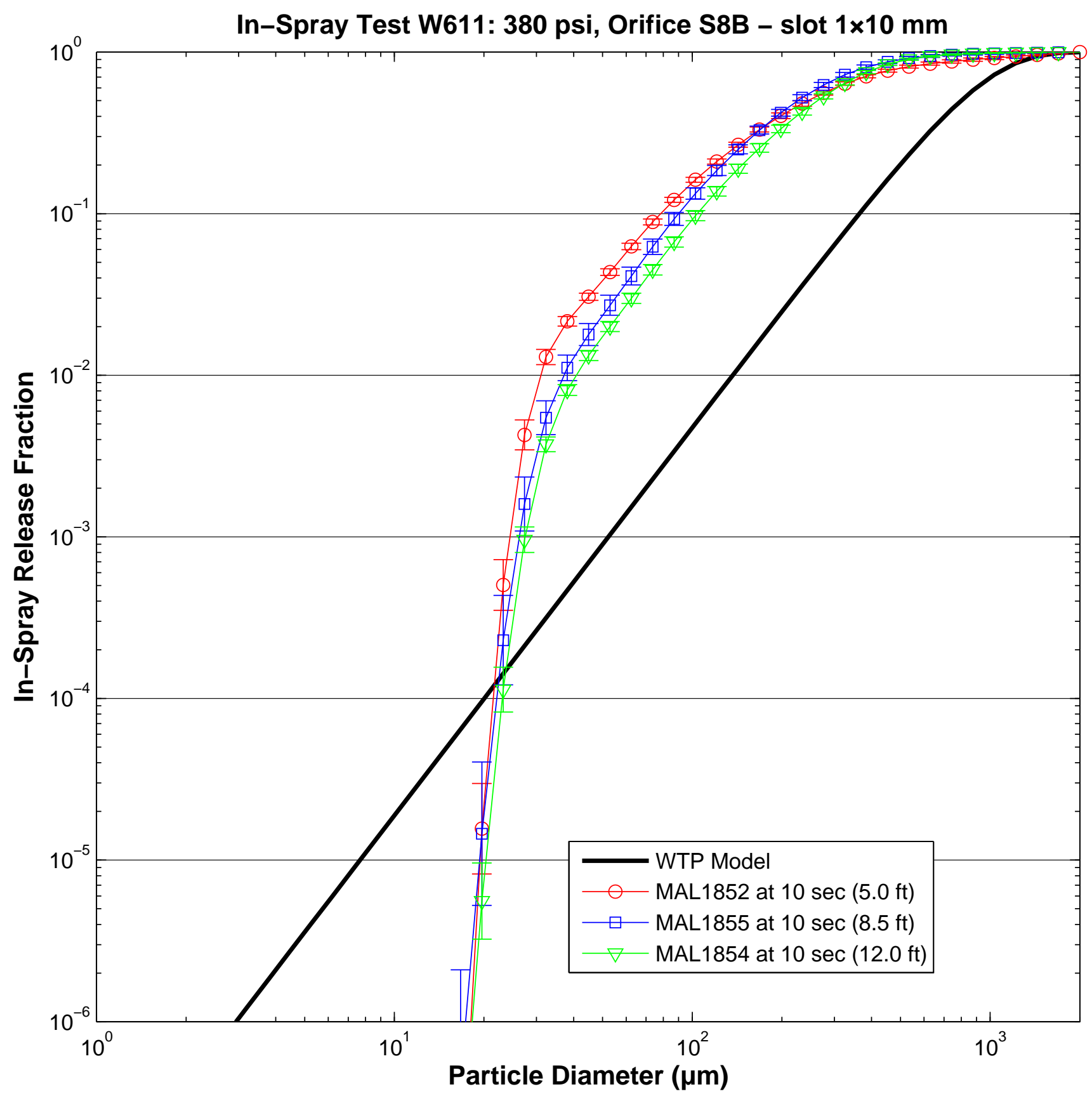




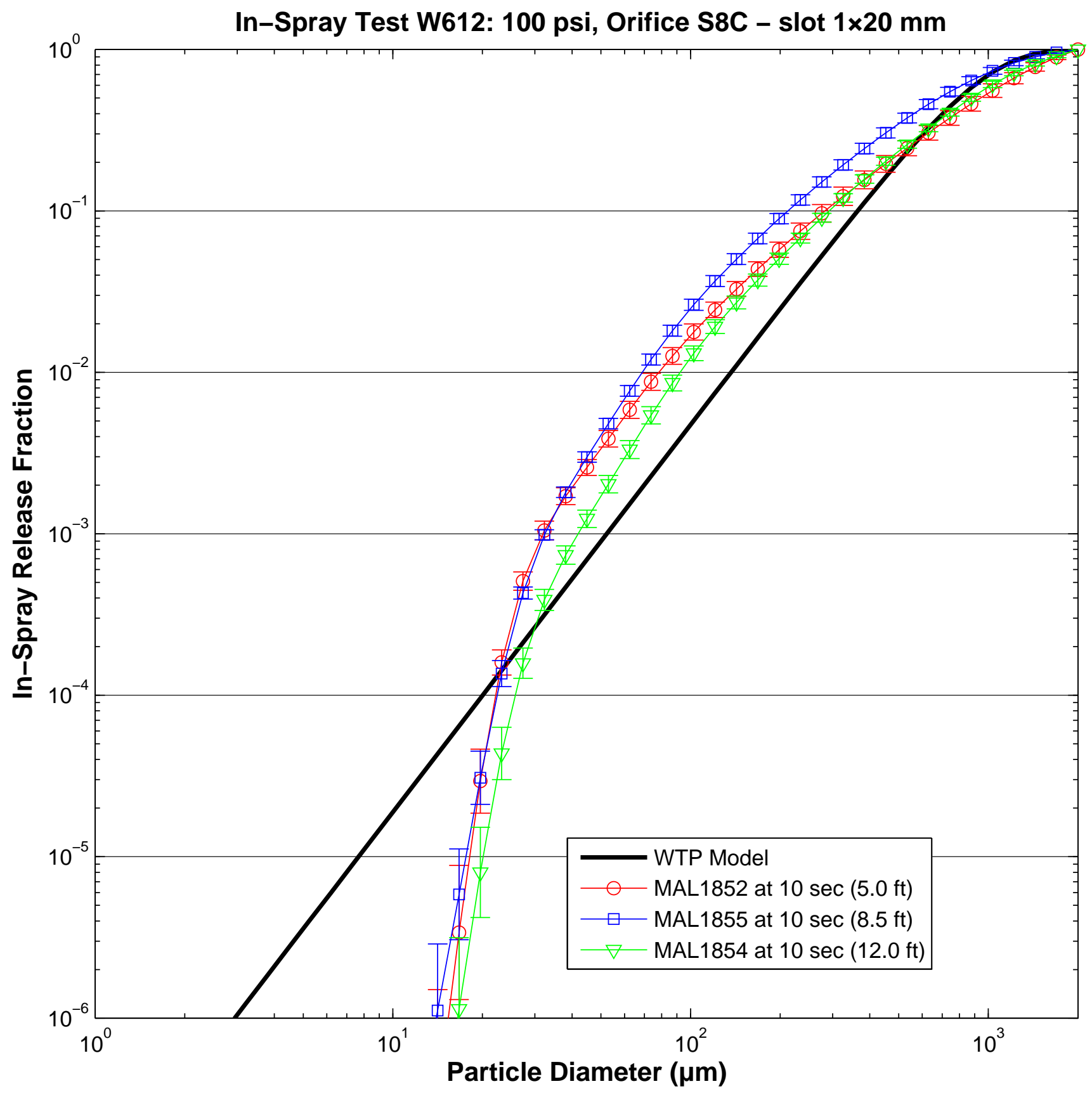




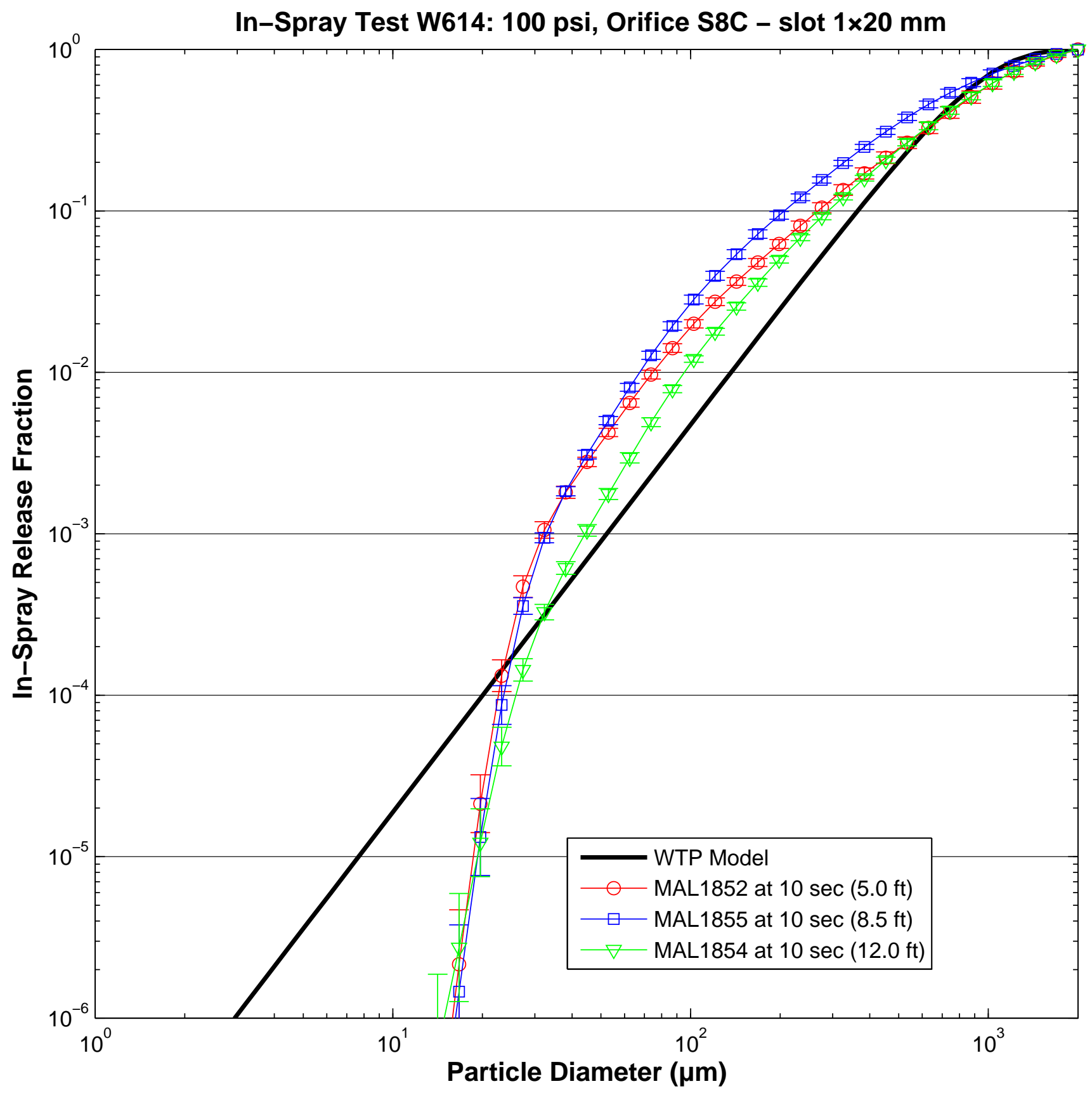

B.382 


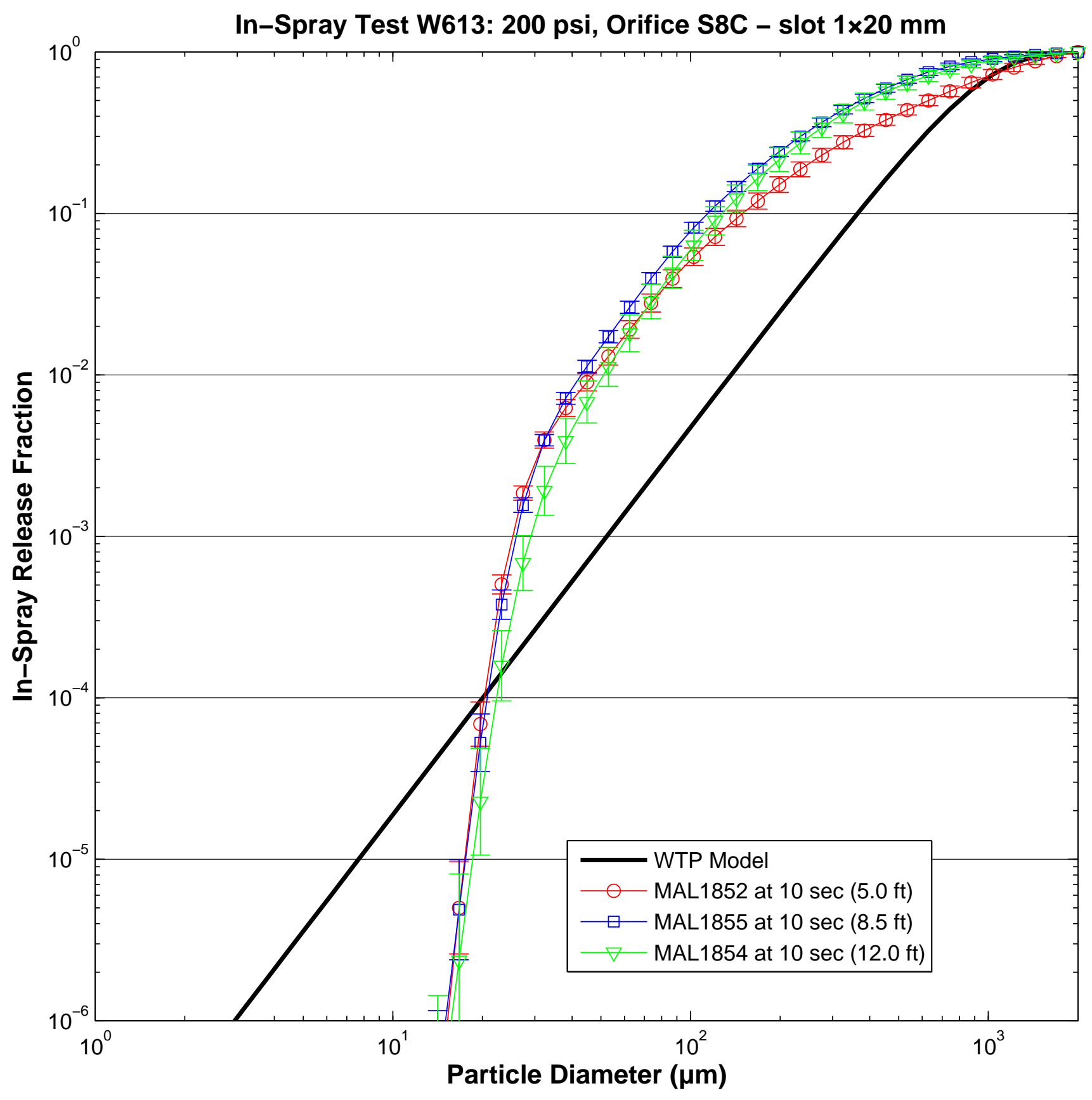




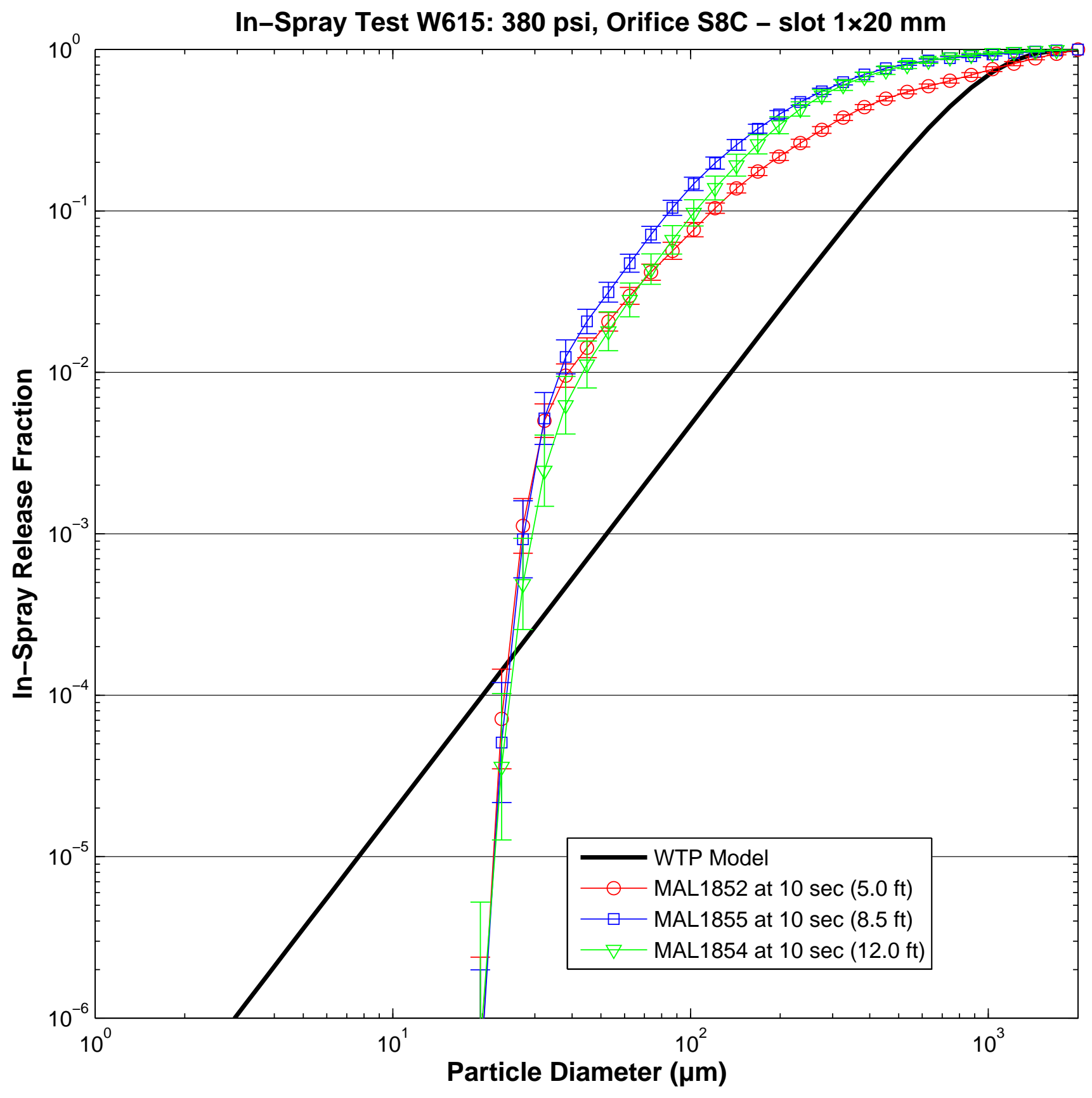




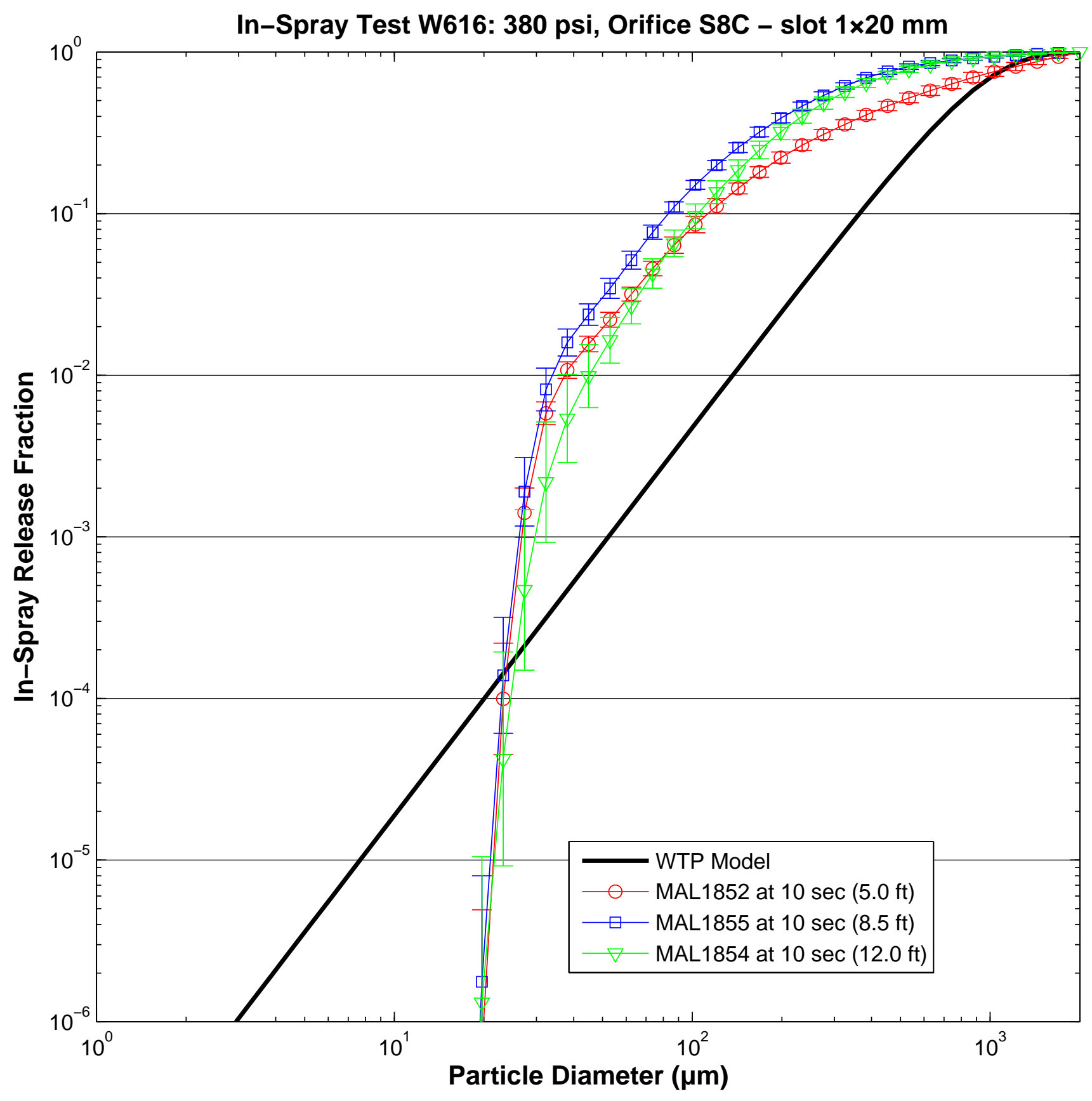




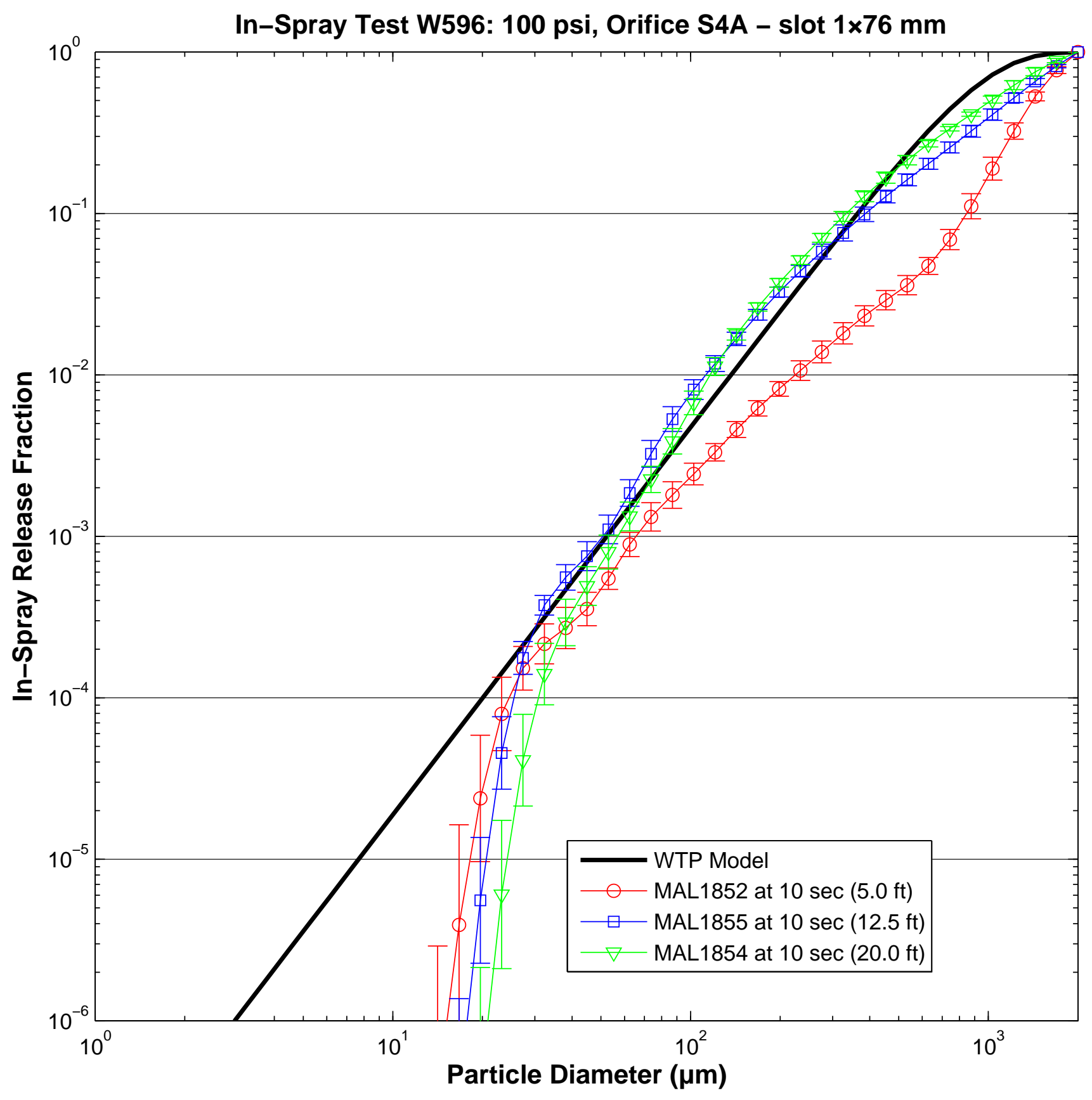




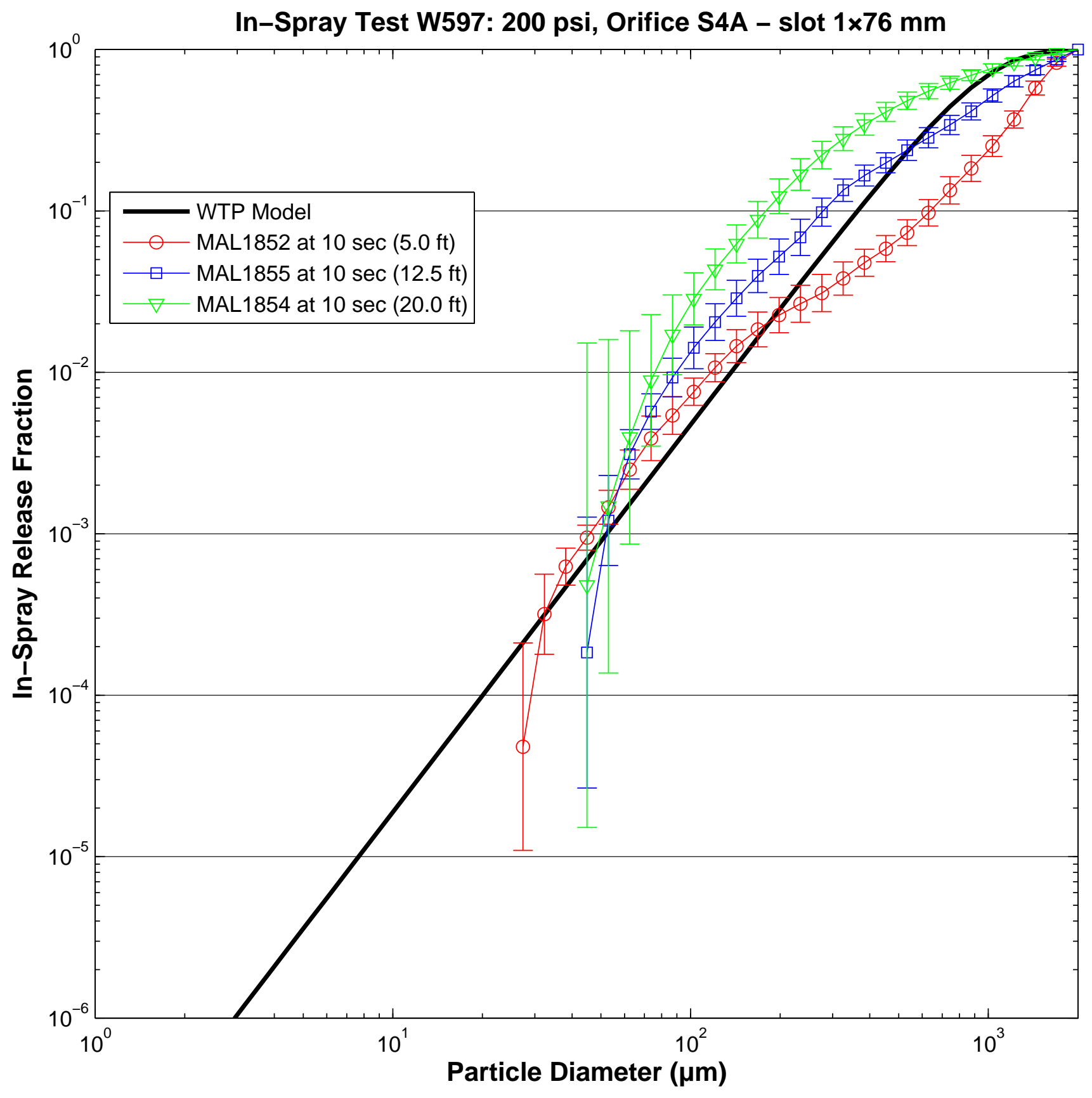




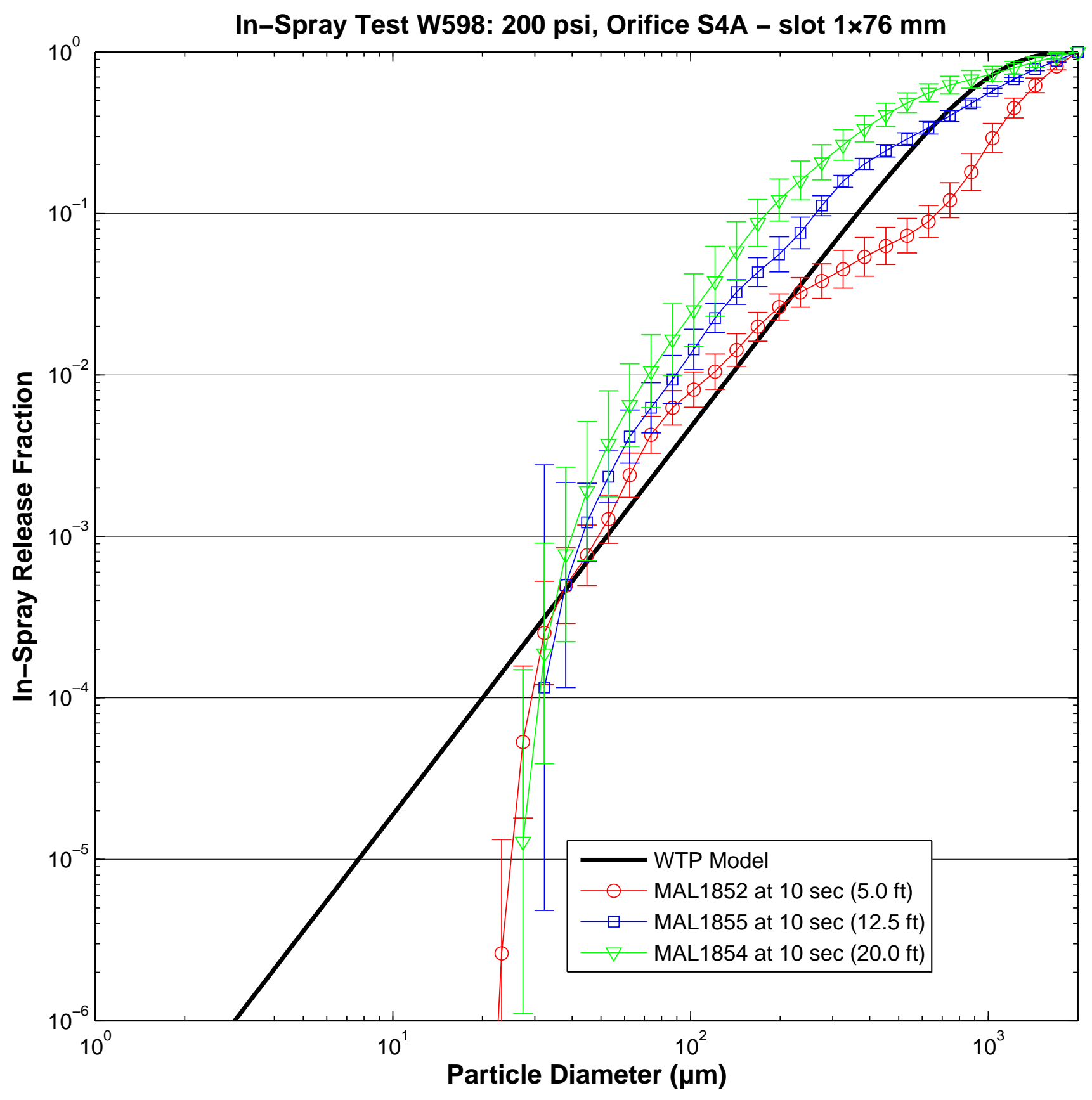




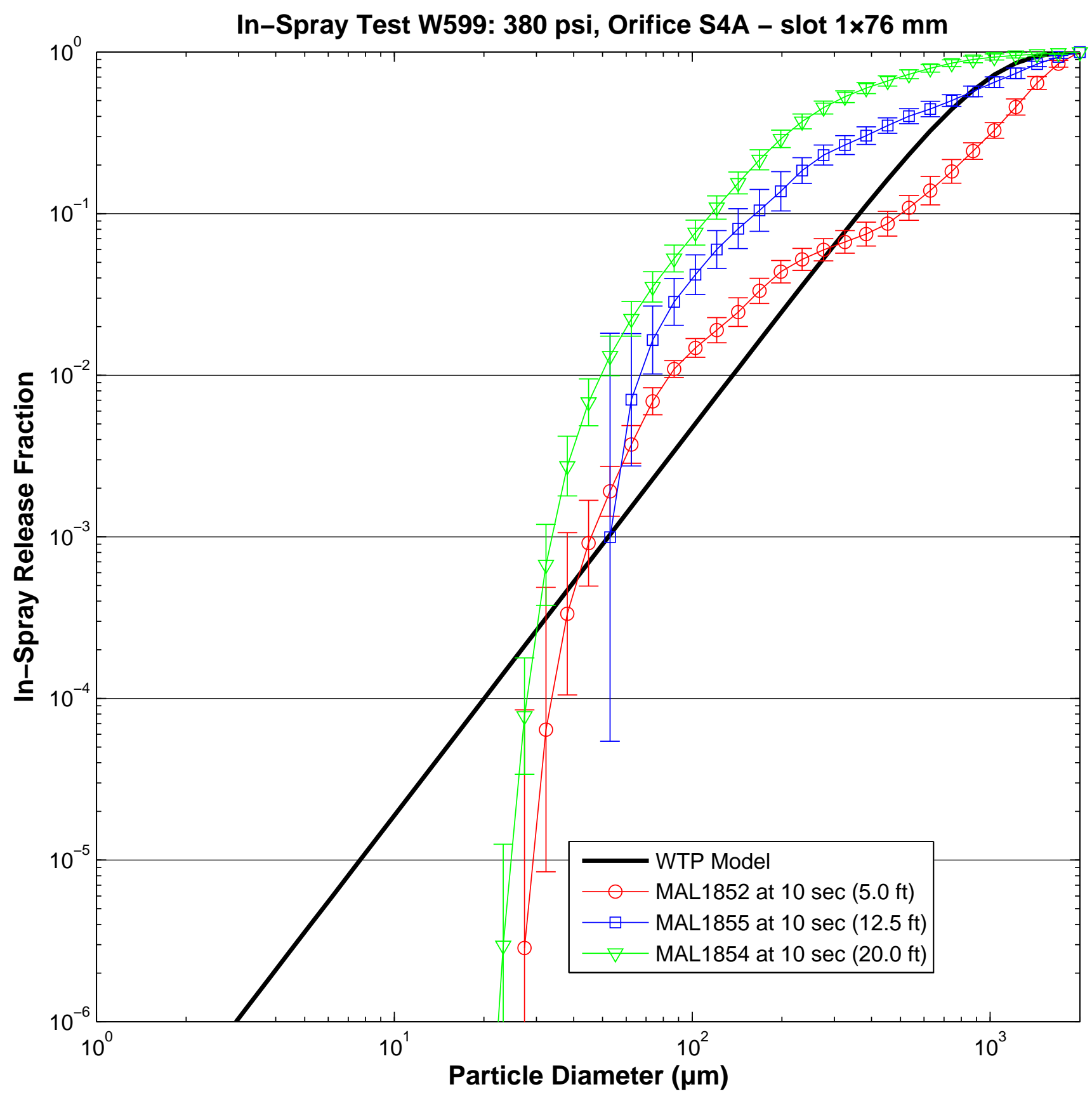




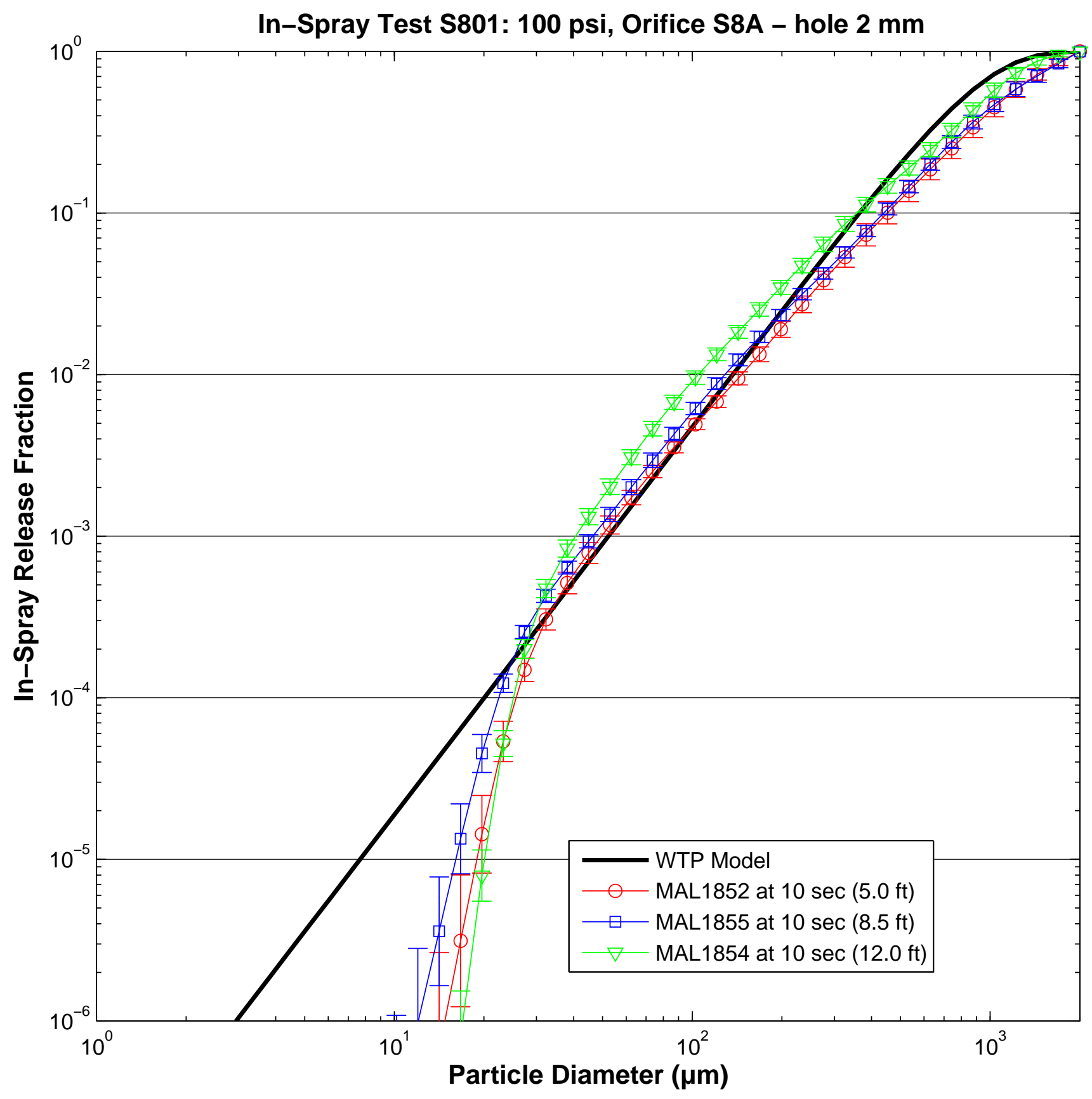




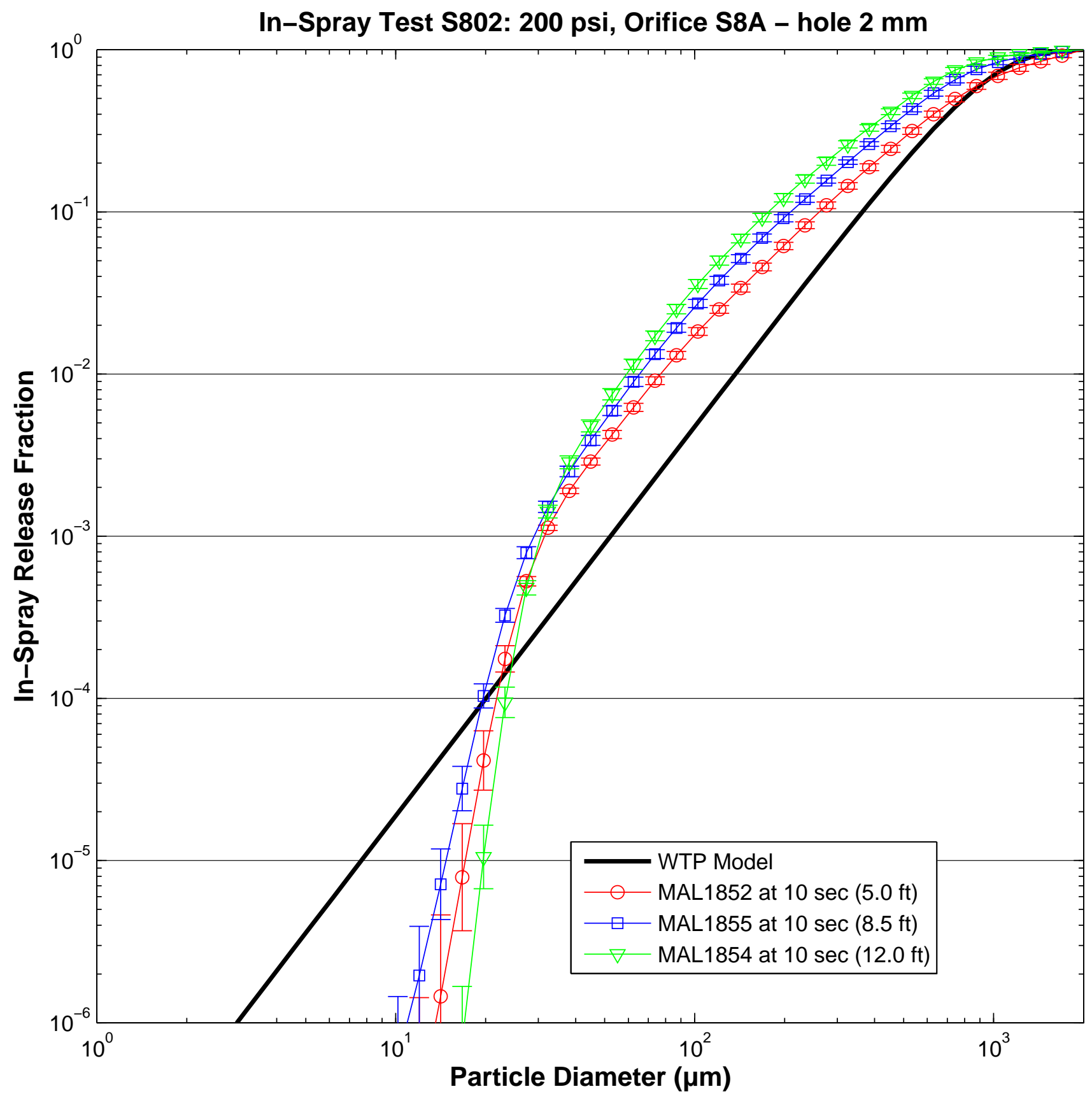




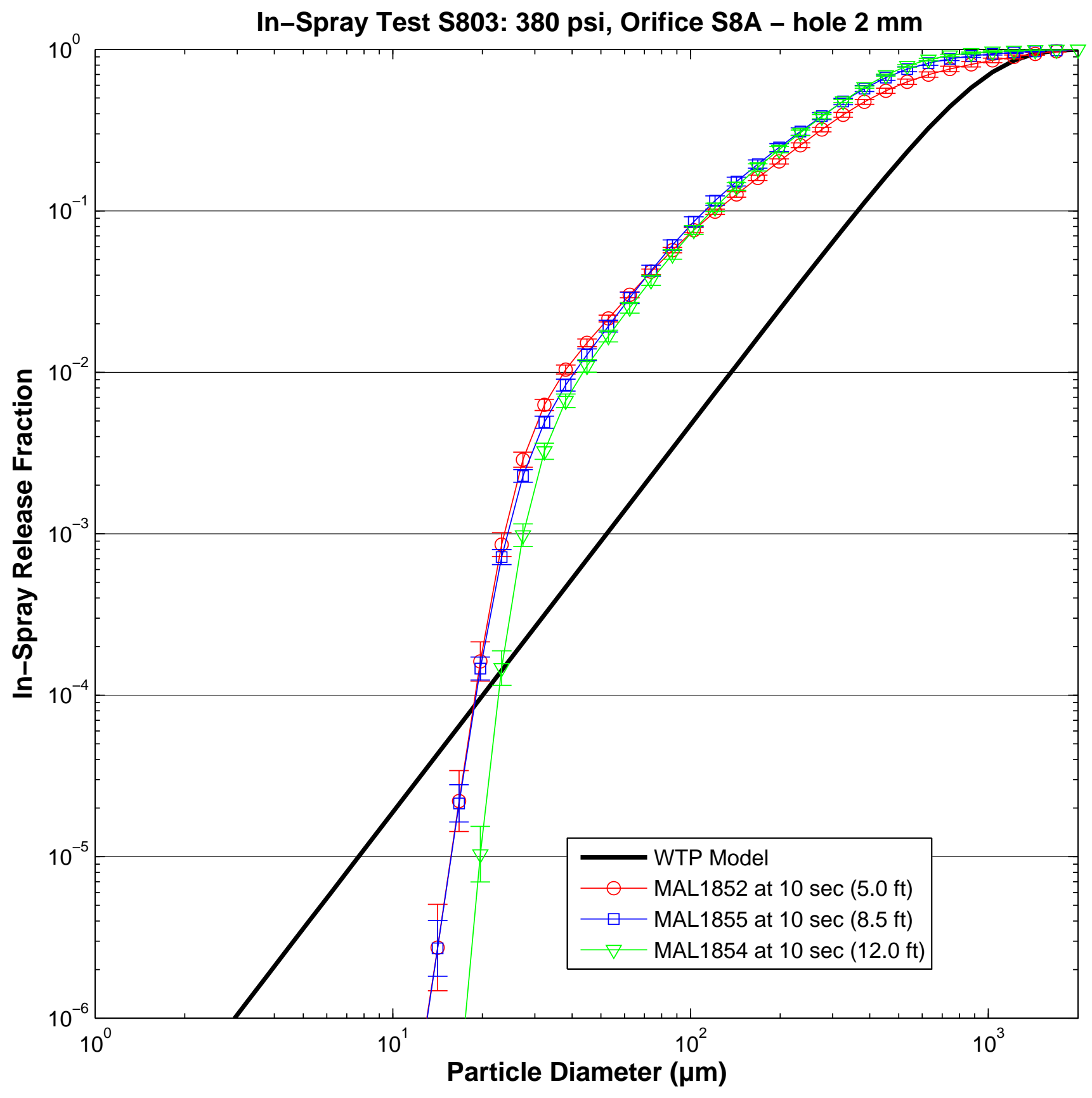




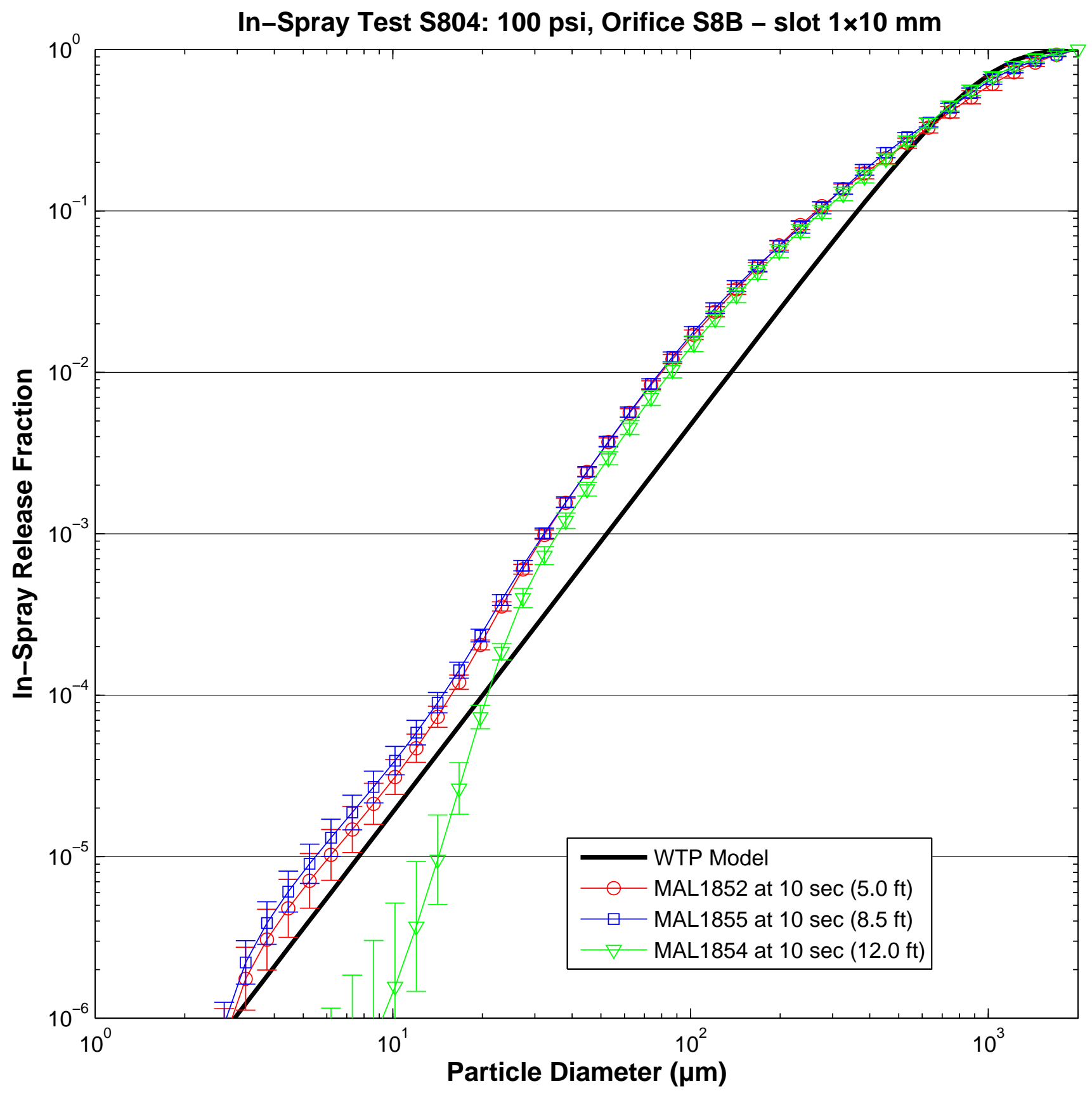




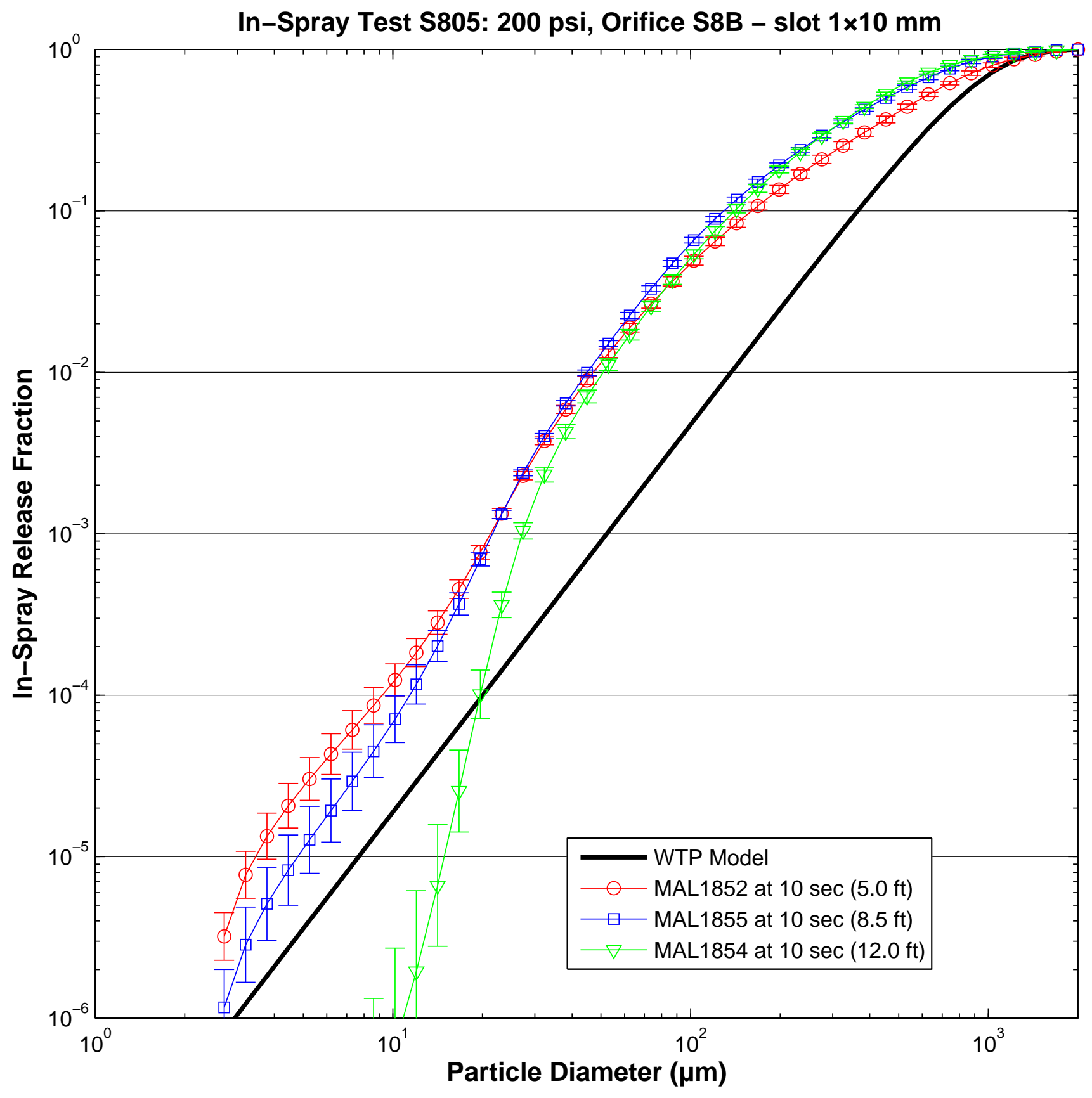




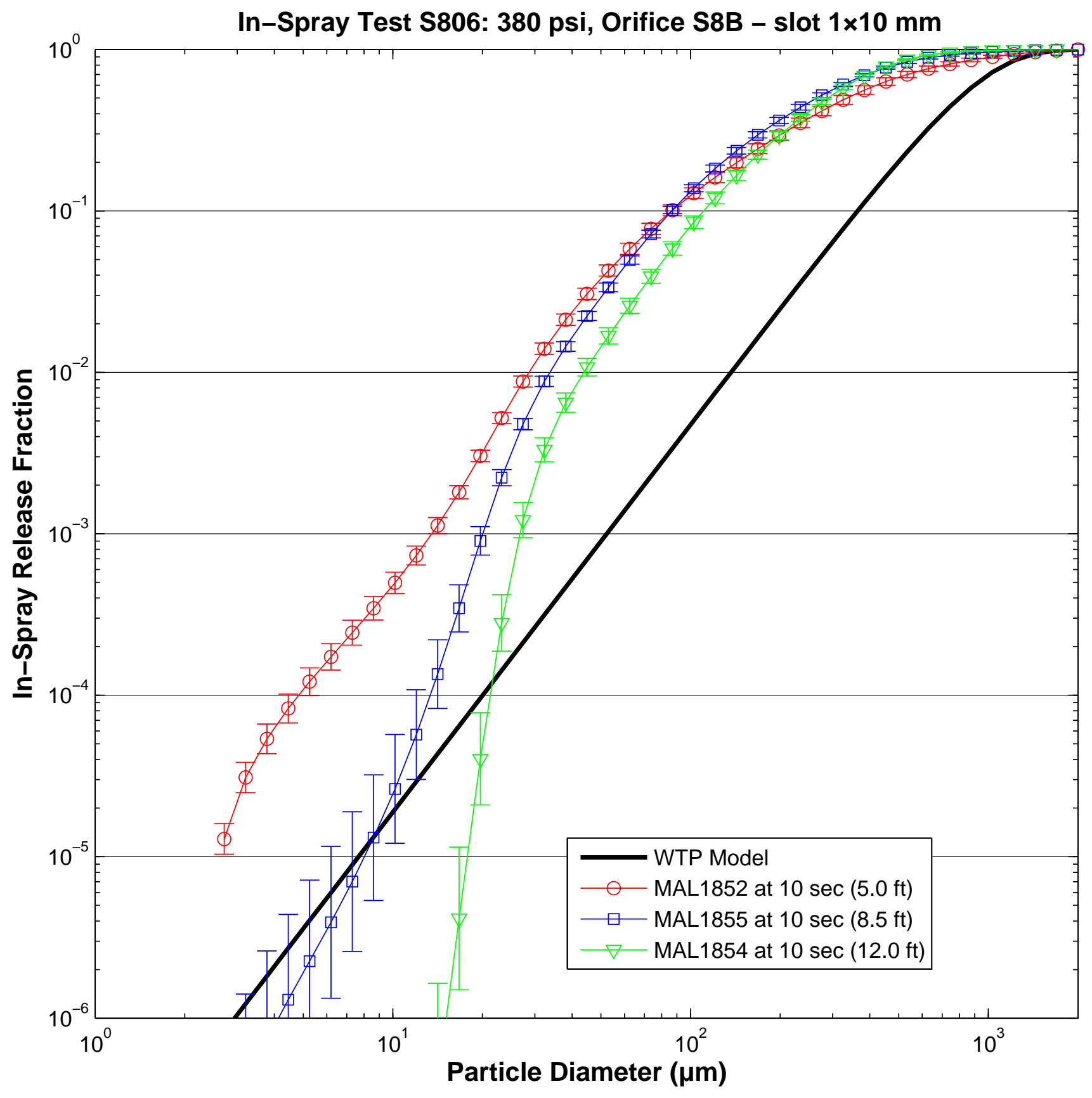




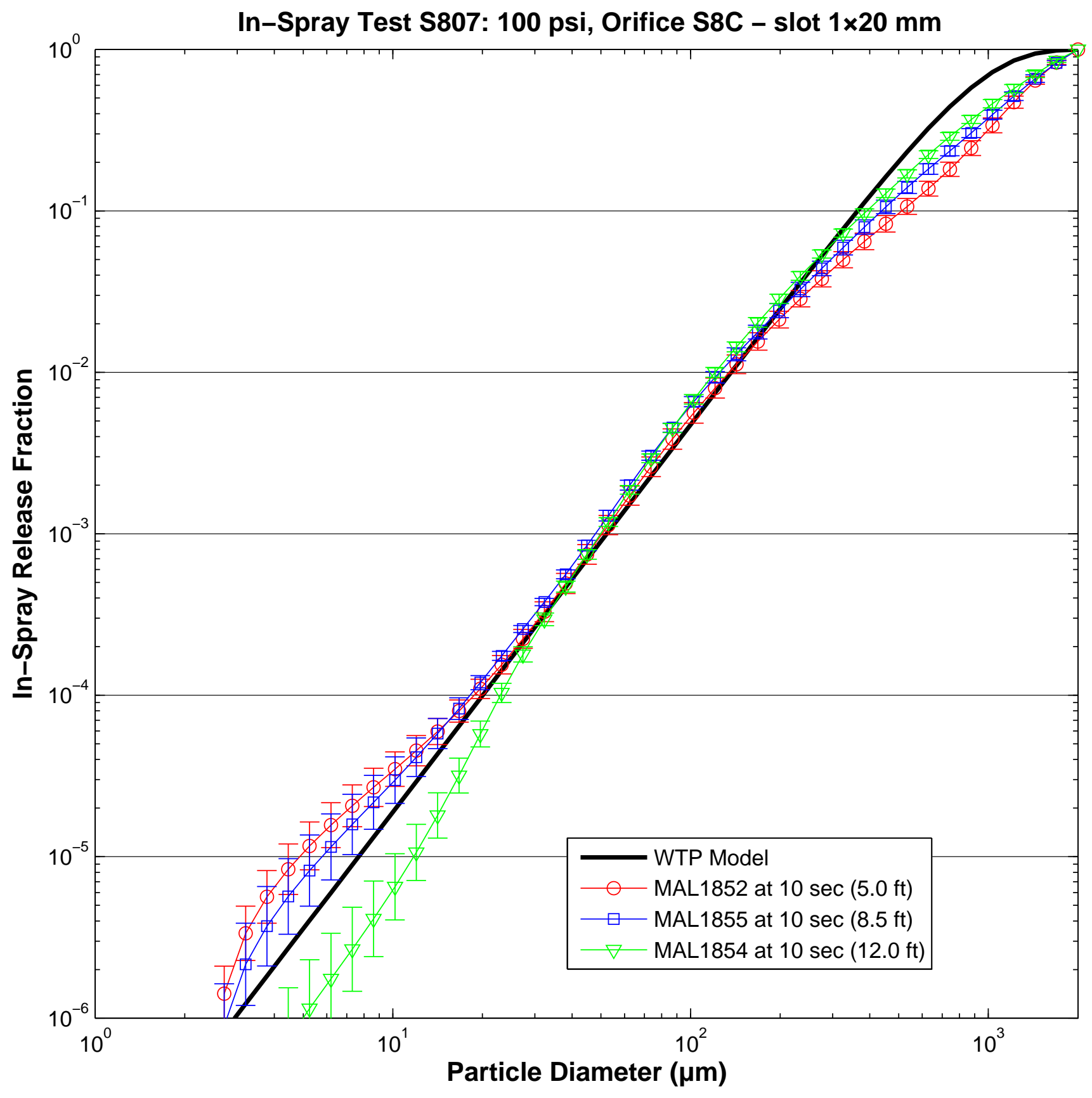




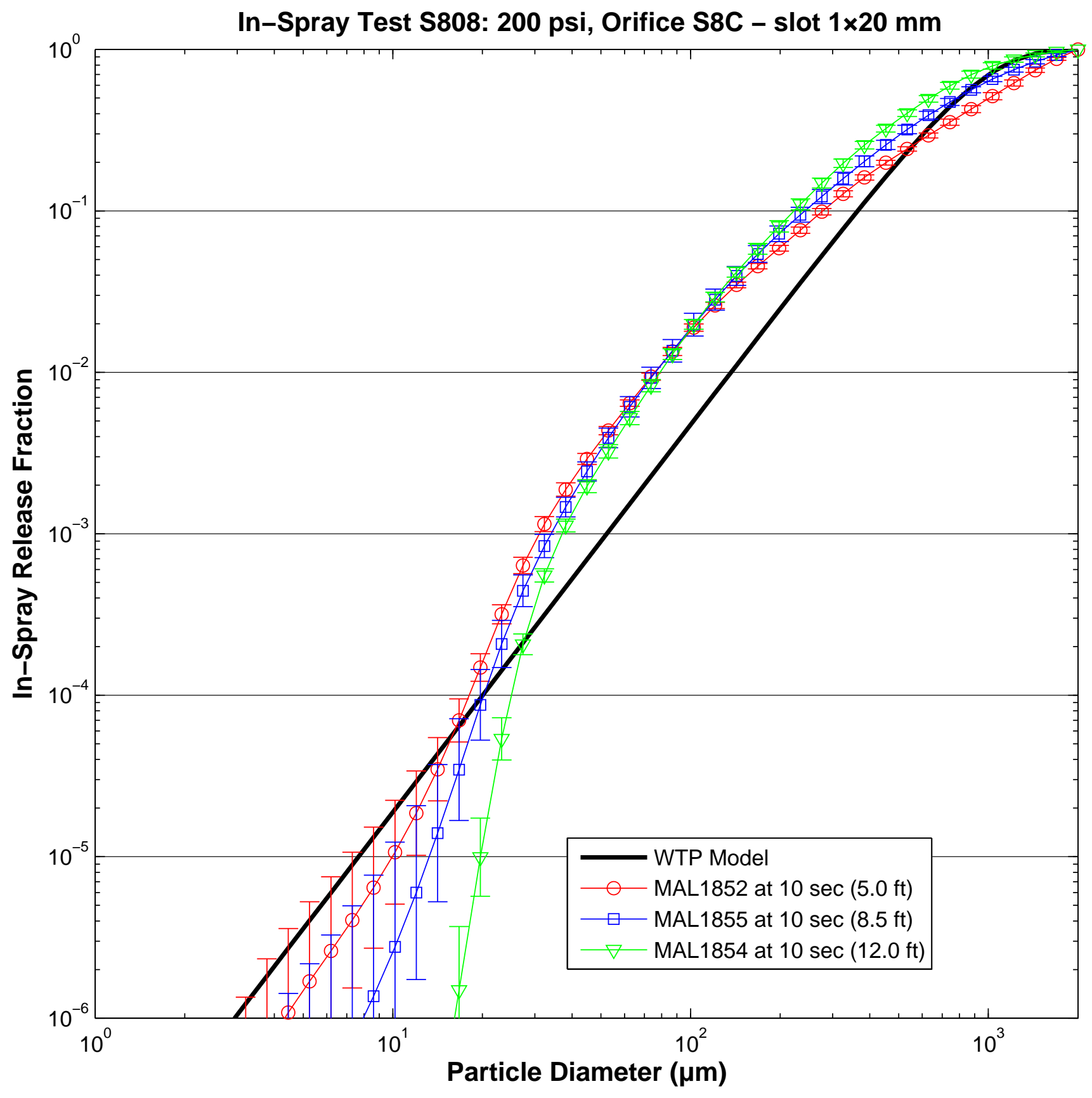




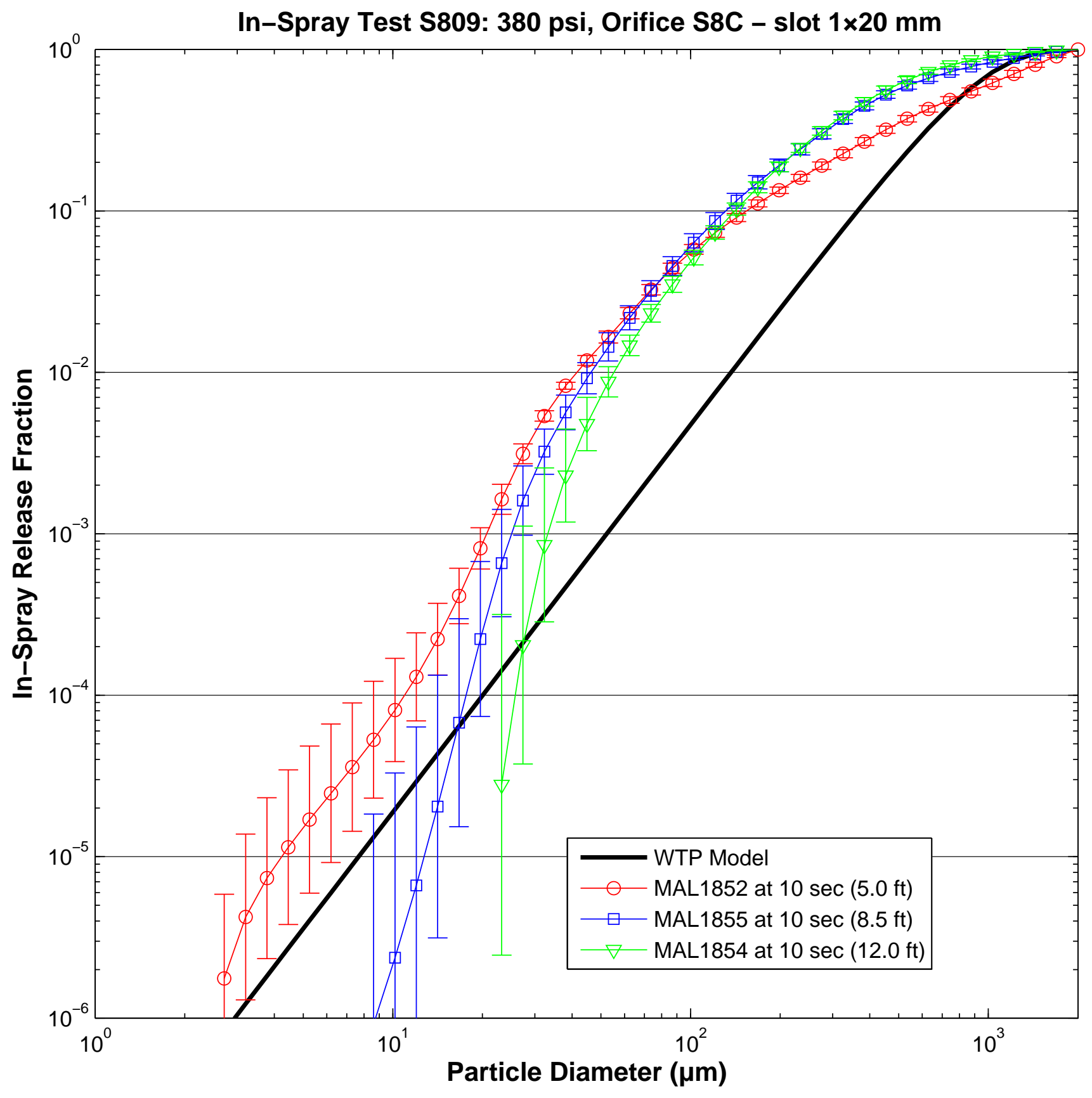




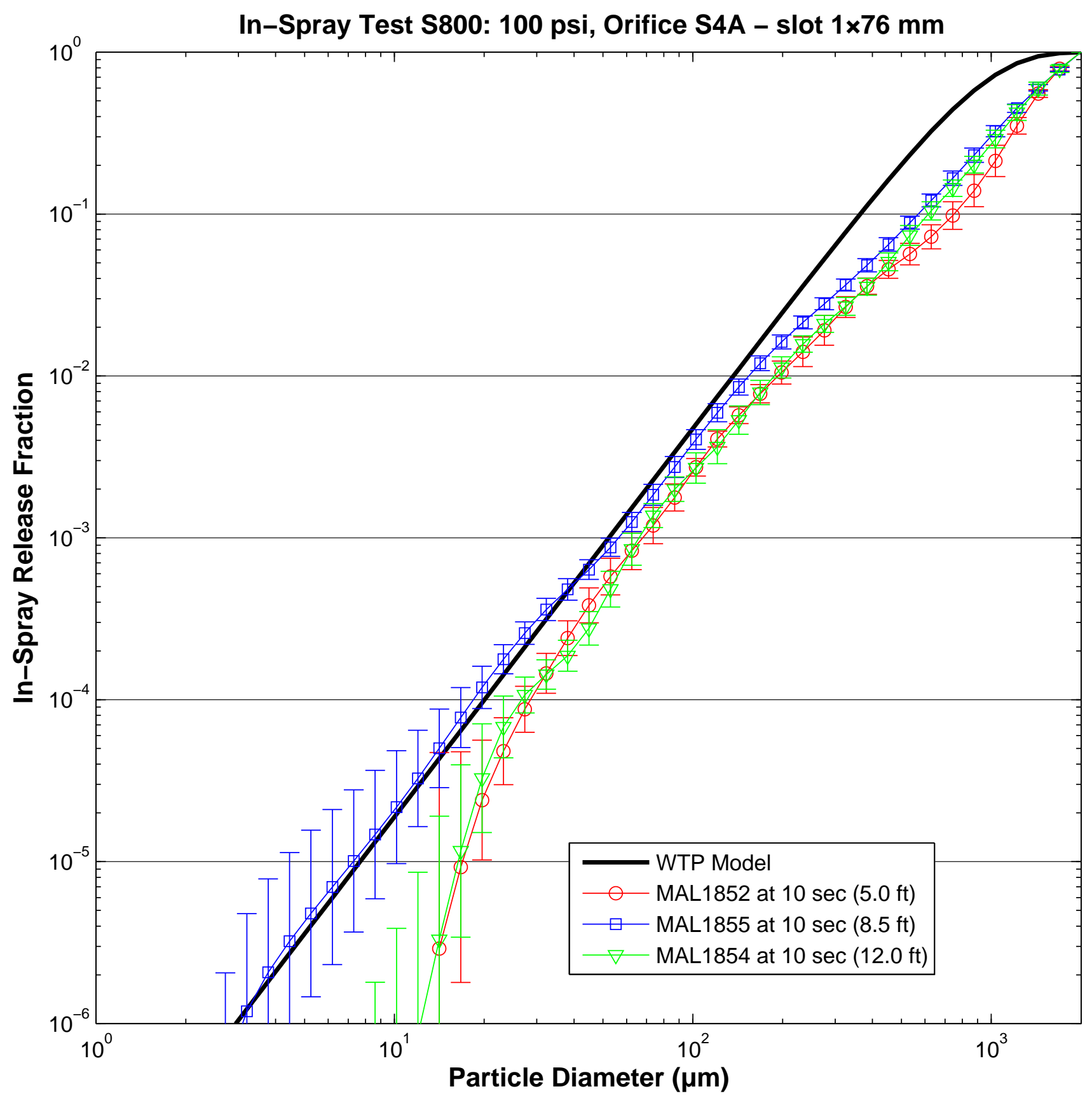




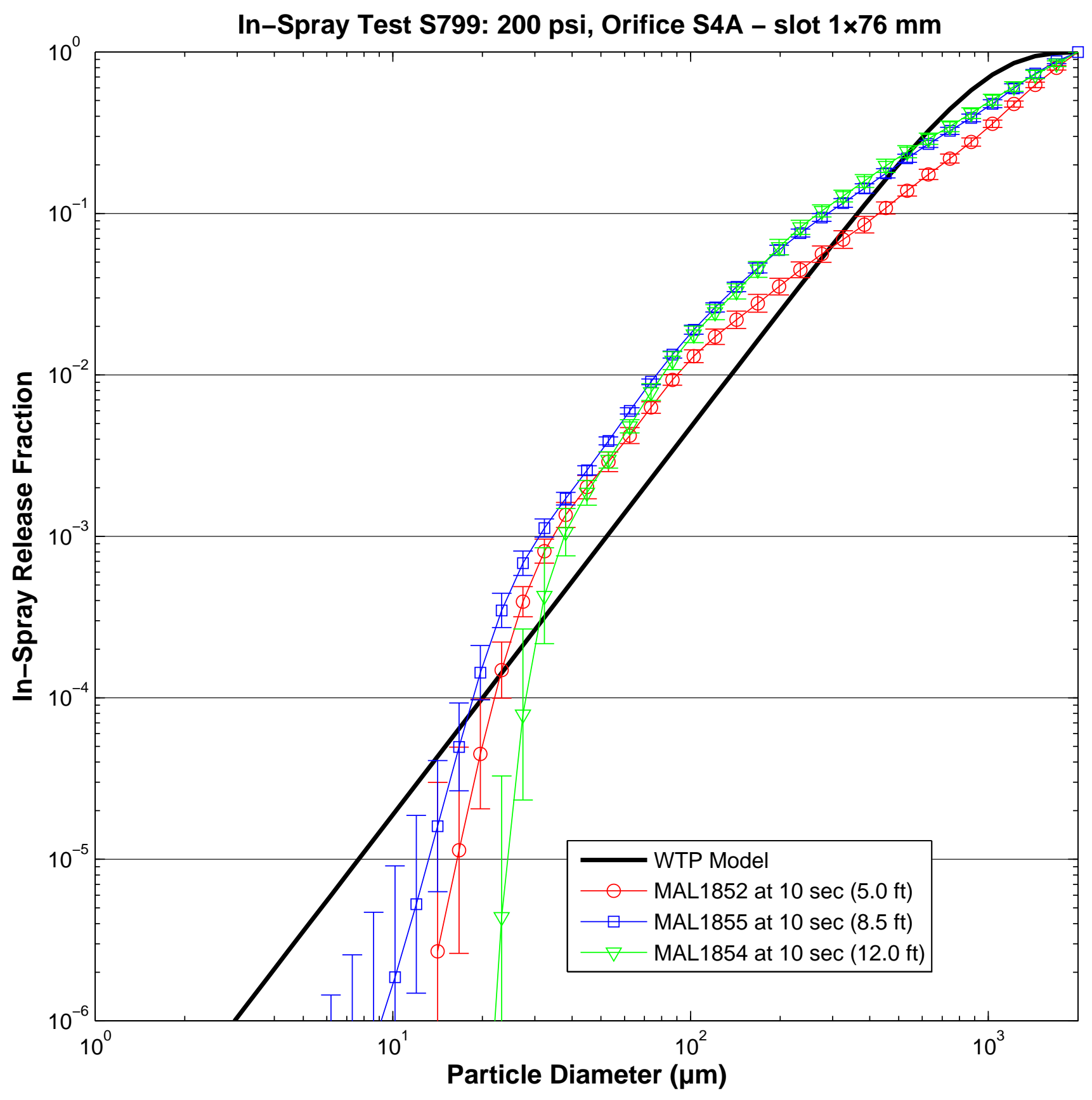




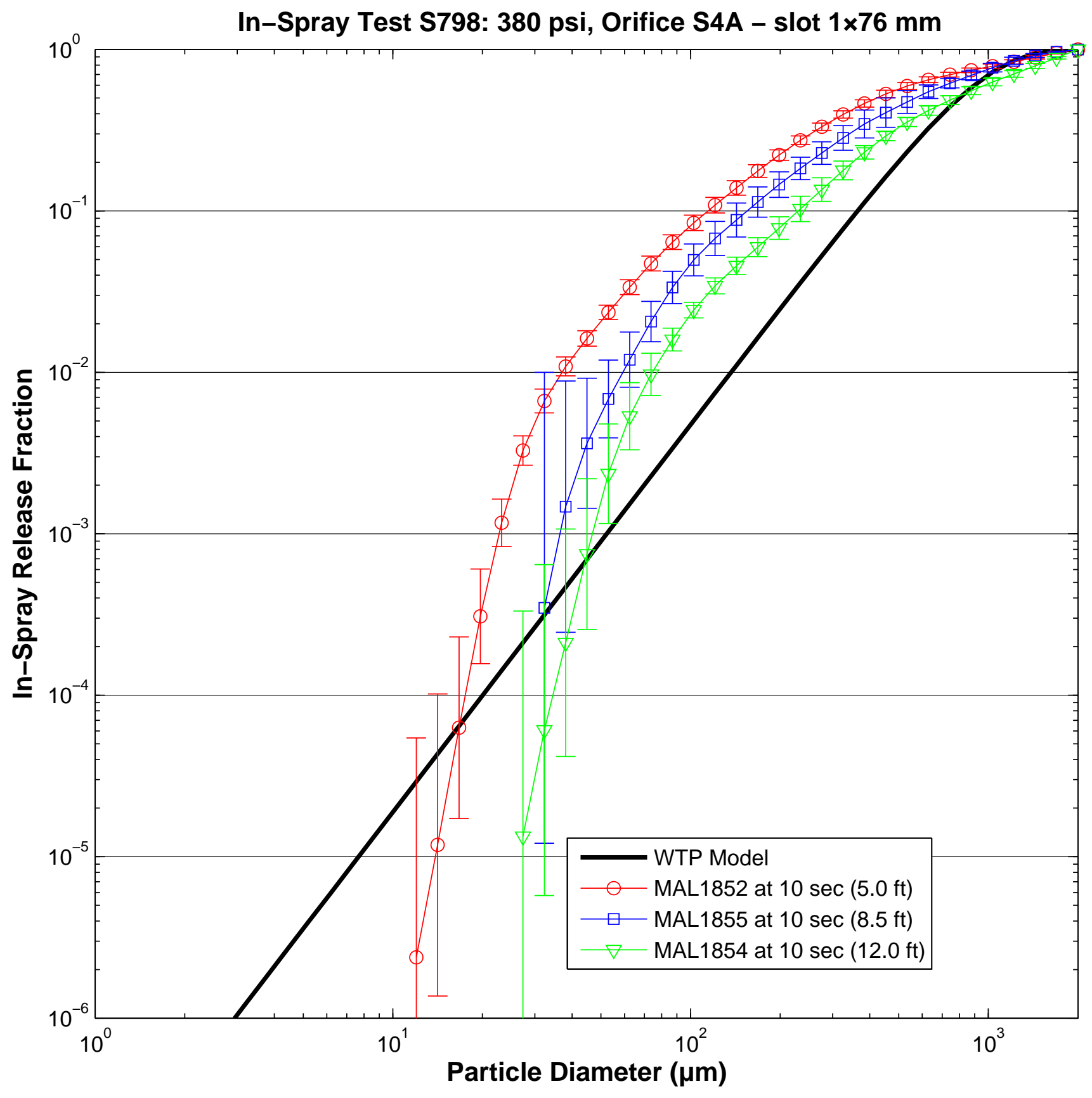




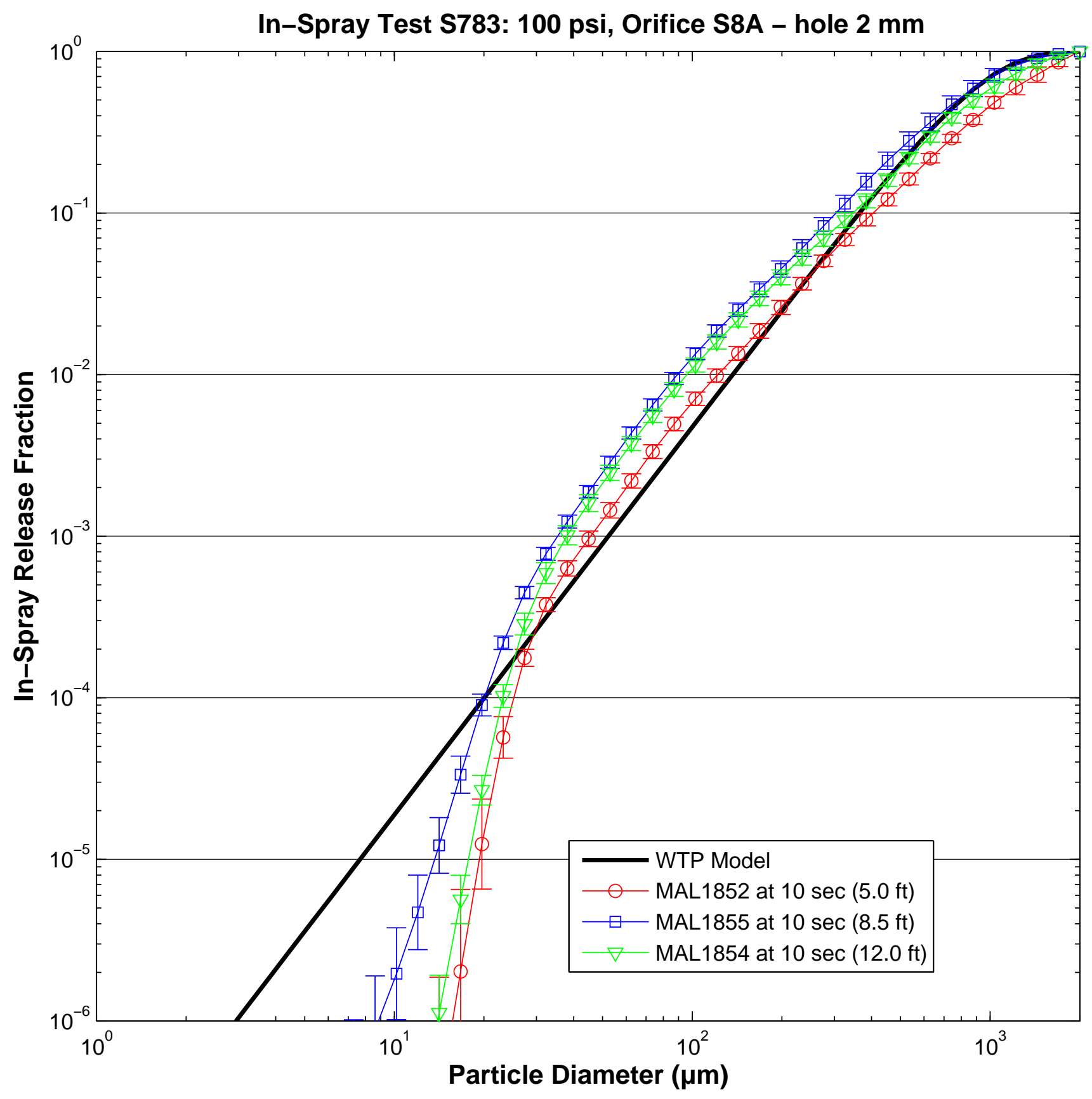




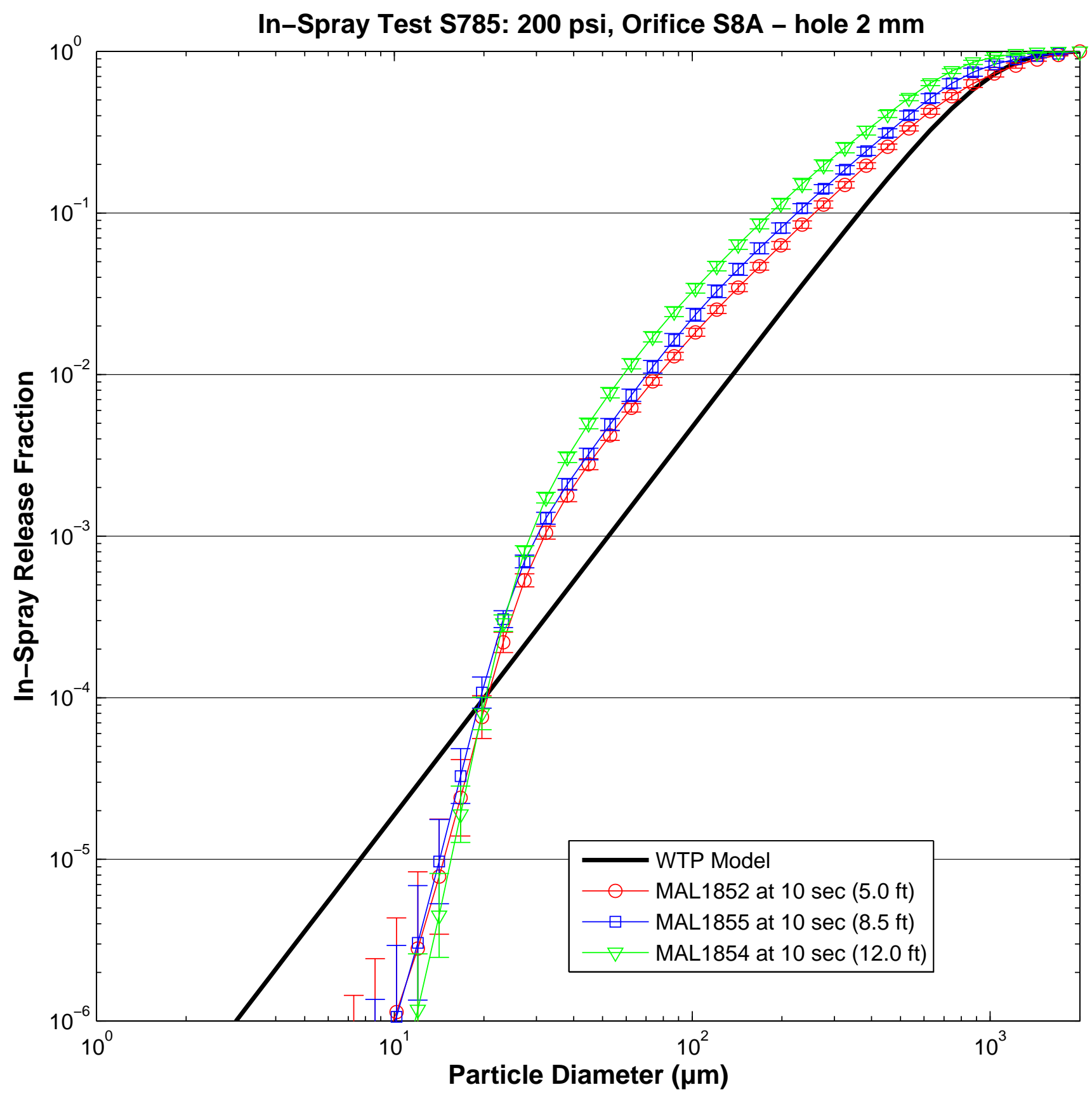




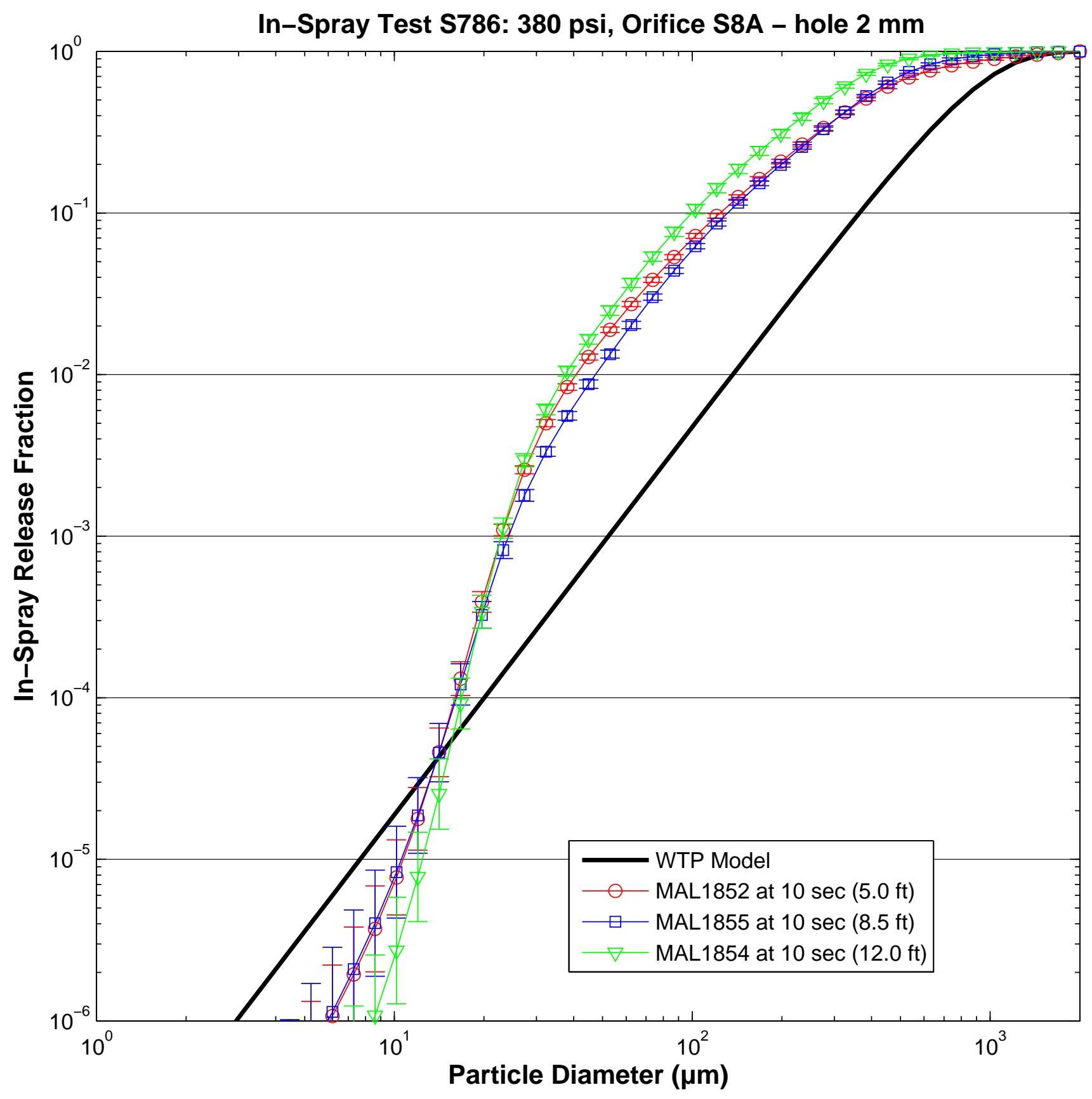




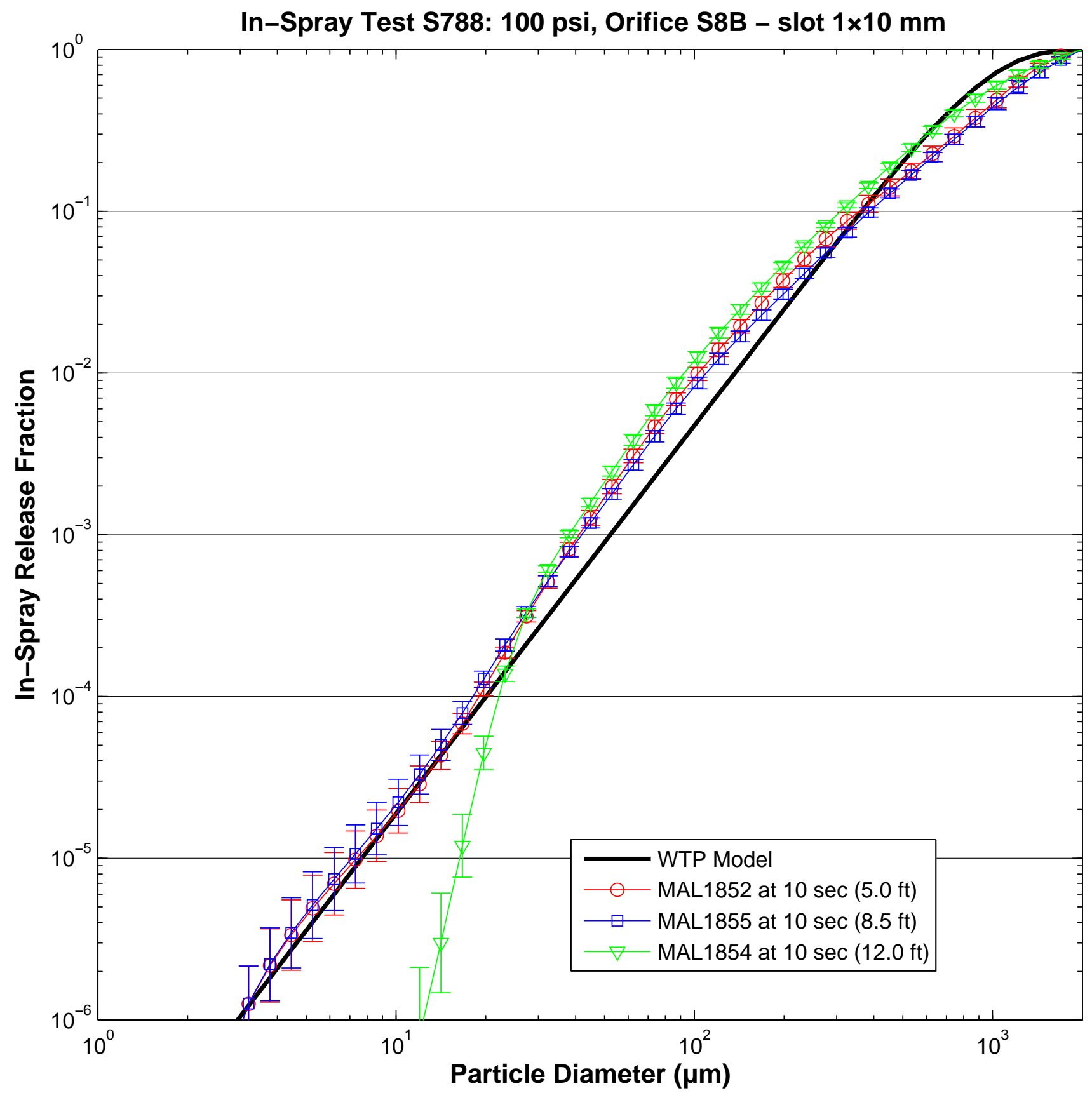




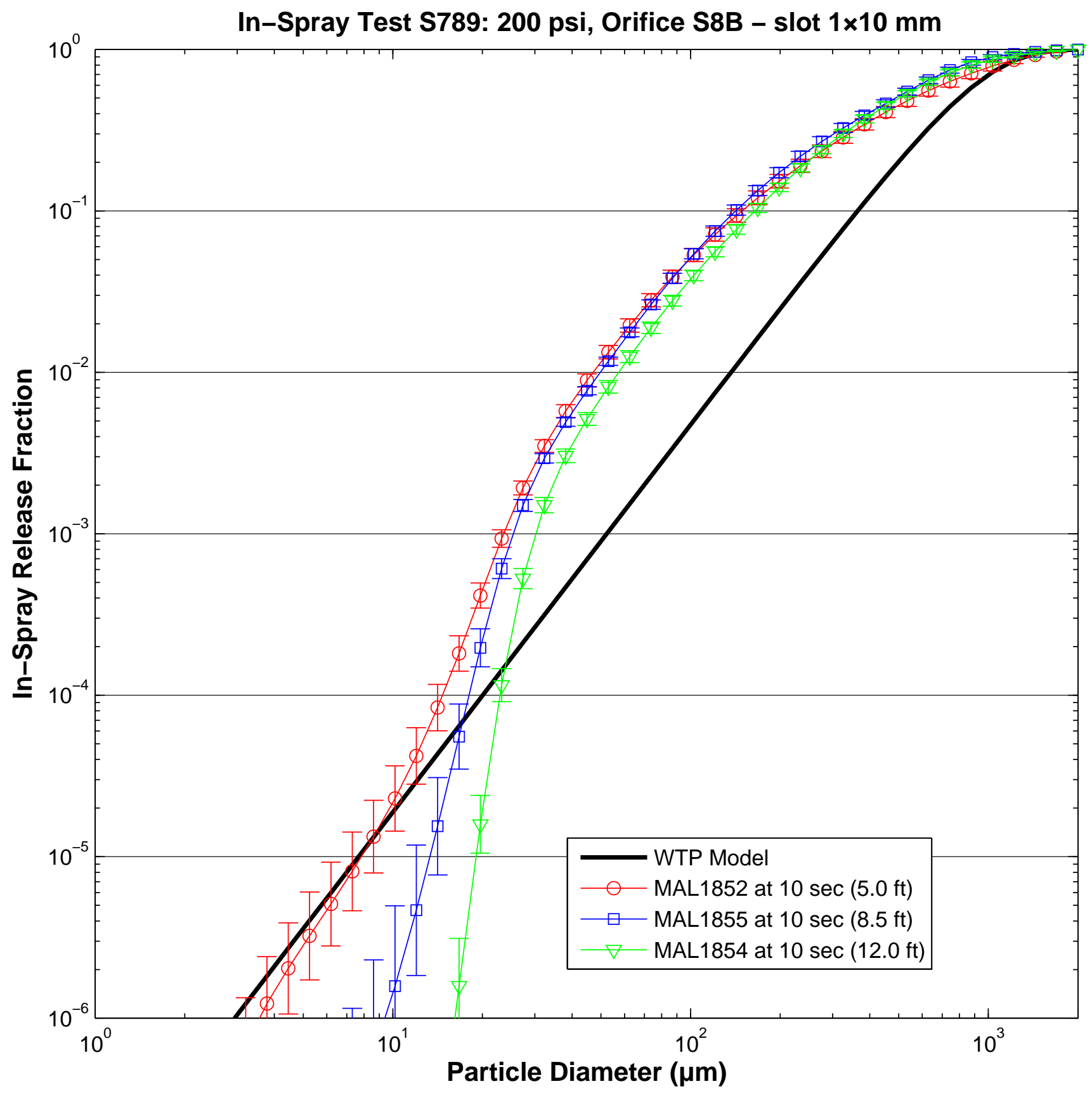




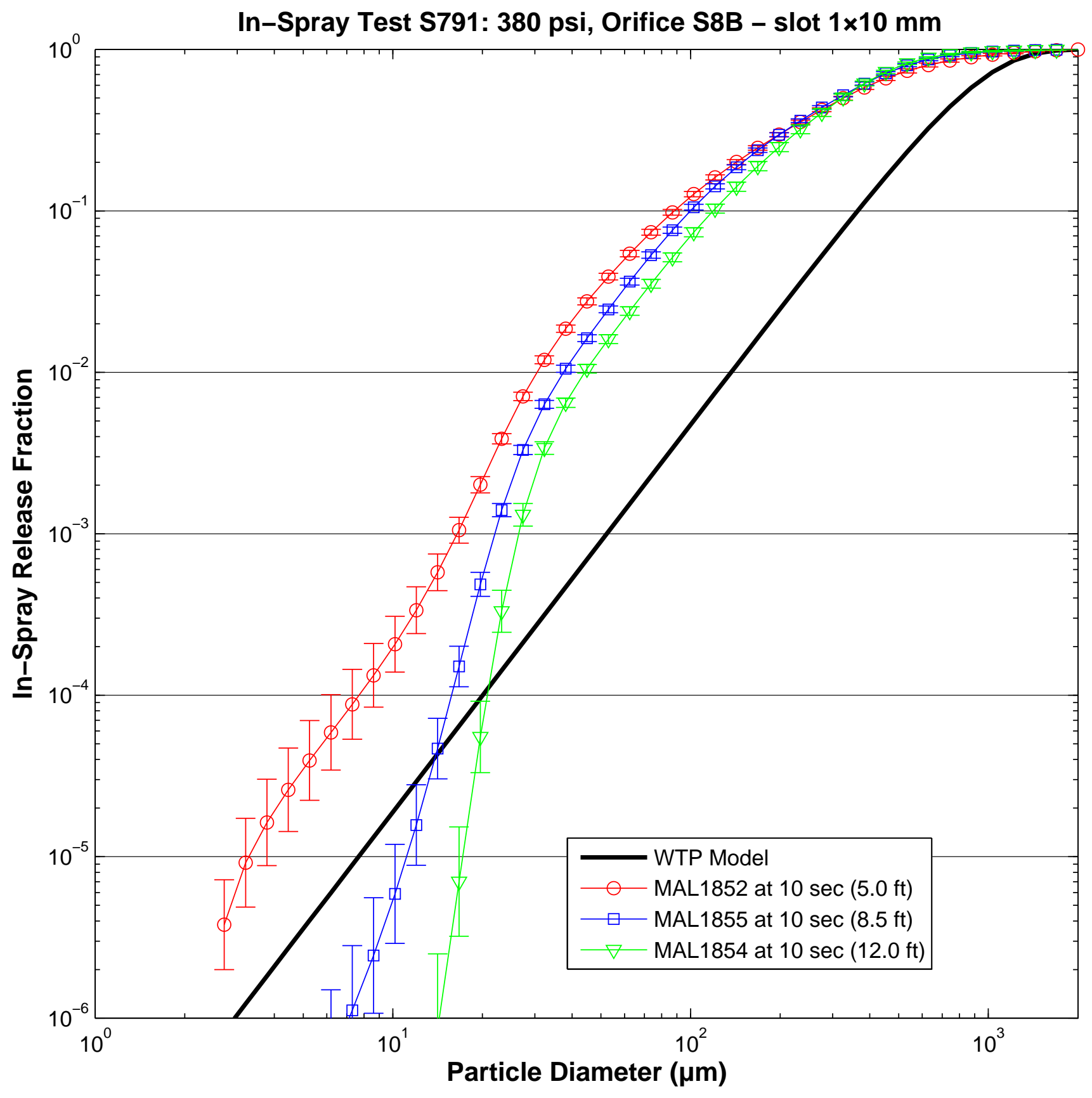




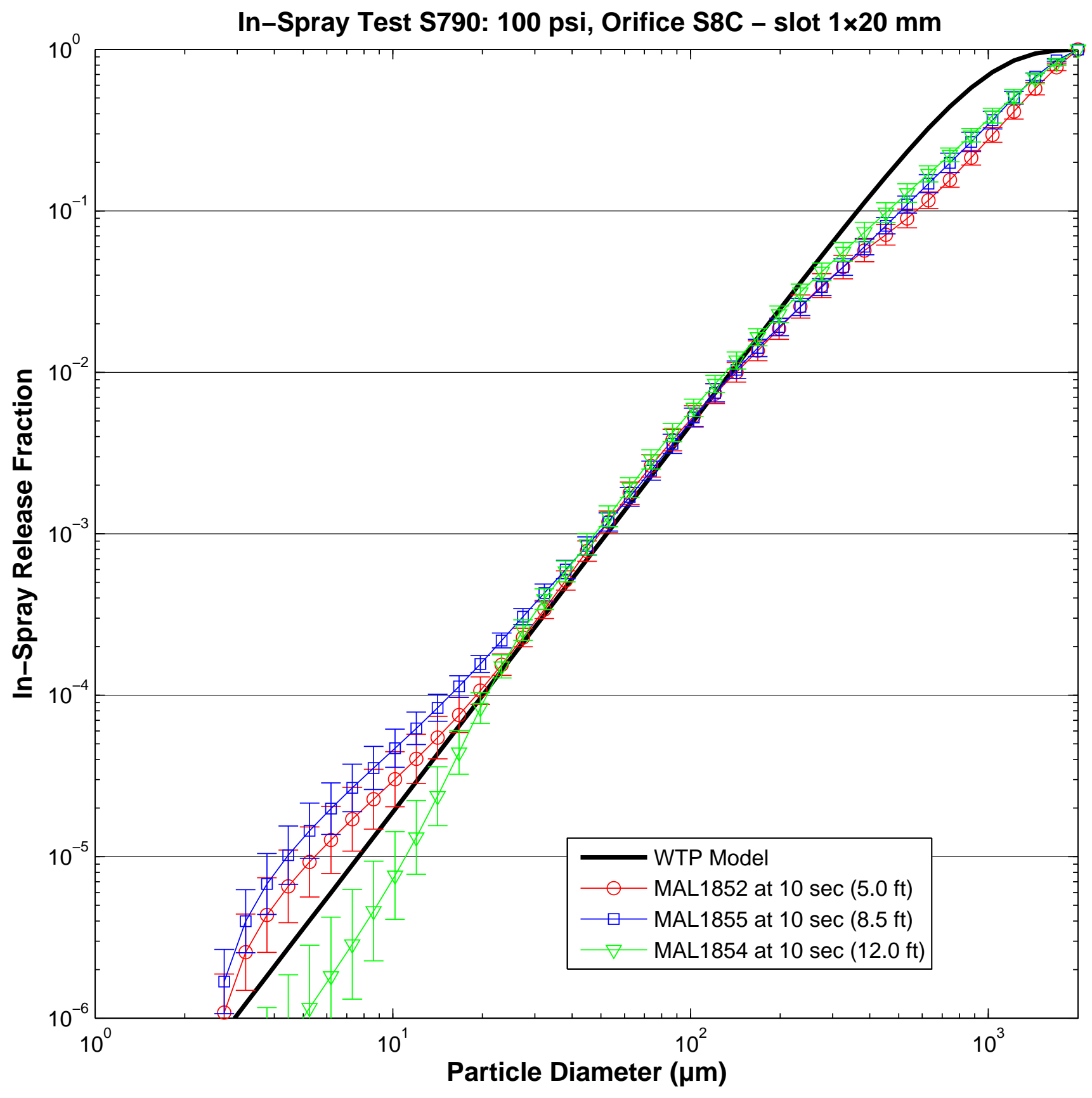




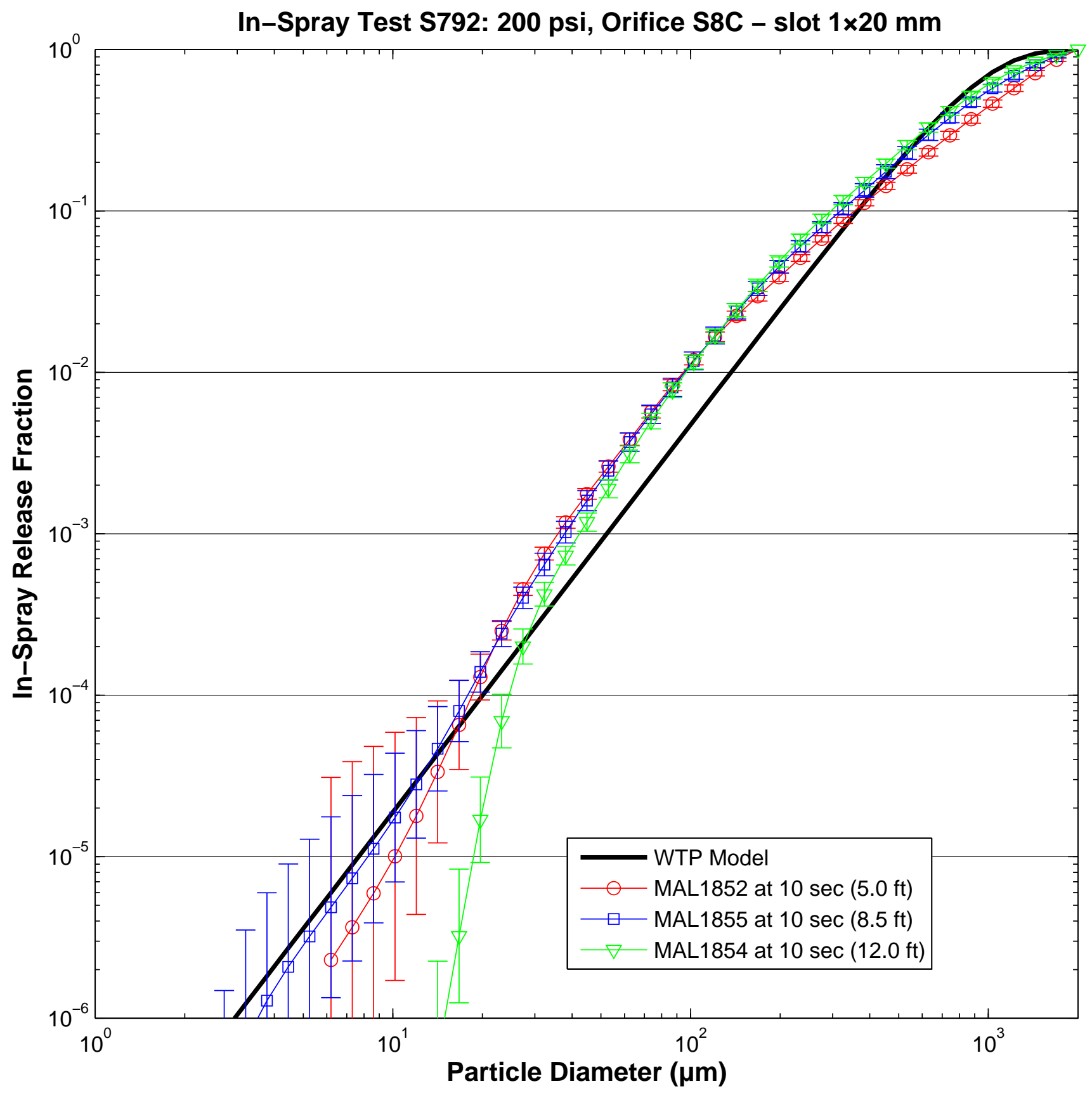




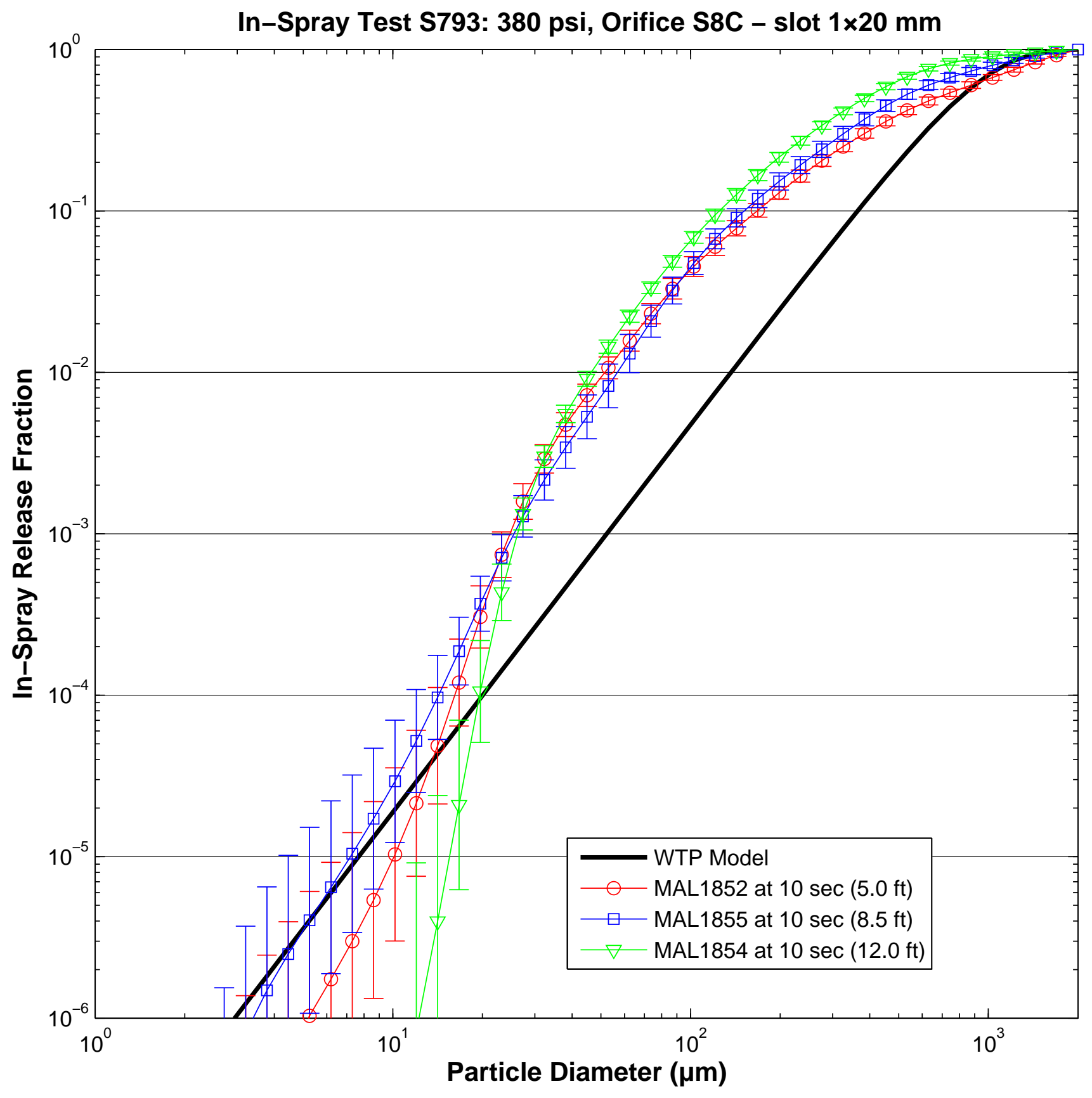




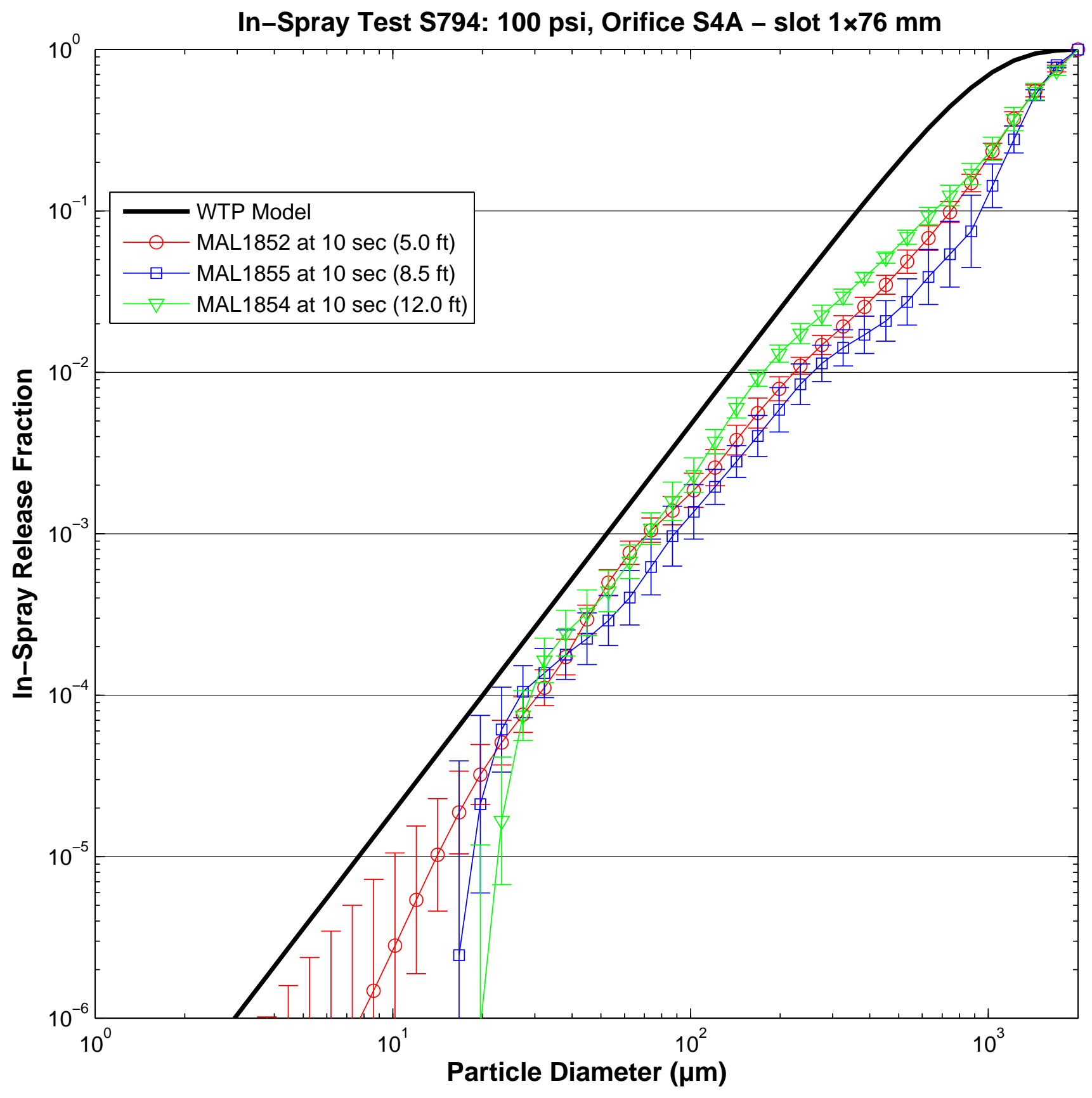




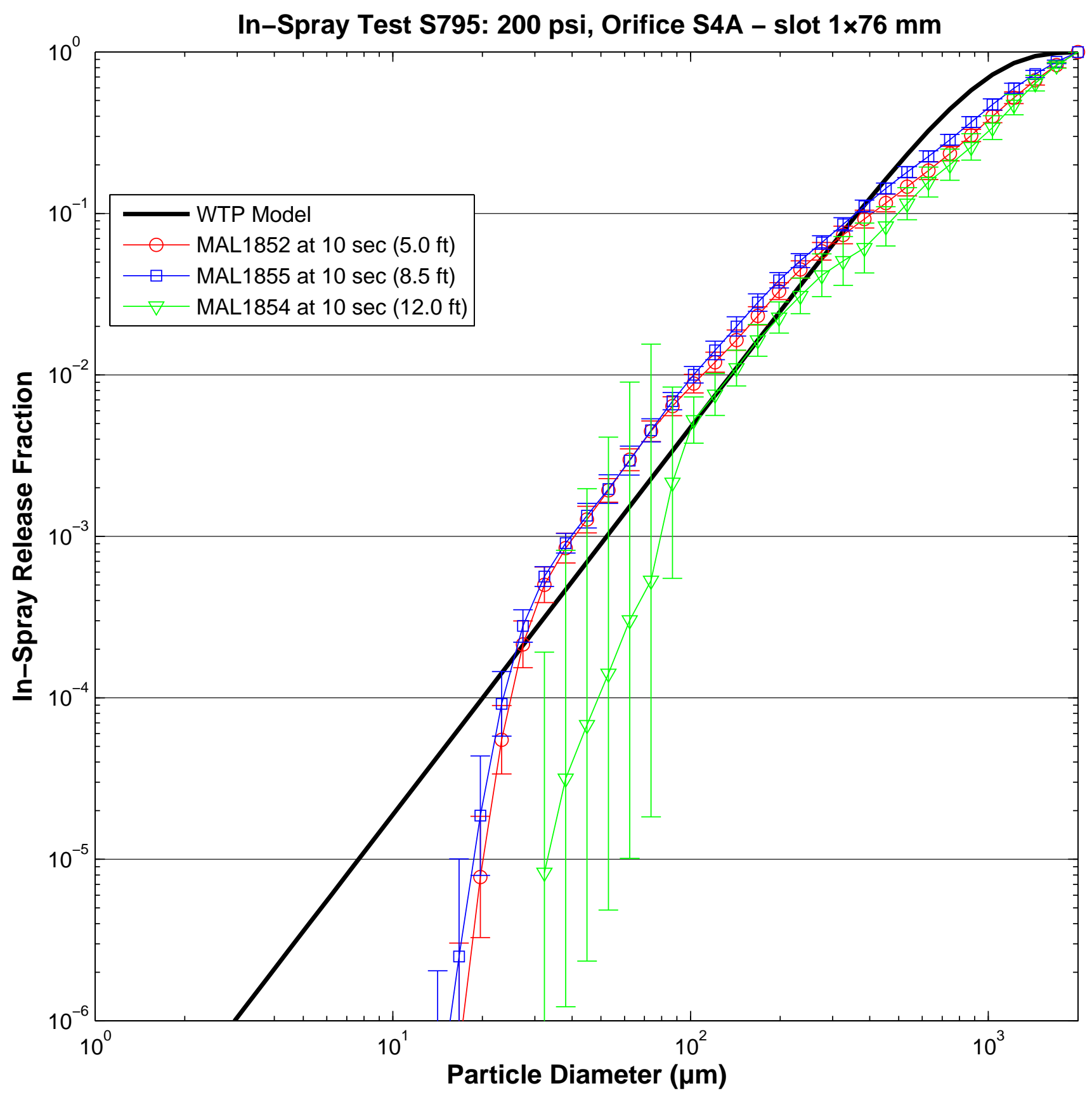




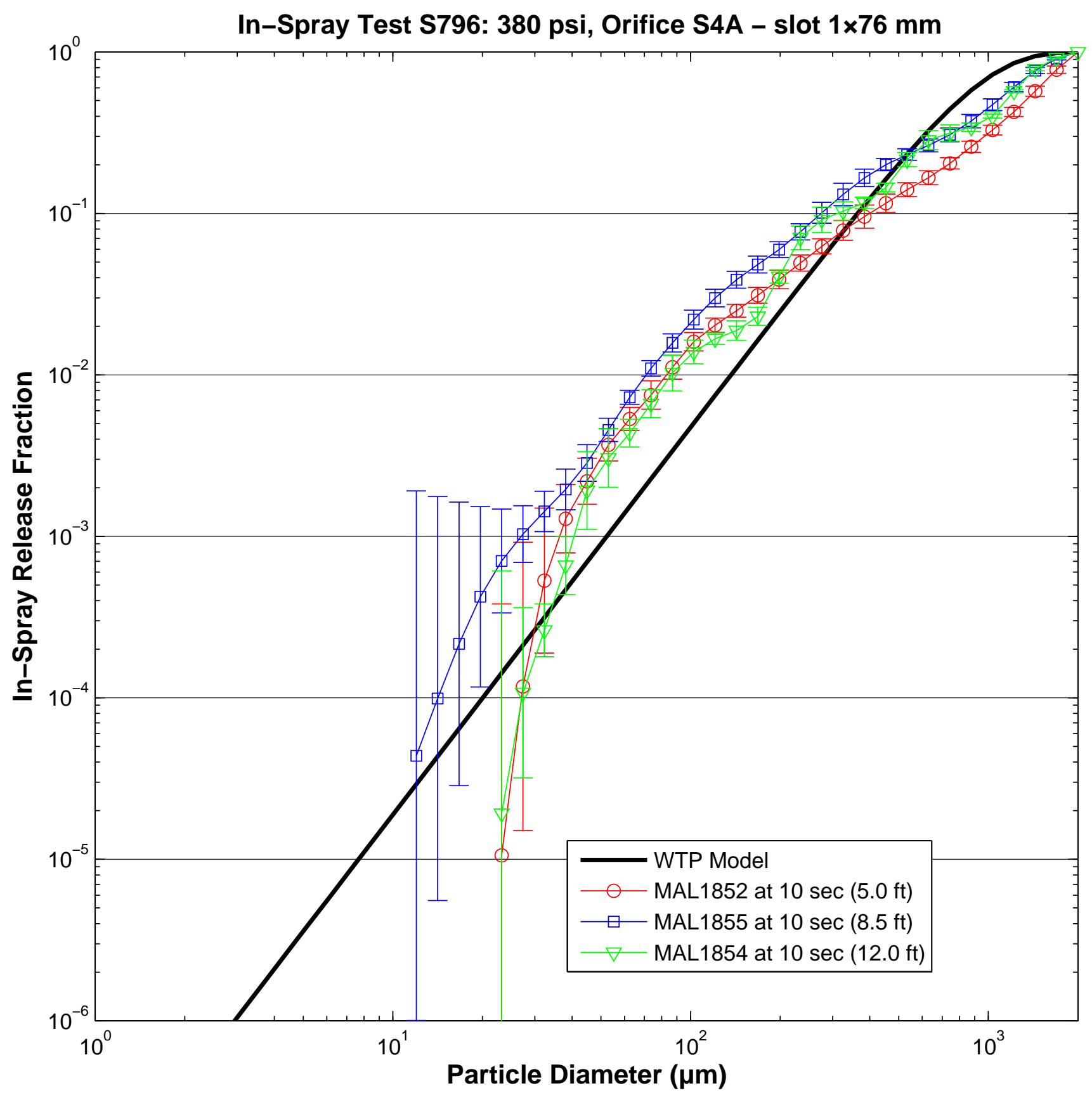




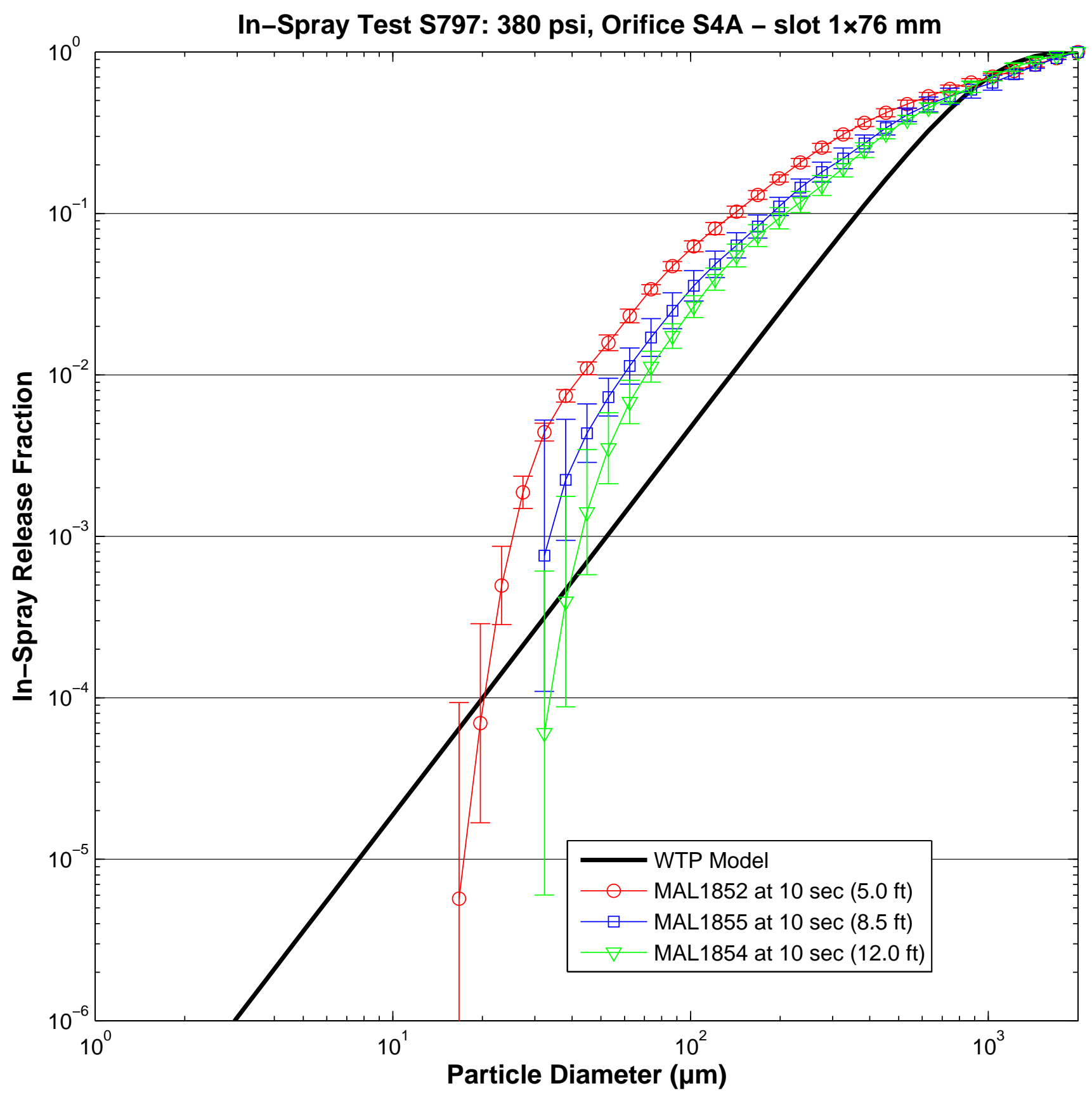




\section{Distribution*}

No. of

$\underline{\text { Copies }}$

ONSITE

4 DOE Office of River Protection

JS Fox

H6-60

CC Harrington

GL Jones

DL Noyes

7 Bechtel National Inc.

A Hassan

HR Hazen

J Mauss

S Omberg-Carro

RJ Van Vleet

JL Weamer

WTP PETD Docs
No. of

Copies

16 Pacific Northwest National Laboratory

HE Adkins, Jr

K7-15

JM Billing

JR Bontha

P7-25

P7-25

GN Brown

CA Burns

RC Daniel

CW Enderlin

MS Fountain

PA Gauglitz

JJ Jenks

ML Kimura

DE Kurath

PP Schonewill

P7-25

P7-25

P7-22

K7-15

P7-25

K7-15

K7-15

K6-28

K3-52

P7-25

P7-25

K6-24

DN Tran

P8-55

Information Release (pdf)

K3-52

*All distribution will be made electronically

Dist. 1 


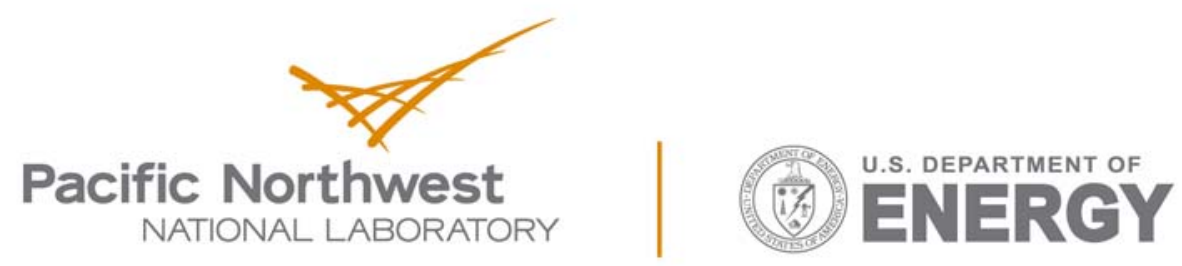

Proudly Operated by Battelle Since 1965

902 Battelle Boulevard

P.O. Box 999

Richland, WA 99352

1-888-375-PNNL (7665)

www.pnnl.gov 